\title{
B ULLE'TIN
}

OF THE

\section{UNITED STATES}

\section{GEOLOGICAL SURVEY}

No. 71

WA SHINGTON

GOVERNMENT PRINTING OFFICE 1891 

UNITED STATES GEOLOGICAL SURVEY

J. W. POWELL, DIRECTOR

\section{INDEX}

TO THE KNOWN

\section{FOSSIL INSECTS OF THE WORLD}

INCLUDING

\section{MYRIAPODS AND ARACHNIDS}

BY

SAMUEL HUBBARD SCUDDER

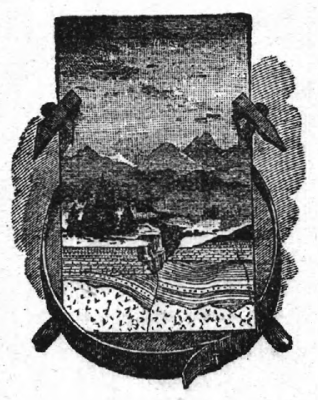

WASHINGTON

GOVERNMENT PRINTING OFFICE 1891 



\section{CONTENTS.}

Letter of transmittal ............................................. 7

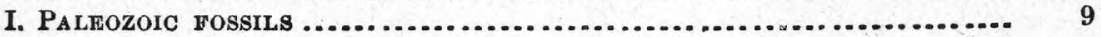

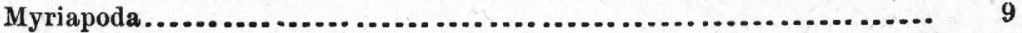

Arachnida ..................................................... 18

Palæodictyoptera .............................................. 30

The orthopteroid series....................................... 30

The neuropteroid series.................................... 70

The hemipteroid series ..................................... 92

The coleopteroid series....................................... 96

Others................................................... 98

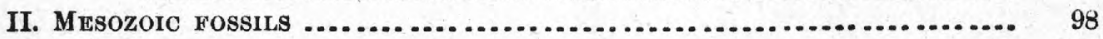

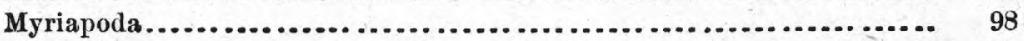

Arachnida ................................................... 99

Hexapoda ................................................ 100

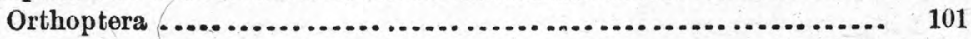

Neuroptera ................................................ 130

Hemiptera............................................. 165

Coleoptera............................................... 177

Diptera .................................................. 221

Lepidoptera ............................................. 227

Hymenoptera............................................ 228

Tracks and foot-prints ........................................... 233

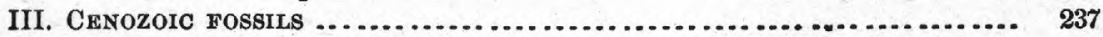

Myriapoda..................................................... 237

Arachnida .................................................... 244

Hexapoda ...................................................... 301

Orthoptera. ............................................ 301

Nenroptera ............................................... 318

Hemiptera.............................................. 380

Coleoptera ............................................ 450

Diptera ............................................. 595

Lepidoptera ............................................ 671

Hymenoptera .............................................. 682

Index of genera .................................................... 735 



\title{
LETTER OF TRANSMITTAL
}

\author{
DEPARTMENT OF THE INTERIOR, \\ U. S. Geological Survey, \\ Cambridge, Mass., July 7, 1890.
}

SIR : With the view of furthering study in the too neglected field of fossil insects, I transmit herewith for publication the card catalogue of described fossil insects which $I$ have used for twenty years and kept constantly up to date, and which has greatly facilitated my own researches. It is believed to be practically complete. At least where insects are figured, and in many other cases where they are the first or only references from a given locality, entries are given where only the genus, the family, or even the order is mentioned. This has been done because the early literature of fossil insects is essentially vague and general, but it should not for that reason be wholly overlooked; and to-day our knowledge of the occurrence of insects of a particular locality, even when of recent discovery, is not infrequently confined to statements of a general nature, which, if not brought to view or mind in a list like this, would be lost sight of, while their recognition may lead to further local investigation, to the earlier benefit of science.

It is in no sense a systematic catalogue, and except occasionally in the notes contains no immediate results of investigation. No questions of synonymy are settled. The entries are made as the authors quoted gave them, with only the corrections of spelling required; it follows that the same insect appears at several points, to which the student is referred by full cross-references, and that more than one insect may (though probably rarely does) figure under one name. In one or two such instances, where the same name has been given by inadvertence to two totally different creatures, the later one is separated from the earlier.

For convenience' sake, the entries are grouped into large sections, nearly identical with those I have employed in my systematic review of fossil insects (Bulletin 31, U. S. Geological Survey), by which method the insects from the Primary, Secondary, and Tertiary rocks are kept distinct; but otherwise the entries are purely alphabetical under the orders. It should be noticed, however, that the vague references to groups higher than genera are brought together at the beginning of each alphabet, first the most vague, and then those which permit some alphabetization. In these cases the primary or secondary alphabet- 
ization is first by authors, second by dates, and in all cases where under one entry there is more than one date the order therein is chronological.

In a previous bulletin (Bulletin $69, \mathrm{U} . \mathrm{S}$. Geological Survey) is given a complete bibliography of the literature from which these entries are taken, and the two works are thus complementary to each other.

I am; very truly, yours,

Hon. J. W. PowELL,

SAMUEL H. ScUdDer.

Director United States Geological Survey. 


\title{
INDEX T0 DESCRIBED FOSSIL INSECTS.
}

\author{
By SAMuel Hubbard Scudder.
}

\section{I.-PALEOZOIC FOSSILS.}

\section{MYRIAPODA.}

1. Acantherpestes brodiei. (Euphoberidæ.)

Locality: Coalbrookdale, England. Horizon: Carboniferous.

1882. Scudder.

1885. " "

1886. " "

1886. "6

1890. " "
Mem. Bost. soc. nat. hist., iii : 156-157. Pl. 11, fig. 5.

Zittel, Handb. palaeont., i, th. ii : 7\%9.

Zittel-Barrois, Traité de paléont., ii : 728.

Bull. U. S. geol. surv., No. 31 : 16.

Foss. ins. N. A., i: 208-209. Pl. 8, fig. 5.

See also Euphoberia ferox, Eurypterus ferox.

2. Acantherpestes inequalis. (Euphoberidæ.)

Locality : Mazon Creek, Ill. Horizon: Carboniferous.

1890. Scudder. Mem. Bost. soc. nat. hist., iv : 424-425. Pl. 33, figs. $2,4$.

1890. " Foss. ins. N. A., i : 400-401. Pl. 25, figs. 2, 4.

3. Acantherpestes major. (Euphoberidæ.)

Locality: Mazon Creek, Ill. Horizon: Carboniferous.

1868. Meek-Worth. Geol. surv. Ill., iii : 559 (hypothetical).

1882. Scudder. Mem. Bost. soc. nat. hist., iii: 150-156. Pl. 10, 11, figs. 1-4, 6-8, 10,11 ; fig. 5 on $p .151$.

1883. Lacoe. List pal. foss. ins., 15-16.

1885. Scudder. Zittel, Handb. palaeont., i, th. ii : 729. Fig. 827 a-c on p. 728.

1886. " Zittel-Barrois, Traité de paléont., ii: 728. Fig. $914 a-c$ on p. 727

1886. " Bull. U. S. geol. surv., No. 31: 16.

1887. Woodward. Geol. mag., (3), iv: 2. Fig. 3.

1889. Lesley. Dict. foss. Penns., i: 1, i. Figs.

1889. Miller. N. A. geol. and pal., 572. Fig. 106\%.

1890. Scudder. Mem. Bost. soc. nat. hist., iv: 424.

1890. " Foss. ins. N. A., i: 202-208, 400. Pl. 7a, 8, figs. 1-4, 6-8, 10, 11; fig. 5 on $p .203$.

See also Euphoberia major.

4. Amynilyspes wortheni. (Euphoberidæ.)

Locality : Mazon Creek, Ill. Horizon: Carboniferous.

1882. Scudder. Mem. Bost. soc. nat. hist., iii : 178. Pl. 13, figs. 1-4, 9.

1883. Lacoe. List pal. foss. ins., 16.

1885. Scudder. Zittel, Handb. palaeont., i, th. ii: 729. Fig. 899.

1886. "Zittel-Barrois, Traité de paléont., ii : 728. Fig. 916. 
1886. Scudder. Bull. U. S. geol. surv., No. 31 : 16.

1889. Lesley.

Dict. foss. Penns., i: 25, v. Fig.

1889. Miller.

N. A. geol. and pal., 57\%. Fig. 1068.

1890. Scudder.

Fess. ins. N. A., i: 230. Pl. 10, figs. 1-4, 9.

\section{Anthracerpes typus.}

Locality: Mazon Creek, Ill. Horizon: Carboniferous.

1865. Meek-Worth. Proc. acad. nat. sc. Philad., 1865 : 51.

1866. " Worth., Geol. surv. Ill., ii: 409-410. Pl. 32, figs. 1, 1 a.

1882. Scudder. Mem. Bost. soc. nat. hist., iii : 180.

1883. Lacoe. List pal. foss.ins., 16.

1889. Lesley. Dict. foss. Penns., i, p. v.

1889. Miller. N. A. geol. and pal., 572.

1890. Scudder. Foss, ins. N. A., i: 232.

6. Archidesmus macnicoli. (Archidesmidæ.)

Locality: Forfarshire, Scotland. Horizon: Lower Devonian.

1882. Peach.

1882. " "

1885. Scudder.

1886. " "

1886. “

Proc. roy. phys. soc. Edinb., vii : 182-186. Pl. 2, fig. 2, 2 a-d.

Foss. myr. Forfarsh., 5-9. Pl., figs. 2, $2 a-d$.

Zittel, Handb. palaeont., i, th. ii: 728. Fig. 896.

Zittel-Barrois, Traité de paléont., ii : 727. Fig. 913.

Bull. U. S. geol, surv., No. 31: 15.

\section{Archiulus - ( 2 sp.). (Archinlidæ.)}

Locality: Joggins, Nova Scotia. Horizon: Carboniferous.

1883. Scudder. Phil. trans., 1882: 649.

\section{Archiulus - (Archiulidæ.)}

Locality: Mazon Creek, Ill. Horizon: Carboniferous.

1890. Scudder. Mem. Bost. soc. nat. hist., iv: 437. Pl. 37, fig. 1.

1890. " Foss. ins. N. A., i: 413 . Pl. 29, fig. 1.

9. Archiulus (Julus) brassi. (Archiulidæ.)

Locality: Lehbach, Germany. Horizon: Lower Dyas.

1885. Scudder. Zittel, Handb. palaeont, i, th.ii: 730. Fig. 901.

1886. " Zittel-Barrois, Traité de paléont., ii: 729. Fig. 917.

See also Julus brassi.

Archiulus dawsoni. See Xylobius dawsoni.

10. Archiulus glomeratus. (Archiulidæ.)

Locality : Mazon Creek, Ill. Horizon: Carboniferous.

1890. Scudder. Mem. Bost. soc. nat. hist., iv: 436-437. Pl. 37, figs. 2, 3.

1890. " Foss. ins. N. A., i: 412-413. Pl. 29, figs.2, 3.

11. Archiulus xylobioides. (Archiulidæ.)

Locality: Joggins, Nova Scotia. Horizon: Carbonifersus.

1868. Scudder.

1869 "

1873. “

1878. " "

1878. "
Dawson, Acad. geol., $2 d$ ed., 496.

Quart. journ. geol. soc. Lond., xxv: 441 (undescr.).

Mem. Bost. soc. nat. hist., ii : 236-238.

Suppl. note foss. Myr., 1. Figs. 5-7.

Mem. Bost. soc. nat. hist., ii : 561-562. Figs. 5-7. 
1878. Dawson.

1880. “"

1883. Lacoe.

1889. Lesley.

1889. Miller.

1890. Scudder.
Acad. geol., suppl. 2d ed., 56. Fig. 11 e-g.

Chain of life, 145 . Fig. $126 b$.

List pal. foss. ins., 16-17.

Dict. foss. Penns., i, p. vii.

N. A. geol, and pal.,573. Fig. 1069.

Foss. ins. N. A., i : 26-28, 31-32.- Figs. 5-7.

\section{Arthropleurion inermis.}

Locality : Saarbrück, Germany. Horizon: Carboniferous.

1877. Goldenberg. Fauna saræp. foss., ii : 48-49 (Athropleurion). Pl. fig. 2, 30. 1882. Scudder. Mom. Bost. soc. nat. hist., iii : 180.

1890. " Foss. ins. N. A., i : 232.

Athropleurion inermis. See Arthropleurion inermis.

13. Chonionotus lithanthracis. (Euphoberidæ.)

Locality: Jägersfreude, Germany. Horizon: Carboniferous.

1854. Jordan. Palaeontogr., iv: 12-13. Pl. 2, fig. 3.

1867. Goldenberg. Vorweltl. fauna steink. Saarbr., 21.

1873. " Faun. saræp. foss., i: 21. Pl. 1, fig.19.

1882. Scudder. Mem. Bost. soc. nat. hist., iii: 180. Pl. 11, fig. 9.

1885. " "Zittel, Handb. palaeont, i, th. ii : 729 (lithanthraca).

1886. " "Zittel-Barrois, Traité de paléont., ii : 728.

1886. " B Bull. U. S. geol. surv., No.31: 16.

1890. " Foss. ins. N. A., i: 23\%. Pl. 8, fig. 9.

14. Eileticus æqualis. (Eoscolopendridæ.)

Locality : Mazon Creek, Ill. Horizon: Carboniferous.

1890. Scudder. $\quad$ Mem. Bost. soc. nat. hist., iv: 421. Pl. 38, figs. 6-9.

1890. " Foss.ins. N.A., i: 397. Pl. 30, figs. 6-9.

15. Eileticus anthracinus. (Eoscolopendridæ.)

Locality : Mazon Creek, Ill. Horizon: Carboniferous.

1882. Scudder. Mem. Bost. soc. nat. hist., iii : 179. Pl. 13, figs. 5,6.

1883. Lacoe. List pal. foss. ins., 17.

1885. Scudder. $\quad$ Zittel, Handb. palæont., i, th. ii: 729.

1886. " " Zittel-Barrois, Traité de paléont., ii : 728.

1886. “ Bull. U. S. geol. surv., No.31: 16.

1889. Miller. $\quad$ N. A. geol. and pal., 573.

1890. Scudder Mem. Bost. soc. nat. hist., iv : 420-421. Pl. 38, fig. 5.

1890. “ Foss. ins. N. A., i : 231, 396-397. Pl. 10, figs.5,6; Pl. 30, fig. 5.

16. Eojulus fragilis. (Archiulidæ?)

Locality: Rakonitz, Bohemia. Horizorr: Carboniferous.

1885. Kušta. Neue foss. arthrop. Rakonitz, 4-5. Fig.

1885. " Sitzungsb. k. böhm. gesellsch. wiss., 1885 : 593-594. Fig.

17. Euphoberia —. (Euphoberidæ.)

Locality: Mazon Creek, Ill. Horizon: Carboniferous.

1890. Scudder. Mem. Bost. soc. nat. hist., iv: 435.

1890. " Foss. ins. N. A., i: 411. 
18. Euphoberia anguilla. (Euphoberidæ.)

Locality : Mazon Creek, Ill. Horizon: Carboniferous.

1882. Scudder.

1883. Lacoe.

1889. Lesley.

1889. Miller.

1890. Scudder.

1890.
Mem. Bost. soc. nat. hist., iii: 17\%. Pl. 12, fig. 20.

List pal. foss. ins., 17.

Dict. foss. Penns., i, p. xxiii.

N. A. geol, and pal., 573.

Mem. Bost. soc. nat. hist., iv : 426, 435-436. Pl. 36, fig. 3.

Foss. ins. N. A., i : 229, 402, 411-412. Pl. 9, fig. $20 ;$ Pl. 28, fig. 3.

19. Euphoberia armigera. (Euphoberidæ.)

Locality : Mazon Creek, Ill. Horizon: Carboniferous.

1868. Meek-Worth. Amer. journ. sc. arts, (2), xlvi: 25-27.

1868. “ Worth., Geol. surv. Ill., iii : 556-558. Figs. A-D.

1876. Roemer. Lethæa geogn. (Euphorberia). Pl. 47, fig. 19.

1879. Nicholson. Manual palæont., 2d ed., i : 404. Fig. 255.

1882. Scudder.

Mem. Bost. soc. nat. hist., iii : 160-167. $P l$. 12, figs. 1-3, 5, 6, 13;

Pl. 13, figs. 7, 8, 10; figs. 6, 7 on p. 161.

1883. Lacoe.

List pal. foss. ins., 17.

1885. Scudder.

1886. “

Zittel, Handb. palaeont, i, th. ii : 729. Fig. 898.

Zittel-Barrois, Traité de paléont., ii: 728. Fig. 915.

1889. Nicholson.

1889. Miller.

Man. palæont., 3d ed., i: 584. Fig. 439.

1890. Scudder.

1890. “

N. A. geol, and pal., 573. Figs. 1070, 1071 (not 1072).

Mem. Bost. soc. nat. hist., iv : 426, 427. Pl. 34, figs. 2, 4, 8; Pl. 35, fig. 3.

Foss. ins. N. A., i: 212-219, 402, 403. Pl. 9, figs. 1-3, 5, 6, $13 ; \mathrm{Pl}$. 10, figs. 7, 8, 10; $P l .26$, figs $2,4,8 ; P l .27$, fig. 3 ; figs. 6,7 on p. 213.

20. Euphoberia brownii. (Euphoberidæ.)

Locality: Kilmaurs, Scotland. Horizon: Carboniferous.

18\%1. Woodward. Geol. mag., viii : 102-104. Pl. 3, fig. 6 a-c.

1882. Scudder. Mem. Bost. soc. nat. hist., iii : 167-168. Pl. 12, figs. 7, 8, 21.

1890. " Foss. ins. N. A., i: 219-220. Pl. 9, figs, 7, 8, 21.

21. Euphoberia carri. (Euphoberidæ.)

Locality: Mazon Creek, Ill. Horizon : Carboniferous.

1882. Scudder. Mem. Bost. soc. nat. hist., iii : 171-174. Pl. 12, figs. 4, 9-12, 1419 ; Pl. 13, figs. 16-18.

1883. Lacoe. List pal. foss. ins., 18.

1889. Lesley. Dict, foss. Penns., i, p. xxiv.

1889. Miller. N. A. geol. and pal., 573.

1890. Scudder. Mem. Bost. soc. nat. hist., iv : 426, 429.

1890. " Foss. ins. N. A., i: 223-226, 402, 405. Pl. 9, figs 4, 9-12, 14-19; Pl. 10, figs. 16-18.

22. Euphoberia cuspidata. (Euphoberidæ.)

Locality: Mazon Creek, Ill. Horizon: Carboniferous.

1890. Scudder. Mem. Bost. soc. nat. hist., iv: 426, 429-430. Pl. 34, figs. 3, 7.

1890. " Foss. ins. N. A., i: 402, 405-406. Pl. 26, figs. 3, 7.

23. Euphoberia ferox. (Euphoberidæ.)

Localities: Tipton, N. Straffordshire, Coalbrookdale, Sedgley, Grassington, Coseley, Yorkshire, England. Horizon: Carboniferous.

1845. Brodie.

Foss. ins. sec. rocks Engl., 105, 115 ("caterpillar"). Pl. 1, fig. 11.

1845. Westwood.

Brodie, Foss. ins. sec. rocks Engl., xvii-xviii ("caterpillar"). 
1868. Meek-Worth. Amer. journ. sc. arts, (2), xlvi : 26-27.

1873. Woodward. Geol. mag., $\mathrm{x}:$ 109-112. Figs. $8,10$.

1873. " Brit. foss. arthr., 6-9. Figs. 8, 10.

1882. Scudder. Mem. Bost. soc. nat. hist., iii: 157-158. Pl. 12, fig. 23.

1887. Woodward. Geol. mag., (3), iv : 6-10. Pl. 1, figs. 1-13; figs. 1-3 in text.

1887. " Geol. mag., (3), iv: 116-117. Figs. 1, 2 in text.

1890. Scudder. Foss. ins. N, A., i : 209-210. Pl. 9, fig. 23.

See also Acantherpestes brodiei, Eurypterus ferox.

24. Euphoberia flabellata. (Euphoberidæ.)

Locality: Mazon Creek, Ill. Horizon: Carboniferous.

1882. Scudder. Mem. Bost. soc. nat. hist., iil: 174-177. Pl. 13, fig. 15.

1883. Lacoe. List pal. foss. ins., 18.

1889. Miller. N. A. geol. and pal., 573.

1890. Scudder Mem. Bost. soc. nat. hist., iv : 426 .

1890. " . Foss. ins. N. A., i : 226-229, 402. Pl. 10, fig. 15.

25. Euphoberia granosa. (Euphoberidæ.)

Locality: Mazon Creek, Ill. Horizon: Carboniferous.

1882. Scudder. Mem. Bost. soc. nat. hist., iii : 168-170. Pl. 12, figs. 22, 24-26; Pl. 13, fig. 13 ; fig. 8 in text.

1883. Lacoe. List pal. foss. ins., 18.

1889. Lesley. Dict. foss. Penns., i, p. xxiv.

1889. Miller. N. A. geol. and pal., 573. Fig. 1072.

1890. Scudder. Mem. Bost. soc. nat. hist., iv : 426, 427-428. Pl. 34, figs. 5, $6 ; \mathrm{Pl}$. 36, fig. 2.

1890. " Foss. ins. N. A., i : 220-222, 402, 403-404. Pl. 9, figs. 22, 24-26; Pl. 10, fig. 13; Pl. 26, figs. 5, 6; Pl. 28, fig. $2 ;$ fig. 8 on p. 220.

26. Euphoberia horrida. (Euphoberidæ.)

Locality: Mazon Creek, Ill. Horizon: Carboniferous.

1882. Seudder. Mem. Bost. soc. nat. hist., iii : 158-160. Pl. 13, figs. 11, 12, 14 .

1883. Lacoe. List pal. foss. ins., 18.

1889. Lesley. Dict. foss. Peuns., i, p. xxiv.

1889. Miller. $\quad$ N. A. geol. and pal., 573.

1890. Scudder. Mem. Bost. soc. nat. hist., iv : 426.

1890. " $\quad$ Foss. ins. N. A., i : 210-212, 402. Pl. 10, figs. 11, $12,14$.

27. Euphoberia hystricosa. (Euphoberidæ.)

Locality: Mazon Creek, Ill. Horizon: Carboniferous.

1890. Scudder. Mem. Bost. soc. nat. hist., iv: 426. Pl. 33, figs. 1, 3.

1890. " Foss. ins. N. A., i : 402. Pl. 25, figs. 1, 3.

28. Euphoberia major. (Euphoberidæ.)

Locality: Mazon Creek, Ill. Horizon: Carboniferous.

1868. Meek-Worth. Amer. journ. sc. arts, (2), xlvi : 25-27.

1868. “ Worth., Geol. surv. Ill, iii : 558-559. Fig.

1872? Woodward. Monogr. Merost., 172. Fig.

1873. " Geol. mag., x: iii. Fig. 11.

See also Acantherpestes major.

29. Euphoberia simplex. (Euphoberidæ.)

Locality: Mazon Creek, Ill. Horizon : Carboniferous.

1890. Scudder. Mem. Bost. soc. nat. hist., iv: 426, 432-433. Pl. 35, figs. 2, 6, 7.

1890. « Foss. ins. N. A., i : 402, 408-409. Pl, 27, figs, 2, 6, 7. 
30. Euphoberia spinulosa. (Euphoberidæ.)

Locality: Mazon Creek, Ill. Horizon: Carboniferous.

1890. Scudder. Mem. Bost. soc. nat. hist., iv: 426, 430-432. Pl. 34, fig. 1 ; Pl. 35, figs. 1, 4, 5; Pl. 36, figs. 7, 8.

1890. “ Foss. ins. N.A., i : 402, 406-408. Pl. 26, fig. 1; Pl. 27, figs. 1, 4, 5; Pl. 28, figs. 7, 8.

31. Euphoberia tracta. (Euphoberidæ.)

Locality: Mazon Creek, Ill. Horizon : Carboniferous.

1890. Scudder. Mem. Bost. soc. nat. hist., iv: 426, 433-435. Pl. 36, figs. 1, 4-6.

1890. " Foss. ins. N. A., i : 402, 409-411. Pl. 28, figs. 1, 4-6.

Euphorberia armigera. See Euphoberia armigera.

32. Eurypterus ferox. (Euphoberidæ.)

Locality: Tipton, N. Staffordshire, England. Horizon: Carboniferous. 1863. Salter. Quart. journ. geol. soc. Lond., xix: 86-87. Fig. 8 on p. 84.

See also Euphoberia ferox, Acantherpestes brodiei.

33. Ilyodes divisa. (Eoscolopendridæ.)

Locality : Mazon Creek, Ill. Horizon: Carboniferous.

1890. Scudder. Mem. Bost. soc. nat. hist., iv: 422-423. Pl. 38, fig. 1.

1890. " Foss. ins. N. A., i: 398-399. Pl. 30, fig. 1.

34. Ilyodes elongata. (Eoscolopendridæ.)

Locality: Mazon Creek, Ill. Horizon: Carboniferous.

1890. Scudder. Mem. Bost. soc. nat. hist., iv : 423. Pl. 38, fig. 2.

1890. " Foss. ins. N. A., i: 399. Pl. 30, fig. 2.

35. Julus brassi. (Archiulidæ?)

Locality: Lehbach, Germany. Horizon: Lower Dyas.

1868. Dohrn. Verhandl. naturh. ver. preuss. Rheinl., (3), v: 335-336. Pl. 6, fig. $2 a-b$.

1877. Goldenberg. Fauna saræp. foss., ii : 33, 51.

1882. Sendder. Mem. Bost. soc. nat. hist., iii : 148.

1885. " $\because \quad$ Zittel, Handb. palaeont., i, th, ii: 730.

1886. " "Zittel-Barrois, Traité de paléont., ii : 729.

1886. “ Bull. U. S. geol. surv., No. $31: 17$.

1890. " Foss. ins. N. A., i: 200.

See also Archinlus (Julus) brassi.

36. Julus constans. (Archiulidæ?)

Locality: Nyřan, Bohemia. Horizon: Permian.

1879. Fritsch. Fauna d. gaskohle Böhmens, i: 31.

37. Julus costulatus. (Archinlidæ?)

Locality : Nyřan, Bohemia. Horizon: Permian.

1879. Fritsch. Fauna d. gaskohle Böhmens, i: 31 .

38. Julus pictus. (Archiulidæ?)

Lecalities: Kounová, Zabor, Bohemia. Horizon: Permian.

1879. Fritsch. Fauna d. gaskohle Böhmens, i : 31. 
39. Kampecaris forfarensis. (Archidesmidæ.)

Locality : Forfarshire, Scotland. Horizon : Lower Devonian.

1856. Page. Adv. text book geol., 135 (genus only). Fig. 4.

[1865. Salt.-Woodw.Chart foss. Crust.]

1882. Peach. Proc. roy. phys. soc. Edinb., vii : 179-182. Pl. 2, figs. 1, 1a-f.

1882. " Foss. myr. Forfarsh., 3-5. Pl., figs. 1, $1 a-f$.

1885. Scudder. Zittel, Handb. palaeont., i, th. ii : 728.

1886. " Zittel-Barrois, Traité de paléont., ii : 727.

1886. " Bull. U.S. geol. surv., No. 31: 15.

40. Latzelia primordialis. (Gerascutigeridæ.)

Locality: Mazon Creek, Ill. Horizon: Carboniferous:

1890. Scudder. Mem. Bost. soc. nat. hist., iv: 419. Pl. 38, fig. 3.

1890. " Foss.ins. N.A., i: 395. Pl. 30, fig. 3.

41. Palæocampa anthrax. (Protosygnatha.)

Locality: Morris, Ill. Horizon: Carboniferous.

1865. Meek-Worth. Proc. acad. nat. sc. Philad., 1865: 52.

1866. " " Worth., Geol. surv. Ill., ii : 410-411. Pl. 32, fig. 3.

1868. " "Worth., Geol. surv. Ill, iii : 565.

1868. Scudder. Geol. mag., v: 218.

1882. " Amer. jour. sc., (3), xxiy : 161-170.

1883. Lacoe. List pal. foss. ins., 18-19.

1884. Scudder. Mem. Bost. soc. nat. hist., iii : 283-291, 293-297. Pl. 26, figs. 1-9.

1885. " Zittel, Handb. palaeont., i; th. ii : 726. Fig. 894ab.

1886. " "Zittel-Barrois, Traité de paléont., ii : 725. Fig. 911ab.

1886. " " Bull. U. S. geol, surv., No. 31 : 14 .

1889. Miller. $\quad$ N. A. geol. and pal., 573.

1889. Nicholson Man. palæont., 3d ed., i: 583. Fig. 438.

1890. Lesley. Dict. foss. Penns., ii : 579. Fig.

1890. Scudder. Foss. ins. N. A., i: 247-255, 257-260. Pl. 12, figs. 1-9.

\section{Palæojulus dyadicus.}

Locality: Chemnitz, Saxony. Horizon: Dyas.

1872. Geinitz. Sitzungsb. naturw. gesellsch. Isis, 1872: 128-131. Pl. 1, fgs. 4-7.

1877. Goldenberg. Fauna saraep. foss., ii : 34, 51.

1878. Sterzel. Neues jahrb. min., 1878: 729-731 (Cyclopteris).

1878. Geinitz. Nenes jahrb. min., 1878: 733.

1878. Sterzel. Zeitschr. dentsch. geol, gesellsch., xxx: 417-426. Pl.19, figs.1-11.

1880. " Zeitschr. deutsch. geol. gesellsch., xxxii : 1-2.

1885. Scudder. Zittel, Handb. palaeont., i, th. ii : 730.

1886. " " Zittel-Barrois, Traité de paléont., ii : 729.

1886. “ Bull. U. S. geol. surv., No. $31: 17$.

[Not a Myriapod.]

43. Palenarthrus impressus. (Eoscolopendridæ.)

Locality: Mazon Creek, Ill. Horizon: Carboniferous.

1890. Scudder. Mem. Bost. soc, nat. hist., iv : 422. Pl. 38, fig. 4.

1890. " Foss. ins. N. A., i: 398. Pl. 30, fig. 4.

44. Trichiulus ammonitiformis.

Locality : Mazon Creek, IIl. Horizon: Carboniferous.

1883. Scudder. MSS.

1883. Lacoe. List pal. foss, ins., 19. 
1884. Scudder. Mem. Bost. soc. nat. hist., iii : 292-293. Pl. 27, fig. 4. 1886. " Mem. Bost. soc. nat. hist., iii : 438 (fern).

1889. Miller. N. A. geol. and pal., 573.

1890. Scudder. Foss. ins. N. A., i : 256-257, 330. Pl. 13, fig. 4.

[Not a Myriapod.]

\section{Trichiulus nodulosus.}

Locality: Mazon Creek, Ill. Horizon : Carboniferous.

1883. Scudder. MSS.

1883. Lacoe. List pal. foss. ins., 19.

1884. Scudder. Mem. Bost. soc. nat. hist., iii : 292. Pl. 27, figs. 1, 3.

1886. " Mem. Bost. soc. nat. hist., iii : 438 (fern).

1889. Miller. $\quad$ N. A. geol. and pal., 573.

1890. Scudder. Foss. ins. N. A., i: 256, 330. Pl. 13, figs. 1, 3.

[Not a Myriapod.]

\section{Trichiulus villosus.}

Locality : Mazon Creek, Ill. Horizon : Carboniferous.

1883. Scudder. MSS.

1883. Lacoe. List pal. foss. ins., 19.

1884. Scudder. Mem. Bost. soc. nat. hist., iii : 291-292. Pl. 27, fig. 2.

1885. " Zittel, Handb. palaeont., i, th. ii : 729. Fig. 900.

1889. Miller. N. A. geol. and pal., 574.

1890. Scudder. Foss. ins. N. A., i : 255-256, 330 (fern). Pl. 13, fig. 2.

[Not a Myriapod.]

\section{Xylobius dawsoni. (Archiulidæ.)}

Locality : Joggins, Nava Scotia. Horizon : Carboniferous.

1868. Scudder. Dawson, Acad. geol., $2 d$ ed., 496.

1869. " "Quart. journ. geol. soc. Lond., xxv: 441 (undeser.).

1873. " " Mem. Bost. soc. nat. hist., ii : 235-236.

1878. " Mem. Bost. soc. nat. hist., ii: 561. Fig. 4.

1878. “ Suppl. note foss. Myr., 1. Fig. 4.

1878. Dawson. Acad. geo!., suppl. $2 d$ ed., 56 (Archinlus). Fig. $11 d$.

1883. Lacoe. List pal. foss. ins., 19.

1889. Miller. N. A. geol. and pal., 574.

1890. Scudder. Foss. ins. N. A., i: 25-26, 31. Fig. 4.

Xylobius farctus. See Xylobius fractus.

48. Xylobius fractus. (Archinlidæ.)

Locality: Joggins, Nova Scotia. Horizon : Carboniferous.

1868. Scudder. Dawson, Acad. geol., $2 d$ ed., 496.

1869. “ Quart.journ. geol. soc. Lond., xxv : 441 (undescr.).

1873. " Mem. Bost. soc. nat. hist., ii : 234-235.

1878. " Mem. Bost. soc. nat. hist., ii : 561. Fig. 3.

1878. “ -Suppl. note foss. Myr., 1. Fig. 3.

1878. Dawson. Acad. geol., suppl. 2d ed., 56 (farctus). Fig. 11 c.

1880. " Chain of life, 145 (farctus). Fig. 126 c.

1882. Scudder. Mem. Bost. soc. nat. hist., iii : 148.

1883. Lacoe. List pal. foss. ins., 19.

1889. Miller. N. A. geol. and pal., 574.

1890. Scudder. Foss. ins. N. A., i : 24-25, 31, 200. Fig. 3 on p. 31. 
49. Xylobius frustulentus. (Archiulidæ.)

Locality: Mazon Creek, Ill. Horizon: Carboniferous.

1890. Scudder. Mem. Bost. soc. nat. hist., iv: 438-439. Pl. 37, figs. 4-6.

1890. " Foss. ins. N. A., i: 414-415. Pl. 29, figs. 4-6.

Xylobius mazonius. See Xylobius mazonus.

50. Xylobius mazonus. (Archiulidæ.)

Locality: Mazon Creek, Ill. Horizon: Carboniferous.

1885. Scudder. Zittel, Handb. palaeont., i, th. ii: 730. Fig. 902b.

1886. " "Zittel-Barrois, Traité de palént., ii : 729. Fig. 918b.

1889. Miller. N. A. geol. and pal., 574 (mazonius).

1890. Scudder. Mem. Bost. soc. nat. hist., iv: 439-440. Pl.37,figs. 7-11.

1890. “ Foss. ins. N. A., i: 415-416. Pl. 29, figs.7-11.

51. Xylobius sigillariæ. (Archiulidæ.)

Localities: Joggins, N. S., Bradley, Cooper's Bridge, Huridersfield, England; Kilmaurs, near Glasgow, Scotland. Horizon : Carboniferous.

1859. Dawson. Quart. journ. geol. soc. Lond., xvi : 271-273. Figs,4-9.

1863. " Can. nat. and geol., viii : 280. Pl. 6, figs. 57-61.

1865. Lyell. Elem. geol., 6th ed., 509. Fig. 562 a-c.

i867. Woodward. Trans. geol, soc. Glasg., ii : 234-237. Pl. 3, figs. 11-13, 11a=13a.

186\%. Binney. Proc. lit. phil. soc. Manch., vi: 59.

1867. " Geol. mag., iv: 132 .

1868. Scudder. Geol. mag., v: 216.

1868. " " Amer. nat., i : 630. Pl. 16, fig. 4.

1868. “ Dawson, Acad. geol., 2 d ed., $495-496$.

1869. " Q Quart. journ. geol. soc. Lond., xxv : 441.

1872. Packard. Guide ins., 3d ed., 78. Pl. 1, fig. 4.

1873. Scndder. Mem. Bost. soc. nat. hist., ii : 232-233.

1877. Goldenberg. Fauna saræp. foss., ii : 34.

1877. Nicholson. Life-hist. earth, 182. Fig. 124 a-c.

1878. Scudder. Mem. Bost. soc. nat. hist., ii : 561. Fig. 1.

1878. " Suppl. note foss. Myr., 1. Fig. 1.

1878. Dawson. Acad. geol., suppl. 2d ed., 56. Fig. 11a.

1879. Nicholson. Man. palæont., 2d ed., i: 403. Fig. 254.

1880. Dawson. Chain of life, 145. Fig. 126a.

188:. Scudder. Mem. Bost. soc. nat. hist., iii: 148.

1883. Lacoe. List pal. foss. ins., 19-20.

1885. Scudder. Zittel, Handb. palaeont., i, th. ii: 730. Fig. 902a.

1886. “ Zittel-Barrois, Traite de paléont., ii: 729. Fig. 918a.

1889. Miller. N. A. geol. and pal., 574. Figs. 1073, 1073a.

1839. Nicholson. Man. palæont., 3d ed., i : 585. Fig. 440.

1890. Scudder. Foss. ins. N. A., i : 22-23, 31, 200. Fig. 1 on p. 31.

See also $\mathbf{X}$. woodwardii.

52. Xylobius similis. (Archiulidæ.)

Locality : Joggins, N. S. Horizon: Carboniferous.

1868. Scudder. Dawson, Acad. geol., $2 \mathrm{~d}$ ed., 496.

1869. " "Quart. journ. geol. soc. Lond., xxv: 441 (undescr.).

1873. " " Mem. Bost. soc. nat. hist., ii : 233-234.

1878. " Mem. Bost. soc. nat. hist., ii : 561. Fig. 2.

1878. " Suppl. note foss. Myr., 1. Fig. 2.

Bull. $71-2$ 
1878. Dawson. Acad. geol, suppl. 2d ed., 56. Fig. $11 b$.

1883. Lacoe.

List pal. foss. ins., 20.

1889. Miller.

N. A. geol. and pal., 574 .

1890. Sendder.

Foss. ins. N. A., i : 23-24, 31. Fig. 2.

53. Xylobius woodwardii. (Archiulidæ.)

Locality : Kilmaurs, Huddersfield, England. Horizon: Carboniferous.

1869. Scudder. Quart. jouru. geol. soc. Lond., xxv : 441 (undeser.).

1873. " Mem. Bost. soc. nat. hist., ii : 238-239.

1882. " " Mem. Bost. soc, nat. hist., iii : 148.

1890. “ Foss. ins. N. A., i: $28-29,200$.

See also X. sigillariæ.

\section{ARACHNIDA.}

54.

Locality: Wettin, Germany. Horizon: Carboniferous.

1886. Kliver. Palaeontogr., xxxii: 112-113. Pl. 14, figs.14, $14 a$-b.

55.

Locality: Bohemia. Horizon: Carboniferous.

1852. Reuss.

Geogn. verhältn. Böhmens, 59.

56.

(Anthracomarti.)

Locality: Steinbachthal, Saarbrück, Germany. Horizon: Carboniferous.

1886. Kliver. Palaeontogr. xxxii: 110-112. Pl. 14, fig. 11.

1886. Karsch. Palaeontogr. xxxii: 111-112.

57.

(Eoscorpioidæ.)

Locality?

Horizon: Carboniferous.

1889. Nicholson. Man. palæont.,3d ed,, i: 573. Fig. 430 c-d.

58.

(Palæophonoidæ.)

Locality : Lesmahagow, Scotland. Horizon: Upper Silurian.

1885. Peach. Nature, xxxi : 297 (scorpion). Fig. 2.

See also Palæophonus _—, Palæophonus caledonicus.

59.

(Scorpiones.)

Locality: Chomle, Bohemia. Horizon: Carboniferous.

1837. Buckland. Geol. and mineral., ii: 79. Pl. $46^{\prime \prime}$, fig. 13.

1838. " Géologie, 6́d. Agassiz, ii : expl. pl. 46",p.6. Pl.46", fig. 13.

60.

(Scorpiones.)

Locality: Scotland. Horizon: Carboniferous.

1882. Peach. Trans. roy. soc. Edinb., $\mathrm{xxx}:$ 406-407. Pl. 22, figs. 4-6; Pl. 23, figs. 13-15, 17-21.

61. - - (Scorniones.)

Locality: Joggins, N. S. Horizon: Carboniferous.

1883. Scudder. Phil.trans, 1882: 650 . 


\section{Anthracomartus —. (Architarboidex.)}

Locality : Petrovic near Rakonitz, Bohemia. Horizon : Carboniferous. 18:8. Kušta. Věstn. král. česke společn. nauk, 1888: 199.

63. Anthracomartus affinis. (Arehitarboidæ.)

Locality : Rakonitz, Bohemia. Horizon: Carboniferous.

1835. Kušta. Sitzungsb. böhnn. gesellsch. wiss., 1884: 399-400. Pl., fig. 2. 188:. " Neue arachuiden, 4-5. Pl., fig. 2.

64. Anthracomartus carbonis. (Architarboidø.)

Locality: Mons, Belgium. Horizon: Carboniferous.

1885. Seudder. Comptes rend. soc. ent. Belg., (3), lxii : 84-85.

1885. " " Anthr, carb., 1-2.

See also Brachypyge carbonis.

Anthracomartus hageni. See Termes hageni, etc.

65. Anthracomartus krejčii. (Architarboidæ.)

Locality : Rakonitz, Bohemia. Horizon: Carboniferous.

1883. Kušta. Sitzungsb. böhm. gesellsch, wiss., 1883: 340-345. Pl., figs. 1 -3.

1883. " Anthracomartus krejěii, 3-7. Pl., figs. 1-3.

1884. Scudder. Proc. Amer. acad. arts sc., $\mathrm{xx}: 17$.

See also A. minor.

\section{Anthracomartus minor. (Architarboidæ.)}

Locality: Rakonitz, Bohemia. Horizon: Carboniferous.

1884. Kušta. Sitzungsb. böhm. gesellsch. wiss., 1884: 191.

1884. " "Thelyphonus bohemicus, 7 (name only).

1884. “ Sitzungsb. böhm. gesellsch. wiss., 1884: 398-399. Pl., fig. 1.

1885. " Neue arachniden, 3-4. Pl., fig. 1 .

1885. “ Neue foss. ar.hrop. Rak., 6 (printed krejčii but corrected in MSS. to minor).

See also A. krejěii.

\section{Anthracomartus pustulatus. (Architarboidæ.)}

Locality: Mazon Creek, Ill. Horizon: Carboniferous.

1884. Scudder. Proc. Amer. acad. arts se, $x x: 18$.

1889. Lesley. Dict. foss. Penu., i, p. v.

1889. Miller. N. A. geol. and pal., 570.

1890. Scudder. Mem. Bost. soc. nat. hist., iv: 452-453. Pl.40, figs. 5, 8.

1890. " Foss, ins. N. A., i : 428-429. Pl. 32, figs. 5,8 .

68. Anthracomartus socius. (Architarboidæ.).

Locality: Rakonitz, Bohemia. Horizon: Carboniferous.

1888. Kušta. Věstn. král. česke společn. nauk, 1888: 198-199, 204-205. Pl. fig. 4 .

69. Anthracomartus trilobitus. (Architarboidæ.)

Locality : Fayetteville, Ark. Horizon: Carboniferous.

1884. Seudder. Proc. Amer. acad. arts se., $\mathrm{xx}: 17-18$.

18 $\div 5$. " Comptes rend, soc. ent. Belg., (3), lxii : 85. Fig. on p. 84.

1885. “ Anthr. carbonis, 2. .Fig. on p.1. 
1886. Harvey.

1889. Lesley.

1889. Miller.

1890. Scudder.

1890. “
Proc. acad.nat. sc. Philad., 1886: 231-232.

Dict. foss. Penns., i, p. v.

N. A. geol. and pal., 570 .

Mem. Bost. soc. nat. hist., iv : 451-452. $\quad P l$. 39, figs. 7-10.

Foss. ins. N. A., i : 427-428. Pl.31, figs. 7-10.

70. Anthracomartus völkelianus. (Architarboidæ.)

Locality : Neurode, Schlesien. Horizon: Carboniferous.

1882. Karsch. Zeitschr. deutsch. geol. gesellsch., 1882: 556-558,560,561. Pl.21, figs. 1,2 .

1884. Scudder. Proc. Amer. acad, arts sc., $\mathrm{xx}: 17$.

1885. " " Zittel, Handb. palaeont, i, th. ii: 736. Fig. 911.

1886. " Zittel-Barrois, Trait€́ de paléont., ii : 735. Fig. 927.

71. Anthracoscorpio juvenis. (Eoscorpioidæ.)

Locality : Rakonitz, Bohemia. Horizon: Carboniferous.

1888. Kušta.

Věstn. král. česke společn. nauk, 1888: 202-203, 206.

See also Cyclophthalmus senior, etc.

\section{Aranea}

Locality: Coalbrookdale, England. Horizon: Carboniferous.

1834. Prestwich. -Lond. Edinb. phil. mag., iv : 376.

73. Architarbus elongatum. (Architarboidæ.)

Locality : Mazon Creek, Ill. Horizon : Carboniferons.

1890. Scudder. Mem. Bost. soc. nat. hist., iv : 449-450. Pl. 40, fig. 4. 1890. " Foss. ins. N. A., i : 425-426. Pl. 32, fig. 4.

74. Architarbus rotundatum. (Architarboidæ.)

Locality: Mazon Creek, Ill. Horizon: Carboniferous.

1868. Scudder. Worth., Geol. surv. Ill., iii : 568 (rotundatus). Fig. 4.

1872. Woodward. Geol. mag., ix : 386. Pl. 9, fig. 2 .

1872. " Arachn. coal meas., 2. Pl. 9, fig. 2.

1882. Karsch. Zeitschr. deutsch. geol. gesellsch., 1882: 560 .

1883. Lacoe. List pal. foss. ins., 20.

1884. Scudder. Proc. Amer. acad. arts sc., $\mathrm{xx}: 17$.

1885. " Zittel, Handb. palaeont, i, th. ii: 736. Fig. 910.

1886. " Zittel-Barrois, Traité de paléont., ii : 735. Fig. 926.

1886. " Bull. U. S. geol. surv., No. 31: 24.

1889. Lesley. Dict. foss. Penns., i, p. vii.

1889. Miller. N. A. geol. and pal., 5\%0. Fig. 1064.

1890. Scudder. Mem. Bost. soc. nat. hist., iv : 449.

1890. " Foss. ins. N. A., i : 425 .

\section{Architarbus silesiacum. (Architarboidæ.)}

Locality: Glatz, Silesia. Horizon: Carboniferous.

1879. Roemer.

1884. Scudder.

1885. " "

$1886 . \quad$ "

1886. “
Jahresb. schles. gesellsch. vaterl. cult., lvi : 54-55 (silesiacus).

Proc. Arner, acad. arts se., xx : 17.

Zittel, Handb. palaeont., i, th. ii : 736.

Zittel-Barrois, Traité de paléont., ii : 735.

Bull. U. S. geol. surv., No. 31 : 24 , 
76. Architarbus subovale. (Arehitarboidæ.)

Localities: Burniey and Padiham, Lancashire, England. Horizon: Middle carboniferous.

1872. Wood ward. Geol. mag., ix : 385-387 (subovalis). Pl. 9, fig. $1 a b$. 187\%. " Arachn. coal meas., 1-3. Pl. 9, fig. $1 \mathrm{ab}$.

1876. Roemer. Lethæa geogn. Pl. 47, fig. 2.

1877. Haughton. Journ. geol. soc. Ireland, n. s., iv: 222-223. 2 figs.

1879. Nicholson. Manual palæont., 2d ed., i : 401. Fig. 252B.

188\%. Karsch. Zeitschr. deutsch. geol. gesellsch., 1882: 560.

1834. Scudder. Proc. Amer. acad. arts sc., $x x: 17$.

1885. " " Zittel, Hasdb. palaeont., i, th. ii: 736.

1886. " Zittel-Barrois, Traite de paleont., ii : 735.

1886. " Bull. U. S. geol. surv., No. 31: 24 .

1889. Nicholson. Man. palæont., 3d ed., i: 576. Fig. $432 b$.

See also Curculioides ansticii, Hevila ansticii.

\section{Arthrolycosa antiqua.}

Locality : Mazon Creek, Ill. Horizon : Carboniferous.

1874. Harger.

1883. Lacoe.

1884. Scudder.

1885. " "

1886. “

1886. “

1889. Beecher.

1889. Lesley.

1889. Miller.

1890. McCook.
Am. journ. sc., (3), vii : 219-223. Fig.

List pal. foss. ins., 20.

Proc. Amer. acad. arts sc., $\mathrm{xx}: 15$.

Zittel, Handb. palaeont., i, th. ii : 735. Fig. 909.

Zittel-Barrois, Traité de paléont., ii : 734. Fig. 925.

Bull. U. S. geol. surv., No. 31 : 24.

Am. journ. sc., (3), xxxviii : 219-223. 3 figs.

Dict. foss. Penn., i : 37, vii. Fig.

N. A. geol. and pal. Fig. 1065.

Amer. spiders, ii : 455-456. Figs. 381-382.

78. Brachypyge carbonis. (Architarboidæ.)

Locality: Mons, Belgium. Horıon : Carboniferous.

1878. Woodward. Geol. mag., (2), v : 433-436. Pl. 11.

1878. " " Fossil crab coal-meas. Belg., 1-4. Pl. 11.

1878. " " Bull, acad. roy. Belg., (2), xlv: 410-415. Pl.

See also Anthracomartus carbonis,

- 79. Buthus carbonarius. (Eoscorpioidæ.)

Locality : Mazon Creek, Jll. Horizon: Carboniferous.

1868. Meek-Worth. Am. journ. sc. arts, (2), xlvi : 22-24.

See also Eoscorpius carbonarius.

Calotermes hageni. See Termes (Calotermes) hageni.

80. Centromachus anglicus. (Eoscorpioidæ.)

Locality: England. Horizon: Carboniferous.

1885. Thorell. K. svensk. vetensk. akad. handl., xxi, No. 9: 25.

See also Eoscorpius anglicus.

81. Centromachus euglyptus. (Eoscorpioidæ.)

Locality : Scotland. Horizon: Carboniferons.

1885. Thorell. K. svensk. vetensk, akad. handl., xxi, No. 9 : 21.

See also Eoscorpius euglyptus. 
82. Centromachus glaber. (Eoscorpioidæ.)

Locality: Scotland. Horizon: Lower Carboniferous.

1885. Thorell. K. svensk. vetensk. akad. handl, xxi, No. 9: 25.

- See also Eoscorpius glaber.

83. Centromachus inflatus. (Eoscorpioidæ.)

Locality : Scotland. Horizon : Lower Carboniferous.

1885. Thorell. K. svensk. vetensk. akad. handl., xxi, No. $9: 25$.

See also Eoscorpius inflatus.

84. Centromachus tuberculatus. (Eoscorpioidæ.)

Locality : Scotland. Horizon: Carboniferous.

1885. Thorell. K. svensk. vetensk. akad. handl., xxi, No. $9: 25$.

See also Eoscorpius tuberculatus.

\section{Curculioides ansticii. (Architarboidæ.)}

Locality: Coalbrookdale, England. Horizon: Carboniferous.

1837. Buckland. Geol, and mineral, ii : 76. Pl. 46" fig. 1.

1838. " " Géologie, éd. Ågassiz, expl. pl. 46", pp. 1-2. Pl. 46", fig. 1.

1838. Agassiz. Buckl., Géologie, éd. Agassiz, expl. pl. 46", p. 2 note.

1854. Mantell. Medals of creation, $2 d$ ed., ii : 555 .

1881. Scudder. Geol, mag., (2), viii : 300 .

1883. " " Mem. Bost. soc. nat. hist., iii: 223.

1886. " " Bull. U. S. geol. surv., No. 31:24 (= Architarbus subovale).

1890. " " Foss. ins. N. A., i: 245.

See also Hevila ansticii, Architarbus subovale.

86. Curculioides prestvicii. (Eophrynoidæ.)

Locality : Coalbrookdale, England. Horizon: Carboniferous.

1837. Buckland. Geol, and mineral., ii : 76-77. Pl. 46" fig. 2.

1838. " Géologie, éd Agassiz, ii, expl. pl. 46": pp. 3-4. Pl. 46", fig: 2.

1854. Mantell. Medals of creation, $2 d$ ed., 555.

1882. Karsch. Zeitschr. deutsch. geol. gesellsch., 1882: 560.

See also Duma prestvicii, Eophrynus prestvicii, Euphryuus prestvicii.

87. Cyclophthalmus __. (Eoscorpioidæ.)

Locality: Bohemia. Horizon : Carboniferous.

1882. Peach. Trans. roy. soc. Edinb., xxx: (copy of fig. of C. senior). Pl. 23, fig. 25.

See also C. senior, etc.

88. Cyclophthalmus kralupensis. (Eoscorpioidæ.)

Locality: Kralup, Bohemia. Horizon: Carboniferous.

1885. Thorell. K. svensk. vetensk, akad. handl., xxi, No. 9: 17 note, 20.

See also C. senior, etc.

89. Cyclophthalmus senior. (Eoscorpioidæ.)

Localities: Stein-Anjezd Kralup, Chomle, Rakonitz, Bohemia. Horizon: Carboniferous.

1835. Corda. Verhandl. gesellsch. vaterl. mus. Böhm., 1835: 38-43. Pl., figs. 1-14.

1837. Buckland. Geol, and mineral, ii : 74-75 (no spec. name). Pl. 46', figs, 1-7. 
1838. Buckland. Géologie, éd. Agassiz, ii : expl. pl. 46' (no spec. name). Pl. 46', figs. 1-7.

1844. Walck.-Gerv. Hist. nat. ins., Apt., iii : 70-72 (no specific name).

185\%. Giebel.

Deutschl. petref., 635 .

1856. " "

Insect. d. vorwelt, 470-471.

1853-'56. Bronn.

Lethæa geogn., $3^{\text {e }}$ anfl., i, th. ii : $681 . \quad P l .9^{3}$, fig. 16.

1874. Fritsch.

Arch. naturw. landesđurchf. Böhm., ii, th. ii, No. 1: 9-14. Pl. 1, fiqs. 1-8; Pl. 2, figs. 1-6, $2 a ; P l .3$, fig. 4.

1876. Roemer.

Lethæa geogn. Pl. 47, fig. 1.

1877. Nicholson.

Life-hist. earth, 181. Fig. 123.

1879. "6

Manual palæont., 2d ed., i: 400. Fig. 251.

1882. Karseh.

Zeitsahr. deutseh. geol. gesellseh., 1882: 560 .

1884. Scudder

1884. Kušta.

Proc. Amer. acad. arts sc., xx : 22.

Vesmír, xiii : 97-98.

1884. " "

Sitzungsb. böhm. gesellsch. wiss., 1884: 48-50.

1884. “ “

Nener fundort Cyclophth., 1-3.

1885. " “

Sitzungsb. böhm gesellsch. wiss., 1884: 401. Pl., fig. 4.

1885. “6

Neue arachuiden, 6. Pl., fig. 4.

1885. Thorell.

K. svensk. vetensk. akad, handl, xxi, No.9: 17 note, 24.

1885. Scudder.

1886. "6

Zittel, Handb. palaeont., i, th. ii: 740.

Zittel-Barrois, Traité de paléont., ii : 739.

1889. Nicholson. Man. palæont., 3d ed., i: 579. Fig. 435.

See also Cyclophthalmus —, C. kralupensis, C. sternbergi, Mierolabis sternbergi, Anthracoscorpio juvenis.

90. Cyclophthalmus sternbergi. (Eoscorpioidæ.)

Locality: Bohemia. Horizou: Carboniferous.

1854. Pictet.

Traité paléont., 2e é̉., ii : 407. Pl.41, fig.1.

1885. Thorell.

K. svensk. vetensk. akad. handl., xxi, No. $9: 17$ note, 24 .

[The same as C. senior?]

See also Microlabis sternbergi, Cyclophthalmus senior, ete.

91. Duma prestvicii. (Eophrynoidæ.)

Locality: England. Horizon: Carboniferous.

1856. Giebel. Insect. d. vorwelt, 137.

See also Curculioides prestvicii, Eophrynus prestvicii, Enphrynus prestvicii.

\section{Eolycosa lorenzi.}

Locality: Rakonitz, Bohemia. Horizon: Carboniferous.

1885. Kušta. Sitzungsb. k. böhm. gesellsch. wiss., 1885: 592-593. Fig.

1885. " "Nene foss. arthr., 3-4. Fig.

1888. “ Věstn. král. česke společn. nauk, 1888: 197.

93. Eophrynus prestvicii. (Eophrynoidæ.)

Locality : Dudley, England. Horizon: Carboniferous.

1871. Woodward.

1872 .

1879. Nicholson.

1884. Scudder.

1885.

1886.

1886.

1889. Lesley.

1889. Nicholson.
Geol. mag., viii : 3£5-38\%. Pl. 11, figs. 1,2.

Rep. Brit. assoc. adv. sc., xli : notic. 113.

Manual palaeont., 2d erl., i: 401. Fig. 252a.

Proc. Amer. acad. arts sc., xx: 19.

Zittel, Handb. palaeont., i, th. ii : 737. Fig. 913 .

Zittel-Barrois, Traité de paléont., ii : 736. Fig. 929.

Bull. U. S. geol. surv., No. $31: 25$.

Dict. foss. Penus., i : 37 (unnamed). Fig.

Man. palæont., 3d ed., i: 576. Fig. 432a.

See also Curculioides prestvicii, Duma prestvicii, Euphrynus prestvicii. 
94. Eophrynus salmi. (Eophrynoidæ.)

Locality : Ostrau, Moravia. Horizon: Carboniferous.

1877. Stur.

1884. Scudder. $1885 . \quad$ " "

1886.

1886. “

Abhandl. geol, reichsanst., viii: no. 2; p.v. note (Euphrynus). Fig.

Proc. Amer, acad, arts sc., xx : 19.

Zittel, Handb. palaeont, , i, th. ii: 737.

Zittel-Barrois, Traité de paléont., ii : 736.

Bull. U. S. geol. surv., No. 31 : 25.

95. Eoscorpius

(Eoscorpioidæ.)

Locality : River Esk, Scotland. Horizon: Lower Carboniferous.

1882. Peach.

Trans. roy. soc. Edinb., xxx : 404-405. Pl. 23, figs. 11, $11 a$.

96. Eoscorpius anglicus. (Eoscorpioidæ.)

Locality : Mansfield, England. Horizon: Carboniferous.

1876. Woodward. Quart. journ. geol. soc. Lond., 1876: 58. Pl. 8, figs. 2,3,3a,5.

1882. Karsch. Zeitschr. deutseh. geol. gesellsch., 1882: 560.

1884. Seudder. Proc. Amer. acad. arts se., $\mathrm{xx}: 22$.

See also Centromachns anglicus.

97. Eoscorpius carbonarius. (Eoscorpioidæ.)

Localities: Mazon Creek, Ill.; Sandwell Park Colliery, near Birmingham, England. Horizon: Carboniferous.

1868. Meek-Worth. Am. journ. sc., (2), xlvi : 22-25.

1868. " Worth., Geol. surv. Ill., iii : 560-562. Figs.

1876. Woodward. Quart. journ. geol. soc. Lond., 1876: 57-59. Pl.8, fig. 1.

1880. Dawson. Dawn of life, 150. Fig. 132 on p. 151.

1882. Peach. Trans. roy. soc. Edinb., $\mathrm{xxx}: 412$. Pl. 23, figs. 23-23b.

1882. Karsch. Zeitschr. dentsch. geol. gesellsch., 1832: 560.

1883. Lacoe. List pal. foss. ins, 21.

1884. Scudder. Proc. Amer. acad. arts sc., xx: 21.

1885. Thorell. K. svensk. vetensk. akad. handl., xxi, No. 9: 25.

1885. Scudder. Zittel, Handb. palaeont., i, th. ii : 739. Fig. 916.

1886. " " Zittel-Barrois, Traité de paléont., ii : 739. Fig.933.

1889. Lesley, Dict. foss. Penns., i: 220 . Fig.

1889. Miller. N. A. geol. and pal., 571. Fig. 1066.

1889. Nicholson. Man. palaeont., 3d ed., i: 578. Fig. 434.

See also Buthus earbonarius.

98. Eoscorpius euglyptus. (Eoscorpioidæ.)

Locality : River Esk, Scotland. Horizon : Lower Carboniferous.

188. Peach. Trans. roy. soc. Edinb., xxx: 402-404. Pl. 22, figs. 3-3d.

1884. Scudder. Proc. Amer, acad, arts sc., $\mathrm{xx}: 22$.

See also Centromachus euglyptus.

99. Eoscorpius glaber. (Eoscorpioidæ.)

Locality : Redhall, River Esk, Dumfrieshire. Horizon: Lower Carboniferous.

1882. Peach. Trans, roy. soc. Edinb., xxx: 400-402. Pl. 22, figs. 2-2l.

1884. Scudder. Proc. Amer. acad. arts sc., $\mathrm{xx}: 22$.

See also Centromachus glaber. 
100. Eoscorpius inflatus. (Eoscorpioidæ.)

Locality : River Esk, Scotland. Horizon: Lower Carboniferous.

1882. Peach.

Traus. roy. soc. Edinb., xxx: 405-406. Pl. 23, figs. 12-12d.

1884. Scudder.

Proc. Amer. acad. arts se., xx : 22.

See also Centromachus inflatus.

101. Eoscorpius tuberculatus. (Eoscorpioidæ.)

Localities: Blair Point, Redhall, Cramond, Scotland. Horizon: Carboniferous.

1882. Peach. Trans. roy. soc. Edinb., xxx: 398-400. Pl. 23, figs. 8-8h.

1884. Scudder. Proc. Amer. acad. arts sc., xx : 22.

See also Centromachus tuberculatus.

102. Eoscorpius woodianus. (Eoscorpioidæ.)

Locality : Illinois. Horizon: Carboniferous.

1882. Peach. Trans. roy. soc. Edinb., xxx: 408-409. Pl. 23, figs. 24, 24a.

1885. Thorell. K. svensk, vetensk. akad. handl, xxi, No. 9: 25.

See also Mazonia woodiana.

103. Eotarbus litorale. (Architarboidæ.)

Locality : Rakonitz, Bohemia. Horizon: Carboniferons.

1888. Kušta. Věstn. král. česke společn. nank, 1888: 198, 204 (litoralis). $P l .$, fig. 3.

104. Euphrynus prestvicii. (Eophrynoidæ.)

Locality: England. Horizon: Carboniferous.

1876. Roemer. Lethaea geogn. Pl. 47, fig. 12.

See also Eophrynus prestvicii, Duma prestvicii, Curculioides prestvicii.

Euphrynus salmi. See Eophrynus salmi.

105. Geralinura bohemica. (Geralinuridæ.)

Locality: Bohemia. Horizon: Carboniferous.

1885. Scudder. Zittel, Handb. palaeont., i, th. ii: 737. Fig.914.

1886. " "Zittel-Barrois, Traité de paléont., ii: 736. Fig.930.

1886. " " Bull. U. S. geol. surv., No. 31: 26.

1890. " " Mem. Bost. soc. nat. hist., iv : 455-456.

1890. " " Foss. ins. N. A., i : 431-432.

See also Thelyphonus bohemicus, G. (Thelyphonus) bohemica.

106. Geralinura (Thelyphonus) bohemica. (Geralinuridæ.)

Locality: Bohemia. Horizon: Carboniferous.

18ธิฮ., Scudder. Zittel, Handb. palaeont., i, th. ii : 737. Fig. 914.

1886. "6 Zittel-Barrois, Traité de paléont., ii : 736. Fig. 930.

See also Geralinura bohemica, etc.

107. Geralinura carbonaria. (Geralinuridæ.)

Locality: Mazon Creek, Ill. Horizon: Carboniferous.

1884. Scudder. Proc. Amer. acal. arts sc., $\mathrm{xx}:$ 19-20.

1885. " " Zittel, Handb. palaeont., i, th. ii: 737 .

1886. " "Zittel-Barrois, Traité de paléont., ii: 737.

1886. “ Bull. U. S. geol. surv., No. 31: 26.

1889. Miller. $\quad$ N. A. geol, and pal., 571.

1890. Scudder. Mem. Bost. soc. nat. hist., iv: 455. Pl. 39, figs. 1, 3, 4.

1890. " Foss. ins. N. A., i: 431. Pl. 31, figs. 1, 3, 4. 
108. Geralinura crassa. (Geralinuridæ.)

Locality : Rakonitz, Bohemia. Horizon: Carboniferous.

1888. Kušta. $\quad$ Věstn. král. česke společn. nauk, 1888: 202, 206.

109. Geralinura noctua. (Geralinuridæ.)

Locality: Rakonitz, Bohemia. Horizon: Carboniferous.

1888. Knšta. Věstn. král. česke společn. nauk, 1888: 200-201, 206. Pl., fig. 6.

110. Geralinura scudderi. (Geralinuridæ.)

Locality: Rakonitz, Bohemia. Horizon: Carboniferous.

1888. Kušta. Věstn. král. česke společn. nauk, 1888: 201-202, 205. Pl., fig. 5.

\section{Geralycosa fričii:}

Locality: Rakonitz, Bohemia. Horizon: Carboniferous.

1888. Kušta Vèstn. král. česke společn. nauk, 18ホ8: 196, 203-204. Pl., fig. 1.

112. Geraphrynus carbonarius. (Architarboidæ.)

Locality: Mazon Creek, Ill. Horizon: Carboniferous.

1884. Scudder. Proc. Amer. acad. arts sc., xx: 16-17.

1885. " " Zittel, Handb. palaeont., i, th. ii: 735 .

1886. " " Zittel-Barrois, Traité de paléont., ii: 735.

1886. " Bull. U. S. geol. surv., No. 31: 24 .

1889. Miller. $\quad$ N. A. geol. and pal., 571.

1890. Scudder. Mem. Bost. soc. nat. hist., iv: 446-447. Pl. 40, figs. 1, 9, 10, 12.

1890. " Foss. ins. N. A., i : 422-423. Pl. 32, figs. 1, 9, 10, 12.

113. Geratarbus lacoei. (Architarboidæ.)

Locality: Mazon Creek, Ill. Horizon: Carboniferous.

1890. Scudder. Mem. Bost. soc. nat. hist., iv: 448. Pl. 40, fig. 11.

1890 “ Foss. ins. N. A., i: 424. Pl. 32, fig. 11.

114. Geratarbus scabrum. (Architarboidæ.)

Locality : Mazon Creek, Ill. Horizon : Carboniferous.

1890. Seudder. Mem. Bost. soc. nat. hist., iv: 448-449. Pl. 39, fig. 5.

1890. " Foss. ins. N. A., i : 424-425. Pl. 31, fig. 5.

115. Graeophonus carbonarius. (Geralinuridæ.)

Locality: Mazon Creek, Ill. Horizon: Carboniferous.

1890. Scudder. Mem. Bost. soc. nat. hist., iv: 454. Pl. 40, figs. 2, 3, 6.

1890. " Foss. ins. N. A., i: 430. Pl. 32. figs. 2, 3, 6.

See also Libellula carbonaria.

116. Hevila ansticii. (Architarboidæ.)

1856. Giebel. Insect. $d$, vorwelt, 143.

See also Architarbus subovale, Curculioides ansticii.

117. Kreischeria wiedei. (Eophrynoidæ.)

Locality: Zwickau, Germany. Horizon: Carboniferous.

1882. Geinitz. Zeitschr. deutsch. geol. gesellsch., 1882: 238-242. Pl. 14, figs. $1,2$.

1882. Karsch. Zeitsehr. deutsch, geol. gesellsch., 1882: 560 . 
1884. Scudder. Proc. Amer. acad. arts sc., xx: 18-19.

1885. " "

1886. “

1886. " “

Zittel, Handb. palaeont., i, th. ii: 736. Fig. 912.

Zittel-Barrois, Traité de paléont., ii : 735. Fig. 928.

Bull. U. S. geol. surv., No. 31: 25.

118. Kustarachne tenuipes. (Architarboidæ.)

Locality : Mazon Creek, Ill. Horizon: Carboniferous.

1890. Scudder. Mem. Bost. soc. nat. hist., iv: 450-451. Pl. 40, fig. 7.

1890. " Foss. ins. N. A., i : $426-42 \%$. Pl. 32, fig. $\%$.

119. Libellula carbonaria. (Geralinuridæ.)

Locality: Cape Breton, Nova Scotia. Horizon: Carboniferous.

1875. Scudder. Proc. Bost. soc. nat. hist., xviii: 113-114 (unnamed).

1876. " Ent. notes, v: 2-3 (unnamed).

1876. " Proc. Amer. assoc. adv. sc., xxiv: B 110-111. Fig. 1.

1876. “ Can. nat., (2), viii: 88-89. Fig. 1 [2].

1876. " Ius. carb. Cape Breton, [1-2]. Fig. 1 [2].

1878. Dawson. $\quad$ Acad. geol., suppl. 2d ed., 55. Fig. 8.

1880. " Chain of life, 148. Fig. 129.

1883. Lacoe. List pal. foss. ins., 9-10.

1885. Scudder. Mem. Bost. soc. nat. hist., iii : 350.

1885. " Zittel, Handb. palaeont., i, th. ii: 736 .

1886. " " Zittel-Barrois, Trait6 de paléont., ii: 735.

1886. “ Bull. U. S. geol. surv., No. 31: 25 .

1889. Lesley. Dict. foss. Penns., i: 336.

1889. Miller. N. A. geol. and pal., 578. Fig. 1074.

1890. Scudder. Foss. ins. N. A., i : 314.

See also Græophonus carbonarius.

120. Mazonia -. (Eoscorpioidæ.)

Locality : Joggins, N. S. Horizon: Carboniferous.

1883. Scudder. Phil. trans,, 1882: 650 .

Mazonia woodana. See Mazonia woodiana.

121. Mazonia woodiana. (Eoscorpioidæ.)

Locality : Mazon Creek, Ill. Horizon: Carboniferons.

1868. Meek-Worth. Worth., Geol. surv. Ill., iii : 563-565. Figs. $A-D$.

1882. Peach. Trans. roy. soc. Edinb., $\mathrm{xxx}:$ 408-409.

1882. Karsch. Zeitschr. deutsch. geol. gesellsch., 1882: 560 .

1883. Lacoe. List pal. foss. ins., 21.

1884. Scudder. Proc. Amer. acad. arts se., xx: 22.

1889. Lesley. $\quad$ Diet. foss. Penns., i : 382.

1889. Miller. N. A. geol, and pal., 571 (woodana).

See also Eoscorpius woodianus.

122. Microlabis sternbergi. (Eoscorpioidæ.)

Compared with Obisium carcinoides, Europe.

Locality: Malikowetz bei Chomle, Bohemia. Horizon: Carboniferous.

1839. Corda. Verhandl. geseliseh. vaterl. mus. Böhm., 1839: 15-18. Pl. 1, figs. 1-5.

1852. Giebel. Dentschl, petref., 635.

1853-'56. Bronn. Lethæa geogn., $3^{\mathrm{e}}$ aufl. i, th. ii : 681.

1856. Giebel. Insect. d. vorwelt, 474. 
1870. Hagen.

1874. Friě.

Proc. Bost. soc. nat. hist., xiii : 269.

Arch. naturw. landesdurchf. Böbm., ii, pt. 2, No. 1: 13-14 (= young Cyclophthalmus senior).

1876. Gerstaecker. Klass. ordn. arthr., Crust., i : 292.

188:. Karsch. Zeitschr. deutsch. geol. gusellsch., 1882: 560.

See also Cyclophthalmus senior, C. sternbergi, -etc.

Palæophoneus nuncius. See Palæophonus nuncius.

123. Palæophonus _- (Palæophonoidæ.)

Locality : Scotland. Horizon: Upper Silurian.

1889. Nicholson. Man. palæont., 3d ed., i: 578. Fig. 433.

See also — $\longrightarrow$ (Palæophonoidæ) Peach, Palæophonus caledonicus.

124. Palæophonus caledonicus. (Palæophonoidæ.)

Locality: Logan Water, Scotland. Horizon: Silurian.

1886. Hunter. Trans. geol. soc. Glasg., viii: 169-170 (undescr.).

1887. “ Trans. geol. soc. Edinb., v: 187-189. Pl. 4 (ined.).

See also —— (Palæophonoidæ) Peach, Palæophonus - - .

125. Palæophonus nuncius. (Palæophonoidæ.)

Locality: Gotland, Sweden. Horizon: Upper Ludlow beds.

1884. Lindström. Comptes rendus, xcix : 984-985 (Palæophoneus).

1884. " La nature, xcix: 33-34. Fig.

1885. Thor.-Lindstr.K. svensk. vetensk. akad. handl., xxi, No.9. Pl.

1885. Scudder. Zittel, Handb. palaeont., i, th. ii : 738. Fig. 915.

1885. Brongniart. Jahrb. geol. reichsanst., $\mathrm{xxxv}: 652$ (Palæophonens).

1886. Seudder. Zittel-Barrois, Traité de paléont., ii : 738. Fig. 931.

1886. " " Bull. U. S. geol. surv., No. 31: 27.

1887. Lindström. Trans. Edinb. geol. soc., v : 184-186. $\quad P l .3$ (ined.).

1890. Lesley. Dict. foss. Penns., ii : 586. Fig.

126. Palæophonus osborni. (Eoscorpioidæ.)

Locality: Waterville, N. Y. Horizon: Lower Helderberg.

1885. Whitfield. Science, vi : 87-88. Fig.

See also Proscorpius osborni.

127. Palaranea borassifolia. (Liphistioidæ.)

Localities: Svina, Radnitz, Bohemia. Horizon: Carboniferous.

[1854. Reuss.

[1864. “

1869. Frič.

1874. “

1884. Sendder.

1884. Kušta.

1885. Scudder.

1886. " "

1886. “
Geogn. verhältn. Bëhmens.]

Ziva, 231-233:]

Feistm., Arch. naturw. landesdurchf. Böhm., i, th. ii, Steink. beck. Radn., 66 (undescr.).

Arch. naturw. landesdurchf. Böhm., ii, th. ii, i: 8 (borassifoliæ). Pl. 2, fig. 7.

Proc. Amer. acad. arts sc., $\mathrm{xx}: 22$.

Vesmír, xiii : 97-98.

Zittel, Haudb. palaeont., i, th. ii: 742 .

Zittel-Barrois, Traité de paléont., ii : 742.

Bull. U. S. geol. surv., No. 31 : 30 .

Palaranea borassifolize. See Palaranea borassifolia. 
128. Poliochera punctulata. (Poliocheridæ.)

Locality: Mazon Creek, Ill. Horizon: Carboniferous.

1884. Scudder. Proc. Amer. acad. arts sc., $\mathrm{xx}: 16$.

1885. " " Zittel, Handb. palaeont., i, th. ii : 735 .

1886. “ "Zittel-Barrois, Traité de pal6ont., ii : 734.

1886. " " Bull. U. S. geol. surv., No. 31 : 24 .

1889. Miller. N. A. geol. and pal., 571.

1890. Lesley. Dict. foss. Penns., ii: 730.

1890. Scudder. Mem. Bost. soc. nat. hist., iv: 444-445. Pl. 39, figs. 2, 6 .

1890. " Foss. ins. N. A., i: 420-421. Pl. 31, figs.2, 6.

Proscorpius osbornei. See Proscorpius osborni.

129. Proscorpius osborni. (Eoscorpioidæ.)

Locality: Waterville, N.Y. Horizon: Lower Helderberg.

1885. Whitfield. Bull. Amer. mus. nat. hist., i: 181-196. Pl. 19, 20.

1885. Scudder. Zittel, Handb. palaeont., i, th. ii : 739. Fig.915a.

1886. " " Zittel-Barrois, Traité de palént., ii : 738. Fig. 932 on p. 739.

1886. " Bull. U. S. geol. surv., No. 31: 28.

1886. Thorell. Amer. nat., xx : 269-274 (osbornei).

1886. Whitfield. Science, vii : 216-217.

1889. Miller. N. A. geol. and pal., 571.

1890. Lesley. Dict. foss. Penn., ii : 773-774. Figs.

See also Palæophonus osborni.

\section{Protolycosa anthracophila. (Liphistioidæ.)}

Locality: Kattowitz, Oberschlesien. Horizon: Carboniferous.

1865. Roemer. Geol. mag., ii : 468.

1866. " Rep. Brit. assoc. adv. sc., 1865 : not., 73.

1866. " Neues jahrb. min., 1866: 136-141. Pl. 3, figs. 1-3.

1870. Thorell. European spiders, 221-2222.

1876. Roemer. Lethæa geogn. Pl. 47, fig. 7.

1877. Goldenberg. Faun. saræp. foss., ii : 31-33, 51.

1882. Karsch. Zeitschr. deutsch. geol. gesellsch., 1882: 559.

1884. Scudder. Proc. Amer. acad. arts sc., xx : 22.

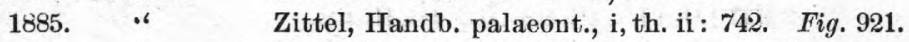

1886. " " Zittel-Barrois, Traité de paléont., ii : 741. Fig. 938 on p. 742.

1886. " Bull. U. S. geol. surv., No. 31: 30 .

1890. MeCook. Amer. spiders, ii : 453-455. Figs. 378-380.

\section{Rakovnicia antiqua.}

Locality: Rakonitz, Bohemia. Horizon : Carboniferous.

1885. Kušta. Sitzungsb. böhm. gesellsch. wiss., 1884: 400-401. Pl., fig. 3.

1885. " Neue arachn., 5-6. Pl., fig. 3.

1885. Scudder. Zittel, Handb. palaeont., i, th. ii: 735.

1886. “ Zittel-Barrois, Traité de paléont., ii : 734.

1886. " Bull. U. S. geol. surv., No. 31: 24 .

132. Scorpio - (Scorpiones.)

Locality: Nyřan, Bohemia. Horizon: Permian.

1879, Fritsch. Fauna d. gaskohle Böhmens, i; 32 . 


\section{Scudderia carbonaria. (Architarboidæ.)}

Locality : Rakonitz, Bohemia. Horizon: Carboniferous.

1888. Kušta. Věstn. král. česke společn. nank, 1888: 196-197,204. Pl., fig. 2.

\section{Termes hageni. (Architarboidæ.)}

Locality : Altenwald, Germany. Horizon: Carboniferous.

1877. Goldenberg. Fauna saræp. foss., ii : 50.

1885. Scudder. Hanỏb. palaeont., i, th. ii : 736.

1886. " "Zitíel-Barrois, Traité de paléont., ii: 735.

1886. “ Bull. U. S. geol. surv., No. 31: 25 (vic. Anthracomartus).

See also T. (Calotermes) hageni.

135. Termes (Calotermes) hageni. (Architarboidæ.)

Locality : Altenwald, Germany. Horizon : Carboniferous.

1854. Goldenberg. Palaeontogr., iv: 37 (unnamed). Pl.6.jig. 8.

1854. " " Foss. ins. Saarbrücken, 21 (unnamed). Pl. 4, fig. 8.

1867. " " Vorweltl. fauna steink. Saarbr., 12. Pl. 2, fig. 7 (ined.).

1873. “ $\quad$ Fauna saræp. foss., i: 12. Pl. 2, fig. 7.

See also Termes hageni.

136. Thelyphonus bohemicus. (Geralinuridæ.)

Locality: Rakonitz, Bohemia. Horizon: Carboniferous.

1884. Kušta. Sitzungsb. böhm. gesellsch. wiss., 1884: 186-191. Pl., figs. 1-4.

1884. " "Thelyphonus bohemicus, 1-7. Pl., figs. 1-4.

1885. " "Sitzungsb. böhm. gesellsch. wiss., 1884: 401-402.

1885. " " Neue arachn., 6-7.

1885. " Nene foss. arthrop. Rak., 5-6.

1885. " Sitzungsb. böhm. gesellsch. wiss., 1885: 594-595.

See also Geralinura bohemica, etc.

\section{PALAEOICTYOPTERA. \\ THE ORTHOPTEROID SERIES.}

137.

Locality : Cusel, Rheinpfalz. Horizon: Carboniferous.

1867. Dohrn. Palaeontogr., xvi: 134. Pl. 8, fig. 4.

See also Termitidium rugosum.

138. (Palæoblattariæ.)

Locality : Löbejün, Saxony. Horizon : Carboniferous.

1886. Kliver. Palaeontgr., xxxii : 114-115. Pl. 14, figs. 13, 13a.

139.

(Palæoblattariæ.)

Compared with Petrablattina sepulta.

Locality : Sydney, Cape Breton. Horizon: Lower Carboniferous.

1879. Scudder. Mem. Bost. soc. nat. hist., iii : 128. Pl. 6, fig. 11.

1890. " Foss. ins. N. A., i : 148. Pl. 6, fig. 11. 
140. (Palæoblattariæ.)

Compared with Archimylacris parallela.

Locality : Cannelton, Pa. Horizon : Lower Carboniferous.

1879. Sendder. Mem. Bost. soc. nat. hist., iii : 128. Pl. 6, fig. 13.

1890. “ Foss, ins. N. A., i : 148. Pl. 6, fig. 13.

141. - (Protophasmida.)

Locality : Claxheugh, Durham, England. Horizon: Carboniferous.

1867. Kirkby. Geol. mag., iv: 389. Pl. 17, fig. 8.

1881. Sendder. Geol. mag., (2), viii : 300.

1883. " "Mem. Bost. soc. nat. hist., iii : 223.

1890. " " Foss. ins. N. A., i : 245.

Acridites —. See I, Neuropt, Idem.

142. Adelophthalmus (Eurypterus) granosus. (Palæoblattariæ.)

Locality : Jägersfreude bei Saarbrïck, Germany. Horizon : Carboniferous. 1854. Jordan. Jordan-Meyer, Palaeontogr., iv: 8-12. Pl. 2, figs. 1-2.

See also Polyzosterites granosus.

143. Fdœophasma anglica. (Protophasmida.)

Locality: Near Liverpool, England. Horizon: Carboniferous.

1885. Scudder. Geol. mag., (3), ii : 265-266.

1885. " Zittel, Handb. palaeont., i, ii : 758. Fig. 941.

1886. " Zittel-Barrois, Traité de paléont., ii: 757. Fig.957.

1886. " " Bull. U.S. geol. surv., No.31: 41.

Anthracoblattina abnormis. See Blattina (Anthracoblattina) abnormis.

144. Anthracoblattina camerata. (Palæoblattariæ.)

Locality: Dudweiler, Germany. Horizon: Carboniferous.

1883. Kliver. Nene blatt. Saarbrück., 3-4. Pl. 1, figs. 1, $1 a$.

1883. “ Palaeontogr., xxix: 251-252. Pl. 34, figs. 1, 1a.

145. Anthracoblattina dresdensis. (Palæoblattariæ.)

Locality : Klein Opitz, Saxony. Horizon: Upper Carboniferous.

1879. Scudder. Mem. Bost. soc. nat. hist., iii : 92-93. Fig.

1890. " -Foss. ins. N. A., i : 112-113. Fig.

See also Blattina dresdensis.

146. Anthracoblattina incerta. (Palæoblattariæ.)

Locality : Saarbrück, Germany. Horizon: Carboniferous.

1883. Kliver. Neue blatt. Saarbrïck., 5-6. Pl. 1, figs. 2, 2a.

1883. " Palaeontogr., xxix : 253-254. Pl. 34, figs. 2, $2 a$.

Anthracoblattina lubnensis. See Blattina (Anthracoblattina) lubnensis.

147. Anthracoblattina porrecta. (Palæoblattariæ.)

Locality: Weissig, Germany. Horizon: Lower Dyas.

1879. Scudder. Mem. Bost. soc. nat. hist., iii : 93-94. Pl. 4, fig. 5.

1890. “ Foss.ins. N. A., i : 11:-114. Pl. 4, fig. 5.

See also Blattina porrecta, B. (Anthracoblattina) porrecta. 
148. Anthracoblattina remigii. (Palæoblattariæ.)

Locality : Cusel, Rheinpfalz. Horizon : Upper Carboniferous.

1879. Scudder. Mem. Bost. soc. nat. hist., iii : 95-96. Pl. 4, fig. 2. 1890. " $\quad$ Foss. ins. N. A., i : 115-116. Pl. 4, fig. 2.

See also Blattina remigii.

149. Anthracoblattina rückerti. (Palæoblattariæ.)

Locality: Stockheim, Oberfranken. Horizon: Dyas.

1879. Scudder. Mem. Bost. soc.nat. hist., iii : 96-97. Pl. 4, fig. 1.

1890. " Foss.ins. N. A., i: 116-117. Pl. 4, fig. 1.

See also Blattina rückerti.

150. Anthracoblattina scudderi. (Palæoblattariæ.)

Locality: Wemmetsweiler, Germany. Horizon: Carboniferous.

1881. Goldenberg. Zeitschr. ges. naturwiss., (3), vi : 186-187. Figs. 1-2, on p. 185.

1883. Kliver.

Nene blatt, Saarbrück., 10. $\quad P l .2, f i g .7$.

1883. " Palaeontogr., xxix: 258. Pl. 35, fig. 7.

1886. " Palaeontogr., xxxii: 104. Pl. 14, fig. 4.

151. Anthracoblattina sopita. (Palæoblattariæ.)

Locality : Weissig, Germany. Horizon : Lower Dyas.

1879. Scudder. Mem. Bost. soc. nat. hist., iii : 89-91. Pl. 4, fig. 8. 1890. " Foss. ins. N. A., i: 109-111. Pl. 4, fig. 8.

See also Blattina didyma, ete.

152. Anthracoblattina spectabilis. (Palæoblattariæ.)

Localities: Löbejün, Weissig?, Germany. Horizon: Upper Carboǹiferous, Lower Dyas?

1879. Scudder. Mem. Bost. soc. nat. hist., iii : 88-89. Pl. 2, fig. 8.

1890. " Foss. ins. N. A., i: 108-109. Pl.2, fig.8.

See also Blattina spectabilis, Blattina (Anthracoblattina) ef. spectabilis.

153. Anthracoblattina wagneri. (Palæoblattariæ.)

Loeality : Löbejün, Saxony. Horizon: Carboniferous.

1886. Kliver. Palaeontogr., xxxii: 114. Pl. 14, figs. 12, $12 a$.

154. Anthracoblattina winteriana. (Palæoblattariæ.)

Locality : Dudweiler, Germany. Horizon: Middle Carboniferous.

1879. Scudder. Mem. Bost. soc. nat. hist., iii : 94-95. Pl. 4, fig. 12.

1890. “ Foss. ins. N. A., i: 114-115. Pl. 4, fig. 12.

See also Blattina winteriana.

155. Archæoptilus ingens. (Protophasmida.)

Loeality: Chesterfield, Derbyshire, England. Horizon: Carboniferous.

1881. Scudder. Geol. mag., (2), viii : 295, 300.

1883. " Mem. Bost. soc. nat. hist., iii: 217-219, 223. Pl. 17, figs. 10-12.

1885. Brongniart. Bull. soc. amis sc. Rouen, (3), xxi: 60.

1885. " " Rev. scient., (3), xxxvi: 277 .

1885. " " Geol. mag., (3), ii: 487.

1885. " " Trans. geol. soc. Manch., xviii : 280 .

1885. " " Foss. ins. prim. rocks, 11.

1885. “ Jahrb. geol. reichsanst., $\mathrm{xxxv}: 656$.

1885. Scudder. Zittel, Handb. palaeont., i, th. ii: 757. 
1886. Scudder. Zittel-Barrois, Traité de paléont., ii : 756.

1886. " "Bull. U. S. geol. surv., No. 31: 40.

1890. " Foss. ins. N. A., i: 239-241, 245. Pl. 11, figs. 10-12.

See also Dictyoneura ingens.

156. Archæoptilus lucasi. (Protophasmida.)

Locality: Commentry, France. Horizon: Carboniforous.

1885. Brongniart. Bull. soc. amis sc. Rouen, (3), xxi : 60.

1885. " $\quad$ Rev. scient., (3), xxxvi: 277.

1885. “ " Geol. mag., (3), ii : 487.

1885. " " Trans. geol. soc. Manch., xviii : 280.

1885. " " Foss, ins, prim, rocks, 11.

1885. " J Jahrb. geol. reichsanst., $\mathrm{xxxv}: 656$.

157. Archegogryllus priscus. (Protophasmida?)

Locality : Tallmadge, Ohio. Horizon: Carboniferous.

1868. Scudder. Proc. Bost. soc. nat. hist., xi : 402-403.

1868. " Entom, notes; i: 8-9.

1883. Lacoe. List pal. foss. ins., 5 .

1885. Scudder. Mem. Bost. soc. nat. hist., iii: 323. Pl. 29, figs, 2, 3.

1885. Brongniart. Bull. soc, amis sc. Rouen, (3), xxi: 60 .

1885. " " Rev. scient., (3), xxxvi: 277.

1885. " " Geol. mag., (3), ii: 487.

1885. " " Trans. geol. soc. Manch., xviii : 279.

1885. " " Foss. ins. prim. rocks, 11.

$1885 . \quad$ J Jahrb. geol. reichsanst., $\mathrm{xx \times v}: 656$.

1885. Scudder. Zittel, Handb. palaeont., i, ii: 758.

1886. " " Zittel-Barrois, Traité de pal6́nt., ii: 758.

1886. “ Bull. U. S. geol. surv., No. 31: 41 .

1886. Brauer. Ann. naturhist. hofmus., i: 113.

1889. Lesley. Dict. foss. Penns., i: p. vi.

1890. Miller. N. A. geol. and pal., 575.

1890. Scudder. Foss. ins. N. A., i : 287. Pl. 15, figs. 2, 3.

Archimulacris acadicus. See Archimylacris acadica.

158. Archimylacris acadica. (Palæoblattariæ.)

Locality: Pictou, N. S. Horizon: Middle Carboniferous.

1868. Scudder.

Dawson, Acad. geol., 2 d ed., 388 (Archimulacris acadicus). Fig. 153.

1868. Scudder.

1868.

Amer. nat., i : $630 . \quad P l .16$, fig. 2 .

1872. Packard.

Geol. mag., v: 177.

1879. Scudder.

Guide ins., 3d ed., 78. Pl. 1, fig. 2.

1879. Nicholson.

1880. Dawson.

1883. Lacoe.

1889. Lesley.

1889. Miller.

1890. Sendder.

Mem. Bost. soc. nat. hist., iii : 84-85. Pl. 6, figs. 8, 14.

Manual palæont., 2d ed., i : 407. Fig. 258.

Dawn of life, 146. Fig. $127 a$.

List pal. foss. ins., 5 .

Dict. foss. Penns, i : p. vi.

N. A. geol. and pal., 575. Fiq. 1075 (not 1074).

Foss. ins. N. A., i: 104-105. Pl. 6, figs. 8, 14.

159. Archimylacris parallela. (Palæoblattariæ.)

Locality : Cannelton, Pa. Horizon: Lower Carboniforous.

1879. Scudder. Mem. Bost. soc. nat. hist., iii : 85-87 (parallelum). Pl. 6, fig. 6 .

1883. Lacoe. List pal. foss. ins, 5.

Bull. $71--3$ 
1889. Lesley. 1889. Miller. 1890. Scudder.
Dict. foss. Penns., i: 37. Fig.

N. A. geol. and pal., 575 .

Foss. ins. N. A., i: 105-107. Pl, 6, fig. 6.

160. Archimylacris paucinervis. (Palrooblattariæ.)

Locality: Mazon Creek, Ill. Horizon: Carboniferous.

1883. Scudder.

18×3. Lacoe.

1889. Lesley. 1890. Scudder. 1890.
Mss. (paucinerve).

List pal. foss. ins., 5.

Dict. foss. Penns., i: p. vi.

Mem. Bost. soc. nat. hist., iv : 411-414. Pl. 31, fig. 5.

Foss. ins. N. A., i : 387-390. Pl. 23, fig. 5.

\section{Blatta - (Palæoblattariæ.)}

Locality: Claxheugh, Durham. Horizon: Carboniferous.

1867. Kirkby.

Geol, mag., iv: 389. Pl. 17, figs, 6-7.

See also Blattidium mantidioides, etc.

162. Blatta americana. (Palæoblattariæ.)

Locality: Bristol, R. I. Horizon: Carboniferous.

1884. Clark. Proc. Newp. nat. hist. soc., ii : 12 (undescr.).

See also Mylacris packardii.

163. Blatta germari. (Palæoblattariæ.)

Locality : Wettin, Saxony. Horizon: Carboniferous.

1856. Giebel. Insect. d. vorwelt, 321.

See also Blattina — Germ., Blattina germari, Gerablattina germari.

164. Blatta gracilis. (Palæoblattariæ.)

Locality: Lebach, Germany. Horizon; Lower Dyas.

1856. Giebel. Insect. d. vorwelt, 321.

See also Blattina gracilis, Petrablattiaa gracilis.

165. Blatta helvetica. (Palæoblattariæ.)

Locality : Erbignon, Switzerland. Horizon : Carboniferous,

1876. Heer. Prim. world Switz., i: 20. Pl. 16C, Figs. a, b. 1877. Woodward. Geol. mag., (2), iv: 79. Figs. $1 a, b$.

See also Blattina helvetica, Progonoblattina helvetica.

166. Blattidium mantidioides. (Palæoblattariæ.)

Locality : England. Horizon: Upper Carboniferous.

1877. Goldenberg. Fauna saræp. foss., ii : 20.

See also Blatta — Kirkby, Etoblattina mantidioides.

167. Blattina - (Palæoblattariæ.)

Locality : Belgium. Horizon: Carboniferous,

1877. Andrä. Sitzungsb, niederrhein, gesellsch., 1876: 28.

168. Blattina - (Palæoblattariæ.)

Locality: Wettin, Saxony. Horizon: Carboniferous.

1851. Germar. Verstein. steink. Wettin, 87. Pl, 31, fig. 9.

Sée also Blatta germari, etc. 
169. Blattina - (Palæoblattariæ.)

Locality: Lubna, Bohemia. Horizon: Carboniferous.

1883. Kušta. Sitzungsb. k. böhm. gesellsch. wiss., 1883: 214. Fig. 3.

1884. " Vesmír, xiii: 97-98. Fig. 5.

Blattina —. See Gerablattina balteata.

170. Blattina (Anthracoblattina) abnormis. (Palæoblattariæ.)

Locality: Weissig, Germany. Horizon: Lower Dyas.

1880. Geinitz. Blatt. unt. dyas Weissig, 3-16. Pl. 39. figs. 1-3.

1880. "6 Verhandl. leop-carol. akad. naturf, xli: 423-436. Pl. 39, figs.1-3.

See also Anthracoblattina sopita, Blattina didyma, etc.

171. Blattina affinis. (Palæoblattariæ.)

Locality : Löbejïn, Saxony. Horizon: Carboniferous.

1869. Goldenberg. Neues jahrb. f. mineral., 1869: 159. Pl. 3, fig. 3.

187\%. " Fauna saræp. foss., ii : 19.

See also Etoblattina affinis.

172. Blattina anaglyptica. (Palæoblattariæ.)

Locality: Germany. Horizon: Carboniferous.

1842. Germar. Münst., Beitr. petref, v: 92. Pl. 13, fig. 2.

1851. " Verstein. steink. Wettin, 84. Pl. 31, fig. 4.

1852. Giebel. Deutschl. petref., 637.

1856. " Insect. d. vorwelt, 314-315.

1864. Heer. Viert. naturf. gesellsch. Zürich, ix : 287.

1877. Goldenberg. Fauna saræp. foss., ii : 19.

See also B. anthracophila, B. anaglyptica var. labachensis, B. labachensis, Etoblattina anaglyptica, Etoblattina anthracophila.

173. Blattina anaglyptica var. labachensis. (Palæoblattariæ.)

Locality: Labach, Germany. Horizon: Carboniferous.

1867. Goldenberg. Vorweltl. fanna steink. Saarbr., 16. Pl. 2, fig. 15 (ined.)

1873. " " Fauna saræp. foss., i: 16. Pl. 2, fig. 15.

1877. " Fauna saræp. foss., ii: 19.

See also B. anaglyptica, etc.

174. Blattina anthracophila. (Palæoblattariæ.)

Locality : Weissig, Germany. Horizon: Lower Dyas.

1842. Germar. Münster, Beitr. z. petref., v : 92-93. $\quad P l .13$, fig. 3.

1852. Giebel. ' Doutschl. petref., 637.

1864. Heer. Viert. naturf: gesellsch. Zürich, ix : 287 (=B. anaglyptica?)

1873. E. Geinitz. Neues jahrb. f. mineral., 1873 : 694 . Pl. 3, fig. 2.

1873. " " Verstein. unter. dyas Weissig, 4. Pl. 3, fig. 2.

1876. Roemer. Lethæa geogn. Pl. 56, fig. $2 a$.

See also B. anaglyptica, etc., Etoblattina anthracophila.

175. Blattina (Etoblattina) bituminosa. (Palæoblattariæ.)

Locality: Lubná, Bohemia. Horizon: Carboniferous.

1883. Kušta. Sitzungsb. k. böhm. gesellsch. wiss., 1883: 213-214. Fig. 1.

1884. " Vesmír, xiii : 97-98. Fig. 3 . 


\section{Blattina bretonensis. (Palæoblattariæ.)}

Locality : Sydney, Cape Breton. Horizon: Carboniferous.

\section{Scudder.}

Can. nat., vii : 271-272. Fig. 1.

1878. Dawson. Acad. geol, suppl. 2d. ed., 55. Fig. 5.

1880. " Dawn of life, 146. Fig. 127 .

See also Mylacris bretonensis.

\section{Blattina carbonaria. (Palæoblattariæ.)}

Compared with Hormetica tuberculata. S. Amer.

Localities: Weissig, Wettin, Germany. Horizon: Carboniferous.

1851. Germar.

1856. Giebel.

Verstein. steink. Wettin, 85-86. Pl. 3i, fig. 6a-b.

1864. Heer.

Insect. d. vorwelt, 315.

1875. E. Geinitz.

1875. " Neue aufschl, dyas Weissig, 5.

1877. Goldenberg. Fauna saræp. foss., ii : 19.

See also Etoblattina carbonaria, etc.; also I, NEUROPT., Blattina carbonaria.

178. Blattina (Etoblattina) carbonaria var. deichmülleri. (Palæoblattariæ.)

Locality: Weissig, Germany. Horizon: Lower Dyas.

1880. Geinitz. Blatt. unter. dyas Weissig, 19-20. Pl. 39, fig.9.

1880. " " Verhandl. leop.-carol. akad. naturf, xli: 439-440. Pl.39. fig.9.

See also Etoblattina carbonaria, etc.

179. Blattina clathrata. (Palæoblattariæ.)

Locality : Manebach, Thüringen. Horizon: Carboniferous.

1864. Heer. Viert. naturf. gesellsch. Zürich, ix : 288, 294-296. Pl., fig. 3. 1877. Goldenberg. Fauna saræp. foss., ii : 19.

See also B. latinervis, Gerablattina clathrata.

Blattina (Etoblattina) deichmülleri. See Blattina (Etoblattina) carbonaria var. deichmülleri.

180. Blattina didyma. (Palæoblattariæ.)

Localities: Weissig, Wettin, Germany. Horizon : Carboniferous.

1842. Germar.

1848. Giebel.

1851. Germar.

1852. Giebel.

1852. Quenstedt.

1854. Pictet.

1856. Giebel.

1864. Heer.

1875. E. Geinitz.

1875. " "

1877. Goldenberg.

1880. E. Geinitz.

1880.
Münster, Beitr. z. petref., v: 92. Pl. 13, fig. 1a-b.

Gæa excurs. germ. $P l .5$, fig. 26.

Verstein. steink. Wettin, 83. (See also 87 and pl. xxxi : 10.) Pl. 31, figs. 2-3.

Deutschl. petref., 637.

Handb. petref. $P l .24$, fig. 16.

Traité de paléont., $2^{e}$ éd., ii : $362 . \quad P l .40$, fig. 2.

Insect. d. vorwelt, 314 .

Viert. naturf. gesellsch. Zürich, ix : 287.

Neues jahrb. f. mineral., $1875: 4-5$. $P l .1$, fig. 1.

Neue aufschl. dyas Weissig, 4-5. Pl. 1, fig. 1.

Fauna saræp. foss., ii : 19.

Blatt. unter. dyas Weissig. $P l .39$, figs. 4-5.

Verhandl. leop.-c arol. akad. naturf, xli. $\quad P l .39$, figs. 4-5.

See also Etoblattina didyma, Anthracoblattina sopita, Dictyopteris didyma, Blattina (Anthracoblattina) abnormis; also I, NkURoPT., Blattina carbonaria, and III, ORThopt., Blatta didyma. 
181. Blattina dresđensis. (Palæoblattariæ.)

Locality: Klein-Opitz, Saxony. Horizon: Upper Carboniferous.

1879. Gein.-Deichm. Sitzungsb. naturw, gesellsch. Isis, 1879: 12-13. 2 figs.

See also Anthracoblattina dresdensis.

182. Blattina (Etoblattina) elongata. (Palæoblattariæ.)

Locality: We1ssig, Germany. Horizon: Lower Dyas.

1880. Geinitz. Blatt. unter. dyas Weissig, 20. Pl. 39, fig. 10.

1880. " Verhandl. leop.-carol. akad. naturf., xli: 440. Pl. 39, fig. 10.

See also Etoblattina elongata, etc.

183. Blattina euglyptica. (Palæoblattariæ.)

Locality: Wettin, Saxony. Horizon: Carboniferous.

1851. Germar. Verstein. steink. Wettin, 86-87. Pl. 31, figs. 7a-b, 8 .

1856. Giebel. Insect. d. vorwelt, 315 .

1864. Heer. Viert. naturf. gesellsch. Zürich, ix : 287.

1869. Goldenberg. Neues jahrb. f. mineral., 1869: 162-163. Pl. 3, figs. 8-9.

1877. " " Fauna saræp, foss., ii: 19.

See also B. euglyptica var. weissiana, Gerablattina producta, Etoblattina englyptica, E. dohrnii.

184. Blattina euglyptica var. weissiana. (Palæoblattariæ.)

Locality: Brïcken, Canton Waldmohr (Rheinpfalz). Horizon: Carboniferous. 1869. Goldenberg. Neue jahrb. f. mineral., 1869 : 163. Pl. 3, fig. 10.

1877. " Fauna saræp. foss., ii : 19. Pl. 1, fig. 12.

See also Blattina euglyptica, etc., B. weissiana, Gerablattina weissiana.

185. Blattina fascigera. (Palæoblattariæ.)

Locality : Pittston, Pa. Horizon: Carboniferous.

1878. Scudder. Proc. Bost. soc. nat. hist., xix: 238-239.

1878. “ Entom. notes, vi : 35-36.

See also Gerablattina fascigera.

186. Blattina flabellata. (Palæoblattariæ.)

Locality: Wettin, Saxony. Horizon : Carboniferous.

1842. Germar. Münster, Beitr. z. petref., v: 92. Pl. 13, fig. $4 a b$.

1851. " Verstein. steink. Wettin, 84-85. Pl. 31, fig. 5.

1852. Giebel. Deutschl. petref., 637.

1856. “ Insect. d. vorwelt, 315.

1864. Heer. Viert. naturf.gesellsch. Zürich, ix: 287.

1877. Goldenberg. Fauna saræp. foss., ii : 19.

1880. E. Geinitz. Blatt. unter, dyas Weissig. Pl. 39, fig. 8.

1880. " Verhandl. leop.-carol. akad. naturf., xli : $P l .39$, fig. 8.

See also Gerablattina münsteri, Etoblattina flabellata, Blattina (Etoblattina)

flabellata var. dyadica, Etoblattina flabellata var. stelzneri.

187. Blattina (Etoblattina) flabellata var. dyadica. (Palæoblattariæ.)

Locality: Weissig, Germany. Horizon: Lower Dyas.

1880. E. Geinitz. Blatt. unter. dyas Weissig, 17-18. Pl. 39, fig. 7.

1880. " Verbandl. leop.-carol. akad, naturf., xli: 437-438. Pl.39, fig. 7.

See also Blattina flabellatr, etc. 


\section{Blattina fritschii. (Palæoblattariæ.)}

Locality: Manebach, Thüringen. Horizon: Carboniferous.

1864. Heer, Viert. naturf. gesellsch. Zürich, ix : 287, 293-294. Pl., fig. 2. 1877. Goldenberg. Fauna saræp. foss., ii : 19.

See also Progonoblattina fritschii.

189. Blattina geinitzi. (Palæoblattariæ.)

Locality: Löbejün, Saxony. Horizon: Carboniferons.

1869. Goldenberg. Neues jahrb. f. mineral., 1869: 160-161. Pl. 3, fig. 5. 1877. " Fauna saræp. foss., ii : 19.

See also Gerablattina geinitzi.

190. Blattina germari. (Palæoblattariæ.)

Locality: Wettin, Saxony. Horizon: Carboniferous.

1864. Heer. Viert. naturf. gesellseh. Zürich, ix : 288.

1877. Goldenberg. Fauna saræp. foss., ii : 19.

See also Blatta germari, etc.

191. Blattina goldenbergi. (Palæoblattariæ.)

Localities: Ilmenau, Manebach, Germany. Horizon : Carboniferous.

1870. Mahr. Neues jahrb. f. mineral., 1870: 282-284. Fig. 1.

1877. Goldenberg. Fauna saræp. foss., ii : 19.

See also Gerablattina goldenbergi.

192. Blattina gracilis. (Palæoblattariæ.)

Locality : Lebach bei Saarbrück, Germany. Horizon : Lower Dyas.

1854. Goldenberg. Palaeontogr., iv: 23. Pl. 3, figs. 3, 3a.

1854. " Foss. insect. Saarbrücken, 7. Pl. 1, figs. 3, 3a.

1864. Heer. Viert. naturf. gesellsch. Zǘrich, ix : 288.

1877. Goldenberg. Fauna saræp. foss., ii: 20, 27-28, 51. Pl. 2, fig. 1 a.

See also Blatta gracilis, Petrablattina gracilis.

193. Blattina heeri. (Palæoblattariæ.)

Locality : Sydney, Cape Breton. Horizon : Carboniferous.

1874. Scudder. Can. nat., vii : 272. Fig, 2, p. 271.

1878. Dawson. Acad. geol., suppl.2d ed., 55. Fig. 6.

1880. " Dawn of life, 146 (hesri). Fig. 127 c.

See also Mylacris heeri.

194. Blattina helvetica. (Palæoblattariæ.)

Locality : Erbignon, Switzerland. Horizon: Carboniferous.

1864. Heer. Viert. naturf. gesellsch. Zürich, ix: 287, 291-293, Pl., fig. 1.

1865. " Urwelt der Schweiz, 592 note.

1872. " Monde prim: Suisse,22. Fig. $16 \mathrm{Cab}$.

1877. Goldenberg. Fauna saræp. foss., ii: 19.

1879. Heer. Urwelt der Sehweiz, $2^{e}$ aufl, 24. Fig. 34ab.

See also Blatta helvetica, Progonoblattina helvetica. 
Blattina hesri. See Blattina heeri.

195. Blattina insignis. (Palæoblattariæ.)

Locality : Skalley-Schacht, Hirschbach, Germany. Horizon : Carboniferous. 1867. Goldenberg. Vorweltl. fauna steink. Saarbr., 17. Pl. 2, fig. 14 (ined.).

1873. " " Fauna saræp. foss., i: 17. Pl. 2, fig. 14.

1877. " Fauna saræp. foss., ii : $20,51$.

1879. Seudder. Mem. Bost. soc. nat. hist., iii : 19 note 4.

1880. " " Areh. sc. phys. nat., (3), iii : 367 note 2.

1887. Woodward. Geol. mag., (3), iv. Pl. 12, fig. 3.

1890. Scudder. Foss.ins. N. A., i : 39 note 4.

See also Etoblattina insignis, etc.

196. Blattina intermedia. (Palæoblattariæ.)

Locality: Wemmetsweiler, Germany. Horizon : Carboniferous.

1877. Goldenberg. Fauna saræp. foss., ii : 19, 24-25, 51. Pl. 1, figs. 10, $10 a$.

See also Gerablattina intermedia, etc.

197. Blattina (Gerablattina) intermedia. (Palæoblattariæ.)

Locality: Wemmetsweiler, Germany. Horizon: Upper Carboniferous.

1883. Kliver. Neue blatt. Saarbr., 9-10. Pl. 2, figs. 2, $2 a$.

1883. " Palaeontogr., xxix: 257-258. Pl. 35, figs. 2, 2a.

See also Gerablattina intermedia, etc.

198. Blattina labachensis. (Palæoblattariæ.)

Locality : Labach, Germany. Horizon : Upper Carboniferous.

1877. Goldenberg. Fa una saræp. foss., ii : 51.

See also Etoblattina labachensis, B. anaglyptica var. labachensis, Blattina anaglyptica, ete.

199. Blattina (Etoblattina) lanceolata. (Palæoblattariæ.)

Locality : Lngau, Saxony. Horizon: Carboniferous.

1881. Sterzel. Ber. naturw. gesellsch. Chemn., vii : 271-273. Pl., figs. 1, 2. See also Etoblattina lanceolata.

200. Blattina latinervis. (Palæoblattariæ.)

Locality : Manebach, Thüringen. Horizon: Upper Carboniferous.

1864. Heer. Viert. naturf. gesellsch. Zürich, ix: 288, 296-297 (=B. clathrata ?). Pl., fig. 4.

1877. Goldenberg. Fanna saræp. foss., ii : 20.

1879. Scudder. Mem. Bost. soc. nat. hist., iii: 127-128. Pl. 4, fig. 3.

1890. "6 Foss. ins. N. A., i: 147-148. Pl. 4, fig. 3.

See also Blattina elathrata, Gerablattina clathrata.

201. Blattina lebachensis. (Palæoblattariæ.)

Locality : Lehbach bei Saarbrück, Germany. Horizon: Lower Dyas.

1852. Goldenberg. Sitzungsb. math.-nat. cl. k. akad. wiss. Wien, ix : 38 (lehbachensis) (undescr.).

1854. " $\quad$ Palaeontogr., iv : 22-23. Pl. 6, fig. 7.

1854. “ Foss. insect. Saarbr., 6-7. P!. 4, fig. 7.

1856. Giebel. Insect. d. vorwelt, 316 .

1877. Goldenberg. Fauna saræp. foss, ii : 20, 27, 51. Pl. 1, fig. 20.

See also Hermatoblattina lebachensis. 
Blattina lehbachensis. See Blattina lebachensis.

202. Blattina leptophlebica. (Palæoblattariæ.)

Locality: Löbejün, Saxony. Horizon: Carboniferous.

1869. Goldenberg. Nenes jahrb. f. mineral., 1869: 158-159. Pl. 3, figs. 1a, 1 A. 1877. "

See also Etoblattina leptophlebica.

203. Blattina ligniperda. (Palæoblattariæ.)

Locality: Lubná, Bohemia. Horizon: Carboniferous.

1883. Kušta. Sitzungsb. k. böhm. gesellsch. wiss., 1883: 214. Fïg. 2.

1884. " Vesmír, xiii : 97-98. Fig. 4.

204. Blattina (Anthracoblattina) lubnensis. (Palæoblattariæ.)

Locality: Lubná, Bohemia. Horizon : Carboniferons.

1883. Kušta. Blatt. lubn. gask., 1-8. Pl., fig. 1.

1883. " " Sitzungsb. k. böhm. gesellsch. wiss., 1882: 430-437. Pl., fig. 1.

1884. " $\quad$ Vesmír, xiii: $97-98$. Fig. 2.

205. Blattina mahri. (Palæoblattariæ.)

Locality : Ilmenau, Mauebach, Thüringen. Horizon : Carboniferous. 1870. Goldenberg. Mahr, Neues jahrb. f. mineral., 1870: 284-285. Fig. 2ab. 1877. " Fauna saræp, foss., ii : 19.

See also Gerablattina mahri.

206. Blattina cf. mahri. (Palæoblattariæ.)

Locality : Weissig, Germany. Horizon: Lower Dyas.

1875. E. Geinitz. Neues jahrb. f. mineral., 1875: 5. Pl. 1, fig. 2.

1875. " Nene anfschl. dyas Weissig, 5. Pl. 1, fig. 2.

See also Etoblattina elongata, etc.

207. Blattina manebachensis. (Palæoblattariæ.)

Locality : Manebach, Thüringen. Horizon: Carboniferous.

1869. Goldenberg. Neues jahrb. f. mineral., 1869: 160. Pl. 3, fig. 4.

1877. " $\quad$ Fauna saræp. foss., ii : 19.

See also Etoblattina manebachensis, Blattina (Etoblattina) manebachensis.

208. Blattina (Etoblattina) manebachensis. (Palæoblattariæ.)

Locality: Manebach, Thüringen. Horizon: Carboniferous.

1883. Kušta. $\quad$ Blatt. lubn. gask., 8. Pl., fig. 2.

1883. " Sitzungsb. k. böhm. gesellsch. wiss., 1882: 43\%. Pl., fig. 2.

See also B. manebachensis, Etoblattina manebachensis.

209. Blattina neuropteroides. (Palæoblattariæ.)

Locality : Horizon : Permian.

1864-'65. Göppert. Foss. flora perm. form., 289. Pl. 28, figs. 17-18.

1864-65. " " Palaeontogr., xii: 289. Pl. 28, figs. 17-18.

210. Blattina parvula. (Palæoblattariæ.)

Locality : Löbejün, Saxony. Horizon: Carboniferous.

1869. Goldenberg. Nenes jahrb. f. mineral., 1869: 161. Pl. 3, fig. 6.

1877. "

See also Etoblattina parvula. 
211. Blattina porrecta. (Palæoblattariæ.)

Locality: Weissig, Germany. Horizon: Lower Dyas.

1875. E. Geinitz. Neues jahrb. f. mineral., 1875: 6. Pl. 1, fig. 4. 1875. " " Neue aufschl. dyas Weissig, 6. Pl. 1, fig. 4.

1877. Goldenberg. Fauna saræp. foss., ii : 20.

See also Anthracoblattina porrecta, Blattina (Anthracoblattina) porrecta.

212. Blattina (Anthracoblattina) porrecta. (Palæoblattariæ.)

Locality: Weissig, Germany. Horizon : Lower Dyas.

1880. Geinitz. Blatt. unter. dyas Weissig, 21. Pl. 39, fig. 12.

1880. " Verhandl. leop.-carol. akad. naturf., xli: 441. Pl. 39, fig. 12.

See also Anthracoblattina porrecta, Blattina porrecta.

213. Blattina primæva. (Palæoblattariæ.)

Localities: Auerswald bei Saarbrück, Gersweiler, Germany. Horizon : Carboniferous.

1852. Goldenberg. Sitzungsb. math.-nat, cl. k. akad. wiss. Wien, ix : 38 (undescr.).

1854. " " Palaeontogr., iv: 22. Pl. 3, fig. 4.

1854. " Foss. insect. Saarbrïcken, 6. Pl. 1, fig. 4.

1854-'56. Bronn. Lethæa geogn., $3^{\mathrm{e}}$ aufl, i, ii: 683. Pl. $9^{3}$, fig. $15 a$.

1856. Giebel. Insect. d. vorwelt, 316.

1864. Heer. Viert. naturf. gesellsch. Zïrich, ix : 288.

1867. Goldenberg. Vorweltl. fauna steink. Saarbr., 16. Pl. 2, fig. 13 (ined.).

1873. " Fauna saræ̉p. foss., i: 16. Pl. 2, fig. 13.

18\%6. Roemer. Lethæa geogn. Pl. 47, fig. 18.

1877. Goldenberg. Fauna saræp. foss., ii : 19,51.

See also Etoblattina primæva.

214. Blattina propria. (Palæoblattariæ.)

Locality : Saarbrück, Germany. Horizon: Carboniferous.

1883. Kliver. Neue blatt. Saarbr. Pl. 2, fig. 3.

1883. " Palaeontogr, xxix. Pl. 35, fig. 3.

See also Etoblattina propria.

215. Blattina rarinervis (piece of wing). (Palæoblattariæ.)

Locality: Horizon: Permian.

1864-65. Göppert. Foss. flora perm. form., 289. Pl. 28, figs. 15-16.

1864-'65. " $\quad$ Palaeontogr., xii : 289. Pl. 28, figs. 15-16.

216. Blattina remigii. (Palæoblattariæ.)

Locality : Cusel, Rheinpfalz. Horizon : Carboniferous.

1867. Dohrn. Palaeontogr., xvi: 133-134. Pl. 8, fig. 3.

1877. Goldenberg. Fauna saræp. foss., ii : 20, 26-27, 51. Pl.1, fig. 13.

See also Anthracoblattina remigii.

217. Blattina reticulata. (Palæoblattariæ.)

Locality: Wettin, Saxony. Horizon: Carboniferous.

1851-53. Germar. Verstein. steink. Wettin, heft $7: 87-88 . \quad P l, 39, f i g, 15 a b$ in heft 8. 1852. Giebel. Deutschl. petref., 637.

1856. " Insect. d. vorwelt, 316.

1877. Goldenberg. Fauna saræp. foss., ii : 19.

See also Oryctoblattina reticulata. 
218. Blattina rückerti. (Palæollatțariæ.)

Locality : Stockheim, Oberfranken. Horizon: Rothliegende.

1869. Goldenberg. Neues jahrb. f. mineral, 1869: 163-164. Pl. 3, fig. 11.

See also Anthracoblattina rïckerti.

219. Blattina russoma. (Palæoblattariæ.)

Locality: Löbejiin, Saxony. Horizon : Carboniferous.

1869. Goldenberg. Neues jahrb. f. mineral., 1869: 159. Pl. 3, figs. 2a, 2A-2B.

1877. " Fauna saræp. foss., ii : 20.

See also Etoblattina russoma.

220. Blattina scaberata. (Palæoblattariæ.)

Locality : Altenwald, Germany. Horizon: Carboniferous.

1877. Goldenberg. Fauna saræp. foss., ii : 19,25,51. Pl. 1, fig. 8.

See also Gerablattina scaberata.

221. Blattina sepulta. (Palæoblattariæ.)

Locality: Cape Breton, Nova Scotia. Horizon: Carboniferous.

1876. Scudder. Proc. Amer. assoc. adv. sc., xxiv B: 111. Fig. 2 on $p .110$.

1876. " Can. nat., n. s., viii : 89-90. Fig. 2 [1].

1876. " Ins, carb. Cape Breton, [2]. Fig. 2 [1].

1878. Dawson. Acad. geol., suppl. $2 d$ ed., 55. Fig. 7.

See also Petrablattina sepulta.

222. Blattina spectabilis. (Palæoblattariæ.)

Localities: Weissig, Löbejün, Germany. Horizon : Carboniferous.

1869. Goldenberg. Nenes jahrb. f. mineral., 1869: 161-162. Pl. 3, figs. 7, 7ab.

1875. E. Geinitz. Neues jahrb. f. mineral., 1875: 6.

1875. " Neue anfschl. dyas Weissig, 6.

1877. Goldenberg. Fauna saræp. foss., ii : 19.

See also Anthracoblattina spectabilis, etc.

223. Blattina (Anthracoblattina) cf. spectabilis. (Palæoblattariæ.)

Locality: Weissig, Germany. Horizon: Lower Dyas.

1880. Geinitz. Blatt. unter. dyas Weissig, 17. Pl. 39, fig. 6.

1880. " Verhandl. leop.-carol. akad. naturf., xli: 437. Pl. 39, fig.6.

See also Anthracoblattina spectabilis, etc.

224. Blattina splendens (abdomen). (Palæoblattariæ.)

Locality :? Horizon: Permian.

1864-65. Göppert. Foss. flora perm. form., 289. Pl. 64, fig. 11.

1864-65. " Palaeontogr., xii : 259. Pl. 64, fig. 11.

225. Blattina tischbeini. (Palæoblattariæ.)

Locality : Hirschbach, Germany. Horizon : Middle Carboniferons.

1867. Goldenberg. Vorweltl. fauna steink. Saarbr., 16-17. Pl. 2, fig. 16 (ined.).

1873. " " Fauna saræp. foss., i: 16-17. Pl. 2, fig. 16.

1877. " Fauna saræp. foss., ii : 19, 51.

1879. Scudder. Mem. Bost. soc. nat. hist., iii: 127. Pl. 4, fig. 10.

1890. " Foss, ins. N. A., i : 147. Pl. 4, fig. 10. 
226. Blattina venosa. (Palæoblattariæ.)

Locality: Wemmetsweiler, Germany. Horizon: Upper Carboniferous.

1877. Goldenberg. Fauna saræp. foss., ii : 19, 25,51. Pl. 1, fig. 7.

1879. Scudder. Mem. Bost. soc. nat. hist., iii : 128. Pl. 4, fig. 6.

1890. " Foss. ins. N. A., i : 148. Pl. 4, fig. 6.

227. Blattina venusta. (Palæoblattariæ.)

Locality: Frog Bayou, Ark. Horizon: Carboniferous.

1860. Lesquereux. Owen, 2d rep. geol. Ark., 314. Pl. 5, fig. 11.

1864. Heer. Viert. naturf. gesellsch. Zürich, ix: 287.

1868. Scudder. Geol. mag., v: 176-177.

18\%7. Goldenberg. Fanna saræp. foss., ii : 19.

See also Etoblattina venusta.

228. Blattina weissiana. (Palæoblattariæ.)

Locality : Brücken, Canton Waldmohr, Rheinpfalz. Horizon : Carboniferous. 1877. Goldenberg. Fauna saræp. foss., ii : 26, 51.

See also B. euglyptica var. weissiana, etc.

229. Blattina weissigensis. (Palæoblattariæ.)

Locality: Weissig, Germany. Horizon: Lower Dyas.

1873. E. Geinitz. Neues jahrb. f. mineral., 1873: 692-693. Pl. 3, fig. 1.

1873. " Verstein. unter. dyas Weissig, 2-4. Pl. 3, fig. 1.

1875. " "Neues jahrb. f. mineral., 1875: 6 .

1875. " Neue aufschl. dyas Weissig, 6.

See also Etoblattina weissigensis, Blattina (Etoblattina) weissigensis.

230. Blattina (Etoblattina) weissigensis. (Palæoblattariæ.)

Locality: Weissig, Germany. Horizon : Lower Dyas.

1880. Geinitz. Blatt. unter. dyas Weissig, 21. Pl. 39, fig. 11.

1880. " Verhandl. leop.-carol. akad. naturf., xli: $441 . \quad P l .39, f i g .11$.

See also Etoblattina weissigensis, Blattina weissigensis.

231. Blattina wemmetsweileriensis. (Palæoblattariæ.)

Locality: Wemmețweiler, Germany. Horizon: Carboniferous.

1877. Goldenberg. Fauna saræp. foss., ii : 19,24, 51. Pl. 1, fig. 9.

See also Hermatoblattina wemmetsweileriensis, etc.

232. Blattina (Hermatoblattina) wemmetsweileriensis. (Palæoblattariæ.)

Locality: Wemwetsweiler, Germany. Horizon: Carboniferous.

1883. Kliver. Nene blatt. Saarbriicken. $P l$. 1, figs. 4, $4 a$.

1883. " Palaeontogr., xxix. Pl. 34, figs. 4, $4 a$.

See also Hermatoblattina wemmetsweiliensis, etc.

233. Blattina winteriana. (Palæoblattariæ.)

Locality : Dudweiler, Germany. Horizon: Carboniferous.

1870. Goldenberg. Neues jahrb. f. mineral., 1870 : 288-289. Figs. 1-4.

1877. " Fauna saræp. foss., ii : 19, 25-26, 51. Pl. 1, fig. 11.

See also Authracoblattina winteriana. 
234. Breyeria borinensis. (Protophasmida.)

Compared with Attacus aurora.

Locality: Mons, Belgium. Horizon: Carboniferous.

\begin{tabular}{|c|c|c|}
\hline 875. & Borre. & Compt. rend. soc. ent. Belg., (2), No. 13: 7-11. \\
\hline 1875. & “ & Ann. soc. ent. Belg., xviii : 6-10. Pl. 5, fig. $2 ;$ Pl. 6, fig. 2. \\
\hline 1875. & “ & Empreint. insect. foss. Mons, 6-10. Pl. 5, fig. 2; Pl. 6, fig. 2. \\
\hline 875. & “ & Gerv., Journ. de zool., iv : 291-297. \\
\hline 1875. & Giard. & Bull. scient. dép. Nord, vii : 121-127. \\
\hline 1876. & Scudder. & Compt. rend. soe. ent. Belg., (2), No. 21 : 2 (not a lepidopteron.) \\
\hline 1876. & Borre. & Compt. rend. soc. ent. Belg., (2), No. 22: 5-6. \\
\hline 1876. & Hagen. & Compt. rend. soc. ent. Belg., (2), No. 22: 6 (a Dictyoneura?). \\
\hline 1876. & Heer. & Compt. rend. soc. ent. Belg., (2), No. 22: 6 (a Libellula?). \\
\hline 1876. & Bar. & Compt. rend, soc. ent. Belg., (2), No. $28: 6$ (homopterous). \\
\hline 1877. & McLachlan. & Compt. reud. soc. ent. Belg., (2), No. $41: 5-6$ (an ephemerid). \\
\hline 1879. & Wallace. & Nature, xix : 501, 582 (lepidopteron). \\
\hline 1879. & McLachlan. & Nature, $\mathrm{xx}: 5-6$ (neuropteron). \\
\hline 1879. & Eaton. & Nature, xx: 315 (ephemerid). \\
\hline 1879. & Borre. & Compt. rend. soc. ent. Belg., (2), No. 65 : 7-12. \\
\hline 1879. & 66 & Note sur Breyeria, 1-6. \\
\hline 1885. & Scudder. & Proc. Amer. acad. arts sc., xx: 171 . \\
\hline 1885. & Brongniart. & Bull. soc. amis sc. Rouen, (3), xxi : 64 . \\
\hline 1885. & “6 & Rev. scient., (3), xxxvi : 278 . \\
\hline 1885. & “ & Geol. mag., (3), ii : 489. \\
\hline 1885. & “ & Trans. geol. soc. Manch., xviii : 284. \\
\hline 1885. & “6 & Foss. ins. prim. rocks, 16. \\
\hline 1885. & 6 & Jahrb. geol. reichsanst., xxxv: 659 . \\
\hline 1885. & Scudder. & Zittel, Handb. palaeont., i, ii : 757. Fig. 940 . \\
\hline 1886. & "6 & Zittel-Barrois, Traité de paléont., ii : 757. Fig. 956. \\
\hline 1886. & 6 & Bull. U. S. geol. surv., No. $31: 40$. \\
\hline
\end{tabular}

See also Pachytylopsis borinensis.

235. Breyeria elongata. (Protophasmida.)

Locality : Dudweiler, Germany. Horizon : Carboniferous.

1885. Scudder. Proc. Amer, acad. arts sc., xx: 171.

See also Dictyoneura elongata, Goldenbergia elongata.

\section{Caloneura dawsonii.}

Locality: Commentry. Horizon: Carboniferous.

1885. Brongniart. Ins. foss. terr. prim., 59. Pl. [4], fig. 2.

1885. " "Bull. soc. amis se. Rouen, (3), xxi: 59.

1885. “ $\quad$ Rev. scient., (3), xxxvi : 276 .

1885. " " Geol. mag., (3), ii: 487. Pl. 12, fig. 2.

1885. “ " " Trans, geol. soc. Manch., xviii. Pl., fig. 2.

1885. " Jahrb. geol, reichsanst, $x \times x v: 655$.

Calotermes affinis. See Termes (Calotermes) affinis.

Calotermes decheni. See Termes (Calotermes) decheni.

237. Calotermes heeri. (Protophasmida.)

Locality : Altenwald, Germany. Horizon : Carboniferous.

1858. Hagen. Catal. Neuropt. Brit. mus., 11 (loc. err.).

See also Termes (Eutermopsis) heeri, etc. 
Calotermes humboldtianus. See Termes (Calotermes) humboldtianus, Dictyoneura humboldtiana.

238. Dictyoneura - (Protophasmida.)

1883. Kliver.

Locality : Saarbrück basin. Horizon: Carboniferous.

1883. " Palaeontogr., xxix: 261. Pl. 35, fig.6.

Dictyoneura absoleta. See Dictyoneura obsoleta.

239. Dictyoneura affinis. (Protophasmida.)

Locality: Germany. Horizon: Carboniferous.

1885. Scudder. Proc. Amer. acad. arts sc., xx: 170.

See also Termes affinis, etc.

240. Dictyoneura anthracophila. (Protophasmida.)

Locality : Gersweiler, Germany. Horizon: Carboniferous.

1854. Goldenberg. Palaeontogr., iv: 35. Pl. 6, fig. 6.

1854. “ Foss. ias. Saarbriicken, 19. Pl. 4, fig. 6.

1854-'56. Bronn. Lethæa geogn., $3^{\text {te }}$ aufl. i, ii : 684 . Pl. $9^{3}$, fig. $15 b$.

1856. Giebel. Insect. d. vorwelt, 267.

1857. Hagen. Verhandl. naturh, ver. preuss. Rheinl. Westph., xiv: 44.

1867. Goldenberg. Vorweltl. fauna steink. Saarbr., 14. Pl. 2, fig. 19 (ined.).

187:. " " Fauna saræp. foss., i : 14. Pl. 2, fig. 19.

1877, " Fauna saræp. foss., ii: 50.

See also Litoneura anthracophila, Goldenbergia anthracophila.

Dictyoneura borinensis. See Breyeria borinensis.

241. Dictyoneura decheni. (Protophasmida.)

Locality: Altenwald, Germany. Horizon: Carboniferous.

1877. Goldenberg. Fauna saræp. foss., ii : 50.

See also Termes (Eutermopsis) decheni, etc.

242. Dictyoneura elegans. (Protophasmida.)

Locality: Dudweiler, Germany. Horizon: Carboniferous.

1877. Goldenberg. Fauna saræp. foss., ii : 9-10,50. Pl. 1, fig. 1.

1885. Braner. Sitzungsb, akad. wiss. Wien, xci : 281.

See alsu Polioptenus elegans; Goldenbergia elegans.

243. Dictyoneura elongata. (Protophasmida.)

Locality: Dndweiler, Germany. Horizon : Carboniferous.

187\%. Goldenberg. Fauna saræp. foss., ii : 10-11,50. Pl. 1, fig. 2.

1885. Brauer. Sitzungsb. akad. wiss. Wien, xci : 281.

See also Breyeria elongata, Goldenbergia elongata.

244. Dictyoneura formosa. (Protophasmida.)

Locality: Gersweiler, Germany. Horizon: Carboniferous.

1877. Goldenberg. Fauna saræp. foss., ii : 50.

See also Termes (Entermopsis) formosus, etc. 
245. Dictyoneura goldenbergi. (Protophasmida.)

Locality : Commentry, France. Horizon : Carboniferous.

1883. Brongniart. Naturaliste, $v: 268$.

1883. " $\quad$ Aperçu ins. foss., 7.

1884. " " Compt. rend, acad. sc, xcviii : 832-833.

1884. " Bull, seances soc. ent.Fr., 1884: 225.

1885. " " Bull. soc. amis sc. Rouen, (3) xxi : 62 (on pl. wrongly as monyi, see Errata). Pl. 2, fig. 3.

1885. " " Rev. scient., (3), xxxvi: 277 .

1885. " Geol. mag., (3), ii: 488.

1885. " "Trans. geol. soc. Manch., xviii : 282.

1885. " " Foss. ins. prim. rocks, 13.

1885. " " Jahrb. geol. reichsanst, $\mathrm{xxxv:657.}$

1885. Scudder. Proc. Amer, acad, arts sc., $\mathrm{xx}: 173$.

246. Dictyoneura gracilis. (Protophasmida.)

Locality : Friedrichsthal, Saarbrück, Germany. Horizon: Carboniferous. 1886. Kliver. Palaeontogr., xxxii: 107-108. Pl. 14, figs. 7, 8.

247. Dictyoneura haplophlebia. (Protophasmida.)

Locality : Cape Breton. Horizon : Carboniferous.

1877. Goldenberg. Fauna saræp. foss., ii: 16.

See also Haplophlebium barnesii.

Dictyoneura humboldtana. See Dictyoneura humboldtiana.

248. Dictyoneura humboldtiana. (Protophasmida.)

Locality: Sulzbach, Germany. Horizon : Carboniferous.

1854. Goldenberg. Palaeontogr., iv: 35-36. Pl. 6, fig. 5.

1854. " Foss. ins. Saarbrücken, 19-20. Pl. 4, fig. 5.

1856. Giebel. Insect. d. vorwelt, 267 (humboldtana).

1857. Hagen. Verhandl, naturh, ver. preuss. Rheinl. Westph., xiv: 44.

1860. " Linn. entom., xii : 296 (a Calotermes).

1876. Roemer. Lethæa geogn. Pl. 47, fig. 17.

1877. Goldenberg. Fauna saræp, foss., ii : 50.

1885. Scudder. Proc. Amer. acad. arts se., $\mathrm{xx}: 170$.

See also Termes (Calotermes) humboldtiana, Goldenbergia humboldtiana.

249. Dictyoneura ingens. (Protophasmida.)

Locality : England. Horizon: Carboniferous.

1884. Brongniart. Bull. séances soc. ent. Fr., 1884 : 226.

See also Archæoptilus ingens.

250. Dictyoneura jucunda. (Protophasmida.)

Locality: Pennsylvania. Horizon: Carboniferous.

1885. Brongniart. Bull. soc. amis sc. Rouen, (3), xxi : 62.

1885. " " Geol. mag., (3), ii : 488.

1885. " "Trans. geol. soc. Mauch., xviii : 282.

1885. " " Foss. ins, prim. rocks, 13.

1885. “ Jahrb, geol. reichsanst., $\mathrm{xx \times V}: 65 \%$.

See also Titanophasma jueunda. 
251. Dictyoneura libelluloides. (Protophasmida.)

Localities: Gersweiler, Auerswald bei Saarbrick, Germany. Horizon : Carboniferous.

1854. Goldenberg. Palaeontogr., iv : 33-35. Pl. 3, fig. 5.

1854. " Foss. ins. Saarbrücken, 17-19. Pl. 1, fig. 5.

1856. Giebel. Insect. d. vorwelt, 266-267.

1867. Goldenberg. Vorweltl. fauna steink. Saarbr., 13-14. Pl. 2, fig. 12 (ined.).

1873. " Fauna saræp. foss., i: 13-14. Pl. 2, fig. 12.

1876. Gerstaecker. Klass. ordn. arthr., Crust., i : 289, 292 (an ephemerid).

187\%. Goldenberg. Fauna saræp. foss., ii : 50.

1885. Brongniart. Bull. soc. amis sc. Rouen, (3), xxi: 62.

1885. "f Geol. mag., (3), ii : 488.

1885. " " Trans. geol, soc. Manch., xriii : 282.

1885. " " Foss. ins. priw. rocks, 13.

1885. " "Jahrb. geol. reichsanst., ХXX₹: 657.

See also Titanophasma libelluloides.

252. Dictyoneura monyi. (Protophasmida.)

Locality : Commentry, France. Horizon: Carboniferous.

1884. Brongniart. Compt. rend. acad. sc., xeviii: 833 .

1884. " Bull. séances soc. ent. Fr., 1884: 225.

1885. Scudder. Proc. Amer. acad. arts sc., xx: 173.

See-also D. goldenbergi, Meganeura monyi, Meganeura (Dictyoneura) monyi.

253. Dictyoneura nigra. (Protophasmida.)

Locality : Frankenhöltz, Bavaria. Horizon: Carboniferous.

1883. Kliver. Neue blatt. Saarbrücken, 12-13. Pl. 2, fig. 5.

1883. " Palaeontogr., xxix : 260-261. Pl. 35, fig. 5.

See also Goldenbergia nigra.

254. Dictyoneura obsoleta. (Protophasmida.)

Locality: Altenwald, Germany. Horizon: Carboniferous.

1877. Goldenberg. Fauna saræp. foss., ii : 11,50 (absoleta in plate). Pl. 1, fig. 4.

See also Litoneura obsoleta, Goldenbergia obsoleta.

255. Dictyoneura schmitzii. (Protophasmida.)

Locality: Altenwald, Germany. Horizon: Carboniferous.

1877. Goldenberg. Fauna saræp. foss., ii: 11,50. Pl. 1, fig. 3.

1885. Scudder. Proc. Amer. acad. arts se, xx: 170.

See also Goldenbergia schmitzii.

256. Dictyoneura sinuosa. (Protophasmida.)

Locality: Saarbriick basin, Germany. Horizon: Carboniferous.. .

1883. Kliver. Neue blatt. Saarbrücken, 11-12. Pl. 2, fig. 4.

1883. " Palaeontogr., xxix: 259-260. Pl. 35, fig. 4.

1885. Scudder. Proc. Amer. acad. arts sc., xx : 170.

See also Goldenbergia sinuosa.

257. Dictyopteris didyma. (Palæoblattariæ.)

Locality: Germany. Horizon: Carboniferous.

1839. Rost. De filicum ectypis, 21.

See also Blattina didyma, etc. 
258. Ftoblattina affinis. (Palæoblattariæ.)

Locality : Löbejün, Saxony. Horizon: Upper Carboniferous.

1879. Scudder. Mem. Bost. soc. nat. hist., iii : 62. Pl. 2, fig. 2.

1890. " Foss. ins. N. A., i : 82. Pl. 2, fig. 2.

See also Blattina affinis.

259. Etoblattina anaglyptica. (Palæoblattariæ.)

Locality: Wettin, Saxony. Horizon: Upper Carboniferous.

1679. Scudder. Mem. Bost. soc. nat. hist., iii: 69-70. Pl. 2, fig. 15.

1890. " Foss. ins. N. A., i: 89-90. Pl. 2, fig. 15.

See also Blattina anaglyptica, etc.

260. Etoblattina anthracophila. (Palæoblattariæ.)

Locality : Wettin, Saxony: Horizon: Upper Carboniferous.

1879. Scudder. Mem. Bost. soc. nat. hist., iii: 64-65. Pl. 2, fig. 1.

1890. " Foss. ins. N. A., i: 84-85. Pl. 2, fig. 1.

See also Blattina anthracophila, Blattina anaglyptica, etc.

261. Etoblattina balteata. (Palæoblattariæ.)

Locality: West Virginia. Horizon: Permo-carboniferous.

1889. Scudder. Proc. Bost. soc. nat. hist., xxiv: $46,48$.

18๕9. Lesley. Dict. foss. Penns., i: p. xxii-xxiii.

See also Gerablattina balteata.

Etoblattina bituminosa. See Blattina (Etoblattina) bituminosa.

262. Etoblattina carbonaria. (Palæoblattariæ.)

Localities: Wettiu, Weissig?, Germany. Horizon: Upper Carboniferous, Dyas?

1879. Scudder. Mem. Bost. soc. nat. hist., iii : 73-74. Pl. 2, fig. 3.

1887. Deichmüller. Ber. senckenb. gesellsch. Frankf., 1886-87: 93-94. Pl. 3, fig. 3.

1890. Scudder. Foss. ins. N. A., i: 93-94. Pl. 2, fig. 3.

See also Blattina carbonaria, B. (Etoblattina) carbonaria var. deichmülleri, Etoblattina carbonaria var.

263. Etoblattina carbonaria var. (Palæoblattariæ.)

Locality: Weissig, Germany. Horizon: Dyas.

1882. Deichmüller. Sitzungsb. gesellsch. Isis, 1882: 38-40. Pl. 1, figs. 2-3.

See also Etoblattina carbonaria, etc.

264. Ftoblattina didyma. (Palæoblattariæ.)

Localities: Wettin, Ilmenau, Germany. Horizon: Upper Carboniferous.

1879. Sendder. Mem. Bost. soc. nat. hist., iii : 75-76. Pl. 2, fig. 13.

1890. " Foss, ins. N. A., i: 95-96. Pl. 2, fig. 13.

See also Blattina didyma, etc.

265. Etoblattina dohrnị. (Palæoblattariæ.)

Locality : Wettin, Saxony. Horizon: Upper Carboniferous,

1879. Scudder. Mem. Bost. soc. nat. hist., iii : 66-67, Pl. 2, fig. 5,

1890. " Foss. ins. N. A., i: 86-87, Pl, 2, fig. 5.

See also Blattina englyptica, etc, 
266. Etoblattina elongata. (Palæoblattariæ.)

Locality: Weissig, Germany. Horizon: Lower Dyas.

1879. Scudder. Mem. Bost. soc. nat. hist., iii : 80-81. Pl. 2, fig. 10.

1890. “ Foss. ins. N. A., i: 100-101. Pl. 2, fig. 10.

See also Blattina cf. mahri, B. (Etoblattina) elongata.

267. Etoblattina euglyptica. (Palæoblattariæ.)

Locality: Wettin, Saxony. Horizon: Upper Carboniferous.

1879. Scudder. Mem. Bost. soc. nat. hist., iii : 60-61. Pl. 2, fig. 16; Pl. 4, fig. 7. 1890. " Foss. ins. N. A., i: 80-81. Pl. 2, fig. 16; Pl. 4, fig. 7.

See also Blattina euglyptica, etc.

\section{Ftoblattina fasciata. (Palæoblattariæ.)}

Localities: Richmond, Ohio; Cassville, W. Va. Horizou : Upper Carboniferous. 1889. Scudder. 1889. Lesley.

Proc. Bost. soc. nat. hist., xxiv : 47-48.

Dict. foss. Penns., i: p. xxiii.

\section{Etoblattina flabellata. (Palæoblattariæ.)}

Localities: Wettin, Weissig, Germany. Horizon : Upper Carboniferous, Dyas. 1879. Scudder. Mem. Bost. soc. nat. hist., iii : 62-64. Pl. 2, fig. 4. 1890. “ Foss. ins. N. A., 1: 82-84. Pl. 2, fig. 4.

See also Blattina flabellata, etc.

270. Etoblattina flabellata var. stelzneri. (Palæoblattariæ.)

Locality: Weissig, Germany. Horizon: Dyas.

1882. Deichmuller. Sitzungsb. gesellsch. Isis, 1882 : 34-38. Pl. 1, fig. 1, 1 a-d.

See also Blattina flabellata, etc., Etoblattina flabellata.

271. Etoblattina hustoni. (Palæoblattariæ.)

Locality: Richmond, Ohio. Horizon : Barren Coal Measures.

1889. Scudder. Proc. Bost. soc. nat. hist., xxiv : 53.

1389. Lesley. Dict. foss. Penns., i : p. xxiii.

272. Etoblattina insignis. (Palæoblattariæ.)

Locality : Saarbrück, Germany. Horizon: Middle Carboniferous.

1879. Scudder. Mem. Bost. soc. nat. hist., iii: 82-83. Pl. 2, fig. 7; Pl. 4, fig. 9. 1890. " Foss. ins. N. A., i: 102-103. Pl. 2, fig. 7; Pl. 4, fig. 9.

See also Blattina insignis, Leptoblattina insignis.

273. Etoblattina intermedia. (Palæoblattariæ.)

Locality : Wemmetsweiler, Germany. Horizon: Middle Carboniferous. 1883. Kliver. Neue blatt. Saarbrück., 9-10. Pl. 2, fig. 2, $2 a$. 188:3. “ Palaeontogr., xxix: 257-258. Pl. 35, fig. 2, 2a.

See also Gerablattina intermedia, etc.

274. Etoblattina johnsoni. (Palæoblattariæ.)

Locality: Coseley, vic. Dudley, England. Horizon: Carboniferous. 1887. Woodward. Geol. mag., (3), iv: 53-55. Pl.2, fig. 1a-b.

Bull. $71-4$ 
275. Etoblattina labachensis. (Palæoblattariæ.)

Locality: Labach, Germany. Horizon: Upper Carboniferous.

1879. Scudder. Mem. Bost. soc. nat. hist., iii : 59-60. Pl. 3 , fig. 5.

1890. " Foss. ins. N. A., i: 79-80. Pl. 3, fig. 5.

See also Blattina labachensis, B. anaglyptica var. labachensis, Blattina anaglyptica, etc.

276. Etoblattina lanceolata. (Palæoblattariæ.)

Locality: Lugau, Saxony. Horizon: Carboniferous.

1881. Sterzel. Ber. naturw. gesellsch. Chemn., vii : 273.

See also Blattina (Etoblattina) lanceolata.

277. Etoblattina leptophlebica. (Palæoblattariæ.)

Locality : Löbejün, Saxony. Horizon: Upper Carboniferous.

1879. Scudder. Mem. Bost. soc. nat. hist., iii: 77-79. Pl. 3, fig. 9 .

1890. “ Foss. ins. N. A., i: 97-99. Pl. 3, fig. 9.

See also Blattina leptophlebica.

278. Etoblattina lesquereuxii. (Palæoblattariæ.)

Locality: Vic. Pittston, Pa. Horizon: Middle Carboniferous.

1879. Scudder. Mem. Bost. soc. nat. hist., iii: 67-69. Pl. 6, figs. 3, 4; fig. on p. 68.

1883. Lacoe. List pal. foss. ins., 6.

1889. Lesley. Dict. foss. Penns., i: p. xxiii.

1889. Miller. $\quad$ N. A. geol. and pal., 576.

1890. Scudder. Foss. ins. N. A., i: 87-89. Pl. 6, figs. 3,4 ; fig. on p. 88.

279. Etoblattina manebachensis. (Palæoblattariæ.)

Locality: Manebach, Germany. Horizon: Upper Carboniferons.

1879. Scudder. Mem. Bost. soc. nat. hist., iii : 79-80. Pl. 2, fig. 14.

1885. " Zittel, Handb. palaeont., i, th. ii: 754. Fig. 932.

1886. “ Zittel-Barrois, Traité de pal6ont., ii: 754. Fig. 949.

1886. Brauer. Ann. naturh. hofmus., i: 115-116. Pl. 7, fig. 8.

1890. Scudder. Foss. ins. N. A., i: 99-100. Pl. 2, fig. 14.

See also Blattina manebachensis, B. (Etoblattina) manebachensis.

280. Etoblattina mantidioides. (Palæoblattariæ.)

Locality: Claxheugh, Durham, England. Horizon: Upper Carboniferous.

1879. Scudder. Mem. Bost. soc. nat. hist., iii : 72-73. Pl. 3, fig. 8; fig. on p. 73.

1881. Scudder. Geol. mag., (2), viii : 300.

1883. " Mem. Bost. soc. nat. hist., iii : 223.

1890. " Foss. ins. N. A., i: 92-93, 245. Pl. 3, fig. 8; fig. on p. 93.

See also Blattidium mantidioides, etc.

281. Etoblattina marginata. (Palæoblattariæ.)

Locality : Richmond, Ohio. Horizon: Barren Coal Measures.

1889. Scudder. Proe. Bost. soc. nat. hist., xxiv : 48-50.

1889. Lesley. Dict. foss. Penns., i: p. xxiii.

282. Etoblattina mazona. (Palæoblattariæ.)

Locality: Mazon Creek, Ill. Horizon: Carboniferous.

1882. Scudder. Mem. Bost. soc. nat. hist., iii : 181. Pl. 10.

1882. " Proc. Bost. soc. nat. hist., xxi : 391-396. 
1883. Lacoe. List pal. foss. ins., 6.

1886. Scudder. Miall, Cockroach, 211. Fig. 120.

1.88\%. Woodward. Geol. mag., (3), iv: 53. Fig. 4.

1889. Lesley. Dict. foss. Penus., i: p. xxiii.

1859. Miller. N. A. geol, and pal, 577 (mazonana).

1s90. Scudder. Foss. ins. N. A., i: 233, Pl. $7 a$.

Etoblattina mazonana. See Etoblattina mazona.

283. Etoblattina occidentalis. (Palæoblattariæ.)

Locality : Lawrence, Kans. Horizon: Carboniferous.

1890. Scudder. Mem. Bost. soc. nat. hist., iv: 410-411. Pl.32, fig. 4.

1890. " Foss.ins. N. A., i: 386-38\%. Pl.24, fig.4.

284. Etoblattina ornatissima. (Palæoblattariæ.)

Locality : Grïgelborn, Germany. Horizon: Carboniferous.

1887. Deichmüller. Ber. senckenb. gesellsch. Frankf., 1886-'87: 90-92. Pl.3, fig. 1.

285. Etoblattina parvula. (Palæoblattariæ.)

Locality: Löbejün, Saxony. Horizon: Upper Carboniferons.

1879. Scudder. Mem. Bost. soc. nat, hist., iii : 81. Pl. 2, fig.9.

1890. " Foss.ins. N. A., i: 101. Pl.2, fig.9.

See also Blattina parṿula.

286. Etoblattina peachii. (Palæoblattariæ.)

Locality: Kilmaurs, Scotland. Horizon: Carboniferous.

1887. Woodward. Geol. mag., (3), iv : 433-435. Pl. 12, fig. 1.

287. Etoblattina primæva. (Palæoblattariæ.)

Locality: Gersweiler, Germany. Horizon: Upper Carboniferous.

1879. Scudder. Mem. Bost. soc. nat. hist., iii: 58-59. Pl. 3, fig. 7.

18๙9. Miller. N. A. geol. and pal. Fig. 1080.

1890. Scudder. Foss. ins. N. A., i: 78-79. Pl. 3, fig. 7.

See also Blattina primæva.

288. Etoblattina propria. (Palæoblattariæ.)

Locality : Saarbrïck basin, Germany. Horizon: Carboniferous.

1883. Kliver. Neue blatt. Saarbrück., 10-11. Pl. 2, fig. 3.

1883. “ Palaeontogr., xxix: 258-259. Pl. 35, fig. 3.

See also Blattina propria.

289. Etoblattina rollei. (Palæoblattariæ.)

Locality : Grügelborn, Germany. Horizon: Carboniferons.

1887. Deichmüller. Ber. senckenù. gesellsch. Frankf., 1886-'87: 92-93. Pl. 3, fig. 2.

290. Etoblattina russoma. (Palæoblattariæ.)

Locality: Löbejün, Saxony. Horizon: Upper Carboniferous.

1879. Scudder. Mem. Bost. soc. nat. hist., iii : 76-77. Pl. 2, fig. 6.

1890. " Foss. ins. N. A., i: 96-97, Pl. 2, fig. 6.

See also Blattina russoma. 
291. Etoblattina steinbachensis. (Palæoblattariæ.)

Locality : Steinbachthal, Germany. Horizon: Carboniferous. 1886. Kliver.

Palaeontogr., xxxii : 100-104. Pl. 14. figs. 2, 3.

292. Etoblattina stipata. (Palæoblattariæ.)

Locality: Richmond, Ohio. Horizon : Barren Coal Measures. 1889. Seudder.

Proc. Bost. soc. nat. hist., xxiv : 50-51.

1889. Lesley.

Dict. foss. Penns., i : p. xxiii.

293. Etoblattina strigosa. (Palæoblattariæ.)

Locality: Richmond, Ohio. Horizon: Barren Coal Measures. 1889. Scudder. Proc. Bost. soc. nat. hist., xxiv : 52-53.

1889. Lesley. Dict. foss. Penns., i : p. xxiii.

294. Etoblattina tenuis. (Palæoblattariæ.)

Locality : Richmond, Ohio. Horizon : Barren Coal Measures.

1889. Scudder.

1889. Lesley.

Proc. Bost. soc. nat. hist., xxiv : 46-47.

Dict. foss. Penns., i : p. xxiii.

295. Etoblattina variegata. (Palæoblattariæ.)

Locality : Richmond, Ohio. Horizon: Barren Coal Measures.

1889. Scudder.

1889. Lesley.
Proc. Bost. soc. nat. hist., xxiv : 51-52.

Dict. foss. Penns., i: p. xxiii.

296. Etoblattina venusta. (Palæoblattariæ.)

Locality : Frog Bajou, Ark. Horizon: Lower Carboniferous. 1879. Scndder. Mem. Bost. soc. nat. hist., iii: 70-72. Pl. 6, fig. 12. 1883. Lacoe. List. pal. foss. ins., 7. 1889. Lesley. Dict. foss. Penns, i: p. xxiii. 1889. Miller. N. A. geol. and pal., 577. Fig. 1081. 190. Scudder. Foss. ins. N. A., i : 90-92. Pl. 6, fig. 12. See also Blattina venusta.

297. Etoblattina weissigensis. (Palæoblattariæ.)

Locality : Weissig, Germany. Horizon : Dyas.

1879. Scudder. Mem. Bost. soc. nat. hist., iii: 65-66. Pl. 6, fig, 5 . 1890. " Foss. ins. N. A., i: 85-86. Pl. 6, fig. 5.

See also Blattina weissigensis, B. (Etoblattina) weissigensis.

Eurypterus granosus. See Adelophthalmus (Eurypterus) granosus

Eutermopsis affinis. See Termes (Eutermopsis) affinis.

Eutermopsis decheni. See Termes (Eutermopsis) decheni.

Eutermopsis formosus. See Termes (Eutermopsis) formosus.

Eutermopsis heeri. See Termes (Eutermopsis) heeri. 
298. Gerablattina balteata. (Palæoblattariæ.)

Localities: Cassville, W. Va., Bellaire, Ohio. Horizon: Upper Carboniferous, Permo-Carboniferous.

1879. Scudder. Mem. Bost. soc. nat. hist., iii : 110-112. Pl. 6, figs. 9, 10.

1880. " " Font.-White, Perm. flora W. Va. (Rep. 2d geol. surv. Penn., PP), 104. $P l .38$, figs. 5, 5a.

Font.-White. Upper carb. flora W. Va. (Blattina sp.). Pl. 22, figs. 16, $16 a$. (ined.).

1883. Lacoe. List. pal. foss. ins., 7, 8.

1889. Lesley. Dict. foss. Penns., i: 253. Figs.

1889. Miller. $\quad$ N. A. geol. and pal., 577. Fig. 1083.

1890. Scudder. Foss. ins. N. A., i : 130-132. Pl. 6, figs. 9, 10.

See also Etoblattina balteata.

\section{Gerablattina clathrata. (Palaeoblattariæ.)}

Locality: Manebach, Thüringen. Horizon: Upper Carboniferous.

1879. Scudder. Mem. Bost. soc. nat. hist., iii : 100-101. Pl. 3, fig. 4.

1890. " Foss. ins. N. A., i: 120-121. Pl. 3, fig. 4.

Seo also Blattina clathrata, B. latinervis.

300. Gerablattina fascigera. (Palæoblattariæ.)

Locality : Pittston, Pa. Horizon: Lower Carboniferous.

1879. Scudder. Mem. Bost. soc. nat. hist., iii : 113-115. Pl. 6, figs. 1, 2. 1883. Lacoe. List pal. foss. ins., 8.

1889. Lesley. Dict. foss. Penns., i: p. xxv, 254. 2 figs.

1889. Miller. N. A. geol. and pal., 577.

1890. Scudder. Foss. ins. N. A., i: 133-135. Pl. 6, fig8. 1, 2.

See also Blattina fascigera.

\section{Gerablattina geinitzi. (Palæoblattariæ.)}

Locality : Löbejün, Saxony. Horizon: Upper Carboniferous.

1879. Scudder. Mem. Bost. soc. nat. hist., iii : 103-104. Pl. 2, fig. 11.

1890. " Foss. ins. N. A., i : 123-124. Pl. 2, fig. 11.

See also Blattina geinitzi.

302. Gerablattina germari. (Palæoblattariæ.)

Locality: Wettin, Saxony. Horizon: Upper Carboniferous.

1879. Scudder. Mem. Bost. soc. nat. hist., iii : 107-108. Pl. 3, fig. 6.

1890. " Foss. ins. N. A., i: 127-128. Pl. 3, fig. 6.

See also Blatta germari, etc.

303. Gerablattina goldenbergi. (Palæoblattariæ.)

Locality: Manebach, Thüringen. Horizon: Upper Carboniferous.

1879. Scudder. Mem. Bost. soc. nat. hist., iii : 98-99. Pl. 3, fig. 13.

1890. " Foss. ins. N. A., i: 118-119. Pl. 3, fig. 13.

See also Blattina goldenbergi.

\section{Gerablattina intermedia. (Palæoblattariæ.)}

Locality: Wemmetsweiler, Germany. Horizon: Middle Carboniferous.

1879. Scudder. Mem. Bost. soc. nat. hist., iii : 101-102. Pl. 3, fig. 11.

1883. Kliver. Neue blatt. Saarbrück., 9-10. Pl. 2, figs. 2, $2 a$.

1883. " Palaeontogr., xxix : 257-258. Pl. 35, figs, 2, 2a.

1890. Scudder. Foss. ins. N. A., i : 121-122. Pl. 3, fig. 11.

See also Blattina intermedia, B. (Gerablattina) intermedia, Etoblattina intermedia. 
305. Gerablattina mahri. (Palæoblattaı iæ.)

Locality : Manebach, Thüringen. Horizon: Upper Carboniferous.

1879. Scudder. Mem. Bost. soc. nat. hist., iii : 108-109. Pl. 3, fig. 14.

1890. " " Foss. ins, N. A., i: 128-129. Pl. 3, fig. 14.

See also Blattina mahri.

306. Gerablattina münsteri. (Palæoblattariæ.)

Locality : Wettin, Saxony. Horizon: Upper Carboniferous.

1879. Scudder. Mem. Bost. soc. nat. hist., iii : 104-106. Pl. 2, fig. 12. 1890. " Foss. ins. N. A., i : 124-126. Pl. 2, fig. 12.

See also Blattina flabellata, Etoblattina flabellata.

307. Gerablattina producta. (Palæoblattariæ.)

Locality: Wettin, Saxony. Horizon: Upper Carboniferous.

1879. Sendder. Mem. Bost. soc. nat. hist., iii : 106-107. Pl. 3, fig. 2.

1890. " Foss. ins. N. A., i: 126-127. Pl. 3, fig. 2.

See also Blattina euglyptica, etc.

308. Gerablattina robusta. (Palæoblattariæ.)

Locality: Wemmetsweiler, Germany. Horizon: Carboniferous.

1883. Kliver.

Neue blatt. Saarbrïck., 7. $P l$. 1, figs. 3, $3 a$.

1883. " Palaeontogr., xxix: 255. Pl. 34, figs. 3, $3 a$.

309. Gerablattina scaberata. (Palæoblattariæ.)

Locality : Altenwald, Germany. Horizon: Middle Carboniferous.

1879. Scudder. Mem. Bost. soc. nat, hist., iii: 102-103. Pl. 3, fig. 3.

1890. " Foss. ins. N. A., i: 122-123. Pl. 3, fig. 3.

See also Blattina seaberata.

310. Gerablattina weissiana. (Palæoblattariæ.)

Locality: Brücken, Rheinpfalz. Horizon: Upper Carboniferous.

1879. Scudder. Mem. Bost. soc. nat. hist., iii : 109-110. Pl. 3, fig. 1.

1890. " Foss. ins. N. A., i: 129-130. Pl. 3, fig. 1.

See also Blattina euglyptica var. weissiana, etc.

\section{Gerephemera simplex.}

Locality: St. John, New Brunswick. Horizon : Deronian.

1865. Scudder. Devon. ins. N. Brunsw., 1 (unnamed).

1865. " "Bailey, Obs. geol. south. N. Brunsw., 140 (unnamed).

1865. “ Amer. journ. sc., (2), xxxix : 357 (unnamed).

1865. “ Can. nat., n. s., ii: 235 (unnamed).

1865. “ " Trans. ent. soc. Lond., (3), ii : 117 (unnamed).

1868. " " Geol, mag., v: 174, 175 .

1880. “ Dev. ins. N. Brunsw. (Anniv. mem. Bost. soc. nat. hist.), 12-16. Pl. 1, figs. 8, 8 a.

1881. Hagen. Bull. mus. comp. zool, viii : $277-278$.

1883. Lacoe. List pill foss. ins., 8 .

1885. Scudder. Larly winged ins. Amer., 2-3. Pl., figs. 8, 9.

1885. Brongniart. Bull. soc. amis se. Ronen, (3), xxi: 56.

1885. Hagen. Nature, xxxii: 54.

1885. " Zool. anz., viii : 298-300.

1885. Brongniart. Geol. mag., (3), ii : 484. 
1885. Brongniart. Trans. geol, soc. Manch., xviii: 275.

1885. “ “

1885.

Foss. ins. prim. rocks, 7 .

1886. Braver.

1889. Miller.

Jahrb. geol. reichsanst., xxxv: 653 .

1890. Sendder.

Ann, naturh. hofmus., i: 111.

N. A. geol. and pal., 577 .

Foss. ins. N. A., i : 164-168, 276-277. Pl. 7, figs. 8, 8a; Pl. 14, figs. 8, 9 .

312. Goldenbergia affinis. (Protophasmida.)

Locality: Germany. Horizon: Carboniferons.

1885. Brongniart. Bull. soc. amis sc. Rouen, (3), xxi : 61.

1885. " Geol. mag., (3), ii: 488.

1885. “ " Trans. geol. soc. Manch., xviii : 281 .

1885. " " Foss. ins. prim. rocks, 13.

1885. “ Jahrb. geol. reichsanst, $\mathrm{xxxv}: 657$.

See also Termes affinis, ete.

\section{Goldenbergia amissa.}

Locality: Altenwald, Germany. Horizon: Carboniferous.

1885. Brongniart. Bull. soc. amis sc. Rouen, (3), xxi : 61.

1885. " " Geol. mag., (3), ii : 488.

1885. " " Trans, geol. soc. Manch, xviii : 281.

1885. " " Foss. ins. prim. rocks, 13.

1885. " " Jahrb, geol, reichsanst., xxxv: 657.

See also Termitidium amissum.

314. Goldenbergia anthracophila. (Protophasmida.)

Locality: Gersweiler, Germany. Horizon: Carboniferous.

1885. Brongniart. Bull. soc. amis se. Rouen, (3), xxi: 61 .

1885. " Geol. mag., (3), ii : 488.

1885. " "Trans. geol, soc. Manch., xviii : 281.

18,5. " " Foss. ins. prim. rocks, 13.

1885. " " Jahrb. geol. reichsanst., $\mathrm{xx \times v}: 657$.

See also Dictyoneura anthracophila, Litoneura anthracophila.

Goldenbergia contusa. See I, Neuropt., Idem.

315. Goldenbergia decheni. (Protophasmida.)

Locality : Altenwald, Germany. Horizon : Carboniferous.

1885. Scudder. Proc. Amer. acad. arts sc., xx : 171.

1885. Brongniart. Bull. soc. amis sc. Rouen, (3), xxi : 61 .

1885. 6 " Geol. mag., (3), ii : 488.

1885. " " Trans. geol. soc. Manch., xviii : 281.

1885. " " Foss. ins. prim. rocks, 13.

885. " " Jahrb. geol, reichsanst., $x \times x v: 65 \%$.

See also Termes (Eutermopsis) decbeni, etc.

316. Goldenbergia elegans. (Protophasmida.)

Locality: Dudweiler, Germany. Horizon: Carboniferous.

1885. Brongniart. Bull. soc. amis sc. Ronen, (3), xxi : 61.

1885. " Geol. mag., (3), ii : 488.

1885. " " Trans. geol. soc. Manch., xviii : 281.

1885. " " Foss. ins. prim. rocks, 13.

1885. " "Jahrb. geol. reichsanst., xxxv: 657.

See also Dictyoneura elegans, Polioptenus clegans. 
317. Goldenbergia elongata, Germany. (Protophasmida.)

Locality : Dudweiler, Germany. Horizon: Carboniferous.

1885. Scudder. Proc. Amer. acad. arts sc., xx : 171.

1885. " Mem. Bost. soc. nat. hist., iii : 322, note.

1885. Brongniart. Bull, soc. amis sc. Rouen, (3), xxi : 61.

1885. " Geol, mag., (3), ii : 488.

1885. " Trans. geol. soc. Manch., xviii : 281.

1885. " " Foss. ins. prim. rocks, 13.

1885. “ Jahrb. geol. reichsanst., xxxv: 657.

1890. Scudder. Foss.ins. N. A., i: 286 note 3.

See also Dictyoneura elongata, Breyeria elongata.

318. Goldenbergia formosa. (Protophasmida.)

Locality: Gersweiler, Germany. Horizon: Carboniferous.

1885. Scudder. Proc. Amer. acad. arts sc., $\mathrm{xx}: 172$.

See also Termes (Eutermopsis) formosus, etc.

319. Goldenbergia heeri. (Protophasmida.)

Locality: Altenwald, Germany. Horizon: Carboniferous.

1855. Scudder. Proc. Amer. acad. arts sc., xx: 172.

1885. Brongniart. Bull. soc. amis sc. Rouen, (3), xxi: 61 .

1885. " " Geol. mag., (3), ii : 488.

1885. " " Trans. geol. soc. Manch., xviii : 281 .

1885. " " Foss. ins. prim. rocks, 13.

1885. " J Jahrb. geol. reichsanst., $\mathrm{xx \times v}: 65 \%$.

See also Termes (Eutermopsis) heeri, etc.

320. Goldenbergia humboldtiana. (Protophasmida.)

Locality: Sulzbach, Germany. Horizon: Carboniferous.

1885. Brongniart. Bull. soc. amis sc. Rouen, (3), xxi : 61.

1885. " " Geol. mag., (3), ii : 488.

1885. " " Trans. geol. soc. Manch., xviii : 281.

1885. " " Foss. ins. prim. rocks, 13.

1885. " "Jahrb. geol. reichsanst., $\mathrm{xxxv}: 657$.

See also Dictyoneura humboldtiana, etc.

321. Goldenbergia laxa. (Protophasmida.)

Locality: Dudweiler, Germany. Horizon: Carboniferous.

1885. Brongniart. Bull. soc. amis sc. Rouen, (3), xxi: 61.

1885. " " Geol. mag., (3), ii : 488.

1885. " " Trans. geol. soc. Manch., xviii: 281.

1885. " " Foss. ins. prim. rocks, 13.

1885. " J Jahrb. geol. reichsanst., $\mathrm{xxxv}: 657$.

See also Termes laxus, etc.

322. Goldenbergia longitudinalis.

Locality: Mazon Creek, Ill. Horizon : Carboniferous.

1885. Brongniart. Bull. soc. amis sc. Rouen, (3), xxi : 61.

1885. " G Geol. mag., (3), ii: 488.

1885. " " Trans. geol. soc. Manch., xviii : 281.

1885. " " Foss. ins. prim. rocks, 13.

1885. " " Jahrb. geol. reichsanst., xxxv : $65 \%$.

See also Termes longitudinalis. 
323. Goldenbergia nigra. (Protophasmida.)

Locality: Bavaria. Horizon: Carboniferons.

1885. Scudder. Proc. Amer. acad. arts sc., xx : 171.

See also Dictyoneura nigra.

324. Goldenbergia obsoleta. (Protophasmida.)

Locality: Altenwald, Germany. Horizon: Carboniferous.

1885. Brongniart. Bull. soc. amis sc. Rouen, (3), xxi: 62.

1885. " " Geol. mag., (3), ii: 488.

1885. “ "Trans. geol. soc. Manch., xviii: 281.

1885. " " Foss. ins. prim. rocks, 13.

1885. " ". Jahrb, geol, reichsanst., $\mathrm{xxxv}: 657$.

See also Dictyoneura obsoleta, etc.

325. Goldenbergia schmitzii. (Protophasmida.)

Locality : Altenwald, Germany. Horizon: Carboniferous. 1885. Brongniart. Bull. soc. amis. sc. Rouen, (3), xxi: 62 (smitzii).

1885. " "Geol. mag., (3), ii: 488.

1885. " " Trans. geol. soc. Manch., xviii : 281.

1885. " ". Foss. ins. prim. rocks, 13.

1885. “ Jahrb. geol, reichsanst., xxxv : 657.

See also Dictyoneura schmitzii.

326. Goldenbergia sinuosa. (Protophasmida.)

Locality: Saarbrück, Germany. Horizon: Carboniferous.

1885. Brongniart. Bull, soc. amis sc. Rouen, (3), xxi : 62.

1885. " Geol. mag., (3), ii : 488.

1885. " Trans. geol. soc. Manch., xviii : 281 .

1885. " " Foss. ins. prim. rocks, 13.

1885. " " Jahrb, geol. reichsanst., xxxv : 657.

See also Dictyoneura sinuosa.

Goldenbergia smitzii. See Goldenbergia schmitzii.

Gryllacris (Corydalis) brongniarti. See I, NeUropt, Idem.

327. Haplophlebium barnesii. (Protophasmida.)

Locality: Cape Breton. Horizon: Carboniferous.

1867. Scudder.

1867. 6

186\%. " "

1868. “

1868.

1868. " "

1872. Packard.

1877. Grildenberg.

1877. Nicholson.

1879.

1880. Dawson.

1883. Lacoe.

1885. Scudder.

1885. Brongniart.

1885.
Proc. Bost. soc. nat. hist., xi : 151.

Geol. mag., iv: 386. Pl. 17, fig. $1 a$.

Can. nat., (2), iii : 202-206.

Dawson, Acad. geol., $2 d$ ed., 380-388. Figs. 152, 152a.

Amer. nat., i: 631. Pl. 16, figs. 8,8a.

Geol. mag., v: 177.

Guide ins., 3d ed., 78. $P l$. 1, figs. 8,8a.

Fauna saræp. foss., ii : 50.

Life hist. earth, 182. Fig. 125.

Manual palæont., 2d ed., i: 406. Fig. 257.

Dawn of life, 147. Fig. 128.

List pal. foss. ins., 8.

Proc. Amer. acad. arts sc., $\mathrm{xx}$ : 172.

Bull. soc. amis sc. Rouen, (3), xxi : 61 .

Geol. mag., (3), ii : 488. 
1885. Brongniart. Trans. geol. soc. Manch., xviii : 281.

1885. " " Foss. ins. prim. rocks, 13.

1885. " " Jahrb. geol. reichsanst., xxxv : 657.

1889. Lesley. Dict. foss. Penus., i: 271-272. Fig.

1889. Miller. N. A. geol. and pal., 577-578. Fig. 1085.

1889. Nicholson. Matu. palæont., 3d ed., i : 594. Fig. 444.

See also Dictyoneura haplophlebia.

\section{Haplophlebium longipennis. (Protophasmida.)}

Locality: Pittston, Pa. Horizon: Carboniferous.

1885. Scudder. Proc. Amer. acad. arts sc., $\mathrm{xx}: 172$.

1885. Brongniart. Bull. soc. amis se. Rouen, (3), xxi : 61.

188:. " Geol. mag., (3), ii : $4 \approx 8$.

1805. " " Trans. geol. soc. Manch., xviii : 281.

1885. " " Foss. ins. prim. rocks, 13.

1885. " " Jahrb. geol. reichsanst., $\mathrm{xxxv:657.}$

1889. Lesley. Dict. foss. Penns., i: 272 (longipinnis).

1889. Miller. $\quad$ N. A. geol. and pal., 578.

Haplophlebium longipinnis. See Haplophlebium longipennis.

329. Hermatoblattina kirkbyi. (Palæoblattariæ.)

Locality: Fifeshire, Scotland. Horizon: Upper Carboniferous.

1887. Seudder. Proc. Bost. soc. nat. hist., xxiii : 357.

See also Lithomylacris kirkbyi.

330. Hermatoblattina lebachensis. (Pælæoblattariæ.)

Locality: Lebach, Germany. Horizon: Dyas.

1879. Scudder. Mem. Bost. soc. nat. hist., iii: 117-118. Pl. 4, fig. 11.

1890. " Foss. ins. N. A., i: 137-138. Pl. 4, fig. 11.

Seo also Blattina lebachensis.

331. Hermatoblattina wemmetsweileriensis. (Palæoblattariæ.)

Locality: Wemmetsweiler, Germany. Horizon: Upper Carboniferous.

1879. Sendder. Mem. Bost. soc. nat. hist., iii : 115-116. Pl. 4, fig. 14.

1883. Kliver. Neue blatt. Saarbr., 8-9. Pl. 1, figs. 4, 4a.

1883. " Palaeontogr., xxix: 256-257. Pl. 34, figs. 4, 4a.

1890. Sendder. Foss. ins. N. A., i: 135-136. Pl. 4, fig. 14.

See also Blattina wemmetsweileriensis, B. (Hermatoblattina) wemmetsweileriensis.

332. Leptoblattina exilis. (Palæoblattariæ.)

Locality: Coseley, vicinity Dudley, England. Horizon: Carboniferous. 1887. Woodward. Geol. mag., (3), iv: 56-58. Pl. 2, figs. 2-3; Pl. 12, fig. 2.

333. Leptoblattina insignis. (Palæoblattariæ.)

Locality : Saarbrïck, Germany. Horizon: Middle Carboniferous.

1887. Woodward. Geol. mag., (3), iv: 58.

See also Etoblattina insignis, etc.

Libellula borinensis. See Breyeria borinensis.

Lithomantis carbonaria. See I, NeURopt, Idem. 
334. Lithomylacris angusta. (Palæoblattariæ.)

Locality: Pittston, Pa. Horizon: Upper Carboniferous.

1879. Scudder. Mem. Bost. soc, nat. hist, iii : 48-50 (angustum). Pl. 5, figs. 2-3.

1883. Lacoe. List pal. foss. ins., 10 .

1885. Scudder. Zittel, Handb. palaeont., i, th. ii: 754. Fig. 931.

1886. " Zittel-Barrois, Traité de paléont., ii: 754. Fig. 948.

1889. Lesley. Dict. foss. Penns., i: 351. 3 figs.

1889. Miller. N. A. geol. and pal., 579. Fig. 1089.

1890. Scudder. Foss. ins. N. A., i: 68-70. Pl. 5, figs. 2-3.

335. Lithomylacris kirkbyi. (Palæoblattariæ.)

Locality : Meithil, Fifeshire, Scotland. Horizon: Upper Carboniferous.

1887. Woodward. Geol. mag., (3), iv: 55-56. Pl. 2, fig. $4 a b$.

See also Hermatoblattina kirkbyi.

336. Lithomylacris pauperata. (Palæoblattariæ.)

Locality : Port Griffith, Pa. Horizon: Carboniferons.

1883. Scudder. Mss. (pauperatum).

1883. Lacoe. List pal. foss. ins., 10.

1889. Lesley. Dict. foss. Penns., i : 352.

1890. Scudder. Mem. Bost. soc. nat. hist., iv : 409-410. Pl. 32, fig. 5.

1890. " $\quad$ Foss. ins. N. A., i : 385-386. Pl. 24, fig. 5.

337. Lithomylacris pittstoniana. (Palæoblattariæ.)

Locality : Pittston, Pa. Horizon: Upper Carboniferous.

1879. Scudder. Mem. Bost. soc. nat. hist., iii: 50-51 (pittstonianum). Pl. 5, figs. 4, 10.

1883. Lacoe. List pal. foss, ins., 10.

1889. Lesley. Dict. foss. Penns., i : 352. 2 figs.

1890. Scudder. Foss. ins. N. A., i : 70-71. Pl. 5, figs. 4, 10.

338. Lithomylacris simplex. (Palæoblattariæ.)

Locality : Vicinity Danville, IIl. Horizon : Lower Carboniferous.

1879. Scudder. Mem. Bost. soc. nat. hist., iii : 51-52. Pl. 5, fig. 5.

1883. Laeoe. List pal. foss. ins., 10.

1889. Lesley. Dict. foss. Penns., i : 351, xxxi.

1889. Miller. $\quad$ N. A. geol. and pal., 579.

1890. Scudder. Foss, ins. N. A., i : 71-72. Pl. 5, fig. 5.

Lithophasma lithanthraca. See I, NeUropt., Idem.

339. Litoneura anthracophila. (Protophasmida.)

Locality : Gersweiler, Germany. Horizon: Carboniferous.

1885. Scudder. Proc. Amer, acad. arts sc., xx : 169.

See also Dictyoneura anthracophila, Golüenbergia anthracophila.

340. Litoneura laxa. (Protophasmida.)

Locality : Dudweiler, Germany. Horizon: Carboniferous.

1885. Scudder. Proc. Amer. acad. arts se., xx: 169.

See also Termes laxus, etc. 
341. Litoneura obsoleta. (Protophasmida.)

Locality : Altenwald, Germany. Horizon: Carboniferous.

1885. Scudder. Proc. Amer. acad. arts. se., xx : 169.

See also Dictyoneura obsoleta, etc.

\section{Mantis ?}

Locality: Mazon Creek, Ill. Horizon: Carhoniferous.

1868. Scudder. Worthen, Geol. surv. Ill., iii : 567-568. Fig. 3.

Megablattina kliveri. See I, HeмıPт., Idern.

343. Meganeura monyi. (Protophasmida.)

Locality: Commentry, France. Horizon: Carboniferous.

1885. Brongniart. Bull. soc. amis se. Rouen, (3), xxi : 60 .

1885. " " Rev. scient., (3), xxxvi: 277 .

$1885 . \quad$ " Geol. mag., (3), ii: 487.

1885. " " Trans. geol, soc. Manch., xviii: 279-280.

1885. “ $\quad$ Foss. ins. prim. rocks, 11.

See also Dictyoneura monyi, etc.

344. Meganeura (Dictyoneura) monyi. (Protophasmida.)

Locality : Commentry, France. Horizon: Carboniferous.

1886. Brauer. Ann. naturhist. hofmus., i: 107-108, 114.

See also Dictyoneura monyi, etc.

\section{Megaptilus blanchardi.}

Locality : Commentry, France. Horizon: Carboniferous.

1885. Brongniart. Bull. soc. amis sc. Rouen, (3), xxi : 61.

1885. “ Rev. scient., (3), xxxvi: 277.

1885. " " Geol. mag., (3), ii : 488.

1885. " " Trans. geol. soc. Manch., xviii : 281.

1885. " " Foss. ins. prim. rocks, 13.

1885. “ Jahrb. geol. reichsanst, xxxv: 657.

1886. Braver. Ann. naturh. hofmus., i: 109, 110.

See also Titanophasma fayoli.

346. Mylacris anthracophila. (Palæobląttariæ.)

Locality : Colchester, Ill. Horizon: Lower Coal Measures.

1868. Seudder. Worthen, Geul. surv. Ill., iii : 568-570. Figs. 5, 6.

1879. " Mem. Bost. soc. nat. hist., iii: 45-47 (anthracophilum). Pl. 5, figs. 6-8.

1883. Lacoe. List pal. foss. ins., 11-12.

1884. Scudder. Mem. Bost. soc. nat. hist., iii: 307.

1885. " "Zittel, Handb. palaeont., i, ii: 754. Fig. 930.

1886. " "Zittel-Barrois, Traité de paléont., ii : 754. Fig. 947.

1886. " " Miall, Cockroach, 212. Fig. 121.

1887. Woodward. Geol. mag., (3), iv: 55. Fig. 5.

1889. Lesley. Dict. foss. Penns., i: 435 . Fig.

1889. Miller. N. A. geol. and pal., 579. Figs. 1091, 1092.

1890. Scudder. Foss. ins. N. A., i : 65-67, 271. Pl. 5, figs. 6-8. 
347. Mylacris antiqua. (Palæoblattariæ.)

Locality : Mazon Creek, Ill. Horizon : Carboniferous.

1884. Scudder. Mem. Bost. soc. nat. hist., iii : 300-301 (antiquum).

1889. Lesley. Dict. foss. Penns., i: 435.

18*9. Miller. N. A. geol, and pal., 579.

1890. Scudder. Foss. ins. N. A., i : 264-265.

348. Mylacris bretonensis. (Palæoblattariæ.)

Locality : Sydney, Cape Breton. Horizon : Middle Carboniferous.

1879. Scudder. Mem. Bost. soc. nat. hist., iii : 41-42 (bretonense). Pl. 5, fig. 1.

1883. Lacoe. List pal. foss. ins., 12.

1884. Scudder. Mem. Bost. soc. nat. hist., iii : 300.

1889. Lesley. Lict. foss. Penns., i: 435.

1889. Miller. N.A.geol. and pal., 579.

1890. Scudder. Foss. ins. N. A., i : 61-62, 264. Pl. 5, fig. 1.

See also Blattina bretonensis.

349. Mylacris carbonum. (Palæoblattariæ.)

Localities: Wilkes Barre, Cannelton, Pa. Horizon : Carboniferous.

1883. Scudder. MSS.

1883. Lacoe. List pal. foss. ins., 12.

1884. Scudder. Mem. Bost. soc. nat. hist., iii : 304-307. Pl. 27, figs. 6,7, 10.

1889. Lesley. Dict. foss. Penns., i: 435.

1889. Miller. $\quad$ N. A. geol. and pal., 579.

1890. Scudder. Foss. ins. N. A., i : 268-271. Pl. 13, figs. 6, 7, 10.

350. Mylacris heeri. (Palæoblattariæ.)

Locality : Sydney, Cape Breton. Horizon : Middle Carboniferous.

1879. Scudder. Mem. Bost, soc. nat. hist., iii : 43-44. Pl. 5 , fig. 11.

1883. Lacoe. List pal. foss. ins.; 12.

1884. Scudder. Mem. Bost. søc. nat. hist., iii : 300 .

1889. Lesley. Dict. foss. Penns., i : 435.

1889. Miller. $\quad$ N. A. geol. and pal., 579.

1890. Scudder. Foss. ins. N. A., i: 63-64, 264. Pl. 5, fig. 11.

See also Blattina heeri.

351. Mylacris lucifuga. (Palæoblattariæ.)

Locality: Pittston, Pa. Horizon: Carboniferous.

1883. Scudder. Mss. (lucifugum).

1883. Lacoe. List pal. foss. ins., 12.

1884. Scudder. Mem. Bost. soc. nat. hist., iii: 301-302. Pl. 27, fig. 8.

1889. Lesley. Dict. foss. Penns., i : 435.

1890. Scudder. Foss. ins. N. A., i: 265-266. Pl. 13, fig. 8.

352. Mylacris mansfieldi. (Palæoblattariæ.)

Locality : Cannelton, Pa. Horizon: Lower Carboniferous.

1879. Scudder. Mem. Bost. soc. nat. hist., iii : 47. Pl. 5, fig. 15.

1883. Lacoe. List pal. foss. ins., 12.

1884. Scudder. Mem. Bost. soc. nat. hist., iii : 308.

1889. Lesley. Dict. foss. Penns., i : 436. Fig.

1890. Seudder. Mem. Bost. soc. nat. hist., iv: 377-378.

1890. “ Foss, ins. N. A., i : 67, 272, 401-402. Pl. 5, fig. 15. 
353. Mylacris ovalis. (Palæoblattariæ.)

Locality : Cannelton, Pa. Horizon: Carboniferous.

1883. Sendder. Mss. (ovale).

1883. Lacoe. List pal. foss. ins., 12.

1884. Scudder. Mem. Bost. soc. nat. hist., iii : 308-309. Pl. 27, fig. 5.

18-9. Lesley. Dict. foss. Penns., i: 436.

1889. Miller. N. A. geol. and pal., 579 .

1890. Scudder. Foss. ins. N. A., i : 272-273. Pl. 13, fig. 5.

354. Mylacris packardii. (Palæoblattariæ.)

Locality: Bristol, R. I. Horizon: Carbouiferous.

1885. — Rand. notes nat. hist., ii : 64 (name only).

See also Blatta americana.

355. Mylacris pennsylvanica. (Palæoblattariæ.)

Locality: Cannelton, Pa. Horizon: Carboniferous.

1879. Scudder. Mem, Bost. soc. nat. hist., iii : 44-45 (pennsylvąnicum). Pl. 5,

1883. Lacoe. figs. 13-14.

1884. Scudder.

List pal. foss. ins., 12-13.

1889. Lesley.

1889. Miller.

1890. Scudder.

Mem. Bost. soc. nat. hist., iii : 302-304. Pl. 27, fig. 11.

Dict. foss. Penns., i: 436. 2 figs.

N. A. geol. and pal., 579.

Foss. ins. N. A., i : 64-65, 266-268. $\quad$ Pl. 5, figs. 13, 14; Pl.13, fig. 11.

356. Mylacris priscovolans. (Palæoblattariæ.)

Locality: Cannelton, Pa. Horizon: Carboniferous.

1883. Scudder. Mss.

1883. Lacoe. List pal. foss. ins., 13.

1884. Scudder. Mem. Bost. soc. nat. hist., iii: 307-308. Pl. 27, fig. 9.

1889. Lesley. Dict. foss. Penns., i: 436.

1889. Miller. N. A. geol. and pal., 579.

1890. Scudder. Foss.ins. N. A., i: 271-272. Pl. 13, fig. 9.

357. Necymylacris heros. (Palæoblattariæ.)

Locality : Cannelton, Pa. Horizon: Lower Carboniferous.

1879. Scudder.

1883. Lacoe.

Mem. Bost. soc. nat. hist., ii : 54-56. Pl. 5, fig. 9.

1889. Miller.

List pal. foss. ins., 13.

1890. Lesley.

N. A. geol. and pal., 579.

1890. Scudder.

Dict. foss. Penns., ii : 448. Fig.

Foss. ins. N. A., i : 74-76. Pl. 5, fig. 9.

358. Necymylacris lacoana.. (Palæoblattariæ.)

Locality: Vicinity Pittston, Pa. Horizon: Lower Carboniferous.

1879. Scudder.

1883. Lacoe.

Mem. Bost. soc. nat. hist., iii : 53-54 (lacoanum). $\quad P l .5$, fig. 12.

1889. Miller.

List. pal. foss. ins., 13.

1890. Lesley.

N. A. geol. and pal., 579 .

1890. Scudder.

Dict. foss. Penns., ii : 448 . Fig.

Foss. ins. N. A., i : 73-74. Pl. 5, fig. 12.

359. Grischia williamsonii.

Locality : Commentry, France. Horizon: Carboniferous.

1885. Brongniart. Bull. soc. amis se. Rouen, (3), xxi : 58-59. Pl. 1, fig. 3. 
360. Oryctoblattina arndti. (Palæoblattariæ.)

Locality : Třemošna, Bohemia. Horizon: Carboniferous.

1888. Kušta. Veštn. král. česke spol. nauk, 1888: 387-393. Fig.

361. Oryctoblattina oblonga. (Palæoblattariæ.)

Locality: Weissig, Germany. Horizon: Dyas.

1882. Deichmiiller. Sitzungsb. gesellsch. Isis, 1882: 41-42. $\quad P l$. 1, fig. 4.

362. Oryctoblattina occidua. (Palæoblattariæ.)

Locality: Mazon Creek, Ill. Horizon: Carboniferous.

1885. Scudder.

Proc. acad. nat. sc. Philad., 1885: 37.

1889. Miller.

N. A. geol. and pal., 579.

1890. Lesley.

Dict. foss. Penns., ii : 503.

1890. Scudder.

1890. " "

Mem. Bost. soc. nat. hist., iv : 414-415. $P l .32, f i g .3$.

Foss, ins. N. A., i : 390-391. Pl. 24, fig. 3.

363. Oryctoblattina reticulata. (Palæoblattariæ.)

Locality: Wettin, Saxony. Horizon: Upper Carboniferous.

1879. Scudder. Mem. Bost. soc. nat. hist., iii : 122-123. Pl. 4, fig. 13.

1890. " Foss. ins. N.A., i: 142-143. Pl. 4, fig. 13.

See also Blattina reticulata.

364. Pachytylopsis borinensis. (Protophasmida.)

Locality: Mons, Belgium. Horizon: Carboniferous.

1875. Borre. Comptes rend. soc. ent. Belg., (2), xii : 17.

1875. " Ann. soc. ent. Belg., xviii: 4-5. Pl. 5, fig. 2; Pl. 6, fig. 2.

1875. " Empr. d'ins. foss., 4-5. Pl. 5, fig. 2; Pl. 6, fig. 2.

1875. Giard. Bull. se. hist. litt. dép. Nord, vii : 122-127.

See also Breyeria borinensis.

Pachytylopsis persenairei. See I, Neuropt., Idem.

Palæoblattina douvillei. See I, NeUropt, Idem.

365. Paolia gurleyi. (Protophasmida.)

Locality: Orange County, Iud. Horizon: Carboniferous.

1885. Scudder. Proc. Amer. acad. arts sc., xx: 173.

1889. Miller. N. A. geol. and pal., 580.

1890. Lesley. Dict. foss. Penns., ii : 595.

366. Paolia lacoana. (Protophasmida.)

Locality : Pittston, Pa. Horizon: Carboniferous.

1885. Scudder. Proc. Amer. acad. arts se., xx: 173.

1889. Miller. N. A. geol. and pal., 580 .

1890. Lesley. Dict. foss. Penns, ii : 595.

367. Paolia superba. (Protophasmida.)

Locality : Mazon Creek, Ill. Horizon: Carboniferous.

1885. Scudder. Proc. Amer. acad. arts se, $x x: 173$.

1889. Miller. $\quad$ N. A. geol. and pal., 580.

1890. Lesley. Dict, foss, Penus, , ii : 595. 
368. Paolia vetusta. (Protophasmida.)

1871. Smith.

Locality : Paoli, Ind. Horizon: Carboniferous.

18\%1. "“

Amer.journ. sc., (3), i : 44-46. Fig.

Notice foss. ins., 1-3. Fig.

1883. Lacoe.

List pal. foss. ins., 13.

1885. Scudder.

1885. " "

Proc. Amer. acad. arts sc., xx: 173.

1886. "“

Zittel, Handb. palaeont., i, th. ii : 758. Fig. 942.

1889. Miller.

Zittel-Barrois, Traité de paléont., ii : 758. Fig.958.

1×90. Lesley.

N. A. geol. and pal., 580 .

Dict. foss. Penns., ii : 596. Fig.

369. Paromylacris ampla. (Palæoblattariæ.)

Locality: Mazon Creek, Ill. Horizon: Carboniferous.

1890. Scudder. Mem. Bost. soc. nat. hist., iv: 408. Pl.31, fig. 7.

1890. " Foss. ins. N. A., i: 384. Pl.23, fig.7.

370. Paromylacris rotunda. (Palæoblattariæ.)

Locality : Mazon Creek, Ill. Horizon: Carboniferous.

1883. Scudder. Mss. (rotundum).

1883. Lacoe. List pal. foss, ins., 13.

1885. Scudder. Proc. acad. nat. sc. Philad., 1885: 35.

1885. " Zittel, Handb. palaeont., i, th. ii: 754.

1886. " " Zittel-Barrois, Traité de paléont., ii: 754 .

1886. " Bull. U. S. geol. surv., No. $31: 38$.

1889. Miller. $\quad$ N. A. geol. and pal., 580.

1890. Lesley. Dict. foss. Penns, ii: 602.

1890. Scudder. Mem. Bost. soc. nat. hist., iv: 406-408. Pl. 32, figs. 1-2.

1890. " Foss, ins. N. A., i : 382-384. Pl. 24, figs. 1-2.

371. Petrablattina gracilis. (Palæoblattariæ.)

Locality: Lebach, Germany. Horizon: Dyas.

1879. Scudder. Mem. Bost. soc. nat. hist., iii: 124-125. Pl. 4, fig. 4.

1890. " $\quad$ Foss.ins. N. A., i: 144-145. Pl. 4, fig. 4.

See also Blattina gracilis, Blatta gracilis.

\section{Petrablattina sepulta. (Palæoblattariæ.)}

Locality: Sydney, Cape Breton. Horizon: Lower Carboniferous.

1879. Scudder. Mem. Bost. nat. hist., iii : 125-126. Pl. 6, fig. 7.

1883. Lacoe. List pal. foss. ins., 14.

1889. Miller. $\quad$ N. A. geol. and pal., 580 .

1890. Lesley. Dict. foss. Penns., ii : 627.

1890. Scudder. Foss. ins. N. A., i: 145-146. Pl. 6, fig. 7.

See also Blattina sepulta.

Petrablattina subtilis. See I, Neuropt., Idem.

373. Polioptenus elegans. (Protophasmida.)

Locality: Dudweiler, Germany. Horizon: Carboniferous.

1885. Scudder. Proc. Amer. acad. arts sc., xx: 170.

1885. " Zittel, Handb. palaeont., i, th. ii: 756. Fig. 938.

1886. " Zittel-Barrois, Traité de paléont., ii : 757. Fig. 954.

1886. “ Bull. U. S. geol. surv., No. 31: 40.

See also Dictyoneura elegans, Goldenbergia elegans. 
374. Polyzosterites granosus. (Palæoblattariæ.)

Locality : Jägersfrende, Saarbriick, Germany. Horizon: Carboniferous. 1867. Goldenberg. Vorweltl, fanna steink. Saarbr., 18. Pl. 1, fig. 17 (ined.). 1873. " " Fanna saræp. foss., i: 18. Pl. 1, fig. 17.

1877. " " Fauna saræp. foss., ii : $20,51$.

1879. Scudder. Mem. Bost. soc. 1uat. hist., iii: 129.

1890. " Foss ins. N. A., i: 149.

See also Adelophthalmus (Eurypterus) granosus.

375. Progonoblattina fritschii. (Palæoblattariæ.)

Locality: Manebach, Thüringen. Horizon: Upper Carboniferous.

1879. Seudder. Mem. Bost. soc. nat. hist., iii : 120-121. Pl. 3, fig. 12.

1885. " Zittel, Handb. palaeont., i, th. ii: 755. Fig. 934.

1886. " " Zittel-Barrois, Traité de paléont., ii: 755. Fig. 951.

1890. " Foss. ins. N. A., i : 140-141. Pl. 3, fig. 12.

See also Blattina fritschii.

376. Progonoblattina helvetica. (Palæoblattariæ.)

Locality: Erbignon, Switzerland. Horizon: Carboniferous.

1879. Scudder. Mem. Bost. soc. nat. hist., iii : 119-120. Pl. 3, fig. 10.

1887. Woodward. Geol. mag., (3), iv: 50. Figs, 1, 2.

1889. Nicholson. Man. palæont., 3d ed., i : 593. Fig. 443.

1890. Scudder. Foss. ins. N. A., i: 139-140. Pl. 3, fig. 10.

See also Blattina helvetica, Blatta helvetica.

377. Promylacris ovalis. (Palæoblattariæ.)

Locality: Mazon Creek, Ill. Horizon: Carboniferous.

1885. Scudder. Proc. acad. nat. sc. Philad., 1885: 34-35 (ovale).

1885. " Zittel, Handb. palaeont., i, th. ii: 754 .

1886. " "Zittel-Barrois, Traité de paléont., ii : 754.

1886. “ Bull. U. S. gool. surv., No. 31: 38 .

1889. Miller. $\quad$ N. A. geol. and pal., $580 . \bullet$

1890. Lesley. Dict. foss. Penus., ii : 772.

1890. Scudder. Mem. Bost. soc. nat. hist., iv : 403, 404 . Pl. 31, figs. 1-4.

1890. " - Foss. ins. N. A., i: 379, 380. Pl. 23, figs. 1-4.

378. Promylacris rigida. (Palæoblattariæ.)

Locality: Mazon Creek, Ill. Horizon : Carboniferous.

1890. Scudder. Mem. Bost. soc. nat. hist., iv : 403, 404-405. Pl. 31, fig. 6.

1890. " Foss. ins. N. A., i : 379, 380-381. Pl. 23, fig. 6.

379. Promylacris testudo. (Palæoblattariæ.)

Locality : Mazon Creek, Ill. Horizon: Carboniferous.

1890. Scudder. Mem. Bost. soc. nat. hist., iv: 403. Pl. 32, fig. 6.

1890. " Foss, ins. N. A., i: 379. Pl. 24, fig. 6.

Protogryllacris brongniarti. See I, Neuropt., Idem.

380. Protophasma dumasii. (Protophasmida.)

Locality: Commentry, France. Horizon: Carboniferous.

1878. Brongniart. Compt. rend. soc. ent. Belg., (2), xlvii : 9-12.

1878. "6 Découv. orth. coureur, 1-4,

Bull. $71-5$ 
1878. Brongniart.

1878.

1878.

1879.

1879.

1879. Scudder.

1879. Girard.

1880. Scudder.

18×3. Brongniart.

1883.

1883.

1885. Seudder.

1885. " "

1886. “

1886. " "

1886. Brauer.

1890. Scudder.
Ann. sc. nat., (6), vii, art. 1. Pl. 6, figs. 1-4,6,9.

Nouv. genre orthop. foss., 1-9. Pl. 6, figs. 1-4,6, 9.

Bull. séanc. soc. ent. France, 1878: 69-70.

Geol. mag., (2), vi : 97-102. $\quad P l .4$, figs. 1-4,6, 9.

New foss. ins. coal-meas., 1-6. Pl. 4, figs., 1-4,6,9.

Mem. Bost. soc. nat. hist., iii : 19.

La nature, vii : 108-110. Fig.

Arch. sc. phys. nat., (3), iii : 365 .

Bull. ś́anc. soc. ent. France, 1883: 20-21.

Naturaliste, v : 267.

Aperçu ins. foss., 3,5 .

Proc. amer. acad. arts sc., $x x: 170$.

Zittel, Handb. palaeont., i, th. ii : 757. Fig. 939.

Zittel-Barrois, Traité de pal6́ont, ii : 756. Fig. 955 on p. 757.

Bull. U. S. geol, surv., No. 31 : 40.

Ann. naturhist. hofmus. i: 116.

Foss. ins. N. A., i : $\$ 9$.

381. Protophasma gaudryi. (Protophasmida.)

Locality : Commentry, France. Horizon: Carboniferous.

1885. Brongniart. Bull. soc. amis sc. Rouen, (3), xxi : 59. Pl. 2, fig. 1.

382. Protophasma woodwardii. (Protophasmida.)

Locality: Commentry, France. Horizon: Carboniferous.

1883. Brongniart. Bull. séanc, soc. ent. France, 1883: 20-21.

1883. " Naturaliste, v: 267.

1883. " " Aperçu ins. foss., 5.

Prototettix lithanthraca. See I, Neuropt., Idem.

\section{Spilaptera libelluloides.}

Locality : Commentry, France. Horizon: Carboniferous.

1885. Brongniart, Bull. soc, amis sc Rouen, (3), xxi : 63.

1885. " " Geol. mag., (3), ii : 489.

1885. " " Trans. geol. soc. Manch., xviii : 283.

1885. “ "Foss. ins. prim. rocks, 15.

1885. “ Jahrb, geol, reichsanst., xxxv: 658 .

384. Spilaptera packardi.

Locality: Commentry, France. Horizon: Carboniferous.

1885. Brongniart. Ins. foss. terr. prim., 63. Pl. [5], fig. 1.

1885. “ Bull. soc. amis sc. Rouen, (3), xxi : 63.

1885. " " Geol. mag., (3), ii: 489.

1885. " " Trans. geol. soc. Manch., xviii : 283.

1885. “ " $\quad$ Foss. ins. prim. rocks, 15.

1885. “ J Jahrb. geol. reichsanst., xxxv: 658.

1886. Brauer. Anp. naturh. hofmus., i: 110. Pl. 8, fig. 16.

385. Spilaptera venusta.

Locality: Commentry, France. Horizon: Carboniferous.

1885. Brongniart. Bull. soc. amis sc. Rouen, (3), xxi: 63.

1885. " " Geol. mag., (3), ii : 489.

1885. “ " Trans. geol. soc. Manch., xviii : 283.

1885. " " Foss. ins. prim. rocks, 15.

1885. “ Jahrb. geol, reichsanst., $x \times x \vee: 658$. 
386. Sthenarocera pachytyloides.

Locality : Commentry, France. Horizon: Carboniferous.

1885. Brongniart. Bull. soc. amis. sc. Rouen, (3), xxi : 59. Pl. 1, figs. 1-2.

1885. " Rev. scient., (3), xxxvi : 276.

\section{Sthenaropoda filholi.}

Locality : Commentry, France. Horizon: Carboniferons.

1885. Brongniart. Bull. soc. amis. sc. Rouen, (3), xxi: 59. Pl. 1, fig. 5.

\section{Sthenaropoda fischeri.}

Locality : Commentry, France. Horizon : Carboniferous.

1885. Brongniart. Bull. soc. amis. sc. Rouen, (3), xxi : 59. Pl. 1, fig. 4.

389. Termes affinis. (Protophasmida.)

Locality: Sulzbach, Germany. Horizon: Carboniferous.

1852. Goldenberg. Sitzungsb. math.-nat. cl. akad. wiss. Wien, ix : 39 (undescr.). 1877. " Fauna saræp. foss:, ii : 50.

See also T. (Eutermopsis) affinis, T. (ralotermes) affinis, Dictyoneura affinis. Goldenbergia affinis; also III, NeuropT., Termes (Calotermes) affinis.

390. Termes (Calotermes) affinis. (Protophasmida.)

Locality : Altenwald, Germany. Horizon: Carboniferous.

1867. Goldenberg. Vorweltl. fanna steink. Saarbr., 11. Pl. 2, fig. 5 (ined.),

1873. " Fauna saræp. foss., i : 11. Pl. 2, fig. 6 (not 5).

See also Termes affinis, etc.; also III, Neuropt, Termes (Calotermes) affinis.

391. Termes (Eutermopsis) affinis. (Protophasmida.)

Locality : Friedrichsthal, Germany. Horizon: Carboniferous.

1854. Goldenberg. Palaeontogr., iv : 31-32. Pl. 6, fig. 1.

1854. " " Foss. ins. Saarbr., 15-16. Pl. 4, fig. I.

1856. Giebel. Insect. d. vorwelt, 298.

1857. Hagen. Verhandl. naturh. ver. prenss. Rheinl. Westph., xiv: 42-43.

See also Termes affinis, etc.

392. Termes dechent. (Protophasmida.)

Locality : Altenwald, Germany. Horizon: Carboniferous.

1885. Brauer. Sitzungsb. akad. wiss. Wien, xci : 281.

See also T. (Eutermopsis) decheni, etc.

393. Termes (Calotermes) decheni. (Protophasmida.)

Localities: Gersweiler, Altenwald, Germany. Horizon: Carboniferous.

1867. Goldenberg. Vorweltl. fanna steink. Saarbr., 10-11. Pl. 2, figs. 3-5 (ined.). 1873. " Fauna saræp. foss., 10-11. Pl. 2, figs. 3-5.

See also T. (Eutermopsis) decheni, etc.

394. Termes (Eutermopsis) decheni. (Protophasmida.)

Locality : Altenwald, Germany. Horizon: Carboniferous.

1854. Goldenberg. Palaeontogr., iv: 31. Pl. 5, fig. 3.

1854. “ Foss. ins. Saarbr., 15. Pl. 3, fig. 3.

1856. Giebel. Insect. d. vorwelt, 297-298.

185\%. Hagen. Verhandl. naturh. xer. preuss. Rheinl. Westph., xiv : 42-43.

See also Termes decheni, Dictyoneura decheni, Goldenbergia decheni, Termes. (Calotermes) decheni, Termes (Eutermopsis) formosus, etc. 
395. Termes (Eutermopsis) formosus. (Protophasmida.)

Locality : Gersweiler, Germang. Horizon : Carboniferous.

1854. Goldenberg. Palaeontogr., iv : 30-31. Pl. 5, fig. 2.

1854. " Foss. ins. Saarbr., 14-15. Pl. 3, fig. 2.

1856. Giebel. Insect. d. vorwelt, 297.

1857. Hagen. Verhandl, natủrh. ver. preuss. Rheinl. Westph., xiv : 42-43.

1860. " Linn. entom., xii: 296.

1867. Goldenberg. Vorweltl. fauna steink. Saarbr., 10 (=T. decheni).

See also Goldenbergia formosa, Dictyoneura formosa, Termes (Eutermopsis) decheni, etc.

396. Termes haidingeri. (Termitina.)

Locality : Flenu, Belgium. Horizon: Carboniferous.

1881. Mourlon. Géol. Belg., ii : 57.

See also I, Neuropt., Termes?_— Borre.

\section{Termes heeri. (Protophasmida.)}

Locality : Sulzpach, Germany. Horizon: Carboniferous.

1852. Goldenberg. Sitzungsb. math.-nat. cl. akad, wiss. Wien, ix : 39 (undeser.).

See also T. (Eutermopsis) heeri, etc.

398. Termes (Calotermes) heeri. (Protophasmida.)

Locality : Altenwald, Germany. Horizon: Carboniferous.

1867. Goldenberg. Vorweltl, fauna steink. Suarbr., 9-10. Pl. 2, fig. 2 (ined.).

1873. " " Fauna saræp. foss., i: 9-10. Pl. 2, fig. 2.

1877. " Fauna saræp. foss., ii: 50.

See also T. (Eutermopsis) heeri, etc.

399. Termes (Eutermopsis) heeri. (Protophasmida:)

Locality : Altenwald, Germany. Horizon: Carboniferous.

1854. Goldenberg. Palaeontugr., iv: 29-30. $\quad P l .4, f i g .5$.

1854. “ Foss. ins. Saarbr., 13-14. Pl. 2, fig. 5.

1856. Giebel. Insect. d. vorwelt., 297.

1857. Hagen. Verhandl. naturh. ver. preuss. Rheinl. Westph., xiv : 42-43.

1860. " Linn. entom., xii : 295-296.

See also Termes heeri, Calotermes heeri, Termes (Calotermes) heeri, Goldenbergia heeri.

Termes (Calotermes) humboldti. See Termes (Caloternes) humboldtianus.

400. Termes (Calotermes) humboldtianus. (Protophasmida.)

Locality : Altenwald, Germany. Horizon : Carbonıferous.

1867. Goldenberg. Vorweltl, fauna steink. Saarbr., 11-12 (humboldtiana). Pl. 2, fig. 8 (ined.).

1873. “ Fanna saræp. foss., 11-12 (humboldti on plate). Pl. 2, fig. 8.

See also Dictyoneura humboldtiana, etc.

401. Termes incertus. (Protophasmida.)

Locality : Dudweiler, Germany. Horizon: Carboniferous.

1880. Kliver.

Palaeontogr., xxxii : 104-106. Pl. 14, fig. 5. 
402. Termes laxus. (Protophasmida.)

Locality: Dudweiler, Germany. Horizon: Carboniferous. 1877. Goldenberg. Fauna saræp. foss., ii : 17,50 (laxa). Pl. 1, fig. 5. 1885. Brauer. Sitzungsb. akad. wiss. Wien, xei: 281.

See also Litoneura laxa, Goldenbergia laxa.

\section{Termes longitudinalis.}

Locality : Mazon Creek, Ill, Horizon: Carboniferous.

1883. Scudder. Mss.

1883. Lacoe. List pal. foss. ins., 15.

1885. Scudder. Mem. Bost. soc. nat. hist., iii : 350.

1890. " Foss. ins. N. A., i: 314.

See also Goldenbergia longitudinalis.

404. Termes parvulus. (Prophasmida.)

Locality: Michelberg, Saarbrück, Germany. Horizon: Carboniferous. 1886. Kliver. Palaeontgr., xxxii : 106-107. Pl. 14, fig. 6.

Termidium amissum. See Termitidium amissum.

405. Termitidium amissum.

Locality: Altenwald, Germany. Horizon: Carboniferous,

1877. Goldenberg. Fauna saræp. foss., ii : 17, 50 (Termidium in pl.). Pl. 1, fig. 6. 1885. Scudder. Mem. Bost. soc. nat. hist., iii : 350 .

1890. “ Foss. ins. N. A., i : 314.

See also Goldenbergia amissa.

406. Termitidium? rugosum.

Locality : Near Cusel, Rheinbayern. Horizon: Carboniferous.

1877. Goldenberg. Fauna saræp. foss,, ii: 17-18,50. Pl. 1, fig. 14.

1885. Scudder. Mem. Bost. soc. nat. hist., iii : 350 .

1890. " Foss. ins. N. A., i: 314.

See - Dohrn.

\section{Titanophasma fayoli. (Protophasmida.)}

Locality : Commentry, France. Horizon: Carboniferous.

1882. Brongniart. Comptes rend. acad. sc., xcv : 1228-1230.

1882. " " Nouv. ins. foss., 1-3.

1883. " " Compt. reud. soc. geol. Fr., 1883: 15-16.

1883. " Bull. soc. géol Fr., (3), xi: 241.

1883. “ Bull. séances soc. ent. Fr., 1883: 20.

1883. Scudder. Science, i: 95-96. Fig.

1883. Brongniart. Bull. soc. géol. Fr., (3), xi : 145-146. Pl. 4, fig. 3.

1883. " Naturaliste, v : 267-268.

1883. " " Aperȩu ins. foss., 6-8.

1885. Scudder. Proc. Amer. acad. arts sc., xx: 169.

1885. " Zittel, Handb. palaeont., i, ii: 756. Fig. 937.

1886. " - Zittel-Barrois, Traité de paléont., ii: 756. Fig. 953.

1888. Vidal. Ins. y plantas, 4, 10.

See also Megaptilns blanchardi. 
408. Titanophasma jucunda. (Protophasmida.)

1885. Scudder. Proc. Amer. acad. arts sc., xx: 169.

Locality: Pittston, Pa. Horizon: Carboniferous.

1889. Miller.

N. A. geol. and pal., 581.

See also Dictyoneura jucunda.

409. Titanophasma libelluloides. (Protophasmida.)

Locality : Germany. Horizon: Carboniferous.

1885. Scudder. Proc. Amer. acad. arts sc., xx: 169.

See also Dictyoneura libelluloides.

THE NEUROPTEROID SERIES.

410.

Locality : Raṿenhead, near Liverpool, England. Horizon : Carboniferous. 1871. Higgins. Pres. address (Liverp. nat. field club), 10,11. Pl., fig. 15.

411.

Locality : Reden, Saarbrück, Germany. Horizon: Carboniferous.

1886. Kliver. Palaeontogr., xxxii : 110. Pl. 14, fig. 10.

412.

Locality: Rakonitz, Bohemia. Horizon: Carboniferous.

1885. Kušta. Sitzungsb. böhm. gesellsch. wiss., 1885: 594.

1885. " Neue foss. arthrop. Rak., 5.

413.

Locality : Alfredgrube near Königshütte, Silesia. Horizon : Carboniferous. 1884. Roemer. Jahresb. schles. gesellsch. vater. cult., xxvi : 226.

414. - - (Homothetidæ.)

Locality: Mazon Creek, Ill. - Horizon : Carboniferous.

1885. Scudder. Mem. Bost. soc. nat. hist., iii : 329. Pl. 30, fig. 7.

1890. “ Foss. ins. N. A., i: 293. Pl. 16, fig. 7.

415. Acridites carbonarius. (Hemeristina.)

Locality : Wettin, Saxony. Horizon: Carboniferous.

1842. Germar. Münst., Beitr. z. petref., v: 93-94. Pl. 13, fig. 5 .

184-. " " Voigt, Naturw. ber. ver. Harzes (carbonatus).

1851. “ " Verstein. steink. Wettin, 87 (=Blattina didyma?). Pl. 31, fig.10.

1856. " " Voigt, Naturw. ber. ver. Harzes, 1840-'46: 26 (carbonatus).

See also Blattina carbonaria, etc., Megathentomum carbonatum.

Acridites carbonatus. See Acridites carbonarius, Megathentomum carbonatum.

416. Acridites formosus. (Gerarina.)

Locality : Fischbach, Saarbrück, Germany. Horizon: Carboniferous.

1867. Goldenberg. Vorweltl. fauna steink. Saarbr., 18-20. Pl. 2, fig. 18 (ined.).

1873. "6 Fauna saræp. foss., i : 18-20. Pl. 2, fig. 18.

1876. Scudder. Proc. Bost. soc. nat. hist., xviii : 359 .

1877. Goldenberg. Fauna saræp. foss., ii : 51.

See also Megathentomum formosum. 
417. Acridites goldenbergi. (Gerarina.)

Locality : Steinbachthal, Saarbrück, Germany. Horizon : Carboniferous. 1886. Kliver. Palaeontogr., xxxii: 108-109. Pl. 14, fig. 9.

418. Acridites priscus. (Homothetidæ.)

Locality: Stradonitz, Bohemia. Horizon : Carboniferous.

1864. Andree. Neues jahrb. min., 1864: 163-164. Pl. 4, fig. 1.

1874. Friě. $\quad$ Arch. naturw. landesdurchf. Böhm., 2, ii, i: 15. , Pl. 3, fig. 3.

1884. Kušta. Vesmír, xiii : 97-98.

1885. Scudder. Mem. Bost. soc. nat. hist., iii : 325.

1885. Brongniart. Bull. soc. anis se. Rouen, (3), xxi : 63 .

$1885 . \quad$ " Geol. mag., (3), ii : 489.

1885. " " Trans. geol. soc. Manch., xviii : 283.

1885. " " Foss. ins. prim. rocks, 15.

1885. " J Jahrb. geol. reichsanst., xxxv : 658.

1885. Scudder. Zittel, Handb. palaeont, i, tb. ii : 759.

1886. " " Zittel-Barrois, Traité de paléont., ii : 759.

1886. “ Bull. U. S. geol. surv., No. 31: 41.

1890. " " Foss. ins. N. A., i: 289.

Adaphlebia lacoana. See Adiphlebia locoana.

419. Adiphlebia lacoana. (Gerarina.)

Locality: Mazon Creek, Ill. Horizon: Carboniferous.

1885. Scndder. Mem. Bost. soc. nat. hist., iii : 345. Pl. 32, fig. 6.

1885. " Zittel, Handb. palaeont, i, th. ii : 763.

1886. " "Zittel-Barrois, Traité de paléont., ii : 763.

1886. “ Bull. U. S. geol. surv., No. 31: 45.

1889. Lesley. Dict. foss. Penns, i: p. ii (Adaphlebia).

1889. Miller. N. A. geol. and pal., 575 .

1890. Scudder. Foss. ins. N. A., i : 309. Pl. 18, fig. 6.

420. \#thophlebia singularis. (Palæopterina.)

Locality : Mazon Creek, Ill. Horizon: Carboniferous.

1885. Scudder. Mem. Bost. soc. nat. hist., iii : 338. Pl. 31, fig. 9.

1886. “ Bull. U. S. geol. surv., No. 31: 43.

1886. Brauer. Ann. naturh. hofmus., i: 113.

1889. Lesleg. Dict. foss. Penns., i : p. ii.

1889. Miller. $\quad$ N. A. geol. and pal, 575.

1890. Scudder. Foss. ins. N. A., i : 302. Pl. 17, fig. 9.

421. Anthracothremma robusta. (Homothetidø.)

Locality : Mazou Creek, Ill. Horizon : Carboniferous.

1885. Scudder. Mem. Bost. soc. nat. hist., iii : 327 . Pl. 30, figs 1,5,6.

1886. " Bull. U. S. geol. surv., No. 31: 42 .

1889. Lesley. Dict. foss. Penns., i: p. v.

1889. Miller. N. A. geol. and pal, 575.

1890. Scudder. Foss. ins. N. A., i : 291. Pl. 16, figs. 1, 5, 6 .

422. Archæoscolex corneus. (Larva.)

Locality : St. John, New Brunswick. Horizon : Cordaite Shales, Lower Devonian 1889. Matthew. Trans. roy. soc. Can., 1888, iv : 59-60. Pl. 4, fig. 11.

Archimantis carbonarius. See Lithomantis carbonarius. 


\section{Blattina carbonaria. (Hemeristina.)}

Locality: Wettin, Saxony. Horizon: Carboniferous.

1864. Heer. Viert. naturf. gesellsch. Zürich, ix : 288, No. 15.

187\%. Guldenberg. Fauna samp. foss., ii : 20.

See also Lithusialis carbonaria, Acridites carbonarius; also I, ORTHOPT., Blattina carbotaria, B. didyma, ete.

\section{Brachyptilus}

Locality : Commentry, France. Horizon : Carboniferous.

1885. Brougniart. Bull. soc. amis sc. Roven, (3), xxi : 66 .

Breyeria borinensis. See I, ORTHOPT., Idem.

425. Brodia priscocincta. (Hemeristina.)

Locality: Tipton, Staffordshire, England. Horizon: Carboniferous.

1881. Scudder. Geol. mag., (2), viii : 293-295, 300. Fig.

18-3. " Mem. Bost. soc. nat. hist., iii : 213-217, 223. Pl. 17, figs. 3-7.

1865. " Mem. Bost. soc. nat. hist., iii : 340.

1885. Bronguiart. Bul'. soe. amis. se. Rouen, (3), xxi : 63.

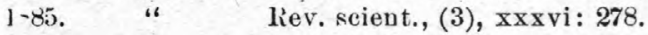

1×85. " " Geol. mag., (3), ii: 489.

1285. " " Traus. Manch. geol. soc., xviii: 284.

1885. " " Foss. ins. prim. rocks, 15.

1835. " " Jahrb. geol. reichsanst., $\mathrm{xxxv}: 658$.

1885. Scudder. Zittel, Handb. palaeont., i, th. ii : 761. Fig. 951.

18sii. " Zittel-Barrois, Traité de paléont., ii : 761. Fig. 967.

1-8ii. Brauer. Ann, naturh. hofmus., i: 113.

1500. Seudder. Foss. ins. N. A., i : 235-239, 245, 304. Pl. 11, figs. 3-7.

Calotermes hageni. See I, Arachnida, Idem.

Calotermes heeri. See I, Orthopt., Idem.

426. Campyloptera

Locality : Commentry, France. Horizon : Carboniferous.

1-85. Brongniart. Bull, soc. amis. sc. Rouen, (3), xxi : 63.

185.). " Rev, scient., (3), xxxvi: 278.

Chelephlebia carbonaria. See Cheliphlebia carbonaria.

Chelephlebia elongata. See Cheliphlebia elongata.

427. Cheliphlebia carbonaria. (Homothetidæ.)

Locality: Mazon Creek, Ill. Horizon: Carboniferous.

18×5. Sendler. Mem. Bost. soc. nat. hist., iii : 328. Pl. 30, fig. 8.

1886. “ Bull. IT. S. geol. surv., No. $31: 42$.

1289. Lesley. Dict. foss. Penns., i : p. xiii (Chelephlebia).

1889. Miller. N. A. geol, and pal., 575 (not the figure).

1890. Sendder. Foss. ins. N. A., i: 292. Pl. 16, fig. 8.

428. Cheliphlebia elongata. (Homothetidæ.)

Locality : Mazon Creek, Ill. Horizon: Carboniferous.

1885. Scudder. Mem. Bost. soe, nat. hist., iii: 328-329. Pl. 29, fig. 7.

1886. " Bull. U. S. geol surv., No. 31: 42 . 
1889. Lesley. 1889. Miller. 1890. Scudder.
Dict. foss. Penns., i : p. xiii (Ćhelephlebia).

N. A. geol. and pal., 575 ,

Foss. ins. N. A., i : 292-293. Pl. 15, fig. 7.

429. Chrestotes danæ. (Gerarina.)

Locality : Mazon Creek, Ill. Horizon : Carboniferous.

1885. Brongniart. Bull. soc: amis. sc. Rouen, (3), xxi: 66 .

1885. " "Geol. mag., (3), ii : 491.

1885. " " Trans. geol. soc. Manch., xviii : 287.

1885. " " Foss. ins. prim. rocks, 18.

1885. " Jahrb, geol, reichsanst, $\mathrm{xx \times v:660.}$

See also Miamia danæ, Gerarus danæ.

\section{Chrestotes lapidea. (Hemeristina.)}

Locality: Mazon Creek, Ill. Horizon: Carboniferous.

1868. Scudder.

1883. Lacoe.

1885. Scudder.

1885. Brongniart.

1885.

1885.

1885.4

1885.

1885. Scudder.

1886. ". "

1886. “

1886. Brauer.

1889. Lesley.

1889. Miller.

1890. Scudder.

Worth., Geol. surv. Ill., iii : 567. Frig.2.

List pal. foss. ins., 6 .

Mem. Bost. soc. nat. hist., iii : 341. $\quad$ Pl. 31, fig. 2.

Bull. soc. amis. sc. Rouèn, (3), xxi : 66.

Geol. mag., (3), ii : 491.

Trans. geol, soc. Manch., xviii : 287.

Foss. ins. prim. rocks, 18.

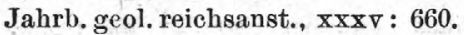

Zittel, Handb. palaeont.; i, th. ii : 762. Fig. 952.

Zittel-Barrois, Traité de pal6ont., ii : 762. Fig. 968.

Bull. U. S. geol. surv., No. 31 : 44.

Ann. naturh. hofmus., i : 114 (Chrestotus).

Dict. foss. Penns., i : 130. Fig.

N. A. geol. and pal., 575-576. Fig. 1076.

Foss. ins. N. A., i: 305. Pl. 17, fig. 2.

431. Chrestotes lugauensis. (Homothetidæ.)

Locality : Lugan, Saxony. Horizon: Carboniferous.

1885. Brongniart. Bull. soc. amis. sc. Rouen, (3), xxi : 66.

1885. “ Geol. mag., (3), ii: 491.

1885. " " Trans. geol. soc. Manch., xviii: 287.

1885. " " Foss. ins. prim. rocks, 18.

1885. " J Jahrb. geol. reichsanst., xxxv: 660 .

See also Mixotermes lugauensis, etc.

Chrestotus lapidea. See Chrestotes lapidea.

432. Corydalis - (Hemeristina.)

Locality: Coalbrookdale, England. Horizon : Carboniferous.

1833. Audouin. Anu. soc. ent. France, bull., 7-8.

1839. Mantell. Wonders of geology, ii : 680 .

1839. Murchison. Silurian system, i: 104-105. Fig. a.

1854. " Siluria, 284. Fig. 1.

1854. Pictet. Traité pal., $2^{\mathrm{e}}$ ed., ii : 377-378 (Sialidae). Pl. 40, fig. 1.

1854. Mantell. Medals of creation, 2d ed., ii : 556 (Purbecks). Fig. $182 a b$.

See also Lithosialis brongniarti, etc. 


\section{Corydalis brongniarti. (Hemeristina.)}

Locality : Coalbrookdale, England. Horizon: Carboniferous.

1844. Mantell. Medals of creation, ii : 575, 578. Lign. 124, fig. 2.

1854. " Medals of creation, $2 \mathrm{~d}$ ed., ii : 551,554. Lign. 181, fig. 2.

1859. Murchison. Siluria, 3d ed., 320. Foss. 81, fig. 1 on p. 321.

1874. Swinton. Geol. mag., (2), i: 3-5.

1874. "6 Foss. orth., 3-5.

See also Lithosialis brongniarti, etc.

434. Corydaloides gracilis.

Locality : Commentry, France. Horizon : Carboniferons.

1885. Brongniart. Bull, soc. amis. sc. Rouen, (3), xxi : 64-65.

1885. “ Rev. scient., (3), xxxvi : 278.

1885. " " Geol. mag., (3), ii: 490.

1885. “ $\quad$ Trans. geol. soc. Manch., xviii : 285.

1885. " " Foss. ins. prim. rocks, 16 .

1885. " Jahrb, geol. reichsanst., xxxv : 659 .

435. Corydaloides scudderi.

Locality : Commentry, Francé. Horizon: Carboniferous.

1885. Brongniart. Bull. ś́ances soc. ent. Fr., 1885: 13.

1885. Scudder. Mem. Bost. soc. nat. hist., iii : 350 .

1885. Brongniart. La nature, ann. xiii, ii : 156. Figs. 1, 2.

1885. " " Ins. foss. terr. prim., 64. Pl. [4], fig. 3.

1885. " " Bull. soc. amis sc. Rouen, (3), xxi : 64-65.

1885. " " Rev. scient., (3), xxxvi : 278.

1885. " " Geol. mag., (3), ii : 490. Pl. 12, fig. 3.

1885. “ " Trans. geol. soc. Manch., xviii: 285. Pl., fig. 3.

1885. " " Foss. ins. prim. rocks, 16.

1885. “ Jahrb. geol. reichsanst., xxxv: 659.

1886. Brauer. Ann. naturh. hofmus., i: 106-107.

1886. - Pop. sc. news, xx: 62. Figs. 1, 2.

1890. Scudder. Foss. ins. N. A., i : 314.

436. Dasyleptus lucasi. (Thysanura.)

Locality: Commentry, France. Horizon: Carboniferous.

1885. Brongniart. Bull. seances soc. ent. Fr., 1885 : 101-102.

1885. " Bull. soc. amis sc. Rouen, (3), xxi : 58-59.

1885. " $\quad$ Rev, scient., (3), xxxvi: 276.

1885. “ Geol. mag., (3), ii : 486.

1885. " Trans. geol. soc. Manch., xviii : 278.

1885. " " Foss. ins. prim. rocks, 9-10.

1885. “ Jahrb. geol. reichsanst., xxxv: 655.

1886. Brauer. Ann. naturh. hofmus., i: 88 (lukasii).

1890. Brongniart. Psyche, iv : 353.

Dasyleptus lukasii. See Dasyleptus lucasi.

437. Diaphanoptera

Locality : Commentry, France. Horizon: Carboniferous.

1885. Brongniart. Bull. soc. amis se. Rouen, (3), xxi : 66 .

Dictyoneura spp. See I, ORTHOPT., Idem. 


\section{Dic:yophlebia protogæa.}

Locality: Malstatt, Germany. Horizon: Carboniferous. 1852. Goldenberg. Sitznngsb. math.-nat. cl. akad. wiss. Wien., ix: 39 (nndescr.).

439. Didymophleps contusa. (Homothetidæ.)

Locality : Vermilion County, Ill. Horizon: Carboniferous.

1885. Seudder.

1885. " "

1886.

1886. " "

1886. Brauer.

1889. Lesley.

18:9. Miller.

1890. Scudder.

Mem. Bost. soc. nat. hist., iii : 330-331. Pl. 29, fig. 6.

Zittel, Handb. palaeont., i, th. ii : 760 .

Zittel-Barrois, Trait6́ de pal6́ont., ii : 759.

Bull. U. S. geol. surv., No. 31: 42.

Ann, naturh. hofinus., i: 113 .

Dict. foss. Penns., i: p. $\mathrm{xx}$.

N. A. geol. and pal, 576.

See also Termes contusus, Goldenbergia (Didymophleps) contusa, Goldenbergia contusa.

440. Dieconeura arcuata. (Palæopterina.)

Locality: Mazon Creek, Ill. Horizon: Carboniferous.

1885. Scudder. Mem. Bost. soc. nat. hist., iii : 336. Pl. 30, fig. 4.

1886. “ Bull. U. S. geol. surv., No. 31: 43.

1889. Lesley. Dict. foss. Penns, i: p. $\mathrm{xx}$.

1889. Miller. N. A. geol. and pal., 576 .

1890. Scudder. Foss. ins. N. A., i : 300. Pl. 16, fig. 4.

441. Dieconeura rigida. (Palæopterina.)

Locality: Pittston, Pa. Horizon: Carboniferuus.

1885. Scudder. Mem. Bost. soc. nat. hist., iii : 336-337. Pl. 29, fig. 10.

1886. " Bull. U. S. geol. surv., No. 31: 43 .

1889. Lesley. Dict. foss. Fenus., i : 202. Fig.

1889. Miller. N. A. geol. and pal., 576.

1890. Scudder. Foss. ins. N.A., i : 300-301. Pl. 15, fig. 10.

\section{Dyscritus vetustus.}

Locality: St. John, New Brunswick. Horizon: Devonian.

1865. Scudder.

1865. " "

1865. " "6

1865.

1865. "6

1868. " "

1880 . "

1881. Hagen.

188:3. Lacoe.

1885. Brongniart.

1855.

1835.

185. " 6

1885.

1880. Brauer.

1889. Lesley.

1889. Miller.

1890. Scudder.
Dev. ins. N. Brunsw., 1 (unnamed).

Bailey, Obs. geol. south. N. Brunsw., 140 (unnamed).

Amer. journ. sc., (2), xxxix: 357 (unnamed).

Can. nat., n. s., ii : 234 (unnamed).

Trans. ent. soc. Lond., (3), ii : 117 (unnamed).

Geol. mag., v: 17\%, 176.

Dev. ins. N. Brunsw. (Anniv. mem. Bost. soc. nat. hist.), 20-22. Pl. 1, fig. 4.

Bull. mus. comp. zool., viii : 281.

List pal. foss. ins., 6 .

Bull. soc. amis sc. Ronen, (3), xxi : 56.

Geol. mag., (3), ii: 485.

Trans. geol, soc. Manch., xviii : 276.

Foss. ins. prim. rock:, 8 .

Jahrb. geol. reichsanst., xxxv: 653.

Ann. naturh. hofmus., i: 112.

Dict. foss. Penns., i: p. $\mathrm{xx}$.

N. A. geol. and pal., 576.

Foss. ins. N. A., i: 172-173. Pl. 7, fig. 4. 
443. Ephemerites affinis.

Locality : Morris, Ill: Horizou: Carboniferous.

1868. Sendder. Worth., Geol. surv. Ill., iii : 572 (Euphemerites). Fig. 10.

1885. " Mem. Bost. soc. nat. hist., iii : 350 .

1889. Miller. $\quad$ N. A. geol. and pal., 576.

1890. Sendder. Foss. ins. N. A., i : 314.

See also Euephemerites affinis.

(Probably not an insect.)

444. Ephemerites gigas.

Locality : Morris, Ill. Horizon : Carboniferous.

1868. Scudder. Worth., Geol. surv. Ill., iii : 571-572 (Euphemerites). Fig. 9. 1885. " Mem. Bost. soc. nat. hist., iii : 350 .

1889. Miller. N. A. geol. and pal., 576.

1890. Scudder. Foss. ins. N. A., i : 314.

See also Euephemerites gigas.

(Probably not an insect.)

445. Ephemerites rückerti. (Palephemeridæ.)

Locality : Reitsch near Stockheim, Germany. Horizon : Dyas.

1865. Geinitz. Nenes jahrb. min., 1865: 385-389. Pl. 2, fig. 1.

1865. Hagen. Neues jahrb. min., 1865: 386-388.

1880. Sendder. Dev. ins. N. Bruusw., 3t, note.

1885. " " Mem. Bost. soc. nat. hist., iii : 324.

1885. “ Zittel, Handb. palaeont., i, th. ii : 758.

1886. " "Zittel-Barrois, Traité de palént., ii : 758.

1886. “ Bull. U. S. geol. surv., No. 31: 41 .

1890. “ Foss. ins. N. A., i: 183 uote, 288.

446. Ephemerites simplex.

Locality : Morris, Ill. Horizon: Carboniferous.

1868. Seudder. Worth., Geol. surv. Ill., iii : 571 (Euphemerites). Fig. 8.

1885. " Mem. Bost. soc. nat. hist., iii : 350 .

1889. Miller. N. A. geol. and pal., 576.

1890. Scudder. Foss. ins. N. A., i: 314

(Probably not an insect.)

See also Euephemerites simplex.

447. Eucænus ovalis. (Homothetidæ.)

Locality : Mazon Creek, III. Horizon: Carboniferous.

1885. Scudder. Mem. Bost. soc. nat. hist., ii : 325. Pl. 29, fig. 4.

1886. " " Bull. U. S. geol. surv., No. $31: 42$.

1889. Lesley. Dict. foss. Penns, i, p. xxiii.

1889. Miller. N. A. geol. and pal., 576 (Encænus).

1890. Scudder. Foss. ins. N. A., i : 289. Pl. 15, fig. 4.

448. Euephemerites affinis.

Locality: Illinois. Horizon: Carboniferous.

1883. Lacoe. List pal. foss. ins., 7.

1889. " Lesley, Dict. foss. Penus., i, p. xxiii.

(Probably not an insect.)

See also Ephemerites affinis. 
449. Euephemerites gigas.

Locality : Illinois. Horizon: Carboniferous.

1883. Lacoe. List pal. foss. ins., 7.

1889. " L Lesley, Dict. foss. Penus., i, p. xxiii.

(Probably not an insect.)

See also Ephemerites gigas.

450. Euephemerites primordialis.

Locality: Pennsylvania? Horizon: Carboniferous.

1878. Scudder. Proc. Bost. soc. nat. hist., xix : 248-249.

1878. " " Entom. notes, vi : $37-38$.

1883. Lacoe. - List pal. foss. ins., 7.

1885. Scudder. Mem. Bost. soc. nat. hist., iii : 350.

1889. Lacoe. Lesley, Dict. foss. Penns., i, p. xxiii.

1890. Scudder. Foss. ins. N. A., i: 314.

(A plant.)

451. Euephemerites simplex.

Locality : Ilinois. Horizon: Carboniferous.

1883. Lacoe. List pal. foss. ins., 7.

1889. " Lesley, Dict, foss. Penns., i, p. xxiii.

(Probably not an insect.)

See also Ephemerites simplex.

Euphemerites affinis. See Ephemerites affinis.

Euphemerites gigas. See Ephemerites gigas.

Euphemerites simplex. See Ephemerites simplex.

Eutermopsis spp. See I, ORThopt., Idem.

452. Genentomum validum. (Homothetidæ.)

Locality: Mazon Creek, Ill. Horizon: Carboniferous.

1885. Scudder. Mem. Bost. soc. nat. hist., iii : 329-330. Pl. 30, figs. 2, 3.

1885. " "Zittel, Handb. palaeont., i, th. ii : 759. Fig. 945.

1886. " " Zittel-Barrois, Traite de pal6ont., ii : 759. Fig. 961.

1886. " " Bull. U. S. geol. surv., No. 31: 42 .

1886. Brauer. Ann. naturh. hofmus., i: 113.

1889. Lesley. Dict. foss. Penns., i: 253, xxv. Fig.

1889. Miller. N. A. geol. and pal., 577. Fig. 1082.

1890. Scudder. Foss. ins. N. A., i : 293-294. Pl. 16, figs. 2, 3.

453. Genopteryx constricta. (Homothetidæ.)

Locality : Mazon Creek, Ill. Horizon : Carboniferous.

1885. Scudder. Mem. Bost. soc. nat. hist., iii: 327-328. Pl. 29, fig. 11.

1886. " Bull. U. S. geol. surv., No. 31: 42.

1889. Lesley. Dict. foss. Penns., i, p. xxv.

1889. Miller. N. A. geol. and pal., 577.

1890. Scudder. Foss. ins. N. A., i : 291-292. Pl. 15, fig. 11. 
454. Genopteryx lithanthraca. (Homothetidæ.)

Locality : Saarbrück, Germany. Horizon: Carboniferous.

1885. Scudder. Mem. Bost. soc. nat. hist., iii : 328.

1885. " "Zittel, Handb. palaeont, i.th.ii: 759. Fig. 944.

1886. " "Zittel-Barrois, Traité de pal6ont., ii: 759. Fig. 960.

1886. “ Bull. U. S. geol. surv., No. 31: 42 .

1886. Brauer. Ann. naturh. hofmus., i: 113.

1890. Scudder. Foss. ins. N. A., i: 292.

See also Gryllacris lithanthraca, etc.

Gerapompus blatinoides. See Gerapompus blattinoides.

455. Gerapompus blattinoides. (Homothetidæ.)

Locality : Mazon Creek, Ill. Horizon: Carboniferous.

1885. Scudder. Mem. Bost. soc. nat. hist., iii : 326. Pl. 29, fig. 1.

1886. " Bull. U. S. geol. surv., No. 31: 42.

1889. Miller. N. A. geol, and pal., 577 (blatinoides).

1890. Scudder. Foss. ins. N. A., i: 290. Pl. 15, fig. 1.

456. Gerapompus extensus. (Homothetidæ.)

Locality: Mazon Creek, Ill. Horizon: Carboniferous.

1885. Scudder. Mem. Bost. soc. nat. hist., iii : 326. Pl. 29, figs. 5, 8.

1886. " Bull. U. S. geol. surv., No. 31: 42 .

1889. Miller. N. A. geol. and pal., 577.

1890. Scudder. Foss. ins. N. A., i: 290. Pl. 15, figs. 5, 8.

\section{Gerarus? - (Gerarina.)}

Locality : Mazon Creek, Ill. Horizon : Carboniferous.

1885. Scudder. Mem. Bost. soc. nat. hist., iii : 344. Pl. 32, fig. 3.

1890. “ Foss. ins. N. A., i: $308 . \quad$ Pl. 18, fig. 3.

458. Gerarus? —. (Gerarina.)

Locality: Mazon Creek, Ill. Horizon: Carboniferous.

1885. " " Mem. Bost soc. nat. hist., iii : 345. Pl. 32, fig. 5

1890. " Foss. ins. N. A., i: 309. Pl. 18, fig. 5.

\section{Gerarus danæ. (Gerarina.)}

Locality: Mazon Creek, Ill. Horizon: Carboniferons.

1885. Scudder. Mem. Bost. soc. nat. hist., iii : $345 . \quad P l .31$, fig. 5.

1885. " Zittel, Handb. palaeont,, i, th. ii: 762. Fig. 953.

1886. " " Zittel-Barrois, Traité de paléont., ii : 762. Fig. 969.

1886. “ Bull. U. S. geol. surv., No. 31: 44.

1889. Miller. N. A. geol. and pal., 577. Fig. 1084.

1890. Scudder. Foss. ins. N. A., i : 309. Pl. 17, fig. 5.

See also Miamia danæ, Chrestotes danæ.

460. Gerarus mazonus. (Gerarina.)

Locality : Mazon Creek, Ill. Horizon: Carboniferous.

1885. Scudder. Mem. Bost. soc. nat. hist., iii : 344-345. Pl. 32, fig. 7.

1886. “ Bull. U. S. geol. surv., No. 31: 44.

1889. Miller. N. A. geol. and pal., 577.

1890. Scudder. Foss. ins. N. A., i : 308-309. Pl. 18, fig. 7. 
461. Gerarus vetus. (Gerarina.)

Locality: Mazon Creek, Ill. Horizon: Carboniferous.

1885. Scudder. Mem. Bost. soc. nat. hist., iii : 344. Pl. 31, fig. 6.

1886. “ Bull. U. S. geol. surv., No. 31: 44.

1889. Miller. N. A. geol. and pal., 577 .

1890. Scudaer. Foss. ins. N. A., i: 308. Pl. 17, fig. 6.

Gerephemera simplex. See I, ORTHоPт., Idem.

462. Geroneura wilsoni.

Locality: Lancaster, N. B. Horizon: Cordaite shales, Lower Devonian.

1889. Matthew. Trans, roy. soc. Can., 1888, iv: 57-59. Pl. 4, fig. 10.

1889. Miller. N. A. geol, and pal., 577.

Goldenbergia spp. See I, ORTHOPT., Idem.

463. Goldenbergia contusa.

Locality : Illinois. Horizon: Carboniferons.

1885. Brongniart. Bull, soc. amis sc. Rouen, (3), xxi : 61.

1885. “ Geol. mag., (3), ii: 488.

1885. “ Trans. geol. soc. Manch., xviii : 281.

1885. “ " Foss. ins. prim. rocks, 13.

1885. “ Jahrb. geol. reichsanst., $\mathrm{x} \times \mathrm{xv}: 657$.

See also Didymophleps contusa, etc.

464. Goldenbergia (Didymophleps) contusa.

1886. Braver. Ann. naturh. hofmus, i: 114.

See also Didymophleps contusa, etc.

465. Gryllacris bohemica.

Locality: Stradonitz, Bobemia. Horizon : Carboniferous.

1880. Novák. Jahrb. geol. reichsanst., xxx: 69-74. Pl. 2, fig. 1-3.

i884. Kušta. Vesmír, xiii : 97-98. Fig. 1.

See also Lithosialis bohemica.

466. Gryllacris brongniarti. (Hemeristina.)

Locality: England. Horizon: Carboniferous.

1880. Nov亿k. Jahrb. geol. reichsanst, $\mathrm{xxx}:$ 73-74. Pl. 2, fig. 4.

See also Lithosialis brongniarti, etc.

467. Gryllacris (Corydalis) brongniarti. (Hemeristina.)

Locality : England. Horizon: Carboniferons.

1874. Swinton. Geol. mag., (2), i: 5. Pl. 14, fig. 3.

1874. " Foss. orth., 5. Pl., fig. 3.

1876. Woodward. Quart. journ. geol. soc. Lond., xxxii: 60. Pl.9, fig. 2.

See also Lithosialis brongniarti, etc.

468. Gryllacris lithanthraca. (Homothetidæ.)

Localities: Russhütte, Fischbach bei Saarbrück, Germany. Horizon: Carboniferous.

1852. Goldenberg. Sitzungsb. math.-nat. cl. akad. wiss. Wien, ix : 39 (undescr.). 
1854. Goldenberg. Palaeontogr., iv: 24-27. Pl. 4, figs. 1, 2. 1854. "

1865. Lyell. Elem. geol., 6th ed., 491. Fig. 549.

1876. Goldenberg. Vorweltl. fauna steink. Saarbr., 20. Pl. 2, fig. 17 (ined.).

1873. " " Fauna saræp. foss, i: 20. Pl. 2, fig. 17.

1877. " Fauna saræp. foss., ii : 51.

See also Prototettix lithanthraca, Genopteryx lithanthraca, Lithophasma lithanthraca.

469. Hemeristia occidentalis. (Hemeristina.)

Locality : Morris, Ill. Horizon: Carboniferous.

1864. Dana.

1865. Scudder.

1865. "6

1866. 66

1866. "

1868. “

1876. Gerstäcker.

1877. Goldenberg.

1883. Lacoe.

1885. Scudder.

1885. Brongniart.

1885.

1885.

1885. “

$1885 . \quad$ "

1885. Scudder.

1886. "6

1886. “

1886. Brauer.

1889. Miller.

1890. Scudder.
Amer. journ. sc., (2), xxxvii : 35. Fig. 2.

Amer. journ. sc., (2), xl: 269-271.

Proc. Bost. soc. nat. hist., $\mathrm{x}$ : 96.

Mem. Bost. soc. nat. hist., i : 191-192. Pl. 6, figs. 1, 3.

Zool. relat. foss. neur. ins., 19-20. $\quad \mathrm{Pl}$., figs. $1,3$.

Geol. mag., v : 217-218.

Klass. ordn. arthr., Crust., i: 292 (an ephemerid).

Fauna saræp. foss., ii : 15-16 (Hemeristica).

List pal. foss. ins., 9.

Mem. Bost. soc. nat. hist., iii : 342 .

Bull. soc. amis. sc. Rouen, (3), xxi : 66.

Geol. mag., (3), ii : 491.

Trans. geol. soc. Manch., xviii : 287.

Foss. ins. prim. rocks, 18.

Jahrb. geol. reichsanst, $\mathrm{xx \times v}: 660$.

Zittel, Handb. paleont., i, th. ii: 762.

Zittel-Barrois, Traité de paléont., ii : 762.

Bull. U. S. geol. surv., No. 31 : 44.

Ann. naturh. hofmus., i: 113 . Pl. 8, fig. 17.

N. A. geol. and pal., 578.

Foss. ins. N. A., i: 19-20, 306. Pl. 1, figs. 1, 3.

Eemeristica occidentalis." See Hemeristia occidentalis.

470. Homaloneura elegans.

Locality : Commentry, France. Horizon: Carboniferous.

1885. Brongniart. Bull. soc. amis sc. Rouen, (3), xxi : 66. Pl. 3, fig. 2.

1886. Brauer. Ann. naturh. hofmus., i: 108-109.

471. Homothetus fossilis. (Homothetidæ.)

Locality: St. John, New Brunswiek. Horizon: Devonian.

1865. Scudder.

1865. " "

1865. "

1865. " "

1865. “

1867. " "

1867. " "

1868. " "

1868. "6

1868. " "

1872. Packard.

1880. Scudder.
Dev. ins. N. Brunsw., 1 (unnamed).

Bailey, Obs. geol, south. N. Brunsw, 140 (unnamed).

Amer. journ. sc., (2), xxxix: 357 (unnamed).

Can. uat., n. s., ii : 235 (unnamed).

Trans. ent. soc. Lond., (3), ii : 117 (unnamed).

Can. nat., (2), iii : 205. . Pl. 3.

Geol. mag., iv : 387. Pl. 17, fig. 3.

Dawson, Acad. geol., 2d ed., 524-525. Fig. 182.

Amer. nat., i: 631. Pl. 16, fig. 7.

Geol. mag., v : 172, 176.

Guide ins., $3 \mathrm{~d}$ ed., 78. $\quad \mathrm{Pl} .1, \mathrm{fig} .7$.

Dev. ins. N. Brunsw. (Anniv. mem. Bost. soc. nat. hist.), 17-20.

Pl. 1, figs. 1, 2. 
18ช0. Dawson. Chain of life, 140. Fig. $123 b$.

1581. Hagen.

Bull. mus. comp. zool., viii : 278-279.

1883. Lacoe.

List pal. foss. ins., 9 .

1885. Scudder.

Mem. Bost. soc. nat. hist., iii : 331.

1885.

1885. Brongniart.

1885 .

Earl. winged ins. Amer., 5-6. Pl., fig. 1 ; fig. on p. 5.

1885 .

Bull. soc. amis sc. Rouen, (3), xxi : 56 .

Geol. mag., (3), ii : 485.

1885. " " Foss. ins. prim. rocks, 8.

1885.

1885. Hagen.

1885. " "

1885. " "

Trans. geol. soc. Manch., xviii : $2 \% 6$.

Jahrb. geol. reichsanst., xxxv : 653.

1885. Scudder.

1886. " "

1886. “ "

Nature, xxxii : 54.

Dev. ins., 2.

Zool. anz., viii : 29\%.

1886. Braner.

1889. Miller.

Zittel, Handb. palaeont., i, th. ii: 760. Fig.946.

1890. Scudder.

Zittel-Barrois, Traité de paleont., ii : 759. Fig. 962.

Bull. U. S. geol. surv., No. 31 : 42.

Ann. naturh. hofmus., i : 111-112.

N. A. geol. and pal., 578. Fig. 1087.

Foss. ins. N. A., i : 169-172, 279-280,295. Pl. 7, figs. 1,2; pl. 14, fig. 1 ; fig. on $p .279$.

472. Lamproptilia elegans.

Locality : Commentry, France. Horizon : Carboniferous.

1885. Brongniart. Bull, soc. amis sc. Rouen, (3), xxi : 63.

1885. " " Geol. mag., (3), ii : 489.

1885. " " Trans. geol. soc. Manch., xviii: 283.

1885. " " Foss. ins. prim. rocks, 15.

1885. " J Jahrb, geol, reichsanst., $\times x \times v: 658$.

\section{Lamproptilia grand'euryi.}

Locality : Commentry, France. Horizon : Carboniferous.

1885. Brongniart. Bull. soc. amis sc. Ronen, (3), xxi : 63. Pl. 3, fig. 1.

1885. " Rev. scient., (3), xxxvi: 277. Fig. 4.

1885. 6: Geol. mag., (3), ii : 489.

1885. " " Trans. geol. soc. Manch., xviii : 283. Fig.

1885. " " Foss. ins. prim. rocks, 15.

1885. " " Jahrb. gęol. reichsanst., xxxv: 658.

1886. Brauer. Ann, naturh, hofmus, i: 109-110. Pl.8, fig.14.

474. Lamproptilia priscotincta.

Locality: Commentry, France. Horizon : Carboniferous.

1885. Brongniart. Bull. soc. amis sc. Rouen, (3), xxi : 63.

1885. " " Geol. mag., (3), ii : 489.

1885. “ " $\quad$ Trans. geol. soc. Manch., xviii : 283.

1885. " " Foss. ins. prim. rocks, 15.

$1885 . \quad$ Jahrb. geol, reichsanst., $\operatorname{xxxv:658.}$

\section{Ieptoneura}

Locality : Commentry, France. Horizon: Carboniferous.

1885. Brongniart. Ins. foss. terr. prim. $\mathrm{Pl}$. [5], fig. 3.

1886. Brauer. Ann. naturh. hofmus., i: 110.

Bull. $71-6$ 
476. Leptoneura delicatula.

Locality : Commentry, France. Horizon: Carboniferous

1885. Brongniart. Bull. soc. amis sc. Rouen, (3), xxi: 62.

1885. " " Geol.mag., (3), ii : 488.

1885. " " Trans, geol. soc. Manch., xviii : 282.

1885. " " Foss. ins. prim. rocks, 14。

1885. " " Jahrb. geol. reichsanst,, xxxv: 657 .

\section{Leptoneura elongata.}

Locality: Commentry, France. Horizon: Carboniferons.

1885. Brongniart. Bull. soc. amis sc. Rouen, (3), xxi : 62.

1885. " Geol. mag., (3), ii: 488.

1885. " " Trans. geol. soc. Manch., xviii : 282,

1885. " " Foss. ins. prim. rocks, 14.

1885. " J Jahrb, geol, reichsanst., $\mathrm{xxxv}: 657$.

478. Ieptoneura oustaleti.

Locality: Commentry, France. Horizon: Carboniferous.

1885. Brongniart. Ins. foss. terr. prim., 62. Pl. [5], fig. 4.

1885. " Bull. soc. amis sc. Rouen, (3), xxi: 62.

1885. " " Geol. mag., (3), ii : 488.

1885. " " Trans. geol. soc. Manch., xviii : 282.

1885. "6 Foss. ins. prim. rocks, 14.

1885. “ Jahrb. geol. reichsanst, $\mathrm{xxxv}: 65 \%$.

1886. Brauer. Ann. naturh. hofmus., i: 110.

\section{Leptoneura robusta.}

Locality : Commentry, France. Horizon: Carboniferous.

1885. Brongniart. Bull. soc. amis sc. Rouen, (3), xxi : 62.

1885. " " Geol. mag., (3), ii : 488.

1885. " " Trans. geol, soc. Manch., xviii : 28\%.

1885. " " Foss. ins. prim. rocks, 14.

1885. " Jahrb. geol, reichsanst, $\mathrm{xxxv}: 657$.

Libellula carbonaria. See I, Arachnida, Ibid.

Lithentomum hartii.. See Lithentomum harttii.

480. Lithentomum harttii. (Hemeristina.)

Locality: St. John, N. B. Horizon : Devonian.

1865. Scudder.

Dev. ins. N. Brunsw., 1 (unnamed).

1865. " "

1865. " "

Bailey, Obs. geol. south. N. Brunsw., 140 (unnamed).

Amer. journ. sc., (2), xxxix : 357 (unnamed).

1865. “ Can. nat., n. s., ii : 235 (unnamed).

1865. “ "Trans. ent. soc. Lond., (3), ii : 117 (unnamed).

1867. " Can nat., (2), iii: 206. Pl. 4.

1867. " Geol. mag., iv: 387. Pl. 17, fig. 4.

1868. " " Dawson, Acad. geol., 2d ed.,525. Fig. 183.

1868. " Amer. nat., i: 630 (hartii). Pl. 16, fig. 5.

1868. “ Geol. mag., v: 172,176.

1872. Packard. Guide ins., 3 d ed., 78 (hartii). Pl. 1 , fig. 5.

1880. Scudder. Dev. ins. N. Brunsw. (Anniv. mem. Bost. soc. nat. hist.), 22-24. Pl. 1, fig. 3.

1880. Dawson. Chain of life, 140. Fig. $123 c$. 
1881. Hagen. Bull. mus. comp. zool, viii: 278.

1883. Lacoe. List pal. foss. ins., 10.

1085. Scudder. Earl. winged ins. Amer., 4. Pl., figs. 2, 3.

1885. " Mem. Bost. soc. nat. hist., iii : 341.

1585, Brongniart. Bull. soc. amis se. Rouen, (3), xxi : 56.

1885. " " Geol. mag., (3), ii : 485.

1885. “ Trans. geol. soc. Manch., xviii: 275-276.

1885. " " Foss. ins. prim. rocks, 7 .

1885. " J Jabrb. geol. reichsanst., xxxv: 653.

1885. Scudder. Zittel, Handb. palaeont., i, th. ii : 761 .

1886. " Zittel-Barrois, Traite de pal6ont., ii : 761.

1886. " Bull. U. S. geol. surv., No. 31: 44.

1886. Brauer. Ann. naturh. hofmus, i: 111, 114.

18๙9. Lesley.

1889. Miller.

Dict. foss. Penns, i : \$51.

1890. Scudder.

N. A. geol. and pal., 578-579. Fig. 1088.

Foss. ins. N. A., i: 174-175, 278,305. Pl. 7, fig. 3 ; Pl. 14, figs. 2, 3.

481. Lithomantis carbonaria. (Hemeristina.)

Compared with Blepharis domina, Abyssinia.

Locality: Scotland. Horizon: Carboniferous.

1876. Woodward. Quart. journ. geol. soc. Lond., xxxii : 60-64 (carbonarius). Pl. 9, fig. 1.

1879. Scudder.

1880. " "6

Mem. Bost. soc. nat. hist., iii : 18 note 2 (Archimantis).

1881. " "

1881. " "

1883. " "

1885. " "

1885.

1886. "

1886. ‘

1886. Brauer.

Areh. se. phys. nat., (3), iii : 363.

Geol. mag., (2), viii : 296-300.

Harv. univ. bull., ii : 175.

Mem. Bost. soc. nat. hist., iii : 219, 220, 223.

Mem. Bost. soc, nat. hist., iii : 340.

Zittel, Handb. palaeont., i, th. ii : 761. Fig. 950.

Zittel-Barrois, Traité de paléont., ii: 761. Fig. 966.

Bull. U. S. geol. surv., No. 31 : 44.

1890. Scudder.

Ann. naturh. hofmus., i : 113.

Foss. ins. N. A., i : 38 note, 241, 242, 245, 304.

482. Iithophasma lithanthraca. (Homothetidæ.)

Locality : Saarbrüek, Germany. Horizon : Carboniferong.

1885. Brongniart. Bull. soc. amis sc. Rouen, (3), xxi : 59.

1885. " " Geol. mag., (3), ii : 487 (lithantraca).

1885. " " Trans. geol. soc. Manch., xviii: 279 .

1885. " " Foss. ins. prim. rocks, 11.

1885. " " Jahrb. geol, reichsanst., xxxv: 656.

See also Gryllacris lithanthraca, etc.

Iithophasma lithantraca. See Lithophasma lithanthraca.

483. Iithosialis bohemica. (Hemeristina.)

Locality: Bohemia. Horizon: Carboniferous.

1881. Scudder. Proc. Bost. soc. nat. hist., xxi : 167.

1881. " " Harv, univ. bull., ii: 175.

1885. " " Mem. Bost. soc. nat. hist., iii : 341 .

1885. " Zittel, Handb palaeont, i, th. ii : 761 .

1886. " "Zittel-Barrois, Traité de paléont., ii : 761.

1886. " Bnll. U. S. geol, surv., No. 31: 44.

1890. " Foss. ins. N. A., i : 304.

See also Gryllacris bohemica. 
484. Lithosialis brongniarti. (Hemeristina.)

Locality: Coalbrookdale, Shropshire, Englend. Horizon: Carboniferous. 1881. Scudder. Proc. Bost. soc. nat. hist., xxi : $16 \%$.

1881. " Harv. univ. bull., ii : 175.

1881. " "Geol. mag., (2), viii : 296-300.

1883. " " Mem. Bost. soc. nat hist., iii : 220-223. Pl. 17, fig. 1, 2,8,9.

1885. " Mem. Bost. soc. nat. hist., iii : 340.

1885. " " Zittel, Handb. palaeont., i, th. ii: 761,822.

1886. " Z Zittel-Barrois, Traité de pal6ont., ii : 761,824.

1886. " "Bull. U. S. geol. surv., No. $31: 44,104$.

1890. " Foss. ins. N. A., i : 242-245, 304. Pl. 11, figs. 1, 2, 8,9.

See also Corydalis — Aud., Corydalis brongniarti, Gryllacris brongniarti, Gryllacris (Corydalis) brongniarti, Protogryllacris brongniarti, Mantispa Keferst.

485. Iithosialis carbonaria. (Hemeristina.)

Locality: Wettin, Saxony. Horizon: Carboniferous.

18\&5. Scudder. Mem. Bost. soc. nat. hist., iii: 340.

1885. " " Zittel, Handb. palaeont., i, th. ii : 761 .

1886. " "Zittel-Barrois, 'Traité de paléont., ii: 761.

1886. " " Bull. U. S. geol. surv., No. 31: 44.

1890. " Foss. ins. N. A., i: 304.

See also Blattina carbonaria, etc.

\section{Mantispa}

Locality : Colebrook, England. Horizon: Carboniferous.

1834. Keferstein. Naturg. erdkörp., ii : 338.

See also Lithosialis brongniarti.

(Very probably Lithosialis brongniarti.)

Meganthentomum formosum. See Megathentomum formosum.

487. Megathentomum carbonatum.

Locality: Germany. Horizon: Carboniferous.

1885. Brongniart. Bull. soc. amis sc. Ronen, (3), xxi : 60 (referring to "Acridites carbonatus Gold.," but there is no such insect described : possibly he refers to A. carbonarius Germ.).

1885. Brongniart. Geol. mag., (3), ii : 487 .

1885. " Trans. geol. soc. Manch., xviii : 280.

1885. " " Foss. ins. prim. rocks, 12.

1885. " J Jahrb. geol. reichsanst., $\mathrm{xxxv}: 656$.

See also Acridites carbonarius.

488. Megathentomum formosum. (Gerarina.)

Locality : Saarbrïck, Germany. Horizon: Carboniferous.

1876. Scudder. Proc. Bost. soc. nat. hist., $x$ viii : 359 .

1885. " Mem, Bost. soc. nat. hist., iii : 347 (Meganthentomum.)

1885. Brongniart. Bull. soc. amis sc. Rouen, (3), xxi : 60 .

1885. " Geol. mag., (3), ii: $48 \%$.

1885. " " Trans. geol. soc. Manch., xviii : 280 .

1885. " " Foss, ins. prim. rocks, 12.

1885. “ Jahrb. geol. reichsanst., $\mathrm{xx \times v}: 656$.

1890. Scudder. Foss. ins. N. A., i: 311.

See also Acridites formosus. 
489. Megathentomum pustulatum. (Gerarina.)

Locality: Morris, Ill. Horizon: Carboniferous.

1868. Scudder.

1868. " "

1868. " "

1872. Packard.

1876. Scudder.

1883. Lacoe.

1885. Scudder.

1885. Brongniart.

1885.

1885. " "

1885. " "

1885.

1885. Scudder.

1886. " "

1886. "

1886. Brauer.

1889. Lesley.

1889. Miller.

1890. Scudder.
1864. Dana.

1865. Scudder.

1865.

1866. " "

1866.

1868. " "

1868. “

1872. Packard.

1874. Dana.

1876. Gerstäcker.

1877. Goldenberg.

1883. Lacoe.

1885. Brongniart.

1885.

1885.

1885 . " 6

1885. Scudder.

1886. "

1886. " "

1886. Braner.

1889. Lesley.

1889. Miller.

1890. Scudder.
1885. Scudder.

1885.

Proc. Bost. soc. nat. hist., xi : 401-402.

Ent. notes, i : 7-8.

Worth., Geol. snrv. Ill., iii : 570. Fig. 7.

Guide ins., 3d ed., 621. Fig. 61\%.

Proc. Bost. soc. nat. hist, xviii : 359.

List pal. foss. ins., 11.

Mem. Bost. soc. nat. hist., iii : 346-347. Pl. 32, figs. 1,9,10.

Bull. soc. amis sc. Rouen, (3), xxi : 60 .

Geol. mag., (3), ii : 487.

Trans. geol. soc. Manch., xviii : 280.

Foss. ins. prim. rocks, 11-12.

Jahrb. geol. reichsaust., $\mathrm{xxxv}: 656$.

Zittel, Handb. palaeont., i, th. ii: 762. Fig. 954.

Zittel-Barrois, Traité de paléont., ii: 762. Fig. 970.

Bull. U. S. geol. surv., No. 31 : 45.

Ann. naturh. hofmus., i: 114.

Dict. foss. Peuns., i: 389. Fig.

N. A. geol, and pal., 579. Fig. 1090.

Foss. ins. N. A., i : 310-311. $\quad P l .18$, figs. 1, 9, 10.

490. Miamia bronsoni. (Palæopterina.)

Locality: Morris, Ill. Horizon: Carboniferous.

Amer. journ. sc., (2), xxxvii: 34-35. Fig. 1.

Amer. journ. se., (2), xl : 269-271.

Proc. Bost. soc. nat. hist., $\mathrm{x}: 96$.

Mem. Bost. soc. nat. hist., i : 190. Pl. 6, figs. 2, 4.

Zool. relat. foss. neur. ins., 18. Pl., figs. $2,4$.

Amer. nat., i: 630. Pl. 16, fig. 1.

Geol. mag., v : 217-218.

Guide ins., 3d ed., 78. $\quad P l .1$, fig. 1.

Man. geol., $2 \mathrm{~d}$ ed., 343. Fig. 669 on $p .335$.

Klass. ordn. arthr., Crust., i : 292 (a Perlarian).

Fauna saræp. foss., ii : 14-15.

List pal. foss. ins., 11.

Mem. Bost. soc. nat. hist., iii : 333 .

Bull. soc. amis se. Rouen, (3), xxi : 62.

Geol. mag., (3), ii : 488.

Trans. geol. soc. Manch., xviii : 282.

Foss. ins. prim. rocks, 14 .

Jahrb. geol. reichsanst., xxxv: $65 \%$.

Zittel, Handb. palaeont., i, th. ii : 760 .

Zittel-Barrois, Traité de paléont., ii : $\mathbf{7 6 0 .}$

Bull. U. S. geol. surv., No. 31 : 43.

Ann. naturh. hofmus., i: 113.

Dict. foss. Penns., i : 402.

N. A. $z \rightarrow \circ$ and pal., 579 .

Foss, i. . ․ A., i: 18, 297. Pl. [1], figs. 2, 4.

491. Miamia danæ. (Gerarina.)

Locality : Mazcu Creek, Ill. Horizon: Carboniferous.

1868. Scudder. Worth., Geol. surv. Ill., iii: 566 . Fig. 1.

1883. Lacoe. List pal. foss. ins., 11.

1874. Dana. Man. geol., 2d ed., 343. Fig. 670 on p. 335.

See also Gerarus danæ, Chrestotes danæ. 


\section{Mixotermes lugauensis. (Homothetidæ.)}

Locality: Lugau, Saxony. Horizon: Carboniferous.

1885. Scudder. Mem. Bost. soc. nat. hist., iii : 331.

1885. " "Zittel, Handb. palaeont., i, th. ii : 760 .

1886. " Zittel-Barrois, Trait6 de paléont., ii: 760.

1886. " " Bull. U. S. geol. surv., No. 31: 42 .

1890. “ " Foss. ins. N. A., i: 295.

See also Termes (Mixotermes) lugauensis, Chrestotes lugauensis.

493. Omalia macroptera. (Homothetidæ.)

Locality: Sars Longchamps, Belgium. Horizon: Carboniferous.

1867. Bened.-Coem. Bull. acad. roy. Belg., (2), xxiii, iv: 384-401. Pl., fig. [5.]

1867. " " Ins. et gast. terr. houill., 11-20. Pl., fig. [5.]

1867. “ Anu. sc. nat., (5), zool., vii : 271-276. Pl. 1, fig. 10.

1877. Goldenberg. Fauna saræp. foss., ii : 16, 50.

18×5. Scudder. Mem. Bost. soc. nat. hist., iii : 331.

1885. Brongniart. Bull. soc. amis sc. Roven, (3), xxi: 66.

1885. " " Geol. mag., (3), ii : 491.

1885. " " Trans. geol. soc. Manch., xviii : 287.

1885. " " Foss. ins. prim. rocks, 18.

1885. Scudder. Zittel, Handb. palaeont., i, th. ii: 760 .

1886. " Zittel-Barrois, Traité de paléont., ii : 760.

1886. “ Bull. U. S. geol. surv., No. 31: 42.

1890. “ Foss. ins. N. A., i: 295.

\section{Oustaletia}

Locality : Commentry, France. IIorizon: Carboniferous.

1885. Brongniart. Bull. soc. amis sc. Rouen, (3), xxi : 66.

Pachytylopsis borinensis. See I, ORThopt, Idem.

495. Pachytylopsis persenairei. (Hemeristina.)

Locality: Mons, Belgium. Horizon: Carboniferous.

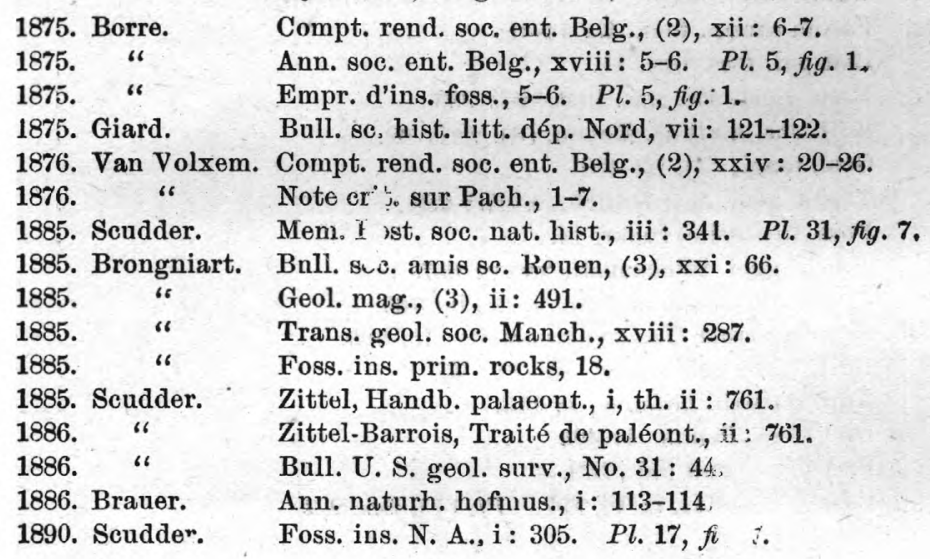

496. Palæoblattina douxillei.

Locality: Jurques, France. Horizon : Middle Silurian.

1885. Brongniart. Compt. rend., xcix : 1164-1165.

1885. " " Bull. séances soc. ent. Fr., 1884 : 236-237.

1885. " La nature, xiii: 116. Figs. 1, 2. 
1885. Brongniart. Bull. soc. amis sc. Rouen, (3), xxi : 55. Pl. 3, figs. 4, 5.

1885. " " Geol. mag., (3), ii: 484.

1885. " "Trans. geol, soc. Manch., xviii : 274 .

1885. " " Foss. ins. prim. rocks, 6.

1885. “ Jahrb. geol. reichsanst., xxxv: 652.

1885. Goss. Ent. monthl. mag., $\mathrm{xxi}: 200$.

1885. Scudder. Mem. Bost. soc. nat. hist., iii : 322 (without name).

1885. " Zittel, Handb. palaeont, i, th. ii : 753,820. Fig. 929.

1886. " Zittel-Barrois, Trạité de paléont., ii: 752,823. Fig. 946.

1886. " " Bull. U. S. geol. surv., No. 31: 46, 103.

1886. “ Miall, Cockroach, 213.

1886. Brauer.

1888. Vidal.

1890. Lesley.

Ann. naturh. hofmus., i: 97, 104. Pl. 8, fig. 20.

Ins. y plantas, 8 .

Dict. foss. Penns., ii : 578. Fig.

1890. Scudder. Foss. ins. N. A., i : 286 (without name).

Palephemera antiqua. See Platephemera antiqua.

497. Palingenia feistmanteli. (Palephemeridæ.)

Compared with P. longicanda.

Locality : Votvovic, Bohemia. Horizon: Carboniferous.

1880. Fritsch. Vesmír, ix : 241-242. Figs. $A, B$.

1882. " Mojs., Beitr. palaeont. Oesterr.-Ung., ii : 1-3. Pl.1, figs. 1-6.

1882. " Foss, arthr. steink. u. kreidef. Böhmens, 1-3. Pl. 1, figs. 1-6.

1884. Kušta. Vesmír, xiii: 98.

1885. Seudder. Mem. Bost. soc. nat. hist., iii : 324.

1885. " Z Zittel, Handb. palaeont., i, th. ii : 758.

1886. " " Zittel-Barrois, Traite de paléont., ii : 758.

1886. " Bull. U. \$. geol, surv., No. 31: 41 .

1886. Brauer. Ann. naturh. hofmus., i: 113.

1889. Brongniart. Ann. géol, univ., v: 1023.

1890. Scudder. Foss. ins. N. A., i : 288.

498. Petrablattina subtilis. (Palæopterina.)

Locality : Schiffweiler, Germany. Horizon: Carboniferous.

1883. Kliver. Neue blatt. Saarbr., 6-7 (Petroblattina.) Pl. 2, figs. 1, 1a.

1883. “ Palaeontogr., xxix: 254-255. Pl. 35, figs. 1, 1a.

See also Strephocladus subtilis.

Petroblattina subtilis. See Petrablattina subtilis.

499. Pictetia -

Locality : Commentry, France. Horizon: Carboniferous.

1885. Brongniart. Bull. soc. amis sc. Rouen, (3), xxi : 66 .

500. Platephemera antiqua. (Palephemeridæ.)

Locality : St. John, N.B. Horizon: Devonian.

1865. Scudder. Dev. ins. N. Brunsw., 1 (nnnamed).

1865. " Bailey, Obs. geol. south. N. Brunsw., 140 (unnamed).

1865. " " Amer. journ. sc., (2), xxxix: 357 (unnamed).

1865. " Can. nat., ii : 235 (unnamed).

1865. " " Trans. ent. soc. Lond., (3), ii: 117 (nnnamed).

1867. " Can. nat., n. s., iii : 205. Fig. 2.

1867. " "Geol. mag., iv: 337. Pl. 17, fig. 2 .

1868. “ Dawson, Acad. geol, $2 \mathrm{~d}$ ed., 524. Fig. 181. 
1868. Scudder.

1868. "،

1876. Roemer.

1877. Nicholson.

1879.

1880. Scudder.

1880. Dawson.

1881. Hagen.

1883. Lacoe.

1885. Scudder.

1885. "6

1885. Brongniart.

1885.

1885.

1885.

1885.

1885. Hagen.

1885. “"

1885.

1885. Scudder.

1886.

1886. “

1886. Brauer.

1889. Miller.

1889. Brongniart.

1889. Nicholson.

1890. Lesiey.

1890. Scudder.
1872. Packard.

Amer. nat., i: $630 . \quad P l .16$, fig. 3.

Geol. mag., v : 173, 175-176.

Guide ins., $3 d$ ed., 78. $\quad P l .1$, fig. 3.

Lethæa geogn. $P l .31$, fig. 9.

Life-hist. earth, $145 . \quad$ Fig. 89.

Manual palæont., 2d ed., i : 405. Fig. 256.

Dev. ins. N. Brunsw. (Anniv. mem. Bost. soo. nat. hist.), 7-11. Pl. 1, figs. 5, 9, 10.

Chain of life, 140. Fig. 123a.

Bull. mus. comp. zool., viii : 276.

List pal. foss. ins., 14.

Earl. winged ins. Amer., $3-4 . \quad P l$., figs. 10,11 ; fig. on p. 3.

Mem. Bost. soc. nat. hist., iii : 323 (Palephemera).

Bull. soc. amis sc. Rouen, (3), xxi : 56.

Geol. mag., (3), ii : 485.

Trans. geol. soc. Manch., xviii : 275.

Foss. ins. prim. rocks, 7.

Jahrb. geol. reichsanst., xxxv: 653 .

Nature, xxxii : 54.

Devon. ins., 2-4.

Zool. anz., viii : 298-299.

Zittel, Handb. palaeont., i, th. ii : 758. Fig. 943.

Zittel-Barrois, Traité de paléont., ii : 758. Fig. 959.

Bull. U. S. geol. surv., No. 31 : 41.

Ann. naturh. hofmus., i : 110-111, 113.

N. A. geol. and pal., 580. Fig. 1094.

Ann. gøol. univ., v: 1021.

Manual palæont., 3d ed., i : 594. Fig. 445.

Dict. foss. Penns., ii : 663 . Fig.

Foss. ins. N. A., i: 159-163, 277, 287. Pl. 7, figs. 5, 9, 10; fig. on p. 277.

501. Polyernus complanatus. (Gerarina.)

Locality: Mazon Creek, Ill. Horizon: Carboniferous.

1885. Scudder. Mem. Bost. soc. nat. hist., iii: 343. $\quad P l .32, f i g s, 8,11$.

1886. “ Bull. U. S.geol. surv., No. 31: 44.

1889. Miller. N. A.geol. and pal., 580.

1890. Lesley. Dict. foss. Penns., ii : 730.

1890. Scudder. Foss. ins. N. A., i: $307 . P l .18, f i g s .8,11$.

502. Polyernus laminarum. (Gerarina.)

Locality : Pittston, Pa. Horizon : Carboniferous.
1885. Scudder.

1886. " "

1889. Miller.

1890. Lesley.

1890. Scudder.

Mem. Bost. soc. nat. hist., iii : 343. Pl. 31, fig. 1.

Bull. U. S. geol. surv., No. 31: 44.

N. A. geol. and pal., 580 .

Dict. foss. Penns, , ii: 730. Fig.

Foss. ins. N. A., i : $307 . \quad P l .17, f i g .1$.

503. Propteticus infernus. (Palæopterina.)

Locality: Little Vermilion River, Ill. Horizon: Carboniferous.

1883. Scudder. MSS.

1883. Lacoe.

1885. Scudder.

1885 .

List pal. foss. ins., 14-15.

Mem. Bost. soc. nat. hist., iii : 334-336. $\quad P l$. 31, figs. 3, 4.

Zituel, Handb. palaeont., i, th. ii : 760. Fig. 947.

1886. o

Zittel-Barrois, Traité de paléont., ii : 760. Fig. 963. 
1886. Scudder. Bull.U. S. geol. surv., No. 31: 43.

1886. Brauer. Ann. naturh. hofmus., i: 113.

1889. Miller. N. A. geol. and pal., 581.

1890. Lesley. Dict. foss. Penns., ii : 772. Fig.

1890. Scudder. Foss, ins. N. A., i: 298-300. Pl. 17, figs. 3, 4.

504. Protagrion - .

Locality: Commentry, France. Horizon: Carboniferons.

1885. Brongniart. Bull. soc. amis sc. Rouen, (3), xxi : 65-66.

1885. “ Rev. scient., (3), xxxvi : 278.

\section{Protascalaphus}

Locality : Commentry, France. Horizon: Carboniferous. 1885. Brongniart. Bull. soc. amis sc. Rouen, (3), xxi : 67.

\section{Protocapnia}

Locality : Commentry, France. Horizon: Carboniferous.

1885. Brongniart. Bull. soc. amis sc. Rouen, (3), xxi : 63.

1885. “ Rev. scient., (3), xxxvi: 278.

\section{Protodiamphipnoa}

Locality: Commentry, France. Horizon: Carboniferous. 1885. Brongniart. Bull, soc. amis se. Rouen, (3), xxi : 66.

508. Protogryllacris brongniarti. (Hemeristina.)

Locality : Coalbrookdale, England. Horizon: Carboniferous.

18\$5. Brongniart. Bull, soc. amis sc. Rouen, (3), xxi : 59.

1885. " Rev. scient., (3), xxxvi: 276.

1885. “ Geol. mag., (3), ii: 486.

1885. " " Trans. geol. soc. Manch., xviii : 278.

1885. " " Foss. ins. prim. rocks, 10.

See also Lithosialis brongniarti, etc.

\section{Protokollaria ingens.}

Locality : Commentry, France. Horizon: Carboniferous. 1885. Brongniart. Bull. soc. amis sc. Rouen, (3), xxi : 66. Pl. 3, fig. 3. 1886. Brauer. Ann, naturh. hofmus., i: 108, Pl. 7, fig. 6.

\section{Protoperla}

Locality: Commentry, France. Horizon: Carboniferous. 1885. Brongniart. Bull. soc. amis sc. Rouen, (3), xxi : 66 .

511. Prototettix lithanthraca. (Homothetidæ.)

Locality : Saarbrück, Germany. Horizon: Carboniferous.

1856. Giebel. Insect. d. vorwelt, 306-307.

See also Gryllacris lithanthraca, etc.

\section{Sphecoptera}

Lncality: Commentry, France. Horizon: Carboniferous. 1885. Brongniart. Bull. soc. amis sc. Rouen, (3), xxi : 63-64.

1885. " Rev. scient., (3), xxxvi: 278. 
513. Strephocladus subtilis. (Palæopteriua.)

Locality: Schiffweiler, Germany. Horizon: Carboniferous.

1885. Scudder. Mem. Bost. soc. nat. hist., iii : 337.

1885. " Zittel, Handb. palaeont., i, th. ii: 760. Fig. 948.

1886. “ Zittel Barrois, Traité de pal6ont., ii: 760. Fig. 964.

1886. " Bull. U. S. geol. surv., No. 31: 43.

1886. Brauer. Ann. naturh. hofmus., i: 113.

1890. Scudder. Foss.ins. N. A., i: 301.

See also Petrablattina subtilis.

Termes spp. See I, ORTHOPt., Idem.

514. Termes?

Locality: Mons, Belgium. Horizon: Carboniferous.

1875. Borre. Compt. rend. soc. ent. Belg., (2), xii : 7.

1875. " Ann. soc. ent. Belg., xviii: 5-6. Pl. 5, fig. 3.

1875. " Empr. d'ins, foss., 5-6. Pl. 5, fig. 3 ,

See also I, Orthopt, Termes haidingeri.

\section{Termes buchi.}

Locality: Jägersfreude, Germany. Horizon: Carboniferous.

1877. Goldenberg. Fauna saræp. foss., ii : 50.

See also Termes (Calotermes) buchi.

\section{Termes (Calotermes) buchi.}

Locality: Jägersfreude, Germany. Horizon : Carboniferous.

1867. Goldenberg. Vorweltl, fauna steink. Saarbr., 12-13. Pl. 2, fig. 9 (ined.)

1873. “ Fauna saræp, foss., i: 12-13. Pl. 2, fig. 9.

See also Termes buchi.

\section{Termes contusus. (Homothetidæ.)}

Locality : Vermilion Co., III. Horizon: Carboniferous.

1878. Scudder. Proc. Bost. soc. nat. hist., xix : 300-301.

1878. " Entom. notes, vi : 54-55.

1883. Lacoe. List. pal. foss. ins., 15.

See also Didymophleps contusa, etc.

Termes hageni. See I, Arachinida, Idem.

518. Termes (Mixotermes) lugauensis. (Homothetidæ.)

Locality: Lugau, Saxony. Horizon: Carboniferous.

1881. Sterzel. Ber. naturw. gesellsch. Chemn., vii: 273-276. Pl., figs. 3-5.

See also Mixotermes lugauensis, etc.

Termitidium spp. See I, ORTHOPT., Idem.

519. Trichaptum

Locality : Commentry, France. Horizon: Carboniferous.

1885. Brongniart. Bull. soc. amis sc. Rouen, (3), xxi : 63.

1885. " Rev. scient., (3), xxxvi: 278. 


\section{Woodwardia longicauda.}

Locality : Commentry, France. Horizon: Carboniferous.

1885. Brongniart. Bull. soc. amis sc. Rouen, (3), xxi : 64 .

1885. " " Rev. scient., (3), xxxvi : 278.

1885. " " Geol. mag., (3), ii : 489.

1885. " "Trans, geol. soc. Manch., xviii : 284.

1885. " " Foss. ins. prim. rocks, 16.

1885. “ Jahrb. geol. reichsanst., $x \times x: 659$.

\section{Woodwardia modesta.}

Locality : Commentry, France. Horizon: Carboniferous.

1885. Brongniart. Bull. soc. amis sc. Rouen, (3), xxi : 64.

1885. " $\quad$ Rev. scient., (3), xxxvi : 278.

1885. “ " Geol. mag., (3), ii: 489.

1885. " " Trans, geol, soc. Manch., xviii : 284.

1885. " " Foss. ins. prim. rocks, 16.

1885. “ Jahrb, geol. reichsanst., $x \times x v: 659$.

\section{Woodwardia nigra.}

Locality: Commentry, France. Horizon: Carboniferous.

1885. Brongniart. Ins. foss. terr. prim., 64. - Pl. 2, fig. 2; Pl. [4], fig. 1.

1885. “ Bull. soc. amis se. Rouen, (3), xxi: 64. Pl. 2, fig. 2.

1885. " Rev. scient., (3), xxxvi: 278.

1885. " Geol. mag., (3), ii: 489. Pl. 12, fig. 1.

1885. " "Trans. geol. soc. Manch., xviii : 284-285. Pl., fig. 1.

1885. " " Foss. ins. prim. rocks, 16.

1885. " Jahrb. geol. reichsanst., $\times \times \times \times$ : 659.

1886. Brauer. Ann. naturh. hofmus., i : 105-106. Pl. 8, fig. 12.

523. Xenoneura antiquorum. (Xenoneuridæ.)

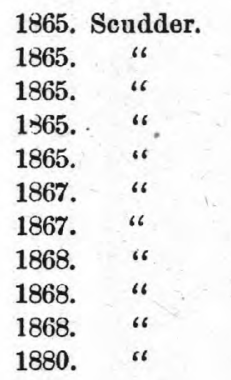

1880. Dawson.

1881. Hagen.

1883. Lacoe.

1885. Scudder.

1885. " "

1885. Brongniart.

1885.

1885.

1885.

1885.

1885. Hagen.
Locality: St. John, N. B. Horizon : Devonian.

Dev. ins. N. Brunsw., 1 (unnamed).

Bailey, Obs. geol. south. N. Brunsw., 140 (unnamed).

Amer. journ. sc., (2), xxxix : 357 (unnamed).

Can. nat., n. s., ii : 235 (unnamed).

Trans. ent. soc. Lond., (3), ii : 117 (unnamed).

Can. nat., n. s., iii : 206. Fig. 5.

Geol. mag., iv: 387-388. Pl. 17, fig. 5.

Dawson, Acad. geol., 2d ed., 525-526. Fig. 184.

Amer. nat., ii: 163 . Fig. 1.

Geol. mag., $v$ : 174, 176.

Dev. ins. N. Brunsw. (Anniv. mem. Bost. soc. nat. hist.), 24-29. Pl. 1, figs. 5, 6, 7.

Chain of life, 140. Fig. $123 d$.

Bull. mus. comp. zool., viii : 279-280.

List pal. foss. ins., 15.

Earl. winged ins. Amer., 6-7, Pl, figs. 5-7.

Mem. Bost. soc. nat. hist., iii : 338.

Bull. soc. amis sc. Rouen, (3), xxi : 57.

Geol. mag., (3), ii : 485.

Trans. geol. soc. Manck., xviii : 276.

Foss. ins. prim. rocks, 8.

Jahrb. geol. reichsanst., xxxv : 653.

Nature, xxxii : 53-54. 
1885. Hagen.

1885. " "

1885. Scudder.

1886. " "

1886. “ "

1886. Brauer.

1889. Miller.

1890. Scudder.
Zool. anz., viii : 297, 300 .

Devon. ins., 1-2.

Zittel, Handb. palaeont., i, th. ii : 760. Fig. 949.

Zittel-Barrois, Traité de paléont., ii : 760. Fig. 965.

Bull. U. S. geol. surv., No. 31 : 43.

Ann. naturh. hofmus., i : 112.

N. A. geol. and pal., 581. Fig. 1096.

Foss. ins. N. A., i: 176-181, 280-281, 302. Pl. 7, figs. 5-7; Pl. 14, figs. 5-7.

524. Zeilleria carbonaria.

Locality : Commentry, France. Horizon: Carboniferous.

1885. Brongniart. Bull. soc. amis sc. Ronen, (3), xxi : 63.

1885. " " Geol. mag., (3), ii : 489.

1885. " " Trans. geol, soc. Manch., xviii : 283.

1885. " " Foss. ins. prim. rocks, 15.

1885. “ Jahrb. geol. reichsanst., xxxv : 658.

\section{Zeilleria formosa.}

Locality: Commentry, France. Horizon: Carboniferous.

1885. Brongniart. Bull. soc. amis se. Rouen, (3), xxi : 63.

1885. " " Geol. mag., (3), ii : 489.

1885. " " Trans. geol. soc. Manch., xviii: 283.

1885. " " Foss. ins. prim. rocks, 15.

1885 " " Jahrb, geol, reichsanst., xxxv: 658.

\section{Zeilleria fusca.}

Locality : Commentry, France. Horizon: Carboniferous.

1885. Brongniart. Ins. foss. terr. prim., 63. Pl. [5], fig. 2.

1885. " "Bull. soc. amis sc. Rouen, (3), xxi : 63.

1885. " " Geol. mag., (3), ii: 489.

1885. " " Trans. geol. soc. Manch., xviii : 283.

1885. " " Foss. ins. prim. rocks, 15.

$1885 . \quad$ "Jahrb. geol, reichsanst., $\mathrm{x \times xv}: 658$.

1886. Brauer. Ann. naturh. hofmus., i: 110.

\section{THE HEMIPTEROID SERIES.}

527. Dictyocicada antiqua.

Locality: Commentry, France. Horizon: Carboniferous.

1885. Brongniart. Bull. soc. amis sc. Rouen, (3), xxi : 67.

1885. " " Rev. scient., (3), xxxvi : 278.

1885. " Geol. mag., (3), ii : 491.

1885. “ " Trans. geol. soc. Manch., xviil : 288.

1885. " " Foss. ins. prim. rocks, 19.

1885. " Jahrb. geol, reichsanst., xxxv: 661 .

528. Eugereon -

Locality: Nyřan, Bohemia. Horizon: Permian.

1879. Fritsch. Fauna d. gaskohle Böhmens, i: 31-32.

529. Eugereon boeckingi.

Locality : Abenteuerhütte, Fürst. Birkenfeld, Germany. Horizon: Todtliegende.

1866. Dohrn. Eugereon boeckingi, 1-7. Pl. 41.

1866. " $\quad$ Palaeontogr., xiii: 333-340. Pl. 41.

1867. “ Palaeontogr., xvi: 129-131. Pl. 8, fig. 1. 
1867. Dohrn. Stett. entom. zeit., xxviii : 145-148. Pl. 1.

1867. Snell. v. Voll. Versl. alg. vergad. nederl. entom. vereen., xxii : 13.

1869. Brauer: Verwandl. insekt. sinne descend. theorie, 19.

1869. " Verhandl. k. k. zool. bot. gesellsch., 1869: 317.

1877. Goldenberg. Fauna saræp. foss., ii : 11-14, 50. Pl. 1, figs. 15, $15 a$.

1879. Scudder.

1885. "6

Mem. Bost. soc. nat. hist., iii : 18.

1885. " "

Mem. Bost. soc. nat. hist., iii : 347.

1885. Brongniart. Bull. soc. amis sc. Rouen, (3), xxi : 60-61.

1885. “ 'Rev. scient., (3), xxxvi: 277.

1885. “ Geol, mag., (3), ii : 487.

1885. " " Trans. geol. soc. Manch., xviii: 280 .

1885. " " Foss. ins. prim. rocks, 12.

1885. “ Jahrb. geol. reichsanst., $\mathrm{xxxv}: 656$.

1885. Brauer. Sitzungsb. akad. wiss. Wien, xci: 275-279.

1886. " Aun. naturhist. hofmus., i: 114.

1886. Scudder. Zittel-Barrois, Traité de paléont., ii : 763. Fig. 971.

1886. " Bull. U. S. geol. surv., No. 31: 45.

1889. Nicholson. Man. palæont., 3d ed., i: 595. Fig. 446.

1890. Seudder. Foss. ins. N. A., i: 38, 311.

530. Eugereon heeri.

Locality : Commentry, France. Horizon: Carboniferous.

1885. Brongniart. Bull. soc. amis sc. Rouen, (3), xxi : 60-61.

1885. " " Rev. scient., (3), xxxvi: 277.

1885. " "Geol. mag., (3), ii : 487.

1885. " " Trans. geol. soc. Manch., xviii: 280 .

1885. " " Foss. ins. prim. rocks, 12.

1885. " "Jahrb. geol, reichsanst., $\mathrm{xxxv}: 656$.

\section{Fulgora ebersi.}

Locality : Saarbrück, Germany. Horizon: Carboniferous.

1867. Dohrn. Palaeontogr., xvi : 131-133. Pl. 8, fig. 2.

1876. Gerstaecker. Klass. ordn. arthr., Crust., i : 292 (belongs to Blattidæ).

1886. Brauer. Ann. naturhist. hofmus., i : 114-115.

See also Fulgorina ebersi.

\section{Fulgorina ebersi.}

Locality: Saarbrück, Germany. Horizon: Carboniferous.

1877. Goldenberg. Fauna saræp. foss., ii : 28-30, 51 . Pl. 1, figs. 16-17.

1885. Scudder. Mem. Bost. soc. nat. hist., iii : 347.

1885. i6 Zittel, Handb, palaeont, i, th. ii: 764. Fig. 956.

1885. Brongniart. Bull. soc. amis sc. Rouen, (3), xxi : 67.

1885. " $"$ Rev. scient., (3), xxxvi: 278 .

1885. " " Geol. mag., (3), ii : 491.

1885. " "Trans. geol. soc. Manch., xviii : 287.

1885. " " Foss. ins. prim. rocks, 19.

18×5. “ Jahrb. geol, reichsanst., xxxv: 661 .

1886. Scudder. Zittel-Barrois, Traité de paléont., ii : 764. Fig. 972.

1886. “ Bull. U. S. geol. surv., No. 31: 45.

1890. " Foss. ins. N. A., i : 311.

See also Fulgora ebersi. 
533. Fulgorina goldenbergi.

Loeality : Commentry, France. Horizon: Carboniforous.

1885. Brongniart. Ins. foss. terr. prim., 67. Pl. [5], fig. 5.

1885. " "Bull. soc. amis sc. Rouen, (3), xxi : 67.

1885. " " Rev. scient., (3), xxxvi: 278.

1885. " Geol. mag., (3), ii : 491 (goldendergi).

1885. " " Trans. geol. soc. Manch, xviii : 287.

1885. " " Foss. ins. prim. rocks, 19.

1885. " Jahrb. geol, reichsanst., xxxv: 661 .

Fulgorina goldendergi. See Fulgorina goldenbergi.

Fulgorina klieveri. See Fulgorina kliveri.

534. Fulgorina kliveri.

Localities: Saarbrïck, Weissig, Michelsberg, Germany. Horizon: Carboniferous. 1869. Goldenberg. Neues jahrb. f. mineral., 1869: 166-167 (klieveri). Pl. 3, figs. 13,134 .

1877. " Fauna saræp. foss., ii : 31,51 . Pl. 1, figs. 18, 18a.

1875. E. Geinitz. Neues jahrb. f. mineral., 1875: 6. Pl. 1, fig. 3.

1875. " Nene aufschl. dyas v. Weissig, 6. Pl. 1, fig. 3.

1883. Kliver. Neue blatt. Saarbr., 10.

1883. " Palaeontogr. xxix: 258.

1885. Scudder. Mem. Bost. soc. nat. hist., iii : 348.

1890. " Foss. ins. N. A., i: 312.

See also Megablattina kliveri.

\section{Fulgorina lebachensis.}

Locality : Lehbach, Germany. Horizon: Lower Dyas.

1377. Goldenberg. Fauna saræp. foss., ii : 30-31,51. Pl. 1, figs, 19, 19a.

1885. Scudder. Mem. Bost. soc. nat. hist., iii : 348.

1885. Brongniart. Bull. soc. amis sc., Ronen, (3), xxi : 67.

1885. "

1885. " " Geol, mag., (3), ii: 491.

1885. “ Trans. geol. soc. Manch., xviii : 287.

1885. " " Foss. ins. prim. rocks, 19.

1885. “ Jahrb. geol, reichsanst., xxxv: 661 .

1890. Scudder. Foss, ins. N. A, i: 312.

536. Fulgorina minor.

Locality : Commentry, France. Horizon: Carboniferous.

1885. Brongniart. Bull. soe. amis sc. Rouen, (3), xxi: 67.

1885. " " Rev. scient., (3), xxxvi: 278.

1885. “ Geol. mag., (3), ii : 491.

1885. “ " Trans. geol. soc. Manch., xviii: 287.

1885. " " Foss. ins. prim.rocks, 19.

1885. “ Jahrb. geol. reichsanst., xxxv : 661 .

537. Fulgorina ovalis.

Locality : Commentry, France. Horizon: Carboniferous.

1885. Brongniart. Bull. soc. amis sc. Rouen, (3), xxi: 78.

1885. " Rev. scient., (3), xxxvi : 278.

1885. " Geol. mag., (3), ii : 491.

1885. " "Trans. geol. soc. Manch., xviii : 287.

1885. " $\quad$ Foss. ins. prim. rocks, 19.

1885. " Jahrb. geol. reichsanst., xxxv: 661. 
538. Macrophlebium hollebeni.

Locality : Manebach, Thüringen. Horizon: Carboniferous.

1869. Goldenberg. Neues jahrb. f. mineral., 1869: 164-166. Pl. 3, figs. 12A, $12 a$.

1885. Scudder. Mem. Bost. soc. nat. hist., iii: 348.

1885. Brongniart. Bull. soc. amis sc. Rouen, (3), xxi : 59.

1885. " " Geol. mag., (3), ii: 48\%.

1885. " " Trans. geol, soc. Manch., xviii : 279 .

1885. " " Foss. ins. prim. rocks, 11.

1885. " J Jahrb. geol. reichsanst., xxxv: 655.

1885. Scudder. Zittel, Handb. palaeont., i, th. ii : 764 (cockroach ?).

1886. “ Z Zittel-Barrois, Traité de paléont., ii : 764 (cockroach ?).

1886. “ Bull. U. S. geol. surv., No. $31: 45$.

1890. " Foss. ins. N. A., i: 312 .

Megablattina klieveri. See Megablattina kliveri.

539. Megablattina kliveri.

Locality: Germany. Horizon: Carboniferous.

1885. Brongniart. Bull. soc. amis sc. Rouen, (3), xxi : 58 (klieveri).

1885. " Geol. mag., (3), ii : 486.

1885. " " Trans. geol, soc. Manch., xviii : 278.

1885. 6. Foss. ins. prim. rocks, 10.

1885. " " Jahrb. geol. reichsanst., xxxv : 655 .

See also Fulgorina kliveri.

\section{Palæocixius antiquus.}

Locality : Commentry, France. Horizon: Carboniferous.

1885. Brougniart. Bull. soc. amis se. Rouen, (3), xxi : 67.

1885. " "Geol. mag., (3), ii : 491 (Protocixius).

1885. " " Trans. geol. soc. Manch., xviii : 288 (Protocixius).

1885. " " Foss. ins. prim. rocks, 19 (Protocixius).

1835. “ Jahrb. geol, reichsanst., Xxxv: 661.

\section{Palæocixius fayoli.}

Locality : Commentry, France. Horizon: Carboniferous.

1885. Brongniart. Bull, soc. amis se. Ronen, (3), xxi : 67.

1885. " "Geol. mag., (3), ii: 491 (Protocixius).

1885. " "Trans. geol. soc. Manch., xviii : 288 (Protocixius).

1885. " " Foss. ins. prim. rocks, 19 (Protocixius).

1885. “ Jahrb. geol. reichsanst., xxxv: 661.

\section{Phthanocoris occidentalis.}

Locality : Kansas City, Mo. Horizon: Carboniferous.

1883. Scudder. Proc. Bost. soc. nat hist., xxii : 58-59

1883. Lacoe. List pal. foss. ins., 14.

1785. Scudder. Mem. Bost. soc. nat. hist., iii: 348-349. Pl. 32, fig. 4.

1885. Bronguiart. Bull. soc. amis sc. Rouen, (3), xxi : 67.

1885. " " Rev. scient., (3), xxxvi : 278.

1885. 66 Geol. mag., (3), ii: 491.

1885. “ "Trans. geol. soe. Manch., xviii : 288.

1885, “ " Foss. ins. prim. rocks, 19.

1885. “ Jahrb. geol, reichsanst., хXXv : 661.

1885. Scudder Zittel, Handb. palaeont., j, th. ii: 764. Fig. 957. 
1886. Scudder.

1886. "“

1889. Miller.

1890. Lesley.

1890. Scudder.
Zittel-Barrois, Traité de paléont., ii : 764. Fig. 973.

Bull. U. S. geol. surv., No. 31 : 45.

N. A. geol. and pal., $5 \Omega 0$.

Dict. foss. Penns., ii : 639. Fig.

Foss. ins. N. A., i: 312-313. Pl. 18, fig. 4.

\section{Protociccus fuscus.}

Locality: Commentry, France. Horizon: Carboniferous.

1885. Brongniart. Bull, soc. amis sc. Rouen, (3), xxi : 67.

1885. " "Geol, mag., (3), ii : 491.

1885. “ Trans. geol. soc. Manch., xviii : 288.

1885. “ " Foss. ins. prim. rocks, 19.

1885. “ Jahrb. geol. reichsanst., xxxv: 661.

\section{Protociccus parvulus.}

Locality: Commentry, France. Horizon: Carboniferous.

1885. Brongniart. Bull. soc. amis sc. Rouen, (3), xxi : 67.

1885. " Geol. mag., (3), ii : 491.

1885. “ “ Trans. geol. soc. Manch., xviii: 288.

1885. " " Foss. ins. prim. rocks, 19.

1885. “ Jahrb. geol. reichsanst., xxxv: 661.

Protocixius antiquus. See Palæocixius antiquus.

Protocixius fayoli. See Palæocixius fayoli.

545. Rhipidioptera elegans.

Locality : Commentry, France. Horizon: Carboniferous.

1885. Brongniart. Bull. soc. amis sc. Rouen, (3), xxi: 67.

1885. " Rev. scient., (3), xxxvi : 278.

1885. “ Geol. mag., (3), ii: 491.

1885. “ Trans, geol. soc. Manch., xviii : 284 (belongs on 288).

1885. " " Foss. ins. prim. rocks, 19.

1885. “ Jahrb. geol, reichsanst., $\mathrm{xxxv}: 661$.

\section{Scudderia}

Locality : Commentry, France. Horizon: Carboniferous.

1885. Brongniart. Bull, soc. amis sc. Rouen, (3), xxi : 61 .

1885. " Rev. scient., (3), xxxvi : 277.

\section{THE COLEOPTEROID SERIES.}

547. (pupa.)

Locality: Bradley, England. Ḧorizon: Carboniferous.

1867. Binney. Proc. lit. phil. soc. Manchester, vi : 59.

1867. " Geol. mag., iv: 132.

548.

Locality: Coalbrookdale, England, Horizon: Carboniferous.

1834. Prestwich. Lond. Edinb. phil, mag, iv : 376 . 
549.

Locality : [Bohemia.] Horizon: Carboniferous.

1836. Sternberg. Verhandi. gesellsch. vaterl. mus. Böhm., 1836: 34-35 (regardèd by him as larval mines of Lepidoptera). $\quad P l .1, f i g .3-4$.

Seg also Xyloryctes septarius.

550.

Locality : Yorkshire, England. Horizon: Coal Slate.

1830. Taylor. Loud., mag. nat. hist., iii: 361.

551.

Locality : Danby coalpits, Yorkshire, England. Horizon: Carboniferous. 1830. Taylor.

Lond., mag. nat. hist., iii : 361.

552.

(Carabidæ.)

Locality : Steinkunzendorf, Schlesien. Horizon: Culmschichte.

1885. Dathe.

1885. Scudder.

1886. " "

1886. " "
Zeitschr. dentsch. geol. gesellsch., 1885: 542-543.

Zittel, Handb. palaeont., i, th. ii : 764 note.

Zittel-Barrois, Traité de paléont., ii : 764 note.

Bull. U. S. geol. surv., No. 31:,106 note.

553.

(Ténebrionidæ.)

Locality: Steinkunzendorf, Schlesien. Horizon: Culmschichte.

1885. Dathe. Keitsehr. dentsch. geol. gesellsch., 1885: 542-543.

1885. Scudder. Zittel, Handb. palaeont, i, th. ii: 764 note.

1886. " "

1886. “

Zittel-Barrois, Trâité de paléont, ii : 764 note.

Bull. U. S. geol. surv., No. 31: 106 note.

Curculioides ansticii. See I, Arachnida, Idem.

Curculioides prestvicii. See I, Arachnida, Idem.

Duma prestvicii. See I, Arachnida, Idem.

Hevila ansticii. See I, ArAchNIDA, Idem.

554. Hylesinus - (borings). (Scolytidæ.)

Locality: Autun, France. Horizon: Carboniferous.

1877. Brongniart. Ann. soc. ent. Fr., (5), vii : 214-217. Pl. 7II, figs. 1-4.

1878. Girard.

La nature, vi : 112. Figs. 1-4.

1885. Scudder. Zittel, Handb. paiaeont., i, th. ii : 764. Fig. 958.

1886. " Zittel-Barrois, Traité de paléont., ii: 764. Fig. 974.

555. Troxites germari.

Localities: Salzłach, Altenwald, Germany. Horizon: Carboniferous.

1854. Goldenberg. Palaeontogr., iv: 36. Pl. 3, fig. 6.

1854. " Foss. ins. Saarbr., 20. Pl. 1, fig. 6.

1856. Giebel. Insect. d. vorwelt, 36-37.

1867. Goldenberg. Vorweltl. fauua steink. Saarbr., 7. Pl. 2, fig. 1 (ined.)

1873. " Fauna saræp. foss., 7. Pl. 2, fig. 1.

1876. Gerstaecker. Klass, ordn. arthr., Crust., i : 289 (coleopteron?).

1877. Goldenberg. Fauna saræp. foss, ii : 5 7.

1879. Scudder. Mem. Bost. soc. nat. hist., iii : 19.

Bull. $71-7$ 
1885. Scudder. Zittel, Handb. palaeont., i, th. ii : 764,794 (fruit?).

1886. " "Zittel-Barrois, Traite de pal6ont., ii : 764, 794.

1886. " "Bull. U. S. geol. surv., No. 31: 46, 73.

1890. " Foss. ins. N. A., i : 39.

(Probably not an insect.)

556. Xyloryctes planus.

Locality : Overhohndorf, Germany. Horizon: Carboniferous.

1855. Geinitz. Verstein. steinkohlenf. Sachsen, 1-2 (unnamed). Pl. 8, fig8. 1, 4. 1874. Frið. Areh. naturwiss. landesdurehf. Böhm., 2, ii, i: 16. Pl. 3, fig. 5. 1884. Kušta. Vesmír, xiii: 97-98.

557. Xyloryctes septarius.

Locality: [Bohemia.] Horizon: Carboniferous.

1874. Fric. Arch. naturwiss. landesdurchf. Böhm., 2, ii, i: 15-16. Pl. 3, fig. 6. 1884. Kušta.

See also Vesmír, xiii : 97-98.

- Sternberg.

"Caterpillar." [Lepidoptera.] See I, Mrriapoda, Euphoberia ferox.

Larval mines. [Lepidóptera.] See I, Coleopt. - - Sternb.

Breyeria borinensis. [Lepidoptera.] See I, ORTHOPT., Idem.

558.

Locality : Near Glasgow, Scotland. Horizon: Carboniferous.

1841. Buckland. Proc. geol. soc. Lond., iii : 505. (Hymenoptera.)

1842. " Ann. mag. nat. hist., ix: 163.

\section{II.-MESOZOIC FOSSILS.}

\section{MYRIAPODA.}

559. Geophilus proavus.

Locality: Kelheim, Bavaria. Horizon: Jura.

1842. Germar. Münst., Beitr. z. petref., v : 89-90. Pl. 9, fig. 9.

1852. Giebel. Deutschl. petref., 634.

18.56. " " Insect d. vorwelt, 489.

1862. Hagen. Palaeontogr., $x$ :- 111 (a nereid worm).

1869. Weyenbergh. Tijdschr. v. ent., (2), iv: 237.

1870? Ehlers. Palaeontogr., xvii : 145.

1885. Scudder. Zittel, Handb. palaeont., i, th. ii: 725 (annelid).

1886. " " Zittel-Barrois, Traité de palént., ii: 724.

1886. " Bull. U. S. geol. surv., No. 31: 13,14.

560. Julopsis cretacea. (Julidæ.)

Locality : Unter Atanekerdluk, Greenland. Horizon: Cretaceous.

1874. Heer.

Kreideflora arct. zone (k. svensk. vetensk. akad. handl., xii ; Flora foss. arct., iii), 120-121. $P l .33, f i g .7$.

1885. Scudder. Zittel, Handb. palneont, i, th. ii : 725,731.

1886. " "Zittel-Barrois, Traité de pa!6́ont., ii : 724, 730.

1886. “ Bull. U. S. geol. surv., No. 31: 13, 18. 


\section{ARACHNIDA.}

\section{1.}

Locality : Hasfield, England. Horizon: Lower Lias.

1873. Brodie. Distr. corr. foss. ins., 17.

562.

Locality : Eichstadt, Bavaria. Horizon: Jura.

1737. Kundmann. Rar. nat. art., 236. Pl. 12, fiq.14.

(Probably not an arachnid.)

\section{Chelifer fossilis.}

Locality : Solenhofen, Bavaria. Horizon : Jura.

1874. Weyenbergh. Periód. zool., i : 89 (fossils) (no description).

1874. " Enum. syst. faune ent. mésoz., 3 (no description).

(Probably not an arachnid.)

Hasseltia primigenius. See Hasseltides primigenius.

564. Hasseltides primigenius. (Agalenidæ.)

Locality: Solenhofen, Bavaria. Horizon: Jura.

1869. Weyenbergh. Arch. mus. Teyl., ii : 253-251. Pl. 34,fig. 1.

1869. " Ins. foss. calc. lithogr., 7-8. Pl. 1, fig. 1.

1869. " "Tijdschr. v. ent., (2), iv: 237 (Hasseltia).

1873. “ Areh. mus. Teyl, iii : 234-236.

1873. " " Notes ins. calc. jurass., 1-3.

1874. " $\quad$ Periód. zool., i: 89.

1874. " Enum. syst. faune ent. mésoz., 3.

\section{Nymphon (vic.) -}

Locality: Solenhofen, Bavaria. Horizon: Jura.

1842. Gray. Buckl., Ann. mag. nat. hist., ix : 162-163.

1842. “ Buckl., Quart. journ. geol. soc. Lond., iii: 504-505.

(Probably, not an arachnid.)

See also Pycnogonites uncinatus.

566. Palpipes cursor.

Locality: Solenhofen, Bavaria. Horizon: Jura..

1851. Roth.

Gel. anz. bayer, akad. wiss., xxxii: 167.

1851. " Neues jahrb. min., 1851: 377 .

1856. Giebel. Insect. d. vorwelt, 469.

1869. Weyenbergh. Tijdschr. $\nabla$. ent., (2), iv: 237 (custor).

1874. " " Periód. zool., i: 88.

1874. " " Enum, syst. faune ent. mésoz., 2.

(Not an arachnid.)

See also Phalangites multipes.

Palpipes custor. See Palpipes cursor.

567. Palpipes priscus.

Locality : Solenhofen, Bavaria. Horizon: Jura.

1851. Roth.

1851. “

1852. Bronn.
Gel, anz. bayer, akad. wiss., xxxii: 166. Fig.

Neues jahrb. min., 1851: 377. Pl. $4 B$, fig. 8.

Lethæa geogn., $3^{e}$ aufl, ii, th. ii : 429-430. Pl. 24, fig. 19. 
1854. Pictet. 1856. Giebel 1861. Meyer. 1863. " 1863. Winkler.

9. Weyenbergh.

1873. Seebach.
Traité pal6́nt., $2^{\ominus} 6$ d., ii : 407-408. Pl. 41, fig. 2.

Insect. $d$. vorwelt, 469.

Neues jahrb. min., 1861: 561 (refers it to Crustacea).

Palaeontogr., x: 299-304. Pl. 1, figs. 1-4.

Cat. syst. coll. pal. mus. Teyl., 421 .

1874. Weyenbergh. Períd. zool., i : 88.

1874. " Enum. syst. faune ent. mésoz., 2.

1890. McCook. Amer. spiders, ii : 457-458. Figs, 385-386.

(Not an arachnid.)

\section{Phalangites multipes.}

1851. Münster.

Locality : Solenhofen, Bavaria. Horizon : Jura.

1851. Roth.

In litt.

1851. " Neues jahrb, min., 1851: 375.

(Not an arachnid.)

See also Palpipes cursor.

\section{Phalangites priscus.}

Locality : Solenhofen, Bavaria. Horizon: Jura.

1839. Münster. Beitr, z. petref., i: 84-85. Pl. 8, figs. 3-4.

1852. Quenstedt. Handb. petref., 308. Pl. 21, figs. 26, 27.

1870. Thorell. European spiders, i : 222-223.

(Not an arachnid)

See also Palpipes prisens.

570. Pycnogonites uncinatus.

Locality : Solenhofen, Bavaria. Horizon: Jura.

1852. Quenstedt. Handb. petref., 308. Pl.21, fig. 28.

(Probably not an arachnid.)

See also Nymphon Gray.

\section{HEXAPODA.}

\section{1. - - (Galls.)}

Localities: Kansas and Nebraska. Horizon: Dakota Group.

1882. Hagen. Nature, $\mathrm{xxv}$ : 265-266 (refers to figs. in Lesquereux, Cret. flora, Hayden Rep.).

572.

Locality: Near Chanda, Central India. Horizon: Kota Maleri Group. 1879. Medl.-Blanf. Geol. India, i : 152, 154.

\section{3.}

Loeality: England. Horizon: Lower Lias.

1845. Murchison. Geol. Cheltenham, 108. Pl.9, fig.1ab.

574.

(Pupa.)

Locality : Siberia. Horizon: ?

1784. Schröter. Neue litt. u. beytr., i : 413-415. Pl.3, figs.14-15. 
575.

Locality: Durdlestone Bay, England. Horizon: Lower Purbecks. 1854. Westwood. Quart.journ. geol. soc. Lond., x: 387,395. Pl.17,fig.7.

576.

Locality: Durdlestone Bay, England. Horizon: Lower Purbecks. 1854. Westwood. Quart. journ. geol. soc. Lond., x: 389,396. Pl. 18,fig.7.

577.

Locality: Durdlestone Bay, England, Horizon: Lower Purbecks. 1854. Westwood. Quart. journ. geol. soc. Lond., $\mathrm{x}: 389,396 . \quad P l .18, f i g .8$.

578.

Locality: Durdlestone Bay, England. Horizon: Lower Purbecks. 1854. Westwood. Quart. journ. soc. Lond., x: 389, 396. Pl. 18, fig. 13.

579.

Locality : Durdlestone Bay, England. Horizon: Lower Purbecks. 1854. Westwood. Quart. journ. geol. soc. Lond, x : 389,396. Pl. 18, fig. 15.

\section{ORTHOPTERA.}

580.

Locality : Dumbleton, England. Horizon: Upper Lias.

1873. Brodie. Distr. corr. foss. ins., 15.

581.

Locality : Grafton, England. Horizon: Lower Lias.

1873. Brodie. Distr. corr. foss, ins., 16.

582.

Locality : Copt Heath near Knowle, England. Horizon: Lower Lias. 1875. Brodie.

Jower Lias Eatington, etc., 10.

583.

Localities: Brown's Wood and Stooper's Wood, near Henley in Arden, England.

Horizon: Lias.

1875. Brodie. Lower Lias Eatington, etc., 10.

584.

Locality : Ilminster, England. Horizon: Upper Lias.

1878. Goss. $\quad$ Proc. geol, assoc., vi : 130 note.

1878. " Ins, fauna mesoz. per, ii : 17 note.

585.

Locality: Durdlestone Bay, England. Horizon: Lower Purbecks. 1854. Westwood. Quart. journ. geol. soc. Lond., $\mathrm{x}: 390,396 . \quad$ Pl. 18, fig. 38.

586.

Locality : Durdlestoue Bay, England. Horizon: Lower Purbecks. 1854. Westwood. Quart. journ. geol. soc. Lond., $\mathrm{x}: 390,396 . \quad P l .18, \mathrm{fig} .40$. 
587.

Locality : Durdlestone Bay, England. Horizon: Lower Purbecks. 1854. Westwood. Quart. journ. geol. soc. Lond., x : 390,396. Pl. 18, fig. 41.

588.

Locality : Durdlestone Bay, England. Horizon : Lower Purbecks. 1854. Westwood. Quart. journ. geol. soc. Lond., $\mathrm{x}: 390,396 . \quad$ Pl. 18, fig. 42.

589. (Grasshopper?)

Locality: Durdlestone Bay, England. Horizon: Lower Purbecks. 1854. Westwood. Quart. journ. geol. soc. Lond., $\mathrm{x}:$ 388, 393. Pl. 14, fig. 5.

590.

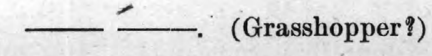

Locality : Durdlestone Bay, England. Horizon: Lower Purbecks. 1854. Westwood. Quart. journ. geol, soc. Lond., $\mathrm{x}: 388,393 . \quad P l .14$, fig. 7. 591. (Grasshopper.)

Locality : Durdlestone Bay, England. Horizon : Middle Purbecks. 1854. Westwood. Quart.journ. geol.soc. Lond., x: 384, 394. Pl. 15, fig. 14 ł. 1886. Scudder. Mem. Bost. soc.nat. hist., iii: 451. Pl. 48, figs. 4, 8 b. 1890. " Foss.ins. N. A., i: 343. Pl. 22, figs. 4,8b.

\section{2.}

Locality : Durdlestone Bay, England. Horizon: Middle Purbecks. 1854. Westwood. Quart. journ. geol, soc. Lond., x: 384, 394 (Neuroptera). Pl. 15, fig. 22.

See also Blattina anceps, Aporoblattina anceps.

593. (Blattariæ?)

Locality : England. Horizon: Wealden.

1845. Brodie. Foss. ins. sec. rocks Engl., 121 (undetermined). Pl. 5, fig. 6. 1886. Scudder. Mem. Bost. soc. nat. hist., iii : 483.

1890. " Foss.ins, N. A., i: 375.

594.

(Blattariæ.)

Locality : Wainlode, Strensham, England. Horizon : Lower Lias.

1845. Brodie.

Foss. ins. sec. rocks Engl., 101 (Westwood, 125). Pl. 8, fig. 17.

595.

Locality : Dumbleton, England. Horizon : Upper Lias.
(Blattariæ.)

1873. Brodie. Distr. corr. foss. ins., 15.

596.

(Blattariæ.)

Locality: Kota, India. Horizon: Lias?

1861. Hislop. Quart. journ. geol. soc. Lond., xvii, i : 354.

597.

(Blattariæ.)

Locality : England. Horizon: Lias.

1871. Phillips. Geology of Oxford, 123. 
598. (2 sp.) (Blattariæ.)

Locality: Fairplay, Colo. Horizon: Trias.

1883. Scudder. Proc. Bost. soc. nat. hist., xxii : 59.

599. (Blattariæ.)

Locality: Durdlestone Bay, England. Horizon: Middle Purbecks. 1854. Westwood. Quart. journ. geol. soc. Lond., x: 384,394. Pl. 15, fig. 19.

600. (Blattariæ.)

Locality : Durdlestone Bay, England. Horizon: Middle Purbecks, 1854. Westwood. Quart. journ. geol. soc. Lond., $\mathrm{x}$ : 384,394. Pl. 15, fig. 20.

601. (Gryllides.)

Localities: Forthampton, Aust, Eng and. Horizon: Lower Lias. 1845. Brodie. Foss. ins. sec. rocks Engl., 101 (Westwood, 125). Pl. 9, fig. 3.

\section{2. - - - (Gryllides.)}

Localities : Wilmcote, Binton, Apperley, Grafton, England. Horizon: Lower Lias. 1873. Brodie. Distr. corr. foss. ins., 16.

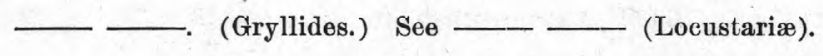

603. - - ( - (Leg.) (Locustariæ.)

Localities: Forthampton, Aust, England. Horizon: Lower Lias.

1845. Brodie. Foss. ins. sec. rocks Engl., 101 (Westwood, 125) (Gryllidæ). Pl. 9, fig. 2.

604. - (Locustariæ.)

Locality: Australia. Horizon : Lower Lias.

1845. Brodie. Foss. ins. sec. rocks Engl., 101 (Gryllidæ). Pl. 9, fig. 14.

Abia duplicata. See II, Neuropt., Idem.

605. Abia sipylus. (Blattariæ.)

Locality : Durdlestone Bay, England. Horizon : Lower Purbecks.

1856. Giebel. Insect. d. vorwelt, 263-264.

See also Sialium sipylus, Pterinoblattina sipylus.

606. Acheta quærula. (Gryllides.)

Locality : Solenbofen, Bavaria. Horizon: Jura.

1869. Weyenbergh. Arch. Mus. Teyl., ii: 276 (Achita). Pl. 36, fig. 29.

1869. " " Ins. foss. calc. lithogr., 30 (Achita). Pl. 3, fig. 29.

1869. " " Tijdschr. v. ent., (2), iv : 234 [134] (Achita).

1874. " $"$ Periód. zool., i: 97 (Achita).

1874. “ Enum. syst. faune ent. mésoz., 11 (Achita).

607. Acheta sedgwicki. (Gryllides.)

Locality: Vale of Wardour, England. Horizon : Purbecks.

1845. Brodie. Foss. ins. sec. rocks Engl., 6, 32, 118. Pl. 2, fig. 4.

See also Gryllus sedgwicki. 
Achita quærula. See Acheta quærula.

608. Acridiites - (Acridii.)

Locality : Dobbertin, Mecklenburg. Horizon : Lower Jura.

1880. E. Geinitz. Zeitschr. deutseh. geol. gesellsch., 1880:522-523. Pl. 22, fig. 5. 1884. " Zeitschr. deutsch. geol. gesellsch., 1884: 572.

609. Acridiites deperditus. (Acridii.)

Locality : Schambelen, Switzerland. Horizon: Lias.

1865. Heer. Urwelt der Schweiz, 84. Pl. 7, fig.4.

1872. " Monde prim. Suisse, 102. Pl. 7, fig. 4.

1876. “ Prim. world Switz., i: 84. Pl. 7, fig. 4.

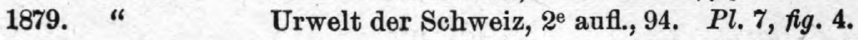

610. Acridiites liasinus. (Acridii.)

1865. Heer.

1872. "

1876. “

1879. “

Locality: Schambelen, Switzerland. Horizon: Lias.

Urwelt der Schweiz, 84.

Monde prim. Suisse, 102.

Prim. world Switz., i : 84.

Urwelt der Schweiz, $2^{\mathrm{e}}$ aufl., 94.

611. Akicera frauenfeldi. (Acridii.)

Locality : England. Horizon: Lower Lías.

1845. Brodie. Foss. ins. sec. rocks Engl., 128 (unnamed). Pl. 8, fig. 18.

1856. Giebel. Insect. $d$, vorwelt, 310.

\section{Akicera heerl. (Acridii.)}

Localities: Wainlode, Streusham, England. Horizon: Lower Lias.

1845. Brodie.

Foss. ins. sec. rocks Engl., 101 (unnamed). $\quad$ Pl. 8, fig. 17.

1856. Giebel.

Insect. $d$. vorwelt, 310 .

\section{Anthracoblattina —- (Palæoblattariæ.)}

Locality : Fairplay, Colo. Horizon: Trias.

1884. Scudder. Amer. journ, sc., (3), xxviii : 200.

See also A. triassica.

614. Anthracoblattina triassica. (Palæoblattariæ.)

Locality : Fairplay, Colo. Horizon: Trias.

1890. Scudder. Mem. Bost. soc. nat. hist., iv: 464-465. Pl. 41, fig. 9.

1890. " Foss. ins. N. A., i: 440-441. Pl. 33, fig. 9.

See also Anthracoblattina -

615. Aporoblattina anceps. (Blattariæ.)

Locality: Durdlestone Bay, England. Horizon: Middle Purbecks.

1886. Scudder. Mem. Bost. soc. nat. hist., iii : 479.

1890. "6 Foss. ins. N.A., i: 371.

See also Blattina anceps, $\_-1$ Westw., 15: 22.

616. Aporoblattina butleri. (Blattariæ.)

Loeality: England. Horizon: Lias.

1886. Scudder, Mem. Bost. soc. nat. hist., iii: 482 . Pl. 48, fig. 15 .

1890. " Foss, ins. N. A., i : 374. Pl.22, fig. 15. 
617. Aporoblattina eatoni. (Blattariø.)

Locality: England. Horizon: Purbecks.

1886. Scudder. Mem. Bost. soc. nat. hist., iii: 479. Pl. 48, fig. 19.

1890. " Foss, ins, N. A., i: 371. Pl. 22, fig. 19.

618. Aporoblattina exigua. (Blattariæ.)

Locality: Durdlestone Bay, England. Horizon: Lower Purbecks.

1854. Westwood. Quart.journ. geol. soc. Lond., x: 390,396 (unnamed). Pl. 18, fig. 38.

1886. Scudder. Mem. Bost. soc. nat. hist., iii : 481-482.

1890. " Foss. ins. N. A., i : 373-374.

619. Aporoblattina incompleta. (Blattariæ.)

Locality: England. Horizon: Lias.

1886. Scudder. Mem. Bost. soc. nat. hist., iii : 481 .

1890. " Foss. ins. N. A., i : 373.

See also Blattina incompleta, Blattidium incompletum.

620. Aporoblattina kollari. (Blattariæ.)

Locality: England. Horizon: Purbecks.

1886. Scudder. Mem. Bost. soc. nat. hist., iii : 480.

1890. " Foss. ins. N. A., i: 372.

See also Blattidium kollari, etc.

621. Aporoblattina maclachlani. (Blattariø.)

Locality: England. Horizon: Purbecks.

1854. Westwood. Quart. journ. geol. soc. Lond., x: 396 (unnamed). Pl. 18, fig. 35. 1886. Scudder. Mem. Bost. soc. nat. hist., iii : 479-480. Pl. 48, fig. 18.

1890. " Foss. ins. N. A., i : 371-372. Pl. 22, fig. 18.

622. Aporoblattina nana. (Blattariæ.)

Locality: Dobbertin, Mecklenburg. - Horizon: Jura.

1886, Scuddər. Mem. Bost. soc. nat. hist., iii : 481.

1890. " Foss. ins. N. A., i: 373.

See also Blattina nana.

\section{Aporoblattina recta. (Blattariæ.)}

Locality: England. Horizon: Wealden.

1886. Scudder. Mem. Bost. sec. nat. hist., iii : 481.

1890. " Foss. ins. N. A., i: 373.

See also Blattina recta, etc.

624. Aporoblattina westwoodi. (Blattariæ.)

Locality : Durdlestone Bay, England. Horizon : Purbecks.

1854. Westwood. Quart. journ. geol. soc. Lond., x: 396 (unnamed). Pl. 18, fig. 28.

1886. Sendder. Mem. Bost. soc. nat. hist, iii: 480 .

1890. " Foss. ins. N. A., i: 372. 
625. Baseopsis forficulina. (Forficulariæ.)

Locality: Schambelen, Switzerland. Horizon: Lias.

1865. Heer. Urwelt der Schweiz, 84. Pl. 7, fig. 5.

1872. " " Monde prim. Suisse, 103. Pl.7, fig. 5.

1876. " Prim. world Switz., i: 84. Pl. 7, fig. 5.

1879. “ Urwelt der Schweiz, 94. Pl. 7, fig. 5.

626. Baseopsis ? sibirica. (Forficulariæ.)

Locality : Ust Balei, Siberia. Horizon: Lias.

1889. (Brauer-Redt-\{Mém. acad. sc. St. Pétersb., (7), xxxvi, No 15: 3-4. Pl. [1], \{enb.-Ganglb.\} figs. 1, 1 b.

627. Bittacus dubius. (Locustariæ.)

Locality: Vale of Wardour, England. Horizon: Purbecks.

1845. Brodie. Foss. ins. sec. rocks Engl., 119 (unnamed). Pl. 5, fig. 21.

1856. Giebel. Insect. d. vorwelt, 258.

1886. Deichm. Ins. lith. schichten dresd. mus., 16-17 (cf. Elcana).

See also Clathrotermes (Elcana) geinitzi, etc.

628. Blabera avita. (Blattariæ.)

Locality: Solenhofen, Bavaria. Horizon: Jura.

1847. Heyden. Palaeontogr., i: 100-101. Pl. 12, fig. 5.

1856. Giebel. Insect. d. vorwelt, 497.

1869. Weyenbergh. Tijdschr. v, ent., (2), iv : 234, [134] (invita).

1885. Scudder. Zittel, Handb. palaeont., i, th. ii : 767 .

1886. " Zittel-Barrois, Traité de paléont., ii : 767.

1886. " Mem. Bost. soc. nat. hist., iii : 483.

1886. Deichm. Ins, lith. schichten dresd. mus., 69 (Hydrophilid?).

1890. Scudder. Foss. ins. N. A., i : 375.

See also Musca lithophila, etc.; also CoLEOPT., Pseudohydrophilus longispinosus, Prodytiscus longispinosus.

\section{Blatta - (Blattariæ.)}

Locality : Vale of Wardour, England. Horizon : Purbecks.

1845. Brodie. Foss. ins. sec. rocks Engl., 32 (Westwood, 118). Pl. 3, fig. 7.

630. Blatta - (Blattariæ.)

Localities: Brown's Wood and Stooper's Wood, near Henley in Arden, England. Horizon : Lias.

1875. Brodie. Lower Lias Eatington, ete., 10.

Blatta dubia. See Gryllites dubius.

631. Blatta elongata. (Blattariæ.)

Locality : Durdlestone Bay, England. Horizon: Middle Purbecks.

1856. Giebel. Insect. d. vorwelt, 322 .

1877. Goldenberg. Fauna saræp. foss., ii: 21.

See also Blattidium elongatum, etc.

632. Blatta kollari. (Blattariæ.)

Locality : England. Horizon: Wealden.

1856. Giebel. Insect. d. vorwelt, 322.

See also Blattidium kollari, etc. 
633. Blatta pinna. (Blattariæ.)

Locality : Vale of Wardour, England. Horizon: Purbecks.

1845. Brodie. Foss. ins. sce. rocks Engl., 118 (unnamed). Pl. 5, fig. 5.

1856. Giebel. Insect. $d$. vorwelt, 322.

See also Blattidium pinna, Ctenoblattina pinna.

634. Blatta pluma. (Blattariæ.)

Locality : Durdlestone Bay, England. Horizon: Middle Purbecks.

1854. Westwood. Quart. journ. geol. soc. Lond., $\mathrm{x}: 394$ (unnamed). Pl. 15, fig. 14 . 1856. Giebel. Insect. d. vorwelt, 322.

1877. Goldenberg. Fauna saræp. foss., ii : 21.

See also Pterinoblattina pluma, Blattidium pluma.

635. Blatta stricklandi. (Blattariæ.)

Locality: Vale of Wardour, England. Horizon: Purbecks.

1845. Brodie.

Foss. ins. sec. rocks Engl., 32, 118. Pl. 4, fig. 11.

1856. Giebel.

Insect. d. vorwelt, 317.

1877. Goldenberg. Fauna saræp. foss.,, ii : 21.

See also Blattidium stricklandi, Rithma stricklandi.

636. Blatta ungeri. (Blattariæ.)

Locality : Durdlestone Bay, England. Horizon: Lower Purbecks.

1854. Westwood. Quart. journ. geol. soc. Lond., x: 387, 395 (unnamed). Pl.17, fiq. 13.

1856. Giebel. Insect. d. vorwelt, 322 .

1877. Goldenberg. Fauna saræp. foss., ii : 21.

See also Blattidium ungeri, Diechoblattina ungeri.

637. Blattaria dunckeri. (Blattariæ.)

Locality: Solenhofen, Bavaria. Horizon: Jura.

1874. Weyenbergh. Períd. zool., i: 86,98. Pl. 3, fig. 12.

1874. “ Enum. syst. faune ent. mésoz., 12.

1886. Seudder. Mem. Bost. soc. nat. hist., iii : 483.

1890. “ Foss. ins. N. A., i : 375.

638. Blattidium - (Blattariæ.)

Locality : England. Horizon: Wealden.

1845. Brodie. Foss. ins. sec. rocks Engl., 119 (unnamed). Pl. 5, fig. 3.

1864. Heer. Viert. naturf. gesellsch. Zürich, ix : 290.

See also Blattina recta, etc.

639. Blattidium ㄴ. (Blattariæ.)

Locality : Vale of Wardour, England. Horizon : Purbecks.

1877. Goldenberg. Fauna saræp. foss., ii : 21, No. 66.

See also Blattina similıs, etc.

640. Blattidium - - (Blattariæ.)

Locality: Durdlestone Bay, England. Horizon: Middle Purbecks.

1854. Westwood. Quart. journ. geol. soc. Lond., x: 394 (unnamed). Pl. 15, fig. 19.

1864. Heer.

Viert. naturf. gesellsch. Zürich, ix : 289

1877. Goldenberg. Fauna saræp. foss., ii : 21, No. 69.

1886. Scudder. Mem. Bost. soc. nat. hist., iii : 483 .

1890. " Foss. ins. N. A., i : 375. 


\section{Blattidium -}

Locality: Durdlestone Bay, England. Horizon: Lower Purbecks.

1854. Westwood: Quart. journ. geol. soc. Lond., $\mathrm{x}: 396$ (unnamed). $\quad P l .18, f i g .35$. 1864. Heer. Viert. naturf. gesellsch. Zïrich, ix : 290.

1877. Goldenberg. Fauna saræp. foss., ii : 21, No. 60 .

\section{Blattidium ——. (Blattariæ.)}

Locality: Durdlestone Bay, England. Horizon : Lower Purbecks.

1854. Westwood. Quart. journ. geol. soc. Lond, x: 396 (innamed). Pl. 18, fig. 38. 1864. Heer.

Viert. naturf. gesellsch. Zürich, ix : 290.

1877. Goldunberg. Fauna saræp. foss., ii: 21, No. 61.

643. Blattidium achelous. (Blattariæ.)

Locality: Durdlestone Bay, England. Horizon: Lower Purbecks.

1854. Westwood. Quart. journ. geol. soc. Lond., x: 390, 396. Pl. 18, fig. 26.

1856. Giebel. Insect. d. vorwelt, 318.

1864. Heer. Viert. naturf. gesellsch. Zürich, ix : 290.

1877. Goldenberg. Fauna saræp. foss., ii : 21.

\section{Blattidium antiquum. (Blattariæ.)}

Locality: Durdlestone Bay, England. . Horizon: Lower Purbecks.

1864. Heer. Viert. naturf. gesellsch. Zürich, ix : 290.

See also Rithma antiqua,etc.

645. Blattidium ? avitum. (Blattariæ.)

Locality: Solenhofen, Bavaria. Horizon: Jura.

1864. Heer. Viert. naturf. gesellsch. Zürich, ix : 289.

1877. Goldenberg. Fauna saræp. foss., ii : 20.

See also Blabera avita, etc., Musca lithophila, etc.

\section{Blattidium beroldingianum. (Blattariæ.)}

Locality : Solenhofen, Bavaria. Horizon: Jura.

1864. Heer. Viert. naturf. gesellsch. Zürich, ix : 289,300-301. Pl., fig. 8.

1877. Goldenberg. Fauna saræp. foss., ii : 20.

See also Musca lithophila, etc.

\section{Blattidium elongatum. (Blattariæ.)}

Locality : Durdlestone Bay, England. Horizon: Middle Purbecks.

1854. Westwood. Quart. journ. geol. soc. Lond., x: 384, 394 (unnamed). Pl. 15, fig. 23.

1864. Heer. Viert. naturf. gesellsch. Zürich, ix : 289.

See also Blatta elongata, Mesoblattina elongata.

648. Blattidium incompletum. (Blattariæ.)

Locality: England. Horizon: Lias.

1864. Heer. Viert. naturf. gesellsch. Züirich, ix : 289.

1877. Goldenberg. Fauna saræp. foss, ii : 20.

See also Blattina incompleta, Aporoblattina incompleta. 
649. Blattidium kneri. (Blattariæ.)

Locality : England. Horizon: Wealden.

1864. Heer. Viert. naturf. gesellsch. Zürich, ix : 291. 1877. Goldenberg. Fauna saræp. foss., ii : 21.

See also Elisama kneri.

650. Blattidium kollari. (Blattariæ.)

Locality: Vale of Wardour, England. Horizon: Purbecks.

1864. Heer.

Viert. naturf. gesellsch. Zürich, ix : 290.

1877. Goldenberg. Fauna saræp. foss., ii : 21.

See also Blatta kollari, Aporoblattina kollari ; also II, NEUropt., Corydalis Brodie.

651. Blattidium liasinum. (Blattariæ.)

Locality : Wainlode, England. Horizon : Lias.

1864. Heer. Viert. naturf. gesellsch. Zürich, ix : 289 (liassinum).

1877. Goldenberg. Fauna saræp. foss., ii : 20.

See also Blattina liasina, etc.

Blattidium liassinum. See Blattidium liasinum.

652. Blattidium medium. (Blattariæ.)

Locality : Schambelen, Switzerland. Horizon: Lias.

1864. Heer. Viert. naturf. gesellsch. Zürich, ix: 289, 300. Pl., fig. 7.

1877. Goldenberg. Fauna saræp. foss., ii : 20.

See also Elisama media.

653. Blattidium minor. (Blattariæ.)

Locality: England. Horizon: Wealden.

1864. Heer. Viert. naturf. gesellsch. Zürich, ix : 291.

See also Elisama minor.

654. Blattidium molossus. (Blattariæ.)

Locality: Durdlestone Bay, England. Horizon:-Middle Purbecks.

1854. Westwood. Quart. journ. geol, soc. Lond., $x: 384,394 . \quad$ Pl. 15, fig. 26.

1864. Heer. Viert. naturf. gesellsch. Zürich, ix : 289.

1877. Goldenberg. Fauna saræp. foss., ii : 21.

See also Nethania molossus, Elisama molossus.

655. Blattidium morrisi. (Blattariæ.)

Locality: Durdlestone Bay, England. Horizon: Lower Purbecks.

1864. Heer. Viert. naturf. gesellsch. Zürich, ix : 290.

See also Rithma morrisi.

656. Blattidium murchisoni. (Blattariæ.)

Locality: Dardlestone Bay, England. Horizon: Lower Purbecks.

1864. Heer. Viert. naturf. gesellsch. Zürich, ix : 290.

See also Rithma murchisoni, etc. 
657. Blattidium nogaus. (Blattariæ.)

Locality : Durdlestone Bay, Engiand. Horizon: Lower Purbecks.

1854. Westwood. Quart. journ. geol. soc. Lond., $\mathrm{x}:$ 390, 396. Pl. 18, fig. 23.

1856. Giebel.

Insect. d. vorwelt, 318.

1864. Heer.

Viert. naturf. gesellsch. Zürich, ix : 290.

1877. Goldenberg. Fauna saræp. foss., ii : 21.

1886. Sendder. Mem. Bost. soc. nat. hist., iii : 474.

1890. " Fosș. ins. N. A., i: 366.

658. Blattidium pinna. (Blattariæ.)

Locality: Vale of Wardour, England. Horizon: Purbecks.

1864. Heer. Viert. naturf. gesellsch. Zürich, ix : 290.

1877. Goldenberg. Fauna saræp. foss., ii : 21.

See also Blatta pinna, Ctenoblattina pinna.

659. Blattidium pluma. (Blattariæ.)

Locality : Durdlestone Bay, England. Horizon: Middle Purbecks.

1864. Heer. Viert. naturf. gesellsch. Zürich, ix : 289.

See also Blatta pluma, Pterinoblattina pluma.

660. Blattidium purbeccensis. (Blattariæ.)

Locality : Durdlestone Bay, England. Horizon: Lower Purbecks.

1864. Heer. Viert. naturf. gesellsch. Zürich, ix : 290.

See also Rithma purbeccensis.

\section{Blattidium ramificatum. (Blattariæ.)}

Locality : Durdlestone Bay, England. Horizon: Middle Purbecks.

1864. Heer. Viert. naturf. gesellsch. Züirich, ix: 289.

See also Rithma ramificata, Elisama ramificata.

662. Blattidium similis. (Blattariæ.)

Locality: Vale of Wardour, England. Horizon: Purbecks.

1864. Heer. Viert. naturf. gesellsch. Zïrich, ix: 289.

See also Blattina similis, etc.

Blattidium simyrus. See Blattidium symyrus.

663. Blattidium stricklandi. (Blattariæ.)

Locality: Vale of Wardour, England. Horizon: Purbecks.

1864. Heer. Viert. naturf. gesellsch. Zürich, ix: 290.

See also Blatta stricklandi, Rithma stricklandi.

664. Blattidium symyrus. (Blattariæ.)

Locality : Durdlestone Bay, England. Horizon: Lower Purbecks.

1854. Westwood. Quart. journ. geol. soc. Lond., x: 390, 396. Pl. 18, fig. 33.

1864. Heer. Viert. naturf. gesellsch. Zürich, ix: 290.

1877. Goldenberg. Fauna saræp. foss., ii : 21.

1885. Scudder. Proc. acad. nat. sc. Philad., 1885: 112.

1885. “ Zittel, Handb. palaeont,, i, th. ii : 766 (simyrus). Fig. 961.

1886. " Zittel-Barrois, Traité de paléont., ii : 766. Fig. 977.

1886. " Miall, Cockroach, 217 (simyrus). Fig. 124.

1886. " Mem. Bost. soc. nat. hist, iii : 474. - Pl. 48, fig. 17.

1886. Deichmmüller. Ins. lith. schichten dresd. mus., 14 (near Chresmoda).

1890. Scudder. Foss. ins. N. A., i: 366. Pl. 22, fig. 17. 
665. Blattidium ungeri. (Blattariæ.)

Locality: Durdlestone Bay, England. Horizon : Lower Purbecks. 1864. Heer.

Viert. naturf. gesellsch. Zürich, ix : 290 .

See also Blatta ungeri, etc.

\title{
666. Blattidium westwoodi. (Blattariæ.)
}

Locality : Durdlestone Bay, England. Horizon: Lower Purbecks. 1864. Heer.

Viert. naturf. gesellsch. Zürich, ix : 290.

See also Rithma westwoodi.

\author{
667. Blattina - (Blattariæ.) \\ Locality : Eichstadt, Bavaria. Horizon: Jura.
}

1886. Deichmüller. Ins. lith. schichten dresd. mus., 9-10.

668. Blattina anceps. (Blattariæ.)

Locality : Durdlestons Bay, England. Horizon : Middle Purbecks.

1854. Westwood. Quart. journ. geol. soc. Lond., x: 394 (unnamed) (wrongly quoted fig. 21 by Giebel). $\quad P l .15$, fig. 22.

1856. Giebel. Insect. d. vorwelt, 317.

See also $\Lambda$ poroblattina anceps, —— Westw., 15: 22.

Blattina angusta. See Blattina angustatia.

669. Blattina angustata. (Blattariæ.)

Locality : Schambelen, Switzerland, Horizon : Lias.

1864. Heer. Viert. naturf. gesellsch. Ziirich, ix : 288,299-300. Pl., fig. 6.

1865. " Urwelt der Schweiz, 83.

1872. " Monde prim. Suisse, 101.

1876. " Prim. world Switz., i: 83.

1877. Goldenberg. Fauna saræp. foss., ii : 20 (angusta).

1879. Heer. Urwelt der Schweiz, $2^{\circ}$ aufl:, 93.

See also Mesoblattina angustata, Blattina (Mesoblattina) angustata.

670. Blattina (Mesoblattina) angustata. (Blattariæ.)

Locality : Schambelen, Switzerland. Horizon: Lias.

1880. E. Geinitz. Zeitschr. deutsch. geol. gesellsch., 1880: 519-520.

See also Blattina angustata, etc.

671. Blattina chrysea. (Blattariæ.)

Locality : Dobbertin, Mecklenburg. Horizon : Lower Jura.

1880. E. Geinitz. Zeitschr. deutsch. geol. gesellsch., 1880: 520. Pl. 22, fig. 2. 1884. " Zeitschr. deutsch. geol. gesellsch., 1884: 570,571.

See also Pterinoblattina chrysea.

672. Blattina (Mesoblattina) dobbertinensis. (Blattariæ.)

Locality: Dobbertin, Mecklenburg. Horizon: Lias.

1884. E. Geinitz. Zeitschr. dentsch. geol. gesellsch., 1884: 570. Pl. 13, fig. 1. See also Mesoblattina dobbertinensis. 
673. Blattina formosa. (Blattariæ.)

Compared with Blatta orientalis $\mathrm{L}$.

Locality : Schambelen, Aargau, Switzerland. Horizon: Lias.

1852. Heer.

1854. Pictet.

1856. Giebel.

1864. Heer.

1865. " "

1872. “

1876. “

1877. Goldenberg

1879. Heer.

Lias-insel Aargau, 15. Pl., figs. 41-42.

Traité de paléont., $2^{\mathrm{e}} 6$ d., ii : $362 . \quad P l .40$, fig. 6.

Insect. d. vorwelt, 498.

Viert. naturf. gesellsch. Zürich, ix : 288, 299.

Urwelt der Schweiz, 83. Pl. 7, figs. 1, 1 .

Monde prim. Suisse, 101. Pl. 7, figs. 1, 1 b.

Prim. world Switz., i: 83. Pl. 7, figs. 1, $1 b$.

Fauna saræp. foss., ii : 20.

Urwelt der Schweiz, 2e aufl., 93. Pl. 7, figs. 1, $1 b$.

See also Rithma formosa.

674. Blattina incerta. (Blattariæ.)

Locality : Dobbertin, Mecklenburg. Horizon : Lias.

1884. E. Geinitz. Zeitschr. deutsch. geul. gesellsch., 1884: 571, 572. Pl. 13, fig. 2. 1886. Seudder. Mem. Bost. soc. nat. hist., iii : 483.

1890. " " Foss, ins. N. A., i : 375.

675. Blattina incompleta. (Blattariæ.)

Locality : England. Horizon: Lias.

1845. Brodie. Foss. ins. sec, rocks Engl. (unnamed). $\quad P l .8$, fig. 13.

1856. Giebel. Insect. d, vorwelt, 317.

See also Aporoblattina incompleta, Blatitidium incompletum.

676. Blattina langfeldti. (Blattariæ.)

Locality : Dobbertin, Mecklenburg. Horizon : Lower Jura.

1880. E. Geinitz. Zeitschr. deutsch. geol. gesellsch., 1880: 521. Pl. 22, fig. 3.

1884. " Zeitschr. deutsch. geol. gesellsch., 1884: 571.

See also Ctenoblattina langfeldti.

677. Blattina liasina. (Blattariæ.)

Locality : Wainlode, Strensham, England. Horizon: Lias.

1845. Brodie. Foss. ins. sec. rocks Engl., 101; Westwood, 125 (unnamed). Pl. 8, fig. 12.

1856. Giebel. Insect. $d$. vorwelt, 317.

See also Blattidium liasinum, Rithma liasina.

678. Blattina mathildæ. (Blattariæ.)

Locality: Dobbertin, Mecklenburg. Horizon: Jura.

1883. E. Geinitz. Flötzform. Mecklenb., 29-30. Pl. 6, fig. 1.

1884. " Zeitschr. deutsch. geol. gesellsch., 1884 : 571.

See also Mesoblattina mathildæ.

679. Blattina media. (Blattariæ.)

Locality: Schambelen, Switzerland. Horizon: Lias.

1865. Heer. Urwelt der Schweiz, 83.

1872. " Monde prim. Suisse, 101.

1876. " " Prim. world Switz., i: 83.

1879. " Urwelt der Schweiz, $2^{\mathrm{e}}$ aufl, 93. 
680. Blattina nana. (Blattariæ.)

Locality: Dobbertin, Mecklenburg. Horizon: Jura.

1883. E. Geinitz. Flötzform. Mecklenb., 30. Pl. 6, fig. 2.

1884. " " Zeitschr. deutsch. geol. gesellsch., 1884: 571 .

See also Aporoblattina nana.

\section{Blattina (Mesoblattina) protypa. (Blattariæ.)}

Locality: Dobbertin, Mecklenburg. Horizon: Lower Jura.

1880. E. Geinitz. Zeitschr, deutsch. geol, gesellsch., 1880: 519-520. Pl. 22, fig. 1.

1884. " Zeitschr. deutsch. geol. gesellsch., 1884: 569-570.

See also Mesoblattina protypa.

682. Blattina recta. (Blattariæ.)

Locality: England. Horizon: Wealden.

1856. Giebel. Insect. d. vorwelt, 318.

See also Blattidium — Brodie, Aporoblattina recta.

683. Blattina (Mesoblattina?) sibirica. (Blattariæ.)

- Locality: Ust Balei, Siberia. Horizon: Lias. 1889. $\left\{\begin{array}{l}\text { Brauer-Red- } \\ \text { tenb.-Ganglb. }\end{array}\right\} \begin{gathered}\text { Mém. acad. sc. St.-Péters., (7), xxxvi, No. 15: 12-13. Pl. [₹], } \\ \text { fig. 15. }\end{gathered}$

684. Blattina similis. (Blattariæ.)

Locality: Vale of Wardour, England. Horizon : Purbecks.

1854. Brodie. Foss. ins. sec. rocks Engl., 119 (unnamed). Pl. 5, fig. 2.

1856. Giebel. Insect. d. vorwelt, 318.

See also Blattidium —_ Goldenb., Blattidium similis, Nannoblattina similis; also II, Neuropt., Corydalis — Brodie.

685. Chresmoda obscura. (Acridii.)

Locality: Solenhofen, Bavaria. Horizon: Jura.

1839. Müuster. MSS.

1839. Germar. Verhandl. leop.-carol. akad. naturf., xix: 201-203. Pl. 22, fig. 4.

1852. Giebel. Dentschl, petref, 637.

1856. " Insect. d. vorwelt, 311.

1×62. Hagen. - Palaeontogr., $x: 110$ (= Locusta prisca.)

1869. Weyenbergh. Tijdschr. v. ent., (2), iv : 234 [134].

187\%. Assmann. Ber. versamml, deutsch. naturf., $1: 192$.

1886. Deichmüller. Ins. lith. schichten dresd. mus., 10-14. Pl. 1, figs. 7-12.

See also Pygolampis gigantea, Halometra gigantea, Propygolampis bronni, Locusta prisca.

Clathrotermes geinitzi. See Elcana (Clathrotermes) geinitzi.

686. Clathrotermes (Elcana) geinitzi. (Locustariæ.)

Locality: Dobbertin, Mecklenburg. Horizon: Lias.

1884. E. Geinitz. Zeitschr. deutsch. geol. gesellsch., 1884: 577-580. Pl. 13, figs. 13-23.

See also Elcana geinitzi, E. (Clathrotermes) geinitzi, E. (Clathrotermes) intercalata, E. tessellata, Bittacus dubius, Panorpidium tessellatum.

Clathrotermes intercalate. See Elcana (Clathrotermes) intercalata.

Bull. $71-8$ 
687. Conocephalus capito. (Locustariæ.)

Locality : Eichștadt, Bavaria. Horizon: Jura.

1886. Deichmüller. Ins. lith. schichten dresd. mus., 24-26. Pl. 2, fig.12.

688. Ctenoblattina arcta. (Blattariæ.)

Locality : England. Horizon: Purbecks.

1886. Scudder. Mem. Bost. soc. nat. hist., iii : 443. Pl. 46, figs. 1, 2 .

1890. " Foss. ins. N. A., i : 335. Pl. 20, figs. 1, 2.

689. Ctenoblattina langfeldti. (Blattariæ.)

Locality : Dobbertin, Mecklenburg. Horizon: Lower Jura.

1886. Scudder.

Mem. Bost. soc. nat. hist., iii : 443.

1890. " Foss. ins. N. A., i: 335 .

See also Blattina langfeldti.

690. Ctenoblattina? pinna. (Blattariæ.)

Locality: Vale of Wardour, England. Horizon: Purbecks.

1886. Scudder. Mem. Bost, soc. nat. hist., iii : 444.

1890. " Foss. ins. N. A., i: 336.

See also Blatta pinna, Blattidium pinna.

\section{Cyrtophyllites rogeri. (Locustariæ.)}

Locality: Eichstadt, Bavaria. Horizon: Jura.

1888. Oppenheim. Palaeontogr., xxxiv: 223. Pl. 30, fig. 5.

692. Decticus speciosus. (Locustariæ.)

Locality: Solenhofen, Bavaria. Horizon: Jura.

1852. Giebel. Dentschl. petref., 637.

1556. " " Insect. d. vorwelt, 304.

See also Locusta speciosa, etc.

693. Diechoblattina ungeri. (Blattariæ.)

Locality : Durdlestone Bay, England. Horizon: Lower Purbecks.

1886. Scudder. Mem. Bost. soc. nat. hist., iii : $47 \%$.

1890. “ Foss. ins. N. A., i: 369.

See also Blatta ungeri, etc.

694. Diechoblattina wallacei. (Blattariæ.)

Locality: England. Horizon: Purbecks.

1886. Scudder. Mem. Bost. soc. nat. hist., iii: 477-478. Pl. 48, fig. 1. 1890. “ Foss. ins. N. A., i: $369-370$. Pl. 22, fig. 1.

695. Dipluroblattina bailyi. (Blattariæ.)

Locality : England. Horizon: Purbecks.

1886. Scudder. Mem. Bost. soc. nat. hist., iii : 476-477. Pl. 48, fig. 5.

1890. " Foss. ins. N. A., i: $368-369 . \quad$ Pl. 22, fig. 5.

696. Elcana amanda. (Locustariæ.)

Locality: Eichstadt, Bavaria. . Horizon: Jura.

1886. Deichmüller. Ins. lith. schichten dresd. mus., 14. Pl. 2, figs. 4-5.

See also Locusta amanda, Elcana (Locusta) amanda. 
697. Ficana (Locusta) amanda. (Locustarix.)

Locality : Eichstadt, Bavaria. Horizon: Jura.

1888. Oppenheim. Palaeontogr., xxxiv: 224. Pl. 30, fig. 17.

See also Elcana amanda, Locusta amanda.

Elcana beyrichi. See II, Neuroptrra, Idem.

698. Elcana geinitzi. (Locustariæ.)

Locality : Dobbertin, Mecklenburg. Horizon: Lower Jura.

1886. Deichmïller. Ins. lith. schichten dresd. mus., 17. Pl. 2, fig. 7.

See also Clathrotermes (Elcana) geinitzi, etc.

699. Elcana (Clathrotermes) geinitzi. (Locustariæ.)

Locality : Dobbertin, Mecklenburg. Horizon: Lower Jura.

1880. Heer. Geinitz, Zeitschr. deutseh. geol. gesellsch, 1880:523-526. Pl. 22, figs. 7-10.

See also Clathrotermes (Elcana) geinitzi, etc.

700. Elcana (Clathrotermes) intercalata. (Locustariæ.)

Locality : Dobbertin, Mecklenburg. Horizon: Lower Jura.

1880. Geinitz. Zeitschr. dentsch. geol. gesellsch., 1880: 526. Pl. 22, fig. 11.

See also Clathrotermes (Elcana) geinitzi, etc.

Elcana sipylus. See Sialium sipylus.

701. Elcana tessellata. (Locustariæ.)

Locality: Durdlestone Bay, England. Horizon: Middle Purbecks.

1856. Giebel. Insect. d. vorwelt, 259 (Westwood, Pl. 15, fig. 17, not Pl. 17, fig. 12).

1886. Deichmiuller. Ins. lith. schichten dresd. mus., $16 . \quad P l .2$, fig. 6.

See also Clathrotermes (Elcana) geinitzi, etc.

702. Elisama bucktoni. (Blattarix.)

Locality : England. Horizon: Purbecks.

1886. Scudder. Mem. Bost. soc. nat. hist., iii: 466-46\%. Pl. 47, figs. 8, 12.

1890. “ Foss. ins. N. A., i: 358-359. Pl. 21, figs. 8, 12.

703. Elisama kirkbyi. (Blattariæ.)

Locality: England. Horizon: Mesozoic.

1886. Scudder. Mem. Bost. soc. nat. hist., iii : 467-468. Pl. 47, fig. 3 .

1890. “ . Foss. ins. N. A., i: 359-360, Pl. 21, fig. 3.

704. Elisama kneri. (Blattariæ.)

Locality : England. Horizon: Wealden.

1845. Brodie. Foss. ins, sec. rocks Engl., 118 (unnamed). Pl. 5, fig. 1.

1856. Giebel. Insect. $d$. vorwelt, 320.

1885. Scudder. Proc. acad. nat. sc. Philad., 1885: 113.

1886. "6 Mem. Bost. soc. nat. bist., iii: 465-466. Pl. 47, fig. 1.

1890. " Foss. ins. N. A., i: 357-358. Pl. 21, fig. 1.

See also Blattidium kneri. 
705. Elisama? media. (Blattariæ.)

Locality : Schambelen, Switzerland. Horizon: Lias.

1886. Scudder. Mem. Bost. soc. nat. hist., iii : 468.

1890. " Foss.ins. N. A., i: 360 .

See also Blattidium medinm.

706. Elisama minor. (Blattariæ.)

1845. Brodie.

Locality: England. Forizon: Wealden.

1856. Giebel.

Foss. ins. sec. rocks Engl., 118 (unnamed). Pl. 5, fig. 20.

1877. Goldenberg. Fauna saræp. foss., ii: 21.

1885. Scudder. Proc. acad. nat. sc. Philad., 1885: 113.

1886. " " Mem. Bost. soc. nat. hist., iii: 466. Pl. 47, fig. 13.

1890. “ Foss, ins. N. A., i: 358. Pl. 21, fig. 13.

See also Blattidium minor.

\section{Elisama molossus. (Blattariæ.)}

Locality: Durdlestone Bay, Eugland. Horizon: Middle Purbecks.

1886. Scudder. Mem. Bost. soc. nat. hist., iii : 464-465.

1890. " Foss. ins, N. A., i: 356-357.

See also Blattidium molossus, Nethania molossus.

708. Elisama? ramificata. (Blattariæ.)

Locality : Durdlestone Bay, England. Horizon: Middle Purbecks.

1886. Scudder. Mem. Bost. soc. nat. hist., iii : 483.

1890. " Foss. ins. N. A., i: 375.

See also Rithma ramificata, Blattidium ramificatum.

709. Etoblattina -. (Palæoblattariæ.)

Locality: Fairplay, Colo. Horizon: Trias.

1883. Scudder. Proc. Bost. soc. nat. hist., xxii: 59.

1884. " " Amer. journ. sc., (3), xxviii : 200.

1890. " Mem. Bost. soc. nat. hist., iv: 460. Pl. 42, fig. 20.

1890. “ Foss. ins. N. A., i: 436. Pl. 34, fig. 20.

See also E. persistens.

710. Etoblattina persistens. (Palæoblattariæ.)

Locality, Fairplay, Colo. Horizon: Trias.

1890. Scudder. Mem. Bost. soc. nat. hist., iv: 459-460. Pl. 41, fig. 7; pl. 42, figs c $10,19$.

1890. " Foss. ins. N. A., i : 435-436. Pl. 33, fig. 7; pl. 34, figs. 10, 19.

See also Etoblattina

711. Forficularia problematica. (Forficulariæ.)

Locality: Solenhofen, Bavaria. Horizon: Jura.

1869. Weyenbergh. Arch. mus. Teyl., ii : 274-275. Pl. 36, figs. 25, 26, $26 a$.

1869. " " Ins. foss. calc. lithogr., 28-29. Pl. 3, figs. 25, 26, 26a.

1869. " " Tijdschr. v. ent., (2), iv: 234 [134].

1874. "6 Periód. zool, i: 97.

1874. “ Énum. syst. faune ent. mésoz., 11, 
712. Gomphocerites bernstorff. (Acridii.)

Locality: Dobbertin, Mecklenburg. Horizon: Lower Jura.

1880. E. Geinitz. Zeitschr. deutsch. geol, gesellsch., 1880: 521-522. Pl. 22, fig. 4. 1884. " " Zeitschr. deutsch. geol. gesellsch., 1884: 572.

713. Gomphocerites bucklandi. (Acridii.)

Locality: Schambelen, Switzerland. Horizon: Lias.

1×52. Heer. Lias-insel Aargau, 15. Pl., fig. 43.

1854. Pietet. Traité paléont., $2^{\circ}$ Gd., ii : 365 . Pl. 40, fig. 5.

1856. Giebel. Insect. d. vorwelt., 498.

1865. Heer. Urwelt der Schweiz, 84,95. Pl. 7, figs. 2-3.

1872. " Monde prim. Suisse, 102,116. Pl. 7, figs. 2,3.

1876. " Prim. world Switz., i: 84,94. Pl. 7, figs. 2,3.

1879. " Urwelt der Schweiz, $2^{\ominus}$ aufl., 94, 105. Pl. 7, figs. 2, 3.

See also Gryllus bucklandi, etc.

714. Gryllacris propinqua. (Locustariæ.)

Locality: Eichstadt, Bavaria. Horizon: Jura.

1886. Deichmüller. Ins. lith. schichten dresd. mus., 26. Pl. 2, figs. 9, 10.

715. Gryllacris schlieffeni. (Locustariæ.)

Locality: Dobbertin, Mecklenburg. Horizon: Lias.

1884. E. Geinitz. Zeitschr. deutsch. geol, gesellsch., 1881: 580-581. Pl. 13, fig. 24.

716. Gryllidium oweni. (Acridii ?)

Locality: Durdlestone Bay, England. Horizon: Lower Purbecks.

1854. Westwood. Quart. journ. geol. soc. Lond., $\mathrm{x}:$ 387,395. Pl. 17, fig.19.

1856. Giebel. Insect. d. vorwelt, 301.

1886. Deichmüller. Ins. lith. schichten dresd. mus., 13 (near Chresmoda).

717. Gryllites? dubius. (Gryllides.)

Locality: Solenhofen, Bavaria. Horizon: Jura.

1842. Germar. Münst., Beitr. z. petref., v: 82-83. Pl. 9, fig. 3; pl. 13, fig. 8.

1852. Giebel. Deutschl. petref., 638.

1862. Hagen. Palaeontogr., $x: 111$ (not a Gryllus).

1869. Weyenbergh. Arch. mus. Teyl., ii: 275 (a Blatta 9 ).

1869. " " Ins. foss. calc. lithogr., 29 (a Blatta.).

1869. " " Tijdschr. v. ent., (2), iv : 234 [134].

1874. " " Periód.zool, i: 98.

1874. " "Enum. syst. faune ent. mésoz., 12.

1877. Assmann. Ber. versamml. deutsch. naturf., 1: 192 (a Cicada).

718. Gryllus - (Locustariæ.)

Locality: Aust, England. Horizon: Rhætic.

1873. Brodie. Distr. corr. foss. ins., 17.

719. Gryllus —. (Locustariæ.)

Locality : Aust, England. Horizon: Lower Lias.

1845. Westwood. Brodie, Foss. ins.sec. rocks Engl., 125. Pl. 9, fig. 14.

1856. Giebel. Insect. d. vorwelt, 309. 
720. Gryllus bucklandi. (Acridii.)

Locality: Grafton, Warwickshire, England. Horizon: Lower Lias. 1845. Brodie. Foss. ins. sec. rocks Engl., 76, 96,101. Pl. 7, fig. 16. 1871. Phillips. Geology of Oxford, 123.

See also Gomphocerites bucklandi, Edipoda bucklandi.

\section{Gryllus dobbertinensis. (Gryllides?)}

Locality : Dobbertin, Mecklenburg. Horizon : Lower Jura.

1880. E. Geinitz. Zeitschr. deutsch. geol. gesellsch., 1880: 523. Pl. 22, fig. 6.

18४4. " Zeitschr. deutsch. geol. gesellsch., 1884: 572.

1885. Scudder. Zittel, Handb. palaeont., i, th. ii : 770.

1886. " Zittel-Barrois, Traité de paléont., ii : 770 .

1885. " Bull. U. S. geol. surv., No. 31: 50 .

722. Gryllus gryllotalpa (recent). (Gryllides.)

1783. Esper.

Locality : Solenhofen, Bavaria. Horizon: Jura.

1784. Schröter. Neue litt. u. beytr., i: 411 (unnamed).

723. Gryllus sedgwicki. (Gryllides.)

Locality : England. Horizon: Purbecks.

1856. Giebel. Insect. d, vorwelt, 300-301.

See also Acheta sedgwicki.

724. Halometra gigantea. (Acridii.)

Locality : Solenhofen, Bavaria. Horizon: Jura.

1883. Oppenheim. Palaeontogr., xxxiv: 230-233. Pl. 31, fig. 18.

See also Chresmoda obscura, etc.

725. Halometra? minor. (Acridii.)

Locality : Eichstadt, Bavaria. Horizon: Jura.

1888. Oppenheim. Palaeontogr., xхxiv: 233. Pl. 31, fig. 4.

726. Humbertiella grandis. (Mantides.)

Compared with H. ceylonica Sauss.

Locality : Ust Balei, Siberia. Horizon: Lias.

1889. $\left\{\begin{array}{l}\text { Braner-Red- } \\ \text { tenb.-Ganglb. }\end{array}\right\}$ Mém. acad. sc. St.-Pétersb., (7), xxxvi, No. 15: 13. Pl. [2], fig. 16.

727. Ledrophora girardi. (Blattariæ.)

Locality: Trebitz, Saxony. Horizon: Buntensandstein.

1864. Heer. Viert. naturf. gesellsch. Zürich, ix. Pl., fig. 5.

1879. Scudder. Mem. Bost. soc.nat. hist., iii : 19.

1880. " " Arch. se. phys. nat., (3), iii : 367.

1890. " " Foss. ins. N. A., i : 39 .

See also Legnophora girardi.

728. Iegnophora girardi. (Blattariæ.)

Locality: Trebitz, Saxony. Horizon: Buntensandstein.

1864. Heer. Viert, naturf. gesellsch. Zürich, ix : 288, 297-299 (on pl. Led-

rophora). Pl., fig. 5 . 
1872. Heer. Monde prim. Snisse, 99.

1877. Goldenberg. Fauna saræp. foss., ii : 20 (Lognophora ;.

1885. Scudder. Zittel, Handb. palaeont., i, th. ii : 766 .

1886. " " Zittel-Barrois, Traité de paléont., ii : 766 .

1856. " " Bull. U. S. geol. surv., No. 31: 47.

See also Ledrophora girardi.

729. Locusta? - (Locustariæ.)

Locality: Strensham, England. Horizon: Lower Lias.

1873. Brodie. Distr. corr, foss. ins., 16.

730. Locusta - - (Locustariæ.)

Locality: Solenhofen, Bavaria. Horizon : Jura.

1834. Keferstein. Naturf, erdkörp., ii : 332 (teste Münster).

731. Locusta? amanda. (Locustariæ.)

Locality: Solenhofen or Eichstadt, Bavaria. Horizon: Jura.

1863. Hagen. Palaeontogr., x: 144-145. Pl. 15, fig. 4.

1 699. Weyenbergh. Tijdschr. v. ent., (2), iv : 234 (134).

1885. Seudder. Zittel, Handb. palaeont, i, th. ii : 769. Fig. 968.

1886. “ Zittel-Barrois, Traité de paléont., ii: 769. Fig. 985.

See also Elcana amanda, Elcana (Locusta) amanda ; also II, DipterA, Asilicus lithophilus.

732. Locusta prisca. (Locustariæ.)

Locality: Solenhofen, Bavaria. Horizon: Jura.

1839. Münster. MSS.

1839. Germar. Verhandl. leop.-carol, akad, naturf, xix: 200-201. Pl.21, fiq.3.

1856. Giebel. Insect. d. vorwelt, 303 .

1862. Hagen. Palaeontogr., $\mathrm{x}: 110$.

1869. Weyenbergh. Tijdschr. v. ent., (2), iv: 234 [134].

1877. Assmann. Ber, versamml. deutsch. naturf., 1: 192.

See also Chresmoda obscura, etc.

733. Locusta speciosa. (Locustariæ.)

Locality : Solenhofen, Bavaria. Horizon: Jura.

1839. Münster. MSS.

1839. Germar. Verhandl. leop.-carol. akad. naturf, xix: 198-200, Pl. 21, figs.1-2.

1852. Giebel. Deutschl. petref., 637.

1862. Hagen. Palaeontogr., $\mathrm{x}: 110$.

1863. Winkler. Cat. syst. coll. pal. mus. Teyl., 425.

1869. Weyenbergh. Arch. mus. Teyl., ii : 276-277. Pl. 36, figs.30-31.

1869. “ Ins. foss. calc. lithogr., 30-31. Pl, 3, figs. 30-31.

1869. “ " $\quad$ Tijdschr. v. ent., (2), iv : 234 [134].

1874. " " Periód.zool., i: 98.

1874. " "E Énum. syst. faune ent. mésoz., 12.

1877. Assmann, Ber. versamml. deutsch. naturf., 1: 192.

1885. Scudder. Zittel, Handb. palaeont, i, th. ii: 769. Fig. 967.

1886. " Zittel-Barrois, Traité de paléont., ii: 769. Fïg. 984.

1886. " Bull. U. S. geol. surv., No. 31: 50 .

See also Decticus speciosus, Pycnophlebia speciosa Pyenoph̀lebia (Locusta) speciosa ; cf. also III, Orthopt., Decticus speciosus.

Iıognophora girardi. See Legnophora giıard.. 
734. Mesoblattina --. (Blattariæ.)

Locality : England. - Horizon : Purbecks.

1886. Scudder.

Mem. Bost. soc. nat. hist., iii : 453. Pl. 46, fig. 3.

1890. " Foss. ins. N. A., i: 345. Pl. 20, fig. 3.

\section{Mesoblattina - - (Blattariæ.)}

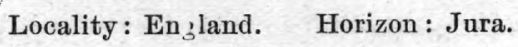

1886. Scudder. Mem. Bost. soc. nat. hist., iii : 453. Pl. 46, fig. 13.

1890. " Foss.ins. N. A., i: 345. Pl.20, fig. 13 ,

736. Mesoblattina angustata. (Blattariæ.)

Locality : Schambelen, Switzerland. Horizon: Lias.

1885. Scudder. Proc. acad. nat. sc. Phiiad., 1885: 114.

1885. "6 Zittel, Handb. palaeont., i, th. ii: 767. Fig. 963.

1886. “ Zittel-Barrois, Traité de paléont., ii: 767. Fig. 979.

1886. " " Mem. Bost. soc. nat. hist., iii: 462.

1890. " " Foss.ins. N. A., i : 354 .

See also Blattina angustata, etc.

737. Mesoblattina antiqua. (Blattariæ.)

Locality : Durdlestone Bay, England. Horizon: Lower Purbecks. 1885. Seudder.

1886. “" Mem. Bost. soc. nat. hist., iii : 463.

1890. " Foss. ins. N. A., i: 355.

See also Rithma antiqua, etc.

738. Mesoblattina bensoni. (Blattariæ.)

Locality: Dumbleton, England. Horizon: Upper Lias.

1886. Scudder. Mem. Bost. soc. nat. hist., iii : 453-454. Pl. 46, fig. 17 .

1890. " Foss. ins. N. A., i : 345-346. Pl. 20, fig. 17.

739. Mesoblattina blakei. (Blattariæ.)

Locality : Alderton, England. Horizon : Upper Lias.

1886. Scudder. Mem. Bost. soc. nat. hist., iii : 452. Pl. 46, fig. 12.

1890. “ Foss. ins. N. A., i : 344-345. Pl. 20, fig. 12.

740. Mesoblattina brodiei. (Blattariæ.)

Locality : England. Horizon: Purbecks.

1886. Scudder. Mem. Bost. soc. nat. hist., iii : 459-460. Pl. 47, fig. 7.

1886. " " Miall, Cockroach, 216. Fig. 123.

1890. “ Foss. ins. N. A., i: 351-352. Pl. 21, fig. 7.

741. Mesoblattina bucklandi. (Blattariæ.)

Locality : England. Horizon: Purbecks?

1886. Scudder. Mem. Bost. soc. nat. hist., iii : 457-458. Pl. 47, fig. 2.

1890. “ Foss. ins. N. A., i : 349-350. Pl. 21, fig. 2.

742. Mesoblattina dobbertinensis. (Blattariæ.)

Locality : Dobbertin, Mecklenburg. Horizon : Lias.

1885. Scudder. Proc. acad. nat. sc. Philad., 1885: 115.

1886. " "Mem. Bost. soc. nat. hist., iii : 455.

1890. " Foss.ins. N. A., i : 347 .

See also Blattina (Mesoblattina) dobbertinensis. 
743. Mesoblattina elongata. (Blattariæ.)

Locality : Durdlestone Bay, England. Horizon: Middle Purbecks. 1885. Scudder. Proc. acad. nat. sc. Philad., 1885: 115. 1886. " " Mem. Bost. soc. nat. hist., iin: 458 . 1890. " " Foss. ins. N. A., i : 350 .

see also Blattidium elongatum, etc.

744. Mesoblattina geikiei. (Blattariæ.)

Locality: Brown's Wood, Moreton Bagot, England. Horizon : Lias. 1886. Scudder. Mem. Bost. soe. nat. hist., iii : 454-455. Pl. 46, fig. 9. 1890. " Foss. ins. N. A., i: $346-347 . \quad$ Pl. 20, fig. 9.

745. Mesoblattina higginsii. (Blattariæ.)

Locality: Eugland. Horizon: Purbecks. 1886. Scudder. Mem. Bost. soc, nat. hist., iii: 455-456. Pl.47, fig. 14. 1890. " Foss. ins. N. A., i : 347-348, Pl.21, fig. 14.

746. Mesoblattina hopei. (Blattariæ.)

Locality: England. Horizon : Purbecks. 1886. Scudder. Mem. Bost. soc. nat. hist., iii : 461. Pl. 47, fig.11. 1890. " Foss. ins. N. A., i :"353. Pl. 21, fig. 11.

747. Mesoblattina lithophila. (Blattariæ.)

Locality: Solenhofen, Bavaria. Horizon: Jura.

1886. Scudder. Mem. Bost. soc. nat. hist., iii : 464.

1886. Deickmüller. Ins. lith. schichten dresd. mus., 6-9. Pl. 1, figs. 1-6.

1888. Oppenheim. Palaentogr., xxxiv: 221. Pl. 30, figs, 6,8. 1890. Scudder. Foss. ins. N. A., i : 356.

See also Musca lithophila, etc.

748. Mesoblattina mantelli. (Biattariæ.)

Locality : England. Horizon: Purbecks.

1886. Scudder. Mem. Bost. soc. nat. hist., iii : 460-461. Pl. 47, fig. 9 . 1890. " $"$ Foss. ins. N. A., i : 352-353. Pl. 21, fig. 9.

749. Mesoblattina mathildæ. (Blattariæ.)

Locality: Dobbertin, Mecklenburg. Horizon: Jura.

1886. Scudder. Mem. Bost. soc. nat. hist., iii : 462-463.

1890. " Foss. ins. N. A., i: 354-355.

See also Blattina mathildæ.

750. Mesoblattina murchisoni. (Blattariæ.)

Locality: Durdlestone Bay, England. Horizon : Lower Purbecks.

1885. Scudder. Proc. acad. nat. sc. Philad., 1885: 113, 115.

1886. " " Mem. Bost. soc. nat. hist., iii : 456-457. Pl. 47. fig. 5.

1890. " Foss. ins. N. A., i: 348-349. Pl. 21, fig. 5.

See also Rithma murchisoni, etc.

751. Mesoblattina murrayi. (Blattariæ.)

Locality: England. Horizon: Purbecks.

1886. Scudder. Mem. Bost. soc. nat. hist., iii : 458-459. Pl. 47, fig. 4. 1890. “ Foss. ins. N. A., i : 350-351. Pl. 21, fig. 4. 


\section{Mesoblattina peachii. (Blattariæ.)}

Locality: England. Horizon: Purbecks.

1886. Scudder. Mem. Bost. soc. nat. hist., iii: 461-462. Pl. 47, fig. 10.

1890. " Foss. ins. N. A., i : 353-354. Pl. $21, f i g .10$.

753. Mesoblattina protypa. (Blattariæ.)

Locality : Dobbertin, Mecklenburg. Horizon: Lower Jura.

1885. Scudder. Proc. acad. nat. sc. Philad., 1885: 114.

1886. " Mem. Bost. soc. nat. hist., iii : 458.

1890. " Foss. ins. N. A., i : 350.

See also Blattina (Mesoblattina) protypa.

Mesoblattina sibirica. See Blattina (Mesoblattina) sibirica.

754. Mesoblattina swintoni. (Blattariæ.)

Locality: England. Horizon : Purbecks.

1886. Scudder. Mem. Bost. soc. nat hist., iii : 454. Pl. 46, fig. 10.

1 90 . " Foss. ins. N. A., i: 346. Pl. 20, fig. 10.

755. Musca lithophila. (Blattariæ.)

Locality: Solenhofen, Bavaria. Horizon : Jura.

1839. Germar. Verhandl. leop.-carol. akad. naturf., xix: 222. $\quad P l .23, f i g .19$.

1852. Giebel. Deutschl, petref., 643.

1856. " Insect. d. vorwelt, 198.

1862. Hagen. Palaeontogr, $\mathrm{x}: 111$ (indeterminable).

1863. Winkler. Cat. syst. coll. pal. mus. Teyl., 422.

1869. Weyenbergh. Arch, mus. Teyl., ii : 256-25\%. Pl. 34, fig. 2.

186). " " Ins. foss. calc. lithogr., 10-11. Pl. 1, fig. 2.

1869. " " Tijdschr. v. ent., (2), iv: 237.

1874. " " Periód. zool., i: 90.

1874. “ Énum. syst. fauna ent. mésoz., 4.

1877. Assmann. Ber. versamml. deutsch. naturf., 1: 192 (= Blattidium bẻroldingianum).

1885. Scudder. Zittel, Handb. palaeont., i, th. ii: 806.

1886. " " Zittel-Barrois, Traité de paléont., ii : 806.

1886. " Bull. U. S. geol, surv., No. 31: 86 .

See also Blattidium beroldingianum, Mesoblattina lithophila, Blabera avita, Blattidium aritum.

756. Nannoblattina prestwichii. (Blattariæ.)

Locality: England. Horizon: Purbecks.

1886. Scudder. Mem. Bost. soc. nat. hist., iii: $475 . \quad P l .48$, fig. 3.

1890. " Foss. ins. N. A., i: 367. "Pl. 22, fig. 3.

757. Nannoblattina similis. (Blattariæ.)

Locality : Vale of Wardour, England. Horizon: Purbecks.

1886. Scudder. Mem. Bost. soc. nat. hist., iii : 475 .

1890. " Foss, ins, N. A., i : 367.

See also Blattina similis, etc.

758. Nannoblattina woodwardi. (Blattariæ.)

Locality: Wiltshire, England. Horizon: Purbecks.

1886. Scudder. Mem. Bost. soc. nat. hist., iii : 476. Pl. 48, fig. 6.

1890. " Foss.ins. N. A., i: 368. Pl. 22, fig. 6. 
759. Neorthroblattina albolineata. (Blattariæ.)

Locality : Fairplas, Colo. Horizon: Trias.

1885. Scudder.

Proc. acad. nat. sc. Philad., 1885 : 109.

1886. " " Mem. Bost. soc. nat. hist., iii : 444.

1890.

1890 . “

Mem. Bost. soc. nat. hist., iv: 467. Pl. 42, figs. 2, 18.

Foss. ins. N. A., i : 336, 443 . $P l .34$, figs. 2, 18.

760. Neorthroblattina attenuata. (Blattariæ.)

Locality : Fairplay, Colo. Horizon: Trias.

1885. Seudder. Proc. acad. nat. sc. Philad., 1885: 110.

1886. " " Mem. Bost. soc. nat. hist.. iii : 444.

1890. " Mem. Bost. soc. nat. hist., iv: 467, 468-469. Pl. 42, fig.1.

1890. " Foss. ins. N. A., i : 336, 443, 444-445. Pl. 34, fig. 1.

761. Neorthroblattina lakesii. (Blattariæ.)

Locality : Fairplay, Colo. Horizon: Trias.

1885. Seudder. Proc. acad. nat. sc. Philad., 1885: 109.

1886. " Mem. Bost. soc. nat. hist., iii : 444.

1886. " Miall, Cockroach, 214. Fig. 122.

1890. " Mem. Bost. soc. nat. hist., iv: 467, 468. Pl. 42, figs. 9, 15 .

1890. " Foss. ins. N. A., i : 336, 443, 444. Pl. 34, figs. 9, 15.

762. Neorthroblattina rotundata. (Blattariæ.)

Locality : Fairplay, Colo. Horizon: Trias.

1885. Scudder. Proc. acad. nat. sc. Philad., 1885: 109-110.

1885. " "Zittel, Handb., palaeont., i, ii: 766. Fig. 960.

1886. " Zittel-Barrois, Traite de paléont., ii : 766. Fig. 976.

1886. " Mem. Bost. soe. nat. hist., iii : 444.

1890. Lesley. Dict. foss. Penns., ii : 449. Fig.

1890. Scudder. Mem. Bost. soc. nat. hist., iv: 467, 468 . Pl. 42, figs. 7, 8.

1890. " Foss. ins. N. A., i : 336, 443, 444. Pl. 34, figs. 7, 8.

763. Nethania molossus. (Blattariæ.).

Locality: Durdlestone Bay, England. Horizon: Middle Purbecks.

1856. Giebel. Insect. d. vorwelt, 321.

See also Blattidium molossus, Elisama molossus.

764. Edipoda bucklandi. (Acridii.)

Locality : Grafton, England. Horizon : Lower Lias.

1856. Giebel. Insect, d. vorwelt, 309.

See also Gryllus bucklandi, etc.

765. Panorpidium -. (Locustariæ.)

Locality : Dobbertin, Mecklenburg. Horizon : Jura

1883. E. Geinitz. Flötzform. Mecklenb., 31 (undescr.).

766. Panorpidium tessellatum.

Locality: Durdlestone Bay, England. Horizon: Lower Purbecks.

1854. Westwood. Quart. journ. geol. soc. Lond., $\mathrm{x}$ : 384,394. Pl. 15, fig. 17.

See also Clathrotermes (Elcana) geinitzi, etc.; also II, NEURopT., Panorpidium tessellatuma var. 
767. Parapleurites gracilis. (Acridii.)

Locality : Ust Balei, Siberia. Horizon: Lias.

1839. $\left\{\begin{array}{l}\text { Braner-Red- } \\ \text { tenb.-Ganglb. }\end{array}\right\}$ Mém. acad. sc. St.-Pétersb., (7), xxxvi, No. 15: 13. Pl. [2], fig. 17.

\section{Periplaneta maculata. (Blattariæ.)}

Compared with P. australasiæ, etc.

Locality : Ust Balei, Siveria. Horizon: Lias.

1889. $\begin{aligned} & \text { Brauer-Red- } \\ & \text { tenb.-Ganglb.S }\end{aligned}$ Mém. acad. se. St.-Pótersb., (7), xxxvi, No. 15: 12. Pl. [2], fig. 14.

769. Petrablattina æqua. (Palæoblattariæ.)

Locality : Fairplay, Colo. Horizon: Trias.

1885. Scudder. Proc. acad, nat. sc. Philad., 1885: 38.

1890. Lesley. Dict. foss. Peuns., ii : 626.

1890. Scudder. Mem. Bost. soc. nat. hist., iv: 465. Pl. 42, fig. 13.

1890. " Foss. ins. N. A., i: 441. Pl. 34, fig. 13.

\section{Petrablattina meieri. (Palæoblattariæ.)}

Locality: Fairplay, Colo. Horizon : Trias.

188.5. Seudder. 1890. Lesley. 1890. Scudder. 1800. " "
Proc. acad. nat. sc. Philad., 1885 : 38.

Dict. foss. Penns., ii : 627.

Mem. Bost. soc. nat. hist., iv: $465 . \quad P l .42$, fig. 17.

Foss. ins. N. A., i: 441 . Pl. 34, fig. 17.

\section{Phaneroptera germari. (Locustariæ.)}

Locality: Solenhofen, Bavaria. Horizon : Jura.

1842. Münster.

1842. Germar.

1852. Giebel.

1854. Pictet.

1856. Giebel.

1862. Hagen.
MSS.

Münster, Beitr. z. petref., v: 81-82. Pl. 9, fig. \&; Pl. 13, fig. 7.

Deutschl. petref., 637.

Traité paléont., $2^{\mathrm{e}}$ éd., ii : 366. Pl. 40, fig. 10.

Insect. $d$. vorwelt, 303 .

Palaeontogr., $\mathrm{x}: 111$.

1869. Weyenbergh. Arch. mus. Teyl., ii : 275.

1869. " " Ins. foss. calc. lithogr., 29.

1869. " " lijdschr. v. ent., (2), $4: 234$ [134].

1874. " " Period. zool., i: 98.

1874. " " Énum. syst. faune ent. mésoz., 12.

1877. Assmann. Ber. versamml. deutsch. naturf., 1: 192.

1885. Scudder. ZitteI, Handb. palaeont., i, th. ii : 768.

1886. " " Ziłtel-Barrois, Traité de paléout., ii : 769.

1886. Deichm. Ins, lith. schichten dresd, mus., 19-20. Pl. 2, fig. 11.

772. Phaneroptera striata. (Locustariæ.)

Locality : Solenhofen, Bavaria. Horizon: Jura.

1869. Weyenbergh. Arch. mus. Teyl., ii : 275-276. Pl. 36, fig. 28 ,

1869. " " Ins. foss. calc. lithogr., 29-30. Pl. 3, fig. 28.

1869. " " Tijdschr. v. ent., (2), iv: 234 [134].

1874. " " Period.zool., i: 98.

1874. “ Enum. syst. faune ent. mésoz, 12. 
773. Poroblattina arcuata. (Palæoblattariæ.)

Locality: Fairplay, Colo. Horizon: Trias.

1885. Scudder.

1890. Lesley.

Proc. acad. nat. se. Philad., 1895 : 39.

1890. Scudder.

1890. "

Diet. foss. Penns., ii : 737.

Mem. Bost. soc. nat. hist., iv : 466 . Pl. 41, fig. 5 .

Foss. ins. N. A., i: 442 . Pl. 33 , fig. 5.

774. Poroblattina lakesii. (Palæoblattariæ.)

Locality: Fairplay, Colo. Horizon: Trias.

1885. Scndder. Proc. acad. nat. sc. Philad., 1885: 39.

1885. " Zittel, Handb. palaeont, i, ii : 755. Fig. 936.

1886. " Zittel-Barrois, Traité de paléont., ii : 755 . Fig. 952.

1890. Lesley. Dict. foss. Penns., ii: 737. Fig.

1890. Scudder. Mem. Bost. soc. nat. hist., iv: 466. Pl. 41, fig. 11.

1890. " Foss. ins. N. A., i: 442 . Pl. 33, fig. 11.

775. Propygolampis bronni. (Acridii.)

Locality: Solenhofen, Bavaria. Horizon: Jura.

1×74. Weyenbergh. Periód. zool., i: 84-85,99. Pl. 3, fig. 3.

1874. " "Enum. syst. faune ent. mésoz., 13.

See also Chresmoda obscura, etc.

776. Pterinoblattina binneyi. (Blattariæ.)

Locality : 'Durdlestone Bay, England. Horizon: Lower Purbecks.

1854. Westwood. Quart. journ. geol. soc. Lond., $\mathrm{x}$ : 390,396 (no name). $\mathrm{Pl}$. 18, fig. 42.

1886. Scudder. Mem. Bost. soc. nat. hist., iii : 473.

1890. “ Foss. ins. N. A., i : 365.

777. Pterinoblattina chrysea. (Blattariæ.)

Locality: Dobbertin, Germany. Horizon: Lias.

1885. Scudder. Proc. acad. nat. sc. Philarl, 1895: 106-107.

1886. " " Mem. Bost. soc. nat. hist., iii : 470-471.

1890. " " Foss. ins. N. A., i: 362-363.

See also Blattina chrysea.

778. Pterinoblattina curtisii. (Blattariæ.)

Locality : Alderton, England. Horizon: Upper Lias.

1886. Scudder. $\quad$ Mem. Bost. soc. nat. hist., iii: 471. Pl. 48, fig. 16.

1890. " Foss. ins. N. A., i: 363. Pl. 22, fig. 16.

779. Pterinoblattina gigas. (Blattariæ.)

Locality : Solenhofen, Bararia. Horizon: Oolite.

1885. Scudder. Proc. acad. nat. se. Philad., 1885: 108.

1886. " " Mem. Bost. soc. nat. hist., iii : 472.

1888. Oppenheim. Palaeontogr., xxxiv: 222.

1890. Scudder. Foss. ins. N. A., i : 364.

See also Ricania gigas. 
780. Pterinoblattina hospes. (Blattariæ.)

Locality: Solenhofen, Bavaria. Horizon: Oolite.

1885. Scudder. Proc. acad. nat. sc. Philad., 1885: 108.

1886. " Mem. Bost. soc. nat. hist., iii : 472.

1883. Oppenheim. Palaeontogr., xxxiv: 221-222. Pl. 31, fig. 29.

1890. Scudder. Foss. ins. N. A., i: 364.

See also Ricania hospes.

781. Pterinoblattina intermixta. (Blattariæ.)

Locality : Alderton, Gloucestershire, England. Horizon: Upper Lias.

1885. Scudder. Proc. acad. nat. sc. Philad., 1885: 107-108.

1 186. " " Mem. Bost. soc. nat. hist., iii : 471-472. $P l .48$, fig. 9.

1886. " " Miall, Cockroach, 218. Fig. 125.

1890. " . Foss. ins. N. A., i: 363-364. Pl. 22, fig. 9.

782. Pterinoblattina penna. (Blattariæ.)

Locality: England. Horizon: Purbecks.

1885. Scudder. Proc. acad. nat. sc. Philad., 1885: 106.

1886. " " Mem. Bost. soc. nat. hist., iii : 470. Pl. A8, fig. 14.

1890. " $\quad$ Foss. ins. N. A., i: 362. Pl. 22, fig. 14.

783. Pterinoblattina pluma. (Blattariæ.)

Locality : Dorset, England. Horizon: Purbecks.

1885. Scudder. Proc. acad. nat. sc. Philad., 1885: 105-106.

1885. " Zittel, Handb. palaeont., i, th. ii: 755. Fig. 935.

1886. " "Zittel-Barrois, Traité de palént., ii: 767. Fiq. 980.

1886. " " Mem. Bost. soc. nat. hist., iii : 469-470. Pl. 48, figs. 7, 8c.

1890. " Foss. ins. N. A., i: 361-362. Pl. 22, figs. 7, 8c.

See also Blatta pluma, Blattidium pluma.

784. Pterinoblattina sipylus. (Blattariæ.)

Locality : Durdlestone Bay, England. Horizon : Lower Purbecks.

1886. Scudder. Mem. Bost. soc. nat. hist., iii : 472-473.

1890. " Foss. ins. N. A., i: 364-365.

Eee also Sialium sipylus, Abia sipylus.

785. Pycnophlebia speciosa. (Locustariæ.)

Locality: Eichstadt, Bavaria. Horizon: Jura.

1886. Deichmüller. Ins. lith. schicht. dresd. mus., 20-24. Pl. 2, figs. 1, 2a, 3.

See also Locusta speciosa, etc.

786. Pycnophlebia (Locusta) speciosa. (Locustariæ.)

Locality: Solenhofen, Bavaria. Horizon: Jura.

1888. Oppenheim. Palaeontogr., xxxiv : 223-224. Pl. 30, fig. 4.

Seo also Locusta speciosa, etc.

787. Pygolampis gigantea. (Acridii.)

Localtty: Solenhofen, Bavaria. Horizon: Jura.

1839. Münster. MSs.

1839. Germar. Verhandl. leop.-carol. akad. naturf, xix : 207-208. Pl. 22, fig.8.

1852. Giebel. Deutsch. petref., 637.

1856. "6 Insect. d. vorwelt, 364 . 
1862. Hagen. Palaeontogr, $\mathrm{x}: 111$.

1869. Weyenbergh. Arch. mus. Teyl., ii : 273. Pl. 35, fig. 21.

1869. " " Ins. foss. calc. lithogr., 27. Pl. 2, fig. 21.

1869. " " Tijdschr. v. ent., (2), iv: 233 [133].

1874. " " $\quad$ Periód. zool., i: 99.

1874. " "Enum. syst. faune ent. mésoz., 13.

187\%. Assmann. Ber. versamml. deutsch. naturf., 1: 192 (=Chresmoda obscura).

1885. Scudder. Zittel, Handb. palaeont., i, th. ii : 783. Fig. 996.

1886. “ Zittel-Barrois, Traité dé paléont., ii : 783. Fig. 1013.

See also Chresmoda obscura, etc.

788. Ricania gigas. (Blattariæ.)

Locality: Sólenhofen, Bavaria. Horizon: Jura.

1869. Weyenbergh. Arch. mus. Teyl., ii: 270-271. Pl. 35, fig. 23.

1869. " " Ins. foss. calc. lithogr., 24-25. Pl. 2, fig. 23.

1869. " " Tijdschr, v. ent., (2), iv: 234 [134].

1874. " " Periód. zool., i: 100.

1874. “ " Énum. syst. faune ent. mésoz., 14.

1885. Scudder. Zittel, Handb. palaeont., i, th. ii: 780 .

1886. " Z Zittel-Barrois, Traité de paléont., ii : 780.

See also Pterinoblattina gigas.

789. Ricania hospes. (Blattariæ.)

Locality: Solenhofen, Bavaria. Horizon: Jura.

1839. Germar. Verhandl. leop.-carol. akad, naturf., xix: 220-221. Pl. 23, fig. 18.

185\%. Giebel. Deutschl. petref., 636.

1856. " " Insect. d. vorwelt, 376.

1862. Hagen. Palaeontogr., $\mathrm{x}: 111$.

1869. Wejenbergh. Tijdschr. v. ent., (2), iv : 234 [134].

1874. " " Peribd. zool., i: 100 .

1874. “ Énum. syst. faune ent. mésoz., 14.

1877. Assmann. Ber. versamml. deutsch. naturf., 1: 192 (Neuropt.).

188.5. Scudder. Zittel, Handb. palaeont., i, ii : 780.

1886. " Zittel-Barrois, Traité de paléont., ii : 780.

See also Pterinoblattina hospes.

790. Rithma - (Blattariæ.)

Locality : England. Horizon: Purbecks.

1886. Scudder. Mem. Bost. soc. nat. hist., iii: 450. Pl. 46, fig. 8.

1890. " Foss. ins. N. A., i: 342. Pl. 20, fig. 8.

791. Rithma antiqua. (Blattariæ.)

Locality : Durdlestone Bay, England. Horizon: Lower Purbecks.

1854. Westwood. Quart. journ. geol. soc. Lond., x : 387, 395 (unnamed). Pl. 17, fig. 10.

1856. Giebel. Insect. d. vorwelt, 319.

187\%. Goldenberg. Fauna saræp. foss., ii : 21.

See also Blattidium antiquum, Mesoblattina antiqua.

792. Rithma daltoni. (Blattariæ.)

Locality: England. Horizon: Purbecks.

1886. Scndder. Mem. Bost. soc. nat. hist., iii : $449 . \quad P l .46, f i g .16$.

1890. " Foss, ins. N. A., i: 341, Pl, 20, fig. 16. 
793. Rithma disjuncta. (Blattariæ.)

Locality: Wiltshire, England. Horizon: Purbecks.

1886. Scudder. Mem. Bost. soo. nat. hist., iii: 446. Pl. 46, fig. 14.

1890. " Foss. ins. N. A., i: 338. Pl. 20, fig. 14.

794. Rithma formosa. (Blattariæ.)

Locality: Schambelen, Switzerland. Horizon: Lias.

1885. Scudder. Proc. acad. nat. se. Philad., 1885: 113, 114.

1885. " " Zittel, Haudb. palaeont., i, th. ii: 767. Fig. 962.

1886. " Zittel-Barrois, Traité de paléont., ii: 767. Fig. 978.

1886. " " Mem. Bost. soc. nat. hist., iii : 447-448.

1890. "6 Foss. ins. N. A., i: 339-340.

See also Blattina formosa.

795. Rithma gossii. (Blattariæ.)

Locality : England. Horizon: Purbecks.

1886. Scudder. Mem. Bost. soc. nat. hist., iii: 445-446. Pl. 46, fig. 15.

1890. " Foss. ins. N. A., i : $337-338$. Pl. 20, fig. 15.

\section{Rithma liasina. (Blattariæ.)}

Locality: Wainlode, Strensham, England. Horizon: Lower Lias.

1885. Seudder. Proc. acad. nat. sc. Philad., 1885: 113, 114.

1886. " Mem. Bost. soc. nat. hist., iii : 447. Pl. 46, fig. 7.

1890. " Foss. ins. N. A., i: 339. Pl. 20, fig. 7.

See also Blattina liasina, etc.

797. Rithma minima. (Blattariæ.)

Locality : Dorset, England. Horizon: Middle Purbecks.

1886. Scndder. Mem. Bost. soc. nat. hist., iii : 450-451. Pl. 48, figs. 2, 8a, 8g, 11. 1890. “ Foss. ins. N. A., i : 342-343. Pl. 22, figs. $2,8 a, 8 g, 11$.

798. Rithma morrisi. (Blattariæ.)

Locality: Durdlestone. Bay, England. Horizon: Lower Purbecks.

1854. Westwood. Quart. journ. geol. soc. Lond., x: 390, 396 (nunamed). Pl. 18, fig. 34.

1856. Giebel. Insect. d. vorwielt, 319.

1877. Goldenberg. Fanua saræp. foss., ii : 21.

1885. Scudder. Proc. acad. nat. sc. Pbilad., 1885: 113, 114.

1886. " Mem. Bost. soc. nat. hist., iii: 448.

1890. " Foss. ins. N. A., i: 340 .

See also Blattidium morrisi.

799. Rithma murchisoni. (Blattariæ.)

Locality : Durdlestone Bay, England. Horizon: Lower Purbecks.

1854. Westwood. Quart. journ. geol. soc. Lond., x: 390,396 (unnamed), $P l, 18$, fig. 43.

1856. Giebel. Insect. d: vorwelt, 319.

1877. Goldenberg. Fauna saræp. foss., ii : 21.

See also Blattidium murchisoni, Mesoblattina murchisoni, 
800. Rithma purbeccensis. (Blattariæ.)

Locality: Durdlestone Bay, Eugland. Horizon: Lower Purbecks.

1854. Westwood. Quart. journ. geol. soc. Lond., $\mathrm{x}: 390,396$ (unnamed). Pl. 18, fig. 32.

1856. Giebel. Insect. d. vorwelt, 319.

1877. Goldenberg. Fauna saræop. foss., ii: 21.

1885. Scudder. Proc. acad. nat. sc. Philad., 1885: 113, 114.

1886. " " Mem. Bost. soc. nat. hist., iii : 448-449.

1890. " Foss. ins. N. A., i: 340-341.

See also Blattidinm purbeccensis.

\section{Rithma ramificata. (Blattariæ.)}

Locality: Durdlestone Bay, England. Horizon: Middle Purbecks.

1854. Westwood. Quart. journ. geol. soc. Lond., x : 394 (unnamed). Pl. 15, fig. 20。

1856. Giebel. Insect. d. vorwelt, 319.

1877. Goldenberg. Fauna saræp. foss., ii : 21.

1885. Scudder. Proc. acad. nat. sc. Philad., 1885: 113.

See also Blattidium ramificatum, Elisama ramificata.

\section{Rithma stricklandi. (Blattariæ.)}

Locality : Vale of Wardour, England. Horizon: Purbecks.

1836. Scudder. Mem. Bost. soc. nat. hist., iii: 445. Pl. 46, figs. 4, 5.

1890. " Foss. ins. N. A., i: 337. Pl.20, figs. 4, 5.

See also Blatta stricklandi, Blattidium stricklandi.

\section{Rithma westwoodi. (Blattariæ.)}

Locality: Durdlestone Bay, England. Horizon : Lower Purbecks.

1854. Westwood. Quart. journ. geol. soc. Lond., x: 390, 396 (unnamed). Pl. 18, fig. 22.

1856. Giebel. Insect. d. vorwelt, 318-319.

1877. Goldenberg. Fauna saræp. foss., ii : 21.

1885. Scudder. Proc. acad. nat. sc. Philad., 1885: 113.

1886. " Mem. Bost. soc. nat. hist., iii : 449-450. Pl. 46, figs. 6?, 11.

1890. " Foss. ins. N. A., i: 341-342. Pl. 20, figs. 6?, 11.

See also Blattidium westwoodi.

804. Scutinoblattina brongniarti. (Blattariæ.)

Locality : Fairplay, Colo. Horizon: Trias.

1885. Scudder. Proc. acad. nat. sc. Philad., 1885: 110-111.

1886. " Mem. Bost. soc. nat. hist., iii : 478.

1890. " Mem. Bost. soc. nat. hist., vi : 469. Pl. 42, fig. 5.

1890. " Foss. ins. N. A., i: 370,445 . Pl. 34, fig. 5.

\section{Scutinoblattina intermedia. (Blattariæ.)}

Locality: Fairplay, Colo. Horizon: Trias.

1885. Seudder. ... Proe. acad, nat. sc. Philad., 1885: 111.

1886. " Mem. Bost. soe. nat. hist., iii : 478 .

1890. " Mem. Bost. soc. nat. hist., iv: 469, 470. Pl. 42, fig. 4.

1.890. " Foss. ins. N. A., i: 370, 445, 446. Pl. 34, fig. 4. 
806. Scutinoblattina recta. (Blattariæ.)

Locality: Fairplay, Colo. Horizon: Trias.

1885, Scudder.

Proc. acad. nat. sc. Philad., 1885: 111.

1886 . " "

Mem. Bost. soc. nat. hist., iii : 478 .

1890. " Mem Bost. soc. nat. hist., iv: 469, 470. Pl. 42, figs. 3, 16.

1-90. “ Foss. ins. N. A., i: $370,445,446 \quad$ Pl. 34, figs. 3, 16.

\section{Sialium sipylus. (Blattariæ.)}

Locality : Durdlestone Bay, England. Horizon: Lower Purbecks.

1854. Westwood. Proc. geol. soc. Lond., $\mathrm{x}: 390,396 . \quad$ Pl. 18, fig. 24. 1886. Deichmïller. Ins. lith. schichten dresd. mus., 17 (cf. Elcana).

See also Abia sipylus, Pterinoblattina sipylus.

808. Spiloblattina gardineri. (Palæoblattariæ.)

Locality : Fairplay, Colo. Horizon: Trias.

1885. Scudder. Proc. acad. nat. sc. Philad., 1885: 36.

1885. " " Zittel, Handb. palaeont., i, th. ii: 754. Fig. 933.

1886. " Z Zittel-Barrois, Traité de paléont.; ii : 754. Fig. 950.

1889. Lesley. Dict. foss. Penns., i: 352. Fig. on p. 351.

1890. Seudder. Mem. Bost. soc. nat. hist., iv: 461-462. Pl. 41, figs. 4, 6, 8, 10.

1890. " Foss. ins. N. A., i : 437-438. Pl. 33, figs. 4, 6, 8, 10.

809. Spiloblattina guttata. (Palæoblattariæ.)

Locality : Fairplay, Colo. Horizon : Trias.

1885. Scudder. Proe. acad, nat, se. Philad., 1885: 36-37.

1890. “ Mem. Bost. soc. nat. hist., iv: 461, 463. Pl. 41, fig. 2; $P l .42$, fig. 14.

1890. " Foss. ins. N. A., i: 437, 439. Pl. 33, fig. 2; Pl. 34, fig. 14.

810. Spiloblattina marginata. (Palæoblattariæ.)

Locality: Fairplay, Colo, Horizon : Trias.

1885. Scudder. 1890 . “ 1890. “

Proc.acad. nat. se. Philad., 1885: 37.

Mem. Bost. soc. nat. hist., iv: 461, 464. Pl. 41, fig. 3 .

Foss. ins. N. A., i : 437, 440. Pl. 33, fig. 3.

\section{Spiloblattina triassica. (Palæoblattariæ.)}

Locality: Fairplay, Colo. Horizon: Trias.

1885. Scudủer. Proc. acad. nat. sc. Philad., $1885: 36$.

1890. " " Mem. Bost. soc. uat. hist., iv: 461, 462-463. Pl. 41, fig.1.

1890. “ Foss. ins. N. A., i : 437, 438-439. Pl. 33, fig. 1.

\section{NEUROPTERA.}

812.

Localities: Hastings, Govers, Cliff End, England. Horizon: Wealden. 1854. Binfield. Quart. journ. geol. soc. Lond., x: 172,173, 174.

813.

Locality: Vale of Wardour, England. Horizon: Purbecks.

1845. Brodie. Foss. ins. see. rocks Engl., 32 (Westw., 116, Staphylinidæ? or

Termes?). $\quad P l .2, f i g .2$.

See also Philonthns kueri, Termes grandævus, etc. 
814.

Locality: Vale of Wardour, England. Horizon: Purbecks.

1845. Brodie. Foss. ins. sec. rocks Engl,, 32 (Westw., 116, Staphylinidæ?). Pl. 2, fig. 3.

See also Prognatha crassa, Termes grandævus, etc.

815.

Locality: Copt Heath near Knowle, England. Horizon: Lower Lias.

1875. Brodie. Lower Lias Eatington, etc., 10.

816.

Localities: Brown's Wood and Stooper's Wood, near Hepley in Arden, England. Horizon. Lias.

18i5. Brodie. Lower Lias Eatington, etc., 10.

817. (abdomen).

Locality: Dobbertin, Mecklenburg. Horizon: Lower Jura.

1880. E. Geinitz. Zeitschr. deutsch. geol. gesellsch., 1880:531. Pl. 22, fig. 23.

818.

Locality : Ilminster, England. Horizon: Upper Lias.

1879. Goss. Proc. geol. assoc., vi : 130.

1879. “ Ins. fauna mesoz. per., ii: 17 .

819.

Compared with Ephemera and Aschna.

Locality: Banks of the Býrka, Siberia. Horizon: Jura.

1818. Müller. Middend., Sib.reise, i, th. i: 261. Pl. 11, fig. 7.

See also Ephemeropsis — Eichw., E. orientalis, E. trisetalis.

820.

Locality: Durdlestone Bay, England. Horizon: Middle Purbecks. 18.34. Westwood. Quart. journ. geol. soc. Lond., x: 384, 394. Pl. 15, fig. 24.

\section{1.}

Locality : Ridgway, England. Horizon: Lower Purbecks. 1854. Westwood. Quart. journ. geol, soc. Lond, x : 385.

822.

Locality: Solenhofen, Bavaria. Horizon: Jura.

1869. Weyenbergh. Arch, mus. Teyl., ii : 265-266. Pl. 35, fig. 17.

1869. " " Ins. foss. calc. lithogr., 19-20. Pl. 2, fig. 17.

1874. " " Períd. zool., i: 95.

1874. " " Ênum. syst. faune ent. mésoz., 9.

823. (Leptoceridæ.)

Locality : Vale of Wardour, England. Horizon: Purbecks. 
824.

(Odonata.)

Localities: Strensham, Brockeridge, Binton, Wilmeote, Grafton, Brown's Wood, Barrow on Soar, England. Horizon: Lower Lias.

1873. Brodie. Distr. corr. foss. ins., 15.

825.

(Odonata.)

Locality : Strensham, Eugland. Horizon: Lower Lias.

1874. Brodie. Distr. corr. foss. ins., 19.

826. (male organ). (Odonata.)

Localities: Brown's Wood and Stooper's Wood, near Henley in Arden, England. Horizon : Lias.

1874. Brodie. 1875. "

Distr. corr. foss. ins., 19.

Lower Lias Eatington, etc., 10.

\section{7.} (Odonata.)

Locality : Pappenheim, Bavaria. Horizon: Jura.

1833. Parkiuson. Organ. rem., $2 d$ ed., iii : 265-266 (unnamed). Pl. 17, fig. 2.

Referred to Eschna antiqua by Gieb., Deutschl. petref., 639. See also Eschna antiqua, etc.

\section{8. —. (Odonata.)}

Locality : Eyeford, England. Horizon: Stonesfield slate.

1854. Westwood. Quart. journ. geol. soc. Lond., $\mathrm{x}: 381,395 . \quad$ Pl. 17, fig. 20.

829. (Middle portion of wing.) (Odonata.)

Locality: Durdlestone Bay, England. Horizon: Middle Purbecks.

1854. Westwood. Quart.journ. geol, soc. Lond., x : 383.

(Odonata.) See II, Hemipt, (Pentatomidæ) Westw.

830. aspasia. (Odonata-Calopterygina.)

Locality: Solenhofen, Bavaria. Horizon: Jura.

- 1862. Hagen. Palaeontogr., x: 105 (undescr.).

See also Anax buchi, etc.

831. abscissa. (Odonata-Libellulina.)

Locality: Bavaria. Horizon: Jura.

1868. Brauer. Verhandl. zool.-bot. gesellsch. Wien, xviii: 738. See also (Libellula) abseissa.

832. antiqua. (Odonata-Libellalina.)

Locality: Bavaria. Horizon: Jura.

1868. Brauer. Verhandl, zool.-bot. gesellsch. Wien, xviii : 738.

See also Asschna antiqua, ete.

833. brodiei. (Odonata-Libellulina.)

Locality: England. Horizon: Upper Lias.

1868. Brauer. Verhandl. zool.-bot. gesellsch. Wien, xviii : 738.

See also Libellula brodiei, etc: 
834. decapitata. (Odonata-Libellulina.)

Locality : England. Horizon: Lias.

1868. Braner. Verhandl. zool.-bot. gesellsch. Wien, xviii: 738 . See also Libellula decapitata.

835. densa. (Odonata-Libellulina.)

Locality : Eichstadt, Bavaria. Horizon: Jura. 1868. Braner. Verhandl. zool.-bot. gesellsch. Wien, xviii : 738. See also Estemoa densa, (Libellula) densa, Estemoa bubas, ete.

836. nævia. (Odonata-Libellulina.)

Locality: Bavaria. Horizon: Jura.

18068. Brauer. Verhandl. zool.-bot. gesellsch. Wien, xviii : 738. See also (Libellula) nævia.

837. valga. (Odonata-Libellulina.)

Locality: Bavaria. Horizon: Jura.

1868. Brauer. Verhandl. zool.-bot. gesellsch. Wien, xviii : 738. See also (Libellula) valga.

838. (Panorpidæ?)

Locality : Dobbertin, Mecklenburg. Horizon : Lower Jura.

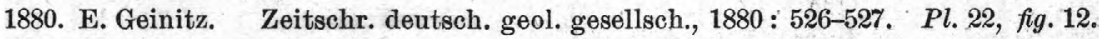

839. (Sialina ?)

Locality : Durdlestone Bay, England. Horizon: Lower Purbecks. 1854. Westwood. Quart. journ. geol, soc. Lond., x: 390, 396. Pl. 18, fig. 35.

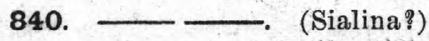

Locality : Durdlestone Bay, England. Horizon : Lower Purbecks. 1854. Westwood. Quart. journ. geol. soc. Lond., x: 390,396. Pl. 18, fig. 37.

841. (Trichoptera.)

Locality: Vale of Wardour, England. Horizon: Purbecks. 1845. Brodie. Foss. ins. sec. rocks Engl, 33 (Westwood, 118-119). Pl. 2, fig.7.

842. (Trichoptera.)

Locality: Grafton, England. Horizon : Lower Lias.

1873. Brodie.

Distr, corr. foss, ins,, 15.

843. (Trichoptera.)

Localities: Brown's Wood and Stooper's Wood, near Henley in Arden, England. Horizon : Lias.

1875. Brodie. Lower Lias Eatington, etc., 10.

844. (Larva.) (Trichoptera.)

Locality : Dobbertin, Mecklenburg. Horizon : Lower Jura.

1880. E. Geinitz. Zeitschr. deutseh. geol. gesellsch., 1880: 531. Pl. 22, fig. 24. 
845. (Trichoptera.)

Locality: Durdlestone Bay, England. Horizon: Middle Purbecks.

1854. Westwood. Quart. journ. geol. soc. Lond., x : 383-384, 394. Pl. 15, fig. $14^{\odot}$. 1886. Scudder. Mem. Bost. soc. nat. hist., iii : 451. Pl. 48, figs. $8 b, 10$.

1890. " Foss. ins. N. A., i: 343. Pl. 22, figs. $8 b, 10$.

846. (Trichoptera.)

Locality : Durdlestone Bay, England. Horizon: Middle Purbecks. 1854. Westwood. Quart. journ. geol, soc. Lond., $\mathrm{x}: 383-384,394 . \quad$ Pl. 15, fig. 14*。 1886. Scudder. Mem. Bost. soc. nat. hist., iii : 451. Pl. 48, figs. 8d, 12. 1890. " Foss, ins. N. A., i: $343 . \quad P l .22$, figs. $8 d, 12$.

\section{7.} (Trichoptera.)

Locality : Durdlestone Bay, England. Horizon : Lower Purbecks. 1854. Westwood. Quart. journ. geol. soc. Lond., x: 390, 396. Pl. 18, fig. 25.

848. (Trichoptera.)

Loeality: Durdlestone Bay, England. Horizon: Lower Purbecks. 1854. Westwood. Qnart. journ. geol. soc. Lond., x: 390,396. Pl. 18, fig. 28.

849. Abia duplicata.

Locality: Durdlestone Bay, England. Horizon : Lower Purbecks. 1854. Westwood. Quart. journ. geol. soc. Lond., x: 396 (unnamed). Pl. 18, fig. 42. 1856. Giebel.

Insect. d. vorwelt, 264.

Abia sipylus. See II, ORThopt., Idem.

$$
\text { 850. Asschna, - (Odonata.) }
$$

Locality: Dumbleton, England. Horizon: Upper Lias.

1873. Brodie. Distr. corr. foss. ins., 15.

\section{Fsschna or Iibellula —. (Odonata.)}

Locality : Solenhofen, Bavaria. Horizon: Jura.

1839. Erichson. v. Buch, Abhandl. akad. wiss. Berl., 1837; phys. abhandl., 135. Pl. 3.

See also Anax buchii, etc.

\section{Fischna, —. (Odonata.)}

Locality: Bavaria. Horizon: Jura.

1826. Koehler. Zeitschr. f. min., 1826: 231-233. Pl. 7, fig. 3.

See also Uropetala koehleri, etc.

853. \#schna (vic.) - (Odonata.)

Locality: Durdlestone Bay, England. Horizon: Lower Purbecks.

1854. Westwood. Quart. journ. geol. soc. Lond., x : 390.

854. \#sschna - (Odonata.)

Locality : Eyeford, England. Horizon: Stonesfield Slate.

1854. Westwood. Quart. journ. geol. soc. Lond., x: 395 (unnamed). Pl.17, fig. 20. 1856. Giebel. Insect. d. vorwelt, 281. 
855. Æschna antiqua. (Odonata.)

Locality : Solenhofen, Bavaria. Horizon: Jura.

1827. Linden.

182\%. "

1839. Brullé.

1850. Hagen.

1852. Giebel.

1856. ".

1862. Hagen.

See also

Nouv. mém. acad. sc. Brux., iv: 247-253. Pl. 1.

Notice empr. ins., 1-9. $\quad P l$.

Gisem. ins. foss., 12.

Rev. odon. (Mém. soc. roy. sc. Liége, vi): 362.

Deutschl. petref., 639.

Insect. d. vorwelt, 279 .

Palaeontoge, $\mathrm{x}$ : 136-137.

Æschna münsteri, Diastatomma mïnsteri, Cordnlegaster mïnsteri, Petalura miinsteri, Asschna wittei, Petalura wittei, Protolindenia wittei, Libella — Schmidel, Aschna schmideli, Agrion latreillei, Heterophlebia latreillei, Isophlebia helle, Heterophlebia helle.

\section{Asschna bavarica. (Odonata.)}

Locality: Bavaria. Horizon: Jura.

1856. Giebel. Insect. d. rorwelt, 280-281.

See alșo Cymatophlebia longialata, etc.

\section{Fschna brodiei. (Odonata.)}

Locality: Dumbleton, England. Horizon: Upper Lias.

1843. Buckman.

1844. "6

1844. 66

1845. Murchison.

1848. Hagen.

1866. Phillips.

Proc. geol. soc. Lond., iv: 212 (undescr.).

Ann. nat hist., xiv: 74 (undescr.).

Lond. Edinb. Dubl. phil. mag., xxiv : 377.

Geol. Cheltenham, 82. Pl. 8, figs. 1, 2.

Stett. entom. zeit., ix : 12.

Geol. mag., iii: 98 . Pl. 6, fig. $d$.

See also Libellula brodiei, etc.

\section{Ifschna buchi. (Odonata.)}

Locality : Solenhofen, Bavaria. Horizon : Jura.

1856. Giebel. Insect. d. vorwelt, 280.

See also Anax buchii, etc.

859. Ifschna charpentieri. (Odonata.)

Locality: Solenhofen, Bavaria. Horizon: Jura.

1848. Hagen. Stett. entom. zeit., ix: 11-12.

1850.

Rev. odon. (Mém. soc. roy. sc. Liége, vi): 362.

1852. Giebel.

Deutschl. petref., 639.

1856. "

Insect. d. vorwelt, 278.

See also Anax buchii, etc.

860. Isschna flindersiensis. (Odonata.)

Locality: Flinder's River, Australia. Horizon: Cretaceous.

1884. Woodward. Geol. mag., (3), i: 337-339. Pl. 11, fig. 1.

1886. Deichmüller. Ins. lith. schichten dresd. mus., 34 (Estemoa?).

861. Zsschna gigantea. (Ođouata.)

Locality: Solenhofen, Bavaria. Horizon: Jura.

1839. Münster. MSS.

1839. Germar. Verhandl. leop.-carol. akad. naturf., xix: 216. Pl. 23, figs. 13, 14, 14a. 
1856. Giebel.

186:. Hageu.

1863. Winkler.
Insect. d. vorwelt, 279 .

Palaeontogr., x: 111 (two species).

Cat. syst. coll. pal. mus. Teyl., 424 .

Ber. vers. deutsch. naturf, 1: 191.

See also Anax gigantens, Petalura gigantea, Esteinoa gigantea, Aschna intermedia, Anax intermedius, Petalura intermedia, Cordulegaster intermedius, Petalura latialata, Petalura eximia; also Anax buchii, Eschna buchi, Asschna or Libellula Erichs., Libellulites — Charp., Eschna charpentieri, Anax charpentieri, aspasia (Odonata, Calopterygina), Isophlebia aspasia, Aspasia gigantea.

\section{Aschna hageni. (Odonata.)}

Louality: Schambelen, Switzerland. Horizon : Lias.

1865. Heer.

1872. " "

1876. “

1879. “

Urwelt der Schweiz, 86. Pl. 7, fig. 9.

Monde prim. Suisse, 105, Pl. 7, fig. 9.

Prim. world Switz., i: 85. $\quad P l .7$, fig. 9.

Urwelt der Schweiz, 96. Pl. 7, fig. 9.

863. Aschna intermedia. (Odonata.)

Locality: Solenhofen, Bavaria. Horizon: Jura.

1839. Münster. MSS.

1839. Germar. Verhandl. leop.-carol. akad. naturf., xix: 216. Pl.23, fig. 13.

1856. Giebel. Insect. d. vorwelt, 280.

1877. Assmann. Ber. vers. deutsch. naturf., 1: 192.

See also A. gigantea, etc.

864. IIschna liassina. (Odonata.)

Localities: Near Evesham, Binton, Bidford, Warwickshire, England. Horizon: Lower Lias, Insect Limestone.

1840. Strickland. Mag. nat. hist., (2), iv : 301-303. Figs. 11-13.

1842. Dale. Ann. mag. nat. hist., ix: 257 (nearer to Cordulegaster and still more to Petalura).

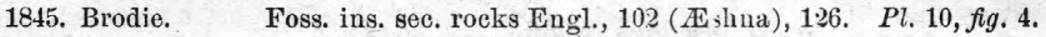

1845. Murchison. Geol. Cheltenham, 82.

1848. Hagen. Stett. entom. zeit., ix : 12.

See also Diastatomma liassina, Petalura liassina, Heterophlebia liassina, Libellula liassica, Libellula hopei, Heterophlebia hopei, Petalura hopei.

865. Aschna longialata. (Odonata.)

Locality: Solenhofen, Bavaria. Horizon: Jura.

1842. Münster. MSS.

1842. Germar. Münst., Beitr. z. petref., v: 79-81. Pl. 9, fig. 1; Pl. 13, figs. 6a-b.

1846. Geinitz. Grundr. versteinerungsk., 187 (longiolata). Pl. 8, figs. 5, $5 a$.

1852. Quenstedt. Handb. petref. (unnamed). Pl. 24, figs. 1-3.

1856. Giebel. Insect. d. vorwelt, 279.

1877. Assmann. Ber. vers. deutsch. naturf, 1: 9:.

See also Cymatophlebia longialata, etc.

\#schna longiolata. See Eschna longialata.

866. Isschna münsteri. (Odonata.)

Locality: Solenhofen, Bavaria. Horizon: Jura.

1839. Germar. Verhandl. leop.-carol. akad. naturf., xix: 215. Pl. 23, fig. 12.

1862. Hagen. Palaeontogr., $\mathrm{x}: 111,137-138$.

1863. Winkler. Cat. syst. coll. pal. mus. Teyl., 423.

1877. Assmann. Ber. vers. deutsch. naturf., 1: 192.

See also Diastatomma münsteri, Cordulegaster münsteri, Petalura mụinsteri, Eschna antiqua, etc. 
867. \#schna multicellulosa. (Odonata.)

Locality: Solenhofen, Bavaria. Horizon: Jura.

1857. Giebel. Zeitschr. ges. naturw., ix: 378-380. Pl. 5, fig. 2.

See also Cymatophlebia longialata, etc.

868. \#schna parkinsoni. (Odonata.)

Locality: Pappenheim, near Solenhofen, Bavaria. Horizon: Jura?

1833. Parkinson. Org. rem., iii : 265-266 (unnamed), Pl. 17, fig. 2a-c.

1850. Selys. Rev. odon. (Mém. soc. roy. se. Lí́ge, vi): 362, note.

1856. Giebel. Insect. d. vorwelt, 279 .

869. Fschna perampla. (Odonata.)

Locality: Vale of Wardour, England. Horizon: Purbecks.

1845. Brodie.

1850. Hagen.

1856. Giebel.

Foss. ins, sec. rocks Engl., 33. Pl. 5, fig.7.

Rev, odon. (Mém. soc. roy. sc. Liége, vi): 362.

Insect. d. vorwelt, 281.

870. Fschna schmideli. (Odonata.)

Locality: Bavaria. Horizon: Jura.

1856. Giebel. Insect. d. vorwelt, 278 .

1862. Hagen. Palaeontogr., x : 141-142 (schmiedeli).

See also Libella _- Schmidel, Eschna antiqua, etc.

\#schna schmiedeli. See Aschna schmideli.

871. Asschna wittei. (Odonata.)

Locality: Solenhofen, Bavaria. Horizon: Jura.

1860. Giebel. Zeitschr. ges. naturw., xvi: 127-131. Pl. 1, fig.1.

See also Petalura wittei, Protolindenia wittei, Aschna antiqua, etc.

872. झschnidium bubas. (Odonata.)

Locality: Durdlestone Bay, England. Horizon: Lower Purbecks.

1854. Westwood. Quart. journ. geol. soc. Lond., x: 387, 393-394. Pl. 15, fig. 5.

See also Estemoa bubas, Libellula antiqua, Estemoa densa, etc.

Fsshna liassina. See Eschna liassina.

\section{Agrion -. (Odonata.)}

Locality: Durdlestone Bay, England. Horizon: Lower Purbecks.

1854. Westwood. Quart. journ. geol. soc. Lond., $\mathrm{x}: 390$.

874. Agrion —. (Odonata.)

Locality: Solenhofen, Bavaria. Horizon: Jura.

1863. Winkler. Cat. syst. coll. pal. mus. Teyl., 424, No. 6358.

1874. Weyenbergh. Period. zool., i: 106.

1874. " Énum. syst. faune ent. mésoz., 20.

875. Agrion buckmani. (Odonata.)

Locality: Dumbleton, England. Horizon: Upper Lias.

1845. Brodie. Foss. ins. sec. rocks Engl., 102, 126. Pl. 8, fig. 2.

1871. Phillips. Geology of Oxford, 123.

See also Heterophlebia dislocata, etc. 
876. Agrion ? eichstættense. (Odonata.)

Localities: Eichstadt, Solenhofen, Bavaria. Horizon: Jura.

1862. Hagen. Palaeontogr., $\mathrm{x}:$ 106, 118-119. Pl. 14, fig. 5.

1863. Winkler. Cat. syst. coll. pal. mus. Teyl., 424.

1869. Weyenbergh. Tijdschr. v. ent., (2), iv: 235.

1874. " Period. zool., i: 93.

1874. “ Enum. syst. faune ent. mésoz., 7.

1886. Deichmïller. Ins. lith. schichten dresd. mus., 58.

877. Agrion exhaustum. (Odonata.)

Localities: Eichstadt, Solenhofen, Bavaria. Horizon: Jura.

1862. Hagen. Palaeontogr., $x: 106$ (undescr.).

1869. Weyenbergh. Tijdschr. v. ent., (2), iv: 235.

1874. " " Peri6d, zool, i: 93.

1874. " "Énum. syst. faune ent. mésoz., 7.

878. Agrion hecticum. (Odonata.)

Locality: Eichstadt, Bavaria. Horizon: Jura.

1862. Hagen. Palaeontogr., $\mathrm{x}$ : 106 (undescr.).

1869. Weyenbergh. Tijdschr. v. ent., (2), iv : 235.

1874. " " Period. zool., i: 92.

1874. " " Enum. syst. faune ent. mésoz., 6.

879. Agrion latreillei. (Odonata.)

Locality: Solenhofen, Bavaria. Horizon: Jura.

1839. Muinst.

MSS.

18:39. Germar. $\quad$ Verhand. leop.-carol. akad, naturf., xix : 218. Pl. 23, fig. 16.

1846. Geinitz. Grundr. versteinerungsk., 187.

1848. Hagen. Stett. entom. zeit., ix: 9 (=Cordulegaster münsteri ?).

1862. " Palaeontogr., x: 111, 138-139.

1877. Assmann. Ber. vers. deutsch. naturf., 1: 192.

1886. Deichmïlıer. Ins. lith. schichten dresd. mus., 44-45. Pl. 4, fig. 13.

Seo also Heterophlebia latreillei, Isophlebia helle etc., Eschna münsteri etc., Eschna antiqua etc.

Agrion venustum. See Agrion vetustum.

880. Agrion vetustum. (Odonata.)

Locality: Solenhofen, Bavaria. Horizon: Jura.

1848. Hagen. Stett. entom, zeit., ix: 7 .

1850. “ Rev. odon. (Mém. soc. roy. sc. Liége, vi): 356.

1852. Giebel. Deutschl. petref., 638 (venustum).

1856. " Insect. d. vorwelt, 272.

1863. Hagen. Palaeontogr., $\mathrm{x}: 106,118$.

1869. Weyenbergh. Tijdschr. v. ent., (2), iv : 235.

1874. " " Period. zool., i: 93.

1874. “ Énum. syst. faune ent. mésoz., 7.

See also Agrionides _- Charpentier.

881. Agrionides —. (Odonata.)

Locality: Solenhofen, Bavaria. Horizon: Jura.

1840. Charpentier. Libell. europ., 170-173. Pl. 48, figs. 2,3.

See also Agrion vetustum. 
882. Agrionidium ætna. (Odonata.)

Locality : Durdlestone Bay, England. Horizon: Lower Purbecks.

1854. Westwood. Quart. journ. geol. soc. Lond., $\mathrm{x}: 396 . \quad$ Pl. 18, fig. 39.

883. Anax buchii. (Odonata.)

Locality : Solenhofen, Bavaria. Horizon: Jura.

1848. Hagen.

Stett. entom. zeit., ix : 11.

1850 .

Rev. odon. (Mém. soc. roy. sc. Liége, vi): 361.

1852. Giebel.

Deutschl. petref., 639 .

1862. Hagen.

Palaeontogr., $\mathrm{x}: 143-144$.

1866. “

Palaeontogr., $\mathrm{xv}:$ 92-96. $\quad P l .3$, fig. 2.

1866. " "

Neur. lithogr. schief., i: 36-40. Pl. 3, fig. 2. .

See also Eschna buchi, Eschna or Libellnla — Erichs., Libellulites

Charp., Eschna charpentieri, Anax charpentieri, _- aspasia (Odonata, Calopterygina), Isophlebia aspasia, Aspasia gigantea; also Anax giganteus, etc.

884. Anax charpentieri. (Odonata.)

Locality: Eichstadt, Bavaria. Horizon: Jura.

1862. Hagen: Palaeontogr., $\mathrm{x}:$ 106,140-141. Pl. 14, fig. 1.

1866. " Palaeontogr., $x v: 95-96$ (=A. buchi ô).

1866. “ Neur. lithogr. schief., i : $39-40$ (=A. buchi $\delta$ ).

1869. Weyenbergh. Tijdschr. $\nabla$. ent., (2), iv : 235.

See also $\mathrm{A}$. buchii, etc.

885. Anax giganteus. (Odonata.)

Locality: Bavaria. Horizon: Jura.

1848. Hagen. Stett. entom. zeit., ix : 10.

1850. “ Rev. odon. (Mém. soc. roy. sc. Liége, vi): 361.

1852. Giebel. Deutschl. petref., 639.

1862. Hagen. Palaeontogr., $\mathrm{x}:$ 142.

See also Æschna gigantea, Petalura gigantea, Estemoa gigantea, Eschna intermedia, Anax iptermedius, Petalura intermedia, Cordulegaster intermedius, Petalura latialata, Petalura eximia ; also Anax buchii, etc.

886. Anax intermedius. (Odonata.)

Locality: Solenhofen, Bavaria. Horizon: Jura.

1848. Hagen. Stett. entom. zeit., ix: 10.

1850. " $\quad$ Rev. odon. (Mém. soc. roy. sc. Liége, vi): 361.

1862. " Palaeontogr., $\mathrm{x}:$ 142-143.

See also Aschna gigantea, etc.

887. Anax longialatus. (Odonata.)

Locality : Solenhofen, Bavaria. Horizon: Jura.

1848. Hagen. Stett. entom. zeit., ix: 11.

1850. " " Rev. odon. (Mém. soc. roy. sc: Liége, vi): 361 (longiolatus).

1852. Giebel. Deutschl. petref., 639.

See also Cymatophlebia longialata, etc.

Anax longiolatus. See Anax longialatus.

Apiaria antiqua. See II, Hymenoptera, Idem.

888. Apochrysa excelsa. (Hemerobina.)

Locality: Eichstadt, Bavaria. Horizon: Jura.

1862. Hagen. Palaeontogr., $x: 108$ (undeser.).

1869. Weyenbergh. Tijdschr. v. ent., (2), iv : 236. 
1885. Scudder.

1886. " "

1888. Oppenhein
Zittel, Handb. palaeont., i, th. ii: $77 \%$.

Zittel-Barrois, 'Traite de paléont., ii : 777.

Palaeontogr., xxxiv: 227. - Pl. 30. fig. 1.

889. Aspasia gigantea. (Odonata.)

Locality: Solenhofen, Bavaria. Horizon: Jura.

1869. Weyenbergh. Tijdschr. v. ent., (2), iv : 234 [134], as of Hagen, but erroneonsly.

See also Anax buchii, etc.

Bittacus dubius. See II, Orthopt., Idem.

890. Blattidium ——. (Odonata.)

Locality : Vale of Wardour, England. Horizon: Purbecks.

1877. Goldenberg. Fauna saræp. foss., ii : 21, No. 51 (refers by mistake to Blattina similis Gieb.).

See also Libellula antiqua.

891. Caloptery $x$ lithographica. (Odonata.)

Locality : Solenhofen, Bavaria. Horizon: Jura.

1857. Giebel. Zeitschr. ges. naturw, ix : 380-382. Pl. 5, fig. 1.

1862. Hagen. Palaeontogr., x: 105.

See also Heterophlebia phryne, etc.

892. Calotermes maculatus. (Termitina.)

Locality: Schambelen, Switzerland. Horizon: Lias.

1865. Heer.

1872. " "

1876. " "

1879. . .
Urwelt der Schweiz, 86. Pl. 7, fig. 7.

Monde prim. Suisse, 104. Pl. 7, fig. 7.

Prim. world Switz., i: 85. Pl. 7, fig. 7.

Urwelt der Schweitz, $2^{\mathrm{e}}$ aufl., 96. Pl. 7, fig. 7.

893. Calotermes obtectus. (Termitina.)

Locality: Schambelen, Switzerland. Horizon: Lias.

1865. Heer.

1872. "

1876. “

1879. "

Urwelt der Schweiz, 86.

Monde prim. Suisse, 105.

Prim. world Switz., i : 85.

Urwelt der Schweiz, $2^{\mathrm{e}}$ aufl., 96.

894. Calotermes plagiatus. (Termitina.)

Locality: Schambelen, Switzerland. Horizon: Lias.

1865. Heer.

1872. " "

Urwelt der Schweiz, 86. Pl. 7, fig. 6.

Monde prim. Suisse, 104. Pl. 7, fig. 6.

Prim. world Switz., i : 85. Pl. 7, fig. 6.

1876. " "

Urwelt der Schweiz, 2e aufl., 96. Pl. 7, fig. 6.

895. Calotermes troglodytes. (Termitina.)

Locality: Schambelen, Switzerland. Horizon: Lias.

1865. Heer.

Urwelt der Schweitz, 86.

1872. " Monde prim. Suisse, 105.

1876. " " Prim. world Switz., i: 85.

1879. " Urwelt der Schweiz, $2^{\circ}$ aufl, 96. 
896. Chauliodes —- (Sialina.)

Localities: Hasfield, Strensham, Bidford, England. Horizon : Lower Lias. 1845. Brodie.

Foss. ins. sec. rocks Engl., 102, 126, Pl. 8, figs. 3, 5,6,14; Pl. 10, figs. 6, 9-12.

See also Orthophlebia - Westwood, 1815 , O. parallela, O. similis, O. intermedia, O. lata, Hagla gracilis, H. similis, H. deleta, H. (Chauliodes) similis, Orthophlebia (Phryganidium) intermedia.

\section{Chauliodes ㄴ. (Sialina.)}

Localities: Brown's Wood, Binton, Copt Heath, Knowle, England. Horizon : Lower Lias.

1873. Brodie. Distr. corr. foss. ins., 15

\section{Chauliodes ㄴ. (Sialina.)}

Locality : England. Horizon: Lias.

1871. Philips. Geology of Oxford, 123.

Chauliodes similis. See Hagla (Chauliodes) similis.

899. Chauliodites helveticus. (Sialina.)

Locality: Mythen, Canton Schwyz, Switzerland. Horizon: Trias.

1877. Heer.

Flora foss. helvet., 77. Pl. 29, figs. 32,32b.

900. Chauliodites minor. (Sialina.)

Locality: Hatham, England. Horizon: Lias, Planorbis Zone.

1876. Blake. Tate and Blake, Yorkshire Lias, 426. Pl.16, fig.12 (quoted as 6).

901. Chauliodites picteti. (Sialina.)

Locality : Gödewitz, bei Salzmunde, Germany. Horizon: Trias, Bunter Sandstein. 1864. Heer. Viert. naturf. gesellsch. Zürich, ix : 279, note. Pl., fig. 11. 1872. " Monde prim. Suisse, 99.

\section{Chauliodites zinkeni. (Sialina.)}

Locality: Gödewitz, bei Salzmunde, Germany. Horizon: Trias, Bunter Sandstein. 1×64. Heer.

Viert. naturf. gesellsch. Zürich, ix : 279, note. Pl., fig. 10. 1872. " Monde prim. Suisse, 99.

903. Chimarrha pytho. (Trichoptera.)

Locality : Durdlestone Bay, England. Horizon: Lower Purbecks.

1856. Giebel.

Insect. d. vorwelt, 269.

See also Phryganidium pytho.

904. Chrysopa excelsa. (Hemerobina.)

Locality : Eichstadt, Bavaria. Horizon: Jura.

1888. Oppenheim. Palaeontogr., xxxiv: 227 (as of Hagen). Pl. 30, fig. 2.

See also Hemerobius priscus.

905. Chrysopa protogæa. (Hemerobina.)

Locality: Eichstadt, Bavaria. Horizon: Jura.

1862. Hagen. Palaeontogr., $x: 108$ (undeser.).

1666. "6 Stett. entom. zeit., xxvii : 396.

1869. Weyenbergh. Tijdschr. v. ent., (2), iv: 236. 
906. Chrysopa? solenhofensis. (Hemerobina.)

Locality: Solenhofen, Bavaria. Horizon: Jura.

1869. Weyenbergh. Arch. mus. Teyl., ii : 264. Pl. 34, figs. 11-12.

1869. " " Ins. foss. calc. lithogr., 18. Pl. 1, figs. 11-12.

$1869 . \quad$ " Tijdschr. v. ent., (2), iv : 236.

1874. " " Periód. zool., 1:96.

1874. “ Énum. syst. faune ent. mésoz., 10.

Clathrotermes (Elcana) geinitzi. See II, ORTHopr., Idem.

907. Clathrotermes signatus. (Termitina.)

Locality: Schambelen, Switzerland. Horizon: Lias.

1865. Heer. Urwelt der Schweiz, 85. Pl. 7, fig. 8.

1872. " Monde prim. Suisse, 104. Pl. 7, fig. 8.

1876. " Prim. world Switz., i: 85. Pl. 7, fig. 8.

1879. “ Urwelt der Schweiz, $2^{e}$ aufl., 95. Pl. 7, fig. 8.

1885. Scudder. Zittel, Handb. palaeont., i, th. ii : 772. Fig. 973.

1886. "Zittel-Barrois, Traité de paléont., ii : 77\%. Fig. 990.

1886. Deichmüller. Ins. lith. schichten dresd. mus., 17-18. Pl. 2, fig. 8,

908. Cordulegaster intermedius. (Odonata.)

Locality: Solenhofen, Bavaria. Horizon: Jura.

1886. Deichmüller. Ins. lith. schichten dresd. mus., 45-48. Pl. 4, fig. 7

See also Eschna gigantea, etc.

Cordulegaster liassinus. See Asschna liassina.

909. Cordulegaster muensteri. (Odonata.)

Locality: Bavaria. Horizon: Jura.

1848. Hagen. Stett. ent. zeit., ix : 8-9.

1850. " Rev. odon. (Mém. soc. roy. sc. Liége, vi): 360.

See also Æsschna münsteri, etc., Æschna antiqua, etc.

\section{Corydalis}

Locality: Vale of Wardour, England. Horizon: Purbecks.

1845. Brodie.

Foss. ins. sec. rocks Engl., 33, 119. Pl. 5, figs. 2, 3, 13,14, 16.

1856. Hagen.

Berendt, Bernst. befindl. organ. reste vorw., ii, th. i : 81 (Brod., $\mathbf{v}:$ 13.)

See also Orthophlebia minuta, Zalmona brodiei; alsp II, ORTHoPT., Blattidium similis, B. kollari, Blattina similis.

\section{Corydalis vetusta. (Sialina.)}

Locality: Eichstadt, Bavaria. Horizon: Jura.

1862. Hagen. Palaeontogr,$x ; 108$ (undescr.).

1869. Weyenbergh. Tijdschr. v. ent., (2), iv : 236.

1888. Oppenheim. Palaeontogr., xxxiv: 227-228. Pl. 30, fig. 3; Pl. 31, fig. 31.

912. Corydalites fecundus. (Sialina.)

Compared with Corydalis cornutus, U. S.

Locality : Crow Creek, near Greeley, Colo. Horizon : Laramie Gronp.

1878. Scudder. Bull. U. S. geol. surv. terr., iv : 537-540 (fecundum).

1879. White.

Ann. rep. U. S. geol. surv. terr., xi : 173-174.

1885. Sendder.

Zittel, Handb. palaeont., i, th. ii : 776. Fig. $981 a b$.

1886 .

Zittel-Barrois, 'Traité de paléont., ii : 776. Fig. 998ab.

1890.

Tert. ins. N. A., 149-153, Pl. 4, figs. 5-7, 13-16, 18-21, 23. 


\section{Cyllonium boisduvalianum.}

Locality: Durdlestone Bay, England. Horizon: Lower Purbecks.

1854. Westwood. Quart. journ. geol. soc. Lond., $\mathrm{x}: 387,395 . \quad$ Pl. 17, fig. 17.

1875. Scudder. Mem. Amer. assoc. adv. sc., i: 88-89. Fig. 2.

1877. Kirby. Synon. catal. diurn. lepid., suppl., 699.

914. Cyllonium hewitsonianum.

Locality: Durdlestone Bay, England. Horizon: Lower Purbecks.

1854. Westwood. Qnart. journ. geol. soc. Lond., $\mathrm{x}: 390,396 . \quad$ Pl. 18, fig. 27.

1875. Scudder. Mem, Amer. assoc. adv. sc., i: 88-89. Fig. 3.

1877. Kirby. Synon. catal. diurn. lepid., suppl., 699.

915. Cymatophlebia longialata. (Odonata.)

Locality: Bavaria. Horizon: Jura.

1886. Deichmüller. Ins. lith. schichten dresil. mus., 49-52 (longiolata). Pl. 3, figs.5-8.

See also Libellula longialata, Eschna longialata, Anax longialatus, Petalia longialata, Gynacantha longialata, Eschna bavariea, A. multicellulosa.

Diastatomma liasina. See Diastatomma liassina.

916. Diastatomma liassina. (Odonata.)

Locality: England. Horizon: Lower Lias.

1856. Giebel. Insect. $d$. vorwelt, 276 (liasina).

See also Aschna liassina, etc.

917. Diastatomma münsteri. (Odonata.)

Locality : Bavaria. Horizon: Jura.

1856. Giebel. Insect. d. vorwelt, 276 .

See also Asschna münsteri, ete., Aschna antiqua, etc.

918. Dictyoneura cellulosa. (Ephemeridæ.)

Locality: Bavaria. Horizon: Jura.

1868. Braner. Verhandl. k.-k. zool.-bot. gesellsch. Wien, xviii : 389, note.

1868. " Verz. bek. neuropt., 31, note.

1871. Eaton. Trans. ent. soc. Lond., 1871: 40.

See also Ephemera cellulosa.

\section{Dictyoneura procera. (Ephemeridæ.)}

Locality: Bavaria. Horizon: Jura.

1868. Brauer. Verhandl. k.-k. zool.-bot. gesellsch. Wien, xviiy: 389, note.

1868. " Verz. bek. neuropt., 31, note.

1871. Eaton. Trans. ent. soc. Lond., 1871: 40.

See also Ephemera procera.

\section{Fla brephos.}

Locality: Durdlestone Bay, England. Horizon : Lower Purbecks.

1856. Giebel. Insect. d. vorwelt, 257.

See also Raphidium brephos.

\section{Elcana beyrichi.}

Locality: Durdlestone Bay, England. Horizon: Lower Purbecks.

1856. Giebel. Insect $d$. vorwelt, 259.

1884. E. Geinitz. Zeitschr. dentsch, geol. gesellsch., 1884: 579.

1886. Deichmiiller. Ins. litb. schichten dresd. mus., 17.

See also Panorpidium tessellatum var. 
Elcana (Clathrotermes) geinitzi. See II, Orthopt., Idem.

Elcana (Clathrotermes) intercalata. See II, ORthopt, Idem.

Elcana liasina. See Rapha liasina.

Elcana tessellata. See II, Orthoptera, Irlem.

922. Ephemera -. (Ephemeridæ.)

Locality: Strensham, England. Horizon: Lower Ljas, Iusect Limestone.

1845. Brodie. Foss. ins. sec. rocks Engl., 102,127. Pl. 10, fig. 14.

See also Rapha liasina.

923. Ephemera -. (Ephemeridæ.)

Localities: Brown's Wood, Binton, Westbury, England. Horizon : Lower Lias. 1873. Brodie. Distr. corr. foss. ins., $\mathbf{1 5}$.

924. Ephemera (vic.) —. (Ephemeridæ.)

Locality: Solenhofen, Bavaria. Horizon: Jura.

1871. Eaton. Trans. ent. soc. Lond., 1871: 158. Pl. 1, fig. 10.

925. Ephemera - (Ephemeridæ.)

Locality: England. Horizon: Lias.

1871. Phillips. Geology of Oxford, 123.

926. Ephemera cellulosa. (Ephemeridæ.)

Compared with E. vulgata.

Locality : Eichstadt, Bavaria. Horizon: Jura.

1862. Hagen. Palaeontogr., $\mathrm{x}:$ 107,115-116. Pl. 15, fig. 3.

1869. Weyenbergh. Tijdschr. v, eut., (2), iv: 236.

See also Dictyoneura cellulosa.

927. Ephemera deposita. (Ephemeridæ.)

Locality : Solenhofen, Bavaria. Horizon: Jura.

1874. Weyenbergh. Period zool., i: 86,96. Pl. 3, fig. 14.

1874. " Énum, syst. faune ent. mésoz., 10.

928. Ephemera meyeri. (Ephemeridæ.)

Locality: Solenhofen, Bavaria. Horizon: Jura.

1874. Weyenbergb. Peri6d. zool., i: 85,96. Pl. 3, fig. 13.

1874. " " Énum. syst. faune ent. mésoz., 10

929. Ephemera mortua. (Ephemeridæ.)

Localities: Eichstadt, Solenhofen, Bavaria. Horizon: Jura.

1862. Hagen. Palaeontogr., $\mathrm{x}:$ 107, 117. Pl. 15, fig. 5.

1869. Weyenbergh. Tijdschr. v. ent., (2), iv : 236.

1874. " " Period. zool., i: 96.

1874. " "Enum. syst. faune ent. mésoz., 10.

1886. Deichmüller. Ins. lith. schichten dresd. mus., 29. I'l, 2, fig. 13.

1888. Oppenheim. Palaeontogr., xxxiv: 225. Pl. 30, fig. 18. 


\section{Ephemera? multinervosa. (Ephemeridx.)}

Locality: Bavaria. Horizon: Jura.

1888. Oppenheim. Palaeontogr., xxxiv: 225.

931. Ephemera prisca. (Ephemeridæ.)

Locality : Solenhofen, Bavaria. Horizon: Jura.

1862. Hagen. Palaeontogr., $x: 108,117-118$.

1869. Weyenbergh. Tijdschr. v. ent., (2), iv: 236.

See also Sciara prisca.

932. Ephemera procera. (Ephemeridø.)

Locality: Eichstadt, Bavaria. Horizon: Jura.

1862. Hagen. Palaeontogr., $\mathrm{x}:-107,116-117 . \quad P l .15, \mathrm{fig} .2$.

1869. Weyenbergh. Tijdschr. v. ent., (2), iv : 236.

1888. Oppenheim. Palaeontogr., xxxiv: 225. Pl. 30, fig. 12.

See alco Dictyoneura procera.

933. Ephemera speciosa. (Ephemeridæ.)

Locality : Eichstadt, Bavaria. Horizon: Jura.

1888. Oppenheim. Palaeontogr., xxxiv: 225. Pl. 30, fig. 13.

\section{Ephemeropsis - - (Ephemeridæ.)}

Locality: Eastern Siberia. Horizon: Jura.

1865. Eichwald. Amtl. ber. vers. deutsch. naturf., xxxix : 170.

See also —_ Müll., Epheineropsis orientalis, E. trisetalis.

935. Ephemeropsis orientalis. (Ephemeridæ.)

Locality : Near Kondnyewskaya on Tourga, Eastern Siberia. Horizon : Purbecks. 1868. Eichwald. Lethæa rossica, ii, pt. ii : 1192-1195. Pl. 37, fig. 8.

1889. Brauer-Redt-\} Mém. acad. sc. St.-Pétersb., (7), xxxvi, No. 15: 5-6. Pl. [1],

See also Ephemeropsis —-, E. trisetalis, _- - Müll.

936. Ephemeropsis trisetalis. (Ephemeridæ.)

Locality: River Towega, distr. Nertschinsk, Siberia. Horizon: Jura.

1864. Eichwald. Bull. soc. gél. France, (2), xxi: 21-22.

1864. “ Quart. journ. geol. soc. Lond., xx: 21.

See also E. orientalis, etc.

\section{Fstemoa bubas. (Odonata.)}

Locality: England. Horizon: Lower Purbecks.

1856. Giebel. Insect. d. vorwelt, $28 \%$.

1886. Deichmüller. Ins. lith. schichten dresd. mus., 34 (=E. densa ?).

See also Eschnidium bubas, Libellnla antiqua, Blattidium —— Goldenb., Estemoa densa, etc.

938. Estemoa densa. (Odonata.)

Locality: Eichstadt, Bavaria. Horizon: Jura.

1886. Deichmüller. Ins. lith. schichten dresd. mús., 34-35. Pl. 3, fig. 4.

See also (Libellnla) densa, — densa (Odonata, Libellnlina), Estemoa bubas, Æschnidium bubas, Libellula antiqua, 13lattidium —- Goldenb,

Bull. $71-10$ 
Estemoa flindersiensis. See Aschna flindersiensis.

939. Estemoa gigantea. (Odonata.)

Locality : Eichstadt, Bavaria. Horizon : Jura.

1886. Deichmüller. Ins, lith. schichten dresd. mns., 35-37. Pl. 3, figs. 1-3.

See also Aeschna gigantea, etc.

940. Euphæa areolata. (Odonata.)

Locality: Eichstadt, Bavaria. Horizon: Jura.

1862. Hagen. Palaeontogr., $\mathrm{x}: 106$ (undescr.).

1869. Weyenbergh. Tijdschr. v. ent., (2), iv : 235.

941. Euphæa filosa. (Odonata.)

Locality: Eichstadt, Bavaria. Horizon: Jura.

1862. Hagen. Palaeontogr., $\mathrm{x}: 106$ (undescr.).

1869. Weyenbergh. Tijdschr, v. ent., (2), iv : 235.

942. Euphæa longiventris. (Odonata.)

Locality : Solenhofen, Bavaria. Horizon : Jura.

1862. Hagen. Palaeontogr., $\mathrm{x}:$ 106, 121-124. Pl. 13, figs. 7, 8.

1863. Winkler. Catal. syst. pal. mus. Teyl., 424.

1869. Weyen bergh. Tijdschr. v. ent., (2), iv : 235.

1874. "

1874. " "Enum. syst. faune ent. mésoz., 6.

See also Tarsophlebia eximia, Heterophlebia eximia.

943. Euphæa ? multinervis. (Odonata.)

Localities: Eichstadt, Solenhofen, Bavaria. Horizon: Jura.

1862. Hagen. Palaeontogr., $\mathrm{x}$ : 106,119-121. Pl. 14, figs. 2-4.

1874. Weyenbergh. Períd. zool., i: 92.

1874. " Énum. syst. faune ent. mésoz., 6.

Eutermes grandævus. See Termes (Eutermes) grandævus.

944. Flata haidingeri. (Termitina.)

Locality : Vale of Wardour, England. Horizon : Purbecks.

1845. Brodie. Foss. ins. sec. rocks Engl., 33, 118 (unnamed). Pl. 2, fig. 6.

1856. Giebel. Insect. d. vorwelt, 375.

1860. Hagen. Linn. entom., xii : 297 (= Termes grandævus).

See also Termes grandævus, etc.

\section{Gomphoides brodiei. (Odonata.)}

Locality: Dumbleton, England. Horizon: Upper Lias.

1850. Selys. Rev. odon. (Móm. soc. roy. se. Liége, vi): 360 , note。

See also Libellula brodiei, etc.

946. Gomphus brodiei. (Odonata.)

Locality: Dumbleton, England. Horizon: Upper Lias.

1850. Hagen. Rev. odon. (Mém, soc, roy, sc. Liége, vi) : 360.

See also Libellula brodiei, etc. 
Gomphus koechleri. See Gomphus koehleri.

\section{Gomphus koehleri. (Odonata.)}

Locality: Bavaria. Horizon: Jura.

1826. Koehler. Zeitschr. f. min., 1826, ii : 231 (unnamed). Pl. 7.

1848. Hagen. Stett. ent. zeit., ix : 8.

1850. " - Rev. odon. (Mém. soc. roy, sc. Liége, vi): 360.

1852. Giebel. Deutschl, petref., 639 (koechleri).

1862. Hagen. Palaeontogr., $x: 139$.

See also Uropetala koehleri, etc.

948. Gomphus petrificatus. (Odonata.)

Locality: Vale of Wardour, England. Horizon: Purbecks.

1850. Hagen. Rev. odon. (Mém. soc. roy. sc. Liége, vi): 359.

Seo also Lindenia —- Brodie, etc.

\section{Gynacantha longialata. (Odonata.)}

Locality: Bavaria. Horizon: Jura.

1848. Hagen. Stett. entom. zeit., ix : 9-10.

1850. " Rev. odon. (Mém. roy. soc. sc. Liége, vi): 361 (longiolata).

1852. Giebel. Deutschl, petref., 639.

See also Cymatophlebia longialata, etc.

Gynacantha longiolata. See Gynacantha longialata.

950. Hagla -. (Sialina.)

Locality : England. Horizon; Lower Lias.

1845. Brodie. Foss. ins. sec. rocks Engl., (unnamed). $P l .10, f i g .5$.

1856. Giebel. Insect. d. vorwelt, 265-266.

951. Hagla deleta. (Sialina.)

Locality : England. Horizon: Lower Lias.

1845. Brodie. Foss, ins. sec. rocks Engl. (Chauliodes sp.). Pl. 8, fig. 3.

1856. Giebel. Insect. d. vorwelt, 265.

See also Chauliodes - Brodie, 1845, etc.

952. Hagla gracilis. (Sialina.)

Locality: England. Horizon: Lower Lias.

1845. Brodie. Foss. ins. sec. rocks Engl. (Chanliodes sp.). Pl. 8, fig. 14. 1856. Giebel. Insect. d. vorwelt, 264-265.

See also Chauliodes — Brodie, 1845, etc.

953. Hagla ignota. (Sialina.)

Locality: Durdlestone Bay, England. Horizon : Middle Purbecks.

1856. Giebel. Insect. d. vorwelt, 265.

See also Termitidium ignotum.

954. Hagla similis. (Sialina.)

Locality : England. Horizon: Lower Lias.

1845. Brodie. Foss. ins. sec. rocks Engl. (Chauliodes sp.). Pl. 8, fig. 6. 1856. Giebel. Insect. d. vorwelt, 265.

See also Chauliodes — Brodie, 1845, etc. 


\section{5. of. Hagla (Chauliodes) similis. (Siaiina.)}

Locality: Dobbertin, Mecklenburg. Horizon: Lias.

1884. E. Geinitz. Zeitschr. dentsch. geol. gesellsch., 1884 : 576-577. Pl. 13, fig. 12. See also Chauliodes — Brodie, 1845, etc.

956. Hemerobioides giganteus. (Hemerobina.)

Locality: Stonesfield, England. Horizon: Stonesfield Slate. 1838. Buckland. Proc, geol, soc. Lond., ii : 688.

1838. " Lond. Edinb. Dubl. phil. mag., (3), xiii: 388.

1844. Mantell. Medals of ereation, ii : 577.

1845. Brodie. Foss. ins. sec. rocks Engl., 45, 123. Pl. 6, fig. 22.

1854. Mantell. Medals of creation, 2 d ed., ii: 553.

1871. Phillips. Geology of Oxford, 173. Digr. 35.

See also Hemerobius giganfens.

957. Hemerobius - (Hemerobina.)

1873. Brodie. Distr. corr. foss, ins., 17.

Locality: Aust, England. Horizon: Rhætic.

958. Hemerobius —-, (Hemerobina.)

Locality: England. Horizon: Lias.

1874. Brodie. Distr. corr. foss, ins., 19.

959. Hemerobius fossilis. (Hemerobina.)

Locality: Solenhofen, Bavaria. Horizon: Jura.

1869. Weyenbergh. Arch. mus. Teyl, ii : 264. Pl. 34, fig. 15.

1869. " Ins, foss. calc. lithogr., 18. Pl. 1, fig. 15.

1869. " " Tijdschr. v. ent., (2), iv: 236.

1874. " " Periód. zool., i; 97.

1874. “ Enum. syst. faune ent. mésoz., 11.

960. Hemerobius giganteus. (Hemerobina.)

Locality: Stonesfield, England. Horizon: Stonesfield Slate.

1854. Morris. 1856. Giebel.

Catal. Brit. foss., $2 \mathrm{~d}$ ed., 117.

Insect. d. vorwelt, 262.

1866. Hagen. Stett. ent. zeit., xxvii : 412 (an hujus generis?).

See also Hemerobioides giganteus.

961. Hemerobius higginsii. (Hemerobina.)

Locality: England. Horizon: Lower Lias, Insect Limestone.

1845. Brodie.

Foss. ins. sec. rocks Engl., 102. Pl. 9, fig. 15.

1856. Giebel.

Insect. $\mathrm{d}$. vorwelt, 262.

962. Hemerobius priscus. (Hemerobina.)

Locality: Solenhofen, Bavaria. Horizon: Jura.

1869. Weyenbergh. Arch. mus. Teyl., ii : 264. Pl. 34, figs. 13, 14.

$1869 . \quad$ " Ins. foss. calc. lithogr., 18. Pl. 1, figs. 13, 14 .

1869. " "Tijdschr. v. ent., (2), iv : 236.

1874. " $\quad$ Peribd. zool., i: 97.

1874. " "Enum. syst. faune ent. mésoz., 11.

1886. Deichraüller. Ins. lith. schichten dresd, mus., 58.

See also Chrysopa excelsa, 


\section{Heterophlebia —. (Odonata.)}

Locality : England. Horizon: Lias.

1848. Westwood. Brodie, Quart. journ. geol. soc. Lond., v: 35.

1818. " Brodie, Foss. ins. sec. rocks Engl., 126, 127. Pl. 8, fig. 4; Pl. 10, fig. 8.

964. Heterophlebia æqualis. (Odonata.)

Localities: Eichstadt, Solenhofen, Bavaria. Horizon: Jura.

1862. Hagen. Palaeontogr., $\mathrm{x}:$ 105, 124-127. Pl. 13, fig8. 4-6.

1869. Weyenbergh. Tijdschr. v. ent., (2), iv : 234 [134].

See also Stenophlebia æqualis.

965. Heterophlebia amphitrite. (Odonata.)

1862. Hagen. Palaeontogr., $x: 105$ (undescr.).

Locality: Eichstadt, Bavaria. Horizon: Jura.

See also Stenophlebia amphitrite.

Heterophlebia brodiei. See Libellula (Heterophlebia) brodiei.

966. Heterophlebia buckmanni. (Odonata.)

Locality: Dumbleton, England. Horizon: Upper Lias.

1856. Giebel. Inseet. d. vorwelt, 288.

See also Heterophlebia dislocata, etc.

\section{Heterophlebia casta. (Odonata.)}

Locality : Eichstadt, Bavaria. Horizon: Jura.

1862. Hagen. Palaeontogr., $x: 106$ (undescr., =Lib. brevialata Munst. ). 1869. Weyenbergh. Tijdsehr. v. ent., (2), iv : 235.

See also Libellula brevialata.

\section{Heterophlebia dislocata. (Odonata.)}

Localities: Dumbleton, Cheltenham, England. Horizon: Upper Lias.

1848. Brodie.

1848. Westwood.

1848.

1849. Hagen.

1850. “ “

1854. Pictet.

1856. Giebel.

1866. Hagen.

1866.

1869. Weyenbergh. Tijdschr. v. ent., (2), iv : 234 [134].

See also Libellula (Heterophlebia) dislocata, Libellnla dislocata, Agrion buckmani, Heterophlebia buckmani.

\section{Heterophlebia eximia. (Odonata.)}

\section{Locality : Eichstadt, Bavaria. Horizon: Jura.}

1862. Hagen.

Falaeontogr., $x$ : 106 (undescr.).

See also Tarsophlebia eximia, Euphæa longiventris. 
970. Heterophlebia helle. (Odonata.)

Locality : Eichstadt, Bavaria. Horizon: Jura.

1862. Hagen. Palaeontogr., $x: 105$ (undeser.).

1869. Weyenbergh. Tijdschr. v. ent., (2), iv: 234 [134] (undescr.).

See also Isophlebia helle, Agrion latreillei, etc., Aschna münsteri, stc., Eschna antiqua, etc.

\section{Heterophlebia hopei. (Odonata.)}

Locality: England. Horizon: Lias.

1850. Selys. Rev. odon. (Mem, soc, roy. sc. Liége, vi): 359, note.

See also Libellula hopei, Æschna liassina, etc.

\section{Heterophlebia latreillei. (Odonata.)}

Locality: Solenhofen, Bavaria. Horizon: Jura.

1862. Hagen. Palaeontogr., $x: 139$.

See also Agrion latreillei, Isophlebia helle, etc., Aschna münsteri, etc., Eschna antiqua, etc.

\section{Heterophlebia liassina. (Odonata.)}

Locality : England. Horizon: Lower Lias.

1850. Selys. Rev. odon. (Mém. soc. roy. sc. Liége, vi) : 359, note.

See also-ÆEschna liassina, etc.

974. Heterophlebia phryne. (Odonata.)

Locality : Eichstadt, Bavaria. Horizon: Jura.

1862. Hagen. Palaeontogr., $x: 105$ (undescr.).

See also Calopteryx lithographica, Stenophlebia phryne.

975. Heterophlebia westwoodi. (Odonata.)

Locality: Cheltenham, England. Horizon: Lower Lias.

1845. Brodie. Foss. ins. sec. rocks Engl. (unnamed). Pl. 10, fig. 8.

1850. Hagen. Rev. odon. (Mém. soc. roy. sc. Liége, vi) : 359.

1856. Giebel. Insect. d. vorwelt, 288.

See also Tarsophlebia westwoodi.

976. Hexagenites weyenberghii. (Ephemeridæ.)

Locality : Solenhofen, Bavaria. Horizon: Jura.

1871. Eaton. Trans. ent. soc. Lond., $1871: 158$ (unnamed). Pl. 1, fig. 10.

1880. Scudder. Dev. ins. N. Brunsw., 6-7.

1890. " Foss. ins. N. A., i: 158-159.

Hydropsyche seebachi. See Phryganidium (Hydropsyche) seebachi.

977. Isophlebia aspasia. (Odonata.)

Localities: Solenhofen, Eichstadt, Bavaria. Horizon: Jura.

1866. Hagen. Palaeontogr., xv: 70-75. Pl. 2, fig. 12; pl. 4, figs. 1-3.

1866. " Nenr. lithogr. schief., i : 14-19. Pl. 2, fig. 12; pl. 4, figs. 1-3.

1869. Weyenbergh. Tijdschr. v. ent., (2), iv : 234 [134].

1874. “ $"$ Periód. zool., i: 91.

1874. “ Enum. syst. fanne ent. mésoz., 5.

1886. Deichmüller. Ins. lith. schichten dresd. mus,, 56-57. Pl. 4, figs, 4-6.

See also Anax buchii, ete. 
978. Isophlebia helle. (Odonata.)

Locality : Solenhofen, Bavaria. Horizon: Jura.

1866. Hagen.

1866. “6

1869. Weye

1874.

1885. Scudder.

1886.
Palaeontogr., xv: 76-79. Pl. 1, fig. 1.

Neur. lithogr. schief., i: 20-23. Pl. 1, fig. 1.

See also Heterophlebia helle, Agrion latreillei, etc., Aschna münsteri, etc., Aschna antiqua, etc.

\section{Libella -. (Odonata.)}

Compared with Libella grandis [ Aschna grandis].

Locality: Solenhofen, Bavaria. Horizon: Jura.

1782. Schmidel. Fortges. vorst. merkw. verstein., 36. Pl. 19, fig. 2.

1784. Schröter. Neue litt. u. beytr., i : 410-411.

See also Aschna schmideli, Eschna antiqua, ete.

980. Libellula —. (Odonata.)

Locality : Strensham, England. Horizon : Lower Lias.

1874. Brodie.

Distr. corr. foss. ins., 19 (Libillula).

\section{Libellula —. (Odonata.)}

Locality: Dobbertin, Mecklenburg. Horizon: Lower Jura.

1880. E. Geinitz. Zeitschr, deutsch. geol. gesellsch., 1880: 529. Pl. 22, fig. 16.

982. Libellula —. (Odonata.)

Locality : Dobbertin, Mecklenburg. Horizon : Jura.

1883. E. Geinitz. Flötzform. Mecklenb., 31. Pl. 6, fig. 5.

\section{Libellula —. (Odonata.)}

Locality: Solenhofen, Bavaria. Horizon: Jura.

1844. Mantell. Medals of creation, ii : 574. Lign. 122.

1854. " Medals of creation, 2d ed., ii : 551. Lign. 174.

984. Libellula? _— (larva). (Odonata.)

Locality: Christian Malford, England. Horizon : Oxford clay. 1845. Westwood. Brodie, Foss. ins. sec. rocks, 36, 122. Pl. 4, fig. 13.

985. (Libellula) abscissa. (Odonata.)

Locality: Eichstadt, Bavaria. Horizon: Jura.

1862. Hagen. Palaeontogr. $x: 107$ (undescr.).

1869. Weyenbergh. Tijdschr. v. ent., (2), iv : 236.

See also — abscissa (Odonata, Libellulina).

986. Libellula antiqua. (Odonata.)

Locality : Vale of Wardour, England. Horizon : Purbecks.

1845. Brodie. Foss. ins. sec. rocks Engl., 33. Pl. 5, fig. 10.

1850. Hagen. Rev. odon. (Mém. soc. roy. sc. Liége, vi) : 363.

1856. Giebel. Insect. d. vorwelt, 285.

1886. Deichmüller. Ins. lith. schichten dresd. mus., 34 (= Estemoa bubas 1).

See also Estemoa bubas, etc., Estemoa densa, ete., Blattidium—Goldenb. 
987. Iibellula brevialata. (Odonata.)

Locality: Solenhofen, Bavaria. Horizon: Jura.

18-. Münster. MSS. Mentioned by Hagen, Palaeontogr., x: 106 (1862) as perhaps belonging to Heterophlebia casta. The species has never been pnblished under either name.

See also Heterophlebia casta.

988. Libeliula brodiei. (Odonata.)

Locality : Dumbleton, England. Horizon: Upper Lias.

1845. Brodie. Foss. ins. sec. rocks Engl., 101. Pl. 8, fig. 1.

1845. Westwood. Brodie, Foss. ins. sec. rocks Engl., 101, 127. Pl. 8, fig. 1.

1853. Buckmann. Aun. nat. hist., (2), xii: 436-438.

1856. Giebel. Insect. $d$. vorwelt, $2 \times 5$.

1871. Phillips. Geology of Oxford, 123.

S.e also Aschna brodiei, Gomphus brodiei, Gomphoides brodıei, Libellula (Heterophlebia) brodiei, L. (Asschna) brodiei, — brodiei (Odonata, Libellulina).

\section{Libellula ( Æsschna) brodiei. (Odonata.)}

Locality: Dobbertin, Mecklenburg. Horizon : Lias.

1884. E. Geinitz. Zeitschr. dentsch. geol. gesellsch., 1884: 581.

See also Libellula brodiei, etc.

990. Libellula (Heterophlebia) brodiei. (Odonata.)

Locality : Dumbleton, England. Horizon: Upper Lias.

1873. Brodie. Distr. corr. foss. ins., 15.

See also Libellula brodiei, etc.

991. Iibellula decapitata. (Odonata.)

Locality: England. Horizon: Lower Lias.

1845. Brodie. Foss. ins. sec. rocks Engl. (unnamed). Pl. 9, fig. 1.

1850. Selys. MSS.

1850. Hagen. Rev. odon. (Mém. soc. roy. sc. Liége, vi) : 364.

1856. Giebel. Insect. d. vorwelt, 285-286.

See also — decapitata (Odonata, Libellulina.)

992. (Libellula) densa. (Odonata.)

Locality: Eichstadt, Bavaria. Horizon: Jura.

1862. Hagen. Palaeontogr., $\mathrm{x}: 107$ (undescr.).

1869. Weyenbergh. Tijdschr. v. ent., (2), iv : 235.

See also Estomoa densa, — densa (Odonata, Libellulina), Estemoa bubas, etc.

993. Libellula dislocata. (Odonata.)

Locality: Dumbleton, England. Horizon: Lias.

1871. Phillips. Geology of Oxford, 123.

See also Heterophlebia dislocata, etc.

994. Libellula (Heterophlebia) dislocata. (Odonata.)

Locality: Dumbleton, England. Horizon: Upper Lias.

1848. Brodie. Quart. journ. geol. soc. Lond., 5: 32-35. Pl. 2.

See also Heterophlebia dislocata, etc. 
995. Libellula hopei. (Odonata.)

Locality: Strensham, Worcestershire, England. Horizon : Lower Lias, Insect Limestone.

1845. Brodie. Foss. ins. see. rocks Engl., 71, 102. Pl. 10, fig. 3. 1871. Phillips. Geology of Oxford, 123.

Soe also Petalura hopei, Heterophlebia hopei, Esschna liassina, etc.

996. Libellula jnrassica. (Odonata.)

Locality: Vale of Wardour, England. Horizon: Purbecks.

1845. Brodie. Foss. ins. sec. rocks Engl. (Lindenia sp.). Pl. 5, fig. 9. 1856. Giebel. Insect. d. vorwelt, 284.

See also Lindenia - _ Brodie, etc.

997. Libellula koehleri. (Odonata.)

Locality: Bavaria. Horizon: Jura.

1856. Giebel. Insect. d. vorwelt, 284-285.

See also Uropetala koehleri, etc.

998. Libellula liassica. (Odonata.)

Locality : England. Horizon: Lias.

1871. Phillips. Geology of Oxford, 123.

See also Aschna liassina, etc.

999. Libellula longialata. (Odonata.)

Locality: Solenhofen, Bavaria. Horizon : Jura.

1839. Münster. MSS.

1839. Germar. Verhandl. leop.-carol. akad. naturf., xix: 216-217. Pl. 23, fig. 15.

1862. Hagen. Palaeontogr., $\mathrm{x}: 111$.

1863. Winkler. Catal. syst. coll. pal. mus. Teyl., 423.

See also Cymatophlebia longialata, etc.

1000. (Libellula) nævia. (Odonata.)

Localities: Solenhofen, Eichstadt, Bavaria. Horizon: Jura.

1862. Hagen. Palaeontogr., $x: 107$ (undescr.).

1869. Weyenbergh. Tijdschr. v. ent., (2), iv : 236.

See also — nævia (Odonata, Libellulina).

1001. Libellula petrificata. (Odonata.)

Locality: Vale of Wardour, England. Horizon: Purbecks.

1845. Brodie. Foss. ins. sec. rocks Engl. (Lindenia sp.). Pl. 5, fig. 8.

1856. Giebel. Insect. d. vorwelt, 284 (petrificatus).

See also Lindenia — Brodie, etc.

1002. (Iibellula) valga. (Odonata.)

Localities: Eichstadt, Solenhofen, Bavaria. Horizon: Jura.

1862. Hagen. Palaeontogr., $x: 107$ (undescr.).

1869. Weyenbergh. Tijdschr. v. ent., (2), iv : 236.

1874. “ Period. zool., i: 95.

1874. " " Énum. syst. faune ent. mésoz., 9.

See also — valga (Odonata, Libellulina). 
1003. Libellula westwoodii. (Odonata.)

Locality : Near Oxford, England. Horizon: Stonesfield Slate.

1866. Phillips. Geol. mag., iii : 97-99. Pl. 6, fig. a-c.

1871. “ Geology of Oxford, 173. Digr. 36.

\section{Libellulites - (Odonata.)}

Locality : Solenhofen, Bavaria. Horizon: Jura.

1840. Charpentier. Libell. europ., 170-173. Pl. 48, fig. 1 .

See also Anax buchii, etc.

\section{Libellulium agrias.}

Locality: Durdlestone Bay, England. Horizon : Lower Purbecks.

1854. Westwood. Quart. journ. geol. soc. Lond., x: 387, 393-394. Pl. 15, fig. 4.

1856. Giebel. Insect. $d$. vorwelt, 286.

\section{Libellulium kaupii.}

Locality: Durdlestone Bay, England. Horizon: Lower Purbecks.

1854. Westwood. Quart. journ. geol. soc. Lond., x: 387, 395. Pl. 17, fig. 21.

1856. Giebel. Insect. d. vorwelt, 286.

\section{Libillula —. See Libellula — Brodie.}

1007. Lindenia —. (Odonata.)

Locality : Vale of Wardour, England. Horizon : Purbecks.

1845. Brodie. Foss. ins. sec. rocks Engl., 33. Pl. 5, figs. 8. 9.

See also Libellula petrificata, Gomphus petrificatus, Libellula jurassica.

1008. Lindenia ? koehleri. (Odonata.)

Locality: Bavaria. Horizon: Jura.

1850. Selys. Rev. odon. (Mém. soc. roy. sc. Liége, vi): 366 (koeleri).

See also Uropetala koehleri, etc.

Lindenia koeleri. See Lindenia koehleri. .

1009. Meloe hoernesi. '(Termitina.)

Locality : England. Forizon: Wealden.

1845. Brodie. Foss. ins. sec. rocks Engl. (unnamed). Pl. 2, fig. 12.

1856. Giebel. Insect, $d$, vorwelt, 106.

See also Termes grandøvus, ete.

1010. Mesobætis sibirica. (Ephemeridæ.)

Locality : Ust Balei, Siberia. Horizon: Lias.

1889. $\left\{\begin{array}{l}\text { Brauer-Redt- } \\ \text { enb.-Ganglb. }\end{array}\right.$ Mém. acad. sc. St.-Pétersb., (7), xxxvi, No. 15: 5. Pl. [1],fig. 3.

1011. Mesoleuctra gracilis (nymph). (Perlina.)

Compared with Leuctra fusciventris.

Locality: Ust Balei, Siberia. Horizon: Lias.

1889. \{Brauer-Redt.\} Mém, acad. sc. St.-Pétersb., (7), xxxvi, No. 15: 9-10. Pl. [2], - enb.-Gauglb.\} figs.11a-d. 


\section{Mesonemura maakii. (Perlina.)}

Compared with Tæniopteryx nebulosa.

Locality : Ust Balei, Siberia. Horizon: Lias.

1889. Brauer-Redt- \}Mém. acad. sc. St.-Pétersb., (7), xxxvi, No. 15: 11. Pl. [2], Aenb.-Ganglb. fig. 13.

1013. Mesoneta antiqua. (Ephemeridæ.)

Compared with Chirotonetes.

Locality: Ust Balei, Siberia. Horizon: Lias.

1889. \{Brauer-Redt- $\}$ Mém. acad. sc. St.-Pétersb., (7), xxxvi, No. 15: 4-5. Pl. [1], (enb.-Ganglb.\} fig. $2 a b$.

1014. Mormolucoides articulatus (larva). (Sialina.)

Locality : Turner's Falls, Mass. Horizon: Trias.

1858: Hitcheock. Ichn. N. Engl., 7-8. Pl. 7, figs. 3, 4 .

1858. Dana. Hitchcock, Ichn. N. Engl., 7. Fig. on p. 8.

1867. Scudder. Proc. Bost. soc. nat. hist., xi : 140.

1868. " " Geol. mag., $\nabla: 218-220$.

1885. " Zittel, Handb. palaeont., i, th. ii : 776, 827. Fig. 980.

1886. " "Zittel-Barrois, Traité de pal6́ont., ii : 776,829. Fig. 997.

1886. " Bull. U. S. geol. surv., No. 31: 56, 109.

1886. " Mem. Bost. soc. wat. hist., iii: 431-438. Pl. 45, figs. 1-16. Fig.

1888. Vidal. Ins. $\mathrm{y}$ plantas, 14.

1889. Lesley. Dict. foss. Penns., i: 423-424. Fig.

1890. Scudder. Foss. ins. N. A., i: $323-330 . \quad P l$. 19. Fig. on $p .323$.

See also Palephemera mediæva.

1015. Myrmeleon —- (Hemerobina.)

Locality : England. Horizon: Lias.

1845. Westwood. Brodie, Foss. ins. sec. rocks Engl., 126. Pl. 10, fig. 5.

1016. Myrmeleon? - - (Hemerobina.)

Locality: Binton, England. Horizon : Lower Lias.

1873. Brodie. Distr. corr. foss. ins., 15.

1017. Myrmeleon extinctus. (Hemerobina.)

Locality : Solenhofen, Bavaria. Horizon: Jura.

1869. Weyenbergh. Arch. mus. Teyl, ii : 265, Pl. 35, figs. 16, $16 a$.

1869. " " Ins. foss. calc. lithogr., 19. Pl. 2, figs. 16, 16a.

1869. " " Tijdschr. v. ent., (2), iv : 236.

1874. "

1874. “ Enum. syst. faune ent. mésoz., 11.

Nemoura —- See Phryganidium (Nemoura) -

1018. Nymphes fossilis. (Hemerobina.)

Locality: Eichstadt, Bavaria. Horizon: Jura.

1862. Hagen. Palaeontogr., $x: 108$ (undescr.).

1866. " Stett. entom. zeit., xxvii : 453.

1869 Weyenbergh. Tijdschr. v. ent., (2), iv : 236. 


\section{Orthophlebia - -. (Panorpidæ.)}

Locality : Brown's Wood and Somersetshire, England. Horizon : Lower Lias. 1873. Brodie. Distr. curr. foss. ins., 15.

1020. Orthophlebia - - (Panorpidæ.)

Locality : England. Horizon: Wealden.

1845. Westwood. Brodie, Foss. ins. sec. rocks Engl., 119. Pl. 5, figs. 12, 18

See also Panorpa gracilis, Orthophlebia bifurcata.

1021. Orthophlebia -. (Panorpidæ.)

Locality : England. Horizon : Lower Lias, Insect Limestone.

1845. Westwood. Brodie, Foss ins. sec. rocks Engl., 126. Pl. 8, figs. 3, 5, 6, 14. See also Chauliodes — Brodie, 1845, etc., Orthophlebia longissima.

1022. Orthophlebia bifurcata. (Panorpidæ.)

Locality: Vale of Wardour, England. Horizon : Purbecks.

1845. Brodie. Foss. ins. sec. rocks Engl. (without specific name). Pl. 5, fig. 12. 1856. Giebel. Insect. d. vorwelt, 260.

See also Orthophlebia — Westw.

1023. Orthophlebia communis. (Panorpidæ.)

Localities: Wainlode, Forthampton, Strensham, Cracombe, Bidford, Copt Heath near Knowle, England. Horizon: Lower Lias, Insect Limestone.

1845. Westwood. Brodie, Foss. ins. sec. rocks Engl., 102, $126 . \quad P l .8$, figs. 7, 8, 9. 1852. Bronn. Lethæa geogn., $3^{\mathrm{e}}$ aufl, ii, th. ii: 430 . Pl. $24^{\prime}$, fig. $19^{\prime}$.

1856. Giebel. Insect. d. vorwelt, 260.

1871. Phillips. Geology of Oxford, 123.

1874. Brodie. Quart. journ. geol. soc. Lond., xxx : 748.

1875. " Lower Lias Eatington, etc., 10.

1885. Scudder. Zittel, Handb. palaeont., i, th. ii : 777. Fig. 983.

1886. "Z Zittel-Barrois, Traité de paléont., ii: 777. Fig. 1000.

1024. Orthophlebia furcata. (Panorpidæ.)

Locality : Aust, England. Horizon: Lower Lias.

1845. Brodie. Foss, ins. sec. rocks Engl., 127 (unnamed). Pl. 9, fig. 16.

1856. Giebel. Insect. d. vorwelt, 261.

See also O. (Phryganidium) furcata, Phryganidium minimum.

1025. Orthophlebia (Phryganidium) furcata. (Panorpidæ.)

Locality: Dobbertin, Mecklenburg. Horizon: Lias.

1884. E. Geinitz. Zeitschr. deutsch. geol. gesellsch., 1884: 573. Pl. 13, figs. 5, 6.

See also O. furcata, Phryganidium minimum.

1026. Orthophlebia intermedia. (Panorpidæ.)

Locality: England. Horizon: Lower Lias.

1845. Brodie. Foss. ins. sec. rocks Engl. (allied to Chauliodes). Pl. 10, fig. 10. 1856. Giebel. Insect. d. vorwelt, 261.

See also Chanliodes — Brodie, 1845, etc.

1027. Orthophlebia (Phryganidium) intermedia. (Panorpidæ.)

Locality : Dobbertin, Mecklenburg. Horizon: Lias.

1884. E. Geinitz. Zeitschr. deutsch. geol. gesellsch., 1884: 574-575 (=O. lata, 0. similis). $\quad P l .13$, fig. 8.

Seo also Chauliodes _-_ Brodie, 1845, etc. 
1028. Orthophlebia lata. (Panorpidæ.)

Locality: England. Horizon: Lower Lias.

1845. Brodie. Foss. ins. sec. rocks Engl. (allied to Chauliodes). Pl. 10, fig. 11. 1856. Giebel. Insect. $d$. vorwelt, 261.

See also Chauliodes — Brodie, 1845, etc.

1029. Orthophlebia liasiana. (Panorpidæ.)

Locality : Aust, England. Horizon : Lower Lias.

1845. Brodie. Foss. ins. sec. rocks Engl., 127 (unnamed). Pl. 9, fig. 17.

1856. Giebel. Insect. d. vorwelt, 261.

1030. Orthophlebia longissima. (Panorpidæ.)

Locality : England. Horizon: Lower Lias.

1845. Brodie. Foss. ins. sec. rocks Engl. (without specific name). Pl.8, fig. 5.

1856. Giebel. Insect. d. vorwelt, 260.

See also Orthophlebia —— Brodie, 1845, etc.

\section{Orthophlebia megapolitana. (Panorpidæ.)}

Locality: Dobbertin, Mecklenburg. Horizon: Jura.

1883. E. Geinitz. Flötzform. Mecklenb., 31. Pl. 6, fig. 3.

1884. " Zeitschr. deutsch, geol, gesellsch., 1884: 572-573, Pl. 13, figs. $3,4$.

1032. Orthophlebia minuta. (Panorpidæ.)

Locality: Vale of Wardour, England. Horizon: Pärbecks.

1845. Brodie. $\quad$ Eoss. ins. sec. rocks Eugl. (Corydalis sp.). Pl. 5, fig. 16.

1856. Giebel. Insect. d. vorwelt, 260.

See also Corydalis — , etc.

1033. Orthophlebia parallela. (Panorpidø.)

Locality: England. Horizon: Lower Lias.

1845. Brodie. Foss. ins. sec. rocks Engl, (allied to Chauliodes), Pl. 10,fig. 9. 1856. Giebel. Insect. $d$. vorwelt, 260.

See also Chauliodes — Brodie, 1845, etc.

1034. Orthophlebia parvula. (Panorpidæ.)

Locality: Dobbertin, Mecklenburg. Horizon: Lias.

1884. E. Geinitz. Zeitschr, deutsch. geol. gesellsch., 1884:573-574. Pl. 13, fig. 7.

1035. Orthophlebia similis. (Panorpidæ.)

Locality : England. Horizon: Lower Lias.

1845. Brodie. Foss. ins. sec. rocks Engl. (allied to Chauliodes). Pl. 10, fig. 12. 1856. Giebel. Insect. d. vorwelt, 261.

See also Chauliodes — Brodie, 1845, etc.

1036. Palæophlebia synlestoides. (Odonata.)

Locality: Ust Balei, Siberia. Horizon : Lias.

1889. $\left\{\begin{array}{l}\text { Brauer - Red-\{ } \\ \text { tenb.-Ganglb. }\end{array}\right.$ Mém. acad. sc. St.-Pétersb., (7), xxxvi, No. 15: 6-7. Pl. [1], fig.5. 
1037. Palephemera mediæva. (Sialina.)

Locality : Turner's Falls, Mass. Horizon: Trias.

1862. Hitchcock. Amer. journ. sc., (2), xxxiii : 452.

1871. Packard. Bull. Essex inst., iii : 1.

See also Mormolucoides articulatus.

1038. Palpares? —. (Myrmeleontidæ.)

Locality: Eichstadt, Bavaria. Horizon: Jura.

1886. Deichmüller. Ins. lith. schichten dresd. mus., 59.

\section{Panorpa -. (Panorpidæ.)}

Locality : England. Horizon: Lower Lias. .

1845. Murehison. Geol. Cheltenham, 82. Pl. 9, fig. 6.

\section{Panorpa? - (Panorpidæ.)}

Locality : Durdlestone Bay, England. Horizon: Lower Purbecks.

1854. Westwood. Quart. journ. geol. soc. Lond., $\mathrm{x}: 388$.

1041. Panorpa gracilis. (Panorpidæ.)

Locality: Vale of Wardour, England. Horizon: Purbecks.

1845. Brodie. Foss. ins. sec. rocks Engl. (Orthoplebia sp.). Pl. 5, fig. 18.

1856. Giebel. Insect. d. vorwelt, 258.

See also Orthophlebia — Westw.

1042. Panorpa hartungi. (Panorpidæ.)

Locality : Ust Balei, Siberia. Horizon: Lias.

1889. $\left\{\begin{array}{c}\text { Brauer-Redt- } \\ \text { enb-Ganglb. }\end{array}\right.$ M6m. acad. sc. St.-Pétersb., (7), xxxvi, No. 15: 16. Pl. 2, fig. 20.

1043. Panorpa liassica. (Panorpidæ.)

Compared with P. germanica.

Locality: Wainlode Ciiff, England. Horizon: Lias.

1844. Mantell. Medals of creation, ii : 576. Lign. 123, figs. 1, 2.

1854. " Medals of creation, 2 d ed., ii : 553. Lign. 180, figs. 1, 2.

Panorpidium —. See II, ORthopt, Idem.

1044. Panorpidium tessellatum var.

Locality : Durdlestone Bay, England. Horizon: Lower Purbecks.

1854. Westwood. Quart. journ. geol. soc. Lond., x: 387, 395. Pl. 17, fig. 12.

See also Elcana beyrichi; also II, ORTHopt., Panorpidium tessellatum.

1045. Petalia longialata. (Odonata.)

Localities: Eichstadt, Solenhofen, Bavaria. Horizon: Jura.

1862. Hagen. Palaeontogr., $\mathrm{x}: 106,127-133 . \quad$ Pl. 13, figs. 1, 2.

1869. Weyenbergh. Tijdschr v. ent., (2), iv: 235.

1874. " " Period.zool., i: 93.

1874. " " Énum. syst. faune ent. mésoz., 7.

1885. Scudder. Zittel, Handb. palaeont., i, th. 1i: 775. Fig. 978.

1886. " Zittel-Barrois, Traité de paléont., ii : 775. Fig. 995.

1889. Nicholson. Man. palæont., 3d ed., i : 598. Fig. 448.

See also Cymatophlebia longialata, etc. 
1046. (Petalura) differenz. (Odonata.)

1862. Hagen. Palaeontogr., $\mathrm{x}: 107$ (undescr.).

Locality: Eichstadt, Bavaria. Horizon: Jura.

1869. Weyenbergh. Tijdschr. v. ent., (2), iv : 235 (undeser.).

\section{Petalura eximia. (Odonata.)}

Locality : Solenhofen, Bavaria. Horizon: Jura.

1862. Hagen. Palaeontogr., x: 107 (undescr.).

1869. Weyenbergh. Tijdschr. v. ent., (2), iv : 235.

See also Aschna gigantea, etc.

1048. Petalura gigantea. (Odonata.)

Localities: Eichstadt, Solenhofen, Bavaria. Horizon : Jura. 1862. Hagen. Palaeontogr., $x: 10 \%$ (undeser.).

1874. Weyenbergh. Period. zool., i : 94.

1874. " Enum. syst. faune ent. mésoz., 8.

See also Esschna gigantea, etc.

\section{Petalura hopei. (Odonata.)}

Locality: Cheltenham, England. Horizon: Lias.

1856. Selys. Rev. odon. (Mém. soc. roy. sc. Liége, vi): 359, note. 1859. Hagen. Palaeontogr., viii : 26.

See also Libellula hopei, Aschna liassina, ete

1050. Petalura intermedia. (Odonata.)

Localities: Eichstadt, Solenhofen, Bavaria. Horizon: Jura.

1862. Hagen. Palaeontogr., $x: 107$ (undescr.)

1869. Weyenbergh. Tijdschr. v. ent., (2), iv: 235.

1874. " " Periód. zool, i: 94.

1874. “ Énum. syst. faune ent. mésoz., 8.

See also Aschna gigantea, etc.

1051. Petalura latialata. (Odonata.)

Locality: Eichstadt, Bavaria. Horizon: Jura.

1862. Münster. MSS.

1862. Hagen. Palaeontogr., $x: 107$ (undescr.).

1869. Weyenbergh. Tijdschr. v. ent., (2), iv: 235.

See also Asschna gigantea, etc.

1052. Petalura liassina. (Odonata.)

Locality : England. Horizon : Lower Lias.

1856. Hagen. Rev. odon. (Mém. soc. roy. sc. Liége, vi): 359-360.

1856. Selys. Rev. odon. (Mém. soc. roy. sc. Liége, vi): 359, note.

See also Aschna liassina, etc.

1053. (Petalura) münsteri. (Odonata.)

Localities: Eichstadt, Solenhofen, Bavaria. Horizon: Jura.

1862. Hagen. Palaeontogr., $\mathrm{x}: 107$.

1869. Weyenbergh. Tijdsehr. v. ent., (2), iv: 235.

1874. "

1874. "4 Enum. syst. faune ent. mésoz., 8.

See also Eschna münsteri, etc., Eschna antiqua, etc. 
1054. (Petalura) varia. (Odonata.)

Localities : Eichstadt, Solenhofen, Bavaria. Horizon : Jura.

1862. Hagen. Palaeontogr., $x: 107$ (undescr.).

1869. Weyenbergh. Tijdschr. v. ent., (2), iv : 235.

1874. " " Period zool., i: 95.

1874. " " Ênum. syst. faune ent. mésoz., 9.

See also Uropetala koehleri, etc.

\section{Petalura? wittei. (Odonata.)}

Locality : Bavaria. Horizon: Jura.

1862. Hagen. Palaeontogr, $\mathrm{x}: 133-136$. Pl. 13, fig. 3.

1862. " Palaeontogr., $\mathrm{x}: 136$ (=Eschna mïnsteri Germ.).

See also Eschna wittei, etc., Asschna antiqua, etc.

1056. Philonthus kneri. (Termitina.)

Loeality : Vale of Wardour, England. Horizon: Purbecks.

1845. Brodie. Foss. ins. sec. rock Engl., 32 (unuamed). Pl. 2, fig. 2.

1856. Giebel. Insect. d. vorwelt, 72-73.

1860. Hagen. Linn. entom., xii : 297 (a Termes).

Sэe also Termes grandævus, ete., —— (Staphylinidæ) Brodie, ii: 2.

Phryganæa micacea. See Phryganea micacea.

1057. Phryganea - ('Trichoptera.)

Locality: Durdlestone Bay, England. Horizon: Lower Purbecks.

1854. Westwood. Quart. journ. geol. soc. Lond., x: 390. Pl. 18, figs. 25, 28.

1058. Phryganea micacea (larval case). (Trichoptera.)

Localities: Kounic, Vyšerovic, Bohemia. Horizon: Cretaceous.

1869. Frič.

1884. "

1882. “

1882. “

Arch. naturf. landesdurchf. Böhm., i, th. ii : 188. Pl. 3, fig. 6.

Vesmír, xiii : 205-206. Fig. 6.

Mojs., Beitr. palaeont. Oesterr.-Ung., ii : 7 (Phryganæa). Pl. 2, fig. 8.

Foss. arthr. steink, u. kreidef. Böhmens, 7. Pl. 2, fig. 8.

Phryganeidium pytho. See Phryganidium pytho.

1059. Phryganidium (Nemoura) —- (Trichoptera.)

Locality : Dobbertin, Mecklenburg. Horizon: Lias.

1884. E. Geinitz. Zeitschr. dentsch. geol. gesellsch., 1884: 576. Pl. 13, fig. 11.

1060. Phryganidium balticum. (Trichoptera.)

Locality : Dobbertin, Mecklenburg. Horizon: Lower Jura.

1880. E. Geinitz. Zeitschr. deutsch. geol. gesellsch., 1880:527-528. Pl, 22, fig. 13.

1884. " Zeitschr. deutsch, geol, gesellsch., 1884: 575 .

1061. Phryganidium balticum var. simplex. (Trichoptera.)

Locality : Doubertin, Mecklenburg. Horizon: Lower Jura,

1880. E. Geinitz. Zeitschr. deutsch. geol, gesellsch., $1880: 528 . \quad P l .22, f i g .14$,

Phryganidium furcatum. See Orthoplebia (Phryganidinm) furcata.

hryganidium intermedium. See Orthophlebia (Phryganichium) intermedia 
1062. Phryganidium minimum. (Trichoptera.)

Locality: Dobbertin, Mecklenburg. Horizon: Jura.

1883. E. Geinitz. Flötzform. Mecklenb,,31. Pl. 6, fig. 4.

See also Orthophlebia (Phryganidium) furcata, Orthophlebia furcata.

1063. Phryganidium (Polycentropus) perlæforme. (Trichoptera.,

Locality: Dobbertin, Mecklenbnrg. Horizon: Lias.

1884. E. Geinitz. Zeitschr. deutsch. geol, gesellsch., 1884: 575-576. Pl. 13, fif. 9.

1064. Phryganidium pytho. (Trichoptera.)

Locality: Durdlestone Bay, England. Horizon: Lower Purbecks.

1854. Westwood. Quart. journ. geol. soc. Lond., x: 390, 396 (Phryganeidium). Pl. 18, fig. 31.

1864. Hagen. Phryg. syuops. synom., 73.

1864. " Verhandl. zool.-bot. gesellsch. Wien, 1864: 871.

See also Chimarrha pytho.

1065. Phryganidium (Hydropsyche) seebachi. (Trichoptera.)

Locality : Dobbertin, Mecklenburg. Horizon : Lias.

1884. E. Geinitz. Zeitschr, deutsch, geol. gesellsch., 1884: 576. Pl. 13, fig. 10.

1066. Platyperla platypoda. (Perlina.)

Compared with Nemura variegata.

Locality: Ust Balei, Siberia. Horizon: Lias.

1889. $\left\{\begin{array}{l}\text { Brauer-Redt- } \\ \text { enb.-Ganglb. }\end{array}\right.$ fém. acad. sc. St.-Pétersb., ( 7 ), xxxvi, No. 15: 10-11. Pl. [2],

Polycentropus perlæformis. See Phryganidium (Polycentropus) perlaforme,

Polystcochetes —. See Polystœchotes —.

1067. Polystøchotes —-

Locality : England. Horizon: Lias.

1874. Brodie. Distr. corr. foss. ins., $19^{\circ}$ (Polystœehetes).

1068. Prognatha crassa. (Termitina.)

Locality : Vale of Wardour, England. Horizon: Purbecks.

1845. Brodie.

F'oss. ins. sec. rocks Engl., 32 (unnamed). $\quad$ Pl. 2, fig. 3.

1856. Giebel. Insect. d. vorwelt, 73.

See also Termes grandævus, etc., _- (Staphylinidæ?) Brodie, it: 3.

1069. Protolindenia wittei. (Odonata.)

Locality: Eichstadt, Bavaria. Horizon: Jura.

1896. Deichmüller. Ins, lith. schiehten dresd, mus., 37-42. Pl. 4, figs. 1, 2, 9, 10.

See also ÆEschna wittei, etc., Asschna antiqua, etc.

1070. Rapha liasina. (Ephemeridæ.)

Locality: Strensham, England. Horizon: Lower Lias.

1856. Giebel. Insect. d. vorwelt, 290.

1886. Deichmiuller. Ins. lith. schichten dresd. mus., 17 (ef. Elcana).

See also Ephemera — Brodie, 1845.

Bull. $71-11$ 
1071. Raphidium brephos. (Sialina.)

Locality: Durdlestone Bay, England. Horizon : Lower Purbecks.

1854. Westwood. Quart. journ, geol. soc. Lond., $\mathrm{x}: 395 . \quad P l .17$, fig. 16. See also Ela brephos.

1072. Samarura angusta (larva). (Odonata.)

Locality : Ust Balei, Siberia. Horizon:-Lias.

1889. $\left\{\begin{array}{l}\text { Brauer-Redt- } \\ \text { enb.-Ganglb. }\end{array}\right\}$ Móm. acad. sc. St.-Pétersb., (7), xxxvi, No. 15: $9 . \quad$ Pl. [1], fig. 9.

1073. Samarura gigantea (larva). (Odonata.)

Locality: Ust Balei, Siberia. Horizon: Lias. 1889. $\begin{aligned} & \text { Braner-Redt \} Mém. acad. sc. St.-Pótersb. (7), xxxvi, No. 15: 7-3. Pl. [1], } \\ & \text { enb.-Ganglb. }\end{aligned}$

1074. Samarura minor (larva). (Odonata.)

Locality: Ust Balei, Siberia. Horizon: Lias.

1889. (Brauer-Redt-\} Móm. acad. sc. St.-Pétersb., (7), xxxvi, No. 15: 8. Pl. [1], figs. enb.-Ganglb. $7 a-c$.

1075. Samarura pulla (larva). (Odonata.)

Locality: Ust Balei, Siberia. Horizon: Lias.

1889. $\begin{aligned} & \text { Brauer-Redt- } \\ & \text { enb.-Ganglb. }\end{aligned} \begin{gathered}\text { Mém. acad. sc. St.-Pétersb., (7), xxxvi, No. 15: 9. } \\ 8 b c .\end{gathered}$

1076. Samarura rotundata (larva). (Odonata.)

Locality: Ust Balei, Siberia. Horizon: Lias.

1889. $\left\{\begin{array}{l}\text { Braner-Redt- } \\ \text { enb.-Ganglb. }\end{array}\right.$ Mém. acad. sc. St.-Pétersb., (7), xxxvi, No. 15: 9. Pl. [1], fig. 10.

1077. Sciara prisca. (Ephemeridæ.)

Locality : Solenhofen, Bavaria. Horizon: Jura.

1839. Müuster. MSS.

1द39. Germar. Verhandl. leop.-carol, acad, naturf, xix: 211-212. Pl. 23, fig. 11.

1852. Giebel. Dentschi. petref., 640.

1856. " Insect. d. vorwelt, 230.

1862. Hagen. - Palaeontogr., $x: 111$ (Ephemera).

1877. Assmann, Ber. versamml. deutsch. naturf., I: 192.

See also Ephemera prisca.

Sialium sipylus. See II, ORthoptera, Idem.

1078. Stenophlebia æqualis. (Odonata.)

Isocality : Solenhofen, Bavaria. Horizon: Jura.

1866. Hagen. PaJaeontogr, $\mathrm{xv}: 86-90$. Pl. 1, figs. 2-4.

1866. “ Neur, lithogr. schief., i: 30-34. Pl. 1, figs. 2-4.

1874. Weyenbergh. Períd. zool., i: 92.

1874. " Énum. syst. faune ent. mésoz., 6.

1885. Sendder. Zittel, Handb. palaeont., i, th. ii: 775. Fig. 977.

1886. " Zițtel-Barrois, Traité de paléont., ii: 775. Fig. 994.

1886. Deichmüller. Ins. lith. schichten dresd. mus., 43-45. Pl. 4, fig.8.

See, also.Heterophlebia æanalis. 


\section{Stenophlebia amphitrite. (Odonata.)}

Iocality : Solenhofen, Bavaria. Horizon: Jura.

1866. Hagen. Palaeontogr., $\mathrm{xv}: 83-86 . \quad P l .3$, fig. 1.

1866. " Neur. lithogr. schief., i: 27-30. Pl. 3, fig. 1.

1869. Weyenbergh. Tijdschr. v. ent., (2), iv: 235.

1886. Deichmüller. Ins. lith. schichten dresd. mus., 43.

See also Heterophlebia amphitrite.

1080. Stenophlebia phryne. (Odonata.)

Locality : Solenhofen, Bavaria. Horizon: Jura.

1866. Hagen. Palaeontogr., xv: 91-92. Pl. 1, fig. 5.

1866. " Neur. lithogr. schief., i: 35-36. Pl. 1, fig. 5.

1886. Weyenbergh. Tijdschr. v. ent., (2), iv : 235.

1886. Deichmïller. Ins. lith. schichten dresd. mus., 43-45. Pl. 4, fig. 4.

See also Heterophlebia phryne, etc.

\section{Tarsophlebia eximia. (Odonata.)}

Locality: Solenhofen, Bavaria. Horizon: Jura.

1866. Hagen. Palaeontogr., $\mathrm{xv}:$ 65-68. Pl. 2, figs. 1-6, 11.

1866. " Neur. lithogr. schief., i: 9-12. Pl. 2, figs. 1-6, 11.

1869. Weyenbergh. Tijdschr. v. ent., (2), iv: 235.

1886. Deichmüller. Ins. lith. schichten dresd. mus., 57-58.

See also Heterophlebia eximia, Euphæa longiventris.

\section{Tarsophlebia westwoodi. (Odonata.)}

Locality: Dumbleton, Englaud. Horizon : Lias.

1866. Hagen. Palaeontogr., xv: 65. Pl. 2, fig. 10.

1866. " Neur. Tithogr. schief., i: 9. Pl. 2, fig. 10.

See also Heterophlebia westwoodi.

\section{Termes?}

Locality: Vale of Wardour, England, Horizon: Purbecks.

1845. Westwood. Brodie, Foss. ins. sec. rocks Engl., 116 (or staphylinid?). Pl. 2, fig. 2.

1084. Termes fossilis. (Termitina.)

Locality: Solenhofen, Bavaria. Horizon: Jura.

1874. Wejenbergh. Period. zool., i: 86, 96. Pl. 3, fig. 15.

1874. "

1085. Termes grandævus. (Termitina.)

Locality: Vale of Wardour, England. Horizon: Purbecks.

1845. Brodíe.

Foss. ins. sec. rocks Engl., 33, 119. Pl. 2, fig. 5.

1858. Hagen. Linn. entom., xii : 181-182, 297

1858. " Catal. neuropt. Brit. mus., 25.

See also Termes (Eutermes) grandævus, Meloe hoernsii, Flata haidingeri, Philon. thus kneri, Prognatha crassa, _- Brodie, ii : 2, ii: 3.

1086 Termes (Futermes) grandævus. (Termitina.)

Locality: Vale of Wardour, England. Horizon: Purbecks.

1856. Giebel. Insect. d. vorwelt, 295-296.

See alse Termes grandævus, etc. 
1087. Termes heros. (Termitina.)

Localities: Eichstadt, Solenhofen, Bavaria. Horizon: Jura.

1862. Hagen.

Palaeontogr., x : 107, 114-115. Pl. 15, fig. 1.

1863. Winkler. Catal. syst. coll. pal. mus. Teyl., 424.

1869. Weyenbergh. Tijdschr. v. ent., (2), iv : 236.

1874. " " Perí́d. zool., i: 95.

1874. “ Énum. syst. faune ent. mésoz., 9.

1895. Scudder. Zittel, Handb. palaeont., i, th. ii : 772.

1886. $\because \quad$ Zittel-Barrois, Traité de paléont., ii : 772.

1886. Deichmüller. Ins. lith. schichten dresd. mus.,-29.

1888. Oppenheim. Palaeontogr., xxxiv : 226.

Termes kneri. See Philonthus kneri.

1088. Termes lithophilus. (Termitina.)

Localities: Eichstadt, Solenhofen, Bavaria. Horizon : Jura.

1862. Hagen. Palaeontogr., $x: 107,115$.

1869. Weyenbergh. Tijdschr. v. ent., (2), iv : 236.

18\%4. " " Peri6d. zool., i: 95.

1874. " " Enum. syst. faune ent. mésoz., 9.

1885. Scudder. Zittel, Handb, palaeont, i, th. ii : 772.

1886. " Zittel-Barrois, Traité de paléont., ii : 772.

1886. Deichmüller. Ins. lith. schichten dresd. mus., 29.

1888. Oppenheim. Palaeontogr., xxxiv: 226.

See also Tineites lithophilus.

1089. Termes plagiatus. (Termitiva.)

Locality : Schambelen, Switzerland. Horizon: Lias.

1858. Heer. Hagen, Linn, entom., xii: 181 (undeser.).

1090. Termes troglodytes. (Termitina.)

Locality: Schambelen, Switzerland. Horizon: Lias.

1858. Heer. Hagen, Linn. entom., xii : 181 (undeser.).

1091. Termitidium ignotum. (Termitina.)

Locality: Durdlestone Bay, England. Horizon: Middle Purbecks.

1854. Westwood. Quart. journ. geol, soc. Lond., $\mathrm{x}: 384,394 . \quad$ Pl. 15, fig. 16.

See also Hagla ignota.

1092. Tineites lithophilus. (Termitina.)

Locality: Eichstart, Bavaria. Horizon : Jura.

1842. Germar. Miinst., Beitr. z. petref., v: 88-89. Pl. 9, fig. 8.

1850. Heer. Verhandl. schweiz. gesellsch. ges. naturw., xxxiv: 81 (refers it to Termes).

1850. " Geschichte d. insekt., 4 .

1850. " Nenes jahrb. f. min., 18:0 18 .

1850. " Quart. journ. geol. soc. Lond., vi : 68.

1852. Giebel. Deutschl. petref., 644.

1856. " Insect. d. vorwelt, 192, 497.

1856. Hagen. Berendt, Bernst. befindl. organ. reste vorw., ii, th. i: 48 (refers it to Termes).

1862. “ Palaeontogr, $\mathrm{x}: 113$ (certainly a Termes).

1877. Assmann. Ber. versamml. deutsch. natnrf., i : 192 (a Termes).

1885. Scudder. - Zittel, Handb. palaeont., i, th. ii : 813 (a Termes).

1886. " "Zittel-Barrois, Traité de paléont., ii : 814 (a Termes).

1886. " " Bull. U. S. geol. surv., No. 31:95

See also Termes lithophilus. 
1093. Trichopteridium gracile.

Locality : Dobbertin, Mecklenburg. Horizon : Lower Jura.

1880. E. Geinitz. Zeitschr. deutsch. geol. gesellsch., 1880: 528. Pl. 22, fig. 15.

1884. " Z Zeitschr. deutsch. geol. gesellsch., 1884: 576.

1094. Uropetala koehleri. (Odonata.)

Locality : Eichstadt, Bavaria. Horizon : Jura.

1886. Deichmüller. Ins. lith. schichten dresd. mus., 52-56. Pl. 4, figs. 3, 11, 12.

See also Gomphus koehleri, Lindenia koebleri, Libellula koehleri, Petalura varia Eschna Koehler.

\section{Zalmona brodiei.}

Locality : Vale of Wardour, England. Horizon : Purbecks.

1845. Brodie.

Foss. ins. sec. rocks Engl. (Corydalis sp.). Pl. 5, fig. 13.

1854. Goldenberg. Palaeontogr,, iv (Corydalis). Pl. 6, fig. 4.

1854. " Foss. ins. Saarbr. (Corydalis). Pl. 4, fig. 4

1856. Giebel. Insect. d. vorwelt, 266.

See also Corydalis —, ete.

\section{HEMIPTERA.}

1096.

Localities: Forthampton, Apperley, Wainlode, England. Horizon: Lower Lias. 1873. Brodie.

Distr. corr. foss. ins., 16.

\section{7.}

Locality : Weissig, Germany. Horizon : Lower Lias.

1873. E. Geinitz. Neues jahrb, min., 1873: 694. Pl. 3, fig. 3.

1873. " Verstein. unter. dyas Weiss., 4. Pl. 3, fig. 3.

1098. — (abdomen).

Locality: Dobbertin, Mecklenburg. Horizon: Lower Jura.

1880. E. Geinitz. Zeitschr. deutsch, geol, gesellsch., 1880: 531. Pl. 22, fig. 22.

1099.

Locality : Durdlestone Bay, England. Horizon: Lower Purbecks.

1854. Westwood. Quart. journ. geol, soc. Lond., $\mathrm{x}: 386,395 . \quad P l .17$, fig. 8.

1100.

Locality : Durdlestone Bay, England. Horizon : Lower Purbecks.

1854. Westwood. Quart. jouru. geol. soc. Lond., x: 386, 395. Pl. 17, fig. 8.

1101.

Locality: Durdlestone Bay, England. Horizon: Lower Purbecks.

1854. Westwood. Quart. journ. geol. soc. Lond., x: 386,395. Pl. 17, fig. 18.

1102. (Cercopidæ?)

Locality: Durdlestone Bay, England. Horizon: Lower Purbecks.

1854. Westwood. Quart.journ. geol. soc. Lond., x: 388,393. Pl. 14, fig. 6. 
1103.

(Cimicidæ.)

Locality: Vale of Wardour, England. Horizon: Purbecks.

1845. Brodie. Foss, ins. sec. rocks Engl., 33 (Westw., 120). Pl. 4, fig. 6.

1104. - - (Cimicidæ.)

Locality: Strensham, England. Horizon : Lower Lias.

1815. Brodie. Foss. ins, sec. rocks Engl., 101 (Westw., 123). Pl. 7, fig. 22.

1105.

(Cimicidæ.)

Locality : England. Horizon: Lias.

1871. Phillips. Geology of Oxford, 123.

1106.

(Cimicidæ.)

Locality : Durdlestone Bay, England. Horizon: Middle Purbecks. 1854. Westwood. Quart.journ. geol, soc. Lond., $\mathrm{x}$ : 384, 394. Pl. 15, fig. 25.

1107.

(Cimicidæ, )

Locality : Durdlestone Bay, England. Horizon : Lower Purbecks.

1854. Westwood. Quart. journ. geol. soc. Lond., $\mathrm{x}:$ 387, $395 . \quad$ Pl. 17, fig. 14.

1108.

(Cimicidæ.)

Locality: Durdlestone Bay, England. Horizon: Lower Purbecks. 1854. Westwood. Quart. journ. geol. soc. Lond., x: 390,396. Pl. 18, fig. 4.

1109.

(Cimicidæ.)

Locality: Durdlestone Bay, England. Horizon: Lower Purbecks.

1854. Westwood. Quart. journ. geol. soc. Lond., $\mathrm{x}: 389,396$ (or Coleoptera). $P l$. $18, f i g .11$.

1110. (Cimicidæ or Homoptera.)

Locality : Durdlestone Bay, England. Horizon : Lower Purbecks. 1854. Westwood. Quart. journ. geol. soc. Lond., $\mathrm{x}: 389,396 . \quad P l .18$, fig. 17.

1111.

Locality : Durdlestone Bay, England. Horizon: Lower Purbecks.

1854. Westwood. Quart. journ. geol. soc. Lond., x: 390,396. Pl.18, fig. 29.

11 i2.

Locality : Hasfield, England. Horizon : Lower Lias.

1845. Brodie Foss. ins. sec. rocks Engl., 101 (Westw., 127). Pl. 7, fig. 15.

1113.

\section{(Homoptera.)}

Locality: Hasfield, England. Horizon: Lower Lias.

1845. Brodie. Foss. ins. sec. rocks Engl., 101 (Westw., 127). Pl. 7, fig. 21.

1114. (larva). (Homoptera.)

Locality : Strensham, England. Horizon : Lower Lias.

1873. Brodie. Distr. corr. foss. ins, 16.

1115. (Homoptera.)

Locality : Binton, England. Horizon : Lower Lias.

1873. Brodie, 
1116.

(Homoptera.)

Locality : England. Horizon: Lias.

1871. Phillips. Geology of Oxford, 123.

1117.

(Homoptera.)

Locality: Durdlestone Bay, England. Horizon: Lower Purbecks.

1854. Westwood. Quart. journ, geol. soc. Lond., $x$ : 387, 395. Pl. 17, fig. 11.

1118.

(Homoptera or Cimicidæ.)

Locality : Durdlestone Bay, England. Horizon: Lower Purbecks.

1854. Westwood. Quart. journ. geol. soc. Lond., $\mathrm{x}: 389,396 . \quad P l .18$, fig. 17.

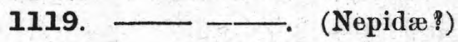

Locality: Durdlestone Bay, England. Horizon : Middle Purbecks.

1854. Westwood. Quart. journ. geol. soc. Lond., x: 384.

1120. (Nepidæ.)

Locality : Ridgway, England. Horizon: Lower Purbecks.

1854. Westwood. Quart. journ. geol. soc. Lond, x: 384, 396. Pl. 18, fig. 14.

1121. (Pentatomidæ.)

Locality : England. Horizon: Lias.

1874. Brodie. Distr. corr. foss. ins., 19.

1122. (Pentatomidæ?)

Locality : England. Horizon: Lias.

1854. Westwood. Quart. journ. geol. soc. Lond., x: 381, 396. Pl. 18, fig. 2.

(Head of larva of Odonata.-S.H.S.)

Actea sphinx. See II, Coleoptera, Idem.

1123. Aphis (Aphidæ.)

Locality : England. Horizon: Wealden.

1845. Westwood. Brodie, Foss, ins, sec. rocks Engl., 120. Pl: 2, fig. 9. See also Aphis dubia.

1124. Aphis dubia. (Aphidæ.)

Locality: England. Horizon: Wealden.

1856. Giebel. Insect. d. vorwelt, 389.

See also Aphis - Westwood.

1125. Aphis plana. (Aphidæ.)

Locality: Vale of Wardour, England. Horizon: Purbecks.

1845. Brodie.

F'oss. ins. sec. rocks Engl., 33, 120. Pl. 2, fig. 10.

1856. Giebel.

Insect. d. vorwelt, 389 .

1126. Aphis valdensis. (Aphidæ.)

Locality: Vale of Wardour, England. Horizon: Purbecks.

1845. Brodie.

1856. Giebel.

1883. Buckton.

1883. Seudder

1886. " "

1886. "
Foss. ins. sec. rocks Engl., 33, 120. Pl. 4, fig. 3.

Insect. d. vorwelt, 388-389.

Monogr. Brit. aphid., iv: 148. Pl. 132, figs. 4, 5.

Zittel, Handb. palaeont., i, th. ii : 780. Fig. 987.

Zittel-Barrois, Traité de paléont., ii : 780. Fig. 1004.

Bull. U. S. greol. surv., No. 31 : 59 . 
1127. Asira egertoni. (Fulgoridæ.)

Locality : Vale of Wardour, England. Horizon: Purbecks.

1856. Giebel. Insect. $d$. vorwelt, 377 (Brodie, iv: 7 ).

See also Asiraca egertoni, Asira kenngotti.

1128. Asira kenngotti. (Fulgoridæ.)

Locality: Vale of Wardonr, England. Horizon: Purbecks.

1856. Giebel.

Insect. d. vorwelt, 377 (Brodie, iv: 8).

See also Asiraca egertoni, Asira egertoni.

1129. Asiraca egertoni. (Fulgoridæ.)

Locality: Vale of Wardour, England. Horizon: Purbecks.

1845. Brodie. Foss. ins. sec. rocks Engl., 33, 120. Pl. 4, figs. 7, 8.

See also Asira kenngotti, A. egertoni.

1130. Belostoma —. (Belostomatidæ.)

1873. Brodie. Distr. corr. foss. ins., 16.

Horizon : Lower Lias.

\section{Belostoma ㄴ. (Belostomatidæ.).}

Locality : England. Horizon: Lias.

1845. Westwood. Brodie, Foss. ins. sec. rocks Engl., 127. Pl. 10, fig. 13.

See also Belostoma liasina.

1132. Belostoma deperdita. (Belostomatidæ.)

Locality: Solenhofen, Bavaria. Horizon: Jura.

1877. Assmann. Ber. versamml. deutsch. naturf., i: 192 (Belostomum).

1886. Deichmüller. Ins. lith. schichten dresd. mus., 61-63. $P l .5$, figs. 1-3.

1888. Oppenheim. Palaeontogr., xxxiv: 233-234. Pl. 31, fig. 3.

See also Belostoma hartingi, Scarabæus deperditus, etc., Scarabæides deperditus, Hyd̀rophilus deperditus.

Belostoma elongata. See II, Hymenoptera, Idem.

1133. Belostoma hartingi. (Belostomatidæ.)

Locality: Solenhofen, Bavaria. Horizon: Jura.

1869. Weyenbergh. Arch. mus. Teyl., ii : 268-270 (Belostomum). Pl. 35, fig. 20.

1869. " " Ins. foss. calc. lithogr., 22-24. Pl. 2, fig. 20.

1869. " " Tijdschr. v. ent., (2), iv: 233 [133].

1874. " " Periód. zool., i: 83, 100.

1874. " "Énum. syst. fanne ent. mésoz., 14.

See also Belostoma deperdita, etc.

1134. Belostoma liasina. (Belostomatidæ.)

Locality : England. Horizon: Lias.

1856. Giebel. Insect. d. vorwelt, 371 (Belostomum).

See also Belostoma — Westwood.

Belostoma schroeteri. See II, Hymenoptera, Idem.

Belostomum deperditum. See Belostoma deperdita.

Belostomum hartingi. See Belostoma hartingi.

Belostomum liasinum. See Belostoma liasina. 


\section{Cercopidium -. (Cercopidæ.)}

Locality : Dobbertin, Mecklenburg. Horizon: Jura.

1883. E. Geinitz. Flötzform. Mecklenb., 32 (name only).

1136. Cercopidium hahnii. (Cercopidæ.)

Locality: Durdlestone Bay, England. Horizon : Lower Purbecks. 1854. Westwood. Quart. journ. geol. soc. Lond., X: 390, 396. Pl. 18, fig. 3. 1856. Giebel.

Insect. d. vorwelt, 388 .

1137. Cercopidium heeri. (Cercopidæ.)

Locality: Dobbertin, Mecklenburg. Horizon: Lower Jura.

1880. E. Geinitz. Zeitschr. deutsch. geol. gesellsch., 1880: 529. Pl. 22, fig. 17. 1885. Sendder. Zittel, Handb. palaeont., i, th, ii: 781 . Fig. 991.

1886. " Zittel-Barrois, Traité de palént., ii : 781. Fig. 1008.

See also Cercopis heeri.

1138. Cercopidium mimas. (Cercopidæ.)

Locality: Durdlestone Bay, England. Horizon : Lower Purbecks.

1854. Westwood. Quart. journ. geol. soc. Lond., x: 390, 393-394. Pl. 15, fig. 3. See also Cercopis mimas.

\section{Cercopidium minutum. (Cercopidæ.)}

Locality: Schambelen, Switzerland. Horizon: Lias.

1865. Heer. Urwelt der Schweiz, 91. Pl. 8, fig. 29.

1872. " Monde prim. Suisse, 111. Pl. 8, fig. 29.

1876. " Prim. world Switz., i: 91. Pl. 8, fig. 29.

1879. “ Urwelt der Schweiz, $2^{\mathrm{e}}$ aufl., 101. Pl. 8, fig. 29.

1140. Cercopidium morio. (Cercopidæ.)

Locality: Schambelen, Switzerland. Horizon: Lias.

1865. Heer.

1872. "6

1876. "6

1879. "6
Urwelt der Schweiz, 91. Pl. 8, fig. 30.

Monde prim. Suisse, 111. Pl. 8, fig. 30.

Prim. world Switz., i: 91 . $P l .8, f i g .30$.

Urwelt der Schweiz, 2e aufl., 101. Pl. 8, fig. 30.

1141. Cercopidium schaefferi. (Cercopidæ.)

Locality: Durdlestone Bay, England. Horizon: Lower Purbecks. 1854. Westwood. Quart. jonrn. geol. soc. Lond., x: 390, 396. Pl. 18, fig. 5. See also Cercopis schaefferi.

\section{Cercopidium signoretii. (Cercopidæ.)}

Locality: Durdlestone Bay, England. Horizon: Lower Purbecks.

1854. Westwood. Quart. journ. geol. soc. Lond., x: 390, 396. Pl. 18, fig. 36. See also Cercopis signoretii.

\section{Cercopidium telesphorus. (Cercopidæ.)}

Locality : Durdlestone Bay, England. Horizon: Middle Purbecks. 1854. Westwood. Quart. journ. geol, soc. Lond., x : 384, 394. Pl. 15, fig. 14**. 1886. Scudder. Mem. Bost. soc. nat. hist., iii : 451. Pl. 48, figs. 8 e, 13. 1890. " Foss. ins. N. A., i: 343. Pl. 22, figs. 8e, 13.

See also Cercopis telesphorus. 
1144. Cercopidium trigonale. (Cercopidæ.)

Locality : Durdlestone Bay, England. Horizon: Lower Purbecks. 1854. Westwood. Quart.journ. geol. soc. Lond., $\mathrm{x}: 396$ (unnamed). $P l .18$, fig. 4. 1856. Giebel.

Insect. d. vorwelt, 388 .

1145. Cercopis —. (Cercopidæ.)

Locality: Vale of Wardour, England. Horizon: Purbecks.

1845. Brodie. Foss. ins. sec. rocks Engil, 33. Pl. 2, fig. $12 ; p l .4, f i g .9$.

1856. Giebel. Insect. d. vorwelt, 382 .

1146. Cercopis heeri. (Cercopidæ.)

Locality: Dobbertin, Mecklenburg. Horizon : Lower Jura.

1884. E. Geinitz. Zeitschr. deutsch. gêol. gesellsch., 1884: 581.

See also Cercopidium heeri.

1147. Cercopis jurassica. (Cercopidæ.)

Locality: Dobbertin, Mecklenburg. Horizon: Lias.

1884. E. Geinitz. Zeitschr. deutsch. geol, gesellsch., 1884: 581-582. Pl. 13, fig. 25.

1148. Cercopis mimas. (Cercopidæ.)

Locality : England. Horizon: Lower Purbecks.

1856. Giebel. Insect. d. vorwelt, 379.

See also Cercopidium mimas.

1149. Cercopis prisca. (Cercopidæ.)

Locality: Solenhofen, Bavaria. Horizon: Jura.

1874. Weyenbergh. Perí6d. zool., i: 86. Pl. 3, fig. 10.

See also Cicada prisca.

1150. Cercopis schaefferi. (Cercopidæ.)

Locality : Durdlestone Bay, England. Horizon : Lower Purbecks.

1856. Giebel. Insect. $d$. vorwelt, 379.

See also Cercopidinm schaefferi.

1151. Cercopis signoretii. (Cercopidæ.)

Locality: Durdlestone Bay, England. Horizon : Lower Purbecks. 1856. Giebel. Insect. d. vorwelt, 379-380.

See also Cercopidium signoretii.

1152. Cercopis telesphorus. (Cercopidæ.)

Locality: Durdlestone Bay, England. Horizon: Middle Purbecks. 1856. Giebel. - Insect. d. vorwelt, 379.

See also Cercopidium telesphorus.

1153. Cercopyllis adolescens. (Cercopidæ.)

Locality: Fairplay, Colo. Horizon: Trias.

1890. Scudder. Mem. Bost. soc. nat. hist., iv : 472. Pl. 42, fig. 12.

1890. " Foss, ins. N. A., i : 448. Pl. 34, fig. 12. 
1154. Cercopyllis delicatula. (Cercopidæ.)

Locality: Fairplay, Colo. Horizon: Trias.

1890. Scndder. Mem. Bost. soc. nat. hist., iv: 471. Pl. 42, fig. 11.

1890. " Foss, ins. N. A., i: 447. Pl. 34, fig. 11.

1155. Cercopyllis justiciæ. (Cercopidæ.)

Locality: Fairplay, Colo. Horizon: Trias.

1890. Scndder. Mem. Bost. soc. nat. hist., iv: 471 . Pl. 42 , fig. 6.

1890. " Foss. ins. N. A., i : 447. Pl. 34, fig. 6.

1156. Cicada - (pnpa). (Stridulantia.)

Locality : Strensham, England. Horizon: Lower Lias.

1873. Brodie. Distr. corr. foss. ins., 8,16 .

\section{Cicada -}

Locality: Strensham, England. Horizon: Lower Lias.

1873. Brodie. Distr. corr. foss. ins., 16 ("like Meloe"!).

1158. Cicada

Locality : England. Horizon: Lias.

1845. Westwood. Brodie, Foss. ins. sec. rocks Engl., 127. Pl. 10, fig. 7.

Cicada dubia. See If, Orthoptera, Gryllites dubius.

1159. Cicada gigantea. (Stridulantia.)

Locality : Solenhofen, Bavaria. Horizon: Jura.

1874. Weyenbergh. Periód. zool., i : 85, 101. Pl. 3, fig. 4.

1874. " Énum. syst. faune ent. mésoz., 15.

1886. Deichmïller. Ins. lith. schichten dresd. mus., 60.

See also Eocicada microcephala.

1160. Cicada murchisoni.

Locality: Hasfield, England. Horizon: Lower Lias.

1845. Brodie. Foss. ins. sec. rocks Engl., 101, 127. Pl. 7, fig. 20.

1856. Giebel. Insect. $d$. vorwelt, 373-374.

1871. Phillips. Geology of Oxford, 123.

1161. Cicada prisca. (Cercopidø.)

Locality : Solenhofen, Bavaria. Horizon: Jura.

1874. Weyenbergh. Periód. zool., i: 101.

1874. " "Enum. syst. faune ent. mésoz., 15.

See also Cercopis prisca.

\section{Cicada proserpina. (Stridulantia.)}

Locality : Solenhofen, Bavaria. Horizon: Jura.

1874. Weyenbergh. Periód. zool., i: 86, 100. Pl. 3, fig. 9.

1974. " Emum. syst. faune ent. mésoz., 14.

1886. Deichmüller. Ins. lith. schichten dresd. mus., 60 .

1163. Cicada psocus. (Cercopidæ.)

Locality : Durdlestone Bay, England. Horizon : Middle Purbecks.

1856. Giebel. Insect. d. vorwelt, 374.

See also Cicadellium psocus. 
1164. Cicada punctata. (Cicadellina.)

Locality: Vale of Wardour, England. Horizon: Purbecks.

1845. Brodie.

Foss. ins. sec. rocks Engl., 33, 120. Pl. 5, fig. 4.

1856. Giebel.

Insect. d. vorwelt, 374 .

1165. Cicadellium dipsas. (Cercopidæ.)

Locality: Durdlestone Bay, England. Horizon: Middle Purbecks.

1854. Westwood. Quart. journ. geol. soc. Lond., $\mathrm{x}: 383,393-394$. Pl. 15, fig. 6, upper left-hand fig.

1166. Cicadellium psocus. (Cercopidæ.)

Locality: Durdlestone Bay, England. Horizon: Middle Purbecks.

1854. Westwood. Quart. journ. geol. soc. Lond., x: 384, 394. Pl. 15, fig. 18. See also Cicada psocus.

1167. Cicadinella (Cercopidæ.)

Locality : Dobbertin, Mecklenburg. Horizon : Lias.

1884. E. Geinitz. Zeitschr. dentsch. geol. gesellsch., 1884: 582 .

1168. Cimex ㄴ. (Cimicidæ.)

Locality: Strensham, England. Horizon: Lower Lias.

1873. Brodie. Distr. corr. foss. ins., 16.

1169. Cimicidium dallasii. (Lyyæidæ.)

Locality: Durdlestone Bay, England. Horizon: Lower Purbecks.

1854. Westwood. Quart. journ. geol. soc. Lond., x: 387,395. Pl. 17, fig. 15.

Seo all Lygæites dallasii.

Cixia maculata. See Cixius macnlatus.

1170. Cixius maculatus. (Fulgoridæ.)

Locality: Vale of Wardour, England. Horizon: Purbecks.

1845. Brodie. Foss. ins. sec. rocks Engl., 33, 120. Pl. 2, fig. 8.

1856. Giebel. Insect. d. vorwelt, 377 (Cixia).

1171. Corixa? - (Notonectidæ.)

Locality : Eichstadt, Bavaria. Horizon: Jura.

1888. Oppenheim. Palaeontogr., xxxiv: 235. Pl. 30, fig. 15.

1172. Corixa mortua. (Notonectidæ.)

Locality: Solenhofen, Bavaria. Horizon: Jura.

1869. Wejenbergh. Arch. mus. Teyl., ii : 268. Pl. 35, figs. 18, $18 a$.

1869.

1869. " " Tijdsehr. v. ent., (2), iv: 233 [133].

1874. " " $\quad$ Períd. zool., i: 99.

1874. 66 Enum. syst. faune ent. mésoz., 13.

1173. Cyclocoris pinguis. (Coreidæ.)

Locality : Schambelen, Switzerland. Horizon : Lias.

1865. Heer. Urwelt der Schweiz, 91. Pl. 8, fig. 27.

1872. " Monde prim. Suisse, 111. Pl. 8, fig. 27.

18i6. " $\quad$ Prim. world Switz., i: 91. Pl. 8, fig. 27.

1879. " Urwelt der Schweiz, $2^{\mathrm{e}}$ aufl., 101. Pl. 8, fig. 27. 
1174. Delphax pulcher. (Fulgoridæ.)

Locality: Vale of Wardour, England. Horizon: Purbecks,

1845. Brodie. Foss. ins, sec. rocks Engl., 33, 120. Pl. 5, fig. 17.

1850. Giebel. Insect. d. vorwelt, 378.

Ditomoptera dubia. See II, Coleoprera, Idem.

1175. Eocicada microcephala. (Stridulantia.)

Locality: Eichstadt, Bavaria. Horizon: Jura.

1888. Oppenheim. Palaeontogr., xxxiv: 229. Pl. 31, fig. 30.

See also Cicada gigantea.

Flata haidingeri. See II, Neuroptera, Idem.

Hagenia schroeteri. See II, Hymknoptera, Idem.

Halometra gigantea. See II, OrThopterA, Idem.

Halometra minor. See II, Orthoptera, Idem.

1176. Hydrometra -. (Hydrometridæ.)

Locality: Vale of Wardour, England. Horizon: Purbecks.

1845. Brodie. Foss. ins. sec. rocks Engl., 33 (Westwood, 121).

1177. Hydrophilus deperditus. (Belostomatidæ.)

Locality: Solenhofen, Bavaria. Horizon: Jura.

1874. Weyenbergh. Period. zool., i : 83-84.

See also Belostoma deperdita, etc.

1178. Ischyopteron suprajurense. (Geocorisa.)

Locality: Eichstadt, Bavaria. Horizon: Jura.

1888. Oppenheim. Palaeontogr., xxxiv: 230. Pl.30, fig. 7.

1179. Kleidocerys or Pachymerus

Locality : Vale of Wardour, England. Horizon: Purbecks.

1845. Brodie. Foss. ins. sec. rocks Engl., 33, 120. Pl. 2, fig. 11.

1180. Lygæites dallasii. (Lygæidæ.)

Locality : Durdlestone Bay, England, Horizon: Lower Purbecks.

1856. Giebel.

Insect. d. vorwelt, 360 .

See also Cimicidium dallasii.

1181. Lygæites furcatus. (Lygæidæ.)

Locality: Durdlestone Bay, England. Horizon: Lower Purbecks.

1854. Westwood. Quart. journ. geol, soc. Lond., $\mathrm{x}: 395$ (unnamed). Pl. 17, fig. 14. 1856. Giebel. Insect. $d$, vorwelt, 360.

1182. Lygæites priscus. (Lygæidæ.)

Locality: Durdlestone Bay, England. Horizon: Lower Purbecks.

1854. Westwood. Quart. journ. geol. soc. Lond., $\mathrm{x}$ : 396 (unnamed). Pl.18, fig. 11.

1856. Giebel. Insect. d. vorwelt, 360. 
1183. Iystra vollenhovenii. (Fulgoridæ.)

Locality: Solenhofen, Bavaria. Horizon: Jura.

1869. Weyenbergh. Arch. mus. Teyl., ii : 271-272. Pl. 36, fig. 24.

1869. " " Ins. foss. calc. lithogr., 25-26. Pl. 3, fig. 24.

1869. " " Tijdschr. v, ent., (2), iv: 234 [134].

1874. " " Peribd.zool., i: 100.

1874. “ Énum. syst. faune ent. mésoz., 14.

\section{Molobius}

Locality: England. Horizon: Lower Parbecks.

1873. Brodie. Distr. corr. foss. ins., 14.

(I do not find any hemipterous genus of this name.)

1185. Naucoris carinata. (Naucoridæ.)

Locality: Eichstadt, Bavaria. Horizon: Jura.

1388. Oppenheim. Palaeontogr., xxxiv: 235. Pl. $30, \mathrm{fig} .14$.

1186. Naucoris lapidarius. (Naucoridæ.)

Locality: Solenhofen, Bavaria. Horizon: Jura.

1869. Weyenbergh. Arch. mus. Teyl, ii : 267-268. Pl. 35, figs. 19, $19 a$.

1869. " Ins. foss. calc. lithogr., 21-22. Pl. 2, figs. 19, 19a.

1869. " " Tijdschr. v. ent., (2), iv: 233 [133].

1874. " " Periód. zool., i: 99.

1874. " " Enum. syst. faune ent. mésoz., 13.

1885. Scudder. Zittel, Handb. palaeont., i, ii: 782.

1886. " Zittel-Barrois, Traité de paléont., ii: 782.

1886. Deichmüller. Ins. lith. schichten dresd. mus., 63-64. Pl. 5, fig. 6.

1888. Oppenheim. Palaeontogr, xxxiv: 234. Pl. 30, fig. 16.

1187. Nepa primordialis. (Nepidæ.)

Locality : Solenhofen, Bavaria. Horizon: Jura.

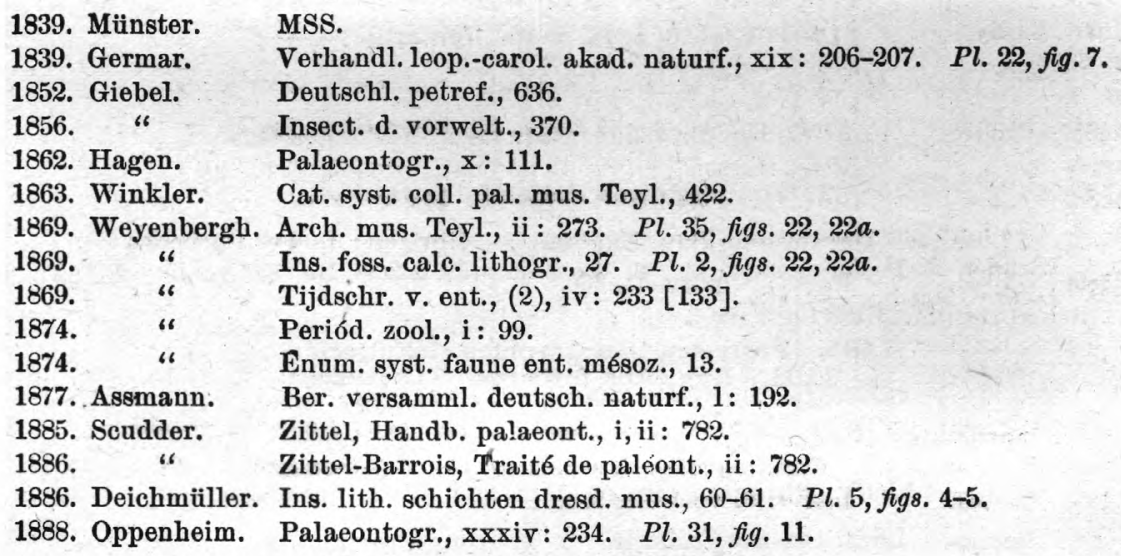

1188. Nepidium stolones. (Nepidæ.)

Locality : Ridgway, England. Horizon: Lower Purbecks.

1854. Westwood. Quart.journ. geol.soc. Lond, $x: 384,396 . \quad P l .18$, fig.9. 
1189. Neurocoris - (Pentatomidæ.)

Locality : Durdlestone Bay, England. Horizon: Lower Purbecks.

1854. Westwood. Quart. journ. geol, soc. Lond., x: 395 (unnamed). Pl. 17, fig. 11. 1856. Giebel.

Insect. d. vorwelt, 339 .

1190. Notonecta elterleini. (Notonectidæ.).

Locality: Eichstadt, Bavaria. Horizon: Jura.

1886. Deichmüller. Ins. lith. schichten dresd. mus., 64. Pl. 5, fig. 7.

Pachymeria —. See Pachymerus —.

1191. Pachymeridium dubium. (Lygæidæ.)

Locality: Dobbertin, Mecklenburg. Horizon : Lower Jura.

1880. E. Geinitz. Zeitsebr. deutsch. geol. gesellsch., 1880: 529-530. Pl. 22, fig. 18. 1884. " Zeitschr. dentsch. geol. gesellsch., 1884: 582.

1192. Pachymerus (or Kleidocerys) ——. (Lygæidæ.)

Locality: Vale of Wardour, England. Horizon: Purbecks.

1845. Brodie. Foss. ins. sec. rocks Engl., 33, 120 (Pachymeria), Pl. 2, fig. 11.

1193. Pachymerus zucholdi. (Lygæidæ.)

Locality: Strensham, England. Horizon: Lower Lias.

1845. Brodie.

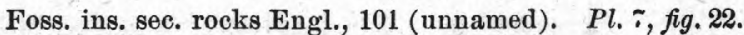

1856. Giebel. Insect. $d$. vorwelt, 356.

1194. Palæontina oolitica. (Stridulantia.)

Locality: Stonesfield, England. Horizon: Stonesfield Slate.

1873. Butler. Lepid. exot., $x v:$ 126-127. $P l .48$, figs. 1-2

1873. " Geol. mag., $\mathrm{x}: 2,3$. Pl. 1; figs. 1,2.

1873. Brodie. Distr. corr. foss. ins., 8 (unnamed).

1874. " Distr. corr. foss, ins., 8-9 (unnamed).

1874. Butler. Geol. mag., (2), i: 446-449. Pl. 19.

1874. Scudder. Proc. Bost. soc. nat. hist., xvi: 112 (unnamed).

1875. " Foss. butt., 89-95. Figs. 4-8.

1877. Kirby. Syn. cat. diurn. Lep., suppl., 717.

1879. Goss. Proc. geol. assoc., vi: $12 \%$.

1879. “ Ins. fauna mésoz. per., ii: 14.

1885. Scudder. Zittel, Handb. palaeont., i, th, ii : 782. Fig. 993.

1886. " "Zittel-Barrois, Traité de paléont., ii : 782. Fig. 1010.

1886. “ Bull. U. S. geol. surv., No. 31:61.

1886. Deichmüller. Ins. lith. schichten dresd. mus., 60.

1889. \{Brauer-Redt-\} Mém. acad. sc. St.-Pétersb., (7), xxxvi, No. 15: 14-15. Pl. [2], \{ enb.-Ganglb.\} fig. 18a.

\section{Prolystra lithographica. (Fulgoridæ.)}

Locality : Eichstadt, Bavaria. Horizon: Jura.

1888. Oppenheim. Palaeontogr., xxxiv: 228. Pl. 31, fig. 1.

\section{Phragmatcoites damesii. (Stridulantia.)}

Locality: East Siberia. Horizon: Brown Jura.

1885. Oppenheim. Berl. entom. zeitschr., xxix: 333. Pl. 10, figs. 2, 3.

1886. Sendder. Zittel-Barrois, Traité de paléont., ii : 814, note.

1886. " Bull. U. S. geol, surv., No. 31: 96 .

1889. \{Brauer-Redt-\} Mém. acad. sc. St.-Pétersb., (7), xxxvi : No. 15,15-16. Pl., [2], 1889. $\{$ enb.-Gangl. $\}$ fig, 19. 
Propygolampis bronni. See II, Orthopt, Idem.

1197. Protocoris insignis. (Coreidæ.)

Locality: Schambelen, Switzerland, Horizon: Lias.

18i5. Heer.

1ั่ว. " "

1876. " "

1879. " "

1885. Seudder.

1836. " "
Urwelt der Schweiz, 91. Pl. 8, fig. 28.

Monde prim. Suisse, 111. $P l .8$, fig. 28.

Prim. world Switz., i: 91. Pl. 8, fig. 28.

Urwelt der Schweiz, 2e aufl., 101. Pl. 8, fig. 28.

Zittel, Handb. palaeont., i, th. ii : 785. Fig. 1001.

Zititel-Barrois, Traité de paléont., ii : 785. Fig. 1018.

1198. Protocoris ovalis. (Coreidæ.)

Locality: Schambelen, Switzerland. Horizon: Lias.

1865. Heer.

1872. "

18:6. " "

1879. “

1852. Heer.

1856. Giebel.

Urwelt der Schweiz, 91.

Monde prim. Suisse, 111.

Prim. world Switz., i: 91.

Urwelt der Schweiz, $2^{\mathrm{e}}$ aufl., 101.

1199. Protocoris planus. (Coreidæ.)

Locality: Aargau, Switzerland. Horizon: Lias.

Lias-insel Aargan, 15. Pl., figs. 44-45.

Insect. d. vorwelt, 498.

Pygolampis gigantea. See II, ORTHOPT., Idem.

1200. Ricania fulgens. (Fulgoridæ.)

Locality: Vale of Wardour, England. Horizon: Purbecks.

1845. Brodie. Foss. ins. sec. rocks Engl., 33, 120. Pl. 4, fig. 12.

1856. Giebel. Insect. $d$, vorwelt, 376.

Ricania gigas. See II, ORThOPT, Idem.

Ricania hospes. See II, ORthopt, Idem.

1201. Scarabæides deperditus. (Belostomatidæ.)

Locality: Solenhofen, Bavaria. Horizon: Jura:

1839. Germar. Verhandl. leop.-carol. akad. naturf., xix : 218-220. Pl. 23, fig. 17.

1852. Quenstedt. Handb. petref., 313. Pl. 24, fig. 5.

1856. Giebel. Insect. d. vorwelt, 36.

1862. Hagen. Palaeontogr., $x: 110$ (not a beetle, resembles Belostoma).

1869. Weyenbergh. Tijdschr. v. ent., (2), iv : 233 [133], (Scarabæoides).

1885. Scudder. Zittel, Handb. palaeont, i, th. ii: 782, 802. Fig.994.

1886. " "Zittel-Barrois, Traité de paléont., ii : 7ð̧. Fig. 1011.

1886. “ Bull. U. S. geol. surv., No. $31: 61,73,82$.

See also Belostoma deperdita, etc.

Scarabæoides deperditus. See Scarabæides deperditus.

1202. Scarabæus deperditus. (Belostomatidæ.)

Locality : Solenhofen, Bavaria. Horizon: Jura.

1873. Weyenbergh. Arch. mus. Teyl., iii : 239-240.

1873. " "Notes ins. calc. jurass., 6-7.

1874. " " Periód. zool., i: 103.

1874. " Énum. syst. faune ent. mésoz., 17.

See also Belostoma deperdita, etc. 
1203. Sphærodema jurassicum.

Locality: Eichstadt, Bavaria. Horizon: Jura.

1888. Oppenheim. Palaeontogr., xxxiv: 235. Pl. 31, fig. 10.

1204. Tettigonia -. (Jassides.)

Locality: Belvaux, Luxembourg. Horizon: Bathonian.

1875. Borre. Comptes rend. soc. ent. Belg., (2), xviii : 17 (belongs to Diptera), 1880. " Mourlon, Geol. Belg., i: 144 .

1205. Tettigonia —_. (Jassides.)

Locality : Wainlode, England. Horizon: Lower Lias.

1873. Brodie. Distr. corr. foss, ins., 16.

\section{Velia -. (Veliidæ.)}

Locality : Vale of Wardour, England. Horizon: Purbecks.

1845. Brodie. Foss. ins. sec. rocks Engl, 33 (Westw., 121).

1207. Velia cornuta. (Veliidæ.)

Locality: Solenhofen, Bavaria. Horizon: Jura.

1874. Weyenbergh. Perí́d. zool,, i : 86, 98. Pl. 3, fig. 11.

1874. " Énum. syst. faune ent. mésoz., 12.

\section{COLEOPTERA.}

1208. (several).

Localities : Bexhill, Gover's Cliff End, East Cliff, England. Horizon : Wealden. 1854. Binfield. Quart. journ. geol. soc. Lond., x: 17\%, 173, 174, 175.

\section{9.}

Compared with Necrodes littoralis.

Locality: Ust Balei, Siberia. Horizon: Lias.

1889. $\left\{\begin{array}{c}\text { Brauer-Redt- } \\ \text { enb-Gangib. }\end{array}\right.$ Mém. acad. sc. St.-Pétersb., (7), xxxvi, No. 15: 20.

$$
\text { 1210. - - (pupa). }
$$

Locality: Vale of Wardour, England. Horizon: Purbecks.

1845. Brodie. Foss. ins. sec. rocks Engl., 32 (Westwood, 116). Pl. 3, fig. 6,

$$
\text { 1211. - (3 sp.). }
$$

Locality : Farleigh, near Bath, England. Horizon: Forest Marble.

1845. Brodie. Foss. ins. sec. rocks Engl., 37.

1873. " Distr. corr. foss. ins., 13.

\section{2.}

Locality : Swindon, England. Horizon: Purbecks?

1846. Brodie. Quart. journ. geol. soc. Lond, iii: 53.

1213.

Locality : Ringstead Bay, England. Horizon: Kimmeridge Clay.

1853. Brodie. Quart. journ. geol. soc. Lond., ix : 52.

1873. " Distr. corr. foss. ins., 13.

Bull. $71-12$ 
1214.

Locality: Hastings Sands, Isle of Wight, England. Horizon: Neocomian. 1853. Brodie. Quart. journ. geol. soc. Lond., ix: 52, note.

\section{5.}

Locality : Hastings, England. Horizon: Wealden (base). 1873. Brodie.

Distr. corr. foss. ins., 12.

1216.

1873. Brodie.

Locality : Isle of Wight, England. Horizon: Wealden.

\section{7.}

Locality : Eastern Moorlands, Yorkshire, England. Horizon : Great Oolite. 1873. Brodie. Distr. corr. foss. ins., 13.

1218.

Localities: Dumbleton, Alderton, England. Horizon: Upper Lias. 1873. Brodie Distr. eorr. foss. ins., 15.

1219.

Locality: The Leigh, England. Horizon: Lima Shales. 1873. Brodie. Distr. corr. foss, ins., 16.

1220. (aquatic).

Locality: Wainlode, England. Horizon: Lower Lias.

1873. Brodie. Distr. corr. foss. ins., 16.

\section{1.}

Locality: Westbury, England.

Horizon: Lower Lias.

1873. Brodie.

Distr. corr. foss. ins., 16.

1222.

Locality: Hotham, Yorkshire, England. Horizon : Lower Lias.

1874. Brodie.

Distr. corr. foss. ins., 20.

1223. (under surface anal segment).

Locality: Hotham, England. Horizon: Lower Lias.

1874. Brodie.

Distr. corr. foss. ins., 20.

1224. (under wings).

Locality : England. Horizon: Lias,

1874. Brodie.

Distr. corr. foss. ins., 20 .

1225.

Locality: Copt Heath, near Knowle, England. Horizon: Lower Lias. 1875. Brodie. Lower Lias Eatington, etc., 10. 
1226.

Localities: Brown's Wood and Stooper's Wood, near Henley, Arden, England. Horizon: Lias.

1875. Brodie. Lower Lias Eatington, etc., 10.

1227. (borings).

Locality : Roschutz, Germany. Horizon: Quadersandstein. 1839. Geinitz. Charakt. sächs. kreidegeb., 13-14. Pl. 6, figs.2-3.

1228.

Locality : Ilminster, England. Horizon: Upper Lias.

1878. Goss.

Proc. geol. assoc., vi : 130.

1878. " "

Ins. fauna mesoz. per., ii : 17 .

1229. (2 or $3 \mathrm{sp.)}$.

Locality : Stonesfield, England. Horizon: Oolite. 1822. Conyb.-Phill. Geol. Engl. Wales, 208.

1230.

Locality : Bohemia. Horizon: Cretaceous.

1869. Frič. $\quad$ Arch. naturw. landesdurchf. Böhmens, i, ii : 188. Pl. 3, fig. 7.

See also Silphites priscus.

\section{1.}

Locality : Dobbertin, Mecklenburg. Horizon: Lower Jura.

1880. Geinitz. Zeitschr. deutsch. geol. gesellsch., 1880:530. Pl.22, fg. 21.

1232.

Locality: Kotá, India. Horizon: Lias?

1861. Hislop. Quart. journ. geol. soc. Lond., xvii, i: 354.

1233.

Loeality: Wateringbury, England. Horizon: Wealden.

1846. Mantell. Quart. journ. geol. soc. Lond., ii : 9 i.

1234.

Locality: Near Stone, Buckinghamshire, England. Horizon : Wealden (?) 1847. Mantell. Geol. exc. Isle of Wight, 400.

1235.

Locality: Kent, England. Horizon: Wealden (?).

1847. Mantell. Geol. exc. Isle of Wight, 400.

1236.

Locality: England. Horizon : Purbecks.

1854. Mantell. Medals of creation, 2d ed., ii : 556. Fig. 182 c.

1237.

Locality : England. Horizon: Purbecks.

1854. Mantell. Medals of creation, 2d ed., ii : 556. Fig. 182d. 
1238.

Locality : Englaud. Horizon: Purbecks.

1854. Mantell. Medals of creation, $2 d$ ed., ii : 556. Fig. $182 e$.

1239.

Locality: England. Horizon: Upper Lias.

1845. Murchison. Geol. Cheltenham, 81, 108. Pl. 8, fig. 4.

1240 (several).

Locality : England. Horizon: Lower Lias.

1845. Murchison. Geol. Cheltenham, 81, 108. Pl. 9, figs. 2, 3, 4b, 5 .

1241. (larva).

Locality: Kehlheim, Bavaria. Horizon: Jura.

1852. Quenstedt. Handb. petref., 318 (Raupe ? oder käfer-larve). Pl. 24, fig. 17.

1242.

Locality : Krälah bei Hildesheim, Germany. Horizon: Rbætic.

1876. Roemer.

Zeitschr. deutsch. geol. gesellsch., xxviii : 352, Fig. 3.

1243.

Locality: Stonesfield, England. Horizon:?

1830. Taylor.

Loud., mag. nat hist., iii : 361.

1244.

Locality: Durdlestone Bay, England. Horizon: Lower Purbecks.

1854. Westwood. Quart. journ. geol. soc. Lond., $\mathrm{x}: 387-388,393$. Pl. 14, fig. 4.

\section{5.}

Locality : Durdlestone Bay, England. Horizon : Lower Purbecks.

1854. Westwood. Quart. journ. geol. soc. Lond., $\mathrm{x}$ : 393. Pl. 14, fig. 9.

\section{6.}

Locality: Durdlestone Bay, England. Horizon : Lower Purbecks. 1854. Westwood. Quart. journ. geol. soc. Lond., $\mathrm{x}: 393 . \quad P l .14, f i g .10$.

1247.

Locality : Durdlestone Bay, England. Horizon: Lower Purbecks.

1854. Westwood. Quart. journ. geol. soc. Lond., x : 386, 393. Pl. 14, fig. 14.

1248.

Locality: Durdlsstone Bay, England, Horizon: Lower Purbecks.

1854. Westwood. Quart. journ. geol. soc. Lond., x: 386, 393. Pl. 14, fig. 15.

1249.

Locality : Durdlestone Bay, England. Horizon: Lower Purbecks. .

1854. Westwood. Quart. journ. geol. soc. Lond., x: 386, 393. Pl. 14, fig. 16.

1250.

Locality: Durdlestone Bay, England. Horizon : Lower Purbecks.

1854. Westwood. Qnart. journ. geol. soc. Lond., $\mathrm{x}: 386,393 . \cdot P l .14$, fig. 17. 


\section{1.}

Locality : Durdlestone Bay, England. Horizon: Lower Purbecks. 1854. Westwood. Quart. journ. geol. soc. Lond., x: 386, 393. Pl. 14, fig. 18.

\section{2.}

Locality: Durdlestone Bay, England. Horizon: Midale Purbecks. 1854. Westwood. Quart. journ. geol. soc. Lond., $x: 383,394 . \quad P l .15$, fig. 10.

\section{3.}

Locality: Durdlestone Bay, England. Horizon: Middle Purbecks. 1854. Westwood. Quart. journ. geol. soc. Lond., x: 383, 394. Pl. 15, fig. 11.

\section{4.}

Locality: Durdlestone Bay, England. Horizon: Middle Purbecks. 1854. Westwood. Quart. journ. geol, soc. Lond., x: 383, 394. Pl. 15, fig. 12.

\section{5.}

Locality: Durdlestone Bay, England. Horizon: Middle Purbecks: 1854. Westwood. Quart. journ. geol. soc. Lond., x: 383, 394. Pl. 15, fig, 13, upper left fig.

\section{6.}

Locality : Durdlestone Bay, England. Horizon: Middle Purbecks. 1854. Westwood. Quart. journ. geol. soc. Lond., x: 383, 394. Pl. 15, fig. 13, upper right fig.

\section{7.}

Locality: Durdlestone Bay, England. Horizon: Middle Purbecks. 1854. Westwood. Quart. journ. geol. soc. Lond., x : 383, 394. Pl. 15, fig. 13, lower right fig.

1258.

Locality : Durdlestone Bay, England. Horizon: Lower Purbecks. 1854. Westwood. Quart. journ. geol. soc. Lond., x: 389, 394. Pl. 16, fig. 2.

1259. (many sp.).

Locality : Ridgway, England. Horizon: Lower Purbecks. 1854. Westwood. Quart. journ. geol. soc. Lond., x: 384-385, 394. Pl. 16, fig. 3.

1260.

Locality: Durdlestone Bay, England. Horizon: Middle Purbecks. 1854. Westwood. Quart. journ. geol. soc. Lond., x: 394. Pl. 16, fig. 7.

\section{1.}

Locality: Durdlestone Bay, England. Horizon: Lower Purbecks. 1854. Westwood. Quart. journ. geol. soc. Lond., x: 390, 395. Pl. 16, fig. 12.

\section{2.}

Locality: Durdlestone Bay, England. Horizon: Lower Purbecks. 1854. Westwood. Quart. journ. geol, soc. Lond., x: 395. Pl. 16, fig. 14. 
1263.

Locality: Durdlestone Bay, England. Horizon: Lower Purbecks. 1854. Westwood. Quart. journ. geol. soc. Lond., x: 389, 395. Pl. 16, fig. 15.

\section{4.}

Locality : Durdlestone Bay, England. Horizon: Lower Purbecks. 1854. Westwood. Quart. journ. geol. soc. Lond., x: 395. Pl. 16, fig. 16.

1265.

Locality: Durdlestone Bay, England. Horizon: Lower Purbecks. 1854. Westwood. Quart. journ. geol. soc. Lond., x: 390, 395. Pl. 16, fig. 17.

1266.

Locality: Durdlestone Bay, England. Horizon: Lower Purbecks. 1854. Westwood. Quart. journ. geol, soc. Lond., x: 395. Pl. 16, fig. 18.

1267.

Locality: Durdlestone Bay, England. - Horizon: Lower Purbecks.

1854. Westwood. Quart. journ. geol. soc. Lond, x : 390, 395. Pl. 16, fig. 19. 1268.

Locality : Durdlestone Bay, England. Horizon: Lower Purbecks. 1854. Westwood. Quart. journ. geol. soc. Lond., x: 395. Pl. 16, fig. 20. 1269.

Locality: Durdlestone Bay, England. Horizon: Lower Purbecks. 1854. Westwood. Quart. jonrn. geol. soc. Lond., x: 395. Pl. 16, fig. 21. 1270.

Locality: Durdlestone Bay, England. Horizon: Lower Purbecks. 1854. Westwood. Quart. journ. geol. soc. Lond., x: 389, 395. Pl. 16, fig. 24. 1271.

Locality: Durdlestone Bay, England. Horizon: Lower Purbecks. 1854. Westwood. Quart. journ. geol. soc. Lond., x: 395. Pl. 16, fig. 25. 1272.

Locality: Durdlestone Bay, England. Horizon: Lower Purbecks. 1854. Wesbwood. Quart. journ. geol. soc. Lond., $\mathrm{x}: 395 . \quad$ Pl. 16, fig. 26. 1273.

Locality: Durdlestone Bay, England. Horizon: Lower Purbecks. 1854. Westwood. Quart. journ. geol. soc. Lond., x: 395. Pl. 16, fig. 27.

\section{4.}

Locality: Durdlestone Bay, England. Horizon: Lower Purbecks. 1854. Westwood. Quart. journ. geol. soc. Lond., x: 395. Pl. 16, fig. 28.

1275.

Locality : Durdlestone Bay, England. Horizon : Lower Purbecks. 1854. Westwood. Quart. journ. geol. soc. Lond., $\mathrm{x}: 386,395 . \quad P l .17, \mathrm{fig} .1$. 
1276.

Locality: Durdlestone Bay, England. Horizon: Lower Purbecks. 1854. Westwood. Quart. journ. geol. soc. Lond., $\mathrm{x}: 386,395 . \quad P l .17$, fig. 2.

\section{7.}

Locality: Durdlestone Bay, England. Horizon: Lower Purbecks. 1854. Westwood. Quart. journ. geol. soc. Lond., x: 386, 395. Pl. 17, fig. 6. 1278.

Locality : Durdlestone Bay, England. Horizon : Lower Purbecks. 1854. Westwood. Quart. journ. geol. soc. Lond., $\mathrm{x}: 386-387,395 . \quad$ Pl. 17, fig. 9.

1279.

Loeality : Durdlestone Bay, England. Horizon : Lower Purbecks. 1854. Westwood. Quart. journ. geol. soc. Lond., $\mathrm{x}: 389,396 . \quad P l .18$, fig. 6.

1280.

Locality: Durdlestone Bay, England. Horizon: Lower Purbecks. 1854. Westwood. Quart. journ. geol. soc. Lond., x : 389,396. Pl. 18, fig. 11. (Or Hemiptera, Cimicidæ.)

1281.

Locality : Durdlestone Bay, England. Horizon : Lower Purbecks. 1854. Westwood. Quart.journ. geol. soc. Lond., x: 389, 396. Pl. 18, fig. 10.

1282.

Locality : Durdlestone Bay, England. Horizon : Lower Purbecks. 1854. Westwood. Quart. journ. geol. soc. Lond., $\mathrm{x}: 389,396 . \quad P l .18$, fig. 16.

1283.

Locality : Durdlestone Bay, England. Horizon: Lower Purbecks. 1854. Westwood. Quart. journ. geol. soc. Lond., x: 389, 396. Pl. 18, fig. 18.

1284.

Locality : Durdlestone Bay, England. Horizon: Lower Pnrbecks. 1854. Westwood. Quart. journ. geol, soc. Lond., x: 389,396. Pl. 18, fig. 19.

1285.

Locality: Swanage Bay, England. Horizon: Hastings Sands, Wealden. 185̆4. Westwood. Quart. journ. geol. soc. Lond., x : 382.

\section{6.}

Locality : Ridgway, England. Horizon : Lower Purbecks.

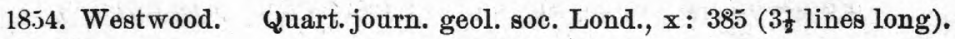
1287. (Blapsidæ.)

Locality: Sevenhampton, England. Horizon: Stonesfield Slate. 1845. Brodie. Foss. ins. sec. rocks Engl., 48. Pl. 6, fig. 16.

1288. (Buprestidæ.)

Locality : Vale of Wardour, England. Horizon : Purbecks. 1845. Brodie. Foss. ins. sec. rocks Engl., 32 (Westw., 116). Pl. 6, fig. 1. 
1289. - - (Buprestidæ.)

Locality: Vale of Wardour, England. Horizon : Purbecks.

1845. Brodie. Foss. ins. sec. rocks Engl., 32 (Westw., 117). Pl. 6, fig. 10.

1290.

(Buprestidæ.)

Localities: Sevenhampton and Eyeford, England. Horizon : Stonesfield Slate. 1845. Brodie. Foss. ins. sec. rocks Engl., 48. Pl. 6, fig. 17.

1291

(Buprestidæ.)

Localities: Sevenhampton and Eyeford, England. Horizon: Stonesfield Slate. 1845. Brodie. $\quad$ Foss. ins. sec. rocks Engl., 48. Pl. 6, fig. 18.

1292.

(Buprestidæ.)

Localities: Sevenhampton and Eyeford, England. Horizon : Stonesfield Slate. 1845. Brodie. Foss. ins. sec. rocks Engl., 48. Pl. 6, fig. 19.

1293. (Buprestidæ or Elateridæ.)

Localities: Wainlode, Apperley, Brockeridge, Churchdown, Aust, England. Horizon: Lower Lias.

1845. Brodie. Foss. ins. sec. rocks Engl., 101 (Westw., 124). Pl. 6, fig. 23.

1294. (Buprestidæ or Elateridæ.)

Localities: Wainlode, Apperley, Brockeridge, Churchdown, Aust, England. Horizon : Lower Lias.

1845. Brodie.

Foss. ins. sec. rocks Engl., 101 (Westw., 124). Pl. 6, fig. 24. 1295.

(Buprestidæ or Elateridæ.)

Localities : Wainlode, Apperley, Brockeridge, Churchdown, Aust, England. Horizon : Lower Lias.

1845. Brodie. $\quad$ Foss. ins. sec. rocks Engl., 101 (Westw., 124). Pl. 6, fig. 25.

1296.

(Buprestidæ or Elateridæ.)

Localities : Wainlode, Apperley, Brockeridge, Churchdown, Aust, England. Horizon : Lower Lias.

1845. Brodie. $\quad$ Foss. ins. sec. rocks Engl., 101 (Westw., 124). Pl. 6, fig. 26. 1297. - (Buprestidæ.)

Locality: Strensham, England. Horizon: Lower Lias.

1845. Brodie. Foss. ins. sec, rocks Engl., 101 (Westw., 124). Pl. 10, fig. 1. 1298. (abdomen). (Buprestidæ?)

Locality : Höganäs, Sweden. Horizon: Rhætic.

1878. Heer.

Förhandl. geol. fören. Stockh., iv: 196. $P l .13$, figs. 7, $7 b$.

1299. (Buprestidæ.)

Locality: England. Horizon: Lias.

1871. Phillips. Geology of Oxford, 123.

1300. - (larva). (Carabidæ?)

Locality: Ust Balei, Siberia. Horizon: Lias.

1889. $\left\{\begin{array}{l}\text { Brauer-Redt- } \\ \text { enb.-Ganglb. }\end{array}\right\}$ Mém. acad. sc. St.-Pétersb., (7), xxxvi, No. 15: 20. 
1301.

(Carabidæ.)

Localities : Apperley, Brockeridge, England. Horizon : Lower Lias.

1845. Brodie.

Foss. ins. sec. rocks Engl., 101 (Westw., 124). Pl. 6, fig. 28.

1302.

(Carabidæ.)

Localities : Apperley, Brockeridge, England. Horizon: Lower Lias. 1845. Brodie. Foss. ins. sec. rocks Engl, 101 (Westw., 124). Pl. 10, fig. 2.

1303. (Carabidæ.)

Locality: The Vallis, near Frome, England. Horizon: Lower Oolite. 1861. Moore. Quart. journ. geol. soc. Lond., xvii : 513.

1304. (Carabidæ.)

1871. Phillips. Geology of Oxford, 123.

Locality: England. Horizon: Lias.

1305. (Carabidæ.)

Locality : Durdlestone Bay, England. Horizon: Lower Purbecks. 1854. Westwood. Quart. journ. geol. soc. Lond., x: 380, 394. Pl. 16, fig. 1.

1306. (Carabidæ.)

Locality: Durdlestone Bay, England. Horizon: Lower Purbecks. 1854. Westwood. Quart. journ. geol. soc. Lond., x: 390, 394. Pl. 16, fig. 10.

1307. . (Carabidæ?)

Locality: Durdlestone Bay, England. Horizon : Lower Purbecks. 1854. Westwood. Quart. journ. geol. soc. Lond., x: 390, 395. Pl. 16, fig. 30.

1308. (Carabidæ-Harpalina, or Chrysomelidø.)

Locality: Vale of Wardour, England. Horizon: Purbecks. 1845. Brodie. Foss. ins. sec. rocks Engl., 32 (Westw., 117). Pl. 6, fig. 3. 1309. (Carabidæ-Harpalina.)

Localities: Binton, Wilmcote, England. Horizon: Lower Lias. 1873. Brodie. Distr. corr. foss. ins., 16. 1310. (Cerambycidæ-Prionini.)

Localities: Sevenhampton and Eyeford, England. Horizon: Stonesfield Slate. 1845. Brodie. Foss. ins. sec. rocks Engl., 47. Pl. 6, fig. 15. 1311. (Cerambycidæ-Prionini.)

Locality : England. Horizon: Stonesfield Slate.

1841. Westwood. Trans. ent. soc. Lond., iv, proc., 40.

See ảlso Buprestis —-Buckland, xlvi" : 4-9.

1312.

Locality: Vale of Wardour, England. Horizon: Purbecks.

1845. Brodie. Foss. ins. sec. rocks Engl., 32 (Westw., 117). Pl. 6, fig. 12.

1313. - - (Chrysomelidæ or Tenebrionidæ-Pimeliinæ.)

Locality : Eyeford, England. Horizon: Stonesfield Slate.

1845. Brodie. Foss. ins. sec. rocks Engl., 48. Pl. 6, fig. 20. 
1314.

(Chrysomelidæ or Curculionidæ.)

Locality : Hasfield, England. Horizon: Lower Lias.

1845. Brodie. Foss. ins. sec. rocks Engl., 101. Pl. 6, fig. 27.

1315.

(Chrysomelidæ or Curculionidæ.)

Locality : Hasfield, England. Horizon : Lower Lias.

1845. Brodie. Foss. ins. sec. rocks Engl., 101 (Westw., 124). Pl. 6, fig. 32.

1316.

(Chrysomelidæ or Curculionidæ.)

Locality: Hasfield, England. Horizon: Lower Lias.

1845. Brodie. Foss. ins. sec. rocks Engl., 101 (Westw., 124). Pl. 6, fig. 33.

1317.

(Chrysomelidæ or Curculionidæ.)

Locality : Hasfield, England. Horizon: Lower Lias.

1845. Brodie. Foss. ins. sec. rocks Engl., 101 (Westw., 124). Pl. 6, fig. 34.

1318.

(Chrysomelidæ.)

Locality : Forthampton, England. Horizon : Lower Lias.

1845. Brodie. Foss. ins. sec. rocks Engl., 101. Pl. 7, fig. 7.

1319.

(Chrysomelidæ or Curculionidæ.).

Locality : Apperley, England. Horizon: Lower Lias.

1873. Brodie. Distr. corr. foss. ins., 16.

1320. - - (Chrysomelidæ.)

Locality : England. Horizon: Lias.

1871. Phillips. Geology of Oxford, 123.

1321. —- (Chrysomelidæ or Curculionidæ.)

Locality : Vale of Wardour, England. Horizon: Purbecks.

1845. Westwood. Brodie, Foss. ins. sec. rocks Engl., 32, 117. Pl. 6, fig. 14.

(Chrysomelidæ or Carabidæ-Harpalina). See Harpalina) Brodie, vi : 3 .

1322.

(Coccinellidæ?)

Locality: Durdlestone Bay, England. Horizon: Middle Purbecks.

1854. Westwood. Quart. journ. geol. soc. Lond., $\mathrm{x}: 383,394$. Pl. 15, fig. 9.

1323.

(Coccinellidæ?)

Locality : Durdlestone Bay, England. Horizon: Lower Purbecks.

1854. Westwood. Quart. journ, geol. soc. Lond., $\mathrm{x}: 389,394$. Pl. 16, fig. 8.

1324.

(Coccinellidæ?)

Locality: Durdlestone Bay, England. Horizon: Lower Purbecks.

1854. Westwood. Quart. journ. geol. soc. Lond., $\mathrm{x}: 389,394 . \quad$ Pl. 16, fig. 9.

1325

(Curculionidæ.)

Locality: Ust Balei, Siberia. Horizon: Lias.

s689. SBrauer - Redt- Mém.ac ad. sc. St.-Pétersb., (7), xxxvi, No. 15: 19. Pl. [2], fig. \{ enb.-Ganglb. 24. 
1326. (Curculionidæ.)

Locality: Vale of Wardour, England. Horizon: Purbecks.

1845. Brodie.

Foss. ins. sec. rocks Engl., 32 (Westw., 117). Pl. 6, fig. 4.

1327.

\section{(Curculionidæ.)}

Locality: Vale of Wardour, England. Horizon: Purbecks.

1845. Brodie.

Foss. ins. sec. rocks Engl., 32 (Westw., 117). Pl. 6, fig. 8.

1328.

(Curculionisæ.)

Localities: Eyeford, Stonesfield, England. Horizon: Stonesfield slate. 1873. Brodie.

Distr. corr. foss. ins., 14.

1329.

\section{(Curculionidæ.)}

Locality : Durdlestone Bay, England. Horizon: Lower Purbecks.

1854. Westwood. Quart. journ. geol. soc. Lond., x: 390, 395. Pl. 16, fig. 33.

(Curculionidæ or Chrysomeidæ.)
Brodie, vi: 14.
$-($ Curculionidæ or Chrysomelidæ.) See
Brodie, vi : 27.

$-\longrightarrow$ (Curculionidæ or Chrysomelidæ.) See - - (Chrysomelidæ)

(Chrysomelidæ)

(Curculionidæ or Chrysomelidæ.) See

Brodie, vi : 33.

(Curculionidæ or Chrysomelidæ.) See
Brodie, vi : 34.

(Curculionidæ or Chrysomelidæ.) See
Brodie, vi : 34.

(Chrysomelidæ)

Brodie, Distr. foss. ins., 16.

(Chrysomelidæ)

1330.

(Dytiscidæ.)

Locality : Durdlestone Bay, England. Horizon: Middle Purbecks.

1854. Westwood. Quart. journ. geol, soc. Lond., x: 383, 394. Pl. 15, fig. 13, large fig. 1331. (Elateridæ.)

Locality: Vale of,Wardour, England. Horizon: Purbecks.

1845. Brodie. Foss. ins. sec. rocks Engl., 32 (Westw., 117). Pl. 6, fig. 6,-

1332.

(Elateridæ.)

Locality: Vale of Aylesbury, England. Horizon : Purbecks.

1345. Brodie. Foss. ins. sec. rocks Engl., 32 (Westw., 117). Pl. 6, fig. 7.

1333.

(Elateridæ.)

Locality: Apperley, England. Horizon: Lower Lias.

1845. Brodie. Foss. ins. sec. rocks Engl, 101. Pl. 7, fig. 1.

1334. (Elateridæ.)

Locality : Apperley, England. Horizon: Lower Lias.

1845. Brodie. Foss. ins. sec. rocks Engl., 101. Pl. 7, fig. 2. 
1335.

(Elateridæ.)

Localities: Wainlode, Westbury, England. Horizon: Lower Lias. 1873. Brodie. Distr. corr. foss. ins., 16.

1336. (Elateridæ or Lampyridæ-Telephorini.)

Localities: Brown's Wood and Stooper's Wood, near Henley in Arden, England. Horizon : Lias.

1875. Brodie. Lower Lias Eatington, etc., 10.

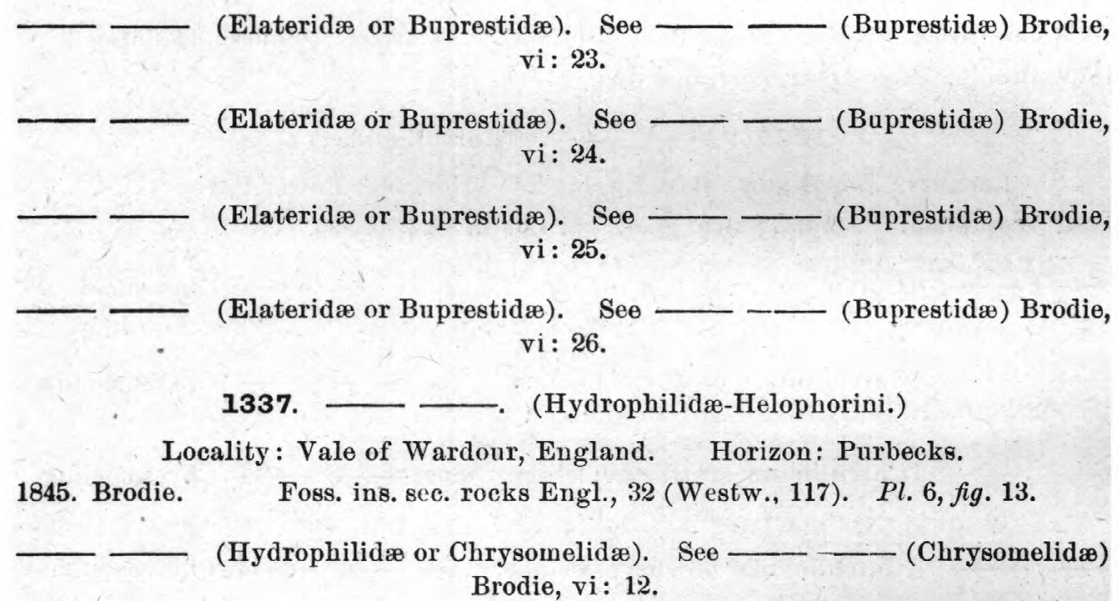

1338. (Lampyridæ-Telephorini.).

Locality: Forthampton, England. Horizon: Lower Lias.

1845. Brodie, Foss. ins. sec. rocks Eugl., 101 (Westw., 124). Pl. 6, fig. 29.

- 1339. —- (Lampyridæ-Telephorini.)

Localities: Wainlode, Brown's Wood, England. Horizon : Lower Lias. 1873. Brodie. Distr. corr. foss, ins., 16.

1340. (Lampyridæ-Telephorini.)

Locality : England. Horizon: Lias.

1871. Phillips. Geology of Oxford, 123.

(Lampyridæ-Telephorini, or Elateridæ). See
teridæ) Brodie, Lower Lias, 10).
1341.
(Meloidæ-Cantharini.)

Locality : Vale of Wardour, England. Horizon: Purbecks.

1845. Brodie. Foss. ins. sec. rocks Engl., 32 (Westw., 117). Pl. 6, fig. 11.

1342. - (Rhynchophora.)

Locality : Vale of Wardour, England. Horizon : Purbecks.

1845. Brodie. Foss. ins. sec. rocks Engl., 32 (Westw., 116). Pl. 3, fig. 4.

- (Staphylinidæ). See II, Neuroptera, Idem. 
1343. - (Tenebrionidæ.)

Locality : Vale of Wardour, England. - Horizon: Purbecks.

1845. Brodie.

Foss. ins. sec. rocks Engl., 32 (Westw., 116). Pl. 6, fig. 2.

(Tenebrionidæ-Pimeliinæ, or Chrysomelidæ). See

(Chrysomelidæ) Brodie, vi : 20.

1344. Actea sphinx. (Buprestidæ.)

Localities: Kelheim, Solenhofen, Bavaria. Horizon: Jura.

1842. Münster. MSS.

1842. Germar. Münster, Beitr. z. petref,, v: 85-87. Pl. 9, fig. 6.

1852. Giebel. Deutschl, petref., $63 \%$.

1862. Hagen. Palaeontogr., $x: 111$ (Hemipteron).

1869. Weyenbergh. Tijdschr. v. ent., (2), iv : 233 [133].

1874. "

1874. " "Enum. syst. faune ent. mésoz., 14.

1877. Assmann. Ber. versamml. deutsch. naturf., 1: 192 (=Chrysobothris veterana).

1885. Seudder. Zittel, Handb. palaeont., i, th. ii : 782.

1886. " Zittel-Barrois, Traité de pal6́ont., ii : 782.

See also Sphenoptera sphinx, etc.

1345. Agrilium cyllabacus. (Buprestidæ.)

Locality: Durdlestone Bay, England. Horizon: Lower Purbecks.

1854. Westwood. Quart. journ. geol. soc. Lond., $\mathrm{x}: 389,395$. Pl. 16, fig. 32.

See also Agrilus cyllabacus.

1346. Agrilium cyllarus. (Buprestidæ.)

Locality: Durdlestone Bay, England. Horizon : Lower Purbecks.

1854. Westwood. Quart. journ. geol. soc. Lond., x: 389,395. Pl. 16, fig. 29. See also Agrilus cyllarus.

\section{Agrilium stomphax. (Buprestidæ.)}

Locality: Durdlestone Bay, England. Horizon: Lower Purbecks.

1854. Westwood. Quart. journ. geol. soc.Lond., $\mathrm{x}: 389,395 . \quad$ Pl. 16, fig. 23.

See also Agrilus stomphax.

1348. Agrilium strombus. (Buprestidæ.)

Locality : Durdlestone Bay, England. Horizon : Lower Purbecks.

1854. Westwood. Quart. journ. geol. soc. Lond., x: 389, 394-395. Pl. 16, fig. 6.

See also Agrilus strombus.

1349. Agrilus cyllabacus. (Buprestidæ.)

Locality: Durdlestone Bay, England. Horizon: Lower Purbecks,

1856. Giebel. Insect. d. vorwelt, 88.

See also Agrilium cyllabacus.

1350. Agrilus cyllarus. (Buprestidæ.)

Locality : Durdlestone Bay, England. Horizon: Lower Purbecks. 
1351. Agrilus stomphax. (Buprestidæ

Locality: Durdlestone Bay, England. Horizon : Lower Purbecks.

1856. Giebel.

Insect. $d$. vorwelt, 88 .

See also Agrilium stomphax.

1352. Agrilus strombus. (Buprestidæ.)

Locality : Dúrdlestone Bay, England. Horizon: Lower Purbecks.

1856. Giebel. Insect. d. vorwelt, 87.

See also Agrilium strombus.

1353. Amara? pseudo-zabrus. (Carabidæ.)

Locality: Eichstadt, Bavaria. Horizon: Jura.

1886. Deichmüller. Ins. lith. schichten dresd. mus., 64-65. $\quad P l .5, f i g .9$.

1354. Ancylocheira -. (Buprestidæ.)

Locality: Gloucestershire, England. Horizon: Lias.

1843. Brodie. $\quad$ Lond. Edinb. Dubl. phil. mag., (3), xxiii: 529.

1843. “ Ann. nat. hist., xi: 509.

1355. Ancylocheira liasina. (Buprestidæ.)

Locality : Strensham, England. Horizon: Lower Lias.

1845. Brodie. Foss. ins. sec. rocks Engl. (unnamed). Pl. 10, fig. 1.

1856. Giebel. Insect. d. vorwelt, 82-83 (Ancylochira).

1356. Ancylocheira teleas. (Buprestidæ.)

Locality : Durdlestone Bay, England. Horizon: Lower Purbecks.

1856. Giebel. Insect. d. vorwelt, 83 (Ancylochira).

See also Buprestium teleas.

Ancylochira liasina. See Ancylocheira liasina.

Ancylochira teleas. See Ancylocheira teleas.

1357. Anisorhynchus? lapideus. (Otiorhynchidæ.)

Locality : Solenhofen, Bavaria. Horizon: Jura.

1869. Weyenbergh. Arch. mus. Teyl., ii: 285-286. Pl. 36, figs. 40, $40 a$.

1869. “ Ins. foss. calc. lithogr., 39-40. Pl. 3, figs. 40,40a.

1869. " " Tijdschr. v. ent., (2), iv: 232 [132].

1874. " " Perí́d zool., i: 104.

1874. “ Énum. syst. faune ent. mésoz., 18.

1888. Oppenheim. Palaeontogr., xxxiv: 241-242. Pl. 31, fig. 7.

1358. Aphodiites protogæus. (Scarabæidæ.)

Locality: Schambelen, Switzerland. Horizon: Lias.

1865. Heer. Urwelt der Schweiz, 90. Pl. 8, fig. 15.

1872. "Monde prim. Suisse, 110. Pl. 8, fig. 15.

1876. " Prim. world Switz., i: 89. Pl. 8, fig. 15.

1879. “Urwelt der Schweiz, $2^{\mathrm{e}}$ anfl., 100. Pl. 8, fig. 15.

1885. Scudder. Zittel, Handb. palaeont., i, th. ii: 794. Fig. 1026.

1886. " Zittel-Barrois, Traité de paléont., ii : 795. Fig. 1044. 
1359. Archiorhynchus angusticollis. (Curculionidæ.)

Locality : Kome, North Greenland. Horizon: Cretaceous.

1874. Heer.

Kreide flora arct. zone (K. sv. vet.-akad. handl., xii ; Flora foss. arct., 3): 91-92. $\mathrm{Pl} .17$, figs. 13 [15].

1883. “

Flora foss. groenl., ii : 143.

Bellengera ovalis. See Bellingera ovalis.

1360. Bellingera laticollis. (Cryptophagidæ.)

Locality: Schambelen, Switzerland. Horizon: Lias.

1865. Heer.

1872. “

1876. “

1879. " "

1885. Seudder.

1886. “
Urwelt der Schweiz. $P l .8, f i g .5$.

Monde prim. Suisse. $P l .8, \mathrm{flg} .5$.

Prim. world Switz., i. $P l .8$, fig. 5.

Urwelt der Schweiz, $2^{\mathrm{e}}$ aufl. (index). Pl. 8, fig. 5.

Zittel, Handb. palaeont., i, th. ii: 800 . Fig. 1050.

Zittel-Barrois, Traité de paléont., ii : 800. Fig. 1067

1361. Bellingera ovalis. (Cryptophagidæ.)

Localities: Aust, England; Schambelen, Aargau, Switzerland ; Dobbertin, Germany. Horizon: Lias (Rhætic).

1852. Heer.

1856. Giebel.

1865. Heer.

1872. "6

1873. Brodie.

1876. Heer.

1879. "

1884. E. Geinitz.
Lias-insel Aargau, 12. Pl., fig. 10.

Insect. d. vorwelt, 497.

Urwelt der Schweiz, 95. Pl. 8, fig. 6.

Monde prim. Suisse, 116. Pl. 8, fig. 6.

Distr. corr. foss. ins., 17 (Bellengera).

Prim. world Switz., i: 94. Pl. 8, fig. 6.

Urwelt der Schweiz, $2^{\ominus}$ aufl., 105. Pl. 8, fig. 6.

Zeitschr. deutsch. geol. gesellsch., 1884: 583 (Bellingeria)

Bellingeria ovalis. See Bellingera ovalis.

\section{Berosus —. See Cercyon (or Berosus) — Brodie}

1362. Berosus - (Hydrophilidæ:)

Locality : Aust, England. Horizon: Lower Lias.

1845. Brodie. Foss. ins. sec. rocks Engl., 101, 125. Pl. 9, fig. 10.

See also Cercyon — Brodie, Berosus liasinns.

1363. Berosus liasinus. (Hydrophilidæ.)

Locality: Aust, England. Horizon: Lower Lias.

1856. Giebel. Insect. d. vorwelt, 52.

See also Berosus — Brodie.

1364. Blaps studeri. (Tenebrionidæ.)

Locality: Sevenhampton, England. Horizon: Stonesfield Slate.

1845. Brodie.

1856. Giebel.

Foss. ins. sec. rocks Engl., 48 (unnamed). $\quad P l .6, f i g .16$.

Insect. d. vorwelt, 108-109.

1365. Blapsidium egertoni. (Tenenrionidæ.)

Locality: England. Horizon: Stonesfield Slate.

1871. Phillips.

Geology of Oxford, 173.

1873. Brodie. Distr. corr. foss. ins., 14.

See also Blapsium egertoni. 
1366. Blapsium egertoni. (Tenebrionidæ.)

Locality: England. Horizon: Stonesfield Slate.

1854. Westwood. Quart. journ. geol. soc. Lond., $\mathrm{x}: 393 . \quad \mathrm{Pl} .14, \mathrm{fig} .13$ 1856. Giebel. Insect. d. vorwelt, 109.

See also Blapsidium egertoni.

\section{Bostrychus - (borings). (Ptinidæ.)}

Compared with B. chalcographus.

Locality : Lottinghem, Pas de Calais, France. Horizon: Gault.

1876. Brongniart. Ann. soc. ent. Fr., (5), vi, bull. 115-116.

1877. “ Ann. soc. ent. Fr., (5), vii : 217-218. Pl. $7^{\prime \prime}$, fig. 5.

1878. Girard. La nature, vi: 112. Fig. 5.

1368. Bothynophora elegans.

Locality: Schambelen, Switzerland. Horizon: Lias.

1865. Heer. Urwelt der Schweiz. Pl. 7, fig. 20.

1872. " Monde prim. Suisse. Pl. 7, fig. 20.

1876. " $\quad$ Prim. world Switz. $P l .7, f i g .20$.

1879. " Urwelt der Schweiz, $2^{\ominus}$ auf. Pl. 7, fig. 20.

1369. Brachinites truncatus. (Carabidæ.)

Locality: Vyšerovic, Bohemia. Horizon: Cretaceous.

1882. Fritsch. Mojs., Beitr. paläont. Oestr.-Ung., ii : 5-6. Pl. 2, fig. 3 (2 figs.).

1882. " Foss. arthr. steink. u. kreidef. Böhmens, 5-6. Pl. 2 fig. 3 (2 figs.).

1884. " Vesmír, xiii : 205-206. Figs. 3,3a.

\section{Bruchus —. (Bruchidæ.)}

Locality: Eyeford, England. Horizon: Stonesfield Slate.

1845. Murchison. Geol. Cheltenham, 68.

1371. Buprestidium —. (Buprestidæ.)

Localities: Stonesfield, Eyeford, England. Horizon: Stonesfield Slate.

1871. Phillips. Geology of Oxford, 173. Digr. 33.

1372. Buprestis or Elater - —. (Buprestidæ.)

Locality : Strensham, England. Horizon: Lower Lias.

1873. Brodie. Distr. corr. foss. ins., 16.

1373. Buprestis - (Buprestidø.)

Locality: Stonesfield, England. Horizon: Stonesfield Slate.

1837. Buckland. Geol. and mineral., ii: 78. Pl. $46^{\prime \prime}$, figs. 4-9.

1838. " Géologie, éd. Agassiz, ii : expl. pl. xlvi", p. 4. Pl. 46", figs. 4-9.

1865. Lyell. Elem. geol., 6th ed., 403. Fig. 410.

See also —— (Cerambycidæ-Prionini) Westw.

1374. Buprestis —. (Buprestidæ.)

Locality: Japan. Horizon: (in chalcedony.)

1837. Buckland. Geol. and mineral., ii : 78.

1838. " Géologie, éd. Agassiz, ii : expl. pl. 46", p. 5.

1375. Buprestis —. (Buprestidæ.)

Locality: Eyeford, England. Horizon: Stonesfield Slate.

1845. Murchison. Geol. Cheltenham,68. Pl. 4, fig. 3. 
1376. Buprestis —. (Buprestidæ.)

Locality : Wainlode Hill, England. Horizon: Lower lias.

1845. Murchison. Geol. Cheltenham, 81. Pl. 9, fig. 4.

1377. Buprestis? - (Buprestidæ.)

Compared with B. variabilis, New Holland.

Locality: Stonesfield, England. Horizon: Stonesfield Slate.

1824. Prévost.

Ann. sc. nat., iv: 417. Pl. 18, fig. 26.

1839. Brulle.

Gisem. ins. foss., 12.

1378. Buprestis bucklandi. (Buprestidæ.)

Locality : Stonesfield, England. Horizon: Stonesfield Slate.

1844. Mantell. Medals of creation, ii : 577-578. Lign. 124, fig. 1.

1854. " Medals of creation, 2 d ed., ii : 554. Lign. 181, fig. 1.

1379. Buprestis lapidelythris. (Buprestidæ.)

Locality: Solenhofen, Bavaria. Horizon: Jura.

1869. Weyenbergh. Arch. mus. Teyl., ii: 283. Pl. 37, figs. 46, 46a.

1869. " " Ins. foss. calc. lithogr., 37. Pl. 4, figs, 46, $46 a$.

1869. " " Tijdschr. v. ent., (2), iv: 232 [132].

1874. " " Periód. zool., i: 103.

1874. " " Enum. syst. faune ent. mésoz., 17.

1380. Buprestites bractoides. (Buprestidæ.)

Locality: Skelton Park Pit, England. Horizon : Lias.

1876. Blake. Tate and Blake, Yorkshire lias, 426. Pl. 16, fig. 5.

1381. Buprestites lyellii. (Buprestidæ.)

Locality: Schambelen, Switzerland. Horizon: Lias.

1865. Heer.

1872. 66

1876. 66

1579. 46

1865. Heer.

1872. " "

1876. 66

1879. “6
Urwelt der Schweiz, 88. Pl. 7, fig. 11.

Monde prim. Suisse, 108. Pl. 7, fig. 11.

Prim. world Switz, i : 83. Pl. 7, fig. 11.

Urwelt der Schweiz, $2^{\mathrm{e}}$ aufl., 98. Pl. 7, fig.' 11.

1382. Buprestites pterophylli. (Buprestidæ.)

Locality: Vadutz, Switzerland. Horizon: Trias.

See also Glaphyroptera pterophylli.

Urwelt der Schweiz, 56.

Monde prim. Suisse, 68.

Prim. world Switz., i: 56.

Urwelt der Schweiz, 2 aufl., 65.

1383. Buprestites rugulosus. (Buprestidæ.)

Locality: Bjuf, Sweden. Horizon: Rhætic.

1878. Heer. Förhandl. geol. fören. Stockh., iv: 194-195. Pl.13, figs. 2, 2b, 20.

1384. Buprestites scabriusculus. (Buprestidæ.)

Locality: Pechgraben, Austria. Horizon: Lias.

1879. Heer. Urwelt der Schweiz, $2^{e}$ anfl., 92.

1385. Buprestites suprajurensis. (Buprestidæ.)

Locality: Eichstadt, Bavaria. Horizon: Jura.

1888. Oppenheim. Palaeontogr., xxxiv: 240. Pl. 31, fig. 21.

Bull. $71-13$ 
1386. Buprestium bolbus. (Buprestidæ.)

Locality : Durdlestone Bay, England. Horizon: Lower Purbecks.

1854. Westwood. Quart. journ. geol. soc. Lond., $\mathrm{x}: 386,395 . \quad P l .17, f i g .5$. 1856. Giebel. Insect. d. vorwelt, 80.

\section{Buprestium dardanus. (Buprestidæ.)}

Locality : Durdlestone Bay, England. Horizon : Lower Purbecks. 1854. Westwood. Quart. journ. geol. soc. Lond., $\mathrm{x}: 389,395 . \quad$ Pl. 16, fig. 38. 1856. Giebel. Insect. d. vorwelt, 80.

\section{Buprestium gorgus. (Buprestidæ.)}

Locality: Durdlestone Bay, England. Horizon: Middle Purbecks. 1854. Westwood. Quart. journ. geol. soc. Lond., $\mathrm{x}: 383,394 . \quad$ Pl. 15, fig. 7. 1856. Giebel. Insect. $d$. vorwelt, 79-80.

1389. Buprestium stygnus. (Buprestidæ.)

Locality: Durdlestone Bay, England. Horizon : Lower Purbecks. 1854. Westwood. Quart. journ. geol. soc. Lond., x: 389, 394-395. Pl. 16, figs. 11, 22. 1856. Giebel.

Insect. d. vorwelt, 80 .

1390. Buprestium teleas. (Buprestidæ.)

Locality: Durdlestone Bay, England. Horizon: Lower Purbecks. 1854. Westwood. Quart. journ. geol. soc. Lond., x: 386, 393. Pl. 14, fig. 19. See also Ancylocheira teleas.

\section{Buprestium valgus. (Buprestidæ.)}

Locality: Durdlestone Bay, England. Horizon: Lower Purbecks. 1854. Westwood. Quart. journ. geol. soc. Lond., $\mathrm{x}: 389,395 . \quad$ Pl. 16, fig. 31.

1392. Buprestium woodlei. (Buprestidæ.)

Locality: Durdlestone Bas, England. Horizon: Lower Purbecks. 1854. Westwood. Quart. journ. geol. soc. Lond., x: 393. Pl. 14, fig.11. 1856. Giebel. Insect. d. vorwelt, 80 .

\section{Byrrhydium arcuatum. (Byrrhidæ.)}

\section{Locality: Schambelen, Switzerland. Horizon: Lias.}

1865. Heer. Urwelt der Schweiz, 89. Pl. 8, fig. 9.

187\%. " Monde prim. Suisse, 109. Pl. 8, fig. У.

1876. “ Prin. world Switz., i: 89. Pl. 8, fig. 9.

1879. “ Urwelt der Schweiz, $2^{\mathrm{e}}$ aufl., 99. Pl. 8, fig. 9.

1394. Byrrhydium morio. (Byrrhidæ.)

Locality: Schambelen, Switzerland. Horizon: Lias.

1865. Heer.

1872. "

1876. " "

1879. “

1885. Scudder.

1886. 6
Urwelt der Schweiz, 89. $\quad P l .8$, fig. 10.

Monde prim. Suisse, 109. $\quad P l$. 8, fig. 10.

Prim. world Switz., i : 89. Pl. 8, fig. 10.

Urwelt der Schweiz., 2e aufl, 99. Pl. 8, fig. 10.

Zittel, Handb. palaeont., i, th. ii : 798. Fig. 1041.

Zittel-Barrois, Traité de palént., ii : 799. Fig. 1058. 
1395. Byrrhydium troglodytes. (Byrrhidæ.)

Locality : Schambelen, Switzerland. Horizon: Lias.

1865. Heer. Urwelt der Schweiz, 64, 89.

1872. " Monde prim. Suisse, 90, 109.

1876. " Prim. world Switz., i: 64, 89.

1879. " Urwelt der Schweiz, $2^{\mathrm{e}}$ aufl., 73, 99.

1396. Camtodontus angliæ. (Carabidæ.)

Locality: Vale of Wardour, England. Horizon: Purbecks.

1845. Brodie. Foss. ins. sec. rocks Engl., 32 (unnamed). Pl, 6, fig. 8.

1856. Giebel. Insect. d. vorwelt, 65.

\section{Carabicina? decipiens. (Carabidæ.)}

Locality: Solenhofen, Bavaria. Horiznn: Jura.

1842. Germar. Münster, Beitr. z. petref., v: 83-84. Pl. 9, fig. 4; Pl. 13, fig. 9.

1062. Hagen. Palaeontogr., $\mathrm{x}: 111$.

1869. Weyenbergh. Arch. mus. Teyl., ii : 288. Pl. 37, fig. 55.

1869. " " Ins. foss. calc. lithogr., 42. Pl. 4, fig. 55.

1869. " " Tijdschr. v. ent., (2), iv : 231 [131.]

1874. " $"$ Period. zool., i: 101.

1874. “ Enum. syst. faune ent. mésoz., 15.

1877. Assmann. Ber. versamml. deutsch. naturf., 1: 192.

1885. Scudder. Zittel, Handb. palaeont., i, th. ii: 815.

1886. " " Zittel-Barrois, Traité de paléont., ii : 817.

1886. " " Bull. U. S. geol. surv., No. 31: 97.

See also II, Hymenoptera, Apiaria lapidea.

1398. Carabidium dejeanianum. (Carabidæ.)

Locality : Durdlestone Bay, England. Horizon : Lower Purbecks.

1854. Westwood. Quart. journ. geol. soc. Lond., $\mathrm{x}: 389,396 . \quad P l .18, f i g .1$.

1856. Giebel. Insect. $d$, vorwelt, 61 .

1399. Carabites —. (Carabidæ.)

Locality : Solenhofen, Bavaria. Horizon: Jura.

1852. Quenstedt. Handb. petref., 313. Pl. 24, fig. $6 a b$.

1400. Carabites anthracinus. (Carabidæ.)

Locality : Aargau, Switzerland. Horizon: Lias.

18i2. Heer. Lias-insel Aargau, 12. Pl., fig. 3.

1856. Giebel. Insect. d. vorwelt, 497.

1401. Carabites bellus. (Carabidæ.)

Localities: Schambelen, Switzerland; Dobbertin, Mecklenburg. Horizon: Lias.

1865. Heer. Urwelt der Schweiz, 90. Pl. 8, fig. 22.

1872. " " Monde prim. Suisse, 110. Pl. 8, fig. 22.

1876. " $\quad$ Prim, world Switz., i: 90. Pl, 8, fig. 22.

1879. " Urwelt der Schweiz, $2^{2}$ aufl, 100. Pl. 8, fig. 22.

1884. E. Geinitz. Zeitschr. deutsch. geol. gesellsch., 1884: 583.

1402. Carabites deplanatus. (Carabidæ.)

Locality : Bjuf, Sweden. Horizon: Rhætic.

1878. Heer. Förhandl. geol, fören. Stockh., iv: 197. Pl. 13, figs. 8-9. 
1403. Carabites depressus. (Carabidæ.)

Locality: Pechgraben, Austria. Horizon : Lias.

1879. Heer.

Urwelt der Schweiz, $2^{\mathrm{e}}$ aufl., 91-92.

1404. Carabites harpalinus. (Carabidæ.)

Locality : Schambelen, Switzerland. Horizon: Lias.

1865. Heer.

Urwelt der Schweiz, 90.

Monde prim. Suisse, 110.

Prim. world Switz., i: 90.

1876. " "

1879. “

Urwelt der Schweiz, $2^{\mathrm{e}}$ aufl., 100.

1405. Carabocera prisca. (Carabidæ.)

Locality: Ust Balei, Siberia. Horizon: Lias.

1889. $\{$ Brauer-Redt- $\}$ Mém acad. sc. St.-Pétersb., (7), xxxvi, No. $15: 18-19 . \quad P l$. [2], \{enb.-Ganglb.\} fig. 23.

1406. Carabus ?

(Carabidæ.)

Locality : Eyeford, England. Horizon : Stonesfield Slate.

1845. Murchison. Geol. Cheltenham, 68. Pl. 4, fig. 2.

1407. Carabus elongatus. (Carabidæ.) *

Locality : Vale of Wardour, England. Horizon : Purbecks.

1845. Brodie.

Foss. ins. sec. rocks Engl., 32 (Westw., 115-116). Pl. 2, fig. 1.

1856. Giebel.

Insect. $d$. vorwelt, 60 .

1408. Carabus westwoodii. (Carabidæ.)

Locality: Durdlestone Bay, England. Horizon: Lower Purbecks.

1854. Westwood. Quart. journ. geol. soc. Lond., x: 390,395 (unnamed). Pl. 16, fig. 30.

1856. Giebel. Insect. d. vorwelt, 60-61.

1409. Carabus winkleri. (Carabidæ.)

Locality: Solenhofen, Bavaria. Horizon: Jura.

1869. Weyenbergh. Arch. mus. Teyl., ii : 278-279. Pl. 37, figs. 56, $56 a$.

$1869 . \quad$ " Ins. foss. calc. lithogr., 32-33. Pl. 4, figs. 56, 56a.

1869. " " Tijdschr. v. ent., (2), iv : 231 [131].

1874. " $\quad$ Periód. zool., i: 101.

1874.

Enum. syst. faune ent. mésoz., 15.

1410. Cassida æquivoca. (Chrysomelidæ.)

Local:ty: Solenhofen, Bavaria. Horizon: Jura.

1869. Weyenbergh. Arch. mus. Teyl., ii : 287-288. Pl. 36, fig. 39.

1869. " " Ins. foss. calc. lithogr., 41-42. Pl. 3, fig. 39.

1869. $\quad$ " Tijdschr. v. ent., (2), iv: 233 [133].

1874. " " Períd. zool., i: 105.

1874. “ Énum. syst. fanne ent. mésoz., 19.

1411. Cerambycinus dubius. (Cerambycidæ.)

Locality: Solenhofen, Bavaria. Horizon: Jura.

1839. Münster. MSS.

1839. Germar. Verhandl. leop.-carol. akad. naturf., xix: 208-210. Pl. 22, fig. 9.

1854. Pictet. Traité de paléont., $2^{\mathrm{e}}$ 6d., ii: 355 . Pl. $40, \mathrm{fig} .8$.

1862. Hagen. Palaeontogr., $\mathrm{x}: 110$.

1877. Assmann. Ber. versamml. deutsch. naturf., 1: 192.

See also Mesosa germari, Cerambycinus germari. 
1412. Cerambycinus germari. (Cंerambycidæ.)

Locality: Solenhofen, Bavaria. Horizon: Jura.

1886. Deichmüiler. Ins. lith, schichten dresd. mus., 75, note.

See also C. dubius, etc.

\section{Cerambycites —- (borings).}

Compared with the borings of Cerambyx luridus; now regarded as borings of a mollusk, Gastrochæna amphisbœna Goldf. Cf. Scudd., Bibl. foss. ins., 13.

Localities: Welschufe, Rippchen, Bannewitz, Saxony. Horizon : Quadersandstein. 1839. Geinitz. Charakt. sächs. kreidegeb., 13. Pls. 3-5, 6, fig. 1. 1856. Giebel. Insect. d. vorwelt, 129.

See also Clytus __ Giebel.

1414. Cerambycites dubius. (Cerambycidæ.)

Locality: Eichstadt, Bavaria. Horizon: Jura.

1886. Deichmüller. Ins. lith. schichten dresd. mus., 75. Pl.5, fig. 19. See also Ditomoptera dubia.

1415. Cerambycites minor. (Cerambycidæ.)

Locality : Eichstadt, Bavaria. Horizon: Jura.

1886. Deichmüiller. Ins. lith. schichten dresd. mus., 74. Pl. 5, fig. 20.

1416. Cerambyx -—. (Cerambycidæ.)

Locality: Solenhofen, Bavaria. Horizou: Jura.

1820. Schlotheim. Petrefactenk., 42-43.

\section{Cercyon (or Berosus) - - (Hydrophilidæ.)}

Loeality: Aust, England. Horizon: Rhætic.

1873. Brotlie. Distr. corr. foss. ins., 17.

1418. Cercyon. (Hyärophilidæ.)

Locality : Aust, England. Horizon: Lower Lias.

1845. Westwood. Brodie, Foss. ins. sec. rocks Engl., 125. $P l .9$, fig. 10.

See also Berosus — Brodie.

\section{Cerylon striatum. (Colydiidæ.)}

Locality : Vale of Wardour, England. Horizon: Purbecks.

1845. Brodie.

1856. Giebel.

1885. Scudder.

1886.
Foss. ins. sec. rocks Engl., 32, 116. Pl. 3, fig. 1.

Insect. d. vorwelt, 125 .

Zittel, Handb. palaeont., i, ii : 800. Fig. 1052.

Zittel-Barrois, Traité de paléont., ii : 800. Fig. 1069.

Cetonia defosfa. See Cetonia defossa.

1420. Cetonia defossa. (Scarabæidæ.)

Locality: Solenhofen, Bavaria. Horizon: Jura.
1869. Weyenbergh. Arch. mus. Teyl., ii : 282-283. Pl. 37, fig. 52.

1869. " " Ins. foss. calc. lithogr., 36-37. Pl. 4, fig. 52.

1869. “ " " Tijdschr. v. ent., (2), iv : 232 [132].

1874. " " Perisd. zool., i: 103 (defosfa).

1874. “ Énum. syst. faune ent. mésoz., 17. 
1421. Chlæenius solitarius. (Carabidæ.)

Compared with C. velutinus, C. prasinus.

Locality: Eichstadt, Bavaria. Horizon: Jura.

1886. Deichmüller. Ins. lith. schichten dresd. mus., 65. Pl. 5, fig. 8.

1422. Chrysobothris veterana. (Buprestidæ.)

Locality: Solenhofen, Bavaria. Horizon : Jura.

1847. Hejden. Palaeontogr., i: 99-100. Pl. 12, fig. 4.

1856. Giebel. Insect. d. vorwelt, 497.

1869. Weyenbergh. Arch. mus. Teyl., ii : 288. Pl. 37, fig. 47.

1869. “ " Ins. foss. calc. lithogr., 42. Pl. 4, fig. 47.

1869. " " Tijdschr. v. ent., (2), iv : 232 [132].

1874. " " Periód. zool., i: 103.

1874. “ Enum. syst. fanne ent. mésoz., 17.

1877. Assmann. Ber. versamml. dentsch. naturf., 1: 192 (=Actea sphinx).

See also Sphenoptera sphinx, etc.

1423. Chrysomela —- (Chrysomelidæ.)

Locality : Durdlestone Bay, England. Horizon : Lower Purbecks.

1854. Westwood. Quart. journ. geol. soc. Lond., x: 395 (unnamed). Pl. 17, fig. 2. 1856. Giebel. Insect. d. vorwelt, 121.

1424. Chrysomela andræi. (Chrysomelidæ.)

Locality: Forthampton, England. Horizon: Lower Lias.

1845. Brodie. Foss. ins. sec. rocks Engl., 101 (unnamed). Pl. 7, fig. 7.

1856. Giebel. Insect. d. vorwelt, 119.

1425. Chrysomela dubia. (Chrysomelidæ.)

Locality: Durdlestone Bay, England. Horizou : Lower Purbecks.

1854. Westwood. Quart. journ. geol. soc. Lond., x: 395 (unnamed). Pl. 16, fig. 24.

1856. Giebel. Insect. d. vorwelt, 120.

1426. Chrysomela dunkeri. (Chrysomelidæ.)

Locality: Durdlestone Båy, England. Horizon : Lower Purbecks.

1854. Westwood. Quart. journ. geol. soc. Lond, x: 395 (unnamed). Pl. 16, fig. 26. 1856. Giebel. Insect. d. vorwelt, 120.

1427. Chrysomela ignota. (Chrysomelidæ.)

Locality : Durdlestone Bay, England. Horizon : Lower Purbecks.

1854. Westwood. Quart. journ. geol. soc. Lond., x: 395 (unnamed). Pl. 16, fig. 15. 1856. Giebel. Insect. d. vorwelt, 120.

1428. Chrysomela liasina. (Chrysomelidæ.)

Locality: Hasfield, England. Horizon : Lower Lias.

1845. Brodie. $\quad$ Foss. ins: sec. rocks Engl., 101 (unnamed). Pl. 6, fig. 33.

1856. Giebel. Insect. d. vorwelt, 120.

1429. Chrysomela lithografica. (Chrysomelidæ.)

Locality : Solenhofen, Bavaria. Horizon: Jura.

1869. Weyenbergh. Arch. mus. Teyl., ii: 287. Pl. 36, figs. 35-36.

$1869 . \quad$ " Ins. foss. ealc. lithogr., 41. Pl. 3, figs. 35-36.

1869. “ " Tijdschr. v. ent., (2), iv : 233 [133].

1874. $\because \quad$ Períd. zool., i: 105.

1874. “ Enum. syst. faune ent. mésoz., 19. 
1430. Chrysomela rara. (Chrysomelidæ.)

Locality : Solenhofen, Bavaria. Horizon: Jura.

1869. Weyenbergh. Arch. mus. Teyl, ii : 287. Pl. 36, figs, 34,34a.

$1869 . \quad$ " Ins. foss. calc. lithogr., 41. Pl. 3, figs. 34, 34a.

$1869 . \quad$ " Tijdschr. v. ent., (2), iv : 233 [133].

1874. " " Period. zool., i: 105.

1874. " E Énum. syst. faune ent, mésoz, 19.

1431. Chrysomelites jurassicus. (Chrysomelidæ.)

Locality : Eichstadt, Bavaria. Horizon: Juıa.

1888. Oppenheim. Palaeontogr., xxxiv : 242. $\quad P l .31$, fig. 25.

1432. Chrysomelites minimus. (Chrysomelidæ.)

Locality: Eichstadt, Bavaria. Horizon: Jura.

1888. Oppenheim. Palaeontogr., xxxiv: 242 (minima). Pl. 31, fig. 23.

1433. Chrysomelites prodromus. (Chrysomelidæ.)

Locality: Schambelen, Switzerland. Horizon : Lias.

1865. Heer. Urwelt der Schweiz, 89,93. Pl. 8, fig. 13.

1872. " Monde prim. Suisse, 109, 114. Pl. 8, fig. 13.

1876. " $\quad$ Prim. world Switz., i: 89,92. Pl. 8, fig. 13.

1879. " Urwelt der Schweiz, $2^{\mathrm{e}}$ aufl, 99, 103. Pl. 8, fig. 13.

1885. Seudder. Zittel, Handb. palaeont., i, th. ii : 792. Fig. 1020.

1886. “ Zittel-Barrois, Traité de paléont., ii : 792. Fig. 1038.

1434. Chrysomelites rothenbachi. (Chrysomelidæ.)

Locality : Rutihard, Basel, Switzerland. Horizon: Trias.

1877. Heer. Flora foss. helvet., 76. Pl. 27, figs. $9 b, 9$ c.

1435. Cistelites insignis. (Cistelidæ.)

Locality : Schambelen, Switzerland. Horizon: Lias.

1865. Heer. Urwelt der Schweiz, 89. Pl. 8, fig. 11.

1872. " Monde prim. Suisse, 109. Pl. 8, fig. 11.

1876. " Prim. world Switz., i: 89. Pl. 8, fig. 11.

1879. " Urwelt der Schweiz, $2^{\circ}$ anfl, 99. Pl. 8, fig. 11.

1885. Scudder. Zittel, Handb. palaeont, i, th. ii : 791. Fig. 1016.

1886. “ "Zittel-Barrois, Traité de paléont., ii: 791. Fig. 1034.

1886. “ Bull. U. S. geol, surv., No. 31: 70.

1436. Clytus -. (Cerambycidæ.)

Locality: Welschufe, Saxony. Horizon: Quadersandstein.

1856. Giebel. Insect. d. vorwelt, 129.

See also Cerambycites — Geinitz.

1437. Cocoinella -. (Coccinellidæ.)

Locality : Eyeford, England. Horizon: Stonesfield Slate.

1845. Murchison. Geol. Cheltenham, 68. Pl.. 4,- fig. 1.

1438. Coccinella —. (Coccinellidæ.)

Locality : England. Horizon: Upper Lias.

1845. Murchison. Geol. Cheltenham, 108. Pl. 8, fig. 5. 
1439. Cocoinella heydeni. (Coccinellidæ.)

Locality: Solenhofen, Bavaria. Horızon: Jura.

1869. Weyenbergh. Arch. mus. Teyl, ii : 288. Pl. 36, figs. 32, 32a.

1869. " " Ins. foss. calc. lithogr., 42. Pl. 3, figs. 32, 32a.

1869. " " Tijdschr. v. ent., (2), iv : 233 [133].

1874. " "

1874. “ Énum. syst. faune ent. mósoz., 19.

1440. Coccinella neptuni. (Coccinellidæ.)

Locality : Durdlestone Bay, England. Horizon : Lower Purbecks.

1854. Westwood. Quart. journ. geol. soc. Lond., x: 394 (unnamed). Pl.16, fig. 9. 1856. Giebel.

1885. Scudder. Zittel, Handb. palaeont., i, th. ii : 800. Fig. 1055.

1886. " "Zittel-Barrois, Traité de paléont., ii : 800. Fig.1072.

1441. Coccinella wittsii. (Coccinellidæ.)

Locality : Sevenhampton, England. Horizon: Stonesfield Slate.

1845. Brodie. Foss. ins. sec. rocks Engl., 48. Pl. 6, fig. 21.

See also Pimelia wittsi.

1442. Colymbetes —. (Dytiscidæ.)

Locality : Vale of Wardour, England. Horizon: Purbecks.

1845. Brodie. Foss. ins. sec. rocks Engl., 32 (Westw., 117). Pl. 6, fig. 5. See also Hydrophilus brodiei.

1443. Colymbetes arcuatus. (Dytiscidæ.)

Locality: Aargau, Switzerland. Horizon: Lias.

1852. Heer. Lias-insel Aargau, 12. Pl., figs. 4-5.

1856. Giebel. Insect. d. vorwelt, 497.

1444. Crypticus ungeri. (Tenebrionidæ.)

Locality : Durdlestonē Bay, Eugland. Horizon : Lower Purbecks.

1854. Westwood. Quart. journ. geol. soc. Lond., $\mathrm{x}$ : 393 (unuamed). Pl. 14, fig. 15. 1856. Giebel. Insect. d. vorwelt, 110.

1445. Cryptocephalus antiquus. (Chrysomelidæ.)

Locality: Solenhofen, Bavaria. Horizon: Jura.

1869. Weyenbergh. Arch. mus. Teyl., ii : 286. Pl. 36, fig. 38.

1869. " " Ins. foss. calc. lithogr., 40. Pl. 3, fig. 38.

1869. " " Tijdschr. v. ent., (2), iv: 233 [133].

1874. " " Periód. zool., i: 104.

1874. " "Enum. syst. faune ent. mésoz., 18.

1446. Cryptocephalus mesozoicus. (Chrysomelidæ.)

Locality: Solenhofen, Bavaria. Horizon: Jura.

1869. Weyenbergh. Arch. mus. Teyl, ii: 286. Pl. 36, fig. 37.

1869. " " Ins. foss. calc. lithogr., 40. Pl. 3, fig. 37.

1869. " " Tijdschr. v. ent., (2), iv: 233 [133].

1874. " " Periód, zool., i: 105.

1874. “ Énum. syst. faune ent. mésoz., 19.

1888. Oppenheim. Palaeontogr., xxxiv: 242-243. Pl. 31, fig. 24. 
1447. Ctenicerium blissus. (Elateridæ.)

Locality : Durdlestone Bay, England. Horizon: Lower Purbecks.

1854. Westwood. Quart. journ. geol, soc. Lond., $\mathrm{x}$ : 389, 395. Pl. 16, fig. 36.

1448. Ctenicerium hylastes. (Elateridæ.)

Lo zality: Durdlestone Bay, England. Horizon: Lower Purbecks.

1854. Westwood. Quart. journ. geol. soc. Lond., x: 389, 395. Pl. 16, fig. 37.

1449. Curculio - (leg). (Cureulionidæ.)

Locality: Stonesfield, England. Horizon: Stonesfield Slate.

1837. Buckland. Geol. and mineral., ii : 78. Pl. $46^{\prime \prime}, f i g .10$.

1833. " "Géologie, éd. Agassiz, ii : expl.pl. 46", pp.4-5. Pl. 46", fig. 10.

1450. Curculionidium - - (Curculionidæ.)

Localities: Stonesfield, Eyeford, England. Horizon: Stonésfield Slate.

1871. Phillips. Geology of Oxford, 173. Digr. 34.

1451. Curculionites carlsoni. (Cureulionidæ.)

Locality: Bjuf, Sweden. Horizon: Rhætic.

1878. Heer. Förhandl. geol. fören. Stockh., iv: 195. Pl. 13, fig. 4, 4b, $4 c$.

1452. Curculionites cretaceus. (Curculionidæ.)

Locality : Kome, North Greenland. Horizon: Cretaceous.

1874. Heer. Kreide-flora arct. zone (K. svensk. vetensk.-akad. handl., xiiFlora foss. arct., 3), 92. $P l .17, f i g .14$.

1883. " Flora foss. grönl., ii : 143.

1453. Curculionites liasinus. (Curculionidæ.)

Locality: Aargan, Switzerland. Horizon: Lias.

1852. Heer.

1856. Giebel.

Lias-insel Aargau, 15. Pl., figs. 39-40.

Insect. d. vorwelt, 498.

1454. Curculionites parvulus. (Curculionidæ.)

Locality: Sofiero, Sweden. Horizon: Rhætic.

1878. Heer. Förhandl. geol. fören. Stockh., iv: 195. Pl. 13, figs. $3,3 b, 3 c$.

1455. Curculionites prodromus. (Curculionidæ.)

Locality: Vadutz, Liechtenstein. Horizon: Trias.

18ธิ3. Heer.

1853. "

1865. “

1872. “

1876. “

1879. “

1885. Scudder.

1886. " "

1886. “
Beschr. angeführt. pflanz. u. ins., 20-21. $\quad P l .7, f i g .13$.

Esch., Geol. bemerk. (Neue denkschr. schweiz. gesellsch., xiii): 134-135. Pl. 7, fig. 13.

Urwelt der Schweiz, 56, 82.

Monde prim. Suisse, 68, 99.

Prim. world Switz., i : 56, 82 .

Urwelt der Schweiz, $2^{\mathrm{e}}$ anfl., 65.

Zittel, Handb. palaeont., i, th. ii : 783. Fig. 1007.

Zittel-Barrois, Traité de paléont., ii : 788. Fig. 1024.

Bull, U. S. geol. surv., No. 31: 66 .

1456. Curculionites striatus. (Curculionidæ.)

Locality: Eichstadt, Bavaria. Horizon: Jura.

1888. Oppenheim. Palaeontogr., xxxiv: 241 (striata). Pl. 31, fig. 27. 
1457. Curculionites syrichthus. (Curculionidæ.)

Locality: Durdlestone Bay, England. Horizon : Lower Purbecks.

1856. Giebel. Insect. d. vorwelt, 147 .

See also Curculium syrichthus.

1458. Curculionites tuberculatus. (Curculionidæ).

Locality: Durdlestone Bay, England. Horizon : Lower Purbecks.

1854. Westwood. Quart. journ. geol. soc. Lond., $\mathrm{x}: 395$ (unnamed). Pl. 16, fig. 33. 1856. Giebel. Insect. d. vorwelt, 148.

\section{Curculionites westwoodi. (Cureulionidæ.)}

Locality: Durdlestone Bay, England. Horizon: Lower Purbecks.

1854. Westwood. Quart. journ. geol. soc. Lond., x : 395 (unnamed). Pl. 16, fig. 12. 1856. Giebel. Insect. d. vorwelt, 147.

1460. Curculium syrichthus. (Curculionidæ.)

Locality: Durdlestone Bay, England. Horizon: Lower Purbecks.

1854. Westwood. Quart.journ. geol. soc. Lond., x: 387,393. Pl. 14, fig. 3.

See also Curenlionites syrichthus.

1461. Cycloderma deplanatum. (Trogositidæ.)

Urwelt der Schweiz, 89. Pl. 8, fig. 4.

Monde prim. Suisse, 108. Pl. 8, fig. 4.

1876. " " Prim. world Switz., i: 88. Pl. 8, fig. 4.

1879. " Urwelt der Schweiz, $2^{\mathrm{e}}$ aufl, 99. Pl. 8, fig. 4.

1885. Scudder. Zittel, Handb. palaeont., i, th. ii : 799. Fig. 1043.

1886. " Zittel-Barrois, Traité de paléont., ii: 799. Fig. 1060.

1462. Cymindis antiqua. (Carabidæ.)

Locality : Vale of Wardour, England. Horizon: Purbecks.

1854. Brodie. Foss. ins. sec. rocks Engl., 117 (unnamed). Pl. 6, fig. 10.

1856. Giebel. Insect. d. vorwelt, 69.

1463. Cymindis beyrichi. (Carabidæ.)

Locality : Durdlestone Bay, England. Horizon: Lower Purbecks.

1854. Westwood. Quart. journ. geol. soc. Lond., $\mathrm{x}: 395$ (unnamed). Pl. 16, fig. 16. 1856. Giebel. Insect. d. vorwelt, 68-69.

1464. Cyphon —. (Dascyllidæ.)

Locality: Vale of Wardour, England. Horizon: Purbecks.

1845. Brodie. Foss. ins. sec. rocks Engl., 32 (Westw., 116). Pl. 3, fig. 3.

See also Cyphon vetustus.

1465. Cyphon vetustus. (Dascyllidæ.)

Localities: Vale of Wardour, England; Dobbertin, Germany. Horizon: Lias.

1856. Giebel. Insect. d. vorwelt, 100.

1884. E. Geinitz, Zeitschr. deutsch. geol. gesellsch., 1884 : 583.

1885. Scudder. Zittel, Handb. palaeont., i, th. ii: 798, Fig. 1038.

1886. " Zittel-Barrois, Traité de paléont., ii : 798. Fig. 1056.

See also Cyphon — Brodie. 
1466. Diaperidium mithrax. (Tenebrionidæ.)

Locality : Durdlestone Bay, England. Horizon: Middle Purbecks.

1854. Westwood. Quart. journ. geol. soc. Lond., x: 383, 394. Pl. 15, fig. 8, righthand fig.

\section{Ditomoptera dubia.}

Locality: Solenhofen, Bavaria. Horizon: Jura.

1839. Germar. Verhandl, leop.-carol. akad, naturf, xix : 203-205 (Hemipt.). Pl. 22, fig. 5.

1852. Giebel. Deutschl. petref., 636.

1862. Hagen. Palaeontogr., $\mathrm{x}: 110$ (Coleoptera).

1869. Weyenbergh. Tijdschr. v. ent., (2), iv : 233 [133].

1877. Assmann. Ber. versamml, deutsch. naturf., 1: 192 (Coleopt.).

1888. Oppenheim. Palaeontogr., xxxiv: 240. Pl. 31, fig. 8.

See also Cerambycites dubius.

1468. Dytiscus - (Dytiscidæ).

Locality : Dorset, England. Horizon: Middle Purbecks.

1854. Westwood. Quart. journ. geol. soc. Lond., x: 282 (unnamed). Pl. 15, fig. 13. 1856. Giebel.

Insect. d. vorwelt, 58 .

1469. Dytiscus lentissimus. (Dysticidæ.)

Locality: Solenhofen, Bavaria. Horizon: Jura.

1874. Weyenbergh. Period. zool., i : 101 (undescribed).

1874. " " Énum. syst. faune ent. mésoz., 15 (undescribed).

Elater or Buprestis —. See Buprestis (or Elater) — Brodie.

1470. Elater angulatus. (Elateridæ.)

Localities : Wainlode, Apperley, Brockeridge, Churchdown, Aust, England. Horizon : Lower Lias.

1845. Brodie. $\quad$ Foss. ins. sec. rocks Engl. (unnamed). Pl. 6, fig. 25.

1856. Giebel. Insect. $d$. vorwelt, 92 .

1471. Elater costeri. (Elateridæ.)

Locality: Solenhofen, Bavaria. Horizon: Jura.

1869. Weyenbergh. Arch. mus. Teyl., ii : 284-285. Pl. 37, fig. 43.

1869. " Ins. foss. calc. lithogr., 38-39. Pl. 4, fig. 43.

1869. " " Tijdschr. v. ent., (2), iv: 232 [132].

1874. " " Period. zool., i : 104.

1874. " E Énum. syst. faune ent. mésoz., 18.

1472. Elater grossus. (Elateridæ.)

Locality: Solenhofen, Bavaria. Horizon: Jura.

1869. Weyenbergh. Arch.mus. Teyl, ii : 285 . Pl. 37, fig. 42.

1869. " " Ins. foss. cale. lithogr., 39. Pl. 4, fig. 42.

1869. “ "Tijdschr. v. ent., (2), iv: 232 [132].

1874. " "

1874. " Enum. syst. faune ent. mésoz., 18.

1473. Elater morrisi. (Elateridæ.)

Locality: Durdlestone Bay, England. Horizon: Lower Purbecks.

1854. Westwood. Quart, journ, geol, soc, Loud, $\mathrm{x}$ : 395 (nnnamed), Pl. 16, fig. 36.

1856. Giebel. Insect. $d$, vorwelt, 93 . 
1474. Elater neptuni. (Elateridæ).

Locality : England. Horizon : Lower Lias.

1845. Brodie.

1856. Giebel.

Foss. ins. sec. rocks Engl. (unnamed). $\quad P l .7$, fig. 3.

Insect. d. vorwelt, 91 .

1475. Elater oweni. (Elateridæ.,

Locality: Durdlestone Bay, England. Horizon: Lower Purbecks.

1854. Westwood. Quart. journ. geol, soc. Lond., x: 395 (unnamed). Pl. 16, fig. 37, 1856. Giebel.

Insect. d. vorwelt, 93 .

1476. Elater purbeccensis. (Elateridæ.)

Locality: Vale of Wardour, England. Horizon : Purbecks.

1845. Brodie. Foss. ins. sec. rocks Engl. 32 (unnamed). Pl. 6, fig. 6. 1856. Giebel. Insect. d. vorwelt, 92.

1477. Elater redtenbacheri. (Elateridæ.)

Localities: Wainlode, Apperley, Brockeridge, Chnrehdown, Aust, England.

Horizon: Lower Lias.

1845. Brodie. Foss. ins. sec. rocks Engl. (unnamed). Pl. 6, fig. 26.

1856. Giebel. Insect. d: vorwelt, 92 .

1478. Elater socius. (Elateridæ.)

Locality: Apperley, England. Horizon: Lower Lias.

1845. Brodie. Foss. ins. sec. rocks Engl., 101 (unnamed). Pl. 7, fig. 2.

1856. Giebel. Insect. $d$. vorwelt, 91.

1479. Elater teyleri. (Elateridæ.)

Locality: Solenhofen, Bavaria. Horizon: Jura.

1869. Weyenbergh. Arch. mus. Teyl., ii : 284. Pl. 37, figs. 44, 44a.

1869. " Ins. foss. calc. lithogr., 38. Pl. 4, figs. 44, 44a.

$1869 . \quad$ " Tijdschr. v. ent., (2), iv : 232 [132].

1874. " " Period. zool., i: 103.

1874. “ Énum. syst. faune ent. mésoz., 17.

1480. Elater vanus. (Elateridæ.)

Localities: Wainlode, Apperley, Brockeridge, Churchdown, Aust, England. Horizon: Lower Lias.

1845. Brodie. Foss. ins. sec. rocks Engl. (unnamed). Pl. 6, fig. 23.

1856. Giebel. Insect. d. vorwelt, 92.

1481. Elater vetustus. (Elateridæ.)

Locality: England. Horizon: Lower Lias, Insect Limestone,

1845. Brodie.

Foss. ins. sec. rocks Engl., 101. Pl. 7, fig. 1.

1856. Giebel.

Insect. d. vorwelt, 91 .

1871. Phillips.

Geology of Oxford, 123.

See also Elaterites vetustus.

1482. Elater werneri. (Elateridæ.)

Locality : Vale of Aylesbury, England. Horizon: Purbecks. 
1483. Flaterites priscus. (Elateridæ.)

Locality: Eichstadt, Bavaria. Horizon: Jura.

1888. Oppenheim. Palaeontogr., xxxiv: 241. Pl. 31, fig. 5.

1484. Elaterites robustus. (Elateridæ.)

Locality : Eichstadt, Bavaria. Horizon : Jura.

1888. Oppenheim. Palaeontogr., xxxiv: 241. Pl. 31, fig. 22.

1485. Elaterites sibiricus. (Elateridæ.)

Locality: Tapka near Irkutsk, Siberia. Horizon: Jura.

1876. Heer. M6́m. acad. sc. St.-Pétersb, xxii, No. 12: 41. Pl. 22, figs.9e,9ee. 1877. " " Flora foss. aret., 4, ii : 41. Pl.22, figs, 9e,9ee.

1486. Flaterites vetustus. (Elateridæ.)

Compared with Ampedus sanguineus Fabr.

Localities: Schambelen, Switzerland; Dobbertin, Germany. Horizon : Lias.

1865. Heer. Urwelt der Sehweiz, 88,95. Pl. 7, fig. 21.

1872. " Monde prim. Suisse, 108, 116. Pl. 7, fig. 21.

1876. " $"$ Prim. world Switz., i: 88,94. Pl. 7, fig. 21.

1879. " Urwelt der Schweiz, $2^{\mathrm{e}}$ aufl., $98,105 . \quad$ Pl.7, fig. 21.

1880. E. Geinitz. Zeitschr. deutsch. geol. gesellsch,, 1880: 530 (cfr.). Pl.22, fig.19.

1884. " Zeitschr. deutsch. geol, gesellsch., 1884: 583.

See also Elater vetustus.

1487. Elaterium barypus. (Elateridø.)

Locality: Durdlestone Bay, England. Horizon: Lower Purbecks.

1854. Westwood. Quart. journ. geol. soc. Lond., x: 389, 394-395. Pl. 16, fig. 5.

1856. Giebel. Insect. d. vorwelt, 93.

1488. Elaterium pronæus. (Elateridæ.)

Locality: Durdlestone Bay, England. Horizon: Lower Purbecks 1854. Westwood. Quart. journ. geol. soc. Lond., $\mathrm{x}: 387,393 . \quad P l .14$, fig. 1. 1856. Giebel. Insect. $d$. vorwelt, 92-93.

1489. Elaterium triopas. (Elateridæ.)

Locality: Durdlestone Bay, England. Horizon: Lower Purbecks. 1854. Westwcod. Quart.journ. geol, soc. Lond., $\mathrm{x}:$ 389, 394-395. Pl. 16, fig. 4. 1856. Giebel. Insect. d. vorwelt, 93.

1490. Elateropsis infraliassica. (Elateridæ.)

- Locality : Krälah vic. Hildesheim, Germany. Horizon : Rhætic. 1876. Roemer. Zeitschr. deutsch. geol. gesellsch., xxviii : 351. Figs. 1,1a. 1885. Scudder, Zittel, Handb. palaeont., i, th. ii : 797. Fig. 1036. 1886. " Zittel-Barrois, Traité de paléont., ii : 798. Fig. 1054.

1491. Elmis brodiei. (Parnidæ.)

Locality: Vale of Wardour, England. Horizon: Purbecks.

1856. Giebel.

Insect. d. vorwelt, 50.

See also Limnius _- Brodie. 
1492. Elytridium angelini.

Locality : Kulla Gunnarstorp, Sweden. Horizon : Rhætic.
1878. Heer.
Förhandl. geol. fören. Stockh,, iv: $196 . \quad$ Pl. 13, figs. 5, 5 b.

\section{Elytridium lævigatum.}

Locality : Höganäs, Sweden. Horizon: Rhætic.

1878. Heer. Förhandl. geol. fören. Stockh., iv: 196. Pl.13, figs. 6,6b.

\section{Elytridium multipunctatum.}

Locality : Ivnanguit, Greenland. Horizon: Komeschichten, Cretaceous. 1883. Heer.

Flora foss. grönl., ii : 143-144. " $P l .109$, fig. 5.

\section{Euchroma liasina. (Buprestidæ.)}

Compared with E. gigantea.

Locality : Schambelen, Aargau, Switzerland. Horizon: Lias.

1852. Heer.

1854. Pictet.

1856. Giebel.

1865. Heer.

1872. "6

1876. “

1879. “
Lias-insel Aargau, 13. Pl., figs. 18, 19.

Traité de paléont., $2^{\mathrm{e}}$ ed., ii : $331 . \quad \mathrm{Pl} .40, \mathrm{fig} .3$.

Insect. d. vorwelt, 498.

Urwelt der Schweiz, 88. $\quad P l .7$, fig. 10.

Monde prim. Suisse, 107. Pl. 7, fig. 10.

Prim. world Switz., i: 87. Pl. ${ }^{\star}$, fig. 10.

Urwelt der Schweiz, 2e autl., 98. Pl. 7, fig. 10.

1496. Eumolpites liberatus. (Chrysomelidæ.)

Locality: Schambelen, Switzerland. Horizon: Lias.

1865. Heer.

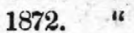

1876. "

1879. “
Urwelt der Schweiz, 89. $\quad P l .8, f i g .14$. .

Moude prim. Suisse, 109. $\quad P l .8$, fig. 14.

Prim. world Switz., i: 89. Pl. 8, fig. 14.

Urwelt der Schweiz, $2^{\ominus}$ aufl, 99. Pl. 8, fig. 14.

1497. Eurythyrea grandis. (Buprestidæ.)

Compared with E. longipennis Heer (Tert.).

Locality : Eichstadt, Bavaria. Horizon: Jura.

1886. Deichmüller. Ins, lithogr. schiefer dresd. mus., 70. Pl. 5, fig. 14.

1888. Oppenheim. Palaeontogr., xxxiv: 240. Pl. 31, fig. 9.

1498. Feronites velenovskyi. (Carabidæ.)

Locality : Pernckých, Bohemia. Horizon: (Cretaceons?)

1884. Friě.

Vesmír, xiii : 205-206. Fig. 8.

1499. Galerucites carinatus. (Chrysomelidæ.)

Locality: Kelheim, Bavaria. Horizon: Jura.

1888. Oppenheim. Palaeontogr., xxxiv : 242 (carinata). Pl. 31, fig. 26.

1500. Geotrupes lithographicus. (Scarabæidæ.)

Compared with G. mutator.

Locality: Eichstadt, Bavaria. Horizon: Jura.

1886. Deichmüller. Ins. lithogr, schiefer dresd. mus., 69. Pl. 5, fig. 13. 
1501. Glaphyroptera brevicollis. (Buprestidæ.)

1865. Heer.

1872. " "

1876. “

1879. “

1852. Heer.

1856. Giebel.

Locality : Schambelen, Switzerland. Horizon: Lias.

Urwelt der Schweiz, 88. Pl. 7, fig. 15.

Monde prim. Suisse, 107. Pl. 7, fig. 15.

Prim. world Switz., i: 87. $\quad P l .7$, fig. 15.

Urwelt der Schweiz, 2e aufl., 98. Pl. 7, fig. 15.

\section{Glaphyroptera depressa. (Buprestidæ.)}

Locality: Aargan, Switzerland. Horizon: Lias.

\section{Lias-insel Aargau, 14. Pl., figs. 23-25*.}

Insect. d. vorwelt, 498.

1503. Glaphyroptera gehreti. (Buprestidæ.).

Localities: Schambelen, Aargau, Switzeriand ; Dobbertin, Mecklenberg. Horizon : Lias.

1852. Heer.

1856. Giebel.

1865. Heer.

1872. “

1876. “

1879. “

1884. E. Geinitz.
Lias-insel Aargau, 14. Pl., figs. 25a, 26-28.

Insect. d. vorwelt, 498.

Urwelt der Schweiz, 88. Pl. 7, fig. 12.

Monde prim. Suisse, 107. Pl. 7, fig. 12.

Prim. world Switz., i: 87. Pl. 7, fig. 12.

Urwelt der Schweiz, $2^{\mathrm{e}}$ aufl, 98. Pl. 7, fig. 12.

Zeitschr. dentsch, geol. gesellsch., 1884 : 583.

1504. Glaphyroptera gracilis. (Buprestidæ.)

Locality : Schambelen, Aargau, Switzerland. Horizon: Lias.

1852. Heer.

1856. Giebel.

1865. Heer.

1872. " "

1876. “

1879. “

Lias-insel Aargan, 14. Pl., figs. 29-32.

Insect. d. vorwelt, 498.

Urwelt der Schweiz, 95. Pl. 7, fig. 14.

Monde prim. Suisse, 116. $\quad P l .7$, fig. 14.

Prim. world Switz., i: 94. Pl. 7, fig. 14.

Urwelt der Schweiz, 2e aufl, 105. Pl. 7, fig. 14.

1505. Glaphyroptera insignis. (Buprestidæ.)

Locality: Schambelen, Switzerland. Horizon: Lias.

1852. Heer.

1856. Giebel.

1865. Heer.

1872. “

1876. " "

1879. “
Lias-insel Aargau, 13-14. $\quad P l$., figs. 20-22.

Insect. d. vorwelt, 498.

Urwelt der Schweiz, 88. Pl. 7, fig. 13.

Monde prim. Suisse, 107. Pl. 7, fig. 13.

Prim. world Switz., i: 87. Pl. 7, fig. 13.

Urwelt der Schweiz, 2e aufl., 98. Pl. 7, fig. 13.

1506. Glaphyroptera pterophylli. (Buprestidæ.)

Locality : Vadutz, Liechtenstein. Horizon: Keuper, Trias.

1853. Heer.

1853. " "

1865. “

1872. " "

1876. " "

1885. Scudder.

1886. “
Esch., Geol. bemerk. (Neue denkschr. allg. schweiz. gesellsch. ges. naturw., xiii): 133-134. Pl. 7, figs. 11a-c, 12.

Beschr. angeführt. pflanz. u. ins., 19-20. Pl. 7, figs. 11a-c, 12.

Urwelt der Schweiz, 82.

Monde prim. Suisse, 99.

Prim. world Switz., 82.

Zittel, Handb. palaeont., i, th. ii : 796. Fig. 1033.

Zittel-Barrois, Traité de paléont., ii : 797. Fig. 1051.

See also Buprestites pterophylli. 
1507. Glaphyroptera spectabilis. (Buprestidæ.)

Locality: Schambelen, Switzerland. Horizon: Lias.

1865. Heer.

1872. " "
1876. "

1879. “

Urwelt der Schweiz, 88.

Monde prim. Suisse, 107.

Prim. world Switz, i : 87.

Urwelt der Schweiz, $2^{2}$ aufl., 98.

1508. Gyrinites antiquus. (Gyrinidæ.)

Locality: Schambelen, Switzerland. Horizon: Lias.

1865. Heer.

Urwelt der Schweiz, 91. Pl. 8, fig. 20.

1872. " Monde prim. Suisse, 110. Pl. 8, fig. 20.

1876. " " Prim. world Switz, i: 90. Pl. 8, fig. 20.

1879. “ Urwelt der Schweiz, $2^{\mathrm{e}}$ anfl., 101. Pl. 8, fig. 20.

1885. Scudder. - Zittel, Handb. palaeont, i, th.ii: 802. Fig. 1065.

1886. " Zittel-Barrois, Traité de paléont, ii : 803. Fig. 1082.

1509. Gyrinites minimus. (Gyıinidæ.)

Localities: Schambelen, Switzerland; Dobbertin, Mecklenburg. Horizon : Lias.

1865. Heer. Urwelt der Schweiz, 91. Pl. 8, fig. 21.

1872. " Monde prim. Suisse, 110. Pl. 8, fig. 21.

1876. " $"$ Prim. world Switz., i: 90. Pl. 8, fig. 21.

1879. “ Urwelt der Schweiz, $2^{\mathrm{e}}$ aufl., 101. Pl. 8, fig. 21.

See also Gyrinus minimus.

1510. Gyrinites troglodytes. (Gyrinidæ.)

Locality : Aargau, Switzerland. Horizon: Lias.

1852. Heer. Lias-insel Aargau, 12. Pl., figs. 6-7.

1856. Giebel. Insect. d. vorwelt, 497.

1865. Heer. Urwelt der Schweiz, 91. Pl. 8, fig. 19.

1872. " Monde prim. Suisse, 110. Pl. 8, fig. 19.

1876. " $\quad$ Prim. world Switz., i: 90 . Pl. 8, fig. 19.

1879. " Urwelt der Schweiz, $2^{\mathrm{e}}$ aul., 101. Pl. 8, fig. 19.

See also Gyrinus troglodytes.

1511. Gyrinus

(Gyrinidæ.)

Locality: Norton, England. Horizon: Lower Lias.

1873. Brodie. Distr. corr. foss. ins., 16 ("like a foreign species").

1512. Gyrinus

(Gyrinidæ.)

Locality : England. Horizon: Lias.

1871. Phillips. Geology of Oxford, 123.

1513. Gyrinus antiquus. (Gyrinidæ.)

Locality: Schambelen, Switzerland. Horizon: Lias.

1865. Heer.

Urwelt der Schweiz, 91. Pl. 8, fig: 20.

1872. " "

1876. “

1879. “

Monde prim. Suisse, 110. Pl. 8, fig. 20.

Prim. world Switz., i: 90. Pl. 8, fig. 20.

Urwelt der Schweiz, 2e aufl., 101. Pl. 8, fig. 20.

1514. Gyrinus atavus. (Gyrinidæ.)

Locality: Schambelen, Switzerland. Horizon: Lias.

1865. Heer.

Urwelt der Schweiz, 64, 90. $\quad$ Pl. 8, fig. 18.

1872. “

1876. “

Monde prim. Suisse, 78, 110. Pl. 8, fig. 18.

Prim. world Switz., i: 64, 90. Pl. 8, fig. 18.

1879. “

Urwelt der Schweiz, $2^{\circ}$ anfl., 73, 100. Pl. 8, fig. 18. 
1515. Gyrinus dubius. (Gyrinidæ.)

- Locality: England, Horizon: Lower Lias.

1845. Brodie. 1856. Giebel.

Foss. ins. sec. rocks Engl. (unnamed). Pl. 7, fig. 6.

Insect. $d$. vorwelt, 56 .

1516. Gyrinus juranus. (Gyrinidæ.)

Locality: Solenhofen, Bavaria. Horizon: Jura.

1869. Weyenbergh. Tijdschr. v. ent., (2), iv : 231 [131].

See also G. jurassicus.

1517. Gyrinus jurassicus. (Gyrinidæ.)

Locality: Solenhofen, Bavaria. Horizon: Jura.

1869. Weyenbergh. Arch. mus. Teyl., ii : 2s0. Pl. 37, figs. 53, 53a.

1869. " Ins. foss. calc. lithogr., 34. Pl. 4, figs. 53, $53 a$.

1874. " " Periód. zool., i: 102.

1874. " "Énum. syst. faune ent. mésoz., 16.

See also G. juranus,

1518. Gyrinus minimus. (Gyrinidæ.)

Locality : Dobbertin, Mecklenburg. Horizon: Lias.

1884. E. Geinitz. Zeitschr. deutsch. geol, gesellsch., 1884: 583 ,

See also Gyrinites minimus.

\section{Gyrinus natans. (Gyrinidæ.)}

Locality : Forthampton, England. Horizon: Lower Lias.

1845. Brodie.

1856. Giebel.

Foss. ins. sec. rocks Engl, 101, 123. Pl. 7, fig. 5.

Insect. d. vorwelt, 56 .

1520. Gyrinus troglodytes. (Gyrinidæ.)

Locality: Schambelen, Switzerland. Horizon: Lias.

1865. Heer. Urwelt der Schweiz, 64.

1872. " Monde prim. Suisse, 78 .

1876. " Prim. world Switz., i: 64.

1879. " Urwelt der Schweiz, $2^{e}$ auf., 73.

See also Gyrinites troglodytes.

\section{Haltica? - - (Chrysomelidæ.)}

Locality : Durdlestone Bay, England. Horizon: Lower Purbecks.

1854. Westwood. Quart. journ. geol. soc. Lond., x : 389, 396. Pl. 18, fig. 12.

1522. Harpalidium anactus. (Carabidæ.)

Locality : Durdlestone Bay, England. Horizon: Lower Purbecks.

1854. Westwood. Quart. journ. geol. soc. Lond., x: 386, 393. Pl. 14, fig. 20. See also Harpalus anactus.

1523. Harpalidium nothrus. (Carabidæ.)

Locality: Durdlestone Bay, England. Horizon : Lower Purbecks.

1854. Westwood. Quart. journ. geol. soc, Lond., $\mathrm{x}: 386,395 . \quad$ Pl. 17, fig. 3.

1856. Giebel. Inseet. d. vorwelt, 63.

Bull $71-14$ 


\section{Harpalus ——. (Carabidæ.)}

Locality : Durdlestone Bay, England. Horizon: Lower Purbecks.

1854. Westwood. Quart. journ. geol. soc. Lond., x : 393 (unnamed). Pl. 14, fig. 16. 1856. Giebel.

Insect. d. vorwelt, 64 .

\section{Harpalus anactus. (Carabidæ.)}

Locality: Durdlestone Bay, England. Horizon: Lower Purbecks. 1756. Giebel. Insect. d. vorwelt, 63.

See also Harpalidium anactus.

1526. Harpalus burmeisteri. (Carabidæ.)

Locality: Durdlestone Bay, England. Horizon : Lower Purbecks.

1854. Westwood. Quart. journ. geol, soe. Lond., $\mathrm{x}$ : 385 (unnamed). Pl. 24, fig. 14. 1856. Giebel.

Insect. d. vorwelt, 63.

\section{Harpalus ewaldi. (Carabidæ.)}

Locality: Durdlestone Bay, England. Horizon: Lower Purbecks.

1854. Westwood. Quart. journ. geol. soc. Lond., $\mathrm{x}$ : 393 (unnamed). Pl. 16, fig. 21. 1856. Giebel. Insect. $d$. vorwelt, 62 .

1528. Harpalus heeri. (Carabidæ.)

Locality : Hasfield, England. Horizon: Lias.

1845. Brodie.

Foss. ins. sec. rocks Engl. (unnamed). Pl. 6, fig. 27.

Insect. $d$. vorwelt, 63 .

1529. Harpalus knorri. (Carabidæ.)

Locality : Durdlestone Bay, England. Horizon : Lower Purbecks.

1854. Westwood. Quart. journ. geol. soc. Lond., x: 395 (unnamed). Pl. 16, fig. 20. 1856. Giebel.

Insect. d. vorwelt, 62 .

\section{Harpalus liasinus. (Carabidæ.)}

Locality: Aust, England. Horizon: Lias.

1845. Brodie. 1856. Giebel.

Foss. ins. sec. rocks Engl. (unnawed). $\quad$ Pl. 9, fig. 11.

Insect. d. vorwelt, 62 .

\section{Harpalus schlotheimi. (Carabidæ.)}

Localities: Apperley, Brockeridge, England. Horizon: Lias.

1845. Brodie.

Foss. ins. sec. rocks Engl. (nnnamed). Pl. 6, fig. $28 ;$ Pl. 10, fig. 2. 1856. Giebel.

Insect. d. vorwelt, 63 .

1532. Helophorus —. (Hydrophilidæ.)

Locality: Vale of Wardour, England. Horizon: Purbecks.

1845. Brodie.

Foss. ins. sec. rocks Engl., $32 . \quad P l .3$, fig. 2.

1845. Westwood. Brodie, foss. ins. sec, rocks Eng., 116. Pl. 3, fig. 2.

See also H. brodiei.

1533. Helophorus antiquus. (Hydrophilidæ.)

Locality : Vale of Wardour, England. Horizon: Purbecks.

1845. Brodie. Foss. ins. sec. rocks Engl., 32 (unnamed). Pl. 6, fig. 13.

1856. Giebel. Insect. d. vorwelt, 51 . 
1534. Helophorus brodiei. (Hydrophilida.)

Locality : Vale of Wardour, England. Horizon: Purbecks.

1856. Giebel. Insect. d. vorwelt, 51.

See also Helophorus — Brodie.

1535. Helopides hildesiensis. (Tenebrionidæ.)

Locality : Krälah bei Hildesheim, Germany. Horizon : Rbaetic.

1876. Roemer. Zeitschr. deutsch. geol. gesellsch., xxviii : 351-352. Figs. 2, 2a.

1536. Helopium agabus. (Tenebrionidæ.)

Locality: Durdlestone Bay, England, Horizon: Lower Purbecks.

1854. Westwood. Quart. journ. geol. soc. Lond., $\mathrm{x}$ : 387,393. Pl. 14, fig. 2.

1856. Giebel. Insect. d. vorwelt, 111.

Helopium ncoridas. See Helopium neoridas.

1537. Helopidium neoridas. (Tenebrionidæ.)

Locality: Durdlestone Bay, England. Horizon: Lower Purbecks.

1854. Westwood. Quart. journ. geol. soc. Lond., x : 389-390, 395 (ncoridas). $P l$. 16, fig. 13.

1885. Scudder. Zitttel, Handb. palaeont, i, th. ii: 791. Fig. 1017.

1886. " Zittel-Barrois, Traité de paléont., ii : 792. Fig. 1035.

1538. Hister relictus. (Histeridæ.)

Locality: Solenhofen, Bavaria. Horizon: Jura.

1869. Weyenbergh. Arch. mus. Teyl., ii : 281. Pl. 37, fig. 50.

1869. " " Ins. foss. calc. lithogr., 35. Pl. 4, fig. 50.

1869. " " Tijdschr. v. ent., (2), iv : 232 [132].

1874. " " Periód. zool., i: 102.

1874. " "Énum. syst. fauné ent. mésoz., 16.

1539. Hydrobiites veteranus. (Hydrophilidæ.)

Locality: Schambelen, Switzerland. Horizon: Lias.

1865. Heer. Urwelt der Schweiz, 91,95. Pl. 8, fig. 23.

1872. “ Monde prim. Suisse, 111, 116. Pl. 8, fig. 23.

1876. " Prim. world Switz., i : 90,94. Pl. 8, fig. 23.

1879. “U Urwelt der Schweiz, $2^{e}$ anfl, 101, 105. Pl. 8, fig. 23.

See also Hydrobius veteranùs.

1540. Hydrobius purbeccensis. (Hydrophilidæ.)

Locality : Vale of Wardour, England. Horizon: Purbecks.

1845. Brodie.

Foss. ins. sec. rocks Engl., 32 (unnamed), Pl. 6, fig. 12.

1856. Giebel.

Insect. $d$. vorwelt, 52 .

1541. Hydrobius veteranus. (Hydrophilidæ.)

Locality: Aargau, Switzerland. Horizon: Lias,

1852. Heer. Lias-insel Aargau, 13. Pl., figs. 15-16.

1856. Giebel. Insect. d. vorwelt, 498.

See also Hydrobiites veteranus. 
1542. Hydrophilites acherontis. (Hydrophilida.)

Locality: Schambelen, Switzerland. Horizon: Lias.

1865. Heer.

1872. "

1876. “

1879. " "

Urwelt der Schweiz, 91, Pl. 8, fig. 25.

- Monde prim Suisse, 111. Pl. 8, fig. 25.

Prim. world Switz., i: 90. Pl. 8, fig. 25.

Urwelt der Schweiz, 2e aufl, 101. Pl. 8, fig. 25.

See also Hydrophilus acherontis.

\title{
1543. Hydrophilites interpunctatus. (Hydrophilidæ.)
}

1865. Heer.

187.. " "

1876. " "

1879. “

Locality : Schambelen, Switzerland. Horizon: Lias.

\author{
Urwelt der Schweiz, 64. \\ Monde prim. Suisse, 78. \\ Prim. world Switz., i: 64. \\ Urwelt der Schweiz, $2^{\mathrm{e}}$ anfl., 73.
}

1544. Hydrophilites nathorsti. (Hydrophilidæ.)

Locality: Bjuf, Sweden. Horizon: Rhaetic.

1878. Heer.

Förhandl. geol. fören. Stockh., iv : 193-194. Pl. 13, figs. 1, 1b, 1c.

1545. Hydrophilites stygius. (Hydrophilidæ.)

Localities: Schambelen, Switzerland; Dobbertin, Mecklenburg. Horizon : Lias. 1865. Heer.

Urwelt der Schweiz, 91. $\quad P l .8, f i g .24$.

1872. "6

Monde prim. Suisse, 111. $\quad P l .8$, fig. 24.

1876. “

Prim. world Switz., i: 90. Pl. 8, fig. 24.

1879. “

Urwelt der Schweiz, $2^{\mathrm{e}}$ aufl., 101. Pl. 8, fig. 24.

1884. E. Geinitz. Zeitschr, dentsch. geol. gesellseh., 1884 : 583.

1546. Hydrophilus

(Hydrophilidæ.)

Locality: England. Horizon: Lias.

1845. Brodie.

1856. Giebel.

Foss. ins. sec. rocks Engl., 124 (unnamed). $\quad P l .6$, fig. 30.

Insect. d. vorwelt, 55 .

\section{Hydrophilus —. (Hydrophilidæ.)}

Locality: Durdlestone Bay, England. Horizon: Middle Purbecks.

1854. Westwood. Quart. journ. geol. soc. Lond., $\mathrm{x}$ : 394 (unnamed). Pl.15, fig. 13. 1856. Giebel. Insect. $d$. vorwelt, 55 .

1548. Hydrophilus - (Hydrophilidæ.)

Locality: Durdlestone Bay, England. Horizon: Lower Purbecks.

1854. Westwood. Quart. journ. geol, soc. Lond, $\mathrm{x}: 394$ (unnamed). Pl. 16, fig. 7. 1856. Giebel. Insect. $d$. vorwelt, 55 .

1549. Hydrophilus acherontis. (Hydrophilidæ.)

Locality : Aargau, Switzerland. Horizon: Lias.

1852. Heer.

1854. Pictet.

1856. Giebel.
Lias-insel Aargau, 12-13. Pl., figs. 12-14.

Traité de paléont., $2^{e}$ éd., ii : $341 . \quad P l .40$, fig. 4

Insect. d. vorwelt, 498 .

See also Hydrophilites acherontis.

1550. Hydrophilus brodiei. (Hydrophilidæ.)

Locality : Vale of Wardour, England. Horizon : Purbecks.

1856. Giebel. Insect. d. vorwelt, 53.

See also Colvmbetes — Brodie. 
Hydrophilus deperditus. See II, Hemiptera, Idem.

1551. Hydrophilus westwoodi. (Hydrophilidæ.)

Locality: Vale of Wardour, England. Horizon: Purbecks.

1845. Brodie. Foss. ins. sec. rocks Engl., 32 (unnamed). Pl. 6, fig. 3 .

1856. Giebel. Insect, $d$, vorwelt, 53 .

1552. Hydroporus neptuni. (Dytiscidæ.)

. Locality: Durdlestone Bay, England. Horizon: Lower Purbecks.

1854. Westwood. Quart. journ. geol. soc. Lond., $\mathrm{x}$ : 395 (unnamed). Pl. 16, fig. 28. 1856. Giebel. Insect. d. vorwelt, 58.

1553. Hydroporus petrefactus. (Dytiscidæ.)

Locality: Solenhofen, Bavaria. Horizon : Jura.

1869. Weyenbergh. Arch. mus. Teyl., ii : 279-280. Pl. 37, fig. 54.

1869. " Ins. foss. calc. lithogr., 33-34. Pl. 4, fig. 54.

1869. " "Tijdschr.v. ent., (2), iv : 231 [131].

1874. " "Periód.zool., i: 101.

1874. “ Énum. syst. faune ent. mésoz., 15.

1554. Hypera antiqua. (Curculionidæ.)

Locality : Vale of Wardour, England. Horizon: Purbecks.

1845. Brodie. Foss. ins. sec. rocks Engl, 32 (unnamed). Pl. 6, fig. 4. (Giebel says Pl. 6, fig. 7; unquestionable mistake for $P l .6$, fig. 4.)

1856. Giebel. Insect. d. vorwelt, 140.

1555. Laccophilus aquaticus. (Dytiscidæ.)

Locality : Hasfield, England. Horizon: Lower Lias, Insect Limestone.

1845. Brodie. Foss. ins. sec. rocks Engl., 101, 124. Pl. 6, fig. 31.

1856. Giebel. Insect. d. vorwelt, 59.

1871. Phillips. Geology of Oxford, 123.

1556. Lacon petrosum. (Elateridæ.)

Locality : Solenhofen, Bavaria. Horizon: Jura.

1869. Weyenbergh. Arch. mus. Teyl., ii : 283-284. Pl. 37, fig. 45.

1869. " Ins. foss. calc. lithogr., 37-38. Pl. 4, fig. 45.

1869. “ " $\quad$ Tijdschr. v. ent., (2), iv : 232 [132].

1874. "

1874. "6 Énum. syst. faune ent. mésoz., 17.

1557. Lamia schrœteri. (Cerambycidæ.)

Locality: Durdlestone Bay, England. Horizon : Lower Purbecks.

1854. Westwood. Quart. journ. geol, soc. Lond., x: 395 (unnamed). Pl. 16, fig. 18. 1856. Giebel. Insect. d. vorwelt, 131.

1558. Lamiites simillimus. (Cerambycidœ.)

Locality: Vyšerovicích, Bohemia. Horizon: Cretaceous.

1888. Fritsch. Vesmír, xviii: 8. Fig. $5 \mathrm{~A}$.

1559. Lathridiites schaumii. (Lathridiidæ.)

Locality: Schambelen, Switzerland. Horizon: Lias.

1865. Heer. Urwelt der Schweiz, 89 (Latridiites). Pl. 8, fig. 1.

1872. " Monde prim. Suisse, 109 (Latridiites). Pl. 8, fig. 1. 
1876. Heer.

1879. "6

1885. Scudder.

1886. " "

1886. " "
Prim. world Switz., i: 89 (Latridiites). Pl. 8, fig. 1.

Urwelt der Schweiz, $2^{\mathrm{e}}$ aufl., 99 (Latridiites). Pl. 8, fig. 1.

Zittel, Handb. palaeont., i, th. ii; 799. Fig. 104\%.

Zittel-Barrois, Traité de paléont., ii : 799. Fig. 1059.

Bull. U. S. geol. surv., No. 31: 78.

Latridiites schaumii. See Lathridiites schaumii.

1560. Leptura primigenia. (Cerambycidæ.)

Locality : Solenhofen, Bavaria. Horizon : Jura.

1869. Weyenbergh. Arch, mus. Teyl., ii : 286. Pl. 36, fig. 33.

1869. " " Ins. foss. eale. lithogr., 40. Pl. 3, fig. 33.

$1869 . \quad$ " $\quad$ Tijdschr. v. ent., (2), iv : 232 [132].

1874. " " Period. zool., i: 104.

1874. “ Énum. syst. faune ent. mésoz., 18.

1561. Limnius - (Parnidæ.)

Locality : Vale of Wardour, England. Horizon : Purbecks.

1845. Brodie. Foss. ins. sec. rocks Engl., 32. Pl. 6, fig. 9.

1845. Westwood. Brodie, Foss. ins. sec. rocks Engl., 117. Pl. 6, fig. 9.

See also Elmis brodiei.

Megacentris tristis. See Megacentrus tristis.

1562. Megacentrus tristis. (Elateridæ.)

Locality: Schambelen, Aargau, Switzerland. Horizon : Lias.

1852. Heer.

1856. Giebel.

1865. Heer.

187\%. " "

1876. " "

1879. “
Lias-insel Aargau, 14-15. Pl., figs 37,38.

Insect. d. vorwelt, 498 (Megacentris).

Urwelt der Schweiz, 88. Pl. 7, fig. 22.

Monde prim. Suisse, 108. Pl. 7, fig. 22.

Prim. world Switz., i: 88. Pl. 7, fig. 22,

Urwelt der Schweiz, 2e aufl., 98. Pl. 7, fig. 22.

1563. Melanophila (vic.) —_- (Buprestidæ.)

Locality : Ust Balei, Siberia. Horizon : Lias.

1889. \{Brauer-Redt-\} Mém. acad. se. St.-Pétersb., (7), xxxvi, No. 15: 19. Pl. [2], fige. enb.-Ganglb.\} 25-27.

1564. Melanophila costata. (Buprestidø.)

Compared with M. decostigma.

t.

1865. Heer.

1872. " "

1876. “

1879. “
Locality : Schambelen, Switzerland. Horizon: Lias.

Urwelt der Sehweiz, 88. Pl. 7, fig. 19.

Monde prim. Suisse, 107. Pl. 7, fig. 19.

Prim. world Switz., i: 87. $\quad P l .7$, fig. 19.

Urwelt der Schweiz, 2e aufl., 98. Pl. 7, fig. 19.

1565. Melanophila sculptilis. (Buprestidæ.)

Locality : Sehambelen, Aargan, Switzerland. Horizon : Lias.

1852. Heêr.

1856. Giebel.

1865. Heer.

1872. "

1876. “

1879.

Lias-insel Aargau, 14. Pl., figs. 33-35.

Insect. d. vorwelt, 498.

Urwelt der Schweiz, 88. Pl. 7, fig. 18.

Monde prim. Suisse, 107. Pl. 7, fig. 18.

Prim. world Switz., i: 87. Pl. 7, fig. 18.

Urwelt der Schweiz, 2e aufl., 98. Pl. 7, fig. 18. 
1566. Meloe bavaricus. (Meloidæ.)

Locality: Solenhofen, Bavaria. Horizon: Jura.

1874. Weyenbergh. Periód. zool., i : 86, 102. Pl. 3, fig. 6.

1874. " Enum. syst. faune ent. mésoz., 16.

1885. Scudder. Zittel, Handb. palaeont., i, th. ii : 790.

1886. " " Zittel-Barrois, Traité de paléout., ii : 790.

1886. " " Bull. U. S. geol. surv., No. $31: 69$.

Meloe hoernesi. See II, Neuroptera, Idem.

1567. Melolontha -. (Scarabæidæ.)

Locality: Aust Cliff, Eugland. Horizon: Lias.

1845. Brodie. Foss. ins. sec. rocks Engl., 124-125 (unnamed). Pl. 9; fig. 7.

1856. Giebel. Insect. d. vorwelt, 32.

1568. Melolontha -. (Scarabæidæ.)

Locality: Eyeford, England. Horizon: Stonesfield Slate.

1845. Murchison. Geol, Cheltenham, 68. Pl. 4, fig. 4.

1569. Melolontha —. (Scarabæidæ.)

Locality: England. Horizon: Lias.

1871. Phillips. Geology of Oxford, 123.

1570. Melolonthidium - - (Scarabæidæ.)

Localities : Eyeford, Stonesfield, England. Horizon : [Jura.]

1871. Phillips. Geology of Oxford, 173.

1571. Mesosa germari. (Cerambycidæ.)

Locality: Solenhofen, Bavaria. Horizon: Jura.

1856. Giebel. Insect. d. vorwelt, 129.

1869. Weyenbergh. Tijdschr, v. ent., (2), iv : 232 [132].

See also Cerambicinus dubius, etc.

1572. Micranthaxia bella. (Buprestidæ.)

Locality : Schambelen, Switzerland. Horizon:- Lias.

1865. Heer. Urwell der Schweiz, 88. Pl. 7, fig, 17.

1872. " Monde prim. Suisse, 107. Pl. 7, fig. 1i.

1876. " $\quad$ Prim. world Switz., i: 88. Pl. 7, fig. 17.

1879. " Urwelt der Schweiz, 2e aufl, 98. Pl. 7, fig. 17.

1885. Scudder. Zittel, Handb. palaeont., i, th. ii : 797. Fig. 1034.

1886. " Zittel Barrois, Traité de paléont., ii: 797. Fig. 1052.

1573. Micranthaxia rediviva. (Buprestiđæ.)

Locality: Schambelen, Aargau, Switzerland. Horizon: Lias.

1852. Heer.

1856. Giebel.

1865. Heer.

18i2. " "

1876. “

1879. “
Lias-insel Aargan, 14. $\quad P l$. fig. 36.

Insect. d. vorwelt, 498.

Urwelt der Schweiz, 88. $P l .7$, fig. 16.

Monde prim. Suisse, 107. Pl. 7, fig. 16.

Prim. world Switz., i : 88. $P l .7$, fig. 16.

Urwelt der Schweiz, 2e autl, 9ð. Pl. 7, fig. 16.

Mormolucoides articulatus. See II, NeUroptera, Idem. 
1574. Nitidulites argoviensis. (Nitidulidæ.)

Localities: Schambelen, Switzerland; Dobbertin, Mecklenburg.

Horizon : Lias.

1865. Heer. Urwelt der Schweiz, 90, 92. Pl. 8, figs. 2, 3.

1872. " $\quad$ Monde prim. Suisse, 110, 113. Pl. 8, figs. 2, 3.

1876. " - Prim. world Switz, i: 89, 91. Pl. 8, figs. 2, 3.

1879. " Urwelt der Schweiz, 2 aufl., 100. Pl. 8, figs. 2, 3.

1880. E. Geinitz. Zeitschr. deutsch. geol. gesellsch., 1880: 530 (cfr.). Pl.22, fig.20.
1884. ") Zeitschr, dentsch. geol. gesellsch., 1884: 583.

1885. Scudder. Zittel, Handb. palaeont., i, th. ii : 799. Fig. 1044.

1886. " "Zittel-Barrois, Traité de paléont., ii: 799. Fig. 1061.

1886. " Bull. U. S. geol. snrr, No, 31: 78 .

1575. Oryctes pluto. (Scarabæidæ.)

Locality : Solenhofen, Bavaria. Horizon: Jura.

1869. Weyenbergh. Arch. mus. Teyl., ii : 282. Pl. 37, fig. 49.

1869. " " Ins. foss. calc. lithogr., 36. Pl. 4, fig. 49.

1869. " " Tijdschr. v. ent., (2), iv : 232 [132].

1874. " "- Period. zool., i: 102.

1874. “ Énum. syst. fanne ent. mésoz., 16.

1576. Oryctites fossilis. (Scarabæidæ.)

Localits: Eichstadt, Bavaria. Horizon: Jura.

1888. Oppenbeim. Palaeontogr., xxxiv: 238-239. Pl. 31, fig, 2.

1577. Otiorhynchites constans. (Otiorhynchidæ.)

Loeality : Mšeno bei Budin, Böbmen. Horizon: Cretaceous.

1882. Fritsch.

$13-2.4$

$1884 . "$. "
Mojs., Beitr. palaeont. Oestr.-Ung., ii: 5. Pl. 2, fig. 2 (2 figs).

Foss, arthr. steink. n. kreidef. Böhmens, 5. Pl. 2, fig. 2 (2 figs).

Vesmir, xiii : 205-206. Fig. 2.

Palephemera mediaeva. See II, Neuroptera, Idem.

1578. Petrorophus truncatus. (Nitidulidø.)

Locality: Schambelen, Aargau, Switzerland. Horizon: Lias.

1852. Heer.

1856. Giebel.

1865. Heer.

1872. “

1876. “

1879. “

1885. Scudder.

1886. " "

1886. " "

Lias-insel Aargau, 12. Pl., figs. 8-9.

Insect. d. vorwelt, 497.

Urwelt der Schweiz, y0. Pl. 8, fig. 12.

Monde prim. Suisse, 109-110. Pl. 8, fig. 12.

Prim. world Switz., i : 89. $\quad \mathrm{Pl} .8, \mathrm{fig} .12$.

Urwelt der Sehweiz, 2 aufl, 100. Pl. 8, fig. 12.

Zittel, Handb. palaeont., i, th. ii: 799.

Zittel-Barrois, Traité de paléont., ii : 799.

Bull. U. S. geol. surv., No. 31 : 78 ,

Philonthus kneri. See II, Neuroptera, Idem.

1579. Pimelia wittsi. (Tenebrionidæ.)

Locality : Sevenhampton, England. Horizon: Stonesfield Slate.

1856. Giebel. Insect. d. vorwelt, 108.

See also Coceinella wittsii.

1580. Pimelia zekelii. (Tenebrionidæ.)

Locality: Eyeford, England, Horizon: Stonesfield Slate.

1845. Brodie. Foss, ins. sec. rocks Engl, 48 (unnamed), $P l .6, f i g .20$.

1856. Giebel. Insect. d. vorwelt, 107 -108. 


\section{Prionidium —. (Cerambycidæ.)}

1871. Phillips. Geology of Oxford, 173

\section{Locality: Stonesfield, England. Horizon : [Jura].}

1582. Prionus antiquus. (Cerambycidæ.)

Loeality: Durdlestoue Bay, England. Horizon: Lower Purbecks.

1854. Westwood. Quart. journ. geol. soc. Lond, x: 395 (unnamed). Pl. 16, fig. 19. 1856. Giebel.

Insect. d. vorwelt, 126 .

1583. Prionus bucklandi. (Cerambycidæ.)

Locality: England. Horizon: Stonesfield Slate.

1854. Morris. Catal. Brit. foss., $2 d$ ed., 117.

See also Prionus ooliticus.

Prionus oolithicus. See Prionus oolitieus.

1584. Prionus ooliticus. (Cerambycidæ.)

Localities: Sevenhampton, Eyeford, England; Dobbertin, Mecklenburg. Horizon: Stonesfield Slate.

1845. Brodie. Foss. ins. sec. rocks Engl., 47. Pl. 6, fig. 15.

1856. Giebel. Insect. d. vorwelt, 126 (oolithicus).

1884. E. Geinitz. Zeitschr. deutsch. geol. gesellsch., 1884: 583 (oolithiens).

1885. Scudder. Zittel, Handb. palaeont., i, th. ii: 793. Fig. 1021.

1886. " Zittel-Barrois, Traité de paléont, ii : 793. Fig. 1039.

See also P. bucklandi.

\section{Procarabus reticulatus. (Carabidæ.)}

Locality: Eichstadt, Bavaria. Horizon: Jura.

1888. Oppenheim. Palaeontogr., xxxiv: 237. Pl. 31, fig. 28.

1586. Procarabus tripartitus. (Carabidæ.)

Locality: Eichstadt, Bavaria. Horizon: Jura.

1888. Oppenheim. Palaeontogr., xxxiv: 237. Pl. 31, fig. 12.

1587. Procarabus zitteli. (Carabidæ.)

Locality: Eichstadt, Bavaria. Horizon: Jura.

1888. Oppenheim. Palaeontogr., xxxiv: 236-237. Pl.31, fig. 14.

1588. Prodytiscus eichstættensis. (Dytiscidæ.)

Locality: Eichstadt, Bavaria. Horizon: Jura.

1888. Oppenheim. Palaeontogr., sxxiv: 238. Pl. 31, figs. 19, 20.

1589. Prodytiscus longispinosus. (Dytiscidæ.)

Locality : Bavaria. Horizon : Jura.

1888. Oppenheim. Palaeontogr., xxxiv: 237-238. Pl. 31, fig. 15.

See also Pseudohydrophilus longispinosus ; also II, ORTHOPTERA, Blabera avita, etc.

1590. Progeotrupes jurassicus. (Searabæidæ.)

Locality: Eichstadt, Bavaria. Horizon: Jura.

1888. Oppenheim. Palaeontogr., xxxiv: 239. Pl. 31, fig. 17.

Prognatha crassa. See II, Neuroptera, Idem. 
1591. Prototoma striata. (Mycetophagidæ.)

1852. Heer.

Locality : Aargau, Switzerland. Horizon: Lias

1856. Giebel.

Lias-insel Aargau, 12. Pl., fig. 11.

1865. Heer.

Insect. d. vorwelt, 498.

1872. "

Urwelt der Schweiz. Pl. 8, fig. 7.

1876. " "

Monde prim. Suisse. Pl. 8, fig. 7.

1879. “

Prim. world Switz. Pl. 8, fig. 7.

1885. Scudder.

Urwelt der Schweitz; 2 auf. Pl. 8, fig. 7 .

1886.

Zittel, Handb. palaeont., i, th. ii : 799. Fig. 1048.

Zittel-Barrois, Traité de paléont., ii : 800. Fig. 1065.

1592. Pseudohydrophilus longispinosus. (Dytiscidæ.)

Locality : Eichstadt, Bavaria. Horizon: Jura.

1886. Deichmüller. Ins. lith. schichten dresd. mus., 67-69. PI. 5, figs. 10-12.

See also Prodytiscus longispinosus; also II, Orthoptrea, Blabera avita, ete.

1593. Pyrochroa brevipes. (Pyrochroidæ.)

Locality : Eichstadt, Bavaria. Horizon: Jura.

1886. Deichmüller. Ins. lith. schicten dresd. mns., 72-73. Pl, 5, figs. 17-18, 18*.

1594. Saperdides cristallosus. (Cerambycidæ.)

Locality: Solenhofen, Bavaria. Horizon: Jura.

1874. Weyenbergh. Periód. zool., i: 85-86, 104. Pl. 3, fig. 5.

187.4. " Énum. syst. faune ent. mésoz., 18.

1595. Scaphidium hageni. (Scaphidiidæ.)

Locality: Solenhofen, Bavaria. Horizon: Jura.

1869. Weyenbergh. Arch. mus. Teyl, ii : 281. Pl. 37, fig. 51.

1869. “ $\quad$ Ins. foss. calc. lithogr., 35. Pl. 4, fig. 51.

$1869 . \quad$ " " Tijdschr. v. ent., (2), iv: 232 [132].

1874. “ " Periód. zool., i: 102 (Scaphiduum).

1874. “ Enum. syst. faune ent. mésoz., 16 (Scaphiduum).

Scaphiduum hageni. See Scaphidium hageni.

Scarabæites deperditus. See II, Hemiptera, Idem.

Scarabæus deperditus. See II, HeMIPTeRA, Idem.

1595a. Silpha - - (Silphidæ.)

Locality : Bohemia. Horizon: Cretaceous.

[1883. Frič. Arch. výsk. čech., đíl. 1, odd. 2, str. 170.]

- See also Silphites priscus, etc.

1596. Silpha tenuilythris. (Silphidæ.)

Locality: Solenhofen, Bavaria. Horizon: Jura.

1869. Weyenbergh. Arch. mus. Teyl., ii : 280-281. Pl. 37, fig. 48.

1869. " " Ins. foss. calc. lithogr., 34-35. Pl, 4, fig. 48 ,

$1869 . \quad$ " Tijdschr. v. ent., (2), iv: 232 [132], (orig. Silphate).

1869. " " Tijdschr. v. ent, (2), iv: 232 [132], (emend. Silpha).

1874. " " Period. zool., i: 102.

1874. " E Enum. syst. faune ent. mésoz., 16.

Silphate tenuilythris. See Silpha tennilythris. 
1597. Silphites angusticollis. (Silphidæ.)

Locality: Eichstadt, Bavaria. Horizon: Jura.

1888. Oppenheim. Palaeontogr., xxxiv: 239. Pl. 31, fig. 6.

1598. Silphites cetoniformis. (Silphidæ.)

Locality: Eichstadt, Bavaria. Horizon: Jura, 1888. Oppenheim. Palaeontogr., xxxiv: 239. Pl. 31, fig. 16.

1599. Silphites priscus. (Silphidæ.)

Locality: Kounic, Bohemia. Horizon: Perucer schichten, Cretaceous.

1882. Fritsch. Mojs., Beitr. palaeont. Oestr.-Ung., ii : 4-5. Pl. 2, fig. 1 (2 figs.).

188. " " Foss. arthr. steink. u. kreidef. Böhmens, 4-5. Pl. 2, fig. 1 (2 figs.).

1884. " - Vesmír, xiii: 205-206. Fig. 1.

See also —— Friě, Silpha — Friě.

1600. Sitonites melanarius. (Curculionidæ.)

1865. Heer. Urwelt der Schwe1z, 90. Pl. 8, fig. 16.

1872. " Monde prim. Suissie, 109. Pl. 8, fig. 16.

1876. “ Prim. world Switz., i: 89. Pl. 8, fig. 16.

1879. “ Urwelt der Schweiz, $2^{\mathrm{e}}$ aufl, 100. Pl. 8, fig. 16.

1601. Sphenoptera sphinx. (Buprestidæ.)

Compared with S. karelini.

Locality : Eichstadt, Bavaria. Horizon: Jura.

1886. Deichmüller. Ins. lith. schichten dresd. mus., 70-72. Pl. 5, figs. 15-16.

See also Actea sphinx, Chrysobothris veterana.

1602. Scrongylites lævigatus. (Nitidulidæ.)

1865. Heer.

1872. " "

1876. "

1879. “

1865. Heer.

1872. " "

1876. “

1879. "

Locality: Schambelen, Switzerland. Horizon: Lias.

Urwelt der Schweiz, 89.

Moude prim. Suisse, 109.

Prim. world Switz., i: 88.

Urwelt der Schweiz, $2^{\text {e }}$ auf., 99.

1603. Strongylites morio. (Nitidulidæ.)

Locality: Schambelen, Switzerland. Horizon: Lias.

Urwelt der Schweiz, 89. Pl. 7, fig. 24.

Monde prim. Suisse, 109. Pl. 7, fig. 24.

Prim. world Switz., i: 88. Pl. 7, fig. 24.

Urwelt der Schweiz, $2^{e}$ aufl., 99. Pl. 7, fig. 24.

1604. Strongylites stygicus. (Nitidulidæ.)

Locality: Schambelen, Switzerland. Horizon: Lias.

1865. Heer.

1872. "

1876. “

1879. “
Urwelt der Suhweiz, 89. Pl. 7, fig. 23.

Monde prim. Suisse, 109. Pl. 7, fig. 23.

Prim. world Switz., i: 88. Pl. 7, fig. 23.

Urwelt der Schweiz, $2^{\mathrm{e}}$ aufl, 99. Pl. 7, fig. 23. 
1605. Telephorium abgarus. (Lampyridæ.)

Locality : Durdlestone Bay, England. Horizon: Lower Purbecks.

1854. Westwood. Quart. journ. geol. soc. Lond., x: 386, 395. Pl. 17, fig. 4.

1856. Giebel. Insect. d. vorwelt, 102.

1885. Scudder. Zittel, Handb. palaeont., i, th. ii: 796.

1886. " " Zittel-Barrois, Traité de paléont., ii : 796.

1886. “ Bull. U. S. geol. surv., No. 31: 76.

\section{Telephorus haueri. (Lampyridæ.)}

Locality : Forthampton, England. Horizon: Lower Lias.

1845. Brodie.

1856. Giebel.

1885. Scudder.

1886. “

Foss. ins. sec. rocks Engl., 101 (unnamed). Pl. 6, fig. 29. Insect. d. vorwelt, 101.

Zittel, Handb. palaeont., i, th. ii : 796. Fig. 1031.

Zittel-Barrois, Traité de paléont., ii : 796. Fig. 1049.

\section{Tenebrio innominatus. (Tenebrionidæ.)}

Locality : Solenhofen, Bavaria. Horizon: Jura.

1869. Weyenbergh. Arch. mus. Teyl., ii : 285. Pl. 37, fig. 41.

1869. " " Ins. foss. cale. lithogr., 39. Pl. 4, fig. 41.

1є69. " " Tijdschr. v, ent., (2), iv : 232 [132].

1874. " " Periód. zool., i: 104.

1874. “ Enum. syst. faune ent. mésoz., 18.

1608. Tenebrio rugosostriatus. (Tenebrionidæ.)

Locality: Vale of Wardour, England. Horizon : Purbecks.

1845. Brodie.

Foss. ins. sec. rocks Engl., 32 (unnamed). Pl. 6, fig. 2.

1856. Giebel.

Insect. $d$. vorwelt, 109.

\section{Tentyridium peleus.}

Locality: Durdlestone Bay, England. Horizon: Lower Purbecks.

1854. Westwood. Quart. journ. geol. soc. Lond., $\mathrm{x}: 393 . \quad$ Pl. 14, fig. 21.

1856. Giebel. Insect. d. vorwelt, 107.

1610. Thurmannia punctulata. (Carabidæ.)

Locality : Schambelen, Aargau, Switzerland. Horizon : Lias.

1852. Heer. Lias-insel Aargau, 11-12. Pl., figs. 1, 2.

1856. Giebel. Insect. d. vorwelt, 497.

1865. Heer. Urwelt der Schweiz, 90. Pl. 8, fig. 17.

1872. " Monde prim. Suisse, 110. Pl. 8, fig. 17.

1876. " Prim. world Switz., i: 90. Pl. 8, fig. 17.

1879. “ Urwelt der Schweiz, $2^{\mathrm{e}}$ aufl, 100 . Pl. 8, fig. 17.

1885. Scudder. Zittel, Handb. palaeont., i, th. ii : 803. Fig. 1068.

1886. "Zittel-Barrois, Traité de paléont., ii : 804. Fig. 1085.

1889. Nicholson. Man. palæont., 3d ed., i : 601. Fig. $449 b$.

1611. Timarchopsis czckanowskii. (Chrysomelidæ.)

Locality: Ust Balei, Siberia. Horizon: Lias.

1869.\{Brauer-Redt- (Mém. acad. sc. St.-Pétersb., (7), xxxvi, No. 15: 17-18. Pl. [2], \{ enb.-Ganglb.\} fig. 22. 
1612. Trixagites floralis. (Throscidæ.)

1865. Heer.

1872. "6

1876. "

1879. “

1885. Scudder.

1886. "6

1886. " "

Locality: Sehambelen, Switzerland. Horizon: Lias.

Urwelt der Schweiz, 90. Pl. \&, fig. 8.

Monde prim. Suisse, 110. Pl. 8, fig. 8 .

Prim. world Switz., i: 89. $\quad P l .8, f g .8$.

Urwelt der Schweiz, $2^{\mathrm{e}}$ aufl., 100. Pl. 8, fig. 8.

Zittel, Handb. palaeont., i, th. ii: 797.

Zittel-Barrois, Traité de paléont., ii: 797.

Bull. U. S. geol. surv., No. 31 : $7 \%$.

\section{Trogulus $\longrightarrow$. See Trogus $\longrightarrow$.}

1613. Trogus ㄱ. (Dytiscidæ.)

Locality: Strensham, England. Horizon: Lower Lias.

1873. Brodie.

Distr. corr. foss. ins., 16 (Trogulus).

1614. Velenovskya inornata.

Locality: Bohemia. Horizon: Cretaceous.

1888. Fritsch. Vesmír, xviii: 8. Fig. $5 B$.

1615. Wollastonia ovalis. (Hydrophilidæ.)

Locality: Aargau, Switzerland. Horizon: Lias.

1852. Heer.

Lias-insel Aargau, 13. Pl., fig. 17.

1856. Giebel.

Insect. d. vorwelt, 498.

see also Wollastonites ovalis.

1616. Wollastonites ovalis. (Hydrophilidæ.)

Locality: Schambelen, Switzerland. Horizon: Lias.

1865. Heer.

1872. “

1876. “

1879. “

1885. Scudder.

1886. " "

Urwelt der Schweiz, 91. Pl. 8, fig. 26.

Monde prim. Suisse, 111. Pl. 8, fig. 26.

Prim. world Switz., i: 90. Pl. 8, fig. 26.

Urwelt der Schweiz, $2^{\mathrm{e}}$ aufl., 101. Pl. 8, fig. 26.

Zittel, Handb. palaeont., i, th. ii : 802. Fig. 1062.

Zittel-Barrois, 'Traité de paléont., ii: 803. Fig. 1079.

See also Wollastonia ovalis.

\section{DIPTERA.}

1617.

Locality : Govers Cliff End, England. Horizon: Wealden.

1854. Binfield. Quart.journ. geol. soc. Lond., $x: 172$.

— - See II, Hemiptera, Tettigonia — Borre.

1618.

Locality: Dumbleton, England. Horizon: Upper Lias.

1873. Brodie. Distr, corr, foss, ins., 15.

1619.

Localities: Wainlode, Apperley, England. Horizon: Lower Lias.

1873. Brodie. Distr. corr, foss. ins., 17. 
1620.

Localities: Brown's Wood and Stooper's Wood, near Henley in Arden, England. Horizon: Lias.

1875. Brodie.

Lower Lias Eatington, etc., 10.

1621.

Locality : Copt Heath, near Knowle, England. Horizon : Lower Lias.

1875. Brodie. Lower Lias Eatington, ete., 10.

$$
1622 .
$$
(larva).

Locality : Vale of Wardour, England. Horizon : Purbecks.

1845. Westwood. Brodie, Foss. ins. sec. rocks Engl., 121. Pl. 4, fig. 1.

\section{- - (Chironomidæ.) See Rhyphus priscus.}

1623. (Empidæ.)

Locality : Vale of Wardour, England. Horizon: Purbecks:

1845. Brodie, Foss, ins. sec. rocks Engl., 34 (Westwood, 121). Pl. 3, fig. 11.

See also Hasmona leo.

1624. (several sp.). (Tipulidæ.)

Locality: Vale of Wardour, England. Horizon : Purbecks.

1845. Brodie.

Foss. ins. sec. rocks Engl., 34.

1625.

(Tipulidæ.)

Locality: Durdlestone Bay England. Horizon: Lower Purbecks.

1854. Westwood. Quart. journ. geol. soc. Lond., $\mathrm{x}: 386,393 . \quad P l .15$, fig. 1.

1626. (Tipulidæ.)

Locality: Durdlestone Bay, England. Horizon : Lower Purbecks.

1854. Westwood. Quart.journ. geol. soc. Lond., $\mathrm{x}$ : 390,396. Pl. 18, fig. 20.

\section{Adonia fittoni. (Mycetophilidæ.)}

Locality : England. Horizon: Purbecks.

1856. Giebel. Insect. d. vorwelt, 239.

See also Platyura fittoni.

1628. Asilicus lithophilus. (Asilidæ.)

Localities: Kelheim, Solenhofen, Bavaria. Horizon: Jura.

1842. Germar. Münst., Beitr. z. petref., v: 87-88. Pl. 9, fig. 7.

1852. Giebel. Deutschl. petref., 642.

1854. Pictet. Traité de paléont., $2^{\circ}$ 6d., ii : $400 . \quad P l .40$, fig. 9.

1856. Giebel. Insect. d. vorwelt, 211.

186\%. Hagen. Palaeontogr., $\mathrm{x}: 111$.

1869. Weyenbergh. Arch. mus. Teyl., ii : 255-256. Pl. 34, fig. 4.

1869. “ Ins. foss. calc. lithogr., 9-10. Pl. 1, fig. 4.

1869. " " Tijdschr. v. ent., (2), iv : 237.

1874. " " Peri6d. zool., i: 89 (littophilus).

1874. " Enum. syst. faune ent. mésoz., 3 (littophilus).

1877. Assmann. Ber. versamml. deutsch. naturf., 1: 192 (Orthopt. near Locusta amanda). 
Asilicus littophilus. See Asilicus lithophilus.

1629. Asilus ignotus. (Asilidæ.)

Locality : Forthampton, England. Horizon: Insect Limestone, Lower Llas. 1845 Brodie. $\quad$ Foss. ins. sec. rocks Engl., 102, $128 . \quad$ Pl. 7, fig. 19.

1856. Giebel. Insect. d. vorwelt, 211.

1871. Phillips. Geology of Oxford, 123.

1630. Asuba brodiei. (Culicidæ.)

Locality : Vale of Wardour, England. Horizon : Purbecks.

1856. Giebel. Insect. d. vorwelt, 253.

See also Tanypns dubius.

\section{Bria prisca. (Rhyphidæ?)}

Locality: Vale of Wardour, England. Horizon : Purbecks.

1856. Giebel Insect. d. vorwelt, 226-227.

Seo also Rhyphus priscus.

1632. Campylomyza grandæva.

Locality: Durdlestone Bay, England. Horizon: Middle Purbecks.

1856 Giebel. Insect, d. vorwelt, 248.

See also Cecidomium grandævum.

1633. Cecidomium grandævum.

Locality Durdlestone Bay, England. Horizon: Middle Purbecks.

1854. Westwood Quart journ geol. soc. Lond., $\mathrm{x}: 384,394 . \quad P l .15$, fig. 21.

1885. Scudder. Zittel, Handb palaeont, i, th. ii : 810 .

1886. Z Zittel-Barrois, Traité de pal6ont., ii : 811 .

1886. “ Bull. U. S. geol, surv., No.31 : 91.

See also Campylomyza grandæva.

Cheilosia dubia. See Chilosia dubia.

1634. Chenesia (or Macropeza) - (Chironomidæ?.)

Locality : Vale of Wardour, England. Horizon : Purbecks.

1845. Brodie. Foss. ins. sec. rocks Engl., 34,121. Pl. 5, fig. 15.

See also Macropeza prisca, etc.

1635. Chilosia dubia. (Syrphidæ.)

Locality: Solenhofen, Bavaria. Horizon: Jura.

1869. Weyenbergh. Arch. mus. Teyl., ii : 259 (Cheilosia). Pl. 34, fig. 3.

1869. " " Ins. foss. calc. lithogr., 13. Pl. 1, fig. 3.

$1869 . \quad$ " Tijdschr. v. ent., (2), iv : 237.

1874. " " Periód.zool., i: 90.

1874. " Enum. syst. faune ent. mésoz., 4.

1636. Chironomus - (Chironomidæ.)

Locality : Vale of Wardour, England. Horizon : Purbecks.

1345. Brodie. Foss. ins. sec. rocks Engl., 34,121. Pl. 3, fig. 14.

See also C. arrogans.

1637. Chironomus arrogans. (Chironomidæ.)

Locality: England. Horizon: Purbecks.

1856. Giebel. Insect. $d$. vorwelt, 250.

See also Chironomns —- Brodie. 
1638. Chironomus extinctus. (Chironomidx.)

Locality: Vale of Wardour, England. Horizon: Purbecks.

1845. Brodie. 1856. Giebel.
Foss. ins. sec. rocks Engl., 34, 121. Pl. 4, fig. 5.

Insect, d. vorwelt, 249-250.

Locality : Durdlestone Bay, England. Horizon: Lower Purbecks.

1854. Westwood. Quart. journ. geol. soc. Lond., $\mathrm{x}: 387,393-394 . \quad$ Pl. 15, fig. 2.

1856. Giebel. Insect. d. vorwelt, 242.

1885. Scudder. Zittel, Handb. palaeont., i, th. ii : 810. Fig. 1082.

1886. " " Zittel-Barrois, Traité de paléont, ii: 811. Fig. 1099.

1886. “ Bull. U. S. geol. surv., No. 31: 91 .

1640. Culex fossilis. (Culicidæ.)

Locality : Vale of Wardour, England. Horizon : Purbecks.

1845. Brodie. Foss. ins. sec. rocks Engl., 34, 121. Pl. 3, fig. 15.

1885. Scudder. Zittel, Handb. palaeont., i, th. ii : 810 .

1886. " " Zittel-Barrois, Traité de paléont., ii : 811.

1886. “ Bull. U. S. geol, surv., No. 31: 92.

See also Dara fossilis.

\section{Dara fossilis. (Culicidæ.)}

Locality: Vale of Wardour, England. Horizon: Purbecks. 1856. Giebel.

Insect. d. vorwelt, 254.

See also Culex fossilis.

1642. Empidia wulpii. (Empidæ.)

Locality: Solenhofen, Bavaria. Horizon: Jura.

1869. Weyenbergh. Arch. mus. Teyl., ii : 258. P! 34, figs. 5,5a.

1869. " $\quad$ Ins. foss. calc. lithogr., 12. Fl. 1, figs. 5, 5a.

1869. " " Tijdschr. v. ent., (2), iv : 237.

1874. " " Peri6d. zool., i: 89.

1874. “ Enum. syst. faune ent. mésoz., 3.

1886. Scudder. Bull. U. S. geol. surv., No. 31: 88 .

\section{Hasmona leo. (Empidæ.)}

Locality : Vale of Wardour, England. Horizon: Purbecks. 1856. Giebel. Insect d. vorwelt, 209.

See also —_ (Empidæ) Brodie.

1644. Macrocera rustica. (Mycetophilidæ.)

Locality: Vale of Wardour, England. Horizon: Purbecks.

1845. Brodie. - Fosf. ins, sec. rocks Engl., 34, 121. Pl. 3, fig. 13.

See also Sama rustica.

\section{Macropeza (or Chenesia) - (Chironomidæ ?.)}

Locality : Vale of Wardour, England. Horizon: Purbecks.

1845. Brodie. Foss. ins. sec. rocks Engl., 34, 121. Pl. 5, fig. 15.

See also Chenesia __, Macropeza prisca.

1646. Macropeza liasina. (Chironomidæ.)

Locality : Dobbertin, Mecklenburg. Horizon: Lias.

1884. E. Geinitz. Zeitschr. deutsch. geol. gesellsch., 1884: 582-583. Pl. 13, fig. 27. 
1647. Macropeza prisca. (Chironomidæ?.)

Locality: Vale of Wardonr, England. Horizon: Purbecks.

1856. Giebel.

Insect. d. vorwelt, 252.

See also Macropeza — Chenesia

1648. Mesopsychoda dasyptera. (Culicidæ.)

Compared with Ulomyia fuliginosa Meig.

Locality : Ust Balei, Siberia. Horizon : Lias.

1889. \{ Brauer-Redt-\} Mém. acad. sc. St.-Pétersb., (7), xxxvi, No. 15: 17. Pl. [2] \{enb.-Ganglb.\} fig. 21.

Musca lithophila. See II, Orthoptera, Idem.

1649. Platyura fittoni. (Mycetophilidæ.)

Locality : Vale of Wardour, England. Horizon : Purbecks.

1845. Brodie. Foss. ins. sec. rocks Engl., 33, $121 . \quad$ Pl. 3, fig. 9.

Seo also Adonia fittoni.

16 0. Protomyia dubia. (Bibionidæ.)

Locality : Dobbertin, Mecklenburg. Horizon: Lias.

1884. E. Geinitz. Zeitschr. deutsch. geol. gesellsch., 1284: 582. Pl. 13, fig. 26.

1885. Scudder. Zittel, Handb. palaeont., i, th. ii : 810 (not to Diptera).

1886. " Zittel-Barrois, Traité de paléont., ii : 812.

1886. " Bull. U. S. geol. surv., No. 31: 92.

\section{Psychoptera - See Ptychoptera -}

1651. Ptychoptera (vic.) - (Tipulidæ.)

Locality : Ust Balei, Siberia. Horizon: Lias.

1889. $\left\{\begin{array}{l}\text { Brauer-Redt- } \\ \text { enb.-Ganglb. }\end{array}\right\}$ Mém. acad. sc. St.-Pétersb., (7), xxxvi, No. 15: 20 (Psychoptera).

1652. Remalia sphinx. (Syrphidæ.)

Locality: England. Horizon: Wealden.

1845. Brodie. Foss. ins. sec. rocks Engl. (unnamed). Pl. 4, fig. 4.

1856. Giebel. Insect. d. vorwelt, 199.

1653. Rhyphus priscus. (Rhyphidæ?)

Locality: Vale of Wardonr, England. Horizon: Purbecks.

1845. Brodie. Foss. ins. sec. rocks Engl., 34, 121. Pl. 4, fig. 10.

1885. Scudder. Zittel, Handb. palaeont., i, th. ii: 809, 810 (Chironomidæ ?)

1886. " Zittel-Barrois, Traité de paléont., ii : $810,811$.

1886. $.6 \quad$ Bull. U. S. geol, surv., No. 31: 90,91.

See also Bria prisca.

1654. Sama rustica. (Mycetophilidæ.)

Locality: Vale of Wardour, England. Horizon: Purbecks.

1856. Giebel.

Insect. d. vorwelt, 238-239.

See also Macrocera rustica.

Sciara prisca. See II, Neuroptera, Idem.

Bull. $71-15$ 


\section{Sciophila -. (Mycotophilidæ.)}

Locality: Vale of Wardour, England. Horizon: Purbecks.

1845. Brodie.

Foss. ins. sec. rocks Engl. (unnamed). $\quad$ Pl. 4, fig. 2.

1856. Giebel.

Insect. d. vorwelt, 254 .

1656. Sciophila defossa. (Mycetophilidæ.)

Locality : Vale of Wardour, England. Horizon: Purbecks.

1845. Brodie.

Foss. ins. sec. rocks Engl., 34, 121. Pl. 3, fig. 12.

See also Thimna defossa.

\section{Simulidium humidum. (Simulidæ.)}

Locality: Vale of Wardour, England. Horizon: Purbecks.

1856. Giebel. Insect. d. vorwelt, 229.

See also Simulium humidum.

1658. Simulidium priscum. (Simulidæ.)

Locality : Durdlestone Bay, England. Horizon: Middle Purbecks.

1854. Westwood. Quart. journ. geol. soc. Lond., $\mathrm{x}: 384,394 . \quad$ Pl. 15, fig. 15.

1856. Giebel. Insect. d. vorwelt, 229.

1885. Scudder. Zittel, Handb. palaeont., i, th.ii : 811. Fig. 1087.

1886. " Z Zittel-Barrois, Traité de paléont., ii: 812. Fig. 1104.

18є6. " Bull. U. S. geol. surv., No. 31: 93.

1659. Simulium humidum. (Simulidæ.)

Locality: Vale of Wardour, England. Horizon: Purbecks.

1845. Brodie. Foss. ins. sec. rocks Engl., 33, 121. Pl. 3, fig. 8.

1886. Scudder. Bull. U. S. geol, surv., No. 31: 93 .

See also Simulidium humidum.

1660. Tanypus dubius. (Culicidæ.)

Locality: Vale of Wardour, England. Horizon: Purbecks.

1845. Brodie.

Foss. ins. sec. rocks Engl., 33, 121. Pl. 3, fig. 10.

1885. Scudder

Zittel, Handb. palaeont,, i, th. ii: 810.

1886. " "

Zittel-Barrois, Traité de paléont., ii : 811.

1886.

Bull. U. S. geol. surv., No. $31: 91$.

See also Asuba brodiei.

1661. Thimna defossa. (Mycetophilidæ.)

Locality: Vale of Warhour, England. Horizon : Purbecks.

1856. Giebel. Insect. d. vorwelt, 235.

See also Sciophila defossa.

1662. Thiras westwoodi. (Mycetophilidæ.)

Locality : Durdlestone-Bay, England. Horizon: Lower Purbecks.

1854. Westwood. Quart. journ. geol. soc. Lond., x: 396 (unnamed). Pl. 18, fig. 20. 1856. Giebel.

Insect. d. vorwelt, 235 .

\section{Tipula -. (Tipulidæ.)}

Locality : Strensham, England. Horizon: Lower Lias.

1873. Brodịe: Distr. corr, foss, ings., 17. 
1664. Tipula -. (Tipulidæ.)

Locality : Dumbleton, England. Horizon: Lias.

1843. Buckman. Quart. jouru. geol. soc. Lond., iv : 212 (undescr.).

1844. " " Ann. nat. hist., xiv : 74 (undeser.).

1665. Tipula —. (Tipulidæ.)

Locality : England. Horizon: Upper Lias.

1845. Murchison. Geol. Cheltenham, new ed., 108. Pl. 8, fig. 3.

1666. Tipula —. (Tipulidæ.)

Locality: Durdlestone Bay, England. Horizon: Lower Purbecks.

1854. Westwood, Quart. journ. geol, soc. Lond, $\mathrm{x}: 393$ (unnamed). Pl. 15, fig. 1. 1856. Giebel.

Insect. d. vorwelt, 242.

1667. Tipularia teyleri. (Tipulidæ.)

Locality: Solenhofen, Bavaria. Horizon : Jura.

1869. Weyenbergh. Arch. mus. Teyl., ii : 257-258. Pl. 34, figs. 6, $6 a$.

1869. “ Ins. foss. calc. lithogr., 11-12. Pl. 1, figs. 6, 6a.

1869. " " Tijdschr. v. ent., (2), iv : $23 \%$.

1874. "6. Periód. zool., i: 89.

1874. " " Énum. syst. faune ent. mésoz., 3.

1885. Scudder. Zittel, Handb, palaeont., i, th. ii : 809 .

1886. " "Zittel-Barrois, Traité de paléont, ii : 810.

1886. “ Bull. U. S. geol. surv., No. 31: 90 .

\section{LEPIDOPTERA.}

1668.

Locality : England. Horizon: Lower Purbecks.

1873. Brodie. - Distr. corr. foss. ins., 15 ("very doubtful").

1669. - - (butterfly).

Locality : Durdlestone Bay, England. Horizon: Lower Purbecks.

1854. Westwood. Quart. journ. geol. soc. Lond, $\mathrm{x}: 390,396 . \quad$ Pl. 18, fig. 30.

1670. - - (larval mines). (Tineidæ and Tortricidæ.)

Locality : Kansas, Nebraska. Horizon: Dakota Group.

1882. Hagen. Nature, xxv: 265-266 (refers to figures of Lesquereux's Cretaceous flora.

$\longrightarrow$ (Tortricidæ). See - - (Tineidæ and Tortricidæ).

Breyeria borinensis. See II, Neuroptera, Idem.

Cyllonium boisduvalianum. See II, Neuroptera, Idem.

Cyllonium hewitsonianum. See II, Neuroptera, Idem.

Fabellovena compressa. See II, Hymenoptera, Idem.

Fabellovena elegans. See II, Hymenoptera, Idem.

Fabellovena karschii. See II, H Ymenoptera, Idem. 
1671. Paiæocossus jurassicus.

Locality: Eastern Siberia. Horizon: Brown Jura.

1885. Oppenheim. Berl. entom. zeitschr., xxix: 333. Pl. 10, figs. 4,6. 1886. Scudder. Zittel-Barrois, Traité de paléont., ii : 814, note.

1886. " Bull. U. S. geol. surv., xxxi : 96.

Palæontina oolitica. See II, Hemiptera, Idem.

Phragmatcoites damesii. See II, Hemiptkra, Idem.

Pseudosirex darwini. See II, Hymenoptera, Idem.

Rhipidorhabdus gracilis. See II, Hymenopteka, Idem.

Rhipidorhabdus minimus. See II, Hymenoptera, Idem.

Rhipidorhabdus schrœteri. See II, HymenopterA, Idem.

Sphinx —. See II, Hymenoptera, Idem.

Sphinx schrcteri. See II, Hymenoptera, Idem.

Sphinx snelleni. See II, Hymenoptera, Idem.

1672. Tinea araliæ (mines).

Locality: Mšeno, Bohemia. Horizon: Cretaceous.

1882. Fritsch.

1884. "
1882. " "

Mojs., Beitr. palaeont. Oestr.-Ung., ii : 6. Pl. 2, fig. 7.

Foss. arthr. steink.- 11. kreidef. Böhmens, 6. Pl. 2, fig. 7.

Vesmír, xiii : 205-206. Fig. 7.

Tineites crystalli. See III, Lepidoptera, Idem.

Tineites lithophilus. See II, Neuroptera, Idem.

\section{HYMENOPTERA.}

1673. Anomalon palæon. (Ichneumonidæ.)

Locality: Solenhofen, Bavaria. Horizon: Jura.

1874. Weyenbergh. Periód. zool., i: 86,91. Pl. 3, figs. 7,8.

1874. " Énum. syst. faune ent. mésoz., 5.

1885. Scudder.

1886. " "

1886. 6

Zittel, Handb. palaeont., i, th. ii : 815 .

Zittel-Barrois, Traité de paléont., ii : 817.

Bull. U. S. geol. surv., No. 31: 97.

\section{Apiaria antiqua.}

1839. Münster.

Locality: Solenhofen, Bavaria. Horizon: Jura.

1839. Germar.

1850. Heer.

MSS.

Verhandl. leop.-carol. acad. naturf., xix : 210-211. Pl. 22, fig. $10 \alpha \beta$.

Verhandl. schweiz. gesellsch. ges, naturwiss., xxxiv: 81-82 (rofers it to Termites).

1850. " " Geschichte d. insekt., 4-5.

1850. " Neues jahrb. min., 1850: 18.

1850. " Quart. journ. geol. soc. Lond., vi, ii: 69.

1852. Giebel. Deutschl. petref., 614.

1856. " Insect. $d$, vorwelt, 184, 497. 
1856. Hagen. Berendt, Berust. befindl. organ. reste vorw., ii, i: 48 (refers it to Termes).

1862. “ Palaeontogr., $\mathrm{x}: 111$ (Hymenopt. not 'Termes).

1869. Weyenbergh. Tijdschr. v. ent., (2), iv: 236.

1885. Scudder. Zittel, Handb. palaeont., i, th. ii : 815.

1886. " Zittel-Barrois, Traité de paléont., ii : 817.

1886. "6 Bull. U. S. geol. surv., No. 31: 97.

See also Sirex antiquus.

1675. Apiaria lapidea.

Locality: Solenhofen, Bavaria. Horizon: Jura.

1842. Germar. Münst., Beitr. z. petref., v: 84-85. Pl. 9, fig. 5; Pl. 13, fig. 10.

1850. Heer. Verhandl. schweiz. gesellsch. ges. naturwiss., xxxiv: 81 .

1850. " Geschichte d. insekt., 4 (calls it an ant).

1850. “ Neues jahrb. min., 1850: 18 .

1850. “ Quart. journ. geol. soc. Lond., vi, ii : 68.

1856. Giebel. Insect. d. vorwelt, 184, 497 .

1862. Hagen. Palaeontogr., $\mathrm{x}: 111$ (perhaps $=$ Carabicina decipiens).

1ะ69. Weyenbergh. Tijdschr. v. ent., (2), iv : 236.

1877. Assmann. Ber. versamml, deutsch. naturf., 1: 192 (=Carabicina decipiens).

1885. Scudder. Zittel, Handb. palaeont., i, th. ii : 815 .

1886. " "Zittel-Barrois, Traité de paléont., ii : 817.

1886. " Bull. U. S. geol. surv., No. 31: 97.

See also II, Coleoptera, Carabicina decipiens.

\section{Apiaria veterana.}

Locality: Solenhofen, Bavaria. Horizon: Jura.

1s69. Weyenbergh. Arch. mus. Teyl., ii : 260. Pl. 34, fig. 8.

1869. " " Ins. foss. ealc. lithogr., 14. Pl. 1, fig. 8.

1869. " " Tijdschr. v. ent., (2), iv : 236.

1874. " " Period. zool., i: 90.

1874. “ Énum. syst. fauue ent. mésoz., 4.

1677. Belostoma elongata. (Uroceridæ.)

Locality: Solenhofen, Bavaria. Horizon: Jura.

1839. Germar. Verhandl. leop.-carol. akad.naturf., xix : 205-206 (Belostomum). Pl. 22, fig. 6.

1852. Giebel. Deutschl. petref., 636.

1856. " Insect. d. vorwelt, 371.

1862. Hagen. Palaeontogr., $\mathrm{x}: 111$.

1869. Weyenbergh. Tijdschr. v. ent., (2), iv : 233 [133].

1873. . " Arch. mus. Teyl., iii: 239.

$1873 . \quad$ " Notes ins. calc. jurass., 6.

1874. " " Periód. zool., i : 83-84 (is a Hymenopt.?).

1877. Assmann. Ber. versamml. deutsch. naturf., 1: 192 (Hymenopt. near Sirex).

1885. Scudder. Zittel, Handb. palaeont, i, th. ii: 815. Fig. 1094.

1886. " "Zittel-Barrois, Traité de paléont., ii : 814 note, 817. Fig. 1111.

1886. "6 Bull. U. S. geol. surv., No. $31: 61,96,97$.

See also Sphinx schrœteri, etc.

1678. Belostoma schrœteri. (Uroceridæ.)

Locality: Solenhofen, Bavaria. Horizon: Jura.

1862. Hagen. Palaeontogr., $\mathrm{x}:$ 109,111-112.

See also Sphinx schrœteri, etc.

Belostomum elongatum. See Belostomum elongata. 
1679. Bombus? conservatus. (Apidæ.)

Locality: Solenhofen, Bavaria. H rizon: Jura.

1869. Weyenbergh. Arch. mus. Teyl., ii : 259-260. Pl. 34, fig. 7.

1869. “ “ Ins. foss. calc. lithogr., 13-14. Pl. 1, fig. 7.

$1869 . \quad$ " Tijdschr. v. ent., (2), iv: 236.

1874. " " Períd. zool., i: 90.

1874. " " Énum. syst. faune ent. mésoz, 4.

1885. Sendder. Zittel, Handb. palaeont., i, th. ii: 815.

1886. " " Zittel-Barrois, Traité de paléont., ii : 817.

1886. " $\quad$ Bull. U. S. geol. surv., No. 31: 97.

1680. Fabellovena compressa. (Uroceridæ.)

Locality: Solenhofen, Bavaria. Horizon: Jura.

1885. Oppenheim. Berl. entom. zeitschr., xxix: 345. Pl.12, fig. 11.

See also Sphinx schroteri, etc.

\section{Fabellovena elegans. (Uroceridw.)}

Locality: Solenhofen, Bavaria. Horizon: Jura.

1885. Oppènheim. Berl. entom. zeitschr., xxix: 345. Pl. 12, fig. 14.

See also Sphinx schrœteri, etc.

1682. Fabellovena karschi. (Uroceridæ.)

Locality: Solenhofen, Bavaria. Horizon: Jura.

1885. Oppenheim. Berl, entom. zeitschr., xxix: 344. Pl. 12, fig. 13.

See also Sphinx schroeteri, etc.

\section{Formicium brodiei. (Formicidæ.)}

Locality : Durdlestone Bay, England. Horizon: Lower Purbecks.

1E54. Westwood. Quart. journ. geol. soc. Lond., x: 388, 393. Pl. 14, fig. 8.

i\$85. Scudder. Zittel, Handb. palaeont., i, th. ii : 815, 816. Fig. 1095.

1896. " "Zittel-Barrois, Traité de paléont., ii : 817. Fig. 1112.

1886. " Bull. U. S. geol. surv., No. 31: 97.

See also Ponera brodiei.

1684. Hagenia schrceteri. (Uroceridæ.)

Locality: Solenhofen, Bavaria. Horizon: Jura.

1869. Weyenbergh. Arch. mus. Teyl., ii : 250,272 .

$1869 . \quad$ "Ins. foss. calc. lithogr., 4, 26.

1869. " " Tijdschr. v, ent., (2), iv: 233 [133].

See also Sphinx schrœteri, etc.

1685. Ichneumon - - (Ichneumonidæ.)

Locality: Solenhofen, Bavaria. Horizon: Jura.

1820. Schlotheim. Petrefactenk., 43.

1686. Myrmica heeri. (Formicidæ.)

Locality: Durdlestone Bay, England. Horizon: Lower Purbecks.

1856. Giebel. Insect. d. vorwelt, 178.

See also Myrmicium heeri. 


\section{Myrmicium heeri. (Formicidæ.)}

Locality: Durdlestone Bay, England. Horizon: Lower Purbecks.

1854. Westwood. Quart. journ. geol. soc. Lond., $\mathrm{x}: 390,396 . \quad P l .18$, fig. 21.

1885. Scudder. Zittel, Handb. palaeont., i, th. ii: 815 .

1886. " " Zittel-Barrois, Traité de paléont., ii : 817.

1886. “ Bull, U. S. geol. surv., No. 31:97.

See also Myrmica heeri.

\section{Nematus cretaceus (eggs).}

Considered by Renger as a cryptogam and called Pteridophyllites sorigerus.

Locality: Bohdankov, Bohemia. Horizon: Cretaceous.

1882. Fritsch. Mojs., Beitr. palaeont. Oestr.-Ung., ii : 6. Pl. 2, figs. 4-6. 1882. " Foss. arthr. steink.-u. kreidef. Böhmen, 6. Pl. 2, figs. 4-6. 1884. " Vesmír, xiii: 205-206. Figs. 4-5.

1689. Palæomyrmex prodromus. (Formicidæ.)

Locality: Schambelen, Switzerland. Horizon: Lias.

1865. Heer. Urwelt der Schweiz, 91. Pl. 8, fiq. 31.

1872. " Monde prim. Suisse, 111. Pl. 8, fiq. 31.

1876. " $\quad$ Prim. world Switz., i: 90. Pl. 8, fig. 31 .

1879. " Urwelt der Schweiz, $2^{\mathrm{e}}$ anfl, 101. Pl. 8, fig. 31.

1879. Goss. Ent. monthl. mag., xvi: 9 (prob. not Hymenopt.).

1880. " Geol. ant. ins., 26 (prob. not Hymenopt.).

1885. Scudder. Zittel, Handb. palaeont., i, th.ii : 815. Fig. 1093.

1886. “ Zittel-Barrois, Traité de paléont., ii : 817. Fig. 1110.

1886. " Bull. U. S. geol. surv., No. 31 : 97.

1690. Ponera brodiei. (Formicidæ.)

Locality : Durdlestone Bay, England. Horizon : Lower Purbecks.

1856. Giebel. Insect. d. vorwelt, 173-174.

See also Formicium brodiei.

1691. Pseudosirex darwini. (Uroceridæ.)

Locality: Solenhofen, Bavaria. Horizon: Jura.

1873. Weyenbergh. Arch. mus. 'Teyl., iii : 238.

1873. " " Notes ins. cale. jurass., 5.

1874. " " Periód. zool., i: 82-83,91. Pl. 3, figs. 1, 2.

1874. " "Enum. syst. faune ent. mésoz., 5.

1878. $\quad 4 \quad$ Pet. nouv. entom., ii : 253.

1885. Scudder. Zittel, Handb. palaeont., i, th. ii : 813.

1886. " " Zittel-Barrois, Traité de palént., ii : 814.

1886. " Bull. U. S. geol. surv., No. 31 : 95 .

See also Sphinx schroteri, etc.

1692. Pseudosirex elongatus. (Uroceridæ.)

Locality: Solenhofen, Bavaria. Horizon: Jura.

1886. Deichmüller. Ins. lith. schichten dresd. mus., 83-84. Pl. 5, figs. 21-22.

See also Sphinx schrœteri, etc.

1693. Pseudosirex minimus. (Uroceridæ.)

Locality: Solenhofen, Bavaria. Horizon: Jura.

1886. Deichmïller. Ins. litb. schichten dresd. mus., 84. Pl. 5, fig. 23.

See also Rhipidorhabdus minimus. 
1694. Pseudosirex schrcteri. (Uroceridæ.)

Locality: Solenhofen, Bavaria. Horizon: Jura.

1886. Deichmüller. Ins. lith schichten dresd. mus., 82-83.

See also Sphinx schrœteri, etc.

1695. Rhipidorhabdus ㄴ. (Uroceridæ.)

Locality: Bavaria. Horizon: Jura.

1888. Oppenheim. Palaeontogr., xxxiv: 243-247. Pl. 30, fig. 10.

\section{Rhipidorhabdus gracilis. (Uroceridæ.)}

Locality: Solenhofen, Bavaria. Horizon: Jura.

1885. Oppenheim. Berl. entom. zeitschr, xxix: 344. Pl. 11, fig. 10.

See also Sphinx schrœteri, etc.

1697. Rhipidorhabdus minimus. (Uroceridæ.)

Locality: Solenhofen, Bavaria. Horizon: Jura.

1885. Oppenheim. Berl. entom. zeitschr., xxix: 344. Pl. 11, fig. 9.

See also Psendosirex minimus.

1698. Rhipidorhabdus schrœteri. (Uroceridæ.)

Locality: Solenhofen, Bavaria, Horizon: Jura.

1885. Oppenheim. Berl. entom. zeitschr., xxix: 344. Pl. 11, fig. 7.

See also Sphinx schroteri, etc.

\section{Sirex antiquus.}

Locality: Solenhofen, Bavaria. Horizon: Jura.

1877. Assmann. Ber, versamml. deutsch. naturf., 1: 192.

See also Apiaria antiqua.

\section{Sphinx —. (Uroceridæ.)}

Locality : Solenhofen, Bavaria. Horizon: Jura.

1820. Schlotheim. Petrefactenk., 42.

See also Sphinx schroteri, etc.

1701. Sphinx schrceteri. (Uroceridæ.)

Locality: Solenhofen, Bavaria. Horizon: Jura.

1784. Schroeter. Nene literatur u. beytr., i : 411-413 (nnnamed, compared by him to S. convolvuli). $\quad P l .3$, fig. 16.

1820. Schlotheim. Petrefuctenk., 42 ("Sphinx").

1839. Germar. Verhandl. leop.-earol. akad. naturf., xix : 193.

1852. Giebel. Deutschl. petref., 644.

1856. " Insect. d. vorwelt, 188.

1877. Assmann. Ber. versamml. deutsch. naturf., 1: 192 ("Holzwespe").

1885. Scudder. Zittel, Handb. palaeont., i, th. ii : 815 (not a Belostoma).

1886. " " Zittel-Barrois, Traité de paléont., ii : 817.

1886. " Bull. U. S. geol. surv., No. 31: 97.

See also Sphinx —- Schlotheim, Belostoma schrœteri, Hagenia schrœteri, Rhipidorhabdus schrœferi, Psendosirex schrœteri, Belostoma elongata, Psendosirex elongata, Sphinx snelleni, Psendosirex darwini, Rhipidorhabdus gracilis, Fabellovena compressa, F. elegans, F. karschi. 
1702. Sphinx snelleni. (Uroceridæ.)

Locality : Solenhofen, Bavaria. Horizon: Jura.

1869. Weyenbergh. Arch. mus. Teyl., ii : 261-263. Pl. 34, figs. 9, 9a, 10, $10 a$.

1869. “ Ins. foss. cale. lithogr., 15-17. Pl. 1, figs. 9, 9a, 10, 10a.

1869. " " Tijdschr. v. ent., (2), iv: 236.

1873. " " Arch. mus. Teyl., iii : 236-239.

1873. " " Notes ins. calc. jurass., 3-6.

1874. " " Periód. zool., i: 91.

1874. “ Enum. syst. faune ent mésoz., 5.

1880. Dawson. Chain of life, 149. Fig. 130.

1885. Seudder. Zittel, Handb. palaeont., i, th. ii, 813.

1886. " " Zittel-Barrois, Traité de paléont., ii : 814.

1886. “ Bull. U. S. geol. surv., No. 31: 95.

See also Sphinx schrœteri. etc.

\section{TRACKS AND FOOTPRINTS.}

[Found in the older rocks, mostly in the Trias, and accredited to insects.]

1703. (unnamed).

Locality: Connecticut River, Mass. Horizon: Trias.

1856. Deane.

Journ, acad. nat. sc. Philad., (2), iii : 176-177, 177. Pl. 19, figs. $e, h, k, l$.

1861. " Ichnogr. Conn. river, 58. Pl. 40, fig. $4 ; \mathrm{Pl}$. 42, figs. 1-3.

1704. Acanthichnus alatus.

Locality: Massachusetts. Horizon: Trias.

1365. Hitchcock. Suppl. ichn. N. Engl., 14, 16. Pl. 6, fig. 6.

1705. Acanthichnus alternans.

Locality: Turner's Falls, Mass. Horizon: Trias.

1865, Hitchcock. Suppl, ichn. N. Engl., 14, 16 (=A. cursorins pars.). Pl. 6, fig. 5

1706. Acanthichnus anguineus.

Locality: Massachusetts. Horizon: Trias.

1865. Hitcheock. Suppl. ichn. N. Engl., 14, 16. Pl. 7, fig. 4.

1707. Acanthichnus cursorius.

Locality : Turner's Falls, Mass. Horizon: Trias.

1856. Deane. Journ, acad, nat. sci. Philad., (2), iii : 176 (without name). $P l$. 19, fig. $f$.

1858. Hitcheock. Ichn. N. Engl., 150-151. Pl. 28, fig. 1; Pl. 31, fig. 1.

1865. " Suppl. ichn. N. Engl., 13,16. Pl.6, figs. 1, 7, 8, 18; Pl. 7, fig. 10.

See also A. alternans.

1708. Acanthichnus divaricatus.

Locality: Massachusetts. Horizon: Trias.

1865. Hitchcock. Suppl. ichn. N. Engl., 15, 16. Pl. 6, fig. 10; Pl. 7, fig. 10.

1709. Acanthichnus punctatus.

Locality : Massachusetts. Horizon: Trias.

1865. Hitchcock.- Suppl. ichn. N. Engl., 15, 16. Pl. 6, fig. 13. 
1710. Acanthichnus rectilinearis.

Locality : Massachusetts. Horizon: Trias.

1865. Hitchcock. Suppl. ichn. N. Engl., 15,16. Pl. 6, fig. 2.

1711. Acanthichnus saltatorius.

Locality : Turner's Falls, Mass. Horizon: Trias.

1858. Hitcheock. Ichn. N. Engl., 151. Pl. 28, figs. 4, 5 .

1861. Deane. Iehnogr. Conn. river, 57. Pl. 40. fig. 1.

1865. Hitchcock. Suppl. ichn. N. Engl., 15, 16. Pl. 6, figs. 9, 12.

1712. Acanthichnus tardigradus.

Locality: Turner's Falls, Mass. Horizon: Trias.

1856. Deane. Journ. acad. nat. sc. Philad., (2), iii : 176 (without name). Pl. 19, fig. $g$.

1858. Hitcheock. Ichn. N. Engl., 151, Pl. 28, fig. 1.

1861. Deane. Ichnogr. Conn. river, 58. Pl. 40, fig. 3.

See also Pterichnus centipes.

1713. Acanthichnus trilinearis.

Locality : Massachusetts. Horizon: Trias.

1865. Hitcheock. Suppl. ichn. N. Engl., 14-15, 16. Pl. 6, fig. 11,

1714. Ampelichnus sulcatus.

Locality: Massachusetts. Horizon: Trias.

1865. Hitchcock. Suppl. ichn. N. Engl., 19-20. Pl. 6, fig. 16.

See also Grammepus unordinatus.

1715. Bifurculapes - -

Locality: Connecticut River, Mass. Horizon: Trias.

1861. Deane. Ichnogr. Conn. river, 58. Pl. 41, fig. 4.

1716. Bifurculapes curvatus.

Locality: Massachusetts. Horizon: Trias.

1865. Hitchcock. Suppl. ichn. N. Engl., 15, 16 (Bifurculipes). Pl. 7, figs. 2, 9.

1890. Lesley.

Dict. foss. Penus., ii : 807 . Fig.

1717. Bifurculapes elachistotatus.

Loeality: Turner's Falls, Mass. Horizon: Trias.

1858. Hitchcock. Ichn. N. Engl., 154-155. Pl. 29, fig. $4 ;$ Pl. 30, fig. 3

1865. " Suppl. ichn. N. Engl, 16.

1718. Bifurculapes laqueatus.

Locality: Turner's Falls, Mass. Horizon: Trias.

1858. Hitcheock. Ichn. N. Engl., 153. Pl. 30, figs. 1-3. (Fig. 2 is marked tardigradus on the plate.)

1861. Deane.

Ichnogr. Conn. river, 58. Pl. 41, figs. 5, 6 .

1865: Hitchcock. Suppl. ichn. N. Engl., 16.

1719. Bifurculapes scolopendroideus.

Locality : Turner's Falls, Mass. Horizon : Trias.

1858. Hitcheock. Ichn. N. Engl., 153-154. Pl. 27, fig. 1.

1865. " " Suppl. ichn. N. Engl., 14, 16.

Bifurculapes tardigradus. See B. laqueatus. 
1720. Bifurculapes tuberculatus.

Localty: Turner's Falls, Mass. Horizon: Trias.

1858. Hitcheock. Ichn. N. Eugl., 153. Pl. 28, fig. 1; Pl. 30, fig. 4.

1861. Deane. Ichnogr. Conn, river, 58. Pl.41, fig. 2.

1865. Hitchcock. Suppl. ichn. N. Engl., 15 (Bifurculipes).

Bifurculipes curvatus. See Bifurculapes curvatus.

Bifurculipes tuberculatus. See Bifurculapes tuberculatus.

1721. Conopsoides curtus.

Locality : Massachusetts. Horizon: Trias.

1865. Hitchcock. Suppl, ichn. N. Engl, 15, 16. Pl.6, fig. 4; Pl. 18, fig. 4.

1722. Conopsoides larvalis.

Localities: Turner's Fal's, Mass.; Wethersfield, Conu. Horizon: Trias.

1856. Deane. Journ. acad. nat. sc. Philad., (2), iii : 176 (unnamed). Pl. 19, figs. $b, c$.

1858. Hitchcock. Ichn. N. Engl., 152. $P l .29, f i g .6$; $P l .30, f i g s .2,4$.

1861. Deane. Ichnogr. Conn. river, 57,58. Pl. 40, fig. 2; Pl. 41, fig. 3.

1865. Hitcheock. Suppl. ichn. N. Engl., 16.

\section{Copeza cruscularis.}

Locality: Massachusetts. Horizon: Trias.

1865. Hitcheock. Suppl. ichn. N. Engl., 15, 16.

See also Lithographus cruscularis.

1724. Copeza propinquata.

Locality: Massachusetts. Horizon: Trias.

1865. Hitcheock. Suppl. ichn. N. Engl., 15, 16 . Pl. 7, figs. 1, 10; Pl. 18, fig. 2. 1890. Lesley. Dict. foss. Penns., ii : 807 (propinquis). Fig.

See also Lithographus hieroglyphicus.

Copeza propinquis. See Copeza propinquata.

1725. Copeza punctata.

Locality: Massachusetts. Herizon: Trias.

1865. Hitchcock. Suppl.ichn. N. Engl., 15,16. Pl.6, figs. 14,15 (?); Pl. 18, fig. 1

1726. Copeza triremis.

Locality: Turner's Falls, Mass. Horizon: Trias.

1858. Hitchcock. Ichn. N. Engl., 159-160. Pl. 31, fig. 4.

1865. " Suppl. ichn. N. Engl., 15, 16.

1727. Grammepus erismatus.

Locality: 'Turner's Falls, Mass. Horizon: Triag.

1858. Hitchcock. Ichn. N. Engl., 155-156. Pl. 29, fig. 1.

1865. $\quad \because \quad$ Suppl. ichn N. Engl., 13, 16.

Grammepus uniordinatus. See Grammepus unordinatus. 
1728. Grammepus unordinatus.

Locality: Turner's Falls, Mass. Horizon: Trias.

1858. Hitchcock. Ichn. N. Engl., 156. Pl. 29, fig. 2.

1865. " " Suppl. ichn. N. Engl., 2 (nniordinatus).

See also Ampelichuus sulcatus.

\section{Haplotichnus indianensis.}

Locality : Orange County, Ind. Horizon: Kaskaskia group.

1889. Miller. N. A. geol. and pal., 578. Fig. 1686.

1730. Harpepus capillaris.

Locality : Massachusetts. Horizon: Trias.

1865. Hitcheock. Suppl, ichn, N. Engl., 16. Pl.7, fig. 6.

\section{Hexapodichnus horrens.}

Locality: Turner's Falls, Mass. Horizon: Trias.

1858. Hiteheock. Ichn. N. Engl., 158. Pl. 30, fig. 1.

1865. " " Suppl. ichn. N. Eugl., 16.

\section{Hexapodichnus magnus.}

Locality : Turner's Falls, Mass. Horizon: Trias.

1858. Hiteheock. Iehn. N. Eugl., 158. Pl. 29, fig. 7.

1861. Deane. Ichnogr. Coun, river, 58. Pl.40, fig. 5.

1865. Hitchcock. Suppl. ichn. N. Engl., 16.

1733. Lithographus cruscularis.

Locality: Turner's Falls, Mass. Horizon : Trias.

1856. Deane. Journ. acad, nat. sc. Phil., (2), iii : 176 (nnnamed). Pl. 19, fig. d.

1858. Hitchcock. Ichn. N. Engl., 157. Pl. 29, fig. 4; Pl. 30, fig. 3.

See also Copeza cruscularis.

1734. Lithographus hieroglyphicus.

Locality: Turner's Falls, Mass. Horizon: Trias.

1858. Hitcheock. Ichn. N. Engl., 156-157. Pl. 27, fig. 2; Pl. 29, fig. 3.

1861. Deane. Ichnogr. Conn, river, 58. Pl. 41, fig. 1.

See also Copeza propinquata.

1735. Iunula obscura.

Locality: Turner's Falls, Mass. Horizon: Trias.

1865. Hitcheock. Suppl, ichn. N. Engl., 17-18. Pl. 2, fig. 6.

\section{Plangtichnus erraticus.}

Locality: Orange County, Ind. Horizon: Kaskaskia group.

1889. Miller. N. A. geol. and pal., 580. Fig. 1093.

\section{Pterichnus centipes.}

Locality: Turner's Falls, Mass. Horizon: Trias.

1865. Hitchcock. Suppl, ichn. N. Engl., 14. Pl. 7, fig. 3.

1890. Lesley. Dict, foss. Peuns., ii : 807. Figs.

see also Acanthichnus tardigradus. 
1738. Sagittarius alternans.

Locality : Massachusetts. Horizon: Trias.

1865. Hitcheock. Suppl. ichn. N. Engi., 16. Pl. 6, fig. $3 ;$ Pl. 18, fig. 5.

\section{Saltator bipedatus.}

Locality : Turner's Falls, Mass. Horizon: Trias.

1858. Hitchcock. Ichn. N. Engl., 137. Pl. 24, fig. 8; Pl. 51, fig. 7. 1865. " " Suppl. ichn. N. Engl., 14.

\section{Saltator caudatus.}

Locality : Turner's Falls, Mass. Horizon: Trias.

1858. Hitcheock. Ichn. N. Engl., 138. Fl. 24 , figs. 9, 11.

1865. " "Suppl, ichn. N. Engl., 14.

1741. Sphærapus larvalis.

Locality : Turner's Falls, Mass. Horizon: Trias.

1858. Hitchcock. Ichn. N. Engl., 164. Pl. 28, fig. 2.

1742. Sphærapus magnus.

Locality: Turner's Falls, Mass. Horizon: Trias.

185\%. Hitchcock. Ichn. N. Engl., 164-165. Pl. 28, fig. 3.

1743. Treptichnus bifurcus.

Locality : Orange County, Ind. Horizon: Kaskaskia group

1889. Miller. N. A. geol. and pal,, 581. Fig. 1095.

\section{III-CENOZOIC FOSSILS.}

\section{MYRIAPODA.}

1744. (Julidæ.)

Locality: Castelnaud, France. Horizon: Tertiary.

1818. Serres. Journ. physique, Ixxxvii: 173.

See also Julus — Serres, etc.

1745. Blaniulus -., (Julidæ.)

Locality: Prussian amber, Horizon: Ligurian.

1856. Menge. Progr. petrischule Danzig, 1856: 6.

1746. Cermatia - - (Cermatiidæ.)

Locality : Prussian amber. Horizon: Ligurian

1845. Berendt. Bernst. befindl. organ. reste vorw., i: 56 .

1747. Cermatia —. (Cermatidæ.)

Locality : Prussian amber. Horizon: Ligurian

1856. Menge. Progr. petrischule Danzig, 1856: 6 . 
1748. Cermatia illigeri. (Cermatiidæ.)

Locality : Prussian amber. Horizon: Ligurian.

1854. Koch-Berendt. Berendt, Bernst. hefindl, organ. reste vorw., i, th. ii: 15-16. Pl. 1, fig. $6 a$.

1854. Menge. Koch-Berendt, Bernst. befindl. organ. reste vorw., $i$, th. ii : 8 . See also Scutigera illigeri.

1749. Cermatia leachii. (Cermatiidæ.)

Locality: Prussian amber. Horizon: Ligurian.

1854. Koch-Berendt. Berendt, Bernst. befindl. organ. reste vorw., i, th. ii : 14-15. Pl. 1, fig. 6.

1854. Menge. Koch-Berendt, Bernst. befindl. organ. reste vorw,, $i$, th. ii : 8. See also Scutigera illigeri.

1750. Craspedosoma -. (Lysiopeltidæ.)

Locality : Prussian amber. Horizon: Ligurian.

1845. Berendt. Bernst. befindl. organ. reste vorw., i: 56.

1751. Craspedosoma - (6 sp.). (Lysiopeltidæ.)

Locality: Prussian amber. Horizon: Ligurian.

1856. Menge. Progr. petrischule Danzig, 1856: 6 .

1752. Craspedosoma aculeatum. (Lysiopeltidæ.)

Locality : Prossian amber. Horizon : Ligurian.

1854. Menge. Koch-Berendt, Bernst. befindl. organ. reste vorw., i, th. ii : 14.

1856. Giebel. Insect. d. vorwelt, 486.

1753. Craspedosoma affine. (Lysiopeltidæ.)

Locality: Prussian amber. Horizon: Ligurian.

1854. Koch-Berendt. Berendt, Bernst. befindl. organ. reste vorw., i, th. ii : 13-14. Pl. 1, fig. $5 a$.

1856. Giebel. Insect. d. vorwelt, 486.

1754. Craspedosoma angulatum. (Lysiopeltidæ.)

Locality : Prússian amber. Horizon: Ligurian.

1854. Koch-Berendt. Berendt, Bernst. befindl. organ. reste vorw., i, th. ii : 13 . Pl. 1 , fig. 5.

1856. Giebel. Insect. d. vorwelt, 486.

1885. Scudder. Zittel, Handb. palaeont, i, th. ii : 731. Fig. 903.

1886. " Zittel-Barrois, Traité de paléont., ii : 730. Fig. 919.

\section{Craspedosoma armatum. (Lysiopeltidæ.)}

1854. Menge. 1856. Giebel.

Locality: Prussian amber. Horizon: Ligurian.

Koch-Berendt, Bernst. befindl. organ. reste vorw., i, th. ii: 14. Insect. d. vorwelt, 486-487.

1756. Craspedosoma cylindricum. (Lysiopeltidæ.)

Locality: Prussian amber. Horizon: Ligurian.

1854. Menge.

1856. Giebel.
Koch-Berendt, Bernst. befindl. organ. reste rorw., i, th. ii : 14.

Insect. d. vorwelt, 487. 
1757. Craspedosoma obtusangulum. (Lysiopeltidæ.)

Locality: Prussian amber. Hurizon: Ligurian.

1854. Menge. 1856. Giebel.

1854. Menge. 1856. Giebel.

1856. Menge.

1854. Menge. 1853-'56. Bronn. 1885. Scudder. 1886. “ " 1886. “ “
Koch-Berendt, Bernst. befindl, organ. reste vorw., i, th. ii : 14. Insect. d. vorwelt, 486 .

1758. Craspedosoma setosum. (Lysiopeltidæ.)

Locality: Prussian amber. Horizon: Ligurian.

Koch-Berendt, Bernst. befindl. organ. reste vorw., i, th. ii : 14. Insect. d. vorwelt, 487.

1759. Euzonus (Lysiopeltidæ.)

Locality: Prussian Amber. Horizon: Ligurian. Progr. petrischule Danzig, 1856: 6.

1760. Euzonus collulum. (Lysiopeltidæ.)

Locality : Prussian amber. Horizon : Ligurian.

Koch-Berendt, Bernst. befindl. organ. reste vorw., i, th. ii : 14. Lethæa geogn., $3^{\mathrm{e}}$ aufl., iii : 623.

Zittel, Handb. palaeont., i, th. ii : 731.

Zittel-Barrois, Traité de paléont., ii : 730.

Bull. U. S. geol. surv., No. 31: 17.

See also E. zonatus.

1761. Euzonus zonatus.. (Lysiopeltiaæ.)

Locality: Prussian amber. Horizon: Ligurın.

1856. Giebel.

See also E. collulum.

Insect. d. vorwelt, 487.

1762. Geophilus (3 sp.). (Geophilidæ.)

1856. Menge.

Locality: Prussian amber. Horizon: Ligurian.

Progr. petrischule Danzig, 1856: 6 .

1763. Geophilus brevicaudatus. (Geophilidæ.)

Locality: Prussian amber. Horizon: Ligurian.

1854. Menge. 1856. Giebel.

1854. Menge. 1856. Giebel.

1854. Menge. 1856. Giebel.

1854. Menge. 1856. Giebel. 1885. Scudder. 1886. " " 1886. " "
Koch-Berendt, Bernst. befindl. organ. reste vorw., i, th. ii : 19. Insect. d. vorwelt, 489.

1764. Geophilus crassicornis. (Geophilidæ.)

: Prussian amber Horizon: Ligurian.

Koch-Berendt, Bernst. befindl. organ, reste vorw., i, th. ii : 19. Insect. $d$. vorwelt, 490.

1765. Geophilus filiformis. (Geophilidæ.)

Locality: Prussian amber. Horizon: Ligurian.

Koch-Berendt, Bernst. befindl. organ. reste vorw., i, th, ii : 19.

Insect. d. vorwelt, 490.

1766. Glomeris denticulata. (Glomeridæ.)

Locality: Prussian amber. Horizon: Ligurian.

Koch-Berendt, Bernst. befindl. organ. reste vorw., i, th. ii : 12.

Insect. d. vorwelt, 485.

Zittel, Handb. palaeont., i, th. ii : 731 .

Zittel-Barrois, Traité de paléont., ii : 730.

Bull. U. S. geol. surv., No. 31: 17. 
1767. Julus

(Julidæ.)

Locality : Prussian amber. Horizon: Ligurian.

1845. Berendt. Bernst. befindl, organ. reste vorw., i : 56.

1768. Julus - (Julidæ.)

Locality : Prussian amber. Horizon: Ligurian.

1835. Gravenhorst. Uebers. schles, gesellsch. vaterl. cult., 1834 : 93.

1769. Julus —. (Julidæ.)

Locality: Aix, France. Horizon: Ligurian.

1847. Hope. Trans. ent. soc. Lond., iv: 253.

1770. Julus - (3 sp.). (Julidæ.)

Locality: Prussian amber. Horizon: Ligurian.

1E56. Menge. Progr. petrischule Danzig, 1856: 6.

1771. Julus -. (Julidæ.)

Locality: Florissant, Colo. Horizon: Oligocene.

1881. Scudder. Bull. U. S. geol. surv. terr., vi : 294.

1883. " Ann. rep. U. S. geol. surv. terr., xii : 288.

Since proved to be a plant.

\section{Julus $\longrightarrow$. (Julidæ.)}

Compared with J. sabulosus.

Locality : Vicinity of Montpellier, France. Horizon: Calcaires lacustres.

1829. Serres. Geogn. terrains tert., 209-210.

1840. Meyer. $\quad$ Ersch. u. Gruber, encycl, wiss., sect. 2, th. xviii : 538.

See also —— (Julidæ) Serres, J. fabulosus, J. sabulosus (vic.)

1773. Julus antiquus. (Julidæ.)

Locality : Rott, Rhenish Prussia. Horizon: Aquitanian.

1878. Heyden. MSS.

1878. Bertkau. Verhandl, naturh, ver. preuss. Rheinl., (4), v: 360 . Fl. 5, fig. 8.

1885. Scudder. Zittel, Handb. palaeont., i, th. ii : 731. Fig.904.

1886. " Zittel-Barrois, Traité de paléont., ii : $730 . \quad$ Fig. 920.

1886. “ Bull. U. S. geol, surv., No, $31: 18$.

1774. Julus badius. (Julidæ.)

Locality: Prussian amber. Horizon: Ligurian.

1854. Menge. 1856. Giebel.
Koch-Berendt, Bernst. befindl. organ. reste vorw., i, th. ii : 13.

Insect. d. vorwelt, 486.

\section{Julus fabulosus. (Julidæ.)}

(By mistake for Julus sp. vic. sabulosus.)

Locality : Near Montpellier, France. Horizon: Süsswasserkalk.

1834. Keferstein. Naturg. erdkörp., i: 370.

1846. Geinitz. Grundr. versteinerungsk., 193

See also Julus - Serres, etc. 
1776. Julus lævigatus. (Julidæ.)

Locality : Prussian amber. Horizon: Ligurian.

1854. Koch-Berendt. Berendt, Bernst. befindl. organ. reste vorw., i, th. ii : 12-13. Pl. 1, fig. 4.

1856. Giebel. Insect. d. vorwelt, 485 .

1777. Julus politus. (Julidæ.)

1854. Menge.

Locality: Prussian amber. Horizon: Ligurian.

1856. Giebel.

Koch-Berendt, Bernst. befindl. organ. reste vorw., i, th. ii : 13.

Insect. d. vorwelt, 485.

1778. Julus rubens. (Julidæ.)

Locality : Prussian amber. Horizon: Ligurian.

1854. Menge. Koch-Berendt, Bernst. befindl. organ. reste vorw., i, th. ii : 13.

1856. Giebel. Insect, d. vorwelt, 486.

1779. Julus sabulosus (vic). (Julidæ.)

Locality: Montpellier, France. Horizon: Lacustrine limestone.

1885. Scudder. Zittel, Handb. palaeont., i, th. ii : 731.

1886. " Zittel-Barrois, Traité de paléont., ii : 730.

1886. " Bull. U. S. geol. surr., No. 31: 18.

See also Julus - Serres, etc.

1780. Julus telluster. (Julidæ.)

Locality: Green River, Wyo. Horizon: Oligocene.

1878. Scudder. Bull. U. S. geol. surv. terr., iv: 776.

1885. " Z Zittel, Handb. palaeont., i, th. ii: 731 .

1886. " Zittel-Barrois, Traité de paléont., ii : 730.

1886. " " Bull. U. S. geol. surv., No. 31: 18.

1890. " Tert. ins. N. A., 44. Pl. 11, fig. 5.

1781. Julus terrestris (recent). (Julidær.)

Localities: Amber (Brulle, Berendt); Tharand, Saxony (Cotta). Horizon: Ligurian, (Berendt); Gneiss seams (Cotta).

[1761. Linn6. Faun. suec., ed. alt., No. 2066.]

1830. Berendt. Insekten im bernstein, 38.

1833. Cotta. Nenes jahrb. min., 1833: 392-394. Pl. 5, figs. 1, 2.

1839. Brulle. Gisem. ins. foss., 19.

1885. Sendder. Zittel, Handb. palaeont., i, th. ii : 731.

1886. " Zittel-Barrois, Traité de paléont., ii : 730.

1782. Lithobius -. (Lithobiidæ.)

Locality: Prussian amber. Horizon: Ligurian.

1845. Berendt. Bernst. befindl. organ. reste vorw., i: 56.

1783. Lithobius - (8 sp.). (Lithobiidæ.)

Locality: Prussian amber. Horizon: Ligurian.

1856. Menge. Progr. petrischule Danzig, 1856: 6 .

1784. Lithobius brevicornis. (Lithobiidæ.)

Locality : Prussian amber. Horizon: Ligurian.

1854. Menge. Koch-Berendt, Bernst. befindl. organ, reste vorw., i, th. ii : 18.

1856. Giebel. Insect. d. vorwelt, 491.

Bull, $71-16$ 


\section{Lithobius longicornis. (Lithobiidæ.)}

Locality : Prussian amber. Horizon: Ligurian.

1854. Koch-Berendt. Berendt, Bernst. befindl. organ. reste vorw., i, th. ii : 18. $\quad P l, 2$, fig. $\mathbf{7 b}$.

1856. Giebel. Insect. d. vorwelt, 490.

1786. Lithobius maxillosus. (Lithobiidæ.)

Locality: Prussian amber. Horizon: Ligurian.

1854. Koch-Berendt. Berendt, Bernst. befindl. organ. reste vorw., i, th. ii : 16-17. Pl. 2, fig. 7 .

1856. Giebel. Insect. d. vorwelt, 490.

1885. Scudder. Zittel, Handb. palaeont., i, th. ii: 727. Fig. 895.

1886. " Zittel-Barrois, Traité de paléont., ii : 726. Fig. 912.

1787. Lithobius octops. (Lithobiidæ.)

Locality: Prussian amber. Horizon: Ligurian.

1854. Menge. Koch-Berendt, Bernst. befindl. organ. reste vorw., i, th.ii : 18.

1788. Lithobius oxylopus. (Lithobiidæ.)

Locality : Prussian amber. Horizon: Ligurian.

1854. Menge. Koch-Berendt, Bernst. befindl. organ. reste vorw., i, th. ii : 18. 1856. Giebel. Insect. d. vorwelt, 490.

1789. Lithobius planatus. (Lithobiidæ.)

Compared with L. vulgaris.

Locality : Prussian amber. Horizon: Ligurian.

1ะ54. Koch-Berendt. Berendt, Bernst, befindl, organ. reste vorw., i, th. ii: 17-18. Pl. 2, fig. 7 a.

1856. Giebel. Insect. d. vorwelt, 490.

\section{Lithobius pleonops. (Lithobiidæ.)}

Locality : Prussian amber. Horizon : Ligurian.

1854. Menge. Koch-Berendt, Benrst. befindl. organ. reste vorw., i, th. ii : 18.

1791. Lithobius scaber. (Lithobiidæ.)

Locality : Prussian amber. Horizon: Ligurian.

1854. Menge. Koch-Berendt, Bernst. befindl, organ. reste vorw., i, th. ii : 18.

1792. Lithobius spinulosus. (Lithobiidæ.)

Locality: Prussian amber. Horizon: Ligurian.

1854. Menge. Koch-Berendt, Bernst, befindl, organ. reste vorw., i, th. ii : 18.

1856. Giebel. Insect. d. vorwelt, 490-491.

1793. Lithobius striatus. (Lithobiidæ.)

Locality : Prussian amber. Horizon: Ligurian.

1854. Menge. Koch-Berendt, Bernst. befindl. organ. reste vorw., i, th. ii : 18. 1856. Giebel. Insect. d. vorwelt, 491.

1794. Lophonotus -. (Polyxenidæ.)

Locality: Prussian amber. Horizon : Ligurian.

1856. Menge. Progr. petrischule Danzig, 1856: 6 . 
1795. Lophonotus hystrix. (Polyxenidæ.)

Locality: Prussian amber. Horizon: Ligurian.

1854. Menge. Koch-Berendt, Bernst. befindl. organ reste vorw., i, th. ii : 12.

1553-56. Bronn. Lethæa geogn., $3^{\mathrm{e}}$ aufl., iii : 622-623.

1856. Giebel. Insect. d. vorwelt, 488.

See also Phryssonotus hystrix.

1796. Phryssonotus hystrix. (Polyxenidæ.)

Locality: Prussian amber. Horizon : Ligurian.

1885. Scudder. Zittel, Handb. palaeont. i, th.ii : 731.

1886. " " Zittel-Barrois, Traité de paléont., i: 730 .

1886. " Bull. U. S. geol. surv., No. 31: 18.

See also Lophonotus hystrix.

Pollyxenus (several species). See Polyxenus, Idem.

1797. Polydesmus —. (Polydesmidæ.)

Locality: Prussian amber. Horizon: Ligurian.

1856. Menge. Progr. petrischule Danzig, 1856: 6.

1798. Polyzenus - - (Polyxenidæ.)

Locality: Prussian amber. Horizon: Ligurian.

1845. Berendt. Bernst. befindl. organ. reste vorw., i: 56 (Pollyxenus).

1799. Polyxenus - (4 sp.). (Polyxenidæ.)

Locality : Prussian amber. Horizon: Ligurian.

1856. Menge. Progr. petrischule Danzig, 1856: 6 (Pollyxenus).

1800. Polyxenus caudatus. (Polyxenidæ.)

Locality: Prussian amber. Horizon: Ligurian.

1854. Menge. Koch-Berendt, Benrst. befindl. organ. reste vorw., i, th. ii: 12 (Pollyxenus).

1856. Giebel. Insect. d. vorwelt, 488.

1801. Polyxenus colurus. (Polyxenidæ.)

Locality : Prussian amber. Horizon: Ligurian.

1854. Menge. Koch-Berendt, Bernst. befindl. organ. reste vorw., i, th. ii : 12 (Pollyxenus).

1856. Giebel. Insect. d. vorwelt, 488.

1802. Polyxenus conformis. (Polyxenidæ.)

Compared with P. lagurus.

Locality: Prussian amber. Horizon: Ligurian.

1854. Koch-Berendt. Berendt, Bernst. befindl. organ. reste vorw., i, th. ii : $11 . P l$.

1856. Giebel. 16, fig. 133 (Pollyxenus).

Insect. $d$. vorwelt, 487.

1803. Polyxenus lophurus. (Polyxenidæ.)

Locality: Prussian amber. Horizon: Ligurian.

1854. Menge. Koch-Berendt, Bernst. befindl. organ. reste vorw., i, th. ii : 12 (Pollyxenus).

1856. Giebel. Insect, d, vorwelt, 488. 
1804. Polyxenus ovalis. (Polyxenidæ.)

Loeality : Prussian amber. Horizon: Lignrian.

1854. Koch-Berendt. Berendt, Berust. befindl. organ. reste vorw., i, th. ii: 12 . Pl. 1, figs. 3, 1*, $2^{*}$ (Pollyxenus).

1856. Giebel. Insect. d. vorwelt, 487-488.

1885. Scudder. Zittel, Handb. palaeont., i, th. ii : 731. Fig. 905.

1886. " Zittel-Barrois, Traité de pal6ont., 1i : 730. Fig. 921.

1805. Scolopendra - (Scolopendridæ.)

Locality: Prussian amber. Horizon: Ligurian.

1835. Gravenhorst. Uebers. schles. gesellsch. vaterl, cult., 1834: 93.

1806. Scolopendra - (Scolopendridæ.)

Locality: Aix, France. Horizon: Ligurian.

1847. Hope. Trans. ent. soc. Lond., iv : 253.

\section{Scolopendra - (2 sp.). (Scolopendridæ.)}

Locality : Prussian amber. Horizon: Ligurian.

1856. Menge. Progr. petrischule Danzig, 1856: 6 .

1808. Scolopendra - (Scolopendridæ.)

Locality : Prussian amber. Horizon: Ligurian.

1742. Sendel. 1825. Guerin. 1829. Serres.
Hist. suec., 17\%. Pl. 6, figs. 6a, b. (Millepedæ).

Dict. class. hist. nat., viii : 580 (Scolopendre).

Geogn. terrains tert., 240.

1809. Scolopendra fossilis. (Scolopendridæ.)

Locality: Near Glarus, Switzerland. Horizon :

1834. Keferstein. Naturg. erdkörp., ii : 370 (undescr.). (Simply quotes Aldrovandi.)

1810. Scolopendra proavita. (Scolopendridæ.)

Locality: Prussian amber. Horizon: Ligurian.

1854. Menge. Koch-Berendt, Bernst. befindl. organ. reste vorw., i, th. ii : 18-19. 1856. Giebel.

Insect. d. vorwelt, 489.

\section{Soutigera illigeri.}

Locality : Prussian amber. Horizon: Ligurian.

1856. Giebel. Insect. d. vorwelt, 491.

See also Cermatia illigeri, C. leachi.

\section{ARACHNIDA.}

1812.

Locality: Aix, France. Horizon : Ligurian.

1837. Buckland. Geol. and min., ii : 79. Pl. $46^{\prime \prime}$, fig. 12.

1838. " Géologie, éd. Agassiz, ii : expl. pl. $46^{\prime \prime}$, p. 6. Pl. 46", fig. 12,

1852. Quenstedt. Handb. petref. Pl, 21, fig. 25,

See also Theridium bucklandi. 
1813.

Locality: Sinigaglia, Italy. Horizon: Tertiary.

1842. Procaccini. Nuov. ann. sc. nat., vii : 449.

$$
1814 .
$$
(2 sp.).

One of them compared with Epeira riparia. Locality: Green River, Wyo. Horizon: Oligocene. 1878. Scudder. Bull. U. S. geol. surv. terr., iv : 776.

See also Epeira — Scudder, 1890, 89.

\section{5.}

Locality : Gurnet Bay, Isle of Wight. Horizon: Bembridge Limestone. 1879. Woodward. Quart. journ. geol, soc. Lond., xxxv: 344. 1879. " Geol. mag., n. s., $\nabla: 89$.

$$
\text { 1816. - (tick). (Acarina.) }
$$

Locality: Green River, Wyo. Horizon: Oligocene.

1882. Scudder. Harv. Univ. bull., ii : 302.

1883. " Ann. rep. U. S. geol. surv. terr., xii : 285.

See also Ixodes tertiarius.

1817. (1 or 2). (Agalenides.)

Locality : Florissant, Colo. Horizon: Oligocene. 1881. Scudder. Bull. U. S. geol. surv. terr., vi : 294.

1818. (Araneides.)

Locality: Prussian amber. Horizon: Ligurian. 1819. Schweigger. Beobacht. naturh. reisen, 111-112. Pl. 8, figs, 68, 68a.

1819. (1 or 2). (Attoidæ.)

Locality : Florissant, Colo. Horizon: Oligocene.

1831. Scudder. Bull. U. S. geol. surv. terr., vi : 294.

$$
1820 .
$$

(Attoidæ.)

Locality: Prussian amber. Horizon : Ligurian.

1868. Smith. Quart. journ. sc., v: 184. Pl., fig. 10.

1821. (several). (Drassides.)

Locality : Florissant, Colo. Horizon: Oligocene.

1881. Scudder. Bull. U. S. geol. surv. terr., vi : 294.

1822. (1 or 2). (Dysderides.)

Locality: Florissant, Colo. Horizon: Oligocene.

1881. Scudder. Bull. U. S. geol. surv. terr., vi : 294.

1823. (several). (Epeirides.)

Locality: Florissant, Colo. Horizon: Oligocene.

Bull. U, S. geol. surv. terr., vi : 294. 
1824. ——. (Theridides.)

Locality: Prussian amber. Horizon: Ligurian.

1835. Gravenhorst. Uebers. schles. gesellsch. vaterl. cult., 1834: 92.

1825. (egg cocoon). (Theridides.)

Localities: Green River, Wyo.; Florissant, Colo. Horizon: Oligocene.

1878. Scudder. Bull. U. S. geol. surv. terr., iv: 776 .

1882. .. Harv. univ. bull., ii: 303 .

See also Aranea coluınbiæ.

1826. (several). (Theridides.)

Łocality: Florissant, Colo. Horizon: Oligocene.

1881. Scudder. Bull. U. S. geol. surv. terr., vi : 294.

1827. (several). (Thomisides.)

Locality : Florissant, Colo. Horizon: Oligocene.

1881. Scudder. Bull. U. S. geol. surv. terr., vi : 294.

1828. Acantholophus —. (Phalangioidæ.)

Locality: Prussian amber. Horizon: Ligurian.

1856. Menge. Progr. petrischule Danzig, 1856: 11 (Acantolopus). (Perhaps identical with Cheiromachus).

1829. Acantholophus nemastomoides. (Phalangioidæ.)

Locality : Prussian amber. Horizon: Ligurian.

1854. Menge. Koch-Berendt, Bernst. befindl. organ. reste vorw., i, th. ii : 102.

See also Gonyleptes nemastomoides.

1830. Acarus -. (Acarina.)

Locality: Prussian amber. Horizon: Ligurian.

1845. Berendt. Bernst. befindl. organ. reste vorw., i: 56.

1831. Acarus - - (Acarina.)

Locality: Oeningen, Baden. Horizon: Tortonian.

1865. Heer. Urwelt der Schweiz, 358 (milben).

1872. " Monde prim. Suisse, 439.

1876. " Prim. world Switz., ii: 12.

1879. “ Urwelt der Schweiz, $2^{\mathrm{e}}$ aufl, 382 (milben).

1832. Acarus? - (leg). (Acarina.)

Locality : Bilin, Bavaria. Horizon: Tripoli earth.

1838. Turpin. Comptes rend. acad. sc. Paris, vii : 502.

1833. Acarus resinosus. (Acarina.)

Locality: Prussian amber. Horizon: Ligurian.

1822. Presl. Del. prag., i: 210.

1834. Acarus rhombeus. (Acarina.)

Locality: Prussian amber. Horizon: Ligurian.

1845. Koch-Berendt. Jabrb. min., 1845: 872.

1854. " Berendt, Bernst. befindl. organ. reste vorw., i, th. ii: 110 . Pl. 13, figs. 113, 114.

1856. Giebel. Insect. d. vorwelt, 484 . 
1835. Actineda - - (Trombididæ.)

Locality: Prussian amber. Horizon: Ligurian.

1845. Berendt. Bernst. befindl. organ. reste vorw., i: 56.

1836. Actineda malleator. (Trombididæ.)

Locality: Prussian amber. Horizon : Ligurian.

1854. Menge. Koch-Berendt, Bernst. befindl. organ reste vorw., i, th. ii: 107.

1856. Giebel. Insect d. vorwelt, 481.

1837. Actineda subnuda. (Trombididæ.)

Locality: Prussian amber. Horizon: Ligurian.

1854. Menge. Koch-Berendt, Bernst. befindl. organ. reste vorw., i, th. ii: 107.

1856. Giebel. Insect. d. vorwelt, 480-481.

1838. Actineda venustula. (Trombididæ.)

Locality : Prussian amber. Horizon : Ligurian.

1845. Koch-Berendt. Jahrb. min., 1845: 872.

1854. " Berendt, Bernst. befindl. organ. reste vorw., i, th. ii: 106-107. Pl. 13, fig. 106.

1856. Giebel. Insect. d. vorwelt, 480.

1839. Agalena —_. (Agalenides.)

Locality: Prussian amber. Horizon: Ligurian.

1845. Berendt. Bernst. befindl. organ. reste vorw., i: 56 (Agelena).

1840. Agalena tabida. (Agalenides.)

Locality: Prussian amber. Horizon: Ligurian.

1845. Koch-Berendt. Jahrb. min., 1845: 871 (Agelena).

1854. " Berendt, Berust. befindl. organ. reste vorw., i, th. ii : 48.

1856. Giebel. $\quad$ Insect. d. vorwelt, 449.

Agelena tabida. See Agalena tabida.

1841. Amaurobius

Locality: Prussian amber. Horizon: Ligurian.

1845. Berendt. Bernst. befindl. organ. reste vorw., i: 56 .

1842. Amaurobius - (3 sp.).

Locality: Prussian amber. Horizon: Ligurian.

1856. Menge. Progr. petrischule Danzig, 1856: 7.

\section{Amaurobius faustus.}

Locality: Prussian amber. Horizon: Ligurian.

1845. Koch-Berendt. Jahrb. min., 1845: 872.

1854. " Berendt, Bernst. befindl. organ. reste vorw., i, th. ii : 57-58. Pl. 6, fig. 47.

1856. Giebel. Insect. d. vorwelt, 453-454.

1844. Amaurobius rimosus.

Locality : Prussian amber. Horizon: Ligurian.

1845. Koch-Berendt. Jahrb. min., 1845: 872.

1854. " Berendt, Bernst. befindl. organ. reste vorw., i, th. ii : 56-57. $P l_{\text {。 }}$ 6, fig. 46.

1856. Giebel. Insect. d. vorwelt, 453. 
1845. Amaurobius spinimanus.

Locality: Prussian amber. Horizon: Ligurian.

1854. Menge. 1856. 6 1856. Giebel.

1887. Gourret.

1856. Menge.

1854. Menge. 1856. Giebel. 1856. Menge.

1854. Menge. 1853-'56. Bronn. 1856. Giebel. 1856. Menge. 1870. Thorell.
Koch-Berendt, Bernst. befindl. organ. reste vorw., i, th. ii: 58. Progr. petrischule Danzig, 1856: 8.

Insect. d. vorwelt, 454.

1846. Amphiclotho breviuscula. (Urocteoidæ.)

Locality: Aix, France. Horizon: Ligurian. Rec. zool. Suisse, iv: 465-467. Pl. 22, fig. 18.

1847. Amphithomisus barbatus. (Thomisides.)

Isocality: Aix, France. Horizon: Ligurian. Rec. zool. Suisse, iv: 447-449. Pl. 21, fig. 12.

1848. Amphitrogûlus sternalis. (Opiliones.) Locality: Aix, France. Horizon: Ligurian. Rec. zool. Suisse, iv : 484-487. Pl. 23, fig. 26.

1849. Anandrus . (Theridides.)

Locality: Prussian amber. Horizon: Ligurian. Progr, petrischule Danzig, 1856: 7 .

\section{Anatone hirsuta. (Drassides.)}

Locality: Prussian amber. Horizon: Ligurian. Progr. petrischule Danzig, 1856: 8 (undescr.). 1851. Anatone marginata. (Drassides.)

Locality: Prussian amber, Horizon: Ligurian.

Koch-Berendt, Bernst. befindl. organ. reste vorw., i, th. ii : 84 . Insect. d. vorwelt, 464 .

Progr. petrischule Danzig, 1856: 8.

\section{Anatone spinipes. (Drassides.)}

Locality : Prussian amber. Horizon : Ligurian.

Koch-Berendt, Bernst. befindl. organ. reste vorw., i, th. ii : 84.

Lethra geogn. $3^{e}$ anfl., iii : 627.

Insect. d. vorwelt, 464.

Progr. petrischule Danzig, 1856: 8.

European spiders, i: 229.

1853. Androgeus - (Epeirides.)

Locality: Prussian amber. Horizon: Ligurian.

1845. Berendt. Bernst. befindl. organ. reste vorw., i: 56.

1854. Androgeus militaris. (Epeirides.)

Locality: Prussian ámber. Horizon: Ligurian.

1845. Koch-Berendt. Jahrb. min., 1845: 871.

1854. " Berendt, Bernst. befindl. organ. reste vorw., i, th. ii : 28. $\mathbf{P l}$. 3, fig. 17.

1856. Giebel. Insect. d. vorwelt, 439.

1890. McCook. Amer. spiders, ii: 452. Fig. 374.

See also Gea militaris, etc. 
1855. Androgeus triqueter. (Epeirides.)

Locality: Prussian amber. Horizon: Ligurian.

1845. Koch-Berendt. Jahrb. min., 1845: 871.

1854. " Berendt, Bernst. befindl, organ. reste vorw., i, th. ii: 29. Pl. 16, fig. 134.

1853-'56. Bronn. Lethæa geogn., $3^{\mathrm{e}}$ aufl., iii : 635-636. $P l .42^{\prime} a$, fig. 11.

1856. Giebel. Insect. d. vorwelt, 439.

1870. Thorell. European spiders, i: 226.

See also Gea triquetra, etc.

1856. Antopia - (3 sp.). (Epeirides.)

Locality: Prussian amber. Horizon: Ligurian.

1856. Menge. Progr. petrischule Danzig, 1856: 7.

1857. Antopia obscura. (Epeirides.)

Locality: Prussian amber. Horizon: Ligurian.

1854. Menge. Koch-Berendt, Bernst. befindl. organ. reste vorw., i, th. ii : 8 See also Gea obscura, etc. note, $24,43$.

1858. Antopia punctulata. (Epeirides.)

Locality: Prussian amber. Horizon: Ligurian.

1854. Menge. Koch-Berendt, Bernst. befindl, organ. reste vorw., i, th, ii : 8, 43. 1853-56. Bronn. Lethæa geogn., $3^{\mathrm{e}}$ aufl., iii : 657. Pl. $42^{\prime} a$, fig. 13.

1856. Giebel. Insect. d. vorwelt, 446-447.

1870. Thorell. European spiders, i : 225-226.

See also Mizalia punctulata.

1859. Antopia tenera. (Epeirides.)

Locality: Prussian amber. Horizon: Ligurian.

1854. Menge. Koch-Berendt, Bernst. befindl, organ. reste vorw., i, th. ii : 43 (undescr.).

1856. Giebel. Insect. d. vorwelt, 447.

\section{Anyphæna -. (Drassides.)}

Loeality: Prussian amber. Horizon: Ligurian.

1845. Berendt. Bernst. befindl. organ, reste vorw., i: 56.

1861. Anyphæna —. (Drassides.)

Locality: Prussian amber. Horizon: Ligurian.

1856. Menge. Progr. petrischule Danzig, 1856: 7.

1862. Anyphæna -. (Drassides.)

Locality: Florissant, Colo. Horizou: Oligocene.

1882. Scudder. Harv. univ. bull., iii : 303.

1882. " " Field nat., i: 62 .

1883. " Ann. rep. U. S. geol, surv. terr., xii : 287.

See also Anyphæna interita. 
1863. Anyphæna fuscata. (Drassides.)

Compared with A. accentuata.

Locality: Prussian amber. Horizon: Ligurian.

1845. Koch-Berendt. Jahrb, min., 1845: 1872.

1854. " Berendt, Bernst. befindl. organ. reste vorw., i, th. ii : 64-65. Pl. 6, fig. 56.

1856. Giebel. Insect. d. vorwelt, 457-458.

1864. Anyphæna interita. (Drassides.)

Locality : Florissant, Colo. Horizon: Oligocene.

1890. Scudder. Tert. ins. N. A., 67-68. Pl. 11, fig. 5.

See also Anyphæna — Scudder.

1865. Aranea - (Theridides.)

Locality: Copal. Horizon: Recent.

1776. Bloch. Beschäft. berl. gesellsch. naturf. freunde, ii: 167. Pl.3, fig. 5.

1866. Aranea - - (Theridides.)

Locality: Prussian amber. Horizon: Ligurian.

1831. Burmeister. Oken, Isis, 1831: 1100.

1867. Aranea ㄴ. (Theridides.)

Locality: Prussian amber. Horizon : Ligurian.

1820. Schlotheim. Petrefactenk., 43.

1868. Aranea (Tegenaria) - (Theridides.)

Locality: Aix, France. Horizon: Ligurian.

1829. Serres. G6́ogn. terrains tert., 220.

See also A. fossilis.

1869. Aranea columbiæ. (Theridides.)

Localities: Quesnel, British Columbia; Green River, Wyo.; Florissant, Colo. Horizon: Oligocene.

1878. Scudder. Rep. progr. geol. surv. Can., 1876-'77: 463-464.

1878. " " Add. ins.-fauna Quesnel, 7-8.

1878. " Rapp. opér. comm. géol. Can., 1876-'77: 521-522.

1890. “ Tert. ins. N. A., 71-73. Pl. 2, figs. 1-2.

1890. McCook. Amer. spiders, ii : 459 (perhaps a Theridiosoma), 387-388.

See also —— (Theridides) Scuẩder, 1878.

1870. Aranea fossilis. (Theridides.)

Locality : Aix, France. Horizon: Ligurian.

1834. Keferstein. Naturg. erdkörp., ii : 370 (undescr.).

See also Aranea (Tegenaria) — Serres.

1871. Aranea fusca pilosa. (Theridides.)

Locality : Copal. Horizon: Recent.

17శ6. Bloch. Beschäft. berl. gesellsch. naturf. freunde, ii : 165. Pl. 3, flg. 2.

1872. Aranea globosa. (Theridides.)

Locality: Prussian amber. Horizon: Ligurian.

1822. Presl. Del. prag., i: 208. 
1873. Aranea oblonga. (Theridides.)

Locality: Prussian amber. Horizon: Ligurian.

1822. Presl. Del. prag., i: 208-209.

1874. Archæa - (Archæoidæ.)

Locality: Prussian amber. Horizon: Ligurian.

1845. Berendt. Bernst. befindl, organ. reste vorw., i: 56.

1875. Archæa - (5 sp.). (Archæoidæ.)

Locality: Prussian amber. Horizon: Ligurian.

1856. Menge. Progr. petrischule Danzig, 1856: 9.

1876. Archæa coniça. (Archæoidæ.)

Locality: Prussian amber. Horizon: Ligurian.

1845. Koch-Berendt. Jahrb. min., 1845: 871.

1854. " Berendt, Bernst. befindl. organ. reste vorw., i, th. ii: 21. Pl. 2, fig. 10.

1856. Giebel. Insect. d. vorwelt, 434.

1877. Archæa hyperoptica. (Archæoidæ.)

Locality: Prussian amber. Horizon: Ligurian.

1854. Menge.

Koch-Berendt, Bernst. befindl. organ. reste vorw., i, th. ii : 22.

1856. Giebel.

Insect. d. vorwelt, 435.

1878. Archæa incomta. (Archæoidæ.)

Locality: Prussian amber. Horizon: Ligurian.

1854. Menge.

Koch-Berendt, Bernst. befindl. organ. reste vorw., i, th.ii : 22.

1856. Giebel.

Insect. d. vorwelt, 435.

1879. Archæa lævigata. (Archæoidæ.)

Locality: Prussian amber. Horizon: Ligurian.

1845. Koch-Berendt. Jahrb. min., 1845: 871.

1854. " Berendt, Bernst. befindl, organ. reste vorw., i, th. ii : 21-22. Pl. 2, fig. 11.

1856. Giebel. Insect. d. vorwelt, 434.

1880. Archæa paradoxa. (Archæoidæ.)

Locality: Prussian amber. Horizon: Ligurian.

1845. Koch-Berendt. Jahrb. min., $1845: 871$.

1854. " Berendt, Bernst. befindl. organ. reste vorw,, i, th. ii : 19-20. Pl 20, figs. 8, 9 .

1853-'56. Bronn. Lethæa geogn., 3e aufl., iii : 638. Pl. 42'a, fig. 14.

1856. Giebel. Insect. d. vorwelt, 433-434.

1870. Thorell. European spiders, i : 232.

1885. Scudder. Zittel, Handb. palaeont., i, th. ii: 742. Fig. 919.

1886. " Zittel-Barrois, Traité de paléont., ii : 741. Fig.936.

1890. McCook. Amer. spiders, ii : 457, 467-468. Figs. 400, 401.

1881. Archæa pougneti. (Archæoidæ.)

Locality : Prussian amber. Horizon: Ligurian.

1884. Simon. Note compl. Arch., 4-5. Figs. 1-2 on p. 2 .

1884. " Ann. mus, eiv. stor. nat. Gen., $x x: 376-377$. Figs. 1-2 on p. 374. 
1882. Archæa sphinx. (Archæoidæ.)

1854. Menge.

Locality: Prussian amber. Horizon: Ligurian.

1856. Giebel.

Koch-Berendt, Bernst. befindl. organ. reste vorw., i, th. ii : 22. Insect. d. vorwelt, 435 .

\section{Argyroneta -. (Agalenides.)}

Loctlity : Aix, France. Horizon : Ligurian.

1841. Buckland. Pro", geol. soc. Lond, iii: 505.

1842. " Ann. mag.nat. hist., ix: 163.

1884. Argyroneta - (Agalenides.)

Compared with A. aquatica.

Locality: Unterkirschburg, Germany. Horizon: Miocene.

1849. Eser. Jahresh. ver, vaterl. naturk. Württ., iv : 265.

1885. Argyroneta antiqua. (Agalenides.)

Localities: Stösschen, Rott, Germany. Horizon: Aquitanian.

1859. Heyden. Palaeontogr., viii: 1-2. Pl. 1, fig. 12.

1878. Bertkau. Verhandl. naturh, ver. preuss. rheinl., (4), v: 352-359. Pl. 5, fig. $1 a-c$.

1878. " Sitzungsb. niederrh. gesellsch. Bonn, 1878: 70-71.

1885. Scudder Ziittel, Handb. palaeont., i, th. ii : 742. Fig. 922.

1886. " "Zittel-Barrois, Traité de paléont., ii: 742. Fig. 939.

1886. " Bull. U. S. geol. surv., No. $31: 30$.

See also Elvina antiqua.

1886. Argyroneta? longipes. (Agalenides.)

Compared with A. aquatica Deg.

Locality : Oeningen, Baden. Horizon: Tortonian.

1865. Heer. Urwelt der Schweiz, 356, 357. Fig. 214.

1870. Thoreli European spiders, i: 224 (not an Argyroneta).

1872. Heer. Monde prim. Suisse, 436, 438. Fig. 214 on $p .437$.

1876. " " Prim. world Switz., ii: 10,11. Fig. 214.

1879. " Urwelt der Schweiz, $2^{\mathrm{e}}$ aufl., $380,382 . \quad F i g .249$ on $p .381$.

1887. Ariames gabrieli. (Theridides.)

Compared with A. nasicus Sim.

Locality : Aix, France. Horizon: Ligurian.

1887. Gourret. Rec. zool. Suisse, iv : 455-457. Pl. 23, fig. 23.

1888. Artamus radiatus. (Thomisides.)

Locality : Prussian amber. Horizon: Ligurian.

1854. Menge. Koch-Berendt, Bernst, befindl, organ, reste vorw., i, th. ii: 81. 1856. Giebel. Insect. d. vorwelt, 464.

See also Syphax radiatus.

1889. Arytæna troguloides. (Trombididæ.)

Locality : Prussian amber. Horizon: Ligurian.

1854. Menge.

Koch-Berendt, Bernst. befindl. organ. reste vorw., i, th. ii : 106.

1853-'56. Bronn.

Lethæa geogn., $3^{\mathrm{e}}$ aufl., iii : 624 .

1856. Giebel

Insect. d. vorwelt, 480 . 
1890. Athera exilis. (Thomisides.)

Locality: Prussian amber. Horizon: Ligurian.

1854. Menge. Koch-Berendt, Bernst. befindl. organ. reste vorw., i, th. ii : 94 (undescr.).

1853-56. Bronn.

Lethæa geogn., $3^{\text {e }}$ aufl., iii : 639.

1856. Giebel.

Insect. d. vorwelt, 469.

1856. Menge.

Progr. petrischule Danzig, 1856 : 9 (undescr.).

1870. Thorell.

European spiders, i : 231.

1891. Attoides eresiformis. (Attoidæ.)

Locality : Aix, France. Horizon: Ligurian.

1877. Brongniart. Ann. soc. ent. Fr., (5), vii : 221-224. Pl. $7^{\mathrm{I}}$, figs. 7-10.

1878. Girard. La nature, vi : 144. Figs. 1-4.

1885. Seudder. Zittel, Handb. palaeont., i, th. ii : 741. Fig. 918.

1886. " " Zittel-Barrois, Traité de pal6ont., ii : 741. Fig. 935.

1886. " Bull. U. S. geol. surv., No. 31: 29.

1892. Attopsis hirsutus. (Attoidæ.)

Locality : Aix, France. Horizon: Ligurian.

1887. Gourret. Rec. zool. Suisse, iv: 441-443. Pl. 23, fig. 27.

1893. Attus fossilis. (Attoidæ.)

Locality: Prussian amber. Horizon: Ligurian.

1805. Walckenaer. Tabl. aran., 25 (name only).

1837. " - Hist. nat. ins., Apt., i: 462.

1894. Bdella —. (Bdellidæ.)

Locality : Prussian amber. Horizon: Ligurian.

1845. Berendt. Bernst. befindl. organ. reste vorw., i: 56.

1895. Bdella bicincta. (Bdellidæ.)

Loeality : Prussian amber. Horiz on: Ligurian.

1854. Menge. 1856. Giebel.

Koch-Berendt, Bernst. befindl, organ. reste vorw., i, th. ii: 108. Insect. d. vorwelt, 482.

Berl, entom. zeitschr., xxviii : 175.

1896. Bdella bombycina. (Bdellidæ.)

Locality : Prussian amber. Horizon: Ligurian.

1854. Menge. Koch-Berendt, Bernst. befindl. organ. reste vorw., i, th. i: 108. 1856. Giebel.

Insect. d. vorwelt, 482.

1897. Bdella lata. (Bdellidæ.)

Locality: Prussian amber. Horizon: Ligurian.

1845. Koch-Berendt. Jahrb. min., 1845: 872.

1854. " Berendt, Bernst. befindl. organ. reste vorw., i, th. ii: 108. Pl. 13, fig. 110.

1856. Giebel. Insect. d. vorwelt, 482.

1898. Bdella obconica. (Bdellidæ.)

Locality: Prussian amber. Horizon: Ligurian.

1854. Menge. Koch-Berendt, Bernst. befindl. organ. reste vorw., i, th. ii : 108, 124.

1856. Giebel. Insect. d. vorwelt, 482.

1884. Karsch. Berl. entom, zeitschr., xxviii : 175. 
INDEX TO DESCRIBED FOSSIL INSEC'TS. [BUL. 71.

1899. Buthus -. (Scorpiones.)

Locality : ? Horizon: Tertiary.

4846. Giebel. . Paläozool., 267.

1900. Cercidiella aquisextana. (Epeirides.)

Locality: Aix, France. Horizon: Ligurian.

1887. Gourret. Rec. zool. Suisse, iv: 452-453. Pl. 21, fig. 8.

Cheiridion hartmanni. See Cheiridium hartmanni.

1901. Cheiridium hartmanni. (Chelonethi.)

Locality: Prussian amber. Horizon: Ligurian.

1855. Menge. Scheerenspinsien, 38. Pl.5, figs.12,13 (given variously as Chelifer and Cheiridion).

1870. Hagen. Proc. Bost. soc. nat. hist., xiii : 264.

See also Chelifer hartmanni.

Cheiromachus ? - See Acantolophus

1902. Cheiromachus coriaceus. (Phalangioidæ.)

Locality : Prussian amber. Horizon: Ligurian.

1854. Menge. Koch-Berendt, Bernst. befindl. organ. reste vorw., i, th. ii : 102. 1853-'56. Bronn. Lethæa geogn., $3^{\mathrm{e}}$ aufl., iii : 624 .

1856. Giebel. Insect. d. vorwelt, 477.

1903. Chelifer —. (Chelonethi.)

Locality: Prussian amber. Horizon: Ligurian.

1845. Berendt. Bernst. befindl. organ. reste vorw., i: 56.

1904. Chelifer —. (Chelonethi.)

Compared with C. hemprichi Koch.

Locality : Prussian amber. Horizon : Ligurian.

1862. Giebel. Zeitschr. ges. naturw., xx : 321 .

1905. Chelifer —. (Chelonethi.)

Locality: Prussian amber. Horizon: Ligurian.

1835. Gravenhorst. Uebers. schles. gesellsch. vaterl. cult., 1834 : 92.

1906. Chelifer —. (Chelonethi.)

Locality : Aix, France. Horizon: Ligurian.

1847. Hope. Trans. ent. soc. Lond., iv : 253.

1907. Chelifer ‥ (Chelonethi.)

Locality : Prussian amber. Horizon : Ligurian.

1819. Schweigger. Beobacht. naturh. reisen, 113.

1834. Keferstein. Naturg. erdkörp., i: 370 (or Obisium).

1908. Chelifer berendtii. (Chelonethi.)

Locality : Prussian amber. Horizon: Ligurian,

1855. Menge.

Scheerenspinnen, 32-33. $\mathrm{Pl}$. 4, fig. 7.

1870. Hagen. Proc. Bost. soc. nat. hist., xiii : 264.

See also Dichela berendtii. 


\section{Chelifer ehrenbergi. (Chelonethi.)}

Compared with C. museorum.

Locality: Prussian amber. Horizon: Ligurian.

1845. Koch-Berendt. Jahrb. min., 1845: 872.

1854. " Berendt, Bernst. befindl. organ. reste vorw., i, th. ii: 95 . Pl. 10, fig. 95.

1856. Giebel. Insect. d. vorwelt, 472.

1870. Hagen. $\quad$ Proc. Bost. soc. nat. hist., xiii : 265.

1910. Chelifer hartmanni. (Chelonethi.)

Locality: Prussian amber. Horizon: Ligurian.

1854. Menge. Koch-Berendt, Bernst. befindl, organ. reste vorw., i, th. ii: 96. 1856. Giebel. Insect. d. vorwelt, 473.

See also Cheiridium hartmanni.

\section{Chelifer hemprichi. (Chelonethi.)}

Compared with C. museorum, C. cancroides.

Locality : Prussian amber. Horizon : Ligurian.

1845. Koch-Berendt. Jahrb. min., 1845: 872.

1854. “ Berendt, Bernst. befindl. organ. reste vorw., i, th. ii : 94-95. Pl. 10, fig. 94.

1855. Menge. Scheerenspinnen, 34. Pl. 4, fig. 8.

1856. Giebel. Insect. d. vorwelt, 472.

1870. Hagen. $\quad$ Proc. Bost. soc. nat. hist., xiii : 266.

1885. Scudder. Zittel, Fandb. palaeont., i, th. ii : 734 (bemprichti). Fig. 908.

1886. " Zittel-Barrois, Traité de paléont., ii : 733 (hemprichtii), Fig. 924.

Chelifer hemprichti. See Chelifer hemprichi.

1912. Chelifer klemanni. (Chelonethi.)

Locality: Prussian amber. Horizon: Ligurian.

1845. Koch-Berendt. Jahrb. min., $1845: 872$.

1854. “ Berendt, Bernst. befindl. organ. reste vorw., i, th. ii : 95-96.

Pl. 16, fig. 143.

1855. Menge. Scheerenspinnen, 21, 34-35. Pl. 5, fig. 9.

1856. Giebel. Insect. d. vorwelt, 472.

18\%0. Hagen. Proc. Bost. soc. nat. hist., xiii : 266.

See also Obisium rathkei.

1913. Chelifer sieboldii. (Chelonethi.)

Locality : Prussian amber. Horizon: Ligurian.

1855. Menge. Scheerenspinnen, 35-36. Pl. 5, fig. 10.

1870. Hagen. Proc. Bost. soc. nat. hist., xiii : $26 \%$.

See also Obisium sieboldii.

Chelifer wiegandi. Sèe Chelifer wigandi.

1914. Chelifer wigandi. (Chelonethi.)

Locality : Prussian amber. Horizon : Ligurian.

1854. Menge. Koch-Berendt, Bernst. befindl. organ. reste vorw., i, th. ii : 96. 1856. Giebel. Insect. d. vorwelt, 472 (wiegandi).

See also Chernes wigandi. 


\section{Chelignathus kochii.}

Locality: Prussian amber. Horizon: Ligurian.

1854. Menge. Koch-Berendt, Bernst. befindl. organ. reste vorw., i, th. ii: 97.

1853-'56. Bronn. Lethæa geogn., $3^{\mathrm{e}}$ aufl, iii : 624-625.

1856. Giebel. Insect. d. vorwelt, 473.

See also Chthonius kochii.

1916. Chernes wigandi. (Chelonethi.)

Locality : Prussian amber. Horizon: Ligurian.

1855. Menge. Scheerenspinnen, 41-42. Pl. 5, fig. 16.

1870. Hagen. Proc. Bost. soc. nat hist., xiii : 268.

See also Chelifer wigandi.

\section{Cheyletus - (Bdellidæ.)}

Locality : Prussian amber. Horizon : Ligurian.

1845. Berendt. Bernst. befindl. organ. reste vorw., i: 56.

1918. Cheyletus portentosus. (Bdellidæ.)

Locality: Prussian amber. Horizon: Ligurian.

1845. Koch-Berendt. Jahrb. min., 1845: 872.

1854. " Berendt, Bernst, befindl, organ, reste vorw., i, th. ii : 109. Pl. 13, fig. 111.

1856. Giebel. Insect. d. vorwelt, 483.

1919. Chthonius kochii. (Chelonethi.)

Locality: Prussian amber. Horizon: Ligurian.

1855. Menge. Scheerenspinnen, 25-26. Pl. 4, fig. 2.

1870. Hagen. Proc. Bost. soc. nat. hist., xiii : 268.

See also Chelignathus kochii.

1920. Clostes priscus. (Theraphosoidæ.)

Locality : Prussian amber. Horizon: Ligurian.

1869. Menge. Scorp. (Schrift. naturf. gesellsch. Danzig, ii: pt. 2), 7. Figs. $a, b$. 1870. Thorell. European spiders, i : 230-231.

1921. Clubiona —- (Drassides.)

Locality: Prussian amber. Horizon: Ligurian.

1845. Berendt. Bernst. befindl. organ. reste vorw., i : 56.

1922. Clubiona - (4 sp.). (Drassides.)

Locality: Prussian amber. Horizon: Ligurian.

1856. Menge. Progr. petrischule Danzig, 1856: 7.

1923. Clubiona $\longrightarrow$ (4 sp.) (Drassides.)

1882. Scudder.

Locality : Florissant, Colo. Horizon: Oligocene.

1882. " "

Harv. univ. bull., ii : 303.

1883. “ Ann. rep. U. S. geol. surv. terr., xii: $28 \%$.

1924. Clubiona arcana. (Drassides.)

Compared with C. sericea, C. lanata.

Locality: Florissant, Colo. Horizon: Oligocene,

1890. Scudder.

Tert. ins. N. A., $64 . \quad P l .11, f i g .4$. 


\section{Clubiona attenuata. (Drassides.)}

Compared with C. amaranta.

Locality: Prussian amber. Horizon : Ligurian.

1845. Koch-Berendt. Jahrb. min., 1845: 872.

1854. " $\quad$ Berendt, Bernst. befindl. organ. reste vorw., i, th. ii : 65-66. Pl. 7, fig. 57.

1856. Giebel. Insect. d. vorwelt, 458.

1890. McClook. Amer. spiders, ii : 467. Fig. 397.

\section{Clubiona eseri. (Drassides.)}

Compared with C. lanata K. and B.

Locality: Oeningen, Baden. Horizon: Tortonian.

1865. Heer.

Urwelt der Schweiz, 356,357. Fig. 213.

1870. Thorell.

European spiders, i : 224 (not a Clubiona).

1872. Heer.

1876. " "

Monde prim. Suisse, 436, 438. Fig. 213, on $p .43 \%$.

Prim. world Switz., ii : 10,12. Fig. 213.

1879. “

Urwelt der Schweiz, $2^{e}$ aufl., 380, 38\%. Fig. 248, on p. 381.

\section{Clubiona eversa. (Drassides.)}

Compared with $\mathrm{C}$. tomentosa $\mathrm{K}$. and $\mathrm{B}$.

Locality: Florissant, Colo. Horizon: Oligocene.

1890. Scudder. Tert. ins. N. A., 63. Pl. 11, fig. 22.

1928. Clubiona lanata. (Drassides.)

Locality: Prussian amber. Horizon: Ligurian.

1845. Koch-Berendt. Jahrb. min., 1845: 872.

1854. " Berendt, Bernst. befindl. organ. reste vorw., i, th. ii : 67-68. Pl. 7, fig. 60 .

1856. Giebel. Insect. d. vorwelt, 459.

1870. Thorell. European spiders, i : 230 (not a Clubiona).

1929. Clubiona latebrosa. (Drassides.)

Compared with C. attenuata K. and B.

Locality: Florissant, Colo. Horizon: Oligocene.

1890. Scudder. Tert. ins. N. A., 65. Pl. 11, fig. 18.

1930. Clubiona latifrons. (Drassides.)

Loeality : Prussian amber. Horizon: Ligurian.

1854. Menge. Koch-Berendt, Bernst. befindl. organ. reste vorw., i, th. ii: 69 (undeser.).

1856. Giebel. Insect. (l. vorwelt, 459.

1931. Clubiona microphthalma. (Drassides.)

Locality: Prussian amber. Horizon: Ligurian.

1845. Koch-Berendt. Jahrb. min., 1845: 872.

1854. " Berendt, Bernst. befindl. organ. reste vorw, i, th. ii: $66 . \quad P l .7$, fig. 58.

1856. Giebel. Insect. d. vorwelt, 458.

Bull. $71-17$ 
1932. Clubiona (stentata. (Drassides.)

Compared with C. microphthalma K. and B.

Locality: Florissant, Colo. Horizon: Oligocene.

1890. Scudder. - Tert. ins. N. A., 65-66. Pl. 11, fig. 24.

1933. Clubiona pubescens. (Drassides.)

Locality: Prussian amber. Horizon: Ligurian.

1845. Koch-Berendt. Jahrb. min., 1845: 872.

1854. " Berendt, Bernst. befindl. organ. reste vorw., i, th, ii: $69 . \quad P l .7$, fig. 62.

1856. Giebe1.

Insect. d. vorwelt, 459.

1934. Clubiona sericea. (Drassides.)

Locality: Prussian amber. Horizon: Ligurian.

1845. Koch-Berendt. Jahrb. min., 1845: 872.

1854. " " Berendt, Bernst. befindl. organ. reste vorw., i, th. ii: $67 . \quad P l .7$, fig. 59.

1856. Giebel. Insect. d. vorwelt, $458-459$.

1935. Clubiona tomentosa. (Drassides.)

Locality: Prussian amber. Horizon: Ligurian.

1845. Koch-Berendt. Jahrb. min., 1845: 872.

1854. " Berendt, Bernst. befindl, organ. reste vorw., i, th. ii: 68. Pl. 7, fig. 61.

1856. Giebel. Insect. d. vorwelt, 459.

1936. Clubionella antiqua. (Drassides.)

Locality: Aix, France. Horizon: Ligurian.

1887. Gourret. Rec. zool. Suisse, iv: 471-474. Pl. 23, fig. 25.

1937. Clya -. (Theridides.)

Locality: Prussian amber. Horizon: Ligurian.

1845. Berendt. Bernst. befindl. organ. reste vorw., i: 56 .

1938. Clya -. (Theridides.)

Locality : Prussian amber. Horizon: Ligurian.

1856. Menge. Progr. petrischule Danzig, 1856: 7.

1939. Clya lugubris. (Theridides.)

Locality: Prussian amber. Horizon: Liguriau.

1845. Koch-Berendt. Jahrb. min., 1845: 871.

1854. " Berendt, Bernst. befindl. organ. reste vorw., i, th. ii : 31. Pl.3, fig. 19.

1853-56. Bropn. Lethæa geogn., $3^{\mathrm{e}}$ aufl., iii : 633-634. Pl. $42^{\prime} a$, fig. 9.

1856. Giebel. Insect. d. vorwelt, 440-441.

1870. Thorell. European spiders, i: 227.

1940. Clythia —. (Thomisides.)

Locality: Prussian amber. Horizon: Liguriap.

1845. Berendt. Bernst. befindl. organ. reste vorw., i: 56.

1941. Clythia - (3.sp.). (Thomisides.)

Locality: Prussian amber. Horizon: Ligurıan.

1856. Menge.

Progr. petrischule Danzig, 1856: 7 . 
1942. Clythia alma. (Thomisides.)

Locality: Prussian amber. Horizon: Ligurian.

1845. Koch-Berendt. Jahrb. min., $1845: 871$.

1854. " Berendt, Bernst. befindl. organ. reste vorw., i, th. ii: 45-46. $P l$. 5, fig. 35.

1856. Giebel. Insect. d. vorwelt, 447.

1870. Thorell. European spiders, i : 231.

1943. Clythia funesta. (Thomisides.)

Locality: Prussian amber. Horizon: Ligurian.

1854. Menge. Koch-Berendt, Bernst. befindl. organ. reste vorw., i, th. ii: 50. See also Textrix funesta.

1944. Clythia gracilenta. (Thomisides.)

Locality: Prussian amber. Horizon: Ligurian.

1854. Menge. Koch-Berendt, Bernst. befindi, organ. reste vorw., i, th.ii : 46.

1856. Giebel. Insect. d. vorwelt, 447.

1945. Clythia leptocarena. (Thomisides.)

Locality: Prussian amber. Horizon: Ligurian.

1854. Menge. Koch-Berendt, Bernst. befindl. organ. reste vorw., i, th. ii: 46. 1856. Giebel. Insect. d. vorwelt, 447.

1946. Clythia lineata. (Thomisides.)

Locality: Prussian amber. Horizon: Ligurian.

1854. Menge. Koch-Berendt, Bernst. befindl. organ, reste vorw., i, th. ii: 49. See also Textrix lineata.

1947. Corynitis —- (2 sp.). (Theridides.)

Locality : Prussian amber. Horizon: Ligurian.

1856. Menge. Progr. petrischule Danzig, 1856: 7 .

1948. Corynitis spinosa. (Theridides.)

Locality: Prussian amber. Horizon: Ligurian.

1854. Menge. Koch-Berendt, Bernst. befindl, organ. reste vorw., $i$, th. ii: 30.

1853-'56. Bronn. Lethæa geogn., $3^{\mathrm{e}}$ aufl., iii : 635.

1856. Giebel. Insect. d. vorwelt, 440.

1870. Thorell. European spiders, i: 226-227.

1949. Corynitis undulata. ('Theridides.)

Locality : Prussian amber. Horizon: Ligurian.

1854. Menge. Koch-Berendt, Bernst. befindl, organ. reste vorw., i, th. ii: 30 .

1853-'56. Bronn. Lethæa geogn., $3^{\mathrm{e}}$ aufl., iii : 635.

1856. Giebel. Insect. d. vorwelt, 440.

1950. Dichela berendtii. (Chelonethi.)

Locality : Prussian amber. Horizon: Ligurian.

1854. Menge. Koch-Berendt, Bernst. befindl. organ. reste vorw., i, th. ii: 96 (undescr.).

1853-'56. Bronn. Lethæa geogn., $3^{\mathrm{e}}$ aufl., iii : 625.

1856. Giebel Insect. d. vorwelt, 473.

See also Chelifer berendtii. 
1951. Dielacata superba. (Theridides.)

Locality: Prussian amber. Horizon: Ligurian.

1854. Menge. Koch.Berendt, Bernst. befindl. organ. reste vorw., i, th. ii: 94 (undescr.).

1853-'56. Bronn. Lethæa geogn., $3^{\text {e }}$ aufl., iii: 638.

1856. Giebel.

Insect. d. vorwelt., 469.

1856. Menge.

Progr. petrischule Danzig, 1856 : 9.

1870. Thorell. European spiders, i: 227.

\section{Drassus - - (Drassides.)}

Locality: Prussian amber. Horizon: Ligurian.

1856. Menge. Progr. petrischule Danzig, 1856: 8 .

1953. Drassus oblongus. (Drassides.)

Locality : Prussian amber. Horizon: Ligurian.

1854. Menge. Koch-Berendt, Bernst. befindl. organ. reste vorw., i, th. ii : 69 (undescr.).

1856. Giebel. Insect. d. vorwelt, 459.

1954. Dysdera —. (Dysderides.)

Locality: Prussian amber. Horizon: Ligurian.

1845. Berendt. Bernst. befindl. organ. reste vorw., i: 56 .

1955. Dysdera glabrata. (Dysderides.)

Locality: Prussian amber. Horizon: Ligurian.

1854. Menge. 1856. Giebel.

Koch-Berendt, Bernst. befindl. organ. reste vorw., i, th. ii: 74 .

Insect. d. vorwelt, 462 .

1956. Dysdera hippopodium. (Dysderides.)

1854. Menge. 1856. Giebel.

1854. Menge. 1856. Giebel.

Locality: Prussian amber. Horizon: Ligurian.

Koch-Berendt, Bernst. befindl. organ. reste vorw., i, th. ii : 74 .

Insect. $d$. vorwelt, 462.

1957. Dysdera scrobiculata. (Dysderides.)

Locality: Prussian amber. Horizon: Ligurian.

Koch-Berendt, Bernst. befindl. organ. reste vorw., i, th. ii : 74 .

Insect. d. vorwelt, 462.

1958. Dysdera tersa. (Dysderides.)

Locality: Prussian amber. Horizon: Ligurian.

1845. Koch-Berendt. Jahrb. min., 1845 : 872.

1854. " Berendt, Bernst. befindl. organ. reste vorw, i, th. ii: $74 . \quad P l .8$, fig. 68.

1856. Giebel. Insect. $d$. vorwelt, 461-462 (thersa).

Dysdera thersa. See Dysdera tersa.

1959. Elvina antiqua. (Agalenides.)

Locality: Rott, Rhenish Prussia. Horizon: Aquitanian.

1870. Thorell. European spiders, i: 223-224.

See also Argyroneta antiqua. 


\section{Entomocephalus formicoides.}

Locality: Prussian amber. Horizon: Ligurian.

1819. Schweigger. Beobacht. naturh, reisen, 111-112. Pl. 8, figs. 68, 68a.

1829. Holl.

Handb. d. petref., 178-179.

1846. Geinitz.

Grundr. versteinerungsk., 192 .

1961. Eoatypus woodwardii. (Theraphosoidæ.)

Locality : Gurnet Bay, Isle of Wight. Horizon: Eocene.

1883. MeCook. Proc. acad. nat. sc. Phila., 1888: 200-202. Figs. 1,2.

1890. " Amer. spiders, ii : 456-457. Figs. 383, 384 .

1962. Fipeira - (7 sp.). (Epeirides.)

1882. Scudder. Harv. univ. bull., ij : 303.

1882. " " Field nat., i: 63 .

1883. " Ann. rep. U. S. geol. surv. terr., xii : 288.

1963. Epeira -. (Epeirides.)

Locality : Florissant, Colo. Horizon: Oligocene.

1890. Scudder. Tert. ins. N. A., 88-89. Pl. 11, fig. 1.

\section{Epeira -. (Epeirides.)}

Compared with E. riparia.

Locality: Green River, Wyo. Horizon: Oligocene.

1890. Scudder. Tert. ins. N. A., 89, first species.

See also —_ Scudder.

1965. Epeira —. (Epeirides.)

Locality: Green River, Wyo. Horizon: Oligocene.

1890. Scudder. Tert. ins. N. A., 89, second species.

See also — Scudder.

1966. Epeira abscondita. (Epeirides.)

Locality: Florissant, Colo. Horizon: Oligocene.

1890. Scudder. Tert. ins. N. A., 84-85. Pl. 11, fig. 7.

1967. Epeira cinefacta. (Epeirides.)

Locality: Florissant, Colo. Horizon: Oligocene.

1890. Scudder. Tert. ins. N. A., 85-86. $\quad P l .11, f i g .16$.

1968. Epeira delita. (Epeirides.)

Locality: Florissant, Colo. Horizon: Oligocene.

1890. Scudder. Tert. ins. N. A., 85. Pl. 11, fig. 6.

1969. Epeira emertoni. (Epeirides.)

Locality: Florissant, Colo. Horizon: Oligocene.

1890. Scudder. Tert. ins. N. A., 87-88. Pl. 11, figs. 15, 19.

1970. Epeira eocæna. (Epeirides.)

Locality : Prussian amber. Horizon : Ligurian.

1856. Giebel.

Insect. d. vorwelt, 437 . 
1971. Epeira eogena. (Epeirides.)

Locality : Prussian amber. Horizon: Ligurian.

1854. Menge. Koch-Berendt, Bernst. befindl. organ. reste vorw., i, th. ii : 24 (undeser.).

1856. " Progr. petrischule Danzig, 1856: 7 .

1972. Epeira krantzii. (Epeirides.)

Locality: Siebengebirge, Germany. Horizon: Aquitanian.

1870. Thorell. European spiders, i: 223.

See also Gea krantzii.

1973. Epeira meekii. (Epeirides.)

Compared with E. tröschelii.

Losality: Florissant. Colo. Horizon: Oligocene.

1890. Scudder. Tert. ins. N. A., 83-84. Pl. 11, figs. 2, 17.

1890. McCook. Amer. spiders, ii : 453. Fig. 375.

1974. Epeira molassica. (Epeirides.)

Locality : Oeningen, Baden. Horizon: Tortonian.

1865. Heer.

1872. "

Urwelt der Schweiz, 356. Fig. 221.

Monde prum. Suisse, 436. Fig. 221 on p. 437.

1876. “

1879. “

Prim. world Switz., ii : 9. Fig. 221 on p. 10.

1890. McCook.

Urwelt der Schweiz, 2e aufl., 380. Fig. 256 on p. 381.

Amer. spiders, ii : 449.

1975. Epeira tröschelii. (Epeirides.)

Locality : Rott, Rhenish Prussia. Horizon: Aquitanian.

1878. Bertkau. Verhandl. naturh.ver. preuss. rheinl., (4), v: 348-349. Pl. 5, fig. 4.

1976. Epeira vulcanalis. (Epeirides.)

Locality : Florissant, Colo. Horizon: Oligocene.

1890. Scudder. Tert. ins. N. A., 86-87.

1977. Epeiridion -—. (Epeirides.)

Locality : Prussian amber. Horizon: Ligurian,

1856. Menge. Progr. petrischule Danzig, 1856: 6 .

1978. Fpeiridion femoratum. (Epeirides.)

Locality: Prussian amber. Horizon: Ligurian.

1854. Menge, Koch-Berendt, Bernst. befindl. organ. reste vorw., i, th. ii : 24 (undeser.).

1856. Giebel. Insect. d. vorwelt, 437 .

1979. Eresoides orbicularis. (Eresoidæ.)

Locality: Aix, France. Horizon: Ligurian.

1887. Gourret. Rec. zool. Suisse, iv : 454-455. Pl. 21, fig. 16.

1980. Eresus —. (Eresuidæ.)

Locality: Prussian amber. Horizon: Ligurian.

1845. Berendt. Bernst. befindl, organ. reste. vorw., i: 56. 
1981. Eresus curtipes. (Eresoidæ.)

Locality: Prussian amber. Horizon: Ligurian.

1845. Koch-Berendt. Jahrb. min., 1845: 872.

1854. " Berendt, Bernst, befindl, organ. reste vorw., i, th. ii: 87-88. Pl. 9, fig. 82.

1856. Giebel. Insect. d. vorwelt. 466.

1982. Eresus monachus. (Eresoidæ.)

Locality: Prussian amber. Horizon: Ligurian.

1845. Koch-Berendt. Jahrb. min., 1845: 872.

1854. " Berendt, Bernst. befindl, organ. reste vorw., i, th. ii: 86-87. Pl. 9, fig. 81 .

1850. Giebel. Insect. d. vorwelt, 466.

1890. McCook. Amer. spiders, ii : 467. Fig. 394.

1983. Erigone -. (Theridides.)

Locality: Prussian amber. Horizon : Ligurian.

1845. Berendt. Bernst. befindl. organ. reste vorw, i: 56 .

1984. Erigone deckenii. (Theridides.)

Locality: Rott, Rhenish Prussia. Horizon: Aquitanian.

1878. Bertkau. Verhandl. naturh. ver. preuss. rheinl., (4), v: 351. Pl.5, figs.3, $3 a$.

1985. Frigone stigmatosa. (Theridides.)

Locality : Prussian amber. Horizon: Ligurian.

1845. Koch-Berendt. Jahrb. min., 1845: 871.

1854. " $\quad$ Berendt, Bernst. befindl. organ. reste vorw., i, th. ii: $38 . \quad P l .16$, fig. 136.
1856. Giebel.

\section{Erithus —. (Drassides.)}

Locality: Prussian amber. Horizon: Ligurian.

1856. Menge. Progr. petrischule Danzig, 1856: 8 .

1987. Erithus applanatus. (Drassides.)

Locality: Prussian amber. Horizon: Ligurian.

1854. Menge. Koch-Berendt, Bernst. befindl, organ. reste vorw., i, th. ii : 69 (undescr.).

1853-56. Bronn. Lethæa geogn., $3^{\text {e }}$ aufl., iii : 630.

1856. Giebel. Insect. d. vorwelt, 459.

1870. Thorell. European spiders, i: 229.

1988. Ero -. (Theridides.)

Locality: Prussian amber. Horizon: Ligurian.

1845. Berendt. Bernst. befindl. organ. reste vorw., i: 56.

1989. Fro - (7 sp.). (Theridides.)

Locality: Prussian amber. Horizon: Ligurian.

1856. Menge.

Progr. petrisclule Danzig, 1856 : 7. 
1990. Ero coronata. (Theridides.)

Locality: Prussian amber. Horizon: Ligurian.

1854. Menge.

1856. Giebel.

Koch-Berendt, Bernst. befindl. organ. reste vorw., i, th. ii : 33 (undescr.).

Insect. d. vorwelt, 441.

Ero exculpta. See Ero exsculpta.

1991. Ero exsculpta. (Theridides.)

Locality: Prussian amber. Horizon: Ligurian.

1854. Menge. Koch-Berendt, Bernst. befindl organ. reste vorw., $i$, th. ii: 33, 124 (undeser.).

1856. Giebel. Insect. d. vorwelt, 441 (exculpta).

1992. Fro quadripunctata. (Theridides.)

Locality: Prussian amber. Horizon : Ligurian.

1854. Menge. Koch-Berendt, Berust. befindl. organ. reste vorw., i, th. ii : 33 (undeser.).

1856. Giebel. Insect. d. vorwelt, 441.

1993. Fro setulosa. (Theridides.)

Locality: Prussian amber. Horizon: Ligurian.

1845. Koch-Berendt. Jahrb. min., 1845: 871.

1854.

Berendt, Bernst. befindl. organ. reste vorw., i, th. ii : 31-32. $\quad P l$. 4, fig. 20.

1856. Giebel.

Insect. d. vorwelt, 441.

1890. McCook.

Amer. spiders, ii: 467. Fig. 395.

1994. Ero sphærica. (Theridides.)

Locality : Prussian amber. Horizon : Ligurian.

1845. Koch-Berendt. Jahrb. min., 1845: 871.

1854.

Berendt, Bernst. befindl. organ. reste vorw., i, th. ii: 32-33. $P l$. 4, fig. 21.

1856. Giebel. Insect. d. vorwelt, 441.

1995. Erythræus —. (Trombididæ.)

Locality: Prussian amber. Horizon: Ligurian.

1845. Berendt. Bernst. befindl. organ. reste vorw., i: 56 .

1996. Erythræus hirsutus. (Trombididæ.)

Locality: Prussian amber. Horizon: Ligurian.

1854. Menge.

1856. Giebel.

Koch-Berendt, Bernst. befindl, organ. reste vorw., i, th. ii : 107.

Insect. d. vorwelt, 481.

1884. Karsch.

Berl. entom. zeitschr., xxviii: 175.

1997. Erythræus lagopus. (Trombididæ.)

Locality: Prussian amber. Horizon: Ligurian.

1854. Menge.

Koch-Berendt, Bernst. befindl, organ. reste vorw., i, th. ii : 107.

1856. Giebel.

Insect. d. vorwelt, 481. 
1998. Erythræus proavus. (Trombididæ.)

1854. Menge. 1856. Giebel. 1884. Karsch.

1854. Menge. 1856. Giebel.

1854. Menge. 1856. Giebel.
Locality : Prussian amber. Horizon : Ligurian.

Koch-Berendt, Bernst. befindl. organ. reste vorw., i, th. ii : 107. Insect. d. vorwelt, 481 .

Berl, entom. zeitschr., xxviii : 175.

1999. Erythræus raripilus. (Trombididæ.)

Locality: Prussian amber. Horizon: Ligurian.

See also Phidippus gibberulus.

Koch-Berendt, Bernst. befindl. organ. reste vorw., i, th. ii: 107. Insect. d. vorwelt, 481.

2000. Euophrys gibberula. (Attoidæ.)

Locality: Prussian amber. Horizon: Ligurian.

Koch-Berendt, Bernst. befindl. organ. reste vorw., i, th. ii : 93.

Insect. d. vorwelt, 463 .

2001. Euryopus -. (Theridides.)

Locality : Prussian amber. Horizon : Ligurian.

1856. Menge. Trogr. petrischule Danzig, 1856: 7 .

2002. Euryopus gracilipes. (Theridides.)

Locality: Prussian amber, Horizon: Ligurian.

1854. Menge. Koch-Berendt, Bernst. befindl. organ. reste vorw., i, th. ii : 40 (undescr.).

1853-'56. Bronn. Lethæa geogn., $3^{\mathrm{e}}$ aufl., iii : 633.

1856. Giebel. Insect. d. vorwelt, 445.

2003. Flegia —. (Theridides.)

Locality: Prussian amber. Horizon: Ligurian.

1845. Berendt. Bernst. befindl. organ. reste vorw., i : 56 .

2004. Flegia -. (Theridides.)

Locality: Prussian amber. Horizon : Ligurian.

1856. Menge. Progr. petrischule Danzig, 1856: 7.

2005. Flegia longimana. (Theridides.)

Locality: Prussian amber. Horizon: Ligurian.

1845. Koch-Berendt. Jahrb. min., 1845: 871.

1854. " Berendt, Bernst. befindl. organ. reste vorw, i, th. ii : 29-30. Pl. 3, fig. 18.

1853-'56. Bronn. Lethæa geogn., 3’e aufl., iii: 634-635. Pls. 42'a, fig. 10.

1856. Giebel. Insect. d. vorwelt, 440.

1870. Thorell. - European spiders, i: 226.

2006. Gea -. (Epeirides.)

Locality: Prussian amber. Horizon: Ligurian.

1845. Berendt. Bernst. befindl. organ. reste vorw,, i : 56 .

2007. Gea - (4 sp.). (Epeirides.)

Locality: Prussian amber. Horizon: Ligurian.

1856. Menge. Progr. petrischule Danzig, 1856: 6-7. 
2008. Gea epeiroidea. (Epeirides.)

Locality : Prussian amber. Horizon: Ligurian.

1845. Koch-Berendt. Jahrb. min., 1845: 871.

1854. " Berendt, Bernst. befindl. organ. reste vorw., i, th. ii: 23-24. Pl. 3, fig. 12.

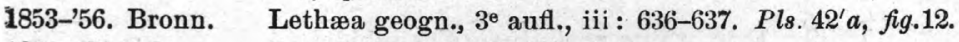

1856. Giebel. Insect. d. vorwelt, 436.

1890. McCook. Amer. spiders, ii: 452. Fig. 373.

See also Græa epeiroidea.

2009. Gea krantzi. (Epeirides.)

Locality : Rott, Rhenish Prussia. Horizon: Aquitanian.

1859. Heyden. Palaeontogr., viii : 2. Pl. 1, fig. 11.

1890. MeCook. Amer. spiders, ii : 452.

See also Epeira krantzi.

2010. Gea militaris. (Epeirides.)

Locality: Prussian amber. Horizon: Ligurian.

1854. Menge. Koch-Berendt, Bernst. befindl, organ. reste vorw., i, th. ii : 28.

See also Androgeus militaris, Græa militaris.

2011. Gea obscura. (Epeirides.)

Locality: Prussian amber. Horizon: Ligurian.

1845. Koch-Berendt. Jahrb. min., 1845: 871.

1854. " Berendt, Bernst. befindl. organ. reste vorw., i, th. ii : 24. Pl.3, fig. 13.

1856. Giebel. Insect. d. vorwelt, 436.

See also Antopia obscura, Græa obscura.

2012. Gea pubescens. (Epeirides.)

Locality : Prussian amber. Horizon: Ligurian.

1854. Menge. Koch-Berendt, Bernst. befindl. organ. reste vorw., 1, th. ii : 24. 1856. Giebel. . Insect d. vorwelt, 436.

See also Græa pubescens.

2013. Gea triquetra. (Epeirides.)

Locality : Prussian amber. Horizon: Ligurian.

1854. Menge: Koch-Berendt, Bernst. befindl. organ. reste vorw., i, th. ii: 29.

See also Androgens triqueter, Græa triquetra.

2014. Gerdia myura. (Hersilioidæ.)

Locality : Prussian amber. Horizon: Ligurian.

1869. Menge. Scorp. (Schrift. naturf. gesellsch. Danzig, ii pt.ii): 8-9. Figs. $a-c$.

1870. Thorell. European spiders, i: 228-229.

1885. Scudder. Zittel, Handb. palaeont., i, th. ii : 743. Fig. 923.

1886. " Zittel-Barrois, Traité de paléont., ii: 742. Fig. 940.

2015. Gonyleptes ——. (Gonyleptidæ.)

Locality: Prussian amber. Horizon: Ligurian.

1845. Berendt. Bernst. befindl. organ. reste vorw., i : 56. 
2016. Gonyleptes nemastomoides. (Gonyleptidæ.)

Locality: Prussian amber. Horizon: Ligurian.

1845. Koch-Berendt. Jahrb. min., 1845: 872.

1854. " Berendt, Bernst. befindl. organ. reste vorw., i, th. ii: 102. Pl. 12, fig. 101.

See also Acantholophus nemastomoides.

2017. Gorgopis - (5 sp.). (Attoidæ.)

Locality: Prussian amber. Horizon: Ligurian.

1856. Menge. Progr. petrischule Danzig, 1856: 9.

2018. Gorgopis fasciata. (Attoidæ.)

Locality: Prussian amber. Horizon: Ligurian.

1854. Menge. Koch-Berendt, Bernst. befindl, organ. reste vorw., i, th, ii: 93.

1853-'56. Bronn. Lethæa geogn., 3' aufl., iii : 625-626. Pls. 42'a, fig.1.

1856. Giebel. Insect. d. vorwelt, 467-468.

See also Phidippus fasciatus, P. formosus.

2019. Gorgopis frenata. (Attoidæ.)

Locality: Prussian amber. Horizon: Ligurian.

1856. Giebel. Insect. d. vorwelt, 466-467.

1870. Thorell. European spiders, i: 232-233.

See also Phidippus frenatus, P. pusillus, P. paululus, Gorgopis torva.

2020. Gorgopis lynx. (Attoidæ.)

Locality : Prussian amber. Horizon: Ligurian.

1854. Menge. Koch-Berendt, Bernst. befindl. organ. reste vorw., i, th. ii : 93.

See also G. melanocephala, etc.

2021. Gorgopis marginata. (Attoidæ.)

Locality: Prussian amber. Horizon : Ligurian.

1854. Menge. Koch-Berendt, Berust. befindl. organ. reste vorw., i, th. ii: 93. 1856. Giebel. Insect. $d$. vorwelt, 467.

See also Phidippus marginatus.

2022. Gorgopis melanocephala. (Attoidæ.)

Locality: Prussian amber. Horizon: Ligurian.

1856. Giebel. Insect. d. vorwelt, 467.

See also Phidippus melanocephalus, P. impressus, Gorgopis lynx.

2023. Gorgopis naumanni. (Attoidæ.)

Locality: Prussian amber. Horizon: Ligurian.

1856. Giebel. Insect. d. vorwelt, 467.

2024. Gorgopis torva. (Attoidæ.)

Locality: Prussian amber. Horizon: Ligurian.

1854. Menge. Koch-Berendt, Bernst. befindl. organ. reste vorw., i, th. ii : 93.

See also G. frenata, etc.

2025. Græa epeiroidea. (Epeirides.)

Locality: Prussian amber. Horizon: Ligurian.

1870. Thorell. European spiders, i: 225.

See also Gea epeiroidea. 
2026. Græa militaris. (Epeirides.)

Locality: Prussian amber. Horizon: Ligurian.

1870. Thorell.

European spiders, i : 225.

See also Gea militaris, etc.

2027. Græa obscura. (Epeirides.)

Locality: Prussian amber. Horizon: Ligurian.

1870. Thorell. European spiders, i: 225.

See also Gea obscura, etc.

2028. Græa pubescens. (Epeirides.)

Locality: Prussian amber. Horizon: Ligurian.

1870. Thorell. European spiders, i: 225.

See also Gea pubescens.

2029. Græa triquetra. (Epeirides.)

Locality: Prussian amber. Horiznn: Ligurian.

1870. Thorell. European spiders, i: 225.

See also Gea triquetra, etc.

Herselioides thanatiformis. See Hersilioides thanatiformis.

2030. Hersilia -. (Hersilioids.)

Locality: Prussian amber. Horizon: Ligurian.

1845. Berendt. Bernst. befindl. organ. reste vorw., i: 56.

2031. Hersilia aquisextana. (Hersilioidæ.)

Compared with Hersilia indica Luc.

Locality: Aix, France. Horizon: Ligurian.

1887. Gourret. Rec. zool. Suisse, iv: 458-460. Pl.21, fig. 14.

2032. Hersilia longipes. (Hersilioidæ.)

Locality: Prussian amber. Horizon: Ligurian.

1856. Giebel. Insect. d. vorwelt, 450.

2033. Hersilia miranda. (Hersilioidæ.)

Locality: Prussian amber. Horizon: Ligurian.

1845. Koch-Berendt. Jahrb. min., $1845: 871$.

1854. " Berendt, Bernst. befindl, organ. reste vorw., i, th. ii: 50 . Pl. 17, fig. 147.

1856. Giebel. Insect. d. vorwelt, 450.

2034. Hersilioides thanatiformis. (Hersilioidæ.)

Locality: Aix, France. Horizon: Ligurian.

1887. Gourret. Rec. zool. Suisse, iv : 460-462 (Herselioides). Pl. 23, fig. 24.

2035. Heteromma intersecta. (Drassides.)

Locality: Prussian amber. Horizon: Ligurian.

1856. Menge.

Progr. petrischule Danzig, 1856: 8.

1870. Thorell.

European spiders, i : 229. 
2036. Histopona? anthracina. (Agalenides.)

Locality : Rott, Rhenish Prussia. Horizon: Aquitanian.

1878. Bertkau. Verhandl. naturh. ver. preuss. rheinl., (4), v: 351-352. Pl. 5, fig. 5 .

2037. Idmonia virginea. (Drassides.)

Locality: Prussian amber. Horizon: Ligurian.

1854. Menge. Koch-Berendt, Bernst. befindl. organ. reste vorw., i, th. il : 94 (undescr.).

1853-56. Bronn. Lethra geogn., $3^{\mathrm{e}}$ aufl., iii : 639 .

1856. Giebel. $\quad$ Insect. d. vorwelt, 469.

1870. Thorell. European spiders, i: 230.

2038. Ixodes tertiarius. (Ixodidæ.)

Locality : Green River, Wyo. Horizon: Oligocene.

1885. Scudder. Zittel, Handb. palaeont., i, th. ii : 733. Fig. 906.

1886. " "Zittel-Barrois, Traité de paléont., ii: 732. Fig. 922.

1890. " Tert. ins. N. A., 47. Pl.6, fig. 12.

2039. Ieda -. (Attides.)

Locality: Prussian amber. Horizon: Ligurian.

1845. Berendt. Bernst. befindl. organ. reste vorw., i: 56.

2040. Leda promissa. (Attides.)

Locality: Prussian amber. Horizon: Ligurian.

1845. Koch-Berendt. Jahrb. min., 1845: 87\%.

1854. " " Berendt, Bernst. befindl, organ. reste vorw., i, th. ii: 93-94. $\quad P l$. 10, fig. 93.

3.856. Giebel. Insect. d. vorwelt, 468-469.

1870. Thorell. European spiders, i: 232.

See also Steneattus promissus.

2041. Leiobunum longipes. (Phalangioidæ.)

Locality : Prussian amber. Horizon: Ligurian.

1854. Menge. Koch-Berendt, Bernst. befindl, organ. reste vorw., i, th. ii: 102.

1856. Giebel. Insect. $d$. vorwelt, $47 \%$.

Iimnochares —. See II, Hemiptera, Idem.

2042. Linoptes —. (Lycosoidæ.)

Locality: Prussian amber. Horizon: Ligurian.

1856. Menge. Progr. petrischule Danzig, 1856: 8.

2043. Linoptes oculeus. (Lycosoidæ.)

Locality : Prussian amber. Horizon : Ligurian.

1854. Menge. Koch-Berendt, Bernst. befindl. organ. reste vorw., i, th. ii: 94 (undescr.).

1853-'56. Bronn. Lethæa geogn., $3^{\mathrm{e}}$ aufl., iii : 639.

1856. Giebel. Insect. d. vorwelt, 469.

1870. Thorell. European spiders, i : 232.

2044. Linyphia -. (Theridides.)

Locality: Prussian amber. Horizon: Ligurian.

1845, Berendt. Bernst. befindl. organ. reste vorw., i : 56. 


\section{Linyphia - (2 sp.). (Theridides.)}

Locality : Prussian amber. Horizon: Ligurian.

1856. Menge. Progr. petrischule Danzig, 1856: 7 .

$$
\text { 2046. Linyphia ——. (Theridides.) }
$$

Locality : Florissant, Colo. Horizon : Oligocene.

1882. Scudder. Harv, univ. bull., ii : -303 .

1882. " " Field nat., i: 62 .

1883. " Ann. rep. U. S. geol. surv. terr., xii : 288.

2047. Linyphia andræi. (Theridides.)

Locality: Rott, Rhenish Prussia. Horizon: Aquitanian.

1878. Bertkau. Verhandl, naturh. ver. preuss. rheinl., (4), v: 350-351. Pl. 5, fig. 6.

2048. Iinyphia cheiracantha. (Theridides.)

Locality : Prussian amber. Horizon : Ligurian.

1845. Koch-Berendt. Jahrb. min., 1845: 871.

1854. " Berendt, Bernst. befindl. organ. reste vorw., i, th. ii : $41 . \quad P l$. 16, fig. 137.

1856. Giebel. Insect. d, vorwelt, 445.

See also L. oblonga.

2049. Linyphia oblonga. (Theridides.)

Locality : Prussian amber. Horizon: Ligurian.

1845. Koch-Berendt. Jahrb. min., 1845: 871.

1854. "6 Berendt, Bernst. befindl, organ. reste vorw., i, th. ii: $\mathbf{4 0 - 4 1}$.

1854. Menge. Ibid., 8,42 (=L. cheiracantha).

See also L. cheiracantha.

2050. Linyphia retensa. (Theridides.)

Compared with L. cheiracantha.

Locality : Florissant, Colo. Horizon: Oligocene.

1890. Scudder. Tert. ins. N. A., 75-76. Pl. 11, figs. 25, 27.

2051. Linyphia rottensis. (Theridides.)

Locality : Rott, Rhenish Prussia. Horizon: Aquitanian.

1878. Bertkan. Verhandl. naturh. ver. preuss. rheinl., (4), v: 349-350. Pl. 5, fig. 2.

2052. Iycosoides hersiliformis. (Lycosoidæ.)

Locality : Célas, Gard, France. Horizon: Oligocene.

1887. Gourret. Rec. zool. Suisse, iy : 443-444. Pl.21, fig. 13.

\section{Macaria —. (Drassides.)}

Locality: Prussian amber. Horizon : Ligurian.

1845. Berendt. Bernst. befindl, organ. reste vorw., i: 56.

$$
\text { 2054. Macaria - (4 sp.). (Drassides.) }
$$

Locality: Prussian amber. Horizon: Ligurian.

1856. Menge. Progr. petrischule Danzig, 1856: 7 . 
2055. Macaria ovata. (Drassides.)

Locality: Prussian amber. Horizon: Ligurian.

1854. Menge. Koch-Berendt, Bernst. befindl. organ. reste vorw., i, th. ii : 64 , 124 (undescr.).

1856. Giebel. Insect. $d$. vorwelt, 457.

\section{Macaria procera. (Drassides.)}

Compared with M. fastuosa.

Locality: Prussian amber. Horizon : Ligurian.

1845. Koch-Berendt. Jahrb. min., 1845: 872.

1854.

1856. Giebel.

Berendt, Bernst. befindl, organ. reste vorw., i, th.ii: 63-64. Pl. 6, fig. 55 .

Insect. d, vorwelt, 457.

2057. Macaria squamata. (Drassides.)

Locality: Prussian amber. Horizon : Ligurian.

1854. Menge. Koch-Berendt, Bernst. befindl. organ. reste vorw., i, th. ii : 64 (undescr.).

1856. Giebel. Insect. d. vorwelt, 457.

2058. Macaria tenella. (Drassides.)

Compared with M. procera K. and B.

Locality : Oeningen, Baden. Horizon: Tortonian.

1865. Heer.

Urwelt der Schweiz, 356, 357. Fig. 218.

187\%. “

1876. " "

Monde prim. Suisse, 436, 438. Fig. 218, on p. 437.

Prim. world Switz., ii : 10, 12. Fig. 218.

1879. " "

Urwelt der Schweiz, $2^{\mathrm{e}}$ aufl, 380,382 . Fig. 253, on p. 381.

2059. Macaria tenuis. (Drassides.)

Locality : Prussian amber. Horizon: Ligurian.

1854. Menge. Koch-Berendt, Bernst. befindl. organ. reste vorw., i, th. ii : 64 (undescr.).

1856. Giebel. Insect. d. vorwelt, 457 .

\section{Mastigusa acuminata.}

Locality : Prussian amber. Horizon : Ligurian.

1854. Menge.

1853-'56. Bronn.

Koch-Berendt, Bernst. befindl. organ. reste vorw., i, th. ii : 94 (undeser.).

1856. Giebel.

1870. Thorell.

Lethæa geogn., $3^{\mathrm{e}}$ anfl., iii.: 639.

Insect. d. vorwelt, 469 .

European spiders, i: 233.

2061. Megameropsis aquensis. (Trombididæ)

Locality: Aix, France. Horizon: Ligurian.

1887. Gourret. Rec. zool. Suisse, iv: 435-437. Pl. 20, fig. 1.

2062. Melanophora - (Drassides.)

Locality : Prussian amber. Horizon : Ligurian.

1845. Berendt. Bernst. befindl. organ. reste vorw., i: 56.

2063. Melanophora (4 sp.). (Drassides.)

Locality : Prussian amber. Horizon : Liguriann.

1856. Menge.

„Progr. petrischule Danzig, i1856: , 7 . 


\section{Melanophora concinna. (Drassides.)}

Locality : Prussian amber. Horizon : Ligurian.

1845. Koch-Berendt. Jahrb. min., 1845: 872.

1854. " Berendt, Bernst. befindl. organ. reste vorw., i, th. ii : 61-62. Pl. 6, fig. 52.

1856. Giebel. Insect. d. vorwelt, 456.

See also M. nobilis.

2065. Melanophora lepida. (Drassides.)

Locality : Prussian amber. Horizon: Ligurian.

1854. Menge. Koch-Berendt, Bernst. befindl. organ. reste vorw., i, th. ii : 63 .

1856. Giebel. Insect. d. vorwelt, 457.

2066. Melanophora mundula. (Drassides.)

Locality : Prussian amber. Horizon: Ligurian.

1845. Koch-Berendt. Jahrb. min. $1845: 872$.

1854. " B Berendt, Bernst. befindl, organ. reste vorw., i, th. ii : 62-63. Pl. 6, fig. 54 .

1856. Giebel. Insect. d. vorwelt, 456-457.

See also Therea hispida.

2067. Melanophora nitida. (Drassides.)

Locality : Prussian amber. Horizon: Ligurian.

1854. Menge. Koch-Berendt, Bernst. befindl. organ. reste vorw., i, th. ii : 63 (undescr.).

1856. Giebel. Insect. d. vorwelt, 457.

2068. Melanophora nobilis. (Drassides.)

Locality : Prussian amber. Horizon : Ligurian.

1845. Koch-Berendt. Jahrb. min., $1845: 872$.

1854. " Berendt, Bernst. befindl. organ. reste vorw., i, th. ii: 62. Pl.6, fig. 53.

1854. Menge.

Ibid., 8 (=M. concinna).

1856. Giebel.

See also M. concinna.

2069. Melanophora regalis. (Drassides.)

Locality : Prussian amber. Horizon : Ligurian.

1845. Koch-Berendt. Jahrb. min., 1845 : 872.

1854. " Berendt, Bernst. befindl. organ. reste vorw., i, th. ii: 60-61. Pl. $6, f i g .51$.

1856. Giebel. Insect. d, vorwelt, 456.

Micriphantes (several species). See Micryphantes, same species.

2070. Micryphantes —-. (Theridides.)

Locality: Prussian amber. Horizon: Ligurian.

1845. Berendt. Bernst. befindl. organ. reste vorw., i: 56 .

2071. Micryphantes - (4 sp.). (Theridides.)

Locality : Prussian amber. Horizon: Ligurian.

1856. Menge. Progr. petrischule Danzig, 1856: 7 (Micriphantes). 
2072. Mioryphantes globulus. (Theridides.)

Locality : Prussian amber. Horizon: Ligurian.

1854. Menge. Koch-Berendt, Bernst. befindl. organ. reste vorw., i, th. ii : 40 (undescr.). (Micriphantes.)

1856. Giebel. Insect. d. vorwelt, 444.

2073. Micryphantes infulatus. (Theridides.)

Locality: Prussian amber. Horizon : Ligurian.

1845. Koch-Berendt. Jahrb. min., $1845: 871$.

1854. "6 Berendt, Bernst. befindl. organ. reste vorw., i, th. ii: 40. $P t_{\alpha}$ 4, fig. 29. (Micriphantes.)

1856. Giebel. Insect. d. vorwelt, 444.

2074. Micryphantes molybdinus. (Theridides.)

Locality : Prussian amber. Horizon: Ligurian.

1845. Koch-Berendt. Jahrb. min., 1845: 871. (Micriphantes.)

1854. " $\quad$ Berendt, Bernst. befindl. organ. reste vorw., i, th. ii: 39 . $P l$. 4, fig. 27.

1856. Giebel. Insect. d. vorwelt, 444 .

2075. Micryphantes regularis. (Theridides.)

Locality: Prussian amber. Horizon: Ligurian.

1845. Koch-Berendt. Jahrb. min., 1845: 871. (Micriphantes.)

1854. " " Berendt, Bernst. befindl, organ. reste vorw., i, th. ii : 38-39. $\quad P$. 4, fig. 28.

1856. Giebel. Insect. d. vorwelt, 444.

2076. Micryphantes turritus. (Theridides.)

Locality: Prussian amber. Horizon: Ligurian.

1854. Menge. Koch-Berendt, Bernst. befindl. organ. reste vorw., i, th. ii : 40 (undescr.). (Micriphantes.)

1856. Giebel. Insect. d. vorwelt, 444.

2077. Mizalia —. (Mizalioidæ.)

Locality : Prussian amber. Horizon: Ligurian.

1845. Berendt. Bernst. befindl, organ. reste vorw., i: 56.

2078. Mizalia - (2 sp.). (Mizalioidæ.)

Locality : Prussian amber. Horizon: Ligurian.

1856. Menge. Progr. petrischule Danzig, 1856: 8 .

2079. Mizalia globosa. (Mizalioidæ.)

Locality: Prussian amber. Horizon: Ligurian.

1845. Koch-Berendt. Jahrb. min., 1845: 871.

1854. " " Berendt, Bernst. befindl. organ. reste vorw., i, th. ii : $43 . \quad P l .5$, fig. 32.

1856. Giebel. Insect. d. vorwelt. 446.

See also Theridium globosum.

Bull. $71-18$ 
2080. Mizalia pilulosa. (Mizalioidæ.)

Locality: Prussian amber. Horizon: Ligurian.

1845. Koch-Berendt. Jahrb. min., 1 $845: 871$.

1854. " Berendt, Bernst, befindl, organ. reste vorw., i, th. ii: 45 . Plo 5, fig. 34; Pl. 16, fig. 138.

1854. Menge. Ibid., 8 (= M. rostrata).

See also Mizalia rostrata.

\section{Mizalia punctulata. (Mizalioidæ.)}

Locality: Prussian amber.' Horizon : Ligurian.

1845. Koch-Berendt. Jahrb. min., $1845: 871$.

1854. " Berendt, Berust. befindl. organ. reste vorw., i, th. ii : $42-43$. See also Antopia punctulata. Pl. 5, fig. 31 .

\section{Mizalia rostrata. (Mizalioidæ.)}

Locality : Prussian amber. Horizon : Ligurian.

1845. Koch-Berendt. Jahrb. min., 1845: 871.

1854. " Berendt, Bernst. befindl. organ. reste vorw., i, th, ii: 44. Pl. 5, fig. 33.

1856. Giebel. Insect. d. vorwelt, 445-440̉.

1870. Thorell. European spiders, i: $2 * 8$.

1885. Scudder. Zittel, Handb. palaeont., i, th. ii: 743. Fig. 924.

1886. " Zittel-Barrois, Traité de paléont., ii : 743. Fig. 941.

See also M. pilulosa.

2083. Mizalia truncata. (Mizalioidæ.)

Locality: Prussian amber. Horizon: Ligurian.

1854. Menge. Koch-Berendt, Bernst. befindl, organ. reste vorw., i, th. ii: 45.

1856. Giebel. Insect. d. vorwelt, 446 .

2084. Mygale ambigua. (Theraphosoidæ.)

Locality : Aix, France. Horizon : Ligurian.

1887. Gourret. Rec. zool. Suısse, iv: 480-482. Pl. 23, fig. 28.

\section{Nemastoma - (Nemastomoidæ.)}

Locality : Prussian amber. Horizon: Ligurian.

1845. Berendt. Bernst. befindl. organ. reste vorw., i : 56.

2086. Nemastoma clavigerum. (Nemastomoidæ.)

Locality : Prussian amber. Horizon: Ligurian.

1854. Menge.

Koch-Berendt, Berust. befindl, organ, reste vorw., i, th. ii: 99.

1856. Giebel.

Insect. d. vorwelt, 475 .

\section{Nemastoma denticulatum. (Nemastomoidæ.)}

Locality: Prussian amber. Horizon: Ligurian.

1845. Koch-Berendt. Jahrb. niu., 1845: 872.

1854. "6 Berendt, Bernst. befindl. organ. reste vorw., i, th. ii: 98-99. $P l_{\text {。 }}$ 11, fig. 98.

1856. Giebel.

Insect. d. vorwelt, 475 . 
2088. Nemastoma incertum. (Nemastomoidæ.)

Locality: Prussian amber. Horizon: Ligurian.

1845. Koch-Berendt. Jahrb. min., 1845: 872.

1854. " Berendt, Bernst. befindl. organ. reste vorw., i, th. ii: 99 . Pl. 17, fig. 149.

1856. Giebel. Insect. d. vorwelt, 475.

2089. Nemastoma tuberculatum. (Nemastomoidæ.)

Locality : Prussian amber. Horizon: Ligurian.

1845. Koch-Berendt. Jahrb. min., 1845: 872.

1854. " $\quad$ Berendt, Bernst. befindl. organ. reste vorw., i, th. ii : 97-98. Pl. 11, fig. 97.

1856. Giebel, Insect. d. vorwelt, 474-475.

2090. Nephila —_. (Epeirides.)

Compared with N. plumipes.

Locality : Florissant, Colo. Horizon: Oligocene.

1881. Scudder. Bull. U. S. geol. surv. terr., vi : 294.

1882. " Harv, univ. bull., ii : 303 .

1882. " Field nat., i: 63.

1883. " Ann. rep. U. S. geol. surv. terr., xii : 288.

See also Nephila pennatipes.

2091. Nephila pennatipes. (Epeirides.)

Compared with N. plumipes.

Locality: Florissant, Colo. Horizon: Oligocene.

1885. Scudder. - Zittel, Handb. palaeont., i, th. ii : 744. Fig. 926.

1886. " "Zittel-Barrois, Traité de paléont., ii : 744. Fig. 943.

1890. " " Tert. ins. N. A., 89-90. Pl. 11, fig. 12.

1890. McCook. Amer. spiders, ii : 450-451. Fig. 372.

See also Nephila —.

2092. Nothrus convexulus. (Oribatidæ.)

Locality: Prussian amber. Horizon: Ligurian.

1884. Karsch. Berl. entom. zeitschr., xxviii: 175.

See also Oribates convexulus.

2093. Nothrus kühli. (Oribatidæ.)

Locality : Prussian amber. Horizon: Ligurian.

1884. Karsch. Berl. entom. zeitschr., xxviii: 176. Figs. 1-3.

2094. Nothrus punctulus. (Oribatidæ.)

Locality : Prussian amber. Horizon : Ligurian.

1884. Karsch. Berl. entom. zeitschr., xxviii: 176.

2095. Nothrus sulcatus. (Oribatidæ.)

Locality: Prussian amber. Horizon : Ligurian.

1884. Karsch. Berl. entom. zeitschr., xxviii : 176.

2096. Obisium -. ('helonethi.)

Locality: Prussian amber. Horizon: Ligurian.

1845. Berendt. Berust, befindl. organ. reste vorw., i : 56 . 
2097. Obisium - (Chelonethi.)

1846. Giebel. Paläozool., 26\%.

\section{Locality Horizon: Tertiary.}

\section{Obisium —. See Chelifer — Schweigger.}

2098. Obisium rathkei. (Chelonethi.)

Locality: Prussian amber. Horizon: Ligurian.

1845. Koch-Berendt. Jahrb. min., 1845 : 872.

1854. " Berendt, Bernst. befindl, organ, reste vorw., i, th. ii : 96-97. Pl. 10, fig. 96.

1855. Menge.

Scheerenspinnen, 28-29. Pl. 4, fig. 4 (rathkii). (Probably young of Chel. kleemanni.)

1856. Giebel. Insect. d. vorwelt, 473.

See also Chelifer kleemanni.

Obisium rathkii. See Obisium rathkei.

2099. Obisium sieboldii. (Chelonethi.)

Locality: Prussian amber. Horizon: Ligurian.

1854. Menge. Koch-Berendt, Bernst. befindl. organ. reste vorw., i, th. ii: 97 (sieboldtii).

1856. Giebel. Insect. $d$. vorwelt, 473 (sieboldi).

1870. Hagen. Proc. Bost. soc. nat. hist., xiii : 270 (sieboldtii).

See also Chelifer sieboldii.

Obisium sieboldtii. See Obisinm sieboldii.

2100. Ocia hirsuta. (Thomisides.)

Locality: Prussian amber. Horizon: Ligurian.

1854. Koch-Berendt. Berendt, Bernst. befindl. organ. reste vorw., i, th. ii : 8 (name only).

2101. Ocypete - (Thomisides.)

Locality : Prussian amber. Horizon: Ligurian.

1845. Berendt. Bernst. befindl. organ. reste vorw., i: 56.

2102. Ocypete angustifrons. (Thomisides.)

Locality: Prussian amber. Horizon: Ligurian.

1854. Menge. Koch-Berendt, Bernst. befindl, organ. reste vorw., i, th. ii: 85 (undescr.).

1856. Giebel. Insect. d. vorwelt, 465 .

2103. Ocypete crassipes. (Thomisides.)

Locality : Prussian amber. Horizon: Ligurian.

1845. Koch-Berendt. Jahrb. min., $1845: 872$.

1854. " $\quad$ Berendt, Bernst. befindl. organ. reste vorw., i, th. ii : 84-85. Pl. 9, fig. 79 .

1856. Giebel. Insect. d. vorwelt, 465.

2104. Ocypete decumana. (Thomisides.)

Locality: Prussian amber. Horizon: Ligurian.

1845. Koch-Berendt. Jahrb. min., 1845: 872.

1854.

Berendt, Bernst. befindl. organ. reste vorw., i, th. ii: 85 . Pl. 9 , fig. 80. 
1856. Giebel.

1856. Menge.
Insect. d. vorwelt, 465.

Progr. petrischule Danzig, 1856: 8 (Oxypete).

2105. Ocypete marginata. (Thomisides.)

Locality: Prussian amber. Horizon: Ligurian.

1854. Menge.

Koch-Berendt, Bernst. befindl. organ. reste vorw., i, th. ii : 85. 1856. Giebel.

\section{Ocypete triguttata. (Thomisides.)}

Locality: Prussian amber. Horizon: Ligurian.

1845. Koch-Berendt. Jahrb. min., $1845: 872$.

1854. " Berendt, Bernst. befindl, organ. reste vorw., i, th. ii: $86 . P l$. 16, fig. 141.

See also Pythonissa triguttata.

$$
\text { 2107. Onca - ( } 2 \text { sp.). (Epeirides.) }
$$

1856. Menge. Progr. petrischule Danzig, 1856: 6.

Locality: Prussian amber. Horizon: Ligurian.

\section{Onca lepida. (Epeirides.)}

Locality: Prussian amber. Horizon: Ligurian.

1854. Menge. Koch-Berendt, Bernst. befindl. organ. reste vorw., i, th. ii: 24 (undescr.).

1856. Giebel. Insect. d. vorwelt, 437.

2109. Onca pumila. (Epeirides.)

Locality: Prussian amber. Horizon: Ligurian.

1854. Menge. Koch-Berendt, Bernst. befindl, organ. reste vorw., i, th. ii: 24 (undeser.).

1856. Giebel. Insect, d. vorwelt, 437.

$$
\text { 2110. Opilio —. (Phalangioidæ.) }
$$

Locality: Prussian amber. Horizon: Ligurian.

1845. Berendt. Bernst. befindl. organ. reste vorw., i: 56.

$$
\text { 2111. Opilio —. (Phalangioidæ.) }
$$

Locality : Prussian amber. Horizon: Ligurian.

1835. Gravenhorst. Uebers. schles. gesellsch. vaterl. cult., 1834: 92.

\section{Opilio corniger. (Phalangioidæ.)}

Locality : Prussian amber. Horizon : Ligurian.

1854. Menge. 1856. Giebel.
Koch-Berendt, Bernst. befindl. organ. reste vorw., i, th. ii : 101. Insect. d, vorwelt, 476 .

2113. Opilio ovalis. (Phalangioidæ.)

Locality : Prussian amber. Horizon: Ligurian.

1845. Koch-Berendt. Jahrb. min., 1845: 872.

1854. " Berendt, Bernst. befindl. organ. reste vorw., i, th. ii: 99-100. Pl. 12 , fig. 99.

1856. Giebel. Inseet. d. vorwelt, 476 . 
2114. Opilio ramiger. (Phalangioidæ.)

Locality: Prussian amber. Horizon: Ligurian.

1845. Koch-Berendt. Jahrb. min., 1845 : 872.

1854. " Berendt, Bernst. befindl. organ. reste vorw., i, th. ii: 100-101. Pl. 12, fig. 100.

1856. Giebel. Insect. d. vorwelt, 476.

2115. Opisthophylax exarata. (Thomisides.)

Locality: Prussian amber. Horizon: Ligurian.

1856. Menge. Progr. petrischule Danzig, 1856: 9 .

1870. Thorell. European spiders, i : 231.

2116. Oribates - (Oribatidæ.)

Locality : Prussian amber. Horizon: Ligurian.

1845. Berendt. Bernst. befindl. organ. reste vorw., i : 56 .

2117. Oribates convexulus. (Oribatidæ.)

Locality: Prussian amber. Horizon: Ligurian.

1845. Koch-Berendt. Jahrb. min., 1845 : 87\%.

1854. " Berendt, Bernst. betindl. organ. reste vorw., i, th. ii: $109 . \quad P l$. 13, fig. 112.

1856. Giebel. Insect. $d$. vorwelt, 483 (convexus).

See also Nothrus convexulus.

Oribates convexus. See Oribates convexulus.

2118. Oribates politus. (Oribatidæ.)

Locality : Prussian amber. Horizon: Ligurian.

1845. Koch-Berendt. Jahrb. min., 1845: 872.

1854. " Berendt, Bernst. befindl, organ, reste vorw., i, th, ii: 110, Pl. 17, fig. 15:.

1856. Giebel. Insect. $d$. vorwelt, 483.

Oxypete decumana. See Ocypete decumana.

2119. Parattus - (3 sp.). (Attoidæ.)

Locality: Florissant, Colo. Horizon: Oligocene.

1882. Scudder. Harv. univ. bull., ii : 303.

1882. " " Field nat., i: 62 .

1883. " " Ann. rep. U. S. geol, surv, terr., xii : 287.

2120. Parattus evocatus. (Atteidæ.)

Locality: Florissant, Colo. Horizon: Oligocene.

1890. Scudder. Tert. ins. N. A., 54-55.

2121. Parattus latitatus. (Attoidæ.)

Locality : Florissant, Colo. Horizon: Oligocene.

1890. Scudder. Tert. ins. N. A., 55-56.

2122. Parattus resurrectus. (Atto1dæ.)

Locality : Florissant, Colo. Horizon: Oligocene.

1890. Scudder. Tert. ins. N. A., 53-54. Pl. 11, fig. 26.

Penthacleus tristiculus. See Penthaleus tristiculus. 


\section{Penthaleus -. (Trombididæ.)}

Locality: Prussian amber. Horizon: Ligurian.

1845. Berendt. Bernst, befindl. organ, reste vorw., i: 56.

\section{Penthaleus tristiculus. (Trombididœ.)}

Locality: Prussian amber. Horizon: Ligurian.

1845. Koch-Berendt, Jahrb. min., 1845: 872 (Penthaclens).

1854. " B Berendt, Bernst. befindl. organ. reste vorw., i, th. ii : 108. Pl. 13, fig. 109.

1856. Giebel. Insect. d. vorwelt, 482.

2125. Phalangillum hirsutum. (Phalangioidæ.)

Locality : Aix, France. Horizon: Ligurian.

1887. Gourret. Rec. zool. Suisse, iv: 487-489. Pl. 21, fig. 15.

2126. Phalangium —. Phalangioidæ.)

Locality: Prussian amber. Horizon: Ligurian.

1820. Schlotheim. Petrefactenk., 43.

2127. Phalangium —_. (Phalangioidæ.)

Compared with $\mathrm{P}$, phaleratum.

Locality: Aix, France. Horizon: Ligurian.

1829. Serres. Geogn, terrains tert., 220.

1847. Walck.-Gerv. Hist. nat. apt., iv : 345.

See also Phryuus fossilis.

2128. Phalangium cancroides (recent). (Phalangioidæ.)

Locality : Prussian amber. Horizon: Ligurian.

[1758. Linné. Syst. nat., ii : 1028.]

1830. Berendt. Insekten im bernstein, 38 .

1839. Brull6,

Gisem. ins. foss., 19.

2129. Phalangium opilio (recent). (Phalangioidæ.)

Locality : Prussian amber. Horizon: Ligurian.

[1758. Linné. Syst. nat., ii : 1027.]

1830. Berendt.

1839. Brullé.

Insekten im bernstein, 38 .

Gisem. ins. foss., 19.

2130. Phalangium succineum. (Phalangioidæ.)

Locality : Prussian amber. Horizon : Ligurian.

1822. Presl.

Del. prag., i : 209 .

Phalangopus subtiles. See Phalangopus subtilis.

2131. Phalangopus subtilis. (Scytodoidæ.)

Locality: Prussian amber. Horizon: Ligurian.

1854. Menge. Koch-Berendt, Bernst. befindl. organ. reste vorw., i, th. ii : 94 (undescr.) (subtiles).

1853-'56. Bronn. Lethæa geogn., $3^{\mathrm{e}}$ anfl., iii : 639.

1856. Giebel. Insect. d. vorwelt, 469.

1870. Thorell. European spiders, i : 228. 


\section{Phidippus - (Attoidæ.)}

Locality : Prussian amber. Horizon: Ligurian.

1845. Berendt. Bernst. befindl. organ. reste vorw., i: 56.

\section{Phidippus fasciatus. (Attoidæ.)}

Locality : Prussian amber. Horizon: Ligurian.

1845. Koch-Berendt. Jahrb. min., 1845: 872.

1854. " " Berendt, Bernst. befindl. organ. reste vorw., i, th. ii : 89-90. $P$. 10, figs. 86-87.

See also Gorgopis faseiata, etc.

2134. Phidippus formosus. (Attoidæ.)

Locality: Prussian amber. Horizon: Ligurian.

1845. Koch-Berendt. Jahrb. min., 1845 : $\$ 72$.

1854. " Berendt, Bernst. befindl. organ. reste vorw., i, th. ii: 90 . Pl. 10, fig. 88.

See also Gorgopis fasciata, ete.

2135. Phidippus frenatus. (Attoidæ.)

Locality: Prussian amber. Horizon : Ligurian.

1845. Koch-Berendt. Jahrb. min., 1845: 872.

1854. " Berendt, Bernst. befindl. organ. reste vorw., i, th. ii: 88-89. Pl. 9, figs. 8:3, 84 .

1890. McСooí. Amer. spiders, ii: 467. Figs. 391-392.

See also Gorgopis frenata, etc.

2136. Phidippus gibberulus. (Attoidæ.)

Locality : Prussian amber. Horizon: Ligurian.

1845. Koch-Berendt. Jahrb. min., 1845: 872.

1854. " Berendt, Berust. befindl, organ. reste vorw., i, th. ii: 92. Pl. 10, fig. 92.

See also Euophrys gibberula.

2137. Phidippus impressus. (Attoidæ.)

Locality: Prussian amber. Horizon: Ligurian.

1845. Koch-Berendt. Jahrb. min., $1845: 872$.

1854. " $"$ Berendt, Bernst. befindl. organ. reste vorw., i, th. ii : 91 . " $l$. 10, fig. 90.

See also Gorgopis melanocephala, etc.

2138. Phidippus marginatus. (Attoidæ.)

Locality: Prussian amber. Horizon : Ligurian.

1845. Koch-Berendt. Jahrb. min., $1845: 87 \%$.

1854. " Berendt, Bernst. befindl. organ. reste vorw., i. th. ii: 92-93. Pl. 16, fig. 142.

See also Gorgopis marginata.

\section{Phidippus melanocephalus. (Attoidæ.)}

Locality: Prussian amber. Horizon: Ligurian.

1845. Koch-Berendt. Jahrb. min., $1845: 872$.

1854. " $\quad$ Berendt, Bernst. befindl. organ. reste vorw., i, th. ii: 89. Pl. 9 , fig. 85.

See also Grorgopis melanocephala, etc. 
2140. Phidippus paululus. (Attoidæ.)

Locality : Prussian amber. Horizon : Ligurian.

1845. Koch-Berendt. Jahrb. min., 1845: 872.

1854. " Berendt, Bernst. befindl. organ. reste vorw., i, th. ii: $91 . \quad P l$. 10, fig. 89.

See also Gorgopis frenata, etc.

2141. Phidippus pusillus. (Attoidæ.)

Locality: Prussian amber. Horizon : Ligurian.

1845. Koch-Berendt. Jahrb. min., 1845: 872.

1854. " Berendt, Bernst. befindl, organ, reste vorw., i, th, ii : 92 . Pl. 10, fig. 91 .

See also Gorgopis frenata, etc.

2142. Philodromus - _. (Thomisides.)

Locality: Prussian amber. Horizon: Ligurian.

1845. Berendt. Bernst. befindl. organ. reste vorw., i: 56.

2143. Philodromus dubius. (Thomisides.)

Locality: Prussian amber. Horizon: Ligurian.

1845. Koch-Berendt. Jahrb. min., 1845: 872.

1854. " " Berendt, Bernst, befindl, organ. reste vorw., i, th. ii: 82. Pl. 8, fig. 76.

See also Pythonissa affinis.

2144. Philodromus griseus. (Thomisides.)

Locality: Prussian amber. Horizon: Ligurian.

1856. Menge. Progr. petrischule Danzig, 1856: 9 (undescr.).

2145. Philodromus microcephalus. (Thomisides.)

Locality: Prussian amber. Horizon: Ligurian.

1845. Koch-Berendt. Jahrb. min., $1845: 872$.

1854. " $\quad$ Berendt, Bernst. befindl, organ. reste vorw., i, th, ii : 81-82. $P l$. 8, fig. 75.

1890. McCook. Amer. spiders, ii : 467. Fig. 396.

See also Pythonissa microcephala.

2146. Philodromus reptans. (Thomisides.)

Locality : Prussian amber. Horizon: Ligurian.

1854. Menge. Koch-Berendt, Bernst. befindl. organ. reste vorw., $i$, th. ii: 84 (undeser.).

1856. " "

1856. Giebel

Progr. petrischule Danzig, 1856 : 9.

Insect. d. vorwelt, 464.

2147. Philodromus retrogradus. (Thomisides.)

Locality : Prussian amber. Horizon: Ligurian.

1854. Menge. Koch-Berendt, Bernst. befindl. organ. reste vorw., i; th. ii: 84 (undescr.).

1856. Giebel. Insect. $d$. vorwelt, 464 . 


\section{Philodromus spinimanus. (Thomisides.)}

Locality : Prussian amber. Horizon: Ligurian.

1845. Koch-Berendt, Jahrb. min., 1845 : 872.

1854. " Berendt. Bernst. befindl. organ. reste vorw, i. th. ii " 83. $P l$. 9, fig. 78 .

See also Pythonissa spinımana.

2149. Philodromus squamiger. (Thomisides.)

Locality : Prussian amber. Horizon: Ligurian.

1845. Koch-Berendt. Jahrb. min., 1845: 872.

1854. " " Berendt, Bernst. befindl, organ, reste vorw,, i, th. ii: 83 . Pl. 9, fig. 77.

See also Pythonissa sericata.

\section{Pholcus - (Scytodoidæ.)}

Locality : Prussian amber. Horizon: Ligurian.

1856. Menge. Progr. petrischule Danzig, 1856:9 (name only).

2151. Phrynus —, (Pedipalpi.)

Locality: Aix, France. Horizon: Ligurian.

1829. Serres. Géogu, terrains tert., 220.

2152. Phrynus fossilis. (Pedipalpi.)

Comparerl with P. phaleratus Panz.

Locality: Aix, France. Horizon: Ligurian.

1834. Keferstein. Naturg. erdkörp., ii : 370 (undescr.).

See also Phalanginm — Serres.

2153. Phrynus marioni. (Pedipalpi.)

Compared with P. reniformis Latr.

Locality: Aix, France. Horizon: Ligurian.

1887. Gourret. Rec. zool. Suisse, iv: 489-491. Pl. 21, fig. 11.

Phytonissa affinis. See Pythonissa affinis.

Phytonissa ambigua. See Pythonissa ambigua.

Phytonissa sericata. See Pythonissa sericata.

2154. Phytoptus antiquus (gall). (Phytoptidæ.)

Localities: Rott, Salzhausen, Germany. Horizon: Aquitanian.

1860. Heyden. Ber. oberh. gesellsch. nat. u. heilk., viii : 63.

1862. " Palaeontogr., $\mathrm{x}: 64 . \quad P l$. 10, fig. 1. (Not on Salix abbreviata Göpp., but on Passiflora brauni Ludw.).

1865. Heyd.-Heyd. Palaeontogr., xiv: 35.

2155. Platybunus —. (Phalangioidæ.)

Locality: Prussian amber. Horizon: Ligurian.

1845. Berendt. Bernst. befindl, organ. reste vorw., i: 56. 
2156. Platybunus dentipalpus. (Phalangioidæ.)

Locality: Prussian amber. Horizon: Ligurian.

1845. Koch-Berendt. Jahrb, min., 1845: 872.

1854. " Berendt, Bernst. befindl. organ, reste vorw., i, th. ii : 101-102. Pl. 15, fig. 125.

1856. Giebel. 1885. Scudder. 1886.
Insect. d. vorwelt, 476-47\%.

Zittel, Handb. palaeont., i, th. ii: 741. Fig. 917.

Zittel-Barrois, Traité de paléont., ii : 740. Fig. 934.

2157. Prodysdera intermedia. (Dysderides.)

Compared with Dysdera parvula, D. erythrina.

Locality: Aix, France. Horizon : Ligurian.

1887. Gourret. Rec. zool. Suisse, iv: 477-480. Pl. 20, figs. 4-6.

2158. Propetes - (5 sp.). (Attoidæ.)

Locality: Prussian amber. Horizon: Ligurian.

1856. Menge.

Progr. petrischule Danzig, 1856: 9.

2159. Propetes argutus. (Attoidæ.)

Locality: Prussian amber. Horizon: Ligurian.

1854. Menge.

1856. Giebel.

Koch-Berendt, Bernst. befindl. organ. reste vorw., i, th. ii : 93 (undeser.).

Insect. d. vorwelt, 468.

2160. Propetes felinus. (Attoidæ.)

Locality : Prussian amber. Horizon: Lignrian.

1854. Menge. Koch-Berendt, Berust, befindl, organ, reste vorw., $i$, th. ii : 93 (undescr.).

1856. Giebel.

Insect. d. vorwelt, 468.

1870. Thorell.

European spiders, i : 233.

2161. Propetes griseus. (Attoidæ.)

Locality: Prussian amber. Horizon: Ligurian.

1854. Menge.

1856. Giebel.

Koch-Berendt, Bernst. befindl. organ. reste vorw., i, th. ii : 93 (undescr.).

Insect. d. vorwelt, 468.

2162. Propetes latifrons. (Attoidæ.)

Locality: Prussian amber. Horizon: Ligurian.

1854. Menge. 1856. Giebel.

Koch-Berendt, Bernst. befindl. organ. reste vorw., i, th. ii: 93.

Insect. d. vorwelt, 468.

2163. Propetes pumilus. (Attoidæ.)

Locality : Prussian amber. Horizon: Ligurian.

1854. Menge.

1856. Giebel.

Koch-Berendt, Bernst, befindl. organ. reste vorw., i, th. ii: 99 (undeser.).

Insect. d. vorwelt, 468 .

2164. Protochersis spinosus. (Eresoidæ.)

Compared with Palpimanus savignyi Aud.

Locality: Aix, France. Horizon: Ligurian.

1887. Gourret. Rec. zool. Suisse, iv: 474-477. Pl. 20, fig. 3. 
2165. Protolachesis annulata. (Enyoidæ.)

Compared with Lachese perversa Sav.

Locality: Aix, France. Horizon: Ligurian.

1887. Gourret. Rec. zool. Suisse, iv : 467-469. Pl. 21, fig. 17; Pl. 22, figs. 21, 22.

2166. Protolycosa attiformis. (Lycosoidæ.)

Compared with Lycosa narbonensis Walck., Attoides eresiformis Brongn.

Locality : Aix, France. Horizon: Ligurian.

1887. Gourret. Rec. zool. Suisse, iv: 444-447. Pl: 20, fig. 7.

2167. Pseudopachygnathus maculatus. (Trombididæ.)

Compared with Pachygnathus villosus Dugès.

Location: Aix, France. Horizon : Ligurian.

1887. Gourret. Rec.zool. Suisse, iv: 437-439. Pl. 20, fig. 2.

2168. Pseudothomisus articulatus. (Thomisides.)

Locality : Aix, France. Horizon : Ligurian.

1887. Gourret. Rec. zool. Suisse, iv : 449-451. Pl. 21, figs. 9-10.

2169. Pythonissa - (Drassides.)

Locality: Prussian amber. Horizon: Ligurian.

1645. Berendt. Bernst. befindl, organ. reste vorw., i : 56 .

2170. Pythonissa - (7 sp.). (Drassides.)

Locality : Prussian amber. Horizon: Ligurian.

1856. Menge. Progr. petrischule Danzig, 1856: 7 .

2171. Pythonissa affinis. (Drassides.)

Locality : Prussian amber. Horizon: Ligurian.

1845. Koch-Berendt. Jahrb. min., 1845: 872 (Phytonissa).

1854. " Berendt, Bernst. befindl. organ. reste vorw., i, th. ii : 58-59. Pl. 6, fig. 48.

1856. Giebel. Insect. d. vorwelt, 454.

Seo also Philodromus dubius.

2172. Pythonissa ambigua. (Drassides.)

Locality : Prussian amber. Horizon : Ligurian.

1845. Koch-Berendt. Jahrb. min. 1845 : 872 (Phytonissa).

1854. " Berendt, Bernst. befindl. organ. reste vorw., i, th. ii: 60. Pl.6, fig. 50.

1856. Giebel. Insect. d. vorwelt, 455.

2173. Pythonissa bipunctata. (Drassides.)

Locality: Prussian amber. Horizon: Ligurian.

1854. Menge. Koch-Berendt, Bernst. befindl. organ. reste vorw., i, th. ii : 60 .

1856. " $\quad$ Progr. petrischule Danzig, 1856: 8.

1856. Giebel. Insect. d. vorwelt, 456. 
2174. Pythonissa discophora. (Drassides.)

Locality: Prussian amber. Horizon: Ligurian.

1854. Menge. 1856. Giebel.

1854. Menge. 1856. Giebel.

1854. Menge. 1856. Giebel.

Koch-Berendt, Bernst. befindl. organ. reste vorw., i, th. ii : 60 . Insect. d. vorwelt, 455-456.

2175. Pythonissa glabra. (Drassides.)

Locality: Prussian amber. Horizon: Ligurian.

Koch-Berendt, Bernst. befindl. organ. reste vorw., i, th. ii : 60 . Insect. $d$. vorwelt, 455 .

2176. Pythonissa microcephala. (Drassides.)

Locality: Prussian amber. Horizon : Ligurian.

Koch-Berendt, Bernst. befindl. organ, reste vorw., i, th. ii: 82. Insect. d. vorwelt, 455.

See also Philodromus microcephalus.

2177. Pythonissa sericata. (Drassides.)

Locality: Prussian amber. Horizon: Ligurian.

1845. Koch-Berendt. Jahrb. min., 1845 : 872 (Phytonissa).

1854. “ Berendt, Bernst. befindl. organ. reste vorw., i, th. ii : 59. Pl.6, fig. 49.

1856. Giebel. Insect. d. vorwelt, 454.

See also Philodromus sqnamiger.

2178. Pythonissa spinimana. (Drassides.)

Locality : Prussian amber. Horizon: Ligurian.

1854. Menge. Koch-Berendt, Bernst. befindl. organ, reste vorw., i, th. ii : 83-84. Pl. 9, fig. 78.

1856. Giebel. Insect. d. vorwelt, 455.

See also Philodromus spinimanus.

2179. Pythonissa triguttata. (Drassides.)

Locality: Prussian amber. Horizon: Ligurian.

1854. Menge. Koch-Berendt, Bernst. befindl. organ. reste vorw., i, th. ii : 86. 1856. Giebel. Insect. $d$. vorwelt, 455.

See also Ocypete triguttata.

2180. Pythonissa villosa. (Drassides.)

1854. Menge. 1856. Giebel.

1845. Berendt.
Locality : Prussian amber. Horizon: Ligurian.

Koch-Berendt, Bernst. befindl. organ. reste vorw., i, th. ii : 60 .

Insect. d. vorwelt, 455.

2181. Rhyncholophus —_. (Trombididæ.)

Locality : Prussian amber. Horizon: Ligurian.

Bernst. befindl. organ. reste vorw., i : 56.

2182. Rhyncholophus bifrons. (Trombididæ.)

Locality : Prussian amber. Horizon: Ligurian.

1854. Menge.

Koch-Berendt, Bernst. befindl. organ. reste vorw., i, th. ii : 106.

Insect. d. vorwelt, 480 . 


\section{Rhyncholophus foveolatus. (Trombididæ.)}

Locality : Prussian amber. Horizon : Ligurian.

1845. Koch-Berendt. Jahrb. min., 1845: 872.

1854. " Berendt, Bernst. befindl. organ. reste vorw., i, th. ii: $104 . \quad P l$. 13, fig. 102.

1856. Giebel. Insect. d. vorwelt, 479.

1885. Scudder. Zittel, Handb. palaeont., i, th. ii : 734. Fig. 907.

1886. " " Zittel-Barrois, Traité de paléont., ii : 733. Fig. 923.

2184. Rhyncholophus illustris. (Trombididæ.)

Locality : Prussian amber. Horizon: Ligurian.

1845. Koch-Berendt. Jahrb. min., 1845: 872.

1854. " Berendt, Bernst. befindl. organ. reste vorw., i, th, ii: $105 . P l$. 13, fig. 104.

1856. Giebel.

Insect. d. vorwelt, 479 .

\section{Rhyncholophus incertus. (Trombididæ.)}

Locality: Prussian amber. Horizon: Ligurian.

1845. Koch-Berendt. Jahrb. min., 1845 : 872.

1854. " Berendt, Bernst. befindl. organ. reste vorw., i, th. ii: 105-106. Pl. 13, fig. 105.

1856. Giebel. Insect. d. vorwelt, 479.

2186. Rhyncholophus longipes. (Trombididæ.)

Locality: Prussian amber. Horizon: Ligurian.

1845. Koch-Berendt. Jahrb. min., 1845: 87\%.

1854. " $\quad$ Berendt, Bernst. befindl. organ. reste vorw., i, th. ii: 104-105. Pl. 13, fig. 103.

1856. Giebel. Insect. d. vorwelt, 479 .

2187. Rhyncholophus procerus. (Trombididæ.)

Locality : Prussian amber. Horizon: Ligurian.

1854. Menge. Koch-Berendt, Bernst. befindl. organ. reste vorw., i, th. ii : 106. 1856. Giebel. Insect. d. vorwelt, 480.

2188. Rhyncholophus rostratus. (Trombididæ.)

Locality: Prussian amber. Horizon: Ligurian.

1854. Menge. Koch-Berendt, Bernst. befindl. organ. reste vorw., i, th. ii : 106. 1856. Giebel. Insect. d. vorwelt, 480.

2189. Rhyncholophus saccatus. (Trombididæ.)

Locality: Prussian amber. Horizon: Ligurian.

1854. Menge. Koch-Berendt, Bernst. befindl. organ. reste vorw., i, th. ii: 104.

1856. Giebel. Insect. d. vorwelt, 479.

See also Trombidium saccatum.

2190. Salticus - (Attoidæ.)

Locality: Prussian amber., Horizon : Ligurian.

1856. Menge. Progr. petrischnle Danzig, 1856: 9. 


\section{Schellenbergia rotundata. (Theridides.)}

Locality: Oeningen, Baden. Horizon: Tortonian.

1865. Heer.

Urwelt der Schweiz, 357. Fig. 211.

1870. Thorell.

European spiders, i : 224.

1872. Heer.

1876. " "

Monde prim. Suisse, 437. Fig. 211.

Prim. world Switz., ii : 11. Fig. 211 on p. 10.

1879. "

Urwelt der Schweiz, $2^{\mathrm{e}}$ aufl, 381. Fig. 246.

1885. Scudder. Zittel, Handb. palaeont., i, th. ii: 743. Fig. 925.

1886. " Zittel-Barrois, Traité de paléont., ii : 743. Fig. 942.

\section{Scorpio schweiggeri. (Scorpiones.)}

Compared with S. americanus.

Locality : Prussian amber. Horizon: Ligurian.

1819. Schweigger. Beobacht. naturh. reisen, 117-118 (with no specific name). $P l$. 8, figs. 69, 69ab.

1829. Holl. Handb. petref, $17 \%-178$.

\section{Segestria —. (Dysderides.)}

Locality : Prussian amber. Horizon: Ligurian.

1845. Berendt. Bernst. befindl. organ. reste vorw., i : 56.

\section{Segestria —. (Dysderides.)}

Locality: Florissant, Colo. Horizon: Oligocene.

1882. Scudder. Harv, univ. bull, ii: 303.

See also Segestria secessa.

2195. Segestria cristata. (Dysderides.)

Locality: Prussian amber. Horizon: Ligurian.

1854. Menge. Koch-Berendt, Bernst. befindl. organ. reste vorw., i, th. ii: 74 .

1856. Giebel. Insect.d. vorwelt, 461.

2196. Segestria cylindrica. (Dysderides.)

Locality: Prussian amber. Horizon: Ligurian.

1845. Koch-Berendt. Jahrb. min., 1845: 872.

1854. “ Berendt, Bernst. befindl. organ. reste vorw., i, th. ii : 73. Pl. 7, fig. 66 .

1856. Giebel. Insect. d, vorwelt, 461.

2197. Segestria elongata. (Dysderides.)

Locality: Prussian amber. Horizon: Ligurian.

1845. Koch-Berendt. Jahrb. min., 1845: 872.

1854. " $\quad$ Berendt, Bernst. befiudl. organ. reste vorw., i, th. ii : 72. Pl. 7, fig. 65.

1856. Giebel. Insect. d, vorwelt, 460-461.

2198. Segestria exarata. (Dysderides.)

Locality: Prussian amber. Horizon: Ligurian.

1854. Menge. Koch-Berendt, Bernst. befindl, organ, reste vorw., i, th. ii: 74.

1856. Giebel. Insect. 'd. vorwelt, 461. 
2199. Segestria nana. (Dysderides.)

Locality: Prussian amber. Horizon: Lignrian.

1845. Koch-Berendt. Jahrb. min., 1845: 872.

1854. " Berendt, Bernst. befindl. organ. reste vorw., i, th. ii : 73-74. Pl 8, fig. 67 .

1856. Giebel. Insect. $d$. vorwelt, 461.

1890. McCook. Amer. spiders, ii: 46\%. Fig. 393.

2200. Segestria pusilla. (Dyøderides.)

Locality : Prussian amber. Horizon: Ligurian.

1854. Menge. Koch-Berendt, Bernst. befindl, organ. reste vorw., i, th. ii : 74 .

1856. Giebel. Insect. d. vorwelt, 461.

2201. Segestria secessa. (Dysderides.)

Compared with S. senoculata, Europe.

Locality : Florissant, Colo. Horizon: Oligocene.

1890. Scudder. Tert. ins. N. A., 61-62. Pl. 11, fig. 28.

See also Segestria — Scndder.

2202. Segestria tomentosa. (Dysderides.)

Compared with S. senoculata, Europe.

Loeality: Prussian amber. Horizon: Ligurian.

1845. Koch-Berendt. Jahrb. min., 1845: 872.

1854. " Berendt, Bernst. befindl. organ, restevorw., i, th. ii: 71-72. Pl. 16, fig. 140 .

1856. Giebel. Insect. d. vorwelt, 460.

2203. Segestria undulata. (Dysderides.)

Locality: Prussian amber. Horizon: Ligurian.

1854. Menge. Koch-Berendt, Bernst. befindl, organ. reste vorw., i, th. ii : 74.

1856. Giebel. Insect. d. vorwelt, 461.

2204. Sejus —. (Gamasidæ.)

Locality : Prussian amber. Horizon: Ligurian.

1845. Berendt. Bernst. befindl, organ. reste vorw., i : 56 .

2205. Sejus bdelloides. (Gamasidæ.)

Locality : Prussian amber. Horizon: Ligurian.

1845. Koch-Berendt. Jahrb. min., 1845 : 872.

i854. " Berendt, Bernst. befindl. organ. reste vorw., i, th. ii: 110-111. Pl. 13, fig. 115.

1856. Giebel. Insect. d. vorwelt, 484.

\section{Siga -. (Epeirides.)}

Locality : Prussian amber. Horizon: Ligurian.

1856. Menge. Progr. petrischule Danzig, 1856: 7.

Siga citrina. See Siga crinita.

2207. Siga crinita. (Epeirides.)

Locality : Prussian amber. Horizon: Ligurian.

1854. Menge. Koch-Berendt, Bernst. befindl. organ. reste vorw., i, th. ii : 27 (undescr.). 
1853-'56. Bronn. Lethæa geogn., $3^{\mathrm{e}}$ aufl., iii : 636 (citrina).

1856. Giebel. Insect. d, vorwelt, 438.

1870. Thorell. European spiders, i : 226.

Sosibius minor. See Sosybius minor.

2208. Sosybius - - (Drassides.)

Locality : Prussian amber. Horizon: Ligurian.

1845. Berendt. Bernst. befindl. organ. reste vorw., i: 56.

2209. Sosybius — (2 sp.). (Drassides.)

Locality: Prussian amber. Horizon: Ligurian.

1856. Menge. Progr. petrischule Danzig, 1856: 8.

2210. Sosybius major. (Drassides.)

Locality: Prussian amber. Horizon: Ligurian.

1845. Koch-Berendt. Jahrb. min., 1845: 872.

1854. " Berendt, Bernst. befindl. organ. reste vorw., i, th. ii: 71. $P l$. 7, fig. 64 .

1853-56. Bronn. Lethæa geogn., 2e aufl, iii : 629-630. Pls. 42'a, fig. 5.

1856. Giebel. Insect. d. vorwelt, 460.

2211. Sosybius minor. (Drassides.)

Locality: Prussian amber. Horizon: Ligurian.

1845. Koch-Berendt. Jahrb. min., $1845: 872$.

1854. " Berendt, Bernst. befindl, organ. reste vorw., i, th. ii: 70. $P l$. 7, fig. 63.

1856. Giebel. Insect. d. vorwelt, 460 .

1870. Thorell. European spiders, i: 229 (Sosibius).

2212. Spheconia brevipes. (Drassides.)

Locality : Prussian amber. Horizon: Ligurian.

1854. Menge. Koch-Berendt, Bernst, befindl, organ reste vorw., i, th. ii: 94 (undeser.).

1853-56. Bronn. Lethæa geogn., $3^{\text {e }}$ aufl., iii : 638.

1856. Giebel. Insect. d. vorwelt, 469.

1870. Thorell. Europeau spiders, i : 230.

2213. Steneattus promissus. (Attoidæ.)

Locality: Prussian amber. Horizon: Ligurian.

1853-'56. Bronn-Roemer. Bronn's Lethæa geogn., $3^{\mathrm{e}}$ aufl, iii : 626-627. Pls. 42'a,

fig. 2 .

See also Leda promissa.

2214. Syphax —_. (Thomisides.)

Locality: Prussian amber. Horizon: Ligurian.

1845. Berendt. Bernst. befindl, organ, reste vorw., i: 56.

2215. Syphax - (3 sp.). (Thomisides.)

Locality: Prussian amber. Horizon: Ligurian.

1856, Menge. Progr. petrischule Danzig, 1856: 9:

Bull. $71-19$ 
2216. Syphax fuliginosus. (Thomisides.)

Locality: Prussian amber. Horizon: Ligurian.

1845. Koch-Berendt. Jahrb. min., 1845 : 872.

1854. " Berendt, Bernst. befindl. organ. reste vorw., i, th. ii : 79-80. Pl. 8, fig. 73 .

1856. Giebel. Insect. d. vorwelt, 463.

1856. Menge. Progr. petrischule Danzig, 1856: 9.

2217. Syphax gracilis. (Thomisides.)

Locality : Prussian amber. Horizon: Ligurian.

1845. Koch-Berendt. Jahrb. min., 1845 : 872.

1854. " " Berendt, Bernst. befindl. organ. reste vorw., i, th. ii : 80. Pl.8, fig. 74.

1856. Giebel.

Insect. d. vorwelt, 464 .

2218. Syphax hirtus. Thomisides.)

Locality : Prussian amber. Horizon: Ligurian.

1854. Menge.

1856. Giebel.

Koch-Berendt, Bernst. befindl. organ. reste vorw., i, th. ii : 81.

Insect. d. vorwelt, 464.

2219. Syphax megacephalus. (Thomisides.)

Locality: Prussian amber. Horizon: Ligurian.

1845. Koch-Berendt. Jahrb. min., 1845 : 872.

1854. " Berendt, Bernst. befindl. organ. reste vorw., $i$, th. ii: 77-78. Pl. 8, fig. 71.

1856. Giebel.

1870. Thorell.

Insect. d. vorwelt, 463.

European spiders, i : 231 .

1890, McCook.

Amer. spiders, ii : 467. Fig. 399.

2220. Syphax radiatus. (Thomisides.)

Locality : Prussian amber. Horizon: Ligurian.

1845. Koch-Berendt. Jahrb. min., 1845: 872 .

1854. " Berendt, Bernst. befindl. organ. reste vorw., i, th, ii : 80-81.

See also Artamus radiatus. Pl. 17, fig. 148.

2221. Syphax thoracicus. (Thomisides.)

Locality : Prnssian amber. Horizon: Ligurian.

1845. Koch-Berendt. Jahrb. min., 1845 : 872.

1854. " Berendt, Bernst. befindl, organ, reste vorw., i, th. ii : 78-79. Pl. 8, fig. 72 .

1853-'56. Bronn. Lethæa geogn., $3^{\text {e }}$ aufl, iii : $628 . \quad P l s .42^{\prime} a$, fig. 3.

1856. Giebel. Insect. d. vorwelt, 463.

2222. Tegenaria - (Agalenides.)

Locality: Prussian amber. Horizon: Ligurian.

1845. Berendt. Bernst. befindl. organ. reste vorw., i : 56.

2223. Tegenaria gracilipes. (Agalenides.)

Locality: Prussian amber. Horizon: Ligurian.

1845. Koch-Berendt. Jahrb. min., 1845: 871.

1854. " Berendt, Bernst, befindl. organ. reste vorw., i, th, ii: 47. Pl. 16, fig. 139.

1856. Giebel. Insect. d. vorwelt, 448. 
2224. Tegenaria lacazei. (Agalenides.)

Locality : Aix, France. Horizon: Ligurian.

1887. Gourret. Rec. zool. Suisse, iv : 469-471. Pl. 22, fig. 20.

2225. Tegenaria obscura. (Agalenides.)

Locality : Prussian amber. Horizon : Ligurian.

1845. Koch-Berendt. Jahrb. min., 1845: 871.

1854. " Berendt, Bernst. befindl, organ. reste vorw., i, th. ii: 46-47. $P l$. 5, fig. 36.

1856. Giebel. Insect. d. vorwelt, 448.

\section{Tegenaria virilis. (Agalenides.)}

Locality: Prussian amber. Horizon: Ligurian.

1854. Menge. 1856. Giebel. 1856. Menge.

Koch-Berendt, Bernst. befindl. organ. reste vorw., i, th. ii : 47.

Insect. d. vorwelt, 448 .

Progr. petrischule Danzig, 1856: 7 .

Tethnæus hentzii. See Tethneus hentzii.

2227. Tethneus - (4 sp.). (Epeirides.)

Locality: Florissant, Colo. Horizon: Oligocene.

1882. Scudder. 1882. " “ 1883. “

Harv, univ, bull., ii : 303.

Field nat., i: 63 .

Ann. rep. U. S. geol. surv. terr., xii : 288.

2228. Tethneus guyoti. (Epeirides.)

Locality: Florissant, Colo. Horizon: Oligocene.

1890. Scudder. Tert. ins. N. A., 78-79. Pl. 11, figs. 8, 10.

\section{Tethneus hentzii. (Epeirides.)}

Locality: Florissant, Colo. Horizon: Oligocene.

1885. Seudder. 1886. " " 1890. “
Zittel, Handb. palaeont., i, th. ii : 744 (Tethnæus). Fig. 928. Zittel-Barrois, Traité de paléont., ii : 743 (Tethnæus). Fig. 945. Tert, ins. N. A., 80-81. Pl. 11, fig. 14.

2230. Tethneus obduratus. (Epeirides.)

Locality : Florissant, Colo. Horizon: Oligocene. 1890. Scudder. Tert. ins. N. A., 79-80. Pl. 11, fig. 31.

2231. Tethneus provectus. (Epeirides.)

Locality: Florissant, Colo. Horizon: Oligocene. 1890. Scudder. Tert. ins. N. A., 81-82. Pl. 11, fig. 21.

\section{Tetragnatha - (Epeirides.)}

Locality: Florissant, Colo. Horizon : Oligocene,

1882. Scudder. 1882. " " Harv. univ. bull, ii : 303 .

Field nat., i : 63.

1883. " Ann. rep. U. S. geol. surv. terr., xii : 288.

See also Tetragnatha tertiaria. 


\section{Tetragnatha tertiaria. (Epeirides.)}

Locality: Florissant, Colo. Horizon: Oligocene.

1885. Scudder. Zittel, Handb. palaeont., i, th. ii: 744. Fig. 927.

1886. " Zittel-Barrois, Traité de paléout., ii: 744. Fig. 944.

1890. " Tert. ins. N. A., 77-78. Pl. 11, fig. 11.

1890. McCook. Amer. spiders, ii : 451.

See also Tetragnatha $\longrightarrow$.

\section{Tetranychus - (Trombididæ.)}

Locality: Prussian amber. Horizon: Ligurian.

1845. Berendt. Bernst. befindl. organ. reste vorw., i: 56.

\section{Tetranychus brevipes. (Trombididæ.)}

Locality: Prussian amber. Horizon: Ligurian.

1854. Koch-Berendt. Berendt, Bernst. befindl. organ. reste vorw., i, th. ii : $10 \%$. Pl. 13, fig. 108.

1856. Giebel. Insect. d. vorwelt, 481.

2236. Tetranychus gibbus. (Trombididæ.)

Locality : Prussian amber. Horizon: Ligurian.

1845. Koch-Berendt. Jahrb. min., 1845: 872.

1854. " Berendt, Bernst. befindl, organ. reste vorw., i, th. ii: 107. Pl. 13, fig. 107.

1856. Giebel. Insect. $d$. vorwelt, 481.

2237. Textrix —. (Agalenides.)

Locality : Prussian amber. Horizon : Ligurian.

1845. Berendt. Bernst. befindl. organ. reste vorw., i: 56.

2238. Textrix funesta. (Agalenides.)

Locality: Prussian amber. Horizon : Ligurian.

1845. Koch-Berendt. Jahrb. min., 1845: 871.

1854. " Berendt, Bernst. befindl. organ. reste vorw., i, th. ii : 49-50. Pl. 17, fig. 146.

1856. Giebel. Insect. d. vorwelt, 449.

1870. Thorell. European spiders, i : 229 (not to Agalenides?).

See also Clythia funesta.

\section{Textrix lineata.}

Locality : Prussian amber. Horizon: Ligurian.

1845. Koch-Berendt. Jahrb. min., 1845: 871.

1854. " Berendt, Bernst. befindl. organ. reste vorw., i, th. ii : 48-49. Pl. 17, fig. 145.

1856. Giebel. Insect. d. vorwelt, 449.

See also Clythia lineata.

\section{Therea —. (Dysderides.)}

Locality: Prussian amber. Horizon: Ligurian.

1845. Berendt. Bernst, befindl. organ, reste vorw., i : 56 , 
2241. Therea hispida. (Dysderides.)

Locality : Prussian amber. Horizon: Ligurian.

1845. Koch-Berendt. Jahrb. min., 1845: 872.

1854. " Berendt, Bernst. befindl, organ. reste vorw., i, th. ii : 76-77. $P l$. 8, fig. 70.

1854. Menge. Ibid., 77 (=Melanophora mundula).

See also Melanophora mundula.

2242. Therea petiolata. (Dysderides.)

Locality : Prussian amber. Horizon : Ligurian.

1845. Koch-Berendt. Jahrb. min., 1845: 872.

1845. " Berendt, Bernst. befindl, organ, reste vorw., i, th. ii : 75-76. $\quad P l$. 8, fig. 69.

1853-56. Bronn. Lethæa geogn., 3e aufl., iii : 628-629. Pls. $42^{\prime} a$, fig. 4.

1856. Giebel. Insect. d. vorwelt, 462 .

1870. Thorell. European spiders, i : 230.

2243. Therea pubescens. (Dysderides.)

Locality : Prussian amber. Horizon : Ligurian.

1854. Menge. Koch-Berendt, Bernst, befindl. organ. reste vorw., i, th. ii : 76.

1856. Giebel. Insect. d. vorwelt, 462 .

Theridion annulipes. See Theridium annulipes.

Theridion globulus. See Theridium globulus.

Theridion maculipes. See Theridium maculipes.

Theridiosoma columbiæ. See Aranea columbiæ.

2244. Theridium —-. (Theridides.)

Locality : Prussian amber. Horizon : Ligurian.

1845. Berendt. Bernst. befindl. organ. reste vorw., i : 56.

2245. Theridium - (8 sp.). (Theridides.)

Locality: Prussian amber. Horizon: Ligurian.

1856. Menge. Progr. petrischule Danzig, 1856: 7.

2246. Theridium - (2 sp.). (Theridides.)

Locality: Florissant, Colo. Horizon : Oligocene. .

1882. Scudder. Harv. univ. bull., ii : 303.

1882. " Field nat, i: 62.

1883. " $\quad$ Ann. rep. U. S. geol. surv. terr., xii : 288.

2247. Theridium alutaceum. (Theridides.)

Locality : Prussian amber. Horizon: Ligurian.

1845. Koch-Berendt. Jahrb. min., 1845: 871.

1854. " Berendt, Bernst. befindl. organ. reste vorw., i, th. ii: $37 . \quad P l$. 16, fig. 135.

1856. Giebel. Insect. d, vorwelt, 443. 
2248. Theridium annulipes. (Theridides.)

1865. Heer. 1872. “ 1876. “ 1879. “

1870. Thorell. 1890. McCook.

See also

1854. Menge.

1856. Giebel.

Locality : Oeningen, Baden. Horizon: Tortonian.

Urwelt der Schweiz, 356 (Theridion). Fig. 212 ("219").

Monde prim. Suisse, 436. Fig. 212, on p. 437.

Prim. world Switz., ii : 9. Fig. 212, on p. 10.

Urwelt der Schweiz, $2^{\mathrm{e}}$ aufl., 380. Fig. 247 ("254"), on p. 381.

2249. Theridium bifurcum. (Theridides.)

Locality : Prussian amber. Horizon: Ligurian.

Koch-Berendt, Bernst. befindl. organ. reste vorw., i, th. ii : 7 (undeser.).

Insect. d. vorwelt, 443.

2250. Theridium bucklandi. (Theridides.)

Locality : Aix, France. Horizon : Ligurian.

European spiders, i : 223.

Amer. spiders, ii : 458. Buckland.

2251. Theridium chorius. (Theridides.)

Locality : Prussian amber. Horizon : Ligurian.

1854. Menge. Koch-Berendt, Bernst. befindl. organ. reste vorw., $i$, th. ii : 7 (undescr.).

1856. Giebel. Insect. d. vorwelt, 443.

2252. Theridium clavigerum. (Theridides.)

Locality : Prussian amber. Horizon: Ligurian.

1854. Menge. Koch-Berendt, Bernst. befindl, organ. reste vorw., i, th. ii: 7 (undescr.).

1856. Giebel. Insect. d. vorwelt, 443.

2253. Theridium crassipes. (Theridides.)

Locality : Prussian amber. Horizon : Ligurian.

1854. Menge. Koch-Berendt, Bernst. befindl. organ. reste vorw., i, th. ii: 7 (undescr.).

1856. Giebel. Insect. $d$, vorwelt, 443 .

2254. Theridium detersum. (Theridides.)

Compared with T. lunatum.

Locality : Prussian amber. Horizon : Ligurian.

1845. Koch-Berendt. Jahrb. min., 1845: 871.

1854. " Berendt, Bernst. befindl, organ. reste vorw., i, th. ii: 37. Pl. 17, fig. 144.

1856. Giebel. Insect, d. vorwelt, 443.

2255. Theridium globosum. (Theridides.)

Locality: Prussian amber. Horizon : Ligurian.

1854. Menge. Koch-Berendt, Bernst. befindl. organ. reste vorw., i, th. ii : 8. See also Mizalia globosa. 
2256. Theridium globulus. (Theridides.)

Locality : Oeningen, Baden. Horizon: Tortonian.

1865. Heer.

187\%. “6

1876. “

1879. “
Urwelt der Schweiz, 356 (Theridion). Fig. 220.

Monde prim. Suisse, 436. Fig. 220, on p. 437.

Prim. world Switz, ii : 9. Fig. 220, on p. 10.

Urwelt der Schweiz, 2 anfl., 380. Fig. 255 , on $p .381$.

2257. Theridium granulatam. (Theridides.)

Locality : Prussian amber. Horizon : Ligurian.

1845. Koch-Berendt. Jahrb. min., $1845: 871$.

1854. " Berendt, Bernst. befindl. organ. reste vorw., i, th. ii : 36 . Pl, 4, fig. 26.

1856. Giebel. Insect. d. vorwelt, 443.

2258. Theridium hirtum. (Theridides.)

Locality : Prussian amber. Horizon: Ligurian.

1845. Koch-Berendt. Jahrb. min., $1845: 871$.

1854. " Berendt, Bernst, befindl, organ. reste vorw., i, th, ii : 35-36. $P l$. 4, fig. 25.

1856. Giebel. Insect. d. vorwelt, 442-443.

1890. MeCook. Amer. spiders, ii: 467. Fïg. 398.

2259. Theridium maculipes. (Theridides.)

Locality : Oeningen, Baden. Horizon: Tortonian.

1865. Heer. Urwelt der Schweiz. (Theridion). Fig. 219 on p. 356.

1872. " Monde prim. Suisse. Fig. 219 on p. 437.

1876. " Prim. world Switz., ii. Fig. 219 on $p .10$.

1870. Thorell. European spiders, i: 224.

1879. Heer. Urwelt der Schweiz, $2^{\mathrm{e}}$ auf. Fig. 254 on $p, 381$.

2260. Theridium opertaneum. (Theridides.)

Compared with T. granulitum.

Locality : Florissant, Colo. Horizon: Oligocene.

1890. Scudder. Tert. ins, N. A., 73-74. Pl. 11, fig. 3.

2261. Theridium ovale. (Theridides.)

Locality : Prussian amber. Horizon : Ligurian.

1845. Koch-Berendt. Jahrb. min., 1845: 871.

1854. " Berendt, Bernst, befindl, organ. reste vorw., i, th. ii : $34 . \quad P l .4$, fig. 23.

1856. Giebel. Insect. d. vorwelt, 442.

2262. Theridium ovatum. (Theridides.)

Locality: Prussian amber. Horizon: Ligurian.

1845. Koch-Berendt. Jahrb. min., 1845: 871.

1854. " Berendt, Bernst, befindl, organ, reste vorw., i, th. ii : 33-34. Pl. 4, fig. 22.

1856. Giebel. Insect. d. vorwelt, 442 .

2263. Theridium seclusum. (Theridides.)

Compared with 'T. hirtum.

Locality: Florissant, Colo, Horizon: Oligocene.

1890. Scudder. Tert. ins. N. A., 74. Pl. 11, fig. 20. 
2264. Theridium setulosum. (Theridides.)

Locality: Prussian amber. Horizon: Ligurian.

1854. Menge. Koch-Berendt, Bernst. befindl, organ. reste vorw., i, th. ii: 7 (undeser.).

1856. Giebel. Insect. d. vorwelt, 443.

\section{Theridium simplex. (Theridides.)}

Locality: Prussian amber. Horizon: Ligurian.

1845. Koch-Berendt. Jahrb. min., $1845: 871$.

1854. " "Berendt, Bernst. befindl. organ. reste vorw., i, th. ii : $35 . \quad P l .4$, fig. 24.

1856. Giebel. Insect. d. vorwelt, 442.

2266. Thomisus - (3 sp.). (Thomisides.)

1882. Scudder. Harv, univ. bull., ii : 303.

1882. " Field nat., i : 62 .

1883. “ Anu. rep. U. S. geol, surv. terr., xii : 287.

2267. Thomisus defossus. (Thomisides.)

Locality: Florissant, Colo. Horizon: Oligocene.

1890. Scudder. Tert. ins. N. A., 59-60. Pl. 11, fig. 23.

2268. Thomisus disjunctus. (Thomisides.)

Locality: Florissant, Colo. Horizon : Oligocene.

1890. Seudder. Tert. ins. N. A., 58-59. Pl. 11, fig. 9 .

\section{Thomisus lividus. (Thomisides.)}

Locality: Ueningen, Baden. Horizon: Tortonian.

1865. Heer.

1872. " "

1876.

1856. Menge.
1879. “

Urwelt der Schweiz, 356. Fig. 216.

Monde prim. Suisse, 436 . Fig. 216 on p. 437.

Prim. world Switz., ii : 9. Fig. 216 on p. 10.

Urwelt der Schweiz, $2^{2}$ aufl., $380 . \quad$ Fig. 251 on $p .381$.

2270. Thomisus matutinus. (Thomisides.)

Locality : Prussian amber. Horizon: Ligurian.

Progr. petrischule Danzig, 1856: 9 (undescr.).

2271. Thomisus œningensis. (Thomisides.)

Locality : Oeningen, Baden. Horizon: Tortonian.

1865. Heer.

1872. "

Urwelt der Schweiz, 356. Fig. 215.

Monde prim. Suisse, 436. Fig. 215 on $p .437$.

Prim. world Switz., ii : 9. Fig. 215 on p. 10.

Urwelt der Schweiz, $2^{\mathrm{e}}$ aufl., 380 . Fig. 250 on $p .381$.

1879. “

1885. Sendder.

1886.

Zittel-Barrois, Traité de paléont., ii : 741. Fig. 937.

See also Xysticus oningensis.

2272. Thomisus resutus. (Thomisides.)

Locality: Florissant, Colo. Horizon : Oligocene. 
2273. Thomisus sulzeri. (Thomisides.)

1865. Heer.

1872. "

1876. " "

1879. “

1856. Menge.

Locality: Oeningen, Baden. Horizon: Tortonian.

Urwelt der Schweiz, 356. Fig. 217.

Monde prim. Suisse, 436. Fig. 217 on $p .437$.

Prim. world Switz., ii : :. Fig. 217 on $p .10$.

Urwelt der Schweiz, 2 e aufl, 380 . Fig. 252 on $p .381$.

2274. Thyelia —. (Theridides.)

Locality: Prussian amber. Horizon: Ligurian.

1845. Berendt. Bernst. befindl. organ. reste vorw., i: 56.

2275. Thyelia - ( 8 sp.). (Theridides.)

Locality: Prussian amber. Horizon : Ligurian.

Progr. petrischule Danzig, 1856 : 7 .

2276. Thyelia anomala. (Theridides.)

Locality: Prussian amber. Horizon: Ligurian.

1845. Koch-Berendt. Jahrb. min., 1845 : 872.

185̄. " Berendt, Bernst. befindl. organ. reste vorw., i, th. ii : $52 . \quad$ Pl. 5. fig. 39.

1856. Giebel. Insect. d. vorwelt, 451.

1870. Thorell. European spiders, i: 227 (probably not a Thyelia).

2277. Thyelia convexa. (Theridides.)

Locality: Prussian amber. Horizon: Ligurian.

1845. Koch-Berendt. Jahrb. min., $1845: 872$.

1854. " " Berendt, Bernst. befindl, organ. reste vorw., i, th. ii : 54-55. Pi. 5, fig. 43.

1856. Giebel.

Insect. d. vorwelt, 452.

2278. Thyelia fossula. (Theridides.)

Locality : Prussian amber. Horizon: Ligurian.

1845. Koch-Berendt. Jahrb. min., 1845: 872.

1854. “ Berendt, Bernst. befindl. organ. reste vorw., i, th. ii : 54. Pl. 5, fig. 42.

1856. Giebel. Insect. d. vorwelt, 451-452.

2279. Thyelia marginata. (Theridides.)

Locality : Prussian amber. Horizon: Ligurian.

1845. Koch-Berendt. Jahrb. min., 1845: 872.

1854. " Berendt, Bernst. befindl. organ. reste vorw., i, th. ii : 56. Pl. 6, fig. 45.

1856. Giebel. Insect. d. vorwelt, 452.

1870. Thorell. European spiders, i : 227 (probably not a Thyelia).

2280. Thyelia mengei. (Theridides.)

Locality: Prussian amber. Horizon: Ligurian.

1856. Giebel. Insect. d. vorwelt, 452-453.

2281. Thyelia pallida. (Theridides.)

Locality : Prussian amber. Horizon : Ligurian.

1845. Koch-Berendt. Jahrb. min., $1845: 872$.

1854. " Berendt, Bernst. befindl. organ. reste vorw., i, th. ii: 55 . Pl. 6 , fig. 44.

1856. Geibel. Insect. d. vorwelt, 452. 
2282. Thyelia pectinata. (Theridides.)

Locality : Prussian amber. Horizon: Ligurian.

1854. Menge.

Koch-Berendt, Bernst. befindl. organ. reste vorw., i, th. ii: 56.

1856. Giebel.

Insect. d. vorwelt, 453

2283. Thyelia scotina. (Theridides.)

Locality: Prussian amber. Horizon: Ligurian.

1845. Koch-Berendt. Jahrb. min., 1845: 872.

1854. " Berendt, Bernst. befindl, organ. reste vorw., i, th. ii : $53 . \quad P l .5$, fig. 41.

1856. Giebel.

Insect. d. vorwelt, 451.

2284. Thyelia spinosa. (Theridides.)

Locality: Prussian amber. Horizon: Ligurian.

1854. Menge. Koch-Berendt, Bernst. befindl, organ. reste vorw., i, th. ii : 56. 1856. Giebel. Insect. d. vorwelt, 453.

2285. Thyelia tristis. (Theridides.)

Locality: Prussian amber. Horizon : Ligurian.

1845. Koch-Berendt. Jahrb. min., 1845: 872.

1854.

Berendt, Bernst. befindl. organ, reste vorw., i, th. ii : 51-52. Pl. 5, fig. 38.

1853-56. Bronn. Lethæa geogn., 3e autl., iii : 630-631. Pls. 42'a, fig. 6.

1856. Giebel.

Insect, d. vorwelt, 451 .

1870. Thorell.

European spiders, i: 227.

2286. Thyelia villosa. (Theridides.)

Locality: Prussian amber. Horizon: Ligurian.

1845. Koch-Berendt. Jahrb, min., 1845: 872.

1854. " Berendt, Bernst. befindl. organ. reste vorw., i, th. ii: 53. Pl. 5, fig. 40 .

1856. Giebel. Insect. d. vorwelt, 451.

2287. Titancea - (2 sp.). (Agalenides.)

1882. Scudder.

Locality: Florissant, Colo. Horizon : Oligocene.

1882. " "

Harv. univ. bull., ii : 303 .

188.3. “ Ann. rep. U. S. geol. surv. terr., xii: 288.

2288. Titanceca hesterna. (Agalenides.)

Locality : Florissant, Colo. Horizon : Oligocene.

1890. Scudder. Tert. ins. N. A., 69-70.

2289. Titancea ingenua. (Agalenides.)

Locality: Florissant, Colo. Horizon: Oligocene.

1890. Seudder. Tert. ins. N.A., 69. Pl. 11, figs. 29, 32.

2290. Tityus eogenus. (Buthoidæ.)

Locality: Prussian amber. Horizon: Ligurian.

1869. Menge. Scorp. (Schrift. naturf. gesellsch. Danzig, ii, th. ii), 3-6. Figs. $a-c$. 
1885. Scudder. Zittel, Handb. palaeont., i, th. ii : $\mathbf{7 4 0 .}$

1886. " "Zittel-Barrois, Traité de paléont., ii: 740.

1886. " Bull. U. S. geol. surv., No. 31: 29.

\section{Trombidium -. (Trombididæ.)}

Locality: Prussian amber. Horizon: Ligurian.

1830. Berendt. Insekten im bernstein, 36 .

1845. " Bernst. beïnỏl. organ. reste vorw., i: 56.

\section{Trombidium aquaticum (recent). ('Trombididæ.)}

Locality : Prussian amber. Horizon: Ligurian.

[1793. Fabricius. Ent. syst., iii : 399.]

1830. Berendt. Insekten im bernstein, 38 .

1839. Brulle. Gisem. ins. foss., 19.

2293. Trombidium clavipes. (Trombididæ.)

Locality: Prussian amber. Horizon: Ligurian.

1845. Koch-Berendt. Jahrb. min., 1845 : 872.

1854. " Berendt, Bernst. befindl, organ. reste vorw., i, th. ii: 103 . Pl. 17, fig. 150.

1856. Giebel. Insect. d. vorwelt, 478.

1884. Karsch. Berl, entom. zeitschr., xxviii : 175.

2294. Trombidium crassipes. (Trombididæ.)

Locality: Prussian amber. Horizon: Ligurian.

1854. Menge. Koch-Berendt, Bernst. befindl. organ, reste vorw., i, th. ii : 104. 1856. Giebel. Insect. d. vorwelt, 478.

1885. Karsch. Berl. entom. zeitschr., xxviii: 175.

2295. Trombidium fossile. (Trombididæ.)

Locality: Prussian amber. Horizon: Ligurian.

1834. Keferstein. Naturg, erdkörp., ii : 371 (undescr.) (Trompidium).

2296. Trombidium granulatum. (Trombididæ.)

Locality : Prussian amber. Horizon: Ligurian.

1854. Menge. Koch-Berendt, Bernst. befindl, organ. reste vorw., i, th. ii : 104. 1856. Giebel. : Insect. d. vorwelt, 478.

2297. Trombidium heterotrichum. (Trombididæ.)

Locality: Prussian amber. Horizon: Ligurian.

1854. Menge. 1856. Giebel.

Koch-Berendt, Bernst. befindl, organ. reste vorw., i, th. ii : 104. Insect. d. vorwelt, 478.

\section{Trombidium saccatum. (Trombididæ.)}

Locality : Prussian amber. Horizon: Ligurian.

1845. Koch-Berendt. Jahrb. min., 1845: 872.

1854. " " Berendt, Bernst. befindl. organ. reste vorw., i, th. ii: $103 . \quad P l$. 17, fig. 151.

See also Rhyncolophus saccatus. 
2299. Trombidium scrobiculatum. (Trombididæ.)

Compared with T. fuliginosum.

Locality : Prussian amber. Horizon : Ligurian.

1£54. Menge. 1856. Giebel.
Koch-Berendt, Bernst. befindl. organ. reste vorw., i, th. ii: 104. Insect. d. vorwelt, 478.

Trompidium fossile. See Trombidium fossile.

2300. Uroctea galloprovincialis. (Urocteoidæ.)

Compared with U. (Clotho) durandi Walek.

Locality: Aix, France. Horizon : Ligurian.

1887. Gourret. Rec. zool. Suisse, iv: 462-464. Pl. 22, fig. 19.

2301. Xysticus annulipes. (Thomisides.)

Locality : Rott, Rheniish Prussia. Horizon: Aquitanian.

1878. Bertkau. Verhandl. naturh. ver. preuss. rheinl., (4), v: 359-360. Pl.5, figs. $7,7 a$.

2302. Xysticus œningensis. (Thomisides.)

Locality: Oeningen, Baden. Horizon: Tortonian.

1070. Thorell. European spiders, i: 224.

See also Thomisus oningensis.

2303. Zilla ㄴ. (Theridides.)

Locality : Prussian amber. Horizon: Ligurian.

1845. Berendt. Bernst. befindl. organ. reste vorw., i: 56 .

2304. Zilla - (3 sp.). (Theridides.)

Locality : Prussian amber. Horizon : Ligurian.

1856. Menge.

Progr. petrischule Danzig, 1856: 7.

2305. Zilla cornumana. (Theridides.)

Locality: Prussian amber. Horizon: Ligurian.

1854. Menge. Koch-Berendt, Bernst. befindl. organ. reste vorw., i, th. ii : 27 (undescr.).

1856. Giebel. Insect. d. vorwelt, 438.

2306. Zilla gracilis. (Theridides.)

Locality : Prussian amber. Horizon : Ligurian.

1845. Koch-Berendt. Jahrb. min., 1845: 871.

1854. " "

Berendt, Bernst. befindl. organ. reste vorw., i, th. ii : 26. Pl. 3 , fig. 15.

1856. Giebel.

Insect. d. vorwelt, 437.

1890. McCook.

Amer. spiders, ii : 467. Fig. 390.

2307. Zilla porrecta. (Theridides.)

Locality: Prussian amber. Horizon: Ligurian.

1845. Koch-Berendt. Jahrb. min., 1845 : 871.

1854.

"6

Berendt, Bernst. befindl. organ. reste vorw., i, th. ii : 25-26. Pl. 3, fig. 14.

1856. Giebel.

Insect. $d$. vorwelt, 437.

1890. MeCook.

Amer. spiders, ii : 467. Fig. 389. 
2308 Zilla spinipalpa. (Theridides.)

Locality : Prussian amber Horizon : Ligurian.

1854. Menge. Koch-Berendt, Bernst. befindl. organ, reste vorw., i, th. ii : 27 (undescr.).

1856. Giebel. Insect. d. vorwelt, 438.

2309. Zilla veterana. (Theridides.)

Locality: Prussian amber. Horizon : Ligurian.

1845. Koch-Berendt. Jahrb. min., 1845: 871.

1854. " Berendt, Bernst. befindl, organ. reste vorw., i, th. ii : 26-27. Pl 3, fig. 16.

1856. Giebel. Insect. $d$. vorwelt, $43 \%^{\%}-438$.

\section{HEXAPODA.}

2310.

Locality : Serzanne, Marne, France. Horizon : Lower Eocene.

1878. Goss.

1878. " “

1879. "

1880 . “
Proc. geol. assoc., v : 214, note.

Ins. fauna tert. per., 36 , note.

Ent. monthl, mag., xvi : 125, note.

Geol, ant. ins., 37, note.

\section{ORTHOPTERA.}

\section{1.}

Locality : Menat, France. Horizon : Aquitanian.

1870. Oustalet. Ann. sc. g6́l., ii : art. 3, 78-79. Pl. 2, fig. 1.

2312.

Locality : Gurnet Bay, Isle of Wight. Horizon: Bembridge Limestone. 1879. Woodward. Geol. mag. (n. s.), v: 88.

2313. (several)。 (Acridii.)

Locality: Florissant, Colo. Horizon: Oligocene.

1881. Scudder. Bull. U. S. geol. surv. terr., vi : 293.

1883. " Ann. rep. U. S. geol. surv. terr., xii : 283.

2314. (Acridii.)

Locality : Gurnet Bay, Isle of Wight. Horizon: Bembridge Limestone. 1879. Woodward. Quart. journ. geol, soc. Lond., xxxv: 344.

2315. (Blattariæ.)

Locality : Rouffach, Alsatia. Horizon: Tongrien.

1881. Bleicher. Bull. soc. géol. Fr., (3), viii : 226-227. (Probably isopod crust., perhaps Blattariæ.)

2316. (Blattariæ.)

Compared with Blatta germanica.

Locality : Prussian amber. Horizon: Ligurian,

1832. Burmeister, Handb. ent., i: 637,

1836. " Man. ent., 579. 
2317. (Blattariæ.)

Compared with Periplaneta americana.

Locality : Brunstatt, Alsatia. Horizon : Middle Oligocene. 1889. Foerster. Mitth. comm. geol. Elsass-Lothr., ii : 101. 2318. (Blattariæ.)

Locality: Prussian amber. Horizon : Ligurian. 1819. Schweigger. Beobacht. naturh. reisen, 113. Pl. 8, figs. 71, 71ab.

2319. (several). (Blattariæ.)

Locality : Florissant, Colo. Horizon: Oligocene.

1881. Seudder. Bull. U. S. geol, surv, terr., vi : 293.

1883. " Ann. rep. U. S. geol. surv. terr., xii : 283.

2320. (several). (Forficulariæ.)

Locality: Florissant, Colo. Horizon: Oligocene. 1881. Scudder. Bull. U. S. geol. surv. terr., vi : 293. 1883. " "

Ann. rep. U. S. geol. surv. terr, xii : 283.

2321. (Locustariæ.)

Locality: Prussian amber. Horizon: Ligurian. 1856. Germ.-Ber. Berendt, Bernst. befindl. organ. reste vorw., 31-32. Pl. 4, figs. $7 a-d$.

2322. (leg). (Locustariæ.)

Compared with Phylloptera.

Locality : Green River, Wyo. Horizon : Oligocene.

1878. Scudder. 1890. ' Bull. U. S. geol. surv. terr., iv : 774 .

Tert. ins. N. A., 234.

2323. (several). (Locustariæ.)

Locality : Florissant, Colo. Horizon : Oligocene.

1881. Scudder. Bull. U. S. geol. surv. terr., vi : 293.

1883. “

Ann. rep. U. S. geol. surv. terr., xii : 282-283.

2324. —- (Phasmida.)

Locality : Florissant, Colo. Horizon : Oligocene.

1881. Scudder. Bull. U. S. geol. surv. terr., vi : 293.

1883. “ Ann. rep. U. S. geol. surv. terr., xii: 283.

2325. Acheta - (Gryllides.)

Locality: Prussian amber. Horizon: Ligurian.

1832. Burmeister. Handb. ent., i: 637 (Acheten).

1836. " Man. ent., 579.

\section{Acheta -. (Gryllides.)}

Compared with A. campestris.

Locality: Aix, France. Horizon : Ligurian.

1829. Serres. Géogn. terr. tert., 226. 
2327. Acheta

(Gryllides.)

Compared with A. italica.

Locality : Aix, France. Horizon: Ligurian.

1829. Serres. Géogn. terr. tert., 226.

\section{Acheta -. (Gryllides.)}

Compared with A. sylvestris.

1829. Serres. Géogn. terr. tert., 226.

Locality: Aix, France, Horizon: Ligurian.

2329. Acridium —. (Acridii.)

Locality : Radoboj, Croatia. Horizon : Mayencian.

1831. Boué. Journ. géol., iii : 105, 143. Pl. 2, fig. 2.

2330. Acridium.

(Acridii.)

Locality: Radoboj, Croatia. Horizon: Mayencian.

1842-43. Unger. Chloris protogæa, 46. Pl. 15, fig. 2.

See also Gryllacris ungeri.

2331. Acridium barthelemyi. (Acridii.)

Locality: Aix, France. Horizon: Ligurian.

1885. Scudder. Zittel, Handb. palaeont., j, th. ii : 768.

1886. " "Zittel-Barroís, Traité de paléont, ii : 768.

1886. " " Bull. U. S. geol. surv., No. $31: 49$.

1890. " Tert. ins. N. A., 220.

See also Acridium (Gryllus) barthelemyí.

2332. Acridium (Gryllus) barthelemyi. (Acridii.)

Locality: Aix, France. Horizon: Ligurian.

1847. Hope. Descr. ins. foss., 5-6 (Grillus). Pl., fig. 2.

See also Acridium barthelemyi.

2333. Agathemera - (Phasmida.)

Locality : Florissant, Colo." Horizon: Oligocene.

1885. Scudder. Zittel, Handb. palaeont., i, th. ii : 767 .

1886. " Zittel-Barroís, Traité de paléont., ii : 768.

1886. " Bull. U. S. geol. surv, No. 31: 49.

See also Agathemera reclusa.

2334. Agathemera reclusa. (Phasmida.)

Locality: Florissant, Colo. Horizon: Oligocene.

1890. Scudder. Tert. ins. N. A., 219-220. Pl. 17, fig. 11.

See also Agathemera $\longrightarrow$.

Arnilia -. See Leptysma

2335. Bacteria -. (Phasmida.)

Locality : Prussian amber. Horizon: Ligurian.

1856. Menge. Progr. petrischule Danzig, 1856: 13.

2336. Blatta -. (Blattariæ.)

Locality: Prussian amber. Horizon: Ligurian.

1830, Berendt. Insekten im bernstein, 36-37. 


\section{Blatta - (Blattariæ.)}

Locality: Prussian amber. Horizon: Ligurian.

1836. Berendt. Ann. soc. ent. Fr., v: 541-542. Pl. 16, fig. 1.

\section{Blatta —. (Blattariæ.)}

Locality: Prussian amber. Horizon: Ligurian.

1836. Berendt. Ann. soc. ent. Fr., v: 543. Pl. 16, fig. 3.

1856. Germ.-Ber. Berendt, Bernst, befindl. organ, reste vorw. Pl. 4, figs. $3 a-d$.

\section{Blatta -. (Blattariæ.)}

Locality : Prussian amber. Horizon : Ligurian.

1836. Berendt. Ann, soc. ent. Fr, v : 543-544. Pl. 16, fig. 4.

\section{Blatta - (Blattariæ.)}

Locality: Prussian amber. Horizon: Ligurian.

1836. Berendt. Ann. soc. ent. Fr., v: 544. Pl. 16, fig. 5.

\section{Blatta -. (Blattariæ.)}

Locality : Prussian amber. Horizon: Ligurian.

1836. Berendt.

$$
\text { Ann. soc. ent. Fr., v: 544-545. Pl. 16, fig. } 6 .
$$

2342. Blatta

(Blattariæ.)

Locality : Prussian amber. Horizon: Ligurian.

1836. Berendt.

1776. Bloch. Ann. soc, ent. Fr., v: 544-545. Pl. 16, fig. 7.

\section{Blatta - (Blattariæ.)}

Locality: Copal. Horizon: Recent.

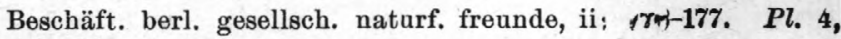
fig. 17.

\section{Blatta - (Blattariæ.)}

Compared with B. germanica.

Locality : Prussian amber. Horizon : Ligurian.

1831. Burmeister. Oken, Isis, 1831: 1100.

1836. " Man. ent., 579.

\section{Blatta - (Blattariæ.)}

Locality : Brunstatt, Alsatia. Horizon : Middle Oligocene.

1890. Foerster. In litt.

$$
\text { 2346. Blatta -. (Blattariæ.) }
$$

Locality : Eisleben, Rhenish Prussia. Horizon: Aquitanian?.

1856. Giebel. Zeitschr. ges, naturw., vii : 385-386. Pl. 5, fig. 3.

\section{Blatta -. (Blattariæ.)}

Locality : Prussian amber. Horizon : Ligurian.

1835. Gravenhorst. Uebers. schles. gesellsch. vaterl. cult., 1834: 93.

2348. Blatta - (Blattariæ.)

Locality : Sicilian amber. Horizon:

1838, Gúfrin. Rev. zool., 1838; 170, 
2349. Blatta - (Blattariæ.)

Locality : Oeningen, Baden. Horizon: Tortonian.

1834. Keferstein. Naturg. erdkörp., ii : 331.

2350. Blatta - - (Blattariæ.)

Locality : Prussian amber. Horizon : Ligurian.

1856. Menge. Progr. petrischule Danzig, 1856: 12-13.

2351. Blatta - - (Blattariæ.)

Locality : Prussian amber. Horizon : Ligurian.

1820. Schlotheim. Petrefactenk., 43.

2352. Blatta baltica. (Blattariæ)

Compared with B. lapponica.

Locality: Prussian amber. Horizon: Ligurian.

1836. Berendt. Ann. soc. ent. Fr., v: 543-546 (unnamed). $P l .16$, figs, 4, 5, 7.

1856. Germ.-Ber. Berendt, Bernst. befindl. organ, reste vorw., ii, i: 32,34 (=Blattina succinea Germ. ?). Pl. 4, fig. 5.

1864. Heer. Viert. naturf. gesellsch. Zürich, ix : 291.

1877. Goldenberg. Fauna saræp. foss., ii : 21.

See also Blattina succinea, Blatta succinea.

2353. Blatta berendti. (Blattariæ.)

Locality: Prussian amber. Horizon: Ligurian.

1856. Giebel. Insect. d. vorwelt, 322-323.

2354. Blatta colorata. (Blattariæ.)

Locality : Oeningen, Baden. Horizon: Tortonian.

1865. Heer. Urwelt der Schweiz. Fig. 229, on $p .366$.

18\%2. " Monde prim. Suisse. Fig. 229, on p. 449.

1876. " " Prim. world Switz, ii. Fig. 229, on p. 21.

1879. " Urwelt der Schweiz, $2^{\mathrm{e}}$ aufl. Fig. 270, on p. 392.

See also Blattidium coloratum.

2355. Blatta didyma. (Blattariæ.)

Compared with B. lapponica.

Locality: Prussian amber. Horizon: Ligurian.

1856. Germ. Ber. Berendt, Bernst. befindl. organ. reste vorw., ii, i: 34-35. Pl. 4, fig. 6.

1864. Heer. Viert. naturf. gesellsch. Zürich, ix : 291.

18\%7. Goldenberg. Fauna saræp. foss., ii : 21.

See also I, Orthopt, Blattina didyma.

2356. Blatta elliptica. (Blattariæ.)

Locality : Prussian amber. Horizon: Ligurian.

1862. Gie’el. Zeitschr. ges. naturw., xx: 315-316.

2357. Blatta gedanensis. (Blattariæ.)

Locality : Prussian amber. Horizon: Ligurian.

1836. Berendt. Ann. soc. ent. Fr., v: 544-546. Pl. 16, fig. 6.

1856. Germ.-Ber. Berendt, Bernst. befindl. organ. reste vorw., ii, i: 33-34. Pl. 4, fig. 4.

1864. Heer. Viert. naturf. gesellsch. Zürich, ix : 291.

1877. Goldenberg. Fauna saræp. foss., ii : 21.

Bull. 71-20 
2358. Blatta hyperborea. (Blattariæ.)

Locality: Cap Staratschin, Spitzbergen. Horizon: Miocene.

1870. Heer. K. svensk. vet.-akad. handl, viii, No. 7: 78. Pl. 16, fig. 43a, d.

2359. Blatta pauperata. (Blattariæ.)

Locality : Rott, Rhenish Prussia. Horizon : Aquitanian.

1862. Heyden. Palaeontogr., x: 74-75. Pl. 10, fig. 22.

2360. Blatta ruficeps. (Blattariæ.)

Locality: Prussian amber. Horizon : Ligurian.

1862. Giebel. Zeitschr. ges. naturw., xx: 314-315.

2361. Blatta succinea. (Blattariæ.)

Locality : Prussian amber. Horizon: Ligurian.

1856. Giebel. Insect. d. vorwelt, 323.

See also Blattina succinea, Blatta baltica.

2362. Blatta tricuspidata. (Blattariæ.)

Locality : Prussian amber. Horizon: Ligurian.

1836. Berendt. Ann. soc. ent. Fr., v: 542-543. Pl. 16, fig. 2.

See also Polyzosteria tricuspidata.

2363. Blattidium coloratum. (Blattariæ.)

Locality : Oeningen, Baden. Horizon : Tortonian.

1864. Heer. Viert. naturf. gesellsch. Zürich, ix: 291, 301-302. Pl., fig.9.

1877. Goldenberg. Fauna saræp. foss., ii : 21.

See also Blatta colorata.

2364. Blattidium fragile. (Blattariæ.)

Locality: Atanekerdluk, N. Greenland. Horizon: Miocene.

1868. Heer. Flora foss. arct., i: 130. Pl. 50, figs. 13, $13 b$.

1883. " Flora foss. grönl., ii : 146.

2365. Blattina succinea. (Blattariæ.)

Compared with Blatta nivea.

Locality: Prussian amber. Horizon: Ligurian.

1813. Germar. Germ., Mag. ent., i: 16.

1864. Heer. Viert. naturf. gesellsch. Zürich, ix : 291 (=Blatta baltica ?).

1888. Schlechtend. Zeitschr. ges. naturw., lxi : 487-489.

See also Blatta succinea, Blatta baltica.

2366. Chæteessa brevialata. (Mantides.)

Locality : Prussian amber. Horizon: Ligurian.

1862. Giebel. Zeitschr. ges. naturw., xx: 316-317 (Chætoessa).

Chætoessa brevialata. See Chæteessa brevialata.

2367. Chimarocephala (vic.) ——. (Acridii.)

Locality : Aix, France. Horizon : Ligurian.

1885. Seudder. Zittel, Handb. palaeont, i, th. ii : 769 .

1886. " Zittel-Barrois, Traité de paléont., ii : 769.

1886. " "Bull. U. S. geol. surv., No. 31: 49.

1890. " " Tert. ins. N. A., 224. 
2368. Chimarocephala fischerì. (Acridii.)

Locality: Oeningen, Baden. - Horizon: Tortonian.

1890. Seudder. Tert. ins. N. A., 224.

See also Edipoda fischeri.

2369. Cymatomera -. (Locustariæ.)

Locality : Florissant, Colo. Horizon: Oligocene.

1885. Scudder. Zittel, Handb. palaeont., i, th. ii : 770 .

1886. " " Zittel-Barrois, Traité de pal6ont., ii : 770.

1886. “ Bull. U. S. geol. surv., No. 31:50.

See also Cymatomera maculata.

2370. Cymatomera maculata. (Locustariæ.)

Locality: Florissant, Colo. Horizon: Oligocene.

1890. Scudder. Tert. ins. N. A., 230-231. Pl. 17, fig. 7.

See also Cymatomera -

2371. Cyrtoxiphus macrocercus. (Gryllides.)

Locality: Aix, France. Horizon: Ligurian.

1890. Scudder. Tert. ins. N. A., 234 (note).

See also Gryllus macrocercus.

2372. Decticus exstinctu. (Locustariæ.)

Locality: Bonn, Germany. Horizon: Aquitanian.

1856. Giebel. Insect. d. yorwelt, 304.

See also Locusta exstincta, ete.

2373. Decticus speciosus. (Locustariæ.)

Compared with D, albifrons $\mathbf{F}$.

Locality: Oeningen, Baden. Horizon: Tortonian.

1865. Heer. Urwelt der Schweiz, 366. Fig. 222.

1872. " Monde prim. Suisse, 449. Fig. 222.

1876. " " Prim. world Switz., ii : 20. Fig. 222 on p. 21.

1879. “U Urwelt der Schweiz, $2^{\mathrm{e}}$ aufl., 391. Fig. 263 on p. 392.

Compare II, ORthoptera, Decticus speciosus.

2374. Decticus umbraceus. (Locustariæ.)

Locality: Freudenheim, Bohemia. Horizon :

1869. Frič. Arch. naturw. landesdnrchf. Böhm., i, th. ii : 276. Fig. 55.

2375. Dissosteira haidingeri. (Acridii.)

Locality: Radoboj, Croatia. Horizon: Mayencian.

1890. Scudder. Tert. ins. N. A., 224 .

See also Edipoda haidingeri.

2376. Forficula - - (Forficulariæ.)

Locality : Prussian amber. Horizon: Ligurian.

1835. Gravenhorst. Uebers. schles. gesellsch. vaterl. cult., 1834: 93.

2377. Forficula - (Forficulariæ.)

Locality: Prussian amber. Horizon: Ligurian.

1834. Keferstein. Naturg. erdkörp., ii : 331 (as of Burmeister, but erroneously, Burmeister mentioning a cricket of the size of Forficula minor). 
2378. Forficula (Forfieulariæ.)

.Compared with F. auricularia.

Locality : Prussian amber. Horizon : Ligurian.

1856. Menge. Progr. petrischule Danzig, 1856: 12.

2379. Forficula -. (Forficulariæ.)

Compared with F. auricularia et parallela, Europe. Locality : Aix, France. Horizon: Ligurian.

1829. Serres. Géogn. terr. tert., 225.

2380. Forficula bolcensis. (Forficulariæ.)

Locality : Monte Bolca, Italy. Horizon: Ligurian.

1855. Massalongo. Nereid. foss., 31 (undescr.).

1856. " " Studii paleont., 15-17. Pl. 1, figs. 5-7.

1876. Scudder. Bull. U. S. geol, surv, terr., ii : 449.

1886. Omboni. Atti r. ist. ven., (6), iv: 1425. Pl. 2, fig. 5.

1886. " Ins. foss. ven., 5. Pl. 2, fig. 5.

1890. Scudder. Tert. ins. N. A., 203.

2381. Forficula minuta. (Forficulariæ.)

Compared with F. minor L.

1865. Heer. Urwelt der Schweiz, 367.

1872. " Monde prim. Suisse, 450.

1876. " $\quad$ Prim. world Switz., ii: 22.

1879. " Urwelt der Schweiz, 2e aufl., 392.

2382. Forficula primigenia. (Forficulariæ.)

Locality: Oeningen, Baden. Horizon: Tortonian.

1865. Heer. Urwelt der Schweiz, 367. Fig. 227 on p. 366.

1872. " Monde prim. Suisse, 450. Fig. 228 on p. 449.

1876. " Prim. world Switz., ii : 21. Fig. 227.

1876. Scudder. Bull. U. S. geol, surv. terr., ii : 448-449.

1879. Heer. Urwelt der Schweiz, $2^{\mathrm{e}}$ aufl., 392. Fig. 268.

1890. Scudder. Tert. ins. N. A., 202.

2383. Forficula recta. (Forficulariæ.)

Compared with F. annulipes Luc.

Locality : Oeningen, Baden. Horizon: Tortonian.

1865. Heer.

1872. “

1876. "

1876. Scudder.

1879. Heer.

1890. Scudder.

Urwelt der Schweiz, 367. Fig. 226 on p. 366.

Monde prim. Suisse, 450. Fig. 226 on p. 449.

Prim. world Switz., ii : 21. Fig. 226.

Bull. U. S. geol. surv. terr., ii : 448-449.

Urwelt der Schweiz, $2^{\mathrm{e}}$ aufl, 392. Fig. 267.

Tert. ins. N. A., 202.

\section{Gomphocerus -. (Acridii.)}

Locality : Aix, France. Horizon: Ligurian.

18-. Heer. MSS.

See also Leptysma 
2385. Gomphocerus abstrusus. (Acridii.)

Locality: Florissant, Colo. Horizon : Oligocene.

1890. Scudder. Tert. ins. N. A., 223. Pl. 17, fig. 6.

2386. Gomphocerus femoralis. (Acridii.)

Locality: Oeningen, Baden. Horizon: Tortonian.

1849. Heer. 1852. Giebel. 1854. Pictet. 1856. Giebel. Insektenf. tertiärg. Oeningen, ii : 20-21. Pl. 1, fig. \% Dentschl. petref., 638.

Traité de pal6́ont., $2^{\mathrm{e}}$ ed., ii: $365 . \quad P l .40, \mathrm{fig} .16$.

1885. Sendder. Zittel, Handb. palaeont., i. th. ii : 768.

1886. "Zittel-Barrois, Traité de paléont., ii : 768.

1886. “ Bull. U.S. geol. surv., No. 31: 49 .

Grillus barthelemyi. See Acridium (Gryllus) barthelemyi.

2387. Gryllacris —. (Locustariæ.)

Locality: Gabbro, Italy. Horizon: Tripoli earth.

1878. Capellini. Atti accad. linc., (3), Mem. sc. fis., ii : 285.

\section{Gryllacris - _. (Locustariæ.)}

Locality: Florissant, Colo. Horizon: Oligocene.

1885. Scudder. - Zittel, Handb. palaeont., i, th. ii: 770.

1886. " "Zittel-Barrois, Traité de paléont, ii : 770.

1886. " Bull. U. S. geol. surv., No. $31: 50$.

2389. Gryllacris charpentieri. (Locustariæ.)

Locality: Radoboj, Croatia: Horizon: Mayencian.

1849. Heer.

1852. Giebel.

Insektenf. tertiärg. Oeningen, ii : 12-15. Pl. 1, fig. 5.

Deutschl. petref., 637 .

1856. " " Insect. d. vorwelt, 305-306.

See also Myrmeleon brevipenne.

2390. Gryllacris cineris. (Locustariæ.)

Locality : Florissant, Colo. Horizon: Oligocene.

1890. Scudder. Tert. ins. N. A., 233-234. Pl. 17, fig. 17.

2391. Gryllacris ungeri. (Locustariæ.)

Locality: Radoboj, Croatia. Horizon: Mayencian.

1849. Heer. Insektenf. tertiärg. Oeningen, ii : 8-12. Pl. 1, fig. 4.

1852. Giebel. Deutschl. petref., 637.

1856. " Insect. d. vorwelt, 305 .

1874. Swinton. Geol. mag., (2), i: 338-339. Pl. 14, fig. 2.

1874. " Foss. Orthoptera, 2-3. Fig. 5.

See also Acridium __ Unger.

\section{Gryllotalpa -. (Gryllides.)}

Compared with G. vulgaris.

Locality: Aix, France. Horizon: Ligurian.

1829. Serres.

Géogn. terr. tert., 225. 
2393. Gryllotalpa (Gryllides.)

Locality : Gurnet Bay, Isle of Wight. Horizon: Bembridge Limestone. 1879. Woodward. Quart. journ. geol. soc. Lond., $\mathrm{xxxv}: 344$. 1879. " Geol. mag. (n. s.), v: 88.

2394. Gryllotalpa stricta. (Gryllides.)

Locality: Oeningen, Baden. Horizon: Tortouian.

1865. Heer. 1872. " 1876. " " 1879. “
Urwelt der Schweiz, $36 \%$.

Monde prim. Suisse, 450.

Prim. world Switz., ii : 20.

Urwelt der Schweiz, $2^{\circ}$ aufl., 391.

2395. Gryllus (Gryllides.)

Locality: Prussian amber. Horizon: Ligurian.

1841. Ehrenberg. Froriep, Neue notizen, xix: 120.

$$
\text { 2396. Gryllus ㄴ. (Gryllides.) }
$$

Locality : Prussian amber. Horizon: Ligurian.

1835. Gravenhorst. Uebers. schles. gesellsch. vaterl, cult., 1834: 93.

2397. Gryllus _-_: (Acridii.)

Locality: Prussian amber. Horizon: Ligurian.

1742. Sendel.

Hist. succ., 104 (nnnamed). Pl. 3, fig. 16ab.

18:25. Guérin.

Dict. class. d'hist. nat., viii : 580 (criquet).

1829. Serres.

Géogn. terr. tert., 241.

\section{Gryllus —. (Acridii.)}

Compared with G. cœrulescens.

Locality: Aix, France. Horizon: Ligurian.

1829. Serres. Géogn. terr. tert., 226.

See also Edipoda -

Gryllus barthelemyi. See Acridium (Gryllus) barthelemyi.

2399. Gryllus domesticus (recent). (Gryllides.)

Locality: Prussian amber. Horizon: Lignrian.

[1758. Linn6.

Syst. nat. ed. $x, i: 428$.]

1784. Meinecke. Naturforscher, xx : $18 \%$.

2400. Gryllus macrocercus. (Gryllides.)

Locality: Prussian amber. Horizon: Ligurian.

1856. Germ.-Ber. Berendt, Bernst. befindl, organ. reste vorw., ii, i: $36 . \quad P l .4$, figs. $8,8 a-c$.

1885. Scudder. Zittel, Handb. palaeont., i, th. ii : 770 (macrocerus) (probably a trigonid.) Fig. 970.

1886. " Zittel-Barrois, Traité de paléont., ii : 770 (probably a trigonid). Fig. 987.

1886. " Bull. U. S. geol. surv., No. 31: 50.

See also Cyrtoxiphus macrocercus.

Gryllus macrocerus. See Gryllus macrocereus. 
2401. Gryllus troglodytes. (Gryllides.)

1865. Heer.

1872. "

1876. " "

1879. " "

1885. Seudder.

1886. " "

1886. “ "

Locality : Oeningen, Baden. Horizon: Tortonian.

See also Nemobius troglodytes.

Urwelt der Schweiz, 367. Fig. 225 on p. 366.

Monde prim. Suisse, 450. Fig. 225 on p. 449.

Prim. world Switz., ii : 21. Fig. 225.

Urwelt der Schweiz, 2e aufl, 391-392. Fig. 266.

Zittel, Handb. palaeont., i, th. ii : 770 (probably a Nemobius).

Zittel-Barrois, Traité de paléont., ii : 770 (probably a Nemobius).

Bull. U. S. geol. surv., No. 31: 50.

2402. Heterogamia antiqua. (Blattariæ.)

Locality: Parschlug, Styria. Horizon: Tortonian.

1849. Heer.

1852. Giebel.

Insektenf. tertiärg. Oeningen, ii : 1-3. Pl. 1, fig. 1.

1856. “ “

Deutsehr. petref., 637.

1864. Heer.

Insect. d. vorwelt, 313.

1877. Goldenberg. Fauna saræp. foss., ii : 21.

2403. Hippiscus melanostictus. (Acridii.)

Locality : Radoboj, Croatia. Horizon: Mayencian.

1890. Scudder. Tert. ins. N. A., 224.

See also CEdipoda melanosticta.

2404. Homcogamia ventriosus. (Blattariæ.)

Locality : Florissant, Colo. Horizon: Oligocene.

1876. Scudder. Bull. U. S geol. surv. terr., i : 447.

1890. " Tert. ins. N. A., 218. Pl. 17, fig. 8.

2405. Labidura lithophila. (Forficulariæ.)

Iocality: Florissant, Colo. Horizon: Oligocene.

1876. Scudder. Bull. U. S geol. surv. terr., ii : 259-260.

See also Labiduromma lithophilum.

2406. Labidura tertiaria. (Forficulariæ.)

Locality: Florissant, Colo. Horizon: Oligocene.

1876. Scudder. Bull. U. S. geol. surv, terr., i : 447-449.

1876. " Bull. U. S. geol. surv, terr., ii : 259.

See also Labiduromma tertiarium.

2407. Labiduromma -. (Forficulariæ.)

Locality: Florissant, Colo. Horizon: Oligocene.

1890. Scudder. Tert. ins. N. A., 214. Pl. 16, fig. 24.

2408. Iabiduromma avia. (Forficulariæ.)

Locality : Florissant, Colo. Horizon: Oligocene.

1890. Scudder. $\quad$ Tert. ins. N. A., 20̈-206. Pl. 16, figs. 3, 5, 11,22, 23.

2409. Labiduromma bormansi. (Forficulariæ.)

Locality: Florissant, Colo. Horizon: Oligocene.

1890. Scudder. Tert, ins. N. A., 206-207. Pl. 16, fig. 1. 
2410. Labiduromma commixtum. (Forficulariæ.)

Locality : Florissant, Colo. Horizon : Oligocene.

1890. Scudder. Tert. ins. N. A., 208-209. Pl. 16, figs. 10, 17.

2411. Labiduromma exsulatum. (Forficulariæ.)

Locality: Florissant, Colo. Horizon: Oligocene.

1885. Scudder.

1886. “ "

1890. “

Zittel, Handb. palaeont., i, th. ii : 766. Fig. 959.

Zittel-Barrois, Traité de palént., ii: 766. Fig. 975

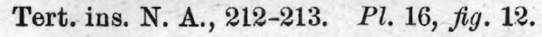

2412. Labiduromma gilberti. (Forficulariæ.)

Locality: Florissant, Colo. Horizon: Oligocene.

1890. Scudder. Tert. ins. N. A., 211. Pl. 16, fig. 14.

2413. Labiduromma infernum. (Forficulariæ.)

Locality: Florissant, Colo. Horizon: Oligocene.

1890. Scudder. Tert. ins. N. A., 214. Pl. 16, fig. 7.

2414. Labiduromma labens. (Forficulariæ.)

Locality: Florissant, Colo. Horizon: Oligocene.

1890. Scudder. Tert. ins. N. A., 214-215. Pl. 16, figs. 9, 13, 16.

2415. Labiduromma lithophilum. (Forficulariæ.)

Locality: Florissant, Colo. Horizon: Oligocene.

1890. Scudder. Tert. ins. N. A., 213. Pl. 16, fig. 19.

Seo also Labidura lithophila.

2416. Labiduromma mortale. (Forficulariæ.)

Locality : Florissant, Colo. Horizon: Oligocene.

1890. Scudder. Tert. ins. N. A., 207-203. Pl. 16, figs. 2, 6, 20.

2417. Labiduromma tertiarium. (Forficulariæ.)

Locality: Florissant, Colo. Horizion: Oligocene.

1890. Scudder. Tert. ins. N. A., 209-211. Pl. 16, figs. 15, 18, 21.

See also Labidura tertiaria.

2418. Leptysma (or Arnilia) _-. (Acridii.)

Locality : Aix, France. Horizon: Ligurian.

1890. Scudder. Tert. ins. N. A., 223.

See also Gomphocerus —

2419. Lithymnetes guttatus. (Locustariæ.)

Locality : Florissant, Colo. Horizon: Oligocene.

1878. Scudder.

1855. " "

1886. " "

1889. Lesley.

1890. Scudder.
Bull. U. S. geol. surv. terr., iv : 533-534.

Zittel, Handb. palaeont., i, th. ii : 769. Fig. 969.

Zittel-Barrois, Traité de paléont, ii : 769. Fig. 986.

Dict. foss. Penns., i: 354. Fig.

Tert. ins. N. A., 229-230. Pl. 17, figs. 14, 15.

2420. Locusta

(Locustariæ.)

Locality: Ruine, Germany. Horizon: Braunkohl.

1831. Goldfuss. Verhandl. leop.-carol. akad. naturf, vii, th. i : 118. 
2421. Locusta - -. (Locustariæ.)

Locality: Prussian amber. Horizon: Ligurian.

1834. Keferstein. Naturg. erdkörp., ii : 332 (as of Burmeister, but erroneously).

2422. Locusta - (Locustariæ.)

Locality : Florissant, Colo. Horizon: Oligocene.

1885. Scudder. Zittel, Handb. palaeont., i, th. ii : 770.

1886. " "Zittel-Barrois, Traité de paléont., ii : 770.

1886. “ Bull. U. S. geol. surv., No.31: 50.

2423. Locusta -. (Locustariæ.)

Compared with L. grisea.

1829. Serres. Géogn. terr. tert., 226.

1890. Scudder. Tert. ins. N. A., 232.

2424. Locusta exstincta. (Locustariæ.)

Compared with L. grisea.

Locality : Rhenish Prussia. Horizon : Aquitanian.

1837. Germar. Fanna insect. Europæ, xix : 16. $\quad P l .16$.

1886. Seudder. Bull. U. S. geol. surv., No. 31: 50.

See also Decticus exstinctus, Locustites exstinctus.

2425. Locusta grönlandica. (Locustariæ.)

Locality: Ober Atanekerdluk, Greenland. Horizon : -

1883. Heer. Flora foss. grönl., ii: (Grönl. geol. unders.) 146-147. Pl. 109, figs: $12,12 b$.

1885. Scudder. Zittel, Handb. palaeont., i, th. ii : 770 .

1886. " "Zittel-Barrois, Traité de paleont., ii: 770.

1886. “ Bull. U. S. geol. surv., No. 31: 50 .

1890. “ Tert. ins. N. A., 232.

2426. Iocusta silens. (Locustariæ.)

Compared with L. occidentalis, United States.

Locality : Florissant, Colo. Horizon: Oligocene.

1890. Scudder. Tert. ins. N. A., 232. Pl. 17, figs. 9, 10.

2427. Locustites exstinctus. (Locustariæ.)

Locality: Bonn, Rhenish Prussia. Horizon: Aquitanian.

1852. Giebel. Deutschl. petref., $63 \%$

See also Locusta exstincta, etc.

2428. Locustites maculatus. (Locustariæ.)

1849. Heer.

1852. Giebel.

1856. “

Locality: Parschlug, Styria. Horizon: Tortonian.

Insektenf. tertiärg. Oeningen, ii : 5-8. Pl. 1, flg.

Deutschl. petref., 637 .

Insect. d. vorwelt, 303.

2429. Mantis (Mantides.)

Locality : Prussian amber. Horizon : Ligurian. 
2430. Mantis protogæa. (Mantides.)

Locality : Oeningen, Baden. Horizon: Tortonian.

1849. Heer.

1852. Giebel.

1856. " "

1885. Scudder.

1886. " "

1886. “
Insektenf. tertiärg. Oeningen, ii : 21-22. $\quad P l .1, f i g .8$.

Deutschl. petref., 638.

Insect. d. vorwelt, 311 .

Zittel, Handb. palaeont., i, th. ii : 767.

Zittel-Barrois, Traité de paléont., ii : 767.

Bull. U. S. geol. surv., No. 31 : 49.

2431. Myrmeleon brevipenne. (Locustariæ.)

Locality : Radoboj, Croatia. Horizon: Mayencian.

1843. Charpentier. Verhandl. leop.-carol. akad. naturf., xx: 406-407. Pl. 22, fig. 1. 1866. Hagen. Stett. ent, zeit., xxvii : 436 (an orthopteron ?).

See also Gryllacris charpentieri.

\section{Nanthacia torpida. (Acridii.)}

Locality: Florissant, Colo. Horizon: Oligocene.

1890. Scudder. Tert. ins. N. A., 224-225.

2433. Nemobius - $(2 \mathrm{sp}$.$) . (Gryllides.)$

Locality : Aix, France. Horizon: Ligurian.

1890. Scudder. Tert. ins. N. A., 234 (undescr.).

2434. Nemobius tertiarius. (Gryllides.)

Compared with N. vittatus (Harr.).

Locality: Green River, Wyo. Horizon: Oligocene.

1878. Scudder. Bull. U. S. geol. surv. terr., iv: 774.

See also Pronemobius tertiarius.

2435. Nemobius troglodytes. (Gryllides.)

Locality: Oeningen, Baden. Horizon : Tortonian.

1890. Scudder. Tert. ins. N. A., 234.

See also Gryllns troglodytes

2436. CEdipoda

Locality : Aix, France.
Tert. ins. N. A., 225.

1890. Scudder. Tert. ins. N.
See also Gryllus

2437. Edipoda fischeri. (Acridii.)

Locality: Oeningen, Baden. Horizon: Tortonian.

1865. Heer. Urwelt der Schweiz, 367. Fig. 224, on p. 366.

1872. " Monde prim. Suisse, 450. Fig. 224, on p. 449.

1876. " Prim. world Switz., ii : 20. Fig. 224, on p. 21.

1879. “ Urwelt der Schweiz, $2^{e}$ aufl, 391. Fig. 265, on $p$. 392.

See also Chimarocephala fischeri.

2438. Edipoda germari. (Acridii.)

Compared with CE. migratoria.

Locality: Oeningen, Baden. Horizon: Tortonian.

1865. Heer. Urwelt der Schweiz, 367.

1872. " Monde prim. Suisse, 450.

1876. " " Prim. world Switz., ii : 20.

1879. “ Urwelt der Schweiz, $2^{\mathrm{e}}$ aufl, 391. 
2439. Edipoda haidingeri. (Acridii.)

Loeality : Radoboj, Croatia. Horizon: Mayencian.

1865. Heer.

Urwelt der Schweiz, 36\%. Fig. 223, on p. 366.

1872. " "

Monde prim. Snisse, 450. Fig. 223, on p. 449.

1876. " "

Prim. world Switz., ii : 20. Fig. 223, on p. 21.

1879. “

Urwelt der Schweiz, $2^{\mathrm{e}}$ aufl., 391. Fig. 264, on $p .392$.

See also Dissosteira haidingeri.

2440. Edipoda melanosticta. (Acridii.)

Locality: Radoboj, Croatia. Horizon: Mayencian.

1840. Unger. Chloris protogæa, i (unnamed). Pl. 5.

1843. Charpentier. Verhandl. leop.-carol. akad. naturf., xx: 405-406. Pl. 21, figs. 1-5.

1846. Geinitz. Grundr. versteinerungsk., 189. Pl. 8, figs. 6-7.

1849. Heer. Insektenf. tertiärg. Oeningen, ii : 15-18. Pl. 2, fig. 1.

1852. Giebel. Deutschl. petref., 637.

1856. " Insect. d. vorwelt, 308.

See also Hippiscus melanostictus.

2441. Edipoda nigro-fasciolata. (Acridii.)

Locality: Radoboj, Croatia. Horizon: Mayencian.

1849. Heer. Insektenf. tertiärg. Oeningen, ii : 18-20. Pl. 2, fig. 2.

1852. Giebel. Dentschl. petref., 63\%.

1856. "6 Insect. d. vorwelt, 308.

1885. Seudder. Zittel, Handb. palaeont., i, th. ii : 768.

1886. " "Zittel-Barrois, Traité de paléont., ii : 768.

1886. " " Bull. U. S. geol. surv., No. 31: 49 .

See also Scyllina nigrofasciolata.

2442. Gidiopda coningensis. (Acridii.)

Locality: Oeningen, Baden. Horizon: Tortonian.

"1755. Knorr. Merkwïrd. d. nat., (unnamed). Pl. 33, fig. 5." Heer.

1849. Heer. Insektenf. tertiärg. Oeningen, ii : 20. Pl. 2, fig. 4.

1852. Giebel. Deutschl. petref., 637.

1856. " Insect. d. vorwelt, 309.

1890. Scudder. Tert. ins. N. A., 225.

2443. CEdipoda præfocata. (Acridii.)

Locality : Florissant, Colo, Horizon : Oligocene.

1890. Scudder. Tert. ins. N. A., 225. Pl. 17, fig. 5.

2444. Orchelimum -. (Locustariæ.)

Locality : Florissant, Colo. Horizon: Oligocene.

1885. Scudder. Zittel, Handb. palaeont., i, th. ii : 770.

1886. " Zittel-Barrois, Traité de paléont., ii : 770.

1886. " Bull. U. S. geol. surv., No. 31: 50 .

See also Orchelimum placidum.

2445. Orchelimum placidum. (Locustariæ.)

Locality : Florissant, Colo. Horizon : Oligocene.

1890. Scudder. Tert. ins. N. A., 231-232. Pl. 17, figs. 16, 18, 19.

See also Orehelimum —. 


\section{Pachytylus - (Acridii.)}

Locality : Oeningen, Baden. Horizon: Torlonian.

1888. Schöberlin. Soc. ent., iii : 51 (Pachytilus).

\section{Paralatindia -. (Blattariæ.)}

Locality : Green River, Wyo. Horizon: Oligocene.

1885. Scudder. Zittel, Handb. palaeont., i, th. ii : 76\%.

1886. " "Zittel-Barrois, Traité de paléont., ii: 767.

1886. " " Bull. U. S. geol. surv., No. 31: 48.

See also Paralatindia saussurei.

2448. Paralatindia saussurei. (Blattariæ.)

Locality: Green River, Wyo. Horizon: Oligocene.

1890. Scudder. Tert. ins. N. A., 216. Pl. 6, fig. 25.

See also Paralatindia -

2449. Phaneroptera vetusta. (Locustariæ.)

Locality: Oeningen, Baden. Horizon: Tortonian.

1849. Heer. Insektenf. tertiärg. Oeningen, ii : 3-5. Pl. 1, fig. 2.

1852. Giebel. Deutschl, petref., 637.

1856. " Insect. d. vorwelt, 302-303.

1885. Scudder. Zittel, Handb. palaeont., i, th. ii: 770 .

1886. " Zittel-Barrois, Traité de paléont., ii : 770.

1886. " Bull. U.S. geol, surv., No. 31: 50 .

2450. Phasma -. (Phasmida.)

Locality : Prussian amber. Horizon: Ligurian.

1856. Hagen. Berendt, Bernst. befindl. organ. reste vorw., ii, th. i: 122 . Pl, 4, fig. 11.

1856. Menge. Progr. petrischule Danzig, 1856: 13.

Phylloptera - _ See _ — (Locustariæ) Scudder.

2451. Polyzosteria - (Blattariæ.)

Locality: Prussian amber. Horizon: Ligurian.

1856. Menge. Progr. petrischule Danzig, 1856: 12-13.

2452. Polyzosteria parvula. (Blattariæ.)

Locality: Prussian amber. Horizon: Ligurian.

1836. Berendt. Aun. soc. ent. Fr., v : 541-542 (unnameđ). Pl. 16, fig. 1.

1856. Germ.-Ber. Berendt, Bernst, befind], organ. reste vorw., ii, th. i: $32,35, \quad P l$. 4, fig. 2.

1864. Heer. Viert. naturf. gesellsch. Zürich, ix : 291.

1877. Goldenberg. Fanna saræp. foss,, ii : 21.

2453. Polyzosteria tricuspidata. (Blattariæ.)

Compared with P. decipiens Burm., Europe.

Locality : Prussian amber. Horizon: Ligurian.

1856. Germ.-Ber. Berendt, Bernst. befindl, organ. reste vorw., ii, th. i : $32,35-36$. Pl. 4, fig. 1.

1861. Heer. Viert. naturf. gesellsch. Zürich, ix : 291.

1877. Goldenberg. Fauna saræp. foss., 21.

See also Blatta tricuspidata. 
2454. Pronemobius induratus. (Gryllides.)

Locality: Green River, Wyo. Horizon: Oligocene.

1890. Scudder. Tert. ins. N. A., 235. Pl. 6, fig. 18.

2455. Pronemobius smithii. (Gryllides.)

Locality: Green River, Wyo. Horizon: Oligocene.

1890. Scudder. Tert. ins. N. A., 236-237. Pl. 6, fig. 22.

2456. Pronemobius tertiarius. (Gryllides.)

Locality: Green River, Wyo. Horizon: Oligocene.

1890. Scudder. Tert. ins. N. A., 235-236. Pl. 6, figs. 13, 21, 23.

See also Nemobius tertiarius.

\section{Pseudoperla gracilipes. (Phasmida.)}

Locality: Prussian amber. Horizon: Ligurian.

1854. Hagen. 1854. Pictet. 1856. “6

1856. Hagen.
Verhandl, zool.-bot. ver. Wien, iv : 228.

Traité de paléont., $2^{\mathrm{e}}$ ed., ii : $364 . \quad P l .40$, fig. 25.

Berendt, Bernst. befindl. organ. reste vorw., ii, th. i : $38 . \quad P l .4$, fig. 9.

Berendt, Bernst. befindl, organ, reste vorw., ii, th, $i$; 39-40.

2458. Pseudoperla lineata. (Phasmida.)

Locality : Prussian amber. Horizon: Ligurian.

1854. Pictet.

1856. “

Traité de paléont., $2^{\mathrm{e}}$ ed., ii : 364 (undescr.).

Berendt, Bernst. befindl. organ. reste vorw., ii, th. i : 38-39. $\quad P l$. 4, fig. 10.

1856. Hagen.

1885. Scudder.

1886. " "
Zittel, Handb. palaeont, i, th. ii : 768. Fig. 964

Zittel-Barrois, Traité de pal6ont, ii : 768. Fig. 981.

2459. Scyllina? nigrofasciolata. (Acridii.)

Locality : Radoboj, Croatia. Horizon: Mayencian.

1890. Scudder. Tert. ins. N. A., 220.

See also Edipoda nigrofasciolata.

2460. Taphacris reliquata. (Acridij.) .

Locality: Florissant, Colo. Horizon: Oligocene.

1890. Scudder. Tert. ins. N. A., 226-227. Pl. 12, figs. 8, 19.

2461. Tetrix gracilis. (Acridii.)

Locality: Oeningen, Baden. Horizon: Tortonian.

1865. Heer.

1872. " "

1876. “

1879. “

1885. Scudder.

1886. “
Urwelt der Schweiz. Fig. 228 on p. 366.

Monde prim. Suisse. Fig. 228 on p. 449.

Prim. world Switz., ii. Fig. 228 on p. 21.

Urwelt der Schweiz, $2^{\mathrm{e}}$ aufl. Fig. 269 on $p$. 392.

Zittel, Handb. palaeont., i, th. ii : 768 (Tettigidea in expl. of cut). Fig. 966.

Zittel-Barrois, Traite de paléont., ii : 768 (Tettigidea in expl. of cut). Fig. 983.

Bull. U.S. geol. surv., No. $31 ; 49$,

$1886 . \quad$ Bull. U. S.
Seo also Tettigidea gracilis 
2462. Tettigidea gracilis. (Acridii.)

Locality : Oeningen, Baden. Horizon: Tortonian.

1890. Scudder. Tert. ins. N. A., 220 .

See also Tetrix gracilis.

\section{Tyrbula multispinosa. (Acridii.)}

Localities: Green River, Wyo.; Florissant, Colo. Horizon: Oligocene. 1890. Scudder. Tert. ins. N. A., 221-222. Pl. 17, fig. 13.

\section{Tyrbula russelli. (Acridii.)}

Locality : Florissant, Colo. Horizon : Oligocene.

1885. Scudder. 1890. " "

1829. Serres: 1886. "

Zittel, Handb. palaeont., i, th. ii : 768. Fig. 965.

Zittel-Barrois, Traité de paléont, ii : 768. Fig. 982.

Tert. ins. N. A., 222-223. Pl. 17, figs. 1-4.

\section{Xya - - (Gryllides.)}

Compared with X. variegata, Europe.

Locality: Aix, France. Horizon: Ligurian.

Géogn. terr. tert., 226.

2466. Zetobora -. (Blattariæ.)

Locality: Florissant, Colo. Horizon: Oligocene.

1885. Scudder. Zittel, Handb. palaeont., i, th. ii: 767.

1886. " "Zittel-Barrois, Traité de paléont., ii: 767.

1886. " Bull. U. S. geol. surv., No. $31: 48$.

2467. Zetobora brunneri. (Blattariæ.)

Locality : Florissant, Colo. Horizon : Oligocene.

1890. Scudder. Tert. ins. N. A., 217. Pl. 17, fig. 12.

\section{NEUROPTERA.}

2468.

- Locality : England. Horizon: Pleistocene.

1888. Bell.

\section{9.}

Locality: Bournemouth, England. Horizon: Middle Eocene.

1878. Goss.

Proc. ent. soc. Lond., 1878: 8.

2470.

Locality : Sinigaglia, Italy. Horizon: Helvetian?.

1842. Procaccini. Nuov. ann. sc. nat., vii : 449.

\section{1.} (larva).

Locality : Florissant, Colo. Horizon: Oligocene.

1881. Scudder. Bull. U. S. geol. surv. terr., vi : 294.

See also Planocephalus aselloides. 
2472

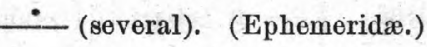

Locality : Florissant, Colo. Horizon: Oligocene.

1881. Scudder. 1883.
Bull. U. S. geol, surv, terr., vi : 293.

Ann. rep. U. S. geol, surv. terr., xii : $2 \Varangle 4$.

2473. (several). (Hydropsychidæ.)

Locality: Florissant, Colo. Horizon: Oligocene. 1881. Seudder. Bull. U. S. geol. surv, terr., vi : 293.

2474. (several). (Leptoceridæ.)

Locality : Florissant, Colo. Horizon: Oligocene. 1881. Scudder.

Bull. U. S. geol. surv, terr,, vi : 293

2475. (under wing). (Odonata.)

Locality: Eisleben, Saxony. Horizon:

1856. Giebel.

Zeitschr. ges. naturw., vii: 386. Pl. 5, fig. 4.

2476. (Odonata-Libellulina).

Locality: Green River, Wyo. Horizon: Oligocene. 1878. Scudder. Bull. U. S. geol. surv, terr., iv : 775.

See also Libellula —_ Scudder.

2477. calypso. (Odonata-Libellulina).

Locality: Oeningen, Baden. Horizon: Tortonian. 1868. Brauer. Verhandl. zool.-bot. gesellsch. Wien, xviii : 738. 1868. “ Verz. bek. Neur., 86.

2478. cassandra. (Odonata-Libellulina).

Locality : Rott, Rhenish Prussia. Horizon: Aquitanian. 1868. Brauer. Verhandl. zool.-bot. gesellsch. Wien, xviii : 738. 1868. " Verz. bek. Neur., 86.

See also Libellula cassandra.

2479. ceres. (Odonata-Libellulina).

Locality: Rott, Rhenish Prussia. Horizon: Aquitanian. 1868. Brauer. Verhand1. zool.-bot. gesellsch. Wien, xviii: 738. 1868. " " Verz. bek. Neur., 86 (= cellulosa ?).

See also Libellula ceres, etc.

2480. doris. (Odonata-Libellulina).

Locality: Oeningen, Baden. Horizon: Tortonian. 1868. Brauer. Verhandl, zool. bot.-gesellsch. Wien, xviii : 738. 1868. " Verz. bek. Neur., 86.

See also Libellula doris.

2481. eurynome. (Odonata-Libellulina).

Locality: Oeningen, Baden. Horizon: Tortonian. 1868. Brauer. Verhandl. zool.-bot. gesellsch. Wien, xviii : 738. 1868. " " Verz. bek. Neur., 86.

See also Libellula eurynome. Libellula —— Scheuchzer. 
2482. melobasis. (Odonata-Libellulina).

Locality : Oeningen, Baden. Horizon: Tortonian. 1868. Brauer. Verhandl. zool.-bot. gesellsch. Wien, xviii : 738. 1868. " $\quad$ Verz. bek. Neur., 86.

See also Libellula melobasis.

2483. - perse. (Odonata-Libellulina).

Locality : Oeningen, Baden. Horizon : Tortonian. 1868. Brauer. Verhandl. zool.-bot. gesellsch. Wien, xviii : 738. 1968. " " Verz. bek. Neur., 86.

See also Libellula perse.

2484. - thetis. (Odonata-Libellulina).

Locality: Oeningen, Baden. Horizon: Tortonian. 1868. Brauer. Verhandl. zool.-bot. gesellsch. Wien, xviii : 738. 1868. " " Verz. bek. Neur., 86.

See also Libellula thetis.

2485. thoe. (Odonata-Libellulina).

Locality: Oeningen, Baden. Horizon: Tortonian.

1868. Brauer. Verhandl. zool.-bot. gesellsch. Wien, xviii: 738. 1868. " " Verz. bek. Neur., 86.

See also Libellula thoe.

2486. (several). (Perlina.)

Locality: Florissant, Colo. Horizon: Oligocene. 1881. Scudder. Bull. U. S. geol. surv. terr., vi : 293.

2487. (several). (Phryganidæ.)

Locality: Florissant, Colo. Horizon: Oligocene.

1881. Soudder. Bull. U. S. geol. surv. terr., vi : 293.

2488. (several). (Rhyacophilidæ.)

Locality: Florissant, Colo. Horizon: Oligocene.

1881. Scudder.

Bull. U. S. geol. surv. terr., vi: 293.

2489. (7 sp.). (Termitina.)

Locality: Oeningen, Baden. Horizon: Tortonian. 1888. Schöberliu. Soc. ent., iii : 51.

2490. (Thysanura.)

Locality : Prussian amber. Horizon : Ligurian. 1819. Schweigger. Beobacht. naturh. reisen, 113. Pl. 8, figs. 71, 71ab. 2491. (Trichoptera.)

Locality: Prussian amber. Horizon: Ligurian. 1841. Ehrenberg. Froriep, Neue notizen, xix : 120.

2492. (larval tubes). (Trichoptera.)

Locality: Gard, France. Horizon: Oligocene.

1884. Marion. Saporta, Organ. probl. anc. mers, 24-26 (on leaves of Nymphma dumasii). Pls. 3, 4, and fig. 4, on p. 25. 
2493. Acreagris - (Thysanura?.)

Locality : Prussian amber. Horizon: Ligurian.

1845. Berendt. Bernst. befindl. organ. reste vorw., i: 56.

2494. Acreagris crenata. (Thysanura?.)

Locality : Prussian amber. Horizon : Ligurian.

1845. Koch-Ber. Jahrb. f. mineral., 1845: 873.

1854. " Berendt, Bernst. befindl. organ. reste vorw., i, th. ii : 123. Rl.17, fig. 157.

1\%54. Menge. Ibid., i, th. ii : 12? (=Monophlebus o ).

18:6. Giebel. Insect. d. vorwelt, 331.

See also III, Hemiptera, Monophlebus _

2495. 㞋schna —. (Odonata.)

Compared with Aschna grandis.

Locality: Oeningen,-Baden. Horizon: Tortonian.

1832. Curtis. Murch., Trans. geol. soc. Lond., (2), iii : 286 (Gschna). Pl. 34, figs. 4-5.

2496. Æschna - (Odonata.)

Locality: Bournemouth, England. Horizon: Middle Eocene, Bagshot Sands.

1878. Goss. Entom., xi : 193-195. Fig.

1878. " Proc. ent. soc. Lond., 1887: 8.

See also Basiæschna -

2497. \#schna - (tip of wing), (Odonata.)

Locality: Prussian amber. Horizon: Ligurian.

1854. Hagen. Verhanäl. zool.-bot. ver. Wien, iv : 227.

See also Gomphoides occulta.

2498. Aschna - (2 sp.). (Odonata.)

Locality: Florissant, Colo. Horizon : Oligocene.

1881. Scudder. Bull. U. S. geol. surv. terr., vi : 293.

1882. " " Proc. Bost. soc. nat. hist., xxi : 409.

1883. “ Ann. rep. U. S. geol. surv. terr., xii : 284.

2499. \#schna - (larva). (Odonata.)

Locality : Florissant, Colo. Horizon: Oligocene.

1881. Scudder. Bull. U. S. geol. surv. terr., vi : 293.

1882. " " Proc. Bost. soc. nat. hist., xxi: 409.

18ะ3. " Aun, rep. U. S. geol, surv. terr., xii : 284.

See also Aschna larvata.

2500. Fschna dido (nymph). (Odonata.)

Locality: Rott, Rhenish Prussia. Horizon: Aquitanian.

1863. Hagen. Palaeontogr., $x:$ 263. Pl. 44, fig. 8.

1870. L. Heyden. Foss. Dipt. Rott, 30.

1870. " Palaeontogr., xvii: 266.

Bull. $71-21$ 
2501. \#schna eudore (larva). (Odonata.)

Locality: Oeningen, Baden. Horizon: Tortonian.

1849. Heer.

1850. Hagen.

1852. Giebel.

1856. " "

- Insektenf. tertiärg. Oeningen, ii : 73-74. Pl. 8, fig. 4. Rev. Odon. (Mém. soc. roy. sc. Liége, vi): 362.

Deutschl. petref., 6.39 .

Insect. d. vorwelt, 278.

2502. Æschna larvata (larva). (Odonata.)

Locality: Florissant, Colo. Horizon: Oligocene.

1890. Scudder. Tert. ins. N. A., 145. Pl. 13, fig. 11.

See also Eschna — Scudder.

2503. Æsschna metis. (Odonata.)

Locality: Radoboj, Croatia. Horizon : Mayencian.

1849. Heer. Insektenf. tertiärg. Oeningen, ii : 68-73. $P l .5, f i g .1$. 1856. Giebel. Insect. d. vorwelt, 277-278.

See also Anax metis.

2504. Æsschna polydore. (Odonata.)

Locality : Oeningen, Baden. Horizon: Tortonian.

1249. Heer. Insektenf, tertiärg. Oeningen, ii : 63-67. Pl. 4, fig. 6.

1850. Hagen. Rev. Odon. (Mém. soc. roy. sc. Liége, vi) : 362 。

1852. Giebel. Deutschl. petref., 639.

1856. “ Insect. d. vorwelt, 277.

See also Eschna (Aschna) polydore.

2505. Aschna (झschna) polydore. (Odonata.)

Locality: Oeningen, Baden. Horizon: Tortonian.

1890. Scudder. Tert. ins. N. A., 142.

See also Aschna polydore.

2506. Ischna (Basiæschna) separata. (Odonata.)

Compared with $\AA$.janata Say.

Locality: Florissant, Colo. Horizon: Oligocene.

1890. Scudder.

Tert. ins. N. A., 144-145. Pl. 13, fig. 15.

2507. \#schna (\#schna) solida. (Odonata.)

Compared with A. constricta.

Locality: Florissant, Colo. Horizon : Oligocene.

1890. Scudder. Tert. ins. N. A., 143-144. Pl. 13, fig. 1.

2508. Aschna tyche (Odonata.)

Locality: Oeningen, Baden. Horizon: Tortonian.

1849. Heer.

1850. Hagen.

1852. Giebel.

1856. “
Insektenf. tertiärg. Oeningen, ii : 67-68. $\mathrm{Pl} .4, \mathrm{fig} . \%$.

Rev. Odon. (Mém. soc. roy, sc. Liége, vi) : 362.

Deutschl. petref., 639 .

Insect. d. vorwelt, $27 \%$.

See also Aschna (Aschna) tyche.

2509. Aschna (Æschna) tyche. (Odonata.)

Locality: Oeningen, Baden, Horizon: Tortonian,

1890. Scudder.

Tert. ins, N. A, 142 .

See also Aschna tyche. 
2510. Agapetus æqualis. (Rhyacophilidæ.)

1856. Hagen.

Locality : Prussian amber. Horizon: Ligurian.

1864. " " Phryg. synopsis synom., 3.

1864. " Verhandl. zool.-bot. gesellsch. Wien, xiv : 801.

2511. Agraylea succinea. (Hydroptilidæ.)

Locality : Prussian amber. Horizon : Ligurian.

1864. Hagen. Verhandl. zool.-bot. gesellsch. Wien, xiv : 4.

1864. " Phryg. synopsis synom., 4.

2512. Agrion -. (Odonata.)

Locality : Prussian amber. Horizon : Ligurian.

1845. Berendt. Bernst. befindl. organ. reste vorw., i : 57 .

\section{Agrion - (Odonata.)}

Compared with A. sanguineum.

Locality: Oeningen, Baden, Horizon: Tortonian.

1832. Curtis. Murch., 'Trans. geol, soc. Lond,, (2), iii : 286. Pl. 34, fig. 6.

See also Agrion aglaope.

\section{Agrion —. (Odonata.)}

Locality: Aix, France. Horizon: Ligurian.

1847. Hope. Trans. ent. soc. Lond., iv : 252.

2515. Agrion - (2 sp.). (Odonata.)

Locality : Florissant, Colo. Horizon : Oligocene.

1881. Scudder. Bull. U. S. geol, surv. terr., vi : 293.

1882. " Proc. Bost. soc. nat. hist., xxi : 409.

1883. " Ann. rep. U. S. geol. surv. terr., xii : 284.

2516. Agrion - (nymph). (Odonata.)

Locality : Florissant, Colo. Horizon : Oligocene.

1881. Scudder. Bull. U. S. geol. surv. terr., vi : 293.

1882. " Proc. Bost. soc. nat. hist., xxi : 409.

Soe also Agrion telluris.

\section{Agrion - (Odonata,)}

Locality : Guruet Bay, Isle of Wight. Horizon: Bembridge Limestone. 1879. Woodward. Quart. journ. geol. soc. Lond, xxxv : 344. $1879 . \quad$ "Geol. mag. (n. s.), v: 89.

\section{Agrion aglaope. (Odonata.)}

Compared with A. elegans Lind.

Locality : Oeningen, Baden. Horizon: Tortonian.

1849. Heer.

1850. Hagen.

1852. Giebel.

1856. " "
Insektenf. tertiärg. Oeningen, ii : 59-62, $\quad P l .4, f i g .4$.

Rev. Odon. (Mém. soc. roy. sc. Liége, vi): 356.

Deutschl. petref., 638.

Insect, d. vorwelt, 272. 
1865. Heer.

$187 \%$. "

1876. " "

1879. " "

1890. Scudder.
Urwelt der Schweiz, 369

Monile prim. Suisse, 453.

Prim. world Switz., ii : 24.

Urwelt der Schweiz, $2^{\circ}$ aufl., 395.

Tert. ins. N. A., 137.

See also Agrion — Curtis.

2519. Agrion aglaopheme. (Odonata.)

Locality: Oeningen, Baden. Horizon: Tortonian.

1849. Heer. 1850. Hagen. 185\%. Giebel. 1856. “

1848. Pictet. 1848. Hagen. 1850. "6 1854. "6 1856. Giebe]. 1856. Pict.-Hag.
Insektenf. tertiärg. Oeningen, ii : 62-63. Pl. 4, fig. 5.

Rev. Odon. (Mém. soc. roy. sc. Liége, vi): 356.

Deutschl. petref., 638.

Insect. d. vorwelt, 273 .

2520. Agrion antiquum. (Odonata.)

Locality: Prussian amber. Horizon : Ligurian.

MSS.

Stett. ent. zeit., ix : 7 .

Rev. Odon. (Mém. soc. roy. sc. Liége, vi) : 356-357.

Verhandl. zool.-bot. ver. Wien, iv : '227.

Insect $d$, vorwelt, 273 .

Berendt, Bernst, befindl. organ. reste vorw., ii, th. i: 78-80. Pl. 6, figs. 4b-d; Pl. 8, fig. 11.

See also Argia antiqua, Platycnemis antiqua.

\section{Agrion (Lestes) coloratụm. (Odonata.)}

Locality : Radoboj, Croatia. Horizon: Mayencian.

1841. Charpentier. Neues jahrb. min., 1841 : 332-337 (unnamed). Pl. 1.

1849. Heer.

1856. Giebel.

Insektenf. tertiärg. Oeningen, ii : 55-56.

Insect. d. vorwelt, 273 .

See also Lestes coloratus.

2522. Agrion exsularis. (Odonata.)

Locality : Florissant, Colo. Horizon: Oligocene.

1890. Scudder. Tert. ins. N. A., 139-140. Pl. 13, fig. 6.

2523. Agrion icarus. (Odonata.)

Locality : Rott, Rhenish Prussia. Horizon: Aquitanian.

1863. Hagen. Palaeontogr., $\mathrm{x}: 260 . \quad P l .44$, fig. 5.

See also Platycnemis icarus.

2524. Agrion iris. (Odonata.)

Compared with A. fasciatum and longicandum.

Locality: Oeningen, Baden. Horizon: Tortonian.

1879. Heer. Urwelt der Schweiz, $2^{\mathrm{e}}$ aufl., 395. Fig. 275.

See also Lestes iris.

\section{Agrion leucosia. (Odonata.)}

Compared with A. longicaudum.

Locality: Oeningen, Baden. Horizon: Tortonian.

1865. Heer.

1872. " "

Urwelt der Schweiz, 369.

1876. " "

Monde prim. Suisse, 453.

1879. " "

Prim. world Switz., ii : 24.

Urwelt der Schweiz, 2e aufl., 395.

See also Agrion (Lestes) leucosia, etc. 
2526. Agrion (Lestes) leucosia. (Odonata.)

Compared with A. longicaudum.

Locality: Oeningen, Baden. Horizon: Tortonian.

1849. Heer. Insektenf. tertiärg. Oeningen, ii : 56-57. Pl. 4, fig. 1. 1856. Giebel. Insect. d. vorwelt, 273-274.

See also Lestes leucosia, Agrion leucosia.

2527. Agrion (Lestes) ligea. (Odonata.)

Locality: Oeningen, Baden. Horizon: Tortonian.

1849. Heer. Insektenf. tertiärg. Oeningen, ii : 57-59. Pl. 4, fig. 2. 1856. Giebel. Insect. d. vorwelt, 274.

See also Lestes ligea.

2528. Agrion mascescens. (Odonata.)

Locality: Florissant, Colo. Horizon: Oligocene.

890. Scudder. Tert. ins. N. A., 138-139. Pl. 13, figs. 8, 9.

2529. Agrion mysis (nymph). (Odonata.)

Locality : Rott, Rhenish Prussia. Horizon: Aquitanian.

1863. Hagen. Palaeontogr., $\mathrm{x}: 269$. Pl. 44, fig. 6.

2530. Agrion parthenope. (Odonata.)

Compared with A. fasciatum, South Africa..

Locality: Oeningen, Baden. Horizon: Tortonian.

1852. Giebe]. Dentschl. petref., 638.

1865. Heer. Urwelt der Schweiz, 369.

1872. " Monde prim. Suisse, 453.

1876. " " Prim. world Switz., ii: 24.

1879. " Urwelt der Schweiz, $2^{\ominus}$ aufl., 395.

See also Agrion (Sterope) parthenope, etc.

\section{Agrion (Sterope) parthenope. (Odonata.)}

Locality: Oeningen, Baden. Horizon: Tortonian.

1849. Heer. Insektenf. tertiärg. Oeningen, ii : 45-55. Pl. 3, fig. 11. 1856. Giebel. Insect. $d$. vorwelt, 274-275.

See also Agrion parthenope, Sympyena parthenope, Sterope parthenope.

2532. Agrion (Lestes) peisinoe. (Odonata.)

Locality: Oeningen, Baden. Horizon: Tortonian.

1849. Heer. Insektenf, tertiärg. Oeningen, ii: 59. Pl. 4, fig. 3. 1856. Giebel. Insect. d. vorwelt, 274.

See also Lestes peisinoe.

2533. Agrion telluris (nymph). (Odonata.)

Locality: Florissant, Colo. Horizon: Oligocene.

1890. Scudder. Tert. ins. N. A., 140-141. Pl. 13, fig. 10.

See also Agrion — Scndder.

2534. Agrion thais (nympl). (Odonata.)

Locality : Rott, Rhenish Prussia. Horizon : Aquitanian.

1863. Hagen. Palaeontogr., $x: 269$ (pupa). Pl. 44, fig. 7. 


\section{Agrypnia - (Trichoptera.)}

Compared with A. pagetana.

Locality: Prussian amber. Horizon : Ligurian.

1856. Germar. Berendt, Bernst. befindl. organ, reste vorw., ii, th. i: 101. See also Limnophilus — Hagen.

2536. Amphientomum —. (Psocina.)

Locality : Prussian amber. Horizon: Ligurian.

1845. Berendt. Bernst. befindl, organ. reste vorw., $i$ : 57 .

2537. Amphientomum paradoxum. (Psocina.)

Locality: Prussian amber. Horizon: Ligurian.

1854. Pictet.

1854. : "

1856. “

1865. Hagen.

1866. “

1866. " "

1882. “

Hagen, Verhandl, zool.-bot. ver. Wien, iv : 225 (undescr.).

Traité de paléont., $2^{e}$ éd., ii : $376 . \quad P l .40$, figs. 27, $27 a b$.

Berendt, Bernst. befindl. organ. reste vorw., ii, th. i: 61-64. Pl. 7, figs. 21b-d; pl. 8, figs. 10a-e.

Ent. monthl. mag., ii : 149.

Verhandl. zool.-bot. gesellsch. Wien, xvi : 204.

Psoc. Emb. synopsis, 4.

Stett. ent. zeit., xliii : 269-276. Pl. 1, fig. 6 (9 figs.).

\section{Anax metis. (Odonata.)}

Locality: Radoboj, Croatia. Horizon: Mayencian.

1850. Hagen.

1852. Giebel.

Rev. Odon. (Mém. soc. roy. sc. Liége, vi): 361.

1890. Seudder.

Deutschl. petref., 639.

See also Eschna metis.

\section{Aph́eilocheira —_. (Hydropsychidæ.)}

Locality : Prussian amber. Horizon: Ligurian.

1845. Berendt. Bernst. befinäl. organ. reste vorw., i: 57 (Aphilocheira).

\section{Apheilocheira fusconigra. (Hydropsychidæ.)}

Locality: Prussian amber. Horizon: Ligurian.

1854. Pictet.

1856. " "

1864. Hagen.

1864. “
Traité de paléont., $2^{\mathrm{e}}$ éd., ii : 376 (undescr.).

Berendt, Bernst, befindl, organ. reste vorw., ii, th. i: 115. Pl. 7, fig. 18.

Phryg. synopsis synom., 7 (= Polycentropus dubins).

Verhandl. zool.-bot. gesellsch. Wien, xiv : 805 (=Polycentropus dubius).

See also Polycentropus fusconiger, etc. See Apheilocheira

2541. Archipsocus puber. (Psocina.)

Locality: Prussian amber. Horizon: Ligurian.

1882. Künow. MSS.

1882. Hagen.

Stett. ent. zeit., xliii : 222-225. Pl. 1, figs. 101-9. 
2542. Argia antiqua. (Odonata.)

1854. Hagen.

1856. "6

Locality : Prussian amber. Horizon : Ligurian.

Verhandl. zool.-bot. ver. Wien, iv: 227 (Argya).

Berendt, Bernst. befindl. organ. reste vorw., ii, th. i: 79-80 (Argya).

See also Agrion antiquum, etc.

Argya antiqua. See Argia antiqua.

2543. Ascalaphus edwardsii. (Hemerobina.)

Compared with A. barbarus, S. Enrope.

Locality: Saint-Gérand le Puy, France. Horizon: Aquitanian.

1870. Oustalet. Ann. sc. g6́ol., ii, art. 3:93-94. Pl. 2, figs. 8, 9 .

2544. Ascalaphus proavus. (Hemerobina.)

Compared with A. subvertens, Venezuela.

Locality : Stösschen, bei Linz, Austria. Horizon: Aquitanian.

1858. Hagen. Palaeontogr., v: 125-126. Pl. 25.

See also Suphalasca proavus.

2545. Aspatherium geinitzii. (Trichoptera.)

Locality : Prussian amber. Horizon : Ligurian.

1851. Kolenati. Abhandl. böhm. gesellsch.wiss., (5), vi: 15 (undescr.).

2546. Atropos succinea. (Psocina.)

Compared with A, divinatoria.

Locality : Prussian amber. Horizon: Ligurian.

1882. Künow. MSS.

1882. Hagen. Stett. ent. zeit., xliii : 231, 289-292. Pl. 2, figs. 31, 1a-c, 2, 3, 36 .

1883. " Stett. ent. zeit., xliv: 296.

See also Troctes succinicus.

2547. Bætis -.. (Ephemeridæ.)

Locality : Prussian amber. Horizon: Ligurian.

1845. Berendt. Bernst. befindl. organ. reste vorw., i: 57.

2548. Bætis anomala. (Ephemeridæ.)

Locality : Prussian amber. Horizon: Lignrian.

1854. Pictet. Hagen, Verhandl. zool.-bot. ver. Wien, iv : 227 (undescr.).

1844. " Traité de paléont., 2 éd., ii : 371 (undescr.).

1856. Pietet-Hagen. Berendt, Bernst. befindl. organ. reste vorw., ii, th. i: 75-76. Pl. 6, fig. $1 b-c$.

See also Cronicus anomalus.

2549. Bætis gigantea. (Ephemeridæ.)

Locality: Prussian amber. Horizon: Ligurian.

1856. Hagen. Berendt, Bernst. befindl. organ. reste vorw., ii, th. i: 75.

2550. Bætis grossa. (Ephemeridæ.)

Locality : Prussian amber. Horizon: Ligurian.

1856. Hagen. Berendt, Bernst. befindl. organ. reste vorw., ii, th. $i: 75$. 
2551. Bætis longipes. (Ephemeridæ.)

Loeality: Prussian amber. Horizon: Ligurian.

1854. Hagen.

Verhaucll. zool. bot. ver. Wien, iv: 2\%7 (undescr.).

1856. “

Berendt, Bernst. befindl. organ. reste vorw., ii, th. i : 76-77.

2552. Basiæschna (Odonata.)

Locality : Bonrnemouth, England. Horizon: Middle Eocene. 1890. Sendder. Tert. ins. N. A., 142.

See also Aschna _ Goss.

Basiæschna separata. See Aschna (Basiæschna) separata.

2553. Bittacus - . (Panorpidæ.)

Locality: Prussiau amber. Horizon: Ligurian.

1845. Berendt. Bernst. befindl. organ. reste vorw., i: $5 \%$.

2554. Bittacus antiquus. (Panorpidæ.)

Compared with B. italicus, Europe.

Locality : Prussiau amber. Horizon: Ligurian.

1854. Pictet. Hagen, Verhandl, zool.-bot. ver. Wien, iv: 229 (undescr.).

1854. “ Traité de paléont., $2^{\circ}$ éd., ii : $379 . \quad P l .40$, fig. 26.

1856. " Berendt, Berust. befindl. organ. reste vorw., ii, th. i: 92. Pl. 7, fig. $23 ;$ pl. 8, fig. 22.

2555. Bittacus reticulatus. (Panorpidæ.)

1849. Heer.

1852. Giebel.

1856. “

Locality : Radoboj, Croatia. Horizon: Mayencian.

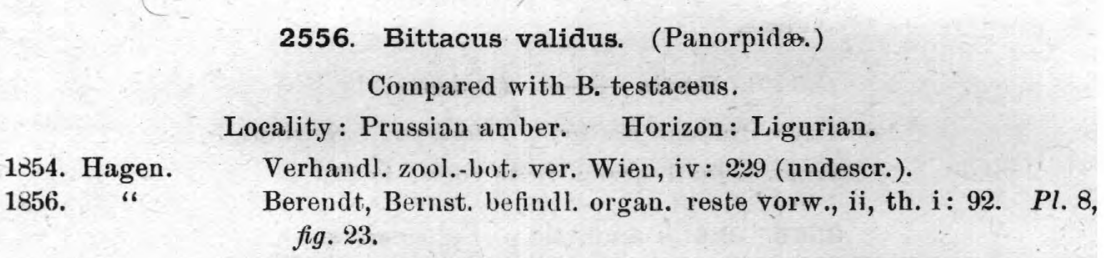

2557. Bothromicromus lachlani. (Hemerobina.)

Compared with Micromus hirtus, Europe.

Locality: Quesnel, British Columbia. Horizon: -

1878. Scudder. Add. ins. fauna Quesnel, 6-7.

1878. " "

1878. “

Rep. progr. geol. surv. Can., 1876-'77: 462-463.

1890. “

Rapp. oper. comm. géol. Can., 1ه76-'77 : 520-521.

Tert. ins. N.A., 164-165. Pl. 2, figs. 7-10.

2558. Brachycentrus labialis. (Sericostomidæ.)

Locality : Prussian amber. Horizon: Ligurian.

1864. Hagen. Phryg. synopsis synom., 9.

1864. " Verhandl, zool.-bot. gesellsch. Wien, xiv: 807.

See also Hydronantia labialis. 
2559. Cæcilius abnormis. (Psocina.)

Locality: Prussian amber. Horizon : Ligurian.

1866. Hagen. Psoc. Emb. syuopsis, 4.

1866. “ Verhandl. zool.-bot. gesellsch. Wien, xvi : 204.

See also Elıpsocus abnormis, etc.

\section{Cæcilius debilis. (Psocina.)}

Compared with C. obsoletus.

Locality : Prnssian amber. Horizon: Ligurian.

1882. Hagen. Stett. ent. zeit., xliii : 284-237. Pl. 1, figs. $4^{1-3}$.

See also Psocus debilis, Epipsocus debilis.

2561. Cæcilius pilosus. (Psocina.)

Locality: Prussian amber. Horizon : Ligurian.

1882. Hagen. Stett. ent. zeit., xliii : 283-284. Pl. 1, figs. $3^{1-2,4 \cdot 7}$.

2562. Cæcilius proavus. (Psocina.)

Compared with C. aridus, C. dimidiatus.

Locality: Prussian amber. Horizon: Ligurian.

1866. Hagen. Stett, ent, zeit., xxvii : 206.

1866. " $\quad$ Psoc. Emb. synopsis, 6.

1882. " " Stett. ent. zeit., xliii : 280-283. Pl. 1, figs. 21-8, 8a, 9-11.

1866. " Verhandl. zool.-bot. gesellsch. Wien, xvi : 206

See also Psocus proavus.

2563. Calopteryx? (nymph). (Odonata.)

Locality : Prussian amber. Horizon: Ligurian.

1854. Hagen. Verhandl. zool.-bot. ver. Wien, iv: 227 (undeser.).

2564. Calotermes affinis. (Termitina.)

Locality : Prussian amber. Horizon: Ligurian.

1854. Hagen. Verhandl. zool. bot. ver. Wien, iv : 222.

1858. " Catal. Nenropt. Brit. mus., 8.

1858. " " Linn. entom., xii : 53-54.

1860. “ Linn. entom., xiv : 100-101.

See also Termes (Calotermes) affinis, ete.

2565. Calotermes berendtii. (Termitina.)

Locality: Prussian amber. Horizon: Ligurian.

1854. Hagen. Verhandl zool.-bot. ver. Wien, iv : 222 (undeser.).

$1 \times 58$.

Catal. Neuropt. Brit. mus., 7.

1858. “ Linn. entom., xii : 49-51.

1860. " Linn. entom., xiv : 100 .

See also Termes berendtii, ete.

2566. Calotermes rhenanus. (Termitina.)

Locality: Rott, Rhenish Prussia. Horizon: Aquitanian.

1863. Hagen. Palaeontogr., $\mathrm{x}$ : 250-251. Pl. 44, figs. 1-2. 


\section{Celithemis cellulosa. (Odonata.)}

Locality : Rott, Rhenish Prussia. Horizon: Aquitanian.

1868. Brauer. Verhanll, zool.-bot. gesellsch. Wien, xviii : 738.

1868. " Verz. bek. Neuropt., 86.

See also Libellula cellnlosa, L. ceres, ete.

2568. Chauliodes —. (Sialina.)

Locality: Prussian amber. Horizon : Ligurian.

1815. Berendt. Bernst. befindl. organ. reste vorw., i : 57.

2569. Chauliodes prisca. (Sialina.)

Locality: Prussian amber. Horizon: Ligurian.

1854. Pictet. Hagen, Verhandl. zool.-bot. ver. Wien, iv: 228 (undescr.).

1854. " Traité de paléout., 2e éd., ii : 377. Pl. 40, figs. 24, 24a.

1856. " Berendt, Bernst, befindl, organ, reste vorw., ii, th. i: 82. Pl. 7, fig. 22.

2570. Chrysopa —. (Hemerobina.)

Compared with C. perla Linn.

Locality: Thalheim, Siebenbiirgen. Horizon :

1855. Andrä. Foss. flora Siebenb., 26. Pl. 5, figs, 3, $3 a$.

1856. Giebel. Iusect. d. vorwelt, 262-263.

1866. Hagen. Stett, ent. zeit., xvi : 399 (a Chrysopa?).

2571. Chrysopa - (Hemerobina.)

Locality : Prussian amber. Horizon: Ligurian.

1845. Berendt. Bernst. befindl. organ. reste vorw., i: 57.

2572. Chrysopa (vic.) —. (Hemerobina.)

Locality: Florissant, Colo. Horizon: Oligocene.

1881. Scudder. Bull. U. S. geol. surv. terr., vi: 293.

See also Palæochrysa - - , Tribochrysa - - .

Chrysopa or Hemerobius —_. See Hemerobius (or Chrysopa) - -

2573. Coniopteryx timida. (Coniopterygidæ.)

Locality: Prussian amber. Horizon: Ligurian.

1866. Hagen. " Stett. ent. zeit., xxvii : 401.

See also Coniortes — Hagen, Coniortes timidus.

2574. Coniortes, (Osmylus, or Nymphes) —_ (larva).

Locality: Prussian amber. Horizon: Ligurian.

1856. Hagen. Berendt, Bernst. befindl. organ. reste vorw., ii, th. i: 90.

See also Coniopteryx timida, etc.

2575. Coniortes timidus. (Coniopterygidæ.)

Locality : Prussian amber. Horizon: Ligurian.

1854. Hagen. Verhandl. zool.-bot. ver. Wien, iv: 228 (undescr.).

1856. " Berendt, Bernst. befindl. organ. reste vorw., ii, th. i: 89.

See also Coniopteryx timida, ete. 
2576. Cordulia platyptera. (Odonata.)

1849. Heer.

1850. Hagen.

1852. Giebel.

1856. " "

Locality: Radoboj, Croatia. Horizon: Mayencian.

Insektenf. tertiärg. Òeningen, ii : 74-79. $\quad P l .5$, fig. 3.

Rev. Odon. (Mém. soc. roy. se. Liége, vi) : 363.

Deutschl. petref., 639.

Insect. d. vorwelt, 288-289.

See also Libellula platyptera.

2577. Cordulia scheuchzeri. (Odonata.)

Locality: Monte Bolca, Italy. Horizon: Ligurian.

[1723. Scheuchzer. Herb. diluv. (unnamed). Pl. 5, fig. 2.]

1855. Massalongo. Nereid. foss., 31 (undescr.).

1856. " " Stud. paleont., 17-18. Pl. 2, fig. 7.

1886. Omboni. Atti r. ist. Ven., (6), iv: 1425-1426. Pl. 2, fig. 6.

1886. " Ins. foss. Ven, 5-6. Pl. 2, fig. 6.

\section{Corydalis? - (leg).}

Locality : Rott, Rhenish Prussia. Horizon: Aquitanian.

1862. Heyden.

1863. Hagen.
Palaeontogr., x: 77. Pl. 10, fig. 26.

Palaeontogr, $x$ : 250 (not Corydalis, perhaps Termes).

2579. Cronicus anomaluis. (Ephemeridæ.)

Locality : Prussian amber. Horizon: Ligurian.

1871. Eaton. Trans. ent. soc. Lond., 1871: 133-134. Pl. 6, fig. 8.

1885. Scudder. Zittel, Handb. palaeont, i, th. ii: 774. Fig. 976.

1886. " " Zittel-Barrois, Traité de paléont., ii : 774. Fig. 993.

See also Brtis anomala.

2580. Cyrnus subatomarius. (Trichoptera.)

Locality: Prussian amber. Horizon: Ligurian.

1851. Kolenati. Abhandl. böhm. gesellsch. wiss., (5), vi : 15 (undescr.).

2581. Derobrochus abstractus. (Hydropsychidæ.)

Locality : Florissant, Colo. Horizon: Oligocene.

1890. Scudder. Tert. ins. N. A., 183.

2582. Derobrochus æternus. (Hydropsychidæ.)

Locality: Florissant, Colo. Horizon: Oligocene.

1890. Sendder. Tert. ins. N. A., 184.

2583. Derobrochus cænulentus. (Hydropsychidæ.)

Locality : Florissant, Colo. Horizon: Oligocene.

1890. Seudder. Tert. ins. N. A., 183.

2584. Derobrochus commoratus. (Hydropsychidæ.)

Locality: Florissant, Colo. Horizon : Oligocene.

1890. Scudder. Tert. ins. N. A., 184.

2585. Derobrochus crateræ. (Hydropsychidæ.)

Locality : Florissant, Colo. Horizon : Oligocene.

1890. Scudder. Tert. ins. N. A., 186. Pl. 13, fig. $13 ;$ Pl. 15, fig. 4. 
2586. Derobrochus frigescens. (Hydropsychidæ̇.)

Locality: Florissant, Colo. Horizon: Oligocene.

1885. Scudder. 1886. " " 1890. " "
Zittel, Handb. palaeont., i, th. ii : 779. Fig. 986.

Zittel-Barrois, Traité de paléout., ii: 779. Fig. 1003.

Tert. ins. N. A., 185-186. Pl. 15, figs. 6, 16.

\section{Derobrochus marcidus. (Hydropsychidæ.)}

Locality: Florissant, Colo. Horizon: Oligocene.

1890. Scudder.

Tert. ins. N. A., $185 . \quad P l .15, f i g .2$.

\section{Diplax? — (Odonata.)}

Compared with D. minuscula Ramb.

Locality: Quesnel, British Columbia. Horizon:- -

1877. Scudder. Rep. progr. geol. surv. Can., 1875-'76: 280.

1877. " Ins. tert. Quesn., 15.

1877. " Rapp. opér. comm. géol, Can., 1875-'76: 310.

2589. Dysagrion - $(3 \mathrm{sp}$.$) . (Odonata.)$

Locality: Green River, Wyo. Horizon: Oligocene.

1882. Scudder. Proc. Bost. soc. nat. hist., xxi : 409.

1883. " Ann. rep. U. S. geol. surv. terr., xii : 284.

See also the following species.

2590. Dysagrion fredericii. (Odonata.)

Locality: Green River, Wyo. Horizon: Oligocene.

1878. Scudder. Bull. U. S. geol. surv. terr., iv : 536-537, 775.

1890. " Tert. ins. N. A., 130-131. Pl. 6, figs. 2,5, 6, 9, 10, 14, 17.

See also Dysagrion

2591. Dysagrion lakesii. (Odonata.)

Locality: Green River, Wyo. Horizon: Oligocene.

1890. Scudder. Tert. ins. N. A., 132.

See also Dysagrion ——.

2592. Dysagrion packardii. (Odonata.)

Locality: Green River, Wyo. Horizon: Oligocene.

1885. Scndder. Zittel, Handb. palaeont, i, th. ii: 776. Fig. 979.

1886. " " Zittel-Barrois, Traité de paléont., ii: 7\%6. Fig. 996.

1890. " Tert. ins. N. A., 132-133. Pl. 6, figs. 1, 3, 11.

See also Dysagrion ——.

\section{Elipsocus abnormis. (Psocina.)}

Compared with E. hyalinus.

Locality : Prussian amber. Horizon: Ligurian.

1882. Hagen. Stett. ent. zeit., xliii : 287-289. Pl.1, fig.7.

See also Psocus abnormis, Cæeilius abnormis, Philotarsns abnormis.

2594. Elipsocus kïhli. (Psocina.)

Locality : Prussian amber. Horizon: Ligurian.

1883, Kolbe, Stett, ent. zeit, xliv : 188-190. 
2595. Embia -. (Embidina.)

Loeality: Prussian amber. Horizon: Ligurian.

1845. Berendt. Bernst. befindl, organ. reste vorw., i: 57.

2596. Embia antiqua. (Embidina.)

Locality: Prussian amber: Horizon: Ligurian.

1854. Pictet. Traité de paléont., $2^{\text {e }}$ éd, ii : 370. Pl. 40, fig. 28.

1854. Hagen. Verhandl. zool.-bot. ver. Wien, iv : 225.

1856. Pictet.

Berendt, Bernst. befindl. organ. reste vorw., ii, th. i : 56-57. Pl. 5, figs. $7 b-f$.

1866. Hagen. Psoc. Emb. synopsis, 21.

1866. " Verhandl. zool.-bot. gesellseh. Wien, xvi : 221.

1885. Scudder. Zittel, Handb. palaeont., i, th. ii : 773.

See also Oligotoma antiqua.

2597. Empheria reticulata. (Psocina.)

Locality : Prussian amber. Horizon : Lignrian.

1854. Hagen. Verhandl. zool.-bot. ver. Wien, iv : 225 (undescr.).

1856. " Berendt, Bernst. befiudl, organ, reste vorw, ii, th, i: 64. Pl. 8, fig. 6.

1865. “ Ent. monthl. mag., ii : 171.

1866. " Psoc. Emb. synopsis, 7.

1866. " " Verhandl. zool.-bot. gesellsch. Wien, xvi : 207.

1882. “ Stett. ent. zeit., xliii: 217-221. Pl. 1, fig. 8 (12 figs.).

2598. Empheria villosa. (Psocina.)

Locality : Prussian amber. Horizon: Ligurian.

1882. Hagen. Stett. ent. zeit., xliii : 221-222. Pl. 1, figs. $9^{1-6}$.

Entermes —. See Eutermes -

2599. Ephemera - - (Ephemeridæ.)

Locality: Prussian amber. Horizon: Ligurian.

1832. Burmeister. Handb. ent., i: $63 \%$.

1836. “ Man, ent, 578.

2600. Ephemera (or Phryganea) -

Locality : Oeningen, Baden. Horizon: Tortonian.

1820. Schlotheim. Petrefactenk., 43.

2601. Ephemera —. (Ephemeridæ.)

Locality : Prussian amber. Horizon : Ligurian.

1742. Sendel. Hist. succ., 60-61 (inter ephemeras minores). $P l .1$, fig. 33.

1825. Guérin. Dict. class. d'hist. nat., viii : 580 (ephemère).

1829. Serres. G6ogu. terr. tert., 241.

1830. Berendt. Insekten im bernstein, 35 .

2602. Ephemera —. (Ephemeridæ.)

Locality: Vegetable Creek, Australia. Horizon :

1883. Wilkinson. Abstr. proc. Linn. soc. N. S. Wales, Ang. 29, 1883: 4. 
2603. Ephemera exsucca. (Ephemeridæ.)

Locality : Florissant, Colo. Horizon: Oligocene.

1890. Scudder. Tert. ins. N. A., 124. Pl. 12, fig. 9.

2604. Ephemera immobilis (larva). (Ephemeridæ.)

Locality : Florissant, Colo. Horizon : Oligocene.

1890. Scudder. Tert. ins. N. A., 121. Pl. 12, fig. 5.

2605. Ephemera interempta (nymph). (Ephemeridæ.)

Locality: Florissant, Colo. Horizon: Oligocene.

1890. Scudder. Tert. ins. N. A., 123-124.

2606. Ephemera macilenta (larva). (Ephemeridæ.)

Locality : Florissant, Colo. Horizon: Oligocene.

1890. Scudder. Tert. ins. N. A., 122. Pl. 12 , figs. 4,10 .

2607. Ephemera œningensis. (Ephemeridæ.)

Locality : Oeningen, Baden. Horizon : Tortonian.

1865. Heer.

1872. " "

Urwelt der Schweiz, 370 .

Monde prim. Snisse, 453.

1876. " " Prim. world Switz, ii: 25.

1879. “ Urwelt der Schweiz, $2^{\mathrm{e}}$ aufl., 395.

2608. Ephemera pumicosa (larva, nymph). (Ephemeridæ.)

Locality: Florissant, Colo. Horizon : Oligocene.

1890. Scudder. Tert. ins. N. A., 122-123. Pl. 12, figs. 7, 15, 16.

2609. Ephemera tabifica (nymph). (Ephemeridx.)

Locality: Florissant, Colo. Horizon: Oligocene.

1890. Scudder. Tert. ins. N. A., 120.

2610. Epipsocus ciliatus. (Psocina.)

Compared with E. delicatus, E. pictus.

Locality : Prussian amber. Horizon: Ligurian.

1866. Hagen.

Psoc. Emb. synopsis, 7.

1866. " "

Verhandl. zool.-bot. gesellsch. Wien, xvi : 207.

18:2. " Stett. ent. zeit., xliii: 276-280. Pl. 1, figs. $5^{1-11}$.

See also Psocus ciliatus.

2611. Epipsocus debilis. (Psocina.)

Locality : Prussian amber. Horizon: Ligurian.

1866. Hageu. Psoc. Emb. synopsis, 7.

1866. " Verhandl. zool.-bot. gesellsch. Wien, xvi ; 207.

See also Psocus debilis, Cæcilius debilis.

2612. Epipsocus tener. (Psocina.)

Locality: Prussian amber. Horizon: Lignrian.

1866. Hagen. Psoc. Emb. synopsis, 7.

1866. " Verhandl. zool.-bot. gesellsch. Wien, xvi : 207,

See also Psocus tener, etc. 


\section{Eutermes - $(2 \mathrm{sp}$.$) . (Termitina.)$}

Locality : Florissant, Colo. Horizon: Oligocene.

1881. Scudder. Harv, univ, bull., ii : 219.

1881. “ Bull. U. S. geol. surv. terr., vi: 294.

1883. " Ann. rep. U. S. geol. surv. terr., xii : 285.

2614. Eutermes - (worker). (Termitina.)

Locality : Florissant, Colo. Horizon : Oligocene.

1890. Scudder. Tert. ins. N. A., 116.

2615. Eutermes antiquus. (Termitina.)

Locality : Prussian amber. Horizon: Ligurian.

1854. Hagen. Verhandl. zool.-bot. ver. Wien, iv: 222.

See also Termes (Eutermes) antiquas.

Eutermes croaticus. See Termes croaticus.

Eutermes debilis. See Termes debilis.

Eutermes diaphanus. See Termes (Eutermes) diaphanus.

2616. Eutermes fossarum. (Termitina.)

Locality: Florissant, Colo. Horizon: Oligocene.

1883. Scudder. Proc. Amer. acad. arts sc., xix : 143-144.

1890. " Tert. ins. N. A., 115. Pl. 12, fig. 20.

2617. Eutermes meadii. (Termitina.)

Locality: Florissant, Colo. Horizon: Oligocene.

1883. Scudder. Proc. Amer. acad. arts se, xix : 144-145.

18.30. " Tert. ins. N. A., 115-116. Pl. 12, figs. 12,17.

Eutermes mœstus. See Termes (Eutermes) mœestus.

Eutermes obscurus. See Termes (Eutermes) obscurus.

Eutermes pristinus. See Termes pristinus.

Eutermes punctatus. See Termes (Eutermes) punctatus.

Ex termes pusillus. See Termes pusillus.

2618. Forbicina - (Thysanura.)

Locality : Prussian amber. Horizon : Ligurian.

1845. Berendt. Bernst. befindl. organ. reste yorw., i: 56.

2619. Forbicina -. (Thysanura.)

Locality : Prussian amber. Horizon : Ligurian.

1856. Menge. Progr. petrischule Danzig, 1856: 11.

2620. Forbicina acuminata. (Thysanura.)

Locality : Prussian amber. Horizon: Ligurian.

1845. Koch-Berendt. Jahrb, mineral, 1845: 872.

1854. " Berendt, Bernst. befindl, organ. reste vorw., i, th. ii : 115-116. Pl. 14, fig. 121.

See also Machilis acuminatus.

Friganea major. See Phryganea major. 


\section{Glessaria -_. (Thysanura.)}

Loeality: Prussian amber. Horizon: Ligurian.

1845. Berendt. Bernst. befindl. organ. reste vorw, i : 56.

2622. Glessaria rostrata. (Thysanura.)

Locality : Prussian amber. Horizon: Ligurian.

1845. Koch-Berendt. Jahrb. mineral., 1845: 87:.

1854. “

Berendt, Bernst. befindl. organ. reste vorw., i, th. ii : 117-118. Pl. 17, figs. $154 A-F$.

1853-56. Bronn. Lethæa geogn. $3^{\mathrm{e}}$ auf., iii : 649-650. Pl. $42^{1 a}$, fig. 15.

1856. Giebel.

Insect. d. vorwelt, 328 .

2623. Glossosoma ——. (Rhyacophilidæ.)

Loeality: Prussian amber. Horizon : Ligurian.

1864. Hagen. Verbandl, zool.-bot. ver. Wien, iv : 230.

2624. Gœra proava. (Sericostomidæ.)

Locality : Prussian amber. Horizon : Ligurian.

1864. Hagen. Phryg. synopsis synom, 16.

1864. " Verhandl. zool.-bot. gesellsch. Wien, xix : 814.

See also Trichostomum proavum.

2625. Gomphoides occulta. (Odonata.)

Locality : Prussian amber. Horizon : Ligurian.

1856. Hagen. Berendt, Bernst. befindl, organ, reste vorw., ii, th. i: 81 .

See also Aschna — Hagen.

\section{Gomphus (Odonata.)}

Locality : Prussian amber. Horizon: Ligurian.

1845. Berendt. Bernst. befindl, organ. reste vorw., i : 57 .

2627. Gomphus - (larva). (Odonata.)

Locality : Prussian amber. Horizon: Ligurian.

1856. Hagen. Berendt, Bernst, befindl. organ. reste vorw., ii, th. i: $80 . \quad P l .8$, fig. 12.

2628. Gomphus resinatus. (Odonata.)

Locality : Prussian amber. Horizon : Ligurian.

1848. Hagen. Stett. ent. zeit., ix : 8.

1850. " Rer. Odon. (Mém. soc. rog. sc. Liége, vi) : 358.

1852. Giebel. Deutschl. petref., 639.

See also Libellula resinata.

2629. Halesus retusus. (Limnophilidæ.)

Compared with II. nigricornis.

Locality: Prussian amber. Horizon : Ligurian.

1854. Hagen. Verhandl. zool.-bot. ver. Wien, iv; 229 (undeser.) (Hallesus).

1856. "

Berendt, Bernst. befindl. organ. reste vorw., ii, th. i : 102 (Hallesus).

1864. "6. Phryg. synopsis synom., 20,

1864. " Verhandl. zool.-bot. gesellsch. Wien, xiv : 818.

Hallesus retusus. See Halesus retusus. 
2630. Hemerobites antiquus. (Termitina.)

Locality: Prussian amber. Horizon: Ligurian.

1813. Germar.

Germ., Mag. entom., i : 16-17.

1×32. Burmeister. Handb. ent., i : 637 (Hemerobius) (is a Termes).

1836. " Man. ent., 578-579 (is a Termes).

1866. Hagen. Stett. ent. zeit., xxvii : 407.

1888. Schlechtend. Zeitschr. gesellsch. naturw., lxi : 489-490.

See also Termes antiquus, etc.

\section{Hemerobius -. (Hemerobina.)}

Locality : Prussian amber. Horizon: Ligurian.

1830. Berendt. Insekten im bernstein, $30,35,37$.

2632. Hemerobius —. (Hemerobina.)

Locality : Copal. Horizon : Recent.

1776. Bloch. Beschäft. berl. gesellsch. naturf. fr., ii: 173-175. Pl. 4, fig. 13.

2633. Hemerobius —-. (Hemerobina.)

Locality: Copal. Horizon : Recent.

1776. Bloch. Beschäft. berl. gesellsch. naturf, fr., ii : 186.

\section{Hemerobius - (Hemerobina.)}

Compared with $\mathrm{H}$. hirtus or $\dot{H}$. pulchellus.

Locality : Prussian amber. Horizon: Ligurian.

1832. Burmeister. Handb. ent., i : 637.

1836. “ Man. ent., 578.

2635. Hemerobius —. (Hemerobina.)

Locality : Prussian amber. Horizon: Ligurian.

1835. Gravenhorst. Uebers. schles. gesellsch. vaterl. cult., 1834: 92.

2636. Hemerobius (or Chrysopa) _- (larva). (Hemerobina.)

Locality : Prussian amber. Horizon : Ligurian.

1856. Hagen. Berendt, Bernst. befindl. organ. reste vorw., ii, th. i: 90.

2637. Hemerobius - (Hemerobina.)

Locality : Gurnet Bay, Isle of Wight. Horizon: Bembridge Limestone. 1879. Woodward. Quart. journ. geol. soc. Lond., xxxv : 344.

1879. " Geol. mag. (n. s.), v: 89.

Hemerobius antiquus. See Hemerobites antiquus.

2638. Hemerobius mœstus. (Hemerobina.)

Compared with H. pygmæus.

Locality : Prussian amber. Horizon : Ligurian.

1854. Hagen. Verhandl. zool.-bot. ver. Wien, iv : 228 (undeser.).

1856. " Berendt, Bernst. befindl, organ, reste vorw., ii, th, i: 88-89, Pl. 8, fig. 18.

1866. " $\quad$ Stett. ent. zeit., xxvii : 415.

Bull, $71-22$ 
2639. Hemerobius resinatus. (Hemerobina.)
1854. Hagen.
1856. " Berendt, Bernst. befindl. organ. reste vorw., ij, th. i: $88 . \quad P l .7$, figs. $24 b-d ; p l .8$, fig. 17.
1866. " Stett. ent. zeit., xxvii: 419.
See also Mucropalpus elegans.

Loeality : Prussian amber. Horizon: Ligurian.

2640. Heterophlebia jucunda. (Odonata.)

Locality: Sieblos, Rhenish Prussia. Horizon: Aquitanian.

1858. Hagen. Palaeontogr., v: 121-123. Pl. 24, figs. 1, 2.

2641. Hodotermes —. (Termitina.)

Locality: Florissant, Colo. Horizon: Oligocene.

1881. Scudder. Harv, univ. bull., ii : 291.

1881. " " Bull. U. S. geol. surv. terr., vi: 294.

1883. “ Ann. rep. U. S. geol. surv, terr., xii : 285.

See also Hodotermes coloradensis.

2642. Hodotermes coloradensis. (Termitiva.)

Locality: Florissant, Colo. Horizon: Oligocene.

1883. Scudder. Proc. Amer. acad. arts sc., xix : 142-143.

1890. “ Tert. ins. N. A., 113-114. Pl. 12, fig. 6.

See also Hodotermes

2643. Hodotermes haidingeri. (Termitina.)

Locality: Radoboj, Croatia. Horizon: Mayencian.

1858. Hagen. Linn. entom., xii : 98-99.

1858. " Catal. Neuropt. Brit. mus., 15.

See also Termes (Termopsis) haidingeri, etc.

2644. Hodotermes heerianus. (Termitina.)

Locality: Schossnitz, Silesia. Horizon: Miocene.

1869. Assmann. Beitr. ins. vorwelt, 45-47. Pl. 1, figs. 7, 7 a.

1869. " Schles. zeitschr. f. ent., (2), i: 45-47. Pl. 1, figs. 7, 7a.

See also Termopsis heeriana, T. heerii.

2645. Hodotermes insignis. (Termitina.)

Locality: Oeningen, Baden. Horizon: Tortonian.

1858. Hagen. Linn, entom., xii: 100-101.

1858. " Catal. Neuropt. Brit. mus., 15.

See also Termes ('Termopsis) insignis, etc.

2646. Hodotermes procerus. (Termitina.)

Locality: Radoboj, Croatia. Horizon: Mayencian.

1858. Hagen. Linn. entom., xii : 97-98.

1858. " Catal. Neuropt. Brit. mus., 15.

See elso Termes pristinus, etc.

2647. Hodotermes spectabilis. (Termitina.)

Locality: Oeningen, Baden. Horizon: Tortonian.

1858. Hagen. Linn. entom., xii: 99-100.

1858. " Catal. Neuropt. Brit. mus., 15.

See also Termes (Termopsis) spectạbilis, etc. 
2648. Holcorpa maculosa. (Panorpidæ.)

1878. Scudder.

1883.

1885. “

1886. “

1890.

1854. Hagen.

1856. “6

1854. Hagen.

1856. "6

Locality : Florissant, Colo. Horizon: Oligocene.

Bull. U. S. geol. surv, terr., iv : 542.

Ann. rep. U. S. geol, surv. terr, xii : 283,

Zittel, Handb. palaeont., i, th. ii, 778. Fig. 984.

Zittel-Barrois, Traité de paléont., ii : 778. Fig. 1001 ,

Tert. ins. N. A., 174-175. Pl. 14, figs. 4, 5.

\section{Hydronautia - - (Sericostomidæ.)}

Locality: Prussian amber. Horizon: Ligurian.

Verhandl. zool.-bot. ver. Wien, iv : 229.

Berendt, Bernst. befindl, organ. reste vorw., ii, th. i: 106.

2650. Hydronautia labialis. (Sericostomidæ.)

Locality: Prussian amber. Horizon : Ligurian.

Verhandl, zool.-bot. ver. Wein, iv : 229 (undeser.).

Berendt, Bernst. befindl. organ, reste vorw., ii, th. i: $106 . \quad P l .8$, fig. 26.

See also Brachycentrus labialis.

\section{Hydropsyche -. (Hydropsychidæ.)}

Locality: Prussian amber. Horizon: Ligurian.

1845. Berendt. Bernst. befindl. organ. reste vorw., i : 57.

2652. Hydropsyche —. (Hydropsychidæ.)

Compared with H. atomaria.

Locality: Prussian amber. Horizon: Ligurian.

1856. Hagen. Berendt, Bernst. befindl. organ. reste vorw., ii, th. i: 119.

2653. Hydropsyche barbata. (Hydropsychidæ.)

Locality : Prussian amber. Horizon: Ligurian.

1854. Pictet. Traité de paléont., 2e éd., ii : 376 (undescr.).

1856. " Berendt, Bernst. befindl. organ. reste vorw., ii, th. i: 112, 119.

See also Polycentropus barbatus.

2654. Hydropsyche marcens. (Hydropsychidæ.)

Locality : Florissant, Colo. Horizon: Oligocene.

1890. Scudder. Tert. ins. N. A., 180. Pl. 15, fig. 7.

2655. Hydropsyche operta. (Hydropsychidæ.)

Locality: White River, Colo. Horizon: Oligocene.

1890. Scudder. Tert. ins. N. A., 180. Pl. 5, figs. 52, 53.

See also Phryganea operta.

2656. Hydropsyche prisca. (Hydropsychidæ.)

Locality : Prussian amber. Horizon: Ligurian.

1854. Pictet. Traité de paléont, '2e éd, ii : 376 (undescr.).

1856. " Berendt, Bernst. befindl, organ. reste vorw., ii, th. i; 116,119.

See also Polycentropus priscus. 
2657. Hydropsyche submaculata. (Hydropsychidæ.)

Locality : Prussian amber. Horizon: Ligurian.

1851. Kolenati. Abhandl. böhm. gesellsch. wiss., (5), vi : 15 (undescr.).

2658. Hydropsyche subvariabilis. (Hydropsychidæ.)

Locality: Prussian amber. Horizon: Ligurian.

1851. Kolenati. Abhandl. böhm. gesellsch. wiss., (5), vi : 15 (undescr.),

2659. Hydropsyche xanthocoma. (Hydropsychidæ.)

Locality : Prussian amber. Horizon: Ligurian.

1864. Kollar. Hagen, Phryg. synopsis synom., 26.

1864. " Hagen, Verhandl, zool.-bot. gesellsch. Wien, xiv: 824.

See also Phryganeolitha vetusta, etc.

2660. Hydroptila - (Hydroptilidæ.)

Locality : Prussian amber. Horizon: Ligurian.

1854. Hagen. Verhandl. zool.-bot. ver. Wien, iv : 229.

1856. "Berendt, Bernst. befindl. organ. reste vorw., ii, th. i: 108 (undescr.).

\section{Hydrorchestria succinica. (Hydroptilidæ,}

Locality: Prussian amber. Horizon: Ligurian.

1854. Hagen. Verhandl. zool.-bot. ver. Wien, iv : 229 (undescr.).

1856. " Berendt, Bernst, befindl organ, reste rorw., ii, th. i: 107-108.

See also Agraylea succinica.

2662. Ictinus fur. (Odonata.)

Locality: Rott, Rhenish Prussia. Horizon: Aquitanian.

1863. Hagen. Palaeontogr., $\mathrm{x}:$ 258-260. Pl. 43, fig. 9.

2663. Indusia calculosa. (Limnophilidæ.)

Locality: Horse Creek, Wyo. Horizon: Upper Green River Group.

1878. Scudder. Bull. U. S. geol. surv. terr., iv : 542-54.3.

1879. " $\quad$ Ann. rep. U. S. geol. surv. terr., xi : $528,633-639$.

1885. " Zittel, Handb. palaeont, , i, th. ii : 778. Fig. 985.

1886. " "Zittel-Barrois, Traité de paléont., ii : 778. Fig. 1002.

1890. " Tert. ins. N. A., 194-195. Pl. 4, fig. 4.

2664. Indusia tubulata. (Limnophilidæ?.)

Locality: Auvergne, France. Horizon: Aquitanian.

1810. Brongniart. Ann. 1nus. hist. nat., xv : 392-39.3.

1822. Defrance. Dict. sc. nat, xxiii : 411-412.

1833. Croizet. Bull. soc. géol. Fr., iv: 25.

1833. St. Hilaire. Rev. encycl., lix : 77-78.

1838. Bronn. Lethrea geogn., ii : 1160-1161. Pl. 36, fig. 15.

1842. Viquesnel. Bull. soc. géol. Fr., xiv: 149.

1843. Burmeister. Geschichte d. schöpfung, 446.

1843. Bronn. Index palaeont., nomencl, 609.

1849. " " Index palaeont, enumerat., 612 .

1853-'56. Bronn. Lethæa geogn., $3^{\text {e }}$ aufl., iii : 615. Pl. 36, fig. 15.

1856. Giebel. Insect. d. vorwelt, 269.

See also Phryganẹ corentiana, P. gerandiana, P. gigantea, Ind, tụbulosạ, 
2665. Indusia tubulosa. (Limnophilidæ?.)

Locality : Auvergne, France. Horizon: Aquitanian.

An XIII, Bose. Journ. d. mines, xvii : 397-400. Pl. 7, figs. C-E. 18:25. Guérin. Dict. class. d'hist. nat., viii : 581 . 18:7. Scrope. Geol. centr. France (without name). 1829. Serres. Géogn. terr. tert., 208.

1840. Meyer.

Ersch u. Gruber, Eneycl. wiss., sect. 2, th. xviii : 136 (no name). 1846. Pictet. Traité élém. paléont., iv: 108 (ńo name).

1854. “" 18j8. Serope. 1864. Hagen. Traité de paléont., $2^{\text {e }}$ éd., ii : 375-376 (no name). Geol. centr. France, $2^{\mathrm{d}}$ ed., 10-13 (without name). Phryg. synopsis synom., 28.

1864. "6 Verhandl. zool.-bot. gesellsch. Wien, xiv : 826. 1865. Lyell. Elem. geol., 6th ed., 223-225 (no name). Fig. 168. 1885. Scudder. 1886. " "Zittel-Barrois, Traité de paléont., ii : 778.

See also Indusia tubulata, etc.

\section{Inocellia - (4 sp.). (Sialina.)}

Locality : Florissant, Colo. Horizon : Oligocene.

1882. Scudder. Proc. Bost. soc. nat. hist., xxi : 407.

1883. " Ann. rep. U. S. geol. surv. terr., xii : 283.

See also the following species.

Inocellia erigena. See Raphidia (Inocellia) erigena.

2667. Inocellia eventa. (Sialina.)

Locality: Florissant, Colo. Horizon: Oligocene. 1890. Scudder. Tert. ins. N. A., 160-161.

See also Inocellia _-

2668. Inocellia somnolenta. (Sialina.)

Locality: Florissant, Colo. Horizon: Oligocene.

1890. Scudder. Tert. ins. N. A., 157-158. Pl. 14, fig. 12.

See also Inocellia -

2669. Inocellia tumulata. (Sialina.)

Locality : Florissant, Colo. Horizon: Oligocene.

1890. Scudder. Tert. ins. N. A., 158-159. Pl. 14, fig. 15.

See also Inocellia $\longrightarrow$.

\section{Inocellia veterana. (Sialina.)}

Locality: Florissant, Colo. Horizon: Oligocene. 1890. Scudder. Tert. ins. N. A., 156-157. Pl. 14, fig. 1.

See also Inocellia

Kalotermes See Calotermes

2671. Lampropholis (2 sp.). (Thysanura.)

Locality: Prussian amber. Horizon: Ligurian. 
2672. Lampropholis argentata. (Thysanura.)

Locality: Pruss an amber. Horizon: Ligurian.

1854. Menge. Koch-Ber., Bernst. befindl. organ. reste vorw., i, th. ii : 117. See also Lepisma mengei.
2673. Lepidion —. (Thysanura.)
Locality : Prussian amber. Horizon : Ligurian.
1856. Menge. Progr, petrischule Danzig, 1856: 11.
2674. Lepidion pisciculus. (Thysanura.)
Locality: Prussian amber. Horizou: Ligurian.
1854. Menge. Koch-Ber., Berust. befindl. organ. reste vorw., i, th. ii : 117. See also Lepisma pisciculus.

\section{Lepidotrix — (2sp.). (Thysanura.)}

1856. Menge. Progr. petrischule Danzig, 11.

Locality: Prussian amber. Horizon: Ligurian.

2676. Lepidotrix piliferum. (Thysanura.)

Locality : Prussian amber. Horizon : Ligurian.

1854. Menge. Koch-Ber, Bernst. befindl, organ. reste vorw, i, th. ii : 117. See also Lepisma pilifera.

\section{Lepisma - (Thysanura.)}

Locality: Prussian amber. Horizon: Ligurian.

1845. Berendt. Bernst. befindl. organ. reste vorw., i: 56 .

2678. Lepisma - (Thysanura.)

Locality: Prussian amber. Horizon: Ligurian.

1820. Schlotheim. Petrefactenk., 43.

\section{Lepisma - (Thysanura.)}

Locality : Florissant, Colo. Horizon : Oligocene.

1881. Scudder. Bull. U. S. geol, surv, terr., vi : 294.

1883. " Anv. rep. U. S. geol. surv. terr., xii : 284.

See also Lepisma platymera.

2680. Lepisma argentata. (Thysanura.)

Locality: Prussian amber. Horizon: Ligurian.

1845. Koch-Berendt. Jahrb. mineral., 1845: 872.

1854. " Berendt, Bernst. befindl. organ, reste vorw., i, th. ii: 117. Pl. 14, fig. 123.

1856. Giebel. Insect. d. vorwelt, 327 .

2681. Lepisma dubia. (Thysanura.)

Compared with L. saccharina.

Locality : Prussian amber. Horizon: Ligurian.

1845. Koch-Berendt. Jahrb. mineral., 1845 : 872.

1854. " Berendt, Bernst, befindl, organ, reste vorw., i, th. ii: 116. Pl. 14, fig. 122.

1856. Giebel. Insect. d. vorwelt, 327. 
2682. Lepisma mengei. (Thysanura.)

Locality : Prussian amber. Horizon : Ligurian.

1856. Giebel. Insect. d. vorwelt, 327 .

See also Lampropholis argentata.

2683. Lepisma pilifera. (Thysanura.)

Locality: Prussian amber. Horizon: Ligurian.

1856. Giebel. Insect. d. vorwelt, 327-328.

See also Lepidotrix piliferum.

2684. Lepisma pisciculus. (Thysanura.)

1856. Giebel. Insect. d. vorwelt, 327.

See also Lepidion pisciculus.

2685. Lepisma platymera. (Thysanura.)

Locality: Florissant, Colo. Horizon: Oligocene.

1890. Scudder. Tert. ins. N. A., 102-103. Pl. 12, fig. 18.

See also Lepisma —— Scudder.

2686. Leptobrochus luteus. (Hydropsychidæ.)

Locality: Florissant, Colo. Horizon: Oligocene.

1890. Seudder. Tert. ins. N. A., 187-188. Pl. 15, figs. 1, 3.

2687. Leptophlebia prisca. (Ephemeridæ.)

Locality : Prussian amber. Horizon: Ligurian.

1871. Eaton.

Monogr. Ephemeridæ, 87.

See also Potamanthus priscus.

\section{Lestes coloratus. (Odonata.)}

Locality: Radoboj, Croatia. Horizon: Mayencian.

1841. Charpentier. Neues jahrb. mineral., 1841 : 332-337 (no name). Pl. 1.

1848. Hagen. Stett. ent. zeit., ix : 7-8.

1850. " Rev. Odon. (Mém. soc. roy. sc. Liége, vi) : 357.

1852. Giebel. Deutschl. petref., 638.

See also Agrion coloratum.

2689. Lestes iris. (Odonata.)
Locality : Oeningen, Baden. Horizon: Tortonian.
1890. Scudder. Tert. ins. N. A., 127
See also Agrion iris.

2690. Lestes leucosia. (Odonata.)

Locality: Oeningen, Baden. Horizon: Tortonian.

1850. Hagen. Rev. Odon. (Mém, soc. roy. sc. Liége, vi): 357.

1852. Giebel. Deutschl. petref., 638.

1890. Scudder. Tert. ins. N. A., 127.

See also Agrion leucosia, etc. 


\section{Lestes ligea. (Odonata.)}

Locality : Oeningen, Baden. Horizon: Tortonian.

1850. Hagen. Rev. Odon. (Mém. soc. roy. sc. Liége, vi): 357.

1852. Giebel. Deutsch. petref., 638.

1890. Scudder. Tert. ins. N. A., 126-127.

See also Agrion ligea.

2692. Lestes peisinoe. (Odonata.)

Locality: Oeningen, Baden. Horizon: Tortonian.

1850. Hagen. Rev. Odon. (Mém. soc. roy. sc. Liége, vi) : 357.

1852. Giebel. Deutschl. petref., 638.

1890. Scudder. Tert. ins. N. A., 127.

See also Agrion peisinoe.

2693. Lestes vicina. (Odonata.)

Locality: Sieblos, Rhenish Prussia. Horizon: Aquitanian.

1858. Hagen. Palaeontogr., v: 123-124. Pl. 24, figs. 3, 4.

\section{Leuctra -. (Perlina.)}

Locality: Prussian amber. Horizon : Liguriau.

1845. Berendt. Bernst. befindl. organ. reste vorw., i: 57.

2695. Leuctra antiqua. (Perlina.)

Compared with L. cylindrica.

Locality: Rott, Rhenish Prussia. Horizon: Aquitanian.

1863. Hagen. Palaeontogr., $\mathrm{x}:$ 251-253. Pl. 44, figs. 3, 4.

2696. Leuctra fusca. (Perlina.)

Locality: Prussian amber. Horizon: Ligurian.

1854. Pictet. Hagen, Verhandl. zool.-bot. ver. Wien, iv : 226 (undescr.).

1856. " Berendt, Bernst. befindl. organ. reste vorw., ii, th. i: 70. Pl. 6 , figs. $10 b-d$.

See also Nemura (Leuctra) fusca.

2697. Leuctra gracilis. (Perlina.)

Compared with L. cylindrica, etc.

Locality: Prussian amber. Horizon : Ligurian.

1854. Pictet. Hagen, Verhandl, zool.-bot. ver. Wien, iv: 226 (undeser.).

1856. " Berendt, Bernst. befindl. organ. reste vorw., ii, th. $i: 69$. Pl. 6, figs. $9 b-f ; p l .8$, fig. 2.

See also Nemura (Leuctra) gracilis.

2698. Leuctra linearis. (Perlina.)

Locality: Prussian amber. Horizon: Ligurian.

1854. Hagen. Verhandl. zool.-bot. ver. Wien, iv : 226 (undescr.).

1856. " Berendt, Bernst. befindl, organ, reste vorw., ii, th. i: 69-70. Pl. 8, fig. 3.

2699. Leuctra minuscula. (Perlina.)

Locality : Prussian amber. Horizon: Ligurian.

1854. Hagen. Verhandl. zool.-bot. ver. Wien, iv : 226 (undescr.).

1856. " Berendt, Bernst, befindl, organ, reste vorw, ii, th, i: 71.

Libella —. See Libellula — Scheuchzer. 
2700. Libellula —. (Odonata.)

Locality : Prussian amber. Horizon: Ligurian.

1830. Berendt. Insekten im bernstein, 35.

2701. Libellula - (larva). (Odonata.)

Localities: Porcarecca, Pane e vino, Limone, etc., Italy. Horizon: Tortonian. 1875. Capellini. Rend. sess. acead. se. ist. Bologna, 1874-'75; 24, 133.

1880. " Atti accad. line., (3), mem. sc. fis., v : 379, 383-385.

\section{Libellula —. (Ordonata.)}

Locality : Fabbro, Italy. Horizon: Tripoli.

1878. Capellini. Atti accad. Iinc., (3), mem. se. fis., ii : 285.

2703. Libellula _ (larva). (Odonata.)

Locality : Oeningen, Baden. Horizon: Tortonian.

1723. Scheuchzer. Herb. diluv., ed. nov., 21 (Libella). Pl. 5, fig. 1.

See also Libellula eurynome, —_ ourynome (Odonata-Libillulina).

2704. Libellula -. (Odonata.)

Locality : Monte Bolea, Italy. - Horizon : Ligurian.

1723. Scheuchzer. Herb. diluv., ed. nov., 21 (Libella). Pl. 5, fig. 2.

\section{Libellula —. (Odoata.)}

Locality: Green River, Wyo. Horizon: Oligocene.

1890. Scudder. Tert, ins. N. A., 146. Pl. 6, figs. 4, 16.

See also —— (Odonata-Libellulina).

\section{Iibellula — (Udonata.)}

Compared with Aschua grandis.

Locality : Aix, France. Horizon: Ligurian.

1829. Serres. Géogn. terr. tert, 228.

2707. Libellula (larva), (Odonata.)

Compared with L. eurynome, Oeningen.

Locality: Montescano presso Stradella, Italy. Horizon: Marne gessifere.

1882. Sordelli. Alc. ins. foss. Lomb., 3-4.

1882. " Bull. soc. ent, ital., xiv : 226-227.

\section{8, Libellula - (larva). (Odonata.)}

Compared with L. doris, Oeningen.

Locality: Montescavo presso Stradella, Italy. Horizon: Marne gessifere. 1882. Sordelli. Alc. ine. foss. Lomb., 4.

1882. "6 Bull. soc. ent. ital., xiv: $22 \%$.

\section{Libellula —, (Odonata.)}

Locality: Gurnet Bay, Isle of Wight. Horizon: Bembridge Limestone. 1879. Woodward. Quart. journ. geol. soc. Lond., xxxv : 344 .

1879. G Geol. mag. (n. s,), v: 89. 
2710. Libellula aglaia. (Odonata.)

1861. Heer. Saporta, Heer, Rech. climat pays tert., 153 (undescr.).

Locality: Aix, France. Horizon: Ligurian.

2711. Libellula calypso (pupa). (Odonata.)

1849. Heer. 1850. Hagen. 1852. Giebel. 1854. Pictet. 1856. Giebel. 1865. Heer. 1872 . “ 1876. “ " 1879 . “

Locality: Oeningen, Baden. Horizon: Tortonian.

\section{Libellula cassandra (pupa). (Odonata.)}

Locality: Rott, Rhenish Prussia. Horizon: Aquitanian.

1863. Hagen.

1870. L. Heyden.

1870.

See also

Palaeontogr., x: 264-267. Pl. 45, figs. 1-4.

Foss. Dipt. Rott, 30.

Palaeontogr., xvii : 266. cassandra (Odonata-Libellulina).

\section{Libellula cellulosa. (Odonata.)}

Compared with L. eponina.

Locality: Rott, Rhenish Prussia. Horizon: Aquitanian.

1863. Hagen. Palaeontogr., $\mathrm{x}:$ 253-258. Pl. 43, figs. 1-8.

1870. L. Heyden. Foss. Dipt. Rot', 29.

1870. “ Palaeontogr., \& rii : 265.

See also Celithemis cellulosa, Lib llula ceres, etc.

\section{Iibellula ceres (pupa). (Odonata.)}

Locality: Rott, Rhenish Prussia. Horizon: Aquitanian.

1863. Hagen. Palaeontogr., $\mathrm{x}:$ 260-264. Pl. 44, figs. 9-11; pl. 45, figs. 5-12.

1870. L. Heyden. Foss. Dipt. Rott, 30.

1870.

Palaeontogr., xvii : 266.

See also _ ceres (Odonata-Libellulina), Libellula cellulosa, Celithemis cellulosa.

\section{Libellula dorii. See Libellula doris.}

2715. Libellula doris (larva, pupa). (Odonata.)

Compared with L. depressa L.

Localities: Limone, near Lavorno, Sogliano, Perticara, Monte Donato nel Bolognese, Ancona, Guarène, Sinigallia, Italy; Girgenti, Sicily; Grasseth, Bohemia ; Oeningen, Baden. Horizon: Tortonian, Helvetian.

1849. Heer.

1850. Hagen.

1852. Giebel.

1856. “

1856. Massalongo.

1857. Jokély.
Insektenf. tertiärg. Oeningen, ii: 81-83, Pl.5, fig. $5 ; p l .6, f i g .4$.

Rev. Odos. (Mém. soc. roy. sc. Liége, vi) : 363-364.

Dentschl. petref., 639.

Insect. d. vorwelt, 282-283.

Stud, paleont., 23. Pl. 1, figs. 12, 13.

Jahrb. geol, reichsanst., viii : 502 (dorii). 
1859. Searabelli. Massal.-Scurab., Stud. flora foss. Senig., 25.

1865. Sismonda.

Mem. accad. sc. Torino, (2), xxii : 470. Pl. 17, fig. 6 .

1865. Heer.

Urwelt der Sehweiz, 368. Fig. 231 on $p .366$.

1872. ". Capellini.

Monde prim. Suisse, 452. Fig. 231 on p. 449.

1875. Heyden.

Mem. accad. sc. ist. Bologna, (3), iv : 539, 557.

Stoehr, Bull. com. geol. ital., 1875 : 286.

1876. Heer.

Prim. world Switz., ii : 23. Fig. 231 on p. 21.

1879. " "

1880. Bosniaski.

1882. Sordelli.

1882. " "

Urwelt der Schweiz, 2 aufl., 394. Fig. 272 on $p .392$.

Atti soc. tosc. sc. nat., $18 € 0: 93$.

Alc. ins. foss. Lomb., 5 , note 3.

See also _ doris (Odonata-Libellulina).

2716. Libellula eurynome (larva, pupa). (Odonata.)

Compared with L. depressa L.

Localities: Sinigallia, Italy ; Girgenti, Sicily ; Oeningen, Baden. Horizon: Tortonıan, Helvetian.

1849. Heer.

1850. Hagen.

1852. Giebel.

1856. " "

1856. Massalongo. Stud. paleont., 23. $\mathrm{Pl}$. 1, figs. 8-11.

1859. Scarabelli. Massal.-Scarab., Stud. flora foss. Senig., 25.

1865. Heer.

1872. “

Urwelt der Schweiz, 368 .

Monde prim. Suisse, 452

1875. Heyden.

1876. Heer.

Stoehr, Bull. com. geol. ital., 1875: 286.

1879. “

Prim. world Switz., ii : 23.

See also

Urwelt der Schweiz, $2^{\mathrm{e}}$ aufl., 394.

eurynome (Odonata-Libellulina), Libellula Scheuchzer.

2717. Libellula kieseli. (Odonata.)

Locality: Schossnitz, Silesia. Horizon: Miocene.

1869. Assmann. Beitr. ins. vorwelt, 52-56. Pl. 1, figs. 10, 10a.

1869. " Schles, zeitschr, f, ent., (2), i: 5:-56. Pl. 1, figs. 10, $10 a$.

2718. Libellula melobasis (pupa). (Odonata.)

Locality: Oeningen, Baden. Horizon: Tortonian.

1849. Heer. Insektenf. tertiärg. Oeningen, ii : 86-87. Pl. 5, fig. 8; pl.6, fig. 6.

1850. Hagen. Rev. Odon (Mém. soc. roy. sc. Liége, vi): 364.

1852. Giebel. Deutschl. petref., 639.

1856. " Insect. d. vorwelt, 283.

See also - melobasis (Odonata-Libellulina).

2719. Libellula minuscula. (Odonata.)

Locality: Corent, France. Horizon: Aquitanian.

1870. Oustalet. Ann. sc. geol., ii, art. 3: 88. Pl. 2, fig. 6.

2720. Libellula ceningensis (larva). (Odonata.)

Locality: Oeningen, Baden. Horizon: Tortonian.

1825. König. Icon. foss. sectiles, 2. Pl. 2, fig. 17.

2721. Libellula cningensis. (Odonata.)

Locality: Oeningen, Baden. Horizon: Tortonian.

1852. Quenstedt. Handb. petref., 317, Pl. 24, fig. 8. 
2722. Libellula pannewitziana. (Odonata.)

Locality : Schossnitz, Silesia. Horizon: Miocene.

1855. Göppert.

Tert. flora Sehossnitz, vii. $\mathrm{Pl} .26$, fig. 55 .

1869. Assmann. Beitr. ins. vorwelt (Schles. zeitschr. f. ent., (2), i): 56-57. Pl. 1, fig. 11.

2723. Libellula perse (pupa). (Odonata).

Loealities : Oeningen?, Baden; Aix, France. Horizon: Tortonian, ? Ligurian. 1849. Heer. Insektenf. tertiärg. Oeningen, ii : 80-81. Pl. 5, fig. 4; pl. 6, fig. 3. 1850. Hagen. 1852. Giebel. Rev, Odon. (Mém. soc. roy, sc. Liége, vi): 363. 1856. “ Deutschl. petref., 639.

1856. Heer. Insect. d. vorweli, 282.

See also _ - perse (Odonata-Libellulina).

2724. Libellula platyptera. (Odonata.)

Locality: Radoboj, Croatia. Horizon: Mayencian.

1843. Charpentier. Verhandl. leop.-carol. akad. naturf., xx : 408 (platyptra). Pl. 22, fig. 3.

1848. Hagen. Stett. ent. zeit., ix : 12.

See also Cordulia platyptera.

Libellula platyptra. See Libellula platyptera.

2725. I ibellula resinata. (Odonata.)

Locality: Prussian amber. Horizon: Ligurian.

1856. Giebel. Insect. d. vorwelt, 284 (resinatus).

See also Gomphus resinatus.

2726. Libellula sieboldiana. (Odonata.)

Locality: Schossnitz, Silesia. Horizon: Miocene.

1855. Göppert. Tert. flora Schossnitz, vii, - Pl. 26, fig. 54.

1869. Assmann. Beitr. ins. vorwelt (Schles. zeitschr. f. ent., (2), i): 48-52. Pl. 1, figs. $9,9 a$.

2727. Libellula thetis (pnpa). (Odonata.)

Locality: Oeningen, Baden. Horizon: Tortonian.

1849. Heer. Insektenf. tertiärg. Oeningen, ii : 83-85. Pl. 5, fig. $6 ;$ pl.6, fig. 5.

1850. Hagen. Rev. Odon. (Mém, soc. roy, sc. Liége, vi): 364 .

1852. Giebel. Deutschl. petref., 639.

1856. " Insect. d. vorwelt, 283.

See also — thetis (Odonata-Libellulina).

2728. Iibellula thoe (pupa). (Odonata.)

Locality : Oeningen, Baden. Horizon: Tortonian.

[1755. Knorr. Samml. v. merkwürd., 1 (unnamed). Pl. 33, figs. 3-4.]

1849. Heer. Insektenf. tetiärg. Oeningen, ii : 79-80. Pl. 6, fig. 2.

1850. Hagen. Rev. Odon. (Mém. soc. roy. sc. Liége, vi): 363.

1852. Giebel. Deutschl. petref., 639.

1856. " Insect. d. vorweit, 282.

See also — thoe (Odonata-Libellulina).

Limnephilus dubius. See Limnophilns dubius.

Limnephilus piceus. See Limnophilus piceus. 


\section{Limnophilus ㄴ. (Limuophilidæ.)}

Locality: Prussian amber. Horizon: Ligurian.

1845. Berendt. Bernst. befindl. organ. reste vorw., i : 57.

\section{Limnophilus - (2 sp.). (Limnophilidæ.)}

Locality : Prussian amber. Horizon: Ligurian.

1854. Hagen. Verhandl. zool.-bot. ver. Wien, iv: 229 (undescr.).

1856. “

Berendt, Bernst. befindl. organ. reste vorw., ii, th. i: 101 (undescr.).

See also Agrypnia

2731. Limnophilus dubius. (Limnophilidæ.)

Locality: Prussian amber. Horizon: Ligurian.

1854. Pictet. Traité de paléont., $2^{e}$ éd., ii : 376 (undeser.) (Limnephilus).

1856. " Berendt, Bernst. befindl. organ. reste vorw., ii, th. i : 100-101 (Limnephilus).

See also Phryganea dubia.

2732. Limnophilus piceus. (Limnophilidæ.)

Locality: Prussian amber. Horizon: Ligurian.

1854. Pictet. Traité de paléont., 2̌éd., ii : 376 (undescr.) (Limnephilus).

1856. " Berendt, Bernst. befindl. organ. reste vorw., ii, th. i: 99 (Limnephilus).

See also Phryganea picea, Neuronia piceà.

2733. Limnophilus soporatus. (Limnophilidæ.)

Locality : Florissaut, Colo. Horizon: Oligocene,

1890. Scudder. Tert. ins. N. A., 193. Pl. $15, f g .5$.

2734. Limnopsyche dispersa. (Phryganidæ.)

Locality: Florissant, Colo. Horizon: Oligocene.

1890. Scudder. Tert. ins. N. A., 199-200. Pl. 13, fig. 2.

\section{Lithagrion - - (Odonata.)}

Locality : Florissant, Colo. Horizon: Oligocene.

881. Scudder. Bull. U. S. geol, surv, terr., vi : 293.

1882. " " Proc. Bost. soc. nat. hist., xxi : 409 (2 sp.).

1883. " Ann. rep. U. S. geol. surv. terr., xii : 284.

See also Lithagrion hyalinum, L. umbratum.

2736. Lithagrion hyalinum. (Odonata.)

Locality: Florissant, Colo. Horizon: Oligocene.

1890. Scudder. Tert. ins. N. A., 135-136. Pl. 13, fig. 4 .

See also Lithagrion $\longrightarrow$.

2737. Lithagrion umbratum. (Odonata.)

Locality : Florissant, Colo. Horizon: Oligocene,

1890. Scudder. Tert. ins. N. A., 136-137. Pl. 13, figs. 12, 14,

See also Lithagrion $\longrightarrow$. 
2738. Litobrochus externatus. (Hydropsychidm.)

Locality : Florissant, Colo. Horizon : Oligocene.

1890. Scudder. Tert. ins. N. A., 186-187. Pl. 15, fig. 10.

2739. Machilis ——. (Thysanura.)

Locality : Prussian amber. Horizon: Ligurian.

1835. Gravenhorst. Uebers. schles. gesellsch. vaterl. cult., 1834 : 93.

2740. Machilis (Forbicina) acuminatus. (Thysanura.)

Locality: Prussian amber. Horizon: Ligurian.

1856. Giebel. Insect. $d$. vorwelt, 326 .

See also Forbicina acuminata.

2741. Machilis (Petrobius) albomaculatus. (Thysanura.)

Locality : Prussian amber. Horizon: Ligurian.

1856. Giebel. Insect. d. vorwelt, 326.

See also Petrobius albomaculatus.

2742. Machilis (Petrobius) angueus. (Thysanura.)

Locality: Prussian amber. Horizon: Ligurian.

1856. Giebel. Insect. d. vorwelt, 326.

See also Petrobius angueus.

2743. Machilis (Petrobius) coruscus. (Thysanura.)

Locality : Prussian amber. Horizon: Ligurian.

1856. Giebel. Insect. d. vorwelt, 324-325.

See also Petrobius imbricatus, etc.

2744. Machilis (Petrobius) electus. (Thysanura.)

Locality: Prussian amber. Horizon: Oligocene.

1856. Giebel.

Insect. d. vorwelt, 325.

See also Petrobius electus, etc.

2745. Machilis (Petrobius) imbricatus. (Thysanura.)

Locality : Prussian amber. Horizon: Ligurian.

1856. Giebel. Insect. d. vorwelt, 325.

See also Petrobius imbricatus, etc.

2746. Machilis (Petrobius) macrura. (Thysanura.)

Locality : Prussian amber. Horizon: Ligurian.

1856. Giebel.

Insect. $d$. vorwelt, 326 .

See also Petrobius macrura.

2747. Machilis polypoda. (Thysanura.)

Locality: Prussian amber. Horizon: Ligurian.

1832. Burmeister. Handb. ent., i: 637.

1836. " Man. ent., 578.

2748. Machilis (Petrobius) saliens. (Thysanura.)

Locality: Prussian amber. Horizon : Ligurian.

1856. Giebel. Insect. d, vorwelt, 326 ,

See also Petrrobius saliens. 
2749. Machilis (Petrobius) seticornis. (Thysanura.)

Locality: Prussian amber. Horizon : Ligurian.

1856. Giebel. Insect. d. vorwelt, 325-326.

See also Petrobius seticornis.

Macropalpus elegans. See Mucropalpus elegans.

2750. Maresa plebeja. (Termitina.)

Locality : Prussian amber. Horizon: Ligurian.

1856. Giebel. Insect. d. vorwelt, 298.

See also Termes antiqua, etc.

2751. Megasemum ronzonense. (Odonata.)

Locality: Le Puy, France. Horizon: -

1854. Aymard. Congr. scient. Fr., sess. xxii : 42 (undeser.).

2752. Mesobrochus imbecillus. (Hydropsychidæ.)

Locality: Florissant, Colo. Horizon: Oligocene. 1890. Scudder. Tert. ins. N. A., 189. Pl. 15, fig. 13.

2753. Mesobrochus lethæus. (Hydropsychidæ.)

Locality : Florissant, Colo. Horizon: Oligocene.

1890. Scudder. Tert. ins. N. A., 188. Pl. 15, fig. 11.

2754. Mormonia - -. (Sericostomidæ.)

Locality: Prussian amber. Horizon : Ligurian.

1845. Berendt. Bernst. befindl, organ. reste vorw, i : 57.

2755. Mormonia ——, (Sericostomidæ.)

Locality : Prussian amber. Horizon: Ligurian.

1854. Hagen. Verhandl. zool.-bot. ver. Wien, iv : 229.

2756. Mormonia tæniata. (Sericostomidæ.)

Locality : Prussian amber. Horizon: Ligurian.

1854. Pictet.

1854. “"

1856. “

1864. Hagen.

1864. " "

Hagen, Verhandl, zool.-bot. ver. Wien, iv : 229 (undeser.).

Traité de paléont., $2^{e}$ éd., ii : 376 (undeser.).

Berendt, Bernst. befindl. organ. reste vorw., ii, th. i: 103-105. Pl. 7, figs. 5b-d.

Phryg. synopsis synom., 50.

Verhandl. zool.-bot. gesellsch. Wien, xiv : 848 .

2757. Mucropalpus elegans. (Hemerobina.)

Locality : Prussian amber. Horizon : Ligurian.

1854. Pictet.

Traité de paléont., 'ze éd., ii : 378 (undescr.) (Macropalpus).

1856. Berendt. Bernst. befindl, organ. reste vorw., ii, th. i: 88 .

See also Hemerobits resinatus.

2758. Myrmecoleon —. (Myrmeleontidæ.)

Locality : Prussian amber. Horizon: Ligurian.

1845, Berendt. Bernst, befindl, organ, reste vorw., i ; 57, 


\section{Myrmecoleon}

Loeality : Prussian amber.
- (Myrmeleontidæ.)

Horizon : Ligurian.

1832. Burmeister. Handb. ent., i : 637.

1836. “ Man. ent, 578.

2760. Myrmecoleon reticulatum. (Myrmeleontidæ.)

Locality: Radoboj, Croatia. Horizon: Mayencian.

1856. Giebel. Insect. $d$. vorweit, 256 .

See also Myrmeleon reticulatum.

\section{Myrmeleon - (Myımeleontidæ.)}

Locality: Prussian amber. Horizon: Ligurian.

1830. Berendt. Insekten im bernstein, 35.

2762. Myrmeleon - (larva). (Myrmeleontidæ.)

Locality: Seeberg, near Gotha, Germany. Horizon: Flötzmuschelnkalkstein. 1823. Schlotheim. Nachtr. z. petrefactenk., ii : 60-61, 85. Pl. 22, fig. 10.

Myrmeleon brevipenne. See III, Orthoptera, Idem.

2763. Myrmeleon reticulatum. (Myrmeleontidæ.)

Locality: Radoboj, Croatia. Horizon : Mayencian.

1843. Charpentier. Verhandl. leop.-carol. akad. naturf., $\mathrm{xx}:$ 407-408. Pl. 22, fig. 2.

1849. Heer.

Insektenf. tertiärg. Oeningen, ii : 92.

1852. Giebel.

Deutschl. petref., 640 .

1866. Hagen. Stett. ent. zeit., xxvii : $44 \tau$ (a Myrmeleon?).

See also Myrmecoleon reticulatum.

2764. Mystacides

(Leptoceridæ.)

Locality: Prussian amber. Horizon: Ligurian.

1831. Burmeister. Oken, Isis, 1831: 1100.

2765. Mystacides —. (Leptoceridæ.)

Compared with M. ferruginea.

Locality : Prussian amber. Horizon: Ligurian.

1856. Hagen. Berendt, Bernst. befindl, organ. reste vorw., ii, th. i: 121.

2766. Mystacides —_. (Leptoce ridæ.)

Locality: Prussian amber. Horizon: Ligurian.

1856. Hagen. Berendt, Bernst. befindl organ. reste vorw., ii, th. i: 121.

2767. Necropsocus

Locality : Florissant, Colo. Horizon : Oligocene.

1883. Scudder. Ann. rep. U. S. geol. surv. terr., xii : 284.

See also III, Hemiptera, Necropsylla rigida.

Nemoura (several sp.). See Nemura, the same species.

2768. Nemura -. (Perlina.)

Locality : Prussian amber. Horizon: Ligurian.

1845. Berendt. Bernst, befindl organ. reste vorw., i: 57 (Nemonra), 
2769. Nemura

(Perlina.)

Locality: Prussian amber. Horizon : Ligurian.

1856. Hagen.

Berendt, Bernst. befindl. organ. reste vorw., ii, th. i: 73 (Nemoura).

2770. Nemura affinis. (Perlina.)

Locality: Prussian amber. Horizon : Ligurian.

1854. Berendt.

Hagen, Verhandl. zool.-bot. ver. Wien, iv : 226 (undeser.) (Nemoura).

1856. " Bernst. befindl. organ. reste vorw., ii, th. i: 72. Pl. 6, figs. 12b-d.

2771. Nemura (Tænioptery $\mathbf{x}$ ) ciliata. (Perlina.)

Locality: Prussian amber. Horizon: Ligurian.

1854. Pietet. Traité de pal6ont., $2^{2} 6 \mathrm{~d}$., ii : 375 (undescr.) (Nemoura).

See also Tæniopteryx ciliata.

2772. Nemura (Leuctra) fusca. (Perlina.)

Locality: Prussian amber. Horizon: Ligurian.

1854. Pictet. Traité de paléont., $2^{\mathrm{e}}$ ed., ii : 375 (undescr.) (Nemoura).

See also Leuctra fusca.

2773. Nemura (Ieuctra) gracilis. (Perlina.)

Locality : Prussian amber. Horizon: Ligurian.

1854. Pictet. Traité de paléont., $2^{\mathrm{e}}$ éd., ii : 375 (undescr.) (Nemoura).

See also Lenctra gracilis.

2774. Nemura lata. (Perlina.)

Locality: Prussian amber. Horizon: Ligurian.

1854. Hagen. Verhandl. zool.-bot. ver. Wien, iv : 226 (undeser.) (Nemoura).

1856. "6 Berendt, Bernst. befindl, organ. reste vorw., ii, th. i: 72.

2775. Nemura ocularis. (Perlina.)

Compared with N. variegata, N. meyeri.

Locality: Prussian amber. Horizon: Ligurian.

1854. Pictet. Hagen, Verhandl. zool.-bot. ver. Wein, iv : 226 (undeser.) (Nemoura).

1856. “

Berendt, Bernst. befindl, organ. reste vorw., ii, th, i: 71-72. $P l$. 6, figs. $11 b-f$.

2776. Nemura puncticollis. (Perlina.)

Locality: Prussian amber. Horizon: Ligurian.

1854. Hagen. Verhandl. zool.-bot. ver. Wien, iv: 226 (undeser.) (Nemoura).

1856. " Berendt, Bernst. befindl. organ. reste vorw., ii, th. i: 73.

2777. Neuronia evanescens. (Phryganidæ.)

Locality: Florissant, Colo. Horizon: Oligocene.

1890. Scudder. Tert. ins. N. A., 196-197. Pl. 13, fig. 3.

2778. Neuronia picea. (Phryganidæ.)

Locality: Prussian amber. Horizon: Ligurian.

1864. Hagen. Phryg, synopsis synom., 54.

1864. " Verhandl. zool.obot. gesellsch. Wien, xiy; 852

See also Phryganea picea, Limnophilus piceus,

Bull, $71-23$ 
2779. Neuropterites deperditus.

Locality: Krottensee, Bohemia. Horizon: Aquitanian.

1877. Novák. Sitzungsb. acad. wiss. Wien, lxxvi: 82-83. Pl. 2, fig. 4.

1877. “ Fauna eger. tertiärb., 12-13. Pl. 2, fig. 4.

2780. Nothochrysa - (Hemerobina.)

Locality: Florissant, Colo. Horizon: Oligocene.

1881. Scudder. Bull. U. S. geol. surv. terr., vi : 293.

See also Palæochrysa — , Tribochrysa —

2781. Nymphes mengeanus. (Hemerobina.)

Locality: Prussian amber. Horizon: Ligurian.

1856 . 6

Verhandl. zool.-bot. ver. Wien, iv: 228 (undescr.).

Berendt, Bernst. befindl. organ. reste vorw., ii, th. i: 85-86. $\quad P l$. 8, fig. 15.

1866. " Stett. ent. zeit., xxvii : 453 (an hujus generis ?).

2782. Nymphon (vic.) - (Hemerobina.)

Locality : Florissant, Colo. Horizon: Oligocene.

1881. Scudder. Bull. U. S. geol. surv. terr., vi: 293.

See also Palæochrysa — Tribochrysa ——.

2783. Odontocerus - (2 sp.). (Leptoceridæ.)

Locality: Prussian amber. Morizon: Ligurian.

1856. Hagen. Berendt, Bernst. befindl-organ. reste vorw., ii, th. i: 121.

Eschna —. See Eschna — Curtis.

2784. Oligotoma antiqua. (Embidina.)

Locality: Prussian amber. Horizon: Ligurian.

1885. Hagen. Monogr. Emb., (2), 6-7.

1885. " Can. ent., xvii: 176-177.

1886. Scudder. Bull. U. S. geol. surr., No. 31: 53.

1886. “ Zittel-Barrois, Traité de paléont., ii : 773.

See also Embia antiqua.

2785. Osmylus —. (Hemerobina.)

Locality: Florissant, Colo. Horizon: Oligocene.

1882. Scudder. Proc. Bost. soc. nat. hist., xxi: 408.

1883. " Ann. rep. U. S. geol, surv. terr., xii: 283.

See also Osmplus requietus.

2786. Osmylus pictus. (Hemerobina.)

Compared with O. chrysops.

Locality: Prussian amber. Horizon: Ligurian.

1854. Hagen.

1856. “6

1866. If
Verhandl. zool.-bot. ver. Wien, iv: 228 (undescr.).

Berendt, Bernst. befindl. organ. reste vorw., ii, th, $i$; 86-87. $\boldsymbol{P} \boldsymbol{l}_{\text {e }}$ 8, fig. 16.

Stetth, ent, zeit., xxvii; 455 , 
2787. Osmylus requietus. (Hemerobina.)

Compared with O. pictus, Amber.

Locality : Florissant, Colo. Horizon: Oligocene.

1890. Scudder. Tert. ins. N. A., 162-163. Pl. 14, figs. 3, 8.

See also Osmylus —_.

\section{Paidium -. (Thysanura.)}

Locality: Prussian amber. Horizon: Ligurian.

1845. Berendt. Bernst. befindl, organ. reste vorw., i: 56 .

2789. Paidium crassicorne. (Thysanura.)

Locality : Prussian amber. Horizon: Ligurian.

1845. Koch-Berendt. Jahrb. min., 1845: 872.

1854. " Berendt, Bernst. befindl, organ. reste vorw., i, th. ii: $122 . \quad P l$. 17, fig. 155.

1856. Giebel. Inseet. d. vorwelt, 331.

Paidium piriforme. See Paidium pyriforme.

2790. Paidium pyriforme. (Thysanura.)

Locality : Prussian amber. Horizon: Ligurian.

1845. Koch-Berendt. Jahrb. min., 1845: 872 (piriforme).

1854. " Berendt, Bernst. befindl, organ. reste vorw., i, th. ii: $123 . \quad P l$. 17, fig. 156.

1856. Giebel. Insect. $d$. vorwelt, 331.

2791. Paladicella eruptionis. (Hydropsychidæ.)

Locality : Florissant, Colo. Horizon: Oligocene.

1890. Scudder. Tert. ins. N. A., 189-190. Pl. 15, fig. 14.

2792. Palæochrysa - (Hemerobina.)

Locality : Florissant, Colo. Horizon: Oligocene.

1882. Scudder. Proc. Bost. soc. nat. hist., xxi : 408.

1883. " A Ann. rep. U. S. geol. surv6 terr., xii: 283.

See also Chrysopa (vic.) ——, Nothochrysa —_, Nymphon (vic.) — , Palæochrysa stricta.

2793. Palæochrysa stricta. (Hemerobina.)

Locality : Florissant, Colo. Horizon: Oligocene.

1890. Scudder. Tert. ins. N. A., 166-168. Pl. 14, figs. 13, 14.

Seo also Palæochrysa -

2794. Palæopsocus tener. (Psocina.)

1883. Kolbe. , Stett. ent. zeit., xliv : 190.

See also Psocus tener, etc.

2795. Palingenia - - (Ephemeridæ.)

Locality: Prussian amber. Horizon: Ligurian.

1845, Berendt, Bernst, befindl, organ, reste vorw, i: 57 . 
2796. Palingenia gigas. (Ephemeridæ.)

Locality: Prussian amber. Horizon: Ligurian.

1854. Hagen. Verhandl. zool.-bot. ver. Wien, iv : 227 (undescr.).

2797. Palingenia macrops. (Ephemeridæ.)

Locality: Prussian amber. Horizon : Ligurian.

1854. Pictet. Hagen, Verhandl. zool.-bot. ver. Wien, iv : 227 (undescr.).

1854. “ Traité de paléont., $2^{e}$ éd., ii : 371 (undeser.).

1856. Pictet-Hagen. Berendt, Bernst. befindl, organ. reste vorw., ii, th. i: 74-75. Pl. 6, fig. $2 b ; p l .8, f i g .5$.

2798. Panorpa $\longrightarrow$ (Panorpidæ.)

Locality: Prussian amber. Horizon: Ligurian.

1830. Berendt. Insekten im bernstein, 35 .

2799. Panorpa brevicauda. (Panorpidæ.)

Locality: Prussian amber. Horizon : Ligurian.

1854. Hagen. Verhandl. zool.-bot. ver. Wien, iv : 229 (undeser.).

1856. " Berendt, Bernst. befindl, organ. reste vorw., ii, th i: 91. Pl. 8, fig. 21.

2800. Panorpa rigida. (Panorpidæ.)

Locality : Florissant, Colo. Horizon : Oligocene.

1890. Scudder. Tert. ins. N. A., 176.

2801. Paropsocus disjunctus. (Psocina.)

Locality : White River, Utah. Horizon : Oligocene.

1890. Scudder. Tert. ins. N. A., 118. Pl. 5, fig. 51.

2802. Parotermes - (3 sp.). (Termitina.)

Locality : Florissant, Colo. Horizon: Oligocene.

1881. Scudder. Harv. univ. bull., ii : 291.

1881. " Bull. U. S. geol. surv. terr., vi : 294.

1883. " Ann. rep. U. S. geol. surv. terr., xii: 285.

See also the following species.

2803. Parotermes fodinæ. (Termitina.)

Locality: Florissant, Colo. Horizon: Oligocene.

1883. Scudder. Proc. Amer. acad. arts sc., xix: 141.

1890. " Tert. ins. N. A., 112-113. Pl. 12, figs. 3, 22.

See also Parotermes $\longrightarrow$.

2804. Parotermes hagenii. (Termitina.)

Locality : Florissant, Colo. Horizon: Oligocene.

1883. Scudder. Proc, Amer, acad, arts sc, xix : 139-140.

1890. " Tert, ins, N. A, 110-112, Pl, 12, fig. 2.

See also Parotermes $\longrightarrow$ 
2805. Parotermes insignis. (Termitina.)

Locality: Florissant, Colo. Horizon: Oligocene.

1883. Scudder. Proc. Amer. acad. arts sc., xix : 137-139.

1885. " " Zittel, Handb. palaeont., i, th. ii: 773. Fig. 974.

1886. " " Zittel-Barrois, Traité de paléont., ii : 773. Fig. 991.

1890. “ Tert. ins. N. A, 108-110, Pl. 12, figs, 13, 14.

See also Parotermes

\section{Perla ㄱ. (Perlina.)}

Locality: Prussian amber. Horizon: Ligurian.

1845. Berendt. Berust. befindl, organ, reste vorw., i : 57.

2807. Perla —. (Perlina.)

Locality: Prussian amber. Horizon: Ligurian.

1742. Sendel. Hist. succ., 30-31 (unnamed). Pl. 1, figs. 5, 5a,6.

1825. Guérin. Dict. class. d'hist. nat., viii : 580 (Perle).

1829. Serres. G6́ogn, terr. tert., 242.

2808. Perla —- (Perlina.)

Locality: Gurnet Bay, Isle of Wight. Horizon: Bembridge Limestone.

1879. Woodward. Quart. journ. geol. soc. Lond., xxxv : 344.

1879. " Geol. mag. (n. s.), v: 89.

2809. Perla prisca. (Perlina.)

Locality: Prussian amber. Horizon: Ligurian.

1854. Pictet. Hagen, Verhandl. zool.-bot. ver. Wien, iv : 226 (undescr.).

1854. " "Traité de paléont., 2 éd., ii : 375 (undescr.).

1856. " Berendt, Berust. befindl, organ. reste vorw., ii, th. i: 65-66. Pl.

6, figs. 7b-d.

2810. Perla resinata. (Perlina,)

Locality: Prussian amber. Horizon: Ligurian.

1854. Hagen. Verhandl. zool.-bot. ver. Wien, iv: 226 (undescr.).

1856. " Berendt, Bernst. befindl, organ, reste vorw., ii, th. i: 66-67. Pl. 8, fig. 1.

\section{Perla succinica. (Perlina.)}

Compared with P. annulicauda.

Locality : Prussian amber. Horizon: Ligurian.

1854. Hagen. Verhandl. zool.-hot. ver. Wien, iv : 226 (undescr.).

1856. " Berendt, Bernst. befindl. organ. reste vorw., ii, th. i: 67.

2812. Petalura acutipennis. (Odonata.)

Locality: Sieblos, Rhenish Prussia. Horizon: Aquitanian.

1859. Hagen. Palaeontogr., viii : 22-26. Pl. 3, figs. 1-4.

2813. Petalura ovatipennis. (Odonata.)

Locality: Sieblos, Rhenish Prussia. Horizon: Aquitanian.

1860. Hagen. Hassencamp, Würzb, naturw. zeitschr., i: 79 (undescr.).

2814. Petrobius ——. (Thysanura.)

Locality: Prussian amber. Horizon: Ligurian.

1845. Berendt. Bernst, befindl, organ. reste vorw., i: 56. 
2815. Petrobius - $(8 \mathrm{sp}$.). (Thysanura.)

1856. Menge. Progr. petrischule Danzig, 1856: 11.

Locality: Prussian amber. Horizon: Ligurian.

2816. Petrobius albomaculatus. (Thysanura.)

Locality: Prussian amber. Horizon: Ligurian.

1854. Menge. Koch-Berendt, Bernst. befindl. organ. reste vorw., i, th. ii : 115. See also Machilis albomaculatus.

Petrobius anguens. See Petrobius angueus.

2817. Petrobius angueus. (T'hysanura.)

Loeality: Prussian amber. Horizon : Ligurian.

1845. Koch-Berendt. Jahrb. mineral, $1845: 872$.

1854. " " Berendt, Bernst. befindl, organ. reste vorw., i, th. ii: 114-115 See also Machilis angueus. (anguens on plate), $P l .14, f i g .120$.

2818. Petrobius confinis. (Thysanura.)

Locality : Prussian amber. Horizon: Ligurian.

1845. Koch-Berendt. Jahrb. mineral., 1845 : 872.

1854. “ Berendt, Bernst. befindl, organ, reste vorw., i, th. ii: 115. Pl. 17, fig. 153.

See also Petrobius electus, etc.

2819. Petrobius coruscus. (Thysanura.)

Locality: Prussian amber. Horizon: Ligurian.

1845. Koch-Berendt. Jahrb. mineral., 1845: 872.

1854. " Berendt, Bernst. befindl, organ. reste vorw., i, th. ii : 111-112. Pl. 14, fig. 116.

See also Petrobius imbricatus, etc.

\section{Petrobius electus. (Thysanura.)}

Locality: Prussian amber. Horizon: Ligurian.

1845. Koch-Berendt. Jahrb. mineral., 1845: 872.

1854. " Berendt, Bernst. befindl. organ. reste vorw, i, th. ii : 113. Pl. 14, fig. 119.

See also Machilis (Petrobius) electus, P. confinis.

\section{Petrobius imbricatus. (Thysanura.)}

Locality: Prussian amber. Horizon: Ligurian.

1845. Koch-Berendt. Jahrb. mineral., $1845: 872$.

1854. " " Berendt, Bernst. befindl. organ. reste vorw., i, th. ii : 112. Pl. 14, fig. 117.

See also Machilis (Petrobius) imbricatus, P. coruscus, Machilis (Petrobius) coruseus, P. longipalpus.

2822. Petrobius longipalpus. (Thysanura.)

Locality: Prussian amber. Horizon: Ligurian.

1845. Koch-Berendt. Jahrb. mineral., 1845: 872.

1854. " Berendt, Bernst. befindl, organ. reste vorw,, i, th. ii: 113. Pl. 14, fig. 118.

See also Petrobius imbricatus, etc. 
2823. Petrobius macrura. (Thysanura.)

Locality: Prussian amber. Horizon: Ligurian.

1854. Menge. Koch-Berendt, Bernst. befindl. organ. reste vorw., i, th. ii : 115. See also Machilis (Petrobius) macrura.

2824. Petrobius saliens. (Thysanura.)

Locality: Prussian amber. Horizon: Ligurian.

1854. Menge. Koch-Berendt, Bernst. befindl. organ. reste vorw., i, th. ii : 115 . See also Machilis (Petrobius) saliens.

2825. Petrobius seticornis. (Thysanura.)

Locality: Prussian amber. Horizon: Ligurian.

1845. Koch-Berendt. Jahrb. mineral., $1845: 872$.

1854. " Berendt, Bernst. befindl. organ. reste vorw., i, th. ii: 114. Pl. 15, fig. 124.

1885. Scudder. Zittel, Hancb. palaeont, i, th. ii : 772. Fig. 971.

1886. " Zittel-Barrois, Traité de paléont., ii : 772. Fig. 988.

See also Machilis (Ptírobius) seticornis.

2826. Philopotamus - (2 sp.). (Hydropsychidæ.)

Locality: Prussian amber. Horizon: Ligurian.

1856. Hagen. Berendt, Bernst. befindl. organ. reste vorw., ii, th. i: 119.

2827. Philotarsus abnormis. (Psocina.)

Locality: Prussian amber. Horizon: Ligurian.

1883. Kolbe. Stett. ent. zeit., xliv : 190.

See also Elipsocus abnormis, etc.

2828. Philotarsus antiquus. (Psocina.)

Locality : Prussian amber. Horizon: Ligurian.

1883. Kolbe. Stett. ent. zeit., xliv : 187-188.

2829. Phryganea - (cases). (Trichoptera.)

1836. Beck. $\quad$ Proc. geol. soc. Lond., ii: 219.

1836. “ Lond, Edinb. phil, mag., (3), viii: 555.

2830. Phryganea - (Trichoptera.)

Locality: Prussian amber. Horizon: Ligurian.

1845. Berendt. Bernst. befindl, organ. reste vorw., i: 57.

2831. Phryganea —. (Trichoptera.)

Locality: Prussian amber. Horizon: Ligurian.

1832. Burmeister. Handb. ent, i : 637.

1836. " Man. ent., 578.

2832. Phryganea —. (Trichoptera.)

Locality : Prussian amber. Horizon: Ligurian.

1835. Gravenhorst. Uebers. schles. gesellsch. vaterl. cult, 1834 : 92 
2833. Phryganea - (Trichoptera.)

Compared with P. grandis.

Locality : Mombach, Rhenish Prussia. Horizon: Middle Tertiary.

1845. Hoeninghaus. Michelin, Ann. soc. ent. Fr., (2), iii, bull, ent., 31.

Seo also P. mombachiana.

2834. Phryganea - (cases). (Trichoptera.)

Locality : Lewes levels, England. Horizon: Alluvium.

1829. Mantell. Trans. geol. soc. Lond., (2), iii : 201.

1888. Bell. Entom., xxi: 2.

2835. Phryganea (or Ephemera)

Locality: Oeningen, Baden. Horizon: Tortonian.

1820. Schlotheim. Petrefactenk., 43.

2836. Phryganea -. (Trichoptera.)

Locality: Prussian amber. Horizon: Ligurian.

1742. Sendel. Hist. succ., 85. Pl. 2, figs. 21, 23.

1852. Guérin. Dict. class. d'hist. nat., viii : 580 (Frigane).

1829. Serres. Géogn. terr. tert., 242.

1830. Berendt. Insekten im bernstein, 35 .

2837. Phryganea -. (Trichoptera.)

Locality : Gurnet Bay, Isle of Wight. Horizon: Bembridge Limestone. 1879. Woodward. Quart. journ. geol. soc. Lond., xxxv : 344. 1879. " Geol. mag. (n. s.), v: 89.

2838. Phryganea-antiqua. (Phryganidæ.)

Locality: Oeningen, Baden. Horizon: Tortonian.

1849. Heer. Insektenf. tertiärg. Oeningen, ii : 89-90. Pl. 5, fig. 10.

1852. Giebel. Deutschl. petref., 639 .

1854. Pictet. Traité de paléont., $2^{\mathrm{e}}$ éd., ii : 376 (undescr.).

1856. " Berendt, Bernst. befindl. organ. reste vorw., ii, th. i : $97-98$ (=P. fossilis Pict. nec antiqua Heer).

1856. Giebel. Insect. d. vorwelt, 268.

1864. Hagen. Phryg. synopsis synom., 59.

1864. " Verhandl. zool.-bot. gesellsch. Wien, xiv : $85 \%$.

See also Phryganea fossilis.

2839. Phryganea aquensis. (Phryganidæ.)

Locality : Aix, France. Horizon : Ligurian.

1883. Heer. Flora foss. grönl, ii : 148. Pl. 109, fig. 15.

2840. Phryganea blumii (larval cases). (Phryganidæ.)

Locality : Leistadt, near Dürkheim, Bavaria. Horizon : -

1844. Hepp. Jahresb. Pollich., ii : 19-23.

2841. Phryganea corentiana (cases). (Phryganidæ.)

Locality: Gergovia, France. Horizon: Aquitanian.

1870. Oustalet. Am. sc. géol, ii, art. 3: 101.

See also Indusia tubulata, etc. 
2842. Phryganea dubia. (Limnophilidœ.)

1854. Pictet.

Locality : Prussian amber. Horizon: Ligurian.

1856. Hagen.

Hagen, Verhandl. zool.-bot. ver. Wien, iv : 229 (undeser.).

Berendt, Bernst. befindl. organ. reste vorw., ii, th. i: 100-101. Pl. 7, figs. $4 b-d$.

See also Limnophilus dubius.

2843. Phryganea fossilis. (Phryganidæ.)

Compared with P. minor.

Locality: Prussian amber. Horizon: Ligurian.

1854. Pictet. Hagen, Verhandl. zool.-bot. ver. Wien, iv: 229 (undescr.).

1854. " Traité de paléont., $2^{e}$ éd., ii : 376 (undeser.).

1856. " Berendt, Bernst. befindl, organ. reste vorw., ii, th. i: 96. Pl. 7, figs. $1 b-d, 2 b-c$.

1864. Hagen. Phryg. synopsis synom., 63.

1864. “ Verhandl. zool.-bot. gesellsch. Wien, xiv: 861.

See also Phryganea antiqua.

2844. Phryganea gerandiana (cases). (Phryganidæ.)

Locality : Chavroches, Allier, France. Horizon: Aquitanian.

1870. Onstalet. Ann. sc. géol., ii, art., 3: 101-102.

See also Indusia tubulata, etc.

2845. Phryganea gigantea (cases). (Phryganidæ.)

Locality: Auvergne, France. Horizon: Aquitanian.

1844. Hepp. Jahresb. Pollich., ii : 23.

See also Indusia tubulata, etc.

2846. Phryganea hyperborea. (Phryganidæ.)

Compared with P. grandis, etc.

Locality : Ober Atanakerdluk, Greenland. Horizon :

1883. Heer. Flora foss. grönl., ii : 147-148. Pl. 109, figs. 13, 13 .

2847. Phryganea labefacta. (Phryganidæ.)

Compared with P. grandis.

Locality : Florissant, Colo. Horizon: Oligocene.

1890. Scudder. Tert. ins. N. A., 197-198. Pl. 13, fig. 5.

2848. Phryganea longirostris. (Phryganidæ.)

Locality : Prussian amber. Horizon: Ligurian.

1854. Hagen. Verhandl. zool.-bot. ver. Wien, iv: 229 (undescr.).

1856. " "Berendt, Bernst. befindl. organ. reste vorw., ii, th. i: 100 .

1864. " " Phryg. synopsis synom., 65.

1864. “ Verhandl, zool.-bot. gesellsch. Wien, xiv: 863 .

2849. Phryganea major. (Phryganidæ.)

Locality: Valnerina, Italy. Horizon: Quaternary.

1880. Verri. Atti soc, ital, sc. nat, xxiii : 289 (Friganea).

Phryganea mombachana. See Phryganea mombachiana. 
2850. Phryganea mombachiana. (Phryganidæ.)

Compared with P. grandis.

Locality: Mombach, Rhenish Prussia. Horizon: Middle Tertiary.

1844. Hoeninghaus. Phryganea mombachiana. Figs.

1848. Bronn. Index palaeont., i, nomencl., 969 (mombachana).

1873. Hagen. Verhandl. zool.-bot. yesellsch. Wien, xxiii : 379.

1873. Hagen. Beitr. kenntn. Phryg., 3.

See also Phryganea — Hoeninghaus.

2851. Phryganea operta. (Phryganidæ.)

Locality: White River, Colo. Horizon: Oligocene.

1877. Scudder. Bull. U. S. geol. surv. terr., iii : 762.

See also Hydropsyche operta.

2852. Phryganea parschlugiana. (Phryganidæ,)

Locality : Parschlug, Styria. Horizon: -

1883. Heer. Flora foss. grönl., ii: 148. Pl. 109, fig. 14.

2853. Phryganea picea. (Phryganidæ.)

Locality: Prussian amber. Horizon: Ligurian.

1854. Pictet. Hagen, Verhandl. zool.-bot. ver. Wien, iv : 229 (undescr.).

1856. Hagen. Berendt, Bernst. befindl, organ, reste vorw., ii, th. i: 98-99. Pl. 7, figs. 3b-c; pl. 8, fig. 24.

See also Limnophilus piceus, Neuronia picea.

2854. Phryganeolitha vetusta. (Trichoptera.)

Compared with Phryganea wæneri Linn.

Locality : Prussian amber. Horizon: Ligurian.

1813. Germar. Germ., Mag. entom., i : 17-18.

1856. Giebel. Insect. d. vorwelt, 268-269.

See also Polycentropus vetustus, P. xanthocoma, Hydropsyche xanthocoma.

2855. Planocephalus aselloides. (Thysanura.)

Locality: Florissant, Colo. Horizon: Oligocene.

1885. Scudder. Descr, artic., 1-6. Figs. 1-3.

1885. " Mem. nat. acad. se., iii: 85-90. Figs. 1-3.

1885. Bertkau. Sitzungsb. niederrhein. gesellsch. natur.- u. heilk., 1885: 298-300.

1885. Scudder. Zittel, Handb. palaeont., i, th. ii: 772. Figs. 972ab.

1886. " Zittel-Barrois, Traité de paléont., ii : 772. Figs. 989ab.

1886. “ Bull. U. S. geol. surv., No. 31: 52 .

1890. “ Tert. ins. N. A., 94-101. Figs. 1-3.

1890. Lesley. Dict. foss. Penns, ii: 651. Figs.

See also —— Scudder.

2856. Platyonemis antiqua. (Odonata.)

Locality: Prussian amber. Horizon: Ligurian.

1890. Scudder. Tert. ins. N. A., 127.

Seo also Agrion antiquum, ete. 
2857. Platyonemis icarus. (Odonata.)

Loeality : Rott, Rhenish Prussia. Horizon: Aquitanian.

1890. Scudder.

Tert. ins. N. A., 127.

See also Agrion icarus.

2858. Podagrion abortivum. (Odonata.)

Locality: Green River, Wyo. Horizon: Oligocene.

1878. Scudder. Bull. U. S. geol. surv. terr., iv : 775-776.

1890. " Tert. ins. N. A., 134. Pl. 6, figs. 7, 8.

2859. Podura -. (Thysanura.)

Locality: Prussian amber. Horizon: Ligurian.

1845. Berendt. Bernst. befindl. organ. reste vorw., i: 56.

2860. Podura -. (Thysanura.)

Locality: Prussian amber. Horizon: Ligurian.

1835. Gravenhorst. Uebers. schles. gesellsch. vaterl. cult., 1834 : 93.

2861. Podura fuscata. (Thysanura.)

Locality: Prussian amber. Horizon: Ligurian.

1845. Koch-Berendt. Jahrb. min., 1845: 872.

1854. " Berendt, Bernst. befindl. organ. reste vorw., i, th. ii : 119. $P l$. 15, fig. 127.

1856. Giebel. Insect. d. vorwelt, 330 .

2862. Podura pilosa. (Thysanura.)

Locality: Prussian amber. Horizon: Ligurian.

1845. Koch-Berendt. Jahrb. min., 1845: 872.

1854. " Berendt, Bernst. befindl. organ. reste vorw., i, th. ii : $120 . P$ Pl. 15, fig. 129.

1856. Giebel. Insect. d. vorwelt, 330.

\section{Podura pulchra. (Thysanura.)}

Locality: Prussian amber. Horizon: Ligurian.

1845. Koch-Berendt. Jahrb. min., 1845 : 872.

1854. " Berendt, Bernst, befindl. organ. reste vorw., i, th. ii: 119-120. Pl. 15, fig. 128.

1856. Giebel. Insect. d. vorwelt, 330.

2864. Podura tæniata. (Thysanura.)

Locality: Prussian amber. Horizon: Ligurian.

1815. Koch-Berendt. Jahrb. min., 1845: 872.

1854. " Berendt, Bernst. befindl. organ. reste vorw., i, th. ii : $\mathbf{1 1 9}$ (tænita). Pl. 15, fig. 126.

1856. Giebel. Insect. d. vorwelt, 329-330.

Podura tænita. See Podnra tæniata.

2865. Polycentropus - (Hydropsychidæ.)

Locality: Prussian amber. Horizon: Ligurian.

1845. Berendt. Bernst. befindl, organ. reste vorw., i: 57. 


\section{Polycentropus affinis. (Hydropsychidæ.)}

1854. Pictet. 1854. " " 1856. “

1864. Hagen. 1864. “

1856. Hagen. 1864. “ 1864. “

1854. Pictet. 1854. " " 1856. " "

1864. Hagen. 1864 .

1854. Pictet. 1856. Hagen.

1864. “ 1864. "

Locality: Prussian amber. Horizon: Ligurian.

Hagen, Verhandl. zool.-bot. ver. Wien, iv: 229 (undescr.).

Traité de pal6ont, $2^{\circ}$ éd., ii: 376 (undescr.).

Berendt, Bernst. befindl. organ. reste vorw., ii, th. i: 114. $\boldsymbol{P l}$. 7 , figs. $7 b-c$.

Phryg. synopsis synom., 75.

Verhandl. zool.-bot. gesellsch. Wien, xvi : 873 .

2867. Polycentropus antiquus. (Hydropsychidæ.)

Locality: Prussian amber. Horizon : Ligurian.

Berendt, Bernst. befindl. organ. reste vorw., ii, th. i: 113.

Phryg. synopsis synom., 75.

Verhandl. zool.-bot. gesellsch. Wien, xiv: 873 .

\section{Polycentropus atratus. (Hydropsychidæ.)}

Locality: Prussian amber. Horizon: Ligurian.

\section{Hagen, Verhandl. zool.-lot. ver. Wien, iv: 229 (undescr.),}

Traité de palént., $2^{\mathrm{e}}$ éd., ii : 376 (undescr.).

Berendt, Bernst. befindl, organ, reste vorw, ii, th. i: 114. Pl. 7 . fig. 10.

Phryg. synopsis synom., 75.

Verhandl. zool,-bot. gesellsch. Wien, xiv: 873 .

2869. Polycentropus barbatus. (Hydropsychidæ.)

Locality: Prussian amber. Horizon: Ligurian.

Hagen, Verhandl. zool.-bot. ver. Wien, iv : 229 (undescr.).

Berendt, Bernst. befindl. organ. reste vorw., ii, th. i: 112. $\quad \boldsymbol{P l}$. 6, fig. $17 b$.

Phryg. synopsis synom., 75.

Verhandl. zool.-bot. gesellsch. Wien, xiv : 873.

See also Hydropsyche barbata.

\section{Polycentropus dubius. (Hydropsychidæ.)}

Locality: Prussian amber. Horizon: Ligurian.

1854. Pictet. Hagen, Verhandl, zool.-bot. ver. Wien, iv: 229 (undescr.).

1854. " " Trait6 de paléont., $2^{\mathrm{e}}$ éd., ii : 376 (undescr.).

1856. " Berendt, Bernst. befindl. organ. reste vorw., i, th. ii: 115 . Pl.7 fig. 14.

1864. Hagen. Phryg. synopsis synom., 75.

1864. " Verhandl. zool.-bot. gesellsch. Wien, xiv: 873.

See also Polycentropus fusconiger, etc.

2871. Polycentropus eviratus. (Fydropsychiāæ.)

Locality: Florissant, Colo, Horizon: Oligocene.

1890. Scudder. Tert. ins. N. A., 182. Pl. 13, fig. 7.

2872. Polycentropus exesus. (Hydropsychidæ.)

Locality : Florissant, Colo. Horizon: Oligocene.

1890. Scudder. Tert. ins. N. A., 181. 
2873. Polycentropus fusconiger. (Hydropsychidæ.)

Locality: Prussian amber. Horizon: Ligurian.

1856. Hagen. Berendt, Bernst. befindl. organ. reste vorw., ii, th. i: 115 (= P. incertus?).

See also Polycentropus incertus, P. dubius, P. macrocephalus, Aphelocheira fusconigra.

2874. Polycentropus guttulatus. (Hydropsychidæ.)

Locality: Prussian amber. Horizon: Ligurian.
1854. Pictet. - Hagen, Verhandl. zool.-bot. ver. Wien, iv: 229 (undescr.).

1854. 6" Traité de paléont., $z^{\circ}$ éd., ii : 376 (undescr.).

1856. " Berendt, Bernst. befindl, organ, reste vorw., ii, th. i: 111-112. Pl. 7, figs. $8 b-c$.

1864. Hagen. Phryg. synopsis synom., 76.

1864. "Verhandl. zool.-bot. gesellsch. Wien, xiv: 874.

2875. Polycentropus incertus. (Hydropsychidæ.)

Locality: Prussian amber. Horizon: Ligurian.

1854. Pictet. Traité de paléont., $2^{\mathrm{e}}$ éd., ii : 376 (undescr.).

1856. " Berendt, Bernst. betindl. organ. reste vorw., ii, th. i: i15. Pl. 7, fig. 13.

1864. Hagen. Phryg. synopsis synom., 76.

1864. " Verhandl. zool.-bot. gesellsch. Wien, xiv: 874.

See also Polycentropus fusconiger, etc.

2876. Polycentropus lævis. (Hydropsychidæ.)

Locality: Prussian amber. Horizon: Ligurian.

1854. Pictet. Traité de paléont., $2^{\mathrm{e}}$ éd., ii : 376 (undescr.).

1856. " Berendt, Bernst. befindl. organ. reste vorw., ii, th. i: 110-111.

1864. Hagen. Phryg. synopsis synom., 76 (= P. latus).

1864. "Verhandl. zool.-bot. gesellsch. Wien, xiv: 874 (= P. latus).

See also Polycentropus latus.

2877. Polycentropus latus. (Hydropsychidæ.)

Locality: Prussian amber. Horizon: Ligurian.

1854. Pictet. Hagen, Verhandl. zool.-bot. ver. Wien, iv: 229 (undescr.).

1854. " " Traité de paléont., $2^{\circ}$ éd., ii: 376 (undescr.).

1856. “ Berendt, Bernst. befindı. organ. reste vorw., ii, th. i: 109-111. Pl. 7, figs. 11b-e; pl. 8, fig. 27.

1864. Hagen. Phryg. synopsis synom., 76.

1864. " Verhandl. zool.-bot. gesellsch. Wien, xiv : 874.

See also Polycentropus lævis.

2878. Polycentropus macrocephalus. (Hydropsychidæ.)

Locality: Prussian amber. Horizon : Ligurian.

1854. Pictet. Traité de paléont., $2^{\mathrm{e}}$ éd., ii : 376 (undeser.).

1856. " " Berendt, Bernst. befindl. organ, reste vorw. ii, th. i : 115.

1E64. Hagen. $\quad$ Phryg. synopsis synom., 76 ( = P. dubius).

1864. "Verhandl. zool.-bot. gesellsch. Wien, xiץ: 874 (= P, dubius),

See also Polycentropus fusconiger, ete, 
2879. Polycentropus priscus. (Hydropsychidæ.)

Locality : Prussian amber. Horizon: Ligurian.

1856. Hagen. Berendt, Bernst. befindl. organ. reste vorw., ii, th. i: 116. Pl. 7, fig. 16.

1864. " Phryg. synopsis synom., 76.

1864. “ Verhandl. zool.-bot. gesellsch. Wien, xiv: 874.

See also Hydropsyche prisca.

2880. Polycentropus vetustus. (Hydropsychidæ.)

Locality: Prussian amber. Horizon: Ligurian.

1854. Germar. Hagen, Verhandl, zool.-bot. ver. Wien, iv: 229 (undescr.).

1856. Hagen. Berendt, Bernst. befindl. organ. reste vorw., ii, th. i: $113 . \quad P l$. 7 , fig. 9.

1864. " " Phryg. synopsis synom., 77.

1864. “ Verhandl. zool.-bot. gesellsch. Wien, xiv: 875.

See also Phryganeolitha vetusta, etc.

2881. Polycentropus xanthocoma. (Hydropsychidæ.)

Locality: Prussian amber. Horizon: Ligurian.

1854. Pictet. Traité de paléont., $2^{e}$ éd., ii : 376 (undescr.)

1856. " Berendt, Bernst. befindl, organ. reste vorw., ii, th. i: 113.

1864. Kollar. Hagen, Phryg. synopsis synom., 77.

1864. " Hagen, Verhandl, zool.-bot. gesellsch. Wien, xiv : 875.

See also Phryganeolitha vetusta, etc.

2882. Potamanthus ㄴ. (Ephemeridæ.)

Locality: Prussian amber. Horizon: Ligurian.

1845. Berendt. Bernst. befindl, organ. reste vorw., i : 57.

2883. Potamanthus priscus. (Ephemeridæ.)

Locality: Prussian amber. Horizon: Ligurian.

1854. Pictet. Hagen, Verhandl, zool.-bot. ver. Wien, iv : 227 (undescr.).

1854. "6 Traité de paléont., $2^{\mathrm{e}}$ éd., ii : 371 (undescr.) (Pothamanthus).

1856. Pictet-Hagen. Berendt, Bernst. befindl. organ. reste vorw, ii, th. i: $77 . \quad P l .6$, fig. $3 b$.

See also Leptophlebia prisca.

Pothamanthus priscus. See Potamanthus priscus.

2884. Psocus - (Psocina.)

Locality : Prussian amber. Horizon : Ligurian.

1845. Berendt. Bernst. befindl. organ. reste vorw., i : 57.

2885. Psocus -. (Psocina.)

Locality: Prussian amber. Horizon: Ligurian.

1831. Burmeister. Oken, Isis., 1831: 1100.

1832. " Handb. ent., i: 637.

1836. “ Man. ent., 579.

2886. Psocus - (Psocina.)

Locality: Prussian amber. Horizon: Ligurian.

1835, Gravenhorst, Uebers, schles, gesellsch, yaterl, cult., 1834; 92, 


\section{Psocus - (Psocina.)}

Locality: Sicilian amber. Horizon:

1838. Guérin. Rev. zool, 1838: 170. Pl. 1, fig. 8.

2888. Psocus abnormis. (Psocina.)

Locality: Prussian amber. Horizon: Ligurian.

1854. Hagen. Verhandl. zool.-bot. ver. Wien, iv : 225 (undeser.).

1856. " Berendt, Bernst. befindl. organ. reste vorw., ii, th. i : $61 . \quad P l .8$, figs. $9 a-c$.

See also Elipsocus abnormis, etc.

\section{Psocus affinis. (Psocina.)}

Compared with P. nervosus, P. bifasciatus.

Locality: Prussian amber. Horizon: Ligurian.

1854. Pictet.

1856. “

1866. Hagen.

1866. " "

1882. “
1854. Pictet. 1856. “

Hagen, Verhandl. zool.-bot. ver. Wien, iv: 225 (undescr.).

Berendt, Bernst. befindl, organ. reste vorw., ii, th. i: $58 . \quad P l .5$, figs. $9 b-c, 12$.

Psoc. Emb. synopsis, 12.

Verhandl. zool.-bot. gesellsch. Wien, xvi : 212.

Stett. ent. zeit., xliii : 232-237. Pl. 1, fig. 1 (22 figs.).

2890. Psocus ciliatus. (Psocina.)

Compared with P. lasiopterus.

Locality: Prussian amber. Horizon: Ligurian.

Hagen, Verhandl. zool.-bot. ver. Wien, iv : 225 (undescr.).

Berendt, Bernst. befindl. organ, reste vorw., ii, th. i: 59-60. Pl. 5, figs. 10b-c.

See also Epipsocus ciliatus.

\section{Psocus debilis. (Psocina.)}

Locality : Prussian amber. Horizon: Ligurian.

1854. Pictet.

Hagen, Verhandl, zool.-bot. ver. Wien, iv: 225 (undeser.).

1856. " "

Berendt, Bernst. befindl. organ. reste vorw., ii, th. i: 60. Pl.5, fig. $11 b$,

See also Cœcilius debilis, Epipsocus debilis.

2892. Psocus proavus. (Psocina.)

Locality: Prussian amber, Horizon: Ligurian.

1854. Hagen. Verhandl. zool.-bot. ver. Wien, iv: 225 (undeser.).

1856. " Berndt, Bernst. befindl. organ. reste vorw., ii, th. i: 59. Pl. 5, fig. $8 b ; p l .8$, fig. 7 .

See also Cæcilius proavus.

2893. Psocus tener. (Psocina.)

\section{Locality: Prussian amber. Horizon: Ligurian.}

1854. Hagen. Verhandl, zool.-bot. ver. Wien, iv : 225 (undescr.).

1856. "B Berendt, Bernst, befindl. organ, reste vorw., ii, th. i: 60. Pl. 8, fig. 8.

1882. “ Stett. ent. zeit., xliii : 225-226.

See also Palæopsocus tener, Epipsocus tener.

Psychomia (several species). See Psychomyia, the same species, 


\section{Psychomyia —-. (Hydropsychidæ.)}

Locality : Prussian amber. Horizon: Ligurian.

1845. Berendt. Bernst. befindl. organ. reste vorw., i: 57.

2895. Psychomyia lata. (Hydropsychidæ.)

Locality : Prussian amber. Horizon: Ligurian.

1856. Hagen. Berendt, Bernst. befindl. organ. reste vorw, ii, th. i: 119 (Psychomia).

See also Psychomyia sericea, etc.

2896. Psychomyia pallida. (Hydropsychidæ.)

Locality: Prussian amber. Horizon : Ligurian.

1854. Pictet.

Traité de paléont., $2^{\mathrm{e}}$ éd, ii : 376 (undęser.) (Psychomia).

1856. " "

Berendt, Bernst. befindl. organ. reste vorw., ii, th. i: 118 (=P. sericea). $P l .7$, figs. $19 b-d$.

See also Psychomyia sericea, etc.

2897. Psychomyia sericea. (Hydropsychidæ.)

Locality: Prussian amber. Horizon: Ligurian.

1854. Pictet. Traité de paléont., $2^{\ominus}$ éd., ii : 376 (undescr.) (Psychomia).

1856. " "

Berenidt, Bernst. befindl. organ. reste vorw., ii, th. i: 118 (Psychomia). Pl. 7, figs. $19 b-e ; p l .8$, fig. 28.

1864. Hagen. Phryg. synopsis synom., 79.

1864. “ Verhandl. zool.-bót. gesellsch. Wien, xiv: 877 .

See also Psychomyia pallida, P. lata.

\section{Raphidia —. (Sialina.)}

Locality: Prussian amber. Horizon: Ligurian.

1830. Berendt. Insekten im bernstein, 35 .

2899. Raphidia - (larva). (Sialina.)

Locality: Prussian amber. Horizon: Ligurian.

1856. Menge. Berendt, Bernst. befindl, organ. reste vorw., ii, th. i: 83-84. $\quad P l$. 8, fig. 31 .

2900. Raphidia —. (Sialina.)

Locality : Florissant, Colo. Horizon : Oligocene.

1881. Scudder. Bull. U. S. geol. surv. terr., vi: 293.

1882. " Proc. Bost. soc. nat. hist., xxi: 407.

1883. " Ann. rep. U. S. geol. surv. terr., xii : 283.

See also Raphidia tranquilla.

2901. Raphidia erigena. (Sialina.)

Locality: Prussian amber. Horizon: Ligurian.

1854. Menge. Hagen, Verhandl. zool.-bot. ver. Wien, iv: 228 (undescr.).

See also Raphidia (Inocellia) erigena.

\section{Raphidia (Inocellia) erigena. (Sialina.)}

Locality : Prussian amber. Horizon: Ligurian.

1856. Menge, Berendt, Bernst, befindl, organ, ryste vorw., ii, th, i: 83. Pl. 8 , fig. 14 .

See also Raphidia erigena, 
2903. Raphidia tranquilla. (Sialina.)

Locality : Florissant, Colo. Horizon: Oligocene.

1890. Scudder. Tert. ins. N.A., 154-155. Pl.14, fig. 2.

See also Raphidia — Scudder.

2904. Rhyacophila - (Rhyacophilidæ.)

Locality : Prussian amber. Horizon : Ligurian.

1845. Berendt. Bernst. befindl. organ. reste vorw., i: 57.

2905. Rhyacophila occulta. (Rhyacophilidæ.)

Locality : Prussian amber. Horizon: Ligurian.

1856. Hagen. Berendt, Bernst. befindl. organ. reste vorw., ii, th. i: 120.

1864. " " Phryg. synopsis synom., 81.

1864. " Verhandl. zool.-bot. gesellsch. Wien, xiv : 879.

2906. Rhyacophila prisca. (Rhyacophilidæ.)

Locality : Prussian amber. Horizon: Ligurian.

1854. Pictet. Traité de paléont., $2^{\mathrm{e}}$ ed., ii : 376 (undeser.).

1856. " Berendt, Bernst, befindl. organ. reste vorw., ii, th. i: 116-117. Pl. 7, fig. 6.

See also Tinodes prisca.

2907. Rhyacophila subumbrosa. (Rhyacophilidæ.)

Locality: Prussian amber. Horizon : Ligurian.

1851. Kolenati. Abhandl. böhm. gesellsch. wiss., (5), vi: 15 (undescr.).

2908. Rhyacophila succinica major. (Rhyacophilidæ.)

Locality: Prussian amber. Horizon: Ligurian.

1851. Kolenati. Abhandl. böhm. gesellsch. wiss., (5), vi: 15 (undescr.).

2909. Rhyacophila succinica media. (Rhyacophilidæ.)

Locality : Prussian amber. Horizon: Ligurian.

1851. Kolenati. Abhandl. böhm. gesellsch. wiss., (5), vi : 15 (undescr.).

2910. Rhyacophila succinica minor. (Rhyacophilidæ.)

Locality : Prusssian amber. Horizon : Ligurian.

1851. Kolenati. Abhandl. böhm. gesellsch. wiss., (5), vi : 15 (undescr.).

Rophalis amissa. See Sisyra (Rophalis) amissa.

2911. Rophalis relicta. (Hemerobina.)

Locality: Prussian amber. Horizon : Ligurian.

1842. Erichson. MSS.; cf. Hagen in Berendt, Bernst. befindl, organ. reste vorw., ii, th. i: 87 .

1854. Pictet.

Traité de paléont., $2^{\mathrm{e}} 6 \mathrm{~d}$., ii : 378 (undescr.).

1866. Hagen. Stett. ent. zeit., xxvii : 459.

See also Sisyra relicta, S. (Rophalis) relicta.

2912. Semblis

(Sialina.)

Locality: Prussian amber. Horizon: Ligurian.

1832. Burmeister. Handb. ent., i: 637.

1836. “ Man. ent., 578.

Bull. $71-24$ 


\section{Semblis —. (Sialina.)}

Locality: Prussian amber. Horizon: Ligurian. 1835. Gravenhorst. Uebers. schles, gesellsch. vaterl. cult., 1834: 92.

2914. Sericostomum —. (Sericostomidæ.)

1854. Hagen. Verhandl. zool.-bot. ver. Wien, iv: 299.

1856. " Berendt, Bernst. befindl. organ. reste vorw., ii, th. i : 107.

2915. Sericostomum hyalinum. (Sericostomidæ.)

Locality : Prussian amber. Horizon: Ligurian.

1854. Hagen. Verbandl. zool.-bot. ver. Wien, iv : 229 (undeser.).

1856. " Berendt, Bernst. befindl. organ, reste vorw., ii, th. i: 106-107.

\section{Setodes abbreviata. (Leptoceridæ.)}

Locality: Florissant, Colo. Horizon: Oligocene.

1890. Scudder. Tert. ins. N. A., 192.

\section{Setodes portionalis. (Leptoceridæ.)}

Locality : Florissant, Colo. Horizon: Oligocene.

1890. Scudder. Tert. ins. N. A., 191-19\%. Pl. 15, fig. 15.

$$
\text { 2918. Sisyra —. (Hemerobina.) }
$$

Locality: Prussian amber. Horizon: Ligurian.

1845. Berendt. Bernst. befindl. organ. reste vorw., i: 57.

\section{Sisyra amissa. (Hemerobina.)}

Locality: Prussian amber. Horizon: Ligurian.

1854. Hagen,

Verhandl. zool.-bot. ver. Wien, iv : 228.

1866. " Stett. ent. zeit., xxvii: 460.

See also Sisyra (Rophalis) amissa.

\section{Sisyra (Rophalis) amissa. (Hemerobina.)}

Locality : Prussian amber. Horizon : Ligurian.

1856. IIagen. Berendt, Bernst. befindl, organ, reste vorw., ii, th. i: 87-88. Pl. 8, fig. 20.

See also Sisyra amissa.

\section{Sisyra relicta. (Hemerobina.)}

\section{Locality: Prussian amber. Horizon: Ligurian.}

1854. Hagen. Verhandl. zool.-bot. ver. Wien, iv: 228 (undescr.),

1866. " Stett. ent. zeit., xxvii : 460.

See also Rophalis relicta, etc.

2922. Sisyra (Rophalis) relicta. (Hemerobina.)

Compared with S. fuscata.

Locality : Prussian amber. Horizon : Ligurian.

1856. Hagen. Berendt, Bernst. befindl. organ, reste vorw., ii, th. i: 87. Pl. 7, figs. $25 b-f ; p l .8$, fig. 19.

See also Rophalis relicta, etc. 
2923. Smynthurus —. ('Thysanura.)

Locality: Prussian amber. Horizon: Ligurian.

1845. Berendt. Bernst. befindl. organ. reste vorw., i: 56.

2924. Smynthurus brevicornis. (Thysanura.)

Locality: Prussian amber. Horizon: Ligurian.

1845. Koch-Berendt. Jahrb. min., 1845: 873.

1854. “ " Bernst. befindl. organ. reste vorw., i, th. ii : 121. Pl. 15, fig. 131. 1856. Giebel. Insect. d. vorwelt, 329 .

2925. Smynthurus longicornis. (Thysanura.)

Locality: Prussian amber. Horizon: Ligurian.

1845. Koch-Berendt. Jahrb. min., 1845: 873.

1854. " " Bernst. befindl. organ. reste vorw., i, th. ii : 121. Pl. 15, fig. 130.

1856. Giebel. Insect. 1 . vorwelt, 329.

2926. Smynthurus ovatulus. (Thysanura.)

Locality : Prussian amber. Horizon: Ligurian.

1845. Koch-Berendt. Jahrb. min., 1845: 872.

1854. " Bernst. befindl. organ. reste vorw, i, th, ii: 121-122. Pl. 15, fig. 132.

1856. Giebel. Insect. d. vorwelt, 329.

2927. Sphæropsocus künowi. (Psocina.)

Locality: Prussian amber. Horizon: Ligurian.

1882. Hagen. Stett. ent. zeit., xliii : 226-231, 300. Pl. 2, figs. 11-14.

1883. "6 Stett. ent. zeit., xliv: 320 .

1885. Scudder. Zittel, Handb. palaeont., i, th. ii: 773. Fig. 975.

1886. " Z Zittel-Barrois, Traité de paléont., ii: 773. Fig. 992.

2928. Sterope parthenope. (Odonata.)

Locality: Oeningen, Baden. Horizon: Tortonian.

1850. Hagen. Rev. Odon. (Mém soc. roy. sc. Liége, vi): 358.

See also Agrion (Sterope) parthenope, etc.

2929. Suphalasca proavus. (Hemerobina.)

Locality: Stösschen, Austria. Horizon: Aquitanian.

1866. Hagen. Stett. ent. zeit., xxvii : 461.

See also Ascalaphus proavus.

2930. Sympycna parthenoe. (Odonata.)

Locality: Oeningen, Baden. Horizon: Tortonian.

1890. Scudder. Tert. ins. N. A., 127.

See also Agrion (Sterope) parthenoe, etc.

2931. Tæniopteryx - (Perlina.)

Locality: Prussian amber. Horizon: Ligurian.

1845. Berendt. Bernst. befindl. organ. reste vorw., i : 57 (Tænioptrix). 
2932. Tæniopteryx ciliata. (Perlina.)

Locality : Prussian amber. Horizon: Ligurian.

1854. Pictet. Hagen, Verhandl, zool.-bot. ver. Wien, iv : 226 (undescr.).

1856. " Berendt, Bernst. befindl. organ. reste vorw., ii, th. i : 68 . pl. 6 , figs. $89 b-g$.

See also Nemoura ('Tæniopteryx) ciliata.

2933. Tæniopteryx elongata. (Perlina.)

Locality : Prussian amber. Horizon: Ligurian.

1854. Hagen. Verhandl. zool.-bot. ver. Wien, iv : 226 (undescr.).

1856. " Berendt, Bernst. befindl, organ. reste vorw., ii, th. i: 68-69. Pl. 8, fig. 4.

Tænioptrix —. See Tæniopteryx -

2934. Termes —. (Termitina.)

Locality : Prussian amber. Horizon : Ligurian.

1845. Berendt. Bernst. befindl, organ. reste vorw., i : 57 .

2935. Termes ‥ (Termitina.)

Locality : Prussian amber. Horizon : Ligurian.

1831. Burmeister. Oken, Isis, 1831: 1100.

\section{Termes —. (Termitina.)}

Locality: Brunstatt, Alsatia. Horizon: Middle Oligocene.

1888. Foerster. Mitth. comm. geol. Elsass-Lothr., i : 164.

1889. “ Mitth. comm. geol. Elsass-Lothr., ii : 103.

2937. Termes —. (Termitina.)

Locality : Prussian amber. Horizon: Ligurian.

1825. Guériu. Dict. class. d'hist, nat., viii : 580.

\section{Termes —. (Termitina.)}

Locality : Prussian amber. Horizon: Ligurian.

1838. Ouchakoff. Bull. soc. imp. Mosc., 1838: 37-42. Pl. 1, figs. 1-2.

1840. “ Ann. sc. nat., (2), xiii: 204-207. Pl. 1, fig. B.

1842. “ Cale. journ. nat. hist., ii : 74-78.

1854. Hagen. Verhandl. zool.-bot. ver. Wien, iv: 224 (=? Eutermes antiquus).

1856. " Berendt,Bernst.befindl.organ. reste vorw., ii, th. i: 55 (=? Termes antiquus).

See also Termes antiquus, etc.

2939. Termes —. (Termitina.)

Locality : Prussian amber. ' Horizon : Ligurian.

1820. Schlotheim. Petrefactenk, 43.

2940. Termes -. (Termitina.)

Locality: Prussian amber. Horizon: Ligurian,

1868. Smith. Quart. journ. sc., v: 184. Pl., fig. 9. 


\section{Termes —. (Termitina.)}

Locality : Gurnet Bay, Isle of Wight. Horizon: Bembridge Limestone. 1879. Woodward. Quart, journ. geol. soc. Lond., xxxv : 344. $1879 . \quad$ "Geol. mag., (n. s.), v: 89.

\section{Termes (Calotermes) affinis. (Termitina.)}

Locality: Prussian amber. Horizon: Ligurian.

1856. Hagen. Berendt, Bernst. betindl. organ. reste vorw., ii, th. i: 50-51 (Kalotermes). $P l .5$, figs, $5 b-e$.

See also Termes obscurus, T. (Eutermes) obscurus (1), T. girardi, T. (Termopsis) girardi ; also I, Neunopt., Termes (Calotermes) affinis, Calotermes affinis.

2943. Termes antiquus. (Termitina.)

Locality: Prussian amber. Horizon: Ligurian.

1858. Hagen. Catal. Neuropt. Brit. mus., 24-25.

1858. " Linn. entom., xii : 180-181.

1860. " Linn. entom., xiv: 107.

See also Termes — Ouchakoff, T. (Eutermes) antiquus, Hemerobites antiquus, Maresa plebeja, Termes gracilis.

\section{Termes (Eutermes) antiquus. (Termitina.)}

1742. Sendel.

Locality: Prussian amber. Horizon: Ligurian.

1856. Hagen. Berendt, Bernst. befindl, organ. reste vorw., ii, th, i: 54-55. $P l$. 5, figs. $6 b-i$.

1856. Giebel. Insect. d. vorwelt, 296-297.

1860. Hagen. Linn. entom., xii : 297 (not Germar's Hemerobites antiquus, as Giebel thought).

See also Termes antiquus, etc.

\section{Termes berendtii. (Termitina.)}

Locality: Prussian amber. Horizon: Ligurian.

1854. Pictet. Traité de paléont., $2^{\mathrm{e}}$ éd., ii: 370 (undescr.).

1856. " Berendt, Bernst. befindl, organ, reste vorw., ii, th. i: 49-50. $P l$. 5, figs, $2 b-e$.

See also Calotermes berendtii, Termes (Calotermes) berendtii.

2946. Termes (Calotermes) berendtii. (Termitina.)

Locality: Prussian amber. Horizon: Ligurian.

1854. Hagen. Berendt, Bernst. befindl, organ, reste vorw., ii, th. i: 49 (Kalotermes).

See also Termes berendtii, etc.

2947. Termes bremii. (Termitina.)

Locality: Prussian amber. Horizon: Ligurian.

1852. Giebel. Deutschl. petref., 638 (bremi).

See also T. (Termopsis) bremii, etc.

2948. Termes (Termopsis) bremii. (Termitina.)

Locality: Prussian amber. Horizon: Ligurian.

1849. Heer. Insektenf. tertiärg. Oeningen, ii : 31-32. Pl. 3, fig. 2.

1852. Giebel. Deutschl. petref., 638. 
1856. Giebel.

1856. Hagen.

Insect. d. vorwelt, 294 .

Berendt, Bernst. befindl. organ. reste vorw., ii, th. i: 51-53. Pl. 5, figs. $3 b-e$.

See also Termes pictetii, T. granulicollis, T. bremii, Termopsis bremii.

2949. Termes büchii. (Termitina.)

Compared with T. lucifugus Latr.

Locality: Oeningen, Baden. Horizon: Tortonian.

1865. Heer.

1872. " 6

1876. "

1879. “
Urwelt der Schweiz, 360, 368.

Monde prim. Suisse, 442, 451.

Prim. world Switz., ii : 14, 22.

Urwelt der Schweiz, $2^{e}$ aufl., 386, 393.

2950. Termes croaticus. (Termitina.)

Locality: Radoboj, Croatia. Horizon: Mayencian.

1852. Giebel.

Deutschl. petref,, 638.

See also Termes (Eutermes) croaticus.

Termes (Entermes) croaticus. See Termes (Eutermes) croaticus.

2951. Termes (Eutermes) croaticus. (Termitina.)

Locality: Radoboj, Croatia. Horizon: Mayencian.

1849. Heer. Insektenf. tertiärg. Oeningen, ii ; $34 . \quad P l .3, f i g .5$.

1852. Giebel. Deutschl. petref., 638.

1856. " Insect. d. vorwelt, 295.

1858. Hagen. Linn. entom., xii : 205.

1858. " Catal. Neuropt. Brit. mus., 28 (Entermes).

See also Termes croaticus.

2952. Termes debilis. (Termitina.)

Locality: Prussian amber. Horizon: Ligurian.

1852. Giebel. Deutschl. petref., 638.

See also Termes (Eutermes) debilis.

2953. Termes (Eutermes) debilis. (Termitina.)

Locality: Prussian amber (Heer); Copal (Hagen). Horizon: Ligurian (Heer); Recent (Hagen).

1849. Heer.

1856. Giebel.

1856. Hagen.

Insektenf. tertiärg. Oeningen, ii : $35 . P l .3, f i g .6$.

Insect. d. vorwelt, 295.

See also Termes debilis.

2954. Termes (Termopsis) deciduus. (Termitina.)

Locality: Prussian amber. Horizon: Ligurian.

1856. Hagen. Berendt, Bernst. befindl. organ. reste vorw., ii, th. i: 52-53.

See also Termopsis decidua.

2955. Termes (Eutermes) diaphanus. (Termitina.)

Locality : Prussian amber. Horizon : Ligurian.

1856. Giebel.

Insect. d. vorwelt, 296.

1860. Hagen.

Linn. entom., xii : 297 (=T. pusillus).

See also Termes pusillus, etc. 
2956. Termes girardi. (Termitina.)

Locality: Prussian amber. Horizon: Ligurian.

1888. Schlechtend. Zeitschr. ges. naturw., lxi : 491.

See also Termes (Calotermes) affinis, etc.

2957. Termes (Termopsis) girardi. (Termitina.)

Localitỹ: Prussian amber. Horizon: Ligurian.

1856. Giebel. Insect. d. vorwelt, 294.

1860. Hagen. Linn. entom., xii : 297 (=T. affinis).

See also Termes (Calotermes) affinis, etc.

2958. Termes gracilicornis. (Termitina.)

Locality : Prussian amber. Horizon: Ligurian.

1856. Pictet. Berendt, Bernst. befindl. organ. reste vorw., ii, th. i: 53-54. Pl. 5, figs. $4 b-d$.

See also Termes (Termopsis) gracilicornis, Termopsis gracilicornis.

2959. Termes (Termopsis) gracilicornis. (Termitina.)

Locality : Prussian amber. Horizon : Ligurian.

1854. Pictet. Traité de paléont., $2^{\mathrm{e}}$ éd., ii : 370 (undescr.).

1856. Hagen. Berendt, Bernst. befindl. organ. reste vorw., ii, th. $i$ : 53-54. Pl. 5, figs. 4, $9 b-d$.

See also Termes gracilicornis, etc.

2960. Termes gracilis. (Termitina.)

Locality: Prussian amber. Horizon: Ligurian.

1854. Pictet. Traité de paléont., $2^{e}$ éd., ii : 370 (undescr.).

1856. " Berendt, Bernst. befindl, organ. reste vorw., ii, th. i: 54-55, 124. Pl. 5, figs. 6b-i.

See also Termes antiquus, etc.

2961. Termes granulicollis. (Termitina.)

Locality : Prussian amber. Horizon: Ligurian.

1854. Pictet. Traité de paléont., $2^{\mathrm{e}}$ éd., ii : 370 (undescr.).

1856. " Berendt, Bernst. befindl. organ. reste vorw., ii, th. i: 51.

See also Termes (Termopsis) s $^{n}$ mii, etc.

3962. Termes haidingeri. (Termitina.)

Lotality : Radoboj, Croatia. Horizon : Mayencian.

1849. Heer. Haidinger, Berichti, v: 87.

1852. Giebel. Deutschl. petref., 638.

See also Termes (Termopsis) haidingeri, etc.

2963. Termes (Termopsis) haidingeri. (Termitina.)

Locality : Radoboj, Croatia. Horizon: Mayencian.

1849. Heer. - Insektenf. tertiärg. Oeningen, ii : 26-28.

1852. Giebel. Dentschl, petref., 638.

1854. Goldenberg. Palaeontogr., iv. Pl. 5, fig. 1.

1854. “ Foss. ins. Saarbr. Pl. 3, fig. 1.

1856. Giebel. Insect. d. vorwelt, 293.

See also Hodotermes haidingeri, Termes haidingeri. 


\section{Termes hartungi. (Termitina.)}

Compared with T. lucifugus Latr.

Localities: Radoboj, Croatia; Oeningen, Baden. Horizon : Mayencian, Tortonian.

1858. Heer.

1865. “

1872. "

1876. “

1879. “
Hagen, Linn. entom., xii: 181 (undeser.).

Urwelt der Schweiz, 360, 368. Fig. 230, on p. 366.

Monde prim. Suisse, 442, 451. Fig. 230, on p. 449.

Prim. world Switz., ii : 14, 22. Fig. 230.

Urwelt der Schweiz, $2^{\circ}$ aufl, 386, 393. Fig. 271, on p. 392.

2965. Termes hassencampi. (Termitina.)

Locality: Sieblos, Rhenish Prussia. Horizon: Aquitanian.

1860. Heer.

Hassencamp, Würzb. naturw. zeitschr., i: 79 (undescr.).

2966. Termes insignis. (Termitina.)

Compared with 'T. fatalis L.

Locality: Oeningen, Baden. Horizon: Tortonian.

1852. Giebel.

1865. Heer.

1872. “

1876. “

1879. "

Deutschl. petref., 638.

Urwelt der Sehweiz, 367.

Monde prim. Suisse, 451.

Prim. world Switz., ii : 22.

See also Termes (Termopsis) insignis, ete.

2967. Termes (Termopsis) insignis. (Termitina.)

1849. Heer.

Locality: Oeningen, Baden. Horizon: Tortonian.

1852. Giebel.

Insektenf. tertiärg. Oeningen, ii: 29-31. $\quad P l .3, f i g .1$.

See also Hodotermes insignis, Termes insignis.

\section{Termes (Eutermes) mœstus. (Termitina.)}

Locality: Prussian amber. Horizon: Ligurian.

1856. Giobel.

Insect. d. vorwelt, 497.

See also Termes (Eutermes) obseurus (2).

\section{Termes obscurus. (Termitina.)}

Locality : Radoboj, Croatia. Horizon: Mayencian.

1852. Giebel.

Deutschl. petref., 638.

1854. Pictet.

Traité de paléont., $2^{\ominus} 6$ d., ii : 370 (undescr.).

1856. 66

Berendt, Bernst. befindl, organ, reste vorw., ii, th. i: 50-51. $\quad P l$. 5, figs. $3 b-e$.

See also Termes (Calotermes) affinis, etc.

Termes (Entermes) obscurus. See Termes (Eutermes) obscurus (1).

2970. Termes (Eutermes) obscurus (1). (Termitina.)

Locality: Radoboj, Croatia. Horizon: Mayencian.

1849. Heer. Insektenf, tertiärg. Oeningen, ii : 33-34. Pl. 3, fig. 4.

1858. Hagen. Linn. entom., xii: 204-205.

1858. “ Catal. Neuropt. Brit. mus., 28 (Entermes).

See also Termes (Calotermes) affinis, etc. 
2971. Termes (Eutermes) obscurus (2). (Termitina.)

Locality: Prussian amber. Horizon: Ligurian.

1856. Giebel. Insect. d. vorwelt, 297.

1858. Hagen. Linn. entom., xii : 205, 297.

See also Termen (Eutermes) mostus.

\section{Termes peccanæ. (Termitina.)}

Locality : Monte Bolca, Italy. Horizon : Ligurian.

1856. Massalongo. Stud. paleont., 19. Pl. 2, figs. 5,6 .

1886. Omboni. Atti r. ist. Ven,, (6), iv: 1426 (peccanœ). Pl. 2, fig. 7.

1886. " Ins. foss. ven., 6. Pl. 2, fig. 7.

Termes peccance. See Termes peccanæ.

2973. Termes pictetii. (Termitina.)

Locality: Prussian amber. Horizon: Ligurian.

1854. Berendt. MSS.

1854. Pictet. Traité de paléont., $2^{\text {e }}$ éd., ii: 370. Pl.40, figs. 23, 23a.

1856. Berendt. Bernst. befindi. organ. reste vorw., ii, th. i: 51-53. Pl. 5, figs. $3 b-e$.

See also Termes (Termopsis) bremii, etc.

\section{Termes pristinus. (Termitina.)}

Locality: Radoboj, Croatia. Horizon: Mayencian.

1843. Charpentier. Verhandl. leop.-carol. akad. naturf., xx: 409-410. Pl. 23, figs. $2-4$.

1847. Unger. Chloris protogæa, 134-135 (without specific name). Pl.44, fig.1. 1849. Heer. Insektenf. tertiärg. Oeningen, ii ; 33, note.

1852. Giebel. Deutschl. petref., 638.

1858. Hagen. Linn. entom., xii : 161-162.

1858. "6 Catal. Neuropt. Brit. mus., 22.

See also Termes (Eutermes) pristinus, T. procerns, T. (Termopsis) procerus, Hodotermes procerus.

2975. Termes (Eutermes) pristinus. (Termitina.)

Locality: Radoboj, Croatia. Horizon: Mayencian.

1849. Heer. Insektenf. tertiärg. Oeningen, ii : 32-33. Pl. 3, fig. 3.

1856. Giebel. Insect. d. vorwelt, 294-295.

See also Termes pristinus, ete.

\section{Termes procerus. (Termitina.)}

Locality: Radoboj, Croatia. Horizon: Mayencian.

1852. Giebel. Deutschl. petref., 638.

See also Termes pristinus, ete.

2977. Termes (Termopsis) procerus. (Termitina.)

Locality: Radoboj, Croatia. Horizon: Mayencian.

1849. Heer. Insektenf. tertiärg. Oeningen, ii : 23-26. Pl. 2, fig. 5.

1856. Giebel. Insect. d. vorwelt, 292-293.

See also Termes pristinus, etc. 
2978. Termes pusillus. (Termitina.)

1852. Giebel. . Dentscbl. petref., 638.

Locality: Prussian amber. Horizon: Ligurian.

See also Termes (Eutermes) pusillus, T. diaphanus, T. (E.) punctatus.

2979. Termes (Eutermes) pusillus. (Termitina.)

Localities: Prussian amber (Heer); Copal (Hagen). Horizon : Ligurian (Heer);

Recent (Hagen).

1849. Heer. 1856. Giebel.

Insektenf. tertiärg. Oeningen, ii : 35-36. $\quad P l .3$, fig. 7. 1856. Hagen. Insect. d. vorwelt, 295.

See also Termes pusillus, etc.

2980. Termes (Eutermes) punctatus. (Termitina.)

Locality: Prussian amber. Horizon: Ligurian.

1856. Giebel.

Insect. d. vorwelt, 296.

1860. Hagen. Linn. entom., xii : 297 (=? T. pusillus).

See also Termes pusillus, etc.

2981. Termes spectabilis. (Termitina.)

Compared with T. fatalis $\mathbf{L}$.

Locality: Oeningen, Baden. Horizon: Tortonian.

1852. Giebel.

Dentschl. petref., 638 .

1865. Heer.

1872. "

Urwelt der Schweiz, 367.

Monde prim. Suisse, 451.

1876. "

Prim. world Switz., ii : 22.

1879. “

Urwelt der Schweiz, $2^{\mathrm{e}}$ aufl., 393.

See also Termes (Termopsis) spectabilis, etc.

2982. Termes (Termopsis) spectabilis. (Termitina.)

Locality : Oeningen, Baden. Horizon: Tortonian.

1849. Heer. Insektenf. tertï̈rg. Oeningen, ii : 28-29. Pl. 2, fig. 6.

1856. Giebel. Insect. d. vorwelt, 293.

See also Hodotermes spectabilis, Termes spectabilis.

2983. Termopsis bremii. (Termitina.)

1854. Hagen. Verhandl. zool.-bot. ver. Wien, iv: 222.

1858. " Linn. entom., xii: 78-80.

1858. “ "Catal. Neuropt. Brit. mus., 12.

1860. " Linn. entom., xiv: 102.

See also Termes (Termopsis) bremii, etc.

2984. Termopsis decidua. (Termitina.)

Locality : Prussian amber. Horizon: Ligurian.

1858. Hagen. Linn. entom., xii : 80-81 (deciduus.)

1858. “ Catal. Neuropt. Brit. mus., 13.

See also Termes (Termopsis) deciduus.

Termopsis. girardi. See Termes (Termopsis) girardi. 
2985. Termopsis gracilicornis. ('Termitina.)

1854. Hagen. Verhandl. zool.-bot. ver. Wien, iv : 222 (undescr.).

1858. " Linn. entom., xii : 80 .

1858. " Catal. Neuropt. Brit. mus., 12-13.

See also Termes gracilicornis, etc.

Termopsis haidingeri. See Termes (Termopsis) haidingeri.

2986. Termopsis heeriana. (Termitina.)

Locality: Schossnitz, Silesia. Horizon: Miocene.

1855. Göppert. Tert. flora Schossnitz. Pl. 26, fig. 53.

See also Termopsis heerii, Hodotermes heerianus.

2987. Termopsis heerii. (Termitina.)

Locality : Schossnitz, Silesia. Horizon: Miocene.

1855. Göppert. Tert. flora Schossnitz, p. vii.

See also Termopsis heeriana, Hodotermes heerianus.

Termopsis insignis. See Termes (Termopsis) insignis.

Termopsis procera. See Termes (Termopsis) procerus.

Termopsis spectabilis. See Termes (Termopsis) spectabilis.

2988. Tinodes grossa. (Hydropsychidæ.)

Locality: Prussian amber. Horizon: Ligurian.

1856. Hagen. Berendt, Bernst. befindl. organ. reste vorw., ii, th. i: 117.

1864. " $\quad$ Phryg. synopsis synom., 89.

1864. " Verhandl. zool.-bot, gesellsch. Wien, xiv : 887.

2989. Tinódes paludigina. (Hydropsychidæ.)

Locality : Florissant, Colo. Horizon: Oligocene.

1890. Scudder. Tert. ins. N. A., 190-191. Pl. 15, fig. 9.

2990. Tinodes prisca. (Hydropsychidæ.)

Locality: Prussian amber. Horizon: Ligurian.

1856. Hagen.

Berendt, Bernst. befiudl. organ. reste vorw., ii, th.i: 117. Pl. 7, figs. $6 b-d ; p l .8$, fig. 29.

1864. “ Phryg. synopsis synom., 90.

1864. " Verhandl, zool.-bot, gesellsch. Wien, xiv : 888.

See also Rhyacophila prisca.

\section{Tribochrysa - (3 sp.). (Hemerobina.)}

Locality: Florissant. Colo. Horizon: Oligocenè.

1882. Scudder. Proc. Bost. soc. nat. hist., xxi : 408.

1883. "6 Ann.rep.U.S. geol. surv. terr., xii : 283.

See also Chrysopa (vic.) —, Nothochrysa —, Nymphon (vic.) — , and the following species.

2992. Tribochrysa firmata. (Hemerobina.)

Locality : Florissant, Colo. Horizon : Oligocene.

1890. Scudder. Tert. ins. N. A., 172. Pl. 14, figs. 6, 7, 10,11.

See also Tribochrysa 
2993. Tribochrysa inequalis. (Hemerobina.)

Locality : Florissant, Colo. Horizon: Oligocene.

1885. Seudder. Zittel, Handb. palaeont., i, th. ii: 777. Fig. 982.

1886. "6 Zittel-Barrois, Traité de paléont., ii: 777. Fig. 999.

1890. " Tert. ins. N. A, 170-171.

See also Tribochrysa

2994. Tribochrysa vetuscula. (Hemerobina.)

Locality: Florissant, Colo. Horizon: Oligocene.

1890. Scudder. Tert. ins. N. A., 170. Pl. 14, fig. 9.

See also Tribochrysa -

2995. Trichostomum proavum. (Sericostomidæ.)

Locality : Prussian amber. Horizon: Ligurian.

1854. Hagen. Verhandl. zool.-bot. ver. Wien, iv: 229 (undescr.).

1856. " Berendt, Bernst. befindl. organ. reste vorw., ii, th. i: 103. Pl. 8, fig. 25.

See also Gœra proava.

2996. Troctes succinicus. (Psocina.)

Locality : Prussian amber. Horizon : Ligurian.

1883. Kolbe. Stett. ent. zeit., xliv : 190.

See also Atropos succinea.

\section{HEMIPTERA.}

\section{7.}

Locality : [Sinigaglia ?], Italy. Horizon:

1841. Bassi.

Atti reun. scienz. ital., iii : 401.

2998.

("Baumwanze.")

Locality : Brunstatt, Alsatia. Horizon: Middle Oligocene.

1885. Foerster. Tagebl. versamml, deutsch. naturforsch., lviii : 392.

1885. " " Mitth. ober-elsässs. tertiär., 7.

1888. " Mitth, comm, geol. Elsass-Lothr., i: 164.

2999.

(10 sp.).

Locality: Brunstatt, Alsatia. Horizon: Middle Oligocene.

1889. Foerster. Mitth. comm. geol. Elsass-Lothr, ii : 102.

3000.

Locality: Sinigaglia, Italy. Horizon: Helvetian?.

1842. Procaccini. Nuov, ann. sc. nat., vii : 449.

3001. (several). ("Water-bugs.")

Locality: Florissant, Colo. Horizon: Oligocene.

1881. Scudder. Bull. U. S. geol. surv. terr., vi : 292.

1883. "6 Ann. rep. U. S. geol. surv. terr., xii : 282.

3002

Locality: Gurnet Bay, Isle of Wight. Horizon: Bembridge Limestone.

1879. Woodward. Quart. journ. geol. soc. Lond., xxxv : 344.

1879. " Geol, mag. (n. s.), v: 88. 
3003.

(Aphides ?.)

Locality: British Columbia. Horizon:

1879. Scudder. 1879.
Rep. progr. geol. surv. Can., 1877-78: 186B.

Ins, tert. Nicola, 11.

3004. (several). (Aphides-Aphidinæ.)

Locality: Florissant, Colo. Horizon: Oligocene.

1881. Scudder. 1883. “
Bull. U. S. geol. surv. terr., vi : 293.

Ann. rep. U. S. geol. surv. terr., xii : 282.

3005.

Locality : Florissant, Colo. Horizon: Oligocene.

1881. Scudder. 1883. "

1881. Scudder. 1883. “
Bull. U. S. geol. surv. terr., vi : 293.

Ann. rep. U. S. geol. surv. terr., xii : 282.

\section{(2 sp.). (Aradidæ.)}

Locality : Florissant, Colo. Horizon: Oligocene.

Ann. rep. U. S. geol. surv. terr., xii : 282.

3007. (several). (Cercopidæ.)

Locality: Florissant, Colo. Horizon : Oligocene. 1881. Scudder. 1883.

Bull. U. S. geol. surv. terr., vi : 292.

Ann. rep. U. S. geol. surv. terr, xii : 282.

3008. ("Cicadariæ.")

Locality: Rouffach, Alsatia. Horizon: Tongrian. 1881. Bleicher. Bull. soc. géol. Fr., (3), viii : 226.

3009. (2 sp.). ("Cicadellidæ.")

Locality: Brunstatt, Alsatia. Horizon: Middle Oligocene. 1889. Foerster. Mitth. comm. geol. Elsass-Lothr., ii : 102.

1796.

$$
\text { 3010. — ("Cimicidæ.") }
$$

Locality : (Italy ?). Horizon: Ittiol. veron., i: 31.

3011. (9 sp.). ("Cimicidæ.")

Locality: Brunstatt, Alsatia. Horizon: Middle Oligocene. 1889. Foerster. Mitth. comm. geol. Elsass-Lothr., ii : 102.

3012. (1 or 2). (Coreidæ-Alydina.)

Locality: Florissant, Colo. Horizon: Oligocene.

1881. Scudder. Bull. U. S. geol. surv. terr., vi : 292. 1883. "

3013.

Locality: Brunstatt, Alsatia. Horizon: Middle Oligocene.

1889. Foerster, Mitth. comm. geol. Elsass-Lothr., ii : 102 . 
3014. (several). (Fulgoridæ.)

Locality : Florissant, Colo. Horizon: Oligocene.

1881. Scudder.

Bull. U. S. geol. surv. terr., vi : 293.

1883. “ Ann. rep. U. S. geol. surv. terr., xii : 282.

3015.

(Jassides.)

Locality: Brunstatt, Alsatia. Horizon: Middle Oligocene.

1889. Foerster. Mitth. comm. geol. Elsass-Lothr., ii : 102.

3016. (several). (Lygæidæ.)

Locality : Aix, France. Horizon: Ligurian.

1829. Curtis. Edinb. new phil. journ., vii : 296.

1829. " Murchison-Lyell, Tert. form. Aix, 12.

3017. (7 sp.). (Lygæidæ.)

Locality : Brunstatt, Alsatia. Horizon: Middle Oligocene.

1889. Foerster. Mitth. comm. geol. Elsass-Lothr., ii : 102.

3018. - (several). (Lygæidæ.)

Locality : Florissant, Colo. Horizon : Oligocene.

1881. Scudder. Bull. U. S. geol. surv. terr., vi : 292.

1883. " A Ann. rep. U. S. geol. surv. terr., xii : 282.

3019. - (several). (Pentatomidæ.)

1881. Scudder. Bull. U.S. geol. surv, terr., vi: 292.

1883. " Ann. rep. U.S. geol, surv. terr., xii: 282.

3020. - (several). (Pentatomidæ-Corimalænidæ.)

Locality: Florissant, Colo. Horizon: Oligocene.

1881. Scudder. Bull. U. S. geol. surv. terr., vi : 292.

1883. " Ann. rep. U. S. geol. surv. terr., xii : 282.

3021. - $(8 \mathrm{sp}$.$) . (Pentatomidæ-Cydnida.)$

Locality: Brunstatt, Alsatia. Horizon: Middle Oligocene.

1889. Foerster. Mitth. comm. geol. Elsass-Lothr., ii : 102.

3022.

(Reduviidæ.)

Locality: Brunstatt, Alsatia. Horizon: Middle Oligocene.

1889. Foerster. Mitth. comm. geol. Elsass-Lothr., ii: 102.

3023. —— (several). (Reduviidæ.)

Locality : Florissant, Colo. Horizon: Oligocene.

1881. Scudder. Bull. U. S. geol. surv. terr., vi : 292.

1883. " Ann. rep. U. S. geol, surv. terr., xii : 282.

3024. Acanthosoma? - (Pentatomidæ.)

Locality: Brunstatt, Alsatia, Horizon: Middle Oligocene.

1890. Foerster. In litt. 
3025. Acanthosoma livida. (Pentatomidæ.)

Locality: Radoboj, Croatia. Horizon: Mayencian.

1853. Heer. Insektenf. tertiärg. Oeningen, iii: 41-42. Pl.3, fig.6; pl.8, fig. 3 . 1856 " 6 1856. Giebel:

Ber. oesterreich. litt., 1850-'53: 200.

Insect. d. vorwelt, 346 .

3026. Acanthosoma maculata. (Pentatomidæ.)

Locality : Radoboj, Croatia. Horizon: Mayencian.

1853. Heer.

1855. "

Insektenf, tertiärg. Oeningen, iii : 42-43. $P l .3$, fig. $7 ; p l .8$, fig. 5 . Ber. oesterreich. litt., 1850-'53: 200.

1856. Giebel.

Insect. d. vorwelt, 346-347.

1885. Scudder. Zittel, Handb. palaeont., i, th. ii : 785. Fig. 1003.

1886. " Zittel-Barrois, Traité de paléont., ii : 785. Fig. 1020.

3027. Acanthosoma morloti. (Pentatomidæ.)

Locality: Radoboj, Croatia. Horizon: Mayencian.

1853. Heer. Insektenf. tertiärg. Oeningen, iii : 39-41. Pl. 3, fig. $5 ;$ pl.8. fig. 4. 1855. " Ber, oesterreich. litt., 1850-'53: 200.

1856. Giebel. Insect. d. vorwelt, 346.

3028. Achrestocoris cinerarius. (Coreidæ.)

Locality: Florissant, Colo. Horizon: Oligocene.

1890. Scudder. Tert. ins. N. A., 413-414. Pl. 22, fig. 1.

3029. Acocephalus adæ. (Jassides.)

Locality: Green River, Wyo. Horizon: Oligocene.

1878. Scudder. Bull. U. S. geol. surv. terr., iv : 771.

1890. "6 Tert. ins. N. A., 311. Pl. 6, fig. 29.

3030. Acocephalus callosus. (Jassides.)

Locality : Florissant, Colo. Horizon: Oligocene.

1890. Scudder. Tert. ins. N. A., 311-312. Pl.19, fig. 15.

3031. Acocephalus crassiusculus. (Jassides.)

Locality : Radoboj, Croatia. Horizon: Mayencian.

1853. Heer. Insektenf. tertiärg. Oeningen, iii : 112. $P l .13, f i g .2$.

1855. " Ber. oesterreich. litt., 1850-53: 203.

1856. Giebel. Insect. d. vorwelt, 385 .

3032. Acocephalus curtulus. (Jassides.)

Locality: Radoboj, Croatia. Horizon: Mayencian.

1853. Heer.

1855. “

1856. Giebel.

Insektenf. tertiärg. Oeningen, iii : 110-112. Pl. 13, fig. 1.

Ber. oesterreich. litt., 1850-'53: 203.

Insect. d. vorwelt, 384-385.

3033. Ælia obsoleta. (Pentatomidæ.)

Locality : Oeningen, Baden. Horizon: Tortonian.

1853. Heer.

Insektenf. tertiärg. Oeningen, iii: $32 . \quad P l .2$, fig. 9.

1856. Giebel.

Insect. d. vorwelt, 343.

See also Pentatoma obsoletum. 
3034. \#thus punctulatus. (Pentatomidæ.)

Locality: Green River, Wyo. Horizon: Oligocene.

1878. Scudder. Bull. U.S. geol. surv. terr., iv: 769-770.

See also Stenopelta punctulata.

\section{Atorhinus - (Capsidæ.)}

Locality: Prussian amber. Horizon: Ligurian.

1890. Scudder. Tert. ins. N. A., 362.

3036. Agallia abstructa. (Jassides.)

1890. Scudder. Tert. ins. N. A., 307. Pl. 19, fig. 5.

3037. Agallia flaccida. (Jassides.)

Locality: Florissant, Colo. Horizon: Oligocene.

1890. Scudder. Tert. ins. N. A., 306. Pl. 19, fig. 18.

3038. Agallia instabilis. (Jassides.)

Locality : Florissant, Colo. Horizon: Oligocene.

1890. Scudder. Tert. ins. N. A., 306-307. Pl. 21, fig. 1.

3039. Agallia lewisii. (Jassides.)

Locality: Florissant, Colo. Horizon: Oligocene.

1890. Scudder. Tert. ins. N. A., 305-306. Pl. 19, figs. 7, 21.

3040. Aleurodes aculeatus. (Coccidæ.)

Compared with A, chelidonii Latr.

1856. Menge.

Locality: Prussian amber. Horizon : Ligurian. Progr. petrischule Danzig, 1856: 18.

3041. Alydus herrichii. (Coreidæ.)

Compared with A. lateralis Germ.

Locality: Oeningen, Baden. Horizon: Tortonian.

1861. Heer. Rech. climat pays tert., 203 (undescr.).

3042. Alydus pristinus. (Coreidæ.)

Compared with A. calcaratus.

Locality : Vic. Bonn, Rhenish Prussia. Horizon: Aquitanian?.

1837. Germar. Fauna insect. Europae, xix : 18. Pl. 18.

1852. Giebel. Deutschl. petref., 636.

1856. " Insect. d. vorwelt, 349 ,

3043. Alydus pulchellus. (Coreidæ.)

Locality: Oeningen, Baden. Horizon: Tortonian.

1853. Heer. Insektenf. tertiärg. Oeningen, iii : 47-49,128. Pl. 4, fig. 2; pl. 8, fig. $9 ; p l .14$, fig. 19.

1856. Giebel. Insect. d. vorwelt, 349 .

3044. Amalancon lutosus. (Aphides.)

Locality : Florissant, Colo. Horizon: Oligocene.

1890, Scudder, Tert, ins, N. A., 270-271. Pl. 18, fig. 13. 
3045. Anasa priscoputida. (Coreidæ.)

1890. Scudder. Tert. ins. N. A., 412-413. Pl. 24, fig. 4.

3046. Anconatus bucktoni. (Aphides.)

Locality : Flori ssant, Colo. Horizon: Oligocene.

1890. Scudder. Tert. ins. N. A., 272-273.

3047. Anconatus dorsuosus. (Aphides.)

Locality: Florissant, Colo. Horizon: Oligocene.

1883. Buckton. Monogr. Brit. aphid., iv: 177-178. Pl. 133, fig. 4.

1890. Scudder. Tert. ins. N. A., 272. Pl. 18, fig. 9.

3048. Aphana atava. (Fulgorina.)

Locality: White River, Colo. Horizon: Oligocene.

1877. Scudder. Bull. U. S. geol. surv. terr., iii : 759-760.

1890. " Tert.ins. N. A., 281-282. Pl. 5, figs. 96,97.

3049. Aphana rotundipennis. (Fulgorina.)

Locality: Green River, Wyo. Horizon: Oligocene.

1878. Scudder. Bull. U. S. geol. surv. terr., iv: 772.

1890. "6 Tert. ins. N. A., 282. Pl. 6, fig. 27.

3050. Aphantaphis exsuca. (Aphides.)

Locality: Florissant, Colo. Horizon: Oligocene.

1890. Scudder. Tert. ins. N. A., 254.

3061. Aphidioides succifera. (Aphides.)

Compared with Aphis pinophila.

Locality: Prussian amber. Horizon: Ligurian.

1856. Motschulsky. Etud. entom., v: 29. Pl., fig. 8.

1883. Buckton. Monogr. Brit. aphid., iv: 164 (Aphioides). Pl. 132, fig. 7.

3052. Aphidopsis _. (Aphides.)

Locality : Florissant, Colo. Horizon : Oligocene.

1890. Soudder. Tert. ins. N. A., 266. $P l .18$, fig. 11.

3053. Aphidopsis dalli. (Aphides.)

Locality : Florissant, Colo. Horizon: Oligocene.

1890. Seudder. Tert. ins. N. A., 264-265.

3054. Aphidopsis emaciata. (Aphides.)

Locality : Florissant, Colo. Horizon: Oligocene.

1890. Scudder. Tert. ins. N. A., 265.

3055. Aphidopsis hargeri. (Aphides.)

Locality : Florissant, Colo. Horizon: Oligoceno.

1890. Scudder. Tert. ins. N. A., 262-263.

3056. Aphidopsis lutaria. (Aphides.)

Locality: Florissant, Colo. Horizon: Oligocene.

1890. Scudder. Tert. ins. N. A., 263.

Bull. $71-25$ 


\section{Aphidopsis margarum. (Aphides.)}

1890. Scudder.

Locality : Florissant, Colo. Horizon: Oligocene. Tert. ins. N. A., $264 . \quad P l .18$, fig. 8.

3058. Aphidopsis subterna. (Aphides.)

Locality : Florissant, Colo. Horizon: Oligocene.

1890. Scudder. Tert. ins. N. A., 261-262.

Aphioides succifera. See Aphidioides succifera.

$$
\text { 3059. Aphis —. (Aphides.) }
$$

Locality: Prussian amber. Horizon: Ligurian.

1845. Berendt. Bernst. befindl. organ. reste vorw., i: 55.

$$
\text { 3060. Aphis —. (Aphides.) }
$$

Locality: Aix, France. Horizon: Ligurian.

1829. Curtis. Edinb. new phil. journ., vii : 296.

1829. " Murchison-Lyell, Tert. form. Aix, 12.

3061. Aphis araneiformis. (Aphides.)

Compared with A. pomi.

Locality: Prussian amber. Horizon: Ligurian.

1856. Germ.-Ber. Berendt, Bernst. befindl. organ. reste vorw., ii, th. i: 6. Pl. 1, fig. 8.

1883. Buckton. Monogr. Brit. aphid., iv: 165 . Pl. 131, fig. 4.

Aphis cimicoides. See Lachnus cimicoides.

3062. Aphis delicatula. (Aphides.)

Locality: Aix, France. Horizon: Ligurian.

1856. Heer.

Viert. naturf. gesellsch. Zïrich, i : 40. Pl. 2, fig. 13.

1883. Buckton.

Monogr. Brit. aphid., iv: 170. Pl. 132, fig. 6.

3063. Aphis hirsuta. (Aphides.)

Compared with A. pieridis.

Locality: Prnssian amber. Horizon: Ligurian.

1856 Germ.-Ber. Berendt, Bernst. befindl. organ. reste vorw., ii, th. i: 6. Pl. 1, fig. 7.

1883. Buckton. Monogr. Brit. aphid., iv: 166. Pl. 131, fig. 5.

3064. Aphis largiflua. (Aphides.)

Locality: Prussian amber. Horizon: Ligurian.

1856. Menge. Progr. petrischule Danzig, 1856: 19 (undescr.).

3065. Aphis longicaudata. (Aphides.)

Locality: Ambérieux, France. Horizon: -

1853. Millière.

Ann. soc. ent. Fr. (\$), i : 9-11 (longicaudatus). $P l .3^{11}$.

1883. Buckton. Monogr. Brit. aphid., descr. pl. exxxii. Pl.132, fig. 1.

3066. Aphis longicornis. (Aphides.)

Locality: Prussian amber. Horizon: Ligurian.

1856, Menge, Progr. petrischule Danzig, 1856: 19 . 
3067. Aphis macrostyla. (Aphides.)

Locality: Radoboj, Croatia. Horizon: Mayencian.

1853. Heer.

1855. " "

1856. Giebel.

1883. Buckton.
Insektenf. tertiärg. Oeningen, iii : 121-122. $\quad P l .15$, fig. 3.

Ber. oesterreich. litt., 1850-53: 203.

Insect. d. vorwelt, 389.

Monogr. Brit. aphid., iv : 169-170. Pl. 132, fig. 2.

3068. Aphis morloti. (Aphides.)

Locality : Radoboj, Croatia. Horizon: Mayencian.

1853. Heer. Insektenf. tertiärg. Oeningen, iii : 122-123. Pl. 15, figs. 5ab.

1855. " " Ber. oesterreich. litt., 1850-53: 203.

1856. Giebel. Insect. d. vorwelt, 389.

1883. Buckton. Monogr. Brit. aphid., iv : 171 (morlotti). Pl. 132, fig. 9.

See also Lachnus — Buckton.

Aphis morlotti. See Aphis morloti.

3069. Aphis pallescens. (Aphides.)

Locality : Radoboj, Croatia. Horizon: Mayencian.

1853. Heer. Insektenf. tertiärg. Oeningen, iii : 122. $P l .15, f i g .4$.

1855. " Ber. oesterreich. litt., 1850-53: 203.

1856. Giebel. Insect. d. vorwelt, 389.

1883. Buckton. Monogr. Brit. aphid., iv : 170-171. Pl. 132, figs. 10, 10a.

3070. Aphis retrolactens. (Aphides.)

Locality: Prussian amber. Horizon: Ligurian.

1856. Menge. Progr. petrischule Danzig, 19 (undescr.).

3071. Aphis transparens. (Aphides.)

Locality: Prussian amber. Horizon: Ligurian.

1856. Germ.-Ber. Berendt, Bernst. befindl. organ. reste vorw., ii, th. i: 7. Pl. 2, fig. 1.

1883. Buckton. Monogr. Brit. aphid., iv: 168. Pl. 131, fig. 10.

3072. Aphrophora -. (Cercopidæ.)

Locality: Prussian amber. Horizon: Ligurian.

1845. Berendt. Bernst. befindl, organ. reste vorw., i: 55.

3073. Aphrophora - (4 sp.). (Cercopidæ.)

Locality: Brunstatt, Alsatia. Horizon: Middle Oligocene.

1890. Foerster. In litt.

3074. Aphrophora - (Cercopidæ.)

Locality: Prussian amber. Horizon: Ligurian.

1835. Gravenhorst. Uebers. schles. gesellsch. vater1. cult., 1834 : 93.

3075. Aphrophora -. (Cercopidæ.)

Locality : Florissant, Colo. Horizon: Oligocene.

1890. Scudder. Tert. ins. N. A., 337. Pl. 19, fig. 10. 
3076. Aphrophora (Ptyelus) carbonaria. (Cercopidæ.)

Compared with A. leucophthalma, Europe.

Locality : Prussian amber. Horizon: Ligurian.

1856. Germ.-Ber. Berendt, Bernst. befindl. organ. reste vorw., ii, th. i: 11-12 (Ptyela). Pl. 2; fig. 3.

3077. Aphrophora electrina. (Cercopidæ.)

Compared with A. (Ptyela) leucophthalma, Europe.

Locality: Prussian amber. Horizon: Ligurian.

1856. Germ.-Ber. Berendt, Bernst. befindl. organ. reste vorw., ii, th. i: 10-11. $P l .1, f i g .15$.

3078. Aphrophora molassica. (Cercopidæ.)

Locality: Greith, Hohe Rhonen, Germany. Horizon: Aquitanian.

1853. Heer.

1856. Giebel.

Insektenf. tertiärg. Oeningen, iii : 107-109. Pl. 12, fig. 9.

Insect. d. vorwelt, 383.

Aphrophora pinguicola. See Aphrophora pinguicula.

3079. Aphrophora pinguicula. (Cercopidæ.)

Locality: Aix, France. Horizon: Ligurian.

1853. Heer.

1856. "

Insektenf. tertiärg. Oeningen, iii : 107. $P l .12$, fig. 8.

Viert. naturf. gesellsch. Zürich, i: 39.

1856. Giebel. Insect. d. vorwelt, 383 (pinguicola).

3080. Aphrophora spumarioides. (Cercopidæ.)

Locality: Oeningen, Baden. Horizon: Tortonian.

1853. Heer.

Insektenf. tertiärg. Oeningen, iii : 106-107. Pl. 12, fig. 7.

1856. Giebel.

Insect. d. vorwelt, 383.

3081. Aphrophora spumifera. (Cercopidæ.)

Localities : Radoboj, Croatia; Aix, France. Horizon : Majencian, Ligurian. 1853. Heer.

1855. “

1856. “"

Insektenf. tertiärg. Oeningen, iii: 104-106, 134. Pl. 12, fig. 6.

Ber. oesterreich. litt., 1850-'53: 202.

1856. Giebel.

Viert. naturf. gesellsch. Zürich, i : 39.

Insect. d. vorwelt, 383.

See also Tettigonia — Curtis, Tettigonia spumaria.

3082. Aphrophora vetusta. (Cercopidæ.)

Compared with Gspona glanca, Brazil.

Locality : Prussian amber. Horizon: Ligurian.

1856. Germ.-Ber, Berendt, Bernst. befindl, organ. reste vorw., ii, th. i: 11 . Pl. 1, fig. 16.

3083. Aporema præstrictum. (Capsidæ.)

Locality: Florissant, Colo. Horizon: Oligocene.

1890. Scudder. Tert. ins. N. A., 370. Pl. 20, fig. 4.

3084. Aradus - (Aradidæ.)

Locality: Prussian amber. Horizon: Ligurian.

1845. Berendt. Bernst, befindl. organ, reste vorw., i: 55 . 
3085. Aradus - (larva). (Aradidæ.)

Locality: Prussian amber. Horizon : Ligurian.

1856. Germ.-Ber. Berendt, Bernst. befindl. organ. reste vorw., ii, th. i: 23. Pl. 3, fig. 17.

\section{Aradus -. (Aradidæ,)}

Compared with A. corticalis.

Locality: Aix, France. Horizon: Ligurian.

1847. Hope.

Trans. ent. soc. Lond., iv : 252.

3087. Aradus -. (Aradidæ.)

Locality : Florissant, Colo. Horizon: Oligocene.

1885. Scudder. Zittel, Handb. palaeont., i, th. ii : 784.

1886. " " Zittel-Barrois, Traité de paléont., ii: 784.

1886. " " Bull. U. S. geol. surv., No. 31: 62.

3088. Aradus - (Aradidæ.)

Locality: Aix, France. Horizon: Ligurian.

1829. Serres. Géogn. terr tert., 227.

3089. Aradus antediluvianus. (Aradidæ.)

1853. Heer.

1855. " "6

Locality: Radoboj, Croatia. Horizon: Mayencian.

1856. Giebel.

Insektenf, tertiärg. Oeningen, iii : 73. Pl.5, fig. 16.

Ber. oesterreich. litt., 1850-'53: 201.

Insect. d. vorwelt, 362 .

3090. Aradus assimilis. (Aradidæ.)

Compared with A. depressus, Europe.

Locality: Prussian amber. Horizon: Ligurian.

1856. Germ.-Ber. Berendt, Bernst, befindl organ. reste vorw., ii, th. i: 22. Pl. 2, fig. 12.

3091. Aradus consimilis. (Aradidæ.)

Compared with A. annulicornis.

Locality: Prussian amber. Horizon : Ligurian.

1856. Germ.-Ber. Berendt, Bernst. befindl. organ. reste vorw., ii, th. i: 23. Pl. 2, fig. 13.

3092. Aradus superstes. (Aradidæ.)

Compared with A. corticalis, Europe.

Locality : Prussian amber. Horizon: Ligurian.

1856. Germ.-Ber. Berendt, Bernst. befindl. organ. reste vorw., ii, th. i: 22. Pl. 2, fig. 11.

1885. Scudder. Zittel, Handb. palaeont., i, th. ii: 784. Fig. 998.

1886. " Zittel-Barrois, Traité de paléont, ii : 784. Fig. 1015.

3093. Archilachnus mudgei. (Aphides.)

Locality: Florissant, Colo. Horizon: Oligocene,

1890. Scudder. Tert. ins. N. A., 247-248. 
3094. Archilachnus pennatus. (Aphides.)

Locality: Florissant, Colo. Horizon: Oligocene.

1883. Buckton. Monogr. Brit. aphid., iv: 177. Pl. 133, fig. 3.

1890. Scudder. 'T'ert. ins. N. A., 247. Pl. 18, fiys. 1, 15-17.

\section{Arma -. (Pentatomidæ.)}

Locality: Brunstatt, Alsatia. Horizon: Middle Oligocene.

1890. Foerster. In litt.

3096. Asira tertiaria. (Fulgoridæ.)

Locality: Aix, France. Horizon : Ligurian.

1856. Giebel. Insect. d. vorwelt, 377.

See also Asiraca ——, A. tertiaria, Cicadellites obseurus.

3097. Asiraca - (Fulgoridæ.)

Locality : Aix, France. Horizon : Ligurian.

1829. Curtis. Edinb. new phil. journ, vii : 296. Pl. 6, fig. 5.

1829. " Murchison-Lyell, Tert. form. Aix, 12 . Pl. 6, fig. 5.

See also Asira tertiaria, etc.

3098. Asiraca tertiaria. (Fulgoridæ.)

Locality: Aix, France. Horizon : Ligurian.

1890. Scudder. Tert. ins. N. A., 295.

See also, Asira tertiaria, etc.

3099. Belostoma —. (Belostomatidæ.)

Locality: Rhine, Germany. Horizon: Aquitanian?.

1831. Goldfuss. Verhandl, leop.-carol. akad, naturf, vii, abth. i: 118.

3100. Belostoma goldfussi. (Belostomatidæ.)

Compared with B. grundi.

Locality: Vicinity Bonn, Rhenish Prussia. Horizon: Aquitanian?.

1837. Germar. Fauna insect. Europæ, xix: 17, Pl. 17.

1852. Giebel. Deutschl. petref., 636.

1856. " Insect. d. vorwelt, 371 (Belostomum).

3101. Belostoma speciosum. (Belostomatidæ.)

Compared with B. grande, Brazil.

Locality: Oeningen, Baden. Horizon: Tortonian.

1865. Heer. Urwelt der Schweiz, 392. Fig. 303, on p. 391.

1872. " Monde prim. Suisse, 481. Fig. 303, on p. 479.

1876. " $\quad$ Prim. world Switz., ii: 51. Fig. 303, on p. 49.

1879. " Urwelt der Schweiz, $2^{e}$ aufl, 418. Fig. 345, on $p .417$.

See also Belostomates speciosa.

3102. Belostomates harrisii. (Belostomatidæ.)

Locality: Oeningen, Baden. Horizon: Tortonian.

1888. Schöberlin. Soc. ent, iii : 61 (undescr.). 
3103. Belostomates speciosa. (Belostomatidæ.)

Locality: Oeningen, Baden. Horizon: Tortonian.

1888. Schöberlin. Soc. ent., iii : 61 .

See also Belostoma speciosum.

Belostomum goldfussi. See Belostoma goldfussi.

3104. Berytopsis femoralis. (Coreidæ.)

Locality: Oeningen, Baden. Horizon: Tortonian.

1853. Heer. Insektenf. tertiärg. Oeningen, iii: 55-56. Pl. 4, fig. 9; pl.9, fig. 3. 1853-'56. Bronn. 1856. Giebel. Lethæa geogn., $3^{\mathrm{e}}$ aufl., iii: $642 . \quad P l .42^{\prime}$, figs. $17 a-c$. Insect. d. vorwelt, 352 .

1885. Scndder. Zittel, Handb. palaeont., i, th. ii: 785. Fig. 1002. 1886. " Zittel-Barrois, Traité de paléont., ii : 785. Fig. 1019.

\section{5, Berytus ——, (Coreidæ.)}

Locality : Prussian amber. Horizon: Ligurian.

1856. Menge. Progr. petrischule Danzig, 1856: 20.

3106. Brachypelta -. (Pentatomidæ.)

Locality: Brunstatt Alsatia. Horizon: Middle Oligocene.

1890. Foerster. In litt.

3107. Brachypelta rotundata. (Pentatomidæ.)

Localities: Krottensee, Aag bei Franzensbad, Bohemia. Horizon: Aquitanian. 1877. Novak. Sitzungsb. akad. wiss. Wien, lxxvi: 80-81. Pl. 3, figs. 5ab. 1877. " Faun. egerer tertiärb., 10-11. Pl. 3, figs. 5ab.

3108. Bythoscopus —. (Jassides.)

Locality : Prussian amber. Horizon : Ligurian.

1845. Berendt. Bernst. befindl, organ. reste vorw., i : 55.

3109. Bythoscopus homousius. (Jassides.)

Compared with B. (Jassus) flavicollis or B. bipustulatus.

Locality: Prussian amber. Horizon: Ligurian.

1856. Germ-Ber. Berendt, Bernst. befindl. organ. reste vorw., ii, th. i: 8. Pl. 1, fig. 10.

3110. Bythoscopus lapidescens. (Jassides.)

Locality: White River, Colo. Horizon: Oligocene.

187\%. Scudder. Bull. U. S. geol. surv, terr., iii : 761.

1890. " Tert. ins. N. A., 305. Pl. 5, fig. 94.

3111. Bythoscopus melanoneurus. (Jassides.)

Locality: Radoboj, Croatia. Horizon: Mayencian.

1853. Heer. Insektenf. tertiärg. Oeningen, iii : 113. Pl. 13, fig. 与.

1855. " Ber. oesterreich. litt., 1850-53: 203.

1856. Giebel. Insect. d. vorwelt, 385 .

3112. Bythoscopus muscarius. (Jassides.)

Locality : Aix, France. Horizon: Ligurian.

1853. Heer. Insektenf, tertiärg. Oeningen, iii : 112-114. Pl. 13, fig. 4.

1856. " Viert. naturf. gesellsch. Zürich, i: 39.

1856. Giebel. Insect, d, vorwelt, 385. 
3113. Cacalydus exstirpatus. (Coreidæ.)

1890. Scudder.

Locality: Florissant, Colo. Horizon: Oligocene.

Tert. ins. N. A., 420. $P l .25, f i g .3$.

3114. Cacalydus lapsus. (Coreidæ.)

Locality : Florissant, Colo. Horizon: Oligocene.

1890. Scudder. Tert. ins. N. A., 419-420. Pl. 25, fig. 12.

3115. Cacoschistus maceriatus. (Pentatomidæ.)

Locality: Florissant, Colo. Horizon: Oligocene.

1890. Scudder. Tert. ins. N. A., 459.

3116. Calothrips scudderii. (Physapodes.)

Locality: Aix, France. Horizon: Ligurian.

1873. Oustalet. Bull. soc. philom. Paris, x : 23-24.

\section{Capsus -. (Capsidæ.)}

- Locality: Prussian amber. Horizon : Ligurian.

1845. Berendt. Bernst. befindl, organ, reste vorw., i : 55 .

3118. Capsus - (Capsidæ.)

Compared with C. spissicornis.

Locality: Prussian amber. Horizon: Ligurian.

1835. Gravenhorst. Uebers. schles. gesellsch. vaterl. cult., 1834: 93.

3119. Capsus —. (Capsidæ.)

Compared with C. triguttatus.

Locality : Prussian amber. Horizon: Ligurian.

1835. Gravenhorst. Uebers. schles. gesellsch. vaterl. cult., 1834 : 93.

3120. Capsus —. (Capsidæ.)

Locality: Prussian amber. Horizon: Ligurian.

1890. Scudder. Tert. ins. N. A., 362.

\section{Capsus lacus. (Capsidæ.)}

Locality : Florissant, Colo. Horizon : Oligocene.

1890. Scudder. Tert. ins. N. A., 369. Pl. 22, fig. 2.

3122. Capsus obsolefactus. (Capsidæ.)

1890. Scudder. Tert. ins. N. A., 368-369. Pl. 23, fig. 13.

3123. Carmelus gravatus. (Capsidæ.)

Compared with C. parvus.

Locality : Florissant, Colo. Horizon: Oligocene.

1890. Scudder. Tert. ins. N. A., 364. Pl. 24, fig. 10.

3124. Camelus sepositus. (Capsidæ.)

Compared with C. parvus.

Locality : Florissant, Colo. Horizon: Oligocene. 
3125. Cataneura absens. (Aphides.)

Locality: Florissant, Colo. Horizon: Oligocene,

1890. Scudder. Tert. ins. N. A., 245.

3126. Cataneura rileyi. (Aphides.)

Locality: Florissant, Colo. Horizon: Oligocene.

1890. Scudder. Tert. ins. N. A., 245-246.

3127. Catopamera augheyi. (Lygæidæ.)

Locality: Florissant, Colo. Horizon: Oligocene.

1890. Scudder. Tert. ins. N. A., 387. Pl. 27, fig. 7.

3128. Catopamera bradleyi. (Lygæidæ.)

Locality : Florissant, Colo. Horizon: Oligocene.

1890. Scudder. Tert. ins. N. A., 387-388. Pl. 26, fig. 12.

3129. Catopsylla prima. (Psyllidæ.)

Locality : Florissant, Colo. Horizon: Oligocene.

1890. Scudder. Tert. ins. N. A., 277-278.

3130. Cephalocoris pilosus. (Lygæidæ.)

Locality: Oeningen, Baden. Horizon: Tortonian.

1853. Heer. Insektenf. tertiärg. Oeningen, iii : 61-62. Pl.4, fig. 16; pl. 9, fig. 7.

1853-'56. Bronn. Lethæa geogn., $3^{\mathrm{e}}$ aufl., iii : 641-642. .Pl. 42', figs. $16 a b$.

1856. Giebel. Insect. d. vorwelt, 355 .

1885. Scudder. Zittel, Handb. palaeont, i, th. ii: 785. Fig. 1000.

1886. " Zittel-Barrois, Traité de paléont., ii : 784. Fig. 1017.

3131. Cercopidium rugulosum. (Cercopidæ.)

Locality : Atanekerdluk, Greenland. Horizon: Miocene.

1870. Heer. Phil. trans., elix (Flora foss. aret., ii): $485 . \quad P l .44, f i g s .9 b, 9 b b$. 1883. " Flora foss, grönl, ii : 148.

3132. Cercopis —. (Cercopidæ.)

Locality : Prussian amber. Horizon: Ligurian.

1845. Berendt. Bernst. befiudl. organ. reste vorw., i: 55 .

3133. Cercopis ——. (Cercopidæ.)

Locality : Brunstatt, Alsatia. Horizon: Middle Oligocene.

1890. Foerster. In litt.

\section{Cercopis - Cercopidæ.)}

Locality : Rhine, Germany. Horizon : Aquitanian.

1831. Goldfuss. Verhandl. leop.-carol. akad. naturf, vii, abth. i: 118.

\section{Cercopis —. (Cercopidæ.)}

Compared with C. gibba.

Locality: Prussian amber. Horizon: Ligurian.

1835. Gravenhorst. Uebers. schles. gesellsch. vaterl. cult, , 1834 : 93. 
3136. Cercopis (Cereopidæ.)

Compared with C. pini.

Locality: Prussian amber. Horizon: Ligurian.

1835. Gravenhorst. Uebers. schles, gesellsch. vaterl. cult., 1834 : 93.

3137. Cercopis ㄴ. (Cercopidæ.)

Locality : Aix, France. Horizon: Ligurian.

1829. Serres. G6́ogn. terrains. tert., 267.

3138. Cercopis astricta. (Cercopidæ.)

Locality: Green River, Wyo. Horizon : Oligocene.

1890. Scudder. Tert. ins. N, A, 318-319. Pl. 7, fig. 15.

\section{Cercopis aurata. (Cercopidæ.)}

Compared with C. rubra, Brazil.

Locality: Prussian amber. Horizon: Ligurian.

1862. Giebel. Zeitschr. ges. naturw., $\mathrm{xx}: 314$.

3140. Cercopis charpentieri. (Cercopidæ.)

Locality: Radoboj, Croatia. Horizon : Mayencian.

1853. Heer. Insektenf. tertiärg. Oeningen, iii : 101-103, Pl. 12, fig. 1.

1855. " " Ber. oøsterreich. litt., 1850-'53: 202.

1856. Giebel. Insect. d. vorwelt, 381-382.

See also Hylotoma cineracea.

3141. Cercopis fasciata. (Cercopidæ.)

Locality : Radoboj, Croatia. Horizon: Mayencian.

1853. Heer. Insektenf, tertiärg. Oeningen, iii : 100-101. Pl.11, fig. 9 (not 10),

1855. “ Ber. oesterreich. litt., 1850-'53: 202.

1856. Giebel. Insect. d. vorwelt, 381.

1890. Scudder. Tert. ins. N. A, 333.

\section{Cercopis germari. (Cercopidæ.)}

Locality: Oeningen, Baden. Horizon: Tortonian.

1865. Heer. Urwelt der Schweiz, 393. Fig. 305, on $p .391$.

1872. " Monde prim. Suisse, 482. Fig. 305, on p. 479.

1876. " Prim. world Switz., ii : 52. Fig. 305, on p. 49.

1879. " Urwelt der Schweiz, $2^{\circ}$ aufl, 419. Fig. 347 , on $p .417$.

3143. Cercopis gigantea. (Cercopidæ.)

Locality: Radoboj, Croatia. Horizon: Mayencian.

1853. Heer.

1855. "،

1856. Giebel.

Insektenf. tertiärg. Oeningen, iii: 94-95. Pl. 11, fig.5.

Ber. oesterreich. litt., 1850-53: 202.

Insect. $d$, vorwelt, 380 .

3144. Cercopis gliickseligi. (Cercopidæ.)

Locality: Grasseth, Bohemia. Horizon: -

1857. Heer.

Jokély, Jahrb. geol, reichsanst., viii : 502 (undescr.). 
3145. Cercopis hageni. (Cercopidæ.)

1865. Heer.

187\%. " "

1876. “

1879. “

Locality: Oeningen, Baden. Horizon: Tortonian.

Urwelt der Schweiz, 393.

Monde prim. Suisse, 482.

Prim. world Switz., ii : 52.

Urwelt der Schweiz, 2e aufl., 419.

3146. Cercopis haidingeri. (Cercopidæ.)

Locality: Radoboj, Croatia. Horizon: Mayencian.

1853. Heer.

1855 . “

Insektenf. tertiärg. Oeningen, iii : 95-96. Pl. 11, fig. 6.

1856. Giebel.

Ber. oesterreich. litt., 1850-53 : 202.

See also Locrites haidingeri.

3147. Cercopis herrichi. (Cercopidæ.)

Locality: Oeningen, Baden. Horizon: Tortonian.

1865. Heer.

1872. "

Urwelt der Schweiz, 393.

1876. " "

Monde prim. Suisse, 482.

1879. "6

Prim. world Switz., ii : 52.

Urwelt der Schweiz, $2^{\ominus}$ aufl., 419.

3148. Cercopis lanceolata. (Cercopidæ.)

1853. Heer.

1855. “

1856. Giebel.

Locality: Radoboj, Croatia. Horizon: Mayencian.

Insektenf. tertiärg. Oeningen, iii : 104. Pl. 12, fig. 3.

Ber. oesterreich. litt., 1850-'53 : 202.

Insect. d. vorwelt, 382 .

3149. Cercopis longicollis. (Cercopidæ.)

Locality : Radoboj, Croatia. Horizon: Mayencian.

1853. Heer.

1855. “

1856. Giebel.

Insektenf. tertiärg. Oeningen, iii : 103-104. $\quad P l .12$, fig, 2.

Ber. oesterreich. litt., 1850-'53: 202.

Insect. d. vorwelt, 382 .

3150. Cercopis melæna. (Cercopidæ.)

Compared with C. sanguinolenta.

Locality: Prussian amber. Horizon: Ligurian.

1856. Germ.-Ber. Berendt, Bernst. befindl, organ. reste vorw., ii, th. i: 12 . Pl. 1 , fig. 17.

1890. Scudder. Tert. ins. N. A., 333.

3151. Cercopis œningensis. (Cercopidæ.)

Locality: Oeningen, Baden. Horizon: Mayencian.

1853. Heer.

1856. Giebel.

Insektenf. tertiärg. Oeningen, iii : 98-99. Pl. 11, fig. 8 (not 9).

1890. Scudder.

Insect. d. vorwelt, 380-381.

Tert. ins. N. A., 333.

3152. Cercopis pallida. (Cercopidæ.)

Locality: Radoboj, Croatia. Horizon: Mayencian.

1853. Heer.

1855.

Insektenf. tertiärg. Oeningen, iii : 97-98. Pl. 11, fig. 7 (not 8).

1856. Giebel.

Ber. oesterreich. litt., 1850-'53: 202.

1890. Sendder.

Insect. d. vorwelt, 380 .

Tert. ins. N. A., 333. 
3153. Cercopis rectelinea. (Cercopidæ.)

Locality: Radoboj, Croatia. Horizon: Mayencian.

1853. Heer.

1855. " "

1856. Giebel.

1890. Scudder.
Insektenf. tertiärg. Oeningen, iii : 99-100. Pl. 11, fig. 10 (not 11).

Ber. oesterreich. litt., 1850-53: 202 (rectilinea).

Insect. d. vorwelt, 381 (rectilinea).

Tert. ins. N. A., 333.

Cercopis rectilinea. See Cercopis rectelinea.

3154. Cercopis selwyni. (Cercopidæ.)

Locality: Nine-Mile Creek, British Columbia. Horizon :

1879. Scudder.

1879. 6

1890 . " "
Rep. progr. geol. surv. Can., 1877-78: B 184-185.

Ins. tert. Nicola, 9-10.

Tert. ins. N. A., 318. Pl. 2, figs. 14, 15.

3155. Cercopis suffocata. (Cercopidæ.)

Locality : Florissant, Colo. Horizon: Oligocene.

1890. Scudder. Tert. ins. N. A., 319. Pl. 19, figs. 2, 3.

3156. Cercopis ungeri. (Cercopidæ.)

Locality : Radoboj, Croatia. Horizon: Mayencian.

1853. Heer.

Insektenf. tertiärg. Oeningen, iii : 96-97. (Heer quotes pl. 11, fig. 7, but this species is not figured at all.)

1855. " "

1856. Giebel.

Ber. oesterreich. litt., 1850-'53: 202.

Insect. $d$. vorwelt, 380 .

3157. Cercopites calliscens. (Cercopidæ.)

Locality: Green River, Wyo. Horizon: Oligocene.

1890. Scudder. Tert. ins. N. A., 317. Pl. 6, fig. 32.

3158. Cercopites umbratilis. (Cercopidæ.)

Locality : Green River, Wyo. Horizon: Oligocene.

1890. Scudder. Tert. ins. N. A., 316-317. Pl. 7, fig. 9.

3159. Cholula triguttata. (Lygæidæ.)

Locality: Green River, Wyo. Horizon: Oligocene.

1890. Scudder. Tert. ins. N. A., 389-390. Pl. 7, fig. 21.

3160. Cicada - (Stridulantia.)

Locality: Prussian amber. Horizon: Ligurian.

1830. Berendt. Insekten im bernstein, 37.

3161. Cicada —. (Stridulantia.)

Locality: Copal. Horizon: Recent.

1776. Bloch. Beschäft. berl. gesellsch, naturf. freunde, ii : 187 .

3162. Cicada - (Stridulantia.)

Locality : Prussian amber. Horizon: Ligurian.

1831. Burmeister. Oken, Isis, 1831: 1100. 


\section{Cicada -. (Stridulantia.)}

Compared with C. plebeja.

Locality: Aix, France. Horizon: Ligurian.

1829. Serres. Géogn. terr. tert., 228.

3164. Cicada - (Stridulantia.)

Compared with C. violacea.

Locality: Aix, France. Horizon : Ligurian.

1829. Serres. Góogn. terr. tert., 228.

3165. Cicada aichhorni. (Stridulantia.)

1853. Heer.

1855. "

1856. Giebel.

Locality: Radoboj, Croatia. Horizon: Mayencian.

Insektenf. tertiärg. Oeningen, iii: 89. Pl. 11, fig. 2.

Ber. oesterreich. litt., 1850-53: 202.

Insect. d. vorwelt, 374 .

3166. Cicada bifasciata. (Stridulantia.)

1853. Heer. 1855. " "

1856. Giebel.

Locality : Radoboj, Croatia. Horizon: Mayencian.

Insektenf. tertiärg. Oeningen, iii : 90. Pl. 11, fig. 4.

Ber. oesterreich. litt. 1850-'53: 202.

Insect. d. vorwelt, 374-375.

3167. Cicada emathion. (Stridulantia.)

Locality: Oeningen, Baden. Horizon: Tortonian.

1853. Heer.

1856. Giebel.

Insektenf. tertiärg. Oeningen, iii : 88. Pl. 11, fig. 1.

1865. Heer.

1872. " "

1876. “

1879. " "

Insect. d. vorwelt, 374 .

Urwelt der Schweiz, 393. Fig. 304, on p. 391.

Monde prim. Suisse, 481. Fig. 304, on p. 479.

Prim. world Switz., ii : 51. Fig. 304, on p. 49.

Urwelt der Schweiz, 2e aufl., 418. Fig. 346, on p. 417.

3168. Cicada ungeri. (Stridulantia.)

Locality : Radoboj, Croatia. Horizon: Mayencian.

1853. Heer.

1855. “

1856. Giebel.

Insektenf. tertiärg. Oeningen, iii : 89-90. Pl. 11, fig. 3.

Ber. oesterrich. litt., 1850-'53: 202.

Insect. d. vorwelt, 374 .

3169. Cicadellites bruckmanni. (Jassides.)

Locality: Oeningen, Baden. Horizon: Tortonian.

1853. Heer.

1856. Giebel.

Insektenf. tertiärg. Oeningen, iii : 120, Pl. 13, fig. 8.

Insect. d. vorwelt, 387 .

3170. Cicadellites nigriventris. (Jassides.)

Locality: Radoboj, Croatia. Horizon: Mayencian.

1853. Heer.

1855. " "

1856. Giebel.

Insektenf. tertiärg. Oeningen, iii : 119-120. Pl. 13, fig. 13.

Ber. oesterreich. litt., 1850-'53 : 203.

Insect. d. vorwelt, 387.

3171. Cicadellites oblongus. (Jassides.)

Locality: Oeningen, Baden. Horizon: Tortonian.

1853. Heer.

1856. Giebel.

Insektenf. tertiärg. Oeningen, iij: 120。 Pl. 13, fig. 9.

Insect, d. vorwelt, $38 \%$. 
3172. Cicadellites obscurus. (Jassides.)

Locality: Aix, France. Horizon: Ligurian.

1856. Heer. Viert. naturf. gesellsch. Zürich, i: 39-40.

See also Asira tertiaria, etc.

3173. Cicadellites pallidus. (Jassides.)

1853. Heer.

Locality: Radoboj, Croatia. Horizon: Majencian.

1855. " "

Insektenf. tertiärg. Oeningen, iii : 119. $P l .13$, fig. 7.

1856. Giebel.

Ber. oesterreich. litt., 1850-'53: 203.

Insect. d. vorwelt, 387.

3174. Cicadula saxosa. (Jassides.)

Compared with C. sexnotata, United States.

Locality: Green River, Wyo. Horizon: Oligocene.

1890. Scudder. Tert. ins. N. A., 310-311. Pl. 6, fig. 26.

\section{Cimex}

Loc\$lity: Unterkirchberg, Germany. Horizon: Miocene.

1849. Eser. Jahresh, ver. vaterl. naturk. Württ., iv : 265.

\section{Cimex $\longrightarrow$.}

Locality: Prussian amber. Horizon : Ligurian.

1820. Schlotheim. Petrefactenk., 43.

\section{Cimex $\longrightarrow$.}

Locality : Prussian amber. Horizon: Ligurian.

1829. Serres. Géogn. terrains tert., 241.

1830. Berendt. Insekten im bernstein, 37.

\section{Cimex}

Locality : Ulverston, England. Horizon: Pleistocene.

1862. Stainton. Bolton, Quart. journ. geol. soc. Lond., xviii, pt. i : 274-275.

1888. Bell.

Entom., xxi : 2.

\section{Cixius —. (Fulgoridæ.)}

Locality: Prussian amber. Horizon: Ligurian.

1845. Berendt. Bernst. befindl. organ. reste vorw., i: 55.

3180. Cixius - (2 sp.). (Fulgoridæ.)

Locality : Brunstatt, Alsatia. Horizon: Middle Oligocene.

1890. Foerster. In litt.

\section{Cixius - (Fulgoridæ.)}

Locality: Prussian amber.' Horizon : Ligurian.

1856. Menge. Progr. petrischule Danzig, 1856 : 19.

\section{Cixius fraternus. (Fulgoridæ.)}

Locality: Prussian amber. Horizon : Ligurian.

1856. Germ.-Ber. Berendt, Bernst. befindl, organ, reste vorw, ii, th. i: 14-15. 
3183. Cixius gracilis. (Fulgoridæ.)

Locality : Prussian amber. Horizon : Ligurian.

1856. Germ.-Ber. Berendt, Bernst. befindl. organ. reste vorw, ii, th. i: 16. Pl. 1, fig. 25.

3184. Cixius? hesperidum. (Fulgoridæ.)

1878. Seudder.

Locality: Green River, Wyo. Horizon: Oligocene.

1890. " Tert. ins. N. A., 287. Pl. 6, fig. 19.

3185. Cixius insignis. (Fulgoridæ.)

Locality: Prussian amber. Horizon: Ligurian.

1856. Germ.-Ber. Berendt, Bernst. befindl. organ, reste vorw., ii, th. i: 13-14. Pl. 1, fig. 20.

3186. Cixius loculatus. (Fulgoridæ.)

Compared with C. seriatus, Brazil.

Locality: Prussian amber. Horizon: Ligurian.

1856. Germ.-Ber. Berendt, Bernst. befindl. organ. reste vorw., ii, th. i: 15-16. Pl. 1, fig. 24 .

3187. Cixius longirostris. (Fulgoridæ.)

Locality: Prussian amber. Horizon: Ligurian.

1856. Germ.-Ber. Berendt, Bernst. befindl, organ. reste vorw., ii, th. i: $15 . \quad P l .1$, fig. 22.

3188. Cixius ? proavus. (Fulgoridæ.)

Locality: Florissant, Colo. Horizon: Oligocene.

1890. Scudder. Tert. ins. N. A., 287. Pl. 19, fig. 14.

3189. Cixius sieboldtii. (Fulgoridæ.)

Locality: Prussian amber. Horizon: Ligurian.

1856. Germ.-Ber. Berendt, Bernst. befindl. organ. reste vorw., ii, th. i: 14. Pl. 1, fig. 21 .

3190. Cixius succineus. (Fulgoridæ.)

Locality : Prussian amber. Horizon : Ligurian.

1856. Germ.-Ber. Berendt, Bernst. befindl. organ. reste vorw., ii, th. i: $15 . \quad P l .1$, fig. 23.

3191. Cixius testudinarius. (Fulgoridæ.)

Locality : Prussian amber. Horizon: Ligurian.

1856. Germ.-Ber. Berendt, Bernst, befindl. organ. reste vorw., ii, th. i: $13 . \quad P l$. 1, fig. 19.

3192. Cixius vitreus. (Fulgoridæ.)

Compared with C. nervosus, Europe.

Locality: Prussian amber. Horizon: Ligurian.

1856. Germ.-Ber, Berendt, Bernst, befindl, organ, reste vorw, ii, th, i; 12-13, $P l_{\text {. }}$ 1, fig. 18. 
3193. Clastoptera comstocki. (Cercopidæ.)

Locality : Florissant, Colo. Horizon: Oligocene. 1890. Scudder. Tert. ins. N. A., 338. Pl. 19, fig. 22.

3194. Closterocoris elegans. (Capsidæ.)

Locality: Florissant, Colo. Horizon: Oligocene. 1890. Scudder. Tert. ins. N. A., 363. Pl. 24, fig. 7.

\section{Coccus avitus. (Coccidæ.)}

Locality: Prussian amber. Horizon: Ligurian.

1856. Menge. Progr, petrischule Danzig, 1856: 17.

3196. Coccus termitinus. (Coccidæ.)

Locality : Prussian amber. Horizon: Ligurian.

1856. Menge. Progr. petrischule Danzig, 1856: 17.

3197. Cœlidia columbiana. (Jassides.)

Locality : Similkameen River, British Columbia. Horizon:

1879. Scudder. Rep. progr. geol. surv. Can., 1877-78: 185B.

1879. " Ins. tert. Nicola, 10.

1890. " Tert. ins. N. A., 313. Pl. 2, fig. 13.

3198. Cœlidia wyomingensis. (Jassides.)

Locality: Twin Creek, Wyo. Horizon: -

1890. Scudder. Tert. ins. N. A., 313-314. Pl. 4, fig. 8.

3199. Cophocoris tenebricosus. (Lygæidæ.)

Locality: Florissant, Colo. Horizon: Oligocene.

1890. Scudder. Tert. ins. N. A., 391.

3200. Coptochromus manium. (Lygæidæ.)

Locality: Florissant, Colo. Horizon : Oligocene.

1890. Scudder. Tert. ins. N. A., 405.

\section{Coræus —. See Coreus}

3201. Coreites crassus. (Coreidæ.)

Locality : Radoboj, Croatia. Horizon: Mayencian.

1853. Heer.

1855. " "

Insektenf. tertiärg. Oeningen, iii : 56-57. Pl. 4, fig. 10.

Ber. oesterreich. litt., 1850-53: 201.

1856. Giebel. Insect. d. vorwelt, 352.

3202. Coreites oblongus. (Coreidæ.)

Locality : Radoboj, Croatia. Horizon : Mayencian.

1853. Heer. 1856. Giebel.
Insektenf. tertiärg. Oeningen, iii : 57. $P l .4$, fig. 11 .

Insect. d. vorwelt, 353.

3203. Coreites redemtus. (Coreidæ.)

Locality : Oeningen, Baden. Horizon: Tortonian.

1853. Heer. 1856. Giebel.
Insektenf. tertiärg. Oeningen, iii : 57-58. $\quad P l .4$, fig. 12.

Insect. $d$. vorwelt, 353 . 
3204. Coreus ——. (Coreidæ.)

Locality: Aix, France. Horizon: Ligurian.

1829. Serres. Géogn. terrains tert., 227 (Coræus).

3205. Corimalæna - (Pentatomidæ.)

Locality: Florissant, Colo. Horizon: Oligocene.

1885. Scudder. Zittel, Handb. palaeont., i, th. ii : 786.

1886. " Zittel-Barrois, Traité de paléont., ii : 786.

1886. “ Bull. U. S. geol. surv., No. $31: 64$.

Corisa fasciolata. See Corixa fasciolata.

3206. Corixa —. (Corixidæ.)

Locality: Florissant, Colo. Horizon: Oligocene.

1881. Scudder. Bull. U. S. geol, surv. terr., vi : 292.

1883. " Ann. rep. U. S. geol. surv. terr., xii : 282.

3207. Corixa fasciolata. (Corixidæ.)

Locality: Oeningen, Baden. Horizon: Tortonian.

1853. Heer. Insektenf. tertiärg. Oeningen, iii : 86-87 (Corisa). Pl. 10, fig. 12.

1856. Giebel. Insect. d. vorwelt, 372 (Corisa).

3208. Coriza immersa. (Corixidæ.)

Compared with C. hellensii Sahlb., Europe.

Locality : Florissant, Colo. Horizon: Oligocene.

1890. Scudder. Tert. ins. N. A., 345-348. Pl. 22, fig. 16.

3209. Corixa pullus. (Corixidæ.)

Locality: Stösschen, Austria. Horizon : Miocene.

1859. Heyden, Palaeontogr., viii : 10-11. Pl. 1, fig. 13.

3210. Corixa vanduzeei. (Corixidæ.)

Compared with C. interrupta Say, United States ; C. præusta Fieb., Europe.

Locality: Florissant, Colo. Horizon: Oligocene.

1890. Scudder. Tert. ins. N. A., 344-345. Pl, 22, fig. 17.

3211. Corizus —_. (Coreidæ.)

Compared with C. byoscyami Linn.

Locality: Aix, France. Horizon : Ligurian.

1829. Curtis. $\quad$ Edinb. new phil. journ., vii : 296.

1829. " Murchison-Lyell, Tert. form. Aix, 12.

3212. Corizus abditivus. (Coreidæ.)

Locality: Florissant, Colo. Horizon: Oligocene.

1890. Scudder. Tert. ins. N. A., 433-434. Pl. 25, fig. 5; pl. 26, fig. 4.

3213. Corizus boyeri. (Coreidæ.)

Locality : Aix, France. Horizon : Ligurian.

1847. Hope. Trans. ent. soc. Lond., iv: 255. Pl. 19, figs. 3, 3*.

See also Pachymerus boyeri.

Bull. $71-26$ 
3214. Corizus celatus. (Coreidæ.)

Locality : Fìorissant, Colo. Horizon: Oligocene.

1890. Scudder. $\quad$ Tert. ins. N. A., 433. Pl. 27, fig. 15:

3215. Corizus guttatus. (Coreidæ.)

Locality: Green River, Wyo. Horizon: Oligocene.

1890. Scudder. Tert. ins. N. A., 434-435. Pl. 7, fig. 11.

See also Reduvius guttatus.

3216. Corizus somnurnus. (Coreidæ.)

Locality : Florissant, Colo. Horizon: Oligocene.

1890. Scudder. Tert. ins. N. A., 434.

3217. Cryptochromus letatus. (Lygæidæ.)

Locality: Flurissant, Colo. Horizon: Oligocene.

1890. Scudder. Tert. ins. N. A., 409.

3218. Ctereacoris primigenus. ( $\left.\mathrm{L}_{\mathcal{J}} \mathrm{g} æ i d æ.\right)$

Locality : Florissant, Colo. Horizon : Oligocene.

1890. Scudder. Tert. ins. N. A., 394-395.

3219. Cydamus robustus. (Coreidæ.)

Locality : Florissant; Colo. Horizon : Oligocene.

1890. Scudder. Tert. ins. N. A., 420-421. Pl. 26, fig. 3.

3220. Cydnopsis atavina. (Pentatomidæ.)

Locality: Oeningen, Baden. Horizon: Tortonian.

1853. Heer.

1856. Giebel.

Insektenf. tertiärg. Oeningen, iii : 18. $\quad P l .1$, fig. 9.

Insect. d. vorwelt, 337 .

3221. Cydnopsis brevicollis. (Pentatomidæ.)

Locality: Radoboj, Croatia. Horizon: Mayencian.

1853. Heer.

1855. “

1866. Giebel.

Insektenf. tertiärg. Oeningen, iii: 21-22. $P l .2, f i g .1 ; p l, 6$, fig. 11.

Ber. oesterreich. litt., 1850-'53: 200.

Insect. $\mathrm{d}$. vorwelt, 338 .

3222. Cydnopsis coleopteroides. (Pentatomidæ.)

1853. Heer.

Locality : Radoboj, Croatia. Horizon: Mayencian.

Insektenf. tertiärg. Oeningen, iii : 16-17. Pl. 1, fig. 7; pl. 6, fig. 6.

1855. “

1856. Giebel.

Ber. oesterreich. litt., 1850-'53: 199.

Insect. d. vorwelt, 337.

3223. Cydnopsis deleta. (Pentatomidæ.)

Locality: Oeningen, Baden. Horizon: Tortonian.

18\%3. Heer.

Insektenf. tertiärg. Oeningen, iii: 17-18. Pl. 1, fig. 8; pl. 6, fig. 7 .

1856. Giebel. Insect. d. vorwelt, 337. 
3224. Cydnopsis exilis. (Pentatomidæ.)

Locality: Oeningen, Baden. Horizon: Tortonian.

1853. Heer. Insektenf. tertiärg. Oeningen, iii : 127. $\quad P l .15$, fig. 7.

1856. Giebel. Insect. d. vorwelt. 338.

\section{Cydnopsis haidingeri. (Pentatomidæ.)}

Localities: Oeningen, Baden; Radoboj, Croatia. Horizons: Tortonian, Mayencian. 1853. Heer.

Insektenf. tertiärg. Oeningen, iii : 15-16, 127. Pl.1, fig.6; pl.6, fig. 5.

1855. “ Ber. oesterreich. litt., 1850-'53: 199.

1856. Giebel. Insect. d. vorwelt, 336-337.

3226. Cydnopsis heeri. (Pentatomidæ.)

Locality: Aix, France. Horizon : Ligurian.

1877. Oustalet. Bull. soc. philom. Paris, (6), xi, 1874: 14-16.

- 3227. Cydnopsis pygmæa. (Pentatomidæ.)

Locality: Oeningen, Baden. Horizon: Tortonian.

1853. Heer. Insektenf. tertiärg. Oeningen, iii : 22. Pl. 2, fig. 2; pl. 6, fig. 12. 1856. Giẹbel. Insect. d. vorwelt, 338.

3228. Cydnopsis sagittifera. (Pentatomidæ.)

Locality: Oeningen, Baden. Horizon: Tortonian.

1853. Heer.

Insektenf. tertiärg. Oeningen, iii : 23. Pl. 2, fig. $3 ;$ pl. 6, fig. 13.

1856. Giebel.

Insect. d. vorwelt, 338-339.

3229. Cydnopsis scutellaris. (Pentatomidæ.)

Locality : Radoboj, Croatia. Horizon: Mayencian.

1853. Heer.

1855. “

Insektenf. tertiärg. Oeningen, iii : 21. Pl. 1, fig. 13; pl. 6, fig. 10.

1856. Giebel.

Ber. oesterreich. litt., 1850-'53: 200.

Insect, d. vorwelt, 338 .

3230. Cydnopsis tertiaria. (Pentatomidæ.)

Localities: Oeningen, Baden; Radoboj, Croatia. Horizons: Tortonian, Mayencian. 1853. Heer.

Insektenf. tertiärg. Oeningen, iii : 18-20. Pl. 1, figs. 10a-l; pl. 6, fig. 9.

1855. " "

1856. Giebel.

Ber. oesterreich. litt., 1850-'53: 199-200.

1853-56. Bronn.

Insect. d. vorwelt, 337-338.

1865. Heer.

1872. “

1876. “

Lethæa géogn., 3e anfl., iii : 643-644. Pl. $42^{\prime}$, fig. 20ab.

1879. “

Urwelt der Sehweiz, 390. Fig. 300, on p. 391.

Monde prim. Suisse, 479. Fig. 300.

Prim. world Switz., ii : 49. Fig. 300.

Urwelt der Schweiz, $2^{\mathrm{e}}$ aufl., 416. Fïg. 342, on p. 417.

3231. Cydnus - (Pentatomidæ.)

Compared with C. albomarginatus Fabr.

Locality : Aix, France. Horizon: Ligurian.

1829. Curtis. Edinb. new phil. journ., vii : 296.

1829. " Murchison-Lyell, Tert. form. Aix, 12.

3232. Cydnus - (19 sp.). (Pentatomidæ.)

Locality: Brunstatt, Alsatia. Horizon: Middle Oligocene.

1890. Foerster. In litt. 
3233. Cydnus (vic.) (Pentatomidæ.).

Locality: Rocky River, Sydney Flats, Australia. Horizon: 1870. Moore. Quart. journ. geol. soc. Lond., xxvi : 263. Pl. 18, fig. 11. 3234. Cydnus costæ. (Pentatımidæ.)

Locality: Aix, France. Horizon: Ligurian.

1847. Hope. Ann. accad. aspir. nat. Napoli, 1847 : ö-7. $\quad$ Pl. 10, fig. 4. 1847. Deser. ins. foss., 6-7. Pl., fig. 4.

3235. Cydnus ? mamillanus. (Pentatomidæ.)

Locality: Green River, Wyo. Horizon: Oligocene.

1878. Scudder. Bull. U. S. geol. surv. terr., iv: 770 .

See also Procydnus mamillanus.

3236. Cydnus œningensis. (Pentatomidæ.)

Locality : Oeningen, Baden. Horizon: Tortonian.

1853. Heer. 1856. Giebel. 1865. Heer. 1872. " " 1876. “ " 1879. “

Insektenf. tertiärg. Oeningen, iii : 12-13. $P l .1$, fig. 5 ; pl. 6, fig. 4.

Insect. d. vorwelt, 336 .

Urwelt der Schweiz, 359.

Monde prim. Suisse, 441.

Prim. world Switz., ii : 14.

Urwelt der Schweiz, 2e aufl., 385.

3237. Cyrtomenus concinnus. (Pentatomidæ.)

Compared with C. mutabilis Perty.

Locality: Green River, Wyo. Horizon: Oligocene.

1878. Scudder. Bull, U. S. geol. surv. terr, iv: 769.

1890. “ Tert. ins. N. A., 451-452. Pl. 7, fig. 14.

3238. Delphax —. (Fulgoridæ.)

Locality : - Horizon :

1846. Giebel. Paläozool., 269.

3239. Delphax senilis. (Fulgoridæ.)

Locality: White River, Colo. Horizon: Oligocene.

1877. Scudder. Bull. U. S. geol. surv. terr., iii: 760.

1890. “ Tert. ins. N. A., 295. Pl. 5, fig. 95.

3240. Deltocephalus —. (Jassides.)

Locality : Brunstatt, Alsatia. Horizon: Middle Oligocene.

1890. Foerster. In litt.

3241. Diaplegma abductum. (Fulgoridæ.)

Locality: Florissant, Colo. Horizon: Oligocene.

1890. Scudder. Tert. ins. N. A., 290-291. Pl. 15, fig. 8.

3242. Diaplegma haldemani. (Fulgoridæ.)

Locality: Florissant, Colo. Horizon: Oligocene.

1890. Scudder. Tert. ins. N. A., 289-290.

3243. Diaplegma obdormitum. (Fulgoridæ.)

Locality: Green River, Wyo. Horizon: Oligocene.

1890. Scudder. Tert. ins. N. A., 292. 
3244. Diaplegma occultorum. (Fulgoridæ.)

1890. Scudder. Tert. ins. N. A., 291-29̄2.

Locality : Florissant, Colo. Horizon: Oligocene.

3245. Diaplegma ruinosum. (Fulgoridæ.)

Locality: Florissant, Colo. Horizon: Oligocene.

1890. Scudder. Tert. ins. N. A., 292.

3246. Diaplegma venerabile. (Fulgoridæ.)

Locality : Florissant, Colo. Horizon: Oligocene.

1890. Scudder. Tert. ins. N. A., 291.

3247. Diaplegma veterascens. (Fulgoridæ.)

Locality : Florissant, Colo. Horizon: Oligocene.

1890. Scudder. Tert. ins. N. A., 290.

3248. Dichrooscytus —-. (Capsidæ.)

Locality: Prussian amber. Horizon: Ligurian.

1890. Scudder. Tert. ins. N. A., 362.

3249. Dictyophara bouvei. (Fulgoridæ.)

Locality: Florissant, Colo. Horizon: Oligocene.

1890. Scudder. Tert. ins. N. A., 286. Pl. 21, fig. 16.

3250. Dictyophorites tingitinus. (Jassides.)

Locality: Radoboj, Croatia. Horizon: Mayencian.

1853. Heer. Insektenf, tertiärg. Oeningen, iii : 115-116. Pl. 13, fig. 6.

1855. “ Ber. oesterreich. litt., 1850-'53: 203.

1856. Giebel. Insect. $d$. vorwelt, 386.

1885. Scudder. Zittel, Handb. palaeont., i, th. ii: 781. Fig. 990.

1886. “ Zittel-Barrois, Traité de paléont., ii: 781. Fig. 1007.

3251. Diplonychus rotundatus. (Nepidæ.)

Locality: Oeningen, Baden. Horizon: Tortonian.

1853. Heer.

1856. Giebel.

Insektenf. tertiärg. Oeningen, iii : 85-86. Pl. 10, fig. 10.

Insect. $d$. vorwelt, 371.

Urwelt der Schweiz, 392.

Monde prim. Suisse, 481.

Prim. world Switz., ii : 51.

Urwelt der Schweiz, 2e aufl., 418.

3252. Discostoma - (Pentatomidæ.)

Locality: Florissant, Colo. Horizon: Oligocene.

1890. Scudder. Tert. ins. N. A., 452. Pl. 22, fig. 6.

3253. Docimus psylloides. (Jassides.)

Locality: Florissant, Colo. Horizon: Oligocene.

1890. Scudder. Tert. ins. N. A., 314-315. Pl. 19, figs. 6,17.

3254. Dorthesia - (Coccidæ.)

Locality: Prnssian amber. Horizon: Ligurian.

1856. Menge. Progr. petrischule Danzig, 1856: 18. 
3255. Dysdercus cinctus. (Lygæidæ.)

Locality : Florissant, Colo. Horizon: Oligocene. 1890. Scudder. Tert. ins. N. A., 410. Pl. 24, figs. 11,13, 14.

3256. Dysdercus unicolor. (Lygæidæ.)

Locality: Florissant, Colo. Horizon: Oligocene.

1890. Scudder. Tert. ins. N. A., 4 to.

3257. Flidiptera regularis. (Fulgoridæ.)

Locality: Florissant, Colo. Horizon: Oligocene. 1890. Scudder. Tert. ins. N. A., 297. Pl. 19, fig. 13.

3258. Eothes elegans. (Reduviidæ.)

Locality: Florissant, Colo. Horizon: Oligocene. 1890. Scudder. Tert. ins. N. A., 355-356. Pl. 26, fig. 5.

3259. Eotingis antennata. (Tingididæ.)

Locality: Florissant, Colo. Horizon: Oligocene. 1890. Scudder. Tert, ins. N. A., 360. Pl. 23, figs. $1,3$.

3260. Eotingis quinquecarinata. (Tingididæ.)

Locality : Prussian amber. Horizon: Ligurian.

1890. Scudder. Tert. ins. N. A., 359-360.

See also Tingis quinquecarinata.

3261. Etirocoris infernalis. (Coreidæ.)

Locality: Florissant, Colo. Horizon: Oligocene.

1890. Scudder. Tert. ins. N. A., 426. Pl. 26, fig. 16.

3262. Eucorites serescens. (Lygæidæ.)

Locality : Florissant, Colo. Horizon: Oligocene.

1890. Scudder. Tert. ins. N. A., 392.

3263. Eurydema arcuata. (Pentatomidæ.)

Locality: Oeningen, Baden. Horizon: Tortonian.

1853. Heer.

Insektenf. tertiärg. Oeningen, iii : 36. $\quad P l .2$, fig. 15; pl. 7, fig. 9. 1856. Giebel.

Insect. d. vorwelt, 344-345.

3264. Eurydema brevicollis. (Pentatomidæ.)

Locality : Oeningen, Baden. Horizon: Tortonian.

1853. Heer.

Insektenf. tertiärg. Oeningen, iii : $36-37 . \quad P l .2$, fig. 17; pl. 7, fig. 10.

1856. Giebel. Insect. d. vorwelt, 345 .

3265. Eurydema effossa. (Pentatomidæ.)

Locality: Oeningen, Baden. Horizon: Tortonian.

1853. Heer. 1856. Giebel.

Insektenf. tertiärg. Oeningen, iii : 37. $P l .2, f i g .16 ; p l .7, f i g .11$.

Insect. d. vorwelt, 345 . 
3266. Eurydema impudica. (Pentatomidæ.)

Locality : Oeningen, Baden. Horizon: Tortonian.

1853. Heer. Insektenf. tertiärg. Oeningen., iii : 35-36,127-128. Pl. 3, fig. 1; pl. 7, fig. $8 ;$ pl. 14 , fig. 21.

1856. Giebel. Insect. d. vorwelt, 344 .

3267. Eurygaster —. (Pentatomidæ.)

Locality: Brunstatt, Alsatia. Horizon: Middle Oligocene.

1890. Foerster. In litt.

3268. Eusarcoris. (5 sp.). (Pentatomidæ.)

Locality: Brunstatt, Alsatia. Horizon: Middle Oligocene. 1890. Foerster. In litt.

3269. Eusarcoris pinguis. (Pentatomidæ.)

Locality : Oeningen, Baden. Horizon: Tortonian.

1853. Heer. Insektenf. tertiärg. Oeningen, iii: 38-39. Pl. 3, fig.4; pl.8, fig. 2. 1856. Giebel. Insect. d. vorwelt, 345-346.

3270. Eusarcoris prodromus. (Pentatomidæ.)

Locality: Oeningen, Baden. Horizon: Tortonian.

1853. Heer.

1856. Giebel.

Insektenf. tertiärg. Oeningen, iii : 37-38. Pl.3, fig. 3; pl.8, fig. 1.

Insect. d. vorwelt, 345 .

3271. Euschistus antiquus. (Pentatomidæ.)

Locality: Quesnel, British Columbia. Horizon: -

1878. Scudder. Add. ins. fauna Quesn., 3-5.

1878. “ Rep. progr. geol. surv. Can., 1876-77: 459-461.

1878. “ Rapp. opér. comm. géol. Can., 1876-'77: 516-518.

See also Teleoschistus antiquus.

3272. Evagoras impressus. (Reduviidæ.)

Locality : Oeningen, Baden. Horizon: Tortonian.

1853. Heer. Insektenf. tertiärg. Oeningen, iii : 83-84. Pl. 5, fig. 27; pl. 10, fig. 8.

1856. Giebel. Insect. d. vorwelt, 368.

3273. Exitelus exsanguis. (Lygæidæ.)

Locality: Florissant, Colo. Horizon: Oligocene.

1890. Scudder. Tert. ins. N. A., 408. Pl. $27, f i g .2$.

3274. Ficarasites stigmaticus. (Fulgoridæ.)

Locality: Green River, Wyo. Horizon: Oligocene.

1890. Scudder. Tert. ins. N. A., 301 (stigmaticum). Pl. 6, fig. 20.

\section{Flata —. (Fulgoridæ.)}

Compared with F. eunicularia.

Locality: Prussian amber. Horizon: Ligurian.

1832. Burmeister. Handb. ent., i: 638.

1836. 6 Man. ent, 579. 
3276. Flata _. (Fulgoridæ.)

Compared with F. wervosa.

Locality: Prussian amber. Horizon: Ligurian.

1835. Gravenhorst. Uebers. schles. gesellsch. vaterl. cult., 1834: 93. 3277. Florissantia elegans. (Fulgoridæ.)

Locality : Florissant, Colo. Horizon: Oligocene. 1890. Scudder. Tert. ins. N. A., 294. Pl. 19, fig. 12.

3278. Fulgora granulosa. (Fulgoridæ.)

Locality: Green River, Wyo. Horizon: Oligocene. 1878. Scudder. Bull. U. S. geol. surv. terr., iv: 771-772.

1890. " Tert. ins. N.A., 284. Pl. 6, fig. 35.

3279. Fulgora obticescens. (Fulgoridæ.)

Locality: Florissant, Colo. Horizon: Oligocene.

1890. Scudder. Tert. ins. N. A., 285. Pl. 19, fig. 1.

3280. Fulgora populata. (Fulgoridæ.)

Locality: Green River, Wyo. Horizon: Oligocene.

1890. Scudder. Tert. ins. N. A., 284-285. Pl. 7, fig. 16.

3281. Fuscus ? fæcatus. (Capsidæ.)

Locality: Florissant, Colo. Horizon: Oligocene.

1890. Scudder. Tert. ins. N. A., 365. Pl. 22, fig. 5.

3282. Geocoris infernorum. (Lygæidæ.)

Locality: Florissant, Colo. Horizon: Oligocene.

1890. Scudder. Tert. ins. N. A., 381. Pl. 23, figs. 17, 26.

3283. Gerancon davisii. (Aphides.)

Locality: Florissant, Colo. Horizon : Oligocene.

1890. Scudder. Tert. ins. N. A., 248-249.

3284. Gerancon petrorum. (Aphides.)

Locality: Quesnel, British Columbia. Horizon:

1890. Scudder. Tert. ins. N. A., 249-250. Pl. 2, fig. 6.

See also Lachnus petrorum.

3285. Gerris - (Veliidæ.)

Compared with G. currens.

Locality : Aix, France. Horizon: Ligurian.

1829. Serres. Géogn. terrains tert., 228.

3286. Gypona cinercia. (Jassides.,

Locality: Florissant, Colo. Horizon: Oligocene.

1890. Scudder. Tert. ins. N. A., 308. Pl. 19, fig. 4.

3287. Hadronema cinerescens. (Capsidæ.)

Locality: Florissant, Colo. Horizon: Oligocene.

1890. Seudder.

Tert. ins. N. A., $370 \quad P l .24, f i g .12$. 


\section{Halobates - (Hydrobatidæ.)}

Locality: Prussian amber. Horizon: Ligurian.

1845. Berendt. Bernst. befindl. organ. reste vorw., i: 55 .

3289. Halobates (or Hydrometra) - (larva). (Hydrobatidæ.)

Locality: Prussian amber. Horizon: Ligurian.

1853. Germ.-Ber. Berendt, Bernst. befindl. organ. reste vorw., ii, th. i: 19. Pl. 2 , figs. 7,8.

3290. Halobates — (Hydrobatidæ.)

Locality: Florissant, Colo. Horizon: Oligocene.

1885. Scudder. Zittel, Handb. palaeont., i, th. ii : 783.

1886. " " Zittel-Barrois, Traité de paléont, ii : 783.

1886. " " Bull. U. S. geol. surv., No. 31: 62.

See also Metrobates æternalis.

3291. Halys bruckmanni. (Pentatomidæ.)

Locality: Oeningen, Baden. Horizon : Tortonian.

1853. Heer. Insektenf. tertiärg. Oeningen, iii : 32-35. Pl. 3, fig. 2; pl.7, fig.7. 1856. Giebel. Insect. d. vorwelt, $343-344$.

See also Pentatoma bruckmanni.

3292. Halys spectabilis. (Pentatomidæ.)

Locality: Oeningen, Baden. Horizon: Tortonian.

1865. Heer. Urwelt der Schweiz. Fig. 299, on p. 391.

1872. " Monde prim. Suisse. Fig. 299, on p. 479.

1876. " Prim. world Switz., ii. Fig. 299, on p. 49.

1879. “ Urwelt der Schweiz, $2^{\mathrm{e}}$ aufl. Fig. 341, on $p .417$.

3293. Hammapteryx reticulata. (Fulgoridæ.)

Locality: Green River, Wyo. Horizon: Oligocene.

1890. Scudder. Tert. ins. N. A., 298-299. Pl. 6, fig. 34.

3294. Harmostites œningensis. (Coreidæ.)

Locality: Oeningen, Baden. Horizon: Tortonian.

1853. Heer. Insektenf. tertiärg. Oeningen, iii : 49-50. pl. 4, fig. 4; pl. 9, fig. 1.

1853-'56. Bronn. Lethæa geogn., $3^{\text {e }}$ aufl, iii : 642-643. Pl. $42^{\prime}$, figs. $18 a b$.

1856. Giebel. Insect. $d$. vorwelt, 350 .

\section{Harpactor —. (Reduviidæ.)}

Locality: Brunstatt Alsatia. Horizon: Middle Oligocene.

1890. Foerster. In litt.

3296. Harpactor bruckmanni. (Reduviidæ.)

Locality: Oeningen, Baden. Horizon: Tortonian.

1853. Heer.

Insektenf. tertiärg. Oeñingen, iii: 82. $\quad P l$. 5, fig. 24.

1856. Giebel. Insect. d. vorwelt, 367 .

3297. Harpactor constrictus. (Reduviidæ.)

Locality: Oeningen, Baden. Horizon: Tortonian.

1853. Heer. Insektenf. tertiärg. Oeningen, iii : 80-81. Pl. 5, fig. 22; pl. 10, fig. 5.

1856. Giebel. Insect. d. vorwelt, 366 . 
3298. Harpactor gracilis. (Reduviidæ.)

Locality: Radoboj, Croatia. Horizon: Mayencian.

1853. Heer.

1855. " "

1856. Giebel.

Insektenf. tertiärg. Oeningen, iii : 81-82. Pl. 5, fig. 23; pl. 10, fig. 6.

Ber. oesterreich. litt., 1850-'53 : 201.

Insect. d. vorwelt, 367 .

3299. Harpactor longipes. (Reduviidæ.)

1853. Heer.

Locality: Oeningen, Baden. Horizon: Tortonian.

1856. Giebel.

Insektenf. tertiärg. Oeningen, iii : 78. Pl. 5, fig. $20 ; p l .10, f i g .3$.

Insect. d. vorwelt, 366 .

3300. Harpactor maculipes. (Reduviidæ.)

Locality: Oeningen, Baden. Horizon: Tortonian.

1853. Heer.

Insektenf. tertiärg. Oeningen, iii : 79-80, 132 . Pl. 5, fig. $21 ; p l$. 10, fig. $4 ; p l .15$, fig. 10.

1856. Giebel.

Insect. d. vorwelt, 366 .

1865. Lyell.

Elem. geol., 6th ed., 255. Fig. 193.

Urwelt der Schweiz. Fig. 306, on p. 391.

Monde prim. Suisse. Fig. 306, on p. 479.

Prim. world Switz., ii. Fig. 306, on p. 49.

Urwelt der Schweiz, 2 aufl. Fig. 348, on p. 417.

1879. “

1885. Sendder.

1886. “

Zittel, Handb. palaeont., i, th. ii : 784. Fig. 997.

Zittel-Barrois, Traité de paléont., ii : 783. Fig. 1014.

3301. Harpactor obsoletus. (Reduviidæ.)

Locality: Oeningen, Baden. Horizon: Tortonian.

1853. Heer.

1×56. Giebel.

Insektenf. tertiärg. Oeningen, iii: 82. $\quad P l .5, f i g .25$.

Insect. $d$. vorwelt, 367 .

3302. Harpocera —. (Capsidæ.)

Locality : Prussian amber. Horizon: Ligurian.

1890. Scudder. Tert. ins, N. A., 362.

3303. Heeria fœda. (Coreidæ.)

Locality: Florissant, Colo. Horizon : Oligocene.

1890. Scudder. Tert. ins. N. A., 432.

3304. Heeria gulosa. (Coreidæ.)

Locality: Florissant, Colo. Horizon : Oligocene.

1890. Scudder.

Tert. ins. N. A., 431. Pl. 27, figs. 5, 12, 18; pl. 28, fig. 17.

3305. Heeria lapidosa. (Coreidæ.)

Locality: Florissant, Colo. Horizon: Oligocene.

1890. Seudder.

Tert. ins. N. A., 432. $P l .27$, figs. 3, 19.

3306. Heliothrips clypeata. (Physapodes.)

Locality : Rott, Rhenish Prussia. Horizon: Aquitanian.

1887. Schlechtend. Zeitschr. ges. naturw., lx: 589-591. Pl. 5, fig. 24.

3307. Heliothrips cucullata. (Physapodes.)

Locality: Rott, Rhenish Prussia. Horizon: Aquitanian.

1887. Schlechtend. Zeitschr. ges, naturw., lx : 582-586. Pl. 5, figs. 21a-d, 22a, 22AB. 
3308. Heliothrips frechi. (Physapories.)

Locality : Rott, Rhenish Prussia. Horizon: Aquitanian.

1887. Schlechtend. Zeitschr. ges. naturw., Ix : 591-592. Pl. 4, figs. 25, 25a.

3309. Heliothrips longipes. (Physapodes.)

Locality : Rott, Rhenish Prussia. Horizon: Aquitanian.

1887. Schlechtend. Zeitschr. ges. naturw., Ix: 586-589. Pl. 4, figs. 23, 23a.

3310. Heterogaster - (2 sp.). (Lygæidæ.)

Locality : Brunstatt, Alsatia. Horizon : Middle Oligocene.

1890. Foerster. In litt.

3311. Heterogaster antiquus. (Lygæidæ.)

Locality: Aix, France. Horizon : Ligurian.

1853. Heer. Insektenf. tertiärg. Oeningen, iii : 68-69. Pl. 5, fig. 11 ; pl. 9, fig. 14 .

1856. “ Viert. naturf. gesellsch. Zürich, i : 39.

1856. Giebel. Insect. d. vorwelt, 359.

3312. Heterogaster pumilio. (Lygæidæ.)

Locality: Aix, France. Horizon : Ligurian.

1853. Heer. Insektenf. tertiärg. Oeningen, iii: $69 . \quad P l .5, f i g .12 ; p l .9, f i g .15$. 1856. " Viert. naturf. gesellsch. Zürich, i: 39.

1856. Giebel. Insect. d. vorwelt, 359.

3313. Heterogaster radobojanus. (Lygæidæ.)

Locality: Radoboj, Croatia. Horizon: Mayencian.

1853. Heer. Insektenf. tertiärg. Oeningen, iii : 69-70. Pl. 5, fig. 13; pl.9, fig. 16.

1855. “ Ber. oesterreich. litt., 1850-'53: 201.

1856. Giebel. Insect. d. vorwelt, 359.

3314. Heterogaster redivivus. (Lygæidæ.)

Locality: Radoboj, Croatia. Horizon: Mayencian.

1853. Heer. Insektenf. tertiärg. Oeningen, iii : 71, 131. Pl. 5, fig. 15; pl.14, fig. 17.

1855. “ Ber. oesterreich. litt., 1850-53: 201.

1856. Giebel. Insect. d. vorwelt, 360 .

3315. Heterogaster tristis. (Lygæidæ.)

Compared with H. urticæ F.

Locality: Ueningen, Baden. Horizon: Tortonian.

1865. Heer.

Urwelt der Schweiz, 392.

Monde prim. Suisse, 480.

Prim. world Switz., ii : 50.

1876. “

Urwelt der Schweiz, $2^{2}$ aufl, 416.

3316. Heterogaster troglodytes. (Lygæidæ.)

Locality : Radoboj, Croatia. Horizon: Mayencian.

1853. Heer. Insektenf, tertiärg. Oeningen, iii : 70-71, $131 . \quad P l .5$, fig. 14; pl. 9, fig. 17; pl. 14, fig. 18 .

1855. " "

Ber. oesterreich. litt., 1850-'53: 201.

1856. Giebel. Insect. d. vorwelt, 360 . 
3317. Homodemus ㄴ. (Capsidæ.)

1890. Scudder. Tert. ins. N. A., 362.

Locality : Prussian amber. Horizon : Ligurian.

3318. Hoplomachus - (2 sp.). (Capsidæ.)

Locality: Prussian amber. Horizon: Ligurian.

1890. Scudder. Tert. ins. N. A., 362.

3319. Hydrometra — (Hydrobatidæ.)

Locality : Prussian amber. Horizon: Ligurian.

1845. Berendt. Bernst. befindl. organ. reste vorw., i: 55.

3320. Hydrometra - (Hydrobatiāæ.)

Locality: Aix, France. Horizon: Ligurian.

1832. Burmeister. Handb. ent., i : 640.

1836. " Man, ent., 581.

See also Ploiaria ——.

\section{Hydrometra -. (Hydrobatidæ.)}

Locality : Prussian amber. Horizon: Ligurian.

1856. Menge. Progr. petrischule Danzig, 1856: 20.

\section{Hydrometra —. See Halobates (or Hydrometra) -}

3322. Hygrotrechus ståli. (Hydrobatidæ.)

Locality: Similkameen River, British Columbia. Horizon: -

1879. Scndder. Rep. progr. geol. surv. Can., 1877-'78: B 183-184.

1879. " Ins. tert. Nicola, 8-9.

See also Telmatrechus ståli.

\section{Hylotoma cineracea. (Cercopidæ.)}

Locality: Radoboj, Croatia, Horizon: Mayencian.

1843. Charpentier. Verhandl. leop.-carol. akad. naturf., xx: 409. Pl. 23, fig. 1.

1856. Giebel. Insect. d. vorwelt, 153.

See also Cercopis charpentieri.

\section{Hypselonotus lavateri. (Coreidœ.)}

Locality: Oeningen, Baden. Horizon: Tortonian.

1853. Heer. Insektenf. tertiärg. Oeningen, iii : 50-51. Pl. 4, fig. $3 ;$ pl. 9, fig. 2.

1856. Giebel. Insect. d. vorwelt, 350 .

3325. Jassopsis evidens. (Jassides.)

Locality : Florissant, Colo. Horizon : Oligocene.

1890. Scudder. Tert. ins. N. A., 312. Pl. 19, fig. 16.

\section{Jassus —_. (Jassides.)}

Locality: Prussian amber. Horizon: Ligurian.

1845. Berendt. Bernst. befindl. organ. reste vorw., i : 55. 


\section{Jassus —_. (Jassides.)}

Locality: Prussian amber. Horizon: Ligurian.

1831. Burmeister. Oken, Isis, 1831: 1100.

1832. " " Handb. ent., i: 638 .

1836. “ Man. ent., 579.

3328. Jassus _-. (Jassides.)

Locality: Prussian amber. Horizon: Ligurian.

1835. Gravenhorst. Uebers. schles. gesellsch. vaterl. cult., 1834 : 93.

3329. Jassus - (Jassides.)

Compared with J. atomarius.

Locality: Prussian amber. Horizon: Ligurian.

1835. Gravenhorst. Uebers. schles. gesellsch. vaterl. cult., 1834 : 93.

3330. Jassus —_. (Jassides.)

Compared with J. lineatus.

Locality: Prussian amber. Horizon: Ligurian.

1835. Gravenhorst. Uebers. schles. gesellsch. vaterl. cult., 1834: 93.

3331. Jassus, (Jassides.)

Compared with J. unifasciatus.

Locality : Prussian amber. Horizon: Ligurian.

1835. Gravenhorst. Uebers. schles. gesellsch. vaterl. cult., 1834: 93.

3332. Jassus immersus. (Jassides.)

Compared with J. subfusculus, Europe.

Locality: Prussian amber. Horizon: Ligurian.

1856. Germ.-Ber. Berendt, Bernst. befindl. organ. reste vorw., ii, th. i: 8-9. Pl.1, fig. 11.

3333. Jassus ? latebræ. (Jassides.)

Locality : Florissant, Colo. Horizon: Oligocene.

1890. Scudder. Tert. ins. N. A., 308. Pl. 20, fig. 19.

3334. Jassus spinicornis. (Jassides.)

Compared with J. fumipennis, Europe.

Locality: Prussian amber. Horizon: Ligurian.

1856. Germ.-Ber. Berendt, Bernst. befindl. organ. reste vorw., ii, th.i: 9. Pl. 1, fig. 12.

\section{Lachnus ——. (Aphides.)}

Locality: Prussian amber. Horizon : Ligurian.

1845. Berendt. Bernst. befindl, organ, reste vorw., i : 55.

\section{Lachnus ? - (Aphides.)}

Locality : Radoboj, Croatia. Horizon: Mayencian.

1883. Buckton. Monogr. Brit. aphid., iv. Pl. 132, fig. 11 (= Heer's figure 5a).

See also Aphis morloti. 
3337. Lachnus bonneti. (Aph̆ides.)

Locality : (Oeningen?), Baden. Horizon: Tortonian.

1853. Heer. 1856. Giebel. 1883. Buckton.

Insektenf. tertiärg. Oeningen, iii : 124-125. Pl. 15, fig. 1.

Insect. d. vorwelt, 390 .

Monogr. Brit. aphid., iv: 170. Pl. 132, fig. 3.

3338. Lachnus cimicoides. (Aphides.)

Locality: Prussian amber. Horizon: Ligurian.

1856. Germ.-Ber. Berendt, Bernst. befindl. organ. reste vorw., ii, th. i : 5-6. $P l .1$, fig. 6.

1883. Buckton. Monogr. Brit. aphid,, iv : 167 (Aphis in expl. pl.).`Pl. 131, fig. 8.

3339. Lachnus dryoides. (Aphides.)

Compared with L. quercus.

Locality: Prussian amber. Horizon: Ligurian.

1856. Germ.-Ber. Berendt, Bernst. befindl. organ. reste vorw., ii, th. i : 4-5. Pl. 1, fig. 4.

1883. Buckton. Monogr. Brit. aphid,, iv : 166-167. Pl. 131, figs. 6-7.

3340. Lachnus glandulosus. (Aphides.)

Locality : Prussian amber. Horizon: Ligurian.

1856. Menge. Progr. petrischule Danzig, 1856: 19.

3341. Lachnus longulus. (Aphides.)

Locality : Prussian amber. Horizon: Ligurian.

1856. Germ.-Ber. Berendt, Bernst. befindl. organ. reste vorw., ii, th. i: 5. Pl. 1, fig. 5.

1883. Buckton. Monogr. Brit. aphid., iv: 167. Pl. 131, fig. 9.

3342. Lachnus pectorosus. (Aphides.)

Losality : Radoboj, Croatia. Horizon: Mayencian.

1853. Heer. Insektenf. tertiärg. Oeningen, iii: 123-124. Pl. 15, fig. 2.

1855. " Ber, oesterreich. litt., 1850-53: 203.

1856. Giebel. Insect. d. vorwelt, 390 .

1883. Buckton. Monogr. Brit. aphid., iv: 171-172. Pl. 132, fig. 8.

3343. Lachnus petrorum. (Aphides.)

Compared with L. pini (Linn.).

Locality : Quesnel, British Columbia. Horizon :

1877. Scudder. Rep. progr. geol. surv. Can., 1875-'76: 279.

1877. " " Ins. tert. Quesn., 14.

1877. “ Rapp. opér. comm. géol. Can., 1875-76 : 309-310.

See also Gerancon petrorum.

3344. Lachnus quesneli. (Aphides.)

Locality : Quesnel, British Columbia. Horizon :

1878. Scudder. Add. ins. fauna Quesn., 5-6.

1878. " Rep. progr. geol. surv. Can., 1876-'77: 461-462.

1878. " Rapp. opér. comm. géol. Can., 1876-"77 : 518-519.

See also Sbenaphis quesneli. 
3345. Ledophora producta. (Jassides.)

1353. Heer.

Locality : Oeningen, Baden. Horizon: Tortonian.

1853-'56. Bronn.

Insektenf. tertiärg. Oeningen, iii : 116-117. Pl. 1\%, fig. 12.

1856. Giebel.

Lethæa geogn., $3^{\mathrm{e}}$ aufl., iii : 641. Pl. $4 \varkappa^{\prime}$, figs. $15 a-d$.

Insect. d. vorwelt, $386-387$.

3346. Leptoscelis humata. (Coreidæ.)

Compared with Pyrrhocoris königi Fabr.

Locality: Sieblos, Rhenish Prussia. Horizon: Aquitanian.

1858. Heyden. Palaeontogr., v : 117-119. Pl. 23, fig. 16.

Ligæus mutilus. See Lygæus mutilus.

3347. Ligyrocoris exsuctus. (Lygæidæ.)

Locality : Florissant, Colo. Horizon: Oligocene.

1890. Scudder. Tert. ins. N. A., 385. Pl. 24, fig. 5.

3348. Limnacis succini. (Hydrobatidæ.)

Locality: Prussian amber. Horizon : Ligurian.

1856. Germ.-Ber. Berendt, Bernst. befindl. organ. reste vorw., ii, th. i: 19. Pl. 3, fig. 18.

3349. Limnobates prodromus. (Limnobatidæ.)

Compared with L. stagnorum L., Europe.

Locality: Oeningen, Baden. Horizon: Tortonian.

1865. Heer. Urwelt der Schweiz, 392.

1872. " Monde prim. Suisse, 481.

1876. " " Prim. world Switz., ii: 51.

1879. “ Urwelt der Schweiz, $2^{\ominus}$ aufl., 418.

3350. Limnochares antiquus. (Galgulidæ.)

Locality : Rott, Rhenish Prussia. Horizon: Aquitanian.

1862. Heyden. Palaeontogr., $\mathrm{x}: 63$. Pl. 10, figs. 27-29.

1878. Bertkau. Verhandl. naturh. ver. preuss. Rheinl., 1878: 346-347, anm.

1885. " Sitzungsb. niederrh, gesellsch. natur- u. heilk., 1885: 298-299.

1890. Scudder. Tert. ins. N. A., 347.

3351. Linnæa abolita. (Lygæidæ.)

Locality : Florissant, Colo. Horizon: Oligocene.

1890. Scudder. Tert. ins. N. A., 398.

3352. Linnæa carcerata. (Lygæidæ.)

Locality : Florissaut, Colo. Horizon: Oligocene.

1890. Scudder. Tert. ins. N. A., 398-399. Pl. 23, fig. 2.

3353. Linnæa evoluta. (Lygæidæ.)

Locality : Florissant, Colo. Horizon: Oligocene。

1890. Scudder. Tert. ins. N. A., 399. Pl. 23, fig. 21.

3354. Linnæa gravida. (Lygæidæ.)

Locality : Florissant, Colo. Horizon: Oligocene.

1890. Scudder. Tert. ins. N. A., 399. Pl. 23, fig. 19. 
3355. Linnæa holmesii. (Lygæidæ.)

Locality : Florissant, Colo. Horizon: Oligocene.

1890. Scudder. Tert. ins. N. A., 397. Pl. 23, fig. 10.

3356. Linnæa putnami. (Lygæidæ.)

Locality : Florissant, Colo. Horizon: Oligocene.

1890. Scudder. Tert. ins. N. A., 397-398. Pl. 23, fig. 4.

3357. Iithadothrips vetusta. (Physapodes.)

Locality: White River, Utah. Horizon: Oligocene.

1875. Scudder. Bull. U. S. geol. surv, terr., i : 222.

1875. " Tert. Phys. Col., 2.

1877. “ Bull. U. S. geol. surv. terr., iii : 761 .

1890. " Tert. ins. N. A., 372. Pl. 5, figs. 88, 89, 102, 103.

3358. Lithaphis diruta. (Aphides.)

Localities: Florissant, Colo.; Green River, Wyo. Horizon: Oligocene.

1890. Scudder. Tert. ins. N. A., 258.

3359. Iithecphora diaphana. (Cercopidæ.)

Locality: Florissant, Colo. Horizon: Oligocene.

1890. Scudder. Tert. ins. N. A., 330-331. Pl. 21, fig.13.

3360. Lithecphora murata. (Cercopidæ.)

Locality : Florissant, Colo. Horizon: Oligocene.

1890. Scudder. Tert. ins. N. A., 331. Pl. 21, figs. 3, 8.

3361. Lithecphora setigera. (Cercopidæ.)

Locality: Florissant, Colo. Horizon: Oligocene.

1890. Scudder. Tert. ins. N. A., 330. Pl. 20, fig. 22.

3362. Iithecphora unicolor. (Cercopidæ.)

Locality: Florissant, Colo. Horizon: Oligocene.

1890. Scudder. Tert. ins. N. A., 331. Pl. 21, figs. 4, 5, 11, 14.

3363. Iithochromus extraneus. (Lygæidæ.)

Locality: Florissant, Colo. Horizon: Oligocene.

1890. Seudder. Tert. ins. N. A., 404. Pl. 26, fig. 6 .

3364. Lithochromus gardneri. (Lygæidæ.)

Locality: Florissant, Colo. Horizon : Oligocene.

1890. Scudder. Tert. ins. N. A., 403. ${ }^{-}$Pl. 26, fig. $10 ; p l .27$, fig. 8.

3365. Lithochromus mortuarius. (Lygæidæ.)

Locality: Florissant, Colo. Horizon: Oligocene.

1890. Scudder. Tert. ins. N. A., 404. Pl. 26, fig. 2.

3366. Lithochromus obstrictus. (Lygæidæ.)

Locality: Florissant, Colo. Horizon: Oligocene.

1890. Scudder. Tert. ins. N. A., 403-404. 
3367. Lithocoris evulsus. (Lygæidæ.)

Locality: Florissant, Colo. Horizon: Oligocene.

1890. Scudder. Tert. ins. N. A., 391.

3368. Lithopsis elongata. (Fulgoridæ.)

Locality: Green River, Wyo. Horizon: Oligocene.

1890. Scudder. Tert. ins. N. A, 301. Pl. 6, fig. 28.

3369. Iithopsis fimbriata. (Fulgoridæ.)

Locality: Green River, Wyo. Horizon: Oligocene.

1878. Seudder.

Bull. U. S. geol, surv. terr., iv : 774.

1885. " " Zittel, Handb. palaeont., i, th. ii: 781. Fig. 989.

1886. " Zittel-Barrois, Traité de pal6ont., ii: 781. Fig. 1006.

1890. " Tert. ins. N. A., 300. Pl. 6, figs.36, 37.

3370. Locrites copei. (Cercopidæ.)

Locality : Florissant, Colo. Horizon : Oligocene.

1890. Scudder. Tert. ins. N. A., 323-324. Pl. 21, fig. 19.

3371. Locrites haidingeri. (Cercopidæ.)

Locality: Radoboj, Croatia. Horizon: Mayencian.

1890. Scudder. Tert. ins. N. A., 323.

See also Cercopis haidingeri.

3372. Locrites whitei. (Cercopidæ.)

Locality: Florissant, Colo. Horizon: Oligocene

1890. Scudder. Tert. ins. N. A., 324. Pl. 21, fig. 17.

\section{Lopus - (Capsidæ.)}

Locality: Prussian amber. Horizon: Ligurian.

1890. Scudder. Tert. ins. N. A., 362.

3374. Lyctocoris terreus. (Acanthiidæ.)

Locality : Green River, Wyo. Horizon: Oligocene.

1890. Scudder. Tert. ins. N. A., 361. Pl. 7, fig. 20.

See also Rhyparochromus terreus.

3375. Iygæites acutus. (Lygæidæ.)

Locality : Oeningen, Baden. Horizon: Tortonian.

1853. Heer.

Insektenf. tertiärg. Oeningen, iii : 131-132. Pl. 14, fig. 15 。

1856. Giebel.

Insect. d. vorwelt, 361 .

3376. Lygæites lividus. (Lygæidæ.)

Locality : Radoboj, Croatia. Horizon: Mayencian.

1853. Heer. Insektenf. tertiärg. Oeningen, iii : 131. Pl. 14, fig. 14.

1855. " Ber. oesterreich. litt., 1850-'53: 201.

1856. Giebel. Insect. d. vorwelt, 361.

3377. Lygæites obsoletus. (Lygæidæ.)

Locality: Oeningen, Baden. Horizon: Tortonian.

1853. Heer. Insektenf, tertiärg. Oeningen, iii : 72, Pl. 14, fig. 12.

1856. Giebel. Insect. d. vorwelt, 361.

Bull. $71-27$ 
3378. Lygæites ovalis. (Lygæidæ.)

Locality : Oeningen, Baden. Horizon: Tortonian.

1853. Heer. 1856. Giebel.

1853. Heer. 1856. Giebel

1845. Berendt.

1829. Curtis. 1829.
Insektenf. tertiärg. Oeningen, iii : 71-72. Pl. 14, fig. 11.

Insect. $d$. vorwelt, 360-361.

3379. Lygæites pusillus. (Lygæidæ.)

Locality: Radoboj, C'roatia. Horizon: Mayencian.

Insektenf. tertiärg. Oeningen, iii : 72-73, $P l$. 14, fig. 13.

Insect. d. vorwelt, 361 .

\section{Lygæus ㄴ.. (Lygæidæ.)}

Locality : Prussian amber. Horizon: Ligurian.

\section{Lygæus —. (Lygæidæ.)}

Compared with L. abietis.

Locality: Aix, France. Horizon: Ligurian.

Edinb, new phil. journ., vii : 296.

Murchison-Lyell, Tert. form. Aix, 12.

3382. Lygæus - (2 sp.). (Lygæidæ.)

Locality: Brunstatt, Alsatia. Horizon: Middle Oligocene.

1890. Foerster. In litt.

\section{Lygæus - (Lygæidæ.)}

Locality: Prussian amber. Horizon: Ligurian.

1835. Gravenhorst. Uebers. schles. gesellsch. vaterl. cult., 1834: 93.

3384. Lygæus - - (Lygæidæ.)

Compared with $I_{\text {. }}$ compressicornis. Locality: Aix, France. Horizon: Ligurian.

1829. Serres. Géogn. terrains tert., $22 \%$.

3385. Lygæus —_. (Lygæidæ.)

Compared with L. errans.

Locality : Aix, France. Horizon: Ligurian.

1829. Serres. G6́ogn. terrains tert., 227.

\section{Lygæus ㄴ. (Lygæidæ.)}

Compared with L. melanocephalus. Locality : Aix, France. Horizon: Ligurian.

1829. Serres. Géogn. terrains tert., 227.

\section{Lygæus —_. (Lygæidæ.)}

Compared with L. punctum.

Locality: Aix, France. Horizon: Ligurian.

1829. Serres. Géogn, terrains tert., 227. 
3388. Iygæus atavinus. (Lygæidæ.)

\section{Locality: Radoboj, Croatia. Horizon: Mayencian.}

1853. Heer.

Insektenf. tertiärg. Oeningen, iii : $60-61 . \quad P l .4$, fig. $14 ; p l .9$, fig. 6.

1856. Giebel. Insect. d. vorwelt, 355 (attavinus).

Lygæus attavinus. See Lygæus atavinus.

3389. Lygæus dasypus. (Lygæidæ.)

Locality: Oeningen, Baden. Horizon: Tortonian.

1853. Heer.

1856. Giebel.
Insektenf. tertiärg. Oeningen, iii : 128-129. Pl. 15, fig. 8.

Insect. d. vorwelt, 354-355.

3390. Lygæus delle-chiaje. (Lygæidæ.)

Locality: Aix, France. Horizon: Ligurian.

1847. Hope. Descr. ins. foss., 6. Pl., fig. 3.

3391. Lygæus deprehensus. (Lygæidæ.)

Locality : Sieblos, Rhenish Prussia. Horizon: Aquitanian.

1859. Heyden. Palaeontogr., viii : 16. Pl. 3, fig. 8.

1860. " Hassenc., Würzb. naturw. zeitschr., i : 79.

3392. Lygæus deucalionis. (Lygæidæ.)

Locality : Radoboj, Croatia. Horizon: Mayencian.

1853. Heer. Insektenf. tertiärg. Oeningen, iii : 59-60. Pl. 4, fig. 15; pl.9, fig. 5.

1855. " Ber. oesterreich. litt., 1850-'53: 201.

1856. Giebel. Insect. d. vorwelt, 354.

3393. Lygæus fæculentus. (Lygæidæ.).

Locality : Florissant, Colo. Horizon: Oligocene.

1890. Soudder. Tert. ins. N. A., 377.

3394. Lygæus fossitius. (Lygæidæ.)

Locality : Sieblos, Rhenish Prussia. Horizon: Aquitanian.

1858. Heyden. Palaeontogr., v: 119. Pl. 23, fig. 17.

3395. Lygæus mutilus. (Lygæidæ.)

Locality: Krottensee, Bohemia. Horizon: Aquitanian.

1877. Novák. Sitzungsb. akad. wiss. Wien, lxxvi : 81 (Ligæus). Pl. 2, fig. 6. 1877. " $\quad$ Faun, egerer tertiärb., 11 (Ligæus), Pl. 2, fig. 6.

1890. Scudder. Tert. ins. N. A., 376 (not a Lygæus).

3396. Lygæus obsolescens. (Lygæidæ.)

Locality: Florissant, Colo. Horizon: Oligocene.

1890. Scudder. Tert. ins, N. A., 37\%. Pl. 24, fig. 15.

3397. Lygæus stabilitus. (Lygæidæ.)

Locality: Florissant, Colo. Horizon: Oligocene.

1890. Scudder. Tert. ins. N. A., 376-377. Pl. 23, fig. 10; pl. 24, fig. 16. 
3398. Lygæus tinctus. (Lygæidæ.)

Compared with L. venustus Böb., Europe.

Locality: Oeningen, Baden. Horizon: Tortonian.

1853. Heer.

1856. Giebel.

1865. Heer.

1872. " "

1876. “

1879. “

Insektenf. tertiärg. Oeningen, iii : 58-59. Pl. 4, fig. $13 ; \cdot p l .9$, fig. 4.

Insect. d. vorwelt, 354.

Urwelt der Schweiz, 362, 391. Fig. 301, on p. 391

Monde prim. Suisse, 444, 480. Fig. 301, on p. 479.

Prim. world Switz., ii : 16, 50. Fig. 301, on $p .49$.

Urwelt der Schweiz, $2^{\circ}$ aufl., 388, 416. Fig. 343, on p. 417.

3399. Lygæus ventralis. (Lygæidæ.)

Locality: Radoboj, Croatia. Horizon: Mayencian.

1853. Heer.

1855. "

Insektenf. tertiärg. Oeningen, iii : 129-130. Pl. 15, fig. 9.

Ber. oesterreich. litt., 1850-'53 : 201.

1856. Giebel. Insect. d. vorwelt, 355 .

3400. Lygus ㄴ. (Capsidæ.)

Locality: Prussian amber. Horizon : Ligurian.

1890. Scudder. Tert. ins. N. A., 362.

3401. Lystra ? leei. (Fulgoridæ.)

Locality: Green River, Wyo. Horizon: Oligocene.

1890. Scudder. Tert. ins. N. A., 283-284. Pl. 7, fig. 2.

3402. Lystra? richardsoni. (Fulgoridæ.)

Locality: Green River, Wyo. Horizon: Oligocene.

1878. Scudder. Bull. U. S. geol, surv. terr., iv: 772.

1890. " Tert. ins. N. A., 283. Pl. 6, figs. 24, 30, 31; pl. 7, figs. 1, 3.

3403. Matæoschistus limigenus. (Pentatomidæ.)

Locality : Florissant, Colo. Horizon: Oligocene.

1890. Scudder. Tert. ins. N. A., 460.

3404. Mecocephala ——. (Pentatomidæ.)

Locality : Florissant, Colo. Horizon : Oligocene.

1890. Scudder. Tert. ins. N. A., 464. Pl. 28, fig. 6.

3405. Melanothrips extincta. (Physapodes.)

Locality : White River, Colo. Horizon: Oligocene.

1875. Seudder.

1875. “

Bull. U. S. geol. surv. terr., i : 221.

1877. 6

Tert. Phys. Colo., 1.

Bull. U. S. geol. surv, terr., iii : 761.

1890. “

Tert. ins. N. A., $371 . \quad$ Pl. 5, figs. 90,91.

3406. Membracites cristatus. (Jassides.)

Locality: Oeningen, Baden. Horizon: Tortonian.

1853. Heer.

Insektenf. tertiärg. Oeningen, iii : 93. Pl. 13, fig. 12 ,

1856. Giebel.

Insect. d. vorwelt, 378. 
3407. Metrobates æternalís. (Hydrobatidæ.)

Locality: Florissant, Colo. Horizou: Oligocene.

1890. Scudder. Tert. ins. N. A., 353-354. Pl. 22, fig. 15.

See also Halobates — Scudder.

3408. Micropus - - Lygæidæ.)

Locality : Stösschen, Austria. Horizon : Miocene.

1859. Heyden. Palaeontogr, viii: 11. Pl. 1, fig. 15.

\section{Miris - (Capsidæ.)}

Locality : Aix, France. Horizon: Ligurian.

1829. Curtis. Edinb. new. phil. journ., vii : 296.

1829. " Murchison-Lyell, Tert. form. Aix, 12.

3410. Miris - (Capsidæ.)

Locality: Prussian amber. Horizon: Ligurian.

1835. Gravenhorst. Uebers. schles. gesellsch. vaterl. cult., 1834: 93.

\section{Miris ㄴ. (Capsidæ.)}

Compared with M. campestris.

Locality: Prussian amber. Horizon: Ligurian.

1835. Gravenhorst. Uebers. schles. gesellsch. vaterl. cult., 1834: 93.

\section{Miris —. (Capsidæ.)}

Compared with M. populi.

Locality: Prussian amber. Horizon: Ligurian. 1835. Gravenhorst. Uebers. schles. gesellsch. vaterl. cult., 1834: 93.

$$
\text { 3413. Miris - (Capsidæ.) }
$$

Compared with M. rufipennis.

Locality: Prussian amber. Horizon: Ligurian.

1835. Gravenhorst. Uebers. schles. gesellsch, vaterl, cult., 1834 : 93.

\section{Miris -. (Capsidæ.)}

Compared with M. tanaceti.

Locality : Prussian amber. Horizon: Ligurian.

1835. Gravenhorst. Uebers. schles. gesellsch. vaterl, cult, 1834 : 93.

3415. Mnemosyne terrentula. (Fulgorid®,)

Locality: Green River, Wyo. Horizon: Oligocene.

1878. Scudder. Bull. U. S. geol, surv. terr., iv: 773.

See also Oliarites terrentulus.

3416. Monanthia flexuosa. (Tingididæ.)

Compared with M. quadrimaculata Wolff, and M. wolffii Fieb., Europe,

Locality: Krottensee, Bohemia. Horizon: Aquitanian.

1877. Novák. Sitzungsb. akad. wiss. Wien, lxxvi : 79-80. Pl. 2, figs, 8-11.

1877. “ Faun. egerer tertiärb., 9-10. Pl. 2, figs. 8-11.

1890. Scudder. Tert. ins. N. A., 358. 
3417. Monanthia veterna. (Tingididæ.)

Locality: Florissant, Colo. Horizon: Oligocene.

1890. Scudder. Tert. ins. N. A., 359. Pl. 23, figs, 5,9.

3418. Monanthia wollastoni. (Tingididæ.)

Compared with M. convergens Klug.

Locality : Oeningen, Baden. Horizon: Tortonian.

1865. Heer. Urwelt der Sehweiz, 392 (in fig. as Tingis wollastoni),

1872. " Monde prim. Suisse, 480 (as above).

1876. " " Prim. world Switz., ii : 50 (as above).

1879. " Urwelt der Schweiz, $2^{\mathrm{e}}$ aufl., 417 (as above).

1890. Sendder. Tert. ins. N. A., 358.

See also Tingis wollastoni.

\section{Monophlebus -. (Coccidæ.)}

Locality: Prussian amber. Horizon: Ligurian.

1845. Berendt. Bernst. befindl. organ. reste vorw., i: 55.

See also III, Neuroptera, Acreagris crenata.

3420. Monophlebus irregularis. (Coccidæ.)

Locality : Prussian amber. Horizon: Ligurian.

1856. Germ.-Ber. Berendt, Bernst. befindl. organ. reste vorw., ii, th. i: 4. Pl.1, fig. 3.

1856. Menge. Progr. petrischule Danzig, 1856: 18 (undescr.).

3421. Monophlebus pinnatus. (Coccidæ.)

Locality: Prussian amber. Horizon: Ligurian.

1856. Germ.-Ber. Berendt, Bernst. befindl. organ. reste vorw., ii, th. i: 3. Pl. 1, fig. 1.

1856. Menge. Progr. petrischule Danzig, 1856: 18.

3422. Monophlebus simplex. (Coccidæ.)

Compared with M. pinnatus, Amber.

Locality : Florissant, Colo. Horizon: Oligocene.

1890. Scudder. Tert. ins. N. A., 242.

3423. Monophlebus trivenosus. (Coccidæ.)

Locality : Prussian amber. Horizon: Ligurian.

1856. Germ.-Ber. Berendt, Bernst. befindl. organ. reste vorw., ii, th. i: 3-4. Pl. 1 , fig. 2.

3424. Nabis —. (Nabidæ.)

Locality: Prussian amber. Horizon: Ligurian.

1845. Berendt. Bernst. befindl, organ. reste vorw, i: 55.

3425. Nabis gracillima. (Nabidæ.)

Locality : Oeningen, Baden. Horizon: Tortonian.

1865. Heer.

187\%. "

1876. " "

1879. “
Urwelt der Schweiz. Fig. 308, on p. 391 .

Monde prim. Suisse. Fig. 308, on p. 479.

Prim. world Switz., ii. Fig. 308, on p. 49.

Urwelt der Schweiz, $2^{\mathrm{e}}$ aufl. Fig. 350 , on $p .417$. 
3426. Nabis livida. (Nabidæ.)

Locality: Radoboj; Croatia. Horizon: Mayencian.

1853. Heer.

1855. " "

1856. Giebel.
Insektenf. tertiärg. Oeningen, iii : 76. $\quad$ Pl. 5, fig. 18.

Ber. oesterreich. litt., 1850-'53: 201.

Insect. d. vorwelt, 365 .

3427. Nabis lucida. (Nabidæe.)

Compared with N. vagans, Europe.

Locality : Prussian amber. Horizon: Ligurian.

1856. Germ.-Ber. Berendt, Bernst. befindl. organ. reste vorw., ii, th. i: 21. Pl. 2 fig. 10 .

3428. Nabis maculata. (Nabidæ.)

Locality: Radoboj, Croatia. Horizon: Mayencian.

1853. Heer. Insektenf. tertiärg. Oeningen, iii : 76-78. Pl. 5, fig. 19; pl. 10, fig. 1.

1855. “ Ber. oesterreich. litt., 1850-'53: 201.

1856. Giebel. Insect. d. vorwelt, 365 .

3429. Nabis prototypa. (Nabidæ.)

Locality : Prussian amber. Horizon: Ligurian.

1356. Menge. Progr. petrisehule Danzig, 1856: 20 (undeser.).

See also Reduvius — Germ.-Ber.

3430. Nabis vagabunda. (Nabidæ.)

Locality : Radoboj, Croatia. Horizon : Mayencian.

1853. Heer. Insektenf. tertiärg. Oeningen, iii : 75-76. Pl. 5, fig. 17; pt. 10, fig. 2.

1855. “ Ber. oesterreich. litt., 1850-53: 201.

1856. Giebel. Insect. d. vorwelt, 364-365.

3431. Naucoris dilatatus. (Naucoridæ.)

Locality : (Oeningen?, Baden). Horizon: Tortonian.

1353. Heer.

Insektenf. tertiärg. Oeningen, iii : 86. $\quad P l .10$, fig. 11.

1856. Giebel. Insect. d. vorwelt, 372.

1885. Scudder. Zittel, Handb. palaeont, i, th.ii: 783. Fig. 995.

1886. " Zittel-Barrois, Traité de paléont., ii : 782. Fig. 1012.

3432. Necrochromus cockerelli. (Lygæidæ.)

Locality: Florissant, Colo. Horizon : Oligocene.

1890. Scudder. Tert. ins. N. A., 407. Pl. 27, fig. 10.

3433. Necrochromus labatus. (Lygæidæ.)

Locality : Florissant, Colo. Horizon: Oligocene.

1890. Scudder. Tert. ins. N. A., 407. Pl. 27, fig. 14.

3434. Necrochromus saxificus. (Lygæidæ.)

Locality: Florissant, Colo. 'Horizon: Oligocene.

1890. Scudder. Tert. ins. N. A., 407-408. 
3435. Necrocydnus amyzonus. (Pentatomidæ.)

Locality : Florissant, Colo. Horizon: Oligocene.

1890. Scudder. Tert. ins. N. A., 446-44\%. Pl. 28, fig. 16.

3436. Necrocydnus gosiutensis. (Pentatomidæ.)

Locality: Green River, Wyo. Horizon: Oligocene.

1890. Scudder. Tert. ins. N. A., 445. Pl. 7, fig. 22.

3437. Necrocydnus revectus. (Pentatomidæ.)

Locality: Florissant, Colo. Horizon: Oligocene.

1390. Scudder. Tert. ins. N. A., 448.

3438. Necrocydnus senior. (Pentatomidæ.)

1890. Scudder. Tert. ins. N. A., 447.

Locality: Florissant, Colo. Horizon: Oligocene.

3439. Necrocydnus solidatus. (Pentatomidæ.)

Locality: Florissant, Colo. Horizon: Oligocene.

1890. Scudder. Tert. ins. N. A., 447. Pl. 28, fig. 13.

3440. Necrocydnus stygius. (Pentatomidæ.)

Locality: Florissant, Colo. Horizon: Oligocene.

1890. Scudder. Tert. ins. N. A., 446.

3441. Necrocydnus torpens. (Pentatomidæ.)

Locality: Florissant, Colo. Horizon: Oligocene.

1890. Scudder. Tert. ins. N. A., 445-446.

3442. Necrocydnus vulcanius. (Pentatomidæ.)

Locality : Florissant, Colo. Horizon: Oligocene.

1890. Scudder. Tert. ins. N. A., 444-445.

3443. Necropsylla rigida. (Psyllidæ.)

Locality : Florissant, Colo. Horizon: Oligocene.

1890. Seudder. Tert. ins. N. A., 276-277. Pl. 12, figs. 11, 21.

See also III, Neuroptera, Necropsocus - - .

3444. Necygonus rotundatus. (Galgulidæ.)

Locality: Green River, Wyo. Horizon: Oligocene.

1890. Scudder. Tert. ins. N. A, 348. Pl. 7, fig. 8 .

3445. Nepa - (Nepidæ.)

Locality : Prussian amber. Horizon : Ligurian.

1830. Berendt. Insekten im bernstein, 36 .

3446. Nepa -. (Nepidæ.)

Locality : Oeningen, Baden. Horizon: Tortonian.

1834. Keferstein. Naturg erdkörp., ii : 340. 
3447. Nepa - (6 sp.). (Nepidæ.)

Locality: Oeningen, Baden. Horizon: Tortonian.

1888. Schöberlin. Soc. ent., iii : 61 .

3448. Nepa
Compared with N, cinerea.
Locality : Aix, France. Horizon : Ligurian.
1829. Serres. $\quad$ Géogn, terrains tert., 228.

3449. Nepa atavina. (Nepidæ.)

Locality: Oeningen, Baden. Horizon: Tortonian.

1853. Heer.

Insèktenf. tertiärg. Oeningen, iii : 85. $P l .10, f i g .9$.

1856. Giebel.

Insect. $d$. vorwelt, 370 (attavina).

Trepa attavina. See Nepa atavina.

3450. Neurocoris elongatus. (Pentatomidæ.)

Locality: Radoboj, Croatia. Horizon: Mayencian.

1853. Heer. 1855. " " 1856. Giebel.

Insektenf. tertiärg. Oeningen, iii : $25 . \quad P l .2$, fig. $5 ; p l .6$, fig. 15. Ber. oesterreich. litt., 1850-'53: 200.

Insect. d. vorwelt, 339.

3451. Neurocoris rotundatus. (Pentatomidæ.)

Locality: Radoboj, Croatia. Horizon: Mayencian.

1853. Heer, Insektenf. tertiärg. Oeningen, iii: 24-25. Pl. 2, fig. 4; pl. 6, - fig. 14.

1853-56. Bronn. Lethæa geogn., $3^{\text {e }}$ aufl, iii : 643. Pl, 42', fig. 19.

1855. Heer. Ber. oesterreich. litt., 1850-'53: 200.

1856. Geibel. Insect. d. vorwelt, 339.

1885. Scudder. Zittel, Handb. palaeont., i, th.ii : 786. Fig. 1004.

1886. " Zittel-Barrois, Traité de palént., ii : 786. Fig. 1021.

3452. Notonecta —. (Notonectidæ.)

Locality : Aix, France. Horizon: Ligurian.

1847. Hope. Trans. ent. soc. Lond., iv : 252.

3453. Notonecta - (Notonectidæ.)

Locality: Oeningen, Baden. Horizon: Tortonian.

1888. Schöberlin. Soc. $\bullet$ nt., iii : 61 .

3454. Notonecta - (Notonectidæ.)

Locality : Florissant, Colo. Horizon : Oligocene.

1885. Scudder. Zittel, Handb. palaeont., i, th. ii : 782.

1886. " Zittel-Barrois, Traité de paléont., ii : 782.

1886. " Bull. U. S. geol. surv., No. $31: 61$.

See also the following.

3455. Notonecta emersoni. (Notonectidæ.)

Locality : Florissant, Colo. Horizon: Oligocene.

1890. Scudder. Tert. ins. N. A., 346-347. Pl. 22, fig. 11.

See also the preceding. 
3456. Notonecta heydeni. (Notonectidæ.)

Compared with N. glauca.

Locality : Kutschlin, Bohemia. Horizon: Miocene.

1881. Deichmüller. Foss, ins. diatomeenseh. Kutschlin, 36-38. Pl. 21, figs. 16-18

1881. " Verhandl. leop,-carol. akad, naturf., xlii: 328-330. Pl. 21, figs. 16-18.

3457. Notonecta primæva. (Notonectidæ.)

Locality: Rott, Rhenish Prussia. Horizon: Aquitånian.

1859. Heyden. Palaeontogr., viii : 11. Pl. 2, fig. 12.

3458. Nyctophylax uhleri. (Fulgoridæ.)

Locality: Florissant, Colo. Horizon: Oligocene.

1890. Scudder. Tert. ins. N. A., 279-280. Pl. 19, fig. 11.

3459. Nyctophylax vigil. (Fulgoridæ)

Locality: Florissant, Colo. Horizon: Oligocene.

1890. Scudder. Tert. ins. N. A., 280. Pl. 19, fig. 8.

3460. Nysius stratus. (Lygæidæ.)

Locality : Florissant, Colo. Horizon : Oligocene,

1890. Scudder. Tert. ins. N. A., 380. Pl. 23, figs. 14, $27 ; p l .25$, figs. $2,8$.

3461. Nysius terræ. (Lygæidæ.)

Locality : Florissant, Colo. Horizon : Oligocene.

1890. Scudder. Tert. ins. N. A., 379. Pl. 23, fig. 31.

3462. Nysius tritus. (Lygæidæ.)

Locality : Florissant, Colo. Horizon : Oligocene.

1890. Scudder. Tert. ins. N. A., 379. Pl. 23, fig. 20.

3463. Nysius vecula. (Lygæidæ.)

Locality : Florissant, Colo. Horizon : Oligocene.

1890. Scudder. Tert. ins. N. A., 378. Pl. 22, fig. 7.

3464. Nysius vinctus. (Lygæidæ.)

Locality : Florissant, Colo. Horizon: Oligocene.

1890. Scudder. Tert. ins. N. A., 378.

3465. Ochyrocoris electrina. (Coccidæ.)

Locality : Prussian amber. Horizon: Ligurian.

1856. Menge. Progr. petrischule Danzig, 1856: 17-18.

3466. Oliarites terrentulus. (Fulgoridæ.)

Locality: Green River, Wyo. Horizon: Oligocene.

1890. Scudder. Tert. ins. N. A., 293 (terrentula). Pl. 7, fig. 17.

See also Mnemosyne terrentula.

3467. Oliarus? lutensis. (Fulgoridæ.)

Locality: Green River, Wyo. Horizon: Oligocene.

1890. Scudder. Tert, ins. N. A., 288. $P l .7, f i g .18$. 
3468. Oncotylus —. (Capsidæ.)

Locality : Prussian amber. Horizon: Ligurian.

1890. Seudder.

r. $=0$.

1890. Scudder.

1899. Scudder.

1890. Scudder.

1890. Scudder.

Tert. ins. N. A., 362.

3469. Orthops - (Capsidæ.)

Locality : Prussian amber. Horizon: Ligurian.

Tert. ins. N. A., 362.

3470. Orthriocorisa longipes. (Coreidæ.)

Locality: Florissant, Colo. Horizon: Oligocene. Tert. ins. N. A., 430. Pl. 26, fig. 1.

3471. Oryctaphis lesueurii. (Aphides.)

Locality : Florissant, Colo. Horizon: Oligocene. Tert. ins. N. A., 267.

3472. Oryctaphis recondita. (Aphides.)

Locality: Florissant, Colo. Horizon: Oligocene. Tert. ins. N. A., 266-267. Pl. 18, fig. 14.

3473. Pachycoris burmeisteri. (Pentatomidæ.)

Locality: Oeningen, Baden. Horizon: Tortonian.

1865. Heer.

18\%2. "

1876. “

1879. “

1853. Heer.

1856. Giebel.

Urwelt der.Schweiz. Fig. 298, on p. 391.

Monde prim. Suisse. Fig. 298, on p. 479.

Prim. world Switz., ii. Fig. 298, on p. 49.

Urwelt der Schweiz, $2^{\mathrm{e}}$ anfl. Fig. 340, on p. 417.

3474. Pachycoris escheri. (Pentatomidæ.)

Locality: Oeningen, Baden. Horizon: Tortonian.

Insektenf. tertiärg. Oeningen, iii : 10-11. Pl. 1, fig. $2 ; p l .6$, fig. 2.

Insect. $d$. vorwelt, 334 .

3475. Pachycoris germari. (Pentatomidæ.)

Locality: Oeningen, Baden. Horizon: Tortonian.

1853. Heer.

1856. Giebel.

Insektenf. tertiärg. Oeningen, iii: 9-10. Pl. 1, fig. 1; pl. 6, fig. 1.

Insect. d. vorwelt, 334 .

3476. Pachycoris protogæus. (Pentatomidæ.)

Locality: Oeningen, Baden. Horizon: Tortonian.

1853. Heer.

1856. Giebel.

Insektenf, tertiärg. Oeningen, iii : 126. Pl. 14, fig. 20 .

Insect. d. vorwelt, 335 (protogneus).

Pachycoris protogneus. See Pachycoris protogæus.

3477. Pachymerus —. (Lygæidæ.)

Locality : Prussian amber. Horizon: Ligurian.

1845. Berendt. Bernst. befindl. organ. reste vorw., i: 55 . 
3478. Pachymerus (4 sp.). (Lygæidæ.)

Locality: Brunstatt, Alsatia. Horizon: Middle Oligocene.

1890. Foerster. In litt.

3479. Pachymerus antiquus. (Lygæidю.)

Locality: Sieblos, Rhenish Prussia. Horizon: Aquitanian.

1859. Heyden.

1860.

Palaeontogr., viii: 16-17. Pl. 3, fig. 9.

Hassenc., Würzb. naturw, zeitschr., i : 79.

3480. Pachymerus bisignatus. (Lygæidæ.)

Locality: Radoboj, Croatia. Horizon: Mayencian.

1853. Heer. Insektenf. tertiärg. Oeningen, iii: 63-64. Pl. 5, fig. 2; pl. 9, fig. 9.

1855. “ Ber. oesterreich. litt., 1850-53: 201.

1856. Giebel. Insect. d. vorwelt, 356 .

Pachymerus bojeri. See Pachymerus boyeri.

3481. Pachymerus boyeri. (Lygæiđæ.)

\section{Locality: Aix, France. Horizon: Ligurian.}

1853. Heer. Insektenf. tertiärg. Oeningen, iii : 64-65 (bojeri). Pl. 5, fig. 1; pl. 9, fig. 10.

1856. " Viert. naturf. gesellsch. Zürich, i: 38 (bojeri).

1856. Giebel. Insect. d. vorwelt, 357 (bojeri).

See also Corizus boyeri.

3482. Pachymerus coloratus. (Lygæidæ.)

Locality: Prussian amber. Horizon: Ligurian.

1856. Germ.-Ber. Berendt, Bernst. befindl. organ. reste vorw., ii, th. i: 30. Pl. 3, fig. 15.

3483. Pachymerus cruciatus. (Lygæidæ.)

Compared with P. luscus H.-S., P. bactris.

1861. Heer.

1865. :

1872. "

1876. “6

1879. "

Locality: Oeningen, Baden. Horizon: Tortonian.

Rech. climat pays tert., 203 (undescr.).

Urwelt der Schwoiz, 391.

Monde prim. Suisse, 480.

Prim. world Switz., ii : 50.

Urwelt der Schweiz, $2^{\circ}$ aufl, 416.

3484. Pachymerus dryadum. (Lygæidæ.)

Locality: Aix, France. Horizon: Ligurian.

1853. Heer.

1856. "6

1856. Giebel.

lnsektenf. tertiärg. Oeningen, iii : 65. Pl. 5, fig. 4.

Viert. naturf. gesellsch. Zürich, i: 38 .

Insect. d. vorwelt, 357.

3485. Pachymerus fasciatus. (Lygæidæ.)

Locality: Aix, France. Horizon : Ligurian.

1853. Heer.

1856. " “

1856. Giebel.

1890. Scudder.
Insektenf. tertiärg. Oeningen, iii : 67. $P l .5, f i g .7 ; p l .9, f i g .12$.

Viert. naturf. gesellsch. Zürich, i : 38.

Insect. d. vorwelt, 358.

Tert. ins. N. A., 397. 
3486. Pachymerus heeri. (Lygæidæ,)

Locality : Aix, France. Horizon : Ligurian.

1856. Giebel. Insect. d. vorwelt, 357.

3487. Pachymerus morio. (Lygæidæ.)

Loeality : Oeningen, Baden. Horizon: Tortonian.

1853. Heer. 1856. Giebel.

1853. Heer.

1856. Heer. 1856. Giebel.
Insektenf. tertiärg. Oeningen, iii : 130. Pl. 14, fig. 16.

Insect: d, vorwelt, 358.

\section{Locality : Aix, France. Horizon: Ligurian.}

Insektenf. tertiärg. Oeningen, iii : 62-63. Pl. 4, fig. 17; pl. 9, fig. 8.

Viert. naturf. gesellsch. Zürich, i : 38 .

Insect. d. vorwelt, 356 .

3489. Pachymerus oblongus. (Lygæidæ.)

Locality: Oeningen, Baden. Horizon: Tortonian.

1853. Heer. Insektenf. tertiärg. Oeningen, iii: 67-58. Pl. 5, fig. $3 ; p l .9$, fig. 13.

1856. Giebel. Insect. d. vorwelt, 358.

3490. Pachymerus obsoletus. (Lygæidæ.)

Locality: Oeningen, Baden. Horizon: Tortonian.

1853. Heer. Insektenf. tertiärg. Oenirgen, iii : $66 . \quad P l .5$, fig. 5.

1856. Giebel. Insect. d. vorwelt, 357.

3491. Pachymerus petrensis. (Lygæidæ.)

Locality: White River; Utah. Horizon : Oligocene.

1877. Scudder. Bull. U. S. geol. surv. terr., iii : 761 .

1890. " Tert. ins. N. A., 401. Pl. 5, figs. 70, 71.

3492. Pachymerus pulchellus. (Lygæidæ.)

Locality: Aix, France. Horizon : Ligurian.

1853. Heer. Insektenf. tertiärg. Oeningen, iii: 66-67. Pl. 5, fig. 6; pl, 9, fig. 11.

1856. " Viert. naturf. gesellsch. Zürich, i: 38.

1856. Giebel. Insest. d. vorwelt, 358.

1890. Scudder. Tert. ins. N. A., 397.

3493. Pachymerus senius. (Lygæidæ.)

Locality : Prussian amber. Horizon : Ligurian.

1856. Germ.-Ber. 'Berendt, Bernst. befindl. organ. reste vorw., ii, th. i: 30 . pl. 3, fig. 14.

3494. Palæocoris spectabilis. (Coreidæ.)

Locality : Radoboj, Croatia. Horizon : Mayencian.

1853. Heer. Insektenf. tertiärg. Oeningen, iii: 46-47. $P l .4, f g .1 ; p l .8$, fig. 11.

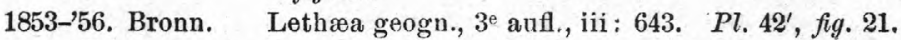

1855. Heer.

1856. Giebel.

Ber. oesterreich. litt., 1850-53: 201.

Insect, d. vorwelt, 348. 
3495. Palæothrips fossilis. (Physapodes.)

Locality: White River, Utah. Horizon: Oligocene.

186\%. Scudder. 1868. " " 1875. Scudder. 1875. “ 1877. “ 1885. “ 1886. “6 1890. Lesley. 1890. Scudder.
1873. Oustalet.

Proc. Bost. soc. nat. hist., xi : 117.

Geol. mag., v : 221.

Bull. soc. philom. Paris, $\mathrm{x}: 27$.

Bull. U. S. geol. surv. terr., i: 222-223.

Tert. Phys. Colo., 2-3.

Bull. U. S. geol. surv. terr., iii : 761.

Zittel, Handb. palaeont., i, th. ii : 784. Fig. 999.

Zittel-Barrois, Traité de paléont., ii : 784. Fig. 1016.

Dict. foss. Penns., ii : 590. Fig.

Tert. ins. N. A., 373-374. Pl. 5, figs. 104, 105, 115.

3496. Palæovelia spinosa. (Veliidæ.)

Locality : Florissant, Colo. Horizon: Oligocene.

1890. Scudder.

Tert. ins. N. A., 349. $\quad P l .22$, fig. 13.

3497. Palaphrodes cincta. (Cercopidæ.)

Locality: Florissant, Colo. Horizon: Oligocene.

1890. Scudder.

Tert. ins. N. A., 334-335. Pl. 20, fig. $16 ;$ pl. 21, fig. 15.

3498. Palaphrodes irregularis. (Cercopidæ.)

Locality: Florissant, Colo. Horizon : Oligocene.

1890. Scudder.

Tert. ins. N. A., 335-336. Pl. 20, figs. 2,18; pl. 21, figs. 6,7.

3499. Palaphrodes obliqua. (Cercopidæ.)

Locality : Florissant, Colo. Horizon: Oligocene.

1870. Scudder.

Tert. ins. N. A., 336. Pl. 21, fig. 10.

3500. Palaphrodes obscura. (Cercopidæ.)

Locality : Florissant, Colo. Horizon : Oligocene.

1890. Scudder.

Tert. ins. N. A., 335. Pl. 21, fig. 18.

3501. Palaphrodes transversa. (Cercopidæ.)

Locality : Florissant, Colo. Horizon: Oligocene.

1890. Scudder. Tert. ins. N. A., 336-337.

3502. Palecphora communis. (Cercopidæ.)

Locality : Florissant, Colo. Horizon: Oligocene.

1890. Scudder.

Tert. ins. N. A., 328. $P l$. 20, figs. 3, 20, 21.

3503. Palecphora inornata. (Cercopidæ.)

Locality : Florissant, Colo. Horizon : Oligocene.

1890. Scudder. Tert. ins. N. A, 329. $P l .20, f i g .15$.

3504. Palecphora maculata. (Cercopidæ.)

Locality : Florissant, Colo. Horizon: Oligocene.

1890. Scudder. Tert. ins. N. A., 326-327. Pl. 20, figs. 10, 17.

3505. Palecphora marvinei. (Cercopidæ.)

Locality: Florissant, Colo. Horizon: Oligocene.

1890. Scudder.

Tert, ins. N. A., 327-328. $\quad P l .20$, figs, 11-13; pl. 21, figs. 9, 12. 
3506. Palecphora patefacta. (Cercopidæ.)

Locality : Green River, Wyo. Horizon: Oligocene.

1890. Scudder. Tert. ins. N. A., 327. Pl. 7, fig. 7.

3507. Palecphora prævalens. (Cercopidæ.)

Locality : Florissant, Colo. Horizon : Oligocene.

1890. Scudder. Tert. ins. N. A., 329. Pl. 20, fig. $1 ; p l .21$, fig. 2.

3508. Parodarmistus abscissus. (Coreidæ.)

Locality : Florissant, Colo. Horizon : Oligocene.

1890. Scudder. Tert. ins. N. A., 422.

3509. Parodarmistus caducus. (Coreidæ.)

Locality: Florissant, Colo. Horizon: Oligocene.

1890. Scudder. Tert. ins. N. A., 422.

3510. Parodarmistus collisus. (Coreidæ.)

Locality: Florissant, Colo. Horizon: Oligocene.

1890. Scudder. Tert. ins. N. A., 422-423. Pl. 25, fig. 13.

3511. Parodarmistus defectús. (Coreidæ.)

Locality : Florissant, Colo. Horizon: Oligocene.

1890. Scudder. Tert. ins. N. A., 423.

3512. Parodarmistus exanimatus. (Coreidæ.)

Locality : Florissant, Colo. Horizon : Oligocene.

1890. Scudder. Tert. ins. N. A., 423-424.

3513. Parodarmistus inhibitus. (Coreidæ.)

Locality : Florissant, Colo. Horizon: Oligocene.

1890. Scudder. Tert, ins. N. A., 424.

3514. Pediopsis - (Jassides.)

Locality : Brunstatt, Alsatia. Horizon: Middle Oligocene.

1890. Foerster. In litt.

\section{Pemphigus bursifex. (Aphides.)}

Compared with P. bursarius L., Europe.

Locality : (Oeningen ?, Baden.) Horizon : Tortonian.

1853. Heer.

1856. Giebel.

1865. Heer.

1872. "

1876. “

1879. "

1883. Buckton.

Insektenf. tertiärg. Oeningen, iii : 125. $\quad P l .15, f i g .6$.

Insect. d. vorwelt, 390 .

Urwelt der Schweiz, 389.

Monde prim. Suisse, 477.

Prim. world Switz., ii : 47.

Urwelt der Schweiz, $2^{\mathrm{e}}$ anfl., 414.

Monogr. Brit. aphid., iv : 172-173. Pl. 132, figs. $12 a, 12 b$.

3516. Pentatoma

(Pentatomidæ.)

Locality : Aix, France. Horizon : Ligurian.

1829. Curtis.

Edinb. new phil. journ., vii : 296.

18:29. “

Murchison-Lyell, Tert. form. Aix, 12. 
3517. Pentatoma - $(4 \mathrm{sp}$.$) . (Pentatomidæ.)$

Locality : Brunstatt, Alsatia. Horizon: Middle Oligocene.

1890. Foerster. In litt.

3518. Pentatoma —- (Pentatomidæ.)

Locality: Prussian amber. Horizon: Ligurian.

1829. Serres. G6́gn. terrains tert., 241.

3519. Pentatoma - (Pentatomidæ.)

Compared with P. grisea.

Locality : Aix, France. Horizon : Ligurian.

1829. Serres. Géogn. terrains tert., 227,271 (on p. 271 called P. grisea). $P l$. 4, figs. 5, 6.

3520. Pentatoma —. (Pentatomidæ.)

Compared with P. oleracea.

Locality : Aix, France. Horizon: Ligurian.

1829. Serres. Géogn. terrains tert., 227.

3521. Pentatoma antiquum. (Pentatomidæ.)

Locality: Oeningen, Baden. Horizon: Tortonian.

1853. Heer. Insektenf. tertiärg. Oeningen, iii: 26-27. Pl. 2, fig. $7 ; p l .7$, fig. 1.

1856. Giebel. Insect. d. vorwelt, 340-341.

3522. Pentatoma appendículatum. (Pentatomidæ.)

Locality : Oeningen, Baden. Horizon: Tortonian.

1853. Heer.

Insektenf. tertiärg. Oeningen, iii : 29. $P l .2$, fig. $11 ; p l .7$, fig. 4. 1856. Giebel.

Insect. d. vorwelt, 341 .

3523. Pentatoma böttgeri. (Pentatomidæ.)

Compared with P. rufipes.

Locality : Salzhausen, Germany. Horizon : Miocene.

1865. Heyd.-Heyd. Palaeontogr., xiv: 34. Pl. 9, fig. 22.

3524. Pentatoma boreale. (Pentatomidæ.)

Locality: Atanekerdluk, North Greenland. Horizon: Miocene.

1868. Heer.

Flora foss. arct., i : 130. Pl. 19, figs. 15, $15 b c$.

1883. " Flora foss. Grönl., ii : 148.

3525. Pentatoma bruckmanni. (Pentatomidæ.)

Locality: Oeningen, Baden. Horizon: Tortonian.

1851. Stizenberger. Uebers. verstein. Baden, 95 (as of Heer; name only. By mistake for some other name, perhaps Halys bruckmanni).

Pentatoma grisea. See Pentatoma- Serres.

3526. Pentatoma lividum. (Pentatomidæ.)

Locality: Radoboj, Croatia. Horizon: Mayencian.

1851. Heer. Insektenf. tertiärg. Oeningen, iii : $30-31 . \quad P l .2, f i g .13 ; p l .7_{s}$ fig. 6.

1855. " Ber. oesterreich. litt., 1850-53:200,

1856. Giebel. Insect. $d$. vorwelt, 342 , 
3527. Pentatoma longiceps. (Pentatomidæ)

Locality: Oeningen, Baden. Horizon: Tortonian.

1853. Heer. Insektenf. tertiärg. Oeningen, iii : 29-30. Pl. 2, fig. 12; pl. 7, fig. 5.

1856. Giebel. Insect. d. vorwelt, 342.

3528. Pentatoma morloti. (Pentatomidæ.)

Locality: Radoboj, Croatia. Horizon: Mayencian.

1853. Heer.

Insektenf. tertiärg. Oeningen, iii : 28-29. $P l .2, f i g .10 ; p l .7$, fig. 3.

1855. “

1856. Giebel.

Ber. oesterreich. litt., 1850-53: 200.

Insect. d. vorwelt, 341 .

3529. Pentatoma obsoletum. (Pentatomidæ.)

Locality: Oeningen, Baden. Horizon: Tortonian.

1853. Heer. Mitth. naturf. gesellsch. Zürich, iii : 191.

1853. "6 Rhynchoten tertiärz., 23.

See also Alia obsoleta.

3530. Pentatoma pictum. (Pentatomidær.)

Locality: Oeningen, Barlen. Horizon: Tortonian.

1865. Heer. Urwelt der Schweiz. Fig. 309 on $p .391$.

1872. " Monde prim. Suisse. Fig. 309 on p. 479.

1876. " $\quad$ Prim. world Switz., ií. Fig. 309 ou p. 49.

1879. " Urwelt der Schweiz, $2^{e}$ anfl. Fig. 351 on p. 417.

3531. Pentatoma schaurothi. (Pentatomidæ,)

Compared with P. dissimile, Europe.

Locality : Prussian amber. Horizon : Ligurian.

1862. Giebel. Zeitschr. gesellsch. naturw, xx: 313-314.

3532. Pentatoma stigmatum. (Pentatomidæ.)

Locality : Oeningen, Baden. Horizon : Tortonian.

1853. Heer. Insektenf. tertiärg. Oeningen, iii: 31-32. Pl. 2, fig. 14.

1856. Giebel. Insect. d, vorwelt, 342.

3533. Pentatoma vetustum. (Pentatomidæ.)

Locality: Oeningen, Baden. Horizon: Tortonian.

1853. Heer.

1856. Giebel.

Insektenf, tertiärg. Oeningen, ii : 27-28. Pl.2, fig.8; pl.7,fig.2. •

Insect, d. vorwelt, 341 .

3534. Pentatomites foliarum. (Pentatomidæ.)

Locality : Florissant, Colo. Horizon: Oligocene.

1890. Scudder. Tert. ins. N. A., 462. Pl. 28, fig. 1.

3535. Petrolystra gigantea. (Cercopidæ.)

Locality : Florissant, Colo. Horizon: Oligocene.

1878. Scudder. Bull. U. S. geol. surv. terr., iv : 531-532.

1885. " Zittel, Haudb. palaeont., i, th. ii: 781. Fig. 992.

1886. " " Zittel Barrois, Traité de paléont., ii : 781. Fig. 1009.

1890. " " Tert. ins, N. A., 321-322. Pl. 20, figs. 5-7.

Bull, $71 \_28$ 
3536. Petrolystra heros. (Cercopidæ.)

1878. Scudder. 1890

Locality : Florissant, Colo. Horizon : Oligocene.

Bull. U. S. geol. surv, terr., iv : 532.

Tert. ins. N. A., 322-323. $\quad P l$. 20, fig. 8.

3537. Phlœocoris monstrosus. (Pentatomide.)

Locality : Radoboj, Croatia. Horizon: Mayencian.

1853. Heer. Insektenf. tertiärg. Oeningen, iii : 25-26. Pl. 2, fig.6.

1855. " Ber. oesterreich. litt., 1850-53: 200.

1856. Giebel. Insect. d. vorwelt, 340 .

3538. Phlœothrips pohligi. (Physapodes.)

Locality : Rott, Rhenish Prussia. Horizon: Aquitanian.

1887. Schlechtend. Zeitschr, gesellsch, naturw., lx: 560-563. Pl. 3, fig. 1.

3539. Phrudopamera chittendeni. (Lygieidæ.)

Locality : Florissant, Colo. Horizon : Oligocene.

1890. Scudder. Tert. ins. N. A., 389. Pl. 26, figs. 7,9 .

3540. Phrudopamera wilsoni. (Lygaidæ.)

Locality: Florissant, Colo. Horizon : Oligocene.

1890. Scudder. Tert. ins. N. A., 388-389. Pl. 27, figs. 9, 16.

3541. Phthinocoris colligatus. (Coreidæ.)

Locality : Florissant, Colo. Horizon: Oligocene.

1890. Scudder. Tert. ins. N. A., 414-415. Pl. 22, fig. 3 .

3542. Phthinocoris languidus. (Coreidæ.)

Locality : Florissant, Colo. Horizon: Oligocene.

1890. Scudder.

Tert. ins. N. A., 415-416. Pl. 27, fig. 6.

3543. Phthinocoris lethargicus. (Coreidæ.)

Locality : Florissant, Colo. Horizon: Oligocene.

1890. Scudder. Tert. ins. N. A., 415. Pl. 26, fig. $17 ; p l .27$, fig. 17.

3544. Phthinocoris petræus. (Coreidæ.)

Locality : Florissant, Colo. Horizon: Oligocene.

1890. Scudder. Tert. ins. N. A., 416.

3545. Phytocoris $\perp$. (Capsidæ.)

Locality : Prussian amber. Horizon: Ligurian.

1845. Berendt. Bernst. befindl, organ, reste vorw., i: 55.

3546. Phytocoris ——. (Capsidæ.)

Locality : Prussian amber. Horizon: Ligurian.

1890. Scudder.

Tert. ins. N. A., 362.

3547. Phytocoris - (larva). (Capsidæ.)

Locality: Prussian amber. Horizon: Ligurian.

1856. Germ.-Ber. Berendt, Berust. befindl, orgau. reste vorw., ii, th, i, 29-30, $P l_{\text {e }}$ 2, fig. 14.

1856. Giebel. Insect, $d$, vorwelt, 362. 
3548. Phytocoris angustulus. (Capsidæ.)

Compared with P. betuleti.

Locality : Prussian amber. Horizon: Ligurian.

1856. Germ.-Ber. Berendt, Bernst. befindl, organ. reste vorw., ii, th. i : 29. Pl. 3 , fig. 11.

3549. Phytocoris balticus. (Capsidæ.)

Compared with $P$. angulatus.

Locality : Prussian amber. Horizon: Ligurian.

1856. Germ.-Ber. Berendt, Bernst. befindl, organ. reste vorw., ii, th. i : 26-27. Pl. $3, f i g .4$.

3550. Phytocoris consobrinus. (Capsidæ.)

Compared with P. sexguttatus, Europe.

Locality: Prussian amber. Horizon: Ligurian.

1856. Germ. Ber. Berendt, Bernst. befindl. organ. reste vorw., ii, th. i: 25-26. Pl. 3, fig. 2.

3551. Phytocoris electrinus. (Capsidæ.)

Compared with $P$. thoracicus.

Locality : Prussiau amber. Horizon : Ligurian.

1856. Germ.-Ber. Berendt, Bernst. befindl. organ. reste vorw., ii, th. i: 28. Pl. 3, fig. 8.

3552. Phytocoris ? euglotta. (Capsidæ.)

Locality: Prussian amber. Horizon: Ligurian.

1856. Germ.-Ber. Berendt, Bernst. befindl. organ. reste vorw., ii, th. i: 24-25. Pl. 2, fig. 16.

3553. Phytocoris gulosus. (Capsiāæ.)

Compared with P. albostriatus.

Locality: Prussian amber. Horizon: Ligurian.

1856. Germ.-Ber. Berendt, Bernst, befindl, organ. reste vorw., ii, th. i: 27-28. Pl. 3, fig. 7.

3554. Phytocoris gummosus. (Capsidæ.)

Locality : Prussian amber. Horizon : Ligurian.

1856. Germ.-Ber. Berendt, Bernst. befindl, organ, reste vorw., ii, th. i: 25. Pl. 3, fig. 1.

3555. Phytocoris involutus. (Capsidæ.)

Compared with P. flavovarius.

Locality: Prussian amber. Horizon: Ligurian.

1856. Germ.-Ber. Berendt, Bernst. befindl, organ. reste vorw., ii, th. i: 28-29. Pl. 3 , fig. 9.

1890. Scudder, Tert. ins. N. A., 363.

3556. Phytocoris merus. (Capsidæ.)

Compared with Capsus capillaris.

Locality: Prussian amber. Horizon : Ligurian.

1856. Germ.-Ber. Berendt, Bernst, befindl, organ, reste vorw., ii, th, i: 27. Pl. 3, fig. 6. 
3557. Phytocoris punctiger. (Capsidæ.)

Compared with $\mathrm{P}$. thunbergi.

Locality: Prussian amber. Horizon: Ligurian.

1856. Germ.-Ber. Berendt, Bernst, befindl. organ. reste vorw., ii, th. i: 27. Pl. 3, fig. 5.

3558. Phytocoris raptorius. (Capsidæ.)

Locality: Prussian amber. Horizon : Ligurian.

1856. Germ.-Ber. Berendt, Bernst. befindl. organ. reste vorw., ii, th. i: 26. Pl.3, fig. 3.

3559. Phytocoris sendelii. (Capsidæ.)

Compared with P. thunbergi.

Locality: Prussian amber. Horizon: Ligurian.

1856. Germ.-Ber. Berendt, Bernst, befindl, organ. reste vorw., ii, th, i: $24 . \quad P l .2$, fig. 15.

3560. Phytocoris vetustus. (Capsidæ.)

Compared with P. ferrugatus.

Locality: Prussian amber. Horizon: Ligurian.

1856. Germ.-Ber. Berendt, Bernst, befindl, organ, reste vorw., ii, th. i : 29. $P l .3$, fig. 10.

3561. Piesma rotunda. (Tingididær.)

Locality: Florissant, Colo. Horizon: Oligocene.

1890. Scudder. Tert. ins. N. A., 358. Pl. 23, fig. 6.

3562. Piezocoris compactilis. (Coreidæ.)

Locality : Florissant, Colo. Hỏrizon: Oligocene.

1890. Scudder. Tert. ins. N. A., 417.

3563. Piezocoris ? peremptus. (Coreidæ.)

Locality : Florissant, Colo. Horizon : Oligocene.

1890. Seudder. Tert. ins, N. A, 417-418. Pl. 26, fig. 14.

3564. Piezocoris peritus. (Coreidæ.)

Locality : Florissant, Colo. Horizon: Oligocene.

1890. Scudder. Tert. ins. N. A., 417. Pl. 25, fig. 15.

3565. Pirates œningensis. (Reduviidæ.)

Locality: Oeningen, Baden. Horizon: Tortonian.

1853. Heer.

Insektenf. tertiärg. Oeningen, iii : 132-134. Pl. 15, fig. 11.

1856. Giebel. Insect, $d$, vorwelt, 368.

3566. Planophlebia gigantea. (Fulgoridæ.)

Locality : Similkameen River, British Columbia. Horizon :

1879. Scudder.

1879. 6

1890.66
Rep. progr. geol. surv. Can., 1877-'78: B 186.

Ins. tert. Nicola, 11.

Tert, ins. N. A., 296-297. Pl.2, fig. 16. 


\section{Platymeris - (Reduviidæ.)}

Locality : Prussian amber. Horizon: Ligurian.

1845. Berendt. Bernst. befindl, organ. reste vorw., i: 55.

3568. Platymeris insignis. (Reduviidæ.)

Compared with P. formicaria, Brazil.

Locality: Prussian amber. Horizon: Ligurian.

1856. Germ.-Ber. Berendt, Bernst, befindl. organ. reste vorw., ii, th. i: 21. $P$. 3, fig. 12.

\section{Ploiaria ——. (Reduviidæ.)}

Locality: Aix, France. Horizon: Ligurian.

1829. Serres. G6́ogn, terrains tert., 228.

See also Hydrometra _— Burmeister.

3570. Pœcilocapsus fremontii. (Capsidæ.)

Compared with P. ornatulus (Stål), Mexico.

Locality: Florissant, Colo. Horizon: Oligocene.

1890. Scudder. Tert. ins. N. A., 365-366. Pl.24, fig. 3.

3571. Pœcilocapsus ostentus. (Capsidæ.)

Locality : Florissant, Colo. Horizon: Oligocene.

1890. Scudder. Tert. ins. N. A., 368. Pl. 24, fig. 2.

3572. Pœcilocapsus tabidus. (Capsidæ.)

Locality : Florissant, Colo. Horizon: Oligocene.

1890. Scudder. Tert. ins. N. A., 367-368. Pl. 24, fig. 8.

3573. Pœcilocapsus veterandus. (Capsidæ.)

Locality : Florissant, Colo. Horizon: Oligocene.

1890. Scudder. Tert. ins. N. A., 366. Pl. 24, fig. 9.

3574. Pœcilocapsus veternosus. (Capsidæ.)

Locality : Florissant, Colo. Horizon : Oligocene.

1890. Seudder. Tert. ins, N. A., 367.

3575. Pœocera - (Fulgoridæ.)

Locality: Prussian amber. Horizon: Ligurian.

1845. Berendt. Bernst. befindl, organ, reste vorw, i : 55.

3576. Pœocera nassata. (Fulgoridæ.)

Locality: Prussian amber. Horizon: Ligurian.

1856. Germ.-Ber. Berendt, Bernst. befindl, organ. reste vorw., ii, th. i : 17-18. $P$ Pl. 2, fig. 5.

3577. Pœocera pristina. (Fulgoridæ.)

Locality: Prussian amber. Horizon: Ligurian.

1856. Germ.-Ber. Berendt, Bernst. befindl, organ. reste vorw., ii, th. i: 18. Pl. 2, fig. 6. 
3578. Pcocera venulosa. (Fulgorids.)

Locality: Prussian amber. Horizon: Ligurian.

1862. Giebel. Zeitschr. gesellsch. naturw., xx: 312-313.

3579. Polioschistus lapidarius. (Pentatomidæ.)

Locality : Florissant, Colo. Horizon : Oligocene.

1890. Scudder. Tert. ins, N. A., 461. Pl. 28, fig. 10.

3580. Polioschistus ligatus. (Pentatomidæ.)

Locality : Florissant, Colo. Horizon: Oligocene.

1890. Scudder. Tert. ins. N. A., 461. Pl. 28, fig. 5 .

3581. Polyclona - (Coccidæ.)

Locality : Prussian amber. Horizon: Ligurian.

1856. Menge. Progr. petrischule Danzig, 1856: 18.

3582. Poteschistus obnubilus. (Pentatomidæ.)

1890. Scudder.

Locality : Florissant, Colo. Horizon: Oligocene.

Tert. ins. N. A., 458. Pl. 28, fig. 18.

3583. Prinecphora balteata. (Cercopidæ.)

Locality : Florissant, Colo. Horizon: Oligocene.

1890. Sendder. Tert. ins. N. A., 332. Pl. 20, fig. 14."

3584. Procoris bechleri. (Lygæidæ.)

Lorality: Florissant, Colo. Horizon: Oligocene.

1890. Scudder. Tert. ins, N. A., 393-394. Pl. 27, fig. 4.

3585. Procoris sanctæjohannis. (Lygæidæ.)

Locality: Florissant, Colo. Horizon: Oligocene.

1890. Scudder. Tert, ins. N. A., 393.

3586. Procrophius communis. (Lygæidæ.)

Loeality: Florissant, Colo. Horizon: Oligocene.

1890. Scudder. Tert. ins. N. A., 382. Pl. 23, figs. 12, 18, 28, 29; pl. 24, fig. 1.

3587. Procrophius costalis. (Lygæidæ.)

Locality: Florissant, Colo. Horizon: Oligocene.

1890. Scudder. Tert. ins. N. A., 382-383. Pl. 23, fig. 8.

3588. Procrophius languens. (Lygæidæ.)

Locality: Florissant, Colo. Horizon: Oligocene.

1890. Seudder. Tert. ins. N. A., 383. Pl. 23, fig. 23.

3589. Procydnus devictus. (Pentatomidæ.)

Locality: Florissant, Colo. Horizon: Oligocene.

1890. Scudder. Tert. ins. N. A., 440. Pl. 28, fig. 4.

3590. Procydnus divexus. (Pentatomidæ.)

Locality: Florissant, Colo. Horizon: Oligocene.

1890. Scudder. Tert, ins. N. A., 440. 
3591. Procydnus eatoni. (Pentatomidæ.)

Locality: Florissant, Colo. Horizon: Oligocene:

1890. Scudder. Tert. ins. N. A., 442.

3592. Procydnus mamillanus. (Pentatomidææ.)

Locality : Green River, Wyo. Horizon: Oligocene. 1890. Scudder. Tert. ins. N. A., 443. Pl. 7, fig. 19.

See also Cydnns mamillanus.

3593. Procydnus pronus. (Pentatomidæ.)

Locality: Florissant, Colo. Horizon: Oligocene.

1890. Scudder. Tert. ins. N. A., 439. Pl. 28, fig. 5.

3594. Procydnus quietus. (Pentatomidæ.)

Locality : Florissant, Colo. Horizon: Oligocene.

1890. Seudder. Tert. ins. N. A., 441.

3595. Procydnus reliquus. (Pentatomidæ.)

Locality: Florissant, Colo. Horizon: Oligocene.

1890. Seudder. Tert. ins. N. A., 441.

3596. Procydnus vesperus. (Pentatomidæ.)

Locality : Florissant, Colo. Horizon: Oligocene.

1890. Scudder. Tert. ins. N. A., 442. Pl. 28, fig. 15.

3597. Prolygæus inundatus. (Lygæidw.)

Locality : Florissant, Colo. Horizon : Oligocene.

1890. Scudder. Tert. ins. N. A., 406. Pl. 27, fig. 13.

3598. Prosigara flabellum. (Corixidæ.)

Locality: Florissant, Colo. Horizon: Oligocene.

1890. Scudder. Tert. ins. N. A., 344, Pl. 22, fig. 12.

3599. Prostemma ceningensis. (Nabilæ.)

Locality: Oeningen, Baden. Horizon: Tortonian.

1853. Heer. Insektenf. tertiärg. Oeningen, ii : $82-83 . \quad P l .5, f i g .26 ; p l .10$, fig. 7 .

1856. Giebel. Insect. d. vorwelt, 367.

3600. Protenor imbecillis. (Coreidæ.)

Locality : Florissant, Colo. Horizon: Oligocene.

1890. Scudder. Tert. ins. N. A., 424-425. Pl. 26, fig. 8.

3601. Pseudophana -. (Fnlgoridæ.)

Locality: Prussian amber. Horizon: Ligurian.

1845. Berendt. Bernst. befindl, organ. reste vorw., $i$; 55 . 
3602. Pseudophana amatoria. (Fulgorides.)

Compared with P. europæa F., Europe.

Locality: Oeningen, Baden. Horizon: Tortonian.

1853. Heer. Insektenf. tertiärg. Oenıngen, iii : 90-91 (Psendophania). Pl. 13, fig. 10.

1856. “

1856. Giebel.

1865. Heer.

1872. “

1876. "

1879. .

Viert. naturf. gesellsch. Zürich, i : 39 .

Insect. d. vorwelt, 376.

Urwelt der Schweiz, 359, 393.

Monde prim. Suisse, 441, 482.

Prim. world Switz., ii: 14,52.

Urwelt der Schweiz, $2^{e}$ aufl., 385, 419.

3603. Pseudophana recticulata. (Fulgoridæ.)

Locality: Prussian amber. Horizon : Ligurıan.

1856. Germ.-Ber. Berendt, Bernst. befindl, organ, reste vorw., ii, th. i: 16-17. Pl. 2, fig. 4 .

Pseudophania amatoria. See Psendophana amatoria.

3604. Pterostigma nigrum. (Aphides.)

Locality: Florissant, Colo. Horizon: Oligocene.

1890. Scudder. Tert. ins. N. A., 275.

3605. Pterostigma recurvum. (Aphides.)

Locality: Florissant, Colo. Horizon: Oligocene.

1883. Buckton. Monogr. Brit. aphid., iv: 178. Pl. 133, fig. 6.

1890. Scudder. Tert. ins. N. A., 274. Pl. 18, fig. 18.

Ptyela carbonaria. See Aphrophora (Ptyelus) carbonaria.

3606. Ptyelus (vic.) - (several). (Cercopidæ.)

Locality: Florissant, Colo. Horizon: Oligocene.

1881. Scudder. Bull. U. S. geol. surv. terr., vi : 292.

1883. " Ann. rep. U. S. geol. surv, terr., xii : 282.

Ptyelus carbonarius. See Apbrophora (Ptyelus) carbonaria.

3607. Ranatra? - (Nepjdæ.)

Locality : Aix, France. Horizon : Ligurian.

1847. Hope. Trans. ent. soc. Lond., iv : 252.

3608. Reduvius - (Reduviidæ.)

Locality : Prussian amber. Horizon: Ligurian.

1845. Berendt, Bernst. befindl, organ. reste vorw, i: 55.

3609. Reduvius - (larva). (Reduviidæ.)

Locality: Prussian amber. Horizon : Ligurian.

1856. Germ.-Ber. Berendt, Bernst. befindl, organ. reste vorw, ii, th. i : 20. Pl. 2, fig. 9.

See also Nabis protypa. 
3610. Reduvius - (Reduviidæ.)

Compared with R. hirticornis.

Locality: Aix, France. Horizon: Ligurian.

1829. Bisres. G6ogn. terrains tert., 228.

3611. Reduvius ? guttatus. (Coreidæ.)

Compared with R. personatus Linn., Enrope.

Locality: Green River, Wyo. Horizon: Oligocene.

1878. Scrjdder. Bull. U. S. geol. surv. terr., iv : 771 .

See also Corizus guttatus.

3612. Rhepocoris macrescens. (Coreidæ.)

Locality : Florissant, Colo. Horizon : Oligocene.

1890. Scusider. Tert. ins. N. A., 427.

3613. Rhepocoris minimus. (Coreidæ.)

Locality : Florissant, Colo. Horizon: Oligocene.

1890. Scudiser. Tert. ins. N. A., 429 (minima).

3614. Rhepocoris prætectus. (Coreidæ.)

Locality: Florissant, Colo. Horizon: Oligocene.

1890. Scudzer. Tert. ins. N. A., 427.

3615. Rhepocoris prævalens. (Coreidæ.)

Locality: Florissant, Colo. Horizon: Oligocene.

1890. Scudder. Tert. ins. N. A., 427-428. Pl. 25, figs. 4, 6, 7, 9, 10,11, 14, $16 ; p l$. 26, fig. 11.

3616. Rhepocoris propinquans. (Coreidæ.)

Locality : Florissant, Colo. Horizon: Oligocene.

1890. Scudder. Tert. ins. N. A., 428-429. Pl. 25, fig. 1; pl. 26, fig. 13.

3617. Rhyparochromus ? terreus. (Lygæidæ.)

Locality: Green River, Wyo. Horizon: Oligocene.

1878. Scudder. Bull. U, S. geol, surv, terr., iv: 770-771.

See also Lyetocoris terreus.

3618. Rhyparochromus verrillii. (Lygæidæ.)

Locality: Florissant, Colo. Horizon: Oligocene.

1890. Scudder. Tert. ins. N. A., 400. Pl. 23, figs. 15, 30.

3619. Ricania multinervis. (Fulgoridæ.)

Locality: Prussian amber. Horizon: Ligurian.

1862. Giebel. Zeitschr. ges. naturw., xx: 313.

$$
\text { 3620. Salda —. (Saldida.) }
$$

Locality: Prussian amber. Horizon: Ligurian.

1845. Berendt. Bernst, befiedl, organ reste vorw, i: 55. 


\section{Salda -. (Saldida.)}

Locality : Prussian amber. Horizon: Ligurian.

1856. Menge. Progr, petrischule Danzig, 1856: 19.

3622. Salda exigua. (Saldidæ.)

Compared with S. zosteræ.

Locality: Prussian amber. Horizou : Ligurian.

1856. Germ.-Ber. Berendt, Bernst. befindl, organ. reste vorw., ii, th. i: 20. Pl, 3, fig. 13.

3623. Sbenaphis lassa. (Aphides.)

Locality : Florissant, Colo. Horizon: Oligocene.

1890. Seudder. Tert. ins. N. A., 253.

3624. Sbenaphis quesneli. (Aphides.)

Localities: Quesnel, British Columbia ; Florissant, Colo. Horizon : Oligocene. 1890. Scudder. Tert. ins. N. A., 250-252. Pl. 2, figs. 4,5; pl. 18, fig. 12.

See also Lachnus quesneli.

3625. Sbenaphis uhleri. (Aphides.)

Locality: Florissant, Colo. Horizon: Oligocene.

1890, Scudder. Tert. ins. N. A., 252.

\section{Schizoneura -. (Aphides.)}

Locality: Prussian amber. Horizon: Ligurian.

1845. Berendt. Bernst. befindl, organ. reste vorw., i : 55.

3627. Schizoneuroides scudderi. (Aphides.)

Locality : Florissant, Colo. Horizon: Oligocene.

1883. Buckton. 1885. Sendder. 1886. " " 1890.
Monogr. Brit. aphid., iv: 178. Pl. 133, fig. 5.

Zittel, Handb. palaeont., i, th. ii : 780 . Fig. 988.

Zittel-Barrois, Traité de paléont., ii : 780. Fig. 1005.

Tert. ins. N. A., 269-270. Pl. 18, fig. $*$.

3628. Siphonophoroides antiqua. (Aphides.)

Locality : Florissant, Colo. Horizon: Oligocene.

1883. Buckton. 1890. Sendder.

Tert. ins. N. A., 255-256. Pl. 18, figs. 3, 5, 7, 10.

3629. Siphonophoroides propinqua. (Aphides.)

Locality: Florissant, Colo, Horizon: Oligocene.

1890. Sendder. Tert. ins, N. A., 257.

3630. Siphonophoroides rafinesquei. (Aphides.)

Locality : Florissant, Colo. Horizon: Oligocene.

1890. Scudder. . Tert. ins. N. A., 256-25\%.

3631. Siphonophoroides simplex. (Aphides.)

Locality : Florissant, Colo. Horizon: Oligocene.

1883. Buckton. Monogr. Brit. aphid., iv: 176-177. Pl. 133, fig. 2.

See also Tephraph1s simplex. 
3632. Spartocerus insignis. (Coreidie)

Locality : Radoboj, Croatia. Horizon: Mayencian.

1853. Heer. Insektenf. tertiärg. Oeningen, iii: 43-45. Pl. 3, fig. 9; pl. 8, fig. 10.

1855. " Ber. oesterreich. litt., 1850-'53: 200.

1856. Giebel. Insect. $d$. vorwelt, $347-348$.

3633. Spartocerus maculatus. (Coreidæ.)

1853. Heer.

1855. “

1856. Giebel.

Locality: Radoboj, Croatia. Horizon : Mayencian.

Insektenf. tertiärg. Oeningen, iii : 45-46. Pl. 3, fig. 10.

Ber, oesterreich. litt., 1850-'53: 200.

Insect. $d$. vorwelt, 348 .

3634. Stenopamera subterrea. (Lygæidæ.)

Locality: Florissant, Colo. Horizon: Oligocene.

1890. Scudder. Tert. ins. N, A., 386. Pl. 23, fig. 7.

3635. Stenopamera tenebrosa. (Lygæidæ.)

Locality: Florissant, Colo. Horizon: Oligocene.

1890. Scudder. Tert. ins. N. A, 386. Pl. 23, figs. 16, 24.

3636. Stenopelta punctulata. (Pentatomid̊r.)

Locality: Green River, Wyo. Horizon: Oligocene.

1890. Scudder. Tert. ins. N. A., 438. Pl. 7, figs. 12, 13.

See also Ethus punctulatus.

3637. Stenopoda gracilis. (Reduviidæ.)

Locality : Oeningen, Baden. Horizon: Tortonian.

1861. Heer. Rech. climat pays tert., 203 (undeser.).

3638. Stenopoda ceningensis. (Reduviidæ.)

Locality: Oeningen, Baden. Horizon: Tortonian.

1861. Heer. Rech. climat pays tert., 203 (undescr.).

3639. Stenovelia nigra. (Veliidæ.)

Locality : Florissant, Colo, Horizon: Oligocene.

1890. Seudder. Tert. ins. N. A., 350. Pl. 22, figs. 8, 14.

3640. Sychnobrochus reviviscens. (Aphides.)

Locality : Florissant, Colo. Horizon : Oligocene.

1890. Sendder. Tert. ins. N. A., 268-269. Pl. 18, fig.6.

3641. Syromastes affinis. (Coreidæ.)

Locality: Oeningen, Baden. Horizon: Tortonian.

1853. Heer.

1856. Giebel.

1865. Heer.

1872. “

1876. " "

1879. "
Insektenf. tertiärg. Oeningen, iii : 52. $P l .4, f i g .6 ; p l .8$, fig. 7.

Insect. d. vorwelt, 351.

Urwelt der Schweiz, 362.

Monde prim. Suisse, 444.

Prim. world Switz, ii : 16.

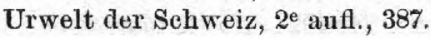


3642. Syromastes buchii. (Coreidæ.)

1853. Heer. 1856. Giebel.

1853. Heer.

1856. Giebel. 1865. Heer. 1872. "6. 1876. " " 1879. “

1853. Heer.

1856. Giebel. 1865. Heer. 18i2. " 1876. “ 1879. " "

1829. Serres.

1890. Seudder.

Locality : Oeningen, Baden. Horizon: Tortonian.

Insektenf. tertiärg. Oeningen, $111: 54 . \mathrm{Pl} .4$, fig. 8 ,

Insect. d. vorwelt, 351.

3643. Syromastes coloratus. (Coreidæ.)

Compared with S. scapha F., Europe.

Locality: Oeningen, Baden. Horizon: Tortonian.

Insektenf. tertiärg. Oeningen, iii : 52-54, 128. $P l .4, f i g .7 ; p l$. 8, fig. 8.

Insect. $d$. vorwelt, 351 .

Urwelt der Schweiz, 391. 'Fig. 302.

Monde prim. Suisse, 479. Fig. 302.

Prim. world Switz., ii : 50. Fig. 302, on p. 49.

Urwelt der Schweiz, $2^{\mathrm{e}}$ aufl, $416 . \quad$ Fig. 344, on $p .417$.

3644. Syromastes seyfriedi. (Coreidæ.)

Locality : Oeningen, Baden. Horizon: Tortonian.

Insektenf, tertiärg. Oeningen, iii : 51-52. Pl. 4, fig. 5; pl. 8, fig. 6.

Insect. d. vorwelt, 351.

Urwelt der Schweiz, 390.

Monde prim. Suisse, 479.

Prim. world Switz, ii : 50.

Urwelt der Schweiz, 2e aufl., 416.

3645. Syrtis —. (Phymatidæ.)

Locality: Aix, France. Horizon: Ligurian.

G6́ogn, terrains tert.; 226-22\%.

3646. Systellonotus ——. (Capsidæ.)

Locality: Prussian amber. Horizon: Ligurian.

1890. Scudder. Tert. ins. N. A., 362 .

3647. Tagalodes inermis. (Reduviidæ.)

Locality: Florissant, Colo. Horizon: Oligocene.

Tert. ins. N. A, 357. Pl. 26, fig. 15.

3648. Teleoschistus antiquus. (Pentatomidæ,)

Locality: Quesnel, British Columbia. Horizon :

1890. Scudder. Tert, ins. N. A., 454-456. Pl. 2, figs. 17-19.

See also Euschistus antiquus.

3649. Teleoschistus placatus. (Pentatomidæ.)

Locality : Florissant, Colo. Horizon: Oligocene.

1890. Scudder. Tert. ins. N. A., 457. Pl. 28, fig. 3.

3650 Teleoschistus rigoratus. (Pentatomidæ.)

Locality: Florissant, Colo. Horizon: Oligocene.

1890. Scudder. Tert. ins. N. A., 456-457. Pl. 28, figs. 2, 14. 
3651. Telmatrechus parallelus. (Hydrobatidæ.)

Locality: Twin Creek, Wyo. Horizon:

1890. Scudder. Tert. ins. N. A., 353. Pl. 4, fig. 1.

3652. Telmatrechus ståli. (Hydrobatidæ.)

Compared with Hygrotrechus remigis (Say).

Locality : Similkameen River, British Columbia. Horizon:

1890. Scudder. Tert. ins. N. A., 351-353. Pl. 2, figs. 11, 12.

See also Hygrotrechus ståli.

3653. Tenor speluncæ. (Coreidæ.)

Locality: Florissant, Colo. Horizon: Oligocene.

1890. Scudder. Tert.ins. N. A., 425.

3654. Tephraphis simplex. (Aphides.)

Locality : Florissant, Colo. Horizon : Oligocene.

1890. Scudder. Tert. ins. N. A., 259-260. Pl. 18 fig. 4.

See also Siphonophoroides simplex.

3655. Tephraphis walshii. (Aphides.)

Locality: Florissant, Colo. Horizos: Oligocene.

1890. Scudder. Tert. ins. N. A., 260. Pl. 18, fig. 19.

3656. Tettigometra debilis. (Fulgoridæ.)

1853. Heer.

Locality : Radoboj, Croatia. Horizon: Mayencian.

1856. Giebel.

Insektenf. tertiärg. Oeningen, iii : 91-92. $\quad P l .13$, fig. 11.

Insect. d. vorwelt, 378.

3657. Tettigonia —_. (Jassides.)

Locality: Prussian amber. Horizon: Ligurian.

1845. Berendt. Bernst. befindl. organ. reste vorw., i: 55.

3658. Tettigonia - (Jassides.)

Compared with T. spumaria.

Locality : Aix, France. Horizon: Ligurian.

1829. Curtis. Edinb. new phil. journ., vii: 296. Pl. $6, f i g .6$.

1829. " Murchison-Lyell, Tert, form. Aix, 12. Pl. 6, fig. 6.

1839. Mantell. Wonders of geology, i : 248. Fig. 1.

See also Aphrophora spumifera, etc.

3659. Tettigonia —. (Jassides.)

Locality : Aix, France. Horizon: Ligurian.

1829. Serres. Géogn. terrains tert., 228.

3660. Tettigonia antiqua. (Jassides.)

Locality : Radoboj, Croatia. Horizon: Mayencian.

1853. Heer.

1855. “

1856. Giebel.
Insektenf. tertiärg. Oeningen, iii : 109-110. $P l$. 12, fig. 5.

Ber, oesterreich. litt., 1850-'53 : 202.

Insect. d. vorwelt, 384. 
3661. Téttigonia morio. (Jassides.)

Locality : Radoboj, Croatia. Horizon: Mayencian.

1853. Heer.

1855 . “

1856. Giebel.

Insektenf. tertiärg. Oeningen, iii: 110. Pl. 12, fig. 4.

Ber. oesterreich. litt., 1850-'53: 203.

Insect. d. vorwelt, 384 .

3662. Tettigonia obtecta. (Jassides.)

Locality: White River, Colo. Horizon: Oligocene.

187\%. Scudder.

1890. " Tert. ins. N. A., 304. Pl. 5, figs. 58,59.

3663. Tettigonia priscomarginata. (Jassides.)

Compared with Aulacipes irroratus (Fabr.).

Locality: Green River, Wyo. Horizon: Oligocene.

1890. Scudder. Tert. ins. N. A., 302-303. Pl. 7, fig. 4.

3664. Tettigonia priscotincta. (Jassides.)

Locality: Florissant, Colo. Horizon: Oligocene.

1890. Scudder. Tert. ins. N, A., 303. Pl. 19, fig. 9.

3665. Tettigonia priscovariegata. (Jassides.)

Locality: Green River, Wyo. Horizon: Oligocene.

1890. Scudder. Tert. ins. N. A., 303-304.

3666. Tettigonia proavia. (Jassides.)

Locality: Prussian amber. Horizon: Ligurian.

1856. Germ.-Ber. Berendt, Bernst. befiudl. organ. reste vorw., ii, th. i: 9-10. Pl. 1, fig. 13.

3667. Tettigonia spumaria. (Jassides.)

Locality: Aix, France. Horizon: Ligurian.

1854. Mantell. Medals of creation, 2 d erl, ii : 558. Fig. 183.1.

See also Aphrophora spumifera, etc.

3668. Tettigonia terebrans. (Jassides.)

Compared with T. viridis, Europe.

Locality : Prussian amber. Horizon : Ligurian.

1856. Germ.-Ber. Berendt, Bernst. befindl. organ. reste vorw., ii, th. i: 10. Pl. 1, fig. 14.

3669. Tetyra hassii. (Pentatomidæ.)

Locality: Oeningen, Baden. Horizon: Tortonian.

1853. Heer. Insektenf. tertiärg. Oeningen, iii: 11-12. Pl. 1, fig. 4; pl. 6, fig. 3.

1856. Giebel. Insect. d. vorweit, 335 .

3670. Thamnotettix fundi. (Jassides.)

Locality : Florissant, Colo. Horizon: Oligocene.

1890. Scudder. Teri. ins. N. A., 310, Pl. 19, fig. 20. 
3671. Thamnotettix gannetti. (Jassides.)

Locality: Green River, Wyo. Horizon: Oligocene.

1890. Scudder. Tert. ins. N. A., 309-310. Pl. 6, fig. $33 ;$ pl. 7, fig. 5.

3672. Thamnotettix mutilata. (Jässides.)

Locality: Green River, Wyo. Horizon: Oligocene.

1890. Scudder. Tert, ins. N. A., 309. Pl. 7, fig. 6.

3673. Thlibomenus limosus. (Pentatomidæ.)

Locality: Florissant, Colo. Horizon: Oligocene.

1890. Scudder. Tert. ins. N. A., 450. Pl. 28, fig. 12.

3674. Thlibomenus macer. (Pentatomidæ.)

Locality : Florissant, Colo. Horizon: Oligocene.

1890. Scudder. Tert. ins. N. A., 451.

3675. Thlibomenus parvus. (Pentatomidæ.)

Locality: Florissant, Colo. Horizon: Oligocene.

1890. Scudder. Tert. ins. N. A., 449-450. Pl.19, fig. 23.

3676. Thlibomenus perennatus. (Pentatomidx.)

Locality: Florissant, Colo. Horizon: Oligocene.

1890. Scudder. Tert. ins. N. A., 450.

3677. Thlibomenus petreus. (Pentatomidæ.)

Locality : Florissant, Colo. Horizon: Oligocene.

1890. Scudder. Tert. ins. N. A., 449.

3678. Thlimmoschistus gravidatus. (Pentatomidæ.)

Locality : Florissant, Colo. Horizon: Oligocene.

1890. Scudder. Tert. ins. N. A., 463-464. Pl. 28, figs. 7, 19.

3679. Thnetoschistus revulsus. (Pentatomidæ.)

Locality: Florissant, Colo. Horizon: Oligocene.

1890. Scudder. Tert. ins. N. A., 458. Pl. 28, fig. 4.

3680. Thrips annosa. (Physapodes.)

Locality : Oeningen, Baden. Horizon: Tortonian.

1865. Heer. Urwelt der Schweiz, 367. Fig. 233 on $p, 366$.

1872. " Monde prim. Suisse, 450. Fig. 233 on p. 449.

1876. " $\quad$ Prim. world Switz., ii : 22. Fig. 233 on $p .21$.

1879. " Urwelt der Schweiz, $2^{e}$ aufl, 392. Fig. 274.

3681. Thrips annulata. (Physapodes.)

Locality : Prussian amber. Horizon: Ligurian.

1856. Menge. Progr. petrischule Danzig, 1856: 11-12.

3682. Thrips antiqua. (Physapodes.)

Locality : Aix, France. Horizon : Ligurian.

18.56. Heer.

Viert, naturf. gesellsch. Zürich, i: 27. $P l, 2$, figs. 9, 10.

1873. Oustalet. Bull, soc, philom, Paris, $x:$ 24-25. 
3683. Thrips breviventris. (Physapodes.)

Locality : Rott, Rhenish Prussia. Horizon : Aquitanian.

1887. Schlechtend. Zeitschr. ges. naturw., lx: 572-574. Pl. 4, figs. 13-15.

3684. Thrips capito. (Physapodes.)

Locality : Rott, Rhenish Prussia. Horizon: Aquitanian.

1887. Schlechtend. Zeitschr. ges. naturw., lx: 579-582. Pl. 5, fig. 20.

3685. Thrips electrina. (Physapodes.)

Locality: Prussian amber. Horizon : Ligurian.

1856. Menge. Progr. petrischule Danzig, 1856: 12.

3686. Thrips excellens. (Physapodes.)

Locality: Rott, Rhenish Prussia, Horizou: Aquitanian.

1887. Schlechtend. Zeitschr. ges. naturw., lx: 564-567. Pl. 3, figs, 2-8.

3687. Thrips formicoides. (Physapodes.)

1873. Oustalet. Bull. soc. philoin. Paris, $\mathrm{x}$ : 25-26.

Locality: Aix, France. Horizon: Ligurian.

3688. Thrips longula. (Physapodes.)

Locality: Rott, Rhenish Prussia. Horizon: Aquitanian.

1887. Schlechtend. Zeitschr. gesellsch. naturw., lx: 568-570. Pl. 3, fig. 9.

3689. Thrips minima. (Physapodes.)

Locality : Rott, Rhenish Prussia. Horizon : Aquitanian.

1887. Schlechtend. Zeitschr. gesellsch. naturw., lx : 574-577. Pl. 4, figs. 16-17.

3690. Thrips obsoleta. (Physapodes.)

Locality: Aix, France. Horizon: Ligurian.

1873. Oustalet. Bull. soc. philom. París, x: 25.

3691. Thrips coningensis. (Physapodes.)

Locality: Oeningev, Baden. Horizon: Tortonian.

1865. Heer. Urwelt der Schweiz, 367.

1872. " Monde prim. Suisse, 450.

1876. "

1879. " " Urwelt der Schweiz, $2^{\mathrm{e}}$ aufl, 392.

3692. Thrips pennifera. (Physapodes.)

Locality : Rott, Rhenish Prussia. Horizon : Aquitanian.

1887. Schlechtend. Zeitschr. gesellsch. naturw., Ix: 570-572. Pl. 3, figs. 10-12.

3693. Thrips pygmæa. (Physapodes.) .

Locality : Rott, Rhenish Prussia. Horizon: Aquitanian.

1887. Schlechtend. Zeitschr. gesellsch. naturw., lx: 577-579. Pl. 4, figs. 18, 19.

3694. Thrips sericata. (Physapodes.)

Locality: Prussian amber. Horizon: Ligurian.

1856. Menge. Progr. petrischule Danzig, 1856:12. 


\section{Tingis —. (Tingididæ.)}

Locality: Prussian amber. Horizon: Ligurian.

1845. Berendt. Bernst. befindl. organ. reste vorw., i: 55.

3696. Tingis —. (Tingididæ.)

Compared with T. cardui.

Locality : Aix, France. Horizon: Ligurian.

1847: Hope. $\quad$ Trans. ent. soc. Lond., iv : 252.

3697. Tingis ——. (Tingididæ.)

Locality: Florissant, Colo. Horizon: Oligocene.

1881. Scudder. Bull. U. S. geol. surv. terr., vi : 292.

3698. Tingis —. (Tingididæ.)

Locality : Aix, France. Horizon : Ligurian.

1829. Serres. Géogn. terrains tert., 227.

3699. Tingis obscura. (Tingididæ.)

Locality: Radoboj, Croatia. Horizon: Mayencian.

153. Heer.

1856. Giebel. Insect. d. vorwelt, 363.

3700. Tingis quinquecarinata. ('Tingididæ.)

Locality : Prussian amber. Horizon : Ligurian.

1856. Germ.-Ber. Berendt, Bernst. befindl. organ, reste vorw., ii, th. i: 23-24. Pl. 3, fig. 19.

See also Eotingis quinquecarinata.

3701. Tingis wollastoni. (Tingididæ.)

Locality : Oeningen, Baden. Horizon: Tortonian.

1865. Heer. Urwelt der Schweiz (Monanthia in text). Fig. 307 on p. 391.

1872. " Monde prim. Suisse. Fig. 307 on p. 479.

1876. " $\quad$ Prim. world Switz., ii. Fig. 307 on $p .49$.

1879. " Urwelt der Schweiz, $2^{\mathrm{e}}$ auf. Fig. 349 on p. 417.

See also Monanthia wollastoni.

3702. Tiroschistus indurescens. (Pentatomidæ.)

Locality : Florissant, Colo. Horizon : Oligocene.

1890. Scudder. Tert. ins. N. A., 463. Pl. 22, fig. 4.

3703. Tiromerus tabifluus. (Lygæidæ.)

Locality : Florissant, Colo. - Horizon: Oligocene.

1890. Scudder. Tert. ins. N. A., 402.

3704. Tiromerus torpefactus. (Lygæidæ.)

Locality: Florissant, Colo. Horizon: Oligocene.

1890. Scndder. Tert. ins. N. A., 402.

3705. Trapezonotus exterminatus. (Lygæidæ.)

Locality: Florissant, Colo. Horizon: Oligocene.

1890. Scudder. Tert. ins. N. A., 395. Pl, 22, fig. 9; pl, 23, figs, 11, 22, 25;

Bull. $71-29$ 
3706. Trapezonotus stygialis. (Lygæidæ.)

Locality: Florissant, Colo. Horizon: Oligocene.

1890. Scndder. Tert. ins. N. A., 396. Pl. 2\%, fig. 11.

Triecophora sanguinolenta. See Triecphora sanguinolenta.

3707. Triecphora sanguinolenta (recent). (Cercopidæ.)

Locality: Gurnet Bay, Isle of Wight. Horizon: Bembridge Limestone.

[1843. Amyot-Serv. Hémiptères, 561-562.]

1879. Woodward. Quart, journ, geol, soc. Lond, xxxv : 344.

1879. " Geol. mag., n. s., v: 88.

1885. Scudder. Zittel, Handb. palaeont., i, th. ii: 782 (Triecophora.)

1886. " "Zittel-Barrois, Traité de paléont., ii : 781.

1886. " Bull. U. S. geol. surv., No. 31: 60 .

3708. Typhlocyba -. (Jassides.)

Locality : Prussian amber. Horizon : Ligurian.

1845. Berendt. Bernst. befindl. organ. reste vorw., i : 55.

3709. Typhlocyba —. (Jassides.)

Locality : Florissant, Colo. Horizon : Oligocene.

1881. Scudder. Bull. U. S. geol. surv, terr., vi: 293 (Typhlocypha).

1883. " Ann. rep. U. S. geol. surv. terr., xii : 282.

3710. Typhlocyba bremii. (Jassides.)

Locality : Radoboj, Croatia. Horizon: Mayencian.

1853. Heer. Insektenf. tertiärg. Oeningen, iii : 117-119. $\quad P l .13, f i g .3$.

1855. “ Ber. oesterreich. litt., 1850-'53: 203.

1856. Giebel. Insect. d. vorwelt, 387.

3711. Typhlocyba carbonaria. (Jassides.)

Locality : Stösschen, Austria. Horizon: Miocene.

1859. Heyden. Palaeontogr., viii: 11. Pl. 1, fig. 14.

3712. Typhlocyba encaustica. (Jassides.)

Locality: Prussian amber. Horizon: Ligurian.

1856. Germ.-Ber. Berendt, Bernst. befindl, organ. reste vorw., ii, th. i: 7. Pl. 1, fig. 9.

3713. Typhlocyba resinosa. (Jassides.)

Compared with T. rosæ.

Locality: Prussian amber. Horizon: Ligurian.

1856. Germ.-Ber. Berendt, Bernst. befindl, organ. reste vorw., ii, th. i: 8 . Pl. 2, fig. 2.

Typhlocypha - - See Typhlocyba

COLEOPTERA.

3714.

Loealities: Le Puy, Auvergne, France.

Horizon: 
3715.

Localities : Lenden, Colchester, England. Horizon : Postglacial drift.

1674. Brodie. Distr. corr. foss. ins., 13.

3716.

Locality: Stanway, England. Horizon: Peat.

1858. Brown. Geologist, 1858: 254.

3717.

Locality: Montagne Ste. Catherine, near Rouen, France. Horizon: "Roche calcaire."

1×26. Desmoulins. Ferr., Bull. sc. nat., ix : 253.

3718.

Locality : Peckham, England. Horizon: Paludina beds, Lower Eocene.

1861. Evans. Geologist, iv: 39. Fig.

3719. (several).

Locality : Nantucket amber, Mass. Horizon :

1879. Goldsmith. Proc. acad. nat. sc. Phil., 1879: 207.

3720.

Locality : Scotland. Horizon: Interglacial.

1880. Goss. $\quad$ Entom. monthl. mag., xvi : 198. (On the authority of Buchanan White.)

3721.

Locality: Oldham, England. Horizon: Pleistocene (peat).

1881. Hollingworth. Quart. journ. geol. soc. Lond., xxxvii : 713.

3722.

Locality: Grasseth, Bohemia. Horizon :

1857. Jokély. Jahrb. geol. reichsanst., viii : 502.

3723.

Locality: Drigg, England. Horizon: Quaternary.

1881. Kendall. Quart. journ, geol, soc. Lond, xxxvii : 34.

3724.

Locality: St. Bees, England. Horizon: Quaternary.

1881. Kendall. Quart. journ. geol. soc. Lond., xxxvii: 35.

3725.

Locality: Parkhurst Barracks, England. Horizon: London clay.

1847. Mantell. Geol. exc. Isle of Wight, 140.

3726. (8 sp.).

Locality : Rocky River, Sydney flats, Anstralia. Horizon:-—.

1870. Moore.

Quart. journ. geol, soc. Lond., xxvi: 263, $P l, 18$, figs, 2-9, 


\section{7}

Locality : Senigaglia, Italy. Horizon : Helvetian?.

1842. Procaccini. Nuov. ann. sc. nat., vii : 449 .

3728. (coprolites of larvæ).

Locality: Westerwald, Germany. Horizon: "Braunkohl." 1852. Quenstedt. Handb, petref., 314. Pl. 24, fig. 7.

3729.

Locality : Prussian amber. Horizon: Ligurian.

1838. Robert. Bull. soc. géol. France, ix : 114.

3730. (borings).

Localities : Auzers, Mauriac, France. Horizon: "Lignite." 1875. Rouchy. Petites nouv, entom., i: 551. 3731. (eggs and larvæ).

Locality: Bodensee, Baden. Horizon: Alluvium.

1851. Stizenberger. Uebers, verstein. Baden, 119.

3732.

Locality : Lincolnshire coast, England, Horizon: Peat beds. 1830. Taylor.

Loud., Mag. nat. hist., iii : 361.

3733.

Locality : Mount's Bay, England. Horizon : "Submarine forest." 1830. Taylor. Loud., Mag. nat. hist., iii : 361.

3734.

Locality : Norfolk coast, England. Horizon: Peat beds.

1830. Taylor. Loud., Mag. nat. hist., iii : 361.

3735.

Locality: Yorkshire coast, England. Horizon : Peat beds.

1830. Taylor. Loud, Mag, nat. hist., iii : 361.

3736.

Locality : Lexden, England. Horizon : Peat beds.

1863. Wollaston. Quart. journ. geol. soc. Lond., xix : 401, No. 10.

3737. ("Schwimmkäfer.")

Locality : Oberelsass. Horizon: Middle Oligocene.

1885. Foerster. Tagebl. versamml. dentsch. naturf, lviii : 392.

1885. “ Mitth. oberelsäss. tertiär, 7 .

3738. (several). ("Water beetles.")

Locality : Florissant, Colo. Horizon : Oligocene.

1881. Scudder. Bull. U. S. geol. surv. terr., vi: 291.

1883, «द Ann. rep. U. S, geol, surv, terr, xii : 281 , 
3739.

("Water beetle.")

Locality: Lexden, England. Horizon: Peat beds.

1863. Wollaston. Quart. journ. geol. soc. Lond., xix : 401 No. 11-12.

- - (Aleocharini). See - - (Staphylinidæ-Aleocharini).

3740.

(Anthicidæ ; "Notoxida.")

Locality: Prussian amber. Horizon: Ligurian.

1856. Menge. Progr. petrischule Danzig, 1856: 21.

(Bostrychini.) See (Ptinidæ-Bostrychini).

3741. (several). (Bruchidæ.)

Locality: Florissant, Colo. Horizon : Oligocene.

1881. Sendder. Bull. U. S. geol. surv. terr., vi : 292.

1883. “ Ann. rep. U. S. geol. surv. terr., xii : 281.

3742.

(Buprestidæ.)

Locality : Dorset, England. Horizon: Bagshot series, Middle Eocene. 1870. Brodie. Geol. mag., vii : 141.

3743. (Buprestidæ.,

Locality : Bournemouth, England. Horizon: Middle Eocene. 1878. Goss.

Proc. ent. soc. Lond., 1878: 8.

3744.

(Buprestidæ.)

Locality: Salzhausen, Germany. Horizon: Miocene.

1856. Heyden. Palaeontogr., iv: 200. Pl. 38, fig. 4.

3745.

(Buprestidæ.)

Locality: Prussian amber, Horizon: Ligurian.

1856. Menge. Progr. petrischule Danzig, 1856 : 22 .

3746. (several). (Buprestidæ.)

Locality : Florissant, Colo. Horizon: Oligocene.

1881. Scudder. Bull. U. S. geol. surv. terr, vi: 291-292.

1883. " Ann. rep. U. S. geol. surv. terr., xii : 281.

3747.

(Buprestidæ.)

Locality : Creech, near Corfe, England. Horizon:

1854. Westwood. Quart. journ. geol. soc. Lond., $\mathrm{x}: 381,395 . \quad P l .16$, fig. 34.

3748.

(Buprestidæ.)

Locality : Lexden, England. Horizon: Peat beds.

1863. Wollaston. Quart.journ. geol. soc. Lond., xix : 401, No. 8.

3749.

(Carabidæ or Rhynchophora.)

Locality : Antrim, Ireland. Horizon: Tertiary basalt beds.

1869. Baily.

Quart. journ. geol. soc. Lond, $\mathrm{xxv}$ : 359-362. Compared to the Entimides, p. 362, to Carabidæ, p. 360. Pl. 14, figs. $14 a b$. 
3750.

(Carabidæ or Rhynchophora.)

Locality : Antrim, Ireland. Horizon: Tertiary basalt beds.

1869. Baily. Quart. journ. geol. soc. Lond., xxv: 359-362. Compared to Apion, p. 362, to Carabidæ, p. 360. $P l .14$, fig. 15.

\section{1.}

\section{(Carabidæ.)}

Locality: Brunstatt, Alsatia. Horizon: Middle Oligocene.

1889. Foerster. Mitth. comm. geol. Elsass-Lothr., ii : 103.

3752.

(Cārabidìe.)

Locality: Florissant, Colo. Horizon: Oligocene.

1881. Scudder. Bull. U. S. geol. surv. terr., vi : 291.

1883. " Ann. rep. U. S. geol. surv. terr., xii : 281.

3753.

(Carabidæ.)

Locality : Caverne de Lunel Vieil, Hérault, France. Horizon : Postpliócene. 1827. Serres. Mém. soe. linn. Paris, v: 457.

3754. —- (Carabidæ.)

Locality : Lexden, England. Horizon: Peat beds.

1863. Wollaston. Quart. journ. geol, soc. Lond, xix : 401, No. 7.

3755. — - (Cerambycidæ.)

Locality: Brunstatt, Alsatia. Horizon: Middle Oligocene.

1889. Foerster. Mitth. comm. geol. Elsass-Lothr., ii : 102.

3756. (several). (Cerambycidæ.)

Locality : Florissant, Colo. Horizon: Oligocene.

1881. Scudder. Bull. U. S. geol, surv. terr., vi : 292.

1883. " Ann. rep. U. S. geol. surv. terr., xii : 281.

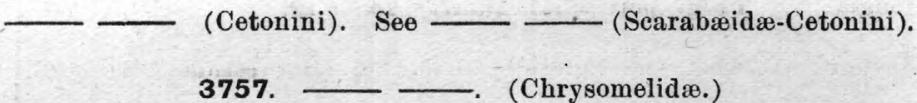

3757. - - (Chrysomelidæ.)

Locality : Schössnitz, Austria. Horizon: Miocene.

1855. Göppert. Tert. flora Schössn̊itz, vii. Pl. 26, fig. 56.

See also Curculionites silesiacus, etc.

3758.

(Chrysomelidæ-Halticini.)

1838. Robert. Bull. soc. géol. France, ix : 114 (Altises).

Locality : Prussian amber. Horizon: Ligurian.

3759. (several). (Chrysomelidæ.)

Locality: Florissant, Colo. Horizon: Oligocene:

1881. Scudder.

Bull. U. S. geol. surv. terr., vi : 292.

1883. " Ann. rep. U. S. geol, surv. terr., xii : 282.

3760.

(Chrysomelidæ.)

Locality: Caverne de Lunel Vieil, Herranlt, Frauce. Horizon: Postpliocene. 1827. Serres. Mém. soc. linn. Paris, v: 457. 
3761. (Chrysomelidæ or Curculionidæ.)

Locality : Creech, near Corfe, Englatid. Horizon: -

1854. Westwood. Quart. journ. geol. soc. Lond., x: 382.

3762.

(Cleridæ.)

Locality : Prussian amber. Horizon : Ligurian.

1856. Menge.

Progr. petrischule Danzig, 1856: 21.

3763.

(Cleridæ.)

Locality : Florissant, Colo. Horizon: Oligocene.

1881. Scudder. Bull. U. S. geol. surv. terr., vi : 292.

1882. " Ann. rep. U. S. geol. surv. terr., xii : 282.

3764. —- (several). (Coccinellidæ.)

Locality: Florissant, Colo. Horizon: Oligocene.

1881. Scudder.

Bull. U. S. geol. surv. terr., vi : 292.

1883. " "

Ann. rep. U. S. geol. surv. terr., xii : 282.

3765.

-. (Curculionidæ.)

Locality : Dorset, England. Horizon: Bagshot series, Middle Eocene.

1870. Brodie. Geol. mag., vii: 141.

3766.

(Curculionidæ.)

Locality : Corfe, England. Horizon:

1874. Brodie, Distr. corr. foss, ins., 13.

3767.

(Curculionidæ.)

Locality: Bournemouth, England. Horizon: Middle Eocene.

1878. Goss.

Proc. ent. soc. Lond., 1878: 8.

3768.

(Curculionidæ.)

Locality : Prussian amber. Horizon: Ligurian.

1856. Menge.

Progr. petrischule Danzig, 1856: 22.

3769.

(Curculionidæ or Chrysomelidæ.)

Locality : Creech, near Corfe, England. Horizon :

1854. Westwood. Quart. journ. geol. soc. Lond., x: 382.

3770.

(Curculionidæ.)

Locality : Creech, near Corfe, England. Horizon :

1854. Westwood. Quart. jonrn. geol soc. Lond., $\mathrm{x}: 382,395 . \quad$ Pl. 16, fig. 35.

3771. - - (Dascyllidx.)

Locality : Prussian amber. Horizon : Ligurian.

1856. Menge. Progr. petrischule Danzig, 1856: 21 (Cyphones).

3772. — - (several). (Dermestidæ.)

Locality : Florissant, Colo. Horizon : Oligocene.

1881. Seudder.

1883 .

Bull. U. S. geol, surv. terr., vi : 292.

Ann, rep, U. S. geol, surv, terr., xii : 282. 
3773.

(Dytiscidæ.)

Locality: Brunstatt, Alsatia. Horizon: Middle Oligocene.

1885. Foerster.

1885. “

1856. Menge.
Tagebl. versamml. dentsch. naturf., 1viii : 392.

Mittb. oberelsäss. tertiär., 7 .

3774

(Elateridæ.)

Locality: Prussian amber. Horizon: Ligurian.

Progr. petrischule Danzig, 1856 : 22.

3775.

(Elateridæ?.)

- Locality: Nicola River, British Columbia. Horizon: -

1879. Scudder. Rep. progr. geol. surv. Can., 1877-78: $182 B$.

1879. " Ins. tert. Nicola, 7.

1890. “ Tert. ins. N. A., 498. Pl. 2, fig. 28.

3776. - (several). (Elateridæ.)

Locality: Florissant, Colo. Horizon : Oligocene.

1881. Scudder. Bull. U. S. geol, surv. terr., vi : 292.

1883. " Ann. rep. U. S. geol. surv. terr., xii : 231.

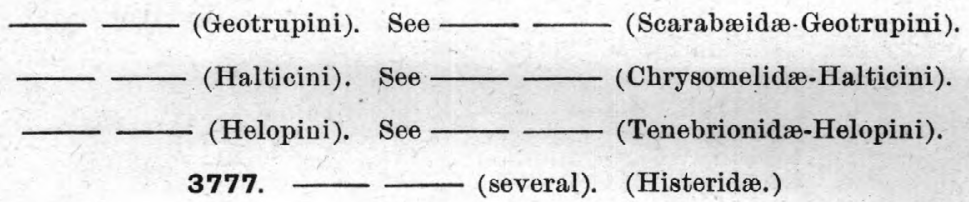

Locality : Florissant, Colo. Horizon: Oligocene.

1881. Scudder. Bull. U. S. geol. surv. terr., vi : 292.

1883. " " Ann. rep. U. S. geol. surv. terr., xii : 28\%.

3778.

(Lampyridæ.)

Locality : Prussian amber. Horizon: Ligurian.

1856. Menge. Progr. petrischule Danzig, 1856: 21.

3779.

(Lucanidæ.)

Locality: Prussian amber. Horizon: Ligurian.

1856. Menge. - Progr. petrischule Danzig, 1856: 23.

3780. (several). (Malacodermata.)

Locality: Florissant, Colo. Horizon: Oligocene.

1881. Seudder. Bull. U. S. geol. surv. terr., vi : $29 \cdot 2$.

1883. " Ann. rep. U.S. geol. surv, terr., xii : 281.

3781. (Meloidæ.)

Compared with Lytta vesicatoria.

Locality: Prussian amber. Horizon: Ligurian.

1847. Hammerschm. Haidinger, Berichte, i : 39.

3782.

(Meloidæ.)

Locality: Prussian amber. Horizon : Ligurian.

1856. Menge. Progr. petrischule Danzig, 1850: 21 . 
3783. (several). (Meloidæ.)

Locality: Florissant, Colo. Horizon: Oligocene.

1881. Scudder. 1883. "

1856. Menge.
Bull. U. S. geol. surv. terr., vi : 292.

Ann. rep. U. S. geol, surv. terr., xii: 281.

\section{(Mordellidæ.)}

Locality: Prussian amber. Horizon: Ligurian. Progr. petrischule Danzig, 1856: 21 .

3785. (several). (Mordellidæ.)

Locality : Florissant, Colo. Horizon : Oligocene.

1881. Scudder. Bull. U. S. geol. surv. terr., vi: 292. 1883. " Ann. rep. U. S. geol. surv. terr., xii : 281.

3786. (Nitidulidæ.)

Locality: Brunstatt, Alsatia. Horizon: Middle Oligocene.

1889. Foerster. Mitth. comm. geol. Elsass-Lothr., ii : 103.

3787. (several). (Nitidulidæ.)

Locality: Florissant, Colo. Horizon: Oligocene.

1881. Scudder. Bull. U. S. geol. surv. terr., vi : 291. 1883. " Ann, rep. U. S. geol, surv, terr., xii : 281.

3788. (Ptinidæ-Bostrychini.)

Locality: Prussian amber. Horizon: Ligurian.

1831. Burmeister. Oken, Isis, 1831: 1100 .

3789. (several). (Ptinidæ.)

Locality : Florissant, Colo. Horizon: Oligocene.

1881. Scudder. Bull. U. S. geol. surv. terr., vi : 292.

1883. " Ann. rep. U. S. geol, surv, terr., xii : 282.

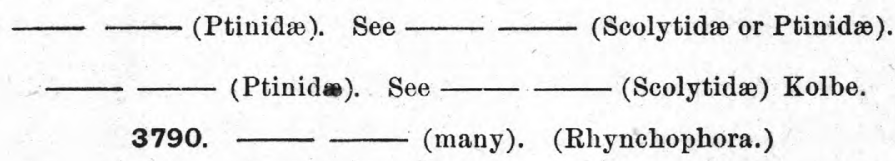

Locality: Florissant, Colo. Horizon : Oligocene.

1881. Scudder. 1883. “

Bull. U: S. geol. surv. terr., vi: 292.

Ann. rep. U. S. geol. surv. terr., xii : 282.

(Rhynchophora). See

$$
\text { Baily. }
$$
(Carabidæ or Rhynchophora)

3791. (Scarabæidæ-Geotrupini.)

Locality: Kutschlin, Bohemia. Horizon: Miocene.

1881. Deichmüller. Foss. ins. diatomeensch. Kutschlin, 12-13, 39. Pl. 21, fig. 3. 1881. " Nova acta acad. leop.-carol., xlii : 305-306, 331. Pl. श1, fig. 3.

3792. (several). (Scarabæidæ.)

Locality : Florissant, Colo. Horizon: Oligocene.

1881. Scudder. 1883 . "
Bull. U. S. geol. surv. terr., vi : 291.

Ann. rep. U. S. geol. surv. terr., xii : 281. 
3793

\section{(Scarabæidæ-Cetonini.)}

Locality : Caverne de Lunel Vieil, Hérault, France. Horizon : Postpliocene. 1827. Serres. Mém. soc. linn. Paris, v : $45 \%$.

3794. - (Scolytidæ or Ptinidæ.)

Locality : Zschipkau, Nieder Lausitz. Horizon: Braunkohl. 1888. Kolbe.

Zeitschr. deutsch. geol. gesellsch, $\mathrm{xl}: 135$. Pl.11, fig.6x.

3795. (Scolytidæ.)

Locality : Scarboro, Canada. Horizon: Interglacial. 1886. Scudder. Cau. ent., xviii : 194-196.

See also Hylastes squalidens.

3796. (several). (Silphidæ.)

Locality : Florissant, Colo. Horizon: Oligocene.

1881. Scudder. Bull. U. S. geol. surv. terr., vi: 292.

1883. "6 Ann. rep. U. S. geol, surv. terr., xii : 282.

3797. (Staphylinidæ-Aleocharini.)

Locality : Prussian amber. Horizon: Ligurian.

1845. Berendt. Bernst, befindl, organ, reste vorw, i : 56 .

3798.

(Staphylinidæ.)

Locality : Brunstatt, Alsatia. Horizon: Middle Oligocene.

1889. Foerster. Mitth. comm. geol. Elsass.-Lothr., ii : 103.

3799. (several). (Staphylinidæ.)

Locality : Florissant, Colo. Horizon: Oligocene.

1881. Scudder. 1883.

Bull. U. S. geol. surv. terr., vi : 291.

Ann. rep. U. S. geol. surv. terr., xii : 281.

3800. - (Tenebrionidæ-Helopini.)

Locality : Dorset, England. Horizon : Middle Eocene, Bagshot series. 1870. Brodie. Geol. mag., vii : 141.

3801. (Tenebrionidæ-Helopini.)

Locality: Corfe, England. Horizon :

1874. Brodie.

Distr. corr. foss. ins., 13.

3802. (several). (Tenebrionidæ.)

Locality: Florissant, Colo. Horizon: Oligocene.

1881. Scudder. 1883.

Bull. U. S. geol. surv. terr., vi : 292.

Ann. rep. U. S. geol, surv, terr, xii : 282.

3803. . (Tenebrionidæ-Helopini.)

Locality : Creech, near Corfe, England. Horizon:

1854. Westwood. Quart. journ. geol. soc. Lond., x : 381-382.

Abax parallela. See Feronia (Abax) parallela.

Abax vetustus. See Pterostichus (Abax) vetustus. 
3804. Acalles icarus. (Curculionidæ.)

Locality : Rott, Rhenish Prussia. Horizon: Aquitanian.

1866. Heyd.-Heyd. Palaeontogr., xv: 152. Pl. 24, fig. 6.

1866. " Käfer u. polypen, 22. Pl. 3, fig. 6.

3805. Acanthoderes lepidus. (Cerambycidæ.)

1865. Heer.

Locality: Oeningen, Baden. Horizon: Tortonian.

1872. " "

Urwelt der Sehweiz, 362 (Acanthodérus).

1876. " "

Monde prim. Suisse, 444 (Acanthoderus).

1879. “

Prim. world Switz., ii: 16 (Acanthoderus).

Urwelt der Sehweiz, 'se aufl., 387 (Acanthoderus).

3806. Acanthoderes phrixi. (Cerambycidæ.)

Locality : Radoboj, Croatia. Horizon: Mayencian.

1847. Heer.

Insektenf. tertiärg. Oeningen, i : 16\%-163. Pl. 5, fig. 13.

1852. Giebel.

Deutschl. petref., 649 .

1856. " "

Insect. d. vorwelt, 130 .

3807. Acanthoderes sepultus. (Cerambycidæ.)

Locality: Oeniugen, Baden. Horizon: Tortonian.

1865. Heer.

1872. "

1876. " "

1879. “

1874. Oustalet.

1862. Heer.

Locality: Oeningen, Baden. Horizon: Tortonian.

3808. Achenium ingens. (Staphylinidæ.)

Locality: Aix, France. Horizon: Ligurian.

Monde prim. Suisse, 444,461 (Acanthoderus).

Prim. world Switz., ii: 16, 31 (Acanthoderus).

Urwelt der Schweiz, 2e aufl., 387, 401 (Acanthoderus).

Acanthoderus lepidus. See Acanthoderes lepidus.

Acanthoderus sepultus. See Acanthoderes sepultus.

Anu. sc. géol., v, art. 2: 142-143. Pl. 2, fig. 18.

3809. Acmæodera antholitha. (Buprestidæ.)

Verhandl, holl, maatsch, wetensch., xvi : 89. Pl.7, fig. 23.

3810. Acmæodera brevicollis. (Buprestidæ.)

Locality: Oeningen, Baden. Horizon : Tortonian.

1862. Heer. Verhandl, holl, maatsch, wetensch., xvi : 89. Pl.7, fig. 22.

3811. Adalia - (Coccinellidæ.)

Locality : Brunstatt, Alsatia. Horizon: Middle Oligocene.

1890. Foerster. In litt.

3812. Adelocera granulata. (Elateridæ.)

Locality: (Oeningen?, Baden). Horizon: Tortonian.

1847. Heer.

Insektenf. tertiärg. Oeningen, i: 139-140, Pl. 8, fig. 7.

1852. Giebel.

Deutschl. petref., 651 .

1856. " Insect, d. vorweit, 96-97.

3813. Adimonia? (Chrysomelidæ.)

Locality : Jarville, France. Horizon: Plenstocene.

1875. Fliche.

Comptes rendus, lxxx : 1234. 
3814. Aggialia rupta. (Scarabæidæ.)

Locality: Green River, Wyo. Horizon: Oligocene.

1890. Scudder. Tert. ins. N. A., 4r9. Pl. 8, fig. 19.

3815. Agabus - (Dytiscidæ.)

Locality : Hösbach, Bavaria. Horizon: Lower Pleistocene.

1884. Flach. Käfer unterpleist. Hösbach, 8.

1884. " Verhandl. physik.-med. gesellsch. Wurzb., n. f., xviii : 292.

3816. Agabus ? - (Dytiscidæ.)

Locality : Prussian amber. Horizou : Ligurian.

1856. Menge. Progr. petrischule Danzig, 1856: 23.

3817. Agabus bipunctatus (recent). (Dytiscidæ.)

Locality: Cambridge, England. Horizon: Peat beds.

[1787. Fabricius. Mant. ins., 190.]

1888. Bell. Entom., xxi: 2.

See also Agabus nebulosus.

3818. Agabus nebulosus (recent). (Dytiscidæ.)

Locality : Cambridge, England. Horizon : Peat beds.

[1781. Forster. Nov. spec. ins., 56.].

See also Agabus bipunctatus.

3819. Agabus reductus. (Djtiscidæ.)

Locality : Rott, Rhenish Prussia. Horizon: Aquitanian.

1866. Heyd.-Heyd. Palaeontogr., xv: 135. Pl. 22, fig. 4.

1866. " Käfer u. polypen, 5. Pl. 1, fig. 4 .

3820. Agatoides carinulatus. (Carabidæ.)

Locality : Prussian amber. Horizon: Ligurian.

1856. Motschulsky. Etudes entom., v : 26.

3821. Agelasa - (Chrysomelidæ.)

Locality : Brunstatt, Alsatia. Horizon: Middle Oligocene.

1890. Foerster. In litt.

3822. Agonum gracile (recent). (Carabidæ.)

Locality: Jarville, France. Horizon: Pleistocene.

[1824. Sturm. Ins., v: 197. Pl. 136, fig. A.]

1875. Fliche. Comptes rendus, $1 \times x x: 1234$.

3823. Agonum sismondæ. (Carabidæ.)

Locality : La Boisse, France. Horizon: Quaternary,

1876. Mortillet. MSS.

1876. Lart.-Chantre. Arch. mus. hist. nat. Lyon, i: 104.

\section{Agrilus - (Buprestidæ.)}

Locality: Prussian amber. Horizon: Ligurian.

1845. Berendt. Bernst, befindl, organ, reste vorw, i; 56. 
3825. Agrilus -. (Buprestidæ.)

Locality : Creech, England. Horizon : Corfe clay.

1854. Westwood. Proc. geol. soc. Lond. 381, 395. Pl. 16, fig. 34.

3826. Agrilus baueri. (Buprestidæ.)

Compared with A. biguttatus Fabr.

Locality : Rott, Rhenish Prussia. Horizon : Aquitanian.

1862. Heyden. Palaeontogr., $\mathrm{x}:$ 68-69. Pl. 10, fig. 21.

3827. Akulosamphus montanus. (Curculionidæ.)

Locality: Le Puy, France. Horizon :

1854. Aymard. Congr. scient. France, sess. xxii : 42 (undescr.).

Alaus spectabilis. See Elater (Alaus) spectabilis.

3828. Aleochara - (Staphylinidæ.)

Locality: Prussian amber. Horizon: Ligurian.

1835. Gravenhorst. Uebers. schles. gesellsch. vaterl. cult., 1834 : 92.

3829. Amara - (2 sp.). (Carabidæ).

Locality : Brunstatt, Alsatia. Horizon: Middle Oligocene.

1890. Foerster. In litt.

3830. Amara aulíca (recent). (Carabidæ.)

Locality : Hösbach, Bavaria. Horizon : Lower Pleistocene.

[1797. Panzer.

1884. Flach.

1884. " "
Faun. germ., xxxviii : 3.$]$

Käfer unterpleist. Hösbach, 6. Pl. 1, fig. 9.

Verhandl. physik.-med. gesellsch. Würzb., n. f., xviii: $290 . \quad P l$. 8, fig. 9.

3831. Amara famelica (recent). (Carabidæ.)

Locality: Hösbach, Bavaria. Horizon: Lower Pleistocene.

[1831\%. Zimmermann. Mon. Carab. i : 36.]

1884. Flach.

1884. " "
Käfer unterpleist. Hösbach, 6-7. $P l .1, f i g .10$.

Verhandl. physik.-med. gesellsch. Würzb., n. f., xviii : 290-291. Pl. 8, fig. 10.

3832. Amara familiaris (recent). (Carabidæ.)

Locality: Hochheim, Germany. Horizon: Kalkmergel.

[1812. Duftschmid. Faun. austr., ii : 119 (Carabus?).]

1840. Meyer.

Ersch u. Griber, Allg. encycl. d. wissensch., sect. 2, th. xviii : 539.

3833. Amara pinguicula. (Carabida.)

Locality: Oeningen, Baden. Horizon: Tortonian.

1862. Heer. Verhandl. holl. maatsch. wetensch., xvi: 26. Pl. 1, figs.14,15.

3834. Amara primigenia. (Carabidæ.)

Locality: Oeningen, Baden. Horizon: Tortonian.

1862, Heør. Verhandl. holl, maatsch, wețensch., xvi: 25-26. $P l, 1, f i g s, 12$, $12 b$. 
3835. Amara princeps. (Carabidæ.)

Locality : Oeningen, Baden. Horizon: Tortonian.

1862. Heer.

Verhandl. holl. maatsch. wetensch., xvi : 24-25. Pl. 1, figs. 13, $13 b-c$.

1865. " Urwelt der Schweiz. Fig. 285 on $p .384$.

1872. " Monde prim. Suisse. Fig. 285 on p. 471.

1876. “ Prim. world Switz., ii. Fig. 285 on p. 42.

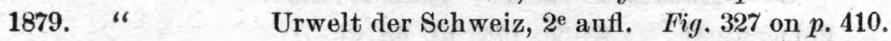

3836. Ammcecius (Scarabæidæ.)

Locality : Brunstatt, Alsatia. Horizon: Middle Oligocene. 1889. Foerster. Mitth. comm. geol. Elsass-Lothr., ii : 10\%.

3837. Ampedus seyfriedii. (Elateridæ.)

Locality: Oeningen, Baden. Horizon: Tortonian.

1847. Heer.

1852. Giebel.

1856. "

Insektenf. tertiärg. Oeningen, i: 131-133. Pl. 4, fig. 2.

1865. Heer.

1872. “

1876. “

1879. “

Deutschl. petref., 651 .

Insect. $d$, vorwelt, 98.

Urwelt der Schweiz, 362.

Monde prim. Suisse, 444.

Prim. world Switz., ii : 16.

Urwelt der Schweiz, $2^{\mathrm{e}}$ aufl., 387.

3838. Amphotis bella. (Nitidulidæ.)

Locality : Radoboj, Croatia. Horizon: Mayencian.

1847. Heer.

1852. Giebel.

Insektenf. tertiärg. Oeningen, i : 38-39. Pl. 7, fig. 22.

1856. " "

Deutschl. petref., 654 .

Insect. d. vorwe]t, 44.

3839. Amphotis œningensis. (Nitidulidæ.)

Locality: Oeningen, Baden. Horizon: Tortonian.

1862. Heer.

Verhandl, holl. maatseh. wetensch., xvi : 53. Pl. 3, fig. 31.

3840. Anaspis —. (Pythidæ.)

Locality: Prussian amber. Horizon: Ligurian.

1845. Berendt. Bernst. befindl. organ, reste vor w., i : 56 .

3841. Anaspis antica. (Pythidæ.)

Locality: Sicilian amber. Horizon: -

1838. Guérin. Rev. zool,, 1838: 170 (undescr.).

3842. Anchomenus - (Carabidæ.)

Locality : Brunstatt, Alsatia. Horizon : Middle Oligocene.

1888. Foerster. Mitth. comm. geol. Elsass-Lothr., i: 165.

3843. Anchomenus - (Carabidæ.)

Locality : Prussian amber. Horizon: Ligurian.

1856. Menge. Progr. petrischule Danzig, 1856: 23.

3844. Anchomenus orphanus. (Carabidæ.)

Locality: Radoboj, Croatia. Horizon: Mayencian.

1847. Heer.

1852. Giebel.

Insektenf. tertiärg. Oeningen, i : 21-22. $\quad P l .1$, fig. 4.

1856, "4

Deutschl. petref., 655 .

Insect, $d$, vorwelt, 66 , 
3845. Ancylocheira alemanica. (Buprestidæ.)

Locality: Oeningen, Baden. Horizon: Tortonian.

1879. Heer. Urwelt der Schweiz, 2e aufl, 384. Figs. 258ab.

3846. Ancylocheira concinna. (Buprestidæ.)

Compared with A. octoguttata Fabr.

Locality: Oeniugen, Baden. Horizon: Tortonian.

1879. Heer. Urwelt der Schweiz, $2^{\mathrm{e}}$ aufl, 38\%, 403. Fig. 259 on $p .384$.

3847. Ancylocheira deleta. (Buprestidæ.)

Localities: Oeningen, Baden; Monte Bolca, Italy. Horizons: Tortonian, Ligurian. 1847. Heer. Insektenf. tertiärg. Oeningen, i : 108 (Ancylochira). Pl. 3, fig. 6. 1852. Giebel. Deutschl. petref., 652 .

1855. Massalongo. Monogr. nereid. foss., 31 ("Comp. faun. foss. bolc., pl. 10, figs. $5-7 ")$.

1856. - 6 Stud̃. paleont., 14. Pl. 1, figs. 1-3.

1856. Giebel. Insect. d. vorwelt, 83-84.

1886. Omboni. Atti r, ist. Ven., (6), iv: 1425. Pl. 2, figs. 2-3.

1886. " Ins. foss. Ven., 5. Pl. 2, figs. 2-3.

3848. Ancylocheira gracilis. (Buprestidæ.)

Locality: Oeningen, Baden, Horizon: Tortonian.

1817. Heer. Insektenf. tertiärg. Oeningen, i: 111-112 (Ancylochira). Pl, 3, fig. 9.

1852. Giebel. Deutschl. petref., 652 .

1856. “ Insect. d. vorwelt, 84 .

3849. Ancylocheira heydenii. (Buprestidæ.)

Locality : Oeningen, Baden. Horizon: Tortonian.

1847. Heer. Inselstenf. tertiärg. Oeningen, i: 106-108 (Ancylochira). Pl. 3, fig. 5 .

1852. Giebel. Deutschl. petref., 65\%.

1856. " Insect. d, vorwelt, 83 .

3850. Ancylocheira pristina. (Buprestidæ.)

Locality : Rott, Rhenish Prussia. Horizon : Aquitanian.

1ะ62. Heyden. Palaeontogr., $\mathrm{x}: 68$ (Ancylochira.)

3851. Ancylocheira redempta. (Buprestidæ.)

Locality : Rott, Rhenish Prussia. Horizon : Aquitanian.

1859. Heyden. Palaeontogr., viii: 4 (Ancylochira). Pl. 1, fig. 1.

3852. Ancylocheira rusticana. (Buprestidæ.)

Locality: Oeningen, Baden. Horizon: Tortonian.

. 1847. Heer.

1852. Giebel.

Insektenf. tertiärg. Oeningen, i : 109-110 (Ancylochira). Pl. 3, fig. 7.

1856. "

Deutschl. petref., 652.

Insect. d. vorwelt, 84 .

3853. Ancylocheira seyfriedii. (Buprestidæ.)

Locảlity : Oeningen, Baden. Horizon: Tortonian.

1847. Heer. Insektenf. tertiärg. Oeningen, i: 110-111 (Ancylochira). Pl. 3, fig. 8.

1852. Giebel. Dentschl. petref., 652.

1856, "4 Insect, $\mathrm{d}$, yorwelt, 84 , 
3854. Ancylocheira tincta. (Buprestidæ.)

Compared with A. octoguttata Fabr.

Locality: Oeningen, Baden. Horizon: Tortonian.

1862. Heer. Verhandl. holl. maatsch. wetensch., xvi : 87 (Ancylochira). $P l$. 7, figs. 19, 20.

1865. “ Urwelt der Schweiz, 362,378. Fig. 258.

1872. " Monde prim. Suisse, 444, 464. Fig. 258, on p. 463.

1876. " Prim. world Switz., ii : 16,35. Fig. 258, on p. 34 .

1879. " Urwelt der Schweiz, $2^{2}$ aufl, 387, 403. Fig. 300, on p. 404.

Ancylochira (various species). See Ancylocheira, the same species.

3855. Anisorhynchus deletus. (Otiorh jnchidæ.)

Compared with A. bajulus and A. hespericus.

Locality : Kutschlin, Bohemia. Horizon: Miocene.

1881. Deichmüller. Foss. ins. diatomeensch. Kutschlin, 21-22. Pl. 21, fig. 7.

1881. " Verhandl. leop--carol. acad. naturf, xlii: 313-314. Pl. 21, fig. 7.

3856. Anisorhynchus effossus. (Otiorhynchidæ.)

Compared with A. bajulus, Europe.

Locality : Corent, France. Horizon: Aquitanian.

1870. Oustalet. Ann. sc. géol., ii, art 3: 72-73. Pl. 1, fig.9.

3857. Anisotoma -. (Silphidæ.)

Locality: Prussian amber. Horizon: Ligurian.

1845. Berendt. Bernst. befindl. organ. reste vorw., i : 56 .

3858. Anobium —. (Ptinidæ.)

Locality: Prussian amber. Horizon: Ligurian.

1845. Berendt. Bernst. befindl. organ. reste vorw., i : 56.

3859. Anobium -. (Ptinidæ.)

Compared with A. pertinax and A. rufipes.

Locality : Prussian amber. Horizon: Ligurian.

1832. Burmeister. Handb, ent., i: 635.

1836. “ Man. ent., 577.

\section{Anobium - (Ptinida.)}

Locality : Gurnet Bay, Isle of Wight. Horizon: Bembridge Limestone.

1879. Woodward. Quart. journ. geol. soc. Lond., xxxv : 344.

1879. " Geol. mag., (n. s.), v: 89.

3861. Anobium? deceptum. (Ptinidæ.)

Locality : Green River, Wṿo. Horizon: Oligocene

1878. Seudder. Bull. U. S. geol. surv. terr., iv : 763.

1890. " Tert. ins. N. A., 492. Pl. 8, fig. 18.

3862. Anobium lignitum. (Ptinidæ.)

Locality: Green River, Wyo. Horizon: Oligocene.

1878. Scudder. Bull. U. S. geol. surv. terr., iv: 763.

1890, " Tert, ins, N, A., 492-493, Pl. 8, fig. 24, 
3863. Anobium? ovale. (Ptinidæ.)

Compared with Endecatomus rugosus LeC.

Locality: Green River, Wyo. Horizon: Oligocene.

1878. Scudder. 1890. "
Bull. U. S. geol. surv., terr., iv: 762-763.

Tert. ins. N. A., 491-492. Pl. 8, fig. 1.

3864. Anomala fugax. (Scarabæidæ.)

Compared with A. julii F., Europe.

Locality: Oeningen, Baden, Horizon: Tortonian.

1862. Heer

1865. " "

1872. “

1876. “

Urwelt der Schweiz, 380.

Monde prim. Suisse, 466.

Prim. world Switz., ii : 37.

Urwelt der Schweiz, $2^{\mathrm{e}}$ aufl., 406.
1879. "6

Verhandl. holl. maatsch. wetensch., xvi : 80. Pl.6, fig. 23.

3865. Anomala primigenia. (Scarabæidæ.)

Locality: Rott, Rhenish Prussia. Horizon: Aquitanian.

1866. Heyd.-Heyd. Palaeontogr., xv : 140-141. Pl. 22, figs. 18, 19.

1866. " Käfer u. polypen, 10-11. Pl. 1, figs. 18, 19.

3866. Anomala thetis. (Scarabæidæ.)

Locality: Rott, Rhenish Prussia. Horizon: Aquitanian.

1866. Heyd.-Heyd. Palaeontogr., xv : 141. Pl. 24, fig. 12.

1866. “ Käfer u. polypen, 11. Pl. 3, fig. 12.

3867. Anomala tumulata. (Scarabæidæ.)

Locality : Rott, Rhenish Prussia. Horizon: Aquitanian.

1866. Heyd.-Heyd. Palaeontogr., xv: 140. Pl. 23, figs.18, 19.

1866. “ Käfer u. polypen, 10. Pl. 2, figs. 18, 19.

3868. Anomalites fugitivus. (Scarabæidæ.)

Locality : Nogent le Rotrou, France. Horizon:

1884. Frið.

Sitzungsber. k. böhm. gesellsch. wiss., 1884: 163-165. Fig.

Anoplites bremii. See Anoplitis bremii.

3869. Anoplitis bremii. (Chrysomelidæ.)

Compared with Anoplitis quadrata F.

Locality: Oeningen, Baden. Horizon: Tortonian.

1852. Giebel.

1872. " "

1876. "

1879. “
1847. Heer.

1856. "6

1865. Heer.

Insektenf." tertiärg. Oeningen, i: 202-204 (Anoplites). Pl. 7, fig. $5 ; p l .8$, fig. 9.

Deutschl. petref., 648 .

Insect. d. vorwelt, 115.

Urwelt der Schweiz, 372. Fig. 241, on p. 371 .

Monde prim. Suisse, 456. Fig. 241, on p. 455.

Prim. world Switz., ii: 28. Fig. 241, on p. 27.

Urwelt der Schweiz, $2^{\circ}$ aufl., 398. Fig. 283, on p. 397.

3870. Anoplognathus rhenanus. (Scarabæidæ.)

Locality: Rott, Rhenish Prussia. Horizon: Aquitanian.

1862. Heyden. Palaeontogr, $\mathrm{x}: 65-66 . \quad P l .10, \mathrm{fig} .19$. 
3871. Anthaxia —. (Buprestidæ.)

Locality : Brunstatt, Alsatia. Horizon: Middle Oligocene.

1885. Foerster. Tagebl. versamml. deutsch. naturf., lviii : 392.

1885. " Mitth. oberelsäss. tertiär., 7.

1888. "6 Mitth. comm. geol. Elsass-Lothr., i: 165.

1889. " Mitth. comm. geol. Elsass-Lothr., ii : 103.

3872. Anthaxia buschi. (Buprestidæ.)

Locality: Naumburg, Prussia. Horizon: Oligocene.

1869. Assmann. Beitr. ins. vorwelt, 61-62. Figs. $2 a, b$.

1869. " Zeitschr. f. ent. ver. schles. insektenk., ii, pt. i : 61-62. Figs. 2a, b.

3873. Anthaxia carbonaria. (Buprestidæ.)

Locality : Salzhausen, Germany. Horizon: Miocene.

1865. Heyd.-Heyd, Palaeontogr., xiv: 32. Pl. 9, figs. 15-16.

3874. Anthaxia crassicollis. (Buprestidæ.)

Locality: Oeningen, Baden. Horizon: Tortonian.

1862. Heer. Verhandl. holl, maatsch. wetensch., xvi: 88. Pl. 7, fig. 16.

3875. Anthaxia deleta. (Buprestidæ.)

Locality: Salzhausen, Germany. Horizon: Miocene.

1865. Heyd.-Heyd. Palaeontogr., xiv: 32.

3876. Anthaxia doris. (Buprestidæ.)

Locality: Oeningen, Baden. Horizon: Tortonìan.

1862. Heer. Verhandl. holl. maatsch. wetensch., xvi: 88-89. Pl. 7, flg. 21.

3877. Anthaxia pallida. (Buprestidæ.)

Locality: Oeningen, Baden. Horizon: Tortonian.

1862. Heer. Verhandl. holl. maatsch. wetensch., xvi: 88. Pl. 7, fig. 25.

3878. Anthasia primæva. (Buprestidæ.)

Locality : Salzhausen, Germany. Horizon : Miocene.

1865. Heyd.-Heyd. Palaeontogr., xiv: 32-33.

3879. Antherophagus priscus. (Cryptophagidæ.)

Compared with A. ochraceus.

Locality: Green River, Wyo. Horizon: Oligocene.

1876. Scudder. Bull. U. S. geol. surv. terr., ii: 79-80.

1878. " Bull. U. S. geol. surv. terr., iv: 762 .

1885. “ Zittel, Handb. palaeont., i, th. ii: 800. Fig. 1051.

1886. " Z Zittel-Barrois, Traité de paléont., ii : 800. Fig. 1068

1890. " Tert. ins. N. A., 501. Pl. 7, figs. 24, 35.

3880. Anthicus - (Anthicidæ.)

Locality: Prussian amber. Horizon: Ligurian.

1845. Berendt. Bernst. befindl. organ. reste vorw., i: 56 , 
3881. Anthicus melancholicus. (Anthicidæ.)

Locality : Aix, France. Horizon: Ligurian.

1874. Oustalet. Ann. sc. géol., v, art. 2: 208-210 (Antichus). Pl. 5, fig. 12.

3882. Anthonomus defossus. (Curculionidæ.)

Compared with A. hæmatopus.

Locality: Florissant, Colo. Horizon: Oligocene.

1876. Scudder. Bull. U. S. geol. surv. terr., ii : 86.

3883. Anthonomus soporus. (Curculionidæ.)

Locality: Green River, Wyo. Horizon: Oligocene.

1890. Scudder. Tert. ins. N. A., 472-473. Pl. 8, fig. 16.

3884. Anthophagus —. (Staphylinidæ.)

Locality: Prussian amber. Horizon: Ligurian.

1845. Berendt. Bernst. befindl. organ. reste vorw., i: 56.

3885. Anthophagus giebeli. (Staphylinidæ.)

Locality : Rott, Rhenish Prussia. Horizon: Aquitanian.

1866. Heyd.-Heyd. Palaeontogr., $\mathrm{xv}:$ 138. Pl. 22, fig. 15.

1866. " Käfer u. polypen, 8. Pl.1, fig. 15.

3886. Anthrenus -. (Dermestidæ.)

Locality : Prussian amber. Horizon: Ligurian.

1845. Berendt. Bernst. befindl. organ. reste vorw., i: 56.

3887. Anthribites moussonil. (Anthribidæ.)

Locality: Oeningen, Baden. Horizon: Tortonian,

1847. Heer. Insektenf. tertiärg. Oeningen, i : 177-178. Pl. 6, fig.7.

1852. Giebel. Deutschl. petref., 649.

See also Anthribus moussoni.

3888. Anthribites pusillus. (Anthribidæ.)

Locality: Oeningen, Baden. Horizon: Tortonian.

1847. Heer. Insektenf. tertiärg. Oeningen, i: 178-179. Pl. 6, fig. 6.

1852. Giebel. Deutschl. petref., 649 .

1856. " Insect. d. vorwelt, 135.

3889. Anthribites rechenbergi. (Anthribidæ.)

Locality: Zschipkau, Nieder Lausitz. Horizon: Braunkohl.

1888. Kolbe. Zeitschr. deutsch. geol. gesellsch., xl: 131-134. 2l.11; figs.1-3, $4 a b$.

3890. Anthribus -

Locality : Prussian amber. Horizon: Ligurian.

1845. Berendt. Bernst. befindl. organ. reste vorw., i: 56.

3891. Anthribus moussoni. (Anthribidæ.)

Locality: Oeningen, Baden. Horizon: Tortonian.

1856. Giebel.

Insect. d. vorwelt, 135.

See also Anthribites monssonii.

Antichus melancholicus. See Anthicus melancholicus. 
3892. Antliarhinites gracilis. (Rhynchitidæ.)

Locality: Oeningen, Baden. Horizon: Tortonian.

1865. Heer.

1872. " "

1876. " "

1879. “

Urwelt der Schweiz, 374. Fig. 246, on p. 371.

Monde prim. Suisse, 458. Fig. 246, on p. 455.

Prim. world Switz., ii : 29. Fig. 246, on p. 27.

Urwelt der Schweiz, 2e aufl., 399. Fig. 288, on p. 397.

$$
\text { 3893. Apate —. (Ptinidæ.) }
$$

Locality : Prussian amber. Horizon: Ligurian.

1832. Burmeister. Handb. ent., i : 635 .

1836. "6 Man. ent., 577.

\section{Apate - (Ptinidæ.)}

Compared with A. capucina.

\section{Locality: Aix, France. Horizon: Ligurian}

1829. Serres. Géogn. terrains tert., 224.

3895. Apate —. (Ptinidæ.)

Locality: Prussian amber. Horizon: Ligurian.

1829. Serres. Géogn. terrains tert., 241.

\section{Aphodius - (Scarabæidæ.)}

Compared with A. fimetarius Fabr.

Locality: Habichtswald, Germany. Horizon: "Polirschiefer."

1843. Landgrebe. Nenes jahrb. f. min., 1843: 141-142.

3897. Aphodius antiquus. (Scarabæidæ.)

Locality: Oeningen, Baden. Horizon: Tortonian.

1847. Heer. Insektenf. tertiärg. Oeningen, i : 66-67. Pl. 7, fig. 28.

1852. Giebel. Deutschl. petref., 653.

1856. " Insect. d. vorwelt, 40 .

3898. Aphodius brevipennis. (Scarabæidæ.)

Locality : Oeningen, Baden. Horizon: Tortonian.

186\%. Heer. Verhandl. holl, maatsch. wetensch., xvi: 77-78. Pl. 6, fig. 21.

3899. Aphodius fossor (recent). (Scarabæidæ.)

Locality : Prussian amber. Horizon: Ligurian.

[1746. Linné.

Faun. suec., 134.]

1838. Robert. Bull. soc. géol. France, ix : 114.

3900. Aphodius krantzi. (Scarabæidæ.)

Locality: Rott, Rhenish Prussia. Horizon : Aquitanian.

1866. Heyd.-Heyd. Palaeontogr., xv: 140. Pl. 22, fig. 24.

1866. " Käfer u. polypen, 10. $P l .1$, fig. 24.

3901. Aphodius meyeri. (Scarabæidæ.)

Locality: Oeningen, Baden. Horizon: Tortonian.

1847. Heer. Insektenf. tertiärg. Oeningen, i: 67. Pl. 7, fig. 27.

1852. Giebel. Dentschl. petref., 653.

1856. "Insect. d. vorwelt, 40. 
3902. Aphodius præcursor. (Scarabæidæ.)

Locality: Port Kennedy, Pa. Horizon: Postpliocene.

1876. Horn.

Trans. Amer. ent. soc., v : 245.

1889. Lesley.

Dict. foss. Penns., i: p. v.

1890. Scudder.

Tert. ins. N. A., 488-489. Pl. 1, fig. 11.

3903. Apion —_. (Curculionidæ.)

Locality : Prussian amber. Horizon: Ligurian.

1845. Berendt. Bernst. befindl. organ. reste vorw., i: 56.

$$
\text { 3904. Apion - (Curculionidæ.) }
$$

Compared with A. trifolii.

Locality: Hösbach, Bavaria. Horizon: Lower Pleistocene.

1884. Flach.

1884. “

Käfer unterpleist. Hösbach, 10. Pl. 2, fig. 9.

Verhandl. physik.-med. gesellsch. Würzburg, n. f., xviii : 294. Pl. 2, fig. 9 .

3905. Apion - (2 sp.). (Curculionidæ.)

Locality: Brunstatt, Alsatia. Horizon: Middle Oligocene.

1889. Foerster. Mitth. comm. geol. Elsass-Lothr., ii: 102.

3906. Apion - (4 sp.). (Cureulionidæ.)

Locality: Brunstatt, Alsatia. Horizon: Middle Oligocene.

1890. Foerster. In litt.

\section{Apion - (Curculionidæ.)}

Locality: Aix, France. Horizon: Ligurian.

1829. Serres. Géogu. terrains tert., 222, 267.

$$
\text { Apion - See - }-(\text { Carabidæ) Baily. }
$$

3908. Apion antiquum. (Curculionidæ.)

Locality : Oeningen, Baden. Horizon: Tortonian.

1865. Heer.

1872. " "

Urwelt der Schweiz. Fig. 242, on p. 371.

Monde prim. Suisse. Fig. 242, on $p .455$.

1876. "6

1879. " "

Prim. world Switz., ii. Fig. 242, on p. 27.

Urwelt der Schweiz, $2^{\mathrm{e}}$ auf. Fig. 284, on $p .397$.

3909. Apion primigenius. (Curenlionidæ.)

Locality : Aix, France. Horizon: Ligurian.

1874. Heer.

MSS. in Oustalet.

See also Cœliodes primigenius.

3910. Apion primordiale. (Curculionidæ.)

Locality: Rott, Rhenish Prussia. Horizon: Aquitanian.

1866. Hөyd-.Heyd. Palaeontogr., xv: 148. Pl. 23, fig. 9.

1866. “ Käfer u. polypen, 18. Pl. 2, fig.9. 
3911. Argutor antiquus. (Carabidæ.)

Locality : Oeningen, Baden. Horizon: Tortonian.

1847. Heer.

Insektenf. tertiärg. Oeningen, i : 22-23. $P l .1, f i g .5$.

1852. Giebel.

Deutschl. petref., 655 .

See also Pterostichus antiquus.

Argutor diligens. See Feronia (Argutor) diligens.

Argutor minutulus. See Pterostichus (Argutor) minutulus.

3912. Argutor vernalis. (Carabidæ.)

Locality: Basel, Switzerland. Horizon: Interglacial.

1886. Seudder. Bull. U. S. geol. surv., No. 31: 84 .

See also Pterostichus vernalis.

3913. Arthropterus kuhlii. (Paussidæ.)

Locality: Prussian amber. Horizon: Ligurian.

1877. Stein. Mittheil. münch. entom, ver,, i: 29.

3914. Asida —. (Tenebrionidæ.)

Compared with A. grisea.

Locality: Aix, France. Horizon : Ligurian.

1829. Serres. G6́ogn. terrains tert., 222, 266-267.

3915. Astynomus tertiarius. (Cerambycidæ.)

Locality : Zschipkau, Nieder Lausitz. Horizon : Braunkohl.

1888. Kolbe. Zeitschr. dentsch. geol. gesellsch., xl: 134-135. Pl. 11, figs. 5, 6.

3916. Atomaria protogæa. (Cryptophagidæ.)

Locality : Oeningen, Baden. Horizon: Tortonian.

1862. Heer. Verhandl. holl. maatsch. wetensch., xvi: 53. Pl. 3, figs.32,32b.

3917. Atopa - (Dascyllidæ.)

Compared with A. cervina.

Locality: Aix, France. Horizon: Ligurian.

1847. Hope. Trans. ent. soc. Lond., iv : 251.

3918. Atopa cervina (recent). (Dascyllidæ.)

Locality : Cambridge, England. Horizon: Peat.

1888. Bell. Entom., xxi: 2.

See also Dascyllus cervinus.

3919. Atractocerus - (Lymexylidæ.)

Locality : Prussian amber. Horizon : Ligurian.

1825. Guérin. Dict. class. d'hist. nat., viii : 580 (Atractocère).

1829. Serres. - Géogn. terrains tert., 241.

3920. Attagenus extinctus. (Dermestidæ.)

Locality : Salzhausen, Germany. Horizon: Miocene.

1865. Heyd.-Heyd. Palaeontogr., xiv: 31-32. Pl. 9, fig. 14.

1885. Scudder. Zittel, Handb. palaeont., i, th.ii: 799. Fig. 1047.

1886. " Zittel-Barrois, Traité de paléont., ii: 799. Fig. 1064. 


\section{Attelabus —. (Attelabidæ.)}

Locality: Copal. Horizon: Recent.

1776. Bloch. Beschäft. berl. gesellsch. naturf. freunde, ii : 168-170. $P l .3$, figs. 7-8.

3922. Attelabus durus. (Attelabidæ.)

Locality: Oeningen, Baden. Horizon : Tortonian.

1865. Heer. Urwelt der Sehweiz, 373. Fig. 244, on p. 371.

1872. “ Monde prim. Suisse, 458. Fig. 244, on p. 455.

1876. “ $\quad$ Prim. world Switz., ii : 29. Fig. 244, on p. 27.

1879. "Urwelt der Schweiz, $2^{\mathrm{e}}$ aufl., 399, Fig. 286, on $p .397$.

3923. Badister debilis. (Carabidæ.)

Locality : (Oeningen ?, Baden). Horizon : Tortonian.

1847. Heer. Insektenf. tertiärg. Oeningen, i: 20-21. Pl. 8, fig. 1.

1852. Giebel. Deutsehl. petref., 655. .

1856. " Insect. d. vorwelt, 68.

3924. Badister fragilis. (Carabidæ.)

Locality: Oeningen, Baden. Horizon : Tortonian.

1862. Heer. Verhandl. holl. maatsch. wetensch., xvi : 21. Pl. 1, figs. 9, 10.

3925. Badister grandis. (Carabidæ.)

Locality : Oeningen, Baden. Horizon: Tortonian.

1862. Heer. Verhandl. holl. maatsch. wetensch., xvi: 19-20. Pl. 1, figs. 5, $5 b, 6,6 b$.

3926. Badister macrocephalus. (Carabidæ.)

Locality: Oeningen, Baden. Horizon: Tortonian.

1862. Heer. Verhandl. holl. maatsch. wetensch., xvi: 19. Pl. 1, fig. 8.

See also Badister macrocephalus var. major.

3927. Badister macrocephalus var. major. (Carabid».)

Locality : Oeningen, Baden. Horizon: Tortonian.

1862. Heer. Verhandl. holl. maatsch. wetensch., xvi: 19. Pl. 1, fig. 7.

See also Badister macrocephalus.

Badister major. See Badister macrocephalus var. major.

3928. Badister prodromus. (Carabidæ.)

Locality: (Oeningen?, Baden). Horizon: Tortonian.

1847. Heèr. Insektenf. tertiärg. Oeningen, i: 18-20. Pl. 1, fig. 3.

1852. Giebel. Deutschl. petref., 655.

1856. "Insect. $d$. vorwelt, $67-68$.

3929. Bagous - (2 sp.). (Curculionidæ.)

Locality: Brunstatt, Alsatia. Horizon : Middle Oligocene.

1890. Foerster. In litt.

3930. Bagous atavus. (Curculionidæ.)

Locality: Corent, France. Horizon: Aquitanian.

1870. Oustalet. Ann. sc. géol., ii, art. 3: $76 . \quad$ Pl. 1, fig 11. 


\section{Balaninus barthelemyi. (Curculionidæ.)}

Locality: Aix, France. Horizon: Ligurian.

1847. Hope.

1856. Giebel.

1874. Oustalet.
Trans. ent. soc. Lond., iv: 254. Pl. 19, figs. 1, 1*.

Insect. d. vorwelt, 147 (Barthelemy).

Ann. sc. géol., v, art. 2 : 294.

3932. Balaninus geinitzi. (Curculionidæ.)

Compared with B. apicalis.

Locality: Kntschlin, Bohemia. Horizon: Miocene.

1881. Deichmüller. Foss. ins. diatomeensch. Kutschlin, 22. Pl. 21, figs. 8, 8a.

1881. " Verhandl. leop.-carol, akad. naturf, xlii: 314. Pl. 21, figs.8,8a.

3933. Baridium —. (Cureulionidæ.)

Locality : Brunstatt, Alsatia. Horizon: Middle Oligocene.

1890. Foerster. In litt.

\section{Baris -. (Curculionidæ.)}

Locality: Aix, France. Horizon: Ligurian.

1854. Pictet. Traité de paléont., $2^{e}$ éd., ii : 351 (fide Serres MSS.).

3935. Bembicidioides inæquicollis. (Staphylinidæ.)

Locality: Prussian amber. Horizon: Ligurian.

1888. Schaufuss. Berl. ent. zeitschr., xxxii : 267-268.

3936. Bembidium (Carabidæ.)

Locality: Jarville, France. Horizon: Pleistocene.

1875. Fliche. Comptes rendus, $1 \times x x: 1234$.

3937. Bembidium —. (Carabidæ.)

Locality: Brunstatt, Alsatia. Horizon: Middle Oligocene.

1889. Foerster. Mitth. comm. geol. Elsass.-Lòthr., ii : 103.

3938. Bembidium absolutum. (Carabidæ.)

Locality: Radoboj, Croatia. Horizon: Mayencian.

1856. Giebel. Insect. d. vorwelt, 64 (refers to "Heer, Insectenfauna i, tf. 10, fig. 91," which does not exist, and moreover Heer describes no Bembidium from Radoboj, and Giebel's short description fits no Radoboj carabid figured by Heer).

3939. Bembidium assimile (recent). (Carabidæ.)

Locality: Hösbach, Bavaria. Horizon: Lower Pleistocene.

[1810. Gyllenhal. Ins, suec., ii : 26.]

1884. Flach.

1884. “
Käfer unterpleist. Hösbach, 7. Pl. 1, fig. 12.

Verhandl. physik.-med. gesellsch. Würzburg, n. f., xviii : 291. Pl. 8, fig. 12.

3940. Bembidium exoletum. (Carabidæ.)

Compared with B. inæquale

1876. Scudder. Bull. U. S. geol, surv, terr., ii : 77-78.

1877. " Bull. U. S. geol. surv. terr., iii: 759.

1890. “ Tert. ins. N. A., 530-531. Pl. 5, figs. 121, 122. 
3941. Bembidium fragmentum. (Carabidæ.)

Compared with B. constrictum Say.

Locality: Scarboro', Ontario. Horizon: Postpliocene.

1890. Scudder. Tert. ins. N. A., 531-532. Pl. 1, fig. 45.

3942. Bembidium glaciatum. (Carabidæ.)

Compared with B. longulum LeC.

Locality : Scarboro', Ontario. Horizon : Postpliocene.

1890. Scudder. Tert. ins. N. A., 531. Pl. 1, fig. 40.

3943. Bembidium infernum. (Carabidæ.)

Locality: Aix, France. Horizon: Ligurian.

1856. Heer. Viert. naturf. gesellsch. Zürich, i: $14 . \quad P l, 1, f i g .1$.

1874, Oustalet. Ann. sc. géol., v, art. 2: 98. Pl. 6, fig. 12.

3944. Bembidium nitidulum (recent). (Carabidæ.)

Locality: Jarville, France. Horizon : Pleistocene.

[1802. Marshall. Ent. brit., 454.]

1875. Fliche. Comptes rendus, Ixxx : 1234.

1886. Scudder. Bull. U. S. geol. surv., No. 31: 84 .

3945. Bembidium obtusum (recent). (Carabidæ.)

Locality: Jarville, France. Horizon: Plestocene.

[18:5. Sturm. 1875. Fliche. 1886. Scudder.

1874. Heer. 1874. Oustalet.

1856. Giebel.
Ins., vi : 165. $P l$. 161, figs. $c, C$. ]

Comptes rendus, lxxx : 1234.

Bull. U. S. geol. surv., No. 31: 84 .

3946. Bembidium saportanum. (Carabidæ.)

Locality: Aix, France. Horizon: Ligurian. MSS.

Ann. sc. géol., v, art. 2: 98-100. Pl. 1, fig. 7.

3947. Bembidium succini. (Carabidæ.)

Locality: Prussian amber. Horizon: Ligurian.

Insect. $d$. vorwelt, 64 .

3948. Berosus sexstriatus. (Hydrophilidæ.)

Compared with B. punctipennis Chevr. (MSS.), Mexico.

Locality: Green River, Wyo. Horizon: Oligocene.

1878. Scudder. Bull. U. S. geol. surv. terr., iv: 760-761.

1890. “- Tert. ins. N. A., 513-514. Pl. 7, fig. 40.

3949. Berosus tenuis. (Hydrophilidæ.)

Compared with B. cuspidatus Chevr., Mexico.

Locality: Green River, Wyo. Horizon : Oligocene.

1878. Seudder. Bull. U. S. geol. surv. terr., iv : 760 .

1890. " Tert. ins. N. A., 514. Pl. 8, fig. 8 .

3950. Bledius —. (Staphylinidæ.)

Locality : Prussian amber. Horizon: Ligurian.

1856. Menge. Progr. petrischule Danzig, 1856: 23. 


\section{Bledius adamus. (Staphylinidæ.)}

Compared with B. annularis LeC.

Locality: Green River, Wyo. Horizon: Oligocene.

1878. Scudder. Bull. U. S. geol. surv. terr., iv: 762.

1890. " Tert. ins. N. A., 504. Pl. 8, fig. 10.

3952. Bledius glaciatus. (Staphylinidæ.)

Compared with B. brevidens LeC.

Locality: Scarboro', Ontario. Horizon: Postpliocene.

1890. Scudder. Tert. ins. N. A., 505. Pl. 1, fig. 35.

3953. Bledius speciosus. (Staphylinidæ.)

Locality: Oeningen, Baden. Horizon: Tortonian.

1862. Heer. Verhandl. holl, maatsch. wetensch., xvi : 46-47. Pl. 3, fig. 2.

1865. " Urwelt der Schweiz, 38.3. Fig. 278, on p. 381.

1872. " " Monde prim. Suisse, 469. Fig. 278, on $p, 467$.

1876. " Prim. world Switz., ii : 40. Fig. 278 , on p. 38.

1879. " Urwelt der Schweiz, $2^{\mathrm{e}}$ aufl, 408. Fig. 320, on p. 407 .

3954. Bolboceras tertiarium. (Scarabæidæ.)

Locality: Kutschlin, Bohemia. Horizon : Miocene.

1881. Deichmüller. Foss. ins. diatomeensch. Kutschlin, 12-14. Pl. 21, figs. 2, $2 a b$.

1881. "6 Verhandl. leop.-carol. akad. naturf, xlii : 304-306. Pl. 21, figs. $2,2 a b$.

\section{Boletophagus —. See Bolitophagus $\longrightarrow$.}

\section{Bolitophagus ——. (Tenebrionidæ.)}

Locality : Prussian amber. Horizon : Ligurian.

1845. Berendt. Bernst. befindl. organ. reste vorw., i: 56 (Boletophagus).

3956. Bolitophagus vetustus. (Tenebrionidæ.)

Locality : Rott, Rhenish Prussia. Horizon: Aquitanian.

1866. Heyd.-Heyd. Palaeontogr., xv: 145. Pl. 22, fig. 23.

1866. " Käfer u. polypen, 15. Pl. 1, fig. 23.

\section{Bostrichus —. See Bostrychus — Hope, Menge.}

3957. Bostrychus ——. (Ptinidæ.)

Locality : Prussian amber. Horizon : Ligurian.

1832. Burmeister. Handb. ent., i: 635.

1836. " Man. ent., 577.

\section{Bostrychus - (Ptinidæ.)}

Locality: Aix, France. Horizon: Ligurian.

1847. Hope. Trans. ent. soc. Lond., iv: 251 (Bostrichus).

3959. Bostrychus - (larva). (Ptinidæ.)

Locality : Prussian amber. Horizon: Ligurian.

1856. Menge. Progr. petrischule Danzig, 1856: 23 (Bostrichus). 
3960. Bothrideres kunowi. (Colydiidæ.)

Compared with B. vittatus, Australia.

Locality: Prussian amber. Horizon: Ligurian.

1881. Stein. Berl. entom. zeitschr., xxv: 221.

3961. Bothrideres succinicola. (Colydiidæ.)

1881. Stein. Berl. entom, zeitschr., xxv: 221.

Brachinus primordialis. See Brachynus primordialis.

3962. Brachycerus ——. (Byrsopidæ.)

Compared with B. algirus.

Locality : Aix, France. Horizon: Ligurian.

1829. Serres. Géogn. terrains tert. 223.

3963. Brachycerus ——. (Byrsopidæ.)

Compared with B. hispanicus.

Locality : Aix, France. Horizon: Ligurian.

1829. Serres. Géogn. terrains tert., 223.

1874. Oustalet. Ann. sc. géol., v, art. 2: 211 (= Hipporhinus heeri q).

See also Hipporhinus heeri.

\section{Brachycerus —. (Byrsopidæ.)}

Compared with B. undatus.

Locality : Aix, France. Horizon : Ligurian.

1829. Serres. Géogn. terrains tert., 223, 272 (Brachyurus). $\quad P l .5$, fig. 8.

3965. Brachycerus exilis. (Byrsopidæ.)

Locality: Aix, France. Horizon: Ligurian.

1837. Germar. Fauna insect. Europæ, xix: 11. Pl. 11.

1856. Giebel. Insect. d. vorwelt, 137.

1874. Oustalet. Ann. se. géol., v, art. 2: 211 (= Phytom. annosus?).

See also Phytonomus annosus.

3966. Brachycerus germanus. (Byrsopidæ.)

Locality: Oeningen, Baden. Horizon: Tortonian.

1847. Heer. Insektenf. tertiärg. Oeningen, i: 180-182. Pl. 6, fig. 9.

1852. Giebel. Deutschl. petref., 649.

1856. " Insect. d. vorwelt, 136-137.

3967. Brachycerus lecoquii. (Byrsopidæ.)

Locality: Gergovia, France. Horizon : Aquitanian.

1870. Oustalet. Ann. se. g6́ol., ii, art. 3: 65. Pl. 1, fig. 4.

3968. Brachycerus nanus. (Byrsopidæ.)

Locality: Oeningen, Baden. Horizon: Tortonian.

1865. Heer.

1872. " "

1876. “

1879. “
Urwelt der Schweiz. Fig. 247, on p. 371.

Monde prim. Suisse. Fig. 247, on $p .455$.

Prim. world Switz., ii. Fig. 247, on p. 27.

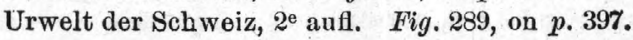


3969. Brachyderes aquisextanus. (Otiorhynchidæ.)

Locality: Aix, France. Horizon: Ligurian.

1874. Oustalet. Ann. sc. géol, v, art. 2: 242-245. Pl. 3, figs. 12, $12 a$.

3970. Brachyderes longipes. (Otiorhynchidæ.)

Locality : Aix, France. Horizon : Ligurian.

1874. Oustalet. Ann. sc. géol., v, art. 2: 235-242. Pl. 3, figs. 22, 23.

See also Hipporhinus longipes, Cleonus — Heer.

3971. Brachymycterus curculionoides. (Byrsopidæ.)

Locality: Rott, Rhenish Prussia. Horizon: Aquitanian.

1866. Heyd.-Heyd. Palaeontogr. xv : 148-149. Pl. 23, figs. 13-15.

1866. “ Käfer u. polypen, 18-19. Pl. 2, figs, 13-15.

1870. L. Heyden. Foss dipt. Rott, 29.

1870. " $\quad$ Palaeontogr, xvii : 265.

3972. Brachynus primordialis. (Carabidæ.)

Locality: Oeningen, Baden. Horizon: Tortonian.

1847. Heer. Insektenf. tertiärg. Oeningen, i: 16 (Brachinus). Pl. 7, fig. 18.

1852. Giebel. Deutschl. petref., 655 .

1856. " Insect. $d$ vorwelt, 70 .

3973. Brachytarsus pristinus. (Anthribidæ.)

Locality : Green River, Wyo. Horizon: Oligocene.

1876. Scudder. Bull. U.S. geol. surv. terr., ii: $8 \%$.

1878. " " Bull. U. S. geol. surv. terr., iv: 769.

1890. " Tert. ins. N. A., 466. Pl. 7, fig. 26.

Brachyurus —. See Brachycerus — Serres.

3974. Bruchus —_. (Bruchidæ.)

Locality: Brunstatt, Alsatia. Horizon: Middle Oligocene.

1889. Foerster. Mitth. comm. geol. Elsass-Lothr., ii : 102.

3975. Bruchus - (2 sp.). (Bruchidæ.)

Locality: Brunstatt, Alsatia. Horizon: Middle Oligocene.

1890. Foerster. In litt.

3976. Bruchus - (Bruchidæ.)

Locality: Aix, France. Horizon: Ligurian.

1829. Serres. Géogn. terrains tert., 222.

3977. Bruchus anilis. (Bruchidæ.)

Locality: White River, Colo. Horizon: Oligocene.

1876. Scudder. Bull. U. S. geol. surv. terr., ii : 82.

1877. " Bull. U. S. geol. surv. terr., iii: 759.

1890. "6 Tert. ins. N. A., 484. Pl.5, fig. 125.

3978. Bruchus bituminosus. (Bruchidæ.)

Locality: Arzburg, Germany. Horizon: Miocene?.

1837. Germar. Fauna insect. Europæ, xix : 10. Pl. 10.

1840. - - Verz. samml. Bayr., 71.

1856. Giebel. Insect. d. vorwelt, 133. 
3979. Bruchus decrepitus. (Bruchidæ.)

Locality: Sieblos, Rhenish Prussia. Horizon: Aquitanian.

1858. Heyden. Palaeontogr., v: 116. Pl. 23, fig. 13.

3980. Bruchus striolatus. (Bruchidæ.)

Locality: Oeningen, Baden. Horizon: Tortonian.

1847. Heer. Insektenf. tertiärg. Oeningen, i: 174-176. Pl. 6, fig. 5 .

1852. Giebel. Deutsehl. petref., 649.

1856. " Insect. d. vorwelt, 133-134.

1865. Heer. Urwelt der Schweiz, 374.

1872. "6 Monde prim. Suisse, 458.

1876. " $\quad$ Prim. world Switz., ii: 29.

1879. " Urwelt der Schweiz, $2^{\mathrm{e}}$ aufl, 399.

3981. Bryaxis - (Pselaphidæ.)

Locality : Prussian amber. Horizon: Ligurian.

1845. Berendt. Bernst. befindl, organ. reste vorw., i : 56 .

3982. Buprestis ——. (Buprestidæ.)

Locality: Lexden, England. Horizon: Pleistocene.

1888. Bell. Entom., xxi : 2 .

3983. Buprestis - (Buprestidæ.)

Locality: Rhine, Germany. Horizon: Aquitanian?.

1831. Goldfuss. Verhandl. leop.-carol. akad, naturf., vii, th. i: 118.

3984. Buprestis - (larva). (Buprestidæ.)

Locality : Prussian amber. Horizon: Ligurian.

1856. Menge. Progr. petrischule Danzig, 1856: 23.
3985. Buprestis
(Buprestidæ.)
Locality: Prussian amber. Horizon: Ligurian.

1838. Robert. Bull. soc. géol. France, ix : 114.
3986. Buprestis —_. (Buprestidæ.)
Compared with B. nana.

Locality : Aix, France. Horizon: Ligurian.

1829. Serres. Géogn. terrains tert., 221.

3987. Buprestis alutacea. (Buprestidæ.)

Compared with B. marianæ.

Locality: Vic. Bonn, Rhenish Prussia. Horizon: Aquitanian.

1837. Germar. Fauna insect. Europæ, xix: $3 . \quad P l .3$.

1852. Giebel. Dentschl. petref., 651.

1856. " Insect. d. vorwelt, 79.

See also Buprestites alutacea. 
3988. Buprestis carbonum. (Buprestidæ.)

Compared with B. mœsta.

Localities: Bonn, Baireuth, Rhenish Prussia. Horizon: Aquitanian.

1837. Germar.

Isis., 1837 : 423 (undescr.).

1837. " Fauna insect. Europæ, xix: 4. Pl. 4.

1840. - Verz. samml. Bayr., 71.

185̃2. Giebel. Dentschl. petref., 651.

See also Dicerca carbonum, Buprestites carbonum.

3989. Buprestis major. (Buprestidæ.)

Compared with B. cariosæ.

Locality: Vicinity Bonn, Rhenish Prussia. Horizon: Aquitanian.

1837. Germar. Fanna insect. Europæ, xix : 2. Pl. 2.

1847. Heer. Insektenf. tertiärg. Oeningen, i: 102.

1852. Giebel. Deutschl. petref., 651.

1856. " Insect. d. vorwelt, 78-79.

See also Buprestites major.

3990. Buprestis meyeri. (Buprestidæ.)

Locality: Sieblos, Rhenish Prussia. Horizon: Aquitanian.

1858. Heyden. Palaeontogr., v : 115. Pl. 23, fig. 11.

3991. Buprestis minnæ. (Buprestidæ.)

Locality : Schwarze Minna, vicinity Eisleben, Saxony. Horizon : 1856. Giebel. Insect. $d$. vorwelt, 79 .

See also Buprestites minnæ.

3992. Buprestis saxigena. (Buprestidæ.)

Locality : Nicola River, British Columbia. Horizon:

1879. Scudder. Rep. progr. geol. surv. Can., 1877-78: B 181.

1879. " Insect. tert. Nicola, 6.

1890. “ Tert. ins. N. A., 494-495. Pl. 2, figs. 24, 25.

3993. Buprestis senecta. (Buprestidæ.)

Locality: Sieblos, Rhenish Prussia. Horizon: Aquitanian.

1858. Heyden. Palaeontogr., v: 116. Pl. 23, fig. 12.

3994. Buprestis sepulta. (Buprestidæ.)

Locality : Nicola River, British Columbia. Horizon:

1879. Scudder. Rep. progr. geol. surv. Can., 1877-'78: B 181.

1879. " Insect. tert. Nicola, 6.

1890. " Tert. ins. N. A., 495. Pl. 2, fig. 26.

3995. Buprestis tertiaria. (Buprestidæ.)

Locality : Nicola River, British Columbia. Horizon :

1879. Scudder. Rep. progr. geol. surv. Can., 1877-'78: B 180-181.

1879. " " Insect. tert. Nicola, $5-6$.

1890. “ Tert. ins. N. A., 493-494. Pl. 2, fig. 23.

3996. Buprestis tradita. (Buprestidæ.)

Locality: Rott, Rhenish Prussia. Horizon: Aquitanian, 1859. Heyden, Palaeontogr,, viii: 3. Pl. 2, fig. 9. 
3997. Buprestis xylographica. (Buprestidæ.)

Locality: Stösschen, vicinity Linz, Austria. Horizon: Miocene. 1849. Germar. Zeitschr. deutsch. geol. gesellsch., i: 55-57. Pl. 2, fig. 1. 1852. Giebel. Deutschl. petref., 651.

See also Chrysobothris xylographica, Buprestites xylographica.

3998. Buprestites agriloides (1). (Buprestidæ.)

Distinct from the next.

Locality: Oeningen, Baden. Horizon: Tortonian.

1862. Heer. Verhandl. holl. maatsch. wetensch., xvi: 90. Pl. 7, fig. 24.

3999. Buprestites agriloides (2). (Buprestidæ.)

Distinct from the preceding.

Locality : Haseninsel, Greenland. Horizon : -

1883. Heer. Flora foss. grönl., ii : 144. Pl. 109, figs. 11, $11 b$.

4000. Buprestites alutacea. (Buprestidæ.)

Locality : Bonn, Rhenish Prussia. Horizon: Aquitanian.

1852. Giebel. Deutschl. petref., 651.

See also Buprestis alutacea.

4001. Buprestites carbonum. (Buprestidæ.)

Locality: Bonn, Rhenish Prussia. Horizon : Aquitanian.

1852. Giebel. Deutschl. petref., 651.

See also Buprestis carbonum, etc.

4002. Buprestites debilis. (Buprestidæ.)

Locality: Sieblos, Rhenish Prussia. Horizon: Aquitanian.

1860. Heer. Hassencamp, Würzb. naturwiss. zeitschr, i : 79 (undescr.).

4003. Buprestites exstinctus. (Buprestidæ.)

Locality : (Oeningen?, Baden). Horizon: Tortonian.

1847. Heer. Insektenf. tertiärg. Oeningen, i: 129-130 (exstincta). Pl. 3, fig。 13.

1852. Giebel. Deutschl. petref., 651 (extincta).

1856. " Insect. d. vorwelt, 79.

Buprestites extincta. See Buprestites exstinctus.

4004. Buprestites falconeri. (Buprestidæ.)

Locality: Bovey Tracey, Devonshire, England. Horizon : Miocene.

1862. Heer. Phil. trans., clii : 1082. Pl. 68, fig. 21.

4005. Buprestites major. (Buprestidæ.)

Locality: Bonn, Rhenish Prussia. Horizon: Aquitanian.

1852. Giebel.

Deutschl. petref., 651 .

See also Buprestis major.

4006. Buprestites minnæ. (Buprestidæ.)

Locality: Vicinity Eisleben, Saxony. Horizon: -

1856. Giebel. Zeitschr. ges, naturwiss., vii: 384-385. Pl. 5, fig. 1.

Șeo also Buprestis minnæ. 
4007. Buprestites œningensis. (Buprestidœ.)

Locality: Oeningen, Baden. Horizon: Tortonian.

1847. Heer. 1852. Giebel. Insektenf. tertiärg. Oeningen, i: 128-129. $\quad \mathrm{Pl}$. 2, fig. 17.

1856. “

Deutschl. petref., 651.

Insect. d. vorwelt, 79.

4008. Buprestites xylographicus. (Buprestidæ.)

Locality : Linz, Austria. Horizon: Miocene.

1852. Giebel.

Deutschl. petref., 651 (xylographica).

See also Buprestis xylographica, etc.

4009. Byrrhus —. (Byrrhidæ.)

Locality: Mundesley, Eugland. Horizon: Pleistocene.

1888. Bell. Entom., xxi: 2.

4010. Byrrhus - (Byrrhidæ.)

Locality: Prussian amber. Horizon: Ligurian.

1845. Berendt. Bernst. befindl, organ. reste vorw., i: 56.

4011. Byrrhus exanimatus. (Byrrhidæ.)

Locality : Rott, Rhenish Prussia. Horizon: Aquitanian.

1866. Heyd.-Heyd. Palaeontogr., xv: 139-140. Pl. 24, fig. 22.

1866. " Käfer u. polypen, 9-10. Pl.3, fig. 22.

4012. Byrrhus lucæ. (Byrrhidæ.)

Loeality: Rott, Rhenish Prussia. Horizon: Aquitanian.

1859. Heyden. Palaeontogr., viii: 3. Pl. 1, fig. 7.

4013. Byrrhus œningensis. (Byrrhidæ.)

Locality: Oeningen, Baden. Horizon: Tortonian.

1847. Heer. Insektenf. tertiärg. Oeningen, i: 44-46. Pl. 2, figs. 5,9,9b-d.

1852. Giebel. Deutschl. petref., 653.

1856. " Insect. d. vorwelt, 49.

1862. Heer. Verdandl. holl. maatsch. wetensch., xvi : 57. Pl. 3, fig. 25.

See also Byrrhus pauper.

4014. Byrrhus pauper. (Byrrhidæ.)

Locality: Oeningen, Baden. Horizon: Tortonian.

1851. Stizenberger. Uebers. verstein. Baden, 101 (as of Heer. Name only; by mistake for B. œningensis ?).

See also Byrrhus œningensis.

4015. Bythinus —. (Pselaphidæ.)

Locality : Prussian amber. Horizon: Ligurian.

1856. Menge. Progr. petrischule Danzig, 1856: 22.

Calænius punctulatus. See Chlænius punctulatus.

4016. Calandra -. (Calandridæ.)

Locality: Aix, France. Horizon: Ligurian.

1854. Pictet. Traité de paléont., 2 éd., ii : 351 (fide Serres MSS.).

Calasposoma _. See Colasposoma 


\section{Calathus -. (Carabidæ.)}

Locality: Prussian amber. Horizon: Ligurian.

1845. Berendt. Bernst. befindl, organ. reste vorw., i: 56.

4018. Callidium -. (Cerambycidæ.)

Locality : Prussian amber. Horizon: Ligurian.

1845. Berendt. Bernst. befindl, organ, reste vorw., i : 56 .

4019. Callidium -. (Cerambycidæe.)

Compared with C. fennicum.

Locality: Utznach, Switzerland. Horizon: Pleistocene?.

1039. Brull6. Gisem. ins. foss., 20.

4020. Callidium - (Cerambycidæ.)

Compared with C. abdominale.

Locality: Aix, France. Horizon: Ligurian.

1829. Serres. Géogn. terrains tert., 225.

4021. Callidium escheri. (Cerambycidæ.)

Compared with C. strepens.

Locality : Oeningen, Baden. Horizon : Tortonian.

1865. Heer.

1872. "

1876. “6

1879. “
1865. Heer.

1872. “

1876. " "

1879. “
Urwelt der Schweiz, 375. Fig. 253, on p. 376.

Monde prim. Suisse, 460. Fig. 253, on p. 461.

Prim. world Switz., ii : 31. Fig. 253, on p. 32.

Urwelt der Schweiz, $2^{\circ}$ anfl, 401. Fig. 295, on p. 402.

4022. Callidium procerum. (Cerambycidæ.)

Compared with C. strepens.

Locality : Oeningen, Badeu. Horizon : Tortonian.

Urwelt der Schweiz, 375.

Monde prim. Suisse, 460.

Prim. world Switz., ii : 31.

Urwelt der Schweiz, 2e aufl., 401.

Callisthenes agassizii. See Calosoma (Callisthenes) agassizii.

4023. Calosoma agassizii. (Carabidæ.)

Locality : Aix, France. Horizon : Ligurian.

1874. Oustalet. La nature, iii: 33. Fig.

See also Calosoma (Callisthenes) agassizii, etc.

4024. Calosoma (Callisthenes) agassizii. (Carabidæ.)

Locality: Aix, France. Horizon: Ligurian.

1874. Oustalet. Ann. sc. g6́ol., v, art. 2: 84-92 (Agassiz). Pl. 1, figs. 2, $2 a b c$.

See also Carabus agassizi, Calosoma saportanum, Calosoma agassizii.

4025. Calosoma caraboides. (Carabidæ.)

Compared with C. longipenne Dej., South America.

Localities: Locle, Switzerland; Oeningen, Baden. Horizon: Tortonian.

1860. Heer. Foss. calosomen, 7-8. Pl., fig. 7.

1865. " Urwelt der Sehweiz, 384, 385.

1872. " Monde prim. Suisse, 471.

1876. " $\quad$ Prim. world Switz., ii : $41,42$.

1879. " Urwelt der Schweiz, $2^{\mathrm{e}}$ aufl., 410 , 
4026. Calosoma catenulatum. (Carabidæ.)

Compared with C. sayi and C. rugosum Dej., America.

Localities: Bonn, Rhenish Prussia; Oeningen, Baden. Horizons: Aquitanian, Tortonian.

1860. Heer.

1865. “

1872. “

1876. “

1879. “

1860. Heer.

1860. Heer.

1860. Heer.

1860. Heer.

1865. “

1872. “

1876. “

1879. “
1860. Heer.

1862. "

1865. "

1872. "

1876. " "

1879. “
Foss. calosomen, 4-5. Pl., fig. 3.

Urwelt der Schweiz, 384.

Monde prim. Suisse, $4 \pi 1$.

Prim. world Switz., ii : 41.

Urwelt der Schweiz, $2^{e}$ aufl, 410.

4027. Calosoma deplanatum. (Carabidæ.)

Locality : Oeningen, Baden. Horizon: Tortonian. Foss. calosomen, 6. Pl., fig. 6.

4028. Calosoma escheri. (Carabidæ.)

Locality: Oeningen, Baden. Horizon: Tortonian.

Foss. calosomen, 7. Pl., fig. 5.

4029. Calosoma escrobiculatum. (Carabidæ.)

Compared with C. brunneum, Peru.

Locality: Oeningen, Baden. Horizon: Tortonian.

Foss. calosomen, 6-7. Pl., figs. $4 a b$.

4030. Calosoma jaccardi. (Carabidæ.)

Compared with C. inquisitor F., Europe.

Locality: Locle, Switzerland. Horizon: Miocene.

Foss. calosomen, 4. Pl., fig. 2.

Urwelt der Schweiz, 384.

Monde prim. Suisse, 471.

Prim. world Switz., ii: 41.

Urwelt der Schweiz, $2^{\mathrm{e}}$ aufl, 410.

4031. Calosoma nauckianum. (Carabidæ.)

Compared with C. maderæ F., Madeira.

Locality : Oeningen, Baden. Horizon: Tortonian.

Foss. calosomen, 5-6. Pl., fig. 3.

Verhandl. holl. maatsch. wetensch., xvi: 17-18. $\quad P l .1$, fig. 4.

Urwelt der Schweiz, 384. Fig. 283.

Monde prim. Suisse, 471 (naukianum). Fig. 283

Prim. world Switz., ii : 41. Fig. 283, on p. 42.

Urwelt der Schweiz, $2^{\mathrm{e}}$ aufl, 410. Fig. 325.

Calosoma naukianum. See Calosoma nauckianum.

4032. Calosoma saportanum. (Carabidæ.)

Locality : Aix, France. Horizon : Ligurian.

1874. Heer. MSS. in Oustalet, Ann. se. géol., v, art. 2: 84, 91-92. (=C. agassizii fide Oust.) $P l .1$, fig. $2 b$.

See also Calosoma (Callisthenes) agassizii, etc. 
4033. Campsosternus atavus. (Elateridæ.)

Compared with C. gemma and C. fulgens.

Locality : Kutschlin, Bohemia. Horizon: Miocene.

1881. Deichmïller. Foss. ins. diatomeensch. Kutschlin, 14-16. Pl. 21, figs. 4a-c.

1881. " Verhandl. leop.-carol. acad. naturf., xlii : 306-308. Pl. 21, figs. $4 a-c$.

4034. Cantharis -. (Meloidæ.)

Locality: Prussian amber. Horizon: Ligurian.

1845. Berendt. Bernst. befindl, organ. reste vorw., i: 56 .

4035. Cantharis - (Meloidæ.)

Compared with C. nigricans Fabr.

Locality: Prussian amber. Horizon: Ligurian.

1832. Burmeister. Handb. ent., i : 635.

1836. " Man. ent., 577.

4036. Cantharis -. (Meloidæ.)

Locality: Rhine, Germany. Horizon: Aquitanian?.

1831. Goldfuss. Verhandl. leop.-carol. akad. naturf., vii, i: 118.

Capnodis abdominalis. See Capnodis puncticollis-abdominalis.

4037. Capnodis antiqua. (Buprestidæ.)

Compared with C. cariosa Pall.

Locality: Oeningen, Baden. Horizon: Tortonian.

1847. Heer. Insektenf. tertiärg. Oeningen, i: 95-102, 221. Pl. 2, fig. 18; pl. 3 , fig. 1 .

1852. Giebel. Deutschl. petref., 652.

1854. Pictet. Traité de paléont., $2^{\circ}$ éd., ii : 331. Pl. 40, fig. 14.

1856. Giebel. Insect. d. vorwelt, 81.

1862. Heer.

1865. "

187\%. "

1876. 66

1879. " "

Verhandl. holl. maatsch. wetenseh., xvi: 83. Pl. 7, figs. 17, 18.

Urwelt der Schweiz, 377. Fig. 260, on p. 378.

Monde prim. Suisse, 463. Fig. 260.

Prim. world Switz., ij : 33. Fig. 260, on p. 34.

See also Capnodis antiqua var. minor.

4038. Capnodis antiqua var. minor. (Buprestidæ.)

Locality: Oeningen, Baden. Horizon: Tortonian.

1847. Heer. Insektenkf. tertiärg. Oeningen, i: 100-101. Pl. 3, fig. 1 b.

See also Capnodis antiqua.

Capnodis compressa. See Capnodis puncticollis-compressa.

Capnodis minor. See Capnodis antiqua var. minor.

4039. Capnodis puncticollis. (Buprestidæ.)

Locaīity: Oeningen, Baden. Horizon : Tortonian.

1847. Heer.

Insektenf. tertiärg. Oeningen, i: 102-104. Pl. 3, fig. 3.

1852. Giebel.

Deutschl. petref., 652 .

1856. " Insect. d. vorwelt, 81-82.

1862. Heer. Verhandl. holl. maatsch, wetensch., xvi : 84. Pl. 7, figs. 15, 16.

See also Capnodis puncticollis-abdominalis, C. puncticollis compressa. 
4040. Capnodis puncticollis-abdominalis. (Buprestidæ.)

Locality: (Oeningen, Baden.) Horizon: Tortonian.

1847. Heer. Insektenf. tertiärg. Oeningen, i: 104-105. Pl. 3, fig. 3c.

See also Capnodis puncticollis, etc.

4041. Capnodis puncticollis-compressa. (Buprestidæ.)

Locality : (Oeningen, Baden.) Horizon: Tortonian.

1847. Heer. Insektenf. tertiärg. Oeningen, i: $104 . \quad P l .3, f i g .3 b$.

See also Capnodis puncticollis, ete.

4042. Capnodis spectabilis. (Buprestidæ.)

Compared with C. cariosa Pall.

Locality: (Oeningen, Baden.) Horizon: Tortonian.

1862. Heer. Verhandl. holl. maatsch. wetensch., xvi: 83-84. Pl. 7, figs. 12-14

1865. “

1872. “

1876. “

1879. “

Urwelt der Schweiz, 377. Fig. 261, on $p .378$.

Monde prim. Suisse, 463. Fig. 261.

Prim. world Switz., ii : 34. Fig. 261.

Urwelt der Schweiz, $2^{\mathrm{e}}$ aufl., $403 . \quad$ Fig. 303, on p. 404.

Caprologus gracilis. See Coprologus grácilis.

4043. Carabites cordicollis. (Carabidæ.)

Locality: Dürnten, Switzerland. Horizon: Interglacial.

1865. Heer. Urwelt der Schweiz, 502. Fig. 359, on p. 501.

1872. "

1876. “

Monde prim. Suisse, 615-616. Fig. 359, on $p .614$.

1879. “

Prim. world Switz., ii : 169. Fig. 359, on p. 168.

Urwelt der Schweiz, $2^{\mathrm{e}}$ aufl., 532. Fig. 402, ou p. 531.

4044. Carabites diluvianus. (Carabidæ.)

Locality: Dürnten, Switzerland. Horizon: Interglacial.

1865. Heer. Urwelt der Schweiz, 502. Figs. $357 a-c$, on p. 501.

1872. " Monde prim. Suisse, 615-616. Figs. $357 a-c$, on p. 614.

1876. " $\quad$ Prim. world Switz., ii : 169. Figs. 357a-c, on p. 168.

1879. “ Urwelt der Schweiz, $2^{e}$ aufl., 532. Fig. 400, on p. 531.

See also Carabites (Harpalus?) diluvianus.

4045. Carabites (Harpalus ?) diluvianus. (Carabidæ.)

Locality : Dürnten, Switzerland. Horizon: Interglacial.

1865. Heer. Urwelt der Schweiz, expl. fig. 357, on p. 501.

1872. " Monde prim. Suisse, expl. fig. 357, on p. 614.

1876. "6 Prim. world Switz., ii : expl. fig. 357 , on p. 168.

See also Carabites diluvianus.

4046. Carabites exilis. (Carabidæ.)

Locality: Oeningen, Baden. Horizon: Tortonian.

1862. Heer. - Verhandl. holl. maatsch. wetensch., xvi: 35. Pl. 1, fig.28.

4047. Carabites feildenianus. (Carabidæ.)

Locality: Watercourse Ravine, near Discovery Harbor, Grinnell Land.

Horizon: Miocene.

1878. Heer. Quart. journ. geol. soc. Lond, xxxiv: 69.

1878. " Miocene flora Grinnell-landes, 38. Pl. 9, figs. 11, 11 .

1878. " Flora foss, arct., $v$, i : 38, Pl. 9, figs. 11, 11 . 
4048. Carabites hyperboreus. (Carabidæ.)

Locality: Cap Staratschin, Spitzbergen. Horizon: Miocene

1870. Heer.

K. svenska vetensk.-akad. handl., viii, No. 7: 73. Pl. 16, figs. 34-35.

4049. Carabites islandicus. (Carabidæ.)

Locality: Iceland. Horizon: Miocene.

1868. Heer.

Flora foss. arct., i: 154. $P l .27$, figs. 21, $21 b$.

4050. Carabites læsicollis. (Carabidæ.)

Locality: Oeningen, Baden. Horizon: Tortonian.

1862. Heer. Verhandl. holl maatsch. wetensch., xvi: 35. Pl. 1, fig. 27.

4051. Carabites nitens. (Carabidæ.)

Locality: Cap Staratschin, Spitzbergen. Horizon: Miocene.

1870. Heer.

K. svenska vetensk.-akad. handl., viii, No. 7: 73. Pl. 16, figs. 19, 20, 20bc.

4052. Carabus -. (Carabidæ.)

Locality : Prussian amber. Horizon: Ligurian.

1835. Gravenhorst. Uebers. schles. gesellsch. vaterl. cult., 1834: 92.

4053. Carabus -_. (Carabidæ.)

Locality : Glücksbrunn, Hesse. Horizon: Braunkohl.

1820. Schlotheim. Petrefactenk., 44.

4054. Carabus agassizi. (Carabidæ.)

Locality: Aix, France. Horizon: Ligurian.

1850. Barthélemy. Car. d'Agass., 1-4.

1851. " " Guór., Rev. et mag. zool., (2), iii : 203.

1856. Giebel. Insect. d. vorwelt, 61.

Sep also Calosoma (Callisthenes) agassizi, etc.

4055. Carabus arvensis (recent). (Carabidæ.)

Locality : Schwerzenbach, Switzerland. Horizon: Glacial clay.

[1787. Fabricius. Mant. ins., i: 196.]

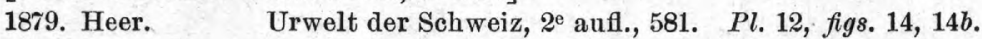

1886. Scudder. Bull, U.S. geol. surv., No. 31: 85 .

4056. Carabus nitens. (Carabidæ.)

Locality: Garvel Park, Clyde, England. Horizon: Pleistocene.

1888. Bell. Entom., xxi : 2 (Carbera).

4057. Carabus novalensis. (Carabidæ.)

Locality : Novale, Italy. Horizon: -

1886. Omboni. Alc. foss. ins. Ven., 10-11. Pl. 3, fig. 15.

1886. " Atti r. ist. ven., (6), iv: 1430-1431. Pl. 3, fig. 15.

4058. Carabus thürachii. (Carabidæ.)

Locality: Hösbach, Bavaria. Horizon: Lower Pleistocene.

1884. Ylach. Käfer unterpleist. Hösbach, 3. Pl. 1, fig. 1.

1884. " Verhandl. physik.-med. gesellsch. Würzb., n. f., xviii : 287. $P l_{\text {. }}$ 8, fig. 1.

Carbera nitens. See Carabus nitens. 
4059. Cardiophorus -. (Elateridæ.)

Locality : Prussian amber. Horizon: Ligurian. 1868. Smith. Quart. journ. sc., v: 184. Pl., fig. 6.

4060. Cardiophorus braunii. (Elateridæ.)

Locality : Oeningen, Baden. Horizon: Tortonian.

1847. Heer. 1852. Giebel. Insektenf. tertiärg. Oeningen, i: 134-135, Pl. 4, fig. 4. Deutschl. petref., 651.

1856. " Insect. d. vorwelt, 97 .

4061. Caryoborus ruinosus. (Bruchidæ.)

Locality : Rott, Rhenish Prussia. Horizon: Aquitanian. 1859. Heyden. Palaeontogr., viii: 8-9. Pl. 2, fig. 1.

4062. Caryoborus striolatus. (Bruchidæ.)

Locality : Oeningen, Baden. Horizon: Tortonian.

1861. Heer. Rech. climat pays tert., 203 (undescr.).

4063. Cassida -. (Chrysomelidæ.)

Compared with C. equestris Fabr.

Locality: Aix, France. Horizon: Ligurian.

1829. Curtis. Edinb. new phil. journ. vii : 295.

1829. " Murchison-Lyell, Tert. form. Aix, 11.

4064. Cassida -. (Chrysomelidæ.)

Compared with C. viridis Fabr.

Locality: Aix, France. Horizon: Ligurian.

1829: Curtis. Edinb. new phil. journ., vii : 295.

1829. " Murchison-Lyell, Tert. form. Aix, 11.

4065. Cassida -. (Chrysomelidæ.)

Locality : Zimmersheim, Alsatia. Horizon : Middle Oligocene.

1888. Foerster. Mitth. comm. geol. Elsass-Lothr., i: 150, 165.

1889. " Mitth. comm. geol. Elsass-Lothr., ii : 102.

4066. Cassida - (3 sp.). (Chrysomelidæ.)

Locality: Brunstatt, Alsatia. Horizon : Middle Oligocene.

1888. Foerster. Mitth. comm. geol. Elsass-Lothr, i: 165.

1889. “ Mitth. comm. geol. Elsass-Lothr., ii : 102.

4067. Cassida -. (Chrysomelidæ.)

Locality: Aix, France. Horizon: Ligurian.

1847. Hope. Trans, ent. soc. Lond., iv : 251.

4068. Cassida - (Chrysomelidæ.)

Locality : Prussian amber. Horizon : Ligurian.

1856. Menge. Progr. petrischule Danzig, 1856: 21 . 


\section{Cassida -. (Chrysomelidæ.)}

Compared with C. meridionalis.

Locality : Aix, France. Horizon : Ligurian.

1829. Serres. Géogn. terrains tert., 225.

See also Chrysomela — Burm.

4070. Cassida - (Chrysomen1dæ.)

Locality: Lexden, England. : Horizon: Peat beds.

1863. Wollaston. Quart. journ. geol. soc. Lond., xix, i: 401, No. 4.

1888. Bell. Entom., xxi : 2.

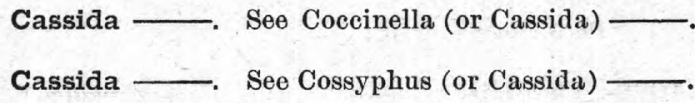

4071. Cassida blancheti. (Chrysomelidæ.)

Compared with C. thoracica Kug.

Localities: Oeningen, Baden; Aix, France. Horizons: Tortonian, Ligurian.

1856. Heer.

1865. ".

1872. " "

1874. Oustalet.

1876. Heer.

1879. “
Viert. naturf. gesellsch. Zürich, i: 25-26. Pl. 1, fig. 17.

Urwelt der Sehweiz, 372. Fig. 239, on p. 371.

Monde prim. Suisse, 456. Fig. 239 , on $p .455$.

Ann. sc. géol., v, art. 2: 340-344. Pl. 4, fig. 15.

Prim. world Switz., ii : 27. Fig. 239.

Urwelt der Schweiz, 2e aufl., 398. Fig. 281, on p. 397.

4072. Cassida hermione. (Chrysomelidæ.)

Compared with C. murræa F.

Locality: Oeningen, Baden. Horizon: Tortonian.

1847. Heer.

1852. Giebel.

Insektenf. tertiärg. Oeningen, i : 205-206. Pl. 7, fig. 6.

1856.

Deutschl. petref., 648 .

1865. Heer.

Insect. d. vorwelt, 114.

1872. " "

Urwelt der Schweiz, 372.

1876. " "

Monde prim. Suisse, 456.

Prim. world Switz., ii : 27.

1879. “6

Urwelt der Schweiz, $2^{\mathrm{e}}$ aufl., 398.

4073. Cassida interemta. (Chrysomelidæ.)

Locality : Rott, Rhenish Prussia. Horizon: Aquitanian.

1862. Heyden. Palaeontogr., x: 74. Pl. 10, fig. 16.

4074. Cassida megapenthes. (Chrysomelidæ.)

Locality: (Oeningen?, Baden). Horizon: Tortonian.

1847. Heer. Insektenf. tertiärg. Oeningen, i: 206-207. Pl. 8, fig. 10.

1852. Giebel. Dentschl. petref., 648.

1856. " Insect. d. vorwelt, 114.

4075. Catops —. (Silphidæ.)

Locality: Prussian amber. Horizon: Ligurian.

1845. Berendt. Bernst. befindl, organ. reste vorw., i: 56. 
4076. Cerambycites Locality: Brunstatt, Alsatia.
(Cerambycidæ.)

Horizon : Middle Oligocene.

1890. Foerster. In litt.

4077. Cerambyx —. (Cerambycidæ.)

Locality : Prussian amber. Horizon: Ligurian.

1830. Berendt. Insekten im bernstein, 30 (Cerambix).

4078. Cerambyx - (Cerambycidæ.)

Locality : Copal. Horizon: Recent.

1776. Bloch. Beschäft. berl. gesellsch. naturf. freunde, ii : 179-180 (Cerambix). Pl. 5, fig. 21.

4079. Cerambyx - (leg). (Cerambycidæ.)

Locality: Prussian amber. Horizon: Ligurian.

1831. Burmeister. Oken, Isis, 18:31: 1100.

4080. Cerambyx —. (Cerambycidæ.)

Locality: Rhine, Germany. Horizon: Aquitanian?.

1831. Goldfuss. Verhandl. leop.-carol. akad. naturf, vii, i : 118.

4081. Cerambyx - (Cerambycidæ.)

Locality: Oeningen, Baden. Horizon: Tortonian.

1834. Keferstein. Naturg. erdkörp., ii : 328.

4082. Cerambyx - (larva). (Cerambycidæ.)

Locality: Prussian amber. Horizon: Ligurian.

1856. Menge. Progr. petrischule Danzig, 1856: 23.

4083. Cerambyx - (Cerambycidæ.)

Locality: Aix, France. Horizon: Ligurian.

1854. Pictet. Traité de paléont., $2^{e}$ éd., ii : 355 (fide Serres MSS.).

4084. Cercyon ㄴ. (Hydrophilidæ.)

Locality: Hösbach, Bavaria. Horizon: Lower Pleistocene.

1884. Flach. Käfer unterpleist. Hösbach, 9. Pl. 2, fig. 3.

1884. " Verhandl. physik,-med. gesellsch. Würzb., n, f., xviii : 293. $\quad P l$. 9, fig. 3.

4085. Cercyon? terrigena. (Hјdrophilidæ.)

Locality: Nicola River, British Columbia. Horizon: -

1879. Scudder. Rept. progr. geol. surv, Can., 1877-'78: B 178.

1879. " Ins. tert. Nicola, 4.

1890. “ Tert. ins. N. A., 510-511. Pl. 2, fig. 21.

4086. Cetonia - (Scarabæidæ.)

Compared with C. hirtellus.

Locality : Aix, France. Horizon: Ligurian.

1829. Curtis. Edinb. new phil. journ., vii : 295.

1829. " Murchison-Lyell, Tert. form. Aix, 11. 


\section{Cetonia - (Scarabæidæ.)}

Compared with C. stictica Fabr.

\section{Locality: Aix, France. Horizon: Ligurian.}

1829. Curtis. Edinb. new phil. journ., vii : 295.

1829. " Murchison-Lyell, Tert. form. Aix, 11.

4088. Ceutorhynohus _ (3 sp.). (Curculionidæ.)

Locality: Brunstatt, Alsatia. Horizon: Middle Oligocene.

1888. Foerster. Mitth. comm. geol. Elsass-Lothr., i: 165.

1889. " Mitth. comm. geol. Elsass-Lothr., ii: 102.

4089. Ceutorhynchus funeratus. (Curculionidæ.)

Locality: Rott, Rhenish Prussia. Horizon: Aquitanian.

1866. Heyd.-Heyd. Palaeontogr., xv: 153. Pl. 24, fig. 7.

1866. 6 Käfer u. polypen, 23. Pl. 3, fig. 7.

4090. Chalcodermus kirschi. (Curenlionidæ.)

Compared with C. ebeninus.

Locality: Kutschlin, Bohemia. Horizon: Miocene.

1881. Deichmüller. Foss. ins. diatomeensch. Kutschlin, 24-26. Pl. 21, figs. 9,9a-c, $10,11$.

1881. “ Verhandl. leop.-carol. akad. naturf, xlii : 316-318. Pl. 21, figs. $9,9 a-c, 10,11$.

4091. Chalcophora lævigata. (Buprestidæ.)

Compared with C. fabricii Rossi,

Locality: Oeningen, Baden. Horizon: Tortonian.

1862. Heer. Verhandl. holl. maatsch. wetensch. Haarl., xvi : 85-86. Pl. 7, figs. 2-9.

1865. " "Urwelt der Schweiz, 362,378. Fig. 259.

187\%. “

1876. "

1879. 66

Monde prim. Suisse, 444, 464. Fig. 259 on p. 463.

Prim. world Switz., ii : 16,35. Fig. 259 on $p .34$.

Urwelt der Schweiz, $2^{\mathrm{e}}$ anfl., 387, 403. Fig. 301 on p. 404.

4092. Chalcophora pulchella. (Buprestidæ.)

Locality: Oeningen, Baden. Horizon: Tortonian.

1862. Heer. Verhandl. holl. maatsch. wetensch. Haarl, xvi : 86-87. Pl. 7, figs. 10, 11.

4093. Chauliognathus pristinus. (Lampýridæ.)

Locality: Florissant, Colo. Horizon: Oligocene.

1876. Scudder.

Bull. U. S. geol. surv. terr., ii : 81.

1885.

Zittel, Handb. palaeont., i, th. ii: 796. Fig. 1032.

1886. " Zittel-Barrois, Traité de paléont., ii: 796. Fig. 1050.

4094. Chilocorus - (2 sp.). (Coccinellidæ.)

Locality : Brunstatt, Alsatia. Horizon : Middle Oligocene.

1890. Foerster. In litt. 
4095. Chlænius (Carabidæ.)

Locality : Prussian amber. Horizon: Ligurian.

1845. Berendt. Bernst. befindl. organ. reste vorw., i: 56.

4096. Chlænius dietzii. (Carabidæ.)

Locality : Hösbach, Bavaria. Horizon: Lower Pleistocene.

1884. Flach. Käfer unterpleist. Hösbach, 4. Pl. 1, fig. 4.

1884. " "

Verhandl. physik.-med. gesellsch. Würzb.,n.f., xviii: 288. $\quad$ Pl. 8, fig. 4.

4097. Chlænius electrinus. (Carabidæ.)

Compared with C. vestitus.

Locality: Prussian amber. Horizon: Ligurian.

1862. Giebel. Zeitschr. ges. naturwiss., xx: 320 .

4098. Chlænius punctulatus. (Carabidæ.)

Compared with C. laticollis, United States.

Locality : Port Kennedy, Pa. Horizon: Postpliocene.

1876. Horn. Trans. Amer, ent. soc., v : 244.

1889. Lesley. Dict. foss. Penns., i: p. xiii (Calænius).

1890. Seudder. Tert. ins. N. A., 517-518. Pl. 1, fig. 7.

4099. Chlænius quadrisulcatus (recent). (Carabidæ.)

Locality : Hösbach, Bavaria. Horizon : Lower Pleistocene.

[1798. Illiger.

1884. Flach.

1884. "
Käfer preuss., i: 176.]

Käfer unterpleist. Hösbach, 4. Pl. 1, figs. $3 a, 3 b$.

Verhandl, physik.-med. gesellsch. Würzb., n. f., xviii : 288. $\quad P l$. 8, figs. $3 a, 3 b$.

4100. Chœridium? ebeninum. (Scarabæidæ.)

Compared with C. histeroides, United States. Locality: Port Kennedy, Pa. Horizon: Postpliocene.

1876. Horn.

Trans. Amer. ent. soc., v : 244-245.

1889. Lesley.

Dict. foss. Penns., i : p. xiii.

1890. Scudder.

Tert. ins. N. A., 490-491. Pl. 1, figs. 18-22.

4101. Choragus fictilis. (Anthribidæ.)

Locality: Green River, Wyo. Horizon: Oligocene.

1890. Scudder. Tert. ins. N. A., 465-466. Pl. 8, fig. 9.

4102. Choragus tertiarius. (Anthribidæ.)

Locality : Rott, Rhenish Prussia. Horizon : Aqnitanian。

1866. Heyd.-Heyd. Palaeontogr., xv: 147. Pl. 23, fig. 8.

1866. " Käfer u. polypen, 17. Pl. 2, fig. 8.

4103. Chrysobothris haydeni. (Buprestidæ.)

Locality: Florissant, Colo. Horizon: Oligocene.

1876. Seudder.

Bull. U. S. geol. surv. terr., ii : 80 . 
4104. Chrysobothris xylographica. (Buprestidæ.)

Locality: Stösschen, vicinity Linz, Austria. Horizon: Miocene.

1856. Giebel.

Insect. d. vorwelt, 87.

See also Buprestis xylographica, etc.

4105. Chrysomela —- (Chrysomelidæ.)

Locality : Prussian amber. Horizon: Ligurian.

1845. Berendt. Bernst, befindl, organ. reste vorw., i: 56.

4106. Chrysomela —- (Chrysomelidæ.)

Locality : Prussian amber. Horizon : Ligurian.

1827. Brongniart. Dict. sc. nat., li : 233 (Chrysomèles).

1829. Serres. Géogn. terrains tert., 241.

4107. Chrysomela —. (Chrysomelidæ.)

Compared with C. cerealis.

Locality: Aix, France. Horizon : Ligurian.

1832. Burmeister. Handb. ent., i : 639 .

1836. " Man. ent., 580.

See also Cassida — Serres.

\section{Chrysomela —. (Chrysomelidæ.)}

Compared with C. banksii Fabr.

Locality: Aix, France. Horizon : Ligurian.

1829. Curtis. Edinb. new phil. journ., vii : 295.

1829. " Murchison-Lyell, Tert. form. Aix, 11.

4109. Chrysomela -. (Chrysomelidæ.)

Locality: Aix, France. Horizon: Ligurian.

1829. Curtis. Edinb. new phil. journ., vii : 295. Pl. 6, fig. 4. 1829. " Murchison-Lyell, Tert. form. Aix, 11. Pl. 6, fig. 4.

See also Chrysomela lyelliana, Chrysomela tertiaria.

\section{Chrysomela —. (Chrysomelidæ.)}

Locality : Prussian amber. Horizon: Ligurian.

1835. Gravenhorst. Uebers. sehles, gesellsch. vaterl. eult., 1834 : 92.

4111. Chrysomela —. (Chrysomelidæ.)

Locality : Prussian amber. Horizon: Ligurian.

1856. Menge. Progr. petrischule Danzig, 1856 : 21.

4112. Chrysomela - (larva). (Chrysomelidæ.)

Locality: Prussian amber. Horizon : Ligurian.

1856. Menge. Progr..petrischule Danzig, 1856: 23.

4113. Chrysomela —. (Chrysomélidæ.)

Localities: Lexden, Bielbecks, England. Horizon: Peat.

1863. Wollaston. Quart. journ. geol. soc. Lond., xix, i: 401, No. 9.

1888. Bell. . Entom., $\mathrm{xxi}: 2$.

Chrysomela —. See Curenlio (or Chrysomela) 
4114. Chrysomela calami. (Chrysomelidæ.)

Compared with C. graminis L.

Locality: Oeningen, Baden. Horizon: Tortonian.

1847. Heer. Insektenf. tertiärg. Oeningen, i: 208-209. Pl. 7, fig. 8.

1852. Giebel. Deutschl. petref., 648.

1856. " Insect. d. vorwelt, 120.

1865. Heer. Urwelt der Schweiz, 361, 362, 372. Fig. 238 on p. 371.

1872. " Monde prim. Suisse, 443, 444, 456. Fig. 238 on p. 455.

1876. " $\quad$ Prim. world Switz., ii: 15, 16, 26. Fig. 238 on p. 27.

1879. “ Urwelt der Schweiz, $2^{\mathrm{e}}$ aufl., 386, 387, 397. Fig. 280.

4115. Chrysomela debilis. (Chrysomelidæ.)

1874. Oustalet. Ann. sc. géol., v, art.2: 334-335. Pl. 6, fig. 1.

4116. Chrysomela lyelliana. (Chrysomelidæ.)

Locality: Aix, France. Horizon: Ligurian.

1856. Heer. Viert. naturf. gesellsch. Zürich, i: 26-27. Pl. 1, fig. 18.

1874. Oustalet. Ann. sc. géol. v, art. 2: 328-331. Pl. 5, fig. 14; pl. 6, fig. 2.

See also Chrysomela — Curtis, vi : 4, ete.

4117. Chrysomela matheroni. (Chrysomelidæ.)

Locality : Aix, France. Horizon: Ligurian.

1874. Oustalet. Ann. sc. géol., v, art.2: 333-334. Pl. 6, fig. 3.

4118. Chrysomela matrona. (Chrysomelidæ.)

Compared with C. banksii, Europe.

Locality: Aix, France. Horizon: Ligurian.

1874. Oustalet. Ann. sc. g6́ol., v, art. 2: 331-332. Pl. 5, fig. 13.

4119. Chrysomela punctigera. (Chrysomelidæ.)

Locality: Oeningen, Baden. Horizon: Tortonian.

1847. Heer. Insektenf. tertiärg. Oeningen, i: 209. Pl. 7, fig. 9.

1852. Giebel. Deutschl. petref., 648.

1856. " Insect. d. vorwelt, 121.

4120. Chrysomela succini. (Chrysomelidæ.)

Locality: Prussian amber. Horizon: Ligurian.

1856. Giebel. Insect. d. vorwelt, 120.

4121. Chrysomela tertiaria. (Chrysomelidæ.)

Locality: Aix, France. Horizon: Ligurian.

1856. Giebel. Insect. d. vorwelt, 121.

See also Chrysomela — Curtis, vi : 4, etc.

4122. Chrysomelites

(Chrysoimelidæ.)

Locality: Brunstatt, Alsatia. Horizon: Middle Oligocene.

1889. Foerster. Mitth. comm. geol. Elsass.-Lothr., ii : 102.

4123. Chrysomelites alaskanus. (Chrysomelidæ.)

Locality : English Bay, Alaska. Horizon:

1869. Heer.

Flora foss. alask. (K. svenska vetensk.-akad. handl., viii; Flora foss. arct.,ii) : 39 . $P l .10$, figs. $6,6 b$. 
4124. Chrysomelites fabricii. (Chrysomelidæ.)

Locality : Atanekerdluk, North Greenland. Horizon : Miocene.

1868. Heer.

1883. “
Flora foss. arct., 129. $\quad P l$. 19, figs. 13-14.

Flora foss. Grönl., ii: 146 .

4125. Chrysomelites lindhageni. (Chrysomelidæ.)

Compared with C. (Lina) tremulæ Fabr.

Localities: Ober-Atanekerdluk, Greenlaud; Cap Staratschin, Spitzbergen.

Horizon: Miocene.

1870. Heer.

K. svenska vetensk.-akad, handl., viii, No. 7: 76. $\quad P l$. 16, figs. $23 a-d$.

1883. “ Flora foss. Grönl., ii: 145-146. Pl. 109, figs. 7, 7b.

4126. Chrysomelites thulensis. (Chrysomelidæ.)

Locality: Cap Staratschin, Spitzbergen. Horizon : Miocene.

1870. Heer.

K. svenska vetensk.-akad. handl., viii, No.7: 76. $\quad P l .16$, figs. 25-27.

4127. Cicindela (Odontocheila) —_- (Cicindelidæ.)

Locality : Prussian amber. Horizon: Ligurian.

1839. Brullé. Gisem. ins. foss., 17.

\section{Cionus -. (Curculionidæ.)}

Compared with C. scrophulariæ.

Locality: Aix, France. Horizon: Ligurian.

1829. Serres. Géogn. terrains tert., 223.

4129. Cionus —. (Curculionidæ.)

Compared with C. verbasci.

Locality : Aix, France. Horizon : Ligurian.

1829. Serres. Géogn. terrains tert., 223, $26 \%$.

4130. Cis ㄴ. (Cioidæ.)

Locality: Prussian amber. Horizon: Ligurian.

1845. Berendt. Bernst. befindl. organ. reste vorw., i: 56 .

4131. Cis krantzi. (Cioidæ.)

Locality: Rott, Rhenish Prussia. Horizon: Aquitanian.

1866. Heyd.-Heyd. Palaeontogr., xv : 144. Pl. 22, fig. 26.

1866. " Käfer u. polypen, 14. $P l .1, f i g .26$.

4132. Cistela - (Cistelidæ.)

Locality: Prussian amber. Horizon: Ligurian.

1845. Berendt. Bernst. befindl. organ. reste vorw., i: 56.

4133. Cistela dominula. (Cistelidæ.)

Locality: (Oeningen ?, Baden). Horizon : Tortonian.

1847. Heer.

1852. Giebel.

1856. "6

Insektenf. tertiärg. Oeningen, i : 160-161, Pl. 5, fig. 8.

Deutschl. petref., 650 .

Insect. d. vorweilt, 112 
4134. Cistelites minor. (Cistelidæ.)

Localities: Puilasok, Aumarutigsat, Greenland. Horizon: Miocene

1874. Heer.

Nachtr. mioc. flora Grönl. (K. svenska vetensk.-akad. handl., xiii ; Flora foss. arct., iii) : 25. Pl. 5, fig. 13.

1883. “

Flora foss. Grönl., ii : 145.

4135. Cistelites punctulatus. (Cistelidæ.)

Localities: Puilasok, Atanekerdluk, Greenland. Horizon : Miocene.

1870. Heer.

Phil. trans., clix (Flora foss. arct., ii) : 484-485. Pl. 56, figs. 14, $14 b c$.

1874. “

Nachtr. mioc. flora Grönl. (K. svenska vetensk.-akad. handl. xiii ; Flora foss. aret., iii) : 25. Pl. 5, fig. 12.

1883. “ “

Flora foss. Grönl., ii : 145.

4136. Cistelites sachalinensis. (Cistelidæ.)

Locality : Mgratsch, Insel Sachalin. Horizon: Miocene.

1878. Heer. Mém. acad. imp. sc. St. Petersb., (7), $x \times v$, vii (Prim. flora foss. sachal.; Flora foss. arct., v, iii): 3. Pl. 15, figs. 12, $12 b$.

4137. Cistelites spectabilis. (Cistelidæ.)

Locality : Oeningen, Baden. Horizon: Tortonian.

1869. Heer. Phil. trans., clix (Flora foss. arct., ii) : 485. Pl. 56, fig. 13.

Citylus varius. See Cytilus varius.

Cleonis —. See Cleonus — Serres.

4138. Cleonolithus antiquus. (Curculionidæ.)

Locality : (Senigaglia ?; Italy). Horizon : Helvetian ?.

1841. Bassi. Atti reun. scienz. ital., iii : 401.

4139. Cleonus? - (Curculionidæ.)

Locality: Brunstatt, Alsatia. Horizon: Middle Oligocene.

1889. Foerster. Mitth. comm. geol. Elsass-Lothr., ii : 102.

4140. Cleonus - (Curculionidø.)

Locality : Aix, France. Horizon: Ligurian.

1874. Heer. MSS. in Oustalet, Ann, sc. géol., v, art. 2: 235-242.

See also Brachyderes longipes, etc.

4141. Cleonus - (7 sp.). (Curculionidæ.)

Locality : Aix, France. Horizon: Ligurian.

1829. Serres. Géogn. terrains tert., 224 (Cleonis).

4142. Cleonus - ("No. 4.") (Curculionirlæ.)

Compared with C. distincta.

Locality: Aix, France. Horizon: Ligurian.

1829. Serres. Géogn. terrains tert., 224, 272 (Cleonis). Pl. 5, fig.9.

4143. Cleonus angusticollis. (Curculionidæ.)

Locality: Oeningen, Baden. Horizon: Tortonian.

1851. Stizenberger. Uebers. verstein. Baden, 99 (as of Heer) name only. ${ }^{1}$ 
4144. Cleonus arvernensis. (Curculionidæ.)

Compared with C. albarius.

Locality : Corent, France. Horizon: Aquitanian.

1870. Oustalet. Ann. sc. g6́ol., ii, art. 3: 67-68. Pl. 1, figs. 5, 6.

4145. Cleonus asperulus. (Curculionidæ.)

1856. Heer.

Locality: Aix, France. Horizon: Ligurian.

1874. Oustalet.

Viert. naturf. gesellsch. Zürich, i: 20. Pl. 1, fig. 15.

Ann. sc. géol., v, art. 2: 259-263. Pl. 3, figs. 20, 21.

4146. Cleonus deucalionis. (Curculionidæ.)

Locality : Oeningen, Baden. Horizon: Tortonian.

1847. Heer.

1852. Giebel.

1856. " "

Insektenf. tertiärg. Oeningen, i: 187. Pl. 6, fig. 12.

Deutschl. petref., 649 .

Insect, $d$, vorwelt, 142.

4147. Cleonus fouilhouxii. (Cureulionidæ.)

Locality: Corent, France. Horizon: Aquitanian.

1870. Oustalet. Ann. sc. g6́l., ii, art. 3: 68-69. Pl. 1, fig. 7.

4148. Cleonus inflexus. (Curculionidæ.)

Locality : Aix, France. Horizon: Ligurian.

1874. Heer. MSS.

1874. Oustalet. Ann. sc. g6́ol., v, art. 2: 263-265. Pl. 3, fig. 14.

4149. Cleonus larinoides. (Curculionidæ.)

Locality : Oeningen, Baden. Horizon: Tortonian.

1847. He⿶r.

Insektenf. tertiärg. Oeningen, i: 183-186. Pl. 6, fig. 15.

1852. Giebel.

Deutschl. petref., 649 .

1856. " Insect. d. vorwelt, 142.

4150. Cleonus leucosiæ. (Cureulionidæ.)

Localities: (Oeningen?, Baden); Aix, France. Horizons: Tortonian, Ligurian.

1847. Heer.

1852. Giebel.

1856. "

1856. Heer.

1874. Oustalet.

See also Cleonus angusticollis.

Insektenf. tertiärg. Oeningen, i: 188-189. Pl. 8, fig. 8.

Deutschl. petref., 649.

Insect. d. vorwelt, 142.

Viert. naturf. gesellsch. Zürich, i : 20.

Ann. sc. géol., v, art. 2 : 254-256.

4151. Cleonus marcelli. (Curculionidæ.)

Locality : Aix, France. Horizon : Ligurian.

1874. Oustalet. Ann. sc. géol., v, art. 2: 256-259. Pl. 3, fig. 13; pl. 5, fig. 5.

4152. Cleonus polii. (Curculionidæ.)

Locality : Aix, France. Horizon: Ligurian.

1847. Hope.

1847. "6

Descr. insect. foss., 5. Pl., fig. 1.

Ann. accad. aspir. nat., $1847:-$ Pl. 10, fig. 1.

4153. Cleonus pygmæus. (Curculionidæ.)

Locality: Aix, Franee. Horizon: Ligurian.

1874. Oustalet.

Ann. sc. géol., v, art. 2: 267. Pl. 3, fig. 10 
1847. Heer. 1852. Giebel. 1856. "

1856. Heer. 1874. Oustalet.

4154. Cleonus pyrrhæ. (Curculionidæ.)

Locality: Oeningen, Baden. Horizon: Tortonian.

Insektenf. tertiärg. Oeningen, i : 1ธ9-190, 221-222. Pl. 6, fig. 13. Deutschl. petref., 649 .

Insect. d. vorwelt, 142-143.

4155. Cleonus sexsulcatus. (Curculionidæ.)

Locality : Aix, France. Horizon : Ligurian.

Viert. naturf, gesellsch. Zürich, i : 20-21. Pl. 1, fig. 9. Ann. se. geol., v, art. 2: 266. Pl. 3, fig. 16; pl. 4, fig. 9 .

4156. Cleonus speciosus. (Cureulionidæ.)

Compared with C. pruinosus Sch.

Locality: Oeningen, Baden. Horizon: Tortonian.

1865. Heer. 1872. " " 1876. “ 1879. “

1847. Heer. 1852. Giebel. 1854. Pictet. 1856. Giebel. 1865. Heer. 1872. " 1876. " 1879. " " 1885. Scudder. 1886. " "

1862. Giebel.

1847. Heer. 1852. Giebel. 1856
Urwelt der Schweiz, 374. Fig. 249.

Monde prim. Suisse, 459. Fig. 249 on p. 455.

Prim. world Switz., ii : 30. Fig. 249 on p. 27.

Urwelt der Schweiz, $2^{\mathrm{e}}$ aufl., 400. Fig. 291 on p. 397.

4157. Clerus adonis. (Cleridæ.)

Compared with C. mutillarius and C. formicarius $\mathrm{L}$.

Locality: Oeningen, Baden. Horizon: Tortonian.

Insektenf. tertiärg. Oeningen, i : 152-154. $\quad P l .5$, fig. 3.

Dentschl. petref., 650 .

Traité de paléont., $2^{e}$ éd., ii : $335 . \quad P l .40$, fig. 15.

Insect. d. vorwelt, 45 .

Urwelt der Schweiz, 377.

Monde prim. Suisse, 462.

Prim. world Switz., ii : 33.

Urwelt der Schweiz, $2^{\circ}$ autl., 402.

Zittel, Handb. palaeont., i, th. ii : 796. Figs. 1030ab.

Zittel-Barrois, Traité de pal6ont., ii : 796. Figs. 1048ab.

4158. Clerus succini. (Cleridæ.)

Compared with C. formicarius, Europe.

Locality : Prussian amber. Horizon: Ligurian.

Zeitschr. gesellsch. naturw., xx : 320 .

4159. Clivina (Carabidæ.)

Locality: Prussian amber. Horizon: Ligurian.

1845. Berendt. Bernst. befindl. organ. reste vorw., i: 56.

4160. Clythra carbonaria. (Chrysomelidæ.)

Locality: Salzhausen, Germany. Horizon: Miocene.

1865. Heyd.-Heyd, Palaeontogr., xiv : 33-34 (larval sack). Pl. 9, fig. 20.

4161. Clythra pandoræ. (Chrysomelidæ.)

Locality: Oeningen, Baden. Horizon: Tortonian.

Insektenf. tertiärg. Oeningen, i: 214-215 (Clytra). Pl. 7, fig. 14.

Deutsebl. petref., 647 .

Insect. d. vorwelt, 117.

Clytra pandoræ, See Clythra pandoræ, 
4162. Clytus —_. (Cerambycidæ.)

Compared with C. detritus.

Locality: Oeningen, Baden. Horizon: Tortonian.

1888. Schöberlin. Soc. entom., iii : 42.

4163. Clytus -. (Cerambycidæ.)

Compared with C. gazella.

Locality: Oeningen, Baden. Horizon: Tortonian.

1888. Schöberlin. Soc. entom., iii : 42.

4164: Clytus leporinus. (Cerambycidæ.)

Compared with Caloclytus semipunetatus (Fabr.), Europe.

Locality: Aix, France. Horizon: Ligurian.

1874. Oustalet. Ann. sc. géol., v, art. 2: 319-321. Pl. 5, fig. 10.

4165. Clytus melancholicus. (Cerambycidæ.)

Locality: Oeningen, Baden. Horizon: Tortonian.

1347. Heer. Insektenf. tertiärg. Oeningen, i: 163-165. Pl. 5, fig. 14.

1×52. Giebel. Deutschl. petref., 649 .

1856. “ Insect. d. vorwelt, 128.

4166. Clytus pulcher. (Cerambycidæ.)

1865. Heer.

Locality: Oeningen, Baden. Horizon: Tortonian.

1872. “

Urwelt der Schweiz. Fig. 252 on p. 376.

Monde prim. Suisse. Fig. 252 on p. 461.

Prim. world Switz., ii. Fig. 252 on p. 32.

1876. “

1879.

Urwelt der Schweiz, $2^{\mathrm{e}}$ aufl. Fig. 294 on $p .402$.

4167. Cocoinella —- (Cocoinellidæ.)

Locality: Lexden, England. Horizon Pleistocene.

1888. Bell. Entom., xxi : 2.

4168. Coccinella —_. (Coccinellidæ.)

Locality: Prussian amber. Horizon: Ligurian.

1845. Berendt. Bernst. befindl. organ. reste vorw., i: 56.

4169. Coccinella

(Coccinellidæ.)

Locality: Aix, France. Horizon: Ligurian.

1847. Hope. Trans. ent. soc. Lond., iv : 251.

4170. Coccinella —. (Coccinellidæ.)

Locality: Oeningen, Baden. Horizon: Tortonian.

1834. Keferstein. Naturg. erdkörp., ii: 328.

4171. Coccinella - (Cocinellidæ.)

Locality : Prussian amber. Horizon : Ligurian.

1856. Menge. Progr. pretrischule Danzig, 1856: 21.

4172. Cocoinella - (larva). (Coccinellidæ.)

Locality: Prussian amber. Horizon: Ligurian.

1856. Menge. Progr. petrischule Danzig, 1856: 23.

Bull. $71-32$ 


\section{Coccinella (or Cassida)} (Coceinellidæ.)

Locality: Lexden, England. Horizon: Peat.

1863. Wollaston. Quart. journ. geol. soc. Lond., xix, i : 400, No. 1.

4174. Coccinella amabilis. (Coccinellidæ.)

Compared with C. ocellata L.

1865. Heer.

1872. "

1876. “

1879. “

Locality: Oeningen, Baden. Horizon: Tortonian.

Urwelt der Schweiz, 371.

Monde prim. Suisse, 455.

Prim. world Switz., ii: 26.

Urwelt der Schweiz, $2^{\text {e }}$ anfl., $3 \Varangle 4,399$. Fig. 261 on p. 384.

4175. Coccinella andromeda. (Coccinellidæ.)

Locality : Oeningen, Baden. Horizon : 'Tortonian.

1847. Heer.

1852. Giebel.

1856. " "

1865. Heer.

1872. “

1876. “

1879. " "
Insektenf. tertiärg. Oeningen, i: 216. $\quad P l .7, f i g .16$.

Deutschl. petref., 647 .

Insect. d. vorwelt, 123.

Urwelt der Schweiz, 371.

Monde prim. Suisse, 455.

Prim. world Switz., ii : 26.

Urwelt der Schweiz, $2^{\mathrm{e}}$ aufl., 396.

4176. Coccinella antiqua. (Coccinellidæ.)

Locality: Rott, Rhénish Prussia. Horizon: Aquitanian.

1862. Heyden. Palaeontogr., x: 74. Pl. 10; fig. 18.

\section{Coccinella bituminosa. (Coccinellidæ.)}

Locality: Rott, Rhenish Prussia. Horizon: Aquitanian.

1866. Heyd.-Heyd. Palaeontogr., xv: 157. Pl. 24, figs. 18, 19.

1866. " Käfer u. polypen, 27. Pl. 3, figs. 18, 19.

4178. Coccinella colorata. (Coccinellidæ.)

Locality : Oeningen, Baden. Horizon: Tortonian.

1865. Heer. Urwelt der Schweiz, 371. Fig. 234.

1872. " Monde prim. Suisse, 455. Fig. 234 .

1876. " Prim. world Switz., ii : 26. Fig. 234 on p. 27.

1879. " Urwelt der Schweiz, $2^{e}$ aufl., 396. Fig. 276 on p. 397.

\section{Coccinella decempustulata. (Coceinellidæ.)}

Locality: Oeningen, Baden. Horizon: Tortonian.

1879. Heer. Urwelt der Sehweiz, $2^{e}$ auf., 397. Fig. 260 on p. 384.

1885. Scudder. Zittel, Handb. palaeont, i, th. ii: 800 . Fig. 1056.

1886. " Zittel-Barrois, Traité de paléont., ii : 801. Fig. 1073.

1889. Nicholson. Man. palæont., 3d ed, i: 601. Fig. 449c.

4180. Coccinella fossilis. (Coccinellidæ.)

Locality: Rott, Rhenish Prussia. Horizon: Aquitanian.

1866. Heyd.-Heyd. Palaeontogr., xv: 157. Pl. 24, fig. 20.

1866. " Käfer u. polypen, 27. Pl. 3, fig. 20. 
4181. Coccinella (Sospita) haagi. (Coccinellidæ.)

Locality : Rott, Rhenish Prussia. Horizon : Aquitanian.

1866. Heyd.-Heyd. Palaeontogr, xv: 155-156 (Sospita haagi on plate). Pl. 24, figs. 15, 16.

1866. " Käfer u. polypen, 25-26 (Sospita haagi on plate). Pl. 3. figs. See also Sospita haagi. $15,16$.

4182. Coccinella hesione. (Coccinellidæ.)

Compared with C. dispar, C. bipunctata.

Locality: Oeningen, Baden. Horizon: Tortonian.

1847. Heer.

1852. Giebel.

1856. " "

1865. Heer.

1872. " "

1876. " "

1879. "

Insektenf, tertiärg. Oeningen, i: 216-217. $\quad P l .7$, fig. 17.

Deutschl. petref., 647.

Inseet. d. vorwelt, 123.

Urwelt der Schweiz, 371.

Monde prim. Suisse, 455.

Prim. world Switz., ii : 26.

Urwelt der Schweiz, $2^{e}$ aufl., 396.

4183. Coccinella kraantzi. (Coccinellidæ.)

Locality : Rott, Rhenish Prussia. Horizon: Aquitanian.

1866. Heyd.-Heyd. Palaeontogx., $\mathrm{xv}:$ 156. Pl. 24 , fig. 17.

1866. " Käfer n. polypen, 26. Pl. 3, fig. 17.

4184. Coccinella perses. (Coccinellidæ.)

Locality : Oeningen, Baden. Horizon: Tortonian.

1847. Heer.

1852. Giebel.

1856. “

Insektenf. tertiärg. Oeningen, i : 217-218. $\quad P l .8$, fig. 11.

Deutschl. petref., 647 .

Insect. d, vorwelt, 123.

4185. Coccinella protogeæ. (Coccinellidæ.)

Locality: Vicinity Bonn, Rhenish Prussia. Horizon : Aquitanian ?.

1837. Germar. Fanna insect. Europæ, xix: 15. Pl. 15.

1852. Giebel. Dentschl. petref,, 647.

See also Escheria protogeæ.

4186. Coccinella spectabilis. (Coccinellidæ.)

Compared with C. marginata, Brazil.

Locality : Oeningen, Baden. Horizon: Tortonian.

1865. Heer.

1872. “

1876. " "

1879. “

Urwelt der Schweiz, 371. Fig. 235.

Monde prim. Suisse, 455. Fig. 235.

Prim. world Switz., ii : 26. Fig. 235 on p. 27.

Urwelt der Schweiz, $2^{e}$ anfl., 396. Fig. 277 on $p .397$.

4187. Cœliodes primigenius. (Curculionidæ.)

Locality: Aix, France. Horizon: Ligurian.

1874. Oustalet. Ann. sc. geol, , v, art. 2: 302-303. Pl. 6, fig. 11.

See also Apion primigenius.

4188. Colasposoma (Chrysomelidæ.)

Locality: Prussian amber. Horizon: Ligurian.

1868. Smith.

Quart. journ. sc., v: 184 (Calasposoma). Pl., fig. 8. 


\section{Colydium - (Colydiidæ.)}

Locality : Prussian amber. Horizon: Ligurian.

1845. Berendt. Bernst. befindl. organ. reste vorw., i : 56.

4190. Colymbetes —. (Dytiscidæ.)

Compared with C. striatus.

Locality: Aix, France. Horizon: Ligurian.

1847. Hope. Trans, ent. soc. Lond., iv : 250.

4191. Colymbetes (Cymatopterus) æmulus. (Dytiscidæ.)

Locality : Oeningen, Baden. Horizon: Tortonian.

1862. Heer. Verhandl. holl. maatsch. wetensch. Haarl., xvi : 41-42. Pl. 2, figs. 16-19.

4192. Colymbetes striatus (recent). (Dytiscidæ.)

Locality : Hösbach, Bavaria. Horizon: Lower Pleistocene.

[1758. Linné. 1884. Flach. 1884. "

Syst. nat., i, 2: 665.]

Käfer unterpleist. Hösbach, 8.

Verhandl. physik.-med. gesellsch. Würzb., n. f., xviii : 292.

4193. Colymbetes ungeri. (DJtiscidæ.)

Locality : Radoboj, Croatia. Horizon: Mayencian.

1847. Heer. 1852. Giebel. 1856. “

Insektenf. tertiärg. Oeningen, i : 27-28. $\quad P l, 1, f i g .8$.

Deutschl. petref., 654 .

Insect, $d$. vorwelt, 58.

Compsidia nephele. See Saperda (Compsidia) nephele.

4194. Coniatus minusculus. (Cureulionidæ.)

Loeality : Aix, France. Horizon: Ligurian.

1874. Oustalet. Ann. sc. géol., v, art. 2: 286-287. Pl. 3, figs. i7, 17a.

4195. Copris druidum. (Scarabæidæ.)

Compared with C. ciliata, Brazil.

Locality : Oeningen, Baden. Horizon: Tortonian.

1862. Heer.

Verhandl. holl. maatseh. wetensch. Haarl., xvi: 73-74. Pl. 6, figs. $3,3 b c$.

1865. " "

1872. "

1876.

1879. “

Urwelt der Schweiz, 379. Fig. 267 on p. 378.

Monde prim. Suisse, 465. Fig. 267 on $p .463$.

Prim. world Switz., ii : 36 . Fig. 267 on $p .34$.

Urwelt der Schweiz, $2^{\mathrm{e}}$ aufl., 405. Fig. 309 on $p .404$.

4196. Copris lunaris (recent). (Scarabæidæ.)

Locality : Mundesley, England. Horizon: Pliocene.

[1758, Linn6. 1840. Curtis.

1843. Morris.

1854. "6

1885. Seudder.

1886. " "

1886. " "

1888. Bell.

Syst. nat., i, 2: 543.]

Lyell, Proc. geol. soc. Lond., iii : 175.

Catal. Brit. foss., 69.

Catal. Brit. foss., $2 d$ ed., 117.

Zittel, Handb. palaeont., i, th. ii : 795.

Zittel-Barrois, Traité de paléont., ii : 795.

Bull. U. S. geol, surv., No. 31 : 74 .

Entom., xxi: 2. 
4197. Copris subterranea. (Scarabæidæ.)

Compared with C. lunaris.

Locality : Oeningen, Baden. Horizon: Tortonian.

1862. Heer. 1865. " " 1872. “ 1876. “ 1879. "

1847. Heer. 1852. Giebel. 1853-'56. Bronn. 1856. Giebel. 1865. Heer. 1872. " " 1876. “ 1879. “

1856. Heer. 1874. Oustalet.

1861. Heer.

Verhandl, holl, maatsch. wetensch. Haarl., xvi : 74. Pl. 6, fig. 4. Urwelt der Schweiz, 379.

Monde prim. Suisse, 465.

Prim. world Switz., ii : 36.

Urwelt der Schweiz, $2^{\mathrm{e}}$ aufl, 405.

4198. Coprologus gracilis. (Scarabæidæ.)

Locality : Oeningen, Baden. Horizon: Tortonian.

Insektenf. tertiärg. Oeningen, i : 60-62. $\quad P l$. 2, fig. 11.

Dentschl. petref., 653 (Caprologus).

Lethæa geogn., $3^{\mathrm{e}}$ aufl., iii : 648. $\quad \mathrm{Pl}$. 42', figs. $25 a b$.

Insect. d. vorwelt, 35.

Urwelt der Schwein, 379.

Monde prim. Suisse, 465.

Prim. world Switz., ii : 36.

Urwelt der Schweiz, 2e aufl., 405.

4199. Corticaria melanophthalma. (Lathridiidæ.)

Locality: Aix, France. Horizon: Ligurian.

Viert. naturf. gesellsch. Zürich, i: 18-19. Pl. 1, fig. 7. Ann. sc. géol., v, apt. 2: 187-188. Pl. 6, fig. 17.

4200. Corymbites sutor. (Elateridæ.)

Compared with C. æneus (Linn.), Europe.

Locality: Oeningen, Baden. Horizon: Tortonian.

Rech. climat pays tert., 204 (undescr.).

4201. Corymbites velatus. (Elateridæ.)

Compared with C. splendens.

Locality: Green River, Wyo. Horizon: Oligocene.

1876. Scudder.

Bull. U. S. geol. surv. terr., ii : 81.

1878. " "

1890 . “

Bull. U. S. geol. surv. terr., iv : 762.

Tert. ins. N. A., 496.

4202. Corynetes -. (Cleridæ.)

Locality: Prussian amber. Horizon: Ligurian.

1845. Berendt. Bernst. befindl. organ. reste vorw., i : 56.

4203. Cossonus marionii. (Calandridæ.)

Locality: Aix, France. Horizon: Ligurian.

1874. Oustalet. Ann. se. g6́ol., v, art. 2: 305-306. Pl. 2, fig. $19 ;$ pl.5, fig. 9.

4204. Cossonus meriani. (Calandridæ.)

Locality: Oeningen, Baden. Horizon: Tortonian.

1847. Heer.

1852. Giebel.

1856. “
Insektenf. tertiärg. Oeningen. i : 196-198. Pl. 7, fig. 2.

Deutschl. petref., 648 .

Insect. d. vorwelt, 145. 
4205. Cossonus spielbergii. (Calandridæ.)

1847. Heer. 1852. Giebel. 1856. “

Locality: Oeningen, Baden. Horizon : Tortonian.

Insektenf. tertiärg. Oeningen, i: 198-199. Pl. $7, \mathrm{fig} .3$.

Deutschl. petref., 648.

Insect. d. vorwelt, 145.

4206. Cossyphus (or Cassida) —_. (Teuebrionidæ.)

Locality: Lexden, England. Horizon: Peat.

1863. Wollaston. Quart. journ. geol. soc. Lond., xix, i: 401, No. 5.

1888. Bell. Entom., xxi: 2.

4207. Cratoparis elusus. (Anthribidæ.)

Compared with C. lunatus Fahr.

Locality: Green River, Wyo. Horizon: Oligocene.

1878. Scudder. Bull. U. S. geol. surv, terr., iv: 768-769.

1890. " Tert. ins. N. A., 467. Pl. 8, fig. 40.

4208. Cratoparis repertus. (Anthribidæ.)

Compared with C. lunatus Fahr.

Locality: Green River, Wyo. Horizon: Oligocene.

1878. Seudder. Bull. U. S. geol surv. terr., iv : 768.

1890. “ Tert. ins. N. A., 466-467. Pl. 8, fig. 4.

4209. Criocerina pristina. (Chrysomelidæ.)

1813. Germar. Germ., Mag. entom., i: 14.

Locality : Prussian amber. Horizon: Ligurian.

See also Crioceris pristina.

\section{Crioceris - (Chrysomelidæ.)}

Locality : Prussian amber. Horizon : Ligurian.

1832. Burmeister. Handb. ent., i: 635.

1836. " Man. ent., $57 \%$.

4211. Crioceris —_. (Chrysomelidæ.)

Loeality : Prussian amber. Horizon : Ligurian.

1856. Menge. Progr, petrischule Danzig, 1856 : 21.

4212. Crioceris margarum. (Chrysomelidæ.)

Locality: Aix, France. Horizon: Ligurian.

1874. Oustalet. Ann. sc. g6́ol, v, art. 2: 324-325. Pl. 5, fig. 11.

4213. Crioceris pristina. (Chrysomelidæ.)

Localıty : Prussian amber. Horizon: Lignrian.

1856. Giebel. Insect. $d$. vorwelt, 116.

See also Criocerina pristina.

4214. Cryptocephalus —. (Chrysomelidæ.)

Locality: Prussian amber. Horizon: Ligurian.

1856. Menge, Progr. petrischule Danzig, 1856: ‘1. 
4215. Cryptocephalus vetustus. (Chrysomelidæ.)

Compared with C. venustus Fabr.

Locality: Green River, Wyo. Horizon: Oligocene.

1878. Scudder. Bull. U. S. geol. surv. terr., iv: 764.

1890. " Tert. ins. N. A., 485-486. Pl. 7, figs. 29, 37.

4216. Cryptohypnus _- (Elateridæ.)

Locality: Prussian amber. Horizon: Ligurian.

1845. Berendt. Bernst. befindl. organ. reste vorw., i: 56.

4217. Cryptohypnus? terrestris. (Elateridæ.)

Compared with C. planatus LeC.

Locality : Nicola River, British Columbia. Horizon:

1879. Scudder.

1879. " "

1890. “

Rep. progr. geol, surv. Can., 1877-'78: B 181-182.

Insect. tert. Nicola, 6-7.

Tert. ins. N. A., 497. $P l .2, f g .30$.

\section{Cryptophagus - - (Cryptophagidæ.)}

Locality : Prussian amber. Horizon: Ligurian.

1845. Berendt. Bernst. befindl. organ. reste vorw., i : 56 .

\section{Cryptorhynchus -. (Curculionidæ.)}

Locality: Brunstatt, Alsatia. Horizon: Middle Oligocene.

1889. Foerster. Mitth. comm. geol. Elsass-Lothr., ii : 102.

4220. Cryptorhynchus annosus. (Curculionidæ.)

Locality: Green River, Wyo. Horizon: Oligocene.

1876. Scudder. Bull. U. S. geol. surv. terr., ii : 86-87.

1878. is Bull. U.S. geol. surv. terr., iv: 767 .

1890. " Tert. ins. N. A., 471. Pl. 8, fig. 3.

4221. Cryptorhynchus gypsi. (Curculionidæ.)

Locality : Aix, France. Horizon: Ligurian.

1974. Oustalet. Ann. sc. géol., v, art. 2: 299-300. Pl. 3, fig. 8.

4222. Cryptorhynchus renudus. (Cureulionidæ.)

Locality: Rott, Rhenish Prussia. Horizon : Aquitanian.

1862. Heyden. Palaeontogr., $\mathrm{x}:$ 71. $\quad P l .10$, fig. 9.

4223. Cucorhinus? (or Strophosomus?) —_. (Otiorhynchidæ.)

Locality: Peckham, England. Horizon: Lower Eocene, Paludina beds.

1861. Smith. Geologist, iv: 40.

4224. Cupes ㄴ. (Cupesidæ.)

Locality : Prussian amber. Horizon: Ligurian.

1845. Berendt. Bernst. befindl organ. reste vorw., i : 56 .

4225. Cupoides tessellatus. (Cupesidø.)

Compared with Cupes atomaria.

Locality: Prussian amber. Horizon: Ligurian.

1856. Motschulsky. Etudes entom., v: 27. 
4226. Curculio - (Curculionidæ.)

Locality: Copal. Horizon: Recent.

1776. Bloch.

Beschäft. berl. gesellsch. naturf. freunde, ii : 189.

4227. Curculio - (Curculionidæ.)

Locality : Prussian amber. Horizon: Ligurian.

1822. Defrance. Dict. sc. nat., xxiii : 524 (charançon).

4228. Curculio? - (Curculionidæ.)

Locality: Schossnitz, Silesia. Horizon: Miocene.

1855. Göppert. Tert. flora Schossnitz. Pl. 26, figs. 56-57.

See also Curculionites silesiacus, etc.

4229. Curculio - (larva). (Curculionidæ.)

Locality: Prussian amber. Horizon: Ligurian.

1856. Menge. Progr. petrischule Danzig, 1856: 23.

4230. Curculio? - (Curenlionìdæ.)

Locality : Nágpur, Central India. Horizon: -

1860. Murray. Quart. journ. geol. soc. Lond., xvi: 184-185, 189. Pl. 10, fig. 38.

4231. Curculio? - (Curculionidæ.)

Locality: Nágpur, Central India. Horizon: -

1860. Murray. Quart.journ. geol. soc. Lond., xvi : 185, 189. Pl. 10, fig. 69.

4232. Cưrculio ? - (Curculionidæ.)

Locality : Nágpur, Central India. Horizon: -

1860. Murray. Quart. journ. geol. soc. Lond., xvi: 185, 189. Pl. 10, fig. 70.

4233. Curculio (or Chrysomela) ——. (Curculionidæ.)

Locality: Lexden, England. Horizon: Peat.

1863. Wollaston. Quart. journ. geol. soc. Lond., xix, i: 401, No. 3.

1888. Bell. Entom., xxi : 2.

\section{Curculio - $\longrightarrow$ (Curculionıdæ.)}

Locality: Gurnet Bay, Isle of Wight. Horizon: Bembridge Limestone. 1879. Woodward. Quart. journ. geol. soe. Lond., $\mathrm{xxxv}$ : 344.

$1879 . \quad$ "Geol. mag., n. s.,v : 89.

4235. Curculionites —. (Curculionidæ.)

Locality: Brunstatt, Alsatia. Horizon: Middle Oligocene.

1889. Foerster. Mitth. comm. geol. Elsass-Lothr., ii : 102.

4236. Curculionites costulatus. (Curculionidæ.)

Locality: Cap Staratschin, Spitzbergen. Horizon: Miocene.

1870. Heer. K. svenska vetensk,-akad. handl, viii, No. vii : $76 . \quad P l .16$, figs. $38 a b, 39,40$.

4237. Curculionites exiguus. (Curculionidæ.)

Locality : Aix, France. Horizon : Ligurian.

1874. Oustalet. Ann. sc. géol., v, art. 2: 310-311. Pl. 4, figs. 10 ["16"]. 
4238. Curculionites lividus. (Curculionidæ.)

Locality: Aix, France. Horizon: Ligurian.

1856. Heer. Viert. naturf. gesellsch. Zürich, i: 24. Pl. 1, fig. 12. 1874. Oustalet. Ann. se. géol., v, art. 2: 308-310. Pl. 6, figs. 8, 10.

4239. Curculionites marginatus. (Curculionidæ.)

Locality: Vicinity Corfe, England. Horizon:

1854. Westwnod. Proc. geol. soc. Lond., 395 (unnamed), Pl. 16, fig. 35.

1856. Giebel. Insect. d. vorwelt, 148.

4240. Curculionites morosus. (Curculionidæ.)

Locality: Aix, France. Horizon : Ligurian.

1856. Heer. Viert. naturf, gesellsch. Zürich, i : 21-55. Pl.1, fig. 13.

See also Liparus — Curtis (ef. punctatus), L. primærus, Hylobius morosus.

4241. Curculionites nitidulus. (Curculionidæ.)

Locality: Cap Staratschin, Spitzbergen. Horizon: Miocene.

1870. Heer. K. svenska vetensk.-akad. handl., viii, No. vii : 77. $P l$. 16, figs. 15-16.

4242. Curculionites obsoletus. (Curculionidæ.)

Locality: Oeningen, Baden. Horizon : Tortonian.

1851. Stizenberger. Urbers, verstein. Baden, 98 (as of Heer, who describes nothing of the kind).

4243. Curculionites ovatus. (Curculionidæ.)

Locality: Corent, France. Horizon: Aquitanian.

1870. Oustalet. Ann. sc. géol., ii, art. 3: 77-78. Pl. 1, fig. 12.

4244. Curculionites parvulus. (Curculionidæ.)

Locality: Aix, France. Horizon: Ligurian.

1856. Heer. Viert. naturf. gesellsch. Zürich, i: 23-24. Pl. 1, fig. 16.

1874. Oustalet. Ann. sc. géol., v, art. 2: 307-308. Pl. 4, fig.11; pl.6, fig. 9.

4245. Curculionites redtenbacheri. (Cureulionidæ.)

Locality: Radoboj, Croatia. Horizon: Mayencian.

1847. Heer. Insektenf. tertiärg. Oeningen, i: 199-200. Pl. 7, fig. 1.

1852. Giebel. Deutschl. petref., 648.

4246. Curculionites senonicus. (Cnrculionidæ.)

Locality: Sahel Alma, Lebanon. Horizon: Senonian.

1888. Kolbe. Zeitschr. dentsch. geol. gesellsch., xl: 135-136. Pl. 11, fig. 8.

4247. Curculionites silesiacus. (Curculionidæ.)

Locality: Schossnitz, Silesia. Horizon: Miocene.

1869. Assmann. Beitr. insect. d. vorwelt, 41. Pl. 1, figs. 4, 4a.

1869. "* Schles. zeitschr. f. ent., (2), i: 41. Pl. 1, figs. 4, $4 a$.

See also Curculio — Göppert, _—_ (Chrysomelidæ) Göppert.

4248. Curculionites taxodii. (Cụrculionidæ.)

Locality: Cap Staratschin, Spitzbergen. Horizon: Miocene.

1870. Heer. K. svenska vetensk.-akad. handl, viii, No. vii : 76-77. Pl. 16, figs. $8 a, 9$. 
4249. Curculionites thoracicus. (Curculionidæ.)

Locality: Cap Staratsehin, Spitzbergen. Horizon: Miocene.

1870. Heer.

K. svenska vetensk.-akad. handl., viii, No. vii : 77. Pl.16, figs. $17-18$.

4250. Cybister agassizi. (Dytiscidæ.)

Compared with C. limbatus F., India ; C. foveatus, Mexico.

Locality: Oeningen, Baden. Horizon: Tortonian.

1862. Heer.

Verhandl. holl. maatsch. wetensch. Haarl., xvi: 37-39. Pl. 2, figs. 1-10.

1865. " Urwelt der Schweiz, 361, 383. Fig. 280 on p. 381.

187\%. " Monde prin. Suisse, 443,470. Fig. 280 on p. 467.

1876. " $\quad$ Prim. world Switz., ii: 15,40. Fig. 280 on p. 38.

1879. " Urwelt der Schweiz, 2 aufl, 387, 409. Fig. 322 on p. 407.

4251. Cybister atavus. (Dytiscidæ.)

Compared with C. africanus Lap., Africa and Mediterranean district.

Locality : Oeningen, Baden. Horizon : Tortonian.

1862. Heer. Verhandl. holl. maatsch. wetensch. Haarl., xvi: 39-40 (Cybistus). Pl. 2, figs. 23, 24 .

1ะ65. " " Urwelt der Schweiz, 383 .

1872. " Monde prim. Suisse, 470.

1876. " " Prim. world Switz., ii : 41.

1879. " Urwelt der Schweiz, $2^{\ominus}$ aufl., 409.

\section{Cybister nicoleti. (Dytiscidæ.)}

Compared with C. costalis Oliv.

Localities: Oeningen, Baden ; Locle, Switzerland. Horizon: Tortonian.

1862. Heer. Verhandl. holl. maatsch. wetensch. Haarl., xvi : 40 . Pl. 2, figs. 21,22 .

1865. " Urwelt der Schweiz, 383.

1872. " Monde prim. Suisse, 470.

1876. " " Prim. world Switz., ii : 40.

1879. " Urwelt der Schweiz, $2^{\mathrm{e}}$ aufl., 409.

Cybistus atavus. See Cybister atavus.

4253. Cychrus (minor). (Carabidæ.)

Compared with C. andrewsii, United States.

Locality: Port Kennedy, Pa. Horizon: Postpliocene.

1876. Horn. Trans. Amer. entom. soc., v : 243.

1889. Lesley. Dict. foss. Penus., i: p. xvii.

1890. Scudder. Tert. ins. N. A., 537-538. Pl. 1, fig. 2.

4254. Cychrus rostratus (recent). (Carabidæ.)

Locality: Hösbach, Bavaria. Horizon: Lower Pleistocene.

[1746. Linń6.

1884. Flach.

1884. "
Fauna suec., no. 823.]

Käfer unterpleist. Hösbach, 3-4. Pl. 1, fig. 2.

Verhandl. physik.-med, gesellsch. Würzb., n. f, xviii : 287-288. Pl. 8, fig. 2. 
4255. Cychrus testeus. (Carabidæ.)

Compared with C. angusticollis Fisch.

Locality : Green River, Wyo. Horizon: Oligocene.

1878. Scudder. Bull. U. S. geol. surv. terr., iv: 758-759.

See also Neothanes testeus.

\section{Cychrus wheatleyi. (Carabidæ.)}

Compared with C. viduus, North America.

1876. Horn. Locality: Port Kennedy, Pa. Horizon : Postpliocene.

1889. Lesley.

Trans. Amer. entom. soc., v : 242-243.

1890. Scudder.

Dict. foss. Penns., i : p. xvii.

Tert. ins. N. A., 536-537. Pl. 1, fig. 1.

4257. Cyclonotum orbiculare (recent). (Hydrophilidæ.)

Localities : Hösbach, Bavaria ; Cambridge, England. Horizon : Pleistocene.

[1775. Fabricius. Syst. ent., 229.]

1884. Flach. Käfer unterpleist. Hösbach, 9. Pl. 2, fig. 2.

1884. " Verhandl. physik.-med. gesellsch. Würzb., n. f., sviii:293. $P l$. 9, fig. 2.

1888. Bell. Entom., $\mathrm{xxi}: 2$.

Cymatbpterus æmulus. See Colymbetes (Cymatopterus) æmulus.

4258. Cymindis —- (Carabidæ.)

Locality: Prussian amber. Horizon: Ligurian.

1831. Burmeister. Oken, Isis, 1831: 1100.

4259. Cymindis aurora. (Carabidæ.)

Compared with C. americana, United States.

Locality: Port Kennedy, Pa. Horizon: Postpliocene.

1876. Horn. Trans. Amer. entom. soc., v: 243.

1889. Lesley. Dict. foss. Penns., i: p. xvii.

1890. Scudder. Tert. ins. N. A., 518. Pl. 1, fig. 6.

4260. Cymindis pulchella. (Carabidæ.)

Locality: Oeningen, Baren. Horizon: Tortonian.

1847. Heer. Insektenf. tertiärg. Oeningen, i: 13-15. Pl. 1, fig. 1.

1852. Giebel. Deutschl. petref., 655.

1856. " Insect. d. vorwelt, 69.

4261. Cymindoides seulptipennis. (Carabidæ.)

Locality: Prussian amber. Horizon: Ligurian.

1856. Motschulsky. Etudes entom., v : 25-26.

4262. Cyphon —. (Dascyllidæ.)

Locality : Prússian amber. Horizon: Ligurian.

1845. Berendt. Bernst. befindI. organ. reste vorw., i: 56 .

4263. Cyphon? - (Dascyllidæ.)

Locality : Rocky River, Sydney Flats, New South Wales. Horizon :

1870. Moore.

Quart. journ. geol. soc. Lond., xxvi : 263. Pl. 18, fig. 11. 
4264. Cytilus varius (recent). (Byrrhidæ.)

Locality: Hösbach, Bavaria. Horizon: Lower Pleistocene.

[1775. Fabricius. 1884. Flach. 1884. " "
Syst. ent., 60.]

Käfer unterpleist. Hösbach, 9 (Citylus), $\quad P l .2$, fig. 5.

Verhandl. physik.-med. gesellsch Würzb., n. f., xviii : 293. $P l$. 9, fig. 5.

4265. Dascyllus cervinus (recent). (Dascyllidæ.)

Locality: Cambridge, England. Horizon: Peat.

[1758. Linné. Syst. nat., ed. 10, i : 376.]

See also Atopa cervina.

4266. Dasytes —. (Malachidæ.)

Locality: Prussian amber. Horizon: Ligurian.

1845. Berendt. Bernst. befindl, organ. reste vorw., i: 56 .

4267. Dermestes —. (Dermestidæ.)

Locality: Prussian amber. Horizon: Ligurian.

1830. Berendt.

1845. " Bernst. befindl. organ. reste vorw., i : 56 .

4268. Dermestes pauper. (Dermestidæ.)

Locality : Oeningen, Baden. Horizon: Tortonian.

1847. Heer. 1852. Giebel. 1856. “

Insektenf. tertiärg. Oeningen, ii: 43-44. Pl. 1, fig. 11.

Deutschl. petref., 654 .

Insect. d. vorwelt, 48.

4269. Dermestes typographus. (Dermestidæ.)

Locality: Copal. Horizon: Recent.

1776. Bloch.

Beschäft. berl. gesellsch. naturf. freunde, ii : 175. Pl. 4, fig. 14.

4270. Diacanthus sutor. (Elateridæ.)

Locality: Oeningen, Baden. Horizon: Tortonian.

1847. Heer.

1852. Giebel.

1856. " "

Insektenf. tertiärg. Oeningen, i; 136-137. Pl. 4, fig. 5.

Dentschl. p̉etref., 651 .

Insect. d. vorwelt, 95 .

4271. Dicælus

(Carabidæ.)

Compared with D. elongatus, United States.

Locality: Port Kennedy, Pa. Horizon: Postpliocene.

1876. Horn.

Trans. Amer. entom. soc., v : 244.

1890. Scudder. Tert. ins. N. A., 525. Pl. 1, fig. 15.

4272. Dicælus alutaceus. (Carabidæ.)

Compared with D. dilatatus, United States.

Locality : Port Kennedy, Pa. Horizon : Postpliocene.

1876. Horn.

Trans. Amer. entom. soc., v : 244.

1889. Lesley.

Dict. foss. Penns., i : p. xix.

1890. Scudder. 
4273. Dicerca bronni. (Buprestidæ.)

Locality: Rott, Rhenish Prussia. Horizon: Aquitanian.

1859. Heyden. Palaeontogr., viii: 4-6. Pl.2, figs.2, 3.

4274. Dicerca carbonum. (Buprestidæ.)

Locality: Boun, Rhenish Prussia. Horizon: Aquitanian?.

1847. Heer. Insektenf. tertiärg. Oeningen, i : 117.

1856. Giebel. Insect. d. vorwelt, 85.

See also Buprestis carbonum, etc.

4275. Dicerca prisca. (Buprestidæ.)

Locality : Oeningen, Baden. Horizon: Tortonian.

1847. Heer. Insektenf, tertiärg. Oeningen, i : 114-117. Pl, 3, fig, 11.

1852. Giebel. Deutschl. petref., 652.

1856. " Insect. d. vorwelt, 86.

4276. Dicerca reticulata. (Buprestidæ.)

Locality: Naumburg, Prussia. Horizon: Oligocene.

1869. Assmann. Beitr. insect. d. vorwelt, 60-61. Figs. $1 a, b$.

1869. " Schles. zeitschr. f. ent., (2), i : 60-61. Figs. 1a, b.

4277. Dicerca taschei. (Buprestidæ.)

Locality : Salzhausen, Germany. Horizon: Miocene.

1856. Heyden. Palaeontogr., iv : 198-199. Pl. 37, figs. 1-4.

1862. " Palaeontogr., $\mathrm{x}: 67-68$.

4278. Dichirotrichus lividus. (Carabidæ.)

Locality: Oeningen, Baden. Horizon: Tortonian.

1862. Heer. Verhandl. holl. maatsch. wetensch. Haarl., xvi : 29-31. Pl. 1, fig. 18.

4279. Dineutes insignis. (Gyrinidæ.)

Compared with D. americanus (Fabr.).

Locality: Oeningen, Baden, Horizon: Tortonian.

1561. Heer. Rech. climat pays tert., 203 (Dineutus) (undescr.).

1862. " Verhandl. holl. maatsch. wetensch. Haarl., xvi: 43-44 (wrong ref. in text to pl. and wrong rame on pl.). Pl. 2, figs. 27,28.

1885. Scudder. Zittel, Handb. palaeont., i, th. ii (wrongly called D. longiventris in text). Fig. 1066.

1886. " Z Zittel-Barrois, Traité de paléont., ii (as above). Fig. 1083.

See also Dineutes longiventris.

4280. Dineutes longiventris. (Gyrinidæ.)

Locality: Oeningen, Baden. Horizon: Tortonian.

1862. Heer. Verbandl, holl. maatsch, wetensch. Haarl, xvi: 44 (Dineutus), Pl. 2, figs. 25, 26.

1865. “ Urwelt der Schweiz. Fig. 281 on $p .381$.

1872. " Monde prim. Suisse. Fig. 281 on p. 467.

1876. " " Prim. world Switz., ii. Fig. 281 on $p .38$.

1879. " Urwelt der Schweiz, $2^{\mathrm{e}}$ aufl. Fig. 32:3 on p. 407.

1885. Scudder. Zittel, Handb. palaeont., i, th. ii : 802 (the fig. belongs to D. insignis).

1886. " Zittel-Barrois, Traité de paléont., ii : 803 (as above).

See also Dinentes insignis.

Dineutus _- (several species). See Dineutes, the same species. 
4281. Diplochila? henshawi. (Carabidæ.)

Compared with D. major, Southern United States.

Locality : Florissant, Colo. Horizon : Oligocene.

1890. Scudder. Tert. ius. N. A., 523-524. Pl. 28, fig. 9.

\section{Donacia - (2 sp.). (Chrysomelidæ.)}

Locality : Mundesley, England. Horizon: Pliocene.

1840. Curtis. Lyell, Proc. geol, soc. Lond., iii : 175.

4283. Donacia -. (Chrysomelidæ.)

Locality: Flanders, Horizon : Postglacial.

1873. Debray. Mém. soc. sc. agric. arts Lille, (3), xi : 451.

1874. " B Bull. soc. géol. France, (3), ii : 48.

\section{Donacia -. (Chrysomelidæ.)}

Locality: Villechetif, Vannes, France. Horizon: Pleistocene.

1876. Fliche. Comptes rendus, Ixxxii : 979.

\section{Donacia - (Chrysomelidæ.)}

Locality: Brunstatt, Alsatia. Horizon: Middle Oligocene.

1890. Foerster. In litt.

\section{Donacia - (Chrysomelidæ.)}

Locality : Nykerk, Holland. Horizon: Peat.

1885. Früh. Jahrb. k. k. geol. reichsanst., $\mathrm{xxxv:679.}$

Donacia appendiculata. See Donacia reticulata. :

4287. Donacia bidens (recent). (Chrysomelidæ.)

Locality : Lefie, Val Gandino, Italy. Horizon: Quaternary.

[1791. Olivier. Encycl. méth., vi: 291.]

1881. Malfatti. Atti soc. ital. sc. nat., xxiv : 91 .

1881. " Bibl. ins. foss. ital., 3.

Donacia clavipes. See Donacia menyanthidis.

4288. Donacia crassipes (recent). (Chrysomelidæ.)

Localities: Vannes, France; Mundesley, England. Horizon: Pleistocene.

[1775. Fabricius. Syst. ent., 195.]

1875. Fliche. Comptes rendus, lxxx: 979.

1888. Bell. Entom., xxi: 2.

4289. Donacia discolor (recent) (1). (Chrysomelidæ.)

Locality: Hösbach, Bavaria. Horizon: Lower Pleistocene.

[1795. Panzer. Ent. germ., 216 (nec Gyll.).]

1884. Flach. Käfer unterpleist. Hösbach, 11. Pl. 2, fig. 14.

1884. " Verhandl, physik.-med. gesellsch. Würzb., n. f., xviii: 295. Pl.

9, fig. 14. 
4290. Donacia discolor (recent) (2). (Chrysomelidæ.)

Localities : Dürnten, Utznach, Lausanne, Switzerland; Chambéry, La Boisse, France; Leffe, Italy. Horizon: Quaternary.

[1813. Gyllenhal. Ins. suec., iii : 660 (nec Panz.).]

1865. Heer. Urwelt der Schweiz, 500,502. Figs. 353-355 on p. 501.

1872. " Monde prim. Suisse, 614,616 . Figs. $353-355$.

1876" " Prim. world Switz., ii : 167. Figs. 353-355 on p. 168.

1876. " Bull. soc. vaud.sc. nat., xiv: 457 .

1876. Lart.-Chantr. Arch. mus. hist. nat. Lyon, i : 104.

1879. Heer: Urwelt der Schweiz, $2^{\mathrm{e}}$ aufl., 530, 532, 533. Figs. 396-398 on p. 531.

1882. Sordelli. Alc. insetti foss. Lomb., 6-9. Fig.

188\%. " Bull. soc. ent. ital., xiv: 230-233. Fig.

4291. Donacia fennica (recent). (Chrysomelidæ.)

Locality : Hösbach, Bavaria. Horizon : Lower Pleistocene.

[1800. Paykull.

Fann. suec., iii : 70.]

1884. Flach.

Käfer unterpleist. Hösbach, 11.

1884. “

Verhandl. physik.-med. gesellsch. Würzb., n. f., xviii : 295.

4292. Donacia genini. (Chrysomelidæ.)

Compared with D. violacea Fabr., D. nigra Latr.

Locality: Sonnaz, Savoy, France. Horizon: Quaternary?.

1850. Mortillet. "Bull. soc. hișt. nat. Savoie, 1850 : 135."

1850. " " "Les Alpes, 1850, No. 5."

1850. " Arch. sc. phys. et nat., xv: 78-79 (genin).

4293. Donacia letzneri. (Chrysomelidæ.)

Locality: Schossnitz, Silesia. Horizon: Miocene.

1869. Assmann. Beitr. insect. d. vorwelt, 42-43. Pl. 1, figs. 5, 5a.

1869. " Schles. zeitschr. f. ent., (2), i: 42-43. Pl. 1, figs. 5, 5a.

4294. Donacia lignitum. (Chrysomelidæ.)

Compared with D. sericea.

Locality: Leffe, Italy. Horizon: Quaternary.

1882. Sordelli. Alc. insetti foss. Lomb., 9-10. Fig. on p. 8.

1882. " Bull. soc. ent. ital., xiv: 233-234. Fig. on p. 232.

4295. Donacia linearis (recent). (Chrysomelidæ.)

Locality : Norfolk forest, Mundesley, England. Horizon: Pleistocene.

[1795. Hoppe. Euum. ins. Erlang., 46. Fig. 10.]

1888. Bell. Entom., $\mathrm{xxi}: 2$.

See also Donacia simplex.

4296. Donacia menyanthidis (recent). (Chrysomelidæ.)

Localities: Chambéry, La Boisse, Savoy, France. Horizon: Quaternary ?

[1792. Fabricins. Ent. sys., i, ii: 117 (= clavipes).]

1865. Heer. Urwelt der Schweiz, 502.

1872. " Monde prim. Suisse, 616.

1876. "6 Prim. world Switz., ii : 170.

1876. Lart. Chantr. Arch. mus. hist. nat. Lyon, i : 104 (as of Mortillet).

1879. Heer. Urwelt der Schweiz, $2^{\circ}$ aufl, 532. 


\section{Donacia obscura (recent). (Chrysomelidø.)}

\section{Locality : Hösbach, Bavaria. Horizon : Lower Pleistocene.}

[1813. Gyllenhal. Ins. suec., iii : 654.]

1884. Flach.

Käfer unterpleist. Hösbach, 11.

1884. " Verhandl. physik.-med. gesellsch. Würzb., n. f., xviii : 295.

4298. Donacia palæmonis. (Chrysomelidæ.)

Locality: Oeningen, Baden. Horizon: Tortonian.

1847. Heer. Insektenf. tertiärg. Oeningen, i: 200-202. Pl. 6, fig. 4.

1852. Giebel. Deutschl. petref., 648 .

1856. “ Insect. d. vorwelt, 116.

1865. Heer. Urwelt der Schweiz, 362.

1872. " Monde prim. Suisse, 444.

1876. " Prim. world Switz., ii: 16.

1879. " Urwelt der Schweiz, $2^{e}$ aufl., 388.

4299. Donacia parvula. (Chrysomelidæ.)

Locality: Cap Staratschin, Spitzbergen. Horizon: Miocene.

1870. Heer. K. svenska vetensk.-akad. handl., viii, No. vii : 75-76. Pl. 16, figs. 32-33.

4300. Donacia pompatica. (Chrysomelidæ.)

Compared with D. pubicollis Suffr.

Locality : Scarboro', Ontario. Horizon : Postpliocene.

1890. Scudder. Tert. ins. N. A., 486-487. Pl.1, figs. 33, 34.

4301. Donacia reticulata (recent). (Chrysomelidæ.)

Locality : Leffe, Val Gandino, Italy. Horizon : Quaternary marl.

[1817. Gyllenhal. Schönh., Syn. ins., App. iii : 37 (= appendiculata).]

1881. Malfatti. Atti soc. ital. sc. nat., xxiv: 91.

1881. " Bibl. insetti foss. ital., 3.

4302. Donacia sagittariæ (recent). (Chrysomelidæ.)

Localities: Leffe, Italy; Hösbach, Bavaria. Horizon : Pleistocene.

[1792. Fabricius. Ent. syst., i, ii: 11\%.]

1882. Sordelli. Alc. insetti foss. Lomb., 6-9. Fig.

1882. " Bull. soc. ent.ital., xiv: 230-233. Fig.

1884. Flach. Käfer unterpleist. Hösbạch, 11. Pl. 2, fig. 12.

1884. “ Verhandl. physik.-med. gesellsch. Würzb., n. f., xviii : 295 . Pl. 9, fig. 12.

\section{Donacia sericea (recent). (Chrysomelidæ.)}

Localities: Dürnten, Schwerzenbach, Ardres, Switzerland ; Hösbach, Bavaria ; Mundesley, England. Horizon: Pleistocene.

[1761. Linu6. 1858. Heer. 1865. " 1872. . "

1876. " "

1878. Debray.

1879. Heer.

1884. Flach.

1884. “

1888. Bell.
Faun. suec., 196.]

Arch. sc. phys. et nat., n. p., ii : 322 .

Urwelt der Schweiz, 500-501. Fig. 352.

Monde prim. Suisse, 614-615. Fig. 352.

Prim. world Switz., ii : 167. Fig. 352 on p. 168.

Ann. soc. géol. nord, v: 127.

Urwelt der Schweiz, 2e aufl., 530,581. Fig. 395 on p. 531.

Käfer unterpleist. Hösbach, 11.

Verhandl. physik.-med, gesellsch. Würzb., n. f., xviii : 295.

Entom., xxi : 2. 


\section{Donacia simplex (recent). (Chrysomelidæ.)}

Locality: England. Horizon: Pleistocene.

[1775. Fabricius. Syst. ent., 195.]

See also Donacia linearis.

4305. Donacia smittiana. (Chrysomelidæ.)

Locality: Cap Staratschin, Spitzbergen. Horizon: Miocene.

1870. Heer. K. svenska vetensk.-akad. handl, viii, No. vii: 76. Pl. 16, figs. 30-31.

4306. Donacia stiria. (Chrysomelidæ.)

Compared with D. porosicollis Lac.

Locality: Scarboro', Ontario. Horizon: Postpliocene.

1890. Scudder. Tert. ins. N. A., 486. Pl. 1, fig. 28.

4307. Donacia thalassina (recent.) (Chrysomelidæ.)

Locality : Hösbach, Bavaria. Hórizon : Lower Pleistocene.

[1811. Germar. Neue schrift. gesellsch. Halle, i, vi : 29.]

1884. Flach. Käfer unterpleist. Hösbach, 11. Pl. 2, fig. 13.

1884. " Verhandl. physik.-med. gesellsch. Würzb., n. f., xviii: 295. Pl. 9, fig. 13.

4308. Dorcadion emeritum. (Cerambycidæ.)

Locality : Rott, Rhenish Prussia. Horizon : Aquitanian.

1862. Heyden. Palaeontogr., $\mathrm{x}:$ 71-72. Pl. 10, fig. 14.

4309. Dorcadionoides subæneus. (Cerambycidæ.)

Loeality: Prussian amber. Horizon: Ligurian.

1856. Motschulsky. Etudes entom., v: 27.

4310. Dorcasoides bilobus. (Lucanidæ.)

Locality: Prussian amber. Horizon: Ligurian.

1856. Motschulsky. Etudes entom., v : 27 (in the pl. called nigrescens, by confusion probably with Scydmænoides nigrescens). Pl., fig. 1.

1885. Scudder. Zittel, Handb. palaeont, i, th. ii: 795. Fig. 1028.

1886. " Zittel-Barrois, Traité de paléont., ii : 795. Fig. 1046.

Dorcasoides nigrescens. See Dorcasoides bilobus.

4311. Dorcatoma —. (Ptinidæ.)

Locality: Prussian amber. Horizon: Ligurian.

1845. Berendt. Bernst. befindl. organ. reste vorw., i : 56.

4312. Dorcatoma -. (Ptinidæ.)

Locality: Brunstatt, Alsatia. Horizon: Middle Oligocene.

1890. Foerster. In litt.

4313. Dorcatoma -. (Ptinidæ.)

Locality: Prussian amber. Horizon: Ligurian.

1856. Menge. Progr. petrischule Danzig, 1856: 21 ,

Bull, $71-33$ 
4314. Dorcus (Lucanidæ.)

Locality: Gurnet Bay, Isle of Wight. Horizon: Bembridge Limestone. 1879. Woodward. Quart. journ. geol, soc. Lond., xxxv : 344. $1879 . \quad$ " Geol. mag., n. s., v: 89.

4315. Dorcus (Eurytrachelus) primigenius. (Lucanidæ.)

Compared with E. saiga and E. platymelus.

Locality : Kutschlin, Bohemia. Horizon: Miocene.

1881. Deichmüller. Foss. ins, diatomeensch. Kutschlin, 11. Pl. 21, figs. 1 ab.

1881. " Verhandl. leop.-carol. akad. naturf., xlii: 303. Pl. 21, figs. 1 ab.

Doritomus —. See Dorytomus — Keferst.

4316. Dorytomus -. (Curculionidæ.)

Locality: Prussian amber. Horizon : Ligurian.

1834. Keferstein. Naturg. erdkörp., ii: 330 (Doritomus):

4317. Dorytomus —. (Curculionidæ.)

Locality: Aix, France. Horizon: Ligurian.

1829. Serres. Géogn. terrains tert., 224.

4318. Dromius -. (Carabidæ.)

Locality: Prussian amber. Horizon: Ligurian.

1845. Berendt. Bernst. befindl. organ. reste vorw., i : 56 .

1845. Motschulsky. Bull. soc. imp. nat. Mosc., xviii, ii: 98.

4319. Dromius -. (Carabidæ.)

Locality : Prussian amber. Horizon: Ligurian.

1832. Burmeister. Handb. ent., i: 635.

1836. " Man. ent., 576.

4320. Dromius - (Carabidæ.)

Compared with D. agilis.

Locality : Prussian amber. Horizon: Ligurian.

1856. Menge. Progr. petrischule Danzig, 1856: 23.

4321. Dromius ——. (Carabidæ.)

Compared with D. quadrimaculatus.

Locality : Prussian amber. Horizon: Ligurian.

1856. Menge. Progr. petrischule Danzig, 1856: 23.

4322. Dromius resinatus. (Carabidæ.)

Locality: Prussian amber. Horizon: Ligurian.

1856. Giebel. Insect. d. vorwelt, 70.

See also Lebina resinata.

4323. Dryocætes carbonarius. (Scolytidæ.)

Locality: Green River, Wyo. Horizon: Oligocene.

1878. Scudder. Bull. U. S. geol. surv. terr., iv : 768.

1890. " Tert. ins. N. A., 470-471. Pl. 8, fig. 6, 
4324. Dryocætes impressus. (Scolytidæ.)

Compared with D. affaber (Mann.).

Locality: Green River, Wyo. Horizon: Oligocene.

1878. Scudder. Bull. U. S. geol. surv, terr., iv: 767-768.

1890. " Tert. ins. N. A., 470. Pl. 8, fig. 28.

See also Trypodendron impressum.

Dyticus —. See Dytiscus — Germ.

4325. Dytiscus -. (Dytiscidæ.)

Locality : Crofthead in Glasgow, Scotland. Horizon: Pleistocene. 1888. Bell.

Entom., xxi : 2.

4326. Dytiscus - (larva). (Dytiscidæ.)

Locality : Vicinity Bonn, Rhenish Prussia. Horizon : Aquitanian?.

1837. Germar. Fauna insect. Europæ, xix: 1 (Dyticus). Pl. 1.

1852. Giebel. Dentschl. petref., 655.

1856. " Insect. d. vorwelt, 58.

4327. Dytiscus - (Dytiscidæ.)

Locality: Rhine, Germany. Horizon: Aquitanian :.

1831. Goldfuss. Verhandl. leop-carol. akad. naturf, vii, i: 118.

4328. Dytiscus … (Dytiscidæ.)

Compared with D. cinereus.

Locality: Aix, France. Horizon: Ligurian.

1829. Serres. Géogn. terrains tert., 221.

4329. Dytiscus —. (Dytiscidæ.)

Locality: Gurnet Bay, Isle of Wight. Horizon: Bembridge Limestone。 1879. Woodward. Geol. mag., n. s., v : 89.

4330. Dytiscus areolatus. (Dytiscidæ.)

Compared with D. marginalis Linn., Europe.

Locality: Oeningen, Baden. Horizon: Tortonian.

1861. Heer. Rech. climat pays tert., 204 (undescr.).

4331. Dytiscus avunculus. (Dytiscidæ.)

Locality: Höhgau, Germany. Horizon: (Miocene?).

1862. Heyden. Palaeontogr., $\mathrm{x}:$ 81-82. Pl. 10, fig. 39.

4332. Dytiscus lavateri. (Dytiscidæ.)

Compared with D. marginalis L., Europe.

Locality: Oeningen, Baden. Horizon: Tortonian.

1847. Heer. 1852. Giebel.

1856. " "

1862. Heer.
Insektenf. tertiärg. Oeningen, i : 24-25, 220-221. Pl. 1, fig. 6.

Deutschl. petref., 654 .

Insect. d. vorwelt, 57 .

Verhandl. holl. maatsch. wetensch. Haarl., xvi: 36-37: Pl. 2, figs, 11-14. 
1865. Heer.

1872. "

1876. " "

1879. " "

1885. Scudder.

1886. “ “

1889. Nicholson.

Urwelt der Schweiz, 361, 383. Figs. $279 a b$ on $p .381$.

Monde prim. Suisse, 443, 470. Figs. 279ab on p. 467.

Prim. world Switz., ii : 15, 40. Figs. $279 a b$ on $p .38$.

Urwelt der Schweiz, $2^{\circ}$ aufl., 387, 409. Fig. 321 on p. 407.

Zittel, Handb. palaeont., i, th. ii: 803. Fig. 1067.

Zittel-Barrois, Traité de paléont., ii: 804. Fig. 1084.

Man. palaeont., $3^{\text {d }}$ ed., i : 601 . Fig. $449 a$.

4333. Dytiscus œningensis. (Dytiscidæ.)

1847. Heer.

1852. Giebel.

Locality : Oeninges, Baden. Horizon: Tortonian.

1856. “

Insektenf. tertiärg. Oeningen, i : 26-27. $P l$. 1, fig. 7.

Deutschl. petref., 654 .

Insect. ๙. vorwelt, 57-58.

4334. Dytiscus zersii. (Dytiscidæ.).

Compared with D. marginalis.

Locality : Morla, vicinity Bergamo, Italy. Horizon: Quaternary.

1882. Sordelli. Alc. insetti foss. Lomb., 10-12. Fig.

1882. " Bull. soc. ent. ital., xiv: 234-236. Fig.

4335. Dytiscus zschokkeanus. (Dytiscidæ.)

Locality : Oeningen, Baden. Horizon: Tortonian.

1847. Heer.

1852. Giebel.

Insektenf. tertiärg. Oeningen, i : 26. $\quad P l .8, f i g .3$.

Dentschl. petref., 654.

1856. " Insect. d. vorwelt, 57.

4336. Ëbæus —. (Malachidæ.)

Locality: Prussian amber. Horizon: Ligurian.

1845. Berendt. Bernst. befindl. organ. reste vorw., i: 56.

4337. Elaphrus irregularis. (Carabidæ.)

Compared with E. viridis Horn.

Locality : Scarboro', Ontario. Horizon : Postpliocene.

1890. Scudder. Tert. ins. N. A., 534. $P l .1, f i g .56$.

4338. Elater - (Elateridæ.)

Locality : Prussian amber. Horizon: Ligurian.

1827. Brongniart. Dict. sc. nat., li : 233 (6laters).

1829. Serres. Géogn. terrains tert., 240 (sternoxes du genre Elater).

1834. Keferstein. Naturg. erdkörp., ii : 329 (sternopes).

\section{Elater - (Elateridæ.)}

Compared with E. æneus.

Locality: Utznach, Switzerland. Horizon: Quaternary。

1839. Brulle. Gisem. ins. foss., 20.

4340. Elater - (Elateridæ.)

Compared with E. cylindricus Gyll.

Locality: Prussian amber. Horizon: Ligurian.

1832. Burmeister. Handb. ent., i: 635.

1836. " Man, ent., 576-577. 
4341. Elater

(Elateridæ.)

Locality: Mundesley, England. Horizon: Pliocene.

1840. Curtis.

Lyell, Proc. geol. soc. Lond., iii: 175.

4342. Elater -. (Elateridæ.)

Locality : Prussian amber. Horizon: Ligurian.

1835. Gravenhorst. Uebers. schles. gesellsch. vaterl. cult., 1834 : 92.

4343. [Elater] - (Elateridæ.)

Locality : Prussian amber. Horizon: Ligurian.

1825. Guérin. Dict. class. d'hist. nat., viii: 580 (taupin).

\section{Elater -. (Elateridæ.)}

Locality : St. Jakob, Basel, Switzerland. Horizon: Interglacial.

1879. Heer.

Urwelt der Schweiz, $2^{\mathrm{e}}$ anfl., 533.

4345. Elater (2 sp.). (Elateridæ.)

Compared with E. niger.

Locality: Aix, France. Horizon: Ligurian.

1847. Hope. Trans. ent. soc. Lond., iv : 251.

\section{Elater - (Elateridæ.)}

Compared with E. æneus.

Locality : Prussian amber. Horizon : Ligurıan.

1829. Serres.

Géogn. terrains tert., 240.

4347. Elater -. (Elateridæ.)

Compared with E. castaneus.

Locality: Prussian amber. Horizon: Ligurian.

1829. Serres. Géogn. terrains tert., 240.

4348. Elater ㄴ. (Elateridæ.)

Compared with E. variabilis, E. pilosus.

Locality: Prussian amber. Horizon: Ligurian.

1829. Serres. Géogn. terrains tert., 240-241.

4349. Elater -. (Elateridæ.)

Locality : Peckham, England. Horizon: Paludina beds, Lower Eocene.

1861. Smith. Geologist, iv: 40.

4350. Elater ehrenwærdi. (Elateridæ.)

Locality: Cap Staratschin, Spitzbergen. Horizon : Miocene.

1870. Heer. K. svenska vetensk.-akad., handl. viii, vii : 74-75. Pl. 16, figs.1a, $2-4,4 b$.

4351. Elater holmgreni. (Elateridæ.)

Locality : Cap Staratschin, Spitzbergen. Horizon: Miocene.

1870. Heer. $\quad$ K. svenska vetensk.-akad. handl., viii, vii : 75. Pl. 16, figs. 5-7. 
4352. Elater linearis. (Elateridæ.)

Locality : Mundesley, England.
1888. Bell.

Horizon: Pleistocene.

4353. Elater naumanni. (Elateridæ.)

Locality : Prussian amber. Horizon: Ligurian.

1856. Giebel. Insect. d. vorwelt, 91.

4354. Flater (Alaus) spectabilis. (Elateridæ.)

Locality: Oeningen, Baden. Horizon: Tortonian.

1865. Heer.

1872. " "

1876. "

1879. “

1776. Bloch.

Urwelt der Schweiz. Fig. 262 on p. 378.

Monde prim. Suisse. Fig. 262 on p. 464.

Prim. world Switz., ii. Fig. 262 on $p .34$.

Urwelt der Schweiz, $2^{\mathrm{e}}$ anfl. Fig. 304 on p. 404.

4355. Flater testaceus. (Elateridæ.)

Locality: Copal. Horizon : Recent.

Beschäft. berl. gesellsch. naturf, freunde, ii : 183-184. $P l .5, f i g$. 24

4356. Elaterites amissus. (Elateridæ.)

Locality: Greith, Hohe Rhönen. Horizon: Aquitanian.

1847. Heer. Insektenf. tertiärg. Oeningen, i: 14\%-143. Pl. 4, figs. 9, $9 b$. 1852. Giebel. Deutschl. petref., 651.

1856. “ Insect. d. vorwelt, 94 .

4357. Elaterites dicrepidioides. (Elateridæ.)

Locality : Kutschlin, Bohemia. Horizon: Miocene.

1881. Deichmüller. Foss. ins. diatomeensch. Kutschlin, 16-19. Pl. 21, figs. 5a-e.

1881. " Verhandl. leop.-carol. akad. naturf., xlii: 308-311. Pl. 21, figs. $5 a-e$.

4358. Elaterites lavateri. (Elateridæ.)

Locality : Oeningen, Baden. Horizon: Tortonian.

1847. Heer.

1852. Giebel.

Insektenf. tertiärg. Oeningen, i: 141-142. Pl. 4, fig. 8.

Deutschl. petref., 651 .

Insect. d. vorwelt, 94 .

4359. Elaterites obsoletus. (Elateridæ.)

Locality : (Oeningen ?, Baden). Horizon: Tortonian.

1847. Heer.

Insektenf. tertiärg. Oeningen, i : 142.

1852. Giebel.

Dentschl. petref., 651 .

1856. " Insect. d. vorwelt, 94 .

4360. Elaterium murchisoni. (Elateridæ.)

Locality : Vicinity Corfe, England. Horizon: -

1854. Westwood. Proc. geol. soc. Lond., 395 (unnamed), Pl. 16, fig. 34.

1856. Giebel.

Insect. d. vorwelt, 93.

Elatior linearis. See Elater linearis. 
4361. Elytridium deplanatum.

Locality : Cap Staratschin, Spitzbergen. Horizon: Miocene.

1870. Heer. $\quad$ K. svenska vetensk.-akad. handl., viii, vii : 77-78. Pl. 16, figs. $41 a, c$.

4362. Elytridium rugulosum.

Locality: Cap Staratschin, Spitzbergen. Horizon: Miocene.

1870. Heer. $\quad$ K. svenska vetensk.-akad. handl., viii, vii: 78. $P l .16$, figs. 36-37.

4363. Elytridium scabriusculum.

Locality: Cap Staratschin, Spitzbergen. Horizon: Miocene.

1870. Heer. $\quad$ K. svenska vetensk.-akad. handl., viii, vii: 78. Pl. 16, figs. 28-29.

4364. Elytridium undecimstriatum.

Locality: Cap Staratschin, Spitzbergen. Horizon: Miocene.

1870. Heer.

K. svenska vetensk.-akad. handl., viii, vii : 77. $\quad P l$. 16, figs. 21-22.

4365. Entimus primordialis. (Otiorhynchidæ.)

Locality : White River, Colo. Horizon: Oligocene.

1876. Scudder. Bull. U. S. geol. surv. terr., ii : 84 .

1877. " Bull. U. S. geol. surv, terr., iii : 759.

1885. " Z Zittel, Handb. palaeont., i, th. ii: 789. Fig. 1011.

1886. " "Zittel-Barrois, Traité de paléont., ii : 789. Fig. 1028.

1890. “ Tert. ins. N. A., 474-475. Pl. 5, figs. 109, 109a.

4366. Epicærus effossus. (Otiorhynchidæ.)

Locality: Green River, Wyo. Horizon: Oligocene.

1878. Scudder. Bull. U. S. geol. surv. terr., iv : 765 .

1890. " Tert. ins. N. A., 480-481. Pl. 8, figs. 7, 35.

See also Eudiagogus effossus.

4367. Epicærus exanimis. (Otiorhynchidæ.)

Locality: Green River, Wyo. Horizon: Oligocene.

1878. Scudder. Bull. U. S. geol. surv. terr., iv : 765.

1890. “ Tert. ins. N. A., 479-480. Pl. 8, figs. 30, 31, 38, 42.

See also Eudiagogus exanimis.

4368. Epicærus saxatilis. (Otiorhynchidæ.)

Compared with E. griseus Schönh., Mexico.

Locality: Green River, Wyo. Horizon: Oligocene.

1878. Scudder. Bull. U.S. geol. surv. terr., iv: 765.

1890. " Tert. ins. N. A., 478-479. Pl. 8, figs. 33, 34, 36.

See also Eudiagogus saxatilis.

4369. Epiphanis deletus. (Elateridø.)

Compared with E. cornutus.

Locality: White River, Utah. Horizon: Oligocene.

1876. Scudder. Bull. U. S. geol. surv. terr., ii : 80-81.

1877. " Bull. U. S. geol. surv. terr., iii : 759.

1890. “ Tert. ins. N. A., 498. Pl. 5, figs. 113, 114. 
4370. Erinnys deleta. (Staphylinidæ.)

Locality: Aix, France. Horizon: Ligurian.

1874. Oustalet. Ann. se. g6́ol., v, art., 2: 145-146. Pl. 2, fig. 16.

See also Lithoplanes

4371. Frinnys elongata. (Staphylinidæ.)

Locality: Aix, France. Horizon: Ligurian.

1874. Oustalet. Ann. sc. géol., $\nabla$, art. 2: 144-145. Pl. 2, fig. 15.

See also Lithoplanes

4372. Erirhinoides cariniger. (Curculionidæ.)

Locality: Prussian amber. Horizon: Ligurian.

1856. Motschulsky. Études entom., v : 27.

4373. Erirhinus chantrei. (Curculionidæ.)

Compared with E. (Notaris) scirpi Fabr., Europe.

Locality : Aix, France. Horizon: Ligurian.

1874. Oustalet. Ann. sc. géol., v, art. 2: 289-290. Pl. 3, fig. 19.

4374. Erycus acridulus (recent). (Curculionidæ.)

Locality : Hösbach, Bavaria. Horizon : Lower Pleistocene.

[1858. Linné. 1884. Flach.

1884. “
Syst. nat., 378.]

Käfer unterpleist. Hösbach, 10. Pl. 2, figs. 8a-c.

Verhandl. physik.-med. gesellsch. Würzb., n. f., xviii : 294. $\quad P l$. 9, figs. $8 a-c$.

\section{Erycus æthiops (recent.) (Curculionidæ.)}

Locality: Hösbach, Bavaria. Horizon: Lower Pleistocene.

[1792. Fabricius.

1884. Flach.

1S84. “
Ent. syst., i, ii : 405.]

Käfer unterpleist. Hösbach, 10. Pl. 2, fig. 7.

Verhandl. physik.-med. gesellsch. Würzb., n. f., xviii : 294. $\quad P l$. 9, fig. 7.

4376. Escheria - (4 sp.). (Hydrophilidæ.)

Locality: Brunstatt, Alsatia. Horizon: Middle Oligocene.

1890. Foerster. In litt.

4377. Escheria bella. (Hydrophilidæ.)

Locality: Oeningen, Baden. Horizon: Tortonian.

1862. Heer.

1865. 66

1872. 6

1876. " "

1879. “

1885. Scudder.

1886.

Verhandl. holl. maatsch. wetensch. Haarl., xvi : 69-70. Pl. 5, fig. 17.

Urwelt der Schweiz. Fig. 273 on $p .381$.

Monde prim. Suisse. Fig. 273 on $p .467$.

Prim. world Switz., ii. Fig. 273 on $p .38$.

Urwelt der Schweiz, $2^{\mathrm{e}}$ aufl. Fig. 315 on $p .407$.

Zittel, Handb. palaeont., i, th. ii : 802. Fig. 1063.

Zittel-Barrois, Traité de paléont., ii : 803. Fig. 1080.

4378. Escheria ovalis. (Hydrophilidæ.)

Locality : (Oeningen?, Baden.) Horizon: Tortonian.

1847. Heer. Insektenf. tertiärg. Oeningen, i: 57-60 (ovata on pl.). Pl. 7, fig. 23. 
1852. Giebel. Deutschl. petref., 653.

1853-56. Bronn. Lethæa geogn., $3^{\mathrm{e}}$ aufl., iii : 648, Pl. 42', figs. $26 a b$.

1856. Giebel. Insect. d. vorwelt, 51-52.

Escheria ovata. See Escheria ovalis.

4379. Escheria ? protogeæ. (Hydrophilidæ.)

Locality: Vicinity Bonn, Rhenish Prussia. Horizon: Aquitanian.

1847. Heer.

Insektenf. tertiärg. Oeningen, i : 60 .

1856. Giebel.

Insect. d. vorwelt, 52 .

See also Coccinella protogeæ.

4380. Eucnemis —. (Elateridæ.)

Locality: Prussian amber. Horizon: Ligurian.

1845. Berendt. Bernst. befindl. organ, reste vorw., i : 56.

4381. Eudiagogus effossus. (Otiorhynchidæ.)

Locality: Green River, Wyo. Horizon: Oligocene.

1876. Scudder. Bull. U. S. geol. surv. terr., ii : 85-86.

See also Epicærus effossus.

4382. Eudiagogus exanimis. (Otiorhynchidæ.)

Locality: Green River, Wyo. Horizon: Oligocene.

1876. Scudder. Bull. U. S. geol. surv. terr., ii : 85.

See also Epicærus exanimis.

4383. Eudiagogus saxatilis. (Otiorhynchidæ.)

Locality: Green River, Wyo. Horizon: Oligocene. 1876. Scudder. Bull. U. S. geol. surv. terr., ii : 84-85.

See also Epicærus saxatilis.

4384. Eudiagogus terrosus. (Otiorhynchidæ.)

Locality: Green River, Wyo. Horizon: Oligocene. 1878. Scudder. Bull. U. S. geol. surv. terr., iv : 766-767.

1890. “ Tert. ins. N. A., 475. Pl. 8, fig. 29.

4385. Eugnamptus decemsatus. (Rhynchitidæ.)

Locality: Green River, Wyo. Horizon: Oligocene.

1878. Scudder. Bull. U. S. geol. surv, terr., iv : 764-765.

1890. " Tert. ins. N. A., 482. Pl. 8, fig. 12.

4386. Eugnamptus grandævus. (Rhynchitidæ.)

Locality: Green River, Wyo. Horizon: Oligocene.

1890. Scudder. Tert. ins. N. A., 481-482. Pl. 8, fig. 20.

See also Sitones grandærus.

4387. Eunectes antiquus. (Dytiscidæ.)

Locality : Corent, France. Horizon: Aquitanian.

1870. Oustalet. Ann. se. géol., ii, art, 3: 56-57. Pl. 1, figs. 1, 2.

4388. Euplectus —_. (Pselaphidæ.)

Locality: Prussian amber. Horizon: Ligurian.

1845. Berendt. Bernst, befindl, organ. reste vorw., i: 56. 
4389. Eupsinoides glabrellus. (Pselaphidæ.)

1856. Motschulsky. Études entom., v: 26-27.

Locality: Prussian amber. Horizon: Ligurian.

4390. Eurhinus occultus. (Curenlionidæ.)

'Locality: Florissant, Colo. Horizon: Oligocene.

1876. Scudder. Bull. U. S. geol. surv. terr., ii : 87.

4391. Furychirus (Otiorhynchus) induratus. (Otiorhynchidæ.)

Loeality: Rott, Rhenish Prussia. Horizon: Aquitanian.

1866. Heyd.-Heyd. Palaeontogr., xv: 149-150. ("Perhaps forms a new genus to which perhaps belong Sphenophorus nægelianus Heer and S. regelianus Heer.") Pl. 23, figs. 16, 17.

1866. “ Käfer u. polypen, 19-20. Pl. 2, figs. 16, 17.

1870. L. Heyden. Foss. dipt. Rott, 29.

1870. “ Palaeontogr., xvii : 265.

4392. Eurythyrea longipennis. (Buprestidæ.)

1847. Heer.

1852. Giebel.

Locality: Oeningen, Baden. Horizon: Tortonian.

1856. " Insect. d. vorwelt, 85.

Eurytrachelus primigenius. See Doreus (Eurytrachelus) primigenius. 4393. Feronia —. (Carabidæ.)

Compared with F. leucophthalma.

Locality: Utznach, Switzerland. Horizon: Quaternary.

1839. Brull6. Gisem. ins. foss., 20.

4394. Feronia (Pœcilus) —- (Carabidæ.)

Locality: Hösbach, Bavaria. Horizon: Lower Pleistocene.

1884. Flach. Käfer unterpleist. Hösbach, 5.

1884. “ Verhandl. physik.-med. gesellsch. Würzb., n. f., xviii : 289.

4395. Feronia (Pterostichus) - (Carabidæ.)

Locality : Hösbach, Bavaria. Horizon : Lower Pleistocene.

1884. Flach, Käfer unterpleist. Hösbach, 5 .

1884. “ Verhandl. physik.-med. gesellsch. Würzb., n.f., xviii : 289.

4396. Feronia (Steropus) æthiops (recent). (Carabidæ.)

Locality: Hösbach, Bavaria. Horizon: Lower Pleistocene.

[1797. Panzer. Faun. germ., xxxvii : 22.]

1884. Flach. Käfer unterpleist. Hösbach, 5. Pl. 1, fig. 7.

1884. “ Verhandl. physik.-med. gesellsch. Würzb., n. f., xviii: 289. Pl. 8, fig. 7.

4397. Feronia (Argutor) diligens (recent). (Carabidæ.)

Locality: Hösbach, Bavaria. Horizon: Lower Pleistocene.

[1824. Sturm. Deutschl. fauna, Ins., v: 81. Pl. 117, figs. a, A.]

1884. Flach. Käfer unterpleist. Hösbach, 6. Pl. 1, fig. 8.

1884. " Verhandl, physik.-med, gesellsch, Würzb,, n. f., xviii : 290. Pl. 8, fig. 8. 
4398. Feronia minax. (Carabidæ.)

Locality: Aix, France. Horizon: Ligurian.

1874. Oustalet. Ann. sc. géol., v, art. 2: 102-104. Pl. 1, fig. 6:

4399. Feronia (Platysma) oblongopunctata (recent). (Carabidæ.)

Locality: Hösbach, Bavaria. Horizon: Lower Pleistocene.

[1801. Fabricias. Syst. eleuth., i: 183.]

1884. Flach. Käfer unterpleist. Hösbach, 5. Pl. 1, fig. 6 ,

1884. " Verhandl. physik.-med. gesellsch. Würzb., n. f., xviii: 289. $P l$. 8, fig. 6.

4400. Feronia (Abax) parallela (recent). (Carabidæ.)

Locality: Hösbach, Bavaria. Horizon: Lower Pleistocene.

[1812. Duftschmid. Faun. austr., ii : 64.]

1884. Flach. Käfer unterpleist. Hösbach, 6.

1884. " Verhandl. physik.-med. gesellsch. Würzb., n. f., xviii : 290.

4401. Feronia provincialis. (Carabidæ.)

Loeality: Aix, France. Horizon: Ligurian.

1874. Oustalet. Ann. sc. g6́ol., v, art. 2: 105-106. Pl. 1, fig. 4.

4402. Fusslinia amœna. (Buprestidæ.)

Locality : Oeningen, Baden. Horizon: Tortonian.

1847. Heer.

1852. Giebel. 1853-56. Bronn. 1856. Giebel. 1885. Scudder. 1886. “
Insektenf. tertiärg. Oeningen, i : 123-128. Pl. 6, fig. 4.

Deutschl. petref., 651.

Lethæa geogn., $3^{e}$ aufl, iii : 64\%. Pl. 42', figs. 28a-c.

Insect, d. vorwelt, 89-90.

Zittel, Haudb. palaeont., i, th. ii: 797. Fig. 1035.

Zittel-Barrois, Traité de paléont., ii : 797. Fig. 1053.

Locality : Prussian amber. Horizon: Ligurian.

1845. Berendt. Bernst. befindl. organ. reste vorw., i: 56 (Galleruca).

4404. Galeruca —. (Chrysomelidæ.)

Locality: Prussian amber. Horizon: Ligurian.

1832. Burmeister. Handb. ent., i: 635 (Galleruca).

1836. “ Man. ent., 577.

4405. Galeruca -. (Chrysomelidæ.)

Locality: Prussian amber. Horizon: Ligurian.

1856. Menge. Progr. petrischule Danzig, 1856: 21.

4406. Galeruca -. (Chrysomelidæ.)

Locality: Prussian amber. Horizon: Ligurian.

1838. Robert. Bull. soc. g6ol. France, ix: 114.

\section{Galeruca aichnorni. (Chrysomelidæ.)}

Locality: Radoboj, Croatia. Horizon: Mayencian.

1858. Heer. Ber. versamml, deutsch. naturf., xxxii : 119 (undescr.). 
4408. Galeruca buchi. (Chrysomelidæ.)

Compared with G. halensis.

Locality: Oeningen, Baden. Horizon: Tortonian.

1865. Heer.

1872. " "

1876. “

1879. “

Urwelt der Schweiz, 372 (Galleruca). Fig. 236 on p. 371.

Monde prim. Suisse, 456. Fig. 236 on $p .455$.

Prim. world Switz., ii : 26. Fig. 236 on p. 27.

Urwelt der Schweiz, $2^{\mathrm{e}}$ aufl., 397. Fig. 278.

4409. Galeruca gemmifera. (Chrysomelidæ.)

Compared with G. (CEdionychus) decemguttata, Cuba, Brazil.

Locality : Oeningen, Baden. Horizon: Tortonian.

1879. Heer. Urwelt der Schweiz, $2^{\mathrm{e}}$ aufl., 397 (Galleruca). Fig. 262 on p. 384.

4410. Galerucella ——. (Chrysomelidæ.)

Locality: Brunstatt, Alsatia. Horizon: Middle Oligocene.

1890. Foerster. In litt.

4411. Galerucella picea. (Chrysomelidæ.)

Compared with G. maritima LeC.

Locality: Nine-mile Creek, British Columbia. Horizon:

1879. Scudder. Rept. progr. geol. surv. Can., 1877-78: B182-183 (Gallerucella).

1879. " " Insect. tert. Nicola, 7-8.

1890. “ "Tert. ins. N. A., 485. Pl. 2, fig. 31.

Galleruca (several species). See Galeruca, the same species.

Gallerucella picea. See Galerucella picea.

4412. Geotrupes - (Scarabæidæ.)

1888. Bell. Entom., xxi: 2.

4413. Geotrupes? - (Scarabæidæ.)

Locality: Aix, France. Horizon: Ligurian.

1847. Hope. Trans. ent. soc. Lond., iv : 251.

4414. Geotrupes atavus. (Scarabæidæ.)

Locality : Aix, France. Horizon: Ligurian.

1874. Oustalet. Ann. sc. géol., v, art. 2: 199-201. Pl. 3, fig. 7.

4415. Geotrupes germari. (Scarabæidæ.)

Locality : Oeningen, Baden. Horizon: Tortonian.

1862. Heer. Verbandl. holl. maatsch. wetensch. Haarl., xvi: 71-72. Pl.6, fig. 10.

1865. " Urwelt der Schweiz, 379.

1872. " Monde prim. Suisse, 465.

1876. " " Prim. world Switz., ii : 36.

1879. “ Urwelt der Schweiz, $2^{\mathrm{e}}$ aufl, 405. 
4416. Geotrupes proævus. (Scarabæidæ.)

Locality : Orsberg, Germany. Horizon: Miocene.

1849. Germar. Zeitsehr. deutsch. geol, gesellsch., i: 57-58. Pl. 2, fig. 2.

1852. Giebel. Deutschl. petref., 653.

1856. " Insect. d. vorwelt, 36.

4417. Geotrupes putridarius (recent). (Scarabæidæ.)

Locality: Vannes, France. Horizon: Pleistocene.

[1846. Erichson. Naturg. ins., iii : 730.]

1876. Fliche. Comptes rendus, lxxxii : 979.

4418. Geotrupes stercorarius (recent). (Scarabæidæ.)

Locality: Hailes Quarry, Edinburgh, Scotland. Horizon: Peat below till.

[1746. Linné. Faun. suec., 135.]

1881. Purves. Geikie, Prehist. Europe, 256.

4419. Geotrupes vernalis (recent). (Scarabæidæ.)

Locality: Vannes, France. Horizon: Pleistocene.

[1746. Linné.

Faun. suec., 136.]

1876. Fliche. Comptes rendus, lxxxii : 979.

4420. Geotrupes vetustus. (Scarabæidæ.)

Compared with G. sylvaticus.

Locality : Vicinity Bonn, Rhenish Prussia. Horizon: Aquitanian?.

1837. Germar. Fauna insect. Europæ, xix : $6 . \quad P l .6$.

1839. Brulle. Gisem. ins. foss., 20.

1852. Giebel. Deutschl. petref., 653.

1856. " Insect. d. vorwelt, 35-36.

4421. Glaphyrus antiquus. (Scarabæidæ.)

Locality: Oeningen, Baden. Horizon: Tortonian.

1862. Heer.

1865. “

1872. "

1876. " "

1879. “
1847. Heer. 1852. Giebel. 1856. - “ 1885. Scudder. 1886. “
Verhandl. holl. maatsch. wetensch. Haarl., xvi: 79. Pl. 6, fig. 20.

Urwelt der Schweiz, 380.

Monde prim. Suisse, 466.

Prim. world Switz., ii : 37.

Urwelt der Schweiz, $2^{\mathrm{e}}$ aufl., 406.

4422. Glenopterus lævigatus. (Carabidæ.)

Locality : Oeningen, Baden. Horizon: Tortonian.

Insektenf. tertiärg. Oeningen, i : 16-18, 219. Pl. 1, fig. 2.

Deutschl. petref., 655 .

Insect. $\boldsymbol{d}$. vorwelt, $\boldsymbol{7 1}$.

Zittel, Handb. palaeont., i, th. ii: 804. Fig. 1069.

Zittel-Barrois, Traité de paléont., ii : 805. Fig. 1086 .

Gnorimus ædilis. See Trichius (Gnorimus) ædilis.

Gnorimus lugubris. See Trichius (Gnorimus) lngubris,

4423. Gonioctena clymene. (Chrysomelidæ.)

Compared with G. pallida Fabr.

Locality: Oeningen, Bader. Horizon: Tortonian.

1847. Heer.

Insektenf. tertiärg. Oeningen, i; 213-214, Pl. 7, fig. 14.

1852. Giebel.

Deutschl. petref., 648 . 
1856. Giebel.

1865. Heer.

1872. " "

1876. " "

1879 . "
Insect. d. vorwelt, 118.

Urwelt der Schweiz, 372.

Monde prim. Suisse, 456.

Prim. world Switz., ii : 26.

Urwelt der Schweiz, $2^{\circ}$ anfl., 397.

4424. Gonioctena curtisii. (Chrysomelidæ.)

Locality: Aix, France. Horizon: Ligurian.

1874. Oustalet. Ann. sc. géol., v, art. 2: 336-337. Pl. 4, fig. 14.

4425. Gonioctena japeti. (Chrysomelidæ.)

Locality: Oeningen, Baden. Horizon: Tortonian.

1847. Heer.

1852. Giebel.

1856. "

Insektenf, tertiärg. Oeningen, i: 212-213. $\quad P l_{\bullet} 7$, fig. 13.

Deutschl. petref., 648.

Insect. d. vorwelt, 117-118.

\section{Gonioctena primordialis. (Chrysomelidæ.)}

Locality : Schossnitz, Silesia. Horizon: Miocene.

1869. Assmann. Beitr.ins. d. vorwelt, 43-44. Pl. 1, figs. 6, $6 a$.

1869. " Schles, zeitschr. f. ent., (2), i: 43-44. Pl. 1, figs. 6, 6a.

4427. Gonocephalum (Hopatrum) pristinum. (Tenebrionidæ.)

Locality : Rott, Rhenish Prussia. Horizon: Aquitanian.

1866. Heyd.-Heyd. Palaeontogr., xv: 144-145 (Opatrum). Pl.22, fig. 27.

1866. “ Käfer u. polypen, 14-15. Pl. 1, fig. 27.

4428. Gymnetron - - (Curculionidæ.)

Locality: Brunstatt, Alsatia. Horizon: Middle Oligocene.

1890. Foerster. In litt.

4429. Gymnetron lecontei. (Curculionidæ.)

Compared with G. teter Schönh.

Locality: Green River, Wyo. Horizon: Oligocene.

1878. Scudder. Bull. U. S. geol. surv. terr., iv : 767.

1890. " Tert.ins. N. A., 471-472. Pl. 8, fig. 26.

4430. Gymnochila obesa. (Trogositidæ.)

Locality: Oeningen, Baden. Horizon: Tortonian.

1862. Heer. Verhandl. holl. maatsch. wetensch. Haarl, xvi: 56-57. Pl.3, figs. $11,11 b$.

1865. " Urwelt der Schweiz, 382.

1872. " Monde prim. Suisse, 469.

1876. " $\quad$ Prim. world Switz., ii: 40.

1879. “ Urwelt der Schweiz, $2^{\mathrm{e}}$ aufl, 408.

4431. Gymnopleurus deperditus. (Scarabæidæ.)

Locality : Oeningen, Baden. Horizon: Tortonian.

1862. Heer. Verhandl. holl. maatsch. wetensch. Haarl., xvi : 73. Pl.6, fig.9. 
4432. Gymnopleurus rotundatus. (Scarabæidæ.)

Locality : Oeningen, Baden. Horizon: Tortonian.

1862. Heer. Verhandl. holl. maatsch. wetensch. Haarl., xvi: 72-73. Pl. 6, fig. 8.

1865. " " Urwelt der Schweiz, 379. Fig. 270 on p. 378.

1872. " Monde prim. Suisse, 465. Fig. 270 on p. 463.

1876. " Prim. world Switz., ii : 36 . Fig. 270 on $p .34$.

1879. " Urwelt der Schweiz, 2 aufl., 405. Fig. 312 on p. 404.

4433. Gymnopleurus sisyphus. (Scarabæidæ.)

Locality: Oeningen, Baden. Horizon: Tortonian.

1847. Heer. 1852. Giebel. 1856. " "

Insektenf. tertiärg. Oeningen, i: 64-66. Pl. 7, fig. 25.

Deutschl. petref., 653 .

Insect. d. vorwelt, 38 .

4434. Gyrinoides limbatus. (Gyrinidæ.)

Compared with Gyrinus minutus.

Locality: Prussian amber. Horizon: Ligurian.

1856. Motschulsky. Études entom., v : 26.

\section{Gyrinus -. (Gyrinidæ.)}

Locality : Prussian amber. Horizon : Ligurian.

1845. Berendt. Bernst. befindl, organ. reste vorw,a i: 56 .

\section{Gyrinus - (Gyrinidæ.)}

Locality: Prussian amber. Horizon: Ligurian.

1856. Menge.

Progr. petrischule Danzig, 1856 : 23.

4437. Gyrinus marinus (recent). (Gyrinidæ.)

Locality: St. Jakob, Basel, Switzerland. Horizon: Interglacial.

[1808. Gyllenhal. Ins. suec., i: 143.]

1s79. Heer.

Urwelt der Schweiz, $2^{\mathrm{e}}$ aufl., 533.

1885. Scudder. Zittel, Handb. palaeont., i, th. ii : 803.

1886. “ Zittel-Barrois, Traite de paléont., ii : 803.

1886. “ Bull. U.S. geol. surv., No. 31: 83.

4438. Gyrinus natator (recent). (Gyrinidæ.)

Locality: Schwerzenbach, Switzerland. Horizon: Glacial clay.

[1746. Linn6.

1879. Heer.

1885. Seudder.

1886. " "

1886. “
Faun. suec., no. 779.]

Urwelt der Schweiz, $2^{\mathrm{e}}$ aufl, 581. Pl. 12, fig. 17.

Zittel, Handb. palaeont., i, th. ii : 803.

Zittel-Barrois, Traité de paléont., ii : 803.

Bull. U. S. geol. surv., No. 31 : 83 .

4439. Gyrophæna saxicola. (Staphylinidæ.)

Compared with G. vinula. .

Locality: White River, Colo. Horizon: Oligocene.

1876. Scudder. Bull. U. S. geol. surv. terr., ii : 78.

1877. " Bull. U. S. geol. surv, terr., iii : 759.

1890. " Tert. ins. N. A., 509. Pl. 5, figs. 123, 124. 
4440. Hæmonia - (Chrysomelidæ.)

Locality: Prussian amber. Horizon: Lígurian.

1845. Berendt. Bernst. befindl, organ. reste vorw., i: 56.

\section{Haliplus ——. (Haliplidæ.)}

Locality: Oeningen, Baden. Horizon : Tortonian.

1888. Schöberlin. Soc. entom., iii : 42.

\section{Hallomenus (or Orchesia) —_. (Melandryidæ.)}

Locality: Prussian amber. Horizon: Ligurian.

1845. Berendt. Bernst. befindl, organ, reste vorw., i : 56 .

\section{Haltica - (Chrysomelidæ.)}

Locality : Prussian amber. Horizon : Ligurian.

1845. Berendt. Bernst. befindl. organ. reste vorw., i: 56 .

4444. Haltica —. (Chrysomelidæ.)

Locality : Prussian amber. Horizon: Ligurian.

1831. Burmeister. Oken, Isis, 1831: 1100.

1832. " Handb. ent., i : 635 .

1836. " Man, ent., 577.

\section{Haltica -. (Chrysomelidæ.)}

Locality: Brunstatt, Alsatia. Horizon : Middle Oligocene. 1889. Foerster. Mitth. comm. geol. Elsass-Lothr., ii : 102.

4446. Haltica - (3 sp.). (Chrysomelidæ.)

Locality : Brunstatt, Alsatia. Horizon: Middle Oligocene.

1890. Foerster. In litt.

\section{Haltica - (Chrysomelidæ.)}

Locality: Prussian amber. Horizon : Ligurian.

1856. Menge. Progr. petrischule Danzig, 1856: 21.

\section{Harpalus —. (Carabidæ.)}

Locality : Prussian amber. Horizon : Ligurian.

1845. Berendt. Bernst. befindl. organ. reste vorw., i : 56.

$$
\text { 4449. Harpalus —_. (Carabidæ.) }
$$

Locality : Aix, France. Horizon: Ligurıa.

1829. Curtis. Edinb. new phil. journ, vii : 295 (perhaps Ophonus)。

1829. " Murchison-Lyell, Tert. form. Aix, 11.

\section{Harpalus (Argutor or Ophonus) ——. (Carabidæ.)}

Locality: Mundesley, England. Horizon: Pliocene,

1840. Curtis. Lyell, Proc. geol. soc. Lond., jii: 175,

1888. Bell, Entom, xxi : 2. 
4451. Harpalus -. (Carabidæ.)

Locality: Bruustatt, Alsatia. Horizon: Middle Oligocene.

1838. Foerster. Mitth. comm. geol. Elsass Lothr., i : 165.

1889. " Mitth. comm. geol. Elsass-Lothr., ii : 103.

4452. Harpalus - - (2 sp.). (Carabidæ.)

Locality : Brunstatt, Alsatia. Horizon: Middle Oligocene.

1890. Foerster. In litt.

4453. Harpalus —. (Carabidæ.)

Compared with $\mathrm{H}$. ruficornis.

Locality: Aix, France. Horizon: Ligurian.

1847. Hope. Trans. ent. soc. Lond., iv : 250.

4454. Harpalus —. (Carabidæ.)

Compared with H. grisens.

Locality: Aix, Franee. Horizon: Ligurian.

1829. Serres. Géogn. terrains tert., 221, 271-272. $\quad P l .5, f i g .7$.

1856. Giebel. Insect. d. vorwelt, 64 .

4455. Harpalus abolitus. (Carabidæ.)

Locality: Rott, Rhenish Prussia. Horizon: Aquitanian.

1866. Heyd.-Heyd. Palaeontogr., xv: 134. Pl. 22, fig. 1.

1866. “ Käfer u. polypen, 4. Pl. 1, fig. 1.

4456. Harpalus bruckmanni. (Carabidæ.)

Locality : Oeningen, Baden. Horizon: Tortonian.

1862. Heer. Verhandl. holl. maatsch. wetensch. Haarl., xvi: 26-27. Pl. 1, figs. 21, $21 b$.

4457. Harpalus constrictus. (Carabidæ.)

Locality: Oeningen, Baden. Horizon: Tortonian.

1862. Heer. Verhandl. holl. maatsch. wetensch. Haarl., xvi: 29. Pl. 1, fig. 24.

4458. Harpalus deletus. (Carabidæ.)

Locality : Aix, France. Horizon: Ligurian.

1874. Oustalet. Ann. sc. géol., v, art. 2: 111-114. Pl. 1, fig. 5.

Harpalus diluvianus. See Carabites (Harpalus) diluvianus.

Harpalus finis. See Harpalus sinis.

4459. Harpalus lævicollis (recent). (Carabidæ.)

Locality: Schwerzenbach, Switzerland. Horizon: Glacial clay.

[1812. Duftschmid. Fanna austr., ii : 163.]

1879. Heer.

1885. Scudder.

1886. " "
Urwelt der Schweiz, $2^{\mathrm{e}}$ aufl., 581. $P l$. 12, figs. $16,16 b$.

Zittel, Handb. palaeont., i, th. ii: 804.

Bull. U. S. geol. surv. No. $31: 84$.

4460. Harpalus nero. (Carabidæ.)

1874. Oustalet. Ann, sc. géol., v, art, 2: 109-111. Pl. 1, fig. 9. 
4461. Harpalus sinis. (Carabidæ.)

Locality: Oeningen, Bacleu. Horizon: Tortonian.

1847. Heer. 1852. Giebel. 1856. " "

1862. Heer.

1862. Heer.

1847. Heer. 185\%. Giebel. 1856. " "

1862. Heer. 1865. " 1872. "6 1876. 66 1879. 6

1862. Giebel.

1847. Hope.

1862. Heer.

1862. Heer.
Insektenf. tertiärg. Oeningen, i : 219-220. Pl. 8, fig. 2.

Deutsehl. petref., 655 (tinis).

Insect. d. vorwelt, 62,64 .

\section{Harpalus stierlini. (Carabidæ.)}

Locality: Oeningen, Baden. Horizon: Tortonian.

Verhandl. holl, maatsch. wetensch. Haarl., xvi : 27. Pl. 1, fig. 23

4463. Harpalus stygius. (Carabidæ.)

Locality : Oeningen, Baden. Horizon: Tortonian.

Verhandl. holl. maatsch. wetenseh. Haarl., xvi : 23. Pl. 1, fig. 22.

4464. Harpalus tabidus. (Carabidæ.)

Locality : Radoboj, Croatia. Horizon: Mayencian.

Insektenf. tertiärg. Oeningen, i : 23-24. Pl. 7, fig. 19.

Deutschl. petref., 655 .

Insect. d. vorwelt, 62 .

4465. Harpalus tardigradus. (Carabidæ.)

Locality : Oeningen, Baden. Horizon : Tortonian.

Verhandl. holl, maatsch. wetensch. Haarl, xvi : 28. Pl. 1, fig. 20.

Urwelt der Schweiz. Fig. 284 on $p .381$.

Monde prim. Suisse. Fig. 284 on p. 471.

Prim. world Switz, ii. Fig. 284 on p. 42.

Urwelt der Schweiz, $2^{\circ}$ anfl. Fig. 326 on p. 410.

4466. Helluomor pha protogæa. (Carabidæ.)

Compared with H. tripustulata, China.

Locality: Prussian amber. Horizon: Ligurian.

Zeitschr. ges. naturw., xx : 316.

4467. Helobia (Carabidæ.)

Locality : Aix, France. Horizon : Ligurian.

Trans. ent. soc. Lond., iv : 250.

4468. Helophorus exilis. (Hydrophilidx.)

Locality : Oeningen, Baden. Horizon: Tortonian.

Verhandl. holl. maatsch. wetensch. Haarl., xvi : 71. Pl.5, fig. 20.

4469. Helophorus magnus. (Hydrophilidæ.)

Locality: Oeningeu, Baden. Horizon: Tortonian.

Verhandl. holl. maatsch. wetensch. Haarl., xvi : 71. Pl. 5, fig. 19.

4470. Helophorus rigescens. (Hydrophilidæ.)

Compared with H. tuberculatus Gyll.

Locality : Scarboro', Ontario. Horizon: Pleistocene.

1890. Scudder. Tert. ins, N. A., 516-517. Pl. 1, fig. 53. 
4471. Helops - (Tenebrionidæ.)

Compared with H. lanipes.

Locality : Eisleben, Saxony. Horizon:

1856. Giebel. Zeitschr. ges. naturw., vii: 385. Pl. 5, fig. 2.

4472. Helops meissneri. (Tenebrionidæ.)

1847. Heer.

Locality : (Oeningen, Baden ?.) Horizon : Tortonian.

1852. Giebel.

Insektenf. tertiärg. Oeningen, i: 161-162. Pl. 5, fig. 9.

1856. " Insect. d. vorwelt, 111 .

4473. Helops molassicus. (Tenebrionidæ.)

Locality: Lausanne, Switzerland. Horizon: Miocene.

1883. Heer. Flora foss. grönl., ii: 145. Pl. 109, fig. 9.

4474. Helops wetteravicus. (Tenebrionidæ.)

Compared with H. lanipes.

Localities: Salzhausen, Germany; Umivik, Greenland. Horizon : Miocene. 1865. Heyd.-Heyd. Palaeontogr., xiv: 33. Pl. 9, fig. 18.

1883. Heer. Flora foss. grönl., ii : 145. Pl. 109, figs. 8, 8b-c.

1886. Scudder. Bull. U. S. geol. surv., No. 31: 71.

4475. Hesthesis antiqua. (Cerambycidæ.)

Locality: Vicinity Bonn, Rhenish Prussia. Horizon: Aquitanian?.

1862. Heyden. Palaeontogr., $x: 73$.

See also Molorchus antiquus.

4476. Hesthesis immortua. (Cerambycidø.)

Locality: Rott, Rhenish Prussia. Horizon: Aquitanian.

1862. Heyden. Palaeontogr., $\mathrm{x}:$ 72-73. Pl. 10, fig. 36.

4477. Hipporhinus brevis. (Curculioṇidæ.)

Locality : Aix, France. Horizon: Ligurian.

1856. Giebel. Insect. d. vorwelt, 146.

4478. Hipporhinus heeri. (Curculionidæ.)

Locality: Aix, France. Horizon: Ligurian.

1849. Germar. Zeitsehr. dentsch. geol. gesellsch., i: 62-64. Pl «, figs, 6, 6a.

1856. Heer. Viert. naturf. gesellsch.Zürich, i: 21-22. Pl. 1, fig. 11.

1856. Giebel. Insect. d. vorwelt, 146.

1874. Oustalet. Ann. sc. géol., v, art. 2: 214-230. Pl. 3, fig. 11; pl. 4, figs. 1-b,

8 ; pl. 5, figs. 1,2; pl. 6, figs. 4,5, 14.

1885. Scudder. Zittel, Handb. palaeont., i, th. ii : 789. Fig. 1008.

1886. “ "Zittel-Barrois, Traité de paléont., ii : 789. Fig. 1026.

1886. " Bull. U. S. geol. surv., No. 31: 68.

See also Brachycerus —- Serres (ef. hispanicus).

4479. Hipporhinus longipes. (Curculionidæ.)

Locality : Aix, France. Horizon: Ligurian.

1874. Heer. MSS. in Oustalet.

See also Brachyderes longipes, etc. 
4480. Hipporhinus reynesii. (Curculionidæ.)

Locality : Aix, France. Horizon : Ligurian.

1874. Oustalet. Ann. sc. géol., v, art. 2: 231-234. Pl. 5, figs. 3, 4.

4481. Hipporhinus schaumii. (Curculionidæ.)

Locality: Aix, France. Horizon: Ligurian.

1856. Heer. Viert. naturf. gesellsch. Zürich, i: 22. Pl. 1, fig. 10. 1874. Oustalet. Ann. sc. géol., v, art. 2: 230-231. Pl. 6, fig. 6.

4482. Hister - - (Histeridæ.)

Locality : Prussian amber. Horizon : Ligurian.

1845. Berendt. Bernst. befindl. organ. reste vorw., i: 56.

4483. Hister - (2 sp.). (Histeridæ.)

Locality: Prussian amber. Horizon: Ligurian.

1856. Menge. Progr. petrischule Danzig, 1856: 21.

4484. Hister æmulus: (Histeridæ.)

Locality: Oeningen, Baden. Horizon: Tortonian.

1862. Heer.

1865. “ "

187\%. " "

1876. " "

1879. “
Verhandl. holl. maatsch. wetensch. Haarl., xvi : 60. Pl. 3, fig. 23.

Urwelt der Schweiz, 382.

Monde prim. Suisse, 468.

Prim. world Switz., ii : 39.

Urwelt der Schweiz, $2^{\mathrm{e}}$ aufl., 407.

4485. Hister antiquus. (Histeridæ.)

Locality: Oeningen, Baden. Horizon: Tortonian.

1862. Heer.

1865. " "

1872. "

1876. " "

1879. “
Verhandl. holl. maatsch. wetensch. Haarl., xvi : 58. Pl. 3, fig.17.

Urwelt der Schweiz, 382.

Monde prim. Suisse, 468.

Prim. world Switz., ii : 39.

Urwelt der Schweiz, $2^{\mathrm{e}}$ aufl., 407.

4486. Hister cadaverinus (recent). (Histeridæ.)

Locality : Oeningen, Baden. Horizon: Tortonian.

[1803. Hoffmann. Ent. hefte, i: 34. Pl. 1, fig. 2.]

1888. Schöberlin. Soc. entom., iii : 42.

4487. Hister coprolithorum. (Histeridæ.)

Locality: Oeningen, Baden. Horizon: Tortonian.

1862. Heer. Verhandl. holl. maatsch. wetensch. Haarl, xvi: 58-59. Pl.3, figs. 19, 20.

1865. " Urwelt der Schweiz, 472.

1865. Lyell. Elem. geol., 6th ed., 249. Fig. 181d.

1872. Heer. Monde prim. Suisse, 575.

1876. " $\quad$ Prim. world Switz., ii : 132.

1879, " " Urwelt der Schweiz, $2^{\mathrm{e}}$ aufl., 499. 
4488. Hister maculigerus. (Histeridæ.)

Locality : Oeningen, Baden. Horizon: Tortonian.

1862. Heer.

Verhandl. holl. maatsch. wetensch. Haarl, xvi : 59-60. Pl. 3, fig. 22.

1865. “

1872. “

1876. "

1879. “

Urwelt der Schweiz, 382.

Monde prim. Suisse, 468.

Prim. world Switz., ii : 39.

Urwelt der Schweiz, $2^{\ominus}$ anfl., 407.

4489. Hister marmoratus. (Histeridæ.)

Locality : Oeningen, Baden. Horizon: Tortonian.

1862. Heer.

Verhandl. holl. maatsch. wetensch. Haarl., xvi: 59. Pl.3, fig. 21.

1885. Seudder.

1886. “

Zittel, Handb. palaeont, i, th. ii : 799. Fig. 1046.

Zittel-Barrois, Traité de paléont., ii : 799. Fig. 1063.

4490. Hister mastodontis. (Histeridæ.)

Compared with H. major L., Southern Europe.

Locality: Oeningen, Baden. Horizon: Tortonian.

1862. Heer.

1865. " "

1872. " "

1876. “

1879. “

Verhàndl. holl. maatsch. wetensch. Haarl., xvi: 57-58. Pl. 3, figs. 16, $16 b$.

Urwelt der Schweiz, 382. Fig. 276, on p. 381.

Monde prim. Suisse, 468. Fig. 276 on p. 467.

Prim. world Switz., ii : 39 . Fig. 276 on p. 38.

Urwelt der Schweiz, $2^{\mathrm{e}}$ aufl., 407. Fig. 318.

\section{Hister morosus. (Histeridæ.)}

Locality: Oeningen, Baden. Horizon: Tortonian.

1862. Heer.

Verhandl. holl. maatsch. wetensch. Haarl., xvi : 60. Pl.3, fig. 24.

4492. Hister vetustus. (Histeridæ.)

Locality: Oeningen, Badeu. Horizon: Tortonian.

1862. Heer. Verhandl. holl. maatsch. wetensch. Haarl., xvi: 58. Pl.3, fig.18.

4493. Homalium —. (Staphylinidæ.)

Locality : Prussian amber. Horizon : Ligurian.

1845. Berendt. Bernst. befindl, organ. reste vorw., i: 56 (Omalium).

4494. Homalium protogeæ. (Staphylinidæ.)

Locality: Radoboj, Croatia. Horizon: Mayencian.

1847. Heer.

1852. Giebel.

1856. “

Insektenf. tertiärg. Oeningen, i : 34-35 (Omalium). Pl. 1, fig. 10.

Deutschl. petref., 654 .

Insect. d. vorwelt, 74 .

4495. Homalota recisa. (Staphylinidæ.)

Locality: Green River, Wyo. Horizon: Oligocene.

1890. Scudder. Tert. ins. N. A., 509-510. Pl. 8, fig. 14.

4496. Hopatrum —. (Tenebrionidæ.)

Compared with H. sabulosum.

Locality: Prussian amber. Horizon : Ligurian.

1832. Burmeister. Handb. ent., i : 635 (Opatrum).

1836. " Man. ent., $57 \%$. 
4497. Hopatrum - (Tenebrionidæ.)

Compared with H. pusillum.

Locality: Aix, France. Horizon: Ligurian.

1829. Serres. Géogn. terrains tert., 222 (Opatrum).

Hopatrum pristinum. See Gonocephalum (Hopatrum) pristinum.

4498. Hopatrum sabulosum (recent). (Tenebrionidæ.)

Locality : Hochheim, Germany. Horjzon : "Kalkmergel."

[1761. Linné. 1840. Meyer. 1885. Scudder. 1886.

Faun. suec., 150. (Opatrum.)]

Ersch u. Grüber, Encycl. d. wissensch., sect. 2, th. xviii : 539.

Zittel, Handb. palaeont., i, th. ii : 791.

Zittel-Barrois, Traité de paléont., ii : 792.

4499. Hormiscus partitus. (Anthribidæ.)

Locality: Green River, Wyo. Horizon: Oligocene.

1890. Scudder. Tert. ins. N. A., 467. Pl. 8, fig. 17.

4500. Hybosorus lividus. (Scarabæidæ.)

Locality: Oeningen, Baden. Horizon: Tortonian.

1862. Heer. Verhandl, holl. maatsch. wetensch. Haarl., xvi : 77. Pl.6, fig. 22.

4501. Hydaticus areolatus. (Dytiscidæ.)

Locality: Oeningen, Baden. Horizon: Tortonian.

1862. Heer. Verhandl. holl. maatsch. wetensch. Haarl., xvi: 40-41. Pl. 2, fig. 20.

4502. Hydatious zschokkeanus. (Dytiscidæ.)

Compared with H. cinereus (Linn.), Europe.

Locality: Oeningen, Baden. Horizon : Tortonian.

1861. Heer. Rech. climat pays tert., 204 (undescr.).

4503. Hydræna riparia (recent). (Hydrophilidæ.)

Locality : Hösbach, Bavaria. Horizon : Lower Pleistocene.

[1794. Kugelann. Schneiders mag., i : 579.]

1884. Flach.

1884. "

Käfer unterpleist. Hösbach, 8. Pl. 2, fig. 1.

Verhandl. physik.-med. gesellsch. Wurzb., n. f., xviii : 292. $P l$. 9, fig. 1.

4504. Hydrobius ——. (Hydrophilidæ.)

Compared with H. fuscipes.

Locality: Aix, Franee. Horizon: Ligurian.
1829. Curtis. 1829. " "

Edinb. new phil. journ., vii : 295.

Murchison-Lyell, Tert. form. Aix, 11.

4505. Hydrobius (Hydrophilidæ.)

Compared with $\mathrm{H}$. fuscipes.

Locality : Aix, France. Horizon: Ligurian.

1847. Hope.

Trans, ent. soc. Lond., iv: 250. 
4506. Hydrobius confixus. (Hydrophilidæ.)

Locality: Green River, Wyo. Horizon: Oligocene.

1890. Scudder: Tert. ins. N. A., 511-512. Pl. 7, fig. 25.

4507. Hydrobius couloni. (Hydróphilida:)

Locality : Oeningen, Baden. Horizon: Tortonian.

1862. Heer. Verhandl. holl. maatsch, wetenseh. Haarl, zvi : 70. Pl. 5, figs. $22,22 b$.

4508. Hydrobius decineratus. (Hydrophilidw،)

Compared with $\mathrm{H}$. fuscipes Curt., California.

Locality: Green River, Wyo. Horizon: Oligocene.

1878. Scudder. Bull. U. S. geol. surv. terr., iv : 761.

1890. " Tert. ins. N. A., ว11. Pl. 8, fig. 27.

4509. Hydrobius fuscipes (recent). (Hydrophilidæ.)

Locality : Hösbach, Bavaria. Horizon : Lower Pleistocene.

[1746. Linué.

1884. Flach.

1884. " "
Faun. suec., no. 766.]

Käfer unterpleist. Hösbach, 8.

Verhandl. physik.-med. gesellsch. Würzb., n. f., xviii : 292.

4510. Hydrobius godeti. (Hydrophilidæ.)

Locality: Oeningen, Baden. Horizon: Tortonian.

1862. Heer. Verhandl. holl. maatsch. wetensch. Haarl, xvi: 70-71. Pl. 5. figs. 21, $21 b$.

\section{Hydrobius longicollis. (Hydrophilidæ.)}

Localits: Radoboj, Croatia. Horizon: Tortonian.

1847. Heer.

1852. Giebel. 1856. " "
Insektenf. tertiärg. Oeningen, i : 56-57 (H. ungeri on pl.). Pl. 2, fig. 6.

Deutschl. petref., 653 .

Insect. d. vorwelt, 53 .

4512. Hydrobius nauckhoff. (Hydrophilidæ.)

1870. Heer. K. svenska vetensk,-akad. handi., viii, No. 7: 74. Pl. 16, figs. $10-11$.

4513. Hydrobius obsoletus. (Hydrophilidæ.)

Locality: Aix, France. Horizon: Ligurian.

1856. Heer. Viert. naturf. gesellsch. Zürich, i: 18. Pl. 1, fig. 19。

1874. Onstalet. Ann. sc. géol., v, art. 2: 128-129.

Hydrobius ungeri. See Hydrobius longicollis.

4514. Hydrochus amictus. (Hydrophilidæ.)

Compared with H. subcupreus Rand.

Locality : Scarbcro', Ontario. Horizon: Pleistocene.

1890. Scudder. Tert. ins. N. A., 515-516. Pl.1, fig. 47. 
4515. Hydrochus relictus. (Hydrophilidæ.)

Locality: Green River, Wyo. Horizon: Oligocene. 1890. Scudder. Tert. ins. N. A., 516. Pl. 8, fig. 11.

4516. Hydronomus (?) nasutus. (Curculionidæ.)

Locality: Aix, France. Horizon : Ligurian.

1874. Oustalet. Ann. sc. géol., v, art. 2: 291-293. Pl. 5, fig. 6.

4517. Hydrophilites naujatensis. (Hydrophilidæ.)

1883. Heer.

Locality: Naujat, Greenland. Horizon: Miocene?.

1883. Heer.

Flora foss. Grönl., ii : 144 (Hydrophylites). Pl. 86, fig. $12 b ; p l$. 109, fig. 10.

4518. Hydrophilopsis elongata. (Hydrophilidæ.)

Locality: Oeningen, Baden. Horizon: Tortonian.

1862. Heer.

Verhandl. holl. maatsch. wetensch. Haarl, xvi : 69. $\quad$ Pl. 5, fig8. 18, $18 b$.

1865. “

1872. " "

1876. “

1879. “

Urwelt der Schweiz. Fig. 274 on p. 381.

Monde prim. Suisse.. Fig. 274 on $p .467$.

Prim. world Switz., ii. Fig. 274 on $p .38$.

Urwelt der Schweiz, 2e aufl. Fig. 316 on $p .407$.

4519. Hydrophilopsis incerta. (Hydrophilidæ.)

Locality: Aix, France. Horizon: Ligurian.

1874. Oustalet. Ann. sc. géol., v, art. 2: 125-126. Pl. 2, fig, 3.

4520. Hydrophilus —. (Hydrophilidæ.)

Locality: Bolca, Italy. Horizon : Ligurian.

1886. Omboni. Alc. ins. foss. ven., 10. Pl. 3, fig. 14.

1886. “ Atti r. ist. Ven., (6), iv: 1430 . Pl. 3, fig. 14.

4521. Hydrophilus ——. (Hydrophilidæ.)

Locality : France. Horizon: Miocene.

1820. Schlotheim. Petrefactenk., 44 (Hydrophylus).

4522. Hydrophilus

(Hydrophilidæ.)

Locality : Florissant, Colo. Horizon: Oligocene.

1881. Scudder. Bull. U. S. geol. surv. terr., vi : 291.

4523. Hydrophilus —. (Hydrophilidæ.)

Locality : Gurnet Bay, Isle of Wight. Horizon: Bembridge Limestone. 1879. Woodward. Geol. mag., n. s., v: 89.

4524. Hydrophilus antiquus. (Hydrophilidæ.)

1874. Heer.

Locality : Aix, France. Horizon: Ligurian.

1874. Oustalet. Ann. sc. géol., v, art. 2: 124-125. Pl. 2, fig. 2. 
4525. Hydrophilus braunii. (Hydrophilidæ.)

Locality: Oeningen, Badeu. Horizon: Tortonian.

1847. Heer.

Insektenf. tertiärg. Oeningen, i: 55-56, 221. Pl. 2, fig. 5; pl. 8, fig. 4.

1852. Giebei. Deutschl. petref., 653.

1856. " Insect. d. vorwelt, 55.

See also Hydrous braunii.

4526. Hydrophilus caraboides (recent). (Hydrophilid:e.)

Locality: St. Jakob, Basel, Switzerland. Horizon: Interglacial.

[1746. 'Linné.

1847. Heer.

1879. "6

Fauna suec., no. 214.]

Insektenf, tertiärg. Oeningen, i: 54, 55. Pl. 2, fig. 4.

Urwelt der Schweiz, 2e anfl,, 533.

4527. Hydrophilus carbonarius. (Hydrophilidæ.)

1847. Heer.

Locality: Parschlug, Styria. Horizon: Tortonian.

1852. Giebel.

Insekteuf. tertiärg. Oeningen, i: 52. Pl. 7, fig. 24.

1856. “

Dentschl. petref., 653 .

Insect. d. vorwelt, 54-55.

4528. Hydrophilus fraternus. (Hydrophilidæ.)

Locality: Rott, Rhenish Prussia. Horizon: Aquitanian.

1859. Heyden. Palaeontogr., viii : 2. Pl. 2, fig. 6.

4529. Hydrophilus gaudini. (Hydrophilidæ.)

Locality: Monod, near Chexbres, Switzerland. Horizon: Aquitanian.

1862. Heєr.

1865. “

1872.

1876. " "

1879. “6

1862. Heer.

1865. 6

1872. "

1876. 6

1879. 6

Verhandl. holl. maatsch. wetensch. Haarl., xvi : 61. Pl. 4, fig. 20.

Urwelt der Schweiz, 381.

Monde prim. Suisse, 467.

Prim. world Switz., ii : 38.

Urwelt der Schweiz, 2 e aufl., 406.

4530. Hydrophilus giganteus. (Hydrophilidæ.)

Locality: Oeningen, Baden. Horizon: Tortonian.

Verhandl. holl. maatsch. wetensch. Haarl, xvi: 62-65. Pl. 4 , figs. 1-12; pl. 5, figs. 1-2.

Urwelt der Schweiz, 381. Fig. 271.

Monde prim. Suisse, 467. Fig. 271.

Prim. world Switz., ii : 38. Fig. 271.

Urwelt der Schweiz, $2^{e}$ anfl., 406 . Fig. 313 on p. 407.

4531. Hydrophilus knorrii. (Hydrophilidæ.)

Locality: Oeningen, Baden. Horizon: Tortonian.

1847. Heer.

Insektenf. tertiärg. Oeningen, i: 51-52. Pl. 2, fig. 2.

1852. Giebel.

1856. “

Deutschl. petref., 653.

Insect. d. vorwelt, 54 .

4532. Hydrophilus noachicus. (Hydrophilidæ.)

Locality: Oeningen, Baden. Horizon: Tortonian.

1847. Heer.

1852. Giebel.

1856. " "
Insektenf. tertiärg. Oeningen, i: 52-53. $\quad P l .1$, fig. 13.

Deutschl. petref., 653.

Insect. d. vorwelt, 55 . 
4533. Hydrophilus piceus (recent). (fIydrophilidæ.)

Locality: Lagozza, vicinity Besnate, Italy: Horizon: Quaternary.

[1746. Limé. Fauna suee., no. 764.]

1882. Sordelli. Alc. ins. foss. Lomb., 2.

1882. " Bull, soc. ent. ital., xir : 225.

1885. Scudder. Zittel, Handb. palaeont, i, th. ii : 802 .

1886. " "Zittel Barrois, Traité de paléont., ii: 803.

1886. " Bull. U.S. geol, surv., No. 31 : 82 .

4534. Hydrophilus rehmanni. (Hydrophilidæ.)

Compared with Hydrochares caraboides.

Locality: Oeningen, Baden. Horizon: Tortonian.

1847. Heer. Insektenf, tertiärg. Oeningen, i: 53-55. Pl. 2, fig. 3.

1852. Giebel. Deutschl. petref., 653.

1856. " Insect. d. vorwelt, 55.

See also Hydrons rehmanni.

4535. Hydrophilus ruminianus. (Hydrophilịdse)

Locality: Monod, near Chexbres, Switzerland. Horizon: Aquitanian.

1865. Heer.

1872. "

1876. “

1879. “
Urwelt der Schweiz, 381.

Monde prim. Suisse, 468.

Prim. world Switz., ii : 38.

Urwelt der Schweiz, $2^{\mathrm{e}}$ aufl., 406.

4536. Hydrophilus spectabilis. (Hydrophilidæ.)

Compared with H. piçeus L., Europe

Locality: Oeningen, Baden. Horizon: Tortonian.

1847. Heer.

1852. Giebel.

1854. Pictet.

1856. Giebel.

1862. Heer.

1865. " "

1872. “

1876 . "

1879. “

Insektenf. tertiärg. Oeningen, i: 49-51. Pl. 2, figs. 1, $1 b$.

Dentschl. petref., 653.

Traité de paléont., 2e éd., ii : 341 . Pl. 40, fig. 12.

Insect. d. vorwelt, 54 .

Verhandl. holl. maatsch. wetenseh. Haarl., xvi : 61. Pl. 4, fig. 21.

Urwelt der Schweiz, 381.

Moude prim. Suisse, 467.

Prim. world Switz., ii : 37.

Urwelt der Schweiz, 2e aufl., 406.

4537. Hydrophilus stenopterus. (Hydrophilidæ.)

Locality: Oeningen, Baden. Horizon: Tortonian.

1862. Heer.

Verhandl. holl. maatsch. wetensch. Haarl., xvi : 65. Pl. 4, fig. 13.

4538. Hydrophilus vexatorius. (Hydrophilidæ.)

1847. Heer.

1852. Giebel.

1856. “

1862. Heer.

Locality : Oeningen, Baden. Horizon: Tortonian.

Insektenf. tertiärg. Oeningen, i: 47-49. Pl.1, figs. 12, $12 b$.

Dentsehl. petref., 653 .

Insect. d. vorwelt, 53-54.

Verhandl. holl. maatsch. wetensch. Haarl., xvi : $62 . \quad$ Pl. 4, figs 14-19.

Fydrophylites naujatensis. See Hydrophilites naujatensis.

Hydrophylus —. See Hydrophilus —_ Schtotheim. 
4539. Hydroporus - (Dytiscida.)

Locality: Hösbach, Bavaria. Horizon: Lower Pleistoceno.

1884. Flach. Käfer unterpleist. Hösbach, 8.

1884. “ Verhandl. physik.-med. gesellsch. Würzb., n. f., xviii : 292.

4540. Hydroporus antiquus. (Dytiscidæ.)

Locality : Oeningen, Baden. Horizon: Tortonian.

1862. Heer. Verhandl. holl. maatsch. wetensch. Haarl., xvi : 42. Pl. 2, fig. 15.

1865. " Urwelt der Schweiz. Fig. 282 on p. 381.

1872. " - Monde prim. Suisse. Fig. 282 on $p .467$.

1876. " Prim. world Switz., ii. Fig. 282 on p. 38.

1879. “ Urwelt der Schweiz, 22 aufl. Fig. 324 on $p .407$.

4541. Hydroporus benzeli, (Dytiscidæ.)

Locality: Oeningen, Baden. Horizon: Tortonian.

1862. Heer. Verhandl. holl. maatseh. wetensch. Haarl., xvi: 42-43. Pl. 2, fig. 29.

4542. Hydrous braunii. (Hydrophilidæ.)

Locality : Oeningen, Baden. Horizon: Tortonian.

1862. Heer. Verhandl. holl. maatsch. wetensch. Haarl, xvi: 67. Pl. 5, figs. 12-14.

See also Hydrophilus braunii.

4543. Hydrous escheri. (Hydrophilidæ.)

Locality : Oeningen, Baden. Horizon: Tortonian.

1862. Heer. Verhandl. holl. maatsch. wetensch. Haarl., xvi: 67-68. Pl. 5, figs. 16, $16 b$.

1865. " Urwelt der Schweiz. Fig. 272 on $p .381$.

1872. " Monde prim. Suisse. Fig. 272 on $p .467$.

1876. " "Prim. world Switz., ii. Fig. 272 on $p .38$.

1879. " Urwelt der Schweiz, $2^{\ominus}$ aufl. Fig. 314 on p. 407.

4544. Hydrous miserandus. (Hydrophilidæ.)

Locality : Rott, Rhenish Prussia. Horizon: Aquitanian.

1859. Heyden. Palaeontogr., viii: 2. Pl. 2, fig. 5.

4545. Hydrous neptunus. (Hydrophilidæ.)

Locality: Rott, Rhenish Prussia. Horizon: Aquitarian.

1866. Heyd.-Heyd. Palaeontogr., xv: 135-136. Pl. 22, fig. 5.

1866. " Käfer u. polypen, 5-6. Pl. 1, fig.5.

4546. Hydrous ovalis. (Hyủrophilidæ.)

Locality: Oeningen, Baden. Horizon: Tortonian.

1862. Heer. Verhandl, holl. maatsch, wetenseh. Haarl, xvi : 68. Pl. 6, fig. 1.

4547. Hydrous rehmanni. (Hydrophiliḋæ.)

Locality : Oeningen, Baden. Horizon: Tortonian.

1862. Heer. Verhandl. holl. maatsch. wetensch. Haarl, xvi : 65-66. Pl. 5, figs. 3-11.

See also Hydrophilus rehmanni. 
4548. Hygronoma deleta. (Staphylinidæ.)

Compared with H. dimidiata Grav., Europe.

Locality: Aix, France. Horizon: Ligurian.

1874. Oustalet. Ann. sc. géol., v, art. 2: 179-180. Pl. 3, fig. 5.

4549. Hylastes ? squalidens. (Scolytidæ.)

Locality: Scarboro', Ontario. Horizon: Postpliocene.

1890. Seudder.

Tert. ins. N. A., 468-469. Pl. 1, figs. 23-25.

See also (Scolytidæ) Scudder.

4550. Hylecœtus cylindricus. (Lymexylidæ.)

Compared with H. dermestoides (L.).

Locality : Oeningen, Baden. Horizon : Tortonian

1865. Heer. Urwelt der Schweiz, 362, 377.

1872. " Monde prim. Suisse, 444, 462.

1876. " " Prim. world Switz., ii : 16,33.

1879. “ Urwelt der Schweiz, $2^{\mathrm{e}}$ aufl., 387, 403.

1886. Seudder. Bull. U. S. geol. surv., No. 31: 75.

4551. Hylesinites electrinus. (Scolytidæ.)

Compared with Hylesinus ligniperda.

Locality : Prussian amber. Horizon : Ligurian.

1813. Germar. Germ., Mag. d. entom., i: 15.

1856. Giebel. Insect. d. vorwelt, 148.

1888. Schlechtend. Zeitschr. ges. naturw., lxi : 486-487.

4552. Hylesinus —. (Scolytidæ.)

Locality: Prussian amber. Horizon : Ligurian.

1845. Berendt. Bernst. befindl, organ. reste vorw., i : 56.

4553. Hylesinus —. (Scolytidæ.)

Locality: Brunstatt, Alsatia. Horizon: Middle Oligocene.

1890. Foerster. In litt.

4554. Hylesinus ——. (Scolytidæ.)

Locality : Prussian amber. Horizon : Ligurian.

1856. Menge.

Progr. petrischnle Danzig, 1856: 22.

4555. Hylesinus - (Scolytidæ.)

Locality: Prussian amber. Horizon: Ligurian.

1829. Serres. Géogn. terrains tert., 241.

4556. Hylesinus facilis. (Scolytidæ.)

Locality : Aix, France. Horizon: Ligurian.

1856. Heer.

1874. Oustalet.

Viert. naturf. gesellsch. Zürich, i: 25. Pl. 1, fig. ૪.

Ann. sc. géol., v, art. 2: 315. Pl.2, fig. 7.

1885. Scudder.

1886. "

Zittel, Handb. palaeont., i, th. ii: 787. Fig. 1006.

Zittel-Barrois, Traité de paléont., ii : 787. Fig. 1023.

Hylobinum tortomanus. See Hylobius tortomanus. 
4557. Hylobius

(Curculionidæ.)

Locality : Prussian amber. Horizon: Ligurian.

1845. Berendt. Bernst. befindl. organ. reste vorw., i: 56.

4558. Hylobius antiquus. (Curculionidæ.)

Locality : Rott, Rhenish Prussia. Horizon : Aquitanian.

1866. Heyd.-Heyd. Palaeontogr., xv: 149, Pl. 23, figs. 11, 12.

1866. " Käfer u. polypen, 19. Pl. 2, figs. 11, 12.

4559. Hylobius caxbo. (Curculionidæ.)

Locality : Aix, France. Horizon : Ligurian.

1874. Oustalet. Ann. sc. g6́ol., v, art.2: 275-277. Pl. 4, fig. 7.

4560. Hylobius deletus. (Curculionidæ.)

Locality: Corent, France. Horizon: Aquitanian.

1870. Oustalet. Ann. sc. géol., ii, art.3: 70-71. Pl. 1, fig.8.

4561. Hylobius morosus. (Curculionidæ.)

Locality: Aix, France. Horizon: Ligurian.

1874. Oustalet. Ann. sc. g6ol., v, art. 2: 271-275. Pl. 3, fig. 18; pl.4, fig. 13.

See also Curculionites morosus, Liparus — Curtis ef. punctatus, L. primævus.

4562. Hylobius provectus. (Curculionidæ.)

Compared with $\mathrm{H}$. picivorus.

Locality: Green River, Wyo. Horizon: Oligocene.

1876. Scudder. Bnll. U. S. geol. surv. terr., ii : 86 .

1878. " ". Bull. U. S. geol. surv. terr., iv: 767.

1890. " Tert. ins. N. A., 473-474. Pl. 8, figs. 37, 41.

4563. Hylobius rugosus. (Curculionidæ.)

Compared with H. pineti.

Locality : Dürnten, Switzerland. Horizon: Interglacial.

1858. Heer.

Arch. sc. phys. nat., n. p.,ii : 322.

1865. " Urwelt der Schweiz, 501. Fig. 356.

1872. : $\quad$ Monde prim. Suisse, 615. Fig. 356 on p. 614.

1876. " " Prim. world Switz., ii: 168 . Fig. 356.

1879. " " Urwelt der Schweiz, $2^{2}$ aufl, 531. Fig. 399.

4564. Hylobius? solieri. (Curculionidæ.)

Locality: Aix, France. Horizon: Ligurian.

1874. Oustalet. Ann. sc. géol., v, art. 2: 277-278.

See also Rhynchænus? solieri.

4565. Hylobius tortomanus (borings in Pinus sylvestris). (Curculionidæ.)

Locality : Monte Vaticano, Italy. Horizon : Tortonian.

1876. Ponzi. Foss. monte Vat., 10 (Hylobinum). Pl. 1, fig. 9.

1876. " " Atti accad. line., (2), iii: 932. Pl. 1, fig. 9.

1876. " Ins. lign. monte Vat., 3.

1876. "6 Atti accad.linc., (2), iii: - 
4566. Hylotrupes senex. (Cerambycidæ.)

Locality : Rott, Rhenish Prussia. Horizon: Aquitanian.

1859. Heyden. Palaeontogr., viii: 10. Pl. 1, fig. 3.

$$
\text { 4567. Hylurgus —- (Scolytidæ.) }
$$

Locality : Aix, France. Horizon: Ligurian.

1829. Serres. G6́ogn. terrains tert., 225.

\section{Hypera —- (Curculionidæ.)}

Locality : Aix, France. Horizon : Ligurian.

1829. Curtis. Edinb. new phil. journ., vii : 295.

1829. " Murehison-Lyell, Tert. form. Aix, 11.

\section{Hypera —. (Cureulionidæ.)}

Locality : Aix, France. Horizon: Ligurian.

1829. Serres. Géogn. terrains tert., 224.

\section{Ilybius - (Dytiscidæ.)}

Locality : Oeningen, Baden. Horizon: Tortonian.

1888. Schöberlin. Soc. entom., iii : 42 .

\section{Ilybius ater? (recent). (Dytiscidæ.)}

Locality: Hösbach, Bavaria. Horizon: Lower Pleistocene.

[1774. DeGeer.

1884. Flach.

1884. “

\section{Ins., iv : 401.]}

Käfer unterpleist. Hösbach, 8.

Verhandl, physik.-med. gesellsch. Wiirzb., n. f., xviii : 292.

\section{Ips - (Nitidulidæ.)}

Locality : Prussian amber. Horizon: Ligurian.

1825. Gúrin.

Dict. class. d'hist. nat., viii : $5 \$ 0$.

1829. Serres.

Géogn. terrains tert., 241.

\section{Ischnodes gracilis. (Elateridæ.)}

Locality: Oeningen, Baden. Horizon: Tortonian.

1847. Heer.

1852. Giebel.

Insektenf. tertiärg. Oeningen, i: 133-134. Pl.4, fig. 3.

1856. " "

Deutschl. petref., 651 .

Insect. d. vorwelt, 97-98.

4574. Labidostomis pyrrha. (Chrysomelidx.)

Locality : Rott, Rhenish Prussia. Horizon: Aquitaniau.

1866. Heyd.-Heyd. Palaeontogr., xv: 154-155. Pl. 24, fig. 11.

1866. " Käfer u. polypen, 24-25. Pl.3, fig. 11.

4575. Laccobius elongatus. (Hydrophilidæ.)

Locality: Green River, Wyo. Horizon: Oligocene.

1878. Sendder. Bull. U. S. geol, surv, terr., iv: 761.

1890. “ Tert. ins. N. A., 513, Pl, 7, figs. 27,28 . 
4576. Laccobius excitatus. (Hydrophilidæ.)

Locality : Rott, Rhenish Prussia. Horizon: Aquitanian.

1866. Heyd.-Heyd. Palaeontogr., xv: 135. Pl.22, fig. 3.

1866. " " Käfer u. polypen, 5. Pl. 1, fig. 3 .

4577. Iaccobius priscus. (Hydrophilidæ.)

Locality: Corent, France. Horizon: Aquitanian.

1870. Onstalet. Ann. sc. géol., ii, art. 3: 59-60. Pl.1, fig. 3.

4578. Laccobius vetustus. (Hydrophilidæ.)

Locality: Aix, France. Horizon : Ligurian.

1874. Oustalet. Ann. se. géol., v, art. 2: 130-131. Pl. 1, fig. 11.

\section{Laccophilus - - (Dytiscidæ.)}

Compared with L. maculosus.

Locality: White River, Utah. Horizon: Oligocene.

1876. Scudder. Bull. U. S. geol. surv, terr, ii : 78.

1877. " Bull. U. S. geol. surv. terr., iii : 759.

1890. “ Tert. ins. N. A., 517. Pl. 5, figs. 116, 117.

4580. Laccophilus parvulus. (Dytiscidæ.)

Locality: Cap Staratschin, Spitzbergen. Horizon: Miocene.

1870. Heer. K. svenska vetensk. akad. handl., viii, No. $7: 73$. Pl. 5, figs. $56 c d$.

4581. Lacon murinus (recent). (Elateridæ.)

Locality: Mundesley, England. Horizon: Pleistocene.

[1758. Linné. 1888. Bell.

Syst. nat., i, pt. $2: 655$.]

Entom., xxi : 2.

4582. Lacon primordialis. (Elateridæ.)

Locality: Oeningen, Baden. Horizon: Tortonian.

1847. Heer.

185\%. Giebel.

Insektenf. tertiärg. Oeningen, i: 138-139. Pl. 4, fig. 7.

1856. "

Deutschl. petref., 651.

Insect. d. vorwelt, 96 .

4583. Lamia - (Cerambycidæ.)

Locality : Prussian amber. Horizon: Ligurian.

1845. Berendt. Bernst. befindl, organ. reste vorw., i: 56.

4584. Lamia antiqua. (Cerambycidæ.)

Locality: Oeningen, Baden. Horizon: Tortonian.

1879. Heer. Urwelt der Schweiz, 2 aufl, 401. Fig. 257 on p. 384.

4585. Lamia petrificata. (Cerambyeidæ.)

Locality : Rott, Rhenish Prussia. Horizon: Aquitanian.

1866. Heyd.-Heyd. Palaeontogr., xv: 154. Pl. 24, fig. 10.

1866. " Käfer u, polypen, 24. Pl. 3, fig. 10. 


\section{Lampyris ? - (Lampyridæ.)}

Locality : Prussian amber. Horizon : Ligurian.

1845. Berendt. Bernst. befindl. organ. reste vorw., i : 56.

4587. Iampyris orciluca. (Lampyridæ.)

Locality : Oeningen, Baden. Horizon: Tortonian.

1865. Heer.

18\%2. " "

1876. " "

1879. “

Urwelt der Schweiz, 377 .

Monde prim. Suisse, 46\%.

Prim. world Switz., ii : 33.

Urwelt der Schweiz, 2e aufl., 403.

4588. Laparocerus wollastoni. (Otiorhynchidæ.)

Compared with L. morio Schönh.

Locality : St. Jorge, Madeira. Horizon: Diluvial.

1857. Heer.

Nene denkschr. allg. schweiz. gesellsch. ges. naturwiss., xv, art. 2: 14, note. Pl. 2, fig. 34 .

4589. Larinus

(Curculionidæ.)

Locality: Aix, France. Horizon: Ligurian.

1832. Burmeister. Handb. ent., i: 639.

1836. “ Man. ent., 580.

See also Rhinobatus — Serres.

4590. Larinus

(Curculionidæ.)

Locality: Brunstatt, Alsatia. Horizon: Middle Oligocene.

1888. Foerster. Mitth. comm. geol, Elsass-Lothr., i : 165.

4591. Iarinus —. (Curculionidæ.)

Locality: - Horizon : -

1846. Giebel. Paläozool., 283.

4592. Larinus bronni. (Curculionidæ.)

Locality : Rott, Rhenish Prussia. Horizon: Aquitanian.

1866. Heyd.-Heyd. Palaeontogr., $\mathrm{x} v:$ 150. Pl. $24 ;$ fig. 1.

1866. “ Käfer $\mathrm{u}$. polypen, 20. $\mathrm{Pl}$. 3, fig. 1.

1885. Scudder. Zittel, Handb. palaeont., i, th. ii : 798. Fig. 1040.

1886. " Zittel-Barrois, Traité de paléont., ii : 788. Fig. 1025.

4593. Larinus ovalis. (Cnrculionidæ.)

Compared with L. jaceæ Fabr., Europe.

Locality : Oeningen, Baden. Horizon: Tortonian.

1861. Heer. Rech. climat pays tert., 204 (undescr.).

4594. Lasia primitiva. (Coccinellidæ.)

Locality : Rott, Rhenish Prussia. Horizon : Aquitanian.

1866. Heyd.-Hejd. Palaeontogr., xv: 157. Pl. 24, fig. 21.

1866. “ Käfer u. polypen, 27. Pl. 3, fig. 21.

4595. Iathridius - (Lathrididæ.)

Locality: Prussian amber. Horizon: Ligurian.

1845. Berendt. Bernst, befindl. organ, reste vorw., i : 56 . 


\section{Lathrobium - (Staphylinidæ.)}

Locality: Prussian amber. Horizon: Ligurian.

1845. Berendt. Bernst. befindl. organ. reste vorw., i: 56.

4597. Iathrobium —. (Staphylinidæ.)

Locality: Aix, France. Horizon: Ligurian.

1829. Curtis. $\quad$ Edinb. new phil. journ., vii: 295. Pl. 6, fig. 1.

1829. " Murchison-Lyell, Tert. form. Aix, 11. Pl. 6, fig. 1.

1839. Mantell. Wonders of geology, i: 248. Fig. 3.

1854. “ Medals of creation, 2d ed., ii : 558 . Fig. 183.3.

See also Xantholinus westwoodianus, Lathrobium provinciale.

4598. Iathrobium abscessum. (Staphylinidæ.)

Compared with L. elongatum.

Locality : Green River, Wyo. Horizon: Oligocene.

1876. Scudder. Bull. U. S. geol. surv. terr., ii : 79.

1878. " Bull. U. S. geol. surv, terr., iv: 762.

1890. " Tert. ins. N. A., 505-506. Pl. 8, figs. 15, 21.

4599. Lathrobium interglaciale. (Staphylinidæ.)

Compared with L. grandis LeC.

Locality : Scarboro', Ontario. Horizon: Postpliocene.

1890. Scudder. Tert. ins. N. A., 506. Pl. 1, fig. 38.

4600. Lathrobium œningense. (Staphylinidæ.)

Locality : Oeningen, Baden. Horizon: Tortonian.

1862. Heer. Verhandl. holl. maatsch. wetensch. Haarl., xvi : 47. Pl. 3, figs. $3,3 b-d$.

4601. Iathrobium provinciale. (Staphylinidæ.)

Locality: Aix, France. Horizon: Ligurian.

1856. Giebel. Insect. d. vorwelt, 72.

See also Lathrobium — Curtis, etc.

4602. Iebia -. (Carabidæ.)

Locality : Prussian amber. Horizon : Liguria

1845. Motschulsky. Bull. soc. imp. nat. Mosc., xviii, ii : 98.

4603. Lebia amissa. (Carabidæ.)

Locality : Salzhausen, Germany. Horizon: Miocene.

1865. Heyd.-Heyd. Palaeontogr., xiv: 31. Pl. 9, fig. 13.

4604. Lebina resinata. (Carabidæ.)

Compared with Lebia quadrimaculata.

Locality : Prussian amber. Horizon: Ligurian.

1813. Germar. Germ., Mag. entom., i : 13.

1888. Schlechtend. Zeitschr. ges. naturw., Ixi: 475-477. Figs. 1, 2.

See also Dromius resinatus.

Bull, $71-35$ 
4605. Lieistotrophus patriarchicus. (Staphylinidæ.)

Locality: White River, Colorado or Utah. Horizon : Oligocene.

1876. Scudder.

Bull. U.S. geol. surv, terr, ii : 78-79.

1877. " " Bull. U. S. geol. surv, terr, iii : 759 .

1890. " Tert. ins. N. A., 507. Pl. 5, fig. 112.

4606. Lema -. (Chrysomelidæ.)

Locality : Riedisheim, Alsatia. Horizon: Middle Oligocene.

1885. Foerster. Tagebl. versamml. deutsch naturf., lviii : 392.

1885. " Mitth, oberelsäss. tertiär, 7.

1888. " " Mitth. comm, geol. Elsass-Lothr., i : 165.

1889. " Mitth. comm. geol. Elsass-Lothr., ii : 102.

4607. Lema tumulata. (Chrysomelidæ.)

Locality: Salzhausen, Germany. Horizon: Miocene.

1865. Heyd.-Heyd. Palaeontogr., xiv: 33. Pl. 9, fig. 19.

4608. Lema vetusta. (Chrysomelidæ.)

Compared with L. merdigera $\mathrm{L}$.

1865. Heer.

Locality : Oeningen, Baden. Horizon: Tortonian.

187\%. " "

1876. “

1879. “6

Urwelt der Schweiz, 372. Fig. 240 on p. 371.

Monde prim. Suisse, 456. Fig. 240 on p. 455.

Prim. world Switz., ii : 28. Fig. 240 on p. 27.

Urwelt der Schweiz, $2^{\circ}$ anfl., 398. Fig. 282 on p. 397.

4609. Lepitrix germanica. (Scarabæidæ.)

Compared with L. lineata F., Southern Africa.

Locality : Oeningen, Baden. Horizon: Tortonian.

1862. Heer.

1865. “

1872. “

1876. “

1879. “

Verhandl. holl. maatsch. wetensch. Haarl., xvi : 80. Pl. 6, fig. 19.

Urwelt der Schweiz, 380. Fig. 264 on $p .378$.

Monde prim. Suisse, 466. Fig. 264 on $p, 463$.

Prim. world Switz., ii : 37. Fig. 264 on $p .34$.

Urwelt der Schweiz, $2^{e}$ aufl., 406. Fig. 306 on p. 404.

4610. Leptura - (Cerambycidæ.)

Locality : Prussian amber. Horizon : Ligurian.

1845. Berendt. Bernst. befindl. organ, reste vorw., i : 56.

4611. Leptura - (Cerambycidæ.)

Locality: Prussian amber. Horizon : Ligurian.

1856. Menge. Progr. petrischule Dauzig, 1856: 21.

4612. Leptura - (larva). (Cerambycidæ.)

Locality: Prussian amber. Horizon: Ligurian.

1856. Menge. Progr. petrischule Danzig, 1856: 23.

4613. Licinus -. (Carabidæ.)

Locality: Lexden, Englaud. Horizon: Pleistocene.

1863. Wollaston. Quart. journ. geol. soc. Lond, xix, i : 401, No. 6.

1889. Bell Entom., xxi:?. 
4614. Limnichus - (Byrrhidæ.)

Locality: Prussian amber. Horizon: Ligurian.

1845. Berendt. Bernst. befindl. organ. reste vorw., i : 56 .

4615. Limonius —_. (Elaterida.)

Locality : Prussian amber. Horizon: Ligurian.

1845. Berendt. Bernst. befindl. organ. reste vorw., i: 56.

4616. Limonius optabilis. (Elateridæ.)

Localities: Oeningen, Baden; Rhine, Germany. Horizons: Tortonian, Aquitanian?. 1847. Heer. Insektenf. tertiärg. Oeningen, i: 137-138. Pl.4, fig. 6.

1852. Giebel. Deutschl. petref., 651.

1856. " " Insect. d. vorwelt, 95-96.

1862. Heyden. Palaeontogr., $\mathrm{x}: 69$.

4617. Lina populeti. (Chrysomelidæ.)

Compared with L. populi.

Localities: Oeningen, Baden; Rhine, Germany. Horizons: Tortonian, Aquitanian?

1847. Heer.

1852. Giebel.

Insektenf. tertiärg. Oeningen, i : 207-208. Pl. 7, fig. 7.

1856. “

Deutschl. petref., 648 .

1858. Heer.

Insect. d. vorwelt, 121-122.

1862. Heyden.

Bull. séances soc. vaud. sc. nat., v : 150.

1865. Heer.

Palaeontogr., $\mathrm{x}:$ 73-74.

1872. “

Urwelt der Schweiz, 362, 372. Fig. 237.

1876. " "

Monde prim. Suisse, 444, 456. Fig. 237 on p. 455.

1879. " "

Prim. world Switz., ii : 16,26. Fig. 237 on p. 27.

Urwelt der Schweiz, $2^{e}$ aufl., 397. Fig. 279.

4618. Lina sociata. (Chrysomelidæ.)

Locality: Rott, Rhenish Prussia. Horizon : Aquitanian.

1866. Heyd.-Heyd. Palaeontogr., xv: 155. Pl. 24, fig. 13.

1866. " Käfer u. polypen, 25. $\quad P l$. 3, fig. 13.

4619. Lina wetteravica. (Chrysomelidæ.)

Localities: Salzhausen, Hessenbruckener Hammers bei Laubach, Germany. Horizon : Aquitanian.

1862. Heyden. Palaeontogr., x: 73. Pl. 10, fig. 24.

1865. Heyd.-Heyd. Palaeontogr., xiv : 35.

\section{Liparus -. (Otiorhynchidæ.)}

Compared with L. anglicanus Marsh.

Locality: Aix, France. Horizon: Ligurian.

1829. Curtis. Edinb. new phil. journ., vii: 295.

1829. " Murchison-Lyell, Tert. form. Aix, 11.

\section{Liparus —_. (Otiorhynchidæ.)}

Compared with L. punctatus.

Locality : Aix, France. Horizon: Ligurian.

1829. Curtis. Edinb. new phil. journ., vii : 295. Pl. 6, fig. 3.

1829. " Murchison-Lyell, Tert. form. Aix, 11. Pl. 6, fig. 3.

1839. Mantell. Wonders of geology, i: 248. Fig. 5.

1854. " Medals of creation, 2d ed., ii : 558. Fig. 183.5.

See also Curculịnịites morosus, Liparus prịmæa ụs, Hỵlobịus morosus. 
4622. Liparus primævus. (Otiorhynchidæ.)

Locality: Aix, France, Horizon: Ligurian.

1856. Giebel. Insect. d. vorwelt, 140.

Seealso Liparus _ Curtis ef. punctatus, Hylobius morosus, Cureulionites morosus.

4623. Listronotus muratus. (Curculionidæ.)

Locality: Green River, Wyo. Horizon: Oligocene.

1890. Scudder. Tert. ins. N. A., 474. Pl. 8, fig. 23.

Lithocaris varicolor. See Lithocharis varicolor.

4624. Iithocharis varicolor. (Staphylinidæ.)

Locality: Aix, France. Horizon: Ligurian.

1856. Heer. Viert. naturf. gesellsch. Zürich, i: 15-16 (Lithocaris). Pl. 1, fig. 2.

1874. Oustalet. Ann. se. géol, v, art. 2: 147-149. Pl.5, fig. 16.

4625. Lithoplanes —. (Staphylinidæ.)

Locality: Aix, France. Horizon: Ligurian.

1886. Scudder. Bull. U. S. geol surv., No. 31 : 81, note.

See also Erynnis deleta, E. elongata.

4626. Lixus —_. (Curculionidæ.)

Locality: Aix, France. Horizon : Ligurian.

1847. Hope. Trans. ent. soc. Lond, iv : 251.

4627. Lixus œningensis. (Curculionidæ.)

Compared with $\mathbf{L}$. angustatus $\mathbf{F}$.

Locality: Ueningen, Baden. Horizon: Tortonian.

1865. Heer. Urwelt der Schweiz, 374.

1872. " Monde prim. Suisse, 459.

1876. " $\quad$ Prim. world Switz., ii : 30.

1879. “ Urwelt der Schweiz, $2^{\mathrm{e}}$ aufl, 400.

4628. Lixus rugicollis. (Curculionidæ.)

Compared with L. gemellatus Sch.

Locality: Oeningen, Baden. Horizon: Tortonian.

1847. Heer.

1852. Giebel.

1856. “

1865. Heer.

1872. "

1876. " "

1879. “
Insektenf. tertiärg. Oeningen, i : 192-193. Pl. 6, fig. 14.

Deutschl. petref., 648 .

Insect. d. vorwelt, 144.

Urwelt der Schweiz, 374.

Monde prim. Suisse, 459.

Prim. world Switz., ii: 30.

Urwelt der Schweiz, $2^{\mathrm{e}}$ aufl., 400.

4629. Lomatus hislopi. (Buprestidæ.)

1860. Murray. Quart. journ. geol. soc. Lond, xvi : 18̨2-183. Pl. 10, fig. 66.

1879. Medl.-Blanf, Geol, India, i; 314, 
4630. Loricera glacialis. (Carabidæ.)

Compared with L. neoscotica LeC.

Locality: Scarboro', Ontario. Horizon: Interglacial.

1877. Scudder. Bull. U. S. geol. surv. terr., iii : 763.

1890. " Tert. ins. N. A., 533. Pl. 1, figs. 50, 57.

4631. Loricera? lutosa. (Carabidæ.)

Locality : Scarboro', Ontario. Horizon: Postpliocene.

1890. Seudder. Tert. ins. N. A., 533-534. Pl. 1, fig. 32.

4632. Loxandrus gelidus. (Carabidæ.)

Compared with $\mathrm{T}_{\mathrm{m}}$ agilis LeC and L. erraticus LeC.

Locality: Scarboro', Ontario. Horizon: Interglacial.

1877. Scudder. Bull. U. S. geol. surv, terr., iii : 763-764.

See also Pterostichus gelidus.

4633. Lucanus - (Lucanidæ.)

Locality : - Horizon :

1846. Giebel. Paläozool., 288.

4634. Lucanus - (Lucanidæ.)

Locality: Rhine, Germany. Horizon: Aquitanian \%.

1831. Goldfuss. Verhandl. leop.-carol. akad, naturf, vii, i : 118.

4635. Iuciola extincta. (Lampyridæ.)

Locality : Rett, Rhenish Prussia. Horizon: Aquitanian.

1862. Heyden. Palaeontogr., $\mathrm{x}: 69 . \quad$ Pl. 10, fig. 15.

4636. Lycoperdina -. (Endomychidæ.) .

Locality : Prussian amber. Horizon: Ligurian.

1845. Berendt. Bernst. befindl, organ. reste vorw., i: 56.

4637. Iycoperdina (vic.) - (Endomychidæ.)

Locality : Prussian amber. Horizon: Ligurian.

1856. Menge. Progr. petrischule Danzig, 1856: 21 .

4638. Lyctus - (Ptinidæ.)

Locality : Prussian amber. Horizon: Ligurian.

1829. Serres. Géogn. terrains tert., 241.

4639. Lycus - . (Lampyridæ.)

Locality: Prussian amber. Horizon: Ligurian.

1845. Berendt. Bernst. befindl. organ. reste vorw., i: 56.

Lymexilon —. See Lymexylon — Berendt.

4640. Lymexylon? - (Lymexylidæ.)

Locality: Prussian amber. Horizon : Ligurian.

1845. Berendt. Berust. befindl, organ, reste vorw., i : 56 (Lymexilon). 


\section{Lymexylon - (larva). (Lymexylidie.)}

1856. Menge.

Locality : Prussian amber. Horizon: Ligurian.

Progr. petrischule Danzig, 1856: 23.

4642. Iytta æsculapii. (Meloid*.)

Compared with $\mathrm{I}_{\text {, }}$ vesicatoria $\mathrm{L}$.

Locality: (Oeningen?, Baden). Horizon: Tortonian.

1847. Heer.

Insektenf. tertiärg. Oeningen, i: 155-159. Pl. 5, figs. 4, 5.

1852. Giebel.

Deutschl, petref., 650 .

1856. " Insect. d. vorwelt, 105-106.

1865. Heer.

Urwelt der Schweiz, 3z6. Fig. 255.

1872. “6

Monde prim. Suisse, 462. Fig. 255 on p. 461.

1876. “

Prim. world Switz., ii : 32. Fig. 255.

1879. “

Urwelt der Schweiz, 2e aufl., 401. Fig. 297 on p. 402.

1885. Scudder. Zittel, Handb. palaeont, , i, th. ii : 790. Fig. 1013.

1886. " Zittel-Barrois, Traité de paléont., ii : 790. Fig. 1031.

See also Meloe proscarabæus.

4643. Magdalinus deucalionis. (Curculionidæ.)

Locality: Rott, Rhenish Prussia. Horizon: Aquitanian.

1866. Heyd.-Heyd. Palaeontogr., x’v: 151-152. Pl. 24, fig. 4.

1866. “ Käfer u. polypen, 21-22. Pl. 3, fig. 4.

4644. Magdalinus protogenius. (Curculionidæ.)

Locality : Rott, Rhenish Prussia. Horizon : Aquitanian.

1866. Heyd.-Heyd. Palaeontogr., xv: 151. Pl. 24, fig. 3.

1866. “ Käfer u. polypen, 21. Pl. 3, fig. 3.

4645. Malachius —. (Malachidæ.)

Locality: Prussian amber. Horizon: Ligurian.

1845. Berendt. Bernst. befindl. organ. reste vorw., i : 56.

4646. Malachius ——. (Malachidæ.)

Locality: Prussian amber. Horizon: Ligurian.

1856. Menge. Progr. petrischule Danzig, 1856: 21.

4647. Malachius vertumni. (Malachidæ.)

Locality: Oeningen, Baden. Horizon: Tortonian.

1847. Heer. Insektenf, tertiärg. Oeningen, $i$ : 150-152 (vertummni). $P l_{0} 5_{\text {, }}$ figs. 1, 2.

1852. Giebel. Dentschl. petref., 650.

1856. " Insect. $d$. vorwelt, 103.

Malachius vertummni. See Malachins vertumni.

4648. Malthinus - (Lampyridæ.)

Locality: Prussian amber. Horizon: Ligurian.

1845. Berendt. Bernst. befindl. organ. reste vorw., i: 56.

4649. Malthodes —_. (Lampyridæ.)

Locality : Brunstatt, Alsatia. Horizon: Middle Oligocene.

1890. Foerster. In litt. 


\section{Meleus ㄱ. (Bytsopidæ.)}

Locality: Aix, France. Forizon: Ligurian:

1829. Serres: Géogn. terrains tèrt., 223:

4651. Meligethes ㄴ.. (Nitidulidæ.)

Locality: Brunstatt, Alsatia. Horizon : Middle Oligocene.

1890. Foerster. In litt.

\section{Meloe … (Meloidæ.)}

Locality : Rhine, Germany. Horižn : Aquitanian?.

1831. Goldfuss. Verhandl. leop-carol. akad. naturf., vii, i : 11\%,

4653. Meloe? (larva). (Meloidæ.)

Locality: Prussian amber. Horizon: Ligurian.

1856. Menge. Progr. petrischule Danzig, 1856: 23-24.

4654. Meloe podalirii. (Meloidæe)

Locality : Radoboj, Croatia. Horizon : Mayencian.

1847. Heer. Insektenf. tertiärg. Oeningen, i: 159-160. Pl. 5, fig. 7.

1852. Giebel. Deutschl. petref., 650.

1856. " Insect. d. vorwelt, 106.

4655. Meloe proscarabæus. (Meloidæ.)

Locality: Oeningeu, Badeu. Horizon: Tortonian.

"1805. Karg. Denkschr. vaterl. gesellsch. ärtze n. naturf. Schwabens, 40."

1847. Heer. Insektenf. tertiärg. Oeningen, i: 159 (may = Lytta æsculapii).

See also Lytta æsculapii.

4656. Melolontha -. (Scarabæidæ.)

Locality : Oeningen, Baden. Horizon : Tortonian.

1834. Keferstein. Naturg. erdkörp., ii : 331 .

4657. Melolontha -. (Scarabæidæ.)

Locality : Aix, France. Horizon : Ligurian.

1829. Serres. Géogn. terrains tert., 221.

4658. Melolontha greithiana. (Scarabæidæ.)

Compared with M. (Catalasis) australis Schönh.

Locality: Greith, Hohe-Rhönen, Germany. Horizon: Aquitanian.

1847. Heer. Insektenf. tertiärg. Oeningen, i: 6ĩ-68. Pl. 8, fig. 5.

1852. Giebel. Dentschl. petref., 652.

1856. “ Insect. d. vorwelt, 32 .

1865. Heer. Urwelt der Schweiz, 380. Fig. 263 on $p .378$.

1872. " Monde prim. Snisse, 466. Fig. 263 on $p .463$.

1876. " $\quad$ Prim. world Switz., ii: 37. Fig. 263, on p. 34.

1879. “ Urwelt der Schweiz, 2e anfl, 406. Fig. 305, on $p .404$.

4659. Melolontha hippocastani (recent). (Scarabæidæ.)

Locality: Schwerzenbach, Switzerland. Horizon: Glacial clay.

[1801. Fabricius. Syst. eleuth., ii : 162.]

1879. Heer. Urwelt der Schweiz, $2^{2}$ aufl., 581.

1885. Sendder. Zittel, Handb. palaeont., i, th. ii : 794.

1886. " Zittel-Barrois, Traité de paleont., ii: 795 .

1886. " Bull, U. S. geol, surv., No. 31: 74 . 
4660. Melolontha solitaria. (Scarabæidæ.)

Locality : Krottensee, Bohemia. Horizon: Aquitanian.

1877. Novák. Sitzungsb. k. akad. wiss. Wien, lxxvi: 92-93. Pl. 3, figs. 7ab. 1877. " "

Fauna egerer tertiärb., 22-23. Pl. 3, figs. 7ab.

4661. Melolonthites aciculatus. (Scarabæidæ.)

1847. Heer. 1852. Giebel. 1856. "

Locality: Oeningen, Baden. Horizon: Tortonian.
Insektenf. tertiärg. Oeningen, i: 71 (aciculata). $\quad$ Pl. 2, fig. 13.
Dentschl. petref,, 652.
Insect. $d$. vorwelt, 33 .

\title{
4662. Melolonthites deperditus. (Scarabæidæ.)
}

1847. Heer. 1852. Giebel. 1856. “ “

Locality : Oeningen, Baden. Horizon : Tortonian.

\begin{abstract}
Insektenf. tertiärg. Oeningen, i: 71-72 (deperdita). Pl. 2, fig. 14.
\end{abstract}
Dentschl. petref., 652 .

Insect. d. vorwelt, 33 .

4663. Melolonthites kollari. (Scarabæidæ.)

Localities: Parschlng, Styria. Horizon: Tortonian.

1847. Heer. Insektenf. tertiärg. Oeningen, i: 72-73. Pl.7, fig.32.

1852. Giebel. Deutschl. petref., 652.

1856. " Insect. d. vorwelt, 33-34.

4664. Melolonthites lavateri. (Scarabæidæ.)

1847. Heer.

1852. Giebel.

1856. " "

Locality: Oeningen, Baden. Horizon: Tortonian.

Insektenf. tertiärg. Oeningen, i : 73-74. Pl. 7, fig. 30.

Deutschl. petref., 652 .

Insect. d, vorwelt, 34 .

4665. Melolonthites obsoletus. (Scarabæidæ.)

Locality: Oeningen, Baden. Horizon: Tortonian.

1347. Heer.

1852. Giebel.

Insektenf. tertiärg. Oeningen, i : 73 (obsoleta). Fl. \&, fig. 15.

- Deutschl. petref., 652.

Insect. d. vorwelt, 34 .

\section{Melolonthites parschlugana. See Melolonthites parschlugianus.}

\section{Melolonthites parschlugianus. (Scarabæidæ.)}

Localities: Parschlug, Stjria; Szántó, Hungary. Horizon : Tortonian.

1847. Heer.

Insektenf. tertiärg. Oeningen, i: 72 (parschlugiana). $\mathrm{Pl} .7$, fig. 31.

1852. Giebel.

1856. " "

1870. Unger.

Deutschl. petref., 652.

Insect. $d$. vorwelt, 33 (parschlngana).

Denkschr. akad. wiss. Wien, math. nat. cl., xxx, i: 3-4. Pl. 1, figs. 13, 13* $14,14^{*}$.

\section{Mengea tertiaria. (Stylopidæ.)}

\section{Locality: Prussian amber. Horizon: Ligurian.}

1886. Grote.

Can. entom., xviii: 100.

See also Triæna tertiaria. 
4668. Meristos hunteri. (Curculionidæ.)

Locality : Nagpur, Central India. Horizon:

1860. Murray. Quart. journ. geol. soe. Lond., xvi: 184. Pl. 10, fig.67.

1879. Medl.-Blanf. Geol. India, i : 314.

4669. Mesosa jasonis. (Cerambycidæ.)

1847. Heer.

1852. Giebel.

1856. " “

1865. Heer.

1872. “

1876. “

1879. “

Locality : Oeningen, Baden. Horizon: Tortonian.
Insektenf. tertï̈rg. Oeningen, i : 165-166. Pl. 5, fig. 12。
Deutschl, petref., 649 .
Insect. d. vorwelt, 129-130.
Urwelt der Schweiz, 376.
Monde prim. Suisse, 461.
Prim. world Switz., ii : 31.
Urwelt der Schweiz, 2e aufl., 401.

4670. Mesosites macrophthalmus. (Cerambycidæ.)

Locality: Kutschlin, Bohemia. Horizon: Miocene.

1881. Diechmiiller. Foss. ins, diatomeensch. Kutsehlin, 27-29. Pl. 21, fig. 12.

1881. " V Verhandl. leop. carol. akad. naturf., xlii : 319-321.

1885. Scudder. Zittel, Handb. palaeont., i, th. ii : 793. Fig. 1022.

1886. "6 Zittel-Barrois, Traité de paléont., ii : 793. Fig. 1040.

4671. Microrhagus —. (Elateridæ.)

Locality: Prussian amber. Horizon: Ligurian.

1845. Berendt. Bernst. befindl. organ. reste vorw., i: 56.

4672. Microzoum veteratum. (Cioidæ.)

Locality: Rott, Rhenish Prussia. Horizon : Aquitanian.

1866. Heyd.-Heyd. Palaeontogr, $\mathrm{xv}:$ 145. Pl. 22, fig. 28.

$1866 . \quad$ “ Käfer u. polypen, 15. Pl. 1, fig. 28.

1885. Scudder. Zittel, Handb, palaeont., i, th. ii : 795. Fig. 1029.

1886. " Zittel-Barrois, Traité de paléont., ii : 796. Fig. 1047.

4673. Molops —. (Carabidæ.)

Locality: Brunstatt, Alsatia. Horizon : Middle Oligocene.

1888. Foerster. Mitth. comm. geol. Elsass-Lothr., i : 165.

1889. “ Mitth. comm. geol. Eisass-Lothr., ii : 103.

4674. Molorchus -. (Cerambycidæ.)

Locality : Prussian amber. Horizon: Ligurian.

1845. Berendt. Bernst. befindl. organ. reste vorw., i : 56.

\section{Molorchus antiquus. (Cerambycidæ.)}

Compared with M. abbreviatus.

Locality: Vicinity Bonn, Rhenish Prussia. Horizon: Aquitanian?.

1837. Germar. Fanna insect. Europæ, xix : 14. Pl. 14.

1839. Brulle. Gisem. ins. foss., 20.

1852. Giebel. Dentsehl. petref., 650.

1856. " Insect. d. vorwelt, 128.

See also Hesthesis antiqua. 
4676. Molytes hassencampi. (Otiorbynchidx.)

Locality : Sieblos, Rhenish Prussia. Horizon: Aquitanian.

1858. Heyden. Palaeontogr., v: 116-11\%. Pl. 23, fig. 14.

4677. Mononychus pseudacori (recent). (Curculionidæ.)

Locality : Jarville, France. Horizon: Pleistocene.

[1792. Fabricius. Ent. syst, i, 2: 408.]

1875. Fliche. Comptes rendus, $1 \times x x: 1234$.

See also Mononychus punctum-album.

4678. Mononychus punctum-album (recent). (Curculionidæ.)

Locality: Jarville, France. Horizon: Pleistocene.

[1784. Herbst. Füssl. arch., 1784: 74. Pl. 24, fig. 17.]

1885. Scudder. Zittel, Handlb. palaeont, i, th. ii: 788.

1886. " "Zittel-Barrois, Traité de paléont, ii : 788.

1886. “ Bull. U.S. geol. surv. No. 31: 67 .

See also Mononychus pseudacori.

4679. Mordella -. (Mordellidæ.)

Locality : Prussian amber. Horizon : Ligurian

1845. Berendt. Bernst. befindl. organ. reste vorw., i: 56 .

4680. Mordella inclusa. (Mordellidæ.)

Locality: Prussian amber. Horizon: Ligurian.

1832. Burmeister. Handb. entom., i: 635 .

1836. “ Man. ent., 577.

1856. Giebel. Insect. d. vorwelt, 112.

1888. Schlechtend. Zeitschr. ges. naturw., 1xi : 479-483.

See also Mordellina inclusa, Ptomaphagus germari.

4681. Mordellina inclusa. (Mordellidæ.)

Compared with Mordella aculeata.

Locality : Prussian amber. Horizon : Ligurian.

1813. Germar. Germar, Mag. entom., i : 14-15.

1885. Sendder. Zittel, Handb. palaeont., i, th. ii: 790.

1886. " "Zittel-Barrois, Traité de paléont., ii : 791

1886. " Bull. U. S. geol. surv., No. 31: 70 .

1888. Schlechtend. Zeitschr. ges. naturw., lxi : 478-479.

See also Mordella inelnsa, Ptomaphagus germari.

4682. Mycetocharoides baumeisteri. (Cistelidæ.)

Locality: Prussian amber. Horizon : Ligurian.

1888. Schanfuss. Berl, ent. zeitschr., xxxii : 269-270.

4683. Mycetoporus —_. (Staphylinidæ.)

Locality: Prussian amber. Horizon: Ligurian.

1845. Berendt. Bernst. befindl. organ. reste vorw., i : 56 . 
4684. Mycotretus binotatus. (Erotylidæ.)

Compared with M. sanguinipennis Lac.

Locality : Green River, Wyo. Horizon: Oligocene.

1878. Sendder. Bull. U. S. geol. surv. terr., iv : 763-764 (binotata). 1885. " Zittel, Handb. palaeont., i, th. ii : 800. Fig. 1053.

1886. " "Zittel-Barrois, Traité de paléont., ii : 800. Fig. 1070.

1890. " Tert. ins. N. A., 502-503. Pl. 7, fig. 30.

4685. Mycterus molassicus. (Melandryidæ.)

Locality: Oeningen, Baden. Horizon: Tortonian.

1847. Heer.

Insektenf. tertiärg. Oeningen, i: 163. $P l .5, f g .10$.

1852. Giebel.

Deutschl. petref., 650 .

1856. " Insect. 凡. vorwelt, 134 .

4686. Mylabris deflorata. (Meloidæ.)

Locality : Rott, Rhenish Prussia. Horizon: Aquitanian.

1866. Heyd.-Heyd. Palaeontogr., $\mathrm{xv}:$ 146. Pl. 23, fig. 5.

1866. “ Käfer u. polypen, 16. Pl. 2, fig. 5.

4687. Myodites meyeri. (Rhipiphoridæ.)

Locality : Rott, Rhenish Prussia. Horizon: Aquitanian.

1866. Heyd.-Heyd. Palaeontogr., xv: 146. Pl. 22, fig. 29.

1866. " Käfer u. polypen, 16. Pl. 1, fig. 29.

4688. Myrmedonia - ( - (Staphylinidæ.)

Locality: Prussian amber. Horizon: Ligurian.

1856. Menge. Progr. petrischule Danzig, 1856: 23.

4689. Nanophyes japetus. (Curculionidæ.)

Locality: Rott, Rhenish Prussia. Horizon: Aquitanian.

1866. Heyd.-Heyd. Palaeontogr., xv: 153. Pl.24, fig.8.

1866. " Käfer u. polypen, 23. Pl. 3, fig. 8.

\section{Naupactus —. (Otiorhynchidæ.)}

Compared with N. Iusitanicus.

Locality : Aix, France. Horizon: Ligurian.

1829. Serres. Géogn. terrains tert., 224.

4691. Naupactus crassirostris. (Otiorhynchidæ.)

Locality : Oeningen, Baden. Horizon: Tortonian.

1865. Heer.

Urwelt der Schweiz, 375. Fig. 245 on $p .371$.

1872. "

Monde prim. Suisse, 460. Fig. 245 on p. 455.

1876. " 6

Prim. world Switz., ii : 30. Fig. 245 on p. 27.

1879. "

Urwelt der Schweiz, 2 aufl., 400. Fig. 287 on p. 397.

\section{Nebria - (Carabidæ.)}

Locality : Prussian amber. Horizon: Ligurian.

1845. Berendt. Bernst, befindl, organ. reste vorw, i: 56. 
4693. Nebria paleomelas. (Carabidæ.)

Compared with N. sahlbergi Fisch.

Locality: Nicola River, British Columbia. Horizon :

1879. Scudder.

1879. "

1890. “

Rep. progr. geol. surv. Can., 1877-78: B 179.

Insect. tert. Nicola, 4 .

Tert. ins. N. A., $532 . \quad P l .2$, fig. 20.

4694. Nebria pluto. (''arabidæ.) -

Locality: Oeningen, Baden. Horizon: Tortonian.

1862. Heer. Verhandl. holl. maatsch. wetensch. Haarl., xvi: 18-19. Pl. 1, figs. 1, 2.

4695. Nebria tisiphone. (Carabidæ.)

Locality: Aix, France. Horizon: Ligurian.

1874. Oustalet. Ann. sc. géol., v, art. 2: 78-80. Pl. 1, fig. 1.

4696. Necticus minutus. (Dytiscidæ.)

Locality: LePuy, France. Horizon:

1854. Aymard. Congr. scient. France, sess. xxii : 42 (undescr.).

4697. Necticus palustris. (Dytiscidæ.)

Locality : LePuy, France. Horizon:

1854. Aymard. Congr. scient. France, sess. xxii : 42 (undescr.).

4698. Necydalis ? - (Cerambycidæ.)

Locality : Prussian amber. Horiznn : Ligurian.

1845. Berendt. Bernst. befindl, organ. reste vorw., i : 56 .

4699. Neothanes testeus. (Carabidæ.)

Locality: Green River, Wyo. Horizon: Oligocene.

1890. Scudder. Tert. ins. N. A., 535-536. Pl. 7, figs. 32, 39.

See also Cychrus testeus.

4700. Nitidula - (Nitidulidæ.)

Locality: Prussian amber. Horizon: Ligurian.

1845. Berendt. Bernst. befindl, organ. reste vorw., i: 56.

4701. Nitidula æmula. (Nitidulidæ.)

Locality: Oeningen, Baden. Horizon: Tortonian.

1862. Heer. Verhandl. holl. maatsch. wetensch. Haarl, xvi: 52. Pl. 3, fig. 29

4702. Nitidula ancora. (Nitidulidæ.)

Locality: Oeningen, Baden. Horizon: Tortonian.

1862. Heer. Verhandl. holl. maatsch. wetensch. Haarl., xvi: 51. Pl. 3, fig. 30.

4703. Nitidula maculigera. (Nitidulidæ.)

Locality: Oeningen, Baden. Horizon: Tortonian.

1862. Heer.

Verhandl. holl. mąatsch. wetensch. Haarl., xvi: 52. Pl.3, fig. 27.

1885. Seudder.

Zittel, Handb, palaeont., i, th. ii : 799. Fig. 1045.

1886. " Zittel-Barrois, Traité de pal6́nt., ii : 799. Fig. 1062. 
4704. Nitidula melanaria. (Nitidulidæ.)

Locality: Oeningen, Baden. Horizon: Tortonian.

1847. Heer. 1852. Giebel. 1856. “

1847. Heer. 1852. Giebel. 1856.

1862. Heer.

Insektenf. tertiärg. Oeningen, i : 36-37. $P l$. 7, fig. 21.

Deutschl. petref., 654 .

Insect. d. vorwelt, 43.

4705. Nitidula pallida. (Nitidulidæ.)

Locality: Oeningen, Baden. Horizon: Tortonian.

Verhandl. holl. maatsch. wetensch. Haarl., xvi: 52-53. Pl. 3, fig. 28.

4706. Nitidula radobojana. (Nitidulidæ.)

Locality: Radoboj, Croatia. Horizon: Mayencian.

Insektenf. tertiärg. Oeningen, i: 37-38. $\quad P l$. 2, fig. 8.

Deutschl. petref., 654 .

Insect. d. vorwelt, 43.

4707. Nosodendron tritavum.- (Byrrbidæ.)

Compared with N. unicolor.

Locality: Green River, Wyo. Horizon: Oligocene.

1890. Scudder. Tert. ins. N. A., 499. Pl. 7, fig. 36.

4708. Notaris? - (Cureulionidæ.)

Locality : Aix, France. Horizon : Ligurian.

1829. Curtis. Edinb. new. phil. journ., vii : 295.

1829. " Murchison-Lyell, Tert. form. Aix, 11.

4709. Notiophilus aquaticus (recent). (Carabidw.)

Locality : Ostend, Norfolk, England. Horizon: Pleistocene.

[1746. Linné. Faun. suec., 752.]

1888. Bell. Entom., xxi : 2(vel palustris).

4710. Notiophilus palustris (recent). (Carabidæ.)

Locality : England. Horizon : Pleistocene.

[1812. Duftschmid. Faun. austr., ii : 192.]

See also Notiophilus aqnaticus.

4711. Oberea præmortua. (Cerambycidœ.)

Locality: Rott, Rhenish Prussia. Horizon: Aquitanian.

1862. Heyden. Palaeontogr., $x:$ 72. Pl.10, fig. 23.

\section{Obrium —. (Cerambycidæ.)}

Compared with O. testaceum.

Locality : Prussian amber. Horizon : Ligurian.

1832. Burmeister. Handb. ent., i: 635.

1836. " Man. ent., 577.

4713. Obrium —. (Cerambycidæ.)

Locality: - Horizon: -

1846. Giebel,

Paläiozool., 283, 
4714. Ochthebius plutonis. (Hydrophilidæ.)

Locality : Rott, Rhenish Prussia. Horizon: Aquitanian.

1866. Heyd.-Heyd. Palaeontogr., $\mathrm{xv}: 136 . \quad P l .22$, figs. 8, 9.

1866. “ Käfer u. polypen, 6. Pl. 1, figs. 8, 9 .

Ocypus atavus. See Staphylinus (Ocypus) atavus.

Ocypus germarii. See Staphylinus (Ocypus) germarii.

Ocypus provincialis. See Staphylinus (Ocypus) provincialis.

Odontocheila —_. See Cicindela (Odontocheila) _-

Odontoda $\longrightarrow$. See Odontota -

4715. Odontota - (Chrysomelidæ.)

Locality: Prussian amber. Horizon: Ligurian.

1856. Menge. Progr. petrischule Danzig, 1856: 21 (Odontoda).

4716. Cademera -. (Edemeridæ.)

Locality : Prussian amber. Horizon: Ligurian.

1845. Berendt. Bernst. befindl, organ, reste vorw., i : 56.

4717. Olibrus - (Phalacridæ.)

Locality: Brunstatt, Alsatia. Horizon: Middle Oligocene.

1890. Foerster. In litt.

Omalium —. See Homalinm —.

Omalium protogeæ. See Homalium protogeæ.

4718. Omaseus nigritus (recent). (Carabidæ.)

Lovality: Dürnten, Switzerland. Horizon: Interglacial.

1886. Scudder. Bull. U. S. geol. surv., No. 31: 84 (nigrita).

See also Pterostichus nigritus.

4719. Oniticellus amplicollis. (Scarabæidæ.)

Compared with O. flavipes F., Europe.

Locality: Oeningen, Baden. Horizon: Tortonian.

1862. Heer. Verhandl. holl. maatsch. wetensch. Haarl., xvi: 76,77. Pl. 6, fig. 11.

1865. " Urwelt der Schweiz, 379. Fig. 269 on $p .378$

1872. " Monde prim. Suisse, 465. Fig. 269 on p. 463.

1876. " Prim. world Switz., ii : 36 . Fig. 269 on $p .34$.

1879. “ Urwelt der Schweiz, $2^{e}$ autl., 405. Fig. 311 on p. 404.

4720. Onitis magus. (Scarabæidæ.)

Locality: Rott, Rhenish Prussia. Horizon: Aquitanian.

1862. Heyden, Palaeontogr., $\mathrm{x}: 65$. Pl. 10, fig. 13. 
4721. Onthophagus bisontinus. (Scarabæidæ.)

Compared with O. affinis St.

1862. Heer. 1865. “ “ 1872. “ 1876. “ 1879. "

1862. Heer. 1865. " 1872. " " 1876. “ 1879. “

1847. Heer. 1852. Giebel. 1856. "6 1865. Heer. 1872. " " 1876. " " 1879. " "

1862. Heer.

1865. " "

187:. “

1876 . “

1879. " "

1885. Scudder. 1886. " "
Locality: Oeningen, Baden. Horizon: Tortonian.

Verhandl. holl. maatsch. wetensch. Haarl., xvi ; 76. Pl.6, fig. 7.

Urwelt der Schweiz, 379.

Monde prim. Suisse, 465.

Prim. world Switz., ii : 36.

Urwelt der Schweiz, $2^{e}$ aufl, 405.

4722. Onthophagus crassus. (Scarabæidæ.)

Compared with O. vacca.

Locality : Oeningen, Baden. Horizon : Tortonian.

Verhandl. holl. maatsch. wetensch. Haarl., xvi : 75, Pl. 6, fig. 5.

Urwelt der Schweiz, 379.

Monde prim. Suisse, 465.

Prim. world Switz., ii: 36.

Urwelt der Schweiz, $2^{\circ}$ aufl, 405.

4723. Onthophagus luteus. (Scarabæidæ.)

Locality : Aix, France. Horizon: Ligurian.

Anu. se. géol., v, art. 2 : 194-196. Pl. 2, fig. 17.

4724. Onthophagus ovaiulus. (Scarabæidæ.)

Compared with O. ovatus L.

Locality: Oeningen, Baden. I Horizon: Tortonian.

Insektenf. tertiärg. Oeningen, i: 64. Pl. 7, fig. 26.

Doutschl. petref., 653 .

Insect. d. vorwelt, 39.

Urwelt der Schweiz, 379.

Monde prim. Suisse, 465.

Prim. world Switz, ii : 36 .

Urwelt der Schweiz, 2e autl., 405.

4725. Onthophagus prodromus. (Scarabæidæ.)

Compared with $\mathrm{O}$, vacea.

Locality : Oeningen, Baden. Horizon: Tortonian.

Verhandl. holl. maatseh. wetensch. Haarl., xvi : 75-76. Pl, 6, fig. 6.

Urwelt der Schweiz, 379. Fig. 268 on $p .378$.

Monde prim. Suisse, 465. Fig. 268 on p. 463.

Prim. world Switz., ii : 36 . Fig. 268 on p. 34.

Urwelt der Sehweiz, 2 aufl., $405 . \quad F i g .310$ on $p .404$.

Zittel, Handb. palaeout., i, th. ii : $795 . \quad$ Fig. 1027.

Zittel-Barrois, Traité de paléout, ii : 795. Fig. 1045.

4726. Onthophagus urus. (Scarabæidæ.)

Compared with O. nuchicornis $\mathrm{L}$.

Locality: Oeningen, Baden. Horizon: Tortonian.

1847. Heer.

1852. Giebel.

Insektenf. tertiärg. Oeningen, i: 62-64. Pl. 2, figs. 10, 10b,

Deutschl. petref., 653 .

Insect, $\mathrm{d}$, vorwelt, 39 , 
1862. Heer.

1865. "

1872. “

1876. “

1879. “
Verhandl. holl. maatseh. wetenseh., xvi : 76.

Urwelt der Schweiz, 379.

Monde prim. Suisse, 465.

Prim. world Switz., ii : 36.

Urwelt der Schweiz, 2e aufl., 405.
Opatrum - See Hopatrum $\longrightarrow$.

Ophonus —. See Harpalus — Curtis.

Ophonus —. See Harpalns (Argutor or Ophonus)

4727. Ophryastes compactus. (Otiorhynchidæ.)

Compared with O. cinereus Schönh., Mexico.

Locality: Green River, Wyo. Horizon: Oligocene.

1878. Scudder. Bull. U. S. geol. surv, terr., iv : 765-766.

1890. " Tert. ins. N. A., 477-478. Pl. 8, fig. 39.

\section{Opilo - (Cleridæ.)}

Locality: Prussian amber. Horizon: Ligurian.

1845. Berendt. Bernst. befindl. organ. reste vorw., i: 56.

4729. Orchesia (or Hallomenus) - _. (Melandryidæ.)

Locality: Prussian amber. Hurizon: Ligurian.

1845. Berendt. $\quad$ Bernst. befindl. organ. reste vorw., i : 56.

4730. Oreina -. (Chrysomelidæ.)

Locality: Brunstatt, Alsatia. Horizon: Middle Oligocene.

1888. Foerster. Mitth. comm. geol. Elsass-Lothr., i: 165.

1889. " " Mitth. comm. geol. Elsass-Lothr., ii : 10 ?.

4731. Oreina? —. (Chrysomelidæ.)

Locality : Lexden, England. Horizon: Peat.

1863. Wollaston. Quart. journ. geol. soc.Loud., xix, i : 400-401, No. 2.

4732. Oreina amphyctionis. (Chrysomelid※.)

1847. Heer.

Locality: Oeningen, Baden. Horizon: Tortonian.

1852. Giebel.

Insektenf. tertiärg. Oeningen, i: 21\%. Pl. 7, fig. 12.

1856. " "

Deutschl. petref,, 648.

Insect. d. vorwelt, 119.

4733. Oreina hellenis. (Chrysomelidæ.)

Locality : Oeningen, Baden. Horizon: Tortonian.

1847. Heer.

Insektenf. tertiärg. Oeningen, i : 209-211. Pl. 7, fig. 10.

1852. Giebel.

Deutschl. petref., 648 .

1856. " Insect. d. vorwelt, 118.

4734. Oreina protogeniæ. (Chrysomelidæ.)

Locality: Oeningen, Baden. Horizon: Tortonian.

1847. Heer.

1852. Giebel.

Insektenf. tertiärg. Oeningen, i : 211-212. $\quad$ Pl. 7, fig. 11.

1856. " "

Dentschl. petref., 648 .

Insect. d, vorwelt, 118-119. 
4735. Oryctoscirtetes protogæum. (Chrysomelidæ.)

Locality : Florissant, Colo. Horizon: Oligocene.

1876. Scudder. Bull. U. S. geol, surv, terr., ii : 82-83.

4736. Otiorhynchus - (Otiorhynchidæ.)

Locality : Garvel Park, Clyde, Scotland. Horizon: Pleistocene. 1888. Bell.

Entom., xxi : 2.

4737. Otiorhynchus —. (Otiorhynchidæ.)

Locality: Brunstatt, Alsatia. Horizon: Middle Oligocene.

1889. Foerster. Mitth. comm. geol. Elsass-Lothr., ii : 102.

4738. Otiorhynchus alpicola (recent). (Otiorhynchidæ.)

Locality: Schwerzenbach, Switzerland. Horizon: Glacial clay.

[1843. Boheman. Schönh., Gen. curc, vii, i: 327.]

1879. Heer. Urwelt der Schweiz, $2^{e}$ aufl, 581. Pl. 12, figs. 20, $20 b$.

4739. Otiorhynchus dubius. (Otiorhynchidæ.)

Locality: Green River, Wyo. Horizon: Oligocene.

1878. Scudder. Bull. U. S. geol. surv. terr., iv: 766 .

See also Otiorhynchus tumbæ.

4740. Otiorhynchus fuscipes (recent). (Otiorhynchidæ.)

Locality : Schwerzenbach, Switzerland. Horizon: Glacial clay. [1808? Olivier. Ent., v : 372. $P l .31$, fig. 464.]

1879. Heer. Urwelt der Schweiz, $2^{\circ}$ anfl., 581.

Otiorhynchus induratus. See Eurychirus (Otiorhynehus) induratus.

Otiorhynchus montanus. See Otiorhynchus niger-montanus.

4741. Otiorhynchus niger (recent). (Otiorhynchidæ.)

Locality : Hösbach, Bavaria. Horizon: Lower Pleistocene.

[1775. Fabricius. Syst. ent., 150.]

1884. Flach. Käfer unterpleist. Hösbach, 10.

1884. “Verhandl. physik.-med. gesellsch. Würzb., n. f., xviii : 294.

See also Otiorhynchus niger-montanus.

4742. Otiorhynchus niger-montanus (recent). (Otiorhynchidæ.)

Locality : Schwerzenbach, Switzerland. Horizon: Glacial clay.

[1843. Boheman. Schönh., Gen. curc., vii, i: 306.]

1879. Heer. Urwelt der Schweiz, $2^{e}$ aufl, 581, Pl. 12, figs. 18, 19, $19 b$.

See also Otiorhynchus niger.

4743. Otiorhynchus perditus. (Otiorhynchidæ.)

Compared with O. sulcatus.

Locality : Green River, Wyo. Horizon: Oligocene.

1876. Scudder. Bull. U. S. geol. surv. terr., ii : 84 .

1878. " Bull. U. S. geol. surv. terr., iv: 766.

1890. “ Tert. ins. N. A., 476-477. Pl. 8, fig. 25.

Bull. $71-36$ 
4744. Otiorhynchus rugifrons (recent). (Otiorñynchidæ.)

Locality : Schwerzenbach, Switzerland. Horizon: Glacial clay. [1813. Gyllenhal. Ins. suec., iii : 319.]

1879. Heer. Urwelt der Schweiz, $2^{2}$ aufl, 581.

4745. Otiorhynchus tumbæ. (Otiorhynchidæ.)

Locality: Green River, Wyo. Horizon: Oligocene.

1890. Scudder. Tert. ins. N. A., 477. Pl. 8, fig. 13.

See also Otiorhynchus dubius.

4746. Oxygonus mortuus. (Elateridæ.)

Locality : White River, Utah. Horizon: Oligocene.

1876. Scudder. Bull. U. S. geol. surv. terr., ii : 81 .

1877. " $\quad$ Bull. U. S. geol, surv. terr., iii: 759 .

1890. " Tert. ins. N. A., 496. Pl. 5, figs. 110, 111.

4747. Oxyporus blumenbachii. (Staphylinidæ.)

Locality: Prussian amber. Horizon: Ligurian.

1806. Gravenhorst. Monogr. col. micr., 235-236 et index.

4748. Oxyporus seuberti. (Staphylinidæ.)

Locality: Oeningen, Baden. Horizon: Tortonian.

1862. Heer. Verhandl, holl. maatsch. wetensch. Haarl., xvi:49. Pl. 3, fig. 6.

4749. Oxyporus stiriacus. (Staphylinidæ.)

Locality : Scarboro', Ontario. Horizon: Postpliocene.

1890. Scudder. Tert. ins. N. A., 505. Pl. 1, fig. 36.

4750. Oxyporus vulcanus. (Staphylinidø.)

Locality: Rott, Rhenish Prussia. Horizon: Aquitanian.

1866. Heyd.-Heyd, Palaeontogr., xy: 137-138. Pl. 22, fig. 14.

1866. " Käfor u. polypen, 7-8. Pl. 1, fig. 14.

4751. Oxytelus - (2 sp.). (Staphylinidæ.)

Locality: Brunstatt, Alsatia. Horizon: Middle Oligocene. 1890. Foerster. In litt.

4752. Oxytelus? (larva). (Staphylinidæ.)

Locality: Rocky River, Sydney flats, New South Wales. Horizon: 1870. Moore. Quart. journ. geol. soc. Lond., xxvi : 263. Pl. 18, fig. 10

4753. Oxytelus pristinus. (Staphylinidæ.)

Compared with O. rugosus.

Locality: White River, Colo. Horizon: Oligocene.

1876. Scudder. Bull. U. S. geol. surv. terr., ii : 79.

1877. " Bull. U. S. geol. surv. terr., iii : 759 .

1890. "6 Tert. ins. N. A., 503-504. Pl. 5, figs. 118-120. 
4754. Oxytelus proævus. (Staphylinidæ.)

Locality: Oeningen, Baden. Horizon: Tortonian.

1862. Heer.

1865. " "

1872. " "

1876. "

1879. "

1829. Serres.

1874. Oustalet.

Verhandl. holl. maatsch. wetensch. Haarl., xvi: 45-46. Pl. 3, fig. 5.

Urwelt der Schweiz, 383.

Monde prim. Suisse, 469.

Prim. world Switz., ii : 40.

Urwelt der Schweiz, $2^{\mathrm{e}}$ aufl., 408.

\section{Pachypus ——. (Scarabæidæ.)}

Compared with P. excavatus.

Locality: Aix, France. Horizon: Ligurian.

Géogn. terrains tert., 22\%.

4756. Panagæus dryadum. (Carabidæ.)

Locality : Aix, France. Horizon: Ligurian.

Ann. sc. géol., v, art. 2: 94-96. Pl. 2, fig.1.

\section{Parandra - - (Spondylidæ.)}

Locality: Rhine, Germany. Horizon: Aquitanian?.

1831. Goldfuss. Verhandl. leop.-carol. akad. naturf., vii, i: 118.

4758. Parandrita vestita. (Cucujidæ.)

Compared with P. cephalotes LeC.

Locality: Green River, Wyo. Horizon: Oligocene.

1890. Scudder. Tert. ins. N. A., 501-502. Pl. 7, fig. 41.

4759. Parolamia rudis. (Cerambycidø.)

Locality: Florissant, Colo. Horizon: Oligocene.

1878. Scudder. Bull. U. S. geol. surv. terr., iv: 530 .

\section{Passandra - (Cucujidæ.)}

Locality: Prussian amber. Horizon: Ligurian.

1856. Menge. Progr. petrischule Danzig, 1856: 21.

4761. Patrobus excavatus (recent). (Carabidø.)

Localities: Jarville, France: Hösbach, Bavaria. Horizon: Pleistocene.

[1790. Paykull. Monogr. carab., 38.]

1875. Fliche. Comptes rendus; Ixxx: 1234.

1884. Flach. Käfer unterpleist. Hösbach, 4-5. Pl. 1, figs. 5a, $5 b$.

1884. " Verhandl. physik.-med. gesellsch. Würzb., n. f., xviii : 288-289. Pl. 8, figs. $5 a, 5 b$.

1886. Scudder. Bull. U. S. geol. surv., No.31: 84 .

4762. Patrobus gelatus. (Carabidæ.)

Compared with P. septentrionis Dej.

Locality: Scarboro,' Ontario. Horizon: Postpliocene.

1890. Scudder. Tert. ins. N. A., 53'. Pl. 1, fig. 48. 
4763. Paussoides mengei. (Paussidæ.)

Locality: Prussian amber. Horizon: Ligurian.

1856. Motschulsky. Etud. entom., v: 26. Pl., figs. $6,6^{\prime}, 6^{\prime \prime}, 6^{\prime \prime \prime}$.

1885. Scudder. Zittel, Handb. palaeont., i, th. ii: 802. Fig. 1060.

1886. " Zittel-Barrois, Traité de paléont., ii: 802. Fig. 1077.

4764. Paussus - (Paussidæ.)

Locality: Prussian amber. Horizon : Ligurian.

1856. Menge. Progr. petrischule Danzig, 1856: 21.

4765. Pelobius cretzschmari. (Dytiscidæ.)

Locality : Rott, Rhenish Prussia. Horizon: Aquitanian.

1866. Heyd.-Heyd. Palaeontogr., xv : 134-135. Pl. 22, fig. 2.

1866. " Käfer u. polypen, 4-5. Pl. 1, fig. 2.

4766. Peltis costulata. (Trogositidæ.)

Locality: Rott, Rhenish Prussia. Horizon: Aquitanian.

1862. Heyden. Palaeontogr., $\mathrm{x}: 65 . \quad$ Pl. 10, fig. 20.

4767. Peltis tricostata. (Trogositidæ.)

Locality: Oeningen, Baden. Horizon: Tortonian.

1847. Heer. Insektenf. tertiärg. Oeningen, i: 39-40. Pl. 7, fig. 34

1852. Giebel. Dentschl, petref., 654 .

1856. "Insect. d. vorwelt, 42.

See also Silpha tricostata.

4768. Pentodon bellerophon. (Scarabæidæ.)

Locality: Rott, Rhenish Prussia. Horizon: Aquitanian.

1866. Heyd.-Heyd. Palaeontogr., $\mathrm{xv}:$ 141-142. $\quad$ Pl. $23, \mathrm{fig} 1$.

1866. “ Käfor u. polypen, 11-12. Pl. 2, fig. 1.

4769. Pentodon proserpinæ. (Scarabæidæ.)

1865. Heer.

Locality: Oeningen, Baden. Horizon: Tortonian.

1872. " Monde prim. Suisse, 465.

1876. " " Prim. world Switz., ii: 36.

1879. “ Urwelt der Schweiz, $2^{\circ}$ aufl., 405.

4770. Perotis bruckmanni. (Buprestidæ.)

Locality: Oeningen, Baden. Horizon: Tortonian.

1862. Heer. Verhandl. holl. maatsch. wetensch. Haarl., xvi: 85. Pl. 7, fig. 1

4771. Perotis hausmanni. (Buprestidæ.)

Locality: Rott, Rhenish Prussia. Horizon: Aquitanian.

1862. Heyden. Palaeontogr., $\mathrm{x}: 66-67$. Pl. 10, fig. 6.

4772. Perotis lævigata. (Buprestidæ.)

Localities: "Oeningen," Baden; Monte Bolea, Italy. Horizon : Ligurian.

1855. Heer. MSS.

1855. Massalongo. Nereid. foss., 32.

1856. " Stud. paleont., 14-15. Pl. 1, fig. 4

1886. Omboni. Atti r. ist. ven., (6), iv: 1425 (løvigata). Pl. 2, fig. 4.

1886. “ Foss. ins. Ven., 5. Pl. 2, fig. 4. 
4773. Perotis lavateri. (Buprestidæ.)

Locality: Oeningen, Baden. Horizon: Tortonian.

1847. Heer. 1852. Giebel. 1856. "6
Insektenf. tertiärg. Oeningen, i: 105-106. Pl. 3, fig. 4。 Deutschl. petref., 652.

Insect. $d$. vorwelt, 82 .

Perotis lœvigata. See Perotis lævigata.

4774. Perotis redita. (Buprestidæ.)

Locality: Rott, Rhenish Prussia. Horizon : Aquitanian.

1862. Heyden. Palaeontogr., $\mathrm{x}: 67$. Pl. 10, fig. 37.

4775. Phalacrus - (Phalacridæ.)

Locality : Prussian amber. Horizon: Ligurian.

1845. Berendt. Bernst. befindl. organ. reste vorw., i : 56.

4776. Phanæus antiquus. (Scarabæidæ.)

Compared with P. pluto, United States.

Locality: Port Kennedy, Pa. Horizon: Postpliocene.

1876. Horn. Trans. Amer. ent. soc., v : 245.

1890. Scudder. Tert, ins. N. A., 489-490. Pl. 1, figs. 12-14.

4777. Phenolia incapax. (Nitidulidæ.)

Compared with P. grossa.

Locality: Green River, Wyo. Horizon: Oligoceno.

1876. Scudder. Bull. U. S. geol. surv. terr., ii : 80.

1878. " "Bull. U. S. geol, surv. terr., iv: 762.

1890. “ Tert.ins. N. A., 499. Pl. 7, fig.23.

4778. Philhydrus — (2 sp.). (Hydrophilidæ.)

Locality: Green River, Wyo. Horizon: Oligocene.

1878. Scudder.

1890. " Tert. ins. N. A., 512.

4779. Philhydrus? morticinus. (Hydrophilidæ.)

Locality : Rott, Rhenish Prussia. Horizon: Aquitanian.

1866. Heyd.-Heyd. Palaeontogr., xv: 136. Pl. 22, figs. 6, 7.

1866. " Käfer u. polypen, 6. Pl. 1, figs. 6, 7.

1885. Seudder. Zittel, Handb. palaeont, i, th. ii: 802. Fig. 1064.

1886. " Zittel-Barrois, Traité de paléont., ii : 803. Fig. 1081.

4780. Philhydrus primævus. (Hydrophilidæ.)

Locality : Green River, Wyo. Horizon: Oligocene。

1876. Scudder. Bull. U. S. geol. surv. terr, ii : 78.

1878. " Bull. U. S. geol. surv. terr., iv: 761.

1890. " Tert. ins. N. A., 512. Pl. 8, fig. 5.

4781. Philonthus —_. (Staphylinidæ.)

Locality : Prussian amber. Horizon: Ligurian.

1845. Berendt. Bernst. befindl. organ. reste vorw., i : 56. 


\section{Philonthus —- (Staphylinidæ.)}

Locality: Hösbach, Bavaria. Horizon: Lower Pleistocene.

1884. Flach.

1884. " Verhandl. physik.-med. gesellsch. Würzb., n. f., xviii : 293.

4783. Philonthus —. (Staphylinidæ.)

Locality: Prussian amber. Horizon: Ligurian.

1856. Menge. Progr. petrischule Danzig, 1856: 23.

4784. Philonthus bituminosus. (Staphylinidæ.)

Locality : Rott, Rhenish Prussia. Horizon: Aquitanian.

1866. Heyd.-Heyd. Palaeontogr., $x v: 137 . \quad P l .22$, fig. 11.

1866. " Käfer $\mathrm{u}$, polypen, 7. $P l .1, f i g .11$.

4785. Philonthus bojeri. (Staphylinidæ.)

Locality: Aix, France. Horizon : Ligurian.

1856. Heer. Viert. naturf. gesellsch. Zürich, i: 17. Pl. 1, fig. 4.

1874. Oustalet. Ann. sc. géol., v, art. 2: 169-170. Pl. 3, fig. 6.

4786. Philonthus marcelli. (Staphylinidæ.)

Locality : Aix, France. Forizon: Ligurian.

1856. Heer.

1874. Oustalet.

Viert. naturf. gesellsch. Zürich, i: 17-18. Fl. 1, fig. 5. Ann. sc. géol., v, art. 2: 170-17i. $P l .5$, fig. 15.

Phosphuga atrata. See Silpha (Phosphuga) atrata.

4787. Phyllobius —. (Otiorhynchidæ.)

Locality: Prussian amber. Horizon: Ligurian.

1832. Burmeister. Handb. ent., i : 635.

1836. " Man, ent., 577.

4788. Phymaphoroides antennatus. (Endomychidæ.)

Locality: Prussian amber. Horizon: Ligurian.

1856. Motschulsky. Etud. entom., v: 27 (Phymophoroides). Pl., figs. 7, 7', 7'. 1885. Scndder. Zittel, Handb. palaeont., i, th. ii : 800. Fig. 1054.

1886. " Zittel-Barrojs, Traité de pal6ont., ii : 800. Fig. 1071.

Phymophoroides antennatus. See Phymaphoroides antennatus.

4789. Phytonomus - (Curculionidæ.)

Locality: Prussian amber. Horizon: Ligurian.

1845. Berendt. Bernst. befindl. organ. reste vorw., i: 56.

4790. Phytonomus annosus. (Curculionidæ.)

1874. Heer.

Locality: Aix, France. Horizon: Ligurian.

1874. Oustalet. Ann. sc. géol., v, art. 2: 283-285. Pl. 3, fig. 9.

See also Brachycerus exilis. 
4791. Phytonomus firmus. (Curenlionidæ.)

Locality : Aix, France. Horizon: Ligurian.

1856. Heer.

1874. Onstalet.

Viert. naturf. gesellsch. Zürich, i: 23. Pl. 1, fig. 14.

Ann. sc. géol., v, art. 2: 282-283. Pl. 6, fig. 7.

\section{Pinitoides scydmæniformis.}

Locality : Prussian amber. Horizon: Ligurian.

1856. Motschulsky. Etud. entom., v: 27. Pl., figs. $2,2^{\prime}, 2^{\prime \prime}$.

4793. Pissodes —. (Curculionidæ.)

Locality: Prussian amber. Horizon: Ligurian.

1845. Berendt. Bernst. befindl. organ. reste vorw., i: 56.

\section{Pissodes —. (Curculionidæ.)}

Locality: Brunstatt, Alsatia. Horizon: Middle Oligocene.

1890. Foerster. In litt.

4795. Pissodes effossus. (Curculionidæ.)

Locality: Sieblos, Rhenish Prussia. Horizon: Aquitanian. .

1858. Heyden. Palaeontogr, v: 117. Pl. 23, fig. 15.

4796. Plagiodera novata. (Chrysomelidæ.)

Compared with P. armoraciæ Linn.

Locality: Rott, Rhenish Prussia. Horizon: Aquitanian.

1866. Heyd.-Heyd. Palaeontogr., xv: 155. Pl. 24, fig. 14.

1866. " Käfer u. polypen, 25. Pl. 3, fig. 14 .

4797. Platycerus - (Lucanidæ.)

Locality: Prussian amber. Horizon: Ligurian.

1845. Berendt. Bernst. befindl. organ. reste vorw., i : 56 .

4798. Platycerus sepultus. (Lucanidæ.)

Compared with P. tenebrioides.

Locality: Vicinity Bonn, Rhenish Prussia. Horizon: Aquitanian?.

1837. Germar. Fauna insect. Europæ, xix: 7. Pl. 7.

1852. Giebel. Deutschl. petref., 653.

1856. " Insect. d. vorwelt, 29.

4799. Platydema geinitzi. (Tenebrionidæ.)

Locality: Rott, Rhenish Prussia. Horizon: Aquitanian.

1866. Heyd.-Heyd. Palacontogr., xv: 145-146. Pl. 22, fig. 22.

1866. “ Käfer u. polypen, 15-16. Pl. 1, fig. 22.

4800. Platynus cæsus. (Carabidæ.)

Locality: Green River, Wyo. Horizon: Oligocene.

1890. Scudder. Tert. ins. N. A., 522-523. Pl. 7, fig. 34 .

4801. Platynus casus. (Carabidæ.)

Compared with P. rubripes Zimm.

Locality: Scarboro', Ontario. Horizon: Pleistocene.

1890. Scudder. Tert. ins. N. A., 519-520. Pl. 1, fig. 42. 
4802. Platynus desuetus. (Carabidæ.)

Compared with P. crenistriatus LeC.

Locality : Scarboro', Ontario. Horizon: Pleistocene. 1890. Scudder. Tert. ins. N. A., 521-522. Pl. 1, figs. 43, 51, 58.

4803. Platynus dissipatus. (Carabidæ.)

Locality : Scarboro', Ontario. Horizon: Pleistocene. 1890. Scudder. Tert. ins. N. A., 521. Pl. 1, fig. 37.

4804. Platynus gracilis (recent). (Carabidæ.)

Locality : Jarville, France. Horizon: Pleistocene.

[1827. Gyllenhal. Ins. suec., iv: 449.]

1886. Scudder. Bull. U. S. geol. surv., No. 31: 84 .

4805. Platynus halli. (Carabidæ.)

Compared with P. crenistriatus LeC.

Locality : Scarboro', Ontario. Horizon: Pleistocene. 1890. Scudder. Tert. ins. N. A., 520-521. Pl. 1, fig. 41.

4806. Platynus harttii. (Carabidæ.)

Locality: Scarboro', Ontario. Horizon: Pleistocene. 1890. Scudder. Tert. ins. N. A., 522. $P l .1$, fig. 31 .

4807. Platynus hindei. (Carabidæ.)

Compared with P. rubripes Zimm.

Locality : Scarboro', Ontario. Horizon: Pleistocene.

1890. Scudder. Tert. ins. N. A., 520. Pl. 1, fig. 54.

4808. Platynus senex. (Carabidæ.)

Compared with P. variolatus LeC., North America.

Locality: Green River, Wyo. Horizon: Oligocene.

1878. Scudder. Bull. U. S. geol. surv. terr., iv: 759.

1890. " Tert. ins. N. A., 519. Pl. 7, fig. 38.

\section{Platypus - (Scolytidæ.)}

Locality : Prussian amber. Horizon: Ligurian.

1832. Burmeister. Handb. ent., i: 635.

1836. " Man. ent., 577.

See also Platypus cylindricus.

\section{Platypus —. (Scolytidæ.)}

\section{Locality: Prussian amber. Horizon: Ligurian.}

1825. Guérin. Dict. class. d'hist. nat., viii : 580 (Platype).

1829. Serres. Géogn. terrains tert., 241.

1845. Motschulsky. Bull. soc. imp. nat. Mose., xviii, ii : 100.

4811. Platypus cylindricus. (Scolytidæ.)

Locality: Prussian amber. Horizon: Ligurian.

1831. Burmeister. Oken, Isis, 1831: 1100.

See also Platypus — Burmeister. 
4812. Platypus maravignæe. (Scolytidæ.)

Locality : Sicilian amber. Horizon :

1838. Guórin.

Rev, zool., 1838: 170. Pl. 1, fig. 7.

Platysma oblongopunctata. See Feronia (Platysma) oblongopunctata.

4813. Plinthus heeri. (Curculionid he.)

Locality : Aix, France. Horizon : Ligurian.

1874. Oustalet. Ann. sc. géol., $\nabla$, art. 2: 279-281. Pl. 4, fig. 12.

4814. Plinthus redivivus. (Curculionidæ.)

Locality: Corent, France. Horizon: Aquitanian

1870. Oustalet. Ann. sc. géol., ii, art. 3: 74. Pl. 1, fig. 10.

Pcecilus —. See Feronia (Pocilus) ——.

4815. Polydrosus - (Otiorhynchidæ.)

Locality : Prussian amber. Horizon: Ligurian.

1832. Burmeister. Handb. ent., i : 635 (Polydrusus).

1836. " Man. ent., 577.

Polydrusus —. See Polydrosus -

4816. Polystichus —. (Carabidæ.)

'Locality: Prussian amber. Horizon: Ligurian.

1845. Berendt. Bernst. befindl. organ. reste vorw., i: 56.

4817. Polystichus hopei. (Carabidæ.)

Locality: Aix, France. Horizon : Ligurian.

1874. Oustalet. Ann. sc. géol., v, art. 2: 119-120. Pl. 1, fig.8.

4818. Prasocuris aucta var. egena (recent). (Chrysomelidæ.)

Locality : Hösbach, Bavaria. Horizon : Lower Pleistocene.

[1827.. Gyllenhall. Ins. suec., iv : 549.]

1884. Flach. Käfer unterpleist. Hösbach, 10-11. Pl. 2, fig. 11.

1884. " Verhandl. physik.-med. gesellsch. Würzb., n.f., xviii : 294-295. Pl. 9, fig. 11.

Prasocuris egena. See Prasocuris aucta var. egena.

4819. Prionus ? - (borings). (Cerambycidæ.)

Locality: Salzhausen, Germany. Horizon: Miocene

1856. Heyden. Palaeontogr., iv : 200. Pl. 38, fig. 3.

4820. Prionus polyphemus. (Cerambycidæ.)

Compared with P. faber $L$.

Locality: Oeningen, Baden. Horizon: Tortonian.

1865. Heer. Urwelt der Schweiz, 375. Fig. 250 on p. 376.

1872. " Monde prim. Suisse, 460. Fig. 250 on p. 461.

1876. " $\quad$ Prim. world Switz., ii: 31 . Fig. 250 on p. 32.

1879. " Urwelt der Schweiz, $2^{\mathrm{e}}$ aufl., 401. Fig. 292 on p. 402. 
4821. Prionus spectabilis. (Cerambyeidæ.)

Compared with P. coriarius L.

Locality : Oeningen, Baden. Horizon: Tortonian.

1865. Heer.

1872. “

1876. “

1879. “
Ërwelt der Schweiz, 375. Fig. 251 on $p .376$.

Monde prim. Suisse, 460. Fig. 251 on $p .461$.

Prim. world Switz., ii : 31. Fig. 251 on $p .32$.

Urwelt der Schweiz, $2^{\circ}$ aufl., 401. Fig. 293 on p. 402.

4822. Prionus umbrinus. (Cerambycidæ,)

Compared with P. coriarius.

Locality: Vicinity Bonn, Rhenish Prussia. Horizon: Aquitanian?.

1837. Germar. Oken, Isis, 1837: 423 (undescr.).

1837. "6

1852. Giebel.

1856. “

Fauna insect. Europæ, xix : 12. Pl. 12.

'Deutschl. petref., 650.

Insect. d. vorwelt, 126.

4823. Pristorhynchus ellipticus. (Otiorhynchidæ.)

Locality: Oeningen, Baden. Horizon: Tortonian.

1847. Heer.

1852. Giebel.

1856. “

1853-'56. Bronn.

1885. Scudder.

1886.
Insektenf. tertiärg. Oeningen, i: 190-192. Pl. 6, fig. 10.

Deutschl. petref., 649 .

Insect. d. vorwelt, 139.

Lethæa geogn., $3^{\mathrm{e}}$ aufl., iii : 647 .

Zittel, Handb. palaeont., i, th.ii : 789. Fig. 1010.

Zittel-Barrois, Traité de paléont., ii : 789. Fig. 1029.

4824. Prometopia depilis. (Nitidulidæ.)

Compared with $P$. sexmaculata.

Locality : Quesnel, British Columbia. Horizon:

1877. Scudder. Rep. progr. geol, surv., Can., 1875-76: 278-279.

1877. " Insect. tert. Quesnel, 13-14.

1877. " Rapp. opér. comm. géol. Can., 1875-'76: 308-309.

1890. “ Tert. ins. N. A., 500. Pl. 2, fig. 29.

4825. Protactus erichsonii. (Staphylinidæ.)

Locality: Oeningen, Baden. Horizon: Tortonian.

1847. Heer. Insektenf. tertiärg. Oeningen, i : 28-34. Pl. 1, figs.9, 9a-c, e, f. 1ช̄̄2. Giebel. Deutschl. petref., 654.

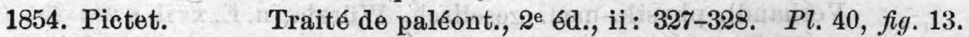

1856. Giebel. Insect. d. vorwelt, 75.

1853-'56. Broun. Lethæa geogn., $3^{\mathrm{e}}$ aufl, iii : 648-649. Pl. $42^{\prime}$, figs. $24 a b$.

1885. Seudder. Zittel, Handb. palaeont., i, th. ii : 801. Fig. 1058.

1886. " Zittel-Barrois, Traité de palént., ii : 801. Fig. 1075.

4826. Protactus minor. (Staphylinidæ.)

Locality: Oeningen, Baden. Horizon: Tortonian.

1862. Heer. Verhandl. holl. maatsch. wetensch. Haarl, xvi : 45. Pl.3, fig. 4.

4827. Protogeñia escheri. (Buprestidæ.)

Locality: Oeningen, Baden. Horizon: Tortonian.

1847. Heer. Insektenf. tertiärg. Oeningen, i: 118-123. Pl. 2, fig. 16; pl. $8, f i g .6$.

1852. Giebel. Deutschl. petref., 652 .

1856. " Insect. d. vorwelt, 88-89.

1853-'56. Bronn. Lethæa geogn., $2^{\mathrm{e}}$ aufl., iii : 647-648. Pl. $42^{\prime}$, figs. $27 a-c$. 
4828. Protoscalidion rugiæ. (Carabidæ.)

Locality : Prussian amber. Horizon: Ligurian.

1888. Schaufuss. Berl, ent, zeitschr, xxxii : 266-267.

4829. Pselaphus ——. (Pselaphidæ.)

Locality: Prussian amber. Horizon: Ligurian.

1245. Berendt. Bernst. befindl. organ. reste vorw., i : 56.

4830. Pseudocistela —. (Cistelidæ.)

Locality : Brunstatt, Alsatia. Horizon : Middle Oligocene.

1890. Foerster. In litt.

\section{Pseudo-elater —_. (Elateridæ?.)}

Locality: (Oeningen?, Baden). Horizon: Tortonian.

1847. Heer. Insektenf. tertiärg. Oeningen, i: 143. Pl. 4, fig. 1.

1851. Stizenberg. Uebers. verstein, Baden, 89,99 (says it is a fruit and $=$ Diachænites Al. Braun).

1852. Giebel. Deutschl. petref., 651.

1856. " Insect. d. vorwelt, 94 (without name).

4832. Pterostichus - (Carabidæ.)

Locality : Prussian amber. Horizon: Ligurian.

1845. Berendt. Bernst. befindl, organ. reste vorw., i : 56.

4833. Pterostichus ? - - (Carabidæ.)

Locality : Port Kennedy, Pa. Horizon: Postpliocene.

1876. Horn. Trans. Amer. ent. soc., v : 243.

1890. Scudder. Tert. ins. N. A., 529. Pl. 1, fig. 5.

4834. Pterostichus -. (Carabidæ.)

Compared with P. coracinus or P. stygicus, United States.

Louality: Port Kennedy, Pa. Horizon: Postpliocene.

1876. Horn. Trans. Amer. entom. soc., v : 243.

See also Pterostichus lævigatus.

4835. Pterostichus - (Carabidæ.)

Locality: Prussian amber. Horizon : Ligurian.

1856. Menge. Progr. petrischule Danzig, 1856: 23.

4836. Pterostichus -. (Carabidæ.)

Locality: Wohlscheid, Germany. Horizon: Peat.

1852. Weber. Palaeontogr., ii : 229. Pl. 25, fig. 17.

See also Pterostichus angustus.

\section{Pterostichus —. (Carabidæ.)}

Locality: Wohlscheid, Germany. Horizon: Peat.

1852. Weber. Palaeontogr., ii : 229. Pl. 25, fig. 18.

See also Pterostichus rhenanus.

Pterostichus —. See Feronia (Pterostichus) - 
4838. Pterostichus abrogatus. (Carabidæ.)

Compared with P. herculaneus Mann.

Locality : Scarboro', Ontario. Horizon: Pleistocene.

1890. Scudder. Tert. ins. N. A., 525. Pl. 1, fig. 39.

4839. Pterostichus angustus. (Carabidæ.)

Locality : Wohlscheid, Germany. Horizon: Peat.

1856. Giebel. Insect. d. vorwelt, 67.

See also Pterostichus — Weber, xxv: 18.

4840. Pterostichus antiquus. (Carabidæ.)

Locality: Oeningen, Baden. Horizon: Tortonian.

1856. Giebel. Insect. d. vorwelt, 66.

See also Argutor antiquus.

4841. Pterostichus destitutus. (Carabidæ.)

Compared with P. sayi Brulle.

Locality: Scarboro', Ontario. Horizon: Postpliocene.

1890. Scudder. Tert. ins. N. A., 526. Pl. 1, fig. 44.

4842. Pterostichus destructus. (Carabidø.)

Compared with P. patruelis.

Locality : Scarboro', Ontario. Horizon: Postpliocene.

1890. Scudder. Tert. ins. N. A., 527. Pl. 1, fig. 46.

4843. Pterostichus dormitans. (Carabidæ.)

Compared with P. lætulus LeC.

Locality : Scarboro', Ontario. Horizon: Postpliocene. 1890. Scudder. Tert. ins. N. A., 526. Pl. 1, figs. 49, 55.

4844. Pterostichus fractus. (Carabidæ.)

Compared with P. destitutus Scudd.

Locality: Scarboro', Ontario. Horizon: Postplioceno.

1890. Scudder. Tert. ins. N. A., 527. Pl. 1, figs. 29, 30.

4845. Pterostichus gelidus. (Carabidæ.)

Compared with $\mathrm{P}$. hudsonicus.

Locality : Scarboro', Outario. Horizon: Postpliocen.

1890. Scudder. Tert. ins. N. A., 527-528. Pl:1, figs. 52, 59-61.

See also Loxandrus gelidus.

4846. Pterostichus lævigatus. (Carabidæ.)

Locality : Scarboro', Ontario. Horizon: Postpliocene.

1876. Horn. MSS.

1890. Scurlder. Tert. ins. N. A., 528-529. Pl. 1, figs. 3, 4.

See also Pterostichus _ Horn, ef. coracinus. 
4847. Pterostichus madidus (recent). (Carabidæ.)

Locality: Norfolk, England. Horizon : Forest bed, Pleistocene.

1888. Bell.

Entom., xxi : 2.

See also Pterostichus maurus.

4848. Pterostichus maurus (recent). (Carabidæ.)

Locality: England. Horizon: Pleistocene.

[1812. Duftschmid. Faun. austr., ii: 160.]

See also Pterostichus madidus.

4849. Pterostichus (Argutor) minutulus. (Carabidæ.)

Locality : Oeningen, Baden. Horizon: Tortonian.

1862. Heer. Verhandl. holl. maatsch, wetensch. Haarl., xvi : 22-23. Pl. 1, fig. 19.

4850. Pterostichus nigritus (recent). (Carabidæ.)

Localities: Dürnten, Schwerzenbach, Switzerland. Horizon: Interglacial clay.

[1792. Fabricius. Ent. syst., i: 158.]

1865. Heer.

1872. " "

Urwelt der Schweiz, 502 (nigrita). Fig. 358 on p. 501.

1876. “

Monde prim. Suisse, 615. Fig. 358 on p. 614.

1879. “

Prim. world Switz., ii : 169. Fig. 358 on p. 168.

Urwelt der Schweiz, $2^{e}$ aufl., 531, 581. Fig. 401 ; pl. 12, figs. 15, $15 b$.

See also Omaseus nigritus.

4851. Pterostichus rhenanus. (Carabidæ.)

Locality: Wohlscheid, Germany. Horizon: Peat.

1856. Giebel. Insect. d. vorwelt, 66-67.

See also Pterostichus - Weber, $\mathrm{xxv}: 17$.

4852. Pterostichus vernalis (recent). (Carabidæ.)

Locality : St. Jakob, Basel, Switzerland. Horizon: Interglacial.

[1796. Panzer. Fauna germ., $\mathrm{xxx}:$ 17.]

1879. Heer. Urwelt der Schweiz, $2^{\mathrm{e}}$ aufl, 533.

See also Argutor vernalis.

4853. Pterostichus (Abax) vetustus. (Carabidæ.)

Locality: Oeningen, Baden. Horizon: Tortonian.

1862. Heer. Verhandl. holl. maatsch, wetensch. Haarl, xvi : 23. Pl. 1, figs. $11,11 b$.

4854. Ptilinus - (Ptinidæ.)

Locality: Prussian amber. Horizon: Ligurian.

1845. Berendt. Bernst. befindl. organ. reste vorw., i: 56 .

4855. Ptilinus? - (borings). (Ptinidæ.)

Locality: Salzhausen, Germany. Horizon: Miocene.

1856. Heyden. Palaeontogr., iv: 199-200. Pl. 38, figs. 1,2. 


\section{Ptilodactyloides stipulicornis. (Dascyllidæ.)}

Locality: Prussian amber. Horizon: Ligurian.

1856. Motschulsky. Etud. entom., v: 26. Pl., figs. $3,3^{\prime}$.

1૪४5. Scudder. Zittel, Handb. palaeont., i, th. ii: 798. Fig. 1039.

1886. " Zittel-Barrois, Traité de paléont., ii: 798. Fig. $105 \%$.

\section{Ptinus —. (Ptinidæ.)}

Locality : Prussian amber. Horizon : Ligurian.

1845. Berendt. Bernst. befindl. organ. reste vorw., i: 56 .

4858. Ptinus? - (Ptinidæ.)

Compared with P. lichenum Marsh.

Locality: Aix, France. Horizon : Ligurian.

1829. Curtis. Edinb. new phil. journ., vii : 295.

18:9. " Murchison-Lyell, Tert. form. Aix, 11.

4859. Ptinus antiquus. (Ptinilæ.)

Locality : Stösschen, Austria. Horizon: Miocene.

1859. Heyden. Palaeontogr., viii: 7. Pl. 1, fig. 8.

4860. Ptinus primordialis. (Ptinidæ.)

Locality: Rott, Rhenish Prussia. Horizon: Aquitanian.

1866. Heyd.-Heyd. Palaeontogr., xv: 143-144. Pl. 23, fig. 4.

1866. " Käfer u. polypen, 13-14. Pl. 2, fig. 4.

4861. Ptinus salinus. (Ptinidæ.)

Locality: Wieliczka, Galicia. Horizon: Miocene.

1844. Schilling. Uebers. arb. u. veränd. schles, gesellsch. vaterl, cult., 1843: 175.

1848. Bronn.

1856. Giebel.

Index palaeont., Nomencl., 1058.

Insect. $\mathbf{d}$. vorwelt, 77.

4862. Ptomaphagus germari. (Silphidæ.)

Locality: Prussian amber. Horizon : Ligurian.

1888. Schlechtend. Zeitschr. ges. naturw., 1xi : 483-486. Fig. 3.

See also Mordella inclusa, Mordellina inclusa.

4863. Pyrochroa - (Pyrochroidæ.)

Locality: Prussian amber. Horizon: Ligurian.

1845. Berendt. Bernst. befindl. organ. reste vorw., i : 56 .

4864. Pythonidium metallicum. (Pythidæ.)

Locality: Spitzbergen. Horizon: Miocene.

1870. Heer. $\quad$ K. svenska vetensk.-akad. handl., viii, No. $7: 75 . \quad P l .16$, figs. $12 a b, 13,14,14 b$.

1885. Scudder. Zittel, Handb, palaeont, i, th. ii : 791. Fig. 1014.

1886. " Zittel-Barrois, Traité de paléont, ii: 791. Fig. 1032.

4865. Quedius —. (Staphylinidæ.)

Locality: Prussian amber. Horizon: Ligurian.

1845. Berendt. Bernst, befindl. organ. reste vorw., i: 56 . 
4866. Quedius - (Staphylinidæ.)

Locality: Prussian amber. Horizon: Ligurian.

1856. Menge. Progr. petrischule Danzig, 1856: 23.

4867. Quedius breweri. (Staphylinidæ.)

Compared with Q. molochinus Grav.

Locality: Florissant, Colo. Horizon : Oligocene.

1890. Scudder. Tert. ins. N. A., 508-509. Pl. 16, fig. 4.

4868. Quedius chamberlini. (Staphylinidæ.)

Locality : Florissant, Colo. Horizon : Oligocene.

1890. Scudder. Tert, ins. N. A., 508. Pl. 16, fig. 8.

4869. Quedius lortetii. (Staphylinidæ.)

Compared with Q. picipes Mann., Europe.

Locality : Aix, France. Horizon: Ligurian.

1874. Oustalet. Ann. sc. géol., v, art. 2: 175-177. Pl. 3, figs. 4, 4a.

4870. Quedius reynesii. (Staphylinidæ.)

Compared with Q. impressus Panz., Europe.

Locality : Aix, France. Horizon: Ligurian.

1874. Oustalet. Ann. sc.g6ol., v, art. 2: 172-175. Pl. 3, fig. 3; pl. 6, fig. 15.

4871. Rhinobatus —. (Curculionidæ.)

Compared with R, cynaræ.

Locality : Aix, France. Horizon: Ligurian.

1847. Hope. Trans. entom. soc. Lond., iv: 251.

4872. Rhinobatus —- (Curculionidæ.)

Locality: Aix, France. Horizon : Ligurian.

1829. Serres. Géogn, terrains tert., 224,267.

See also Larinus _ Burm.

4873. Rhinocyllus improlus. (Curculionidæ.)

Locality : Rott, Rhenish Prussia. Horizon: Aquitanian.

1866. Heyd.-Heyd. Palaeontogr., xv: 151. Pl. 24, fig. 2.

1866. " Käfer u. polypen, 21. Pl. 3, fig. 2.

4874. Rhipidius primordialis. (Rhipiphoridæ.)

Locality : Prussian amber. Horizon: Ligurian.

187\%. Stein. Mittheil. münch. entom. ver., i: 29-30.

4875. Rhipiphorus —- (Rhipiphoridæ.)

Locality: Prussian amber. Horizon: Ligurian.

1845. Berendt. Bernst. befindl, organ. reste vorw., i: 56 (Ripiphorus),

4876. Rhipiphorus —. (Rhipiphoridæ.)

Locality : Prussian amber. Horizon: Liguriant

1856. Menge. Progr. petrischule Danzig, 1856: 21. 


\section{Rhipiphorus geikiei. (Rhipiphoridæ.)}

Locality: Florissant, Colo. Horizon: Oligocene.

1890. Scudder. Tert. ins. N. A., 482-483. Pl. 27 , fig. 1.

4878. Rhizophagus 그. (Nitidulidæ.)

Locality: Prussian amber. Horizon: Ligurian.

1845. Berendt. Bernst. befindl. organ. reste vorw., i: 56.

4879. Rhizotrogus longimanus. (Scarabæidæ.)

Compared with R. paganus Oliv., Southern Europe.

Locality : Oeningen, Baden. Hörizon: Tortonian.

1847. Heer. Insektenf. tertiärg. Oeningen, i: 69-71. Pl. 7, fig. 29.

1852. Giebel. Deutschl. petref., 652.

1856. " Insect. d. vorwelt, $32-33$.

1865. Heer. Urwelt der Schweiz, 380.

1872. " Monde prim. Suisse, 466.

1876. " Prim. world Switz., ii: 37.

1879. " Urwelt der Schweiz, $2^{\mathrm{e}}$ aufl., 406.

4880. Rhizotrogus solstitialis (recent). (Scarabæidæ.)

Locality : Flanders. Horizon: Peat.

[1746. Linné. Faun, suec., 137.]

1873. Debray. Mem soc. sc. agric. arts Lille, (3), xi : 451.

1885. Scudder. Zittel, Handb. palaeont., i, th. ii : 794.

1886. " Zittel-Barrois, Traité de paléont., ii : 795.

1886. " Bull. U. S. geol. surv., No. 31: 74.

4881. Rhynchænus solieri. (Curculionidæ.)

Locality : Aix, France. Horizon: Ligurian.

1847. Hope. Trans. ent. soc. Lond., iv: 255. Pl. 19, figs. 2, 2*.

1856. Giebel. Insect. d. vorwelt, 146-147.

See also Hylobius? solieri.

4882. Rhynchites - (Rhynchitidæ.)

Locality : Prussian amber. Horizon: Ligurian.

1845. Berendt. Bernst. befindl. organ. reste vorw., i: 56.

4883. Rhynchites - (Rhynchitidæ.)

Locality: Aix, France. Horizon: Ligurian.

1847. Hope. Trans. ent. soc. Lond., iv : 251.

4884. Rhynchites dionysus. (Rhyrchitidæ.)

1865. Heer.

Locality : Oeningen, Baden. Horizon: Tortonian

1872. " "

1876. “

1879. “

Urwelt der Schweiz. Fig. 243 on $p .371$.

Monde prim. Suisse. Fig. 243 on p. 455.

Prim. world Switz., ii. Fig. 243 on p. 27.

Urwelt der Schweiz, $2^{\mathrm{e}}$ aufl. Fig. 285 on p. 397.

4885. Rhynchites hageni. (Rhynchitidæ.)

Locality : Rott, Rhenish Prussia. Horizon: Aquitanian.

1866. Heyd.-Heyd. Palaeontogr., $\mathrm{xv}:$ 147. Pl. 23, fig. 6.

1866. " Käfer u. polypen, 17, Pl, 2, fig. 6 . 
4886. Rhynchites orcinus. (Rhynchitidæ.)

Locality: Rott, Rhenish Prussia. Horizon: Aquitanian.

1866. Heyd.-Heyd. Palaeontogr., xv: 147-148. Pl. 23, fig. 7.

1866. “ Käfer u. polypen, 17-18. Pl. 2, fig. 7.

1870. L. Heyden. Foss. dipt. Rott, 29.

1870. " " Palaeontogr., xvii : 265.

4887. Rhynchites silenus. (Rhynchitidæ.)

Locality: Oeningen, Baden. Horizon: Tortonian.

1847. Heer. Insektenf. tertiärg. Oeningen, i: 180. Pl. 6, fig. 8.

1852. Giebel. Deutschl, petref., 649.

1856. " Insect. d. vorwelt, 135-136.

1865. Heer. Urwelt der Schweiz, 362,373.

1872. " " Monde prim. Suisse, 444, 458.

1876. " " Prim. world Switz., ii : 16,29.

1879. “ Urwelt der Schweiz, $2^{\mathrm{e}}$ aufl., 387, 399.

Ripiphorus —. See Rhipiphorus — Berendt.

4888. Saperda —. (Cerambycidœ.)

Locality: Prussian amber. Horizon: Ligurian.

1845. Berendt. Bernst. befindl, organ. reste vorw., i: 56.

4889. Saperda -. (Cerambycidæ.)

Locality: Prussian amber. Horizon : Ligurian.

1856. Menge. Progr. petrischule Danzig, 1856: 21.

4890. Saperda - (larva). (Cerambycidæ.)

Locality : Prussian amber. Horizon : Ligurian.

1885. Scudder. Zittel, Handb. palaeont, i, th. ii : 793. Figs. 1023, 1023a.

1886. “ Zittel-Barrois, Traité de paléont., ii: 793. Figs. $1041 a b$.

4891. Saperda absyrti. (Cerambycidæ.)

Locality : Radoboj, Croatia. Horizon: Mayencian.

1847. Heer.

1852. Giebel.

1856. “
Insektenf. tertiärg. Oeningen, i: 171-172. $\quad P l .6$, fig. 2.

Deutschl. petref., 650 .

Insect. $d$. vorwelt, 132 .

4892. Saperda lata. (Cerambycidæ.)

Compared with S. carcharias.

Locality: Vicinity Bonn, Rhenish Prussia. Horizon: Aquitanian?.

1837. Germar.

185\%. Giebel.

1856. "
Fauna insect. Europæ, xix: $13 . \quad P l .13$.

Deutschl. petref., 649.

Insect. d. vorwelt, 131.

4893. Saperda nephele. (Cerambycidx.)

Locality : Oeningen, Baden. Horizon: Tortonian.

185\%. Giebel.

1856. "6

1865. Heer.

1872 . " "

1876. "

1879. "
Deutschl. petref., 649 .

Insect, d. vorwelt, 131-132.

Urwelt der Schweiz, 375.

Monde prim. Suisse, 460.

Prim. world Switz., ii : 31.

Urwelt der Schweiz, $2^{\mathrm{e}}$ aufl., 401.

See also Saperda (Compsidia) nephele.

Bull. $71 \longrightarrow 37$ 
4894. Saperda (Compsidia) nephele. (Cerambycidæ.)

Locality : Oeningen, Baden. Horizon: Tortonian.

1847. Heer. Insektenf. tertiärg. Oeningen, i: 168-171. Pl.6, fig.1.

See also Saperda nephele.

4895. Saperda valdensis. (Cerambycidæ.)

1865. Hөеr.

Locality : Rovereaz, (Switzerland?). Horizon: Miocene.

1872. " "

1876. " "

1879. “

Urwelt der Schweiz. Fig. 254 on p. 376.

Monde prim. Suisse. Fig. 254 on $p .461$.

Prim. world Switz., ii. Fig. 254 on p. 32.

Urwelt der Schweiz, $2^{\mathrm{e}}$ aufl. Fig. 296 on $p .402$.

\section{Scaphidium —. (Scaphidiidæ.)}

Locality: Prussian amber. Horizon: Ligurian.

1845. Berendt. Bernst. befindl. organ. reste vorw., i: 56.

4897. Scaphidium deletum. (Scaphidiidæ.)

Locality: (Oeningen, Baden). Horizon: Tortonian.

1847, Heer. 1852. Giebel. 1856. " " 1862. Heer.

Insektenf. tertiärg. Oeningen, i: 35-36. $\quad P l .7$, fig. 20.

Deutschl. petref., 654 .

Insect. d. vorwelt, 47.

Verhandl. holl, maatsch. wetensch. Haarl., xvi : 50.

4898. Scaphisoma gracile. (Scaphidiidæ.)

Locality : Oeningen, Baden. Horizon : Tortonian.

1862. Heer.

Verhandl. holl. maatsch. wetensch. Haarl., xvi: 49-50. Pl. 3, fig. 26.

4899. Scarabæus - (Scarabæidæ.)

Locality : Glaris, Switzerland. Horizon: -

1763. Bertrand. Dict. univ. foss., i : 259.

4900. Scarabæus proserpinæ. (Scarabæidæ.)

Locality: Oeningen, Baden. Horizon : Tortonian.

1862. Heer. Verhandl. holl. maatsch. wetensch. Haarl., xvi : 78, Pl. 6, fig.2.

4901. Scarites haidingeri. (Carabidæ.)

Locality : Radoboj, Croatia. Horizon: Mayencian.

1861. Heer. Rech. climat pays tert., 205 (undescr.).

4902. Scolytus - (Scolytidæ.)

Locality: Aix, France. Horizon: Ligurian.

1829. Serres. Géogn. terrains tert., 224-225.

4903. Scraptia ovata. (Melandryidæ.)

Locality: Sicilian amber. Horizon:-

1838. Guérin. Rev. zool., 1838: 170. Pl. 1, fig. 6.

1885. Scudder. Zittel, Handb. palaeont, i, th. ii: 791. Fig. 1015.

1886. " Zittel-Barrois, Traité de paléont., ii : 791. Fig. 1033. 
4904. Scydmænoides nigrescens. (Scydmænidæ.)

Locality : Prussian amber. Horizon : Ligurian.

1856. Motschulsky. Etud. entom., v : 27.

See also Dorcasoides bilobus.

4905. Scydmænus -. (Scydmænidæ.)

Locality: Prussian amber. Horizon: Ligurian.

1845. Berendt. Bernst. befindl, organ. reste vorw., i : 56.

4906. Scydmænus heerii. (Scydmænidæ.)

Compared with Chevrolatia insignis (Jacq. du Val), Europe.

Locality: Aix, France. Horizon: Ligurian.

1874. Oustalet. Ann. sc. géol., v, art. 2: 183-184. Pl. 1, fig. 10.

4907. Scymnus? —. (Coccinellidæ.)

Locality: Prussian amber. Horizon : Ligurian.

1845. Berendt. Bernst. befindl. organ. reste vorw., i : 56.

4908. Scymnus - (Coccinellidæ.)

Locality: Brunstatt, Alsatia. Horizon: Middle Oligocene.

1890. Foerster. In litt.

4909. Scyrtes —. (Dascyllidæ.)

Locality: Prussian amber. Horizon: Ligurian.

1845. Berendt. Bernst. befindl. organ. reste vorw., i : 56.

4910. Seniaulus scaphioides. (Scaphidiidæ.)

Locality : Rott, Rhenish Prussia. Horizon: Aquitanian.

1866. Heyd.-Heyd. Palaeontogr., $\mathrm{xv}:$ 139. Pl. 22, fig. 17.

1866. " " Käfer u. polypen, 9. Pl. 1, fig. 17.

1885. Scudder. Zittel, Handb. palaeont., i, th. ii: 801. Fig. 1057.

1886. " Zittel-Barrois, Traité de paléont., ii: 801. Fig. 1074.

4911. Sepidium —. (Tenebrionidæ.)

Compared with S. hispanicum.

Locality: Aix, France. Horizon: Ligurian.

1829. Serres. Géogn. terrains tert., 222.

4912. Serica -. (Scarabæidæ.)

Locality: Prussian amber. Horizon: Ligurian.

1862. Giebel. Zeitschr. ges. naturwiss, $x x: 321$.

4913. Serica minutula. (Scarabæidæ.)

Compared with S. strigosa Dej.

Locality: Oeningen, Baden. Horizon: Tortonian.

1862. Heer. Verhandl. holl. maatsch. wetenscis. Haarl., xvi: 79-80. Pl. 6, fig. 12.

1865. " " Urwelt der Schweiz, 380.

1872. " Monde prim. Suisse, 466.

1876. " Prim. world Switz., ii : 37.

1879, “ Urwelt der Schweiz, 2 auf., 406. 
4914. Sibynes melancholicus. (Curculionidæ.)

Compared with S. primitus Herbst, Europe.

Locality: Aix, France. Horizon: Ligurian.

1874. Oustalet. Ann. sc. géol., v, art. 2: 296-297 (Sybines). Pl. 5, fig. 8.

\section{Silicernius spectabilis. (Elateridæ.)}

Locality: Rott, Rhenish Prussia. Horizon : Aquitanian.

1859. Heyden. Palaeontogr., viii: 6-7. Pl. 1, fig. 9.

1885. Scudder. Zittel, Handb. palaeont., i, th. ii : 797. Fig. 1037.

1886. " Zittel-Barrois, Traité de paléont., ii : 798. Fig. 1055.

\section{6. [Omitted.]}

4917. Silpha (Phosphuga) atrata (recent). (Silphidæ.)

Locality : Hösbach, Bavaria. Horizon : Lower Pleistocene.

[1746. Linné.

1884. Flach.

1884. "6
Faun. suec. no. 451.]

Käfer unterpleist. Hösbach, 9. Pl. 2, fig. 6.

Verhandl. physik.-med. gesellsch. Würzb., n. f., xviii : 293. Pl. $9, f i g .6$.

\section{Silpha? deplanata. (Silphidæ.)}

\section{Locality : Cap Staratschin, Spitzbergen. Horizon: Miocene.}

1870. Heer. K. svenska vetensk.-akad. handl., viii, No. 7: 73-74. Pl. 16, fig. 42.

4919. Silpha dispar (recent). (Silphidæ.)

Localities: Schwerzenbach, Switzerland; Norfolk, England. Horizon: Glacial clay, Forest bed, Pleistocene.

[1793. Herbst. Käfer, v : 204. Pl. 52, fig. 1.]

1879. Heer. Urwelt der Schweiz, $2^{\mathrm{e}}$ aufl., 581.

1886. Scudder. Bull. U. S. geol. surv., No 31: 82.

1888. Bell. Eutom., xxi: 2.

4920. Silpha obsoleta. (Silphidæ.)

1847. Heer.

185\%. Giebel.

1856. " "
Locality: Radoboj, Croatia. Horizon: Mayencian.

Insektenf. tertiärg. Oeningen, i: 36. $\quad P l .2, f i g .7$.

Deutschl. petref., 654 .

Insect. d. vorwelt, 46 .

\section{Silpha stratuum. (Silphidæ.)}

Compared with S. littoralis.

Locality: Vicinity Bonn, Rhenish Prussia. Horizon : Aquitanian?.

1837. Germar.

1847. Heer.

1852. Giebel.

1856. "
Fauna insect. Europæ, xix : 5. Pl. 5.

Insektenf. tertiärg. Oeningen, i: 33-34.

Deutschl. petref., 654 .

Insect. d. vorwelt, 46. 
4922. Silpha tricostata. (Silphidæ.)

Compared with S. carinata Heer.

Locality: Oeningen, Baden. Horizon: Tortonian.

1862. Heer. Verhandl. holl. maatsch. wetensch. Haarl., xvi: 50-51. Pl. 3, figs. $7,7 b, 8$.

1865. “ Urwelt der Schweiz, 382. Fig. 275 on p. 381.

1872. " Monde prim. Suisse, 468. Fig. 275 on p. 467.

1876. " Prim. world Switz., ii : 39. Fig. 275 on p. 38.

1879. “ Urwelt der Schweiz, $2^{\circ}$ anfl, 408. Fig. 317 on p. 407.

1885. Scudder. Zittel, Handb. palaeont., i, th. ii: 802. Fig. 1061.

1886. “ Zittel-Barrois, Traité de pal6ont., ii : 802. Fig. 1078.

See also Peltis tricostata.

4923. Silvanus - (3 sp.). (Cucnjidæ.)

Locality : Prussian amber. Horizon: Ligurian.

1856. Menge. Progr. petrischuie Danzig, 1856: 21.

4924. Sinis brevicollis. (Carabidæ.)

Locality : Oeningen, Baden. Horizon: Tortonian.

1862. Heer.

1865. “

187\%. "

1876. " "

1879. “

Verhandl. holl. maatsch. wetensch, Haarl, xvi : 32-33. Pl. 1, figs. 16,17 .

Urwelt der Schweiz, 385. Fig. 286 on p. 384.

Monde prim. Suisse, 472. Fig. 286 on p. 471.

Prim. world Switz., ii : 42. Fig. 286.

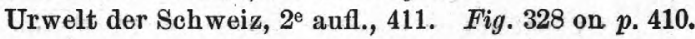

4925. Sisyphus —. (Scarabæidæ.)

Compared with S. schæfferi.

Locality: Aix, France. Horizon : Ligurian.

1829. Serres. Géogn. terrains tert., 222.

4926. Sitodrepa defuncta, (Ptinidæ.)

Locality: Green River, Wys. Horizon: Oligocene.

1876. Scudder.

Bull. U. S. geol. surv. terr., ii : 82.

1878. " "

1890. " "

Bull. U. S. geol. surv. terr., iv : 762.

Tert. ins. N. A., 493.

Sitona - See Sitones — Curtis.

Sitona attavina. See Sitones atavinus.

4927. Sitones —. (Curculionidæ.)

Locality: Prussian amber. Horizon: Ligurian.

1845. Berendt. Bernst. befindl. organ. reste vorw., i: 56 .

4928. Sitones ——. (Curculionidæ.)

Locality : Aix, France. Horizon: Ligurian.

1829. Curtis. Edinb. new phil.journ., vii : 295 (Sitona). Pl. 6, fig. 2. 1829. " Murchison-Lyell, Tert. form. Aix, 11. Pl. 6, fig. 2.

See also Sitones margarum, S. antiquus. 
4929. Sitones antiquus. (Curculionidæ.)

Locality: Aix, France. Horizon: Ligurian.

1856. Giebel. Insect. d. vorwelt, 141 (Sitona antiqua).

See also Sitones — Curtis, etc.

4930. Sitones atavinus. (Curculionidæ.)

Localitý : Oeningen, Baden. Horizon: Tortonian.

1847. Heer. Insektenf. tertiärg. Oeningen, i: 182-183 (Sitona attavina). $P l$. 6, fig. 11.

1852. Giebel. Deutschl. petref., 649 (Sitona attavina).

1856. " " Insect. d. vorwelt, 141.

1865. Heer. Urwelt der Schweiz, 375 (Sitona). Fig. 248 on p. 371.

1872. " " Monde prim. Suisse, 459. Fig. 248 on p. 455.

1876. “ Prim. world Switz., ii : 30. Fig. 248 on p. 27.

1879. “ Urwelt der Schweiz, $2^{\circ}$ aufl., 400. Fig. 290 on p. 397.

4931. Sitones grandævus. (Curculionidæ.)

Locality: Green River, Wyo. Horizon: Oligocene.

1876. Scudder. Bull. U. S. geol. surv. terr., ii : 83-84.

1878. " Bull. U. S. geol. surv. terr., iv : 767.

See also Eugnamptus grandævus.

\section{Sitones margarum. (Cnrculionidæ.)}

Locality: Aix, France. Horizon: Liguriau.

1849. Germar. Zeitschr. deutsch. geol. gesellsch., i: 61-62 (Sitona). Pl. 2, fig.5.

1856. Heer.

1856. Giebel.

Viert. naturf. gesellsch. Zürich, i: 19 (Sitona).

1674. Oustalet.

1874. Oustalet. Ann. sc. géol., v, art. 2: 246-253. Pl. 3, fig. 15.

See also Sitones — Curtis, etc.

4933. Sitones venustulus. (Curculionidæ.)

Locality : Rott, Rhenish Prussia. Horizon : Aquitanian.

1866. Heyd.-Heyd. Palaeontogr., $\mathrm{xv}$ : 149. Pl. 23, fig. 10.

1866. " Käfer u. polypen, 19. Pl. 2, fig. 10.

1870. L. Heyden. Foss. dipt. Rott, 29.

1870. " Palaeontogr., xvii : 265.

4934. Smicronyx —- (Curculionidæ.)

Locality: Brunstatt, Alsatia. Horizon : Middle Oligocene.

1889. Foerster. Mitth. comm. geol. Elsass-Lothr., ii: 102.

4935. Sospita haagi. (Coccinellidæ.)

Locality : Rott, Rhenish Prussia. Horizon : Aquitanian.

18i0. L. Heyden. Foss. dipt. Rott, 29.

1870. “ $\quad$ Palaeontogr., xvii : 265.

See also Coccinella (Sospita) haagi.

4936. Spermophagus vivificatus. (Bruchidæ.)

Locality : Florissant, Colo. Horizon : Oligocene.

1876. Scudder. Bull. U. S. geol. surv. terr., ii : 82.

1885. " Zittel, Handb. palaeont., i, th.ii: 792. Fig. 1019.

1886. “ Zittel-Barrois, Traité de paléont., ii : 792. Fig. 1037. 
4937. Sphenophorus nægelianus. (Calandridæ.)

Locality : (Oeningen?, Baden.) Horizon: Tortonian.

1847. Heer. Insektenf. tertiärg. Oeningen, i: 193-195. Pl. 6, fig. 16. 1852. Giebel. Deutschl. petref., 648.

1856. " " Insect. $d$. vorwelt, 138-139.

See also Eurychirus (Otiorhynchus) induratus:

4938. Sphenophorus proluviosus. (Calandridæ.)

Logality: Rott, Rhenish Prussia. Horizon: Aquitanian.

1866. Heyd.-Heyd. Palaeontogr., xv: 153-154. Pl. 24, fig. 9 .

1866. " Käfer u. polypen, 23-24. Pl. 3, fig. 9.

4939. Sphenophorus regelianus. (Calandridæ.)

Locality: (Oeningen \%, Baden.) Horizon: 'Tortonian.

1847. Heer. Insektenf. tertiärg. Oeningen, i: 195-196. Pl. 6, fig. 17. 1852. Giebel. Deutschl. petref., 648.

1856. " Insect. d. vorwelt, 139.

See also Eurychirus (Otiorhynchus) induratus.

4940. Sphenoptera gigantea. (Buprestidæ.)

Locality : Oeningen, Baden. Horizon: Tortonian.

1847. Heer. Insektenf. tertiärg. Oeningen, i: 117-118. Pl. 3, fig. 12.

1852. Giebel. Deutschl. petref., 652.

1856. “ Insect. d. vorwelt, 86-87.

4941. Sphenoptera knopi. (Buprestidæ.)

Loeality : Salzhausen, Germany. Horizon: Miocene.

1865. Heyd.-Heyd. Palaeontogr, xiv: 33. Pl. 9, fig. 17.

\section{Spondylis - (larva). (Spondylidæ.)}

1885. Scudder. Zittel, Handb. palaeont., i, th. ii : 794. Figs. 1025, 1025a 1886. " Zittel-Barrois, Traité de paléont., ii : 794. Figs. 1043ab.

4943. Spondylis crassicornis. (Spondylidæ.)

1856. Giebel. Insect. d. vorwelt, 127.

4944. Spondylis tertiarius. (Spondylidæ.)

Locality: - Horizon: -

1849. Germar. Zeitschr. deutsch. geol. gesellsch., i: 58-60. Pl. 2, fig. 3。 1852. Giebel. Deutschl. petref., 651 (Spondylis ?).

1856. " Insect. d. vorwelt, 127 (tertiaria).

1885. Scudder. Zittel, Handb. palaeont., i, th. ii: 794. Fig. 1024.

1886. " Zittel-Barrois, Traité de paléont., ii: 794. Fig. 1042.

4945. Staphylinites obsoletus. (Staphylinidæ.)

Locality: Green River, Wyo. Horizon: Oligocene.

1876. Sendder. Bull. U. S. geol. surv. terr., ii : 78 (obsoletum).

1878. " " Bull. U. S. geol. surv. terr., iv: 762.

1890. “ Tert. ins. N. A., 510. Pl. 8, fig. 32. 
4946. Staphylinus ——. (Staphylinidæ.)

Compared with S. œnocephalus.

Locality : (Senigaglia?, Italy.) Horizon : Helvetian?.

1841. Bassi.

Atti reun. scienz. ital., iii : 401.

\section{Staphylinus —. (Staphylinidæ.)}

Locality: Prussian amber. Horizon: Ligurian.

1830. 3erendt. Insekten im bernstein, 34.

4948. Staphylinus - (Staphylinidæ.)

Locality: Prussian amber. Horizon: Ligurian.

1835. Gravenhorst. Uebers. schles. gesellsch. vater?. cult., 1834: 92.

4949. Staphylinus? (Staphylinidæ.)

Locality: Sicilian amber. Horizon:

1838. Guérin.

Rev. zool., 1838: 170.

4950. Staphylinus (larva). (Staphylinidæ.) Locality: Rott, Rhenish Prussia. Horizon: Aquitanian.

1866. Heyd.-Heyd. Palaeontogr., xv: 138-139. Pl. 22, fig. 16.

1866. “ Käfer u. polypen, 8-9. Pl. 1, fig. 16.

4951. Staphylinus —_. (Staphylinidæ.)

Locality: Aix, France. Horizon: Ligurian.

1829. Serres. Géogn. terrains tert., 221.

4952. Staphylinus —. (Staphylinidæ.)

Locality : Gurnet Bay, Isle of Wight. Horizon: Bembridge Limestone. 1879. Woodward. Quart. journ. geol. soc. Lond, xxxv: 344.

1879. “ Geol. mag., n. s., v: 89.

4953. Staphylinus aquisextanus. (Staphyliničø.)

Compared with S. hirtus, Europe.

Locality: Aix, France. Horizon: Ligurian.

1874. Oustalet. Ann. se. géol., v, art.2: 160-162. Pl. 2, fig. 14.

4954. Staphylinus atavus. (Staphylinidæ.)

Locality: Oeningen, Baden. Horizon: Tortonian.

1862. Heer. Verhandl. holl. maatsch. wetensch. Haarl., xvi: 48-49 (distinct from Oustalet's species). $P l .3, f i g .1$.

4955. Staphylinus (Ocypus) atavus. (Staphylinidæ.)

Compared with Ocypus pedator Grav., Europe.

Locality: Aix, France. Horizon: Ligurian.

1874. Oustalet. Ann. sc. géol., v, art. 2: 162-163 (distinct from Heer's species). Pl. 2, fig. 8.

4956. Staphylinus calvus. (Staphylinidæ.)

Locality: Aix, France. Horizon: Ligurian.

1874. Oustalet. Ann. se. géol., v, art.2: 155-157. Pl. 2, fig. 9. 
4957. Staphylinus (Ocypus) germarii. (Staphylinidæ.)

Locality: Aix, France. Horizon : Ligurian.

1874. Oustalet. Ann. sc. géol., v, art.2: 157-159. Pl. 2, fig. 13.

4958. Staphylinus priscus. (Staphylinidæ.)

Locality : Aix, France. Horizon: Ligurian.

1874. Oustalet. Ann. sc. géol., v, art. 2: 166-167. Pl. 2, fig. 11.

4959. Staphylinus prodromus. (Staphylinidæ.)

Locality : Aix, France. Horizon : Ligurian.

1874. Heer. MSS.

1874. Oustalet. Ann. sc. géol., v, art. 2: 163-166. Pl. 2, figs. 10, 12.

4960. Staphylinus (Ocypus) provincialis. (Staphylinidæ.)

Locality: Aix, France. Horizon: Ligurian.

1874. Oustalet. Ann. se. géol., v, art. 2: 159-160. Pl. 3, fig. 2.

4961. Statira (vic.) —. (Lagriidæ.)

Locality: Prussian amber. Horizon: Ligurian.

1868. Smith. Quart. journ. sc., v: 184. Pl., fig. 7.

4962. Stenus —. '(Staphylinidæ.)

Locality: Prussian amber. Horizon: Ligurian.

1845. Berendt. Bernst. befiudl. organ. reste vorw., i: 56.

4963. Stenus —. (Staphylinidæ.)

Locality : Hösbach, Bavaria. Horizon: Lower Pleistocene.

1884. Flach. Käfer unterpleist. Hösbach, 9.

1884. " Verhandl, physik.-med. gesellsch. Wiurzb., n. f., xviii : 293.

4964. Stenus —. (Staphylinidæ.)

Compared with S. juno.

Locality : Hösbach, Bavaria. Horizon: Lower Pleistocene.

1884. Flach. Käfer unterpleist. Hösbach, 9. Pl. 2, fig. 4.

1884. “ Verhandl. physik.-med. gesellsch. Würzb.,n. f., xviii: 293. $P l$. 9, fig. 4.

4965. Stenus —. (Staphylinidæ.

Locality: Brunstatt, Alsatia. Horizon: Middle Oligocene.

1888. Foerster. Mitth. comm. geol. Elsass-Lothr., i : 165.

1889. " Mitth. comm. geol. Elsass-Lothr., ii : 103.

4966. Stenus -. (Staphylinidæ.)

Locality : Prussian amber. Horizon: Ligurian.

1856. Monge. Progr. petrischule Danzig, 1856: 23.

4967. Stenus gypsi. (Staphylinidæ.)

Locality: Aix, France. Horizon: Lignrian.

1874. Oustalet. Ann. sc. géol., v, art. 2: 139-140. Pl. 2, fig. 5. 


\section{Stenus prodromus. (Staphylinidæ.)}

Locality : Aix, France. Horizon : Ligurian.

1856. Heer. Viert. naturf. gesellsch. Zürich, i: 14-15. Pl. 1, fig. 3.

1874. Oustalet. Ann. sc. géol., v, art. 2: 138-139. Pl. 6, figs. 16, 16a.

\section{Stenus scribai. (Staphylinidæ.)}

Locality : Rott, Rhenish Prussia. Horrizon: Aquitanian.

1866. Heyd.-Heyd. Palaeontogr., xv: 137. Pl. 22, fig. 13. 1866.

Käfer u. polypen, 7. Pl. 1, fig. 13.

Steropus æthiops. See Feronia (Steropus) æthiops.

4970. Stilicus - (Staphylinidæ.)

Locality: Prussian amber. Horizon: Ligurian.

1845. Berendt. Bernst. befindl. organ. reste vorw., i: 56 .

4971. Stomis elegans. (Carabidæ.)

Locality : Aix, France. Horizon : Ligurian.

1874. Oustalet. Ann. sc. géol., v, art. 2: 115-116. Pl. 1, fig. 3.

4972. Strongylus ? - (Nitidulidæ.)

Locality: Prussian amber. Horizon: Ligurian.

1845. Berendt. Bernst. befindl, organ. reste vorw., i : 56.

\section{Strophosomus? (or Cucorhinus?) —. (Otiorhynchidæ.)}

Locality : Peckham, England. Horizon: Paludina beds, Lower Eocene. 1861. Smith. Geologist, iv : 40.

4974. Sunius demersus. (Staphylinidæ.)

Locality : Rott, Rhenish Prussia. Horizon: Aquitanian.

1866. Heyd.-Heyd. Palaeontogr., xv: 137. Pl. 22, fig. 12.

1866. " Käfer u. polypen, 7. Pl. 1, fig. 12.

Sybines melancholicus. See Sibynes melancholicus.

4975. Sylvanus? - (Cucujidæ.)

Locality: Prussian amber. Horizon: Ligurian.

1845. Berendt. Bernst. befindl. organ. reste vorw., i: 56.

4976. Tachinus - (Staphylinidæ.)

Locality: Prussian amber. Horizon: Ligurian.

1845. Berendt. Bernst. befindl. organ. reste vorw., i : 56.

$$
\text { 4977. Tachinus —-. (Staphylinidæ.) }
$$

Locality : Prussian amber. Horizon: Ligurian.

1856. Menge. Progr. petrischule Danzig, 1856: 23.

4978. Tachyporus - (Staphylinidæ.)

Locality: Prussian amber. Horizon: Ligurian.

1845. Berendt. Bernst. befindl. organ. reste vorw., i: 56. 
4979. Tachyporus (Staphylinidæ.)

Locality: Prussian amber. Horizon: Ligurian. 1856. Menge. Progr. petrischule Danzig, 1856: 23.

4980. Tachyporus sepultus. (Staphylinidæ.) Locality : Rott, Rhenish Prussia. Horizon: Aquitanian. 1866. Heyd.-Heyd. Palaeontogr., xv: 136. $\quad P l .22, f i g .10$. 1866. " Käfer u. polypen, 6. Pl. 1, fig. 10.

4981. Tagenopsis brevicornis. (Tenebrionidæ.)

Locality : Oeningen, Baden. Horizon: Tortonian. 1865. Heer. Urwelt der Schweiz, 377. Figs. $257,257 b$ on $p .376$. 187.2. " Monde prim. Suisse, 462. Figs. 257, 25ib on p. 461. 187i. " $\quad$ Prim. world Switz., ii: 33 . Figs. 257, $257 b$ on $p .32$. 1-79. “ Urwelt der Schweiz, $2^{\mathrm{e}}$ aufl, 402. Figs. 299, 2996. 1885. Scudder. Zittel, Handb. palaeont., i, th. ii: 791. Fig. 1018. 1886. " Zittel-Barrois, Traité de paléont., ii: 792. Fig. 1036.

4982. Tanymecus seculorum. (Otiorhynchidæ.)

Locality: Green River, Wyo. Horizon: Oligocene. 1890. Scudder. Tert. ins. N. A., 475-476. Pl. 8, fig. 22.

4983. Tanysphyrus deletus. (Curculionidæ.)

Locality: Aix, France. Horizon: Ligurian.

1874. Oustalet. Ann. sc. géol., v, art. 2: 268-269. Pl. 5, fig. 7.

4984. Telephorus atavinus. (Lampyridæ.)

Locality : Radoboj, Croatia. Horizon : Mayencian.

1847. Heer. Insektenf. tertiärg. Oeningen, i: 149-150 (attavinus). Pl. 4, fig. 15.

1852. Giebel. Deutschl. petref., 650 .

1856. " Insect. d. vorwelt, 102.

Telephorus attavinus. See Telephorus atavinus.

4985. Telephorus brodiei. (Lampyridæ.)

Locality : Rott, Rhenish Prussia. Horizon: Aquitanian.

1866. Heyd.-Heyd. Palaeontogr., xv : 143. Pl. 22, fig. 25.

1966. “ Käfer u. polypen, 13. Pl. 1, fig. 25.

4986. Telephorus caducus. (Lampyridæ.)

Locality : Rott, Rhenish Prussia. Horizon: Aquitanian.

1866. Heyd.-Heyd. Palaeontogr., xv : 143. Pl. 22, fig. 20.

1866. " Käfer u. polypen, 13. Pl. 1, fig. 20.

4987. Telephorus carbonarius. (Lampyridæ.)

Locality : Rott, Rhenish Prussia. Horizon: Aquitanian.

1866. Heyd.-Heyd. Palaeontogr., xv : 142. Pl. 23, fig. 2.

1866. “ Käfer u. polypen, 12. Pl. 2, fig. 2. 
4988. Telephorus exauctaratus. (Lampyridæ.)

Locality: Rott, Rhenish Prussia. Horizon: Aquitanian.

1866. Heyd.-Heyd. Palaeontogr., xv: 142-143. Pl. 23, fig. 3.

1866. “ Käfer u. polypen, 12-13. Pl. 2, fig. 3.

1870. L. Heyden. Foss, dipt. Rott, 29.

1870. “ Palaeontogr., xvii: 265.

4989. Telephorus fragilis. (Lampyridæ.)

Locality : Oeningen, Baden. Horizon: Tortonian.

1847. Heer. Insektenf. tertiärg. Oeningen, i: 148-149. Pl.4, fig. 14.

1852. Giebel. Deutschl. petref., 650.

1856. " Insect. $d$, vorwelt, 102.

4990. Telephorus germari. (Lampyridæ.)

Locality: Oeningen, Baden. Horizon : Tortonian.

1847. Heer.

1852. Giebel.

Insektenf. tertiärg. Oeningen, i : 143-145. Pl. 4, fig. 10.

1856. "

Deutschl. petref., 650 .

Insect. d. vorwelt, 101.

4991. Telephorus macilentus. (Lampyridæ.)

Locality: Oeningen, Baden. Horizon: Tortonian.

1865. Heer.

1872. " "

Urwelt der Schweiz. Fig. 256 on p. 376.

Monde prim. Suisse. Figs. 256, 256b-d on p. 461.

1876. " Prim. world Switz., ii. Fig. 256 on $p .32$.

1879. “ Urwelt der Schweiz, $2^{\mathrm{e}}$ aufl. Fig. 298 on $p .402$.

Telephorus œningensis. See Telephorus tertiarius œningensis.

Telephorus radobojanus. See Telephorus tertiarius radobojanus.

4992. Telephorus tertiarius. (Lampyridæ.)

Localities: Oeningen, Baden; Radoboj, Croatia. Horizons: Tortonian, Mayencian.

1847. Heer. Insektenf. tertiärg. Oeningen, i: 145. Pl. 4, figs. 11-13.

1852. Giebel. Deutschl. petref., 650.

1856. " Insect. d. vorwelt, 101-102.

See also Telephorus tertiarius œningensis, T. tertiarius radobojanus.

4993. Tekephorus tertiarius œningensis. (Lampyridæ.)

Locality : Oeningen, Baden. Horizon: Tortonian.

1847. Heer.

Insektenf. tertiärg. Oeningen, i: 145-146. Pl. 4, fig. 11.

See also Telephorus tertiarius, etc.

4994. Telephorus tertiarius radobojanus. (Lampyridæ.)

Locality: Radoboj, Croatia. Horizon: Mayencian.

1847. Heer. Insektenf. tertiärg. Oeningen, i: 147-148. Pl. 4, fig. 12.

See also Telephorus tertiarius, etc.

4995. Tenebrio effossus. ('Tenebrionidæ.)

Compared with T. molitor.

Locality: Vicinity Bonn, Rhenish Prussia. Horizon: Aquitanian ?.

1837. Germar. Fanna insect. Europæ, xix: 8. $P l .8$.

1856. Giebel. Insect. d. vorwelt, 109-110. 
4996. Tenebrio primigenius. (Tenebrionidæ)

Compared with T. molitor Linn.

Locality : Nine Mile Creek, British Columbia. Horizon :

1879. Scudder. Rep. progr. geol, surv, Can., 1877-78: B183.

1879. " Insect. tert. Nicola, 8.

1890. “ Tert. ins. N. A., 483-484. Pl. 2, fiq. 32.

4997. Tenebrio senex. (Tenebrionidæ.)

Locality : Rott, Rhenish Prussia. Horizon: Aquitanian.

1859. Heyden. Palaeontogr., viii: 7-8. Pl. 1, fig. 6.

4998. Throscus -. (Throscidæ.)

Locality: Prussian amber. Horizon: Ligurian.

1845. Berendt. Bernst. befindl. organ. reste vorw., i: 56.

4999. Thylacites —- (Otiorhynchidæ.)

Locality : Prussian amber. Horizon: Ligurian.

1832. Burmeister. Handb. ent., i : 635.

1836. " Man. ent., 577.

5000. Thylacites rugosus. (Otiorhynchidæ.)

Compared with T. guinardi, T. araneiformis.

Locality: Kutschlin, Bohemia. Horizon: Miocene.

1 2 81. Deichmüller. Foss. ius. diatomeensch. Kutschlin, 19-21. Pl. 21, figs. 6, 6a.

1Ł81. " Verhandi. leop.-carol. akad. naturf., xlii ; 31l-313. Pl.21, figs. 6, $6 a$.

1885. Scudder. Zittle, Handb. palaeont., i, th. ii: 789. Fig. 1009.

1886. " Zittle-Barrois, Traité de paléont., ii : 789. Fig. 1027.

5001. Tillus —. (Cleridæ.)

Locality: Prussian amber. Horizon: Ligurian.

1856. Berendt. Bernst. befindl. organ. reste vorw., i: 56.

5002. Timarcha -. (Chrysomelidæ.)

Locality : Norfolk, England. Horizon : Forest bed, Pleistocene.

1888. Bell. Entom., xxi : 2.

5003. Timarcha metallica (recent). (Chrysomelidæ.)

Locality : Hösbach, Bavaria. Horizon: Lower Pleistocene.

[1781. Laichart.

1884. Flach.

18,4. "
Verz. tyrol. ins., 144.]

Käfer unterpleist. Hösbach, 10. Pl. 2, fig. 10.

Verhandl. physik.-med, gesellsch. Würzb., n. f., xviii : 294. $\quad P l$. 9, fig. 10.

5004. Tmesiphoroides cariniger. (Pselaphidæ.)

Locality: Prussian amber. Horizon: Ligurian.

1856. Motschulsky. Etud. entom., v : 26. Pl., figs. $5,5^{\prime}, 5^{\prime \prime}$.

1885. Scudder. Zittel, Handb. palaeont, i, th. ii: 802. Fig. 1059.

1886. " Zittel-Barrois, Traité de paléont., ii : 802. Fig. 1076. 
5005. Tophoderes depontanus. (Anthribidæ.)

Locality: Rott, Rhenish Prussia. Horizon: Aquitanian.

1859. Heyden. Palaeontogr., viii: 9-10. Pl. 1, fig. 2.

1885. Scudder. Zittel, Handb. palaeont., i, th. ii: 787. Fig. 1005.

18z6. " Zittel-Barrois, Traité de paléont., ii: 787. Fig. 1022.

5006. Trachyderes bustiraptus. (Cerambycidæ.)

Locality : Sieblos, Rhenish Prussia. Horizon: Aquitanian.

1859. Heyden. Palaeontogr., viii: 15-16. Pl. 3, fig.7.

See also Trachyderes bustonaptus.

5007. Trachyderes bustonaptus. (Cerambycidæ.)

Locality : Sieblos, Rhenish Prassia. Horizon: Aquitanian.

1860. Heyden. Hassenc, Würzb. naturw, zeitschr, i : 79 (undescr.). See also Trachyderes bustiraptus.

5008. Trechinites clairvillii. (Carabidæ.)

Locality: Oeningen, Baden. Horizon: Tortonian.

1862. Heer. Verhandl. holl. maatsch. wetensch. Haarl., xvi: 34 . Pl. 1, fig. 25.

5009. Trechinites oblongus. (Carabidæ.)

Locality : Oeningen, Baden. Horizon: Tortonian.

1862. Heer, Verhandl. holl. maatsch. wetensch. Haarl., xvi : 34. Pl. 1, fig. 26.

5010. Trechoides fasciatus. (Carabidæ.)

Locality : Prussian amber. Horizon: Ligurian.

1856. Motschulsky. Etud. entom., v : 26.

5011. Trechus —. (Carabidæ.)

Locality : Brunstatt, Alsatia. Horizon : Middle Oligocene.

1890. Foerster. In litt.

5012. Trechus rivularis (recent). (Carabidæ.)

Locality : Hösbach, Bavaria. Horizon : Lower Pleistocene.

[1810. Gyllenhal. Ins. suec., ii : 32.]

1884. Flach. Käfer unterpleist. Hösbach, 7. Pl. 1, fig. 11.

1884. “ Verhanc̈l. physik.-med. gesellsch. Würzb., n. f., xviii : 291. $\quad P l$. 8, fig. 11.

5013. Triæna tertiaria. (Stylopidæ.)

Locality: Prussian amber. Horizon: Ligurian.

1866. Menge. Rhipidopteron (Schrift. naturf. gesellsch. Danzig, (2), i, iii-iv): 2-3. Figs. 1-3.

1885. Scudder. Zittel, Handb. palaeont., i, th. ii : 790. Fig. 1012.

1886. " Zittel-Barrois, Traité de paléont., ii: 790. Fig. 1030.

See also Mengea tertiaria, 
5014. Trichius -. (Scarabæidæ.)

Locality : Caverne de Lunel Vieil, dép. de l'Hérault, France.

Horizon : Postpliocene.

1827. Serres. Móm. soc. linn. Paris, v: 457.

\section{Trichius ædilis. (Scarabæidæ.)}

Compared with T. nobilis L.

1865. Heer.

1872. "

1876. “

1879. “

See also Trichius (Gnorimus) ædilis.

Locality: Oeningen, Baden. Horizon: Tortonian.

\section{Trichius (Gnorimus) ædilis. (Scarabæidæ.)}

\section{Locality : Oeningen, Baden. Horizon: Tortonian.}

1862. Heer. Verhandl. holl. maatsch. wetensch. Haarl., xvi: 81-82. Pl.6, figs. 14, 15.

Seo also Trichius ædilis.

Urwelt der Sehweiz, 380. $\quad$ Fig. 226 on $p .378$.

Monde prim. Suisse, 466. Fig. 226 on p. 463.

Prim. world Switz., ii : 36. Fig. 226 on p. 34 .

Urwelt der Schweiz, $2^{\mathrm{e}}$ aufl., 405. Fig. 308 on p. 404.

5017. Trichius amœnus. (Scarabæidæ.)

Campared with T. fasciatus F.

Locality : (Oeningen ?, Baden.) Horizon : Tortonian.

1847. Heer.

1852. Giebel.

1856. “

1865. Heer.

1872. "

1876. "6

1879. “

Insektenf. tertiärg. Oeningen, i: 74-75. $\quad P l .7$, fig. 53.

Deutschl. petref., 652.

Insect. d. vorwelt, 30 .

Urwelt der Schweiz, 380.

Monde prim. Suisse, 466.

Prim. world Switz., ii : 36.

Urwelt der Schweiz, $2^{\ominus}$ aufl., 405.

5018. Trichius lugubris. (Scarabæidæ.)

Compared with $\mathbf{T}$. variabilis $\mathbf{L}$.

Locality : Oeningen, Baden. Horizon: Tortonian.

1865. Heer.

1872. "

Urwelt der Schweiz, 380.

1876. " "

Monde prim. Suisse, 466.

1879. " "

Prim. world Switz., ii : 36.

See also Trichius (Gnorimus) lugubris.

5019. Trichius (Gnorimus) lugubris. (Scarabæidæ.)

Locality : Oeningen, Baden. Horizon : Tortonian.

1862. Heer.

Verhandl. holl. maatsch. wetensch. Haarl., xvi : 81. Pl. 6, fig. 13.

See also Trichius lugubris.

5020. Trichius rotundatus. (Scarabæidæ.)

Locality: Oeningen, Baden. Horizon: Tortonian.

1862. Heer. Verhandl. holl. maatsch. wetensch. Haarl., xvi: 82. Pl. 6, fig. 18. 


\section{Trichius unifasciatus. (Scarabæidæ.)}

Isocality: Oeningen, Baden. Horizon: Tortonian.

1862. Heer. Verhandl. holl. maatsch. wetenseh. Haarl., xvi : 82. Pl. 6, figs. $16,17$.

5022. Triphyllus heerii. (Mycetophagidæ.)

Locality : Aix, France. Horizon: Ligurian.

1874. Oustalet. Ann. sc. géol., v, art. 2: 189-190. Pl. 6, fig. 13.

1885. Scudder. Zittel, Handb. palaennt., i, th. ii: 799. Fig. 1049.

1886. " Zittel-Barrois, Traité de paléont., ii : 800. Fig. 1066.

5023. Trogosita —. (Trogositidæ.)

Compared with T. corulea.

Locality : Aix, France. Horizon: Ligurıan.

1829. Serres. G6́ogn. terrains tert., 225.

5024. Trogosita amissa. (Trogositidæ.)

Locality : Oeningen, Baden. Horizon: Tortonian.

1862. Heer.

Verhandl. holl. maatsch. wetensch. Haarl., xvi: 55. Pl. 3, figs. 13, 14.

5025. Trogosita assimilis. (Trogositidæ.)

Compared with T. mauritanica L.

Locality: Oeningen, Baden. Horizon: Tortonian.

18C2. Heer.

Verhandl. holl. maatsch. wetensch. Haarl., xvi : 56. Pl. 3, figs. $12,12 b c$.

1865. “

18\%?. “

1876. “

1879. “

Urwelt der Schweiz, 382.

Monde prim. Suisse, 469.

Prim. world Switz., ii: 39.

Urwelt der Schweiz, $2^{\mathrm{e}}$ aufl. $40 \varepsilon$.

5026. Trogosita bella. (Trogositidæ.)

Locality : Oeningen, Baden. Horizon: Tortonian.

1862. Heer.

Verhandl. holl. maatsch. wetensch. Haarl., xvi : 55. $\quad P l .3$, figs. $15,15 b$.

5027. Trogosita emortua. (Trogositidæ.)

Locality : Orsberg, (Rhenish Prussia ?). Horizon: Miocene.

1849. Germar.

1852. Giebel.

Zeitschr. deutsch. geol. gesellsch., i : 60-61. Pl. 2, fig. 4.

1856. “

Deutschl. petref., 654 .

Insect. $d$. vorwelt, 42 .

5028. Trogosita insignis. (Trogositidæ.)

Compared with T. caraboides.

Locality : Atanekerdluk, North Greenland. Horizon: Miocene.

1868. Heer.

1883. “
Flora foss. aret., 129. $\quad P l .50$, figs. 12, $12 b c$.

Flora foss. groenl., ii: 144.

5029. Trogosita kœllikeri. (Trogositidæ.)

Locality : (Oeningen, Baden.) Horizon: Tortonian.

1847. Heer.

1852. Giebel.

1856. “"

1862. Heer.
Insektenf. tertiärg. Oeningen, i: 40-43. Pl. 6, fig. 3.

Dentschl, petref., 654.

Insect. d. vorwelt, 42.

Verhandl. holl. maatsch. wetensch. Haarl., xvi : 54. 
5030. Trogosita longicollis. (Trogositidæ.)

Locality : Oeningen, Baden. Horizon: Tortonian.

1862. Heer. Verhandl. holl. maatsch. wetensch. Haarl., xvi: 54-55. Pl. 3, figs. 10, $10 b$.

5031. Trogosita sculpturata. (Trogositidæ.)

Locality: Oeningen, Baden. Horizon: Tortonian.

1862. Heer. Verhandl. holl. maatsch. wetensch. Haarl., xvi: 53-54. Pl. 3, fig. 9.

1865. “

1872. “

1876. “

1879. “

Urwelt der Schweiz, 382. Fig. 277 on $p$. 381 .

Monde prim. Suisse, 468. Fig. 277 on $p .467$.

Prim. world Switz., ii : $39 . \quad F i g . .277$ on $p .38$.

Urwelt der Schweiz, $2^{\mathrm{e}}$ aufl., 408. Fig. 319 on p. 407.

5032. Trogosita tenebrioides. (Trogositidæ.)

Compared with T. cærulea.

Locality : Vicinity Bonn, Rhenish Prussia. Horizon: Aquitanian 9

1837. Germar. Fauna insect. Europæ, xix : 9. Pl.9.

1852. Giebel. Deutschl. petref., 654 .

1856. “ Insect. d. vorwelt, 42 .

5033. Tropisternus saxialis. (Hydrophilidæ.)

Compared with T. binotatus Walk., Mexico.

Locality: Green River, Wyo. Horizon: Oligocene.

1878. Scudder.

Bull. U. S. geol, surv, terr., iv: 759-760.

1890. “ Tert. ins. N. A., 515. Pl. R, fig. 2.

5034. Tropisternus sculptilis. (Hydrophilidæ.)

Compared with T. mexicanus Castln.

Locality: Green River, Wyo. Horizon: Oligocene.

1878. Scudder. Bull. U. S. geol. surv, terr., iv: 760 .

1890. “ Tert. ins. N. A., 514-515.

5035. Trox oustaleti. (Scarabæidæ.)

Locality: Nine Mile Creek, British Columbia. Horizon:

1879. Scudder. Rep. progr. geol. surv. Can., 1877-'78: B 179-180.

1879. " "Insect. tert. Nicola, 4-5.

1890. " $\quad$ Tert. ins. N. A., 48\%. Pl. 2, fig. 22.

5036. Trypodendron impressum. (Scolytidæ.)

Compared with T. retusum.

Locality : Green River, Wyo. Horizon: Oligocene

1876. Scudder. Bull. U. S. geol. surv, terr., ii : 83 (impressus).

See also Dryocætes impressus.

5037. Tychius -. (Curculionidæ.)

Locality: Brunstatt, Alsatia. Horizon: Middle Oligocene.

1890. Foerster. In litt.

Bull. $71-38$ 
5038. Tychius manderstjernai. (Curculionidæ.)

Compared with T. sparsutus Oliv.

Locality: Rott, Rhenish Prussia. Horizon: Aquitanian.

1866. Heyd.-Heyd. Palaeontogr., xv: 152. Pl. 24, fig. 5.

1866. “ Käfer u. polypen, 22. $\quad P l .3, f i g .5$.

5039. Uloma avia. (Tenebrienidæ.)

Locality: Rott, Rhenish Prussia. Horizon : Aquitanian.

1862. Heyden. Palaeontogr., $\mathrm{x}: 70 . \quad \mathrm{Pl} .10, \mathrm{fig} .7$.

5040. Urodon - (Bruchidæ.)

Locality : Brunstatt, Alsatia. Horizon: Middle Oligocene.

1890. Foerster. In litt.

5041. Urodon priscus. (Bruchidæ.)

Locality : Rott, Rhenish Prussia. Horizon : Aquitanian.

1862. Heyden. Palaeontogr., $\mathrm{x}:$ 70. $P l .10$, fig. 17.

5042. Valgus cningensis. (Scarabæidæ.)

Compared with V. hemipterus (L.).

Locality: Oeningen, Baden. Horizon: Tortonian.

1862. Heer. Verhandl. holl, maatsch. wetensch, Haarl., xvi: 82-83. P’l. 6, figs. $12 a-a a$.

1865. " Urwelt der Schweiz, 380. Fig. 265 on $p .378$.

1872. " Monde prim. Suisse, 466. Fig. 265 on p. 463.

1876. " Prim. world Switz., ii: 36 . Fig. 265 on p. 34.

1879. “ Urwelt der Schweiz, 2e aufl., 405-406. Fig. 307 on p. 404.

5043. Xantholinus westwoodianus. (Staphylinidæ.)

Locality : Aix, France. Horizon : Ligurian.

1856. Heer. Viert. naturf. gesellsch. Zürich, i: 16-17. $P l$. 1, fig. 6. 1874. Oustalet. Ann. sc. g6ol., v, art.2: 151-152. Pl. 2, fig. 6.

See also Lathrobium — Curtis, etc.

5044. Xyletinites tumbicolus. (Ptinidæ.)

Locality : Rott, Rhenish Prussia. Horizon : Aquitanian.

1866. Heyd.-Heyd. Palaeontogr., xv : 144 (tumbicola). Pl. 22, fig. 21 .

1866. “ Käfer u. polypen, 14. Pl. 1, fig. 21.

Zonites vetusta. See Zonitis vetusta.

5045. Zonitis vetusta. (Meloidæ.)

Compared with Z. præusta, Southern Europe.

Locality: Oeningen, Baden. Horizon: Tortonian.

1865. Heer. Urwelt der Schweiz, 376 (Zonites).

1872. " Monde prim, Suisse, 462.

1876. " Prim, world Switz., ii : 33.

1879. “ Urwelt der Schweiz, $2^{e}$ aufl., 402. 


\section{DIPTERA.}

5046.

Locality : (Sinigaglia ?, Italy.) Horizon:

1841. Bassi.

Atti reun. scienz.ital, iii : 401.

5047.

Losality : Radoboj, Croatia. Horizon: Mayencian.

1831. Boué. Journ. géol., iii : 105, 143. $P l .2$, fig. 1.

5048.

Locality: Nantucket amber. Horizon:

1879. Goldsmith. Proc. acad. nat. sc. Philad., 1879: 207.

5049.

Locality : Wilhelmsfund, Nassau. Horizon: "Braunkohl."

1856. Heyden. Palaeontogr., iv: 201. Pl. 37, fig. 8.

5050.

Locality : Sinigaglia, Italy. Horizon: Helvetian ?.

1842. Procaccini. Nuov. ann. sc. nat., vii: 449.

\section{1.}

Localities : Bodensee u. Rheine, Germany. Horizon: Alluvium.

1851. Stizenberg. Uebers. verstein. Baden, 119.

\section{2.}

Locality: Gurnet Bay, Isle of Wight. Horizon: Bembridge Limestone. 1879. Woodward. Quart. journ. geol. soc. Lond., xxxv : 344.

$$
5053 .
$$

(Anthomyidæ.)

Locality : Prussian amber. Horizon: Ligurian.

1850. Loew. Bernstein fauna, 43.

5054.

\section{(Asilidæ.)}

Locality : British Columbia. Horizon :

1879. Scudder.

1879.

Rep. progr. geol. surv. Can., 1877-'78: B 186.

1890.

Ins. tert. beds Nicola, 11.

Tert. ins. N. A., 565 .

5055. (several). (Asilidæ.)

Locality : Florissant, Colo. Horizon: Oligocene

1881. Scudder.

1883. "

1890.
Bull. U. S. geol. surv. terr., vi : 291.

Ann. rep. U. S. geol. surv. terr., xii : 281.

Tert. ins. N. A., 29.
5056.

Locality : Italy?.
(Asilidæ.)

Horizon :

1796.

Ittiol. veron., i : 31 . 
5057. (Bibionidæ.)

Iocality: Aix, France. Horizon : Ligurian.

1829. Curtis. Edinb. new phil. journ., vii: 296. Pl. 6, fig. 10. 1829. “ Murchison-Lyell, Tert. form. Aix, 12. Pl. 6, fig. 10.

See also Protomyia elegans.

5058. — - (Bibionidæ.)

Locality : Corent, France. Horizon : Aquitanian.

1870. Oustalet. Ann. se. g6́ol., ii, art. $3: 152,1^{\text {er }}$ spec.

5059.

(Bibionidæ.)

Locality : Corent, France. Horizon: Aquitanian.

1870. Oustalet. Ann. sc. géol., ii, art. 3: 153, $2^{\circ}$ spec.

5060. (Bibionidæ.)

Locality: Authezat, France. Horizon : Aquitanian.

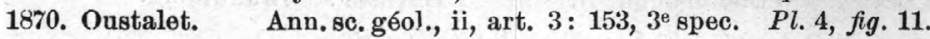

5061. (Bibionidæ.)

Locality: Corent, France. Horizon: Aquitanian.

1870. Oustalet Ann. sc. géol. , ii, art. 3: 153, $4^{\mathrm{e}}$ spec. $P l .3$, fig. 17.

5062. (Beveral). (Bibionidæ.)

Locality : Florissant, Colo. Horizon: Oligocene.

1881. Sendder. 1883. " " 1890. "

Bull. U. S. geol. surv. terr., vi : 291.

Ann. rep. U.S. geol. surv. terr. xii : 281

Tert. ins. N. A., 28-29.

5063. (several). (Bombylidæ.)

Locality: Florissant, Colo. Horizon: Oligocene.

18R1. Scudder. 1883. " "

Bull. U. S. geol. surv, terr., vi : 291.

Ann. rep. U. S. geol, surv, terr., xii : 281. 1890.

Tert. ins. N. A., 29.

5064.

(Chironomidæ.)

Locality : Quesnel, British Columbia. Horizon :

1877. Scudder. 1877. " 187\%. “
Rep. geol. surv. Can., 1875-'76: 270-271.

Ins, tert. Quesnel, 5-6.

Rapp. op6́r. comm. géol. Can., 1875-'76: 299.

5065.

(Chironomidæ.)

Locality: White River, Colo. Horizon: Oligocene.

1877. Scudder. 1890.
Bull. U. S. geol. surv. terr., iii : 745, lines 25-29.

Tert. ins. N. A., 581. Pl. 5, fig. 32, 33.
5066.

Locality: White River, Colo. Horizon: Oligocene.
1877. Scudder. 1890. "
Bull. U. S. geol. surv. terr., iii : 745, lines 30-33.

Tert. ins. N. A.. 581. Pl. 5, fig. 24. 
5067.

(Chironomidæ?.)

Locality: White River, Utah. Horizon: Oligocene.

1877. Scudder. 1890. " "

Locality : Florissant, Colo. Horizon: Oligocene.

1881. Scudder. 1883. “ 1890.

Bull. U. S. geol. surv. terr., vi : 291.

Ann. rep. U. S. geol. surv. terr., xii : 280.

Tert. ins. N. A., 28.

5069.

$$
\text { (Conopidæ-Myopina.) }
$$

Locality: Prussian amber. Horizon: Ligurian.

1850. Loew.

Bernstein fauna, 43.

5070.

(Culicidæ.)

Locality : Florissant, Colo. Horizon: Oligocene.

1881. Scudder.

1883. " "

1890.
Bull. U. S. geol. surv. terr., vi : 291.

Ann. rep. U.S. geol. surv. terr., xii : 280.

Tert. ins. N. A., 28.

\section{1. — - (Culicidæ.)}

Locality : Gurnet Bay, Isle of Wight. Horizon: Bembridge Limestone. 1879. Woodward. Geol. mag., n. s., v: 88.

5072. - (several). (Hermoneuridæ.)

1881. Scudder. Bull. U. S. geol. surv. terr., vi : 291.

1883. " “ Ann. rep. U. S. geol. surv. terr., xii : 281.

1890. " " Tert. ins. N. A., 29.

5073. - (several). (Midasidx.)

Locality: Florissant, Colo. Horizon: Oligocene.

1881. Scudder.

Bull. U. S. geol. surv. terr., vi : 291.

1883. " " Ann. rep. U.S. geol. surv. terr., xii: 281.

1890. " " Tert. ins. N. A., 29.

5074. —- (Muscidæ.)

Locality: Prussian amber. Horizon: Ligurian. 1835. Gravenhorst. Uebers. schles. gesellsch. vaterl. cult., 1834 : 92.

5075. —- (Muscidæ.)

Locality: Prussian amber. Horizon: Ligurian.

1850. Loew. Bernsteinfauna, 43.

5076. - - (Mnscidæ.)

Locality: Green River, Wyo. Horizon: Oligocene.

1870. Scudder. Hayden, Sun pictures, 98. 
5077. (many). (Muscidæ.)

Locklity: Florissant, Colo. Horizon: Oligocene.

1881. Scudder. 1883 . 1890.

Bull. U. S. geol. surv. terr., vi : 291.

Ann. rep. U. S. geol. surv. terr., xii : 281.

Tert. ins. N. A., 29.

5078.

\section{(Mycetophilidæ.)}

Locality : Sicilian amber. Horizon :

1838. Guérin. 1840. Rondani. 1840. Guérin.

\section{Rev. zool., 1838: 170 (Dipt. or Hymen.). $\quad$ Pl. 1, fig. 17.} Rev, zool., 1840 : 369 (Dipt.).

Rev. zool., 1840: 370 .

5079. (mine). (Mycetophilidæ.)

Locality: Rott, Rhenish Prussia. Horizon: Aquitanian.

1862. Heyden. Palaeontogr., $\mathrm{x}: 81$ (in Polyporus foliatus Ludw.). Pl. 10, fig. 3.

5080. (3 sp.). (Mycetophilidæ.)

Locality : Green River, Wyo. Horizon: Oligocene.

1878. Scudder. 1890. " "
Bull. U. S. geol. surv. terr., iv : 750-751.

Tert. ins. N. A., 599. Pl. 10, fig. 12.

5081. (Mycetophilidæ.)

Locality: Florissant, Colo. Horizon: Oligocene.

1881. Seudder. 1883. " " 1890.
Bull. U. S. geol. surv. terr., vi : 291.

Ann, rep. U. S. geol. surv. terr., xii : 281.

Tert. ins. N. A., 28. $\longrightarrow$ (Myopina). See

5082. (several). (Ortalidæ).
1881. Scudder. 1883. " 1890. “

Locality : Florissant, Colo. Horizon: Oligocene.

Bull. U. S. geol. surv. terr., vi : 291.

Ann. rep. U. S. geol. surv. terr., xii : 281.

Tert, ins. N. A., 29 .

5083. (several). (Stratiomyidæ.)

Locality: Florissant, Colo. Horizon: Oligocene.

1881. Scudder. 1883. " " 1890. “
Bull. U. S. geol. surv. terr., vi : 291.

Ann. rep. U. S. geol, surv. terr., xii : 281.

Tert. ins. N. A., 29.

\section{4.}

Locality: Prussian amber.

Berusteinfauna, 42-43.
(Syrphidæ.)

Horizon : Ligurian.

1850. Loew.

5085.

Locality: Green River, Wyo.
(Syrphirlæ.)

Horizon : Oligocene. 
5086. (several). (Syrphidæ.)

Locality : Florissant, Colo. Horizon: Oligocene.

1881. Scudder. - Bull. U. S. geol. surv. terr., vi: 291.

1883. “ "Ann. rep. U.S. geol. surv. terr., xii: 281.

1890. “. "Tert. ins. N. A., 29.

5087.

(Sy-phidæ.)

Locality : Green River, Wyo. Horizon: Oligocene.

1890. Șcudder. Tert. ins. N. A., 562. Pl. 10, fig. 9.

5088.

(Tabanidæ.)

Locality: Grone, Italy. Horizon: Quaternary.

1881. Malfatti.

1881. “ Atti soc. ital. sc. nat., xxiv, 90.

Bibl. ins. foss, ital., 2 .

5089. (several). (Therevidæ.)

Locality : Florissant, Colo. Horizon: Oligocene.

1881. Scudder.

1883.

1890.

Bull. U. S. geol. surv. terr., vi : 291.

Ann. rep. U. S. geol. surv. terr., xii : 281.

Tert. ins. N. A., 29.

5090.

(Tipulidæ.)

Locality : Radoboj, Croatia. Horizon: Mayencian.

1831. Bou6. Journ. géol., iii : 105, $143 . \quad P l$. 2, fig. 3.

\section{1. - - (Tipulidæ.)}

Locality : Prussian amber. Horizon: Ligurian.

1835. Gravenhorst. Uebers. schles. gesellsch. vaterl, cult., 1834: 92.

5092. - (several). (Tipulidæ.)

Locality: Florissant, Colo. Horizon: Oligocene.

1881. Seudder. Bull. U. S. geol. surv. terr., vi : 291.

1883. " $"$ Ann. Rep. U. S. geol, surv. terr., xii : 281.

1890. " Tert. ins. N. A., 28.

5093. — - (Tipulidæ.)

Locality: Gurnet Bay, Isle of Wight. Horizon : Bembridge Limestone. 1879. Woodward. Quart. journ. geol. soc. Lond., xxxv: 344.

1879. " Geol. mag. (n. s.), v: 88.

5094. Aclada - - (Mycetophilidæ.)

Locality: Prussian amber. Horizon: Ligurian.

1850. Loew. Bernsteinfauna, 35 (undescr.).

1856. Giebel. Insect. d. vorwelt, 238.

5095. Acrocera hirsuta. (Cyrtidæ.)

Locality: White River, Utah. Horizon: Oligocene.

1877. Scudder. Bull. U. S. geol. surv. terr., iii : 755.

1890. " Tert. ins. N. A., 563. Pl. 5, fig. 5 .

5096. Adetus - (Tipulidæ.)

Locality : Prussian amber. Horizon : Ligurian.

1845. Berendt. Bernst. befindl, organ. reste vorw., i: 57. 
5097. Imoaipus bornensis. (Tabanidæ.)

Locality: Le Puy, France. Horizon :

1854. Aymard. Congr. scient. France, sess. $22: 42$ (undescr.).

5098. Agromyza - (Agromyzidæ.)

Locality : Schossnitz, Silesia. Horizon: Miocene.

185ら. Goeppert. Tert. flora Schossnitz, vii (mine on Ulmus pyramidalis). $P l .26$, fig. 52.

5099. Agromyza protogæa. (Agromyzidæ.)

Locality : Radoboj, Croatia. Horizon: Mayencian.

1849. Heer. Insektenf. tertiärg. Oeningen, ii : 253-254. Pl. 17, fig. 22.

185\%. Giebel. Dentschl. petref., 643.

1856. " Insect. d. vorwelt, 196-197.

5100. Allarithmia palpata. (Tipulidæ.)

Locality: Prussian amber. Horizon: Ligurian.

1850. Loew. Bernsteinfauna, 38 (undescr.).

1856. Giebel. Insect. d. vorwelt, 247.

See also Eriocera palpata.

5101. Anaclinia? - (Mycetophilidæ.)

Locality: Green River, Wyo. Horizon: Oligocene.

1890. Scudder. Tert. ins. N. A., 597. Pl. 9, fig. 12.

See also Gnoriste (vic.) _ Scudder.

5102. Anatella tacita. (Mycetophilidæ.)

Locality: Green River, Wyo. Horizon: Oligocene.

1890. Scudder. Tert. ins. N. A., 589. Pl. 10, fig. 13.

5103. Anisomera —. (Tipulidæ.)

Locality : Prussian amber. Horizon : Ligurian.

1845. Berendt. Bernst. befindl. organ. reste vorw., i : 57.

5104. Anisomera succini. (Tipulidæ.)

Locality: Prussian amber. Horizon: Ligurian.

1850. Loew. Bernsteinfauna, 37 (undescr.).

1856. Giebel. Insect. d. vorwelt, 244.

See also Eriocera succini.

5105. Anisopus —. (Tipnlidæ.)

Compared with A. fuscus.

1829. Serres. Géogn. terrains tert., 231.

5106. Anthomyia -. (Anthomyidæ.)

Locality: Prussian amber. Horizon: Ligurian.

1831. Burmeister. Oken, Isis, 1831: 1100.

1832. " " Handb. ent., i: 636.

1836. “ Man. ent., 578. 


\section{Anthomyia -. (Anthomyidæ.)}

Locality: Brunstatt, Alsatia. Horizon: Middle Oligocene.

1890. Foerster. In litt.

5108. Anthomyia atavina. (Anthomyidæ.)

Locality: Radoboj, Croatia. Horizon: Mayencian.

1849. Heer. 1852. Giebel. 1856. “
Insektenf. tertiärg. Oeningen, ii : 248-249. Pl. 17, fig. 18.

Deutschl. petref., 643.

Insect. d. vorwelt, 197 (attavina).

Anthomyia attavina. See Anthomyia atavina.

5109. Anthomyia burgessi. (Anthomyidæ.)

Locality : Quesnel, British Columbia. Horizon: -

1877. Scudder. Rep. geol. surv. Can., 1875- $76: 274-275$.

1877. " Ins. tert. Quesnel, 9-10.

1877. “ Rapp. opér. comm. géol. Can., 1875-’76: 303-304.

1890. “ Tert. ins. N. A., 549-550. Pl. 3, fig. 34.

\section{Anthomyia heymanni. (Anthomyidæ.)}

Locality : Rott, Rhenish Prussia. Horizon: Aquitanian.

1870. L. Heyden. Foss. dipt. Rott, 27-28. Pl. 2, fig. 30.

1870. “ Palaeontogr., xvii: 263-264. Pl. 45, fig. 30.

5111. Anthomyia inanimata. (Anthomyidæ.)

Locality: Quesnel, British Columbia. Horizon: -

1877. Scudder. Rep. geol. surv. Can., 1875-'76: 273-274.

1877. " " Ins. tert. Quesnel, 8-9.

1877. “ Rapp. opér. comm. géol. Can., 1875-76: 302-303.

1890. “ Tert. ins. N. A., 548-549. Pl. 3, fig. 19.

5112. Anthomyia latipennis. (Anthomyidæ.)

Locality : Radoboj, Croatia. Horizon: Mayencian.

1849. Heer. Insektenf. tertiärg. Oeningen, ii : 249. $\quad P l .17, f i g .19$.

1852. Giebel. Deutschl. petref., 643.

1856. " Insect. d. vorwelt, 197.

\section{Anthomyia morio. (Anthomyidæ.)}

Locality : Radoboj, Croatia. Horizon: Mayencian.

1849. Heer.

1852. Giebel.

Insektenf. tertiärg. Oeningen, ii: 249-250. Pl.17, fig. 20.

1856. "

Deutschl. petref., 643.

Insect. d. vorwelt, 197.

5114. Anthracida xylotona. (Bombylidæ.)

Locality : Orsberg, (Rhenish Prussia?) Horizon: Miocene.

1849. Germar. Zeitschr. dentsch. geol. gesellsch., i: 64-65. Pl. 2, figs. 7, 7a.

1856. Giebel. Insect. d. vorwelt, 213-214.

1885. Scudder. Zittel, Handb. palaeont, i, th. ii: 808. Figs. $1075 a b$.

1886. " " Zittel-Barrois, Traité de paléont., ii : 809, Figs. 1092ab.

1886. " " Bull. U. S. geol. surv., No. 31: 87 


\section{Anthrax - (Bombylidæ.)}

Compared with A. semiatra.

Locality : Prussian amber. Horizon: Ligurian.

1832. Burmeister. Handb. ent., i: 636.

1836. " Man. ent., 578.

5116. Anthrax —. (Bombylidæ.)

Locality: Rhine, Germany. Horizon: Aquitanian?.

1831. Goldfuss. Verhandl. leop.-carol. deutschl. akad. naturf., vii, i: 118.

5117. Anthrax - (Bombylidæ.)

Locality: Oeningen, Baden. Horizon: Tortonian.

1834. Keferstein. Naturg. erdkörp., ii : 337.

5118. Apedilia - (Chironomidæ.)

Locality: Prussian amber. Horizon: Ligurian.

1850. Loew. Bernsteinfauna, 30.

5119. Aphritis -. (Syrphidæ.)

Compared with A. auro-pubescens.

Locality : Aix, France. Horizon: Ligurian.

1829. Serres. G6ogn. terrains tert., 233.

See also Microdon $\longrightarrow$ Burm.

5120. Arthropeas nana. (Acanthomeridæ.)

Compared with A. sibirica.

Locality: Prussian amber. Horizon: Ligurian.

1850. Loew. Berrsteinfauna, 40 (undeser.).

1856. Giebel. Insect. d. vorwelt, 214.

1861. Loew. Dipt. fauna bernsteins, 7.

1864. " Dipt. amber, 9 .

5121. Asarcomyia cadaver. (Stratiomyidæ.)

Locality: Green River, Wyo. Horizon: Oligocene.

1890. Scudder. Tert. ins. N. A., 567-568. Pl. 9, fig. 17.

\section{Asilus - - (Asilidæ.)}

Locality : Prussian amber. Horizon: Ligurian.

1845. Berendt. Bernst. befindl. organ. reste vorw., i: 57.

\section{Asilus —. (Asilidæ.)}

Locality: Rott, Rhenish Prussia. Horizon: Aquitanian.

1870. L. Heyden. Foss. dipt. Rott, 23, No.1.

1870. " Palaeontogr., xvii: 259.

5124. Asilus —. (Asilidæ.)

Locality: Aix, France. Horizon: Ligurian.

1829. Serres. G6́ogn. terrains tert., 232. 
5125. Asilus angustifrons. (Asilidæ.)

1850. Loew.

Locality: Prussian amber. Horizon: Ligurian.

1856. Giebel.

Bernsteinfauna, 40 (undescr.).

Insect. d. vorwelt, 212.

5126. Asilus antiquus. (Asilidæ.)

Locality: Oeningen; Baden. Horizon: Tortonian.

1849. Heer. 1852. Giebel. 1856. “6

1849. Heer. 1852. Giebel. 1856. " "

1849. Heer. 1852. Giebel. 1856. " "

1850. Loew. 1856. Giebel.

1850. Loew.

1850. Loew.

1845. Berendt. 1850. Loew.

1846. Giebel.
Insektenf. tertiärg. Oeningen, ii : 239-240. Pl. 17, fig. 7.

Deutschl. petref., 64\%.

Insect. d. vorwelt, 211-212.

\section{Asilus bicolor. (Asilidæ.)}

Locality: Radoboj, Croatia. Horizon: Mayencian.

Insektenf. tertiärg. Oeningen, ii : 241. $\quad P l .17, f i g .9$.

Deutschl. petref., 642 .

Insect. d. vorwelt, 212.

5128. Asilus deperditus. (Asilidæ.)

Locality : Oeningen, Baden. Horizon: Mayencian.

Insektenf. tertiärg. Oeningen, ii : 240. Pl. 17, fig. 8.

Deutschl. petref., 642.

Insect. d. vorwelt, 212.

5129. Asilus trichurus. (Asilidæ.)

Locality : Prussian amber. Horizon: Ligurian.

Bernsteinfauna, 40 (undescr.).

Insect. d. vorwelt, 212.

5130. Asynapta (3 sp.). (Cecidomyidæ.)

Locality : Prussian amber. Horizon: Ligurian. Bernsteinfauna, 32.

5131. Ataracta (8 sp.). ('Tipulidæ.)

Locality: Prussian amber. Horizon: Ligurian. Bernsteinfauna, 36, 38.

\section{Atherix -. (Leptidæ.)}

Locality : Prussian amber. Horizon: Ligurian.

Bernst. befindl. organ. reste vorw., i : 57.

Bernsteinfauna, 40 (4 sp.).

\section{Beris —. (Stratiomyidæ.)}

Locality :

$$
\text { Horizon: - }
$$

Paläozool., 27\%.

\section{Bibio —. (Bibionidæ.)}

Locality: Aix, France. Horizon : Ligurian.

1837. Buckland. Geology and mineral., ii : 78-79. Pl. 46", fig. 11.

1838. " Géologie, ed. Agassiz, ii : expl. pl. 46", pp. 5-6. Pl. 46", fig. 11. See also Protomyia bucklandi, etc. 
5135. Bibio? - (Bibionidæ.)

Locality: Prussian amber. Horizon: Ligurian.

1832. Burmeister. Handb. ent., i : 637.

1836. " Man. ent., 578.

5136. Bibio - (several). (Bibionidæ.)

Locality: Aix, France. Horizon: Ligurian.

1832. Burmeister. Handb. ent., i : 639.

1836. " $\quad$ Man. ent., 580.

See also Hirtea — Serres (3 sp.).

5137. Bibio -. (Bibionidæ.)

Locality: Gabbro, Italy. Horizon: Tripoli earth.

1878. Capellini. Atti accad. linc., (3), mem. sc. fis., ii : 285.

5138. Bibio —. (Bibionidæ.)

Compared with B, venosus Meig.

Locality : Aix, France. Horizon : Ligurian.

1829. Curtis. Edinb. new phil. journ., vii : 296.

1829. " Murchison-Lyell, Tert. form. Aix, 12.

5139. Bibio — ( 2 or 3 sp.). (Bibionidæ.)

Locality: Brunstatt, Alsatia. Horizon: Middle Oligocene.

1888. Foerster. Mitth. comm, geol. Elsass-Lothr., i: 164.

1889. " Mitth. comm. geol. Elsass-Lothr., ii : 103.

5140. Bibio - (Bibionidæ.)

Locality: Prussian amber. Horizon: Ligurian.

1742. Sendel. Historia succin., 44 (unnamed). Pl. 1, fig. 18.

1825. Guérin. Dict. class. d'hist. nat., viii : 580 (Bibion).

1829. Serres. Géogn. terrains tert., 242.

5141. Bibio alacris. (Bibionidæ.)

Locality : Corent, France, Horizon: Aquitanian.

1870. Oustalet. Ann. sc. géol., ii, art. 3: 127-128. Pl. 3, fig. 15.

5142. Bibio angustatus. (Bibionidæ.)

Locality: Oeningen, Baden. Horizon: Tortonian.

1849. Heer.

Iusektenf. tertiärg. Oeningen, ii : 215-216. Pl. 16, fig. 4.

1852. Giebel.

Deutschl. petref., 641 .

1856. “ Iusect. d. vorwelt, 217.

5143. Bibio antiquus. (Bibionidæ.)

Locality: Wilhelmsfund, Nassau. Horizon: "Braunkohl。"

1856. Heyden. Palaeontogr., iv: 201. Pl. 37, fig. 7.

5144. Bibio brevis. (Bibionidæ.)

Locality: Oeningen, Baden. Horizon : Mayencian.

1849. Heer.

1852. Giebel.

1856
Insektenf. tertiärg. Oeningen, ii : 225-226. Pl. 16, fig. 16.

Deutschl. petref., 641 .

Insect. d. vorwelt, 220-221. 
5145. Bibio curtisii. (Bibionidæ.)

Locality : Aix, France. Horizon: Ligurian.

1829. Curtis. 1829.

1856. Heer.

Edinb. new. phil. journ., vii : 296 (unnamed). Pl. 6, fig.12. Murchison-Lyell, Tert. form. Aix, 12 (unnamed). Pl. 6, fig. 12. Viert. naturf. gesellsch. Zürich, i: 34-35. Pl. 2, figs. 7, 14.

5146. Bibio cylindratus. (Bibionidæ.)

Locality : Corent, France. Horizon: Aquitanian.

1870. Oustalet. Ann. se. géol., ii, art. 3 : 133, 134. Pl. 4, fig. 12.

5147. Bibio deletus. (Bibionidæ.)

Locality : Rott, Rhenish Prussia. Horizon: Aquitanian.

1859. Herden. Palaeontogr., viii: 14. Pl. 2, fig. 13.

1865. Heyd.-Heyd. Palaeontogr., xiv : 29-30.

5148. Bibio dubius. (Bibionidæ.)

Locality: Vicinity Bonn, Rhenish Prussia. Horizon: Aquitanian.

1856. Giebel.

Insect. d. vorwelt, 221-222.

See also Phthiria dubia.

5149. Bibio edward̄sii. (Bibionidæ.)

Locality : Corent, France. Horizon: Aquitanian.

1870. Oustalet. Aun. se. géol., ii, art. 3: 130-133. Pl. 5, figs. 1-11.

1874. " La Nature, iii : 35. Fig. on p. 33.

See also Plecia edwardsii.

5150. Bibio elegantulus. (Bibionidæ.)

Locality: Krottensee, Bohemia. Horizon: Aquitanian.

1877. Novák. Sitzungsb. k. akad. wiss. Wien, lxxvi : 85-86. Pl. 1, fig. 5.

187\%. " Fauna egerer tertiärb., 15-16. Pl. 1, fig. 5.

5151. Bibio elongatus. (Bibionidæ.)

Locality: Oeningen, Baden. Horizon: Tortonian.

1849. Heer.

1852. Giebel.

Insektenf. tertiärg. Oeningen, ii : 214. $\quad P l$. 16, fig. 2.

Deutschl. petref., 641.

1856. " Insect. d. vorwelt, 216.

1865. Heer. Urwelt der Schweiz, 396. Fig. 311 on p. 394.

187\%. " Monde prim. Suisse, 485. Fig. 311 on $p .484$.

1876. " " Prim. world Switz., ii : 55. Fig. 311 on p. 54.

1879. "Urwelt der Schweiz, 2 aufl., 421 . Fig. 353 on p. 420.

5152. Bibio enterodelus. (Bibionidæ.)

Locality : Radoboj, Croatia. Horizon: Mayencian.

1841. Unger.

Verhandl, leop.-carol. deutschl. akad. naturf., xix: 428. Pl. 72, fig. 7.

1841. " "

1849. Heer.

1850 . "

Foss. insecten, 16. Pi. 72, fig. 7 .

Insektenf. tertiärg. Oeningen, ii : 222-223. Pl. 16, fig. 14.

Neue denkschr. schweiz. gesellsch. wiss., xi: 222-223. Pl. 16, fig. 14.

1852. Giebel. Deutschl. petref., 641.

1856. " Insect. d. vorwelt, 220 . 
5153. Bibio firmus. (Bibionidæ.)

Locality: Radoboj, Croatia. Horizon: Mayencian.

1849. Heer.

1852. Giebel.

1856. "

Insektenf. tertiärg. Oeningen, ii : 226-227. Pl. 16, fig. 17.

Deutschl. petref., 641.

Insect. d, vorwelt, 221.

5154. Bibio formosus. (Bibionidæ.)

Locality: Krottensee, Bohemia. Horizon: Aquitanian.

1877. Novák.

$187 \%$.
Sitzungsb. k. akad. wiss. Wien, lxxvi: 85. Pl. 1, fig. 4.

Fauna egerer tertiärb., $15 . \quad P l .1, f i g .4$.

5155. Bibio fusiformis. (Bibionidæ.)

Localities: Oeningen, Baden; Aix, France; Tك́llya, Hungary. Horizons: Mayencian, Ligurian.

1849. Heer. 1852. Giebel. 1856. " 1856. Heer. 1872. “
Insektenf. tertiärg. Oeningen, ii : 219. $\quad P l .16, f i g .9$.

Deutschl. petref., 641 .

Insect. d. vorwelt, 218-219.

Viert. naturf. gesellsch. Zürich, i : 33.

Mitth. jahrb. ung. geol. anst., ii, i : 8.

5156. Bibio giganteus. (Bibionidæ.)

Locality : Radoboj, Croatia. Horizon : Mayencian.

1841. Unger.

1841. “

1849. Heer.

1852. Giebel.

1856. "6

Verhandl. leop.-carol. deutschl. akad. naturf., xix : 427. Pl. 72, fig. 6.

Foss. insect., 15. $\quad \mathrm{Pl}$. 72, fig. 6.

Insektenf. tertiärg. Oeningen, ii : 212-213. $\quad P l .16$, fig. 1.

Dentschl. petref., 641 .

Insect. d. vorwelt, 216.

\section{Bibio gigas. (Bibionidæ.)}

Locality: Corent, France. Horizon: Aquitanian.

1870. Oustalet. $\quad$ Ann. sc.géol., ii, art. 3: 122-123. Pl. 1, fig8. 2-4.

5158. Bibio gracilis. (Bibionidæ.)

Locality: Radoboj, Croatia. Horizon: Mayencian.

1841. Unger.

Verhandl. leop.-carol. deutschl. akad. naturf., xix: 426. Pl.72, fig. 4.

1841. “

1849. Heer.

1850. “

1852. Giebel. Deutschl. petref., 641.

1856. " Insect. d. vorwelt, 218.

See also Bibio gracilis minor.

Foss. insect., 14. Pl. 72, fig. 4

Insektenf. tertiärg. Oeningen, ii : 217-218. $\quad P l$. 16, fig. 7.

Neue denkschr. schweiz. gesellsch. wiss., xi: 217-218. Pl. 16, fig. 7.

5159. Bibio gracilis minor. (Bibionidæ.)

Locality : Corent, France. Horizon: Aquitanian.

1870. Oustalet. Ann. sc. géol., ii, art. 3: 134-135. Pl. 3, fig. 16.

Seo also Bibio gracilis. 
5160. Bibio incrassatus. (Bibionidæ.)

1849. Heer. 1852. Giebel. 1856. " "

Locality : Radoboj, Croatia. Horizon: Mayencian.

Insektenf. tertiärg. Oeningen, ii : 221-222. $\quad P l .16, f i g .12$.

Deutschl. petref., 641.

Insect. d. vorwelt, 219.

5161. Bibio janus. (Bibionidæ.)

Locality: Rott, Rhenish Prussia. Horizon: Aquitanian.

1870. L. Heyden. Foss. dipt. Rott, 21-22. Pl. 2, fig. 25.

1870. “ Palaeontogr., xvii : 257-258. Pl. 45, fig. 25.

5162. Bibio kochii. (Bibionidæ.)

Locality: Felek, near Klausenburg, Transylvania. Horizon :

1883. Staub. Mitth. jahrb. k. ung. geol. anst., vi : 280-281. Pl. 18, fig. 13.

5163. Bibio lartetii. (Bibionidæ.)

Locality: Corent, France. Horizon: Aquitanian.

1870. Oustalet. Ann. sc. géol., ii, art. 3: 137-138. Pl. 4, fig. 10, 14.

See also Plecia larteti.

\section{Bibio lignarius. (Bibionidæ.)}

Compared with B. marci.

Localities: Rott, Rhenish Prussia; Radoboj, Croatia. Horizons: Aquitanian, Mayencian.

1837. Germar. Fauna insect. Europæ, xix : 23. Pl. 23.

1841. Unger. Verhandl. leop.-carol. deutschl. akad. naturf., xix: $42 \%$. Pl. 72, figs. $5 a b$.

1841. “ Foss. insect., 15. Pl. 72, figs.5ab.

1852. Giebel. Deutschl. petref., 641.

1856. " Insect. d. vorwelt, 218.

1859. Heyden. - Palaeontogr., viii: 14. Pl. 1, fig. 4.

Heer considers Unger's figures distinct and to belong to two species, referring one to his Bibio ungeri, the other to Protomyia lygæoides.

See also Protomyia lignaria, Bibio ungeri, Protomyia lygæoides, Plecia lygæoides.

5165. Bibio linearis. (Bibionidæ.)

Locality: Radoboj, Croatia. Horizon : Mayencian.

1849. Heer. Insektenf. tertiärg. Oeningen, ii : 214-215. Pl. 16, fig. 3.

1852. Giebel. Deutschl. petref., 641.

1853-'56. Bronn. Lethæa geogn., $3^{\text {e }}$ aufl, iii : 640. Pl. $42^{\prime}$, fig. 11 c.

1856. Giebel.

Insect. d. vorwelt, 217.

5166. Bibio lividus. (Bibionidæ.)

Locality : Radoboj, Croatia. Horizon : Mayencian.

1849. Heer. 1852. Giebel. 1856. “
Insektenf, tertiärg. Oeningen, ii : 223-224. Pl. 15, figs. $23 b, d$. Deutschl. petref., 641.

Insect. d. vorwelt, 220.

5167. Bibio macer. (Bibionidæ.)

Locality : Corent, France. Horizon: Aquitanian.

1870. Oustalet. Ann. sc. géol., ii, art. $3: 126-127 . \quad$ Pl. 4, fig. 6. 
5168. Bibio maculatus. (Bibionidæ.)

Locality : Radoboj, Croatia. Horizon: Mayencian.

1849. Heer.

1852. Giebel.

1856. “

Insektenf. tertiärg. Oeningen, ii : 219-220. $\mathrm{Tl} .16$, fig. 10.

Deutschl. petref., 641.

Insect. d. vorwelt, 219.

Bibio marginatus. See Bibio ungeri marginatus.

5169. Bibio martinsi. (Bibionidæ.)

Locality: Aix, France. Horizon : Ligurian.

1861. Heer. Saporta, Heer, rech. climat pays tert., 153 (undescr.).

5170. Bibio mimas. (Bibionidø.)

Locality : Rott, Rhenish Prussia. Horizon : Aquitanian.

1870. L. Heyden. Foss. dipt. Rott, 22. Pl. 2, fig. 26.

1870. “ Palaeontogr., xvii: 258. Pl. 45, fig. 26.

Bibio minor. See Bibio gracilis minor.

5171. Bibio mœestus. (Bibionidæ.)

Compared with B. pomonæ L.

Localities: Oeningen, Baden ; Aix, France. Horizons: Mayencian, Ligurian.

1849. Heer. Insektenf. tertiärg. Oeningen, ii: 224-225. Pl. 16, fig. 15.

1852. Giebel. Deutschl. petref., 641.

1856. " Insect. d. vorwelt, 220.

1856. Heer. Viert. naturf. gesellsch. Zürich, i : 33-34.

1865. " " Urwelt der Schweiz, 396.

1872. " Monde prim. Suisse, 485.

1876. " $\quad$ Prim. world Switz, ii: 55.

1879. " Urwelt der Schweiz, $2^{\mathrm{e}}$ aufl., 421.

5172. Bibio morio. (Bibionidæ.)

Localities: Radoboj, Croatia; Aix, France. Horizons: Mayencian, Ligurian.

1849. Heer. Insektenf. tertiärg. Oeningen, ii : 222. $\quad P l .16, f i g .13$.

1852. Giebel. Deutschl. petref., 641 .

1856. " Insect. d. vorwelt, 219-220.

1856. Heer. Viert. naturf. gesellsch. Zürich, i: 33.

5173. Bibio murchisonis. (Bibionidæ.)

Locality: Radoboj, Croatia. Horizon: Mayencian.

1841. Unger. Verbandl.leop.-carol. deutschl. akad.naturf., xix : 426. Pl. 71, fig. 3.

1841. “ Foss. insect., 14. Pl. 71, fig. 3.

See also Bibiopsis murchisenii, etc.

5174. Bibio oblongus. (Bibionidæ.)

Locality: Oeningen, Baden. Horizon: Tortonian.

1849. Heer. Insektenf. tertiärg. Oeningen, ii : 22\%. $\quad P l .16, f i g .18$.

1852. Giebel. Deutschl. petref., 641.

1856. " Insect. d. vorwelt, 221 . 
5175. Bibio obsoletus. (Bibionidæ.)

Localities: Oeningen, Baden; Corent, France. Horizons: Tortonian, Aquitanian.

1849. Heer.

1852. Giebel.

1856. “ “

1870. Oustalet.
Insektenf, tertiärg. Oeningen. ii : 227-228. $\quad P l .16, f i g .19$.

Deutschl. petref., 641.

Insect. d. vorwelt, 221.

Ann. sc. géol., ii, art. 3: 136. Pl. 4, fig. 13.

Bibio œningensis. See Bibio pınguis ceningensis.

5176. Bibio pannosus. (Bibionidæ.)

Compared with B. lividus Heer.

Locality : Rott, Rhenish Prussia. Horizon : Aquitanian.

1865. Heyd.-Heyd. Palaeontogr., xiv: 20. Pl. 8, fig. 1.

See also Bibio tertiarius.

5177. Bibio partschii. (Bibionidæ.)

Lncality : Radoboj, Croatia. Horizon: Mayencian.

1849. Heer. Insektenf. tertiärg. Oeningen, ii : 216. Pl. 16, fig. 5.

1852. Giebel. Deutschl. petref., 641.

1856. " Insect. d. vôrwelt, 217.

5178. Bibio pinguis. (Bibionidæ.)

Localities: Oeningen, Baden; Radoboj, Croatia. Horizons: Tortonian, Mayencian. 1849. Heer. Insektenf. tertiärg. Oeningen, ii : 220-221. Il. 16, figs. 11a-o. 1852. Giebel. Dentschl. petref., 641.

1856. " Insect. d. vorwelt, 219.

See also Bıbio pinguis œningensis, B. pinguis radobojanus.

5179. Bibio pinguis œningensis. (Bibionidæ.)

Locality : Oeningen, Baden. Horizon : Tortonian.

1849. Heer. Insektenf. tertiärg. Oeningen, ii : 221. Pl. 16, fig. $11 b$.

See also Bibio pinguis, etc.

5180. Bibio pinguis radobojanus. (Bibionidæ.)

Locality : Radoboj, Croatia. Horizon : Mayencian.

1849. Heer. Insektenf. tertiärg. Oeningen, ii : 220-221. Pl. 16, fig. 11a.

See also Bibio pinguis, etc.

5181. Bibio pulchellus. (Bibionidæ.)

Locality : Oeningen, Baden. Horizon: Tortonıan.

1849. Heer.

1852. Giebel.

Insektenf. tertiärg. Oeningen, ii: 217. Pl.16, fig.6.

1856. “

Deutschl. petref , 641.

Insect. d. vorwelt, 217.

Bibio radobojanus. See Bibio pinguis radobojanus.

5182. Bibio robustus. (Bibionidæ.)

Localities: Corent, Authezat, France. Horizon: Aquitanian.

1870. Oustalet. Ann. sc. géol., ii, art. 3; 128-129, Pl. 4, figs. 7-9.

Bull. $71-39$ 
5183. Bibio sereri. (Bibionidæ.)

Locality: Monte Bolca, Italy. Horizon: Ligurian.

1856. Massalongo. Stud. paleont., 21. Pl. 2, fig. 3, 4.

1886. Omboni. Atti r. ist. ven., (6), iv: 1427. Pl. 2, figs. 10-11.

1886. " Ins. foss. Ven., 7. Pl. 2, figs. 10-11.

5184. Bibio tertiarius. (Bibionidæ.)

Locality : Rott, Rhenish Prussia. Horizon: Aquitanian.

1862. Heyden. Palaeontogr., $\mathrm{x}:$ 78. Pl. 10, fig. 38.

1865. Heyd.-Heyd. Palaeontogr., xiv: 30.

See also Bibio pannosus.

5185. Bibio ungeri. (Bibionidæ.)

Localities: Radoboj, Croatia ; Côte Ladoux, France. Horizon : Mayencian. 1849. Heer. Insektenf. tertiärg. Oeningen, ii : 218-219. Pl. 16, fig. 8. 1852. Giebel. Deutschl. petref., 641. 1856. " Insect. $d$. vorwelt, 218.

1870. Oustalet. Ann. sc. géol. ii, art. 3: 123-124. Pl. 1, figs. $16 a b c$.

See also Bibio lignarius, etc., Bibio ungeri marginatus.

5186. Bibio ungeri marginatus. (Bibionidæ.)

Locality: Corent, France. Horizon: Aquitanian.

1870. Oustalet. Ann. sc. géol., ii, art. 3: 125-126. Pl. 3, fig. 14 ; pl. 4, fig. 5.

See also Bibio ungeri.

\section{Bibio xylophilus. (Bibionidæ.)}

Compared with B. marci.

Locality: Bonn, Rhenish Prussia. Horizon: Aquitanian.

1837. Germar. 1852. Geibel. 1856. “

Fauna insect. Europæ, xix : 22. $\quad P l .22$.

Deutschl. petref., 641 .

Insect. d. vorwelt, 218.

5188. Bibiopsis brevicollis. (Bibionidæ.)

Locality : Radoboj, Croatia. Horizon: Mayencian.

1849. Heer.

1852. Giebel. 1853-56. Bronn. 1856. Giebel.
Insektenf. tertiärg. Oeningen, ii : 231. $\quad$ Pl. 15, fig. 26.

Deutschl. petref., 642.

Lethæa geogn, $3^{\mathrm{e}}$ aufl, iii : $640 . \quad P l .42^{\prime}, f i g .11 b$.

Insect. d. vorwelt, 223 .

See also Penthetria brevicollis.

5189. Bibiopsis carbonum. (Bibionidæ.)

Locality: Salzhausen, Germany. Horizon: Miocene 1865. Heyd.-Heyd. Palaeontogr., xiv: 34-35. Pl. 9, fig. 21.

5190. Bibiopsis cimicoides. (Bibionidæ.)

Locality: Radoboj, Croatia. Horizon: Mayencian.

1849. Heer. 1852. Giebel. 1856. "
Insektenf. tertiärg. Oeningen, ii : 229, $\quad P l_{0}$ 15, fig. 24.

Deutschl. petref., 641 .

Insect. $d$. vorwelt, $22 \%$.

See also Penthetria cimicoides. 
5191. Bibiopsis egerana. (Bibionidæ.)

Locality: Krottensee, Bohemia. Horizon: Aquitanian.

1877. Novák. Sitzungsb. k. akad. wiss. Wien, lxxvi: 83. Pl. 1, fig. 3.

1877. “ Fauna egerer tertiärb., 13. Pl. 1, fig. 3.

5192. Bibiopsis funebris. (Bibionidæ.)

Locality: Aix, France. Horizon: Ligurian.

1861. Heer. Saporta, Heer, Rech. climat pays tert., 153 (undescr.).

5193. Bibiopsis imperialis. (Bibionidæ.)

Locality : Krottensee, Bohemia. Horizon: Aquitanian.

1877. Novák. Sitzungsb. k. akad. wiss. Wien, lxxvi : 84. Pl. 2, fig. 3.

1877. "6 Fauna egerer tertiärb., 14. Pl. 2, fig. 3.

5194. Bibiopsis murchisonii. (Bibionidæ.)

Locality : Radoboj, Croatia. Horizon: Mayencian.

1849. Heer. Insektenf. tertiärg. Oeningen, ii : 230-231. Pl. 15, fig. 25.

1852. Giebel. Leutschl. petref., 642.

1853-'56. Bronn. Lethæa géogn., $3^{\mathrm{e}}$ anfl, iii : 640. Pl. $42^{\prime}$, fig. $11 a$.

1856. Giebel. Insect. d. vorwelt, 2222-223.

See also Bibio murchisonis, Penthetria murchisoni.

5195. Bibiopsis volgeri. (Bibionidæ.)

Locality: Rott, Rhenish Prussia. Horizon: Aquitanian.

1859. Heyden. Palaeontogr., viii: 15. Pl. 1, fig. 5.

See also Protomyia volgeri.

5196. Bolbomyia -. (Xylophagidæ.)

Locality: Prussian amber. Horizon: Ligurian.

1850. Loew. Bernsteinfauna, 39 (undescr.).

1856. Giebel. Insect. d. vorwelt, 204 (undescr.).

1862. Loew. Monogr. diptera N. A., i: 17 (undeser.).

5197. Boletina —. (Mycetophilidæ.)

Locality: Brunstatt, Alsatia. Horizon: Middle Oligocene.

1890. Foerster. In litt.

5198. Boletina paludivaga. (Mycetophilidæ.)

Locality : Green River, Wyo. Horizon: Oligocene.

1890. Scudder. Tert. ins. N. A., 594. Pl. 10, fig. 7.

5199. Boletina philhydra. (Mycetophilidæ.)

Locality: Rott, Rhenish Prussia. Horizon: Aquitanian.

1870. L. Heyden. Foss. dipt. Rott, 10. Pl. 1, fig. 11.

1870. " Palaeontogr., xvii: 246. Pl. 44, fig. 11.

5200. Boletina sepulta. (Mycetophilidæ.)

Locality: Quesnel, British Columbia. Horizon:

1877. Scudder. Rep. geol. surv. Can., 1875-'76: 271.

1877. “ Ins. tert. Quesnel, 6.

1877. " Rapp. opér. comm. géol. Can., 1875-'76: 299-300.

1890. “ Tert. ins. N. A., 593. Pl. 3, fig. 9. 
5201. Boletina umbratica. (Mycetophilidæ.)

Locality: Green River, Wyo. Horizon: Oligocene.

1890. Scudder. Tert. ins. N. A., 593-594. Pl. 10, fig. 3.

5202. Boletophila ——. (Mycetophilidæ.)

Locality: Prussian amber. Horizon: Ligurian.

1832. Burmeister. Handb. ent., i : 637.

1836. " Man. ent, 578 .

Bombylis —. See Bombylins _ _ Sehlotheim.

5203. Bombylius ——. (Bombylidæ.)

Locality: Prussian amber. Horizon: Ligurian.

1830. Berendt. Insekten im bernstein, 30 .

$$
\text { 5204. Bombylius ——. Bombylidæ.) }
$$

Locality : Oeningen, Baden. Horizon: Tortonian.

1820. Schlotheim. Petrefactenkunde, 43 (Bombylis).

5205. Brachypeza abita. (Mycetophilidæ.)

Locality : Quesnel, British Columbia. Horizon:

1877. Scudder. Rep. geol. surv. Can., 1875-'76 : 271-272.

1877. " Insect. tert. Quesnel, 6-7.

1877. “ Rapp. opér. comm. géol. Can., 1875-76: 300.

1890. “ Tert. ins. N. A., 591. Pl. 3, figs. 7, 8.

5206. Brachypeza procera. (Mycetophilidæ.)

Locality: Quesnel, British Cólumbia. Horizon:

1877. Scudder. Rep. geol. surv. Can., 1875-'76: 272.

1877. “ Insect. tert. Quesnel, 7.

1877. “ Rapp. opér. comm. géol. Can., 1875-76: 300-301.

1890. “ Tert. ins. N. A., 591-592. Pl. 3, fig. 14.

5207. Brachystoma —. (Empidæ.)

Locality: Prussian amber. Horizon: Ligurian.

1845. Berendt. Bernst. befindl. organ. reste vorw., i : 57.

5208. Brachystoma spinulosa. (Empidæ.)

Compared with B. vesciculosa.

Locality: Prussian amber. Horizon : Ligurian.

1850. Loew.

Bernsteinfauna, 41 (undescr.).

1856. Giebel.

Insect. d. vorwelt, 209.

5209. Callomyia torporata. (Platypezidæ.)

Locality: Green River, Wyo. Horizon: Oligocene.

1890. Scudder. Tert. ins. N. A., 555. Pl. 9, fig. 11.

5210. Calobamon —. (Tipulidæ.)

Locality : Prussian amber. Horizon: Ligurian.

1850. Loew.

Ternsteinfauna, $36,38$. 


\section{Calobata -. (Mieropezidæ.)}

Locality : Prussian amber. Horizon : Ligurian.

1850. Loew. Bernsteinfauna, 44.

5212. Campylomyza - (Cecidomyidæ.)

Locality: Prussian amber. Horizon: Ligurian.

1845. Berendt. Bernst. befindl. organ. reste vorw., i : 57.

5213. Campylomyza (vic.) - - (Cecidomyidæ.)

Locality: Prussian amber. Horizon: Ligurian.

1870. Giebel. Zeitschr. ges. naturwiss., (2), i: 87 .

5214. Campylomyza monilifera. (Cecidomyidæ.)

Locality : Prussian amber. Horizon: Ligurian.

1850. Loew.

Bernsteinfauna, 32.

Insect. d. vor welt, 248.

5215. Cecidomyia —- (Cecidomyidæ.)

Locality : Prussian amber. Horizon: Ligurian.

1845. Berendt. Bernst. befindl, organ. reste vorw., i: 57.

5216. Cecidomyia - (Cecidomyidæ.)

Locality: Prussian amber. Horizon : Ligurian.

1831. Burmeister. Oken, Isis, 1831: 1100.

1832. " "Handb. ent., i: 637.

1836. " Man. ent, 578.

5217. Ceciōomyia - $(2$ sp. $)$. (Cecidomyidæ.)

Locality : Sicilian amber. Horizon : -

1838. Guérin. Rev. zool., 1838: 170.

5218. Cecidomyia bremii. (Cecidomyidæ.)

Compared with C. salicis.

Locality: Oeningen, Baden. Horizon: Tortonian.

1865. Heer. Urwelt der Schweiz. Fig. 322 on $p .394$.

1872. " Monde prim. Suisse. Fig. 322 on p. 484.

1876. " " Prim. world Switz, ii. Fig. 322 on $p .54$.

1879. " Urwelt der Schweiz, $2^{\mathrm{e}}$ anfl. Fig. 364 on p. 420.

5219. Cecidomyia? dubia (gall). (Cecidomyidæ.)

Locality : Rott, Rhenish Prussia. Horizon: Aquitanian.

1862. Heyden. Palaeontogr., $\mathrm{x}$ : 80-81 (on leaf of Juglans acuminata Braun). Pl. 10, fig. 4.

5220. Cecidomyia protogæa. (Cecidomyidæ.)

Locality: Aix, France. Horizon : Ligurian.

1856. Heer. Viert. naturf. gesellsch. Zürich, i: 32-33. Pl. 2, fig. 4.

5221. Ceratopogon —_ (Chironomidæ.)

Locality: Prussian amber. Horizon: Ligurian.

1845. Berendt. Bernst. befiudl. organ. reste vorw., i : 57.

1850. Loew. Bernsteinfauna, 30 (26 sp.). 


\section{Ceratopogon ㄴ. (Chironomidæ.)}

Locality : Prussian amber. Horizon: Ligurian.

1831. Burmeister. Okên, Isis, 1831: 1100.

1832. " Handb. ent., i: 637.

1836. “ Man. ent., 578.

\section{Ceratopogon -. (Chironomidæ.)}

Locality : Prussian amber. Horizon: Ligurian.

1841. Ehrenberg. Froriep, Neue notizen, xix : 120.

\section{Ceratopogon -. (Chironomidæ.)}

Locality : Sicilian amber. Horizon : -

1840. Rondani. Rev. zool., $1840: 369$.

1840. Erichson. Arch. f. naturg., (5), ii : 309.

1840. " Ber. wiss. leist. entom., 1838: 29.

See alsu Dasypogon — Guérin.

\section{Ceratopogon -. (Chironomidæ.)}

Locality : Aix, France. Horizon : Ligurian.

1829. Serres. Géogn. terrains tert., 231.

5226. Ceratopogon alpheus. (Chironomidæ.)

Locality: Rott, Rhenish Prussia. Horizon : Aquitanian.

1870. L. Heyden. Foss. dipt. Rott, 15-16. Pl. 1, fig. 20.

1870. “ Palaeontogr., xvii: 251-252. Pl. 44, fig. 20.

5227. Ceratopogon anomalicornis. (Chironomidæ.)

1850. Loew.

Locality: Prussian amber. Horizon: Ligurian.

1856. Giebel.

Bernsteinfauna, 30.

Insect. d. vorwelt, 252.

5228. Ceratopogon clunipes. (Chironomidæ.)

1850. Loew.

Locality: Prussian amber. Horizon: Ligurian.

1856. Giebel.

Bernsteinfauna, 30.

Insect. d. vorwelt, 252.

\section{Ceratopogon escheri. (Chironomidæ.)}

Locality : Prussian amber. Horizon : Ligurian.

1856. Giebel. Insect. d. vorwelt, 252 .

5230. Ceratopogon spiniger. (Chironomidæ.)

1850. Lnew.

Locality : Prussian amber. Horizon: Ligurian.

1856. Giebel.

Bernsteinfauna, 30.

Insect. d. vorwelt, 252.

Cheilosia —. See Chilosia — Scudder, 1878.

Cheilosia ampla. See Chilosia ampla. 
5231. Chilosia -. (Syrphidæ.)

Locality: Green River, Wyo. Horizon: Oligocene.

1878. Scudder. Bull. U. S. geol. surv. terr., iv: 754 (Cheilosia).

1890. " Tert. ins. N. A., 561. Pl. 9, fig. 8 .

5232. Chilosia? - (Syrphidæ.)

Locality: Green River, Wyo. Horizon: Oligocene.

1890. Scudder. Tert. ins. N. A., 561. Pl. 9, fig. 26.

5233. Chilosia —- (Syrphidæ.)

Locality : Florissant, Colo. Horizon: Oligocene.

1886. Williston. Syn. N. A. Syrph., 282.

5234. Chilosia ampla. (Syrphidæ.)

Locality: Green River, Wyo. Horizon: Oligocene.

1878. Scudder. Bull. U. S. geol. surv. terr., iv: 753-754 (Cheilosia).

1886. Williston.. Syn. N. A. Syrph., 281, 283.

1890. Scudder. Tert. ins. N. A., 559-560. Pl. 9, figs. 14, 27.

5235. Chironomus —. (Chironomidæ.)

Locality : Prussian amber. Horizon : Ligurian.

1845. Berendt. Bernst. befindl. organ. reste vorw., i : 57.

1850. Loew. Bernsteinfauna, 29 (40 sp.).

5236. Chironomus —. (Chironomidæ.)

Locality : Prussian amber. Horizon : Ligurian.

1832. Burmeister. Handb. ent., i: 637.

1836. " Man. ent., 578.

5237. Chironomus - (pupa). (Chironomidæ.)

Locality : Rott, Rhenish Prussia. Horizon : Aquitanian.

1870. L. Heyden. Foss. dipt. Rott, 13-14,30 (No. 6). Pl. 1, fig. 17. 1870. " Palaeontogr., xvii : 249-250, 266. Pl. 44, fig. 17.

5238. Chironomus - (pupa). (Chironomidæ.)

Locality : Rott, Rhenish Prussia. Horizon: Aquitanian. 1870. L. Heyden. Foss. dipt. Rott, 14, 30 (No. 7). Pl. 1, fig. 18.

1870. “ Palaeontogr., xvii: 250, 266. Pl. 44, fig. 18.

5239. Chironomus - (pupa). (Chironomidæ.)

Locality : Rott, Rhenish Prussia. Horizon: Aquitanian.

1870. L. Heyden. Foss. dipt. Rott, 14, 30 (No. 8).

1870. " Palaeontogr., xvii : 250, 266.

5240. Chironomus - (pupa). (Chironomidæ.)

Locality: Rott, Rhenish Prussia. Horizon: Aquitanian.

1870. L. Heyden. Foss. dipt. Rott, 15 (No. 9). Pl. 1, fig. 19.

1870. " Palaeontogr., xvii : 251. Pl. 44, fig. 19. 
5241. Chironomus - (pupa.) (Chironomidæ.)

Locality : Rott, Rhenish Prussia. Horizon : Aquitanian. 1876. L. Hey den. Foss. dipt. Rott, 15 (No. 10). 1870.

Palaeontogr., xvii : 251.

5242. Chironomus (pupa). (Chironomidæ.)

Locality: Rott, Rhenish Prussia. Horizon: Aquitanian. 1870. L. Heyden. Foss. dipt. Rott, 15 (No. 11). 1870.

Palaeontogr., xvii : 251.

\section{Chironomus -. (Chironomidæ.)}

Locality : Green River, Wyo. Horizon: Oligocene.

1878. Seudder. Bull. U. S. geol. surv, terr., iv: 749.

1890. " Tert. ins. N. A., 581.

5244. Chironomus antiquus. (Chironomidæ.)

Locality : Rott, Rhenish Prussia. Horizon: Aquitanian. 1859. Heyden. Palaeontogr., viii : 13. Pl. 2, fig. 10.

5245. Chironomus bituminosus. (Chironomidæ.)

Locality: Rott, Rhenish Prussia. Horizon : Aquitanian. 1870. L. Heyden. Foss. dipt. Rott, 11. Pl. 1, fig. 12.

1870. " Palaeontogr., xvii: 247. Pl. 44, fig. 12.

5246. Chironomus brevirostris. (Chironomidæ.)

Locality : Prussian amber. Horizon : Ligurian.

1856. Giebel. Insect. d. vorwelt, 251.

5247. Chironomus decrepitus. (Chironomidæ.)

Locality : Rott, Rhenish Prussia. Horizon: Aquitanian.

1870. L. Heyden. Foss. dipt. Rott, 12. Pl. 1, fig. 15.

1870. " Palaeontogr., xvii: 248. Pl. 44, fig. 15.

\section{Chironomus depletus. (Chironomidæ.)}

Locality : White River, Colo. Horizon: Oligocene.

1877. Scudder. Bull. U. S. geol. surv. terr., iii : 744.

1890. “ Tert. ins. N. A., 579-580. Pl. 5, fig. 62.

5249. Chironomus dorminans. (Chironomidæ.)

Locality: Rott, Rhenish Prussia. Horizon: Aquitanian.

1870. L. Heyden. Foss. dipt. Rott, 12. Pl. 1, fig. 14.

1870. “ Palaeontogr., xvii: 248. Pl. 44, fig. 14.

\section{Chironomus gaudini. (Chironomidæ.)}

Locality: Oeningen, Baden. Horizon: Tortonian.

1865. Heer. Urwelt der Schweiz, 395. Fig. 316 on p. 394.

1872. " Monde prim. Suisse, 483. Fig. 316 on p. 484.

1876. " Prim. world Switz, ii : 53. Fig. 316 on p. 54.

1879. “ Urwelt der Schweiz, 2e aufl, 420. Fig. 358. 
5251. Chironomus meyeri. (Chironomidæ.)

Locality : Prnssian amber. Horizon : Ligurian.

1849. Heer.

1850 . “

1852. Giebel.

1856. " "

1885. Scudder.

1886. " "

5252. Chironomus microcephalus. (Chironomidæ.)

Locality : Prussian amber. Horizon: Ligurian.

1856. Giebel.
Insektenf. tertiärg. Oeningen, ii: 188-189. Pl. 14, fig. 13.

Nene denkschr. schweiz. gesellsch. wiss., xi : 188-189. Pl. 14, fig. 13.

Deutschl. petref., 640 .

Insect. d. vorwelt, 250-251.

Zittel, Handb. palaeont., i, th. ii : 810. Figs. 1083, 1084.

Zittel-Barrois, Traité de paiéont., ii : 8:1. Figs. 1100, 1101.
1849. Heer.

1850. " "

1852. Giebel.

1856. “

1849. Heer.

1850. “

1852. Giebel.

1856. " "

5253. Chironomus obsoletus. (Chironomidæ.)

Lọcality : Oeningen, Baden. Horizon: Tortonian.

Insektenf, tertiärg. Oeningen, ii : 190. $P l .14$, fig. 15.

Nene denkschr. schweiz. gesellsch. wiss., xi : 190. Pl. 14, fig. 15.

Deutschl. petref. 640 .

Insect. d. vorwelt, 250 .

\section{Chironomus cningensis. (Chironomidæ.)}

Locality : Oeningen, Baden. Horizon: Tortonian.

Insektenf. tertiärg. Oeningen, ii : 189-190. Pl. 14, fig. 14.

Nene denkschr. schweiz. gesellsch. wiss., xi: 189-190. Pl. 14, fig. 14.

Deutschl. petref., 640 .

Insect. d. vorwelt, 250 .

5255. Chironomus palæmon. (Chironomidæ.)

Locality : Rott, Rhenish Prussia. Horizon : Aquitanian.

1870. L. Heyden. Foss. dipt. Rott, 13. Pl. 1, fig. 16.

1870. “ Palaeontogr., xvii: 249. Pl. 44, fig. 16.

\section{Chironomus patens. (Chironomidæ.)}

Locality: White River, Colo. Horizon : Oligocene.

1877. Scudder. Bull. U. S. geol. surv. terr., iii : 744-745.

1890. " Tert. ins. N. A., 580-581. Pl. 5, figs. 18, 19, 28.

\section{Chironomus perditus. (Chironomidæ.)}

Locality: Rott, Rhenish Prussia. Horizon: Aquitanian.

1870. L. Heyden. Foss. dipt. Rott, 11-12. Pl. 1, fig. 13.

1870. " Palaeontogr., xvii : 247-248. Pl. 44, fig. 13.

5258. Chironomus septus. (Chironomidæ.)

Locality : Green River, Wyo. Horizon: Oligocene.

1890. Scudder. Tert. ins. N. A., 578-579. Pl. 10, fig. 8. 
5259. Chironomus sepultus. (Chironomidæ.)

Locality: Radoboj, Croatia. Horizon: Mayencian.

1849. Heer.

1850. “

1852. Giebel.

1856. “

1850. Loew.

Insektenf. tertiärg. Oeningen, ii : 190-191. Pl. 11, fig. 14; pl. 14, fig. 16.

Neue denkschr. schweiz. gesellsch. wiss., xi : 190-191. Pl. 11, fig. $14 ; p l .14$, fig. 16.

Deutschl. petref., 640 .

Insect. d. vorwelt, 250.

\section{Chlorops —. (Oscinidæ.)}

Locality: Prussian amber. Horizon: Ligurian.

\section{Bernsteinfauna, 43.}

5261. Chrysogaster —. (Syrphidæ.)

Locality : Florissant, Colo. Horizon : Oligocene.

1886. Williston. Syn. N. A. Syrphidw, 281.

5262. Chrysothemis - - (Xylophagidæ.)

Locality : Prussian amber. Horizon: Ligurian.

1845. Berendt. Bernst. befindl. organ. reste vorw., i: 57.

5263. Chrysothemis speciosa. (Xylophagidæ.)

Locality : Prussian amber. Horizon : Ligurian.

1850. Loew.

1856. Giebel.

Bernsteinfauna, 39 (undeser.).

Insect. d. vorwelt, 226 (undeser.).

5264. Chrysotus —. (Dolichopodidæ.)

Locality: Prussian amber. Horizon: Ligurian.

1845. Berendt.

1850. Loew.

Bernst. befindl. organ. reste vorw., i : 57.

Bernsteinfauna, $4 \%$.

\section{Chrysotus —. (Dolichopodidæ.)}

Locality: Prussian amber. Horizon: Ligurian.

1870. Giebel. Zeitschr. ges, naturw., (2), i: 87 .

5266. Chrysotus setosus. (Dolichopodidæ.)

1856. Giebel. Insect. d. vorwelt, 205.

Locality: Prussian amber. Horizon: Ligurian.

\section{Conops - (Conopidæ.)}

Locality: Copal. Horizon: Recent.

1776. Bloch. Beschäft. berl.ges. naturf. freunde, ii: 178. Pl. 5, fig.19.

5268. Cordyla antiqua. (Mycetophilidæ.)

Locality: Rott, Rhenish Prussia. Horizon: Aquitanian.

1870. L. Heyden. Foss. dipt. Rott, 8-9. Pl. 1, fig. 9.

1870. “ Palaeontogr., xvii : 244-245. Pl. 44, fig.9. 
5269. Cordyla limnoria. (Mycetophilitææ.)

Locality : Rott, Rhenish Prussia. Horizon: Aquitanian.

1870. L. Heyden. Foss. dipt. Rott, 9-10. Pl.1, fig. 10.

1870. " $\quad$ Palaeontogr., xvii : 245-246. Pl. 44, fig. 10.

5270. Cordyla renuda. (Mycetophilidæ.)

Locality: Rott, Rhenish Prussia. Horizon: Aquitanian.

1870. L. Heyden. Foss. dipt. Rott, 9. Pl. 1, fig.9.

1870. “ Palaeontogr., xvii : 245. Pl. 44, fig.9.

5271. Cordyla subaptera. (Mycetophilidæ.)

Locality: Rott, Rhenish Prussia. Horizon: Aquitanian.

1870. L. Heyden. Foss. dipt. Rott, 8. Pl. 1, fig.8.

1870. " Palaeontogr., xvii: 244. Pl.44, fig.8.

5272. Cordyla vetusta. (Mycetophilidæ.)

Locality : Rott, Rhenish Prussia. Horizon: Aquitanian.

1870. L. Heyden. Foss. dipt. Rott, 7-8. Pl.1, fig.7.

1870. “ Palaeontogr., xvii : 243-244. Pl.44, fig.7.

5273. Cordylura vetusta. (Cordyluridæ.)

1849. Heer. Insektenf. tertiärg. Oeningen, ii: 250. Pl.17, fig. 21.

1852. Giebel. Deutschl. petref., 643.

1856. “ Insect. d. vorwelt, 195.

5274. Corethra - (Culicidæ.)

Locality: Aix, France. Horizon: Oligocene.

1847. Hope. Trans. ent. soc. Lond., iv: 252.

5275. Corethra —. (Culicidæ.)

Locality: Aix, France. Horizon: Ligurian.

1829. Serres. Géogn. terrains tert., 268.

5276. Corethra exita. (Culicidæ.)

Locality : White River, Colo. Horizon : Oligocene.

1877. Scudder. U. S. bull. geol. surv. terr., iii : 744.

1890. “ Tert. ins. N. A., 583. Pl. 5, figs. 22, 23.

5277. Corsomyza crassirostris. (Bombylidæ.)

1850. Loew.

Locality: Prussian amber. Horizon: Ligurian.

1856. Giebel.

1861. Loew.

1869. "

Bernsteinfauna, 40 (undeser.).

Insect. d. vorwelt, 213 (undescr.).

Dipt. faun. bernst., 6.

Dipt. amber, 7.

\section{Criorhina - See Criorrhina -}

5278. Criorrhina -. (Syrphidæ.)

Locality: Prussian amber. Horizon: Ligurian.

1870. Giebel. Zeitschr. ges. naturw., (2), i : 87 (Criorhina). 
5279. Critoneura longipes. (Tipulidæ.)

Locality : Prussian amber. Horizon: Ligurian.

1850. Loew. 1856. Giebel.

Bernsteinfauna, 38 (undescr.).

Insect. d. vorwelt, 246 (undeser.).

5280. Critoneura pentagonalis. (Tipulidæ.)

Locality: Prussian amber. Horizon: Ligurian.

1850. Loew.

Bernsteinfauna, 38 (undeser.).

1856. Giebel. Insect. $d$. vorwelt, 247 (undeser.).

$$
\text { 5281. Ctenophora? — (Tipulidæ.) }
$$

Locality: Brunstatt, Alsatia. Horizon: Middle Oligocene.

1889. Foerster. Mitth. comm. geol. Elsass-Lothr., ii : 103.

5282. Ctenophora decheni. (Tipulidæ.)

Locality: Rott, Rhenish Prussia. Horizon: Aquitanian.

1859. Heyden. Palaeontogr., viii : 13-14. Pl. 2, figs. 7, 8.

\section{Culex ㄴ. (Culicidæ.)}

Locality: Brunstatt, Alsatia. Horizon: Middle Oligocene.

1899. Foerster. In litt.

\section{Culex —. (Culicidæ.)}

Locality : Rott, Rhenish Prussia. Horizon: Aquitanian.

1870. L. Heyden. Foss. dipt. Rott, 16 (No. 2).

1870. " Palaeontogr., xvii: 252.

\section{Culex —. (Culicidæ.)}

Locality: Prussian amber. Horizon: Ligurian.

1845. Motschulsky. Bull. soc. imp. nat. Mosc., xviii, ii : 98.

\section{Culex —. (Culicidæ.)}

Locality: Prussian amber. Horizon: Ligurian.

18\%0. Schlotheim. Petrefactenk., 43.

\section{Culex ceyx. (Culicidæ.)}

Locality: Rott, Rhenish Prussia. Horizon: Aquitanian.

1870. L. Heyden. Foss. dipt. Rott, 16, 30. Pl. 1, fig. 21.

1870. " Palaeontogr., xvii : 252, 266. Pl. 14, fig. 21.

1885. Scudder. Zittel, Handb. palaeont, i, th. ii : 810. Fig. 1085.

1886. “ Zittel-Barrois, Traité de paléont., ii : 812. Fig. 1102.

5288. Culex ciliaris. (Culicidæ.)

Locality: Copal. Horizon: Recent.

1776. Bloch. Beschäft. berl, ges. naturf, freunde, ii : 164-165. Pl. 3, fig. 1.

5289. Culex damnatorum. (Culicidæ.)

Locality: Green River, Wyo.' Horizon: Oligocene.

1890. Scudder. Tert. ins. N. A., 582. Pl. 10, fig. 14. 
5290. Culex loewi. (Culicidæ.)

1862. Giebel.

Locality: Prussian amber. Horizon: Ligurian.

Zeitschr. ges. naturw., xx : 317-318.

5291. Culex proavitus. (Culicidæ.)

Locality : White River, Utah. Horizon : Oligocene.

1877. Scudder. Bull. U. S. geol. surv. terr, iii: 744.

1890. “ Tert. ins. N. A., 582-583. Pl. 5, figs. 8, 9.

5292. Culicites tertiarius. (Culicidæ.)

Locality : Rott, Rhenish Prussia. Horizon: Aquitanian.

1862. Heyden. Palaeontogr., $\mathrm{x}:$ 79-80. Pl. 10, figs. 30-35.

5293. Curtisimyia eximia. (Stratiomyidæ.)

Locality : Aix, France. Horizon: Ligurian.

1829. Curtis. Edinb. new phil. journ., vii: 296. Pl. 6, fig. 12. 1829. " Murch.-Lyell, Tert. form. Aix, 12. Pl. 6, fig. 12. 1856. Giebel. Insect. d. vorwelt, 203.

5294. Cylindrotoma -. (Tipnlidæ.)

Locality: Prussian amber. Horizon: Ligurian.

1845. Berendt. Bernst. befindl. organ. reste vorw., i: 57.

5295. Cylindrotoma brevicornis. (Tipulidæ.)

Locality: Prussian amber. Horizon: Ligurian.

1850. Loew. Bernsteinfauna, 37 (undescr.).

1856. Giebel.

Insect. d. vorwelt, 243 (undescr.).

5296. Cylindrotoma longicornis. (Tipulidæ.)

Locality: Prussian amber. Horizon: Ligurian.

1850. Loew.

1856. Giebel.

Bernsteinfauna, 37 (undeser.).

Insect. d. vorwelt, 243 (undescr.).

5297. Cylindrotoma longipes. (Tipulidæ.)

Locality: Prussian amber. Horizon: Ligurian.

1850. Loew.

Bernsteinfauna, 37 (undescr.).

1856. Giebel.

Insect, d. vorwelt, 243 (undescr.).

5298. Cylindrotoma succini. (Tipulidæ.)

Locality : Prussian amber. Horizon: Ligurian.

1850. Loew.

Bernsteinfauna, 37 (undeser.).

1856. Giebel.

Insect. d. vorwelt, 243 (undeser.).

5299. Cyttaromyia fenestrata. (Tipulidæ.)

Locality : White River, Utah. Horizon: Oligocene.

1877. Scudder.

Bull. U. S. geol. surv. terr., iii : 751-752.

1890. " "

Tert. ins. N. A., 575-576. Pl.5, fig. 78.

$$
\text { 5300. Dasypogon —. (Asilidæ.) }
$$

Locality : Prussian amber. Horizon: Ligurian.

1845. Berendt. Berust. befindl, organ. reste vorw, i : 57. 
5301. Dasypogon -. (Asilidæ.)

Locality : Sicilian amber. Horizon: - .

1838. Gú́rin. Rev. zool., 1838: 170. Pl. 1, figs. 15-16.

See also Ceratopogon $\longrightarrow$ Rondani.

5302. Dasypogon (Holopogon) pilipes. (Asilidæ.)

1850. Loew.

1856. Giebel.

Locality: Prussian amber. Horizon: Ligurian.

\section{Bernsteinfauna, 40 (undescr.).}

Insect. d. vorwelt, 212 (undescr.).

\section{Diadocidia parallela. (Mycetophilidæ.)}

Compared with D. flavicans.

Locality: Prussian amber. Horizon: Ligurian.

1850. Loew.

Bernsteinfauna, 35 (undescr.).

1856. Giebel.

Insect. d. vorwelt, 238 (undeser.).

5304. Diadocidia? terricola. (Mycetophilidæ.)

Locality: Green River, Wyo. Horizon: Oligocene.

1878. Scudder. 1890. "6

Bull. U. S. geol. surv. terr., iv : 750 .

Tert. ins. N. A., 598-599. Pl. 10, figs. 10, 11.

\section{Dianepsia - (2 sp.). (Mycetophilidæ.)}

Locality: Prussian amber. Horizon : Ligurian.

1850. Loew.

Bernsteinfauna, 34.

\section{Dicæra -. (Tipulidæ.)}

Locality: Wigtonshire, Scotland. Horizon: Pleistocene.

1888. Bell.

Entom., xxi : 2.

\section{Dichaneurum infossum. (Tipulidø.)}

Locality : LePuy, France. Horizon :

1854. Aymard. Congr. scient. France, sess. xxii : 42 (undescr.).

5308. Dichaneurum primævum. (Tipulidæ.)

Loeality : LePuy, France. Horizon :

1854. Aymard. Congr. scient. France, sess. xxii : 42 (undeser.).

5309. Dicranomyia primitiva. (Tipulidæ.)

Compared with D. pubipennis.

Localities: White River, Utah ; Green River, Wyo. Horizon : Oligocene.

1877. Scudder. Bull. U. S. geol. surv. terr., iii : 748.

1878. " " Bull. U. S. geol. surv, terr., iv: 749.

1890. “ Tert. ins. N. A., 570-571. Pl. 5, figs. 20, 21, 65-67.

5310. Dicranomyia rostrata. (Tipulidæ.)

Locality: White River, Utah. Horizon: Oligocene.

1877. Scudder. Bull. U. S. geol. surv. terr., iii : 749.

1890. " ' Tert, ins. N. A., 571-572. Pl. 5, figs. 40, 41, 63, 64. 
5311. Dicranomyia stigmosa. (Tipulidæ.)

Localities: White River, Utah and Colorado. Horizon: Oligocene.

1877. Seudder.

Bull. U. S. geol. surv. terr., iii : 746-748.

1890. " Tert. ins. N. A., 568-570. Pl. 5, figs. 16, 17, 25-27, 42, 43, 68, 69.

5312. Dilophus —_. (Bibionidæ.)

Locality : Prussian amber. Horizon: Ligurian.

1845. Berendt. Bernst. betindl. organ. reste vorw., i: 57.

5313. Dilophus - (Bibionidæ.)

Compared with D. marginatus.

Locality: Aix, France. Horizon: Ligurian.

1829. Serres. Géogn. terrains tert., 232.

5314. Dilophus krantzii. (Bibionidæ.)

Locality : Rott, Rhenish Prussia. Horizon: Aquitanian.

1870. L. Heyden. Foss. dipt. Rott, 19-21. Pl. 2, fig. 24.

1870. " Palaeontogr., xvii : 255-257. Pl.45, fig. 24.

5315. Dilophus priscus. (Bibionidæ.)

Locality : Prussian amber. Horizon: Ligurian.

1850. Loew. Bernsteinfauna, 38.

1856. Giebel. . Insect. d. vorwelt, 228.

\section{Diplonema -. (Psychodidæ.)}

Locality: Prussian amber. Horizon: Ligurian.

1845. Berendt. Bernst. befindl. organ. reste vorw., i : 57.

5317. Diplonema brevicornis. (Psychodidæ.)

Locality: Prussian amber. Horizon : Ligurian.

1850. Loew.

Bernsteinfauna, 31 (undeser.).

1856. Giebel. Insect. d. vorwelt, 249.

5318. Diplonema longicornis. (Psychodidø.)

Compared with D. buceras, Copal.

Locality : Prussian amber. Horizon: Ligurian.

1850. Loew.

Bernsteinfauna, 31 (undescr.).

Insect. d. vorwelt, 249 (undescr.).

Dipt. fauna bernstein, 8 .

1861. Loew.

1861. " "

1869. “

Ber. versamml. dentsch. naturf., xxxv : 93.

Amber diptera, 11.

5319. Diplosis —. (Cecidomyidæ.)

Locality : Prussian amber. Horizon: Ligurian.

1850. Loew.

Bernsteinfauna, 32 .

5320. Dipterites angelinii.

Locality: Monte Bolca, Italy. Horizon : Ligurian.

1855. Massalongo. Nereide foss., 31 ("Comp. faun. flor. foss. bolcensis. Pl. 6, figs. $2-3$, , ined.).

1856. " " Stud. palaeont., 20. Pl. 2, figs. 1-2.

1885. Scuddor. Zittel, Handb, palaeont., i, th. ii : 806. 
1886. Scudder.

1886. "6

1886. Omboni.

1886. Omboni. 1886. “

1865. Heer.

1872. " "

1876. " "

1879. “

18:5. Scudder.

1886. "6

1886. "
1886. "

Zittel-Barrois, Traité de paléont., ii : 807.

Bull. U. S. geol. surv., No. 31. 87.

Alc. ins, foss. Ven., 6-7. $\quad P l .2$, figs. 8-9.

Atti r. ist. ven., (6), iv : 1426-1427. Pl. 2, figs. 8-9.

\section{Dipterites catulloi.}

Locality : Cniavon, Italy. Horizon:

Alc. ins. foss. Ven., 8-9. Pl. 3, fig. 13.

Atti r. ist. ven., (6), iv : 1428-1429. $\quad$ Pl. 3, fig. 13.

5322. Dipterites obovatus. (Estridæ?.)

Locality: Oeningen, Baden. Horizon: Tortonian.

Urwelt der Schweiz. Fig. 323 on $p .394$.

Monde prim. Suisse. Fig. 323 on $p .484$.

Prim. world Switz, ii. Fig. 323 on p. 54.

Urwelt der Schweiz, $2^{\mathrm{e}}$ aufl. Fig. 365 on $p .420$.

Zittel, Handb. palaeont, i, th. ii : 806. Fig. 1071.

Zittel-Barrois, Traité de paléont., ii : 807. Fig. 1088.

Bull. U. S. geol. surv., No. 31 : 82 .

5323. Dipterites obsoletus. (Cordyluridæ?.)

Locality: Radoboj, Croatia. Horizon: Mayencian.

1849. Heer.

Insektenf. tertiärg. Oeningen, ii : 254-255 (obsoleta). Pl. 1\%, fig. 23.

1852. Giebel.

1886. Scudder.

Deutschl. petref., 643 .

Bull. U. S. geol. surv., No. 31 : 87.

5324. Diorhiza

(Cecidomyidæ.)

Locality: Prussian amber. Horizon: Ligurian.

1850. Loew.

Bernsteinfauna, 32 .

5325. Dixa - (Tipulidæ.)

Locality: Prussian amber. Horizon: Ligurian.

1845. Berendt.

1850. Loew.

Bernst. befindl. organ, reste vorw., i : 57.

Bernsteinfauna, 37 (4 sp.).

5326. Dolichopus -. (Dolichopodidæ.)

Locality: Prussian anber. Horizon: Ligurian.

1832. Burmeister. Handb. eut., i: 637.

1836. " Man. ent., 578.

5327. Dolichopus —. (Dolichopodidæ.)

Locality : Brunstatt, Alsatia. Horizon: Middle Oligocene.

1890. Foerster. In litt.

5328. Dolichopus ? - (larva). (Dolichopodidæ.)

Locality: Sieblos, Rhenish Prussia. Horizon: Aquitanian.

1858. Heyden. Palaeontogr., v: 120.

5329. Dolichopus - - (Dolichopodidæ.)

Locality: Prussian amber. Horizon: Ligurian.

1850. Loew,

Bernsteinfauna, 42 . 
5330. Dolichopus? (Dolichopodidæ.)

Locality: Quesnel, British Columbia. Horizon:

1877. Scudder. Rep. geol. surv. Can., 1875-'76: 272-273.

1877. " Ins. tert. Quesnel, 7-8.

1877. " Rapp. opér. comm. géol. Can., 1875-'76: 301.

\section{Dolichopus -. (Dolichopodidæ.)}

Locality: Green River, Wyo. Horizon: Oligocene.

1878. Scudder. Bull. U. S. geol. surv, terr., iv: 756.

1890. " Tert. ins. N. A., 562.

\section{Drapetis - (Empidæ.)}

Locality: Prussian amber. Horizon: Ligurian.

1850. Loew. Bernsteinfauna, 42 .

5333. Drosophila -. (Drosophilidæ.,

1850. Loew. Bernsteinfauna, 43.

Locality: Prussian amber. Horizon: Ligurian.

5334. Echinomyia —-. (Tachinidæ.)

Locality : Prussian amber. Horizon: Ligurian.

1868. Smith. Quart. journ. sc., v: 183. Pl., fig. 2.

5335. Echinomyia antiqua. (Tachinidæ.)

Compared with E. echinata Meig.

Locality: Oeningen, Baden. Horizon: Tortonian.

1849. Heer.

1852. Giebel.

1856. “

1865. Heer.

1872. "

1876. " "

1879. “

1850. Loew. 1856. Giebel.

Insektenf. tertiärg. Oeningen, ii : 247-248. Pl.17, fig.17.

Deutschl. petref., 642 .

Insect. d. vorwelt, 198.

Urwelt der Schweiz, 396.

Monde prim. Suisse, 486.

Prim. world Switz., ii: 55.

Urwelt der Schweiz, 2 aufl., 422.

5336. Electra -. (Xylophagidæ.)

Locality: Prussian amber. Horizon: Ligurian.

1845. Berendt. Bernst. befindl. organ. reste vorw., i: 57.

5337. Electra formosa. (Xylophagidæ.)

Locality: Prussian amber. Horizon: Ligurian.

Bernsteinfauna, 39 (undescr.).

Insect. d. vorwelt, 226 (undescr.).

Elephantomyia brevipalpa. See Toxorhina brevipalpa.

Elephantomyia longirostris. See Toxorhina longirostris.

Elephantomyia pulchella. See Toxorhina pulchella.

5338. Empis -. (Empidæ.)

Locality : Prussian amber. Horizon: Ligurian.

1845. Berendt. Bernst. befindl, organ, reste, vorw., i: 57.

1850. Loew. Bernsteinfauna, 41 (16 sp.).

Bull. $71-40$ 
5339. Empis - (Empidæ.)

Locality : Prussian amber. Horizon : Ligurian.

1831. Burmeister. Oken, Isis, 1831: 2000 [1100].

5340. Empis -. (Empidæ.)

Locality : Aix, France. Horizon : Ligurian.

1829. Curtis. Edinh. new thil. journ., vii : 296. $P l, 6, f i g .11$. 1829. " Murch.-Lyell, Tert. form. Aix, 12. Pl. 6, fig. 11.

1856. Giebel. Insect. d, vorwelt, 208.

See also Protomyia livida.

\section{Empis - (Empidø.)}

Locality: Brunstatt, Alsatia. Horizon: Middle Oligocene.

1890. Foerster. In litt.

5342. Empis —. (Empidæ.)

Locality : Prussian amber. Horizon: Ligurian.

1820. Schlotheim. Petrefactenk., 43.

5343. Empis - - (Empidx.)

Locality: Prussian amber. Horizon: Ligurian.

1742. Sendel. Hist. succ., 44-45 (unnamed). Pl. 1 , fig. 19.

1825. Guérin. Dict. class. d'hist, nat., viii : 580 .

5344. Empis —_. (Empidæ.)

Compared with E. tessellata.

Locality: Aix, France. Horizon: Ligurian.

1829. Serres. G6́ogn. terrains tert., 232.

5345. Empis carbonum. (Empidæ.)

Compared with E. morio.

Locality : Baireuth, Bavaria. Horizon: Miocene?.

1837. Germar. Fauna insect. Europæ, xix: 21. Pl. 21.

1840. - Verz. samml. Bayr., 71.

1852. Giebel. Deutschl. petref., 642.

1856. " Insect. d. vorwelt, 207.

5346. Empis melia. (Empidæ.)

Locality : Rott, Rhenish Prussia. Horizon: Aquitanian.

1870. L. Heyden. Foss. dipt. Rott, 23-24. Pl. 2, fig. 27.

1-70. " Palaeontogr., xvii: 259-260. Pl. 45, fig. 27.

1885. Scudder. Zittel, Handb. palaeont., i, th. ii: 807. Fig. 1074.

1886. " Zittel-Barrois, Traité de paléont., ii : 808. Fig. 1091.

5347. Empis poeppigi. (Empiđ̄æ.)

Locality : Prussian amber. Horizon : Ligurian.

1856. Giebel. Insect. d. vorwelt, $20 \%$.

5348. Empis pulvillata. (Empidæ.)

Locality : Prussian amber. Horizon: Ligurian.

1850. Loew.

Bernsteinfauna, 41.

1856. Giebel.

Insect. d. vorwelt, 208. 
5349. Ephydra —. (Ephydridæ.)

Locality: Prussian amber. Horizon: Ligurian.

1850. Loew.

Bernsteinfauna, 43.

5350. Epicypta - (2 sp.). (Mycetophilidæ.)

Locality: Brunstatt, Alsatia. Horizon : Middle Oligocene. 1890. Foerster. In litt.

5351. Epidosis incompleta. (Cecidomyidæ.)

Locality: Prussian amber. Horizon: Ligurian.

1850. Loew.

Bernsteinfanna, 32.

1856. Giebel.

Insect. d. vorwelt, 247.

5352. Epidosis minutissima. (Cecidomyidæ.)

Locality : Prussian amber. Horizon: Ligurian.

1850. Loew. Bernsteinfauna, 32.

1856. Giebel. Insect. d. vorwelt, 24\%.

5353. Epiplecia joannis. (Bibionidæ.)

Locality: Corent, France. Horizon: Aquitanian.

1878. Giard. Bull. scient. dép. nord, (2), i : 13-16.

See also Protomyia joannis, etc.

5354. Friocera palpata. (Tipulidæ.)

Locality: Prussian amber. Horizon: Ligurian.

1869. Osten Sacken. Monogr. dipt. N. A., iv : 251.

See also Allarithmia palpata.

\section{Eriocera succini. (Tipulidæ.)}

Lncality: Prussian amber. Horizon: Ligurian.

1869. Osten Sacken. Monogr. dipt. N. A., iv : 252.

See also Anisomera succini.

5356. Erioptera - (14 sp.). (Tipulidæ.)

1850. Loew. Bernsteinfauna, 37.

Locality: Prussian amber. Horizon: Ligurian.

5357. Erioptera danæ. (Tipulidæ.)

Locality: Rott, Rhenish Prussia. Horizon: Aquitanian.

1870. L. Heyden. Foss. dipt. Rott, 16-17. Pl. 2, fig. 22.

1870. " Palaeontogr., xvii : 252-253. Pl. 45, fig. 22.

5358. Friphia setosa. (Anthomyidæ.)

Locality: Prussian amber. Horizon: Ligurian.

1862. Giebel. Zeitschr. ges. naturw., xx : 319 ,

5359. Eristalis lapideus. (Syrphidæ.)

Locality : White River, Colo. Horizon: Oligocene.

1877. Scudder. Bull. U. S. geol, surv. terr., iii : 756.

1886. Williston. Syn. N. A. Syrphidæ, 281, 283 (probably not Eristalis).

1kgo. Scudder. Tert. ins. N. A., 558-559. Pl. 5, figs. 48, 49. 


\section{Eupedilia —. (Chironomidæ.)}

Locality: Prussian amber. Horizon: Ligurian.

1850. Loew. Bernsteinfauna, 30 .

5361. Glaphyroptera - (3 sp.). (Mycetophilidæ.)

Locality: Brunstatt, Alsatia. Horizon: Middle Oligocene.

1890. Foerster. In litt.

5362. Gloma -- (Empidæ.)

Locality: Prussian amber. Horizon: Ligurian.

1845. Berendt. Bernst. befindl. organ, reste vorw., i: 57.

1850. Loew.

1850. Loew.

1856. Giebel.

Bernsteinfauna, 41 (3 sp.).

\section{Gloma acuticornis. (Empidæ.)}

Locality: Prussian amber. Horizon: Ligurian.

Bernsteinfauna, 41.

Insect. $d$. vorwelt, 208.

5364. Gloma hirta. (Empidæ.)

Compared with G. fuscipennis.

Locality: Prussian amber. Horizon: Ligurian.

1850. Loew. Bernsteinfauna, 41.

1856. Giebel. Insect. d. vorwelt, 208.

5365. Gloma palpata. (Empidæ.)

1850. Loew.

Locality : Prussian amber. Horizon: Ligurian.

1856. Giẹbel.

\section{Bernsteinfauna, 41.}

Insect. d. vorwelt, 208.

5366. Gnoriste —. (Mycetophilidæ.)

Locality: Aix, France. Horizon: Ligurian.

1829. Curtis. Edinb. new phil. journ., vii: 296. Pl. 6, fig. 8. 1829. “ Murchison-Lyell, Tert. form. Aix, 12. Pl. 6, fig. 8. See also Mycetophila meigeniana, M. crassa.

5367. Gnoriste (vic.) -. (Mycetophilidæ.)

Locality: Green River, Wyo. Horizon: Oligocene. 1890. Scudder. Tert. ins. N. A., 597. Pl. 9, fig. 12.

See also Anaclinia? -

5368. Gnoriste dentoni. (Mycetophilidæ.)

Compared with G. megarhina O. S.

Locality : White River, Utah. Horizon: Oligocene.

1877. Scudder. Bull. U. S. geol. surv, terr., iii : 755.

1890. " Tert. ins. N. A., 592-593. Pl. 5, figs: 6, 7.

5369. Habrosoma antiqua. (Xylophagidæ.)

Locality : Prussian axber. Horizon: Ligurian.

1850. Loew.

Bernsteinfauna, 39.

1856. Giebel. Insect. d. vorwelt, 204. 


\section{Haploneura - (4 sp.). (Tipulidæ.)}

1850. Loew.

Locality : Prussian amber. Horizon: Ligurian.

Bernsteinfauna, 36, 38.

5371. Helomyza - - (Helomyzida.)

Locality : Prussian amber. Horizon: Ligurian.

1850. Loew. Bernsteinfauna, 43.

5372. Helophilus primarius. (Syrphidæ.)

Locality: Vicinity Bonn, Rhenish Prussia. Horizon: Aquitanian.

1837. Germar. Fauna insect. Europæ, xix: 25. $\quad$ Pl. 25.

1852. Giebel. Deutschl. petref., 642.

1856. " Insect. d. vorwelt, 201-202.

5373. Hemerodromia —. (Empidæ.)

Locality : Prussian amber. Horizon: Ligurian.

1831. Burmeister. Oken, Isis, 1831: 1100.

\section{Hemerodromia - (3 sp.). (Empidæ.)}

Locality: Prussian amber. Horizon : Ligurian.

1850. Loew. Bernsteinfauna, 41.

5375. Heteromyza detecta. (Helomyzidæ.) .

Locality: White River, Colo. Horizon: Oligocene.

1877. Scudder. Bull. U. S. geol. surv. terr., iii : 758-759.

1890. “ Tert. ins. N. A., 548. Pl. 5, fig. 76.

5376. Heteromyza senilis. (Helomyzidæ.)

-Locality : Quesnel, British Columbia. Horizon: -

1877. Scudder. Rep. geol. surv. Can., 1875-76: 275.

1877. " Ins. tert. Quesnel, 10.

1877. “ Rapp. oper. comm. géol. Can., 1875-76: 304-305.

1890. “ Tert. ins. N. A., 547. Pl. 3, figs. 1, 2.

5377. Heterotricha hirta. (Mycetophilidæ.)

1850. Loew.

Locality: Prussian amber. Horizon: Ligurian.

1856. Giebel. Insect. d. vorwelt, 232.

5378. Hexatoma? œningensis. (Tabanidæ.)

Locality: Oeningen, Baden. Horizon: Tortonian.

1865. Heer.

1872. “

1876. "

1879. "

1885. Scudder.

1886. " "

1856. Heer.
Urwelt der Schweiz, 396. Fig. 321 on p. 394.

Monde prim. Suisse, 486. Fig. 321 on p. 484.

Prim. world Switz., ii : 55. Fig. 321 on p. 54.

Urwelt der Schweiz, $2^{\mathrm{e}}$ aufl, 422. Fig. 363 on p. 420.

Zittel, Handb. palaeont., i, th. ii : 808. Fig. 1078.

Zittel-Barrois, Traité de paléont., ii : 809. Fig. 1095.

5379. Hilarites bellus. (Empidæ.)

Locality: Aix, France. Horizon: Ligurian.

Viert. naturf. gesellsch. Zürich, i : 38. Pl. 2, fig. 5 . 
5380. Hirtea - (Bibionidæ.)

Compared with $\mathrm{H}$. funebris.

Locality: Aix, France. Horizon: Ligurian.

1829. Serres. Géogn. terrains tert., 231.

See also Bibio — Burm., 1: 639.

\section{Hirtea - (Bibionidæ.)}

Compared with $\mathrm{H}$. johannis.

Locality : Aix, France. Horizon: Ligurian.

1829. Serres. Géogn. terrains tert., 231.

See also Bibio —— Burm, 1: 639 .

\section{Hirtea —. (Bibionidæ.)}

Compared with $\mathrm{H}$. febrilis.

Locality: Aix, France. Horizon: Ligurian.

1829. Serres. Géogn. terrains tert., 232.

See also Bibio — Burm., 1: 639 .

Holopogon pilipes. See Dasypogon pilipes.

5383. Hybos ㄴ. (Empidæ.)

Locality : Prussian amber. Horizon: Ligurian.

1845. Berendt. Bernst. befindl. organ. reste vorw., i: 57.

1850. Loew. Bernsteinfauna, $41(2 \mathrm{sp}$.).

5384. Lasioptera —- (Cecidomyidæ.)

Locality : Prussian amber. Horizon: Ligurian.

1832. Burmeister. Handb. ent., i: 637 .

1836. “ Man. ent, 578.

5385. Lasioptera recessa. (Cecidomyidæ.)

Localities: White River, Colorado and Utah. Horizon: Oligocene.

1877. Scudder. 1890.
Bull. U. S. geol. surv. terr., iii: 745-746.

Tert, ins. N. A., 600. Pl. 5, figs. 29-31.

\section{Leia - See Leja $\longrightarrow$.}

5386. Leja -. (Mycetophilidæ.)

Locality: Prussian amber. Horizon: Ligurian.

1832. Burmeister. Handb. ent., i : 637 (Leia).

1836. “ Man. ent., 578 (Leia).

5387. Leja frequens. (Mycetophilidæ.)

Locality : Prussian amber. Horizon: Ligurian.

1850. Loew.

1856. Giebel.

Bernsteinfauna, 34 (undeser.).

Insect. $d$. vorwelt, 232 (undescr.).

5388. Leja interrupta. (Mycetophilidæ.)

Locality: Prussian amber. Horizon: Ligurian.

1850. Loew. Bernsteinfauna, 34 .

1856. Giebel. Insect. d, vorwelt, 232. 
5389. Leja platypus. (Mycetophilidæ.)

1850. Loew.

Locality: Prussian amber. Horizon: Ligurian.

1856. Giebel.

Bernsteinfauna, 34 .

Insect. d. vorwelt, 232 .

\section{Leptis —. (Leptidæ.)}

Locality : Prussian amber. Horizon: Ligurian.

1845. Berendt.

Bernst. befindl, organ, reste vorw., i: 57.

1850. Loew:

Bernsteinfauna, 40 (5 sp.).

5391. Leptis

\section{(Leptidæ:)}

Compared with L: aurata.

Locality: Prussian amber. Horizon: Ligurian.

1832. Burmeister. Handb. ent.; i : 636.

1836. " Man, ent., 578.

5392. Leptis -. (Leptidæ.)

Locality: Prussian amber. Horizon: Ligurian.

1868. Smith. Quart. journ. sc., v : 183, Pl., fig. 1.

5393. Leptogaster hellii. (Asilidæ.)

Locality : Radoboj, Croatia. Horizon: Mayencian.

1841. Ünger.

1841. "؛

Verhandl. leop.-carol. akad, naturf,, xix : 428, Pl. 72, fig. 8.

Foss. insecten, $16 . \quad P l .72, f i g .8$.

Insektenf. tertiärg. Oeningen, ii : 241-242. $\quad P l .17$, fig. 10.

1849. Heer.

Neue denkschr. schweiz. gesellsch. wiss., xi : 241-242. Pl. 17, fig. 10.

1852. Giebel. Deutschl. petref., 642.

1856. “ Insect, d. vorwelt, 212-213.

1885. Scudder. Zittel, Handb، palaeont., i, th, ii : 808. Fig. $107 \%$.

1886. “ Zittel-Barrois, Traité de paléont., ii : 809. Fig. 1094.

5394. Leptopeza —. (Empidæ.)

Locality: Prussian amber. Horizon: Ligurian.

1845. Berendt.

1850. Loew.

Bernst. befindl, organ. reste vorw., i: 57 .

Bernsteinfauna, 41 (3 sp.).

5395. Leptopeza clavipes. (Empidæ.)

Locality: Prussian amber. Horizon: Ligurian.

1850. Loew.

Bernsteinfauna, 41.

1856. Giebel. Insect. d. vorwelt, 208.

5396. Limnobia - (several). (Tipulidæ.)

Compared with L. pulchella.

Locality: Prussian amber. Horizon: Ligurian.

1832. Burmeister. Handb. ent., i: 637.

1836. " Man, ent., 578.

5397. Limnobia -. (Tipulidæ.)

Locality : Aix, France. Horizon: Ligurian.

1829. Curtis. Edinb. new phil. journ., vii: 296. Pl. 6, fig. 7.

1829. " Murchison-Lyell, Tert. form. Aix, 12. Pl. 6, fig. 7.

Sec also Limnobia murchisoni, L. curtisi. 
5398. Limnobia cingulata. (Tipulidæ.)

Locality: Radoboj, Croatia. Horizon: Mayencian.

1849. Heer. 1852. Giebel. 1856

Insektenf. tertiärg. Oeningen, ii : 199-200. $\quad$ Pl. 15, fig. 8. Deutschl. petref., 640 .

Insect. d. vorwelt, 244-245.

5399. Limnobia curtisi. (Tipulidæ.)

Locality : Aix, France. Horizon: Ligurian.

1856. Giebel.

Insect. d. vorwelt, 245.

See also Limnobia — Curtis, etc.

5400. Limnobia debilis. (Tipulidæ.)

Locality : Radoboj, Croatia. Horizon : Mayencian.

1849. Heer. 1852. Giebel.

Insektenf. tertiärg. Oeningen, ii : 201. Fl. 15, fig. 11. 1856 .

Deutschl. petref., 640 .

Insect. d. vorwelt, 245.

5401. Limnobia deleta. (Tipulidæ.)

Locality : Prussian amber. Horizon: Ligurian.

1856. Giebel.

Insect. d. vorwelt, 246.

5402. Limnobia extincta. (Tipulidæ.)

Locality: Radoboj, Croatia. Horizon: Mayencian.

1868. Loew.

Zeitschr. ges, naturw., xxxii : 190-191.

See also Rhipidia extincta.

5403. Limnobia formosa. (Tipulidæ.)

Locality: (Oeningen?, Baden). Horizon: Tortonian.

1849. Heer.

1852. Giebel. 1856.

1856. Giebel.

Insektenf. tertiärg. Oeningen, ii : 198-199. $\quad P l .15$, fig. 7.

Deutschl. petref., 640 .

Insect. d. vorwelt, 244.

5404. Iimnobia furcata. (Tipulidæ.)

Locality : Prussian amber. Horizon: Ligurian.

Insect. d. vorwelt, 245-246.

5405. Limnobia jaccardi. (Tipulidæ.)

Locality : (Locle?, Switzerland). Horizon: Tortonian.

1865. Heer.

1872. "

1876. “

1879. “

Urwelt der Schweiz. Fig. 320 on p. 394.

Monde prim. Suisse. Fig. 320 on p. 484.

Prim. world Switz., ii. Fig. 320 on p. 54.

Urwelt der Schweiz, $2^{\mathrm{e}}$ aufl. Fig. 362 on $p .420$.

5406. Limnobia murchisoni. (Tipulidæ.)

Locality: Aix, France. Horizon: Ligurian.

1856. Heer. Viert. naturf. gesellsch. Zürieh, i : 30-31. Pl. 2, fig. 12.

See also Limnobia — Curtis, ete.

5407. Limnobia picta. (Tipulidæ.)

Locality : Radoboj, Croatia. Horizon: Mayencian.

1868. Loew. Zeitschr. ges. naturw., xxxii : 190-191.

See also Rhipidia picta. 
5408. Iimnobia propinqua. (Tipulidæ.)

Locality : Radoboj, Croatia. Horizon: Mayencian.

1868. Loew. Zeitschr, ges. naturw., xxxii: 190-191.

See also Rhipidia propinqua.

5409. Iimnobia sturi. (Tipulidæ.)

Locality: Rott, Rhenish Prussia. Horizon: Aquitanian.

1870. L. Heyden. Foss. dipt. Rott, 17-19. Pl. 2, fig. 23.

1870. “ Palaeontogr., Xvii: 253-255. Pl. 45, fig. 23.

5410. Limnobia tenuis. (Tipulidæ.)

Locality: Radoboj, Croatia. Horizon: Mayencian.

1849. Heer. Insektenf. tertiärg. Oeningen, ii : 200. Pl. 15, fig. 9.

1852. Giebel. Deutschl. petref., 640 .

1856. “ Insect. d. vorwelt, 245 .

5411. Limnobia vetusta. (Tipulidæ.)

Locality : Radoboj, Croatia. Horizon: Mayencian.

1849. Heer. Insektenf. tertiärg. Oeningen, ii : 200-201. Pl. 15, fig. 10.

1852. Giebel. Deutschl. petref., 640.

1856. "6 Insect. d. vorwelt, 245.

Limnobiorhynchus brevipalpus. See Toxorhina brevipalpa.

Limnobiorhynchus longirostris. See Toxorhina longirostris.

Limnobiorhynchus pulchellus. See Toxorhina pulchella.

5412. Lithomyza condita. (Cecidomyidæ.)

Localities: White River, Colo. Horizon: Oligocene.

1877. Scudder. Bull. U. S. geol, surv. terr., iii : 746.

1885. " Zittel, Handb. palaeont., i, th. ii: 812. Fig. 1089.

1886. “ “ Zittel-Barrois, Traité de paléont., ii: 813. Fig. 1106.

1890. “ Tert. ins. N. A., 601. Pl. 5, figs. 34-36.

5413. Lithophysa tumulata. (Stratiomyidø.)

Locality: Green River, Wyo. Horizon: Oligocene.

1890. Scudder. Tert. ins. N. A., 566-567. Pl. 9, fig. 31.

5414. Lithortalis picta. (Ortalidæ.)

Locality: Quesnel, British Columbia. Horizon:-

1877. Scudder. Rep. progr. geol. surv. Can., 1875-'76: 277.

1877. "6 Ins. tert. Quesnel, 7.

1877. “ Rapp. opér. comm. géol. Can., 1875-76: 306-307.

1890. " Tert.ins. N. A., 541. Pl. 3, figs. 10, 16.

5415. Lomatia gracilis. (Bombylidæ.)

Compared with L. elongata and L. sabæa.

Locality: Prussian amber. Horizon: Ligurian.

1862. Giebel. Zeitschr. ges. naturw., xx: 318-319. 
5416. Lonchæa senescens. (Lonchæidæ.)

Compared with L. vaginalis Fall.

Locality: Quesuel, British Colnmbia: Horizon:

18'77. Scudder. Rep. progr. geol. surv. Can., 1875-76: 277-278. 1877. " “ Ins. tert. Quesn., 12-13.

1877. “ Rapp. opér. comm. géol. Can., 1875-'76: 307.

1890. “ Tert. ins. N. A; 539. Pl. 3, fig. 18.

5417. Macrocera - (6 sp.): .(Mycetophilidæ,)

1850. Loew. Bernsteinfauna, 35.

Locality : Prussian amber. Horizon: Ligurian.

5418. Macrochile —. (Tipulidæ.)

Locality : Prussian amber. Horizon: Ligurian.

1845. Berendt. Bernst. befindl. organ, reste vorw., i; 57.

5419. Macrochíle spectrum. (Tipulidæ.)

Locality: Prussian amber. Horizon: Ligurian.

1850. Loew. Bernsteinfauna, 37 (undescr.).

1856. Giebel. Insect. d. vorwelt, 242 (undescr.).

5420. Macroura - (Mycetophilidæ.)

Locality : Prussian amber. Horizon : Ligurian.

1845. Berendt. Bernst. befindl. organ. reste vork., i : 57 .

5421. Medeterus - (Dolichopodidæ.)

Locality: Prussian amber. Horizon: Ligurian.

1845. Berendt. Bernst. befindl. organ. reste vorw., i: 57.

1859. Loew. Bernsteinfanna, 42.

5422. Medeterus - (Dolichopodidæ.)

Locality: Prussian amber. Horizon: Ligurian.

1832. Burmeister. Handb. ent., i: 637 .

1836. " Man. ent., 578.

5423. Medeterus frauenfeldi. (Dolichopodidæ.)

Locality: Prussian amber. Horizon: Ligurian.

1856. Giebel. Insect. d. vorwelt, 205-206.

5424. Merodon germari. (Syrphidæ.)

Locality : Rott, Rhenish Prussia. Horizon: Aquitanian.

1862. Heyden. Palaeontogr., $\mathrm{x}:$ 78-79. Pl. 10, fig. 5.

5425. Microdon -. (Syrphidæ.)

Locality: Aix, France. Horizon : Ligurian.

1832. Burmeister. Handb. ent., i: 639.

1836. " Man. ent., 580.

See also Aphritis —. 
5426. Milesia quadrata. (Syrphidæ.)

Loeality: Green River, Wyo. Horizon : Oligocene.

18:8. Scudder.

1886. Williston. 1890. Scudder.

See also

1845. Berendt.

1861. Loew.

1861. "

1869. “6

See also Loew, Bernsteinfauna, 29 (1850).
Bull. U. S. geol. surv. terr., iv: 752-753.

Syn. N. A. Syrphidæ, 281, 283 (probably not Milesia).

Tert. ins. N. A., 557-558. $P l .9, f i g .13$. - (Syrphidæ) Scudder, 1870.

5427. Mochlonyx - (Culicidæ.)

Locality : Prussian amber. Hoxi: Ligurian.

5428. Mochlonyx atavus. (Culicidæ.)

Locality: Prussian amber. Horizon: Ligurian.

1850. Loew.

1856. Giebel.

1776. Bloch.

1776. Bloch.
5429. Monodicrana terminalis. (Cecidomyidæ.)

Dipt. fauna bernstein, 8 .

Ber. versamml. deutsch. naturf., xxxv : 95.

Amber diptera, 11.

Locality: Prussian amber. Horizon: Ligurian.

Insect. d. vorwelt, 247 .

5430. Musca (Muscidæ.)

Locality : Copal. Horizon : Recent.

Beschäft. berl. gesellsch. naturf. freunde, ii : 166-167. $P l_{.}$, fig. 4.

$$
\text { 5431. Musca -. (Muscidiæ.) }
$$

Locality: Copal. Horizon: Recent.

Beschäft. berl. gesellsch. naturf. freunde, ii: 171-172. $P l_{0} 4$, fig. 10.

\section{Musca - (Muscidø.)}

Locality: Prussian amber. Horizon: Ligurian.

1831. Burmeister. Oken, Isis, 1831: 1100.

1832. " Handb. ent., i: 636.

1836. “ Man. ent., 578.

\section{Musca -. (Muscidæ.)}

Locality: Prussian amber. Horizon: Ligurian.

1820. Schlotheim. Petrefactenk., 43.

5434. Musca -. (Muscidæ.)

Locality: White River, Colo. Horizon: Oligocene.

1877. Seudder. Bull. U. S. geol. surv. terr., iii : 757.

1890. “ Tert. ins. N. A., 553. Pl. 5, figs. 106, 108.

5435. Musca -. (Muscidæ.)

Locality: White River, Colo. Horizon: Oligocene.

1890. Scudder. Tert. ins. N. A., 554. Pl. 5, figs. 80, 81, 99, 100. 
5436. Musca - (Muscidæ.)

1829. Serres. Géogn, terrains tert., 242.

Horizon : Ligurian.

5437. Musca ascarides. (Muscidæ.)

Locality: White River, Colo. Horizon : Oligocene.

1877. Scudder. Bull. U. S. geol. surv. terr., iii : 756-757.

1890. “ Tert. ins. N. A., 551-552. Pl. 5, figs. 74, 75, 79, 82-87, 98, 101.

5438. Musca bibosa. (Muscidæ.)

Locality: White River, Colo. Horizon: Oligocene.

1877. Scudder. Bull. U. S. geol. surv. terr., iii : 757.

1890. " Tert. ins. N. A., 552. Pl. 5, fig. 73.

5439. Musca cellaris. (Muscidæ.)

Locality: Copal. Horizon: Recent.

1776. Bloch. Beschäft. berl. gesellsch. naturf. freunde, ii : 173. Pl.4, fig. 12.

5440. Musca hydropica. (Muscidø.)

Locality: White River, Colo. Horizon: Oligocene.

1877. Scudder. Bull. U. S. geol. surv. terr., iii : 757-758.

1890. “ Tert. ins. N. A., 553-554. Pl. 5, figs, 72, 92, 93, 107.

5441. Musca longipes. (Muscidæ.)

Locality : Prussian amber. Horizon: Ligurian.

1822. Presl. Deliciж pragenses, i: 206.

5442. Musca meridiana. (Muscidæ.)

Locality : Copal. Horizon: Recent.

1776. Bloch. Beschäft. berl. gesellsch. naturf. freunde, ii: 184. Pl. 5, fig. 24.

5443. Musca pilosa. (Muscidø.)

Locality: Copal. Horizon: Recent.

1776. Bloch. Beschäft. berl. gesellsch. naturf. freunde, ii : 176. Pl.4, fig. 16.

1776. " Beschäft. berl. gesellsch. naturf. freunde, ii: 184-185. Pl. 5, fig. 26.

5444. Musca resinosa. (Muscidæ.)

Locality : Prussian amber. Horizon: Ligurian.

1822. Presl. Deliciæ pragenses, i: 207.

5445. Musca roralis. (Muscidæ.)

Locality: Copal. Horizon: Recent.

1776. Bloch. Beschäft. berl. gesellsch. naturf. freunde, ii: 170-171. Pl. 3, fig. 9.

5446. Musca setosa. (Muscidæ.)

Locality: Prussian amber. Horizon: Ligurian.

1822. Presl. Deliciæ pragenses, i: 205. 
5447. Musca venosa. (Muscidæ.)

Locality: Prussian amber. Horizon: Ligurian.

1822. Presl.

Deliciæ pragenses, i : 203-204.

5448. Musca vinculata. (Muscidæ.)

Locality : White River, Colo. Horizon : Oligocene.

1877. Scudder. 1890. “
Bull. U. S. geol. surv. terr., iii : 758.

Tert. ins. N. A., 554. Pl. 5, fig. 77.

5449. Muscaria - (Muscidæ.)

Locality: - Horizon: -

1846. Giebel. Paläozool., 278.

5450. Muscidites deperditus. (Muscidæ.)

Locality: Nieder-Flörsheim in Rhein-Hessen. Horizon: Aquitanian ?. 1866. Heyd.-Heyd. Palaeontogr., xv : 29. Pl. 23, fig. 22.

i866. " Käfer u. polypen, 29. Pl. 2, fig. 22.

5451. Mycetobia - (4 sp.). (Mycetophilidæ.)

Locality: Prussian amber. Horizon: Ligurian.

1850. Loew. Bernsteinfauna, 35.

5452. Mycetobia defectiva. (Mycetophilidø.)

Locality : Prussian amber. Horizon : Ligurian.

i850. Loew. Bernsteinfauna, 35.

5453. Mycetophila -. (Mycetophilidæ.)

Locality : Prussian amber. Horizon: Ligurian.

1845. Berendt. Bernst. befindl. organ. reste vorw., i: 57.

1850. Loew.

Bernsteinfauna, 34 (23 sp.).

5454. Mycetophila - (Mycetophilidæ.)

Locality : Prussian amber. Horizon : Ligurian.

1831. Burmeister. Oken, Isis, 1831: 1100.

1832. "6 Handb. ent., i: 637.

1836. “ Man. ent., 578.

5455. Mycetophila -. (Mycetophilidæ.)

Localitj: Aix, France. Horizon : Ligurian.

1829. Curtis. Edinb. new phil. journ., vii : 296. Pl. 6, fig. 9.

1829. " Murchison-Lyell, Tert. form. Aix, 12. Pl. 6, fig.9.

1839. Mantell. Wonders of geology, i : 248. Fig. 2.

1854. " Medals of creation, 2d ed., ii : 558. Fig. 183.2.

See also Mycetophilia morio, M. dubia.

5456. Mycetophila - (2 or 3 sp.). (Mycetophilidæ.)

Locality: Brunstatt, Alsatia. Horizon : Middle Oligocene.

1889. Foerster. Mitth. comm. geol. Elsass-Lothr., ii : 103.

5457. Mycetophila -. (Mycetophilidæ.)

Locality: Auvergne, France. Horizon: Aquitanian.

1870. Oustalet. Ann. sc. geol., ii, art. 3: 153-154. Pl. 3, fig. 18. 
5458. Mycetophila amoena. (Mycetophilidæ.)

Locality: Radoboj, Croatia. Horizon: Mayencian.

1849. Heer. 18ฐ2. Giebel. 1856. "

1849. Heer.

1852. Giebel. 1856. “

1850. Loew. 1856. Giebel.
Insektenf. tertiärg. Oeningen, ii : 203. Pl. 15, fig. 14.

Deutschl. petref., 641 .

Insect. d. vorwelt, 233.

\section{Mycetophila antiqua. (Mycetophilidæ.)}

Locality: Radoboj, Croatia. Horizon: Mayencian. Insektenf. tertiärg. Oeningen, ii : 203-205. Pl. 11, fig. 15e; pl. 15, figs. 15, $15 b$.

Dentschl. petref., 641.

Insect. d. vorwelt, 233 .

5460. Mycetophila compressa. (Mycetophilidæ.)

Locality: Prussian amber. Horizon: Ligurian. Bernsteinfauna, 34. Insect. d. vorwelt, 234 .

5461. Mycetophila crassa. (Mycetophilidø.)

Locality: Aix, France. Horizon: Ligurian.

1856. Giebel. Insect. d. vorwelt, 234.

See also Gnoriste — Curtis, etc.

5462. Mycetophila dubia. (Mycetophilidæ.)

Locality: Aix, France. Horizon: Ligurian.

1856. Giebel.

Insect. d. vorwelt, 234.

See also Mycetophila — Curtis, etc.

5463. Mycetophila latipennis. (Mycetophilidæ.)

Locality : Radoboj, Croatia. Horizon: Mayencian.

1849. Heer. Insektenf. tertiärg. Oeningen, ii : 205-2206. Pl. 15, fig. 17.

1852. Giebel. Deutschl. petref., 641.

1856. " Insect. d. vorwelt, 234.

5464. Mycetophila leptocera. (Mycetophilidæ.)

Locality: Prussian amber. Horizon: Ligurian.

1850. Loew.

Bernsteinfauna, 34.

1856. Giebel. Insect. d. vorwelt, 234.

.5465. Mycetophila macrostyla. (Mycetophilidæ.)

Locality: Prussian amber. Horizon: Ligurian.

1850. Loew. Bernsteinfauna, 34 .

1856. Giebel.

Insect. d. vorwelt, 234 .

5466. Mycetophila meigeniana. (Mycetophilidæ.)

Localities: Aix, France; Radoboj, Croatia. Horizons: Ligurian, Mayencian.

1856. Heer. Viert. naturf. gesellsch. Zürich, i: 32.

See also Gnoriste — Curtis, etc. 
5467. Mycetophila morio. (Mycetophilidæ.)

Locality : Aix, France. Horizon : Ligurian.

1856. Heer. Viert. naturf. gesellsch. Zürich, i: 32.

See also Mycetophila — Curtis, etc.

5468. Mycetophila nana. (Mycetophilidæ.)

Locality: Radoboj, Croatia. Horizon: Mayencian.

1849. Heer.

1852. Giebel.

1856. " 6

1849. Heer.

1852. Giebel.

1856. "

1865. Heer.

1872. “

1876. 6.

1879. 6

1856. Heer.

1850. Loew. 1856. Giebel.

1849. Heer.

1852. Giebel. 1856.

1877. Scudder.

Insektenf. tertiärg. Oeningen, ii : 202-203. Pl. 15, fig. 13.

Deutschl. petref., 641.

Insect. d. vorwelt, 233 .

5469. Mycetophila nigritella. (Mycetophilidæ.)

Locality : Radoboj, Croatia. Horizon: Mayencian.

Insek̄tenf. tertiärg. Oeningen, ii : 205. $\quad P l$. 15, fig. 16.

Deutschl. petref., 641 .

Insect. d. vorwelt, 233.

5470. Mycetophila occultata. (Mycetophilidæ.)

Locality: White River, Colo. Horizon: Oligocene.

Bull. U. S. geol. surv. terr., iii : 753.

Tert. ins. N. A., 588-589. Pl. 5, figs. 44, 45, 54, 55 .

5471. Mycetophila orci. (Mycetophilidæ.)

Locality : Oeningen, Baden. Horizon : Tortonian.

Urwelt der Schweiz. Fig. 317 on p. 394.

Monde prim. Suisse. Fig. 317 on p. 484.

Prim. world Switz., ii. Fig. 317 on $p .54$.

Urwelt der Schweiz, 2e aufl. Fig. 359 on p. 420.

5472. Mycetophila pallipes. (Mycetophilidæ.)

Locality : Aix, France. Horizon : Ligurian.

Viert. naturf. gesellsch. Zürich, i: 31. Pl.2, fig. 3.

5473. Mycetophila pulchella. (Mycetophilidæ.)

Locality : Radoboj, Croatia. Horizon: Mayencian.

Insektenf. tertiärg. Oeningen, ii : 201-202. Pl. 15, fig. 12.

Deutsehl. petref., 641 .

Insect. d. vorwelt, 232-233.

5474. Mycetophila pulvillata. (Mycetophilidæ.)

Locality : Prussian amber. Horizon: Ligurian.

Bernsteinfauna, 34.

Insect. d. vorwelt, 234.

5475. Mycetophila pumilio. (Mycetophilidæ.)

Locality: Radoboj, Croatia. Horizon: Mayencian.

1849. Heer.

Insektenf. tertiärg. Osningen, ii: 206. Pl. 15, fig. 18.

1852. Giebel. 1856.

Deutschl. petref., 641 .

Insect. d. vorwelt, 234 . 
5476. Mycetophila pusillima. (Mycetophilidæ.)

Locality: Oeningen, Baden. Horizon: Tortonian.

1865. Heer.

1872. “

1876. “

1879. “
Urwelt der Schweiz. Fig. 318 on $p .394$.

Monde prim. Suisse. Fig. 318 on p. 484.

Prim. world Switz., ii. Fig. 318 on $p .54$.

Urwelt der Schweiz, $2^{\circ}$ aufl. Fïg. 360 on p. 420.

5477. Mycetophilites - (Mycetophilidæ.)

Locality: Brunstatt, Alsatia. Horizon: Middle Oligocene. 1890. Foerster. In litt.

5478. Myiolepta (vic.) —- (Syrphidæ.)

Locality : Florissant, Colo. Horizon: Oligocene.

1886. Williston. Syn. N.A. Syrphidæ, 282.

5479. Nemestrina - (Nemestrinidæ.)

Compared with N. recticulata.

Locality : Aix, France. Horizon : Ligurian.

1829. Serres. G6ogn. terrains tert., 232.

5480. Nemotelus - (Stratiomyidæ.)

Locality : Aix, France. Horizon: Ligurian.

1829. Serres. Géogn. terrains tert., 232.

5481. Nephrotoma —- (Tipulidæ.)

Compared with N. dorsalis.

Locality: Aix, France. Horizon: Ligurian.

1829. Serres. Géogn. terrains tert., 231.

\section{Ochtera -. (Ephydridæ.)}

Compared with O. mantis.

Locality : Aix, France. Horizon: Ligurian.

1829. Serres. Géogn. terrains tert., 233.

5483. Odontomyia herichsonii. (Stratiomyidæ.)

Locality : Aix, France. Horizon : Ligurian.

1847. Hope. - Descr. ins. foss., 7. Pl., fig. 5.

\section{Cistrus - (Estridæ.)}

Locality : Prussian amber. Horizon: Ligurian.

1830. Berendt. Insecten im bernstein, 34 .

5485. CEstrus - (Estridæ.)

Locality : - Horizon:

Palaeozool, 278.

5486. Oxycera - (Stratiomyidæ.)

Locality : Aix, France. Horizon: Ligurian.

1329. Serres. Géogn. terrains tert., 232. 


\section{Palembolus florigerus. (Nemestrinidæ.)}

Locality : Florissant, Colo. Horizon: Oligocene.

1878. Scudder. Bull. U. S. geol. surv. terr., iv : 528-529.

1885. " Zittel, Handb. palaeont,, i, th. ii : 808. Fig. 1076.

1886. " Zittel-Barrois, Traité de pal6ont., ii: 809. Fig. 1093.

1890. Lesley. Dict. foss. Penns., ii : 591. Fig.

5488. Palloptera morticina. (Lonchæidæ.)

Locality: Quesnel, British Columbia. Horizon : -

187\%. Scudder. Rep. progr. geol. surv. Can., 1875-'76: 278.

18\%. " " Ins. tert. Quesnel, 13.

1377. " Rapp. opér. comm. géol. Can., 1875-76: 307-308.

1890. “ Tert. ins. N. A., 540. Pl. 3, fig. 15.

5489. Penthetria (vic.) - (Bibionidæ.)

Compared with P. holosericea.

Locality: Aix, France. Horizon: Ligurian.

1829. Curtis. Edinb. new phil. journ. mag., vii: 296. Pl. 6, fig. 10.

1829. " Murchison-Lyell, Tert. form. Aix, 12.

1839. Mantell. Wonders of geology, i : 248. Fig. 4.

5490. Penthetria (vic.) —. (Bibionidæ.)

Compared with P. holosericea.

Locality: Krottensee, Bohemia. Horizon: Aquitanian.

1852. Kollar. Reuss, Abhandl. k.-k. geol. reichsanst., i : 58. Fig. 20.

5491. Penthetria —. (Bibionidæ.)

Locality: Aix, France. Horizon: Ligurian.

1854. Mantell. Medals of creation, 2d ed., ii : 558. Fig. 183.4.

5492. Penthetria - (Bibionidæ.)

Campared with P. funebris.

Locality: Aix, France. Horizon : Ligurian.

1829. Serres. Géogn. terrains tert., 231.

5493. Penthetria brevicollis. (Bibionidæ.)

Locality : Radoboj, Croatia. Horizon : Mayencian.

1868. Loew. Zeitschr. ges. naturw., xxxii : 186-189.

See also Bibiopsis brevicollis.

5494. Penthetria cimicoides. (Bibionidæ.)

Locality: Radoboj, Croatia. Horizon: Mayencian.

1868. Loew. Zeitschr. ges. naturw., xxxii : 186-189.

See also Bibiopsis cimicoides.

5495. Penthetria murchisoni. (Bibionidæ.)

Locality : Radoboj, Croatia. Horizon: Majencian.

1868. Loew. Zeitschr. ges. naturw., xxxii: 186-189.

See also Bibiopsis murchisoni, etc.

Bull. 71-41 
5496. Penthetria similkameena. (Bibionidæ.)

Locality : Similkameen River, British Columbia. Horizon : 1879. Scudder. Rept. progr. geol. surv. Can., 1877-'78: B 177-179. 1879. "I Ins. tert. Nicola, 2-4.

See also Plecia similkameena.

5497. Penthetria vaillantii. (Bibionidæ.)

Locality: Corent, France. Horizon: Aquitanian. 1870. Oustalet. . Ann, sc. géol., ii, art. 3: 112-113. Pl. 3, figs. 1, 2.

5498. Phalænomyia -. (Psychodidæ.)

1815. Berendt.

Locality : Prussian amber. Horizon: Ligurian.

\title{
5499. Phalænomyia - (9 sp.). (Psychodidæ.)
}

Locality: Prussian amber. Horizon: Ligurian.

1850. Loew.

1856. Giebel.

Bernsteinfauna, 31 (description of genus only).

Insect. d. vorwelt, 248-249.

\section{Phora -. (Phoridæ.)}

Locality: Prussian amber. Horizon: Ligurian.

1845. Berendt.

1850. Loew.

Bernst. befindl. organ. reste vorw., i: 57.

Bernsteinfauna, 44 (11 sp.).

5501. Phthiria dubia. (Bombylidæ.)

Locality: Vicinity Bonn, Rhenish Prussia. Horizon: Aquitanian.

1837. Germar. Fauna insect. Europæ, xix: 24. $P l .24$.

1852. Giebel. Deutschl. petref., 642.

See also Bibio dubius.

\author{
5502. Pipiza -. (Syrphidæ.) \\ Locality : Florissant, Colo. Horizon: Oligocene.
}

1886. Williston. Syn. N. A. Syrphidæ, 282.

\section{Pipiza venilia. (Syrphidæ.)}

Locality: Rott, Rhenish Prussia. Horizon: Aquitanian.

1870. L. Heyden. Foss. dipt. Rott, 24-25. Pl. 2, fig. 28.

1870. "6 Palaeontogr., xvii : 260-261. Pl. 45, fig. 28.

5504. Pipunculus —. (Pipunculidæ.)

Locality : Prussian amber. Horizon: Ligurian.

1845. Berendt. Bernst. befindl. organ, reste vorw., i : 57.

\section{Pipunculus succini. (Pipunculidæ.)}

Compared with P. auctus.

Locality : Prussian amber. Horizon: Ligurian.

1850. Loew. Bernsteinfauna, 42 .

18v6. Giebel. Insect. d. vorwelt, 206. 
5506. Platyura - (Mycetophilidæ.)

Locality: Prussian amber. Horizon: Ligurian.

1845. Berendt. Bernst. befindl. organ. reste vorw., i: $5 \%$.

1850. Loew.

Bernsteinfauna, 35 (16 sp.).

\section{Platyura —- (Mycetophilidœ.) \\ Compared with P. cingulata.}

Locality: Aix, France. Horizon: Ligurian.

1829. Serres. Géogn. terrains tert., 231.

5508. Platyura conjuncta. (Mycetophilidæ.)

1850. Loew.

Locality : Prussian amber. Horizon: Ligurian.

1856. Giebel.

Bernsteinfauna, 35.

Insect. d. vorwelt, 237 .

5509. Platyura ehrhardti. (Mycetophilidæ.)

1850. Loew.

Locality : Prussian amber. Horizon: Ligurian.

1856. Giebel.

Bernsteinfauna, 35.

Insect. d. vorwelt, 237.

\section{Plecia —. (Bibionidæ.)}

Locality: Prussian amber. Horizon: Ligurian.

1845. Berendt. Bernst. befindl, organ. reste vorw., i: 57.

1850. Loew. Bernsteinfauna, 39 (2 sp.).

5511. Plecia - (12 sp.). (Bibionidæ.)

Locality: Brunstatt, Alsatia. Horizon : Middle Oligocene.

1890. Foerster. In litt.

5512. Plecia affinis. (Bibionidø.)

Locality: Oeningen, Baden. Horizon: Tortonian.

1838. Loew. Zeitschr. ges. naturw., xxxii : 184-186.

See also Protomyia affinis.

5513. Plecia amcna. (Bibionidø.)

Locality: Oeningen, Baden. Horizon: Tortonian.

1868. Loew. Zeitschr. ges. naturw., xxxii : 184-186.

See also Protomyia amœna.

\section{Plecia anthracina. (Bibionidæ.)}

Locality: Radoboj, Croatia. Horizon: Mayencian.

1868. Loew. Zeitschr. ges, naturw., xxxii: 184-186.

See also Protomyia anthracina.

5515. Plecia blanchardi. (Bibionidæ.)

Locality : Corent, France. Horizon: Aquitanian.

1878. Brongniart. Bull. sc. dép. nord, (2), i: 75.

1878. " "Bull. séances soc. ent. France, 1878, No. 6: 61.

1878. " $6 \quad$ Ann. soc. ent. France, (5), viii : 48.

See also Protomyia blanchardi. 
5516. Plecia bucklandi. (Bibionidæ.)

Localities: Radoboj, Croatia ; Aix, France. Horizons: Mayencian, Ligurian. 1868. Loew. Zeitschr. ges. naturw., xxxii : 184-186.

See also Protomyia bucklandi, etc.

5517. Plecia dejecta. (Bibionidæ.)

Locality: Green River, Wyo. Horizon: Oligocene. 1890. Scudder. Tert. ins. N. A., 586. Pl. 10, fig. 17.

5518. Plecia edwardsii. (Bibionidæ.)

Locality: Corent, France. Horizon: Aquitanian.

1878. Brongniart. Bull. sc. dep. nord, (2), i : 75-76.

1878. " "Bull, sćances soc. ent. France, 1878, No. 6: 61.

1878. " " Ann. soc. ent. France, (5), viii : 48.

See also Bibio edwardsii.

5519. Plecia? heroica. (Bibionidæ.)

Locality : Rott, Rhenish Prussia. Horizon : Aquitanian.

1866. Heyd.-Heyd. Palaeontogr., xiv : 29. Pl. 8, fig. 16.

5520. Plecia hilaris. (Bibionidæ.)

Locality: Oeningen, Baden. Horizon: Tortonian.

1849. Heer. Insektenf. tertiärg. Oeningen, ii : 211. $\quad P l .17, f i g .6$.

1852. Giebel. Dentschl. petref., 641.

1856. " Insect. d. vorwelt, 227-228.

1865. Heer. Urwelt der Schweiz, 396. Fig. 313 on p. 394.

1868. Loèw. Zeitschr. ges. naturw., xxxii : 183-184.

1872. Heer. Monde prim. Suisse, 485. Fig. 313 on $p .484$.

1876. 66 Prim. world Switz., ii: 55. Fig. 313 on p. 54.

1879. " Urwelt der Schweiz, $2^{\circ}$ aufl, 421 . Fig. 355 on $p .420$.

5521. Plecia joannis. (Bibionidæ.)

Locality : Corent, France. Horizon: Aquitanian.

1878. Brongniart. Bull. sc. d6́p. nord, (2), i : 75.

1878. 6i Bull, śances soc. ent. France, 1878, No. 6: 61.

1878. " Ann. soc. ent. Frauce, (5), viii: 48.

See also Protomyia joannis, etc.

5522. Plecia jucunda. (Bibionidæ.)

Localities: Oeningen, Baden; Parschlug, Styria. Horizon: Tortonian.

1868. Loew. Zeitschr. ges. naturw. xxxii : 184-186.

See also Protomyia jucunda, etc.

5523. Plecia jucunda parschlugiana. (Bibionidæ.)

Locality : Parschlug, Styria. Horizon : Miocene.

1868. Loew. Zeitschr. ges. naturw., xxxii: 184-186.

See also Protomyia jucunda, etc.

5524. Plecia lapidaria. (Bibionidæ.)

Locality: Rott, Rhenish Prussia. Horizon : Aquitanian.

1878. Brongniart. Bull. séances soc. ent. France, 1878, No. 6: 61 .

1878. " Ann. soc. ent. France, (5), viii : 48.

See also Protomyia lapidaria. 
5525. Plecia larteti. (Bibionidœ.)

Locality: Corent, Frauce. Horizon: Aquitanian.

1878. Brongniart. Bull. sc. dép. nord, (2), i : 75.

1878. " " Bull. séances soc. ent. France, 1878, No. 6: 61.

1878. " a Ann. soc. ent. France, (5), viii : 48.

See also Bibio larteti.

5526. Plecia latipennis. (Bibionidæ.)

Locality: Radoboj, Croatia. Horizon: Mayencian.

1868. Loew. Zeitschr. ges. naturw., xxxii : 184-186.

See also Protomyia latipennis.

5527. Plecia longa. (Bibionidæ.)

Locality: Radoboj, Croatia. Horizon: Mayencian.

1866. Loew. Zeitschr. ges. naturw., xxxii : 184-186.

See also Protomyia longa.

5528. Plecia lugubris. (Bibionidæ.)

Locality: Radoboj, Croatia. Horizon: Mayencian.

1849. Heer. Insektenf, tertiärg. Oeningen, ii: 209-211. Pl. 14, fig. 20.

1852. Giebel. Dentschl. petref., 641.

1856. " Insect. d. vorwelt, 227.

1868. Loew. Zeitschr. ges. naturw., xxxii : 183.

5529. Plecia lygæoides. (Bibioniđæ.)

Localities: Radoboj, Croatia; Aix, France. Horizons: Mayencian, Ligurian. 1868. Loew. Zeitschr. ges. naturw., xxxii : 184-186.

See also Bibio lignarius, etc.

5530. Plecia major. (Bibionidæ.)

Locality : Corent, France. Horizon: Aquitaniau.

1870. Oustalet. Ann. sc. géol., ii, art. 3: 114-115. Pl. 2, fig. 19; pl. 3, figs. 3, 4.

5531. Plecia nigxescens. (Bibionidæ.)

Compared with P. funebris, P. velutina, Brazil.

Locality: Corent, France. Horizon: Aquitanian.

1870. Oustalet. Ann. sc. géol., ii, art. 3: 115-117. Pl. 3, figs. 5-10.

5532. Plecia oustaleti. (Bibionidæ.)

Locality: Auvergne, France. Horizon: Aquitanian.

1878. Brongniart. Bull. sc. d6p. nord, (2), i: 75-80.

1878. " Bull. séances soc. ent. France, 1878, No. 6: 60-61.

1878. Giard. Bull. sc. dep. nord, (2), i : 12-16.

See also Protomyia oustaleti.

5533. Plecia pallida. (Bibionidæ.)

Locality: Corent, France. Horizon: Aquitanian.

187e. Onstalet. Ann. sc. géol., ii, art. 3: 118-119. Pl. 3, figs. 11-13.

Plecia parschlugiana. See Plecia jucunda parschlugiana. 
5534. Plecia pealei. (Bibionidæ.)

Locality: Twin Creek, Wyo. Horizon: Oligoceno.

1890. Scndder. Tert. ins. N. A., 「85-586. Pl. 4, figs, 2, 3, 10-12.

5535. Plecia quæsita. (Bibionidæ.)

Locality: Krottensee, Bohemia. Horizon: Aquitanian.

1877. Novak. Sitzungsb. k. akad. wiss. Wien, lxxvi : 86-87. Pl. 2, fig. 5.

1877. " Fauna egerer tertiärb., 16-17 Pl. 2, fig. 5.

5536. Plecia rhenana. (Bibionidæ.)

Locality : Rott, Rhenish Prussia. Horizon: Aquitanian.

1865. Heyd.-Heyd. Palaeontogr., xiv: 28-29. Pl. 9, fig. 9.

5537. Plecia sauvagei. (Bibionidæ.)

Locality: Corent, France. Horizon: Aquitanian.

1878. Brongniart. Bull. sc. dép. nord, (2), i: 75.

1878. " "Bull. séances soc. ent. France, 1878, No. 6: 61.

1878. “ Ann. soc. ent. France, (5), viii : 48.

See also Protomyia sauvagei.

5538. Plecia similkameena. (Bibionidæ.)

Locality : British Columbia. Horizon: - .

1885. Scudder. Zittel, Handb. palaeont., i, th. ii: 811. Fig. 1086.

1886. " Zittel-Barrois, Traité de palént., ii: 812. Fig. 1103.

1886. " " Bull. U. S. geol. surv., No. 31: 92 .

1890. " " Tert. ins. N. A., 583-585. Pl. 3, figs. 20-22.

See also Penthetria similkameena.

5539. Poliomyia recta. (Conopidæ.)

Locality: Green River, Wyo. Horizon: Oligocene.

1878. Scudder.

1885. "6

1886. " "

1890. “
Bull. U. S. geol. surv. terr., iv : 755.

Zittel, Handb. palaeont, , i, th. ii : 807. Fig. 1072.

Zittel-Barrois, Traité de paléont., ii : 807. Fig. 1089.

Tert. ins. N. A., 556-557. Pl. 9, figs. 19, 21.
1845. Berendt. 1850. Loew.

\section{Porphyrops - (Dolichopodidæ.)}

Locality: Prussian amber. Horizon: Ligurian.

Bernst. befindl, organ. reste vorw., i : 57.

Bernsteinfauna, 42.

5541. Porphyrops - (Dolichopodidæ.)

Locality : Prussian amber. Horizon : Ligurian.

1832. Burmeister. Handb. ent., i: 637.

1836. “ Man. ent., 578.

5542. Porphyrops

(Dolichopodidæ.)

Locality: Prussian amber. Horizon: Ligurian.

1870. Giebel. Zeitschr. ges. naturw, (2), i: 87 .

$$
\text { 5543. Posthon -. (Psychodidæ.) }
$$

Locality : Prussian amber. Horizon : Ligurian.

1845. Berendt. Bernst. befindl, organ. reste vorw., i: 57 . 
5544. Posthon gracilis. (Psychodiriæ.)

Locality : Prussian amber. Horizon: Ligurian.

1850. Loew.

Bernsteinfauna, 31.

1856. Giebel. Insect. d. vorwelt, 249.

5545. Pronophlebia rediviva. (Tipulidæ.)

Locality : White River, Colorado or Utah. Horizon: Oligocene. 1878. Scudder.

1890. " Tert. ins. N. A., 574. Pl. 5, fig. 39.

5546. Protomyia - ( 2 or 3 sp.). (Bibionidæ.)

Locality : Brunstatt, Alsatia. Horizon: Middle Oligocene.

1888. Foerster. Mitth. comm. geol. Elsass-Lothr., i: 164.

1889. “ Mitth. comm. geol. Elsass-Lothr., ii : 103.

5547. Protomyia abava. (Bibionidæ.)

Locality: Rott, Rhenish Prussia. Horizon: Aquitanian. 1865. Heyd.-Heyd. Palaeontogr., xiv : 20-21. Pl. 8, figs. 2 A, B.

5548. Protomyia adusta. (Bibionidæ.)

Locality : Corent, France. Horizon: Aquitanian.

1870. Oustalet. Ann. sc. géol., ii, art. 3: 145-146. $\quad P l .5$, fig. 18.

5549. Protomyia affinis. (Bibionidæ.)

Locality : Oeningen, Baden. Horizon: Tortonian,

1819. Heer. Insektenf. tertiärg. Oeningen, ii : 235-236. $\quad P l .17, f i g .3$.

1852. Giebel. Deutschl. petref., 642.

1853-56. Bronn. Lethæa geogn., $3^{\text {e }}$ aufl., iii: 641. Pl. $42^{\prime}$, figs. 13a-d.

1856. Giebel. Insect. d. vorwelt, 224-225.

See also Plecia affinis.

5550. Protomyia amœna. (Bibionidæ.)

Locality: Oeningen, Baden. Horizon: Tortonian.

1849. Heer. Insektenf. tertiärg. Oeningen, ii: 237. $P l .17, f g .4$.

1852. Giebel. Deutschl. petref, 642.

1856. " Insect. d. vorwelt, 225.

See also Plecia amœna.

Protomyia antenata. See Protomyia antennata.

5551. Protomyia antennata. (Bibionidæ.)

Locality : Rott, Rhenish Prussia. Horizon: Aquitanian.

1865. Heyd.-Heyd. Palaeontogr., xiv: 26 (antenata). Pl. 8, fig. 9.

5552. Protomyia anthracina. (Bibionidæ.)

Locality: Radoboj, Croatia. Horizon: Mayencian.

1849. Heer. Insektenf, tertiärg. Oeningen, ii : 236-237. Pl. 16, fig. 21.

1852. Giebel. Deutschl. petref., 642.

1856. " Insect. d. vorwelt, 225.

See also Plecia anthracina. 
5553. Protomyia blanchardi. (Bibionidæ.)

Locality: Corent, France. Horizon: Aquitanian.

18\%0. Oustalet. Ann. sc. géol., ii, art. 3: 148-149. Pl. 6, fig. 5. See also Plecia blanchardi.

5554. Protomyia bohemica. (Bibionidæ.)

Locality : Krottensee, Bohemia. Horizon: Aquitanian.

1877. Novák.

1877. "

Sitzungsb. k. akad. wiss. Wien, lxxvi: 86. Pl. 2, fig. 2.

Fauna egerer tertiärb., 16. $P l .2$, fig. 2.

5555. Protomyia brevipennis. (Bibionidæ.)

Locality: Aix, France. Horizon: Ligurian.

1856. Heer. Viert. naturf. gesellsch. Zürich, i: $35 . \quad$ Pl. 2, fig. 1.

5556. Protomyia bucklandi. (Bibionidæ.)

Localities: Radoboj, Croatia; Aix, France. Horizons : Ligurian, Mayencian.

1849. Heer. Insektenf. tertiärg. Oeningen, ii : 238-239 (buklandi). Pl. 16, fig. 22.

1852. Giebel. Deutschl. petref., 642.

1856. " " Insect. d. vorwelt, 225 .

1856. Heer. Viert. naturf. gesellsch. Zürich, i: 35.

1876. Brongniart. Bull. soc. géol. France, (3), iv: 459-460. Pl. 13, fig. 7.

See also Bibio — Buckland, Plecia bucklandi.

Protomyia buklandi. See Protomyia bucklandi.

Protomyia collossea. See Protomyia colossea.

5557. Protomyia colossea. (Bibionidæ.)

Locality: Rott, Rhenish Prussia. Horizon: Aquitanian.

1865. Heyd.-Heyd. Palaeontogr., xiv: 21 (collossea). Pl. 8, fig. 3.

5558. Protomyia elegans. (Bibionidæ.)

Locality : Aix, France. Horizon : Ligurian.

1856. Heer. Viert. naturf. gesellsch. Zürich, i: 36.

See also —_ (Bibionidæ) Curtis.

5559. Protomyia elongata. (Bibionidæ.)

Locality: Rott, Rhenish Prussia. Horizon: Aquitanian. 1855. Heyd.-Heyd. Palaentogr., xiv: 27-28. Pl. 8, fig. 14.

5560. Protomyia exposititia. (Bibionidæ.)

Locality: Rott, Rhenish Prussia. Horizon: Aquitanian.

1865. Heyd.-Heyd. Palaeontogr., xiv: 24. Pl. 9, figs. 7-8.

1870. L. Heyden. Foss. dipt. Rott, 29.

1870. " $\quad$ Palaeontogr., xvii: 265.

5561. Protomyia formicoides. (Bibionidæ.)

Locality: Corent, France. Horizon: Aquitanian.

1870. Oustalet. Ann. sc. géol., ii, art. 3: 150-151. Pl. 4, fig. 18; pl. 5, fig. 19. 
5562. Protomyia fusca. (Bibionidæ.)

Locality: Corent, France. Horizon: Aquitanian.

1870. Oustalet. Ann. sc. géol., ii, art.3: 145. Pl. 4, fig. 15.

5563. Protomyia globularis. (Bibionidæ.)

Locality : Corent, France. Horizon: Aquitanian.

1870. Oustalet. Ann. se. géol., ii, art.3: 147-148. Pl. 6, fig.7.

5564. Protomyia gracilenta. (Bibionidæ.)

Locality: Rott, Rhenish Prussia. Horizon: Aquitanian.

1865. Heyd.-Heyd. Palaeontogr., xiv: 28. Pl. 9, fig. 12.

5565. Protomyia gracilis. (Bibionidæ.)

Locality: Aix, France. Horizon: Ligurian.

1856. Heer. Viert. naturf. gesellsch. Zürich, i : 36. Pl. 2, fig. $2 a$.

5566. Protomyia grandæva. (Bibionidæ.)

Locality: Rott, Rhenish Prussia. Horizon: Aquitanian.

1865. Heyd.-Heyd. Palaeontogr., xiv: 25-26. Pl. 8, figs. 17-18.

1870. L. Heyden. Foss. dipt. Rott, 29.

1870. “ Palaeontogr., xvii: 265.

5567. Protomyia grossa. (Bibionidæ.)

Compared with P. winnertzii Heyd., Aquitanian.

Locality: Rott, Rhenish Prussia. Horizon: Aquitanian.

1865. Heyd.-Heyd. Palaeontogr., xiv: 22. Pl. 8, fig. 5.

5568. Protomyia heeri. (Bibionidæ.)

Locality: Rott, Rhenish Prussia. Horizon : Aquitanian. 1865. Heyd.-Heyd. Palaeontogr., xiv: 28. Pl. 8, fig. 15.

5569. Protomyia hypogæa. (Bibionidæ.)

Locality: Rott, Rhenish Prussia. Horizon : Aquitanian.

1865. Heyd.-Heyd. Palaeontogr., xiv: 23. Pl. 9, figs. 10-11.

5570. Protomyia incerta. (Bibionidæ.)

Locality : Corent, France. Horizon: Aquitanian.

1870. Oustalet. Ann. sc. g6ol., ii, art. 3: 151-152.. Pl. 1, fig. 16d; pl. 5, figs. 20, 21 .

5571. Protomyia inflata. (Bibionidæ.)

Locality: Corent, France. Horizon: Aquitanian.

1870. Oustalet. Ann. sc. géol., ii, art. 3: 142. Pl. 5, fig. 17.

5572. Protomyia joannis. (Bibionidæ.)

Locality: Corent, France. Horizon: Aquitanian.

1870. Oustalet. Ann. se. géol., ii, art. 3: 143-144. Pl. 6, figs. 4, 14 (bis).

1876. Brongniart. Bull. soe. gél. France, (3), iv: 459-460. Pl. 13, fig. 8.

See also Epiplecia joannis, Plecia joannis. 
5573. Protomyia juounda. (Bibionidæ.)

Localities : Oeningen, Baden; Parschlug, Styria. Horizon : Tortonian.

1849. Heer.

1852. Giebel.

1853-'56. Bronn.

1856. Giebel.
Insektenf. tertiärg. Oeningen, ii : 234-235. $\quad P l .17$, fig. 2.

Deutschl. petref., 642.

Lethæa geogn. $3^{e}$ aufl., iii : $641 . \quad P l .42^{\prime}$, fig. 14.

Insect. d. vorwelt, 224 .

See also Protomyia jucunda parschlugiana, Plecia jucunda, Plecia jucunda parschlugiana.

5574. Protomyia jucunda parschlugiana. (Bibionidæ.)

Locality : Parschlug, Styria. Horizon: Tortonian.

1849. Heer. Insektenf. tertiärg. Oeningen, ii : 235. Pl. 17, figs. 2a-g.

See also Protomyia jucunda, etc.

5575. Protomyia lapidaria. (Bibionidæ.)

Locality : Rott, Rhenish Prussia. Horizon: Aquitanian.

1865. Heyd.-Heyd. Palaeontogr., xiv : 25. Pl. 9, fig. 6.

1870. L. Heyden. Foss. dipt. Rott, 29.

1870. " 4 Palaeontogr., xvii: 265.

See also Plecia lapidaria.

5576. Protomyia latipennis. (Bibionidæ.)

Locality: Radoboj, Croatia. Horizon: Mavencian.

1849. Heer. Insektenf. tertiärg. Oeningen, ii : 237-238. Pl. 17, fig. 5.

1852. Giebel. Dentschl. petref., 642.

1856. " Insect. d. vorwelt, 225.

See also Plecia latipennis.

5577. Protomyia lignaria. '(Bibionidæ.)

Locality : Rott, Rhenish Prussia. Horizon: Aquitanian.

1865. Heyd.-Heyd. Palaeontogr., xiv: 29-30.

See also Bibio lignarius, etc.

5578. Protomyia livida. (Bibionidæ.)

Locality : Aix, France. Horizon : Ligurian.

1849. Heer. Insektenf, tertiärg. Oeningen, ii : 239.

1856. " Viert. naturf. gesellsch. Zürich, $\mathrm{i}: \mathbf{3 5}$.

1856. Giebel. Insect. d. vorwelt, 226.

See also Empis — Curtis.

5579. Protomyia longa. (Bibionidæ.)

Localities: Radoboj, Croatia; Corent, France. Horizons: Mayencian, Aquitanian. 1849. Heer. Insektenf. tertiärg. Oeningen, ii : 233-234. Pl. 16, fig. 20. 1852. Giebel. Deutschl, petref., 642.

1853-'56. Bronn. Lethæa geogn., $3^{\mathrm{e}}$ aufl., iii : 641. Pl. 42', figs. $12 a b$.

1856. GiebeI. Insect. d. vorwelt, 224.

1870. Oustalet. Ann. sc. géol., ii, art. 3: 139-140. Pl. 5, fig. 16.

See also Plecia longa.

5580. Protomyia longipennis. (Bibionidæ.)

Locality : Gorent, France. Horizon: Aquitanian.

1870. Onstalet. Ann. sc. génl., ii, art. 3: 141. Pl. 6, fig. 1. 
5581. Protomyia luctuosa. (Bibionidæ.)

Locality: Rott, Rhenish Prussia. Horizon: Aquitanian.

1865. Heyd.-Heyd. Palaeontogr., xiv : 22. $\quad P l .8$, fig. 6.

1870, L. Heyden. Foss. dipt. Rott, 29.

1870. " Palaeontogr., xvii : 265.

5582. Protomyia lugens. (Bibionidæ.)

Locality: Corent, France. Horizon: Aquitanian.

1870. Oustalet. Ann. se. géol. ii, art. $3: 142-143 . \quad$ Pl. 6, figs. 2, 3.

5583. Protomyia luteola. (Bibionidæ.)

Locality: Rott, Rhenish Prussia. Horizon: Aquitanian.

1865. Heyd.-Heyd. Palaeontogr., xiv: 26-27. Pl. 8, fig. 11.

5584. Protomyia lygæoides. (Bibionidæ.)

Localities: Radoboj, Croatia; Aix, France. Horizon: Mayencian, Ligurian. 1849. Heer. 1852. Giebel.

Insektenf. tertiärg. Oeningen, ii : 232-233. Pl. 17, fig. 1.

1856. " "

Deutschl. petref., 642.

1856. Heer.

Insect. d. vorwelt, 223-224.

See also Bibio lignarius, etc.

5585. Protomyia macrocephala. (Bibionidø.)

Locality: Rott, Rhenish Prussia. Horizon: Aquitanian.

1865. Heyd.-Heyd. Palaeontogr., xiv: 23. Pl. 8, fig. 8.

1870. L. Heyden. Foss. dipt. Rott, 29.

1870. 6 Palaeontogr., xvii : 265.

5586. Protomyia matheroni. (Bibionidæ.)

Locality: Aix, France. Horizon: Ligurian.

1861. Heer. Saporta, Heer, Rech. climat pays tert., 153 (undescr.).

5587. Protomyia oustaleti. (Bibionidæ.)

Locality : Chadrat, Anvergne, France. Horizon: Aquitanian. 1876. Brongniart. Bull. soc. géol. Fr., (3), iv: 459-460. Pl. 13, figs. 5-8. 1876. “ Ann. sc. géol., vii, art. 4: 1-2.

See also Plecia oustaleti.

Protomyia parschlugiana. See Protomyia jucunda parschlugiana.

5588. Protomyia pinguis. (Bibionidæ.)

Locality: Rott, Rhenish Prussia. Horizon: Aquitanian.

1865. Heyd.-Heyd. Palaeontogr., xiv: 24. Pl. 9, figs. 4-5.

5589. Protomyia proserpina. (Bibionidæ.)

Locality: Rott, Rhenish Prussia. Horizon: Aquitanian.

1865. Heyd.-Heyd. Palaeontogr., xiv: 22-23. Pl. 8, fig. 7.

5590. Protomyia rubescens. (Bibionidæ.)

Locality: Corent, France. Horizon: Aquitanian.

1870. Oustalet. Ann. se. géol., ii, art. 3: 149-150. Pl. 4, figs. 16, 17. 
5591. Protomyia sauvagei. (Bibionidæ.)

Locality: Corent, France. Horizon: Aquitanian.

1870. Oustalet. Anu. sc. g6ol., ii, art. 3: 146-147. Pl. 6, fig. 6.

See also Plecia sauvagei.

5592. Protomyia schineri. (Bibionidæ.)

Locality: Rott, Rhenish Prussia. Horizon: Aquitanian.

1865. Heyd.-Heyd. Palaeontogr., xiv: 27. Pl. 8, figs. 12, 13.

5593. Protomyia speciosa. (Bibionidæ.)

1865. Heer.

1872. "6

1876. "

1879. 6

\section{Locality: Oeningen, Baden. Horizon: Tortonian.}

Urwelt der Schweiz, 395. Fig. 312 on $p .394$.

Monde prim. Suisse, 485. Fig. 312 on p. 484.

Prim. world Switz., ii : 55. Fig. 312 on $p .54$.

Urwelt der Schweiz, $2^{\circ}$ aufl, 421 . Fig. 354 on $p .420$.

5594. Protomyia stygia. (Bibionidæ.)

Locality: Rott, Rhenish Prussia. Horizon : Aquitanian.

1865. Heyd.-Heyd. Palaeontogr., xiv: 24. Pl. 9, figs. 1-3.

1870. L. Heyden. - Foss. dipt. Rott, 29.

1870. "6 Palaeontogr., xvii: 265.

5595. Protomyia veterana. (Bibionidæ.)

Locality : Rott, Rhenish Prussia. Horizon: Aquitanian. 1865. Heyd.-Heyd. Palaeontogr., xiv: 25. Pl. 8, fig. 10.

5596. Protomyia volgeri. (Bibionidæ.)

Locality : Rott, Rhenish Prussia. Horizon: Aquitanian.

1865. Heyd.-Heyd. Palaeontogr., xiv : 29-30.

See also Bibiopsis volgeri.

5597. Protomyia winnertzi. (Bibionidæ.)

Compared with P. latipennis Heer, Radoboj.

Locality: Rott, Rhenish Prussia. Horizon: Aquitanian.

1865. Moyd.-Heyd. Palaeontogr., xiv: 21-22. Pl. 8, fig. 4.

1870. L. Heyden. Foss. dipt. Rott, 29.

1870. "6 Palaeontogr., xviii : 265.

\section{Psila —. (Psilidæ.)}

Locality: Oeningen, Baden. Horizon: Tortonian.

1888. Schöberlin. Soc. entom., iii: 68 .

5599. Psilites bella. (Psilidæ.)

Locality: Radoboj, Croatia. Horizon: Mayencian.

1849. Heer. Insektenf. tertiärg. Oeningen, ii : 250-252. $\quad P l .17, f i g .16$.

1852. Giebel. Deutschl. petref., 643.

1856. " Insect. d. vorwelt, 196.

1885. Scudder, Zittel, Handb. palaeont., i, th. ii: 806. Fig. 1070.

1886. “ Zittel-Barrois, Traité de paléont., ii: 807. Fig. 1087. 
5600. Psilopus - (Dolichopodidær.)

Locality : Prussian amber. Horizon: Ligurian.

1850. Loew. Bernsteinfauna, 42.

5601. Psilota tabidosa. (Syrphidæ.)

Locality : Green River, Wyo. Horizon : Oligocene.

1890. Scudder. Tert. ins. N. A., 561-562. Pl. 9, fig. 9.

5602. Psychoda - (Psychodidæ.)

Locality: Prussian amber. Horizon: Ligurian.

1845. Berendt. Bernst. befindl. organ. reste vorw., i: 57.

5603. Psychoda - - (Psychodidæ.)

Locality: Prussian amber. Horizon: Ligurian.

1832. Burmeister. Handb. ent., i: 637.

1836. " Man. ent., 578.

5604. Psychoda oxyptera. (Psychodidæ.)

1850. Loew.

Locality : Prussian amber. Horizon: Ligurian.

1856. Giebel.

Bernsteinfauna, 31.

Insect. d. vorwelt, 248 .

5605. Ptychoptera deleta. (Tipulidæ.)

Locality: Krottensee, Bohemia. Horizon: Aquitanian.

1877. Novák. Sitzungsb. k. akad. wiss. Wien., lxxvi: 88-89. Pl. 2, fig. 1.

1877. " Fauna egerer tertiärb., 18-19. Pl. 2, fig. 1.

5606. Pulex -. (Pulicidæ.)

Locality : Aix, France. Horizon : Ligurian.

1834. Keferstein. Naturg. erdkörp., ii: 336 (two species as of Serres; but Serres merely says: "Peut-être des Aptères de l'ordre des Suceurs." Cf. Walck. and Gervais, Apt. iv : 356. Pictet in the first edition of his Traite de paleontologie (1846) makes the same reference as Keferstein, but omits it in the second).

5607. Rhamphidia —. (Tipulidø.)

1845. Berendt.

Locality: Prussian amber. Horizon: Ligurian.

1850. Loew.

Bernst. befindl, organ. reste vorw., i : 57.

Bernsteinfauna, 37 (4 sp.).

5608. Rhamphidia minuta. ('Tipulidæ.)

Locality: Prussian amber. Horizon: Ligurian.

1850. Loew.

Bernsteinfauna, 37.

1856. Giebel.

1850. Loew.

Insect. d. vorwelt, 242.

5609. Rhamphidia pulchra. (Tipulidæ.)

Locality : Prussian amber. Horizon: Ligurian,

1856. Giebel.

Bernsteinfauna, 37.

Insect. d. vorwelt, 242. 
5610. Rhaphium (Dolichopodidæ.)

Locality: Prussian amber. Horizon: Ligurian.

1832. Burmeister. Handb. ent., i: 637.

1836. " Man. ent., 579.

5611. Rhaphium —- (Dolichipodidæ.)

Locality: Prussian amber. Horizon: Ligurian.

1850. Loew. Bernsteinfauna, $4 \%$.

5612. Rhamphomyia - (Empidæ.)

Locality : Prussian amber. Horizon: Ligurian.

1845. Berendt. Bernst. befindl. organ. reste vorw., i: 57 (Rumphomyia).

1850. Loew.

Bernsteinfauna, 41 (21 sp.).

5613. Rhamphomyia antipedalis. (Empidæ.)

Locality: Prussian amber. Horizon: Ligurian.

1850. Loew.

Bernsteinfauna, 41 (undescr.).

1856. Giebel. Insect. d. vorwelt, 208 (undescr.).

5614. Rhamphomyia crinitarsis. (Empidæ.)

Locality : Prussian amber. Horizon : Ligurian.

1850. Loew.

Berasteinfauna, 41.

1856. Giebel.

Insect. d, vorwelt, 208.

5615. Rhamphomyia distans. (Empidæ.)

Locality : Prussian amber. Horizon : Ligurian.

1850. Loew.

Bernsteinfanna, 41.

1856. Giebel. Insect. d. vorwelt, 208.

5616. Rhamphomyia formosa. (Empidæ.)

1850. Loew.

Locality: Prussian amber. Horizon: Ligurian. 1856. Giebel.

Bernsteinfauna, 41 (undescr.).

Insect. d. vorwelt, 208 (undescr.).

5617. Rhamphomyia pteropa. (Empidæ.)

Locality: Prussian amber. Horizon : Ligurian.

1850. Loew.

Bernsteinfauna, 41 (undescr.).

1856. Giebel.

Insect. d. vorwelt, 208 (undescr.).

5618. Rhamphomyia ptilopa. (Empidæ.)

Locality: Prussian amber. Horizon: Ligurian.

1850. Loew.

Bernsteinfauna, 41 (undeser.).

1856. Giebel.

Insect. d. vorwelt, 208 (undescr.)-

5619. Rhamphomyia remitarsis. (Empidæ.)

Locality: Prussian amber. Horizon: Ligurian.

1850. Loew.

Bernsteinfauna, 41 (undescr.).

1856. Giebel.

Insect. d. vorwelt, 208 (undescr.). 
5620. Rhamphomyia ungulina. (Empidæ.)

1850. Loew.

Locality : Prussian amber Horizon: Ligurian.

1856. Giebel.

Bernsteinfauna, 41.

Insect. d. vorwelt, 208.

\section{Rhingia - - (Syrphidæ.)}

Locality : Aix, France. Horizon : Ligurian.

1847. Hope.

Trans, ent. soc. Lond., iv : 252.

\section{Rhingia -. (Syrphidø.)}

Locality: Florissant, Colo. Horizon : Oligocene.

1886. Williston. Syn. N. A. Syrphidae, 282.

5623. Rhipidia extincta. (Tipulidæ.)

Locality: Radoboj, Croatia. Horízon: Mayencian.

1841. Unger. Verhandl. leop.-carol. akad. naturf., xix: 425. Pl. 71, fig. 1.

1841. " Foss. insect., 13. Pl. 71, fig. 1.

1849. Heer. Insektenf. tertiärg. Oeningen, ii : 196. Pl. 14, fig. 17.

1850. " Nene denkschr. allg. schweiz. gesellsch. wiss., xi : 196. $P l$. 14, fig. 17.

1852. Giebel. Deutschl. petref., 640.

1856. " Insect. d. vorwelt, 243.

1885. Scudder. Zittel, Handb. palaeont, i, th. ii : 809. Fig. 1081.

1886. " Z Zittel-Barrois, Traité de pal6́ont., ii: 811. Fig. 1098.

See also Limnobia extincta.

5624. Rhipidia maior. (Tipulidæ.)

Locality : Radoboj, Croatia. Horizon: Mayencian.

1841. Unger. Verhandl. leop.-carol. akad. naturf., xix: 425-426. $P l .71, f i g .2$. 1841. " Foss. insecten, 13. Pl. 71, fig. 2.

See also Tipula ungeri, etc.

\section{Rhipidia picta. (Tipulidæ.)}

Locality : Radoboj, Croatia. Horizon: Mayencian.

1849. Heer. Insektenf. tertiärg. Oeningen, ii : 197. Pl. 14, fig. 18.

1850. " Neue denkschr. allg. schweiz. gesellsch. wiss., xi : 197. Pl. 14, fig. 18.

1852. Giebel. Deutschl. petref., 640.

1856. " Insect. d. vorwelt, 243.

See also Limnobia picta.

5626. Rhipidia propinqua. (Tipulidæ.)

Locality: Radoboj, Croatia. Horizon: Mayencian.

1849. Heer. Insektenf. tertiärg. Oeningen, ii : 198. Pl. 14, fig. 19.

1852. Giebel. Dentschl. petref., 640.

1856. " Insect. d. vorwelt, 243.

See also Limnobia propinqua.

5627. Rhyphus - (Rhyphidæ.)

Locality: Prussian amber. Horizon: Ligurian.

1845. Berendt. Bernst. befindl. organ. reste vorw., i: 57.

1850. Loew. Bernsteinfauna, 39 (4 sp.). 
5628. Rhyphus - - (Rhyphidæ.)

Locality: Sicilian amber. Horizon : - .

1838. Guérin. Rev. zool., 1838: 170 (Ryphus). Pl. 1, fig. 14.

5629. Rhyphus maculatus. (Rhyphidæ.)

Locality: Radoboj, Croatia. Horizon: Mayencian.

1849. Heer.

1852. Giebel.

1856. "

1885. Scudder.

1886. " "

Insektenf. tertiärg. Oeningen, ii : 208-209. Pl. 15, figs. 22, 23a, c. Deutsehl. petref., 641 .

Insect. r. vorwelt, 226.

Zittel, Handb. palaeont., i, th. ii : 809. Fig. 1080.

Zittel-Barrois, Traité de paléont., ii: 810. Fïg. 1097.

Rumphomyia —. See Rhamphomyia ——.

5630. Rymosia strangulata. (Mycetophilidæ.)

Locality: Green River, Wyo. Horizon: Oligocene.

1890. Scudder. Tert. ins. N. A., 590. Pl. 10, fig. 2.

Ryphus —. See Rhyphus

5631. Sackenia -. (Mycetophilidæ.)

Locality: Green River, Wyo. Horizon: Oligocene.

1878. Scudder. Bull. U. S. geol. surv. terr., iv: 750.

1990. " Tert. ins. N. A., 596-597.

5632. Sackenia? - (Mycetophilidø.)

Locality: White River, Utah. Horizon: Oligocene.

1890. Scudder. Tert. ins. N. A., 596. Pl. 5, fig. 50.

5633. Sackenia arcuata. (Mycetophilidæ.)

Locality: White River, Colo. Horizon : Oligocene.

187\%. Scudder.

1885. " "

Bull. U. S. geol. surv. terr., iii : 754-755.

1886. " "

1890. " "

Zittel, Handb. palaeont., i, th.ii : 811. Fig. 1088.

Zittel-Barrois, Traité de paléont., ii : 813. Fig. 1105.

Tert. ins. N. A., 595-596. Pl. 5, figs. 3, 4, 12, 13.

5634. Sapromyza - (Sapromyzidæ.)

1850. Loew.

Locality : Prussian amber. Horizon: Ligurian.

Bernsteinfauna, 43.

5635. Sargus (vic.) —. (Stratiomyidæ.)

Locality: Aix, France. Horizon: Ligurian.

1829. Curtis. Edinb. new phil. journ., vii: 296. Pl. 6, fig. 12.

1829. " Murchison-Lyell, Tert. form. Aix, 12. Pl. 6, fig. 12.

5636. Sargus - -. (Stratiomyidæ.)

Locality: Aix, France. Horizon: Ligurian.

1829. Serres, Géogn. terrains tert., 232. 
5637. Scatophaga -. (Cordyluridæ.)

Locality: Prussian amber. Horizon: Ligurian.

1832. Burmeister. Handb. ent., i: 636.

1836. " Man. ent., 578.

Scatops —- See Scatopse - Serres.

5638. Scatopse —. (Bibionidæ.)

Locality : Prussian amber. Horizon : Ligurian.

1845. Berendt. Bernst. befindl, organ. reste vorw., i : 57.

1850. Loew.

Bernsteinfauna, 39 (3 sp.).

5639. Scatopse —. (Bibionidæ.)

Locality : Aix, France. Horizon: Ligurian.

1829. Serres. Géogn. terrains tert., 231 (Scatops).

5640. Sciara —. (Mycetophilidæ.)

Locality : Prussian amber. Horizon : Ligurian.

1845. Berendt. Bernst. befindl, organ. reste vorw., i: $5 \%$.

1850. Loew.

Bernsteinfauna, 33 (21 sp.).

5641 Sciara -. (Mycetophilidæ.)

Locality : Prussian amber. Horizon : Ligurian.

1832. Burmeister. Handb. ent., i: 637.

1836. " Man. ent., 578.

5642. Sciara -. (Mycetophilidæ.)

Locality: Rott, Rhenish Prussia. Horizon: Aquitanian.

1870. L. Heyden. Foss. dipt. Rott, 7, No. 6.

1870. "6 Palaeontogr., xvii: 243 , No. 6.

5643. Sciara - (Mycetophilidæ.)

Locality: Rott, Rhenish Prussia. Horizon: Aquitanian.

1870. L. Heyden. Foss. dipt. Rott, 7, No. 7.

1870. “ Palaeontogr., xvii: 243, No. 7.

5644. Sciara —. (Mycetophilidæ.)

Locality: Rott, Rhenish Prussia. Horizon: Aquitanian.

1870. L. Heyden. Foss. dipt. Rott, 7, No. 8.

1870.

Palaeontogr., xvii: 243, No. 8.

5645. Sciara —. (Mycetophilidæ.)

Compared with s. florilega.

Locality: Aix, France. Horizon: Ligurian.

1829. Serres. Gégn. terrains tert., 231 (Sciaris).

5646. Sciara acuminata. (Mycetophilidæ.)

Locality: Radoboj, Croatia.' Horizon: Mayencian.

1849. Heer. Insektenf. tertiärg. Oeningen, ii : 207-208. Pl. 15, fig. 20.

1852. Giebel. Deutschl. petref, 641.

1856. " Insect. $d$. vorwelt, 231.

See also Sciophila acuminata.

Bull. 71- 42 
5647. Sciara atavina. (Mycetophilidæ.)

Locality : Rott, Rhenish Prussia. Horizon: Aquitanian.

18\%0. L. Heyden. Foss. dipt. Rott, 5. Pl. 1, fig. 4. 1870.

Palaeontogr., xvii: 241. Pl. 44, fig. 4.

5648. Sciara defossa. (Mycetophilidæ.)

Locality : Rott, Rhenish Prussia. Horizon; Aquitanian.

1870. L. Heyden. Foss. dipt. Rott, 5. Pl. 1, fig. 3.

1870. " Palaeontogr., xvii: 241. Pl. 44, fig. 3.

5649. Sciara deleta. (Mycetophilidæ.)

Locality: Oeningen, Baden. Horizon: Tortonian.

1865. Heer.

Urwelt der Schweiz. Fig. 319 on $p .394$.

1872. " Monde prim. Suisse. Fig. 319 on p. 484.

1876. " Prim. world Switz., ii. Fig. 319 on p. 54.

1879. " Urwelt der Schweiz, $2^{\circ}$ aufl. Fig. 361 on $p .420$.

5650. Sciara deperdita. (Mycetophilidæ.)

Compared with S. ungulata, Europe.

Locality: Quesnel, British Columbia. Horizon:

1878. Scudder. Add. ins. fauua Quesnel, 1-2.

1878. " $\quad$ Rep. progr. geol. surv. Can., 1876--77: 457-458.

1878. “ Rapp. opér. comm. géol. Can., 1876-'77: 514-515.

1890. " Tert. ins. N. A., 586-587. Pl.3, fig. 17.

5651. Sciara hirtella. (Mycetophilidæ.)

Loeality: Radoboj, Croatia. Horizon: Mayencian.

1849. Heer. Insektenf. tertiärg. Oeningen, ii: 207. $P l .2, f i g .1 a^{* * * *} ; p l .15$, jig. 19.

1852. Giebel. Deutschl. petref., 641.

1856. " Insect. d. vorwelt, 231.

See also Sciophila hirtella.

5652. Sciara hirticornis. (Mycetophilidæ.)

$\begin{array}{ll}\text { 1850. Loew. } & \text { Bernsteinfauna, 34. } \\ \text { 1856. } " & \text { Insect. d. vorwelt, } 231 .\end{array}$

Locality: Prussian amber. Horizon : Ligurian.

5653. Sciara janassa. (Mycetophilidæ.)

Locality: Rott, Rhenish Prussia. Horizon: Aquitanian.

1870. L. Heyden. Foss. dipt. Rott, 4. Pl. 1, fig. 2.

1870. " Palaeontogr., xvii: 240. Pl. 44, fig. 2.

5654. Sciara martii. (Mycetophilidæ.)

Locality: Krottensee, Bohemia. Horizon: Aquitanian.

1877. Novak. $\quad$ Sitzungsb. k. akad. wiss. Wien, lxxvi: 89-90, Pl. 3, fig. 6,

1877. " Fauna egerer tertiärb., 19-20, Pl, 3, fig. 6. 
5655. Sciara minutula. (Mycetophilidæ.)

Localities: Radoboj, Croatia; Aix, France. Horizons: Mayencian, Ligurian. 1849. Heer. Insektenf. tertiärg. Oeningen, ii : 208. Pl. 15, fig. 21. 1852. Giebel. Dentschl. petref., 641. 1856. " Insect. d. vorwelt, 231. 1861. Saporta. Heer, Rech. climat pays tert., 153.

See also Sciophila minutula.

5656. Sciara rottensis. (Mycetophilidæ.)

Locality : Rott, Rhenish Prussia. Horizon : Aquitanian.

1870. L. Heyden. Foss. dipt. Rott, 6. Pl. 1, fig. 5.

1870. “ Palaeontogr., xvii : 242. Pl. 44, fig. 5.

5657. Sciara scopuli. (Mycetophilidæ.)

Locality : Green River, Wyo. Horizon: Oligocene.

1890. Scudder. Tert. ins. N. A., 588. Pl. 10, fig. 16.

5658. Sciara troglodytes. (Mycetophilidæ.)

Locality: Aix, France. Horizon: Ligurian.

1861. Heer. Saporta, Heer, Rech. climat pays tert., 153 (undescr.).

5659. Sciara winnertzii. (Mycetophilidæ.)

Locality : Rott, Rhenish Prussia. Horizon: Aquitanian.

1870. L. Heyden. Foss. dipt. Rott, 6-7. Pl. 1, fig. 6.

1870. " Palaeontogr., xvii : 243-243. Pl. 44, fig. 6.

Sciaris —. See Sciara —— Serres.

5660. Sciobia — (19 sp.). (Mycetophilidæ.)

Locality: Prussian amber. Horizon: Ligurian.

1850. Loew. Bernsteinfauna, 34.

5661. Sciobia peduncularis. (Mycetophilidæ.)

Locality: Prussian amber. Horizon: Ligurian.

1850. Loew. Bernsteinfauna, 34.

5662. Sciobia quadrangularis. (Mycetophilidæ.)

Locality: Prussian amber. Horizon: Ligurian.

1850. Loew. 'Berusteinfauna, 34.

5663. Sciobia spinosa. (Mycetophilidæ.)

Locality: Prussian amber. Horizon: Ligurian.

1850. Loew. Bernsteinfauna, 34.

5664. Sciomyza —- (Sciomyzidæ.)

Locality : Green River, Wyo. Horizon: Oligocene.

1890. Scudder. Tert. ins. N.A., 546. Pl. 10, fig. 5 .

5665. Sciomyza? disjecta. (Sciomyzidæ.)

Locality: Green River, Wyo. Horizon: Oligocene.

1878. Scudder. Bull. U. S. geol. surv. terr., iv: 758.

1890. " Tert. ins. N. A., 546. Pl. 9, figs. 7, 22, 25, 30, 32, 33. 
5666. Sciomyza ? manca. (Sciomyzidæ.)

Locality: Green River, Wyo. Horizon: Oligocene.

1878. Seudder. Bull. U. S. geol. surv. terr., iv : 756-758.

1890. 6

Tert. ins. N. A., 543-545. Pl. 4, fig. $9 ;$ pl. 9, figs. 1-6, 15, 16, 18, $20,23,24,28,29$.

5667. Sciomyza revelata. (Sciomyzidæ.)

Locality: Quesnel, British Columbia. Horizon:

1877. Scudder.

1877. 66

1877. "

1878. " "

1878. “

1878. "

1890. " "
Rep. progr. geol. surv. Can., 1875-'76 : 275-276.

Ins. tert. Quesnel, 10-11.

Rapp. opér. comm. gøol. Can., 1875-'76: 305-306.

Add. ins. fauna Quesnel, 2-3.

Rep. progr. geol, surv. Can., 1876-'77: 458-459.

Rapp. opér. comm. géol. Can., 1876-'77: 515-516.

Tert. ins. N. A., 542-543. Pl. 3, figs. 3-6.

5668. Sciophila —. (Mycetophilidæ.)

Locality : Prussian amber. Horizon: Ligurian.

1845. Berendt. Bernst. befindl. organ. reste vorw., i: 57.

1850. Loew.

5669. Sciophila acuminata. (Mycetophilidæ.)

Locality : Radoboj, Croatia. Horizon: Mayencian.

1852. Giebel. Deutschl. petref., 641.

See also Sciara acuminata.

5670. Sciophila atra. (Mycetophilidæ.)

Locality : Prussian amber. Horizon: Ligurian.

1856. Giebel. Insect. d. vorwelt, 237.

5671. Sciophila dilatata. (Mycetophilidæ.)

1850. Loew.

Locality: Prussian amber. Horizon: Ligurian.

1856. Giebel.

Berusteinfauna, 34 .

Insect. d. vorwelt, 237.

5672. Sciophila hirtella. (Mycetophilidæ.)

Locality: Radoboj, Croatia. Horizon: Mayencian.

1852. Giebel. Deutschl. petref., 641.

See also Sciara hirtella.

5673. Sciophila hyattii. (Mycetophilidæ.)

Locality : Green River, Wyo. Horizon: Oligocene.

1890. Scudder, Tert, ins. N. A., 597-598. Pl. 10, fig. 6 .

5674. Sciophila loewi. (Mycetophilidæ.)

Locality: Prussian amber. Horizon: Ligurian.

2856. Giebel. Insect. d. vorwelt, 236.

5675. Sciophila minutula. (Mycetophilidæ.)

Locality: Radoboj, Croatia. Horizon: Mayencian.

1852. Giebel.

Deutschl, petref., 641.

See also Sciara minutula. 
5676. Sciophila socialis. (Mycetophilidæ.)

1856. Giebel.

Locality : Prussian amber.' Horizon : Ligurian.

Insect. d. vorwelt, 236-237.

5677. Sciophila tenera. (Mycetophilidæ.)

Locality: Prussian amber. Horizon: Ligurian.

1850. Loew. Bernsteinfauna, 34 (undescr.).

1856. Giebel.

Insect, d. vorwelt, 237 (undescr.).

5678. Sciophila vetusta. (Mycetophilidæ.)

Locality: Parschlug, Styria. Horizon: Tortonian.

1849. Heer. Insektenf. tertiärg. Oeningen, ii : 206-207. Pl. 15, fig. 27.

1852. Giebel. Deutschl. petref., 641.

1856. " Insect. d. vorwelt, 236.

5679. Sendelia mirabilis. (Chironomidæ.)

Locality: Prussian amber. Horizon: Ligurian.

1868. Duisburg. Schrift. k. phys.-ökon. gesellsch. Königsb., ix : 23.

5680. Silvius - (Tabanidæ.)

Locality : Prussian amber. Horizon: Ligurian.

1845. Berendt. Berust. befindl, organ, reste vorw., i: 57.

5681. Silvius laticornis. (Tabanidæ.)

Locality: Prnssian amber. Horizon: Ligurian.

1850. Loew.

Berusteinfauna, 40 (undeser.).

1856. Giebel.

Insect. d. vorwelt, 215 (undescr.).

Simulia Berendt, Foerster, Heyden.

Simulia pasithea. See Simulium pasithea.

5682. Simulium (Simulidæ.)

Locality: Prussian amber. Horizon: Ligurian.

1845. Berendt. 1850. Loew.

Bernstein. befindl. organ. reste vorw., i : 57 (Simulia). Bernsteinfauna, 38 ( 6 sp.) (Simulia).

5683. Simulium -. (Simulidæ.)

Locality: Brunstatt, Alsatia. Horizon: Middle Oligocene. 1890. Foerster. In litt. (Simulia).

5684. Simulium - (Simulidæ.)

Locality : Sicilian amber. Horizon:

1838. Guérin. Rev. zool., 1838: 170. Pl. 1, fig. 13.

5685. Simulium —_. (Simulidæ.)

Locality : Rott, Rhenish Prussia. Horizon : Aquitanian.

1870. L. Heyden. Foss. dipt. Rott, 3 (Simulia).

1870. 66 Palaeontogr., xvii : 239 (Simulia). 
5686. Simulium pasithea. (Simulidre.)

Locality: Rott, Rhenish Prussia. Horizon: Aqnitanian.

1870. L. Heyden. Foss. dipt. Rott, 2-3 (Simulia). Pl.1, fig. 1.

1870. " Palaeontogr., xvii: 238-239 (Simulia). Pl. 44, fig. 1.

5687. Sphegina -. (Syrphidæ.)

Locality: Florissant, Colo. Horizon: Oligocene.

1886. Williston. Syn. N. A. Syrphidæ, 281.

5688. Spiladomyia simplex. (Tipulidæ.)

Locality : White River, Colo. Horizon: Oligocene.

1877. Scuddér. Bull. U. S. geol. surv. terr., iii: 750 .

1 90 . " Tert. ins. N. A., 573. Pl. 5, figs. 37, 38.

5689. Stenocinclis ? - (Asilidæ.)

Locality : Green River, Wșo. Horizon: Oligocene.

1890. Scudder. Tert. ins. N. A., 565. Pl. 10, fig. 15.

5690. Stenocinclis anomala. (Asilidæ.)

Locality: Green River, Wyo. Horizon: Oligocene.

1878. Seudder. Bull. U. S. geol. surv. terr, iv: 751-752.

1890. " Tert. ins. N. A., 564-565. Pl. 9, fig. 10.

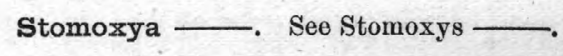

5691. Stomoxys - (Muscidæ.)

Locality : - Horizon: -

1846. Giebel. Paläozool., 278 (Stomoxya).

5692. Stratiomys - (larva). (Stratiomyida.)

Locality: Bernouville, Eure, France. Horizon : Quaternary.

1880. Brongniart. Note tufs Bernouv., 2.

1080. " Bull. soc. géol. France, 1880: 419.

5693. Stratiomys ——. (Stratiomyidæ.)

Locality : - Horizon :

1846. Giebel. Paläozool., 277.

5694. Stratiomys? - (larva). (Stratiomyidæ.)

Locality: Rott, Rhenish Prussia. Horizou : Aquitanian.

1870. L. Heyden. Foss. dipt. Rott, 28. Pl. 2, fig. 31.

1870. " Palaeontogr., xvii: 254. Pl. 45, fig. 31.

5695. Stratiomys heberti. (Stratiomyidæ.)

Locality : Pontary, Auvergne, France. Horizon : Aquitanian.

1870. Oustalet. Ann. se. géol., ii, art. 3: 156-157. Pl. 6, figs. 11-14.

Styringia —. See Styringomyia ——.

5696. Styringomyia —. (Tipulidø.)

Locality: Prussian amber. Horizon: Ligurian.

1845. Berendt. Bernst. luefindl. organ. reste vorw., i: 57 (Styringia). 
5697. Styringomyia gracilis. (Tipulidæ.)

Compared with S. venusta, Copal.

Locality : Prussian amber. Horizon: Ligurian.

1850. Loew.

Bernsteinfauna, 38 (undescr.).

1856. Giebel.

Insect. d. vorwelt, 247.

1861. Loew.

Dipt. fauna bernsteins, 6,8 .

1861. “

Ber. versamml. deutsch. naturf., xxxv : 91, 93.

1869. “

Dipt. amber, 7,11.

5698. Syrphus - (2 sp.). (Syrphidæ.)

Locality : Brunstatt, Alsatia. Horizon: Middle Oligocene.

1890. Foerster. In litt.

5699. Syrphus —. (Syrphidø.)

Locality: Rott, Rhenish Prussia. Horizon: Aquitanian.

1870. L. Heyden. Foss. dipt. Rott, 27 (No. 2). Fig.

1870. " Palaeontogr., xvii : 263 (No. 2). Fig.

5700. Syrphus —_. (Syrphidæ.)

Locality; Green River, Wyo. Horizon : Oligocene.

1878. Scudder. Bull. U. S. geol. surv. terr., iv: 755.

1890. " Tert. ins. N. A., 559.

\section{Syrphus - (Syrphidæ.)}

Locality : Florissant, Colo. Horizon : Oligocene.

1886. Williston. Syn. N. A. Syrphidæ, 282.

5702. Syrphus bremii. (Syrphidæ.)

Locality: Oeningen, Baden. Horizon: Tortonian.

1865. Heer.

1872. "6

1876. “

1879. "6

Urwelt der Schweiz. Fig. 314 on p. 394.

Monde prime Suisse. Fig. 314 on p. 484.

Prim. world Switz., ii. Fig. 314 on $p .54$.

Urwelt der Schweiz, $2^{\text {e }}$ aufl. Fig. 356 on $p .420$.

5703. Syrphus euphemus. (Syrphidæ.)

Locality: Rott, Rhenish Prussia. Horizon: Aquitanian.

1870. L. Heyden. Foss. dipt. Rott, 26. Pl. 2, fig. 29.

1870. " Palaeontogr., xvii: 262. Pl. 45, fig. 29.

5704. Syrphus freyeri. (Syrphidæ.)

Localities: Radoboj, Croatia; Senigaglia, Italj. Horizon: Mayencian. 1849. Heer. Insektenf. tertiärg. Oeningen, ii : 244-245. Pl. 17, fig. 12.

1852. Giebel. Deutschl. petref., 642.

1856. “ Insect. d. vorwelt, 200.

1859. Scarabelli. Mass.-Scarab., Stud. flora foss. Senig., 25.

5705. Syrphus geminatus. (Syrphidæ.)

Locality: Radoboj, Croatia. Horizon: Mayencian.

1849. Heer. Insektenf. tertiärg. Oeningen, ii : 245-246. Pl. 17, fig. 13.

1852. Giebel. Deutschl. petref., 642.

1856. "6 Insect. d. vorwelt 201. 
5706. Syrphus haidingeri. (Syrphidæ.)

Locality : Radoboj, Croatia. Horizon: Mayencian.

1849. Heer.

1852. Giebel.

1849. Heer.

1852. Giebel.

1856. “

1885. Scudder.

1886. " "
1856. "6

Insektenf., tertiäry. Oeningan, ii : 243-244. $\quad \mathrm{Pl} .17, \mathrm{fig} .11$.

Deutschl. petref., 642.

Insect. d. vorwelt, 200.

\section{Syrphus infumatus. (Syrphidæ.)}

Locality: Radoboj, Croatia. Horizon: Mayencian.

Insektenf. tertiärg. Oeningen, ii : 246. $\quad P l .17$, fig. 14.

Deutschl. petref., 64\%.

Insect. $d$. vorwelt, 201.

Zittel, Handb. palaeont., i, th. ii : E07. Fig. 1073.

Zittel-Barrois, Traité de paléont., ii : 807. Fig. 1090.

5708. Syrphus schellenbergi. (Syrphidø.)

Locality : Oeningen, Baden. Horizon: Tortonian.

1865. Heer.

1872. "

1876. “

1879. "
Urwelt der Schweiz. Fig. 315 on $p .394$.

Monde prim. Suisse. Fig. 315 on p. 484.

Prim. world Switz., ii. Fig. 315 on $p .54$.

Urwelt der Schweiz, 2e anfl. Fig. 357 on p. 420.

5709. Tabanus -. (Tabanidæ.)

Locality : Rhine, Germany. Horizon : Aquitanian ?.

1831. Goldfuss. Verhandl. leop.-carol. akad. naturf., vii, i: 118.

5710. Tabanus -. (Tabanidæ.)

Locality : Prussian amber. Horizon: Ligurian.

1834. Keferstein. Naturg. erdkörp., ii : 337 (as of Berendt, but erroneously).

5711. Tabanus —. (Tabanidæ.)

Locality: Aix, France. Horizon: Ligurian.

1829. Serres. G6́ogn. terrains tert., 232.

5712. Tachina - (Tàchinidæ.)

Locality : Prussian amber. Horizon: Ligurian.

1831. Burmeister. Oken, Isis, 1831 : 1100 (Tachinns).

5713. Tachina - (Tachinidæ.)

Locality: Oeningen, Baden. Horizon: Tortonian.

1888. Schöberlin. Soc. entom., iii : 68.

\section{Tachina —. (Tachinidæ.)}

Locality: Green River, Wyo. Horizon: Oligocene.

1878. Scudder. Bull. U. S. geol. surv. terr., iv: 756.

1890. " $\quad$ Tert. ins. N. A., 554-555.

\section{Tachina - (Tachinidæ.)}

Locality: Prussian amber. Horizon: Ligurian.

1863. Smith. 
5716. Tachina succini. (Tachinidæ.)

Compared with T. fastuosa, etc.

Locality: Prussian amber. Horizon: Ligurian.

1862. Giebel. Zeitschr. ges. naturw., xx : 319.

\section{Tachinus - See Tachina — Burmeister, Smith.}

\section{Tachydromia -. (Empidæ.)}

Locality : Prussian amber. Horizon: Ligurian.

1845. Berendt. Bernst. befindl. organ. reste vorw., i: 57.

5718. Tachydromia -. (Empidæ.)

Locality: Prussian amber. Horizon: Ligurian.

1832. Burmeister. Handb. ent., i: 636.

1836. " Man. ent., 578.

5719. Tachydromia - (7 sp.). (Empidæ.)

Locality : Prussian amber. Horizon: Ligurian.

1850. Loew. Bernsteinfanna, 42.

5720. Tachypeza - (5 sp.). (Empidø.)

Locality: Prussian amber. Horizon : Ligurian.

1850. Loew. Bernsteinfauna, 41-42.

5721. Tanymera - (4 sp.). (Tipulidæ.)

Locality: Prassian amber. Horizon: Ligurian.

1850. Loew. Bernsteinfauna, 36, 38.

5722. Tanypus -. (Chironomidæ.)

Locality: Prussian amber. Horizon: Ligurian.

1845. Berendt. Bernst. befindl. organ. reste vorw., i: 57.

1850. Loew. Bernsteinfauna, 29 (7 sp.).

5723. Tanypus -. (Chironomidæ.)

Locality : Prussian amber. Horizon : Ligurian.

1832. Burmeister. Handb, ent., i: 637 .

1836. " Man. ent., 578.

5724. Tanysphyra - (Tipulidæ.)

Locality : Prussian amber. Horizon : Ligurian.

1845. Berendt. Bernst. befindl. organ. reste vorw., i: 57.

5725. Tanysphyra gracilis. (Tipulidæ.)

1850. Loew.

Locality: Prussian amber. Horizon: Ligurian.

1856. Giebel.

Bernsteinfauna, 38.

Insect. d. vorwelt, 247. 
5726. Tephritis antiqua. (Trypetidæ.)

Locality: Radoboj, Croatia. Horizon: Mayencian.

1849 Heer. Insektenf. tertiärg. Oeningen, ii : 252-253. Pl. 17, fig. 15.

1852. Giebel. Deutschl. petref., 643.

See also Trypeta antiqua.

5727. Tetanocera - (2 sp.). (Seiomyzidæ.)

Locality : Brunstatt, Alsatia. Horizon: Middle Oligocene.

1890. Foerster. In litt.

Thereua pinguis. See Thereva pinguis.

5728. Thereva -. (Therevidæ.)

Locality: Prussian amber. Horizon: Ligurian.

1845. Berendt. Bernst. befindl. organ. reste vorw., i: 57.

1850. Loew. Bernsteinfauna, 40 (3 sp.).

5729. Thereva carbonum. ('Therevidæ.)

Locality: Wilhelmsfund, Nasssu. Horizon: "Braunkohl."

1856. Heyden. Palaeontogr., iv: 200-201. Pl. 37, fig.6.

5730. Thereva pinguis. (Therevidæ.)

Locality : Prussian amber. Horizon: Ligurian.

18:0. Loew. Bernsteinfauna, 40 (Thereua).

1856. Giebel. Insect. d. vorwelt, 214.

5731. Thirza naumanni. (Empidæ.)

Locality : Prussian amber. Horizon: Ligurian.

1856. Giebel. Insect. d. vorwelt, 210.

5732. Tipula —. (Tipulidæ.)

Locality : Prussian amber. Horizon: Ligurian.

1845. Berendt. Bernst. befindl. organ. reste rorw., i: 57.

1850. Loew. Bernsteinfauna, 36-37 (16 sp.).

5733. Tipula —. (Tipulidæ.)

Compared with $\mathrm{T}$. pratensis.

Locality: Prussian amber. Horizon: Ligurian.

1831. Burmeister. Oken, Isis, 1831: 1100.

1832. " " Handb. eut., i: 637.

1836. " $\quad$ Man. ent., 578.

5734. Tipula -. (Tipulidæ.)

Locality: Prussian amber. Horizon: Ligurian.

1822. Defrance. Dict. se. nat., xxiii : 524 (Tipules).

5735. Tipula $=(2$ sp.). (Tipulidæ.)

Locality: Brunstatt, Alsatia. Horizon : Middle Oligocene.

1890. Foerster. In $l^{*}$ tt. 
5736. Tipula —. (Tipulidæ.)

Locality : Sicilian amber. Horizon: -

1838. Guérin. Rev. zool., 1838: 170. Pl. 1, fig. 18.

5737. Tipula? - (larva). (Tipulidæ.)

Loca.ity: Sieblos, Rhenish Prussia. Horizon: Aquitanian.

1862. Heyden. Palaeontogr., v: 119-120. Pl. 23, fig. 19.

\section{Tipula ? - (Tipulidæ.)}

Compared with T. rivosa.

Locality : Aix, France. Horizon: Ligurian.

1847. Hope. Trans, ent. soc. Lond., iv : 253.

$$
\text { 5739. Tipula —. (Tipulidæ.) }
$$

Locality: Prussian amber. Horizon: Ligurian.

18\%0. Schlotheim. Petrefactenk., 43.

\section{Tipula - (Tipulidæ.)}

Compared with T. ochracea.

Locality: Oeningen, Baden. Horizon: Tortonian.

1888. Schöberlin. Soc. entom., iii : 69.

\section{Tipula -. (Tipulidæ.)}

Locality : Prussian amber. Horizon: Ligurian.

1742. Sendel. Hist. succ., 32, 66-68, 70, 76, 78, 260. Pl. 1, fig. 8; pl. 2, figs. 1-3, $5-7,11,12,14,16 ; p l .6, f i g .34 ; p l .7$, figs. 2-3.

1825. Guérin. Dict. class. hist. nat., viii: 580 . (Tipule).

1829. Serres. Géogn. terrains tert., 242.

5742. Tipula æmula. (Típulidæ.)

Locality: Radoboj, Croatia. Horizon: Mayencian.

1849. Heer.

1850. "6

Insektenf. tertiärg. Oeningen, ii : 193. Pl. 15, fig. 2.

1852. Giebel.

Neue denkschr. allg. schweiz. gesellsch., xi: 193. Pl. 15, fig. 2.

1856. 6

Deutschl. petref., 640 .

Insect. d. vorwelt, 240 .

5743. Tipula angustata. (Tipulidæ.)

Locality: Krottensee, Bohemia. Horizon: Aquitanian.

1877. Novák. Sitzungsb. k. akad. wiss. Wien, lxxvi: 87-88. Pl. 1, fig. 1.

1877. " Fauna egerer tertiärb., 17-18. Pl.1, fig.1.

5744. Tipula antiqua. (Tipulidæ.)

Locality : Prussian amber. Horizon : Ligurian.

1822. Presl. Del. prag., i : 202-203.

5745. Tipula brevirostris. (Tipulidæ.)

Locality: Prussian amber. Horizon: Ligurian.

1850. Loew.

Bernsteinfauna, 37 (undeser.).

1856. Giebel.

Insect. d. vorwelt, 241 (undescr.). 
5746. Tipula curvicornis. (Tipulidæ.)

Locality: Prussian amber. Horizon: Ligurian.

1822. Presl.

Del. prag., i : 200-201.

5747. Tipula decrepita. (Tipulidæ.)

Localities: White River, Colorado and Utah. Horizon: Oligocene.

1877. Scudder. 1890.

1850. Loew. 1856. Giebel.
Bull. U. S. geol. surv. terr., iii : 752.

Tert. ins. N. A., 576-577. $P l .5$, figs. 56,57.

5748. Tipula eucera. (Tipulidæ.)

Locality: Prussian amber. Horizon: Ligurian.

Bernsteinfauna, 37 (undeser.).

Insect. $d$. vorwelt, 241 (undescr.).

5749. Tipula expectans. (Tipulidæ.)

Locality: Krottensee, Bohemia. Horizon: Aquitanian.

1877. Novák.

1877. “

Sitzungs. k. akad. wiss. Wien, lxxvi: 88. Pl. 1, fig. 2.

Fauna egerer tertiärb., 18. $\quad P l .1$, fig. 2.

5750. Tipula fusca. (Tipulidæ.)

Locality: Copal. Horizon: Recent.

1776. Bloch.

Beschäft. berl. gesellseh. naturf. freunde, ii: 175-176. Pl. 4, fig. 15.

5751. Tipula goliath. (Tipulidæ.)

Locality: Prussian amber. Horizon: Ligurian.

1850. Loew.

Bernsteinfauna, 37 (undeser.).

1856. Geibel.

Insect. d. vorwelt, 241 (undescr.).

5752. Tipula infernalis. (Tipulidæ.)

Locality: Aix, France. Horizon : Ligurian.

1861. Heer.

Saporta, Heer, Rech. climat pays tert., 153 (undescr.).

5753. Tipula lineata. (Tipulidæ.)

Localities: Radoboj, Croatia; Gabbro, Italy. Horizon: Mayencian.

1849. Heer.

1850. “6

1852. Giebel.

1856. “"

1878. Capellini.
Insektenkf, tertiärg. Oeningen, ii : 194-195. Pl. 15, fig. 4 .

Neue denkschr. schweiz. gesellsch. wiss., xi: 194-195. $P l .15$, fig. 4.

Deutschl. petref., 640 .

Insect. d. vorwelt, 241.

Atti accad. linc., (3), mem. sc. fis., ii : 285.

5754. Tipula maculipennis. (Tipulidæ.)

Locality: Radoboj, Croatia. Horizon: Mayencian.

1849. Heer.

1850. “

1852. Giebel.

1856. “6
Insektenf. tertiärg. Oeningen, ii : 191-193. Pl. 15, fig. 1.

Neue denkschr. schweiz. gesellsch. wiss., xi: 191-193. Pl. 15, fig. 1.

Deutschl. petref., 640 .

Insect. d. vorwelt, 240. 
5755. Tipula major. (Tipulidæ.)

Locality: Radoboj, Croatia. Horizon: Mayencian.

1856. Giebel. Insect. d. vorwelt, 241.

See also Tipula ungeri, etc.

5756. Tipula obtecta. (Tipulidø.)

Locality : Radoboj, Croatia. Horizon : Mayencian.

1849. Heer.

1850. “

1852. Giebel.

1856. “

1822. Presl.
Insektenf. tertiärg. Oeningen, ii : $195 . \quad P l .15$, fig. 5.

Neue denkschr. schweiz. gesellsch. wiss., xi : 195. Pl. 15, fig. 5.

Deutschl. petref.; 640.

Insect. $d$. vorwelt, 241 .

5757. Tipula protogæa. (Tipulidæ.)

Locality: Prussian amber. Horizon: Ligurian.

Del. prag., i : 201-202.

5758. Tipula sepulchri. (Tipulidæ.)

Locality: Green River, Wyo. Horizon: Oligocene.

1890. Scudder. Tert. ins. N. A., 578. Pl. 10, fig. 1.

5759. Tipula spoliata. (Tipulidæ.)

Locality: Green River, Wyo. Horizon: Oligocene. 1890. Scudder. Tert. ins. N. A., 577-578. Pl. 10, fig. 4 .

5760. Tipùla tecta. (Tipulidæ.)

Locality : White River, Utah. Horizon: Oligocene.

1877. Seudeler. Bull. U. S. geol. snrv. térr., iii : 752-753.

1890. " Tert. ins. N. A., 577. Pl. 5, figs. 46, 47.

5761. Tipula ungeri. (Tipulidæ.)

Locality: Radoboj, Croatia. Horizon: Mayencian.

1849. Heer. Insektenf. tertiärg. Oeningen, ii : 195-196. Pl. 15, fig. 6.

1852. Giebel. Deutschl. petref., 640.

See also Rhipidia major, Tipula major.

5762. Tipula varia. (Tipulidæ.)

Locality: Radoboj, Croatia. Horizon: Mayencian.

1849. Heer.

1850. “

Insektenf. tertiärg. Oeningen, ii : 193-194. . Pl. 15, fig. 3.

Neue denkschr. schweiz. gesellsch. wiss., xi: 193-194. Pl. 15, fig. 3.

1852. Giebel.

1867. "6

Deutschl. petref., 640 .

Insect. d. vorwelt, 240-241.

5763. Tipula zignoi. (Tipulidæ.)

Locality: Chiavon, Italy. Horizon:

1886. Omboni. Alc. ins. foss. Ven., 8. Pl. 3, fig. 12.

1886. " Atti r. ist. ven., (6), iv: 1428. Pl. 3, fig. 12.

5764. Toxorhina -. (Tipulidæ.)

Locality: Prussian amber. Horizon: Ligurian.

1845. Berendt. Bernst. befindl, organ. reste vorw., i: 57. 
5765. Toxorhina brevipalpa. (Tipulidæ.)

Locality : Prussian amber. Horizon : Ligurian.

1850. Loew.

1851. “ “

1856. Giebel.

1859. Osten Sacken. Proc. acad. nat. sc. Philad., 1859: 221.

1860 .

1869.
Bernsteinfauna, 37 (undeser.).

Linn. entom., v : 400-401. Pl.2, fig. 21.

Insect. d. vorwelt, 242. =Limnobiorhynchus.)

Proc. acad. nat. sc. Philad., 1860: 17. (No spec. mentioned,

Monogr. dipt. N. A., iv: 107-109. (No spec. mentioned, = Elephantomyia.)

\section{Toxorhina longirostris. (Tipulidæ.)}

Locality: Prussian amber. Horizon: Ligurian.

1850. Loew.

1851. “

1856. Giebel.

1859. Osten Sacken. Proc. acad. nat. sc. Philad., 1859: 221.

1860.

“

1869.

66

1850. Loew.

1851. "6

1856. Giebel.

1859. Osten Sacken. Proc. acad. nat. sc. Philad., 1859: 221.

1860.

66

1869.

66

1850. Loew.

1829. Serres.

1845. Berendt. 1850. Loew.

1850. Loew.

1856. Giebel.

Bernsteinfauna, 37 (undescr.).

Linn. entom., v : 400. Pl. 2, figs. 20, 23.

Insect. d. vorwelt, 242. =Limnobiorhynchus.) phantomyia.)

Bernsteinfauna, 37 (undescr.).

Linn. entom., v: 400. Pl.2, fig. 19.

Insect. d. vorwelt, 242 . = Limnobiorhynchus.) phantomyia.) Bernsteinfauna, 37.

G6ogn. terrains tert., 231.

5770. Trichoneura

Bernst. befindl. organ. reste vorw., i: 57.

Bernsteinfauna, 37-38 (3 sp.).

Bernsteinfauna, 37-38.

Insect. d: vorwelt, 246.
Proc. aca nat. sc. Philad,, 1860: 17. (No spec. mentioned,

Monogr. dipt. N. A., iv: 107-109. (No spec. mentioned, = Ele-

5767. Toxorhina pulchella. (Tipulidæ.)

Locality : Prussian amber. Horizon: Ligurian.

Proc. acad. nat. sc. Philad., 1860: 17. (No spec. mentioned,

Monogr. dipt. N. A., iv: 107-109. (No spec. mentioned, = Ele-

5768. Trichocera - (2 sp.). (Tipulidæ.)

Locality: Prussian amber. Horizon: Ligurian.

. 5769. Trichocera —. (Tipulidæ.)

Locality: Aix, France. Horizon : Ligurian.

(Tipnlidæ.)

Locality : Prussian amber. Horizon: Ligurian.

5771. Trichoneura vulgaris. (Tipulidæ.)

Locality: Prussian amber. Horizon: Ligurian. 
5772. Trichonta dawsoni. (Mycetophilidæ.)

Lc cality : Quesnel, British Columbia. Horizon :

1877. Scudder. Rep. progr. geol. surv. Can., 1675-76: 272.

1877. "6

1877 . "

1890. 6

Ins. tert. Quesnel, 7.

Rapp. opér. comm. géol. Can., 1875-'76: 301.

Tert. ins. N. A., 590. Pl. 3, figs. 12, 13.

5773. Trypeta antiqua. (Trypetidæ.)

Locality : Radoboj, Croatia. Horizon: Mayencian.

1852. Giebel. Dentschl. petref., 643 (Tyrpeta).

1856. " Insect. d. vorwelt, 195.

See also Tephritis antiqua.

Tyrpeta antiqua. See Trypeta antiqua.

5774. Xylophagus —- (Xylophagidæ.)

Compared with $\mathrm{X}$. ater.

Locality : Aix, France. Horizon: Ligurian.

1829. Serres. Géogn. terrains tert., 232.

5775. Xylophagus mengeanus. (Xylophagidæ.)

Locality : Prussian amber. Horizon: Ligurian.

1850. Loew. Bernsteinfauna, 39 (undescr.).

1856. Giebel. Insect. d. vorwelt, 204 (undescr.).

5776. Xylophagus pallidus. (Xylophagidæ.)

1856. Heer. Viert. naturf: gesellsch. Zürich, i: 36-37. Pl. 2, fig. 11.

1885. Scudder. Zittel, Handb. palaeont., i, th. ii : 809. Fig. 1079.

1886. " Zittel-Barrois, Traité de paléont., ii : 810. Fig. 1096.

5777. Zygoneura —. (Mycetophilidæ.)

Locality : Prussian amber. Horizon: Ligurian.

1850. Loew. Bernsteinfauna, 32 .

\section{IEPIDOPTERA.}

5778. - (larva).

Locality: Florissant, Colo. Horizon: Oligocene.

1886. Minot.

Arch, mikr. anat, xxviii : 46-47.

5779.

Locality : Sinigaglia, Italy. Horizon: (Helvetian §).

1842. Procaccini. Nuov. ann. sc. nat., vii : 449.

5780. (larva). (Lycænidæ.)

Compared with Papilio w-album.

Locality: Prussian amber. Horizon: Ligurian.

1835. Gravenhorst. Uebers. schles. gesellech, vaterl, cult., 1834 : 93. 
5781. (larva case). (Psychidæ.)

Locality : Prussian amber. Horizon : Ligurian.

1856. Menge. Progr. petrischule Danzig, 1856: 27-28, No. 1.

5782. (larva case). (Psychidæ.)

Locality: Prussian amber. Horizon: Ligurian.

1856. Menge. Progr. petrischule Danzig, 1856: 28, No. 2.

5783. (larva case). (Psychidæ.)

Locality : Prussian amber. Horizon : Ligurian. 1856. Menge. Progr. petrischule Danzig, 1856: 28, No. 3. 5784. (larva case). (Psychidæ.)

Locality : Prussian amber. Horizon : Ligurian. 1856. Menge. Progr. petrischule Danzig, 1856 : 28, No. 4. 5785. (larva case). (Psychidæ.)

Locality: Prussian amber. Horizon: Ligurian. 1856. Menge. Progr. petrischule Danzig, 1856 : 28, No. 5. 5786. (larva case). (Psychidæ.)

Locality : Prussian amber. Horizon: Ligurian. 1856. Menge. Progr. petrischule Danzig, 1856: 28, No. 6.

5787. (larva case). (Psychidæ.)

Locality : Prussian amber. Horizon: Ligurian. 1856. Menge. Progr. petrischule Danzig, 1856: 28, No. 7.

5788. (several). (Pyralidæ or Tortricidæ.)

Locality: Florissant, Colo. Horizon: Oligocene.

1881. Scudder. 1883. “

1881. Scudder. 1883. " "

1856. Menge.
Bull. U. S. geol. surv. terr., vi: 290.

Ann. rep. U. S. geol. surv. terr., xii : 280.

5789. (several). (Rhopalocera).

Locality : Florissant, Colo. Horizon : Oligocene. Bull. U. S. geol. surv. terr., vi : 290. Ann. rep. U. S. genl. surv. terr., xii : 280 .

5790. (Tineidæ.)

Locality : Prussian amber. Horizon : Ligurian. Progr. petrischule Danzig, 1856: 28-29.

5791. (Tortricidæ,)

Locality : Prussian amber. Horizon: Ligurian. 1856. Menge. Progr. petrischule Danzig, 1856: 29.

5792. (2 sp., pupæ). (Tortricidæ.)

Lncality : Prussian amber. Horizon: Ligurian. 1856. Menge.
Progr. petrischule Danzig, 1856 : 29. 
5793. (4 sp., larvæ). ('Tortricidæ.)

Locality: Prussian amber. Horizon: Ligurian.

1856. Menge. Progr. petrischule Danzig, 1856: 28-29.

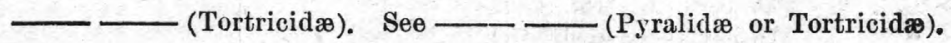

Angerona electrica. See Angerona electrina.

5794. Angerona electrina. (Phalænidæ.)

Locality: Prussian amber. Horizon: Ligurian.

1862. Giebel. Zeitschr. ges. naturw., $x x: 317$.

1885. Oppenheim. Berl. entom, zeitschr., xxix: 347 (electrica).

5795. Antirrhæa? sepulta. (Nymphalidæ.)

Locality: Aix, France. Horizon: Ligurian.

1871. Kirby. Synon. catal. diurn. lepid., 39.

1877. " Synon. catal. diurn. lepid., suppl., 698.

See also Cyllo sepulta, etc.

5796. Apanthesis —. (Nymphalidæ.)

Locality: Florissant, Colo. Horizon : Oligocene.

1889. Scudder. Butt. N. E., i : 758-759 (name only).

See also Apanthesis lence.

5797. Apanthesis leuce. (Nymphalidæ.)

Locality : Florissant, Colo. Horizon : Oligocene.

1889. Scudder. Ann. rep. U. S. geol. surv., viii : 461. Pl. 52, figs. 12-13.

See also Apanthesis

\section{Araschnia vetula. (Hesperidæ.)}

Locality : Rott, Rhenish Prussia. Horizon: Aquitanian.

1871. Kirby. Synon. catal. diurn. lepid., 179.

See also Vanessa vetula, Thanatites vetulus.

5799. Argynnis pluto. (Papilionidæ.)

Locality: Radoboj, Croatia. Horizon: Mayencian.

1868. Edwards. Butt. N.A., i, Argynnis, i. Fig.

1871. Kirby. Synon. catal, diurn. lepid., 155.

See also Vanessa pluto, etc.

5800. Barbarothea - (Nymphalidæ.)

Locality : Florissant, Colo. Horizon: Oligoceno.

1890. Scudder. Tert. ins. N. A., 29.

5801. Bombycites büichii. (Bombycidæ.)

Locality: Oeningen, Baden. Horizon : Tortonian.

1865. Heer. Urwelt der Schweiz, 397. Fig. 310 on p. 394.

1872. " Monde prim. Suisse, 486. Fig. 310 on p. 484.

1876. " Prim. world Switz., ii : 56. Fig. 310 on p. 54.

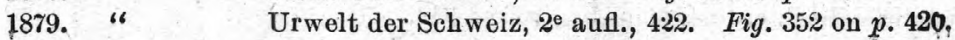

Bull. $71-43$ 
5802. Bombycites œningensis. (Bombycidœ.)

Locality : Oeningen, Baden. Horizon: Tortonian.

1849. Heer.

1852. Giebel.

1856. “

1865. Heer.

1872. "

1876. “

18і9. “

Insektenf. tertiärg. Oeningen, ii : 183-188. Pl. 14, fig. 7.

Dentschl. petref., 644.

Insect. d. vorwelt, 189.

Urwelt der Schweiz, 397.

Monde prim. Suisse, 486.

Prim. world Switz., ii : 56.

Urwelt der Schweiz, $2^{\mathrm{e}}$ aufl., 422.

1885. Oppenheim. Berl. entom. zeitschr., xxix : 349.

5803. Bombyx —. (Bombycidæ.)

Locality : Prussian amber. Horizon: Ligurian.

1742. Sendel. Hist. succin., 104, 116 (unnamed). $P l .3$, figs. 16, 17, 28.

1834. Keferstein. Naturg. erdkörp., ii : 333.

\section{Bombyx (or Cossus) —- (Bombycidæ.)}

Locality : Aix, France. Horizon: Ligurian.

1829. Serres. Gégn. terrains tert., 230 .

5805. Coliates proserpina. (Papilionidæ.)

Locality: Aix, France. Horizon: Ligurian.

1875. Scudder. Mem. Amer. assoc. adv. sc., i: 52-53. Pl. 2, fig. 5.

1877. Kirb5. Synon. catal. diurn. lepid., suppl., 801.

1885. Oppenheim. Berl. entom. zeitschr., xxix : 346.

\section{Cossus —. See Bombyx (or Cossus) $\longrightarrow$.}

\section{Cyllo? —. (Nymphalidæ.)}

Locality: Aix, France. Horizon: Ligurian.

1839. Boisduval. Ann. soc. ent. France, viii, bull. entom., 11-12.

See also Cyllo sepulta, etc.

5807. Cyllo sepulta. (Nymphalidæ.)

Locality: Aix, France. Horizon: Ligurian.

1840. Boisduval. Ann. soc. ent. France, ix: 371-374. Pl. 8.

1843. Serres.

Actes linn, soc. Bord., xiii : 172. Pl. 2 (ined.?).

1851. Lefebvre. Ann. soc. ent. France, (2), ix : 71-88. $\quad P l .3^{11}$.

1851. Boisduval. Ann. soc. ent. France, (2), ix, bull. ent., 96-98.

1851. Westwood. Donbleday-Westwood, Gen. diurn. Lepid., ii : 361.

1854. Pictet. Traité de paléont., ii : 393. Pl. 40, fig. 11.

1868. Butler. Catal. satyr. Brit. mus., 189-190.

1874. Oustalet. La nature, iii: 36. Fig.

See also Cyllo —_, Nymphalis —_, Antirrhæa sepulta, Neorinopis sepulta, Papilio (Satyrus)

5808. Eugonia atava. (Nymphalidæ.)

Locality : Radoboj, Croatia. Horizon: Mayencian.

1875. Scudder. Mem. Amer. assoc. adv.sc., i: 41-44. Pl. 1, figs.1, 3, 7,

1885. Oppenheim. Berl. entom, zeitschr., xxix: 348 ,

Seo also Sphinx atava, oto. 
5809. Junonia piuto. (Papilionidæ.)

Locality: Radoboj, Croatia. Horizon: Mayencian.

1873. Butler. Lepid. exot., 127-128. Pl. 48, fig. 7.

1873. " Geol.mag., x: 3-4. Pl.1, fig.7.

See also Vanessa pluto, etc.

5810. Jupiteria - (Nymphalidø.)

Locality: Florissant, Colo. Horizon: Oligocene.

1883. Seudder. Ann. rep. U. S. geol. surv. terr., xii : 280 (name only).

1885. " "Zittel, Handb. palaeont., i, th.ii : 814 (name only).

1886. " Zittel-Barrois, Traité de paléont., ii : 815 (name only).

1886. " Bull. U. S. geol. surv., No. 31 : 95 (name only).

See also Jupiteria charon.

5811. Jupiteria charon. (Nymphalidæ.)

Locality: Florissant, Colo. Horizon : Oligocene.

1889. Scudder. Ann. rep. U. S. geol surv., viii : 450-452. Pl. 52, figs. 14-15.

See also Jupiteria $\longrightarrow$.

Lethites renuesii. See Lethites reynesii.

5812. Iethites reynesii. (Nymphalidæ.)

Locality: Aix, France. Horizon: Ligurian.

1875. Scudder. Mem. Amer. assoc. adv. sc., i: 37-40. Pl. 1, figs. 2, 5.

1877. Kirby. Synon. catal. diurn. lepid., suppl,, 699.

1885. Oppenheim. Berl. entom. zeitschr., xxix : 346 (renuesii).

See also Satyrites reynesii.

5813. Lithopsyche -. (Nymphalidæ.)

Locality : Florissant, Colo. Horizon: Oligocene.

1883. Scudder. Ann. rep. U. S. geol. surv. terr., xii : 280 (name only).

1885. " "Zittel, Handb. palaeont., i, th. ii : 814 (name only).

1886. " " Zittel-Barrois, Traité de paléont., ii: 815 (name only).

1886. “ Bull. U. S. geol. surv., No. 31: 95 (name only).

See also Lithopsyche styx.

5814. Lithopsyche antiqua. (Phalænidæ.)

Compared with Calospira spp., Craspedosis, Præsos.

Locality: Gurnet Bay, Isle of Wight. Horizon: Bembridge Limestone.

1889. Butler. Proc, zool. soc. Lond., 1889: 294. Pl. 31, figs. 3, 6.

5815. Lithopsyche styx (Nymphalidæ.)

Locality: Florissant, Colo. Horizon : Oligocene.

1889. Scudder. Ann. rep. U.S.geol. surv., viii: 4544-457. Pl. 52, figs. 11, 16, 17.

See also Lithopsyche —_.

\section{Lithosia - (Bombycidæ.)}

Locality: Gurnet Bay, Isle of Wight. Horizon: Bembridge Limestone.

1879. Woodward. Quart. journ. geol. soc. Lond., Xxx : 344,

1879 , of Geol. mag, $(\mathrm{n}, \mathrm{s}),, \mathrm{v} ; 88$, 
5817. Macroglossa - (larva). (Sphingidæ.)

Compared with M. fuciformis.

Locality : Oeningen, Baden. Horizon: Tortonian.

1888. Schöberlin. Soc. entom., iii : 69.

5818. Mylothrites pluto. (Papilionidæ.)

Locality : Radoboj, Croatia. Horizon: Mayencian.

1875. Scudder. Mem. Amer. assoc. adv. sc., i: 45-51. Pl. 2, figs. 2,7, 15 ?, 17 ; fig.

.1 on $p .50$.

18i7. Kirby. Synon. catal. diurn. lepid., suppl., 793.

1885. Oppenheim. Berl. entom. zeitschr., xxix : 348.

See also Vanessa pluto, etc.

5819. Neorinopis sepulta. (Nymphalidæ.)

Locality: Aix, France. Horizon: Ligurian.

1873. Butler. Lepid. exot., 127. Pl. 48, fig. 3.

1873. " Geol. mag., $\mathrm{x}: 3 . \quad \mathrm{Pl} .1$, fig. 3.

1875. Scudder. Mem. Amer. assoc. adv. sc., i : 14-33. Pl. 1, figs. 8-17.

1885. Oppenheim. Berl, entom, zeitschr, xxix : 346.

See also Cyllo sepulta, etc.

5820. Nepticula fossilis (mine). (Tineidæ.)

Localities: Rott, Salzhausen, Germany. Horizon: Aquitanian.

1862. Heyden. Paleontogr., $\mathrm{x}:$ 77-78 (in leaf of Juglans acuminata Br.). Pl. 10, fig. 2.

1865. Heyd.-Heyd. Paleontogr., xiv: 35.

1885. Scudder. Zittel, Handl. palaeont., i, th. ii: 813. Fig. 1090.

1886. " Zittel-Barrois, Traité de paléont., ii : 815. Fig. 110\%.

5821. Noctuites deperditus. (Noctuidæ.)

Locality: Aix, France. Horizon : Ligurian.

1856. Heer. Viert. naturf. gesellsch. Zürich, i: $30 . \quad P l .2$, fig. 8.

1885. Oppenheim. Berl. entom, zeitschr., xxix : 346.

5822. Noctuites effosus. (Noctuidæ.)

Locality: Radoboj, Croatia. Horizon: Mayencian.

1849. Heer. Insektenf. tertiärg. Oeningen, ii : 185-186 (effosa). Pl.14, fig. 10.

1852. Giebel. Deutschl. petref., 644.

1856. " Insect. d. vorwelt, 190.

1885. Oppenheim. Berl. entom. zeitschr., xxix: 348.

5823. Noctuites haidingeri. (Noctuidæ.)

Locality : Radoboj, Croatia. Horizon: Mayenciàn.

1849. Heer. Insektenf, tertiärg. Oeningen, ii : $185 . \quad P l .14$, fig. 9.

1852. Giebel. Dentschl. petref., 644 .

1856. " Insect. d. vorwelt, 190.

1885. Oppenheim. Berl, entom. zeitschr., xxix : 348.

5824. Noctuites incertissimus. (Noctuidæ.)

Locality: Auvergne, France. Horizon: Aquitanian.

1870. Oustalet. Ann. sc. géol., ii, art. 3,158 (incertissima). Pl. 1, fig. 18.

1885. Oppenheim Berl, entom, zeitschr., xxix : 348-349. 
5825. Nymphalis?

(Nymphalidæ.)

Locality : Aix, France. Horizon: Ligurian.

1838. Duponchel. Ann. soc. ent. France, vii, bull. ent., 51-52.

See also Cyllo sepulta, etc.

5826. Nymphalis atava. (Nymphalidæ.)

Locality: Radoboj, Croatia. Horizon: Mayoncian

1877. Kirby. Synon. catal. diurn. lepid., suppl., 733.

See also Sphinx atava, etc.

5827. Nymphalis atavina. (Nymphalidæ.)

Locality : Radoboj, Croatia. Horizon: Mayencian.

1872. Kirby. Synon. catal. diurn. lepid., 648 (atovina).

See also Sphinx atava, etc.

Nymphalis atovina. See Nymphalis atavina.

5828. Nymphalites —. (Nymphalidæ.)

Locality: Florissant, Colo. Horizon: Oligocene.

1889. Seudder. Butt. N. Engl., i: 758 (name only).

See also Nymphalites obscurus.

5829. Nymphalites obscurus. (Nymphalidæ.)

Locality: Florissant, Colo. Horizon: Oligocene.

1889. Scudder. Ann. rep. U. S. geol. surv., viii : 457-459 (obscurum). $P l_{.} 53$, figs. 10-13.

See also Nymphalites

5830. Ocnerites macroceraticus. (Bombycidæ.)

Locality: Rott, Rhenish Prussia. Horizon: Aquitanian.

1885. Oppenheim. Berl. entom. zeitschr., xxix: 347. Pl. 12, fig. 15.

5831. Pamphilites abditus. (Hesperidæ.)

Locality: Aix, France. Horizon: Ligurian.

1875. Scudder. Mem. Amer. assoc. adv. sc., i : 68-69 (abdita). Pl. 3, figs. 14, 17, 18. 1877. Kirby. Synon. catal. diurn. lepid., suppl., 828.

1885. Oppenheim. Berl. entom. zeitschr., xxix : 346.

5832. Papilio (Satyrus) - (Nymphalidæ.)

Locality : Aix, France. Horizon: Ligurian.

1829. Serres. Géogn. terrains tert., 230.

See also Cyllo sepulta, etc.

5833. Phalæena? - (Phalænidæ.)

Locality : Aix, France. Horizon: Ligurian.

1829. Curtis. Edinb, new phil, journ., vii : 295.

1829. " Murchison-Lyell, Tert. form. Aix, 11.

5834. Phalæna geometra (recent). (Phalænidæ.)

Locality : Copal. Horizon : Recent.

1776. Bloch. Beschüft. berl. gesellsch. naturf. freunde, ii: 180-183. Pl. 5, fig. 22. 
5835. Phalænites crenatus. (Phalænidæ.)

Locality : Radoboj, Croatia. Horizon : Mayencian.

1849. Heer.

Insektenf. tertiärg. Oeningen, ii : 186-187 (crenata). Pl. 14, fig. 11.

1852. Giebel. Deutschl. petref., 644.

1856. " Insect. d. vorwelt, 190.

1885. Oppenheim. Berl. entom. zeitschr., xxix: 348.

5836. Phalænites obsoletus. (Phalænidæ.)

Locality: Radoboj, Croatia. Horizon: Mayencian.

1849. Heer.

Insektenf. tertiärg. Oeningen, ii: 187 (obsoleta). $\quad P l .14$, fig. 12.

1852. Giebel.

Deutschl. petref., 644 .

1856. " " Insect. d. vorwelt, 190-191.

1885. Oppehheim. Berl. entom. zeitschr., xxix: 348.

5837. Phalænites proserpinæ. (Phalænidæ.)

Locality: Aix, France. Horizon : Ligurian.

1861. Heer. Saporta, Heer, Rech, climat pays tert., 153 (undescr.).

5838. Pierites freyeri. (Papilionidæ.)

Locality : Radoboj, Croatia. Horizon: Mayencian.

1849. Heer. Insektenf. tertiärg. Oeningen, ii : 182-183. Pl. 14, fig. 6.

1850. " Nouv. mém soc. helv., xi: 182-183. Pl. 14, fig. 6.

1852. Giebel. Deutschl. petref., 644.

1856. " Insect. d. vorwelt, 187.

1877. Kirby. $\quad$ Synon. catal. diurn. lepid., suppl., 806

See also Pontia freyeri.

5839. Pontia freyeri. (Papilionidæ.)

Locality: Radoboj, Croatia. Horizon : Mayencian.

1875. Scudder. Mem. amer. assoc. adv. sc., i : 54-56. Pl. 2, figs. 16, 18. 1885. Oppenheim. Berl. entom, zeitschr., xxix : 348.

See also Pierites freyeri.

5840. Prodryas persephone. (Nymphalidæ.)

Locality: Florissant, Colo. Horizon: Oligocene.

1878. Scudder. 1880. Dawson. 1885. Scudder. 1886. " " 1889. "6 1889. " " 1890. Lesley.
Bull. U. S. geol. surv. terr., iv : 524-526.

Chain of life, 149. Fig. 131 on $p .150$.

Zittel, Handb. palaeont., i, th. ii : 814. Fig. 1092.

Zittel-Barrois, 'Traité de paléont., ii : 815. Fig. 1109.

Butt. N. E., i: 758, 760. 'Pl. 16, fig. 6.

Ann. rep. U. S. geol. surv., viii : 443-448. Pl. 52, fig. 1-10.

Dict. foss. Penns., ii : 746. Fig.

5841. Prolibythea vagabunda. (Nymphalidæ.)

Locality: Florissant, Colo. Horizon: Oligocene.

1889. Scudder. Butt. N. E., i: 759 (name only).

1889. 6

Ann. rep. U. S. geol. surv., viii : 465-467. Pl. 53, figs. 4-9.

5842. Psecadia mortuella. (Tineidæ.)

Locality: Florissant, Colo. Horizon: Oligocene.

1890. Scudder. Tert. ins. N. A., 603. Pl. 15, figs. 12, 17. 
Psyche pincella. See Psyche pineela.

5843. Psyche pineela. (Bombycidæ.)

1849. Heor. 1852. Giebel. 1856. "6 1865. Heer. 1872. " " 1876. “ 1879. “ 1885. Oppenheim. Berl. entom. zeitschr., xxix : 349.

Dentschl. petref., 644.

Insect. d. vorwelt. 189 (pincella).

Urwelt der Schweiz, 397.

Monde prim. Suisse, 486.

Prim. world Switz., ii : 56.

Urwelt der Schweiz, $2^{e}$ aufl., 422.

\section{Locality : Oeningen, Baden. Horizon: Tortonian.}

Insektenf. tertiärg. Oeningen, ii : 184-185. Pl. 14, fig. 8.

5844. Pyralites obscurus. (Pyralidæ.)

Locality : Aix, France. Horizon: Ligurian.

1856. Heer. Viert. naturf. gesellsch. Zürich, i: $30 . \quad P l .2$, fig. 6. 1885. Oppenheim. Berl, entom, zeitschr., xxix : 346.

Satyrites beynesii. See Satyrites reynesii.

5845. Satyrites incertus (larva). (Nymphalidø.)

Locality : Aix, France. Horizon : Ligurian.

1876. Daudet. Rev. mag. zool., (3), iv: 415-424. Pl. 17, figs. 1-4.

1876. " Petites nouv, entom., No. 145.

1885. Oppenheim. Berl. entom. zeitschr., xxix : 346.

1885. Scudder. Zittel, Handb. palaeont., i, th. ii: 814. Fig. 1091.

1886. " Zittel-Barrois, Tr̉aité de paléont., ii : 815. Fig. 1108.

5846. Satyrites reynesii. (Nymphalidæ.)

Locality : Aix, France. Horizon: Ligurian.

1872. Seudder. Rev. mag. zool., 1871-'72: 66-72. Pl. 7.

187\%. " "Descr. nouv. pap. foss., 1-7. Pl.

1872. "6 Geol. mag., ix: 532-533. Pl. 13, figs. 2, 3.

1872. " Descr. new foss. butt., 1-2. Pl., figs. 2, 3.

1873. Brodie. Distr. corr. foss. insects, 8-9 (beynesii).

See also Lethites reynesii.

\section{Satyrus —. See Papilio (Satyrus) -}

5847. Sesia —. (Sphingidæ.)

Compared with S. brosiformis.

Locality: Aix, France. Horizon: Ligurian.

1829. Serres. G6́ogn. terrains tert. 230.

\section{Sesia —. (Sphingidæ.)}

Compared with S. vespiformis.

Locality: Aix, France. Horizon: Ligurian.

1829. Serres. G6́ogn. terrains tert., 230.

5849. Sphinx —. (Sphingidæ.)

Locality: Prussian amber. Horizon: Ligurian.

1830. Berendt. Insekten im bernstein, 37. 


\section{Sphinx atava. (Nymphalidæ.)}

Locality : Radoboj, Croatia. Horizon: Mayencian.

1843. Charpentier. Verhandl. leop.-carol. deutsche akad., xx: 408-409. Pl. 2\&, fig.4. See also Vanessa atavina, Nymphalis atavina, N. atava, Eugonia atava.

\section{Stolopsyche}

\section{(Papilionidæ.)}

\section{Locality: Florissant, Colo. Horizon: Oligocene.}

1889. Scudder. Butt. N. Engl., i: 759 (name only).

See also Stolopsyche libytheoides.

5852. Stolopsyche libytheoides. (Papilionidæ.)

Locality: Florissant, Colo. Horizon: Oligocene. 1889. Scudder. Ann, rep. U. S. geol, surv., viii : 468. Pl. 53, figs. 1-3. See also Stolopsyche $\longrightarrow$.

5853. Thaites ruminianus. (Papilionidæ.)

Locality : Aix, France. Horizon: Ligurian.

1861. Saporta. Heer, Rech. climat pays tert., trad. Gandin, 153 (ruminiana) (without descr.).

1861. Heer.

Rech. climat pays tert., trad. Gaudin, 205 (without deser.).

1872. Saporta.

1872. “

Anv. sc. nat., (5), bot., xv: 343 (without descr.).

Rev. flore gypses d'Aix, i: 70 (without deser.).

1875. scudder.

1877. Kirby.

Mem. amer. assoc. adv, sc., i : 60-62. Pl. 3, figs. 1, 3, 6-10.

1879. Strecker.

Synon. catal. diurn. lepid., suppl., 809.

1885. Oppenheim. Berl. entom. zeitschr., xxix: 346.

Thanatites juvenalis. See Thanatites vetulus.

5854. Thanatites vetulus. (Hesperidæ.)

Locality : Rott, Rbenish Prussia. Horizon: Aquitanian.

1875. Scudder. Mem. Amer. assoc. adv. sc., i : 63 (vetula) (plate marked Thanatites juvenalis by error). $\quad P l .3$, figs. 12,16 .

1877. Kirby.

Synon. catal, diurn. lepid., suppl., 835.

1885. Oppenheim. Berl. entom. zeitschr., xxix : 347.

See also Vanessa vetula, Araschnia vetula.

\section{Tinea -. (Tinөidæ.)}

Compared with T. culmella and T. pellionella.

Locality : Prussian amber. Horizon: Ligurian.

1835. Gravenhorst. Uebers. schles. gesellsch. vaterl. cult., 1834 : 92.

5856. Tinea -. (Tineidæ.)

Compared with T. pellionella.

Locality: Prussian amber. Horizon: Ligurian.

1856. Menge. Progr. petrischule Danzig, 1856: 29.

\section{Tinea - (larva). (Tineidæ.)}

Locality: Prussian amber. Horizon: Ligurian.

1856. Menge.

Progr. petrischule Danzig, 1856 : 29. 
5858. Tinea? - (pnpa). (Tineidæ.)

1856. Menge.

Loeality: Prussiar amber. Horizon: Ligurian.

Progr. petrischule Danzig, 1856: 29.

5859. Tinea antiqua. (Tineidæ.)

Locality: Prussian amber. Horizon: Ligurian.

1822. Presl. Deliciæ pragenses, i : 199-200.

5860. Tineites crystalli. (Tineidæ.)

Locality : Ufalei, Siberia. Horizon: "Bergkrystall."

1876. Kawall. Bull. soc. imp. nat. Mosc., 1876: 171-172.

5861. Tortrix —. (Tortricidæ.)

Compared with T. lecheana, etc.

Locality: Prussian amber. Horizon: Ligurian.

1835. Gravenhorst. Uebers. schles. gesellsch. vaterl. cult., 1834: 92.

5862. Triphæna - (pupa). (Noctuidæ.)

Locality : Quercy, France. Horizon: -

1877. Gervais. Journ. de zool., vi: 68.

5863. Vanessa atavina. (Nymphalidæ.)

Locality : Radoboj, Croatia. Horizon: Mayencian.

1849. Heer. Insektenf. tertiärg. Oeningen, ii : 177-179 (attavina). Pl. 14, fig، 3.

1850. " Nouv. mém. soc. helv., xi: 177-179. Pl. 14, fig. 3.

1852. Giebel. Deutschl. petref., 644 .

1856. " Insect. $d$. vorwelt, 186.

1872. Kirby. Synon. catal. diurn. lepid., 185 (atovina).

See also Sphinx atava, etc.

Vanessa atovina. See Vanessa atavina.

Vanessa attavina. See Vanessa atavina.

5864. Vanessa pluto. (Papilionidæ.)

Locality: Radoboj, Croatia. Horizon: Mayencian.

1849. Heer. Insektenf. tertiärg. Oeningen, ii: 179-182. Pl. 14, figs. 4, 5.

1850. “ Nouv. mém. soc. helv., xi : 179-182. Pl. 14, figs. 4, 5.

1852. Giebel. Dentschl. petref., 644.

1854. Pictet. Traité de paléont., $2^{e}$ 6́d, ii : 393. Pl. 40, fig. 21.

1856. Giebel. Insect. d. vorwelt, 186-187.

1865. Lyell. Elem. geol., 6th ed., 243. Fig. 179.

See also Argynnis pluto, Junonia plnto, Mylothrites pluto.

5865. Vanessa vetula. (Hesperidæ.)

Locality: Rott, Rhenish Prussia. Horizon: Aquitanian.

1859. Heyden. Palaeontogr., viii: 12-13. Pl. 1, fig. 10.

See also Araschnia vetnla, Thanatites vetulus.

5866. Ypsolophus insignis. (Tineidæ.)

Locality : Vicinity Bonn, Rhenish Prussia. Horizon: Aquitanian

1837. Germar. Fauna insect. Europæ, xix : 20. Pl. 20.

1852. Giebel. Deutschl. petref., 644:

1856. " Insect. d. vorwelt, 191. 
5867. Zygæna - (Bombycidæ.)

1829. Serres.

Locality: Aix, France. Horizon: Ligurian.

Géogn. terrains tert., 230 .

\section{HYMENOPTERA.}

5868.

Locality : Rouffach, Alsatia. Horizon: Tongrien.

1881. Bleicher. Bull. soc. géol. France, (3), viii : 226.

\section{9.}

Locality : Prussian amber. Horizon: Ligurian.

1841. Ehrenberg. Froriep., Neue notizen, xix : 120.

5870.

Locality : Sinigaglia, Italy. Horizon: Helvetian ?.

1842. Procaccini. Nuov. ann. se. nat., vii : 449.

\section{1.}

Locality: Gurnet Bay, Isle of Wight. Horizon: Bembridge Limestone.

1879. Woodward. Quart. journ, geol, soc. Lond, xxxv: 344.

- - See III, Dirtera —— — (Mycetophilidæ) Gú́rin.

5872. (several). (Andrenidæ.)

Locality : Florissant, Colo. Horizon: Oligocene.

1881. Scudder. Bull. U. S. geol. surv, terr., vi : 290.

1883. " Ann. rep. U. S. geol. surv. terr., xii, 280.

5873.

(Apidæ.)

Locality: Prussian amber. Horizon: Ligurian.

1831. Burmeister. Oken, Isis, 1831: 1100.

5874. (Apidæ.)

Locality : Chiavon, Italy. Horizon : -

1881. Malfatti. Atti. soc. ital. sc. nat., xxiv: 98.

1881." Bibl. ins, foss, ital., 10.

5875. (several). (Apidæ.)

Locality: Florissant, Colo. Horizon: Oligocene.

1881. Scudder. 1883. “ Bull. U. S. geol. snrv. terr., vi : 290. Ann. rep. U. S. geol. surv. terr., xii : 280.

5876. (several). (Braconidæ.)

Locality: Florissant, Colo. Horizon: Oligocene.

1881. Scudder. 1883. 4

Bull. U. S. geol. surv. terr., vi : 290.

Ann. rep. U.S. geol. surv. terr., xii: 280.

5877. (Braconidæ.)

Locality: Green River, Wyo. Horizon: Oligocene.

1890. Scudder. Tert. ins. N. A, 607. Pl. 10, fig. 18. 
5878.

(Braconidæ.)

Locality : Green River, Wyo. Horizon: Oligocene.

1890. Scudder. Tert. ins. N. A.,607-608. Pl. 10, fig. 28.

5879. (several). (Chalcididæ.)

Locality: Florissant, Colo. Horizon: Oligocene.

1881. Scudder. 1883. "

Bull. U. S. geol. surv. terr., vi : 290.

Ann. rep. U. S. geol. surv, terr., xii: 280.

5880. (Chrysididæ.)

Locality: Prussian amber. Horizon: Ligarian.

1856. Menge.

Progr. petrischule Danzig, 1856: 26.

5881. (several). (Chrysididæ.)

Locality: Florissant, Colo. Horizon : Oligocene.

1881. Seudder. 1883. "6

1856. Menge.

Bull. U.S. geol. surv, terr., vi : 290.

Ann. rep. U. S. geol. surv. terr., xii : 280.

5882. (Crabronidæ.)

Locality: Prussian amber. Horizon: Ligurian. Progr. petrischule Danzig, 1856 : 25.

5883. (Cynipidæ.)

Locality: Prussian amber. Horizon: Ligurian.

1856. Menge. Progr. petrischule Danzig, 1856 : 25.

5884. (several). (Cynipidæ.)

Locality : Florissant, Colo. Horizon: Oligocene.

1881. Scudder. Bull. U. S. geol. surv. terr., vi : 290. 1883. Scudder. Ann. rep. U. S. geol. surv. terr., xii : 280.

5885. (Formicidæ.)

Locality: Brunstatt, Alsatia. Horizon: Middle Oligocene.

1885. Foerster. Tagebl. versamml. dentsch. naturf., lviii : 392 (ameisen). 1885. " Mitth. oberelsäss. tertiär., 7.

5886. (Formicidæ.)

Locality: Nantucket amber. Horizon : 1879. Goldsmith. Proc. acad. nat. sc. Phil., 1879: 207 (ants).

5887. (Formicidæ.)

Locality: Prussian amber. Horizon: Ligurian. 1835. Gravenhorst. Uebers. schles. gesellsch. vaterl. cult., 1834 : 92.

5888. (Formicidæ.)

Locality: Prussian amber. Horizon: Ligurian. 1819. Schweigger. Beobacht. naturh. reisen, 119. Pl. 8, figs. 70, 70ab. 5889. (several). (Formicidæ.)

Locality : Florissant, Colo. Horizon: Oligocene.

1881. Scudder. Bull. U. S. geol. surv. terr., vi : 290.

1883. Scudder.

Ann. rep. U. S. geol. surv. terr., xii : 280. 


$$
\text { 5890. - (several). (Formicidæ-Myrmicinæ.) }
$$

1881. Scudder. 1883. "6

\section{Locality: Florissant, Colo. Horizon: Oligocene.}

\section{Bull. U. S. geol. surv. terr., vi: 290.}

Ann. rep. U. S. geol. surv, terr., xii : 280.

$$
5891 .
$$

\section{(Ichneumonidæ.)}

Compared with Bassus (Euceros) crassicornis Grav.

Locality: Prussian amber. Horizon : Ligurian.

1832. Burmeister. Handb. entom., i : 636.

1836. "6 Man, entom., 57\%.

See also Bassus — Keferstein.

5892. - —. (Ichneumonidæ.)

Locality : Prussian amber. Horizon: Ligurian.

1856. Menge. Progr. petrischule Danzig, 1856: 24.

5893. (several). (Ichneumonidæ.)

Locality: Florissant, Colo. Horizon: Oligocene.

1881. Scudder. Bull. U. S. geol. surv. terr., vi : 290.

1883. "

Ann. rep. U. S. geol. surv. terr., xii : 280.

5894.

(Mutillidæ.)

Locality: Prussian amber. Horizon: Ligurian.

1856. Menge.

Progr. petrischule Danzig, 1856: 25.

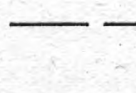

(Myrmicidæ). See (Formicinæ-Myrmicinæ).

5895. (2 sp.). (Pompilidæ.)

Locality : Florissant, Colo. Horizon: Oligocene.

1881. Scudder. 1883.
Bull. U. S. geol. surv. terr., vi : 290.

Ann. rep. U. S. geol. surv. terr., xii : 280.

5896. (several). (Poneridæ.)

Locality : Florissant, Colo. Horizon: Oligocene.

1881. Scudder. 1883. “

\section{Bull. U. S. geol. surv. terr., vi : 290.}

Ann. rep. U. S. geol. surv. terr., xii: 280.

5897. (Proctrotrupidæ.)

Locality: Prussian amber. Horizon : Ligurian.

1886. Brischke. 1886 .

Schrift. naturf. gesellsch. Danzig, n.f., vi, iii : 279.

Iym. bernst., 2.

5898.

$$
\text { - (Proctrotrupidæ.) }
$$

Locality: Sicilian amber. Horizon:-

1881. Malfatti, Atti acad. linc., (3), trans., v: 82. Fig. 1 on p. 83.

5899. (Pteromalidæ.)

Locality: Prussian amber. Horizon: Ligurian. 
5900. ——. (Sphegidæ.)

Locality: Prussian amber. Horizon: Ligurian.

1856. Menge. Progr. petrischule Danzig, 1856: 26 . 5901. (several). (Sphegidæ.)

Locality: Florissant, Colo. Horizon: Oligocene.

1881. Scudder. 1883. “

\section{Bull. U. S. geol. surv. terr., vi : 290.}

Ann. rep. U. S. geol. surv. terr., xii : 280 .

5902. - (several). (Tenthredinidæ.)

Locality : Florissant, Colo. Horizon: Oligocene. 1881. Scudder. 1883. "6 Bull. U. S. geol. surṿ. terr., vi : 290. Ann. rep. U. S. geol. surv, terr., xii : 280.

5903. (Uroceridæ.)

Locality: Florissant, Colo. Horizon: Oligocene. 1881. Scudder. 1883. Bull. U. S. geol. surv. terr., vi : 290. Ann. rep. U. S. geol. surv. terr., xii : 280. 5904. (Vespidæ.)

Locality: Prussian amber. Horizon : Ligurian.

1886. Brischke. 1886. “

Schrift. naturf. gesellsch. Danzig, n. f. vi, iii : 278 . Hym. bernst., 1.

5905. (several). (Vespidæ.)

Locality: Florissant, Colo. Horizon: Oligocene.

1881. Scudder. 1883. "6

1849. Heer. 1852. Giebel. 1856. 6

\section{Bull. U. S. geol. surv. terr., vi : 290.}

Ann. rep. U. S. geol. surv. terr., xii : 280.

Accenites lividus. See Aconitus lividus.

5906. Acœnitus lividus. (Ichneumonidæ.)

Locality : Radoboj, Croatia. Horizon: Mayencian.

Insektenf. tertiärg. Oeningen, ii : 169-170. Pl. 13, fig. 14.

Deutschl. petref., 647.

Insect. d. vorwelt, 156-15\% (Acœnites). 5907. Agathis (Braconidæ.)

Locality: Aix, France. Horizon : Ligurian.

1829. Serres. Géogn. terrains tert., 229.

5908. Amblyteles —. (Ichneumonidæ.)

Locality: Oeningen, Baden, Horizon: Tortonian.

1888. Schöberlin. Soc. entom., iii : 61 .

5909. Ammophila - (Sphegidæ.)

Locality: Horizon :

1846. Giebel.

Paläozool, 280.

\section{Ammophila} (Sphegidæ.)

Locality: Florissant, Colo. Horizon: Oligocene.

1881. Scudder.

Bull. U. S. geol. surv, terr., vi : 290.

1883. “
Ann. rep. U. S. geol. surv. terr., xii : 280 , 
5911. Ammophila annosa. (Sphegidæ.)

Compared with A. sabulosa.

1865. Heer.

1872. "

1876. " "

1879. "

Locality : Oeningen, Baden. Horizon; Tortonian.

\section{Urwelt der Schweiz, 387.}

Monde prim. Suisse, 473.

Prim. world Switz., ii : 44.

Urwelt der Schweiz, $2^{\circ}$ anfl., 412.

5912. Ammophila gigantea. (Sphggidæ.)

Locality: Oeningen, Baden. Horizon: Tortonian.

1888. Schöberlin. Soc. entom., iii : 61 (undescr.).

\section{Ammophila inferna. (Sphegidæ.)}

1865. Heer.

1872. "

1876. "

1879. “

Locality : Oeningen, Baden. Horizon: Tortonian.

Urwelt der Schweiz, 387. Figs. 290a, b on p. 386.

Monde prim. Suisse. 474. Figs. 290a, $b$.

Prim. world Switz, ii, 45. Figs. $290 a, b$ on p. 44.

Urwelt der Schweiz, $2^{\circ}$ anfl, 412 . Figs. 332a, $b$.

5914. Ammophila minima. (Sphegidæ.)

Locality: Oeningen, Baden. Horizon: Tortonian.

1888. Schöberlin. Soc. entom., iii : 61 (undescr.).

\section{Andrena - (Apidæ.)}

Locality : Prussian amber. Horizon: Ligurian.

1886. Brischke. Schrift. naturf. gesellsch. Danzig, n. f. vi, iii : 278.

1886. " Hym. bernst., 1 .

\section{Anomalon - (Ichneumonidæ,)}

Compared with A. variegatum.

Locality: Aix, France. Horizon: Ligurian.

1829. Serres. Géogn. terrains tert., 229,26\%.

5917. Anomalon protogæum. (Ichneumonidæ.)

Locality : Oeningen, Baden. Horizon: Tortonian.

1849. Heer. 1852. Giebel. 1856.
Insektenf. tertiärg. Oeningen, ii: 167-168. Pl. 13, fig. 12.

Deutschl. petref., 647.

Insect. d. vorwelt, 156.

5918. Anomma rubella (recent). (Formicidø.)

Locality: Prussian amber. Horizon: Ligurian.

[1850. Savage. 1868. Smith.

Proc. gcad. nat. sc. Philad., 1850 : 196.]

Quart. journ. sc., v: 184 (or an allied species). $\quad$ Pl., fig. 3.

$$
\text { 5919. Anthophora? ——. (Apidæ.) }
$$

Locality: Prussian amber. Horizon: Ligurian.

1886. Brischke. 1886. “
Schrift. naturf. gesellsch. Danzig, n. f., vi., iii : 278. Hym. bernst., 1.

5920. Anthophora? (Apidæ.)

Locality: Prussian amber. Horizon : Ligurian, Progr, petrischule Danzig, 1856; 26, 
5921. Anthophora effossa. (Apidæ.)

Locality: Rott, Rhenish Prussia. Horizon: Aquitanian.

1862. Heyden. Palaeontogr., $\mathrm{x}:$ 76. Pl. 10, fig. 10.

5922. Anthophorites gaudryi. (Apidæ.)

Compared with A. parietaria.

Locality: Corent, France. Horizon: Aquitanian.

1870. Oustalet. Ann. sc. g6́ol., ii, art. 3: 104-105. Pl. 2, figs. 11-13.

1871. "6 Bibl. hautes étud., sc. nat., iv, art. 7, 104-105. Pl. 2, figs. 11-13.

5923. Anthophorites longævus. (Apidæ.)

Localities: Oeningen, Baden; Radoboj, Croatia. Horizons: Tortonian, Mayencian. 1867. Heer. Neue denkschr. schweiz. gesellsch., xxii, mem. 4 : 5 (longæva). Pl. 3, figs. 12, 13.

1867. " Foss. hymen., 5. Pl. 3, figs. 12, 13.

5924. Anthophorites mellona. (Apidæ.)

Locality: Oeningen, Baden. Horizon: Tortonian.

1849. Heer.

1852. Giebel.

Insektenf. tertiärg. Oeningen, ii : 97-98. $\quad P l .7$, fig. 4.

1856. " Insect. d. vorwelt. 183.

5925. Anthophorites thoracicus. (Apidæ.)

Locality : Radoboj, Croatia. Horizon: Mayencian.

186\%. Heer. Neue denkschr. schweiz. gesellsch., xxii, mem. 4: 6 (thoracica). $P l, 3$, fig. 14.

1867. “ Foss. hymen., 6. Pl. 3, fig. 14 .

5926. Anthophorites titania. (Apidæ.)

Locality : Oeningen, Baden. Horizon: Tortonian.

1849. Heer.

1852. Giebel.

Insektenf. tertiärg. Oeningen, ii: 99. $\quad P l .7, f i g .5$.

1856. "

Deutschl. petref., 645 .

Insect. d. vorwelt, 183.

5927. Anthophorites tonsus. (Apidæ.)

Locality: Oeningen, Baden. Horizon: Tortonian.

1849. Heer.

1852. Giebel.

Insektenf. tertiärg. Oeningen, ii: 99-100 (tonsa). Pl. 7, fig. 6.

1856. " "

Deutschl. petref., 645 .

Insect. d. vorwelt, 183-184.

5928. Anthophorites veteranus. (Apidæ.)

Locality: Oeningen, Baden. Horizon: Tortonian.

1849. Heer. 1852. Giebel. 1856. "6
Insektenf. tertiärg. Oeningen, ii : 100 (veterana). Pl. 7, fig. 7.

Deutsehl. petref., 645 .

Insect. d. vorwelt, 184.

5929. Aphænogaster berendti. (Formicidæ.)

Locality : Prussian amber. Horizon: Ligurian,

Ameis, balț. bernst, 82. $\mathrm{Pl}, 4$, figs. 78-79, 
5930. Aphænogaster fuliginosa. (Formicidæ.)

Locality: Radoboj, Croatia. Horizon: Mayencian.

186\%. Mayr. 1867. " 1867. Heer.
Jahrb, k.-k. geol. reichsanst., xvii : 57 (fulingiosa).

Stud. Rad. form., 11.

Neue denkschr. schweiz. gesellsch., xxii, mem. 4: 42 (says it can not be an Aphænogaster).

1867. " Foss. hymen., 42.

See also Ponera fuliginosa, etc.

Aphænogaster fulingiosa. See Aphænogaster fuliginosa.

5931. Aphænogaster livida. (Formicidæ.)

Locality: Radoboj, Croatia. Horizon: Mayencian.

1867. Mayr.

1867. “

Jahrb. k.-k. geol. reichsanst., xvii : 57 (quotes Heer's sp. as "Ponera" livida).

See also Poneropsis livida.

5932. Aphænogaster longaeva. (Formicidæ.)

Compared with A. berendti Mayr.

Locality: Quesnel, British Columbia. Horizon:

1877. Seudder.

Rep. geol. surv. Can., 1875-'76: 267.

187\%. " "

1877. “

1890. “
Rapp. opér. comm. géol. Can., 1875-'76: 295-296.

Tert. ins. N. A., 615. Pl. 3, fig. 28.

5933. Aphænogaster lugubris. (Formicidæ.)

Locality: Radoboj, Croatia. Horizon: Mayencian.

1867. Mayr.

1867. “

Jahrb. k.-k. geol. reichsanst., xvii: 57.

See also Poneropsis lugubris, etc.

5934. Aphænogaster sommerfeldti. (Formicidæ.)

1868. Mayr.

Locality: Prussian amber. Horizon: Ligurian.

Ameis. balt. bernst., 81-82. Pl. 4, fig. 76-77.

5935. Apiaria dubia. (Apidæ.)

Locality: Orsberg, (Rhenish Prussia?). Horizon: Miocene.

1849. Germar. Zeitschr. deutsch. geol. gesellsch., i: 66. Pl. 2, fig. 8. See also Osmia dubia.

5936. Apis adamitica. (Apidæ.)

Compared with A. mellifica L.

Loeality: Oeningen, Baden. Horizon: Tortonian.

1865. Heer.

1867. "6

1867. "

187\%. "

1876. " "

1879, “6
Urwelt der Schweiz, 386. Fig. 287.

Neue denkschr. schweiz. gesellsch., xxii, mem. 4 : 4. Pl. 3, fig. 11.

Foss. hymen., 4. Pl. 3, fig. 11.

Monde prim. Suisse, 473. Fig. 287 on p. 474.

Prim. world Switz., ii : 43. Fig. 287 on p. 44.

Urwelt der Schweiz, 2e aufl, 412. Fig. 329, 
5937. Apis dormitans. (Apida.)

Locality: Rott, Rhenish Prussia. Horizon: Aquitanian.

1862. Heyden. Palaeontogr., $\mathrm{x}: 76-77 . \quad P l .10, \mathrm{fig} .8$.

\section{Apis proava. (Apidæ.)}

Compared with A. mellifica.

Locality: Prussian amber. Horizon: Ligurian.

1856. Menge. Progr. petrischule Danzig, 1856: 26-27.

\section{Ascogaster —. (Braconidæ.)}

Locality : Prussian amber. Horizon: Ligurian.

1886. Brischke. Schrift. naturf. gesellsch. Danzig, n. f., vi, iii : 279 .

1886. "6 Hym. bernst., 2.

5940, Attopsis - (several). (Formicidx.)

Locality : Brunstatt, Alsatia. Horizon: Middle Oligocene.

1888. Foerster. Mitth. comm. geol. Elsass-Lothr., i: 164.

1889. " Mitth. comm. geol. Elsass-Lothr., ii : 103.

5941. Attopsis anthracina. (Formicidæ.)

Locality : Radoboj, Croatia. Horizon: Mayencian.

1849. Heer.

1852. Giebel.

1856. “"

186\%. Heer.

1867. " "

1867. Mayr.

1867. "

Insektenf. tertiärg. Oeningen, ii : 156-157. Pl. 12, fig. 12.

Deutschl. petref., 646 .

Insect. d. vorwelt, 177.

Neue denkschr. schweiz. gesellsch., xxii, mem. 4 : 29.

Foss. hymen., 29.

Jahrb. k.-k. geol, reichsanst., xvii : 58.

Stud. Rad. form., 12.

5942. Attopsis longipennis. (Formicidæ.)

Locality : Radoboj, Croatia. Horizon: Mayencian.

1849. Heer. 1852. Giebel. 1854. Pictet. Insektenf. tertiärg. Oeningen, ii : 155-156. Pl. 12, fig. 11. Deutschl. petref., 646.

1853-56. Bronn.

Traité de paléont., $2^{\mathrm{e}} 6$ d., ii : $383 . \quad P l .40$, fig. 22.

1856. Giebel.

Lethæa geogn., $3^{\text {e }}$ aufl., iii : $646-647 . \quad P l$. $42^{\prime}$, figs. $23 a b$.

Insect. d. vorwelt, 177.

5943. Attopsis longipes. (Formicidæ.)

Locality: Radoboj, Croatia. Horizon: Mayencian.

186\%. Heer. Nene denkschr. schweiz. gesellsch., xxii, mem. 4 : 29-30. Pl. $\mathbf{z}_{\star}$ figs. $15,15 b$.

1867. “

Foss. hymen., 29-30. Pl. 2, figs. 15, $15 b$.

5944. Attopsis nigra. (Formicidæ.)

Locality : Radoboj, Croatia. Horizon : Mayencian.

1849. Heer.

1852. Giebel.

1856. "

1867. Heer.

1867. "6
Insektenf, tertiärg. Oeningen, ii : 157-158. $\quad P l .12$, fig. 13.

Deutschl. petref., 646 .

Insect. d. vorwelt, 177-178.

Neue denkschr. schweiz. gesellsch., xxii, mem, $4: 29,42$.

Foss. hymen., 29, 42.

See also Catanlacus niger.

Bull. $71-44$ 
5945. Bassus

(Ichnenmonidæ.)

Locality: Prussian amber. Horizon: Ligurian.

1834. Keferstein. Naturg, erdkörp., ii : 332 (as of Burmeister, but erroneously. Burmeister, Handb., i : 636, only says an Ichneumon like Bassus, etc.).

See also —- (Ichneumonidæ) Burm.

5946. Bombus —. (Apidæ.)

Locality : Prussian amber. Horizon: Ligurian.

1886. Brischke. 1886. 6 Schrift. naturf. gesellsch. Danzig, n. f., vi, iii : 278 . Hym. bernst., 1.

5947. Bombus -. (Apidæ.)

Locality : Florissant, Colo. Horizon : Oligocene.

1881. Scudder.

Bull. U. S. geol. surv. terr., vi : 290.

1883. “

Ann. rep. U. S. geol. surv. terr., xii : 280.

5948. Bombus abavus. (Apidæ.)

Locality : Oeningen, Baden. Horizon: Tortonian.

1867. Heer. Neue denkschr. schweiz. gesellsch., xxii, mem. 4: 5 . Pl. 3, figs. 9, 10.

1867. “

Foss. hymen., 5. $\quad P l .3$, figs. 9, 10.

5949. Bombus antiquus. (Apidæ.)

Locality: Rott, Rhenish Prussia. Horizon: Aquitanian.

1859. Heyden. Palaeontogr., viii : 12. Pl. 2, fig. 4.

5950. Bombus carbonarius. (Apidæ.)

Locality: Prussian amber. Horizon: Ligurian.

1856. Menge. Progr. petrischule Danzig, 1856: 27.

5951. Bombus crassipes. (Apidæ.)

Locality: Krottensee, Bohemia: Horizon: Aquitanian.

1877. Novak. Sitzungsb. k. akad. wiss. Wien, lxxvi : 92. Pl. 3, fig. 4.

1877. "6 Fauna egerer tertiärb., 22. Pl. 3, fig. 4.

5952. Bombus grandævus. (Apidæ.)

Localities: Radoboj, Croatia; Oeningen, Baden. Horizons: Mayencian, Tortonian, 1849. Heer. 1852. Giebel.

1856. “

1867. Heer.

1867. “

Insektenf. tertiärg. Oeningen, ii : 96-97. $\quad P l .7$, fig. 3.

Deutschl. petref., 645 .

Insect. d. vorwelt, 182-183.

Neue denkschr. schweiz. gesellsch., xxii, mem. 4: 5. Pl. 3, figs. 6,7 .

Foss. hymen., 5. Pl. 3, figs. 6, 7.

5953. Bombus jurinei (Apidæ.)

Locality: Oeningen, Baden. Horizon: Tortonian.

1865. Heer.

1867. 66

1867. "

187\%. "6

1876. "6

1879.
Urwelt der Schweiz, 386. Fig. 296.

Neue denkschr. schweiz. gesellsch., xxii, mem. 4: 4-5. Pl. 3, fig. 8.

Foss. hymen., 4-5. $P l, 3, f i g .8$.

Monde prim. Suisse, 473. Fig. 296 on p. 474.

Prim. world Switz., ii : 43. Fig. 296 on p. 44.

Urwelt der Schweiz, $2^{e}$ aufl., 411 . Fig. 338 on p. 412. 
5954. Bombus pusillus. (Apidæ.)

1856. Menge.

Locality : Prussian amber. Horizon: Ligurian. Progr. petrischule Danzig, 1856: 27.

5955. Bombusoides mengei. (Apidæ.)

Locality: Prussian amber. Horizon: Ligurian. 1856. Motschulsky. Étud. entom., v : 28.

5956. Brachygaster —. (Evaniidæ.)

Compared with B. minor.

Locality: Prussian amber. Horizon: Ligurian.

1886. Brischke. Schrift. naturf. gesellsch. Danzig, n. f., vi, iii : 278. 1886. "6 Hym. bernst., 1.

5957. Bracon -. (Braconidæ.)

Locality : Brunstatt, Alsatia. Horizon : Middle Oligocene.

1890. Foerster. In litt.

\section{Bracon —. (Braconidæ.)}

Locality: Prussian amber. Horizon: Ligurian.

1835. Gravenhorst. Uebers. schles. gesellsch. vaterl. cult., 1834 : 92.

5959. Bracon -. (Braconidæ.)

Locality: Sicilian amber. Horizon :

1838. Guérin. Rev. zool., 1838: 170.

5960. Bracon —. (Braconidæ.)

Locality: Aix, France. Horizon: Ligurian.

1854. Pictet. Traité de pal6́ont., $2^{e}$ 6́d, ii: 382 (fide Serres MS.).

5961. Bracon -. (Braconidæ.)

Locality : Similkameen River, British Columbia. Horizon: -

1879. Seudder. Rep. progr. geol. surv. Can., 1877-'78: B 177.

1879. " Ins. tert. Nicola, 2.

1890. “ Tert. ins. N. A., 607. Pl. 3, fig. 33.

5962. Bracon laminarum. (Braconidæ.)

Locality: Green River, Wyo. Horizon: Oligocene.

1878. Scudder. Bull. U. S. geol. surv. terr., iv : 748.

1890. 66 Tert. ins. N. A., 606-607. Pl. 10, fig. 29.

5963. Bracon macrostigma. (Braconidæ.)

Locality : Sieblos, Rhenish Prussia: Horizon : Aquitanian.

1858. Heyden. Palaeontogr., v: 119. Pl. 23, fig. 18.

5964. Bradoponera meieri. (Formicidæ.)

Locality : Prussian amber. Horizon: Ligurian.

1868. Mayr. Ameis. balt. bernst., 74-75. Pl. 4, figs. 70-71. 
5965. Calyptites antediluvianus. (Braconidæ.)

Locality: Quesnel, British Columbia. Horizon:

1877. Scudder. Rep. geol. surv. Can., 1875-76: 270 (antediluvianum).

187\%. " Ins. tert. Quesn., 5.

1877. “ Rapp. opér. comm. géol. Can., 1875-76: 299.

1885. " Zittel, Handb. palaeont., i, th. ii: 817. Fig. 1100.

1886. " Zittel-Barrois, Traité de paléont., ii: 819. Ifig. 1117.

1890. “ Tert. ins. N. A., 606. Pl. 3, fig. 32 .

\section{Camponotus - (3 sp.). (Formicidæ.)}

Locality: Brunstatt, Alsatia. Horizon: Middle Oligocene.

1890. Foerster. In litt.

5967. Camponotus —. (Formicidæ.)

Locality: Gurnet Bay, Isle of Wight. Horizon : Bembridge Limestone. 1879. Woodward. Quart. journ. geol. soc. Lond., xxxv: 344. 1879. " Geol. mag., (n. s.), v: 89.

5968. Camponotus constrictus. (Formicidæ.)

1868. Mayr.

Locality: Prussian amber. Horizon: Ligurian.

1867. Mayr. Ameis. balt. bernst., 29-30. Pl. 1, fig. 11.

5969. Camponotus heraclea. (Formicidæ.)

Locality: Oeningen, Baden. Horizon: Tortonian. 1867. "6 Stud. Rad. form., 6.

See also Formica heraclea, etc.

5970. Camponotus igneus. (Formicidæ.)

1868. Mayr.

Locality: Prussian amber. Horizon: Ligurian. Ameis. balt. bernst., 28-29. Pl. 1, figs. 9-10.

5971. Camponotus induratus. (Formicidæ.)

Compared with C. variegatus Sm.

Locality: Radoboj, Croatia. Horizon: Mayencian.

1867. Mayr. Jahrb. k.-k. geol. reichsanst., xvii : 52.

1867. " Stud. Rad. form., 6.

See also Formica indurata.

5972. Camponotus lignitum. (Formicidæ.)

Locality : Oeningen, Baden. Horizon: Tortonian.

1867. Mayr. Jahrb. k.-k. geol, reichsanst., xvii : 51-52.

1867. " Stud. Rad. form., 5-6.

See also Formica heraclea, etc.

5973. Camponotus mengei. (Formicidæ.)

Locality : Prussian amber. Horizon: Ligurian.

1868. Mayr.

Ameis. balt. bernst., 27-28. $\quad P l .1$, figs. 1, 8. 
5974. Camponotus pinguiculus radobojanus. (Formicidæ.)

Locality: Radoboj, Croatia. Horizon: Mayencian.

1867. Mayr. Jahrb. k.-k. geol. reichsanst, xvii: 52. 1867. " Stud. Rad. form., 6.

See also Formica pinguicula, etc.

5975. Camponotus pinguis radobojanus. (Formicidæ.)

Locality : Radoboj, Croatia. Horizon: Mayencian.

1867. Mayr.

1867. “6

Jahrb. k.-k. geol. reichsanst., xvii : 51.

See also Formica pinguis, etc.

Camponotus radobojanus. See Camponotus pinguiculus radobojannई, Camponotus pinguis radobojanus.

5976. Camponotus vetus. (Formicidæ.)

Locality: White River, Colorado or Utah. Horizon: Oligocene.

1877. Scudder. Bull. U. S. geol. surv. terr., iii : 742.

1890. "6 Tert. ins. N. A., 619. Pl. 5, figs. 1, 2.

See also Formica —— Scudder.

Campoplex —. See Ophion (or Campoplex) — Sordelli.

5977. Cataulacus niger. (Formicidæ.)

Locality : Radoboj, Croatia. Horizon: Mayencian.

1867. Mayr.

Jahrb. k.-k. geol. reichsanst., xvii : 58.

1867. " Stud. Rad. form., 12.

1867. Heer.

1867. "6

Neue denkschr. schweiz. gesellsch., xxii, mem. 4: 42.

See also Attopsis nigra.

5978. Cemonus - (Crabronidæ.)

Locality : Prussian amber. Horizon: Ligurian.

1886. Brischke. Schrift. naturf. gesellsch. Danzig, n. f., vi, iii: 278.

1886. " Hym. bernst., 1.

5979. Cephites fragilis. (Tenthredinidæ.)

Locality: Oeningen, Baden. Horizon: Tortonian.

1849. Heer.

1852. Giebel.

1854. Pictet.

1856. Giebel.

1885. Scndder.

1886. “

Insektenf. tertiärg. Oeningen, ii: 174-175. Pl. 14, fig. 1.

Deutschl. petref., 647 .

Traité de paléont., 2 éd., ii : 381. $P l .40, f i g .20$.

Insect. d. vorwelt, 153.

Zittel, Handb. palaeont., i, th. ii: 816. Fig. 1096.

Zittel-Barrois, Traité de pal6́nt., ii : 818. Fig. 1113.

5980. Cephites oeningensis. (Tenthredinidæ.)

Locality: Oeningen, Baden. Horizon: Tortonian.

1849. Heer.

1852. Giebel.

1856. “
Insektenf. tertiärg. Oeningen, ii : 173-174. Pl. 13, fig. 17.

Deutschl. petref., 647.

Insect. d. vorwelt, 152. 
5981. Cephus —. (Tenthredinidæ.)

1856. Menge. Progr. petrischule Danzig, 1856: 24.

Loøality : Prussian amber. Horizon: Ligurian.

5982. Ceraphron —-. (Proctotrupidæ.)

Locality : Prussian amber. Horizon: Ligurian.

1831. Burmeister. Oken, Isis, 1831: 1100.

5983. Cerceris? - - (Crabronidæ.)

Locality: Prussian amber. Horizon: Ligurian.

1886. Brischke. Schrift. naturf. gesellsch. Danzig, n. f., vi, iii : 278.

1886. “ Hym. bernst., 1.

5984. Chalcites debilis. (Chalcididæ.)

Locality: Aix, France. Horizon: Ligurian."

1856. Heer. Viert. naturf. gesellsch. Zürich, i: 29-30. Pl. 2, fig. 16.

1885. Seudder. Zittel, Handb. palaeont, i, th. ii: 816. Fig. 1098.

1886. “ Zittel-Barrois, Traité de paléont., ii : 818. Fig. 1115.

5985. Chalicodoma - (Apidæ.)

Locality: Prussian amber. Horizon: Ligurian.

1886. Brischke. Schrift. naturf. gesellsch. Danzig, n. f., vi, iii : 278. 1886. " Hym. bernst., 1 .

5986. Chelonus —. (Braconidæ.)

Locality: Prussian amber. Horizon : Ligurian.

1886. Brischke. Schrift. naturf. gesellsch. Danzig, n. f., vi, iii : 279.

5987. Chelonus —. (Braconidæ.)

Locality : Prussian amber. Horizon: Ligurian.

1835. Gravenhorst. Uebers. schles. gesellsch. vaterl. cult., 1834: 92.

5988. Chrysis ——. (Chrysididæ.)

Locality: Prussian amber. Horizon: Ligurian.

1886. Brischke. Schrift. naturf. gesellsch. Danzig, n. f., vi, iii : 278. 1886. "6 Hym. bernst., 1.

5989. Chrysis —_- (Chrysididæ.)

Locality : Brunstatt, Alsatia. Horizon: Middle Oligocene.

1890. Foerster. In litt.

5990. Chrysis —. (Chrysididæ.)

Locality: Florissant, Colo. Horizon: Oligocene.

1881. Scudder. Bull. U. S. geol. surv. terr., vi : 291.

1883. " Ann. rep. U. S. geol. surv. terr., xii: 280.

5991. Chrysis cyanea. (Chrysididæ.)

Locality: Copal. Horizon: Recent.

1776. Bloch.

Beschäft. berl. gesellsch. naturf. freunde, ii : 187-188. 
5992. Chrysis viridicyanea. (Chrysididø.)

Compared with C. distinctissima.

Locality : Prussian amber. Horizon: Ligurian.

1862. Giebel. Zeitschr. ges. naturw., xx: 319-320.

5993. Cimbex - (larva). (Tenthredinidæ.)

Compared with C. variäbilis.

Locality: Prussian amber. Horizon: Ligurian.

1856. Menge. Progr. petrischule Danzig, 1856: 24.

5994. Cleptes —. (Chrysididæ.)

Locality : Prussian amber. Horizon: Ligurian.

i886. Brischke. Schrift. naturf. gesellsch. Danzig, n.f., vi, iii: 278.

1886. " Hym. bernst., 1.

5995. Cleptes steenstrupii. (Chrysididæ.)

Locality: Jutland. Horizon: (Pliocene?)

1836. Beck. $\quad$ Proc. geol. soc. Lond., ii : 219 (undescr.) (Cleptis stenstrupii).

1836. “ Lond. Edinb. phil. mag., (3), viii: 555 (undeser.).

Cleptis stenstrupii. See Clepies steenstrupii.

Crematogaster pusilla. Seo Myrmica pusilla.

5996. Crossocerus —. (Crabronidæ.)

Locality: Prussian amber. Horizon: Ligurian.

1886. Brischke. Schrift. naturf. gesellsch. Danzig, n. f., vi, iii : 278.

1886. " Hym. bernst., 1.

5997. Cryptus —. (Ichneumonidæ.)

Locality: Prussian amber. Horizon: Ligurian.

1886. Brischke. Schrift. naturf. gesellsch. Danzig, n. f., vi, iii : 278.

1886. " Hym. bernst., 1.

5998. Cryptus —. (Ichneumonidæ.)

Locality: Prussian amber. Horizon: Ligurian.

1835. Gravenhorst. Uebers. schles. gesellsch. vaterl. cult., 1834 ! 92.

5999. Cryptus —. (Ichnenmonidæ.)

Locality: Oeningen, Baden. Horizon: Tortonian.

1888. Schöberlin. Soc. entom., iii : 61 .

6000. Cryptus —. (Ichneumonidæ.)

Compared with C. rosæ.

Locality: Aix, France. Horizon : Ligurian.

1829. Serres. G6́ogn. terrains tert., 229.

6001. Cryptus antiquus. (Ichneumonidæ.)

Locality : Oeningen, Baden. Horizon: Tortonian.

1849. Heer.

1852. Giebel.

1856. ‘
Insektenf. tertiärg. Oeningen, ii : 168-169. $\quad P l .13$, fig. 13.

Dentschl. petref., 647 .

Insect. d. vorwelt, 155. 
6002. Cynips? - (Cynipidæ.)

Locality: Salzhausen, Germany. Horizon: Miocene.

1865. Heyd.-Heyd. Palaeontogr., xiv: 35 (or Pterowalus?). (Holes in Juglans acuminata Br.).

See also Pteromalus — Heyden.

$$
\text { 6003. Cynips —. (Cynipidæ.) }
$$

Locality : Prussian amber. Horizon: Ligurian.

1820. Schlotheim. Petrefactenk., 43.

6004. Cynips succinea. (Cynipidæ.)

Locality: Prussian amber. Horizon: Ligurian.

1822. Presl. Del. prag., i: 195.

6005. Dasypoda (vic.) —. (Andrenidæ.)

Locality: Prussian amber. Horizon: Ligurian.

1856. Menge. Progr. petrischule Danzig, 1856: 26.

6006. Decatoma antiqua. (Chalcididæ.)

Locality: Green River, Wyo. Horizon: Oligocene.

1878. Scudder. Bull. U. S. geol. surv. terr., iv : 749.

1890. " Tert. ins. N. A., 604-605. Pl. 10, figs. 20, 31.

6007. Didineis solidescens. (Sphegidæ.)

Compared with D. lunicornis (Fabr.).

Locality: Green River, Wyo. Horizon: Oligocene.

1890. Scudder. Tert. ins. N. A., 620. Pl. 10, fig. 30.

\section{Diplolepis - (Cynipidæ.)}

Locality: Prussian amber. Horizon: Ligurian.

1835. Gravenhorst. Uebers. sehles. gesellsch. vaterl. cult., 1834: 92.

6009. Dolerus —. (Tenthredinidæ.)

Locality: Brunstatt, Alsatia. Horizon : Middle Oligocene.

1890. Foerster. In litt.

6010. Dolerus —. (Tenthredinidæ.)

Locality: Oeningen, Baden. Horizon: Tortonian.

1888. Schöberlin. Soc. entom., iii : 61 .

6011. Eclytus lutatus. (Ichneumonidæ.)

Locality: Green River, Wyo. Horizon: Oligocene.

1890. Scudder. Tert. ins. N. A., 614. Pl. 10, fig. 24.

6012. Ectatomma europæum. (Formicidæ.;

1868. Mayr.

Locality: Prussian amber. Horizon: Ligurian.

Ameis. balt. bernst., 76-77. $\quad P l .4$, figs. 72, 73.

6013. Emphytus —. (Tenthredinidæ.)

Locality: Prussian amber. Horizon: Ligurian.

1856. Menge. Progr. petrischule Danzig, 1856: 24. 
6014. Enneamerus reticulatus. (Formicidæ.)

Locality: Prussian amber. Horizon: Ligurian.

1868. Mayr. Ameis. balt. bernst., 100. Pl. 5, figs. 102, 103.

\section{Evania - (Evaniidæ.)}

Compared with E. minuta Fabr.

Locality: Prussian amber. Horizon: Ligurian.

1831. Burmeister. Oken, Isis, 1831: 1100.

183\%. "6 Handb. ent., i: 636.

1836. " Man. ent., 577.

6016. Formica —. (Formicidæ.)

Locality: Thalheim (or Sotzka ?), Austria. Horizon:

1355. Andrae. Foss. flora Siebenb., 26. Pl. 4, figs. $6 a b, 8 b$.

6017. Formica —. (Formicidæ.)

Locality: Copal. Horizon: Recent.

1776. Bloch. Beschäft. berl. gesellsch. naturf. freunde, ii: 183 . Pl.5, fig, 23.

6018. Formica - (Formicidæ.)

Locality : Copal. Horizon: Recent.

1776. Bloch. Beschäft. berl, gesellsch. naturf. freunde, ii : 188-189.

6019. Formica —. (Formicidæ.)

Locality: Prussian amber. Horizon: Ligurian:

1831. Burmeister. Oken, Isis, 1831: 1100.

1832. " Handb. ent., i: 636.

1836. “ Man. ent., 577.

6020. Formica -. (Formicidæ.)

Locality: Gabbro, Italy. Horizon: Tripoli earth.

1878. Capellini. Atti accad. linc., (3), mem. sc. fis., ii : 285.

6021. Formica -. (Formicidæ.)

Locality: Aix, France. Horizon: Ligurian.

1829. Curtis. $\quad$ Edinb. new phil. journ., vii : 295.

1829. " Murchison-Lyell, Tert. form. Aix, 11.

6022. Formica - (several). (Formicidæ.)

Locality: Brunstatt, Alsatia. Horizon: Middle Oligocene.

1888. Foerster. Mitth. comm. geol. Elsass-Lothr., i: 164.

1889. " Mitth. comm. geol. Elsass-Lothr., ii : 103.

6023. Formica - - (Formicidæ.)

Locality: Sicilian amber. Horizon :-

1838. Guérin. Rev. zool., 1838: 170. Pl. 1, fig. 9.

See also Psendomyrme — Scudder, etc.

6024. Formica - (Formicidæ.)

Locality : Sicilian amber. Horizon: -

1838. Guérin. Rev. zool., 1838: 170. Pl. 1, fig. 10.

See also Pseudomyrme — Scudder, etc. 
6025. Formica

(Formicidæ.)

Locality: Sicilian amber. Horizon :

1838. Guérin.

Rev. zool., 1838: 170. Pl. 1, fig. 11.

6026. Formica -. (Formicidæ.)

Locality : Sicilian amber. Horizon :-

1838. Guérin. Rev. zool, 1838: 170. Pl. 1, fig. 12.

6027. Formica -. (Formicidæ.)

Locality : Rott, Rhenish Prussia. Horizon: Aquitanian.

1859. Heyden. Palaeontogr., viii : 12. Pl.2, fig. 11.

6028. Formica —. (Formicidæ.)

Locality : Prussian amber. Horizon: Ligurian.

1856. Menge. Progr. petrischule Danzig, 1856: 25.

6029. Formica —. (Formicidæ.)

Locality : Prussian amber. Horizon : Ligurian.

1820. Schlotheim. Petrefactenk., 43.

6030. Formica - (Formicidæ.)

Locality: Green River, Wyo. Horizon: Oligocene.

1867. Scudder. Proc. Bost. soc. nat. hist., xi : 117.

See also Camponotus vetus.

6031. Formica - (Formicidæ.)

Locality: Prussian amber. Horizon: Ligurian.

1742. Sendel.

1825. Guérin.

Hist. succ., 126-127. Pl. 4, figs. 18-21.

Dict. class. d'hist. nat., viii : 580 (fourmi).

1829. Serres.

G6́ogn. terrains tert., 242.

6032. Formica -. (Formicidæ.)

Compared with F. subterranea.

Locality : Aix, France. Horizon : Ligurian.

1829. Serres.

Géogn. terrains tert., 230.

6033. Formica —. (Formicidæ.)

Locality : Gurnet Bay, Isle of Wight. Horizon: Bembridge Limestone.

1879. Woodward. Quart. journ. geol. soc. Lond., xxxv : 344.

1879. “ Geol. mag., n. s., v: 89.

6034. Formica acuminata. (Formicidæ.)

Locality: Radoboj, Croatia. Horizon: Mayencian.

1849. Heer.

1852. Giebel.

1856. 6

1867. Heer.

1867. “

1867. Mayr.

1867. "
Insektenf. tertiärg. Oeningen, ii : 142. Pl. 11, figs. 13,14g.

Dentschl. petref., 646.

Insect. d. vorwelt, 172.

Neue denkschr. schweiz. gesellsch., xxii, mem. $4:$ ir. $P l .1$, fig. $17 b$.

Foss. hymen., 17. Pl. 1, fig. $17 b$.

Jahrb. k.-k. geol. reichsanst., xvii : 56.

Stud. Rad. form., 10. 
6035. Formica æmula. (Formicidæ.)

Locality : Radoboj, Croatia. Horizon: Mayencian.

1867. Heer. Nene denkschr. schweiz. gesellsch., xxii, mem. 4: 18. Pl. 1, figs. 19,197 .

1867. “ Foss. hymen., 18. Pl. 1, figs. 19, $19 b$.

186\%. Mayr. Jahrb. k.-k. geol. reichsanst., xvii: 56.

1867. " Stud. Rad. form., 10.

6036. Formica arcana. (Formicidæ.)

Locality: Quesnel, British Columbia. Horizon :

1877. Scudder. Rep. geol. surv. Can., 1875-76: 266-267.

1877. " Ins. tert. Quesn., 1-2.

1877. " Rapp. opér. comm. géol. Can., 1875-76: 294-295.

1890. " Tert. ins. N. A., 618. Pl. 3, fig. 24.

6037. Formica atavina. (Formicidæ.)

Locality: Radoboj, Croatia. Horizon: Mayencian.

1849. Heer. Insektenf. tertiärg. Oeningen, ii : 143-144. Pl. 11, fig. 10.

1852. Giebel. Dentschl. petref., 646 (attavina).

1856. " Insect. d. vorwelt, 172-173.

1867. Heer. Nene denkschr. schweiz. gesellsch, xxii, mem. 4 : 18.

1867. " Fose. hymen., 18.

1867. Mayr. Jahrb. k.-k. geol, reichsanst., xvii : 56.

1867. " Stud. Rad. form., 10.

Formica attavina. See Formica atavina.

6038. Formica buphthalma. (Formicidæ.)

Locality : Krottensee, Bohemia. Horizon: Aquitanian.

187\%. Novák. Sitzungsb. k. akad. wiss. Wien, lxxvi: 91-92. Pl. 3, fig. 2.

1877. “ Fauna egerer tertiärb., 21-22. Pl. 3, fig. \%.

6039. Formica capito. (Formicidæ.)

Localities : Aix, France; Radoboj, Croatia. Horizons: Ligurian, Mayencian.

1856. Heer. Viert. naturf. gesellsch. Zürich, i: 29.

1867. “ Neue denkschr. schweiz. gesellsch., xxii, mem. $4: 14-15$. Pl. 1,

figs. 13, 13b.
1867. is $\quad$ Foss. hymen., 14-15. Pl. 1, figs. 13, $13 b$.

6040. Formica cephalica. (Formicidæ.)

Locality: Prussian amber. Horizon : Ligurian.

1831. Burmeister. Oken, Isis, 1831: 1100.

6041. Formica cordata. (Formicidæ.)

Locality: Copal. Horizon: Recent.

1819. Schweigger. Beobacht. naturh. reisen, 119. Pl. 8, figs. 70, 70a, b.

1829. Holl. Handb. d. petref., 140.

1856. Giebel. Insect. d. vorwelt, 173. 
6042. Formica demersa. (Formicidæ.)

Locality: Oeningen, Baden. Horizon: Tortonian.

1849. Heer. 18:2. Giebel. 1856. "6 1867. Heer.

1867. “6

1868. Mayr.

1867. Heer.
1867. “

Insektenf. tertiärg. Oeningen, ii : 140-141. Pl. 11, fig. 7.

Deutschl. petref., 646 .

Insect. d. vorwelt, 171.

Nene denkschr. schweiz. gesellsch., xxii, mem. 4: 16. Pl. 1, fig. 16.

Foss. hymen., 16. Pl. 1, fig. 16.

6043. Formica flori. (Formicidæ.)

Locality: Prussian amber. Horizon: Ligurian.

Ameis. balt. bernst., 48-50. $\quad \mathrm{Pl} .2$, figs. 35-37.

6044. Formica fragilis. (Formicidio.)

Compared with F. longicornis Latr., Africa.

Locality: Radoboj, Croatia. Horizon: Mayencian.

Neue denkschr. schweiz. gesellsch., xxii, mem. 4: 8-9. $\quad$ Pl. 1, figs. $4,4 b, 5 a b$.

Foss. hymen., 8-9. Pl. 1, figs. 4, 4b, 5ab.

See also Lasius fragilis.

6045. Formica freyeri. (Formicidæ.)

Locality : Radoboj, Croatia. Horizon: Mayencian.

1867. Heer. Neue denkschr. schweiz. gesellsch., xxii, mem. 4 : 10-11. Pl. 1 , fig. 9.

1867. “ Foss. hymen., 10-11. Pl.1, fig. 9.

See also Lonchomyrmex freyeri.

6046. Formica gibbosa. (Formicidæ.)

Locality: Prussian amber. Horizon: Ligurian.

1822. Presl.

Del. prag., i : 197-198.

6047. Formica globiventris. (Formicidæ.)

Locality : Oeningen, Baden. Horizon: Tortonian.

1849. Heer. Insektenf. tertiärg. Oeningen, ii : 131-132. $\quad P l .9$, fig. 13.

1852. Giebel. Deutschl. petref., 646 .

1856. " Insect. d. vorwelt, 169 .

6048. Formica globularis. (Formicidæ.)

Localities: Radoboj, Croatia; Gabbro, Italy. Horizon: Mayencian.

1849. Heer.

Insektenf. tertiärg. Oeningen, ii : 131. Pl.10, fig.3.

1852. Giebel.

Deutschl. petref., 645 .

1856. " Insect. $d$. vorwelt, 168.

1867. Heer. Nene denkschr. schweiz. gesellsch., xxii, mem. 4 : 14.

1867. " Foss. hymen., 14.

1878. Capellini. Atti accad. linc., (3), mem. sc. fis., ii : 285.

See also Lasins globularis, Liometopum antiquum.

6049. Formica gracilis. (Formicidæ.)

Locality : Radoboj, Croatia. Horizon: Mayencian.

1867. Heer. Neue denkschr. schweiz. gesellsch., xxii, mem. 4 : 7-8. $P \boldsymbol{P l}$. , fig. $3 a b$.

1867. “ Foss. hymen., 7-8. Pl. 1, fig. $3 a b$, 
6050. Formica gravida. (Formicidæ.)

Locality: Oeningen, Baden. Horizon: Tortonian.

1849. Heer.

1852. Giebel.

Insektenf. tertiärg. Oeningen, ii : 114-115. $P l .9$, fig. 1.

1856. "6

Deutschl. petref., 645 .

Insect. d. vorwelt, 163.

6051. Formica heraclea. (Formicidæ.)

Locality : Oeningen, Baden. Horizon : Tortonian.

1349. Heer.

1852. Giebel.

Insektenf. tertiärg. Oeningen, ii : 116-118. Pl. 11, fig. 3.

1856. “

Deutschl. petref., 645 .

Insect. d. vorwelt, 164.

1865. Heer.

Urwelt der Schweiz, 386, expl. fig. 292 (=F. lignitum).

1867. “6

Neue denkschr. schweiz. gesellsch., xxii, mem. 4: 9-10. Pl. 1, fig. 7.

186\%. " Foss. hymen., 9-10 (see note about pl. 11, fig. 15c). Pl. 1, fig.7.

See also Formica lignitum, Camponotus heraclea, Camponotus lignitum.

6052. Formica imhoffii. (Formicidæ.)

Locality: Radoboj, Croatia. Horizon: Mayencian.

1849. Heer. Insektenf. tertiärg. Oeningen, ii : $138 . \quad P l .10, f i g .10$.

1852. Giebel. Deutschl. petref., 646.

1856. " Insect. d. vorwelt, 170-171.

See also Liometopum imhoffii, etc.

6053. Formica immersa. (Formicidæ.)

Locality: Oeningen, Baden. Horizon: Tortonian.

1849. Heer. Insektenf. tertiärg. Oeningen, ii: 122-123. $P l .9, f i g .5$.

1852. Giebel. Dentschl. petref., 645 .

1856. “ Insect. d. vorwelt, 166.

6054. Formica indurata. (Formicidæ.)

Locality : Radoboj, Croatia. Horizon: Mayencian.

1849. Heer. - Insektenf. tertiärg. Oeningen, ii : 116. Pl. 11, fig. 2.

1852. Giebel. Deutschl. petref., 645 .

1856. " I Insect. d. vorwelt, 164.

1867. Heer. Neue denkschr. schweiz. gesellsch., xxii, mem. 4: 9. Pl. 1, figs. $6 a b$.

1867. " Foss. hymen., 9. Pl. 1, figs. 6ab.

See also Camponotus induratus.

6055. Formica kollari. (Formicidæ.)

Locality : Radoboj, Croatia. Horizon : Mayencian.

1867. Heer. Neue denkschr, schweiz. gesellsch., xxii, mem. 4: 15. $P l .1$, figs. 14, $14 b$.

1867. “6 Foss. hymen., 15. Pl. 1, figs. 14, $14 b$.

6056. Formica lavateri. (Formicidæ.)

Locality: Oeningen, Baden. Horizon: Tortonian.

1849. Heer.

Insektenf, tertiärg. Oeningen, ii : 127. $\quad P l, 9, f i g .11$,

1852. Giebel.

Deutschl. petref., 645.

1856. " "

Insect. d. vorwelt, 167. 
1867. Heer.

Neue denkschr. schweiz. gesellsch., xxii, mem. 4: 11. Pl. 1, fig. $10 a$.

1867. "

Foss. hymen., 11. Pl. 1, fig. $10 a$.

1867. Mayr.

1867.

Jahrb. k.-k. geol. reichsanst., xvii : 53.

Stud. Rad. form., 7 .

See also Formica lavateri major, F. obtecta.

6057. Formica lavateri major. (Formicidæ.)

\section{Locality : Radoboj, Croatia. Horizon: Mayencian.}

1867. Heer. Neue denkschr. schweiz. gesellsch., xxii, mem. 4: 11. Pl. 1, fig. $10 b$.

1867. "6 Foss. hymen., 11. Pl. 1, fig. $10 b$.

See also Formica lavateri, etc.

6058. Formica lignitum. (Formicidø.)

Compared with F. herculeana L.

Localities: Oeningen, Baden; vicinity Boun, Rhenish Prussia. Horizons: Tortonian, Aquitanian?.

1837. Germar. Fauna insect. Europæ, xix: $19 . \quad P l .19$.

1849. Heer. Insektenf. tertiärg. Oeningen, ii : 112-114. Pl. 8, fig. 6.

1852. Giebel. Deutschl. petref., 645.

1856. “ Insect. d. vorwelt, 163.

1865. Lyell.

1865. Heer.

Elem geol., 6th ed., 250. Fig. $181 c$ on $p .249$.

1867. “

Urwelt der Schwèz, 360, 387, 470. Figs. $292 a-c$ on $p .386$.

Neue denkschr. schweiz. gesellsch., xxii, mem. 4: 7. Pl. 1, figs. $2 a b$.

186\%. " Foss. hymen., 7. Pl. 1, figs. 2ab.

1872. " Monde prim. Suisse, 442, 475, 573. Figs. 292a-c on p. 474.

1876. " Prim. world Switz., ii : 14, 45, 131. Figs. 292a-b on $p .44$.

1879. "Urwelt der Schweiz, $2^{\ominus}$ aufl., 386, 413, 497. Fig. 334 on p. 412.

See also Formica heraclea, etc.

6059. Formica longæva. (Formicidæ.)

Locality : Radoboj, Croatia. Horizon: Mayencian.

1849. Heer. Insektenf. tertiärg. Oeningen, ii: 132. Pl. 10, fig. 4.

1852. Giebel. Deutschl. petref., 645.

1856. " Insect. d. vorwelt, 169.

1867. Heer. Neue denkschr. schweiz. gesellseh., xxii, mem. 4 : 14.

1867. " Foss. hymen., 14.

See also Lasius longævus.

6060. Formica longicollis. (Formicidæ.)

Locality: Oeningen, Baden. Horizon: Tortonian.

1849. Heer.

1852. Giebel.

1856.

Insektenf. tertiärg. Oeningen, ii : 115-116. $P l .11, f i g .1$.

Deutschl. petref., 645.

Insect. d. vorwelt, 164.

6061. Formica longipennis. (Formicidæ.)

Locality: Radoboj, Croatia. Horizon: Mayencian.

1849. Heer. 1852. Giebel. 1856. "6 1867. Mayr. 1867. “
Insektenf. tertiärg. Oeningen, ii : 136. Pl. 10, fig.7.

Deutschl. petref., 646.

Insect. d. vorwelt, 170.

Jahrb. k.-k. geol. reichsanst., xvii: 55.

Stud. Rad. form., 9. 
6062. Formica longiventris. (Formicidæ.)

1849. Heer. 1852. Giebel. 1856. "6

1856. Giebel.

1822. Presl.

Locality: Radoboj, Croatia. Horizon: Mayencian. Insektenf. tertiärg. Oeningen, ii: 123. $\quad P l .9$, fig. 6.

Deutschl. petref., 645 .

Insect. d. vorwelt, 166.

6063. Formica Iucida. (Formicidæ.)

Locality : Prussian amber. Horizon : Ligurian.

Insect. d. vorwelt, 163-164.

6064. Formica luteola. (Formicidæ.)

Locality: Prussian amber. Horizon: Ligurian.

Del. prag., i : 197.

6065. Formica macrocephala. (Formicidæ.)

Localities: Oeningen, Baden ; Radoboj, Croatia. Horizons: Tortonian, Mayencian. 1849. Heer. 1852. Giebel. Insektenf. tertiärg. Oeningen, ii : 125-127. Pl. 9, fig. 10. 1856. " " Deutschl. petref., 645 .

1867. Heer. Insect. d. vorwelt, 167.

Neue denkschr. schweiz. gesellsch., xxii, mem. 4 : 12-13. $P l .1$, fig. 11.

1867. " " Foss. hymen., 12-13. Pl. 1, fig. 11.

1867. Mayr. Jahrb. k.-k. geol. reichsanst., xvii : 53. 1867. " Stud. Rad. form., 7.

See also Formica macrocephala oeningensis, F. macrocephala radobojana.

6066. Formica macrocephala oeningensis. (Formicidæ.) Locality: Oeningen, Baden. Horizon: Tortonian.

1849. Heer. Insektenf. tertiärg. Oeningen, ii : 126. $\mathrm{Pl}$. 9, fig. $10 \mathrm{c}$. See also Formica macrocephala, etc.

\section{Formica macrocephala radobojana. (Formicidæ.)}

Locality : Radoboj, Croatia. Horizon: Mayencian.

1849. Heer. Insektenf. tertiärg. Oeningen., ii : 126-127. Pl. 9, fig.10ab.

See also Formica macrocephala, etc.

6068. Formica macrognatha. (Formicidæ.)

Locality: Prussian amber. Horizon: Ligurian.

1822. Presl.

Del. prag., i: 198-199.

6069. Formica macrophthalma. (Formicidø.)

Localities: Oeningen, Baden; Sinigallia, Italy. Horizons : Tortonian, Helvetian. 1849. Heer. 1852. Giebel. 1856. "6 Insektenf. tertiärg. Oeningen., ii : 124-125. Pl. 9, fig. 8.

Deutschl. petref., 645 . Insect. $d$. vorwelt, 166 .

1859. Scarabelli. Mass.-Scarab., Flora foss. Senig., 25.

Formica major. See Formica lavateri major.

6070. Formica minutula. (Formicidæ.)

Localities: Radoboj, Croatia; Aix, France. Horizons: Mayencian, Ligurian. 1849. Heer. Insektenf. tertiärg. Oeningen, ii : 136. Pl. 10, fig. 8. 1852. Giebel.

Deutschl. petref., 646. 
1856. Giebel. Insect. d. vorwelt, 170.

1856. Heer. Viert, naturf. gesellsch. Zürich, i: 28. Pl. 2, fig.2b.

1867. " Neue denkschr. schweiz. gesellsch., xxii, mem. $4: 16 . \quad P l .1, f i g$. 15.

1867. " Foss. hymen., 16. Pl. 1, fig. 15.

See also Lasius minutulus.

\section{Formica nigra. (Formicidæ.)}

Localities: Copal (Bloch), Prussian amber (Presl). Horizons: Recent, Ligurian. 1776. Bloch. Beschäft. berl. gesellsch. naturf. freunde, ii: 168. Pl.3, fig.6. 1822. Presl. Del. prag., i: 196.

6072. Formica obesa. (Formicidæ.)

Loealities: Oeningen, Baden; Radoboj, Croatia. Horizons: Tortonian, Mayencian. 1849. Heer. Insektenf, tertiärg. Oeningen, ii : 108-110. Pl. 8, fig. 1.

1852. Giebel. Dentschl. petref., 645.

1856. “ Insect. d. vorwelt, 162.

1865. Heer. Urwelt der Schweiz, 360.

1872. " " Monde prim. Suisse, 442.

1876. " Prim. world Switz., ii : 14.

1879." " Urwelt der Schweiz, $2^{\mathrm{e}}$ aufl., 386.

See also Formica obesa radobojana, F'. obesa œuingensis, Ecophylla obesa radobojana, Myrmica pusilla.

\section{3: Formica obesa œningensis. (Formicidæ.)}

Locality: Oeningen, Baden. Horizon: Tortouian.

1849. Heer. Insektenf. tertiärg. Oeningen, ii: 110. Pl. 8, figs. $2 a b$.

See also Formica obesa, etc.

6074. Formica obesa radobojana. : (Formicidæ.)

Locality: Radoboj, Croatia. Horizon: Mayencian.

1849. Heer. Insektenf. tertiärg. Oeningen, ii : 108-109. Pl. 8, figs. 1a-e.

See also Formica obesa, etc.

6075. Formica oblita. (Formicidæ.)

Locality : Radoboj, Croatia. Horizon: Mayencian.

1867. Heer. Nene denkschr. schweiz. gesellsch., xxiii, mem. $4: 12,13-14$. $P l$. 1, figs. $12,12 b$.

1867. " Foss. hymen., 12, 13-14. Pl. 1, figs.12, $12 b$.

1867. Mayr. Jahrb. k.-k. geol. reichsanst., xvii : 56.

1867. " Stud. Rad. form., 10.

6076. Formica obliterata. (Formicidæ.)

Locality : Radoboj, Croatia. Horizon: Mayencian.

1849. Heer. 1852. Giebel.

1856. “

1867. Heer.

1867.

See also Lasius obliteratus.

Insektenf. tertiärg. Oeningen, ii : 144-145. Pl. 11, figs. 11e, 12.

Deutschl. petref., 646 .

Insect. d. vorwelt, 173.

Neue denkschr. schweiz. gesellsch., xxii, mem. 4 : 18.

Foss. hymen., 18, 
6077. Formica obscura. (Formicidæ.)

Locality: Radoboj, Croatia. Horizon: Mayerician.

1849. Heer. Insektenf. tertiärg. Oeningen, ii : 119-120. Pl.9, fig. 2.

1852. Giebel. Deutschl, petref., 645.

1856. " Insect. $d$. vorwelt, 165.

See also Lasius obscurus.

6078. Formica obtecta. (Formicidæ.)

Locality : Radoboj, Croatia. Horizon: Mayencian.

1849. Heer. Insektenf, tertiärg. Oeningen, ii: 123-124. Pl. 9, fig. 7.

1852. Giebel. Deutschl. petref., 645.

1856. " Insect. d. vorwelt, 166.

1867. Heer. Foss. hymen., 11 (=F. lavateri).

See also Formica lavateri, etc.

6079. Formica obvoluta. (Formicidæ.)

Locality: Radoboj, Croatia. Horizon: Mayencian.

1849. Heer. Insektenf. tertiärg. Oeningen, ii : 141-142. Pl.10, fig. $9 f$.

1852. Giebel. Deutschl. petref., 646.

1856. " Inseet. d. vorwelt, 172.

1867. Heer. Neue denkschr. schweiz. gesellsch., xxii, mem. 4: 16-17. Pl. 1, fig. $17 a$.

1867. " Foss. hymen., 16-17. Pl.1, fig. 17a.

1867. Mayr. Jahrb. k.-k. geol. reichsanst., xvii: 56.

1867. “ Stud. Rad. form., 10.

6080. Formica occultata. (Formicidæ.)

Locality: Radoboj, Croatia. Horizon: Mayencian.

1849. Heer. Insektenf. tertiärg. Oeningen, ii: 134-135. $P l .10, f i g .6 ; p l .11$, fig. 11.

1852. Giebel. Deutschl, petref,, 646.

1856. “ Insect, d. vorwelt, 169-170.

1858. Heer. Bull. séances soc. vaud. sc. nat., $\nabla: 150$.

1867. " Neue denkschr. schweiz. gesellsch., xxii, mem. 4: 15-16. Pl.3, fig. $15 b$.

1867. " Foss. hymen., 15-16. Pl. 3 , fig. $15 b$.

See also Formica occultata parschlugiana, Lasius occultatus, Hypoclinea haueri.

6081. Formica occultata parschlugiana. (Formicidæ.)

Locality: Parschlng, Styria. Horizon: Tortonian.

1849. Heer. Insektenf. tertiärg. Oeningen, ii : 135. Pl. 10, fig. 6 e.

See also Formica occultata, etc.

6082. Formica ocella. (Formicidæ.)

Locality : Radoboj, Croatia. Horizon: Mayencian.

1849. Heer. Insektenf. tertiärg. Oeningen, ii: 133-134. Pl. 10, fig. 5; pl. 11, fig. 14.

1852. Giebel. Deutschl. petref., 646.

1856. " Insect. d. vorwelt, 169.

1867. Mayr. Jahrb. k.-k. geol. reichsanst., xvị : 54.

1867. " Stud. Rad, form., 8.

Bull. 71-45 
6083. Formica oculata. (Formicidæ.)

Localities: Radoboj, Croatia; Aix, France. Horizons: Mayencian, Ligurian.

1849. Heer.

1852. Giebel.

1856. “

1856. Heer.

1867. “

1867. 4.

1867. Mayr.

1867. " "
Insektenf. tertiärg. Oeningen, ii : 143. $P l_{\bullet} .10, f i g .9 d$.

Deutschl. petref., 646 .

Insect. d. vorwelt, 172.

Viert. naturf, gesellsch: Zürich, i : 28.

Neue denksehr. schweiz. gesellsch., xxii, mem. 4: 17-18. Pl. 1, fig. 18.

Foss. hymen., 17-18. Pl. 1, fig. 18.

Jahrb. k.-k. geol. reichsanst., xvii : 56.

Stud. Rad. form., 10.

Formica œningensis. See Formica macrocephala œningensis, Formica obesa œningensis, Formica pinguicula ceningensis, Formica pinguis œningensis.

6084. Formica opthalmica. (Formicidæ.)

Locality: Radoboj, Croatia. Horizon: Mayencian.

1849. Heer.

Insektenf. tertiärg. Oeningen, ii : 125 . $\quad P l .9$, fig. 9.

1852. Giebel.

Deutschl. petref., 645.

1856.

Insect. d. vorwelt, $16 \%$.

1867. Heer.

Neue denkschr. schweiz. gesellsch., xxii, mem. 4 : 11-12.

1867. " Foss. bymen., 11-12.

1867. Mayr.

Jahrb. k.-k. geol. reichsanst., xvii : 52-53.

1867. " Stud. Rad. form., 6-7.

6085. Formica orbata. (Formicidæ.)

Locality : Oeningen, Baden. Horizon: Tortonian.

1849. Heer.

Insektenf, tertiärg. Oeningen, ii: 141. $\quad P l$. 11, fig. 8.

1852. Giebel.

Deutschl. petref., 646 .

1856. Giebel.

Insect. d. vorwelt, 171.

Formica parschlugiana. See Formica occultata parschlugiana.

6086. Formica parvula. (Formicidæ.)

Locality : Prussian amber. Horizon : Ligurian.

1822. Presl. Del. prag., i: 196-197.

6087. Formica pinguicula. (Formicidæ.)

Localities: Oeningen, Baden; Radoboj, Croatia. Horizons: Tortonian, Mayencian. 1849. Heer. Insektenf. tertiärg. Oeningen, ii : 118-119. Pl. 11, figs. 4, 9. 1852. Giebel. Deutschl. petref., 645.

1856. " Insect. d. vorwelt, 165.

1867. Heer. Neue denkschr. schweiz. gesellsch., xxii, mem. 4: 10. Pl. 1, fig.8. 1867. " Foss. hymen., 10. Pl. 1, fig. 8.

See also Formica pinguicula œningensis, F. pinguicula radobojana, Camponotus . pinguiculus radobojanus.

6088. Formica pinguicula œningensis. (Formicidø.)

Locality : Oeningen, Baden. Horizon: Tortonian.

1849. Heer. Insektenf. tertiärg. Oeningen, ii: 118. Pl. 11, fig. 4s

See also Formica pinguicula, etc. 
6089. Formica pinguicula radobojana. (Formicidæ.)

Loeality : Radoboj, Croatia. Horizon : Mayencian.

1 49. Heer. Insektenf. tertiärg. Oeningen, ii : 118-119. Pl. 9, fig. 9 b.

See also Formica pinguicula, etc.

6090. Formica pinguis. (Formividæ.)

Compared with F. herculeana.

Localities: Oeningen, Baden; Radoboj, Croatia. Horizons: Tortonian, Mayenciar 1849. Heer. Insektenf. tertiärg. Oeningen, ii : 110-111. Pl. 8, figs. 3, 4.

1852. Giebel. Deutschl. petref., 645.

1856. " Insect. d. vorwelt, 162.

1865. Heer. Urwelt der Sehweiz, 470.

1872. " Monde prim. Snisse, 573.

1876. " " Prim. world Switz., ii: 131.

1879. " Urwelt der Schweiz, $2^{\circ}$ aufl, 497.

See also Formica pinguis radobojana, F. pinguis œningensis, Camponotus pinguis radobojanus.

\section{Formica pinguis œningensis. (Formicidæ.)}

Locality: Oeningen, Baden. Horizon: Tortonian.

1849. Heer. Insektenf. tertiärg. Oeningen, ii: 111. Pl. 8, fig. 4ab.

See also Formica pinguis, etc.

\section{Formica pinguis radobojana. (Formicidæ.)}

Locality: Radoboj, Croatia. Horizon : Mayencian.

1849. Heer. Insektenf. tertiärg. Oeningen, ii: 110-111. Pl. 8, fig. 3.

See also Formica pinguis, etc.

6093. Formica primitiva. (Formicidæ.)

Locality: Oeningen, Baden. Horizon: Tortonian.

1849. Heer.

1852. Giebel.

1856. " "6

1867. Heer.

1867. "6

Insektenf. tertiärg. Oeningen, ii : 139-140. Pl. 11, fig. 6.

Deutschl. petref., 646.

Insect. d. vorwelt, 171.

Neue denkschr. schweiz. gesellsch., xxii, mem. 4: 16.

Foss. hymen., 16.

6094. Formica primordialis. (Formicidæ.)

1849. Heer.

1852. Giebel.

1854. Pictet.

1856. Giebel.

Locality: Oeningen, Baden. Horizon: Tortonian.

Insektenf. tertiärg. Oeningen, ii : 120-121. $\quad P l, 9$, figs. 3, 4.

Deutschl. petref., 645.

Traité de paléont., $2^{\mathrm{e}}$ éd., ii : 383. Pl. 40, fig. 18.

Insect. d, vorwelt, 165.

\section{Formica procera. (Formicidæ.)}

Locality: Oeningen, Baden. Horizon: Tortonian.

1849. Heer.

1852. Giebel.

1856. “

1865. Heer.

1867. "6
Insektenf. tertiärg. Oeningen, ii: 111-112. Pl. 8, figs. 5a-0.

Dentschl. petref., 645 .

Insect. d. vorwelt, 163.

Urwelt der Schweiz, 360.

Nene denkschr. schweiz. gesellsch., xxii, mem. 4: 6-7. Pl. 1, figs. $1 a b$. 
1867. Heer. 1872. "

1876. "

1879. “

Foss. hymen., 6, 7. Pl. 1, figs. $1 a b$.

Monde prim. Suisse, 442.

Prim. world Switz., ii : 14.

Urwelt der Sehweiz, $2^{\circ}$ aull., 386.

6096. - Formisa pulchella. (Formicidæ.)

Locality: Oeningen, Baden. Horizon: Tortonian.

1849. Heer.

1852. Giebel. 1856. " " 1867. Heer. 1867. "

Insektenf. tertiärg. Oeningen, ii : 142-143. Pl. 11, fig. 9.

Deutschl. petref,, 646.

Insect. d. vorwelt, 172.

Neue denkschr. schweiz. gesellsch., xxii, mem. 4: 17.

Foss. hymen., 17.

6097. Formica pumila. (Formicidæ.)

Locality: Radoboj, Croatia. Horizon: Mayencian.

1849. Heer.

1852. Giebel.

1856. “

1867. Heer.

1867. “

1867. Mayr.

1867. “

Insektenf. tertiärg. Oeningen, ii : 137. $P l .11$, fig. 15.

Deutschl. petrè., 646 .

Insect. d. vorwelt, $\mathbf{1 7 0}$.

Neue denkschr. schweiz. gesellsch., xxii, mem. 4: 16.

Foss. hymen., 16.

Jahrb. k.-k. geol. reichsanst, xvii : 55.

Stud. Rad. form., 9.

6098. Formica quadrata. (Formicidæ.)

Locality : Prussian amber (Holl), Copal (Giebel). Horizon : Ligurian, recent. 1829. Holl.

Handb. d. petref., 140.

1856. Giebel.

Insect. $d$. vorwelt, 173 .

Formica radobojana. See Formica macrocephala radobojana, Formica obesa radobojana, Formica pinguicula radobojana, Formica pinguis radobojana.

6099. Formica redtenbacheri. (Formicidæ.)

Locality: Radoboj, Croatia. Horizon: Mayencian.

1849. Heer. Insektenf. tertiärg. Oeningen, ii : 129-131. Pl. 10, fig. 2.

185\%. Giebel. Deutschl. petref., 645 .

1856. " Insect. d, vorwelt, 168.

1867. Heer. Neue denkschr. schweiz. gésellsch., xxii, mem. $4: 12,13$.

1867. " Foss. hymen., 12, 13.

See also Lasius redtenbacheri.

6100. Formica rufa (recent). (Formicidæ.)

Locality: Prussian amber. Horizon : Ligurian.

[1758. Linn6. Syst. nat., ed. x., i: 580.]

1831. Burmeister. Oken, Isis, 1831 : 1100.

6101. Formica saccharivora. (Formicidæ.)

Locality : Copal. Horizon: Recent.

1776. Bloch.

Beschäft. berl. gesellsch. naturf. freunde, ii : 178-179. Pl. 5, fig. 20.

\section{Formica salomonis. (Formicidæ.)}

Locality : Copal, Horizon : Recent.

1776. Bloch.

Beschäft. berl, gesellsch. naturf. freunde, ii: 172 178. Pl. 4, fig. $11 ; p l .5$, fig. 20. 


\section{Formica schmidtii. (Formicidæ.)}

Locality : Radoboj, Croatia. Horizon: Mayencian.

1849. Heer.

1852. Giebel.

1856. " "
Insektenf. tertiärg. Oeningen, ii : 138-139. $P l .11, f i g .5$.

Deutschl. petref., 646.

Insect. d. vorwelt, 171.

See also Liometopum imhoffii, etc.

6104. Formica seuberti. (Formicidæ.)

Localities: Oeningen, Baden; Tallyá, Hungary. Horizon : Tortonian.

1849. Heer. Insektenf. tertiärg. Oeningen, ii : 127-128. Pl. 9, fig. 12.

1852. Giebel. Dentschl. petref., 645.

1856. " Insect. d. vorwelt, 167-168.

1872. Heer. Mitth. jahrb. k. ung. geol. anstalt, ii, th. i: 8.

6105. Formica surinamensis (recent). (Formicidæ.)

Locality : Prussian amber. Horizon: Ligurian.

1830. Berendt. Insect. $d$. bernst., 37 (given on the authority of Schweigger as a recent species, but none such is quoted in Mayr's Index, 1883).

6106. Formica trigona. (Formicidæ.)

Locality : Prussian amber. Horizon : Ligurian.

1822. Presl. - Del. prag., i: 198.

6107. Formica ungeri. (Formicidæ.)

Locality : Radoboj, Croatia. Horizon: Mayencian.

1849. Heer.

1852. Giebel.

1856. “

1867. Heer.

1867. "6

1867. Mayr.

1867. "

1868. Mayr.

886. Brischke.

1886. " "

1890. Scudder.

Locality: Green River, Wyo.

Horizon : Oligocene. .

Dentschl, petref., 645.

Insect. d. vorwelt, 168.

Nene denkschr. schweiz. gesellsch., xxii, mem. 4: 12, 13.

Foss. hymen., 12, 13.

Jahrb. k.-k. geol, reichsanst., xvii : 53 (says two species are confounded, one a Formica, the other a Lasius).

Stud. Rad. form., 7.

6108. Gesomyrmex hörnesi. (Formicidæ.)

Locality : Prussian amber. Horizon: Ligurian.

Ameis. balt. bernst., 52-53. $\quad P l .2$, figs. 38-41.

6109. Glypta transversalis. (Ichneumonidæ.)

Tert. ins. N. A., 6̊13-614. Pl. 10, fig. 25.

6110. Gorytes? - _. (Crabronidæ.)

Locality: Prussian amber. Horizon: Ligurian.

Schrift. naturf. gesellsch. Danzig, n. f., vi, iii : 278.

Hym. bernst., 1 .

6111. Hemiteles —. (Ichneumonidæ.)

Locality : Prussian amber. Horizon: Ligurian.

1886. Brischke.

1886. “
Schrift. naturf. gesellsch. Danzig, n. f., vi, iii : 279.

Hym. bernst., 2 
6112. Hemiteles fasciata. (Ichneumonidæ.)

Locality : Radoboj, Croatia. Horizon: Mayencian.

1849. Heer. 1852. Giebel. 1856. “
Insektenf. tertiärg. Oeningen, ii : 170-171. Pl. 13, fig. 15.

Deutschl. petref., 647 .

Insect. d. vorwelt, 157 .

Hylotoma cineracea. See III, Hemiptera, Idem.

6113. Hymenopterites deperditus.

Locality: Spitzbergen. Horizon: Miocene.

1870. Heer. $\quad$ K. svensk. vetensk. akad. handl., viii, 7: 78. Pl. 16, figs. 44, 45.

6114. Hypoclinea - (Formicidæ.)

Locality: Brunstatt, Alsatia. Horizon: Middle Oligocene.

1890. Foerster. In litt.

6115. Hypoclinea anthracina. (Formicidæ.)

Locality: Radoboj, Croatia. Horizon: Mayencian.

1867. Mayr. Jahrb. k.-k. geol. reichsanst., xvii: 57 (quotes Heer's species as "Ponera" anthracina).

1867. " Stud. Rad. form., 11.

See also Poneropsis anthracina.

6116. Hypoclinea baltica. (Formicidø.)

Locality: Prussian amber. Horizon : Ligurian.

1868. Mayr. Ameis. balt. bernst., 64-67. Pl. 3, figs. 61-63; pl. 4, fig. 64.

6117. Hypoclinea constricta. (Formicidæ.)

Locality: Prussian amber. Horizon: Ligurian.

1868. Mayr. Ameis. balt. bernst., 60-61. Pl. 3, figs. 50-51.

6118. Hypoclinea cornuta. (Formicidæ.)

Locality: Prussian amber. Horizon: Ligurian.

1868. Mayr. Ameis. balt. bernst., 61. Pl. 3, fig. 52.

6119. Hypoclinea geinitzi. (Formicidø.)

Locality: Prussian amber. Horizon : Ligurian.

1868. Mayr. Ameis. balt. bernst., 58-60. Pl. 3, figs. 42-46.

6120. Hypoclinea goepperti. (Formicidæ.)

Locality : Prussian amber. Horizon: Ligurian.

1868. Mayr. Ameis. balt. bernst., 56-58. Pl. 1, figs. 3, 7; pl. 3, figs. 42-46.

6121. Hypoclinea haueri. (Formicidæ.)

Locality: Radoboj, Croatia. Horizon: Mayencian.

1867. Mayr.

Jahrb. k.-k. geol. reichsanst., xvii : 60-61 (named by Heer as

Formica occultata). $\quad P l .1$, fig. 11.

1867. “ Stud. Rad. form., 14-15 Pl. 1, fig. 11.

See also Formiea occultata, etc. 
6122. Hypoclinea kutschlinica. (Formicidæ.)

Compared with H. 4-denticulata.

Locality : Kutschlin, Bohemia. Horizon: Miocene.

1881. Deichmüller. Foss. ins. diatomeensch. Kutschlin, 30-32. Pl. 21, fig. 13.

1881. " Verhandl. leop.-carol. deutsche akad. naturf., xlii : 322-324. Pl. 21, fig. 13.

6123. Hypoclinea longipennis. (Formicidæ.)

Locality: Prussian amber. Horizon: Ligurian.

1868. Mayr. Ameis. balt. bernst., 67-68. Pl. 4, fig. 65.

6124. Hypoclinea nitida. (Formicidæ.)

Locality: Radoboj, Croatia. Horizon: Mayencian.

1867. Mayr. Jahrb. k.-k. geol. reichsanst., xvii : $5 \%$.

1867. " Stud. Rad. form., 11.

See also Ponera nitida, etc.

6125. Hypoclinea obliterata. (Formicidæ.)

Locality : Quesnel, British Columbia. Horizon: -

187\%. Seudder. Rep. geol. surv. Can., 1875-'76: 267.

1877. “ Ins. tert. Quesn., 2.

1877. “ Rapp. opér. comm. g6́ol. Can., 1875-'76: 295.

1890. “ Tert. ins. N. A., 616. Pl. 3, figs. 25, 26.

6126. Hypoclinea sculpturata. (F'ormicidæ.)

1868. Mayr.

Locality : Prussian amber. Horizon : Ligurian.

Ameis. balt. bernst., 62. Pl. 3, figs. 53-55.

6127. Hypoclinea tertiaria. (Formicidæ.)

Locality: Prussian amber. Horizon: Ligurian.

1868. Mayr. Ameis. balt. bernst., 62-64. Pl. 3, figs. 56-60.

6128. Ichneumon -. (Ichneumonidæ.)

Locality: Prussian amber. Horizon: Ligurian.

1886. Brischke. Schrift. naturf, gesellsch. Danzig, n. f., vi, iii : 278.

1886. " $\quad$ Hym. bernst., 1 .

6129. Ichneumon - (Ichneumonidæ.)

Locality : Aix, France. Horizon: Ligurian.

1829. Curtis. Edinb. new. phil. journ., vii: 295.

1829. " Murchison-Lyell, Tert. form. Aix, 11.

6130. Ichneumon - (Ichneumonidæ.)

Locality : Prussian amber. Horizon: Ligurian.

1822. Defrance. Dict. sc. nat., xxiii : 524 (des Irhneumons).

1829. Serres. G6ogn. terrains tert., 242.

1830. Berendt. Ins. bernstein, 37.

6131. Ichneumon —. (Ichneumonidæ.)

Locality: Prussian amber. Horizon: Ligurian.

1835. Gravenhorst. Uebers. schles. gesellsch. vaterl. cult., 1834 : 92. 


\section{Ichneumon —. (Ichneumonidæ.)}

Locality : Prussian amber. Horizon: Ligurian. 1820. Schlotheim. Petrefactenk., 43.

6133. Ichneumon - (Ichneunะonidæ.)

Locality: Aix, France. Horizon: Ligarian.

1829. Serres. Géogn. terrains tert., 229.

6134. Ichneumon aquensis. (Ichnenmonidæ.)

1861. Heer. Rech. climat pays tert., 153 (undescr.).

6135. Ichneumon ferrugineus. (Ichneumonidæ.)

Locality: Copal. Horizon: Recent.

1776. Bloch. Beschäft. berl. gesellsch. naturf. freunde, ii : 165-166. Pl. 3, fig. 3.

6136. Ichneumon infernalis. (Ichneumonidæ.)

Locality: Oeningen, Baden. Horizon: Tortonian.

1865. Heer.

18г2. " "

1876. “

1879. “

Urwelt der schweiz. Fig. 294 on p. 386.

Monde prim. Suisse. Fig. 294 on $p .474$.

Prim. world Switz., ii. Fig. 294 on p. 44.

Urwelt der Schweiz, $2^{\mathrm{e}}$ aufl. Fig. $3: 36$ on $p .412$.

6137. Ichneumon longævus. (Ichneumonidæ.)

Locality: Radoboj, Croatia. Horizon: Mayencian.

1849. Heer.

1852. Giebel.

1856. "6

Insektenf, tertiärg. Oeningen, ii : 166-167. Pl. 13, fig. 11.

Deutschl. petref., 647 .

Insect. d. vorwelt, 154.

6138. Ichneumon petrinus. (Ichneumonidæ.)

Locality: White River, Colo. Horizon: Oligocene.

1877. Seudder. Bull. U. S. geol. surv. terr., iii : 743.

1890. " Tert. ins. N.A., 608. Pl. 5, figs. 14, 15.

6139. Ichneumonites bellus. (Ichneumonidæ.)

Locality: Oeningen, Baden. Horizon : Mayencian.

1867. Heer. Neue denkschr. schweiz. gesallsch., xxii, mem. 4: 35. Pl. 3, figs. 19, 20.

1867. “ Foss. hymen., 35. Pl. 3, figs. 19, 20.

1885. Scudder. Zittel, Handb. palaeont, i, th. ii : 817. Fig. 1101.

1886. " Zittel-Barrois, Traité de paléont., ii : 819. Fig. 1118.

6140. Ichneumonites (Trogus?) fusiformis. (Ichneumonidæ.)

Locality: Radoboj, Croatia. Horizon: Mayencian.

1867. Heer. Neue denkschr. schweiz. gesellsch., xxii, mem. 4 : 35-36. Pl. 3, fig. 16.

1867. “ Foss. hymen., 35-36. Pl. 3, fig. 16. 
6141. Imhoffia nigra. (Formicidæ.)

1849. Heer. 1852. Giebel. 1854. Pictet. 1853-'56. Bror n. 1856. Giebel.

Locality: Oeningen, Baden. Horizon : Tortonian.

Insektenf. tertiärg. Oeningen, ii: 153-155. Pl. 12, fig. 10.

Deutschl. petref., 646.

Traité de paléont., $2^{e}$ éd., ii: $383 . \quad P l .40, f i g .17$.

Lethæa geogn., $3^{\mathrm{e}}$ aufl., iii : $646 . \quad P l .42^{\prime}$, figs. $22 a b$.

Insect. d. vorwelt, 176-17\%.

6142. Imhoffia pallida. (Formicidæ.)

Locality: Oeningen, Baden. Horizon: Tortonian.

1865. Heer.

1867. “

1867. “

1872. "

1876. " "

1879. “
Urwelt der Schweiz. Fig. 291 on p. 386.

Neue denkschr. schweiz. gesellsch., xxii, mem. 4 : 28-29. Pl. 2, figs. $9,9 b c$.

Foss. hymen., 28-29. Pl. 2, figs, 9, 9bc.

Monde prim. Suisse. Fig. 291 on $p .474$.

Prim. world Switz., ii. Fig. 291 on $p .44$.

Urwelt der Schweiz, $2^{\mathrm{e}}$ aufl. Fig. 333 on p. 412.

Laccophrys (vic.). _— See__—_ (Braconidæ) Scudder, x: 18.

6143. Lampromyrmex gracillimus. (Formicidæ.)

Locality : Prussian amber. Horizon : Ligurian.

1868. Mayr.

Ameis. balt. bernst., 95. Pl. 5, figs. 97-98.

6144. Lasius edentatus. (Formicidæ.)

Locality: Prussian amber. Horizon: Ligurian.

1868. Mayr. Ameis. balt. bernst., 46-47.

6145. Lasius fragilis. (Formicidæ.)

Locality: Radoboj, Croatia. Horizon: Mayencian.

186\%. Mayr. Jahrb. k.-k. geol. reichsanst., xvii : 56.

1867. “ Stud. Rad. form., I0.

See also Formica fragilis.

6146. Lasius globularis. (Formicidø.)

Locality : Radoboj, Croatia. Horizon : Mayencian.

1867. Mayr. Jahrb. k.-k. geol. reichsanst., xvii : 54.

1867. "6 Stud. Rad. form., 8.

See also Formica globularis, Liometopum antiquum.

6147. Lasius longævus. (Formicidæ.)

Locality : Radoboj, Croatia. Horizon: Mayencian.

1867. Mayr. Jahrb.k.-k. geol, reichsanst., xvii: 54.

1867. " Stud. Rad. form., 8.

See also Formica longæva.

6148. Lasius minutulus. (Formicidæ.)

Locality: Radoboj, Croatia. Horizon: Mayencian.

1867. Mayr. Jahrb. k.-k. geol. reischsanst., xvii : 55.

1867. "6 Stud. Rad. form., 9.

See also Formica minutula. 
6149. Lasius obliteratus. (Formicidæ.)

Locality: Radoboj, Croatia. Horizon: Mayencian.

1867. Mayr.

1867. “

Jahrb. k.-k. geol. reichsanst., xvii : 56.

See also Formica obliterata.

6150. Lasius oblongus. (Formicidæ.)

Locality: Schossnitz, Silesia. Horizon: Miocene.

1869. Assmann. Beitr. insektenf. d. vorwelt (Schles. zeitschr. f. ent., (2), i), 38-39. Pl. 1, figs. 1, 1 a.

6151. Iasius obscurus. (Formicidæ.)

Compared with L. niger L.

Locality : Radoboj, Croatia. Horizon: Mayencian.

1867. Mayr. Jahrb. k.-k. geol. reichsanst., xvii : 52.

1867. " Stud. Rad. form., 6.

See also Formica obscura.

6152. Iasius occultatus. (Formicidæ.)

Locality : Radoboj, Croatia. Horizon: Mayencian.

1867. Mayr. Jahrb. k.-k. geol. reischsanst., xvii : 54-55.

1867. " Stud. Rad. form., 8-9.

See also Formica occultata, etc.

6153. Lasius pumilus. (Formicidæ.)

Locality : Prussian amber. Horizon: Ligurian.

1868. Mayr. Ameis. balt. bernst., 46. Pl. 2, fig. 33.

6154. Iasius punctulatus. (Formicidæ.)

Locality: Prussian amber. Horizon: Ligurian.

1868. Mayr. Ameis. balt. bernst., 46. Pl. 2, fig. 34.

6155. Lasius redtenbacheri. (Formicidæ.)

Locality : Rødoboj, Croatia. Horizon: Mayencian.

1867. Mayr. Jahrb. k,-k. geol, reichsanst., xvii: 54.

1867. " Stud. Rad. form., 8.

See also Formica redtenbacheri.

6156. Lasius schiefferdeckeri. (Formicidæ.)

Locality : Prussian amber. Horizon: Ligurian.

1868. Mayr. Ameis. balt. bernst., 44-46. Pl. 2, figs. 27-32.

6157. Lasius terreus. (Formicidæ.)

Locality: Green River, Wyo. Horizon: Oligocene.

1878. Scudder. Bull. U. S. geol. surv. terr., iv : 747-748.

1890. " Tert. ins. N. A., 618-619. Pl. 10, fig. 23.

Lasius ungeri. See Formica ungeri.

6158. Ieptalea - (2 sp.). (Formicidø.)

Locality: Sicilian amber. Horizon:-

1840. Erichson. Arch. naturg., $\nabla, 2: 309$.

1840. “ Ber. wiss. leist. entom., 1838: 29.

See also Pseudomyrme - $\longrightarrow$ Scudder, etc. 
6159. Leptothorax gracilis. (Formicidæ.)

1868. Mayr.

Locality : Prussian amber. Horizon: Ligurian.
Ameis. balt. bernst., 89-90. Pl. 5, figs. 89-92.

Leptothorax pusillus. See Myrmica pusilla.

6160. Liometopum antiquum. (Formicidæ.)

Localitý : Radoboj, Croatia. Horizon: Mayencian.
1867. Mayr. Jahrb.k.-k. geol. reichsanst., xvii: 60 (named by Heer as Formica globularis). $P l .1$, fig. 10.
1867. “ Stud. Rad. form., 14. Pl.1, fig. 10.
See also Lasius globularis, Formica globularis.

\section{Liometopum imhoffii. (Formicidæ.)}

Locality: Radoboj, Croatia. Horizon: Mayencian.

$\begin{array}{ll}\text { 1867. “ Jahrb. k.-k. geol, reich } & \text { Stud. Rad. form., } 9 .\end{array}$

See also Formica imhoffi, Poneropsis imhoffi, Liometopum schmidtii, Formica schmidtii, Poneropsis schmidtii.

6162. Liometopum pingue. (Formicidæ.)

Locality: White River, Utah. Horizon: Oligocene.

1877. Scudder. Bull. U. S. geol. surv. terr., iii : 742-743.

1890. “ Tert. ins. N. A., 617. Pl. 5, fig. 10.

See also Myrmica — Scudder, 1867.

6163. Liometopum schmidtii. (Formicidæ.)

Locality: Radoboj, Croatia. Horizon: Mayencian.

1867. Mayr. Jahrb. k.-k. geol. reichsanst., xvii: 55-56 (Schmidti) (=L. imhoffii).

1867. " Stud. Rad. form., 9-10.

See also Liometopum imhoffii, etc.

6164. Lithotorus cressoni. (Ichneumonidæ.)

Locality: Green River, Wyo. Horizon: Oligocene.

1890. Scudder. Tert. ins. N. A., 609. Pl. 10, fig. 21.

6165. Lonchomyrmex freyeri. (Formicidæ.)

Locality: Radoboj, Croatia. Horizon : Mayencian.

1867. Mayr. Jahrb. k.-k. geol, reichsanst., xvii: 56,61. Pl. 1, fig. 12.

1867. “ Stud. Rad. form., 10,15. Pl.1, fig. 12.

1885. Scudder. Zittel, Handb. palaeont., i, th. ii: $818 . \quad$ Fig. 1104.

1886. " Zittel-Barrois, Traite de paléont., ii: 820. Fig. 1120.

See also Formica freyeri.

6166. Lonchomyrmex nigritus. (Formicidæ.)

Locality : Schossnitz, Silesia. Horizon: Miocene.

1869. Assmann. Beitr. insektenf. d. vorwelt (Schles. zeitschr. f. ent., (2), i), 39-40. Pl. 1, figs. 2, $2 a$.

6167. Lophyrus —. (Tenthredinidæ.)

Locality: Prussian amber. Horizon: Ligurian.

1886. Brischke. Schrift. naturf. gesellsch. Danzig, n. f., vi, iii : 279.

1886. “ Hym. bernst., 2. 
6168. Lophyrus? (larva). (Tenthrèdinidæ.)

Locality: Prussian amber. Horizon : Ligurian. 1856. Menge. Progr. petrischule Danzig, 1856: 24.

6169. Lyda (larva). (Tenthredinidæ.)

Locality: Prussian amber. Horizon: Ligurian. 1856. Menge. Progr. petrischule Danzig, 1856: 24.

6170. Macrocentrus (Braconidæ.)

Locality : Prussian amber. Horizon: Ligurian. 1886. Brischke. Sehrift. naturf. gesellsch. Danzig, n. f., vi, iii : 279. 1886. "6 Hym. bernst., 2.

Macrocentrus (vic.) - See- - $\longrightarrow$ (Braconidø) Scudder, x: 18.

6171. Macromischa beyrichi. (Hormicidæ.)

1868. Mayr.

Locality: Prussian amber. Horizon: Ligurian. Ameis. balt. bernst., 84. Pl. 4, figs. 80-81.

6172. Macromischa petiolata. (Formicidæ.)

1868. Mayr.

Locality: Prussian amber. Horizon : Ligurian. Ameis. balt. bernst., 85. Pl. 4, figs. 83-84.

6173. Macromischa rudis. (Formicidæ.)

Locality: Prussian amber. Horizon: Ligurian.

1868. Mayr.

Ameis. balt. bernst., 85-86. Pl. 4, fig. 85 .

6174. Macromischa rugosostriata. (Formicidø.)

1868. Mayr.

Locality: Prussian amber. Horizon: Ligurian. Ameis. balt. bernst., 84. Fl. 4, fig. 82.

6175. Melipona (vic.) —. (Apidæ.)

Locality: Prussian amber. Horizon: Ligurian.

1886. Brischke. Schrift. naturf. gesellsch. Danzig, n. f., vi, iii : 278. 1886. “ Hym. bernst., 1.

\section{Mellinus —. (Crabronidæ.)}

Locality : Prussian amber. Horizon: Ligurian.

1886. Brischke. 1886.

Schrift. naturf. gesellsch. Danzig, n. f., vi, iii : 278.

Hym. bernst., 1.

\section{Mesochorus (vic.) - (Ichneumonidæ.)}

Locality: Prussian amber. Horizon: Ligurian.

1886. Brischke. Schrift. naturf. gesellsch. Danzig, n. f., vi, iii : 279. 1886. " Hym. bernst., 2.

\section{Mesoleptus (vic.) —. (Ichneumonidæ.)}

Locality : Prussian amber. Horizon: Ligurian.

1886. Brischke. 1886.
Schrift. naturf. gesellsch. Danzi, Hym. bernst., 2. 
6179. Meteorus ㄱ. (Braconidæ.)

Locality: Prussian amber. Horizon: Ligurian.

1886. Brischke. Schrift. naturf. gesellsch. Danzig, n. f., vi, iii : 279.

1886. " Hym. bernst., 2.

Meteorus (vic.) - See $\longrightarrow-$ (Braconidæ) Sendder, x: 28.

Mimesa —. See Psen — Brischke.

6180. Monomorium pilipes. (Formicidæ.)

Locality: Prussian amber. Horizon: Ligurian.

1868. Mayr. Ameis. balt. bernst., 91-92. Pl. 5, figs. 93-94.

6181. Mutilla —. (Mutillidæ.)

Locality : Prussian amber. Horizon : Ligurian.

1886. Brischke. Schrift. naturf. gesellsch. Danzig, n.f, vi, iii : 278.

1886. 6 Hym. bernst., 1 .

6182. Mutilla - (Mutillidæ.)

Locality: Prussian amber. Horizon : Ligurian.

1827. Brongniart. Dict. sc. nat., li : 234 (mutilles).

6183. Mutilla —. (Mntillidæ.)

Locality: Brunstatt, Alsatia. Horizon: Middle Oligocene.

1890. Foerster. In litt.

6184. Myrmar —. (Proctotrupidæ.)

Locality : Prussian amber. Horizon: Ligurian.

1868. Duisburg. Schriften k. physik.-ökon. gesellsch. Königsberg, ix: 24-28. Figs. 1, 2.

See also Myrmar duisburgi.

6185. Myrmar duisburgi. (Proctotrupidæ.)

Locality: Prussian amber. Horizon: Ligurian.

1877. Stein. Mitth. münch. entom. ver., i: 30.

1885. Scudder. Zittel, Handb. palaeont., i, th. ii: 817. Fig. 1099.

1886. " Zittel-Barrois, Traité de paléont, ii : 819. Fig. 1116.

See also Myrmar — Duisburg.

6186. Myrmica - (Formicidæ.)

Locality: Prussian amber. Horizon: Ligurian.

1832. Burmeister. Handb. ent., i: 636.

1836. " Man. ent., 577.

6187. Myrmica -. (Formicidæ.)

Locality: Prussian amber. Horizon: Ligurian.

1856. Menge. Progr. petrischule Danzig, 1856: 25.

6188. Myrmica —. (Formicidæ.)

Locality: Green River, Wyo. Horizon: Oligocene.

1867. Seudder. Proc. Bost. soc. nat. hist., xi : 117.

See also Liometopnm pingue. 
6189. Myrmica - (Formicidæ.)

Locality : Green River, Wyo. Horizon: Oligocene.

1878. Scudder. Bull. U. S. geol. surv. terr., is: 748.

1890. " Tert. ins. N. A., 615-616. Pl. 10, fig. 22.

\section{Myrmica —. (Formicidæ.)}

Locality: Gurnet Bay, Isle of Wight. Horizon : Bembridge Limestone. 1879. Woodward. Quart. journ. geol. soc., Lond., xxxv: 344. 1879. “ Geol.mag., n. s., $\nabla: 89$.

\section{Myrmica æmula. (Formicidæ.)}

Locality : Parschlug, Styria. Horizon : Tortonian.

1849. Heer. Insektenf. tertiärg. Oeningen, ii: $161 . \quad P l .13$, fig. 4.

1852. Giebel. Dentsehl. petref., 646.

1856. " Insect. d. vorwelt, 179.

\section{Myrmica angusticollis. (Formicidæ.)}

Locality: Oeningen, Baden. Horizon: Tortonian.

1849. Heer.

1852. Giebel.

Insektenf. tertiärg. Oeningen, ii : 162-163. Pl. 13, fig. 7.

Deutschl. petref., 646 .

1856. "Insect. d. vorwelt, 180.

6193. Myrmica bicolor. (Formicidæ.)

Locality : Radoboj, Croatia. Horizon : Mayencian.

1867. Heer. Neue denkschr. schweiz. gesellsch., xxii, mem. 4: $31 . \quad$ Pl. 2, figs. 11, $11 b$.

1867. “ Foss. hymen., 31. Pl. 2, figs. 11, 11 .

1867. Mayr. Jahrb. k.-k. geol. reichsanst., xvii : 60.

1867. “ Stud. Rad. form., 14 (= M. tertiaria radobojana).

Seo also Myrmica tertiaria, etc.

6194. Myrmica bremii. (Formicidæ.)

Locality : Radoboj, Croatia. Horizon: Mayencian.

1849. Heer.

1852. Giebel. 1856. "
Insekteuf. tertiärg. Oeningen, ii: 161-162. $\quad P l .13$, fig. 5.

Deutschl. petref., 646.

Insect. d. vorwelt, 179-180.

6195. Myrmica concinna. (Formicidæ.)

Locality : Radoboj, Croatia. Horizon: Mayencian.

1867. Heer.

1867. “

1867. Mayr. 1867. “

Nene denkschr. schweiz. gesellsch., xxii, mem. 4: 32. $\mathrm{Pl} .2$, figs. 13, $13 b$.

Foss. hymen., 32. Pl. 2, figs. 13, $13 b$.

Jahrb. k.-k. geol. reichsanst., xvii : 59-60.

Stud. Rad. form., 13-14.

6196. Myrmica duisburgi. (Formicidæ.)

Locality: Prussian amber. Horizon: Ligurian.

1868. Mayr. Ameis. balt. bernst., 87-88. Pl. 5, figs. 87-88. 
6197. Myrmica jurinei. (Formicidæ.)

Locality : Radoboj, Croatia. Horizon: Mayencian.

1849. Heer. 1852. Giebel. 1856. " 1867. Mayr. 1867. “

1868. Mayr.

1849. Heer. 1856. “

1849. Heer. 1852. Giebel. 1856. “ 1852. Giebel.

Insektenf. tertiärg. Oeningen, ii : 163-164. $P l .13$, fig. 8. Deutschl. petref., 646.

Insect. d. vorwelt, 180.

Jahrb. k.-k. geol. reichsanst., xvii : 59.

Stud. Rad. form., 13.

6198. Myrmica longispinosa. (Formicidæ.)

Locality : Prussian amber. Horizon : Ligurian. Ameis. balt. bernst., 87. Pl. 4, fig. 86 .

6199. Myrmica macrocephala. (Formicidæ.)

Locality: Oeningen, Baden. Horizon: Tortônian.

Insektenf. tertiärg. Oeningen, ii : 158-159. Pl. 12, fig. 14.

Deutschl. petref., 646.

Insect. d. vorwelt, 178.

6200. Myrmica molassica. (Formicidæ.)

Locality: Oeningen, Baden. Horizon: Tortonian.

Insektenf. tertiärg. Oeningen, ii: 162. Pl. 13, fig. 6.

Deutschl, petref., 646.

Insect. d. vorwelt, 180.

6201. Myrmica? nebulosa. (Formicidø.)

Locality: Krottensee, Bohemia. Horizon: Aquitanian.

1877. Novák.

1877. "

Sitzungsb. k. acad. wiss. Wien, 1xxvi : 91. Pl. 3, fig. 1.

Fauna egerer tertiärb., 21. $\quad P l$. 3, fig.1.

6202. Myrmica obsoleta. (Formicidæ.)

Locality : Parschlug, Styria. Horizon: Tortonian.

1849. Heer. 1852. Giubel. 1856. “ 1867. Heer. 1867. "
Insektenf. tertiärg. Oeningen, ii: 160. $\quad P l .13, f i g .3$.

Deutschl. petref., 646.

Insect. d. vorwelt, 179.

Nene denkschr. schweiz. gesellsch., xxii, mem. 4 : 30.

Foss. hymen., 30 .

Myrmica œningensis. See Myrmica tertiaria œningensis.

6203. Myrmica pusilla. (Formicidæ.)

Locality : Radoboj, Croatia. Horizon: Mayencian.

1849. Heer. 1852. Giebel. 1856. “ 1867. Heer.

1867. “ 186\%. Mayr.

1867. "
Insektenf. tertiärg. Oeningen, ii : 164-165. Pl. 13, fig. 9.

Deutschl. petref., 646 .

Insect. d. vorwelt, 180.

Neue denkschr. schweiz. gesellsch., xxii, mem. 4: 32-33. Pl. 2, fig. 14.

Foss. hymen., 32-33. $\quad P l .2$, fig. 14.

Jahrb. k.-k. geol, reichsanst., xvii : 59 (one specimen is probably a Leptothorax, another perhaps a Pheidole).

Stud. Rad. form., 13.

See also Formica obesa, etc.

Myrmica radobojana. See Myrmica tertiaria radobojana.

Myrmica ruficeps. See Myrmica rugiceps. 
6204. Myrmica rugiceps. (Formicidæ.)

Loealities: Oeningen, Baden; Schossnitz, Silesia. Horizon: Tortonian. 1849. Heer. Insektenf. tertiärg. Oeningen, ii : 160-161. Pl. 13, fig. 2. 1852. Giebel. Deutschl. petref., 646.

1855. Goeppert. Tert. flora Schossnitz, p. vii (ruficeps). Pl. 26, figs. 50-51. 1856. Giebel. Insect. d. vorwelt, 179.

See also Pheidologeton schossnicensis.

6205. Myrmica tertiaria. (Formicidæ.)

Localities: Oenfngen, Baden: Radoboj, Croatia. Horizons: Tortonian, Mayencian.

1849. Heer.

1852. Giebel.

1856. " "

1865. Heer.

1867. "6

186\%. “

1872. "6

1876. "

1879. " "
Insektenf. tertiärg. Oeningen, ii : 159-160. Pl. 13, figs. 1a-c.

Deutschl. petref., 646 .

Insect. d. vorwelt, 179.

Urwelt der Schweiz. Fig. 293 on $p .386$.

Neue denkschr. schweiz. gesellsch., xxii, mem. 4 : 30 . Pl. 2, figs. $10,10 b$.

Foss. hymen., 30. Pl. 2, figs. 10, $10 b$.

Monde prim. Suisse. Fig. 293 on $p .474$.

Prim. world Switz., ii. Fig. 293 on p. 44.

Urwelt der Schweiz, $2^{\circ}$ aufl. Fig. 335 on p. 412.

See also Myrmica tertiaria œningensis, M. tertiaria radobojana, M. bicolor.

6206. Myrmica tertiaria œningensis. (Formicidæ.)

Locality : Oèningen, Baden. Horizon: Tortonian.

1849. Heer. Insektenf. tertiärg. Oeningen, ii : 160. Pl. 13, fig. 1 c.

See also Myrmica tertiaria, etc.

\section{Myrmica tertiaria radobojana. (Formicidæ.)}

Locality: Radoboj, Croatia. Horizon: Mayencian.

1849. Heer.

1867. Mayr.

1867. " "
Insektenf. tertiärg. Oeningen, ii : 159. Pl. 13, figs. $1 a b$.

Jahrb. k.-k. geol, reichsanst., xvii: 58-59.

Stud. Rad. form., 12-13.

See also Myrmica tertiaria, etc.

6208. Myrmica venușta. (Formicidæ.)

Locality : Radoboj, Croatia. Horizon: Mayencian.

1867. Heer. Neue denkschr. sehweiz. gesellsch., xxii, mem. 4 : 31-32. Pl. 2, figs. 12, $12 b$.

186\%. “ Foss. hymen., 31-32. Pl. 2, figs. $12,12 b$.

See also Solenopsis? venusta.

\section{Myrmicites —. (Formicidæ.)}

Locality: Brunstatt, Alsatia. Horizon: Middle Oligocene.

1890. Foerster. In litt.

6210. Myrmicium boreale. (Formicidæ.)

Locality: Spitzbergen. Horizon: Miocene.

1870. Heer. K. svensk. vetensk, akad. handl., viii, 7: 78. Pl. 16, figs. 46, 46 .

6211. Neuropachys —. (Uroceridæ.)

Locality: Radoboj, Croatia. Horizon: Mayencian.

1858. Heer, Ber, versamml. deutsch. naturf, xxxii : 118 (undeser.). 
6212. Crcophylla ——. (Formicidæ.)

Locality: Brunstatt, Alsatia. Horizon: Middle Oligocene.

1890. Foerster. In litt.

6213. Grophylla brischkei. (Formicidæ.)

Locality: Prussian amber. Horizon: Ligurian.

1868. Mayr. Ameis. balt. bernst., 31-32. Pl. 1, figs. 12-13.

6214. Gcophylla obesa radobojana. (Formicidæ.)

Compared with O. smaragdina.

Locality: Kutschliu, Bohemia. Horizon: Miocene.

1867. Mayr. Jahrb. k.-k. geol. reichsanst., xvii : 50-51.

1867. " Stud. Rad. form., 4-5.

1881. Deichmüller. Foss. ins. diatomeensch.Kutschlin, 33-35. Pl. 21, figs. 14, 15, 15a. 1881. " Verhandl, leop.-carol. deutsch. akad. naturf., xlii: 325-327. $P l$. 21, figs. 14, 15, 15 a.

See also Formica obesa, etc.

Ecophylla radobojana. See Ecophylla obesa radobojana.

6215. Ophion -. (Ichneumonidæ.)

Locality : Aix, France. Horizon: Ligurian.

1829. Serres. Géogn. terrains tert., 229.

6216. Ophion (or Campoplex) __. (Ichneumonidæ.)

Locality: Pianico, Italy. Horizon: Quaternary.

1882. Sordelli. Alc. ins. foss. Lombardia, 5-6. Fig.

1882. " Bull. soc. ent. ital., xiv: 228-229. Fig.

6217. Osmia - (Apidœ.)

Locality : Prussian amber. Horizon: Ligurian.

1856. Menge. Progr. petrischule Danzig, 1856: 26.

6218. Osmia antiqua. (Apidæ:)

Locality: Oeningen, Baden. Horizon: Tortonian.

1849. Heer. Insektenf. tertiärg. Oeningen, ii: 95-96. Pl. 7, fig. 2.

185\%. Giebel. Deutschl. petref., 645.

1856. " Insect. d. vorwelt, 182.

6219. Osmia carbonum. (Apidø.)

Loeality: Rott, Rheñish Prussia. Horizon: Aquitanian.

1862. Heyden. Palaeontogr., $\mathrm{x}:$ 75. Pl. 10, figs. 11, 12.

6220. Osmia dubia. (Apidæ.)

Locality : Orsberg (Rhenish Prussia?). Horizon: Miocene. 1856. Giebel. Insect. d. vorwelt, 182.

See also Apiaria dubia.

6221. Passalœcus —. (Crabronidæo)

Locality: Prussian amber. Horizon : Lignrian.

1886. Brischke. Schrift. naturf. gesellsch. Danzig, n. f., vi, iii : 278. 1886. " Hym. bernst., 1.

Bull. $71-46$ 
6222. Pepsis - (Pompilidæ.)

Locality: Prussian amber. Horizon: Ligurian. 1832. Burmeister. Handb. ent., i: 636.

1836. “ Man. ent., 577.

\section{Perilampus ㄴ. (Chalcididæ.)}

Locality: Prussian amber. Horizon: Ligurian.

1886. Brischke. Schrift. naturf. gesellsch. Danzig, n. f., vi : iii : 279.

1886. " Hym. bernst., 2.

\section{Pezomachus —. (Ichneumonidæ.)}

Locality: Prussian amber. Horizon: Ligurian.

1886. Brischke. Schrift. naturf. gesellsch. Danzig, n. f., vi, iii : 279.

1886. "6 Hym. bernst., 2.

Pheidole pusilla. Seo Myrmica pusilla.

6225. Pheidologeton antiquus. (Formieidx.)

Locality: Prussian amber. Horizon: Ligurian.

1868. Mayr.

Ameis. balt. bernst., 93. Pl. 5, figs. 95-96.

6226. Pheidologeton bohemicus. (Formicidæ.)

Locality : Krottensee, Bohemia. Horizon: Aquitanian

1877. Novák. Sitzungsb. k. akad. wiss. Wien, lxxvi: 90-91. Pl. 3, fig. 3.

1८77. " Fauna egerer tertiärb., 20-21. Pl. 3, fig. 3.

6227. Pheidologeton schossnicensis. (Formicidæ.)

Locality : Schossnitz, Silesia. Horizon: Miocene.

1869. Assmann. Beitr. insectenf. d. vorwelt (Schles. zeitschr. f. ent., (2), i), 40. Pl. 1, figs. $3,3 a$.

See also Myrmica rugiceps.

\section{Phygadeuon —. (Ichneumonidæ.)}

1886. Brisehke. Schrift. naturf. gesellsch. Danzig, n. f., vi, iii : 279.
1836. "6

Locality : Prussian amber. Horizon: Ligurian.

$$
\text { 6229. Pimpla —. (Ichneumonidæ.) }
$$

Locality: - Horizon: - .

1846. Giebel. Paläozool., 281.

6230. Pimpla —. (Ichneumonidæ.)

1854. Pictet. Traité de paléont., $2^{\circ} 6$ d., ii : 382 (fide Serres, MS.).

6231. Pimpla —. (Ichneumonidæ.)

Locality: Florissant, Colo. Horizon: Oligocene.

1881. Scudder. Bull. U. S. geol. surv. terr., vi : 290. 
6232. Pimpla antiqua. (Ichneumonidæ.)

Locality : Aix, France. Horizon : Ligurian.

1852. Sanssure.

Rev. mag. zool., iv: 580. Pl. 23, fig. 5.

1852. “ Nouv, hym. foss., 1-2. Pl. 23, fig. 5.

6233. Pimpla (Rhyssa) antiqua. (Ichneumonidæ.)

Compared with Pimpla (Rhyssa) persuasoria (L.).

Locality : Radoboj, Croatia. Horizon: Mayencian.

1867. Heer. Neue denkschr. gesellsch., xxii, mem. 4: $36 . \quad$ Pl. 3, fig. 18.

186\%. 66 Foss. hymen., 36. Pl. 3, fig. 18.

6234. Pimpla decessa. (Ichnenmonidæ.)

Compared with P. instigator Fabr.

Locality: Quesnel, British Columbia. Horizon:

1877. Scudder. Rep. geol. surv. Can., 1875-'76: 269.

1877. " Ins. tert. Quesnel, 4.

1877. “ Rapp. opér. comm. géol. Can., 1875-76: 297-298.

1890. " Tert. ins. N. A., 612-613. Pl. 3, fig. 27.

6235. Pimpla saussurii. (Ichneumonidæ.)

Locality : Aix, France. Horizon: Ligurian.

1856. Heer. Viert. naturf. gésellsch. Zürich, i: 29. Pl. 2, fig. 15.

6236. Pimpla saxea. (Ichneumonidæ.)

Locality: Quesnel, British Columbia. Horizon:-

1877. Scudder. Rep. geol. surv. Can., 1875-76: 268.

1877. " Ins. tert. Quesnel, 3.

1877. “ Rapp. opér. comm. géol. Can., 1875-76 : 296-297.

1890. “ Tert. ins. N. A., 610-611. Pl. 3, fig. 23.

6237. Pimpla senecta. (Ichneumonidæ.)

Locality: Quesnel, British Columbia. Horizon:

1877. Scudder. Rep. geol. surv. Can., 1875-76: 268-269.

1877. " Ins. tert. Quesnel, 3-4.

1877. " Rapp. opér. comm. géol. Can., 1875-'76: 297.

1890. " Tert. ins. N. A., 611-612. Pl. 3, figs. 29-31.

6238. Pimpla succini. (Ichneumonidæ.)

Locality: Prussian amber. Horizon: Ligurian.

1856. Giebel. Insect. d. vorwelt, 155.

6239. Plagiolepis klinsmanni. (Formicidæ.)

Locality: Prussian amber. Horizon: Ligurian.

1868. Mayr. $\quad$ Ameis. balt. bernst., 37-38. Pl. 1, figs. 19, 20.

6240. Plagiolepis kiunowi. (Formicidæ.)

Locality: Prussian amber. Horizon: Ligurian.

1868. Mayr. Ameis. balt. bernst., 39. Pl.1, figs. 22-23.

6241. Plagiolepis singularis. (Formicidæ.)

I.ocality : Prussian amber. Horizon : Ligurian.

1868. Mayr. Ameis. balt. bernst., 38-39. 


\section{Plagiolepis solitaria. (Formicidas.)}

1868. Mayr.

Locality : Prussian amber. Horizon: Ligurian. Ameis. balt. bernst., 40-41.

6243. Plagiolepis squamifera. (Formicidæ.)

Locality : Prussian amber. Horizon: Ligurian.

1868. Mayr. Ameis. balt. bernst,, 40. $P l .1$, fig. 24.

\section{Polistes - (Vespidæ.)}

Loeality: Chaumerac, France. Horizon: - -

1815. Latreille. Faujas, Mém. mus. hist. nat., ii : $457 . \quad P l .15$, fig. 4.

\section{Polistes —. (Vespidæ.)}

Locality: Florissant, Colo. Horizon: Oligocene.

1881. Scudder. 1883. "6

Bull. U. S. geol. surv, terr., vi : 290.

Ann. rep. U. S. geol, surv. terr., xii : 280.

\section{Polistes - (Vespidæ.)}

Compared $w^{i}{ }^{1} \mathrm{~h}$ Vespa gallica.

Locality : Aix, France. Horizon: Ligurian.

1829. Serres.

Géogn. terrains tert., 229.

\section{Polistes —. (Vespidæ.)}

Compared with P. morio.

Locality: Aix, France. Horizon: Ligurian.

1829. Serres. Géogn. terrains tert., 230.

6248. Polistes primitiva. (Vespidæ.)

Locality: Oeningen, Baden. Horizon: Tortonian.

1865. Heer.

1872. "

1876. “

1879. "6

Urwelt der Schweiz, 387.

Monde prim. Suisse, 473.

Prim. world Switz., ii : 43.

Urwelt der Schweiz, $2^{e}$ anfl., 412.

6249. Polyrhachis

(Formicidæ.)

Locality: Prussian amber. Horizon: Ligurian.

1868. Smith.

Quart. journ, sc., v: 184. Pl., fig. 4.

\section{Pompilus} (Pompilidæ.)

Locality: Prussian amber. Horizon: Ligurian.

1886. Brischke. 1886. “

Schrift. naturf. gesellseh. Danzig, n. f., vi, iii : 278.

Hym. bernst., 1.

6251. Pompilus induratus. (Pompilidæ.)

Locality: Oeningen, Baden. Horizon: Tortonian.

1849. Heer.

1852. Giebel.

1856.

1885. Scudder.

1886 .
Insektenf. tertiärg. Oeningen, ii : 165-166. Pl. 13, fig. 10.

Deutschl. petref., 647 .

Insect. d. vorwelt, 159.

Zittel, Handb. palaeont, , i, tb, ii : 819. Fig. 1106.

Zittel-Barrois, Traité de paléont., ii : 821. Fig. 1123 , 
6252. Ponera affinis, (Formicidæ.)

Localities: Radoboj, Croatia; Oeningen, Baden. Horizons: Mayencian, Tortonian, 1849. Heer.

Insektenf. tertiärg. Oeningen, ii : 147-148. Pl. 12, fig. 2.

1852. Giebel.

Deutschl. petref., 646.

1856. “

Insect. d. vorwelt, 174 .

1865. Heer.

Urwelt der Schweiz, 388 .

1872. " Monde prim. Suisse, 475.

1876. “ Prim. world Switz., ii : 45.

See also Poneropsis affinis.

Ponera anthracina. See Hypoclinea anthracina.

6253. Ponera atavia. (Formicidæ.)

Locality: Prussian amber. Horizen: Ligurian.

1868. Mayr. Ameis, balt. bernst., 72. Pl. 4, figs. 66-69.

6254. Ponera crassinervis. (Formicidæ.)

Locality: Parsehlug, Styria. Horizon : Tortonian.

1849. Heer. Insektenf. tertiärg. Oeningen, ii : 150 (crassi-nervis). Pl. 12, fig. 6.

1852. Giebel. Deutschl. petref., 646 .

1856. " Insect. d. vorwelt, 175.

6255. Ponera croatica. (Formícidæ.)

Locality: Radoboj, Croatia. Horizon: Mayencian.

1849. Heer.

1852. Giebel.

Insektenf. tertiärg. Oeningen, ii : 148. $\quad \mathrm{Pl}$. 12, fig. 3.

1856. " "6

1867. Mayr.

Deutschl. petref., 646.

Iusect. d. vorwelt, 174-175.

Jahrb. k.-k. geol. reichsanst., xvii : 57.

1867. " Stud. Rad. form., 11.

6256. Ponera elongatula. (Formicidæ.)

Localities: Radoboj, Croatia; Oeningen, Baden. Horizons: Mayencian, Tortonian.

1849. Heer.

1852. Giebel.

1856. "6

1865. Heer.

1867. Mayr.

1867.

1872. Heer.

1876. “
Insektenf. tertiärg. Oeningen, ii : 150-151. Pl. 12, fig. 7.

Deutschl, petref., 646.

Insect. d. vorwelt, 175 .

Urwelt der Schweiz, 388.

Jahrb. k.-k. geol, reichsanst, xvii : 57.

Stud. Rad. form., 11 (does not belong to Ponera).

Monde prim. Suisse, 475 (elongatulus).

Prim. world Switz., ii : 45.

See also Poneropsis elongatula.

6257. Ponera fuliginosa. (Formicidæ.)

Localities: Oeningen, Baden; Radoboj, Croatia. Horizons: Tortonian, Majencian.

1849. Heer.

1852. Giebel.

1856. " "

1865. Heer.

1872. "

1876. "
Insektenf. tertiärg. Oeningen, ii: 145-147. Pl. 12, fig. 1.

Deutschl. petref., 646.

Insect. d, vorwelt, 174.

Urwelt der Schweiz, 388.

Monde prim. Suisse, 475 (fuliginosus).

Prim. world Switz., ii : 45.

See also Ponera fuliginosa oeningensis, P. f. radobojana, Aphænogaster fuliginosa, Poneropsis fuliginosa. 
6258. Ponera fuliginosa oeningensis. (Formicidæ.)

Locality: Oeningen, Baden. Horizon: Tortonian.

1849. Heer. Insektenf. tertiärg. Oeningen, ii : 145-146. Pl. 12, figs $1 a b$.

See also Ponera fuliginosa, etc.

Ponera fuliginosa radoboj. See Ponera fuliginosa radobojana.

6259. Ponera fuliginosa radobojana. (Formicidæ.)

Locality: Radoboj, Croatia. Horizon: Mayencian.

1849. Heer. Insektenf. tertiärg. Oeningen, ii : 146-147 (radoboj). $P l .12$, figs. $10 d$.

See also Ponera fuliginosa, etc.

6260. Ponera globosa. (Formicidæ.)

Locality: Oeningen, Baden. Horizon : Tortonian.

1849. Heer. 1852. Giebel. 1856. "6

1849. Heer. 1852. Giebel. 1856. “

1868. Mayr.
Insektenf, tertiärg. Oeningen, ii : 151-153. Pl. 12, fig. 9.

Deutschl. petref., 646.

Insect. d. vorwelt, 176.

6261. Ponera graciltcornis. (Formicidæ.)

Locality: Prussian amber. Horizon: Ligurian.

Ameis. balt. bernst., 72-73.

Ponera livida. See Aphænogaster livida.

6262. Ponera longæva. (Formicidæ.)

Locality : Oeningen, Baden. Horizon : Tortonian.

Insektenf. tertiärg. Oeningen, ii: 148-149. Pl. 12, fig. 5.

Dentschl. petref., 646 .

Insect. d. vorwelt, 175 .

Ponera lugubris. See Poneropsis lugubris.

Ponera morio. See Poneropsis morio.

6263. Ponera nitida. (Formicidæ.)

Locality : Radoboj, Croatia. Horizon: Mayencian.

1849. Heer. Insektenf. tertiärg. Oeningen, ii: 149-150. Pl. 12, fig. 4.

1852. Giebel. Deutschl. petref., 646.

1856. " Insect. d. vorwelt, 175.

See also Poneropsis nitida, Hypoclinea nitida.

Ponera oeningensis. See Ponera fuliginosa oeningensis.

Ponera radobojana. See Ponera fuliginosa radobojana.

6264. Ponera succinea. (Formicidæ.)

Locality : Prussian amber. Horizon: Ligurian.

1868. Mayr.

Ameis. balt. bernst., 72.

6265. Ponera tenuis. (Formicidæ.)

Locality: Radoboj, Croatia. Horizon: Mayencian.

1867. Mayr. Jahrb. k.-k. geol. reichsanst., xvii : 57-58.

1867. "6 Stud. Rad. form., 11-12.

See also Poneropsis tenuis. 
6266. Ponera veneraria. (Formicidæ.)

Locality: Ueningen, Baden. Horizon: Tortonian.

1865. Heer.

187\%. "

18\%6. “

1879. “
Urwelt der Schweiz, 359,388. Figs. $288 a b$ on $p .386$.

Monde prim. Suisse, 441, 475 (venerarius). Figs. $288 a b$ on p. 474.

Prim. world Switz., ii : 14, 45. Figs. $288 a b$ on p. 44.

Urwelt der Schweiz, 2e anfl., 385. Fig. 330 on p. 412.

See also Poneropsis veneraria.

6267. Ponera ventrosa. (Formicidæ.)

Locality : Oeningen, Baden. Horizon: Tortonian.

1849. Heer.

Insektenf, tertiärg. Oeningen, ii: 151. $\quad P l .12$, fig. 8.

1852. Giebel.

Dentschl. petref., 646.

1856. “

Insect. d. vorwelt, 175.

6268. Poneropsis affinis. (Formicidæ.)

Localities: Radoboj, Croatia ; Oeningen, Baden. Horizons: Mayencian, Tortonian. 1867. Heer. Neue denkschr. schweiz. gesellsch., xxii, mem. 4: 19. 1867. " Foss. hymen., 19.

1879. " Urwelt der Schweiz, $2^{\circ}$ aufl., 413.

See also Ponera affinis.

6269. Poneropsis anthracina. (Formicidæ.)

Locality: Radoboj, Croatia. Horizon: Mayencian.

1867. Heer. Neue denkschr. schweiz. gesellsch., xxii, mem. 4: 22. Pl. 2 , figs. 8, 8 b.

1867. “ Foss. hymen., 22. Pl. 2, figs. 8, 8 b.

See also Hypoclinea anthracina.

\section{Poneropsis brunascens. (Formicidæ.)}

\section{Locality :- Horizon: -}

1867. Heer. Neue denkschr. schweiz. gesellsch., xxii, mem. 4: 27 (compared with Poneropsis globosa, undescr.). Pl. 2, figs. 7, 7bc.

1867. “

Foss. hymen., 27. Pl. 2, figs. 7, 7bc.

6271. Poneropsis elongata. (Formicidæ.)

Locality : Radoboj, Croatia. Horizon: Mayencian.

1867. Heer. Nene denkschr. schweiz. gesellsch., xxii, mem. 4: 19-20. $P l$. 1, fig. 21.

1867. “ Foss, hymen., 19-20, Pl. 1, fig. 21.

6272. Poneropsis elongatula. (Formicidæ.)

Localities: Radoboj, Croatia ; Oeningen, Baden. Horizons: Mayencian, Tortonian. 1867. Heer. Nene denkschr. schweiz. gesellsch., xxii, mem. 4 : 22.

1867. " Foss. hymen., 22.

1879. “ Urwelt der Schweiz, $2^{\circ}$ aufl., 413.

See also Ponera elongatula.

6273. Poneropsis escheri. (Formicidæ.)

Locality : Oeningen, Baden. Horizon : Tortonian.

1867. Heer. Neue denkschr. schweiz, gesellsch., xxii, mem. 4: 20. Pl. 1, figs. 22, 22bc.

1867. ¿ Foss. hymen., 20. Pl. 1, figs. 22, 22bo. 
6274. Poneropsis fuliginosa. (Formicidæ.)

Localities: Radoboj, Croatia; Oeningen, Baden. Horizons: Mayencian, Tortonian. 1867. Heer. 1867. “ Neue denkschr. schweiz. gesellsch., xxii, mem. 4 : 19. 1879. " Foss. hymen., 19.

See also Ponera fuliginosa, etc.

Poneropsis globosa. See Poneropsis brunascens.

6275. Poneropsis imhoffil. (Formicidæ.)

Locality : Radoboj, Croatia. Horizon: Mayencian.

1867. Heer. Nene denkschr. schweiz. gesellsch., xxii, mem. 4 : 24. Pl. 2, figs. $3 a b$.

1867. "6 Foss. hymen., 24. Pl. 2, figs. $3 a b$.

See also Liometopum imhoffii, etc.

6276. Poneropsis livida. (Formicidæ.)

Locality: Radoboj, Croatia. Horizon: Mayencian.

1867. Heer. Neue denkschr. schweiz. gesellsch., xxii, mem. 4: 25-26. Pl. 2, figs. $5,5 b$.

186\%. “ Foss. hymen., 25-26. Pl. 2, figs. 5, 5 b.

See also Aphænogaster livida.

6277. Poneropsis lugubris. (Formicidæ.)

Locality: Radoboj, Croatia. Horizon: Mayencian.

1867. Heer. Neue denkschr. schweiz. gesellsch., xxii, mem. 4: 21. Pl. 1, figs. 23, 23bcd.

1867. “ Foss. hymen., 21. Pl. 1, figs. 23, 23bcd.

1867. Mayr. Jahrb. k.-k. geol, reichsanst., xvii: 57.

1867. " Stud.Rad. form.,11 (quotes Heer's species as "Ponera" lugubris, and says it belongs to Formicinæ).

See also Aphænogaster lugubris, Poneropsis lugubris minor.

6278. Poneropsis lugubris minor. (Formicidæ.)

Locality: Radoboj, Croatia. Horizon: Mayencian.

1867. Heer. Neue denkschr. schweiz. gesellsch., xxii, mem. 4 : 21.

1867. " Foss. hymen., 21.

See also Poneropsis lugubris, ete.

Poneropsis minor. See Poneropsis lugubris minor.

6279. Poneropsis morio. (Formicidæ.)

Locality : Radoboj, Croatia. Horizon : Mayencian.

1867. Heer. Neve denkschr. schweiz. gesellsch., xxii, mem. 4: 26. Pl. 2, figs. $6,6 b$.

1867. " Foss. hymen., 26.

1867. Mayr. Jahrb. k.-k. geol. reichsanst., xvii : 57.

1867. " Stud. Rad. form., 11, (quotes Heer's species as "Ponera" morio and says it is one of the Formicinø).

See also Poneropsis morio pallens. 
6280. Poneropsis morio pallens. (Formicidæ.)

Locality: Radoboj, Croatia. Horizon: Mayencian.

1867. Meer. Neue denkschr, schweiz. gesellsch., xxii, mem. 4: 26-27. Pl. 2, fig. 6 c.

1867. “ Foss. hymen., 26-27. Pl. 2, fig.60.

See also Poneropsis morio.

6281. Poneropsis nitida. (Formicidæ.)

Locality: Radoboj, Croatia. Horizon: Mayencian.

1867. Heer. Neue denkschr. schweiz. gesellsch., xxii, mem. 4: 20.

1867. " Foss. hymen., 20.

See also Ponera nitida, etc.

Poneropsis pallens. See Poneropsis morio pallens.

6282. Poneropsis pallida. (Formicidæ.)

Locality : Radoboj, Croatia. Horizon: Mayencian.

1867. Heer. Nene denkschr. schweiz. gesellsch., xxii, mem. 4: 23-24. Pl. 2, figs. 2, $2 b$.

1867. “ Foss. hymen., 23-24. Pl. 2, figs. 2, $2 b$.

6283. Poneropsis schmidtii. (Formicidæ.)

Locality: Radoboj, Croatia. Horizon: Mayencian.

1867. Heer. Neue denkschr. schweiz. gesellsch., xxii, mem. 4: 24-25. Pl. 2, figs. $4,4 b$.

1867. " Foss. hymen., 24-25. Pl. 2, figs. 4, 4 b.

See also Liometopum imhoffii, etc.

6284. Poneropsis stygia. (Formicidæ.)

Locality: Oeningen, Baden. Horizon: Tortonian.

1867. Heer. Neue denkschr. schweiz. gesellsch., xxii, mem. $4:$ 27-28. Pl. 1, figs. 20, 20 .

1867. “ Foss. hymen., 27-28. Pl. 1, figs. 20, $20 b$.

6285. Poneropsis tenuis. (Formicidæ.)

Locality : Radoboj, Croatia. Horizon: Mayencian.

1867. Heer. Neue denkschr. schweiz. gesellsch., xxii, mem. 4: 22-23. Pl. 2, - figs. $1,1 b$.

1867. " Foss. hymen., 22-23. Pl. 2, figs. 1, 1 b.

See also Ponera tenuis.

6286. Poneropsis veneraria. (Formicidæ.)

Locality : Oeningen, Baden. Horizon: Tortonian.

1879. Heer. Urwelt der Schweiz, $2^{e}$ aufl, 413. Figs. $330 a b$ on p. 412.

See also Ponera veneraria.

6287. Porizon -. (Ichneumonidæ.)

Locality : Prussian amber. Horizon: Ligurian.

1886. Brischke. Schrift. naturf. gesellsch. Danzig, n. f., vi, iii: 279.

1886. " Hym, bernst., 2. 
6288. Prenolepis henschei. (Formicidæ.)

1863. Mayr.

Locality: Prussian amber. Horizon: Ligurian. Ameis. balt. bernst., 34-35. Pl. 1, figs. 14-17.

6289. Prenolepis pygmæa. (Formicidæ.)

Locality : Prussian amber. Horizon: Ligurian.

1868. Mayr. Ameis. balt. bernst., 36. $\quad P l .1$, fig. 18.

$$
\text { 6290. Priocnemis ㄴ. (Pompilidæ.) }
$$

Locality : Oeningen, Baden. Horizon: Tortonian. 1888. Schöberlin. Soc. entom., iii : 61 .

6291. Prionomyrmex longiceps. (Formicidæ.)

Locality: Prussian amber. Horizon: Ligurian.

1868. Mayr.

1885. Seudder. 1896. “
Ameis. balt. bernst., 78. Pl. 4, figs. 74-75.

Zittel, Handb. palaeont., i, th. ii : 817. Fig. 1103.

Zittel-Barrois, Traité de paléont., ii : 820. Fig. 1121.

6292. Psen (or Mimesa) —_. (Crabronidæ.)

Locality : Prussian amber. Horizon: Ligurian.

18\&6. Brischke. Schrift. naturf. gesellsch. Danzig, n. f., vi, iii : 278. 1886. “ Hymn. bernst., 1 .

\section{Pseudomyrme —. (Formicidæ.)}

Locality : Sicilian amber. Horizon:

1886. Scudder. Zittel-Barrois, Traite de palént., ii : 820. 1886. “ Bull. U. S. geol. surv., No. 31: 100.

See also Leptalea — Erichson, Formica — Guérin, i: 9, 10.

\section{Psilus —. (Proctotrupidæ.)}

Locality: Prussian amber. Horizon: Ligurian.

1835. Gravenhorst. Uebers. schles. gesellsch. vaterl. cult., 1834 : 92.

\section{Pteromalinites œningensis. (Pteromalidæ.)}

Locality : Oeningen, Baden. Horizon: Tortonian.

1865. Heer.

1872. “

18\%6. “

1879. “
Urwelt der Schweiz, 388.

Monde prim. Suisse, 476.

Prim. world Switz., ii : 46.

Urwelt der Schweiz, $2^{\ominus}$ autl., 414.

6296. Pteromalus ?

(Pteromalidæ.)

Locality: Salzhausen, Germany. Horizon: Miocene.

1865. Heyd.-Heyd. Palaeontogr., xiv : 35 (or Cynips?).

See also Cynips — Heyden.

6297. Pteromus (Tenthredinidæ.)

1829. Serres.

Locality: Aix, France. Horizon: Ligurian.

Géogn. terrains tert., 229. 
6298. Rhopalomyrmex pygmæus. (Formicidæ.)

1868. Majr.

Locality: Prussian amber. Horizon: Ligurian. Ameis. balt. bernst., 42. $P l .2$, figs. 25-26.

Rhyssa antiqua. See Pimpla (Rhyssa) antiqua.

6299. Rhysssa juvenis. (Ichneumoni(tæ.)

Locality: Green River, Wyo. Horizon: Oligocene.

1890. Scudder. Tert. ins. N. A., 609-610. Pl. 10, figs. 19.

6300. Sapyja - (Mutillidæ.)

Locality: Prussian amber. Horizon: Ligurian.

1886. Brischke. Schrift. naturf. gesellsch. Danzig, n. f., vi, iii : 278. 1886. " Hym. bernst., 1.

6301. Scolia -. (Scoliadæ.)

Locality: Florissant, Colo. Horizon: Oligocene.

1881. Scudder. 1883. “ Bull. U. S. geol. surv. terr., vi: 290.

Ann. rep. U. S. geol, surv, terr., xii : 280.

6302. Scolia saussureana. (Scoliadæ.)

Locality: Oeningen, Baden. Horizon: Tortonian.

1865. Heer.

1872. " "

1876. "

1879. “

1885. Scudder.

1886.
Urwelt der Schweiz. Fig. 297 on $p .386$.

Monde prim Suisse. Fig. 297 on p. 474.

Prim. world Switz., ii. Frg. 297 on p. 44.

Urwelt der Schweiz, $2^{\mathrm{e}}$ aufl. Fig. 339 on p. 412.

Zittel, Handb. palaeont., i, th. ii : 818. Fig. 1105.

Zittel-Barrois, Traité de palént., ii : 821. Fig. 1122.

6303. Selandria

(Tenthredinidæ.)

Locality: Prussian amber. Horizon: Ligurian.

1886. Brischke. Schrift. naturf. gesellsch. Danzig, n. f., vi, iii : 279. 1886.

Hym. bernst., 2.

Selandria —. See Tenthredo Curtis.

6304. Sima angustata. (Formicidø.)

Locality : Prussian amber. Horizon: Ligurian.

1868. Mayr.

1868. Mayr.

Locality : Prussian amber. Horizon: Ligurian. Ameis. balt. bernst., 101-102. Pl. 5, figs. 104-105.

6306. Sima simplex. (Formicidæ.)

Locality: Prussian amber. Horizon: Ligurian.

1868. Mayr. Ameis. balt. bernst., 102.

6307. Solenopsis? venusta. (Formicidæ.)

Locality: Radoboj, Croatia. Horizon: Mayencian.

1867. Mayr. 1867.

See also Myrmica venusta.
Jahrb. k.-k. geol. reichsanst., xvii: 60.

Stud. Rad. form., 14. 
6308. Sphex —. (Sphegidæ.)

1846. Giebel. Paläozool., 280.

Locality : - Horizon : $\longrightarrow$.

6309. Sphex —. (Sphegidæ.)

Locality: Oeningen, Baden. Horizon: Tortouian.

1888. Schöberlin. Soc. entom., iii : 61 .

6310. Sphex gigantea. (Sphegidæ.)

1867. Heer.

1867. “6

1885. Scudder.

1886. "

\section{Locality: Radoboj, Croatia. Horizon: Mayencian.}

Neue denkschr. schweiz. gesellsch., xxii, mem. 4: 33-34. Pl. 3, figs. $4,5,5 b$.

Foss. hymen., 33-34. Pl. 3, figs. 4, 5, $5 b$.

Zittel, Handb. palaeont., i, th. ii: 819. Fig. 1107.

Zittel-Barrois, Traité de paléont., ii : 821. Fig. 1124.

6311. Stigmomyrmex robustus. (Formicidæ.)

Locality: Prussian amber. Horizon: Ligurian.

1868. Mayr.

1885. Scudder.

1886. 66

Ameis. balt. bernst., 97-98. $\quad P l .5$, fig. 101.

Zittel, Handb. palaeont., i, th. ii : 817. Fig. 1102.

Zittel-Barrois, Traité de paléont., ii : 8:0. Fig. 1119.

6312. Stigmomyrmex venustus. (Formicidæ.)

Locality: Prussian amber. Horizon: Ligurian.

1868. Mayr.

Ameis. balt. bernst., 97. Pl. 5, figs. 99-100.

6313. Tapinoma? - (Formicidæ.)

Locality: Sicilian amber. Horizon:-

1881. Malfatti. Atti accad. linc., (3), trans., v: 82-83. Fig. 2.

6314. Taxonus nortoni. (Tenthrediniđ̋æ.)

Locality: Green River, Wyo. Horizon: Oligoceno.

1890. Scudder. Tert. ins. N. A., 604. Pl. 10, figs. $26,27$.

\section{Tenthredo ——. (Tenthredinidæ.)}

Locality: Prussian amber. Horizon: Ligurian.

1886. Brischke. Schrift. naturf. gesellsch. Danzig, n.f., vi, iii: 279.

1886. " 6 Hym. bernst., 2 .

\section{Tenthredo - (Tenthredinidæ.)}

Compared with Selandria fuliginosa.

Locality: Aix, France. Horizon: Ligurian.

1829. Curtis. Edinb. new. phil. journ., vii : 295 .

1829. " Murchison-Lyell, Tert. form. Aix, 11.

6317. Tenthredo —. (Tenthredinidæ.)

Locality: Prussian amber. Horizon: Ligurian.

1835. Gravenhorst. Uebers. schles. gesellsch. vaterl. cult., 1834: 92. 
6318. Tenthredo ——. (Tenthredinidæ.)

Locality: Prussian amber. Horizon: Ligurian. 1829. Schlotheim. Petrefactenk., 43.

6319. Tenthredo - ( 2 sp.). (Tenthredinidæ.)

Locality: Oeningen, Baden. Horizon: Tortonian.

1888. Schöberlin. Soc. entom., iii : 61 .

6320. Tenthredo —_. (Tenthredinidæ.)

Compared with T. viridis.

Locality: Aix, France. Horizon: Ligurian.

1829. Serres. Géogn. terrains tert., 229 (Thenthredo).

6321. Tenthredo gervaisi. (Tenthredinidæ.)

Locality: Aix, France. Horizon: Ligurian.

1861. Heer. Saporta, Heer, Rech. climat pays tert., 153 (undescr.).

6322. Tenthredo vetusta. (Tenthredinidæ.)

Locality : Oeningen, Baden. Horizon: Tortonian.

1849. Heer. Insektenf. tertiärg. Oeningen, ii: 172-173. Pl.13, fig. 16.

185\%. Giebel. Deutschl. petref., 647.

1856. " Insect, d. vorwelt, 152.

Thenthredo —. See Tenthredo — Serres.

6323. Torymus —. (Chalcididæ.)

Locality : Brunstatt, Alsatia. Horizon : Middle Oligocene.

1890. Foerster. In litt.

\section{Trigona? - (Apidø.)}

Locality: Prussian amber. Horizon: Ligurian.

1832. Burmeister. Handb. ent., i: 636 .

1836. " Man. ent., 577.

Trogus fusiformis. See Ichneumonites (Trogus) fusiformis.

6325. Tryphon - (Ichneumonidæ.)

Locality: Prussian amber. Horizon: Ligurian.

1886. Brischke. Schrift. naturf. gesellsch. Danzig, n. f., vi, iii : 278.

1886. " Hym. bernst., 1.

6326. Typhia? - (Mutillidæ.) .

Locality : Prussian amber. Horizon: Ligurian.

1886. Brischke. Sehrift. naturf. gesellsch. Danzig, n. f., vi, iii : 278.

1886. “ Hym. bernst., 1 .

6327. Urocerites spectabilis. (Uroceridæ.)

Locality: Radoboj, Croatia. Horizon: Mayencian.

1867. Heer. Neue denkschr, schweiz. gesellsch., xxii, mem, $4: 38-39$. Pl. 3, figs. $1,2,2 b, 3$.

1867. “ Foss. hymen., 38-39. Pl. 3, figs. 1,2,2b,3.

1885. Scudder. Zittel, Handb. palaeont., i, th. ii: 816. Fig. 1097.

1886. " Zittel-Barrois, Traité de paléont., ii: 818. Fig. 1114. 


\section{Vespa -. (Vespidæ.)}

Locality : Prussian amber. Horizon: Ligurian.

1835. Gravenhorst. Uebers. schles. gesellsch. vaterl. cult., 1834 : 92.

6329. Vespa -. (Vespidæ.)

Locality : Aix, France. Horizon: Ligurian.

1847. Hope. Trans. ent. soc. Lond., iv : 252.

6330. Vespa ㄴ. (Vespidæ.)

Locality : Oeningen, Baden. Horizon: Tortonian.

1888. Schöberlin. Soc. entom., iii : 61.

6331. Vespa atavina. (Vespidæ.)

Localities: Parschlng, Styria; Moudon, Switzerland. Horizon: Tortonian.

1849. Heer.

1852. Giebel.

1856. “

1865. Heer.

1872. “

1876. "6

1879. 6
Insektenf. tertiärg. Oeningen, ii : 101 (attavina). $\quad$ Pl. 7, fig. 8. Deutsehl. petref., 645 (attavina).

Insect. d. vorwelt, 160 (attavina).

Urwelt der Schweiz, 387. Fig. 289 on p. 386.

Monde prim. Suisse, 473. Fig. 289 on p. 474.

Prim. world Switz., ii: $44 . \quad$ Fig. 289.

Urwelt der Schweiz, 2e aufl, 412. Fig. 331.

Vespa attavina. See Vespa atavina.

\section{Vespa crabroniformis. (Vespidø.)}

Locality: Radoboj, Croatia. Horizon: Mayencian.

1867. Heer.

Neue denkschr. schweiz. gesellsch., xxii, mem. $4: 6$. Pl. 3, fig. $15 a$.

1867. "6

Foss. hymen., 6. Pl. 3, fig. 15a.

1885. Scudder. Zittel, Handb. palaeont., i, th. ii : 819. Fig. 1108.

1886. 6

Zittel-Barrois, Traité de paléont., ii : 821. Fig. 1125.

6333. Vespa dasypodia. (Vespidæ.)

Locality: Prussian amber. Horizon: Ligurian.

1856. Menge.

Progr. petrischule Danzig, 1856: 26.

6334. Xylocopa senilis. (Apidæ.)

Locality: Oeningen, Baden. Horizon: Tortonian.

1849. Heer.

1852. Giebel.

Insektenf. tertiärg. Oeningen, ii: 93-95. $\quad P l .7$, figs. 1.

1856.

Deutschl. petref., 645 .

Insect. d. vorwelt, 181-182.

Urwelt der Schweiz, 386. Fig. 295.

Monde prim. Suisse, 473. Fig. 295 on $p .474$.

Prim. world Switz., ii : 43. Fig. 295 on p. 44.

Urwelt der Schweiz, 2e aufl., 411. Fig. 337 on p. 412.

1879.

1885. Scudder.

1886. “
Zittel, Handb. palaeont., i, th. ii : 819. Fig. 1109.

Zittel-Barrois, Traité de paléont., ii: 821. Fig. 1126. 


\section{INDEX OF GENERIC NAMES.}

Abax, 458.

Abia, 103, 134.

A calles, 459 .

A cantherpestes, 9.

Acanthichnus, 233.

Acanthoderes, 459.

Acanthoderus, 459.

A cantholophus, 246.

A canthosoma, 382.

Acarus, 246.

Achenium, 459.

Acheta, 103, 302.

Achita, 104.

Achrestocoris, 383.

Aclada, 599.

Acmæod ra, 459.

A cocephalus, 383.

Accenites, 685.

Acœnitus, 685.

Acreagris, 321.

Acridiites, 104.

A.cridites, 31, 70.

Acridium, 303.

Acrocera, 599.

Actea, 167, 189.

Actineda, 247.

Adalia, 459.

Adaphlebia, 71.

Adelocera, 459.

Adelophthalmus, 31.

Adetus, 599.

Adimonia, 459.

Adiphlebia, 71.

Adonia, 222.

Edøophasma, 31.

AEgialia, 460.

सElia, 383.

Amoaipus, 600.

Eschna, 134, 321.

Aethophlebia, 71.

Ethus, 384.

Etorhinus, 384.

Agabus, 460.

Agalena, 247.

Agallia, 384 .

Agapetus, 323.

A gathemera, 303.

A gathis, 685 .

A gatoides, 460 .

Agelasa, 460.

Agelena, 247.

Agonum, 460.

Agraylea, 323.
A grilium, 189.

Agrilus, 189, 460.

Agrion, 137, 323.

Agrionides, 138.

Agrionidium, 139.

A gromyza, 600.

A grypnia, 326.

Akicera, 104.

Akulosamphus, 461.

Alaus, 461.

Aleochara, 461.

Aleurodes, 384.

Allarithmia, 600 .

Alydus, 384.

Amalancon, 384.

Amara, 190, 461.

Amaurobius, 247.

Amblyteles, 685 .

Ammocius, 462.

Ammophila, 685.

Ampedus, 462.

Ampelichnus, 234.

Amphiclotho, 248.

Amphientomum, 326.

Amphithomisus, 248.

Amphitrogulus, 218.

Amphotis, 462.

Amynilyspes, 9.

Anaclinia, 600 .

Anandrus, 248.

Anasa, 385.

Anaspis, 462.

Anatella, 600.

Anatone, 248.

Anax, 139, 326.

Anchomenus, 462.

Anconatus, 385.

Ancylocheira, 190, 463.

Ancylochira, 190, 464.

Andræna, 686.

Androgeus, 248.

Angerona, 673.

Anisomera, 600 .

Anisopus, 600.

Anisorhynchus, 190, 464.

Anisotoma, 464 .

Anobium, 464.

Anomala, 465 .

Anomalites, 465.

Anomalon, 228, 686 .

Anomma, 686.

Anoplites, 465 .

Anoplitis, 465.
Anoplognathus, 465.

Anthaxia, 466.

Antherophagus, 466.

Anthiens, 466.

Anthomyia, 600 .

Anthonomus, 467.

Anthophagus, 467.

Anthophora, 686.

Anthophorites, 687.

Anthracerpes, 10.

Anthracida, 601.

Anthracoblattina, 31, 104

Anthracomartus, 19.

Anthracoscorpio, 20.

Anthracothremma, 71 .

Anthrax, 602.

Anthrenus, 467.

Anthribites, 467.

Anthribus, 467.

Antichus, 467.

Antirrhæa, 673.

Antliarhinites, 468.

Antopia, 249.

Anyphæna, 249.

Apanthesis, 673 .

Apate, 468.

Apedilia, 602 .

Aphænogaster, 687.

Aphana, 385.

Aphantaphis, 385.

Apheilocheira, 326.

Aphidioides, 385.

Aphidopsis, 385 .

Aphilocheira, 326.

Aphis, 167, 386.

A.phodiites, 190.

Aphodius, 468.

Aphritis, 602.

A phrophora, 387.

Apiaria, 139, 228, 688.

Apion, 469.

Apis, 688.

Apochrysa, 139.

Aporema, 388.

Aporoblattina, 104.

Aradus, 388.

Aranea, 20, 250.

Araschnia, 673.

Archæa, 251.

Archæoptilus, 32.

Archæoscolex, 71.

Archegogryllus, 33.

Archidesmus, 10. 
Archilachnus, 389.

A rehimantis, 71.

Arehimulacris, 33.

Archimylacris, 33.

Archiorhynchus, 191.

Archipsocus, 326.

A rchitarbus, 20.

Arehiulus, 10.

Argia, 327.

Argutor, 469.

Argya, 327.

Argynnis, 673.

Argyroneta, 252.

Ariamnes, 252.

Arma, 390.

Arnilia, 303.

Artamus, 252.

Arthrolycosa, 21.

Arthropeas, 602.

Arthropleurion, 11.

Arthropterus, 470.

Arytæna, 252.

Asarcomyia, 602.

Ascalaphus, 327.

Ascogaster, 689.

Asida, 470.

Asilicus, 105, 222.

Asilus, 223, 602.

Asira, 168, 390 .

Asiraca, 168, 390.

Aspasia, 140.

Aspatherium, 327.

Astynomus, 470 .

Asuba, 223.

Asynapta, 603.

Ataracta, 603 .

Athera, 253.

Atherix, 603.

Athropleurion, 11.

Atomaria, 470 .

Atopa, 470.

Atractocerus, 470.

Atropos, 327.

Attagenus, 470.

Attelabns, 471.

Attoides, 253.

Attopsis, 253, 689.

Attus, 253.

Bacteria, 303.

Badister, 471.

Bætıs, 327.

Bagous, 471.

Balaninus, 472.

Barbarothea, 673.

Baridium, 472.

Baris, 472.

Baseopsis, 106.

Basiæschna, 328.

Bassus, 690.

Bdella, 253.

Bellengera, 191.

Bellingera, 191.

Belostoma, 168, 229, 390.

Belostomates, 390.

Belostomum, 168, 229, 391.
Bembicidioides, 472 .

Bembidium, 472.

Beris, 603.

Berosue, 191, 473.

Berytopsis, 391.

Berytus, 391.

Bibio, 603.

Bibiopsis, 610.

Bifurculapes, 234.

Bifurculipes, 235.

Bittacus, 106, 140, 328.

Blabera, 106.

Blaniulus, 237.

Blaps, 191.

Blapsidium, 191.

Blapsium, 192.

Blatta, 34, 106, 303.

Blattaria, 107.

Blattidium, 34, 107, 140, 306.

Blattina, 34, 72, 111, 306 .

Bledius, 473.

Bolboceras, 474.

Bolbomyia, 611.

Boletina, 611.

Boletophagus, 474.

Boletophila, 612.

Bolitophagus, 474.

Bombus, 230, 690 .

Bombusoides, 691.

Bombycites, 673.

Bombylius, 612.

Bombylis, 612.

Bombyx, 674.

Bostrichus, 474.

Bostrychus, 192, 474.

Bothrideres, 475.

Bothromicromus, 328.

Bothynophora, 192.

Brachinites, 192.

Brachinus, 475.

Brachycentrus, 328 .

Brachycerus, 475.

Brachyderes, 476.

Brachygaster, 691 .

Brachymycterns, 476 .

Brachynus, 476.

Brachypelta, 391.

Brachypeza, 612.

Brachyptilus, 72.

Brachypyge, 21.

Brachystoma, 612 .

Brachytarsus, 476.

Brachyurus, 476.

Bracon, 691.

Bradoponera, 691.

Breyeria, 44, 72, 227.

Bria, 223.

Brodia, 72.

Bruchus, 192, 476.

Bryaxis, 477.

Buprestidium, 192.

Buprestis, 192, 477.

Buprestites, 193, 479.

Buprestium, 194.

Buthus, 21, 254.

Byrrhus, 480.
Byrrhydium, 194.

Bythinus, 480.

Bythoscopus, 391.

Cacalydus, 392.

Cacoschistus, 392.

Cæcilius, 329.

Calænins, 480.

Calandra, 480.

Calasposoma, 480.

Calathus, 481.

Callidium, 481.

Callisthenes, 481.

Callomyia, 612.

Calobamon, 612.

Calobata, 613.

Caloneura, 44.

Calopteryx, 140, 329.

Calosoma, 481.

Calotormes, 21, 44, 72, 140, 329.

Calothrips, 392.

Calyptites, 692.

Camponotus, 692.

Campoplex, 693.

Campsosternus, 483.

Campylomyza, 223, 613.

Campyloptera, 72.

Camtodontus, 195.

Cantharis, 483.

Capnodis, 483 .

Caprologus, 484.

Capsus, 392.

Carabicina, 195.

Carabidium, 195.

Carabites, 195, 484.

Carabocera, 196.

Carabus, 196, 485.

Carbera, 485.

Cardiophorus, 486.

Carmelus, 392.

Caryoborus, 486.

Cassida, 196, 486.

Cataneura, 393.

Cataulacus, 693.

Catopamera, 393.

Catops, 487.

Catopsylla, 393.

Cecidomium, 223.

Cecidomyia, 613.

Celithemis, 330.

Cemonus, 693.

Centromachus, 21.

Cephalocoris, 393.

Cephites, 693.

Cephus, 694.

Cerambix, 487.

Ceramby cinus, 196.

Cerambycites, 197, 488.

Cerambyx, 197, 488.

Ceraphron, 694.

Ceratopogon, 613.

Cerceris, 694.

Cercidiella, 254.

Cercopidium, 169, 693.

Cercopis, 170, 393.

Cercopites, 396. 
Cercopyllis, 170.

Cercyon, 197, 488.

Cermatia, 237.

Cerylon, 197.

Cetonia, 197, 488.

Ceut orhynchus, 489.

Chæteesa, 306.

Chætoessa, 306.

Chalcites, 694.

Chalcodermus, 489.

Chalcophora, 489.

Chalicodoma, 694.

Chanliodes, 141, 330.

Chaniodites, 141.

Chauliognathus, 489.

Cheilosia, 223, 614.

Cheiridion, 254.

Cheiridium, 254.

Cheiromachus, 254.

Chelephlebia, 72.

Chelifer, 99, 254.

- Chelignathus, 256.

Cheliphlebia, 72.

Chelonus, 694.

Chenesia, 223.

Chernes, 256.

Cheyletus, 256.

Chilocorus, 489.

Chilosia, 223, 615.

Chimarocephala, 306.

Chimarrha, 141.

Chironomus, 223, 615.

Chlænius, $198,490$.

Chlorops, 618.

Chœridium, 490.

Cholula, 396.

Chonionotus, 11.

Choragus, 490.

Chresmoda, 113.

Chrestotes, 73.

Chrestotus, 73.

Chrysis, 694.

Chrysobothris, 198, 490.

Chrysogaster, 618.

Chrysomela, 198, 491.

Chrysomelites, 199, 492.

Chrysopa, 141, 330.

Chrysothemis, 618.

Chrysotus, 618.

Chthonius, 256.

Cicada, 171, 396.

Cicadellites, 397.

Cicadellium, 172.

Cicadinella, 172.

Cicadula, 398.

Cicindela, 493.

Cimbex, 695.

Cimex, 172, 398.

Cimicidium, 172.

Cionus, 493.

Cis, 493.

Cistela, 493.

Cistelites, 199, 494.

Citylus, 494.

Cixia, 172.

Cixius, 172, 398.
Clastoptera, 400.

Clathrotermes, 113, 142.

Cleonolithus, 494.

Cleonis, $49 \pm$.

Cleonus, 494.

Cleptes, 695.

Cleptis, 695.

Clerus, 496.

Clivina, 486.

Closterocoris, 400.

Clostes, 256.

Clubiona, 256.

Clubionella, 258.

Clya, 258.

Clythia, 258.

Clythra, 496.

Clytra, 496.

Clytus, 199, 497.

Coccinella, 199, 497.

Cocens, 400 .

Cœlidia, 400.

Cœliodes, 499.

Colasposoma, 499.

Coliates, 674.

Colydium, 500.

Colymbetes, 200, 500.

Compsidia, 500 .

Coniatus, 500.

Coniopteryx, 330 .

Coniortes, 330.

Conocephalus, 114.

Conops, 618.

Conopsoides, 235.

Copeza, 235.

Cophocoris, 400 .

Copris, 500.

Coprolugus, 501.

Coptochromis, 400.

Coræus, 400.

Cordulegaster, 142.

Cordulia, 331.

Cordyla, 618.

Cordylura, 619.

Coreites, 400.

Corethra, 619.

Corethrium, 224.

Coreus, 401.

Corimalæna, 401.

Corisa, 401.

Corixa, 172, 401.

Corizus, 401.

Corsomyza, 619.

Corticaria, 501.

Corydalis, 73, 142, 331.

Corydalites, 142.

Corydaloides, 74 .

Corymbites, 501.

Corynetes, 501.

Corynitis, 259.

Cossonus, 501.

Cossus, 674.

Cossyphus, 502.

Craspedosoma, 238.

Cratoparis, 502.

Crematogaster, 695.

Criocerina, 502.
Crioceris, 502

Criorhina, 619.

Criorrhina, 619.

Critoneura, 620.

Cronicus, 331.

Crossocerus, 695 .

Crypticus, 200.

Cryptocephalus, 200, 502.

Cryptochromus, 402.

Cryptohypnus, 503.

Cryptophagus, 503.

Cryptorhynchus, 503.

Cryptus, 695.

Ctenicerium, 201.

Ctenoblattina, 114.

Ctenophora, 620.

Ctereacoris, 402.

Cucorhinus, 503.

Culex, 224, 620.

Culicites, 621.

Cupes, 503.

Cupoides, 503.

Curculio, 201, 504.

Curculioides, 22, 97.

Curculionidium, 201.

Curculionites, 201, 504.

Curculium, 202.

Curtisimyia, 621.

Cylister, 506.

Cychrus, 506.

Cyclocoris, 172.

Cycloderma, 202.

Cyclonotum, 507.

Cyclophthalmus, 22.

Cydamus, 402.

Cydnopsis, 402.

Cydnus, 403.

Cylindrotoma, 621.

Cyllo, 674.

Cyllonium, 143, 227.

Cymatomera, 307.

Cymatophlebia, 143.

Cymatopterus, 507.

Cymindis, 202, 507.

Cymindoides, 507.

Cynips, 696.

Cyphon, 202, 507.

Cyrnus, 331.

Cyrtomenus, 104.

Cyrtophyllites, 114.

Cyrtoxiphus, 307.

Cytilus, 508.

Cyttaromyia, 621.

Dara, 224.

Daseyllus, 508.

Dasyleptus, 74 .

Dasypoda, 696.

Dasypogon, 621.

Dasytes, 508.

Decatoma, 696.

Decticus, 114, 307.

Delphax, 173, 404.

Deltocephalus, 404 .

Dermestes, 508.

Derobrochus, 331. 
Diacanthus, 508.

Diadocidia, 622.

Dianepsia, 622.

Diaperidium, 203.

Diaphanoptera, 74.

Diaplegma, 404.

Diastatomma, 143.

Dicælus, 508.

Dicæra, 622.

Dicerca, 509.

Dichaneurum, 622.

Dichela, 259.

Dichirotrichus, 509.

Dichrooseytus, 405.

Dicranomyia, 622.

Dictyocicada, 92.

Dictyoneura, 45, 74, 143.

Dictyophara, 405.

Dict 50 phlebia, 75.

Dictyophorites, 405.

Dictyopteris, 47.

Didineis, 696.

Didymophleps, 75.

Diechoblattin?, 114

Dieconeura, 75.

Dielacata, 260.

Dilophus, 623.

Dineutes, 509.

Dineutus, 509.

Diorhiza, 624 .

Diplax, 332.

Dipluchila, 510.

Diplolepis, 696.

Diplonema, 623.

Diplonychus, 405.

Diplosis, 623 .

Dipluroblattina, 114.

Dipterites, 623.

Discostoma, 405.

Dissosteira, 307.

Ditomoptera, 173, 203.

Dixa, 624.

Docimus, 405 .

Dolerus, 696.

Dolichopus, 624.

Donacia, 510.

Dorcadion, 513.

Dorcadionoides, 513.

Dorcasoides, 513.

Dorcatoma, 513.

Dorcus, 514.

Doritomus, 514.

Dorthesia, 405 .

Dorytomus, 514.

Drapetis, 625.

Drassus, 260.

Dromins, 514.

Drosophila, 625.

Dryocætes, 514.

Duma, 23, 97.

Dysagrion, 332.

Dyscritus, 75.

Dysdera, 260.

Dysdercus, 406.

Dytiens, 515.

Dytiscus, 203, 515 .
Ebæus, 516.

Echinomyia, 625.

Eclytus, 696.

Ectatomma, 696.

Eileticus, 11.

Ela, 143.

Elaphrus, 516.

Elater, 203, 516.

Elaterites, 205, 518.

Elaterium, 205, 518.

Elateropsis, 205.

Elatior, 518.

Elcana, 114, 143.

Electra, 625 .

Elephantomyia, 625 .

Elidiptera, 406.

Elipsocus, 332.

Elisama, 115.

Elmis, 205.

Elvina, 250.

Elytridium, 206, 519.

Embia, 333.

Empheria, 333.

Emphytus, 696.

Empiria, 224.

Empis, 625.

Encænus, 75.

Enneamerus, 697.

Entermes, 333.

Entimus, 519.

Entomocephalus, 261.

Eoatypus, 261.

Eocicada, 173.

Eojulus, 11.

Eolycosa, 23.

Eophrynus, 23.

Eoscorpius, 24.

Eotarbus, 25.

Eothes, 406.

Eotingis, 406.

Epeira, 261.

Epeiridion, 262.

Ephemera, 144, 333:

Ephemerites, 76.

Ephemeropsis, 145.

Ephydra, 627.

Epicærus, 519.

Epicypta, 627.

Epidosis, 627.

Epiphanis, 519.

Epiplecia, 627.

Epipsocus, 334.

Eresoides, 262.

Eresus, 262.

Erigone, 263.

Erinnys, 520.

Eriocera, 627.

Erioptera, 627.

Eriphia, 627.

Erirhinoides, 520.

Erirhinus, 520.

Eristalis, 627.

Erithus, 263.

Ero, 263.

Eryeus, 520.

Erythræus, 264.
Escheria, 520.

Estemoa, 145.

Etirocoris, 406.

Etoblattina, 48, 116.

Eucænus, 76.

Euchroma, 206.

Eucnemis, 521.

Eucorites, 406.

Eudiagogus, 521.

Euephemerites, 76.

Engereon, 92.

Eugnamptus, 521.

Engonia, 674 .

Eumolpites, 206.

Eunectes, 521.

Euophrys, 265.

Eupedilia, 628.

Euphæa, 146.

Euphemerites, 77.

Euphoberia, 11.

Euphorberia, 14.

Euphrynus, 25.

Euplectus, 521.

Eupsinoides, 522.

Eurhinus, 522.

Eurychirus, 522.

Eurydema, 406.

Eurygaster, 407.

Euryopus, 265.

Eurypterus, 14, 52.

Eurythyrea, 206, 522.

Eurytrachelus, 522.

Eusarcoris, 407.

Euschistus, 407.

Eutermes, 146, 335.

Eutermopsis, 52, 77.

Euzonus, 239.

Evagoras, 407.

Evania, 697.

Exitelus, 407.

Fabellovena, 227, 230.

Feronia, 522.

Feronites, 206.

Ficarasites, 407.

Flata, 146, 173, 407.

Flegia, 265 .

Florissantia, 408.

Forbicina, 335 .

Forficula, 307.

Forficularia, 116.

Formica, 697.

Formicium, 230.

Friganea, 335 .

Fulgora, 93, 408.

Fulgorina, 93.

Fuscus, 408.

Fusslinia, 523.

Galeruca, 523.

Galerucella, 524.

Galerucites, 206.

Galleruca, 524

Gallerucella, 524.

Gea, 265.

Genentomum, 77. 
Genopteryx, 77.

Geocoris, 408.

Geophilus, 98, 239.

Geotrupes, 206, 524.

Gerablattina, 53.

Geralinura, 25.

Geralycosa, 26.

Gerancon, 408.

Gerapompus, 78.

Geraphrynus, 26.

Gerarus, 78.

Geratarbus, 26.

Gerdia, 206.

Gerephemera, 54, 79.

Geroneura, 79.

Gerris, 408.

Gesomyrmex, 709.

Glaphyroptera, 207, 628 .

Glaphyrus, 525.

Glenopterus, 525.

Glessaria, 336.

Gloma, 628.

Glomeris, 239.

Glossosoma, 336.

Glypta, 709.

Gnorimus, 525.

Gnoriste, 628.

Gœra, 336 .

Goldenbergia, 55, 79.

Gomphocerites, 117.

Gomphocerus, 308.

Gomphoides, 146, 336.

Gomphus, 146, 336.

Gonioctena, 525.

Gonocephalum, 526.

Gonyleptes, 266.

Gorgopis, 267.

Gorytes, 709.

Græa, 267.

Græophonus, 26.

Grammepus, 235.

Grillus, 309.

Gryllacris, 57, 79, 117, 309.

Gryllidium, 117.

Gryllites, 117.

Gryllotalpa, 309.

Gryllus, 117, 310.

Gymnetron, 526.

Gymnocheila, 526.

Gymnopleurus, 526.

Gynacantha, 147.

Gypona, 408,

Gyrinites, 208.

Gyrinoides, 527.

Gyrinus, 208, 527.

Gyrophæna, 527.

Habrosoma, 628.

Hadronema, 408.

Hæmonia, 528.

Hagenia, 173, 230.

Hagla, 147.

Halesus, 336.

Haliplus, 528.

Hallesus, 336.

Hallomenus, 528 .
Halobates, 409.

Halometra, 118, 173.

Haltica, 209, 528.

Halys, 409 .

Hammapteryx, 409.

Haploneura, 629.

Haplophlebium, 57.

Haplotichnus, 236.

Harmosites, 409.

Harpaclor, 409.

Harpalidium, 209.

Harpalus, 210, 528.

Harpocera, 410.

Harpepus, 236.

Hasmona, 224.

Hasseltia, 99.

Hasseltides, 99.

Heeria, 410.

Heliothrips, 410.

Helluomorpha, 530.

Helobia, 530 .

Helomyza, 629.

Helophilus, 629.

Helophorus, 210, 530

Helopides, 211.

Helopidium, 211.

Helopiam, 211.

Helops, 531 .

Hemeristia, 80 .

Hemeristica, 80.

Hemerobioides, 148.

Hemerobites, 337.

Hemerobins, 148, 337.

Hemerodromia, 629.

Hemiteles, 709.

Hermatoblattina, 58.

Herselioides, 268.

Hersilia, 268.

Hersilioides, 268.

Hesthesis, 531.

Heterogamia, 311.

Heterogaster, 411.

Heteromma, 268.

Flsteromjza, 629.

Heterophlebia, 149, 338.

Heterotricba, 629.

Hevila, 26, 97.

Hexagenites, 150.

Hexapodichuus, 236.

Hexatoma, 629.

Hilarites, 629.

Hippiscus, 311.

Hipporhinus, 531.

Hirtea, 630.

Hister, 211, 532.

Histopona, 269.

Hodotermes, 338.

Holcorpa, 339.

Holopogon, 630 .

Homalium, 533.

Homaloneura, 80 .

Homalota, 533.

Homodemns, 412 .

Homøogamia, 311.

Homothetus, 80.

Hopatrum, 533.
Hoplomachus, 412.

Hormiscus, 534.

Humbertiella, 118.

Hybos, 630 .

Hybosorus, 534.

Hydaticus, 534.

Hydræna, 534.

Hydrobiites, 211.

Hydrobius, 211, 534.

Hydrochus, 535 .

Hydrometra, 173, 412 .

Hydronautia, 339 .

Hydronomus, 536.

Hydrophilites, 212, 536.

Hydrophilopsis, 536.

Hydrophilus, 173, 212, 536.

Hydrophylites, 538.

Hydrophylus, 538.

Hydroporus, 213, 539.

Hydropsyche, 150, 339.

Hydroptila, 340.

Hydrorchestria, 340 .

Hydrous, 539.

Hygronoma, 540.

Hygrotrechus, 412.

Hylastes, 540 .

Hylecœtus, 540.

Hylesinites, 540.

Hylesinus, 97, 540 .

Hylobius, 541.

Hylotoma, 412, 710.

Hylotrupes, 542.

Hylurgus, 542.

Fymenopteritos, 710 .

Hypera, 213, 542.

Hypoclinea, 710 .

Hspselonotus, 412.

Ichneumon, 230, 711 .

Ichneumonites, 712 .

Ictinus, 310.

Idmonia, 269.

Ilybius, 542.

Ilyodes, 14.

Imhoffla, 713.

Indusia, 340 .

Inocellia, 341.

Ips, 542.

Ischnodes, 542.

Ischyopteron, 173.

Isophlebia, 150.

Ixodes, 269.

Jassopsis, 412.

Jassus, 412.

Julopsis, 98.

Julus, 14, 240.

Junonia, 675.

Jupiteria, 675.

Kaloterines, 341.

Kampecaris, 15.

Kleidocerys, 173.

Kreischeria, 26.

Kustarachne, 27. 
Labidostomis, 542.

Labidura, 311.

Labiduromma, 311.

Laccobius, 542.

Laccophilus, 213, 543.

Laccophrys, 713.

Lachnus, 413.

Lacon, 213, 543 .

Lamia, 213, 543.

Lamiites, 213.

Lampromyrmex, 713 .

Lampropholis, 341.

Lamproptilia, 81.

Lampyris, 544.

Laparocerus, 544.

Larinus, 544

Lasia, 544.

Lasioptera, 630.

Lasius, 713.

Lathridiites, 213.

Lathridius, 544.

Lathrobium, 545.

Latridiites, 214.

Latzelia, 15.

Lebia, 545.

Lebina, 545.

Leda, 269.

Ledophora, 415.

Ledrophora, 118.

Legnophora, 118.

Leia, 630.

Leiobunum, 269.

Leistotrophus, 546.

Leja, 630.

Lema, 546.

Lepidion, 342.

Lepidotrix, 342.

Lepisma, 342.

Lepitrix, 546.

Leptalea, 714.

Leptis, 631.

Leptoblattina, 58 .

Leptobrochus, 343.

Leptogaster, 631.

Leptoneura, 81.

Leptopeza, 631.

Leptophlebia, 343.

Leptoscelis, 415.

Leptothorax, 715 .

Leptura, 214, 546.

Leptysma, 312.

Lestes, 343.

Lethites, 675.

Leuctra, 344.

Libella, 151, 344.

Libellula, 27, 58, 82, 151, 345 .

Libellulites, 154.

Libellulium, 154.

Libillula, 154.

Licinns, 546.

Ligæus, 415 .

Ligyrocoris, 415.

Limnaeis, 415.

Limnephilus, 348 .

Limnichus, 547.

Limnius, 214
Limnobates, 415.

Limnobia, 631.

Limnobiorhynchus, 633.

Limnochares, 269, 415.

Limnophilus, 349.

Limnopsyche, 349.

Limonius, 547.

Lina, 547.

Lindenia, 154.

Linnæa, 415.

Linoptes, 269.

Linyphia, 269.

Liometopum, 715 .

Liparus, 547.

Listronotus, 548.

Lithadothrips, 416.

Lithagrion, 349.

Lithaphis, 416.

Lithecphora, 416.

Lithentomum, 82.

Lithobius, 241.

Lithocharis, 548.

Lithochromus, 416.

Lithocoris, 417.

Lithographus, 236.

Lithomantis, 58, 83.

Lithomylacris, 59.

Lithomyza, 633.

Lithophasma, 59, 83.

Lithophysa, 633.

Lithoplanes, 548.

Lithopsis, 417.

Lithopsyche, 675 .

Lithortalis, 633.

Lithosia, 675.

Lithosialis, 84.

Lithotorus, 715.

Lithymnetes, 312 .

Litobrochus, 350.

Litoneura, 59.

Lixus, 548.

Locrites, 417.

Locusta, 119, 312.

Locustites, 313 .

Lognophora, 119.

Lomatia, 633.

Lomatus, 548.

Lonchæa, 634.

Lonchomyrmex, 715 .

Lophonotus, 242.

Lophyrus, 715 .

Lopus, 417.

Loricera, 549.

Loxandrus, 549.

Lucanus, 549.

Luciola, 549.

Lunula, 236.

Lycoperdina, 549.

Lycosoides, 270.

Lyetocoris, 417.

Lyetus, 549.

Lyeus, 549.

Lyda, 716.

Lygæites, 173, 417.

Lygæus, 418.

Lygus, 420.
Lymexilon, 549.

Lymexylon, 549 .

Lystra, 174, 420.

Lytta, 550.

Macaria, 270.

Machilis, 350.

Macrocentrus, 716.

Macrocera, 224, 634.

Macrochile, 634.

Macroglossa, 676.

Macromischa, 716.

Macropalpus,'351.

Macropeza, 224.

Macrophlebium, 95.

Macroura, 634.

Magdalinus, 550.

Malachius, 550 .

Malthinus, 550.

Malthodes, 550.

Mantis, 60, 313.

Mantispa, 84.

Maresa, 351.

Mastigusa, 271.

Matæoschistus, 420.

Mazonia, 27.

Mecocephala, 420 .

Medeterus, 634.

Megablattina, 60, 95.

Megacentris, 214.

Megacentrus, 214.

Megameropsis, 271.

Maganeura, 60.

Megaptilus, 60.

Megasemum, 351.

Megathentomum, 81.

Melanophila, 214.

Melanophora, 271.

Melanothrips, 420.

Melens, 551.

Meligethes, 551.

Melipona, 716.

Mellinus, 716.

Meloe, 154, 214, 551.

Melolontha, 215, 551.

Melolonthidium, 215 .

Melolonthites, 552.

Membracites, 420.

Mengea, 552.

Meristos, 553.

Merodon, 634.

Mesobætis, 154.

Mesoblattina, 120.

Mesobrochus, 351.

Mesochorus, 716.

Mesoleptus, 716.

Mesolenctra, 154.

Mesonemura, 155.

Mesoneta, 155.

Mesopsychoda, 225.

Mesosa, 215, 553.

Mesosites, 553.

Meteorus, 717.

Metrobates, 421.

Miamia, 85.

Micranthaxia, 215. 
Micriphantes, 272.

Mierodon, 634.

Microlabis, 27.

.Micropus, 421.

Microrhagus, 553.

Microzoum, 553.

Micryphantes, 272.

Milesia, 635.

Mimesa, 717.

Miris, 421.

Mixotermes, 86.

Mizalia, 273.

Mnemosyne, 421.

Mochlonyx, 635.

Molobins, 174.

Molops, 553.

Molorchus, 553.

Molytes, 554.

Monanthia, 421.

Monodierana, 635.

Monomorium, 717.

Mononjchus, 554 .

Monophlebus, 422.

Mordella, 554.

Mordellina, 554.

Mormolncoides, 155, 215.

Mormonia, 351.

Mucropalpus, 351.

Musca, 122, 225, 635.

Muscaria, 637.

Muscidites, 637.

Mutilla, 717.

Mycetobia, 637.

Mycetocharoides, 554.

Mycetophila, 637 .

Mycetophilites, 640.

Mycetoporus, 554.

Mycotretus, 555.

Mycterus, 555.

Mygale, 274.

Myiolepta, 640.

Mrlabris, 555.

Mylacris, 60.

Mylothrites, 676.

Myodites, 555.

Myrmar, 717.

Myrmecoleon, 351.

Myrmedonia, 555.

Myrmeleon, 155, 314, 352.

Myrmica, 230, 717.

Myrmicites, 720.

Myrmicium, 231, 720.

Mystacides, 352.

Nabis, 422.

Nannoblattina, 122.

Nanophyes, 555.

Nanthacia, 314.

Naucoris, 174, 423.

Naupactus, 555.

Nebria, 555.

Necrochromus, 423.

Necrocydnus, 424.

Necropsocus, 352.

Necropsylla, 424.

Necticus, 556.
Necydalis, 556.

Necygonus, 424.

Necymylacris, 62.

Nemastoma, 274.

Nematus, 231.

Nemestrina, 640.

Nemobius, 314.

Nemotelus, 640 .

Nemoura, 155, 352.

Nemura, 352.

Neorinopis, 676.

Neorthroblattina, 123.

Neothanes, 556.

Nepa, 174, 424.

Nephila, 275.

Nephrotoma, 640.

Nepidium, 174.

Nepticula, 676.

Nethania, 123.

Neurocoris, 175, 425 .

Neuronia, 353.

Neuropachys, 720.

Neuropterites, 354 .

Nitidula, 556.

Nitidulites, 216.

Noctuites, 676.

Nosodendron, 557.

Notaris, 557.

Nothochrysa, 354.

Nothrus, $27 \overline{5}$.

Notiophilus, 557.

Notonecta, 175, 425.

Nyctophylax, 426.

Nymphalis, 677.

Nymphalites, 677.

Nymphes, 155, 354.

Nymphon, 99, 354.

Nysins, 426.

Oberea, 557.

Obisium, 275.

Obrium, 557.

Uchtera, 640 .

Ochthebius, 558.

Ochyrocoris, 426.

Ocia, 276.

Ocnerites, 677.

Ocypete, 276.

Ocypus, 558.

Odontocerus, 354.

Odontocheila, 558.

Odontoda, 558.

Odontomyia, 640.

Odontota, 558.

đecophylla, 721 .

CEdemera, 558.

Edipoda, 123, $31 \mathrm{~m}$

Edischia, 62 .

Eschna, 354.

CEstrus, 640.

Oliarites, 426.

Oliarus, 426.

Olibrus, 558.

Oligotoma, 354.

Omalia, 86.

Omalium, 558.
Omaseus, 558.

Onca, 277.

Oncotylus, 427.

Oniticellus, 558.

Onitis, 558.

Onthophagus, 559.

Opatrum, 560.

Ophion, 721.

Ophonus, 560 .

Ophryastes, 560.

Opilio, 277.

Opilo, 560.

()pisthophylax, 278.

Orchedimum, 315.

Orchesia, 560 .

Oreina, 560 .

Oribates, 278.

Orthophlebia, 156,

Orthops, 427.

Orthriocorisa, 427.

Oryctaphis, 427.

Oryetes, 216.

Oryctites, 216.

Oryctoblattina, 63.

Oryctoscirtetes, 561 .

Osmia, 721.

Osmylus, 354.

Otiorhynchites, 216.

Otiorhynchus, 561.

Oustaletia, 86.

Oxycera, 640.

Oxygonus, 562.

Oxypete, 278.

Oxyporus, 562.

Oxytelus, 562.

Pachycoris, 427.

Pachymeria, 175.

Pachymeridium, 175.

Pachymerus, 175, 427.

Pachypus, 563.

Pachytilus, 315.

Pachytylopsis, 63, 86.

Pachytylus, 316.

Paidium, 355.

Paladicella, 355.

Palæoblattina, 63, 86.

Palæocampa, 15.

Palæochrysa, 355.

Palæocixins, 95.

Palæocoris, 429.

Palæocossus, 228.

Palæojulus, 15.

Palæomyrmex, 231.

Palæontina, 175, 228.

Palæophlebia, 157.

Palæophoneus, 28.

Palæophonus, 28.

Palæopsocus, 355.

Palæothrips, 430.

Palæovelia, 430.

Palaphrodes, 430.

Palaranea, 28.

Palecphora, 430.

Palembolus, 611 .

Palenarthrus, 15. 
Palephemera, 87, 158, 216.

Paliugenia, 87, 355 .

Palloptera, 641 .

Palpares, 158.

Palpipes, 99.

Pamphilites, 677.

Panagæus, 563.

Panorpa, 158, 356.

Panorpidium, 123, 158.

Paolia, 63.

Papilio, 677.

Paralatindia, 316.

Parandra, 563.

Parandrita, 563.

Parapleurites, 124.

Parattus, 278.

Parodarmistus, 431.

Parolamia, 563.

Paromylacris, 64.

Paropsocus, 356.

Parotermes, 356.

Passalœcus, 721.

Passandra, 563.

Patrobus, 563.

Paussoides, 564.

Paussus, 564.

Pediopsis, 431.

Pelobius, 564.

Peltis, 564.

Pemphigus, 431.

Pentatoma, 431.

Pentatomites, 433.

Penthacleus, 278.

Penthaleus, 279.

Penthetria, 641.

Pentodon, 564.

Pepsis, 722.

Perilampus, 722.

Periplaneta, 124.

Perla, 357.

Perotis, 564.

Petalia, 158 .

Petalura, 159, 357.

Petrablattina, 64, 87, 124

Petrobius, 357.

Petroblattina, 87.

Petrolystra, 433.

Petrorophus, 216.

Pezomachus, 722.

Phalacrus, 565 .

Pbalæna, 677.

Phalænites, 678.

Phalænomyia, 642.

Phalangillum, 279.

Phalangites, 100.

Phalangium, 279.

Phalangopus, 279.

Phanæus, 565.

Phaneroptera, 124, 316.

Phasma, 316.

Pheidole, 722.

Pheidologeton, 722.

Phenolia, 565.

Phidippus, 280 .

Philhydrus, : 65.

Philodronus, 281.

Philonthus, 160, 216, 565.
Philopotamus, 359.

Philotarsus, 359.

Phloocoris, 434.

Phlœothrips, 434.

Pholeus, 282.

Phora, 642.

Phosphuga, 566.

Phragmatœeites, 175, 228.

Phrudopamera, 434.

Phryganæa, 160.

Phryganea, 160, 359.

Phryganeidium, 160.

Phryganeolitha, 362.

Phryganidiam, 160.

Phrynus, 282.

Phryssonotus, 243.

Phthanocoris, 95.

Phthinocoris, 434.

Phthiria, 642.

Phygadeuon, 722.

Phyllobius, 566.

Phylloptera, 316.

Phymaphoroides, 566.

Phymophoroides, 566.

Phytocoris, 434.

Phytonissa, 282.

Phytonomus, 566.

Phytoptus, 282.

Pictetia, 87.

Pierites, 678.

Piesma, 436.

Piezocoris, 436.

Pimelia, 216.

Pimpla, 722.

Pinitoides, 567.

Pipiza, 642.

Pipunculus, 642.

Pirates, 436.

Pissodes, 567.

Plagiodera, 567.

Plagiolepis, 723.

Plangtichnus, 236.

Planocephalus, 362.

Planophlebia, 436.

Platephemera, 87.

Platybunus, 282.

Platycerus, 567.

Platycnemis, 362.

Platydema, 567.

Platymeris, 437.

Platynus, 567.

Platyperla, 161.

Platypus, 568.

Platysma, 569.

Platyura, 225, 643.

Plecia, 643.

Plinthus, 569.

Ploiaria, 437.

Podagrion, 363.

Podura, 363.

Pœcilocapsus, 437.

Pœcilus, 569.

Poocera, 437.

Poliochera, 29.

Poliomyia, 646.

Polioptenus, 64.

Polioschistus, 438.
Polistes, 724.

Pollyxenus, 243.

Polycentropus, 161, 363.

Polyclona, 438.

Polydesmus, 243.

Polydrosus, 569.

Polydrusus, 569.

Polyernus, 88.

Polyrhachis, 724.

Polystichus, 569.

Polystcechetes, 161.

Polystœchotes, 161.

Polyxenus, 243.

Polyzosteria, 316.

Polyzosterites, 65.

Pompilus, 724.

Ponera, 231, 725.

Poneropsis, 727.

Pontia, 678.

Porizon, 729.

Poroblattina, 125.

Porphyrops, 646.

Posthon, 646.

Potamanthus, 366.

Poteschistus, 438.

Prasocuris, 569.

Prenolepis, 730.

Prinecphora, 438.

Priocnemis, 730.

Prionidium, 217.

Prionomyrmex, 730.

Prionus, 217, 569.

Pristorhynchus, 570.

Procarabus, 217.

Procoris, 438.

Procrophins, 438.

Procydnus, 438.

Prodryas, 678.

Prodysdera, 283.

Prodytiscus, 217.

Progeotrupes, 217.

Prognatha, 161, 217.

Progonoblattina, 65.

Prolibythea, 678.

Prolygæus, 439.

Prolystra, 175.

Prometopia, 570.

Promylacris, 65.

Pronemobius, 317.

Pronophlebia, 617.

Propetes, 283.

Propteticus, 88.

Propygoblattina, 125.

Propygolampis, 176.

Proscorpius, 29.

Prosigara, 439.

Prostemma, 439.

Protactus, 570.

Protagrion, 89.

Protascalaphus, 89.

Protenor, 439.

Protocapnia, 89.

Protochersis, 283.

Protocicens, 96.

Protocixius, 96.

Protocoris, 176.

Protodiamphipnoa, 89. 
Protogenia, 570 .

Protogryllacris, 65, 89.

Protokollaria, 89.

Protolachesis, 284.

Protolindenia, 161.

Protoly cosa, 29, 284.

Protomyia, 225, 647.

Protoperla, 89.

Protophasma, 65.

Protoscalidion, 571.

Prototettix, 66, 89 .

Prototoma, 218.

Psecadia, 678.

Pselaphus, 571.

Psen, 730.

Pseudocistela, 571.

Pseudoelater, 571.

Pseudohydrophilus, 218.

Pseudomyrme, 720.

Pseudopachygnathus, 284.

Pseudoperla, 317.

Pseudophana, 439.

Pseudophania, 440.

Pseudosirex, 228, 231.

Pseudothomisus, 284.

Psila, 652 .

Psilites, 652.

Psilopus, 653.

Psilota, 653.

Psilus, 730.

Psocus, 366.

Psyche, 679.

Psychoda, 653.

Psychomia, 367.

Psychomyia, 368.

Psychoptera, 225.

Pterichnus, 236.

Pterinoblattina, 125.

Pteromalinites, 730.

Pteromalus, 730.

Pteromus, 730.

Pterostichus, 571.

Pterostigma, 440.

Ptilinus, 573.

Ptilodactyloides, 574.

Ptinus, 574.

Ptomaphagus, 574.

Ptychoptera, 225, 653.

Ptyela, 440.

Ptyelus, 440.

Pulex, 653.

Pycnogonites, 100.

Pycnophlebia, 126.

Pygolampis, 126, 176.

Pyralites, 679.

Pyrochroa, 218, 574.

Pythonidium, 574.

Pythonissa, 284.

Quedius, 574.

Ranatra, 440.

Rapha, 161.

Raphidia, 368.

Raphidium, 162.

Reduvins, 440.

Remalia, 225.
Rhamphidia, 653.

Rhamphomyia, 654 .

Rhaphium, 654 .

Rhepocoris, 441.

Rhingia, 655.

Rhinobatus, 575.

Rhinocyllus, 575.

Rhipidia, 655.

Rhipidioptera, 96.

Rhipidius, 575.

Rhipidorhabdus, 228, 232.

Rhipiphorus, 575.

Rhizophagus, 576.

Rhizotrogus, 576.

Rhopalomyrmex, 731 .

Rhyacophila, 369.

Rhynchænus, 576.

Rhynchites, 576.

Rhyncholophus, 285.

Rhyparochromus, 441.

Rhyphus, 225, 655 .

Rhyssa, 731.

Ricania, 127, 176, 441.

Ripiphorus, 577.

Rithma, 127.

Rophalis, 369.

Rumphomyia, 656 .

Rymosia, 656.

Ryphus, 656.

Sackenia, 656.

Sagittarius, 237.

Salda, 441.

Saltator, 237.

Salticus, 286.

Sama, 225.

Samarura, 162.

Saperda, 577.

Saperdides, 218.

Sapromyza, 656.

Sapyja, 731.

Sargus, 656.

Satyrites, 679.

Satyrus, 679 .

Sbenaphis, 442.

Scaphidium, 218, 578.

Scaphiduum, 218.

Scaphisoma, 578.

Scarabæides, 176.

Scarabæites, 218.

Scarabæoides, 176.

Scarabæus, 176, 218, 578.

Scarites, 578.

Scatuphaga, 657.

Scatops, 657.

Scatopse, 657.

Schellenbergia, 287.

Schizoneura, 442.

Schizoneuroides, 442

Sciara, 162, 225, 657.

Sciaris, 659.

Sciobia, 659.

Seiomyza, 659 .

Sciophila, 226, 660.

Scolia, 731 .

Scolopendra, 244.

Scolytus, 578.
Scorpio, 29, 287.

Scraptia, 578.

Scudderia, 30, 96.

Scutinoblattina, 129.

Scydmænoides, 579 .

Scydmænus, 579.

Scyllina, 317.

Seymnus, 579.

Scyrtes, 579.

Segestria, 287.

Sejus, 288.

Selandria, 731.

Semblis, 369 .

Sendelia, 661.

Seniaulus, 579.

Sepidium, 579.

Serica, 579.

Sericostomum, 370.

Sesia, 679.

Setodes, 370.

Sialium, 130, 162.

Sibynes, 580.

Siga, 288.

Silicernius, 580 .

Silpha, 218, 580.

Silphate, 218.

Silphites, 219.

Silvanus, 581.

Silvius, 661.

Sima, 731.

Simulia, 661 .

Simulidium, 226.

Simulium, 226, 661.

Sinis, 581 .

Siphonophoroides, 442.

Sirex, 232.

Sisyphus, 581.

Sisyra, 370.

Sitodrepa, 581.

Sitona, 581.

Sitones, 581.

Sitonites, 219.

Smicronyx, 582.

Smynthurus, 371.

Solenopsis, 731 .

Sosibius, 289.

Sospita, 582.

Sosybius, 289 .

Spartocerus, 443.

Spermophagus, 582.

Sphærapus, 237.

Sphærodema, 177.

Sphæropsocus, 371.

Spheconia, 289.

Sphecoptera, 89.

Sphegina, 662.

Sphenophorus, 583.

Sphenoptera, 219, 583.

Sphex, 732.

Sphinx, 228, 232, 679.

Spiladomyia, 662 .

Spilaptera, 66.

Spiloblattina, 130.

Spondylis, 583.

Staphylinites, 583.

Staphylinus, 58 t.

Statira, 585. 
Steneattus, 289.

Stenocinclis, 662.

Stenopamera, $\mathbf{4 4 3}$.

Stunopelta, 443.

Stenophlebia, 162.

Stenopoda, 443.

Stenovelia, 443.

Stenus, 585.

Sterope, 371.

Steropus, 586.

Sthenarocera, 67.

Sthenaropoda, 67.

Stigmomyrmex, 732.

Stilicus, 586.

Stolopsyche, 680.

Stomis, 586.

Stomoxya, 662 .

Stomoxy8, 662 .

Stratiomys, 662.

Strephocladus, 90.

Strongylites, 219.

Strongylus, 586.

Strophosomus, 586.

Styringia, 662.

Sty ringom yia, 662.

Sunius, 586.

Suphalasca, 371.

Sybines, 586.

Sychnobrochus, 443.

Sylvanue, 586.

Sympycna, 371.

Syphax, 289.

Syromaste8, 443.

Syrphus, 663.

Syrtis, 444.

Systellonotus, 444.

Tabanus, 664.

Tachina, 664.

Tachinus, 586, 665 .

Tachydromia, 665 .

Tachypeza, 665.

Tachyporus, 586.

Tæniopteryx, 371 .

Tænioptrix, 372.

Tagalodes, 444.

Tagenopsis, 587.

'Tanymecus, 587.

Tanymera, 665 .

Tanypus, 226, 665 .

Tanysphyra, 665 .

Tanysphyrus, 587.

Taphacris, 317.

Tapinoma, 732.

Tarsophlebia, 163.

Taxonus, 732.

Tegenaria, 290.

Teleoschistus, 444.

Telephorium, 220.

Telephorus, 220, 587.

Telmatrechus, 445.

Tenebrio, 220, 588.

Tenor, 445.

Tenthredo, 732.

Tentyridium, 220.

Tephraphis, 445.
Tephritis, 666 .

Termes, 30, 67, 90, 163, 372.

Termidium, 69.

Termitidium, 69, 90, 164.

Termopsis, 378.

Tetanocera, 666.

Tethnæus, 291.

Terhneus, 291.

Tetragnatha, 291.

Tetranychus, 292.

Tetrix, 317.

Tettigidea, 318.

Tettigometra, 445.

Tettigonia, 177, 445.

Tetyra, 446.

Textrix, 292.

Thaites, 680 .

Thamnotettix, 446.

Thanatites, 680 .

Thelyphonus, 30 .

Thenthredo, 733 .

Therea, 292.

Thereua, 666.

Thereva, 666.

Theridion, 293.

Theridiosoma, 293.

Theridinm, 293.

Thimna, 226.

Thiras, 226.

Thirza, 666.

Thlibomenus, 447 .

Thlimmosehistus, 447.

Thnetoschistus, 447.

Thomisus, 296.

Thrips, 447.

Throseus, 589.

Thurmanuia, 220.

Thyelia, 297.

Thylacite8, 589.

Tillus, 589.

Timarcha, 589.

Timarchopsis, 220.

Tinea, 228, 680 .

Tineites, 164, 228, 681 .

Tingis, 449.

Tinodes, 379 .

Tipula, 226, 666.

Tipularia, 227.

Tiromerus, 449.

Tiroschistus, 449.

Titanœca, 298.

Titanophasma, 69.

Tityus, 298.

Tmesiphoroides, 589.

Tophoderes, 590.

Tortrix, 681.

Torymu8, 733.

Toxorhina, 669.

Trachyderes, 590.

Trapezonotus, 449.

Trechinites, 590.

Trechoides, 590.

Trechus, 590.

Treptichnus, 237.

Triæna, 590.

Tribochrysa, 379.
Trichaptum, 90.

Trichiulus, 15.

Trichius, 591.

Trichocera, 670.

Trichoneura, 670.

Trichonta, 671.

Trichopteridium, 165.

Trichostomum, 380.

Triecophora, 450.

Triecphora, 450.

Trigona, 733.

Triphæna, 681.

Triphyllus, 592.

Trixagites, 221.

Troctes, 380.

Trogosita, 592.

Trogulus, 221.

Trogns, 221, 733.

Trombidinm, 299.

Tropisternus, 593.

Trox, 593.

'Troxites, 97

Trypeta, 671.

Tryphon, 733 .

Trypodendron, 593.

Tyehius, 593.

Typhia, 733.

Typhloeyba, 450.

Typhlocypha, 450.

Tyrbula, 318.

Tyrpeta, 671 .

Uloma, 594.

Urocerites, 733.

Uroctea, 300.

Urodon, 594.

Uropetala, 165.

Valgnd, 594.

Vanessa, 681.

Velanovskya, 221.

Velia, 177.

Vespa, 734.

Wollastonia, 221.

Wollastonites, 221.

Woodwardia, 91.

Xantholinus, 594.

Xenoneura, 91.

Xya, 318.

Xyletinites, 594.

Xylobius, 16.

Xylocopa, 734.

Xylophagns, 671.

Xyloryctes, 98.

Xysticus, 300.

Ypsolophus, 681.

Zalmona, 165.

Zeilleria, 92.

Zetobora, 318.

Zilla, 300.

Zonites, 594.

Zonitis, 594.

Zygæna, 682.

Zygoneura, 671. 